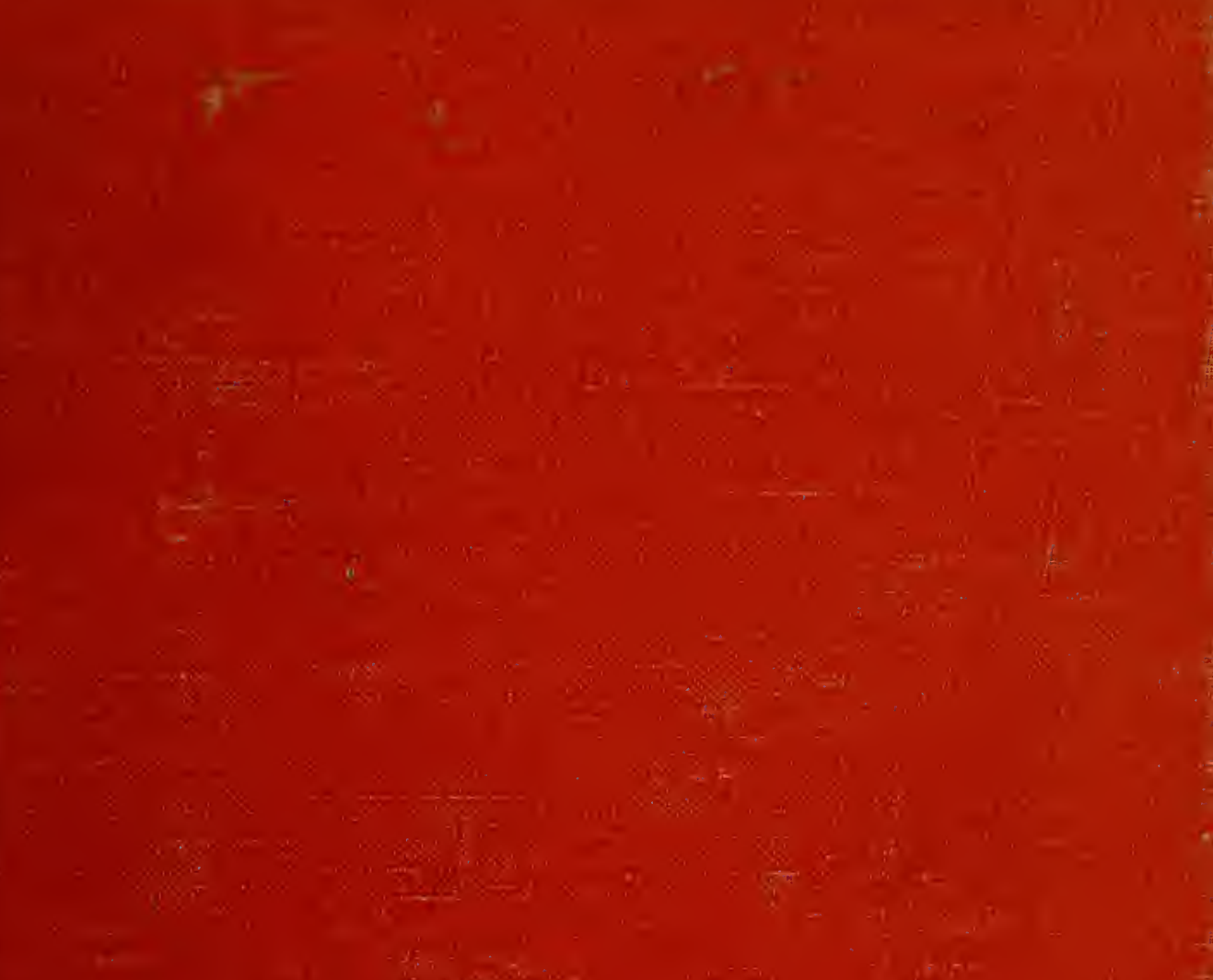

t.

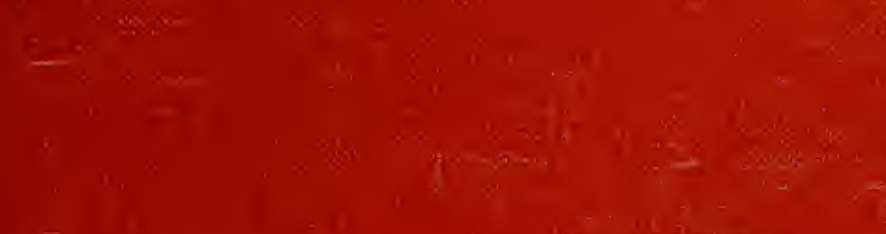

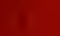

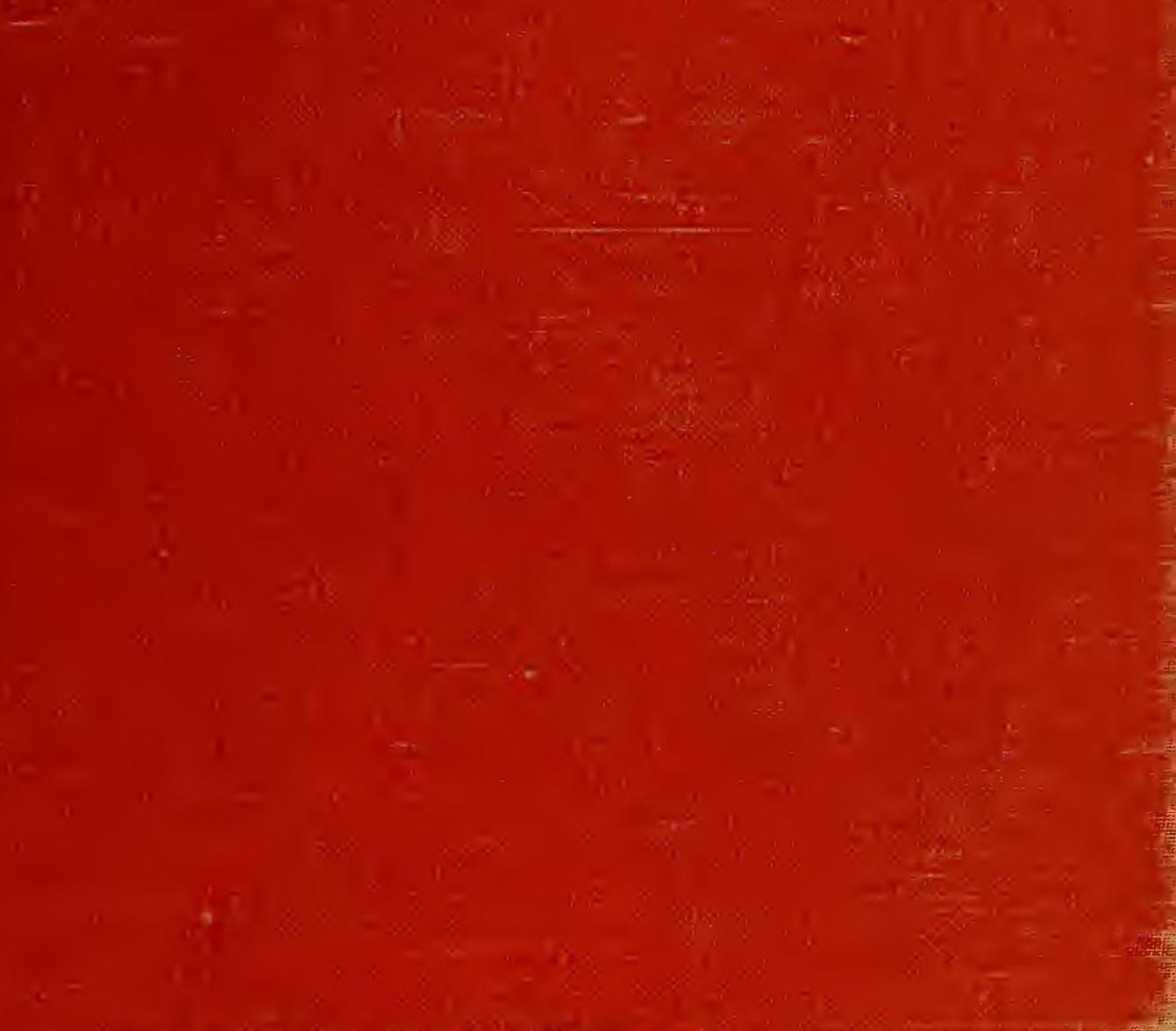







\section{LIGHT AND LIFE}


Contribution No. 302 of the McCollum-Pratt Institute 
A SYMPOSIUM ON
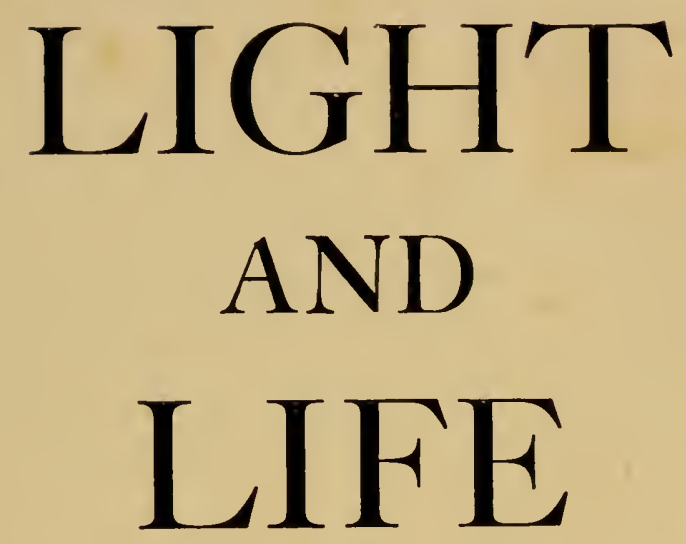

Sponsored by

THE MaCOLLUM-PRATT INSTITUTE

OF

THE JOHNS HOPKINS UNIVERSITY

with support from

The National Science Foundation

Edited by

WILLIAM D. MaELROY AND BENTLEY GLASS

BALTIMORE

THE JOHNS HOPKINS PRESS

1961

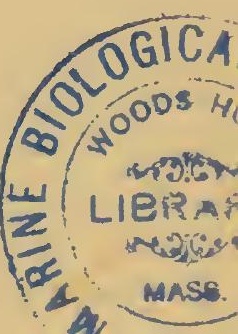




\section{Previous Simposia of THE MCCOLlun-P'RATI INSTITUTE}

Copper Metabolism-A Symposium on Animal, Plant and Soil Relationships.

Phosphorus Metabolism-A Symposium on the Role of Phosphorus in the Metabolism of Plants and Animals, V'ol. $I$.

Phosphorus Metabolism-A Symposimm on the Role of Phosphorus in the Metabolism of Plants and Animals, I'ol. II.

The Mechanism of Enzyme Action.

Amino Acid Metabolism.

Inorganic Nitrogen Metabolism.

The Chemical Basis of Heredity.

The Chemical Basis of Development.

(c) 1961 by The Johns Hopkins Press, Baltimore is, Mal.

Distributed in Creat Britain by Oxford University Press, London

printed in the Cnited States of America by the Horn-Shafer Company, Baltimore

Library of Congress Calalog Card Number 60-16.54 


\section{PREFACE}

A Symposium on "Light and Life" was held at The Johns Hopkins University under the sponsorship of the McCollum-Pratt Institute on March 28-31, 1960. This volume contains the papers and informal discussions presented during the Symposium.

In the planning of the present Symposium we attempted, as in previous symposia, to bring together scientists from a number of different disciplines. Unfortunately time and printing limitations prevented the consideration of a number of interesting photobiological problems.

I would like to acknowledge the active participation of the members of the McCollum-Pratt Institute and of the Department of Biology in the planning of the Symposium. Many of the speakers on the program were helpful in suggesting areas or specific topics that should be discussed. It is also a pleasure to acknowledge the valuable contributions of the following moderators: Dr. Albert Szent-Györgyi, Dr. James Franck, Dr. William Arnold, Dr. C. B. van Niel and Dr. $\mathrm{H}$. K. Hartline.

The support of a limited number of foreign investigators as participants in the Symposium was made possible through the generous aid of the National Science Foundation. Unfortunately, insufficient funds prevented the attendance of a larger number of participants from great distances.

We hope, however, that the published book will be of value to all investigators interested in photobiological processes.

W. D. McElroy

Director, McCollum-Pratt Institute 


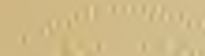

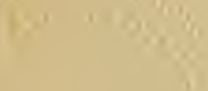

I)

1)

6.

Yn $110=1$

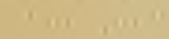




\section{Preface}

Additional Participants

Introduction

Niels Bohr

I. MOLECULAR STRUCTURE AND EXCITED STATES

Introductory Comments

Albert Szent-Györgyi

Electronic Excited States of Simple Molecules

G. Wilse Robinson

The Nature and Significance of $n \rightarrow \pi^{*}$ Transitions

Michael Kasha

Discussion

The Triplet State in Fluid Solution . . . . . . . 69

George Porter

Discussion

II. EXCITED STATES OF MOLEGULES OF

\section{BIOLOGICAL INTEREST}

Introductory Comments

James Franck

Excited States of Proteins

G. Weber

Discussion

Spectra and Structure in Enzyme Complexes of

Pyridine and Flavin Nucleotides

Sidney F. Velick

Discussion

Interactions in Pyridine Nucleotides

Sidney Shifrin and Nathan O. Kaplan

On the Mechanism of the Dihydrothioctyl

Dehydrogenase Reaction

Robert L. Searls and D. R. Sanadi

Some Comments on Flavin and Flavoprotein Complexes

and Semiquinones

Helmut Beinert

\section{LIGHT EMISSION BY CHEMICAL REACTIONS}

Chemiluminescence in Porphyrin-Catalyzed Decomposition

of Peroxides

Henry Linschitz 
The Chemiluminescence of Luminol . . . . . . . . 183

Emil H. White

Discussion

Some Aspects of the Luminol Light Reaction . . . . . 200

H. $H$. Seliger

The Luciferin-Luciferase Reaction

Frank H. Johnson, Edward H.-C. Sie, and Y. Haneda

Mechanisms of Bioluminescent Reactions . . . . . 219

W. D. McElroy and H. H. Seliger

Cypridina Luciferin Oxidation as a Function of $p \mathrm{H}$,

and Reduction of Luciferin by Ascorbic Acid . . . . 258

A. M. Chase, J. H. Ball, C. E. Cornelius, and

R. J. Lederman

Characteristics of Cell-Free Fungal Bioluminescence . . . 262

R. L. Airth

Biochemistry of Renilla reniformis Luminescence . . 274

Milton J. Cormier

Ionic Effects upon Bioluminescence in Gonyaulax Extracts . 291

J. Woodland Hastings and Vemon C. Bode

Discussion . . . . . . . . . . . . . . . 306

\section{PHOTOSYNTHESIS}

Introductory Comments

C. B. van Niel

Some Photochemical and Photophysical Reactions

of Chlorophyll and Its Relatives . . . . . . . 317

Melvin Calvin

Electron Spin Resonance Studies of Photosynthetic Systems 356

Barry Commoner

Different Forms of Chlorophyll a in vivo and

Their Photochemical Function . . . . . . . . 378

Eugene Rabinowitch and Govindjee

Discussion . . . . . . . . . . . . . . . . . . 386

Lipid Function in the Photosynthetic Structure . . . . 392

A. A. Benson

Spectral Changes in Photosynthesis

Bessel Kok and (ieorge Hoch

Discussion

The Nature and Possible Function of Chloroplast

Cytochromes

Robert Hill and Walter I). Bouner, $J r$. 
Reactions of Cytochrome Pigments in Photosynthetic

Bacteria

Lucile Smith

Discussion

Light, Pigments, and Photosynthesis

C. S. French

Discussion

On the Long-wave Decline of the Quantum Yield of Photosynthesis in the Red Alga Porphyridium cruentum . .

J. B. Thomas and Govindjee

Eviclence for Pigments Absorbing at 705-710 $\mathrm{m} \mu$ in

Photosynthetic Organisms . . . . . . . . . . . 479

Masy Belle Allen

Comments on the Function of HAEM Proteins as Related to

Primary Photochemical Processes in Photosynthesis 483

Martin D. Kamen

Cell-Free Photosynthesis and the Energy Conversion Process

Daniel I. Arnon

Discussion

Photoproduction of Molecular Hydrogen from Thiosulfate

by Chromatium Cells

M. Losada, M. Nozaki, and Daniel I. Arnon

Oxidation-Reduction Reactions in Chloroplasts . . . . 576

Andre T. Jagendorf and Giorgio Forti

Reflections on Recent Theories Concerning the Mechanism

of Bacterial Photosynthesis . . . . . . . . 587

Albert W. Frenkel

On the Reductive Dephosphorylation Hypothesis of

Photosynthetic Reductant Formation . . . . . . 593

D. D. Hendley and B. L. Strehler

Continuous Measurement of Photosynthetic Phosphorylation with the Firefly Luminescence Assay System . . . . 601 B. L. Strehler and D. D. Hendley

The Effect of Oxygen on Photophosphorylation and the Effect of Carbonate on the Hill Reaction . . . . . 609

Birgit Vemesland, T. Nakamoto, and Babette Stern

Oxidative Photophosphorylation and the Mehler Reaction

David W. Krogmann 
Photophosphorylation and Photohydrolysis in Cell-Free

Preparations of Blue-Green Alga

Barbara Petrack and Fritz Lipmann

Photochemical Reduction of Triphosphopyridine

Nucleotide by Illuminated Chloroplasts

Anthony San Pietro

\section{BIOLOGICAL RESPONSES TO LIGHT}

Introductory Comments

K. Hartline

Phototropism

Kenneth V. Thimann and George M. Curry

Discussion

Photoperiodism, Chelating Agents, and Flowering of Lemna

perpusilla and L. gibba in Aseptic Culture

William S. Hillman

Comparative Studies on the Growth and Light Sensitivity of

Green and Etiolated Pea Stem Sections . . . . .

A. W. Galston and Ravindar Kaur

The Intensity Factor in Vision

W. A.H. Rushton

Discussion

The Molecular Organization of Visual Systems

George Wald

Discussion

Speculations on the Interrelations and Evolution of Photic

Organs

John Buck

The Color Vision of Insects

Timothy H. Goldsmith

Electrophysiological Evidence for a Mechanism of Color

Vision in the Goldfish

E. F. MacNichol, Jr., M. L. Wolbarsht, and

H. G. Wagner

Discussion

VI. SUMMARY

Bentley Glass

Author Index of Participants Index 


\section{ADDITIONAL PARTICIPANTS}

E. W. Abrahamson

JAMES AdYE

M. I. H. Aleem

ETtORE Appella

Ruth Aranow

William ARNold

Leroy Augustine

ROBERT BALlentine

T. BANNISTER

Rolf Benzinger

J. A. Bergeron

M. J. Bessman

NoRMAN BISHOP

L. R. BLINKS

H. A. BorthWick

ROBERT BOWMAN

Allan H. Brown

VICTOR G. BRUCE

DEAN BURK

WV. L. ButLeR

B. Chance

George M. Gurry

Max Delbruck

D. R. Evans

HENRY FrEISER

IRviN Fridovich

Herbert C. Friedmann

M. G. F. FUORTES

HaNs GafFron

Michael Garrick

Martin Gibbs

Bentley Glass

SOLON A. GORDON

Philip E. Hartiran
Bernhari) Hassenstein

Roger M. Herriott

H. G. Hovenkamp

P. Hovingh

J. S. KaHN

VARDA KAHN

BARBARA KALCKAR

Herman M. Kalckar

Donald L. Keister

C. Kitzinger

A. R. KRall

Hans Laufer

JOHN C. LOPER

WARner E. Love

Maurice Margulies

C. L. MARKERT

James Mathewson

Frank MCCAPRa

E. V. McCollum

Guy C. MCLeOD

J. L. MEgo

T. MERZ

K. J. MONTy

JACK MYERS

Alvin Nason

M. Nishimura

G. B. PINCHOT

C. S. Pittendrigh

John R. Platt

E. RACKER

SHERWOOD M. REICHARD

William Rhodes

Curt P. Richter

P. ROGERS 
Claud S. Rupert

Robert S. SCHUTZ

David Shapiro

Samuel Shibko

WALT SHROPSHIRE

H. W. Siegelman

JOHN D. SPIKES

Malcoli STEInberg

Hirake Takebe

GORDON TOLLIN

Hans Trurnit
Sidney UdENFRIEND

H. VAN DER LOOS

LEO P. VERNON

WOLF VISHNIAC

Michael Vozick

J. WEINMAN

Herbert Wiesheyer

B. H. Willier

THeOdore Wright

MichaEL YARMOLINSKY

L. P. ZILL 


\section{LIGHT AND LIFE}





\title{
INTRODUCTION
}

\section{PHYSICAL MODELS AND LIVING ORGANISMS ${ }^{1}$}

\author{
Niels BOHR \\ Copenhagen, Denmark
}

The discussion of the position of living organisms in a general description of physical phénomena has, in the development of science, passed through a number of stages. In Antiquity, the obvious difficulties inherent in a comparison between organisms and primitive machinery deeply influenced the attitude towards mechanical problems and even led to the attribution of vital characteristics to all matter. With the abandonment of these views, at the time of the Renaissance, through the clarification of the principles of classical mechanics, the problem entered into another stage, stimulated by the great anatomical and physiological discoveries at that period.

Recent advances in technology, and especially the development of automatic control of industrial plants and calculation devices, have given rise to a renewed discussion of the extent to which it is possible to construct mechanical and electrical models with properties resembling the behavior of living organisms. Indeed, it may be feasible to design models reacting in any prescribed manner, including their own reproduction, provided that they have access to the necessary materials and energy sources. Still, quite apart from the suggestive value of such comparisons, we must realize that, in the study of models of given structure and functions, we are very far from the situation in which we find ourselves in the investigation of living organisms, where our task is gradually to unravel their constitution and potentialities.

As regards this problem it is essential to realize from the very beginning that in organic life we are dealing with further resources of nature than in the construction of machines. Indeed, for this

\footnotetext{
${ }^{1}$ Except for a few small additions the substance of these remarks was presented in a contribution to the symposium on Models in Biology, Bristol, 1959.
} 
purpose we can essentially disregard the atomic constitution of matter and confine ourselves to the account of the mechanical and electrical properties of the materials used and to the application of the simple laws governing the interaction between the parts of the machine. From biological research, however, it is eviclent that fundamental characteristics of living organisms, and in particular genetic reproduction, depend primarily on processes on the atomic scale, where we are faced with new problems.

On the ground of classical physics, the very question of maintaining a high degree of order of such immensely complicated systems presents serious difficulties. In fact, the incessant encounters between the atoms with a more or less liquid phase like the cytoplasm would lead to rapidly increasing disorder. Doubts have even been expressed about the compatibility of the existence and stability of living organisms with the laws of thermodynamics, but thorough investigation of the exchanges of energy and entropy accompanying the metabolism and movements of the organisms has never disclosed any departure from these laws.

A whole new background has, however, been created by the development of quantum physics, which, at the same time as it has revealed an essential limitation of the deterministic description of classical mechanics, has offered a proper basis for the account of the stability of atomic and molecular structures. As is well known, no picture on classical lines can be given of the electronic constitution of atoms or of the behavior of the electrons responsible for the bindings between atoms in chemical combinations. Owing to the large masses of the atomic nuclei compared with that of the electron, it is possible, however, effectively to retain a pictorial representation of the relative positions of the atoms in accordance with the structure formulae of chemistry, which have proved so adequate even for the highly complicated molecules with which we are concerned in organic metabolism.

In spite of the multifarious enzymatic processes involved in this metabolism, the problem of the stability of the organisms presents a fundamental simplicity, since, in the range of temperatures within which life can be upheld, the thermal fluctuations in the states of vibration and rotation of the molecules are in general far from sufficient to break the chemical bonds. Such fluctuations rather effect the rapid disappearance of correlations between all secondary characters of the states of the reacting systems and permit us to account for the primary features of their constitution merely by a specification 
of the atoms of which they are composed and the configuration in which these atoms are bound together.

The discoveries in recent years of the specific molecular structures carrying genetic information, and the increasing insight into the processes by which this information is transferred, have opened quite new prospects for the gradual clucidation of biological regularities on the basis of rell-established principles of chemical kinetics. In particular, the almost unlimited possibilities of probing our metabolic transformations lend support to the view that the formation and regeneration of the structural constituents of the organisms are to be regarded as processes of not immediately reversible character, which at any step secure the greatest possible stability uncler the conditions maintained by nutrition and respiration.

Thus, there appears to be no reason to expect any inherent linitation of the application of elementary physical and chemical concepts to the analysis of biological phenomena. Yet, the characteristic properties of living organisms, which have resulted from the whole history of organic evolution, reveal potentialities of inmensely complicated material systems, which have no parallel in the comparatively simple phenomena studied under reproducible experimental conditions. It is on this background that notions referring to the behavior of organisms as entities, and apparently contrasting with the account of the properties of inanimate matter, have found fruitful application in biology.

Even though we are here concerned with typical complementary relationships as regards the use of appropriate terminology, it must be stressed that the argumentation differs in essential aspects from that concerning exhaustive objective description in quantum physics. Indeed, the distinction demanded by this description between measuring apparatus and object under investigation, which implies mutual exclusion of the strict application of space-time coordination and energy-momentum conservation laws in the account of individual quantum processes, is already taken into account in the use of chemical kinetics and thermodynamics. Thus, the dual approach in biology does not seem to be conditioned by an interference with the properties of the specific molecular structures, inherently involved in their identification, but is rather required by the practically inexhaustible potentialities of living organisms entailed by the immense complexity of their constitution and functions. 



\section{Part I}

MOLEGULAR STRUCTURE AND EXCITED STATES 



\title{
INTRODUCTORY COMMENTS
}

\author{
Albert Szent-GyörgYi \\ The Institute for Muscle Research, Marine Biological Laboratory, \\ Woods Hole, Massachusetts
}

It is common knowledge that the ultimate source of all our energy and negative entropy is the radiation of the sun. When a photon interacts with a material particle on our globe it lifts one electron from an electron pair to a higher level. This excited state, as a rule, has but a very short lifetime and the electron drops back within $10^{-7}$ to $10^{-8}$ seconds to the ground state, giving off its excess energy in one way or another. Life has learned to catch the electron in the excited state, uncouple it from its partner and let it drop back to the ground state through its biological machinery, utilizing its excess energy for life processes.

There is little doubt in my mind that Life was driven, at the beginning, by this electronic energy and must have walked a long and tortuous road perfecting its biological machinery, step by step, by developing the substances fit to deal with the electron. But, however this machinery may have been perfected, it had to retain two major shortcomings: (l) the electron and its energy were linked to the energy-producing machinery and could not be taken out of it; (2) while Life is continuous, radiation is intermittent and the possibilities of storing high-energy electrons are very limited.

The story of the storage and transportation of electronic energy consists of a series of discoveries made by Nature. One of the most important of these was the discovery that it is possible to preserve the electronic free energy by linking two orthophosphate molecules together by an anhydride link, producing pyrophosphate, P-O-P. We meet the P-O-P today in a rather sophisticated form, as part of the ATP molecule. This discovery solved the problem of energy transport and alleviated the problem of energy storage. Energy stored in the form of P-O-P could be transported to different loci, allowing for the development of new organs or processes, and relegating the process of photosynthesis proper to special little factories, chromato- 
phores or chloroplasts. As far as we know, from studies of photosynthetic bacteria, the simplest P-O-P producing system consists of very few components. The excited electron passes in it from chlorophyll to a quinone (Vitamin $\mathrm{K}$ or its equivalent), from here to a cytochrome and then back again to chlorophyll to replace its lost electron. All that is left from the electrons circling around a cyclic pathway are the terminal phosphates on ATP. This is a "closed circle" from which nothing leaks out but energy invested into $\sim$ P's, (high-energy phosphate bonds) .

The next discovery of Nature was that of pyridine nucleotides, TPN, which can take over the excited electrons from chlorophyll, and then send them back to it over riboflavin and cytochromes. This new, somewhat more sophisticated circle is also a "closed" one and may involve oxygen as a catalyst. The energy liberated by the electron dropping down to its ground level again produces ATP only.

The next discovery involved water, the mater (mother) and matrix of life. The electron, taken over by TPN can be stabilized by binding protons. This means the decomposition of water, the details of which still need clarification. The simplest assumption is to suppose that the chlorophyll regains its lost electrons over a cytochrome from the $\mathrm{OH}^{-}$ions, left unbalanced after the $\mathrm{H}^{+}$was linked to pyridine. The remaining $(\mathrm{OH})$ radicals can then be dismutated into water and $\mathrm{O}_{2}$.

By introducing ATP and TPN the storage problem was not yet completely solved, for neither of these two substances can be stored by the cell in quantity. The solution was achieved, and the way to higher forms of life was opened, by involving $\mathrm{CO}_{2}$. Once the cell had ATP and reduced TPN, it could reduce with them $\mathrm{CO}_{2}$ to carbohydrate. The carboliydrate could further be transformed into fat, substances which could be stored in almost unlimited quantity. To mobilize their energy the process of energy storage had simply to be reversed, the $\mathrm{H}$ from carbohydrates and fats being now transferred back onto TPN or DPN. The "closed" circle of photosynthesis was thus opened up by the introduction of the two extraneous substances, $\mathrm{H}_{2} \mathrm{O}$ and $\mathrm{CO}_{22}$. These remarks made no pretence of originality. They are based chiefly on the work emanating from the laboratory of D. Armon.

Until recently, the classical objects of study in photosynthesis were green leaves or chloroplasts belonging to the lighest levels of organization. Only lately were the bacterial chromatophores introduced as material for study. With this, research went back on the phylogen- 
tic scale by many millions of years. These chromatophores are still very far from the origin of life, but this is as far as we can go in this direction at present, so a few remarks on these chromatophores may be permitted. As mentioned earlier, the electrons go round in them in a relatively simple closed circle, going from chlorophyll to quinone, from quinone to a cytochrome, and from cytochrome back to chlorophyll. The electrons seem to go it one by one. In any case, they are excited one by one. They are also transmitted one by one from cytochrome to chlorophyll, which makes it most probable that the whole circle is a one-electron business. As to the mechanism of this passage of the electron we know from experiments of Commoner and Calvin that the illuminated chloroplasts give an electron spin resonance signal. This means that an electron pair has been uncoupled, a purely physical process. It has also been discovered lately by Chance and Nishimura that when cells of the photosynthetic sulfur bacterium, Chromatium are illuminated at liquid $\mathrm{N}_{2}$ temperatures, cytochromes become oxidized, suggesting that in chromatophores electrons pass from cytochrome to chlorophyll. This means that no diffusion or classic chemical reaction is involved. It seems highly probable that the whole circle consists only of quantum mechanical changes, physics and no chemistry, that is, no collisions and rearrangement of covalent bonds. It involves physical concepts, as molecular excitation, semiconduction, $n-p$ junctions, charge transfer, etc. A single electron, passing from one orbital to the other, is not expected to cause any major rearrangement in bond structure.

I have personal reasons to emphasize this point. My own problem is: how is the animal body driven? How does it produce the various forms of work, $w$, mechanic, electric, or osmotic, the $w$ 's by which we know life from death. My research has always been led by the conviction that the whole of living Nature is built on but a very few basic principles. Accordingly, I find that my own body reflects the same basic principles by which life was driven at its beginning. I take my energy from the plants, mainly in the form of fat and carbohydrate. What I do with these is to take off their $\mathrm{H}$ atoms, and put them on TPN or DPN and send down electrons over riboflavin and cytochromes in the oxidative chain, as the bacterial chromatophores do, producing ATP by the energy of the dropping electron which reaches its lowest level on the $\mathrm{O}_{2}$, released in the open circles of photosynthesis. I also strongly believe that ATP has two different functions in my body. The one is to supply energy for endergonic synthesis. This it does by "group transfer," that is, by transmitting its 
energy together with its P-O-P, in relatively simple classic chemical reactions. Its other function is to produce w's. This it does, (according to the theory which dominates my research) by converting the bond-energy of its P-O-P's into electronic energy again, the same electronic energy which was invested in it, which was driving life at its beginning, endless millions of years ago when it captured the first photons.

We can approach these fascinating problems by studying the various reactions which may be involved, one by one. Our present meeting will be devoted to the type of reaction which initiates photosynthesis and probably led to the appearance of life on this globe. 
ELECTRONIC EXCITED STATES OF SIMPLE MOLECULES ${ }^{1}$

\author{
G. WiLSE ROBINSON \\ Gates and Crellin Laboratories of Chemistry \\ California Institule of Technology \\ Pasadena, Califomia
}

\title{
I. INTRODUCTION
}

In an approximate way the electronic structure of a large molecule is a superposition of the electronic structures of the small entities of which it is comprised. If the large molecule is made up of parts which do not interact among themselves, and if the large molecules are themselves randomly oriented so that dichroic effects are absent, the spectrum corresponding to the absorption or radiation of energy in the form of light by such a molecule will be precisely that given by a superposition of the randomly oriented parts. This means that the intensity of absorption or emission is merely summed as a function of wavelength over the various parts of the large molecule.

In reality, interactions between parts of the large molecule do occur. ${ }^{3}$ If certain interactions are very large, because of conjugation, for example, these parts should be considered together as the basic molecular entity in terms of which the large molecule is described. Weaker but non-zero interactions affect the energy levels in a less spectacular manner. The problem is analogous to that of electrons in a set of potential wells of various sizes and shapes. If the potential barriers between the wells are infinitely high, electrons cannot delocalize into the neighboring wells and the energy levels are those of the isolated parts. As the barriers are lowered to allow interaction between parts, electrons can delocalize by an amount depending upon the energy of the electrons and the width of the barriers ("distance" between

${ }^{1}$ Supported in part by Office of Ordnance Research and the National Science Foundation.

${ }^{2}$ Contribution No. 2569.

${ }^{3}$ This has been discussed in some detail for double molecules (refs. 2 and 19). References are given here to other pertinent papers on this subject. See ref. 16a and literature cited there for the more general case. 
interacting parts). If there is a large number of parts having very nearly the same energies, a general broadening and lowering of electronic transitions to such a group of states results, since the interaction tends to separate the multiple energy levels of the unperturbed system (16a). The excited states, because they correspond to higher electronic energy and thus are closer to the top of the barriers, will feel such interactions more than the ground state of the molecule. In addition to the effect on the energies, the interactions enable electronic energy transfer to take place within the molecule.

The electronic energy states of small molecules, because of their relative simplicity, form a good starting point for the understanding of detailed effects in large molecules: Many problems of current interest in molecular biology appear to be associated with exactly the same problems which are of current interest to the molecular physicist. The reason for this, of course, is partly due to the increased amount of interaction between these scientific groups and partly, it is to be hoped, to the fact that these are important problems in both fields. Some of the problems are (1) intermolecular and intramolecular energy transfer, (2) long-lived states, (3) electron density in molecules and its change with electronic excitation, (4) molecular geometry and its change with electronic excitation, and (5) the effect of inter- and intra-molecular enviromment upon the ground and excited electronic states of molecules. Some of these effects as they pertain to small molecules will be discussed.

\section{Electronic Orbitals, States, and Transitions}

Molecular stationary states are characterized by solutions of the time-independent Schrödinger equation for the "many electron-many nucleus" system. Important contributions to the energy come from electrostatic attractive interactions between electrons and nuclei, and electrostatic repulsive interactions between electrons and between nuclei. Smaller but no less interesting contributions arise because of oscillation of the nuclear masses about their equilibrium positions, rotation of the molecule as a whole, and magnetic interactions among the magnetic moments of the spinning electrons and those associated with rotating electronic and nuclear charges in the molecule. The approximation is often made that electronic orbital, electronic spin, and nuclear motions can be treated separately, and in this approximation molecular clectronic states are characterized by solutions to the approximately separable electronic part of the wave equation with nuclei fixcd in their equilibrium positions. It is just the cross terms 
between electronic orbital and electronic spin or molecular vibrational motion which are known to caluse the appearance of certain weak but important molecular spectra in the visible and near ultraviolet regions. Thus, one must go beyond the initial approximation if interesting molecular phenomena are to be explained.

The solutions to the electronic wave equation give not only the electronic energy levels but also, and of equal importance, the electronic charge distribution in the molecular state. When a molecule absorbs or enrits light and undergoes an electronic transition from one state to another, it is important to realize that not only the energy but also the electronic charge distribution in the molecule changes. Since the chemical properties depend not only upon the energy but also upon the charge density at various sites, essentially a new chemical species is formed by the electronic excitation. With rare exceptions excited states are more chemically reactive than ground states of molecules.

The equilibrium nuclear geometry, being dependent upon the charge density in a rather sensitive way, is also expected to differ in the various electronic states of the molecule. While the FranckCondon principle states that the most probable electronic transition is one in which the nuclear positions do not change, it often happens that much of this excess nuclear energy (vibrational) in the final electronic state is rapidly transferred to the environment before the molecule undergoes a chemical reaction or a transition to a new electronic state. Hence the final state of an electronic transition may be a molecule having different geometry and different chemical properties from those of the initial state. Because of the large amount of empirical data available $(13,14,26,29)$ for simple molecules in addition to the existence of more refined molecular quantum mechanical calculations $(23,34)$, it is possible to predict fairly accurately the nature of these changes for many simple molecules $(22,38)$.

It is convenient as part of the initial approximation, but not rigorously correct, to place the molecular electrons into what are known as molecular orbitals (analogous to atomic orbitals), and to consider transitions between molecular electronic states to be the process of electrons "jumping" from one orbital to another. Some orbitals may be highly localized in the neighborhood of one or two atoms in the molecule while others may be delocalized over a large number of atoms. The ground electronic orbital configuration is that where all the electrons of the molecule have filled the orbitals of lowest energy without violation of the Pauli exclusion principle. 
Excited configurations arise when one or more electrons are promoted to orbitals of higher energy. A single electronic configuration often results in more than one electronic state. For example, if the configuration contains, besides the filled orbitals, two half-filled nondegenerate orbitals (one electron each), singlet and triplet states result depending upon whether the electron spins are respectively anti-parallel or parallel. Certain configurations in highly symmetrical molecules give rise to more than two states of various multiplicities. The various states derived from a single configuration, primarily because of different electron-electron repulsion contributions, may have energies differing by as much as two electron volts $(1$ e.v. $=23$ $\mathrm{kcal} /$ mole). These concepts are partially illustrated in Fig. 1, where the formaldehyde low-lying orbitals and configurations are schematically represented.

Non-bonding orbitals. In molecules there are often electrons,

\section{FORMALDEHYDE}

ORBITALS

$\sigma^{*}$-ontibonding

$\pi^{*}$-ontibonding

$\sum_{2}^{1}$
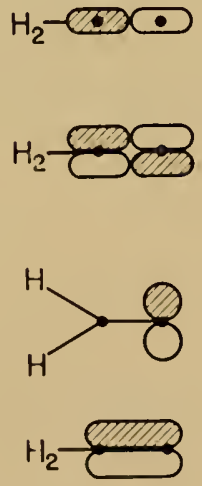
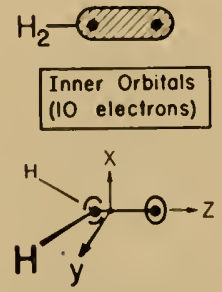

CONFIGURATIONS o non-bonding
品 lone poir
$\sum_{w}$

$\pi$-bonding

$\sigma$-bonding
-
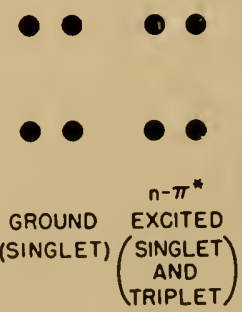

Fig. 1. Molecular Orbitals and Configurations in Fomaldehyde. The cross-hatching is the part of the molecular orbital formed from atomic orbitals with negalive signs. 
termed non-bonding electrons (33), which take a very small part in the actual bonding together of the nuclei. These electrons are in orbitals which concentrate little electron clensity between the nuclei. Examples are the lone pair electrons on a nitrogen atom in the organic azines or those on oxygen in a carbonyl group. Such electrons are characterized by the relatively small amount of ionization energy necessary to remove them completely from the molecular system, and by the insensitivity to the removal of such an electron of the molecular geometry and vibrational constants of the resulting positive ion. Because of their loose coupling to the rest of the molecule, non-bonding electrons, when present, are very often involved in the lowest energy electronic transition.

Bonding and antibonding $\pi$-orbitals. Molecular electrons which are similar to atomic p-orbitals except that they extend over at least two atoms are called $\pi$-electrons. The electron density has a node along the line joining the nuclei over which the $\pi$-orbital extends. A $\pi$-orbital may be approximately constructed by adding together or by substracting atomic p-orbitals on the atoms involved in the $\pi$-bond. In the first case electron charge density is "piled up" between the nuclei and the orbital is bonding, while in the second case a node of electronic charge is formed between the nuclei in a plane perpendicular to the internuclear axis. ${ }^{*}$ This latter type of orbital is termed antibonding, since the resulting electrostatic repulsion causes the bond to become weaker. An electron added to such an orbital results in an increased bond length and a much lower vibrational frequency associated with that part of the molecule.

$n-\pi^{*}$ Transitions. Transitions, termed $n-\pi^{*}$, caused by electrons promoted from a non-bonding orbital $(n)$ to a $\pi$-antibonding orbital $\left(\pi^{*}\right)$, are important in molecules containing either the azine or carbonyl groups (33). These transitions, which occur in the near ultraviolet or visible portions of the spectrum, are very often the lowest energy transitions in such molecules. They are relatively weak as molecular transitions go. The molecular orbitals important for the discussion of the lowest lying $n-\pi *$ electronic transitions in formaldehyde were given previously in Fig. 1. Those for pyridine are shown in Fig. 2. The $n-\pi^{*}$ singlet-singlet transition in formaldehyde is $2.4 \times 10^{-4}$ (oscillator strength, $\left.f=2.4 \times 10^{-4}\right)^{5}$ as intense $(9)$ as

"In the case of conjugated ring hydrocarbons this axis need not be a chemical bond axis, but may cross the ring. See, for example, Fig. 2 and ref. 17.

${ }^{5} f$ (dimensionless) $=4.32 \times 10^{-9} \epsilon d_{\nu}$, where the molar extinction coefficient $\epsilon=1 / c d \log _{10}(100 / T) ; c$ in moles/liter, $d$ in $\mathrm{cm}, \nu$ in $\mathrm{cm}^{-1}, T=\%$ transmis. sion. The integration is over the absorption band. 


\section{PYRIDINE (BENZENE)}

ORBITALS

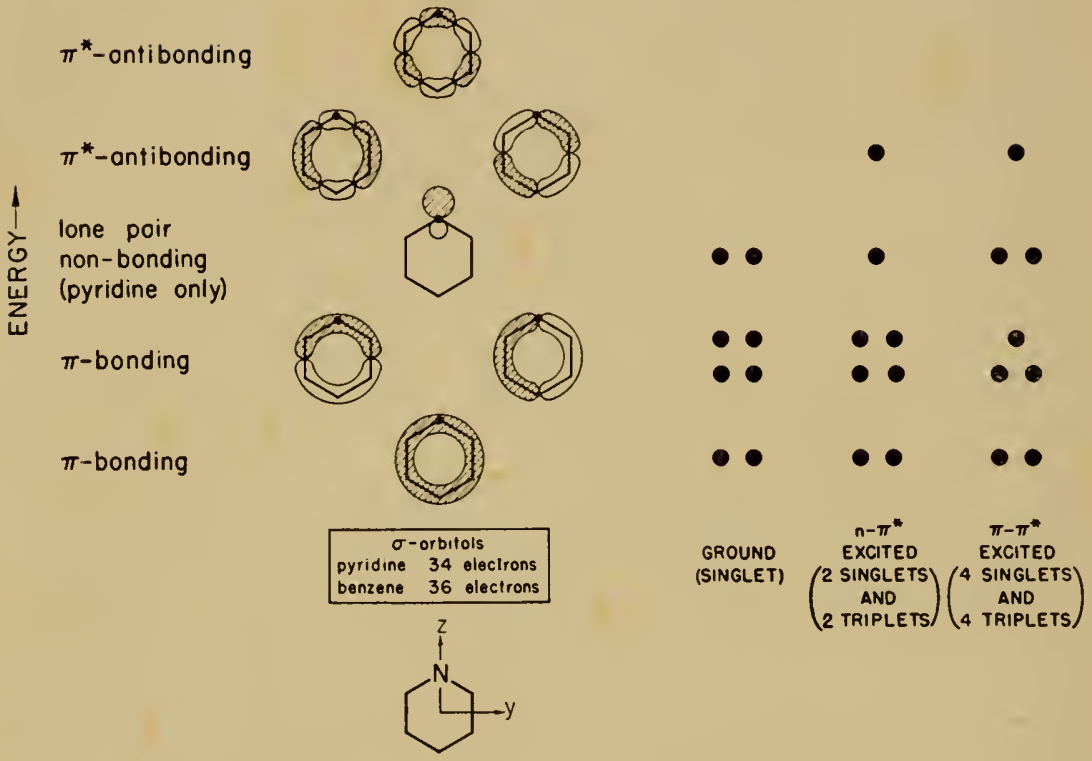

CONFIGURATIONS
Fig. 2. Molecular Orbitals and Configurations in Pyridine (Benzene). The crosshatching is the part of the molecular orbital formed from atomic orbitals with negative signs. The orbitals, originally doubly degenerate in benzene, do not have the same energies in pyridine. The $\pi$ and $\pi^{*}$ orbitals have a nodal plane coincident with the plane of the molecule. (The author wishes to thank Professor M. Kasha for pointing out an error originally present in this figure.)

a fully allowed electric dipole transition, such as the transition corresponding to the "D-lines" of the sodium atom $(f=.976)$ (3). Most of the intensity contribution in the formaldehyde $n-\pi^{*}$ spectrum comes from the unsymmetrical distortion of the electronic charge clouds by unsymmetrical molecular vibrations (27a). Interestingly cnough, there is about a 3 per cent contribution from a magnetic dipole transition (31). The singlet-triplet $n-\pi^{*}$ transitions in these molecules are much weaker in absorption and consequently the lifetime of the upper triplet state is much longer than that of the upper singlet state. In the formaldehyde $n-\pi^{*}$ transition, which has a relatively large singlet-triplet transition probability compared with most molecules containing only first-row atoms, the transition is only 
$1 / 200$ as intense (8) as the singlet-singlet, which itself is of relatively low intensity. This lact illustrates the extreme weakness of such intercombination bands. The lifetime of the excited triplet state of formaldehyde is about .002 seconds.

The $n-\pi^{*}$ singlet-singlet transition probability in pyridine has an oscillator strength of about .003 (36), larger by a lactor of about ten than the $n-\pi^{*}$ transition in formaldehyde. Unlike formaldehyde, the major part of this intensity seems not to be caused by antisymmetric vibrations. ${ }^{6}$ The two singlet-singlet $n-\pi^{*}$ transitions in pyridine should be particularly noted (Fig. 2). Electric dipole transitions between mutually perpendicular $2 p$-orbitals on the same atom can be shown to have zero transition probability while $2 s-2 p$ transitions are extremely intense. Any s-character in the non-bonding orbital will therefore show up as an increased intensity in the $n-\pi^{*}$ transition. In the molecular orbital approximation only combinations of atomic orbitals having the proper local molecular symmetry properties can be mixed to form the molecular orbitals between which electrons undergo transitions. Because of this symmetry restriction, the most loosely bound non-bonding orbital in aldehydes and ketones, being derived from a p-orbital on oxygen, antisymmetric with respect to the $\mathrm{xz}$ plane (Fig. 1), cannot gain local s-character, symmetric with respect to the $\mathrm{xz}$ plane, from oxygen or from the adjacent carbon atom. In better approximations where the configurations are allowed to mix, the excited states of keto groups may show the effect of additional $s$-character in $n-\pi^{*}$ transitions.

$\pi-\pi^{*}$ Transitions. In conjugated hydrocarbons of which ethylene, benzene, and pyridine are prototypes, important transitions are caused by an electron jump between a $\pi$ and a $\pi^{*}$ orbital. The lowest energy singlet-singlet $\pi-\pi^{*}$ transitions occur at about $260 \mathrm{~m}_{\mu}$ in benzene and in pyridine and at a slightly shorter wavelength in ethylene. They are progressively shifted to longer wavelength as the size of the molecule increases (e.g., in carotene). The transition in benzene is symmetry-forbidden but, like the $n-\pi^{*}$ transition in formaldehyde, is made weakly allowed $(f=.0014)$ through vibrational-electronic interaction (24). The lowest $\pi-\pi^{*}$ transition in pyridine is allowed by symmetry but is intrinsically weak $(f=.04)(36)$, since the electronic structure of benzene, where the transition is forbidden, is approximately retained. It is notable that the spectra of many substituted benzenes can be described in terms of relatively small perturbations on the parent molecule $(11,25)$. Fig. 2 shows schematically the molecu-

\footnotetext{
"But one must be careful in assigning (0-0) bands!
} 
lar orbitals pertinent for discussion of low-lying pyridine transitions. The $n$-orbital is shown in addition to the bonding and antibonding $\pi$-orbitals, which are similar to those in benzene.

The $6 \pi$-orbitals in benzene can be represented analytically by

$$
\begin{aligned}
& \psi_{1}=\sqrt{\frac{1}{6}}\left(\phi_{1}+\phi_{2}+\phi_{3}+\phi_{4}+\phi_{5}+\phi_{6}\right) \\
& \psi_{2}=\sqrt{\frac{1}{3}}\left(-\phi_{1}-\frac{1}{2} \phi_{2}+\phi_{3}+\phi_{4}+\frac{1}{2} \phi_{5}-\frac{1}{2} \phi_{6}\right) \\
& \psi_{3}=\frac{1}{2}\left(\phi_{2}+\phi_{3}-\phi_{5}-\phi_{6}\right) \\
& \psi_{4}=\sqrt{\frac{1}{3}}\left(\phi_{1}-\frac{1}{2} \phi_{2}-\frac{1}{2} \phi_{3}+\phi_{4}-\frac{1}{2} \phi_{5}-\frac{1}{2} \phi_{6}\right) \\
& \psi_{5}=\frac{1}{2}\left(-\phi_{2}+\phi_{3}-\phi_{5}+\phi_{6}\right) \\
& \psi_{6}=\sqrt{\frac{1}{6}}\left(-\phi_{1}+\phi_{2}-\phi_{3}+\phi_{4}-\phi_{5}+\phi_{6}\right)
\end{aligned}
$$

in increasing order of energy, where the $\phi$ 's are $2 p_{z}$ orbitals on the six carbon atoms. As indicated in Fig. 2, the pairs 2,3 and also 4,5 have the same energy in benzene, "persubstituted," and symmetrically trisubstituted benzene derivatives, but they have different energy if the symmetry axis is reduced below three-fold, as in pyridine or the mono- or di-substituted benzene derivatives. The reader may easily verify that the analytical functions do indeed approximately represent the $\pi$ and $\pi^{*}$ orbitals in Fig. 2. In the schematic representation, however, no attempt was made to show details of the nonumiformities of "orbital density," and thus electron density, around the ring.

\section{Geometry of Excited States of Molecules}

It was mentioned earlier that the geometry of an excited state of a molecule is expected to be different from that corresponding to the ground state if the bonding electron configuration is significantly different in the two states. The geometry of the excited state may be important in biochemical problems since the transfer of energy is expected to be very sensitive to the crossing of potential energy surfaces, and the surlaces are defined in terms of an equilibrimm geometry of the molecule. The geometry of very simple molecules can be found directly from an analysis of the rotational part of the electronic spectrum of the gas-phase molecule. If the molecule has more than three or four atoms of the mass of carbon or greater, the direct evaluation of excited-state geometry from the rotational structure becomes impractical, since the structure is not easily resolved. In a recent paper (6), however, the rotational contours in the electronic spectrum of naphthalene were "analyzed", and some inferences may be possible concerning the geometry of the upper state. 
For more complicated molecules, geometry changes can be found through a stucly of (i) the magnitude of the vibrational frequencies (I0) and (ii) the relative intensities of the vibrational parts of the clectronic transition (4). In both cases an analysis of the vibrational structure must be made. The first method relies on the fact that the frequencies of vibrations vary approximately as the square root of the force constants, and the lorce constants in turn are roughly related to the bond orders and thus the interatomic distances. In this sense, relative bond distances can be inferred from changes in vibrational frequencies between states. The second method relies on the magnitudes of the so-called Franck-Condon overlap integrals (16), which are related to the displacement of the vibrational origins about which small oscillations of the nuclei are described. Displacements of these origins are caused by geometry changes. Assuming harmonic oscillations, it is possible to compute the relative intensities of vibrational bands for various excited state geometries and to compare them with the experimental intensities. Since the intensity patterns are rather sensitive to geometry changes, reasonable estimates may be made of the geometry of the excited state molecule. Both kinds of calculations have been carried out for benzene $(4,10)$, and the two results agree fairly well. They show that the C-C bond increases by $.037 \mathrm{~A}$ in the excited singlet state of the $\pi-\pi^{*}$ transition. One of the $18 \mathrm{C}$-G bonding electrons is promoted to an antibonding orbital. The effect is spread over six bonds, so that an effective loss per bond of $\frac{1}{3}$ of a bonding electron occurs. One would predict on this basis that the $\mathrm{C}-\mathrm{C}$ bond increases by $.035 \mathrm{~A}$ in this transition. This is in excellent agreement with the observation.

Almost without exception chemical bonds are weaker and longer in excited state molecules. The greatest change expected for a oneclectron transition is that which occurs when a bonding electron is excited to an antibonding orbital. This kind of a transition essentially causes a triple bond to become double, a double bond to become single, or a single bond to disrupt. The bond distance may increase by 15 per cent or more. Bond angles may change by as much as $80^{\circ}$ because of changes in the degree of $s$ - $p$ hybridization, but no generalization can be made concerning the direction of such changes without a better knowledge of the electronic eigenfunctions. ${ }^{\top}$

'Molecules (ref. 13) such as CO. C., HCN, and aceiylene are linear in their ground states but bent in their low excited states. Molecules such as $\mathrm{NH}_{2}$. HCO. and possibly $\mathrm{NO}_{2}$ are bent in their ground state but linear in their lowest excited states. Often the change in bond angle is from around $100^{\circ}$ to $180^{\circ}$, or vice versa. 
Little is known about geometry differences in polyatomic molecules between singlet states and triplet states having the same electron configuration, except what can be implied from the vibrational part of the electronic spectrum. The difference between the states is caused by the fact that, in the triplet state, the electron spins are parallel, while in the singlet they are antiparallel. One could hardly believe that small magnetic effects can have very much to do directly with the equilibrium geometry of the molecule, and on the basis of the orbital approximation it is expected that the geometry differences would be insignificant. Actually, the orbital approximation does not take into account the repulsive interactions between electrons, and it is just this contribution which causes by far the major differences in energy and geometry between states having the same configuration. Electron-electron repulsion energy is greater in the singlet state than the triplet essentially because of the restrictions brought about by the Pauli exclusion principle. In effect, this principle requires that in the triplet state, unlike the singlet, two electrons which have the same spin cannot simultaneously occupy the same space part of the electron cloud. Thus electron-electron repulsion energy is decreased. Because of this, the triplet state lies below the corresponding singlet, and bonding properties and geometry are affected to some extent.

In the simple molecule formaldehyde, the geometry of the lowestlying singlet and triplet excited states was ascertained from an analysis of the rotational structure in the electronic transition $(8,32)$. These states are the excited states of the $n-\pi^{*}$ singlet-singlet and singlettriplet transitions. Since, in the transition, one non-bonding electron becomes antibonding in the $\mathrm{C}=\mathrm{O}$ bond, this bond becomes essentially a 3 -electron rather than a 4-electron bond. The bond length $(\sim 1.3$ A) is expected, and found, to be half-way between single ( $1.2 \mathrm{~A})$ and double $(\sim 1.4 \mathrm{~A})$ bond carbon-oxygen distances. The molecule is planar in the ground state but non-planar (about half-way toward tetrahedral bonding) in both excited states. In the triplet state the carbon-oxygen bond distance is $1.312 \mathrm{~A}$ while it is $1.326 \mathrm{~A}$ in the singlet state, so there is slightly tighter bonding in the triplet state. This is further characterized by the higher carbon-oxygen vibrational frequency in the triplet state. The non-planarity is greater in the triplet state, and the energy difference between the planar and equilibrium non-planar forms is also greater in the triplet than the excited singlet state.

While formaldehyde is the only case where a really precise de- 
termination of the triplet state geometry has been made, vibrational data in other molecules seen to confirm that singlet and triplet states having the same electron configuration have very nearly the same geometrical properties. These may, however, differ greatly from the geometry characteristic of slates derited from other configurations. The importance of the lowest triplet state of a molecule lies not in its geometry, but in the fact that it is the excited state of very lowest encrgy. Thus in any excitation process, followed by rapid internal energy transfer, it is likely to be the only excited electronic state which retains for a sufficient time any electronic excitation energy for use in chemical reactions (12) or other processes especially characteristic of an excited electronic state.

\section{Environmental Perturbations on Electronic and Vibrational States}

Reasonably large environmental perturbations may be caused by van der Waals', dipolar, or ionic fields in liquids, in solids, or in gases at high pressures. In gases and liquids the perturbations must be averaged over the molecular fuctuations. Intramolecular perturbations between various parts of a molecule may be loosely classed as environmental perturbations, if such perturbations remain reasonably small. Because of the variations of electronic density in different electronic states, an interaction with the enviromment affects differently the various electronic states of a molecule. Since the effect on the energy is different in the two combining states a shift in the spectrum results. Activation energies for chemical reactions depend upon energy differences between molecular states, just as spectra do, and it is expected that rates of chemical reactions should generally be increased in those cases where the spectrum is shifted to lower energy (red shift). Reactions should be slowed down in those cases where the spectrum is blue-shifted.

Non-polar solvents. Effects of non-polar neighbors are perhaps the simplest to understand quantitatively, since angular dependent forces, if present, are fairly small and can be neglected to a first order of approximation. The potential between two spherically symmetric molecules is then approximately given by the Lennard-Jones potential (15) ,

$$
V=4 \epsilon\left(\frac{\sigma^{12}}{r^{12}}-\frac{\sigma^{6}}{r^{6}}\right)
$$


The first term in the parenthesis is repulsive and the second is attractive. The spectral shift is accordingly given by

$$
\Delta E=\sum\left(\frac{\Delta A}{r^{12}}-\frac{\Delta B}{r^{6}}\right)
$$

where $\triangle A$ and $\triangle B$ are differences of repulsive and attractive coefficients in the two electronic states, and the sum is over all neighbors. In Eq. $1, \epsilon$ is the intermolecular binding energy and $\sigma$ is a parameter of size at which attractive and repulsive contributions are equal. The sum is justified here since the interactions are known to be approximately additive. The binding energy in the liquid or solid is thereby increased by a factor of 8 to 14 over that of the binary interaction. For ground states of molecules, the parameters for pure substances can be determined from solid state data, thermodynamic virial coefficients or transport properties of gases. ${ }^{8}$ For two different interacting partners (solvent and solute), the parameters are approxinately obtained as mean values of the parameters for each of the pure substances. The attractive interactions are derived from the London dispersion forces. For spherically symmetric molecules as a first approximation these are given by induced-dipole-induced-dipole interactions. They depend upon the molecular polarizability which increases with the electron density in the molecule. In large molecules it is the local polarizability and not the overall molecular polarizability which is important in the discussion of spectral shifts. The repulsive interactions are derived primarily from exchange forces (non-bonded repulsion) as the electrons of one molecule try to crowd into the filled orbitals of a neighboring molecule. Attractive dispersion interactions generally have a larger coefficient in the excited state than in the ground state because of the larger polarizability of the more diffuse electron cloud. Repulsive interactions have a larger coefficient in excited states because of the increase in orbital "size." The interaction coefficients for the excited states of simple nolecules can be estinated quantitatively by extending the London theory to excited molecules (18). Because of the increased magnitude of the excited state intermolecular parameters, solvent-solute attractive interactions result in a shift of the spectrum to lower energy (red shift), while repulsive interactions canse a blue shift. The latter situation is not too common. It might resule when a large molecule is dissolved in a small-molecule solvent, the strong intermolecular solvent-solvent interactions essentially squeezing the solute molecule

${ }^{8}$ For details sec ref. 15 . 
to produce the repulsive interaction and the blue shilt. These blue shilts can be produced artificially by "trapping" molecules in a solid composed of smaller molecules (18), or by high pressure techniques (1). Since bands remain sharp in certain low temperature solids, precise measurements of "solvent shifts" can be macle by that technique. Naphthalene in solid xenon, for example, shows blue shifts of $50 \pm 10 \mathrm{~cm}^{-1}$ in its two lowest-lying $\pi-\pi^{*}$ singlet-singlet electronic transitions. ${ }^{9}$ Much larger shifts to the blue are expected in smaller atom "solvents" such as krypton and argon. ${ }^{10}$

It is important to realize that the electronic origin $[(0-0)$ band ] of a transition is shifted a different amount than the absorption maximum. This is explained by the Franck-Condon principle applied to the molecule plus environment. A change in equilibrium structure of the local environment upon molecular excitation caused by different $\epsilon$ and $\sigma$ in the excited state will cause an additional shift corresponding to the extra lattice energy of the excited molecule in an unstable envirommental state. These shifts are probably much smaller than the electronic shifts themselves because of the strong tendency of the solvent molecules to resist local expansion or compression. This is indicated by the study of sufficiently simple systems where individual vibrational bands of the spectrum can be resolved and accurately measured.

It should be remarked that attractive intermolecular interactions decrease the electron density in the molecule and also in its interacting partner since electronic charge is transferred to the "intermolecular bond." Repulsive interactions increase the electron density in both interacting species. Such effects can be observed upon vibrational energy levels of the molecules, decreasing the vibrational stretching force constants in the first case and increasing them in the second to produce vibrational red and blue shifts respectively. ${ }^{11}$

${ }^{9}$ The ribrational analysis (ref. 35 ) of the $2900 \mathrm{~A}{ }^{1} \mathrm{~B}_{\mathrm{2u}}-{ }^{1} \mathrm{~A}_{1 \mathrm{~g}}$ transitoin in naphthalene is apparently in error. Vibrational bands assigned to exciled vibrational letels in the ground state were found at $4.2^{\circ} \mathbf{K}$. If the original assignment were accepled, the solid xenon permubation would have to shift the spectrum over 1300 $\left(\mathrm{m}^{-1}\right.$ to the red. This is much more difficult to explain than a slight blue shift of similar magnitude as the blue shife in the ${ }^{3} B_{2 u}-{ }^{3} A_{1 g}$ transition. The large red shift $\left(\sim 2250 \mathrm{~cm}^{-1}\right)$ reported for the ${ }^{3} B_{\mathrm{zu}}-{ }^{3} \mathrm{~A}_{1 \mathrm{~g}}$ transition in pure crystalline naphthalene (ref. 20) becomes $754 \mathrm{~cm}^{-3}$, more in keeping with the $462 \mathrm{~cm}^{-1}$ red shift of the ${ }^{1} B_{3 u}-{ }^{1} A_{12}$ transition in the crystal.

${ }^{\text {to }}$ Recent work has shown the shifts in argon to be $243 \mathrm{~cm}^{-1}$ and $348 \mathrm{~cm}^{-1}$ to the blue for the ${ }^{1} B_{3 u}-{ }^{1} A_{1 g}$ and ${ }^{1} B_{2 u}-{ }^{1} A_{1 g}$ transitions respectively.

${ }_{11}$ The situation for bending ibrations is more complex, owing to the fact that the aterage dipole moment of a molecule such as $\mathrm{H}_{2} \mathrm{O}$ or $\mathrm{NH}_{3}$ decreases with 
Taking the force constants (which vary as the square of the frequencies) to be roughly proportional to the overall bonding electron density, one can estimate the gain or loss of such electrons because of the interaction. A situation uncluttered by more complicated effects is the vibrational perturbations on molecules in crystalline rare gases. For the simple molecular fragment $\mathrm{NH}$, the bonding electron density loss $^{12}$ in the bond is $.9 \%, 1.4 \%$, and $2.4 \%$ respectively in solid argon, krypton, and xenon. Usual vibrational shifts are a bit smaller than these since the "local polarizabilities" of normal solvent molecules are smaller than those of the rare gases.

Charge transfer interactions. Many solvent-solute interactions are somewhat more complex than the ones discussed thus far. In certain cases the affinity of the solvent molecule for electrons is anomalously large if there are low-lying unfilled orbitals in the solvent. If the solute is a better donor in the ground state than the excited state, then the ground state of the solute is depressed in energy more than the excited state and a blue shift results even though attractive forces are dominant. Such "charge transfer" complexes (2l) are common, the iodine-pyridine 1:1 complex being a good example (30). They bridge the gap between the weaker van cler Waals' complexes and molecules bound together by strong valence forces. Since the vibrational force constant of the donor molecule is decreased, the vibrational spectrum is expected to be red shifted (27), characteristic of attractive interactions. In the iodine-pyridine complex, the $I_{2}$ vibrational frequency is decreased from $213 \mathrm{~cm}^{-1}$, the gas phase value, to $184 \mathrm{~cm}^{-1}$ in the complex. Ignoring any possible effective mass change, about $7 \%$ of the bonding electron density is removed from the $\mathrm{I}_{2}$ bond. In halogen-benzene systems (where multiple interactions are probably important) $2.8 \%, 2.6 \%$, and $2.1 \%$ of the bonding electron density of $\mathrm{Cl}_{2}, \mathrm{Br}_{2}$, and $\mathrm{I}_{2}$, respectively, appear to be donated to the environment. The decrease here, instead of an expected increase, may in part be accomnted for by repulsive interactions which become increasingly important for the larger halogen molecules.

Interactions involving dipole moments. Another important class of spectral shifts results if the dipole moment of a molecule is different in the two combining electronic states. Since charge density changes with electronic excitation, changes of dipole moment are to be expected. In liquids or solids the point dipole approximation is poor, but a rough description of the interaction is dipole-induced-dipole

"On the assumplion that the reduced mass for vibration is unaltered. 
or dipole-dipole depending upon whether the solvent molecules are themselves polar. A striking confirmation of the role played by the dipole moment in solvent interactions is afforded in the case of trapped $\mathrm{NH}_{2}$ in crystalline rare gases (IS). The electronic properties of this free radical in its ground state are almost identical to those of $\mathrm{H}_{2} \mathrm{O}$ except that one of the lone-pair electrons is missing in $\mathrm{NH}_{2}$. The dipole moment in the ground state is probably of the orcler of 1.0 Debye. In the lowest excited state the molecule, unlike $\mathrm{H}_{2} \mathrm{O}$, is linear and the dipole moment vanishes. Consequently the ground state is depressed not only because of the attractive clispersion forces but also because of dipole-induced-dipole interactions. The excited state of $\mathrm{NH}_{2}$ is depressed only by virtue of the dispersion forces. Blue shifts are expected unless the dispersion forces in the excited state are sufficiently large to cancel the sum of the two ground state interactions. The electronic shifts of $\mathrm{NH}_{2}$ in argon, krypton, and xenon are $27.4 \mathrm{~cm}^{-1}$ to the blue, $7.1 \mathrm{~cm}^{-1}$ to the red, and $41.6 \mathrm{~cm}^{-1}$ to the red, respectively. This shows that the dipole-induced-dipole contribution to the shift is dominant in argon, approximately equal but a little smaller than the dispersion contribution in krypton, and much smaller than the dispersion contribution in xenon.

The effect of the dipole term is apparently somewhat larger in $n-\pi^{*}$ transitions. The blue shift of these transitions in polar and non-polar solvents has been known for some time ${ }^{13}$ and is an important characteristic of $n-\pi^{*}$ transitions. The major part of this shift is very probably caused by an energy change of the states themselves (the $0-0$ band), rather than by the Franck-Condon effect on the local environment. This is borne out by the fact that individual vibrational bands in the formaldehyde $n-\pi^{*}$ transition in solid argon (a non-polar "solvent") can be measured to within $\pm 30 \mathrm{~cm}^{-1}$ and these all show relatively large shifts to the blue of from 220 to 320 $\mathrm{rm}^{-1}$ ! This shift is of similar magnitude as the $n-\pi^{*}$ shift in other non-polar solvents. The behavior of $n-\pi^{*}$ transitions in polar solvents is very analogous to that in these simple non-polar solvents. The energy shifts are expected to be much greater because of dipole-dipole interactions, and strong "charge transfer" or "hydrogen-bonded" complex formation.

\section{ENERgy TRANSFER}

Energy transfer may take place only if total energy is conserved. In an isolated molecule in a radiation field, transfer between states of identical total energy may take place, but transfer between states of

${ }^{13}$ See, for example, ref. 33, pp. 30. 
different energy may occur only if energy can be radiated or absorbed. Only in a few special cases is this a very probable process compared with others which may occur when an environment is present. The case of a molecule in a collisionless environment is important only in the sparse density of interstellar space. Normally, radiationless energy transfer takes place without the above energy restrictions, since units of energy may be transferred rapidly to or from translational, rotational, vibrational, or electronic states of the environment.

Criteria for non-radiative energy transfer. For a non-radiative transition to take place between two electronic states of a polyatomic molecule, a number of considerations should be especially stressed. Assume that the environment acts only as an energy reservoir. Experiments have shown that rapid transfer of energy to the environment can take place only if the energy spacing in the environment is approximately of the same magnitude as the units of energy which are to be lost by the molecule. In other words, many near resonances should occur between envirommental energy levels and the molecular ones. Because of this condition, large molecules, with many closely spaced vibrational levels, nearly always lose excess vibrational energy to an environment in a time short compared with radiative deactivation or chemical processes. For very small molecules such is often not the case. As a corollary to the above, transfer of a large unit of electronic energy to the closely spaced vibrational levels of the environment is an improbable process.

A second condition important for cross-over from one electronic state to another is that potential energy surfaces corresponding to the two electronic states in question should have an available region of mutual "intersection." This means in particular that non-radiative processes between excited states and the ground state of a molecule have relatively small probability, since these states do not in general cross. The precise meaning of the term "intersection" is important, and it will be discussed more fully in the next paragraph.

A third, and very important criterion for non-radiative transfer of energy between electronic states deals with the question of perturbations. Even though an envirommental energy reservoir is available, some perturbation must be present to "mix" the two states between which energy transfer is to take place. It is known from quantum mechanical perturbation theory that a perturbation of this kind is most important in the region where the two states have nearly the same energy and geometry, i.e., in the region of potential surface intersection. Perturbation theory also shows that the interaction be- 
tween two electronic states causes the energy difference between the states to increase (the surlates "repel each other"), depending upon the magnitude of the perturbation. This means that an extremely strong perturbation is likely to caluse such a divergence of the potential energy surfaces that the first criterion above can no longer be met. On the other hand, states can exactly cross only if there is no perturbation which mixes the states, but the transition probability for non-radiative transfer between such states is exactly zero. There therefore appears to be a third important criterion for rapid non-radiative transfer of energy. The perturbation which mixes two electronic states between which non-radiative energy transfer is to occur must be of the correct magnitude, not too large and not too small, so that the energy difference between the interacting states is of the order of environmental energy states. If the environment does not act as a "catalyst," the perturbation must be an intramolecular one. The effect of zero, weak, and strong interactions upon the energy transfer mechanism is depicted in Fig. 3. Perhaps the spin-orbit perturbation operator which mixes states of different multiplicities is of optimum magnitude to promote energy transfer between states which approximately cross. ${ }^{1+}$ It is known experimentally that energy transfer between excited singlet and triplet states in certain rigid environments occurs in an average time much shorter than $10^{-8}$ seconds. On the other hand, non-radiative transfer from the first excited triplet to the ground singlet can be extremely slow in the same environments. In benzene, the geometries and energies of the triplet and ground singlet are different, and there is likely to be no potential surface intersection (in the absence of environmental perturbations); in addition, the zero-order spin orbit perturbation, because of symmetry, vanishes between these two states. Similar statements can be made about other conjugated ring hydrocarbons. It is no wonder such processes are rare until a fluctuating environment is introduced. On the other hand, the excited singlet and triplet probably have nearly the same geometries, have similar energies, and the probability of potential surface intersection is high. Furthermore, in

"One possible example (ref. 7 ) which has been observed but not fully discussed is in the spectrum of the HNO molecule near $750 \mathrm{~m} \mu$. Rolational lines (normally single) are split by a perturbation in the excited state of HNO. Since the electonic structure of HNO is very simple at this low energy, it can be surmised that the only possible perturbing state is a triplet state. The maximum energy splittings are of the order of $1 \mathrm{~cm}^{-1}$ and it is expected that the singlet-triplet radiationless intercombination process would be very rapid from these perturbed rotational levels. For larger molecules the region of interaction may be rery extensive. 


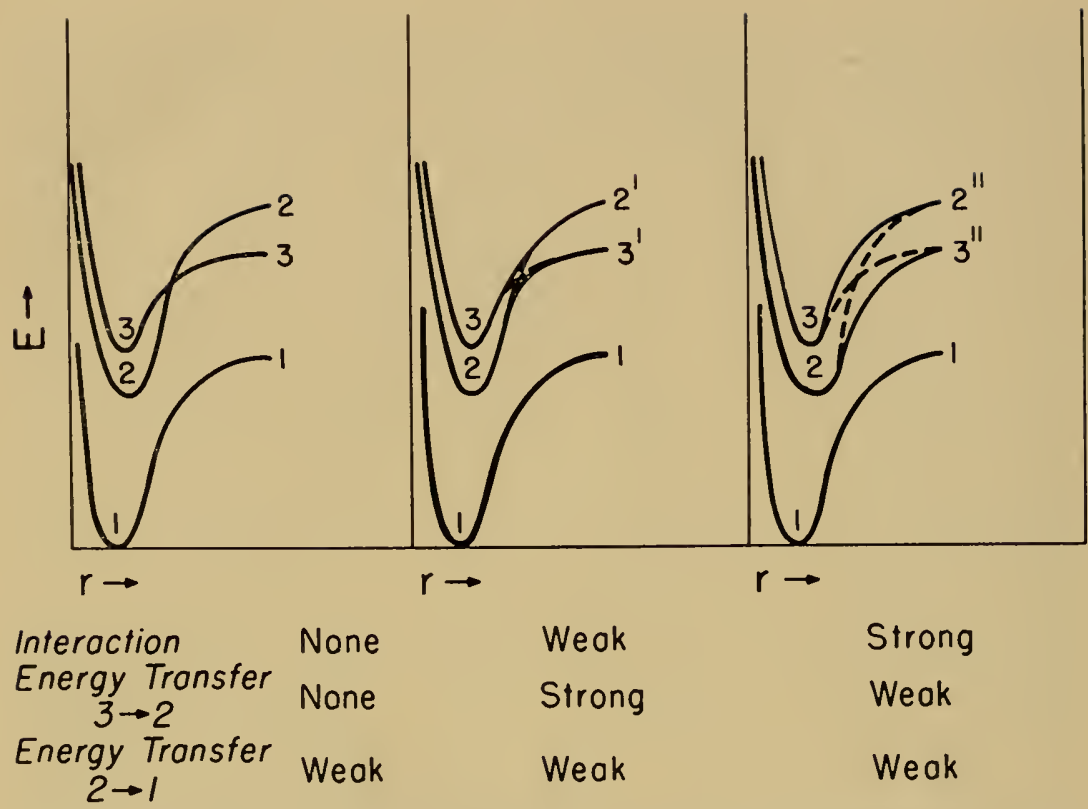

Fig. 3. Zero, weak, and strong interactions between electronic states of a diatomic molecule showing how strong interactions inhibit energy transfer while weak interactions, because the states remain close together, may favor rapid energy transfer through closely spaced environmental levels. Similar considerations should apply for more complex molecules. A fluid environment introduces a fluctuating (time(lependent) perturbation. This can cause states 2 and 1 to spend a small, but significant, part of the time in a region of mutual overlap, whereby the non-radiative transfer of energy from $2 \rightarrow 1$ is greatly enhanced.

benzene and similar molecules, one component of the spin orbit operator has the correct symmetry to mix excited singlet and triplet states (2la).

Effect of enviromment upon energy transfer. It is known that the rate of non-radiative processes between excited states and the ground state is very sensitive to the environment. In rigid media, this process is usually very slow (lifetime $\gg 10 \mathrm{sec}$ for the benzene triplet to ground singlet state), while in liquids or gases the process is very rapid (lifetime of the order of $10^{-4}$ to $10^{-8} \sec$ or less). In certain cases the non-radiative process can be catalyzed by a special environment. This is illustrated by the known quenching effect of paramagnetic molecules on the lifetime of the triplet state (28). In the absence of such catalyzing agents it is the fluctuations in fluid media which quench the radiative processes. A possible mechanism can be con- 
ceived if it is again realized that envirommental perturbations affect the energy levels and electronic charge densities in a molecule. If these perturbations arise from a rigid medium it is probable that many of the molecules have rather uniform environnents, so that no large unsymmetrical distortions of the electron density functions are realized. In fluids the perturbations are changing with time because of the rapid molecular fluctuations in the local environment. Statistically there will always be some large fluctuations which greatly influence the potential energy surfaces, energies, charge clouds (and "mix" clectronic states), and vibrational motions of the molecule and its enviromment. Because of the random time-dependent environmental perturbations in fluid media, all the criteria for energy transfer can be easily met. One can think of the molecule "scanning" the fluctuating perturbations with time until just the proper conditions are found for the non-radiative process to occur. Once the crossover between electronic states has been realized, then the loss to the environment of vibrational energy in the new electronic state is again very rapid.

\section{REFERENCES}

1. Babb, S. E., Jr., Robinson, J. M., and Robertson, W. W., J. Chem. Phys., 30 427 (1959).

2. Coffman, R., and Mcclure, D. S., Can. I. Chem., 36, 48 (1958).

3. Condon, E. U. and Shortley, G. H., Theory of Atomic spectra, p. I49, Cambridge University Press, Cambridge, England, 1935.

4. Craig, D. P.. J. Chem. Soc., 2146 (1950).

5. Craig, D. P., Hollas, J. M., and King, G. W., J. Chem. Phys., 29, 974 (1958).

6. Craig, D. P., Hollas, J. M., Redres, M. F., and Wait, S. C., Proc. Chem. Soc., (London). 361 (1959).

7. Dalbv, F. W., Can. J. Phys., 36, 1356 (1958).

8. Di Giorgio, V. E.. and Robinson, G. W., J. Chem. Phys., 3I, 1678 (1959).

9. Duncan, 1. B. F., and House, E. H., as quoted by J. W. Sidman, J. Chem. Phy's., 29, 644 (1958).

10. Garforth, F. M., Ingold, C. K., and Poole, H. G., J. Chem. Soc., 508 (19-18).

11. Goodman, L., and Shull, H., I. Chem. Phys., 27, 1388 (1957).

12. Hammond, G. S., and Moore, W. M., I. Am. Chem. Soc., 81. 6334 (1959).

13. Herzberg, G., Ann. Rev. Phys. Chem., 9, 315 (1958).

14. Herzberg, G., Proc. Chem. Soc. (London), 116 (1959).

15. Hirschfelder, J. O., Curtiss, C. F., and Bird, R. B., Molecular Theory of Gases and Liquids, John Wiley and Sons, Inc., New York (1954).

16. Hutchisson, E., Phys. Rev., 36, 410 (1930).

16a.Kasha, M., Rev. Modern Phys., 31, 162 (1959).

17. Longuet-Higgins, H. C., and Dewar, M. J. S., Proc. Phys. Soc. (London), A67, 795 (1954).

18. McCarty, M., Jr., and Robinson, G. W., Mol. Phys., 2, 415 (1959).

19. MicClure, D. S., Can. J. Chem. 36, 59 (1958.

20. McClure, D. S., and Schnepp, O., J. Chem. Phys., 23, 1575 (1955).

21. McGlynn, S. P., Chem. Revs., 58, 1113 (1958). 
22. Mulliken, R. S., Can. J. Chem., 36, 10 (1958).

23. Mulliken, R. S., Platt, J. R., Roothaan C. C. J., et al., Laboratory of Molecular Structure and Spectra, Univ. of Chicago, Tech. Report (1957-1959).

24. Murrell, J. N., and Pople, J. A., Proc. Phys. Soc. (London), A69, 245 (1956).

25. l'etruska, J., Ph.D. dissertation, Univ. of Chicago (1960).

26. Platt, J. R., Ann. Rew. Phys. Chem., 10, 349 (1959).

27. Plyler, E. K., and Mulliken, R. S., J. Am. Chem. Soc., 81, 823 (1959).

27a.Pople, J. A., and Sidman, J. W., J. Chem. Phys., 27, I270 (1957).

28. Porter, G., and Wright, M. R., J. chim. phy's., 55, 705 (1958); Discussions Faraday Soc., 27, 18 (1959).

29. Ramsay, D. A., Advances in Spectroscopy, 11, 1 (1959).

30. Reid, C., and Mulliken, R. S., J. Am. Chem. Soc., 76, 3869 (1954).

31. Robinson, G. W., unpub. This, as a possible interpretation, was first suggested to me by the late J. W. Sidman.

32. Robinson, G. W., and Di Giorgio, V. E., Can. J. Chem., 36, 31 (1958).

33. Sidman, J. W., Chem. Revs., 58, 689 (1958).

34. Slater, J. C., et al., Quarterly Progress Reports, Solid State and Molecular Theory Group, Massachusetts Institute of Technology (1955-present).

35. Sponer, H., and Cooper, D. D., J. Chem. Phys., 23, 646 (1955).

36. Stephenson, H. P., J. Chem. Phys., 22, 1077 (1954).

37. Thompson, H. W., Proc. Roy Soc. (London), A254, I (1960).

38. Walsh, A. D., J. Chem. Soc., 2260-233I (1953). 


\title{
THE NATURE AND SIGNIFICANCE OF $n \rightarrow \pi^{*}$ TRANSITIONS
}

\author{
MICHAEL KASHA ${ }^{1}$ \\ Departments of Chemistry \\ Haruard University, Cambridge, Massachusetts \\ and \\ Florida State University, Tallahassee, Florida ${ }^{2}$
}

\section{Biological Function and Electronic Structure}

Biological function as related to electronic structure of molecules, and optical phenomena manifested as a consequence of electronic structure, should be carefully distinguished in such a symposium as this one on "Light and Life." It is the purpose of this paper to present a comprehensive though qualitative treatment of a topic in molecular electronic spectroscopy which has broad biological applications: the nature and significance of $n \rightarrow \pi^{*}$ transitions in polyatomic molecules. Special reference will be made to the properties of deoxyribonucleic acids (DNA) and the chlorophylls. In the latter case, the optical phenomena and biological function are intricately interwoven; in DNA, the optical phenomena bear most directly on the structural problem, and on the effects of radiation on the DNA molecule.

The treatment given will be phenomenological. However, since the experimental work clescribed is based on decluctions derived from spectroscopic theory, pertinent aspects of the latter will be surveyed. Although use is made of one application of Symmetry Groups in classifying electronic states and optical transitions, the presentation presupposes no previous knowledge of this subject.

${ }^{1}$ On leave from Florida State University, academic year 1959-1960.

2 This work was supported by a grant from the Petroleum Research Fund. American Chemical Society. 


\section{Definition of $n \rightarrow \pi^{*}$ Transitions}

\section{A. Empirical Classifications (Burawoy's $K$ and $R$ bands)}

Burawoy (4, 4a) developed a very general classification of electronic absorption bands in polyatomic molecules based on empirical classification of band displacements due to solvent effects and substitution effects. According to Burawoy, K-bands (konjugierte) arose from conjugated electronic systems, and could be characterized by (a) high intensity, (b) red-shifts (bathochromic) in solvents of higher dielectric constant, and (c) red-shifts upon the introduction of conjugative electron-donating substituents $\left(-\mathrm{NH}_{2},-\mathrm{N} R_{2},-\mathrm{OH},-\mathrm{SH}\right)$ on the molecule. This broad group of electronic transitions is now associated with orbital promotions of $\pi$-electrons in conjugated molecules, or $\pi \rightarrow \pi^{*}$ transitions (17).

According to Burawoy, R-bands (radikal-artige) were associated with a radical-like state asserted to be present in the ground state for certain molecular groupings. These bands were characterized empirically by (a) low intrinsic intensity $(\epsilon<2000)$, (b) blue-shifts (hypsochromic) in solvents of higher dielectric constant, (c) disappearance of the absorption bands in acid media, and (d) blueshifts upon introduction of conjugative electron-donating substituents $\left(-\mathrm{NH}_{2},-\mathrm{N} R_{2},-\mathrm{OH},-\mathrm{SH}\right)$ on the molecule. It is now known that Burawoy's interpretation of the origin of ' $R$ '-bands is incorrect, and that electronic transitions corresponding to this latter set of empirical classifications arise in most cases from the promotion of "lone-pair" electrons ( $n$ ) to antibonding $\pi$-orbitals in polyatomic molecules $\left(n \rightarrow \pi^{*}\right.$ transitions, 17$)$.

Nevertheless, Burawoy's empirical classification is a very useful

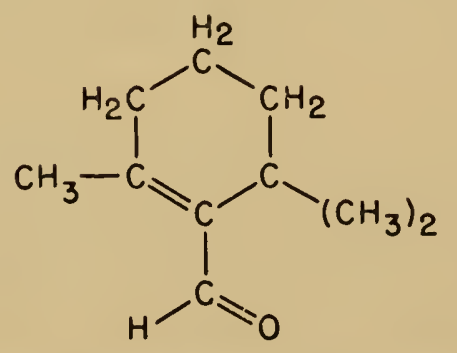

$\beta-$ Cyclocitral

one, and was applied by him to many compounds containing carbonyl groups (aldehydes, ketones) and thiocarbonyl groups (thioketones), as well as azo-, nitro-, nitroso-, and nitrite-groups. Fig. 1 shows ab- 
sorption curves for $\beta$-cyclocitral in hexane and ethanol, showing a blue-shift of the weak long wavelength band $\left(n \rightarrow \pi^{*}\right)$ and a redshift of the strong second absorption band $\left(\pi \rightarrow \pi^{*}\right)$, both being singlet-singlet transitions.

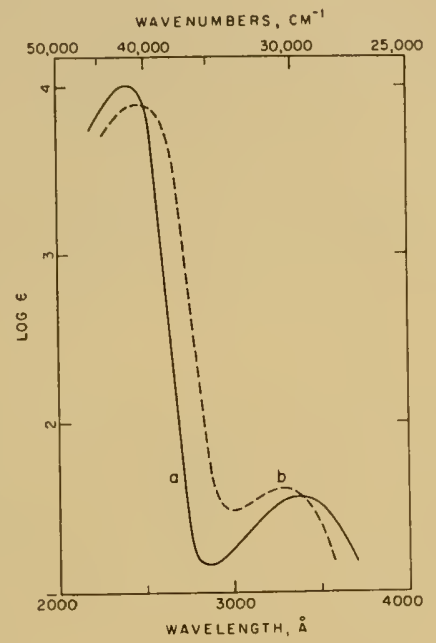

Fig. 1. Absorption spectra of $\beta$-cyclocitral in (a) hexane and

(b) ethanol, after Burawoy (4, ta).

Burawoy did not seem to consider quinones, which also could be included among compounds showing ' $\mathrm{R}$ '-bands. No N-heterocyclic molecules were studied by him. On the other hand, he included the visible absorption band (a doublet $\rightarrow$ doublet transition) of triarylmethyl radical (discussion given below), as well as what may be a singlet $\rightarrow$ triplet ( $\pi$-electron) absorption band in trimethylethylene ( $\lambda$ 2340, $\epsilon=0.06$ ), among the ' $R$ ' bands.

It is instructive to classify the types of "lone-pair" orbitals in polyatomic molecules, and to examine the origin of Burawoy's error. This will be done in the subsequent section.

\section{B. Types of "Lone Pair" Orbitals in Molecules}

In writing classical structures of hetero-molecules the "lone-pair" (doublet-libre) electrons are frequently omitted. Nevertheless, it is these electrons which usually give rise to the lowest singlet excited states of the molecules considered. Moreover, these excited states frequently determine the principal optical and photochemical properties of most hetero-molecules. 
For convenience, the "Ione-pair" electrons may be classified as originating from one of two types of orbitals: n-orbitals (non-bonding), which cannot conjugate with a $\pi$-electron system; and $l$-orbitals ("lone-pair"), which can conjugate with a $\pi$-electron system.

Fig. 2 illustrates several cases of these classifications. In the carbonyl group, Fig. 2 (l) (e.g. formaldehycle, acetone), the $n$-orbital is a $2 p$ orbital on the $\mathrm{O}$ atom, in the plane of the carbonyl group with attached atoms (and is approx. non-bonding), (the second, more tightly bound $n$-orbital is not shown and is probably an (approx.) $s p$-hybrid orbital on the $\mathrm{O}$ atom). On an aza-nitrogen, Fig. 2 (2) (e.g. pyridine), the $n$-orbital is a hybridized (approx.) $s p^{2}$ orbital on the $\mathrm{N}$ atom, in the plane of the molecular ring, as required by

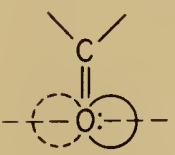

(1)

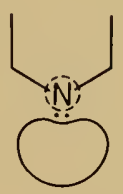

(2)

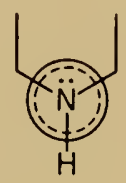

(3)

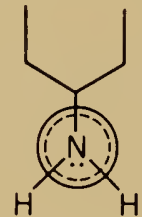

(4)

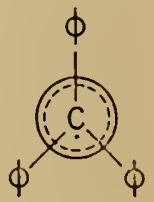

(5)

$\ell_{2 p, c}$

$n_{2 p, 0}$

$n s p^{2}, N$

$\ell_{2 p, N}$

$\ell_{2 p, N}$

$\ell$-orbital (can conjugate)

n-orbital (non-bonding)

Fig. 2. Types of "lone-pair" orbitals in heteroatom molecules.

the ring skeleton binding. Both of these $n$-orbitals are symmetric with respect to the plane of the molecular skeleton at the heteroatom, thus cannot conjugate with the $\pi$-orbital electrons, which are always antisymmetric to this plane (the $\pi$-orbital wavefunction changes sign upon reflection in the skeletal plane). (N.B. Solid contour line designates $(+)$ sign of orbital wavefunction, dashed contour line designates (-) sign of orbital wavefunction.)

The l-orbitals shown in Figs. $2(3), 2(4), 2(5)$ are $2 p \pi$ orbitals on an $\mathrm{N}$ or $\mathrm{C}$ atom, and are antisymmetric to the skeletal plane at the given $\mathrm{N}$ or $\mathrm{C}$ atom (solid contour line outside designates $(+)$ region of orbital wavelunction above skeletal plane; dashed contour inside designates (-) region of orbital wavefunction below skeletal plane) . Fig. 2 (3) represents, e.g., a pyrrole "Ione-pair," which is conjugated with the $2 p \pi$ orbitals on the four carbon atoms of the ring. Fig. 2 (4) represents, e.g., a "Ione-pair" of the aniline molecule. If the amino 
group is coplanar with the ring, the l-orbital is fully conjugated and only $\pi \rightarrow \pi^{*}$ transitions are expected. If the amino group is twisted $90^{\circ}$ about the $\mathrm{C}-\mathrm{N}$ bond, no conjugation of the l-orbital clectrons and the $\pi$-orbital electrons would be possible, because in this molecular conformation the orbitals differ in symmetry. In the $90^{\circ}$ form, an $n \rightarrow \sigma^{*}$ transition (see next section) might be found at higher energies, but an $n \rightarrow \pi^{*}$ transition in aniline would not be expected. At intermediate angles of twist, an intramolecular charge-transfer transition (23) from the "lone-pair" l-orbital to an empty antibonding phenyl $\pi$-orbital could occur: it may be desirable to designate this as $l \rightarrow a_{\pi}$, since the "lone-pair" orbital is not non-bonding in the twisted conformation, nor is $\pi$-antisymmetry preserved for the molecule as a whole. To label such a transition in aniline (or diphenylamine and triphenylamine) as $n \rightarrow \pi^{*}$ would be inaccurate and confusing. It is however worth pointing out that in one sense, viz., the addition of a proton to the hetero-"lone-pair" causes spectral changes in the $l \rightarrow a_{\pi}$ bands of such molecules, which resemble the behavior of bona-fide $n \rightarrow \pi^{*}$ transitions. This is clearly demonstrated in the recent study of absorption bands in triarylarsines by Hochstrasser and Cullen (14). The $l \rightarrow a_{\pi}$ designation seems appropriate, as far as orbital labelling is concerned, for describing such intramolecular charge transfer transitions of high intensity.

The aryl Group V-compounds discussed above have some electronic orbital similarities to the triarylmethyl radicals. Murrell (28) has assigned the long-wavelength absorptions in these molecules as intramolecular charge-transfer transitions, which could again be clesignated as $l \rightarrow a_{\pi}$. Burawoy's interpretation $(4,4 \mathrm{a})$ of the origin of ' $\mathrm{R}$ '-bands was based on the fact that the solvent and substitution effects in triarylmethyl radicals were similar to corresponding effects found in heteroatom molecules. He came to the conclusion that the common ingredient in all these cases was the presence of "radical-like character." Actually, we now can recognize that the common ingredient is an n-orbital or l-orbital "lone-pair" electron, promotion of which shows some similarities with respect to solvation and conjugative substitution effects. However, the polarizations and intensities of $n \rightarrow \pi^{*}$ and $l \rightarrow a_{\pi}$ transitions contrast sharply. The theoretical bases for the empirical classifications will be outlined in a later section.

\section{Interpretation of the Carbonyl Absorption Bands}

A theoretical study, based on the molecular orbital theory, by Mulliken and McMurry in 1941 (27, 22) suggested a quite novel 
explanation of the origin of the characteristic absorption bands associated with carbonyl and thiocarbonyl groups in organic molecules. For an understanding of their interpretation, it is necessary to examine the symmetry properties and spatial distributions of the various molecular orbitals involved.

Fig. 3 shows schematically the optically important orbitals on the formaldehyde, $\mathrm{H}_{2} \mathrm{CO}$, skeleton (solid contour line designates $(+)$ region, dashed contour line (-) region of orbital). The orbitals are arranged in order of decreasing binding energy, left to right. The $\sigma$-orbital and the $\sigma^{*}$-orbital (with a node perpendicular to the C-O axis) are cylindrically symmetrical about the C-O bond, and are shown as made of hybridized C (approx. $s p^{2}$ ) and hybriclized
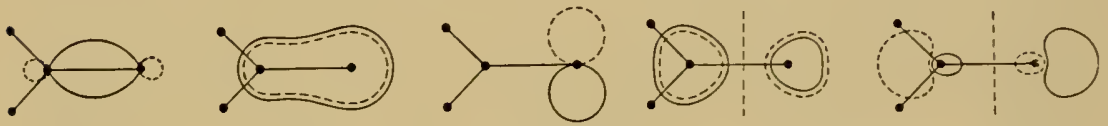

$\sigma$

$\pi$

$n\left(2 p_{0}\right)$

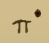

$\sigma$

Fig. 3. Classification of molecular orbitals (schematic contour diagrams, formaldehyde skeleton).

O (probably approx $s p$ ) orbitals. (The hybridization at the O-atom is unknown. If the $\sigma$-orbitals are as shown, the second, more tightlybound lone-pair on the $\mathrm{O}$ atom would also be $s p$; otherwise it would be $2 s$ if non-hybridized. The perturbation produced by the $\mathrm{C}$ atom upon bond formation probably mixes the $2 s$ and the $2 p$ orbital of the $\mathrm{O}$ atom that lies along the $\mathrm{C}-\mathrm{O}$ axis.)

The $\pi$-orbital and $\pi^{*}$-orbital are both antisymmetric with respect to reflection in the molecular plane; in addition, the $\pi^{*}$-orbital has a nodal plane perpendicular to the C-O axis. (Since the $\pi$-orbital has its center of gravity shifted toward the $\mathrm{O}$ atom because of its electronegativity relative to the $\mathrm{C}$ atom, the $\pi^{*}$-orbital, in order to be orthogonal to the former, must have its center of gravity shifted toward the C-atom.) The n-orbital is a non-bonding "lone-pair" $2 p$ orbital on the $\mathrm{O}$ atom. It is symmetrical with respect to reflection in the molecular plane. In the ground state of formaldehyde, the electronic configuration is . . $\sigma^{2} \pi^{2} n^{2}$ (neglecting other filled orbitals).

Mulliken and McMurray $(27,22)$ recognized that formaldehyde has a strong absorption band at about $1650 \mathrm{~A}$ which must correspond 
to the $\pi \rightarrow \pi^{*}$ transition at $1700 \mathrm{~A}$ of ethylene. Consequently, they investigated the possibility that an $n \rightarrow \sigma^{*}$ and $n \rightarrow \pi^{*}$ transition could account for the weaker absorption bands occurring in formaldehyde $(1900 \mathrm{~A}, \epsilon<2000 ; 2900 \mathrm{~A}, \epsilon=18)$. (The orbital promotion designations $\pi \rightarrow \pi^{*}, n \rightarrow \pi^{*}$, etc., were introduced in 1950 by Kasha, 17). Since McMurry was able to calculate approximately the observed intensity of the $1900 \mathrm{~A}$ band of formaldehycle considering an $n \rightarrow \sigma^{*}$ transition, this band was so assigned. (However, Simpson and Barnes (34a) have revised this assignment.) Although a calculation could not be made at the time to establish the nature of the very weak $2900 \mathrm{~A}$ absorption band, it was assigned as an $n \rightarrow \pi^{*}$ transition essentially by the process of elimination of possibilities; however, the assignment agreed with the fact that the unique $n \rightarrow \pi^{*}$ (singletsinglet) transition in formaldehyde should be formally forbidden on symmetry grounds. In a subsequent section the excited state configurations, selection rules, and polarizations will be developed. It is of interest to recall that no direct physical evidence for the assignment of the weak long wavelength band of carbonyl compounds as an $n \rightarrow \pi^{*}$ transition was available at the time the assignment was made.

\section{The $n \rightarrow \pi^{*}$ Transitions in N-Heterocyclics}

The low ionization potential of the lone-pair or $n$-orbital electrons on the $\mathrm{O}$-atom was one of the plausibility arguments adduced by Mulliken and McMurry $(27,22)$ for the assignment of the longest wavelength ultraviolet absorption band of carbonyl compounds, as a transition of essentially $n \rightarrow \pi^{*}$ type. In the case of $\mathrm{N}$-heterocyclic aromatic molecules, it was considered generally that the expected higher ionization potential of lone-pair electrons on the aza-nitrogen would preclude the occurrence of lowest electronic transitions of $n \rightarrow \pi^{*}$ type. There were several spectroscopic studies in the literature which seemed to indicate that in $\mathrm{N}$-heterocyclics the lowest singlet-singlet electronic transitions involved only $\pi \rightarrow \pi^{*}$ promotion. Thus, the spectrum of pyridine as studied by Sponer and Stücklen (37) and later by Sponer and Rush (35), and the spectra of the diazines as studied by Maccoll (20, cf. 32) and by Halverson and Hirt (11) were assumed to fit in with such an interpretation.

However, on the basis of studies of the emission characteristics and solvent effects, in 1950 Kasha (17) proposed that the longest wavelength singlet-singlet absorption bands or absorption shoulders in several $\mathrm{N}$-heterocyclics (especially pyridine, pyrazine, phenazine) 
were of $n \rightarrow \pi^{*}$ type. This suggestion was quickly confirmed in subsequent studies by Halverson and Hirt (12) on the diazines and by Sponer and Rush (36) on the vapor spectra of picolines, and by Stephenson (38) on solution spectra of pyridine and various derivatives. In the last few years many additional researches on $n \rightarrow \pi^{*}$ transitions in $\mathrm{N}$-heterocyclics have been published. Sidman (34) has recently reviewed the general topic of $n \rightarrow \pi^{*}$ transitions, stressing vibrational and electronic symmetry assignments. Goodman and Harrell (9a) have recently given a useful semi-empirical calculation of energy levels for this case.

Before a summary is made of the principal characteristics of $n \rightarrow \pi^{*}$ transitions in N-heterocyclics, some of the features of the molecular orbitals of these molecules will be analyzed.

The $\pi$-molecular orbitals and the (mainly) non-bonding lone-pair or n-orbital of pyricline are shown schematically in Fig. 4. There are three bonding $\pi$-orbitals, and three antibonding $\pi$ or $\pi^{*}$-orbitals. The orbitals are arranged in order of decreasing binding energy, left to right. The lowest orbital has only the characteristic node in the plane of the molecule (solid contour outside means $(+)$ part of the orbital wavefunction above; dashed contour inside means (-) part of the orbital wavefunction below the plane of the molecule). In typical quantum mechanical fashion, with increasing energy of the $\pi$-orbitals, there is an increase in the number of nodes (perpendicular to the molecular plane). The orbitals $\pi_{2}$ and $\pi_{3}$ have the same energy

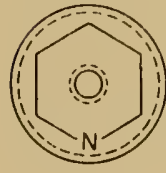

$\pi_{1}$

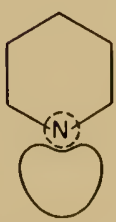

$n\left(s p^{2}, N\right)$

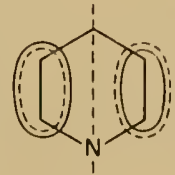

$\pi_{3}$

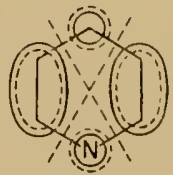

$\pi_{4}^{*}$

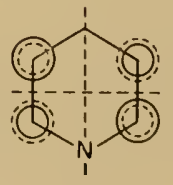

$\pi_{5}^{*}$

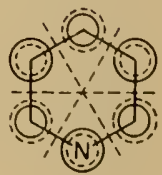

$\Pi_{6}^{*}$

Fig. 1. Schematic contour diagrams of the $\pi$-orbitals and the "-orbital of the pyricline molecule. 
in the benzene molecule, but in pyridine the electronegativity of the $\mathrm{N}$-atom makes $\pi_{2}$ lower in energy than $\pi_{3}$; an analogous result holds for the orbitals $\pi^{*}{ }_{4}$ and $\pi^{*}{ }_{5}$. This $\pi$-orbital order is in accord with the theoretical calculations of McWeeny and Peacock (24). In the diagrans of Fig. \& the orbital distortion due to the electronegativity of the $\mathrm{N}$-atom is not shown.

The $n$-orbital is an approx. $s p^{2}$ hybricl lone-pair orbital on the $\mathrm{N}$ atom, and is symmetric with respect to reflection in the molecular plane.

In the section which follows the symmetry classification of molecular orbitals will be considered. This will be done in order that the full electronic state configurations may be described. From the state symmetries it will be a simple matter to deduce electronic selection rules and polarizations for the electronic transitions. The results will have some important applications to biologically important N-heterocyclics.

\section{Syminetry Classifications of Molecular Orbitals and Electronic States}

\section{A. The Symmetry Requirement}

One of the fundamental quantum mechanical theorems requires that proper wavefunctions for a system should conform to the symmetry operations of the system. It is worthwhile to apply this theorem to classification of the electronic orbitals, configurations, and states of a molecule. Not only will this lead to a concrete picture of the nature of molecular electronic transitions, but the results will be directly useful in our further discussion.

The concept of symmetry is a loose one in ordinary usage. The proper use of molecular symmetry involves the rather abstract subject of Finite Point Groups, the utilization of which assures one that a full closed set of symmetry operations is considered, with which there is associated a complete set of symmetry species suitable for the classification of molecular wavefunctions. In this presentation only the role of symmetry operators will be stressed, circumventing the development of the formal basis of the subject. This will suffice to illustrate the methods used in some simple cases, and to permit some analysis of the main molecules of interest.

\section{B. Classification of Molecules According to Point Groups}

Coordinate axes are chosen for the molecules of interest, as shown 
in Fig. 5. It should be noted that in each case $y z$ is taken to be the molecular plane, and the $x$-axis is taken to be the out-of-plane axis.

It will be noted that the three molecules (a) formaldehyde, (b) pyridine, and (c) pyrimidine have identical symmetry properties as far as their molecular skeletons are concerned. Each of these has the identity operation $E$ ('leave-it-alone'-operator), the two-fold rotation axis $C_{2}$ (rotation by one half-turn about the z-axis), the reflection plane $\sigma_{v}^{\prime}$ (reflection in $\mathrm{xz}$ plane), and the reflection plane $\sigma_{v}^{\prime \prime}$ (reflection in yz plane); both of these are considered to be vertical planes, i.e., containing the $C_{2}$ axis. The unique point group which includes these four (and no other) symmetry operators is labelled the $C_{2 v}$ Point Group (Schoenflies notation).

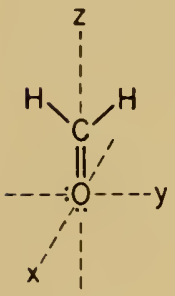

a

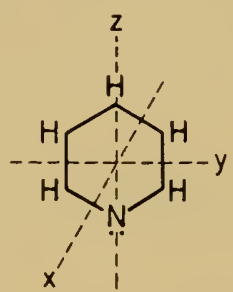

b

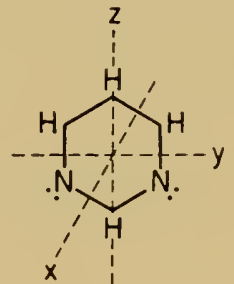

C

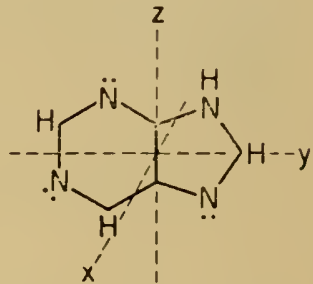

d

Fig. 5. Coordinate axes for the (a) formaldehyde, (b) pyridine,

(c) pyrimidine, and the (d) purine molecules.

The purine molecule, Fig. 5 (d), has a lower symmetry than the previous three molecules. It possesses the identity operation $E$ (included in all point groups by definition), which may be considered to be synonymous with a one-fold axis $C_{1}$ (rotation by a full turn about the $x$-axis). The only other "covering" symmetry operation (which brings the molecular skeleton into itself) is a reflection plane containing the molecular plane, $\sigma_{h}$ (reflection in $y z$ plane: called a horizontal plane because it is perpendicular to the highest-fold axis - $\left.C_{1}\right)$.

\section{Classification of Orbitals According to Symmetry Species}

All molecular orbitals which correspond to proper wavefunctions must, under the symmetry operators of the appropriate point group, satisfy a simple symmetry eigenvalue equation. This is illustrated in Fig. 6 for the $\pi$-orbital and the highest $n$-orbital of formaldehyde $\left(C_{2 v}\right.$ point group). The general equation is given at the top of 


$$
S \psi=S \psi
$$

$$
\begin{aligned}
E(\pi) & =1(\pi) & E(n) & =1(n) \\
C_{2}(\pi) & =-1(\pi) & C_{2}(n) & =-1(n) \\
\sigma_{v}^{\prime}(\pi) & =1(\pi) & \sigma_{v}^{\prime}(n) & =-1(n) \\
c_{v}^{\prime \prime}(\pi) & =-1(\pi) & \sigma_{v}^{\prime \prime}(n) & =1(n) \\
\therefore \pi & \sim b_{1} & \therefore n & \sim b_{2}
\end{aligned}
$$

Fig. 6. Eigenvalue equations for symmetry operators of the $C_{2 v}$ point group acting on the $\pi$-orbital and highest $n$-orbital of the formaldehyle molecule.

Fig. 6 and states that when the operator $S$ acts on the wave function $\psi$, the result should be uniquely a product of a constant $s$ and the wavefunction $\psi$. Applying the four operators $E, C_{2}, \sigma_{v}{ }^{\prime}, \sigma_{v}{ }^{\prime \prime}$ to the $\pi$-orbital of formaldehyde (Figs. 3 and 5), it is evident that the values of the constants $s$ are in turn $+1,-1,+1,-1$. In other words, the $\pi$-orbital is symmetric under the operator $E$ and $\sigma_{v}{ }^{\prime}$, but antisymmetric under $C_{2}$ and $\sigma_{v}^{\prime \prime}$. A different set of eigenvalues (or constants) $s$ is obtained for the same set of four operators applied to the $n$-orbital of formaldehyde (Figs. 3 and 5, where the $2 p_{\mathrm{y}}$-lone pair is taken as the highest $n$-orbital) .

Comparing this set of eigenvalues $s$ with the sets (horizontal rows) corresponding to the symmetry species $A_{1}, A_{2}, B_{1}, B_{2}$ of the $C_{2 v}$ Character Table (Fig. 7 , left) allows a unique symmetry classification. Thus, the $\pi$-orbital of formaldehyde is said to "transform as the symmetry species $B_{1}$ " or to "belong to the representation $B_{1}$." The convention is used, that for orbitals, lower case Schoenflies species symbols are used, in this case $b_{1}$ (Fig. 6, bottom); while the upper case Schoen-

\begin{tabular}{c|c|cccc}
$T$ & $C_{2 v}$ & $E$ & $C_{2}$ & $\sigma_{v}^{\prime}(x z)$ & $\sigma_{v}^{\prime \prime}(y z)$ \\
\hline$z$ & $A_{1}$ & 1 & 1 & 1 & 1 \\
& $A_{2}$ & 1 & 1 & -1 & -1 \\
$x$ & $B_{1}$ & 1 & -1 & 1 & -1 \\
$y$ & $B_{2}$ & 1 & -1 & -1 & 1
\end{tabular}

\begin{tabular}{c|c|cc}
$T$ & $C_{1 h}$ & $E$ & $\sigma_{h}(y z)$ \\
\hline$y, z$ & $A^{\prime}$ & 1 & 1 \\
$x$ & $A^{\prime \prime}$ & 1 & -1
\end{tabular}

Fig. 7. Character tables for the $C_{2 v}$ and $C_{1 n}$ point groups, with transformation properties of translations. 
flies symbol is reserved for molecular states (see below). Similarly, the highest n-orbital of formaldehyde is said to "transform as symmetry species $b_{2 .} . "$ Both of these classifications depend on the axis choice as given in Figs. 5 and 7.

Using the $C_{2,}$, character table of Fig. 7, together with the coordinate choices shown in Fig. 5, the reader should be able to classify all of the molecular orbitals shown in Fig. 3 for the formaldehycle molecule, and Fig. 4 for the pyridine molecule. The results are tabulated in Fig. 8.

Formaldehyde $\left(C_{2 v}\right)$ Pyridine $\left(C_{2 v}\right)$ Pyrimidine $\left(C_{2 v}\right)$ Purine $\left(C_{1 n}\right)$
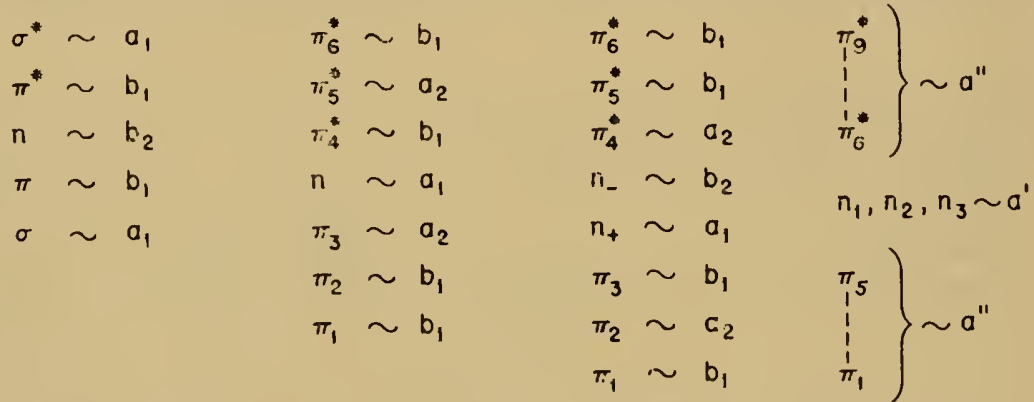

Fig. 8. Symmetry species classification of molecular orbitals

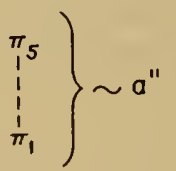

(cf. Figs. 3, 4, 5, 7, and 9).

For pyrimidine, and the other cliazines, a unique problem appears in relation to the symmetry classification of the $n$-orbitals. Referring to the coorclinate axis choice of Fig. 5, it is evident that neither lonepair orbital $n_{a}$ nor $n_{b}$ for pyrimidine (Fig. 9) has proper symmetry properties by itself. For example, if $n_{a}$ is subjected to the $C_{2}$ or $\sigma_{v}^{\prime}(x z)$ operation, it fails to go into $n_{a}$ or $(-) n_{a}$; instead it goes into $n_{b}$ under these operations, and vice versa. Thus, neither the orbital $n_{a}$ nor $n_{b}$ would satisfy the symmetry eigenvalue equation of Fig. 6 (top). However, if one consiclers the linear combinations of $n_{a}$ and $n_{b}$ as shown in the lower part of Fig. 9, then it is found that the resultant orbitals $n^{+}$and $n^{-}$do transform either symmetrically or antisymmetrically under all four operations of the $\mathrm{C}_{2 v}$ point group, to which pyrinidine belongs. These classifications are entered into the third columm of Fig. 8. The interaction between the orbitals $n_{a}$ and $n_{b}$ is exaggerated somewhat in the schematic drawing of Fig. 9. However, the interaction is physically significant (26) and will be discussed further, below. 
The orbital order for the $\pi$-orbitals of pyrimidine is not the same as that for pyridine, in so lar as the nodal planes of the $\pi_{2}$ and $\pi_{3}$ and also the $\pi_{f}{ }^{*}$ and $\pi_{-}^{*}$ pairs are concerned; this explains the different symmetry classifications of these pairs of orbitals for the two molecules, as recorded in Fig. 8 (in pyrimidine, e.g., the $\pi$-orbital with a nodal plane containing the $z$-axis is lower in energy than the $\pi$-orbital with a nodal plane perpendicular to the $z$-axis, of. $\pi_{2}$ and $\pi_{3}$ of Fig. 4). The orbital order for the pyrinicline $\pi$-orbitals was taken from the work of McWeeny and Peacock. In both pyridine

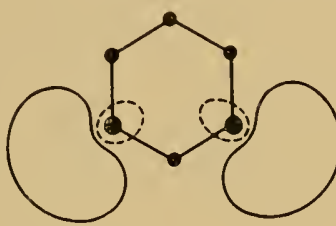

$n_{a}$

$n_{b}$
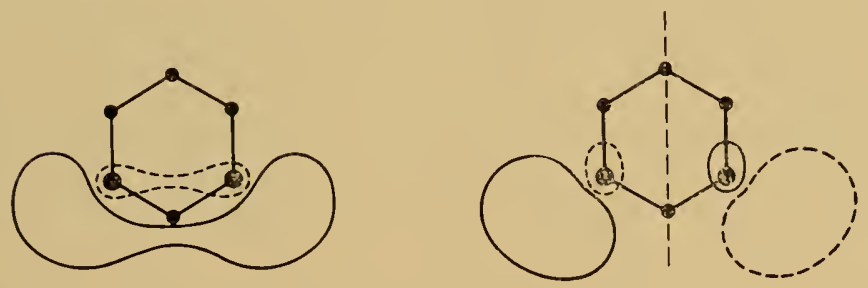

$$
n_{+}=\frac{1}{\sqrt{2}}\left(n_{a}+n_{b}\right)
$$

$$
n_{-}=\frac{1}{\sqrt{2}}\left(n_{a}-n_{b}\right)
$$

Fig. 9. Schematic contonr diagrams of the $n$-orbitals of pyrimidine.

and pyrimidine that orbital lies lowest, of otherwise equivalent pairs, which results in the greatest electron density on the more electronegative $\mathrm{N}$-atoms versus $\mathrm{C}$-atoms.

For purine, with only two symmetry operations, it is immediately evident that the $\pi$-orbitals are all antisymmetric with respect to the $\sigma_{h}$ reflection plane, and therefore all $\pi$-orbitals of purine transform as $a^{\prime \prime}$ species. On the other hand, all $n$-orbitals of purine are symmetric with respect to the reflection, therefore are classified as $a^{\prime}$. There are nine ring atoms with $2 p \pi$ atomic orbitals which build up the molecular $\pi$-orbitals upon molecular formation: there are consequently 9 independent molecular $\pi$-orbitals for the purine molecule. In the next section the electronic configurations and states of the 
molecules under discussion will be described. Not all available orbitals will be populated in the ground state of a molecule. For example, in the purine molecule ten $\pi$-electrons are available, so that in the ground state the lowest $5 \pi$-orbitals will be filled, with two electrons per orbital.

One additional set of symmetry classifications can be derived by the use of the character tables of Fig. 7. It is important to be able to deduce how a vector, or arrow, pointing along each of the coordinate axes, transforms in both the $C_{2 v}$ and the $C_{1 h}$ point groups. These vectors can be considered to be translations $T_{y}, T_{y}, T_{z}$, (or displacements) along the coordinate axes. The symmetry species classification is straightforward and is given in the left column of the two tables of Fig. 7. The physical importance of these symmetry classifications lies in the fact that the electric dipole moment incluced in a molecule by a light wave will have identical symmetry propertics to the translation vectors.

Furthermore, it is of interest to classify the transformation properties of the magnetic dipole moment induced in a molecule by a light wave. It turns out that these components transform analogously to the rotations $R_{s}, R_{y}, R_{z}$ about the coordinate axes.

The above coordinate transformations are important in deducing the intensity of electronic transitions (selection rules) and the optical rotatory strength of electronic transitions, respectively, and will be discussed in the following section.

The molecular orbitals of Fig. 3 for formaldchyde and Fig. 4 for pyridine were presented in order to illustrate the use of symmetry propertics of electronic distribution functions (orbital wavefunctions). In addition, the contour diagrams for the molecular orbitals serve to give a more explicit idea of the nature of the process of electronic excitation, once the molecular state electronic configurations are describel. However, the methods by which these orbitals are derived, and the mathematical form of the wavefunctions, are omitted from this presentation, partly because these are more complicated matters which require much more involved analysis.

\section{1). Electronic Configuration, State, and Transitions}

The order of the molecular orbitals, the number of available electrons, and the Pauli principle may next be used to designate the electronic configuration of a molecule in any desired electronic state. In the molecules under consideration, all of the molecular orbitals are single or of mique energy ("non-degenerate"), and therefore can be occupied by only two electrons with opposed spins. 
For formaldehyde, in which only the C-O $\sigma$-orbitals, the $\pi$-orbitals, and only the highest n-orbital are considered as being important in presently observable ultraviolet electronic transitions, the lowest four electronic state configurations are given in the top portion of Fig. 10. Only singlet states are considered here, as indicated by the superscript prefix on the general state symbol $\mathrm{r}$. The molecular state symmetry would in general replace $r$, when established. The three different

STATE CONFIGURATION

SYMMETRY

SELECTION RULE, POLARIZATION

Formaldehyde $\left(\mathrm{C}_{2 v}\right)$
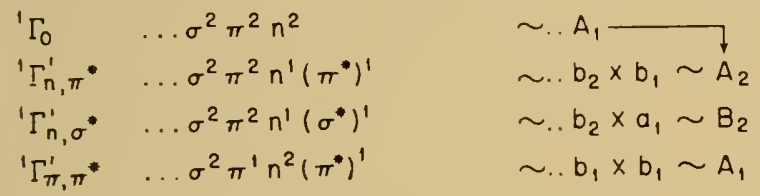

forbidden allowed, $y$ allowed, $z$

\section{Pyridine $\left(C_{2 v}\right)$}

$\begin{array}{llll}{ }^{1} \Gamma_{0} & \ldots \pi_{1}^{2} \pi_{2}^{2} \pi_{3}^{2} n^{2} & \sim \ldots A_{1}-7 & \\ { }^{\prime} \Gamma_{n, \pi^{*}}^{\prime} & \ldots \pi_{1}^{2} \pi_{2}^{2} \pi_{3}^{2} n^{\prime}\left(\pi_{4}^{*}\right)^{\prime} & \sim \ldots a_{1} \times b_{1} \sim B_{1} & \text { allowed, } x \\ { }^{\prime} \Gamma_{n, \pi^{*}}^{\prime \prime} & \ldots \pi_{1}^{2} \pi_{2}^{2} \pi_{3}^{2} n^{\prime}\left(\pi_{5}^{*}\right)^{\prime} & \sim \ldots a_{1} \times a_{2} \sim A_{2} & \text { forbidden } \\ { }^{\prime} \Gamma_{\pi, \pi^{*}}^{\prime} & \ldots \pi_{1}^{2} \pi_{2}^{2} \pi_{3}^{1} n^{2}\left(\pi_{4}^{*}\right)^{\prime} & \sim \ldots a_{2} \times b_{1} \sim B_{2} & \text { allowed, y } \\ { }^{\prime} \Gamma^{\prime \prime} \pi_{1} \pi^{*} & \ldots \pi_{1}^{2} \pi_{2}^{2} \pi_{3}^{1} n^{2}\left(\pi_{5}^{*}\right)^{\prime} & \sim \ldots a_{2} \times a_{2} \sim A_{1} & \text { allowed, } z\end{array}$

\section{Purine $\left(C_{1 h}\right)$}

$$
\begin{aligned}
& { }^{1} \Gamma_{0} \quad \ldots \pi_{1}^{2} \ldots \pi_{5}^{2} n_{1}^{2} n_{2}^{2} n_{3}^{2} \\
& { }^{\prime} \Gamma_{n, \pi^{*}}^{\prime} \quad \ldots \pi_{1}^{2} \ldots \pi_{5}^{2} n_{1}^{2} n_{2}^{2} n_{3}^{1}\left(\pi_{6}^{*}\right)^{\prime} \sim \ldots a^{\prime} \times a^{\prime \prime} \sim A^{\prime \prime} \\
& { }^{\prime} \Gamma_{\pi, \pi^{*}}^{\prime} \ldots \pi_{1}^{2} \ldots \pi_{5}^{1} n_{1}^{2} n_{2}^{2} n_{3}^{2}\left(\pi_{6}^{*}\right)^{\prime} \sim \ldots a^{\prime \prime} \times a^{\prime \prime} \sim A^{\prime}
\end{aligned}
$$

Fig. 10. State symmetries and electronic dipole transition selection rules for several heteromolecules.

types of orbital promotion, $n \rightarrow \pi^{*}, n \rightarrow \sigma^{*}$, and $\pi \rightarrow \pi^{*}$ are indicated by corresponding electronic configurations as shown in the second column of Fig. 10. The subscript suffix on the state symbol is used as a convenient index of the orbital promotion type. The derivation of the overall state symmetry will be given below.

For pyridine, in which only the six $\pi$-orbitals and the $n$-orbital are involved in near ultraviolet spectra, two different low-energy $n \rightarrow \pi *$ and $\pi \rightarrow \pi^{*}$ promotions are considered, each leading to different state symmetries, as will be described below.

For purine, with its lower symmetry, all $n \rightarrow \pi^{*}$ promotions lead to excited configurations and states of the same symmetry, and all 
$\pi \rightarrow \pi^{*}$ promotions lead to excited states of the same symmetry, but differing significantly from the former.

The state symmetry is a compound symmetry which is a product of the orbital symmetries, the latter taken in the product as many times as the orbital is used, i.e., as many times as there are electrons. Group theory offers a simple and powerful tool for evaluating compound symmetry in the Direct Product. The direct product of, e.g., $A_{2} \times A_{2}$ in $C_{2 v}$ is obtained (Fig. 7) by taking the separate products of the eigenvalues or characters under each symmetry operation for the symmetry species involved, and comparing the new set of characters with the available sets in the table. Thus, $A_{2} \times A_{2}$ leads to the set of products (I) (I), (I) (I), (-I) (-I), (-1) (-1) which yield the set of characters $1,1,1,1$; thus $A_{2} \times A_{2}=A_{1}$. It is evident that here the square of the characters of any symmetry species leads to the characters of the $A_{1}$, or totally symmetric species (symmetric under all symmetry operations.) Thus, all closed shell electronic configurations will be totally symmetric $\left(A_{1}\right.$ in $C_{2 v} ; A^{\prime}$ in $\left.C_{1 h}\right)$.

The symmetry classification of an excited state thus can be readily obtained. In formaldehyde, the $n \rightarrow \pi^{*}$ promotion results in the $n$-orbital and the $\pi^{*}$-orbital being singly occupied. The respective orbital symmetries are (cf. Fig. 10) $b_{2}$ and $b_{1}$; thus $b_{2} \times b_{1}=A_{2}$ (where the upper case Schoenflies symbol is used for state symmetry classification, and lower case for orbital symmetry), and the remaining filled orbitals all have $a_{1}$ symmetry, which has no effect on the state symmetry. In this fashion, all of the state symmetries recorded in column 3 of Fig. 10, for the corresponding electronic states of column 1, may be clecluced by consulting Fig. 8 and the electronic configurations of column 2 of Fig. 10, and then obtaining the clirect product of the corresponding symmetry species for each state.

\section{Probability of Electronic Transitions; \\ Optical Rotatory Strength}

\section{A. Transition Moment Integral}

In order to evaluate the probability of an electronic transition in a molecule, and the polarization of the transition with respect to the molecular axes, the appropriate quantum mechanical integral, the "transition moment integral," will be discussed briefly (7). This integral allows one to gain a physical insight into the nature of the light absorption process, and is given in the top part of Fig. 11. The probability, $P$, of an electric dipole transition is proportional to 
the square of a volume integral, orer the entire space occupied by the molecular electronic distribution. The integrand consists of three parts: $\Psi_{i}$, the total wavefunction of the ground state of the molecule; $E_{x \cdot y \cdot:}$ which is an abbreviation for the sum of the electric dipole operator components in the $x, y$, and $z$, directions; and $\Psi_{E}$, the total wavefunction of an excited state of the molecule. The total wavefunctions for a state of a molecule are taken as those products, of the oneelectron orbital wavefunctions, which correspond to the electronic configuration for the state of the molecule; thus, the symmetry species for a state is readily determined from the symmetry species for the orbitals involved, as shown in the previous section. Physically, one can visualize the total electronic distribution of a molecule as a superposition of each of the one-electron molecular orbital distribu-

$P \sim\left(\int_{-\infty}^{\infty} \Psi_{G}^{*} E_{x, y, z} \Psi_{E} d v\right)^{2}$

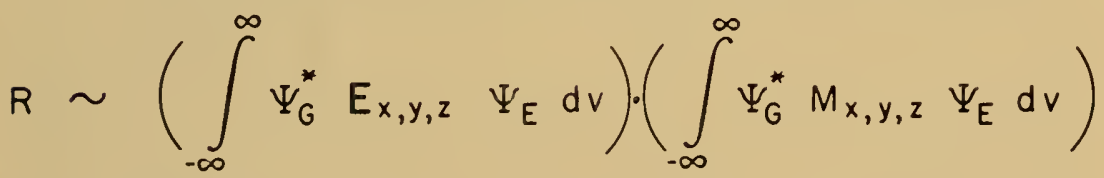

Fig. 11. Quantum mechanical expressions relating to probability, $P$, of an electric dipole transition, and optical rotatory strength, $R$, due to an electronic transition.

tions which make up the configuration. As stated at the end of section III C, the symmetry properties of the components of the electric dipole operator are identical with the symmetry properties of the vectors representing coordinate displacements, or translations. The electric vector of the impinging light wave may be directed along the $x, y$, or $z$ axis of an oriented molecule, and may induce in it a transitory clectric dipole moment, which may lead to an excitation or electronic transition. Whether it does or does not depends on the non-zero or zero value, respectively, of the square of the transition moment integral, and the frequency of the light wave. If the integrand is antisymmetric with respect to any symmetry operation, then integration from minus infunity to plus infinity over the molecular coordinates will give a zero value of the integral. If the integrand is symmetric with respect to all symmetry operations, the integral over all space of the molecule will not vanish, and the transition will be 
allowed (for electric dipole radiation). Thus, to establish whether an electronic transition is allowed from one state of a molecule to another, it is sufficient to show that the complete integrand belongs to (or contains) the totally symmetric species. Thus, by taking the direct product of the species of the ground state symmetry, the coordinate translation symmetries, and the excited state symmetry, one can establish the allowedness of an electronic transition. Furthermore, if only, e.g., a $y$ translation vector causes the integrand to transform totally symmetrically, then only light with its electric vector oriented along the $y$-axis of the molecule (for the chosen coordinate scheme), will be absorbed.

As an example, consider the $n \rightarrow \sigma^{*}$ promotion in formaldehyde, Fig. 10. The ground state total wavefunction has symmetry $A_{1}$. The coordinate vectors $x, y, z$ transform as $B_{1}, B_{2}, A_{1}$ respectively (cf. Fig. 7), for the axis choice of Fig. 5. The excited state corresponding to $n \rightarrow \sigma^{*}$ promotion transforms as $B_{2}$ (third row of Fig. 10). Thus the direct products are:

for the $x$-polarized transition $A_{1} \times B_{1} \times B_{2}=A_{2}$ (forbidden);

for the $y$-polarized transition $A_{1} \times B_{2} \times B_{2}=A_{1}$ (allowed);

for the $z$-polarized transition $A_{1} \times A_{1} \times B_{2}=B_{2}$ (forbidden).

Thus, only if the electric vector of the impinging light wave is polarized along the $y$-axis of formaldehyde (i.e., according to Fig. 5, along a line perpendicular to the $\mathrm{C}-\mathrm{O}$ axis and in the plane of the $\mathrm{H}_{2} \mathrm{CO}$ skeleton), will light be absorbed, even if of the right frequency or wavelength. Column 4 of Fig. 10 tabulates the selection rules and polarization directions for several molecules.

It is especially to be noted that lor singlet-singlet transitions, the transitions of $n \rightarrow \pi^{*}$ type are always polarized out-of-plane ( $x$-ixis, Fig. 5), if not forbidden. Conversely, the transitions of $\pi \rightarrow \pi^{*}$ type are always polarized in-plane ( $y$-axis or z-axis, Fig. 5), if not forbidden. This characteristic polarization not only permits a qualitative distinction to be made between these transitions, but also leads to differences in physical interactions between molecules, as will be discussed later.

\section{B. Rotatory Strength}

The optical rotatory strength (contribution to optical rotatory dispersion) of an electronic transition is given by a scalar product of two different vector integrals (7), as shown in the lower part of Fig. 11. The first integral is the transition moment integral which 
was discussed in the preceding section, and whose square is related to the intensity of an electronic transition.

The second integral involves the three components of the magnetic dipole operator, and again the total wavefunctions for the ground and excited states. As stated in section III-C, the magnetic dipole opcrator has the symmetry properties of a rotation, $R_{x}, R_{y}$, or $R_{\tilde{r}}$. These have not been tabulated in the character table, but are given in standard sources (Appendix, 7).

The translations $T_{x}, T_{y}$, and $T_{z}$ and the rotations $R_{x}, R_{y}$, and $R_{z}$ transform in a complementary manner if there is a plane of symmetry or a center of inversion in the molecule. In this case, since the rotatory strength is a scalar product of the two (vector) integrals, and the two integrals involving a given excited state wavefunction will not involve the same coordinate component of the electric dipole and the magnetic dipole operator simultaneously, the rotatory strength would be zero. This is the quantum mechanical basis for the well-known rule that to show optical activity, a molecule must possess neither a plane of symmetry nor a center of symmetry (inversion).

On the quantitative side, if a molecule does possess optical rotatory power, the fact that two different integrals are involved, only one of which is related to the intensity of a transition, leacls to the possibility that weak bands may in principle contribute more strongly to optical rotatory porver at a given wavelength, than strong bands contribute. In particular, $n \rightarrow \pi^{*}$ bands, which as we shall see are inherently weak even if formally allowed, usually contribute strongly to optical rotatory power. This confirms an empirical observation which occurs frequently in the early literature on the importance of weak bands in optical rotatory dispersion, although the spectral assignment of the bands as $n \rightarrow \pi^{*}$ transitions in heteromolecules in general is more recent.

\section{Characteristics of $n \rightarrow \pi^{*}$ Transitions}

After the Mulliken-McMurry assignment $(27,22)$ of the carbonyl absorption band was published, no immediate development of the general topic ensucl, partly because the abstract treatment was not at once related to direct experimental proof, even though some of this was already in the literature: and partly because in molecules with higher ionization potentials for lone-pair electrons on heteroatoms, the possibility of long wavelength $n \rightarrow \pi^{*}$ transitions was not immediately apparent.

However, a lone-pair on a heteroatom carries immediate chemical 
implications. Exploring these, a set of empirical criteria for characterizing $n \rightarrow \pi^{*}$ transitions was developed by Kasha (17), which correlated most of the 'R'-band cases of Burawoy (4, 4a) with the Mulliken-McMurry interpretation $(27,22)$. These characterizing criteria were later extended further by Sidman (34).

The characteristics of $n \rightarrow \pi^{*}$ transitions may be usefully enumerated as follows:

\section{A. Absence in Hydrocarbon Analogs}

An obvious useful check on the identity of a band as an $n \rightarrow \pi^{*}$ band may sometimes be made by a comparison of the spectrum of a hydrocarbon analog of a molecule: formaldehyde may be compared with ethylene: pyridine or the diazines may be compared with benzene; phthalazine and quinoxaline may be compared with naphthalene; ctc. To observe a long wavelength $n \rightarrow \pi^{*}$ band a necessary but not sufficient condition is that an electron lone-pair should occur on a heteroatom which is $\pi$-conjugated to other atoms.

\section{B. Disappearance in Acid Media}

If only one lone-pair is available in the molecule, then in the cation or protonated molecule the $n \rightarrow \pi^{*}$ bands may shift far out of range of study and effectively disappear. However, the $\pi \rightarrow \pi^{*}$ transitions also may shift considerably, obscuring the region of observation. A variation on the use of acidic solvents is to study the spectra of the methhalide salts (12). If two or more heteroatoms are present in the molecule, effectively only one lone-pair is bound by a proton in general, so that although considerable changes in the spectrum may result-sometimes clearly showing the removal of an $n \rightarrow \pi^{*}$ band —other $n \rightarrow \pi^{*}$ transitions may still be present.

\section{Blue Shift in Polar Solvents}

In comparing bands of different orbital promotion type in hydroxylic solvents like ethanol and water, versus hydrocarbon solvents, a relatively large blue shilt (to higher liequency) is observed for $n \rightarrow \pi^{*}$ transitions, whereas a relatively small $\pi \rightarrow \pi^{*}$ red-shift (to lower frequencies) is olsserved. McComnell (21) has tabulated a large collection of such shifts, demonstrating their utility in classification of transitions by orbital type. Brealey and Kasha (3) demonstrated that a major cause of the $n \rightarrow \pi^{*}$ blue shift is the formation of hydrogen-bonded solvates at the heteroatom lone-pair of the molecule. Fig. 12 illustrates the sensitivity of the phenomenon for the case of pyridazine in hexane-chanol solvents. 


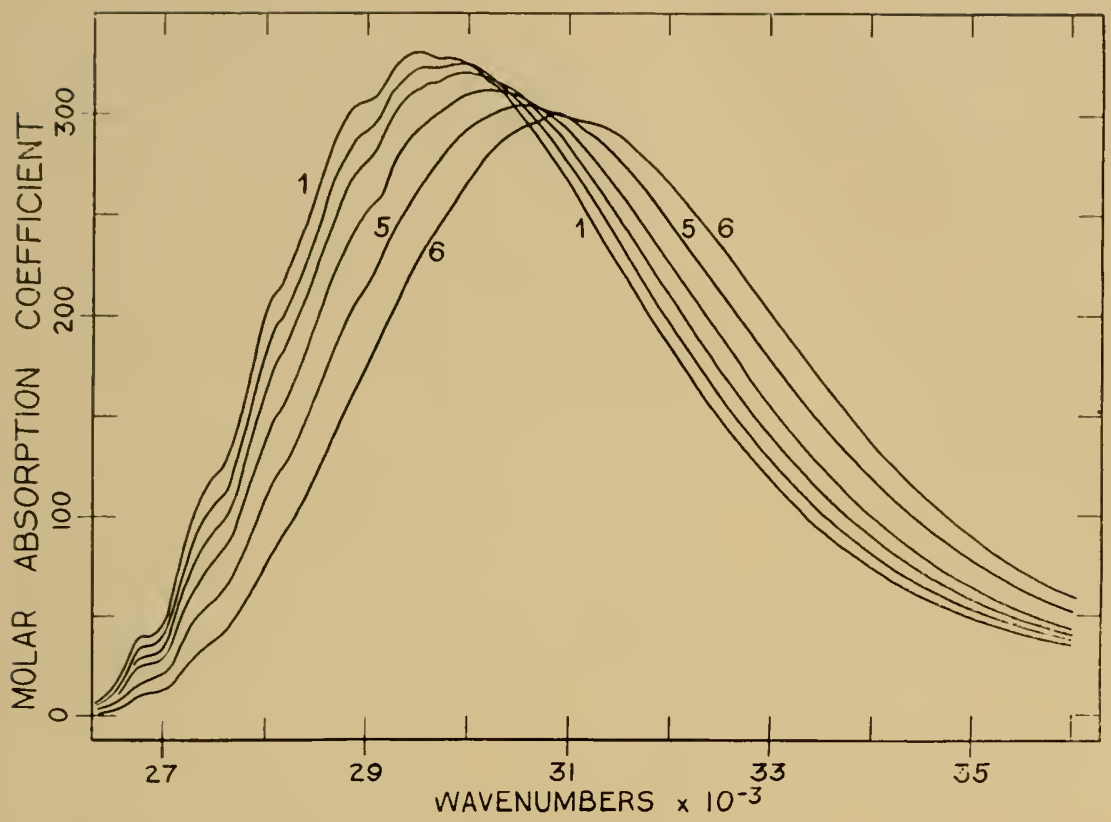

Fig. 12. Blue shift of first $n \rightarrow \pi^{*}$ singlet-singlet absorption band of pyridazine after Brealey and Kasha (3). Solutions in hexane, with added ethanol, from curve 1 , 0 percent ethanol to curve 6. 3.2 percent ethanol by volume.

\section{Conjugative-Substituent Blue-Shift}

As mentioned earlier, Burawoy (4, 4a) had used the effect of elertron-donating substituents such as $-\mathrm{OH},-\mathrm{SH},-\mathrm{NH}_{2},-\mathrm{N} R_{2}$, to classify electronic band types in molecules. A theoretical treatment of these effects was supplied by Nagakura (29), for the case of replacement of a methyl group of acetone by the substituents - $\mathrm{Cl}$, $-\mathrm{OC}_{2} \mathrm{H}_{5}$, $-\mathrm{N}\left(\mathrm{CH}_{3}\right)_{2}$, using an LCAO-Molecular Orbital calculation. In the $n \rightarrow \pi^{*}$ transition in carbonyl compounds, electronic charge is, in effect, transferred from the oxygen to the carbon of the carbonyl group. Nagakura (29) showed that substituent groups which donate charge to the carbonyl carbon raise the energy of the antibonding $\pi$-orbital, causing a blue-shift of the $n \rightarrow \pi^{*}$ transition in carbonyl compounds, proportional to the electron-donating ability of the substituent group. Goodman and Shull (10a) made a general prediction of the blue-shift effect of (purely conjugative) electrondonating substituents on $n \rightarrow \pi^{*}$ transitions. However, their discussion considers the effect on corresponding state energies, and occasionally predicts a zero-shift effect (cf. the 5-methylpyrimidine case, Goodman and Harrell, 9a) . 


\section{E. Low Absorption Intensity}

It is observed empirically that allowed $\pi \rightarrow \pi^{*}$ bands in polyatomic molecules may have oscillator strengths from 0.1 to approx. 1 , with molar absorption coefficients frequently in the range 10,000 to 100,000 or more.

On the other hand, $n \rightarrow \pi^{*}$ transitions, even if symmetry allowed, have oscillator strengths of the order of magnitude of $10^{-2}$, with molar absorption coefficients in the range 300 to 2000. A study of the spatial clistributions for $\pi$-orbitals and $n$-orbitals (Fig. 3, 4) will inclicate one cause of this low intensity: the relatively good orbital overlap for $\pi \rightarrow \pi^{*}$ promotion and the relatively poor overlap for $n \rightarrow \pi^{*}$ promotion. Orgel (30) has examined other sources of low intensity in $n \rightarrow \pi^{*}$ transitions in addition to spatial overlap, including the effect of charge redistribution upon $n \rightarrow \pi^{*}$ promotion, and the climinution of intensity arising from hybrid lone-pair orbitals, in which only one of the hybrid components (s-orbitals in the calse of $s p^{2}$ hybrid orbitals on the $\mathrm{N}$ of $\mathrm{N}$-heterocyclics) contributes to the intensity of an $n \rightarrow \pi^{*}$ transition.

\section{F. Unique Polarization}

In section IV-A the polarization of $n \rightarrow \pi^{*}$ singlet-singlet transitions was shown to be out-of-plane for the $\mathrm{N}$-heterocyclic molecules, if allowed (cf. Fig. 10), and the $\pi \rightarrow \pi^{*}$ transitions in-plane, by the use of group theory. For singlet-triplet transitions, these polarizations are reversed, as shown by Clementi and Kasha (5) .

Physically it is easy to see that the singlet-singlet $n \rightarrow \pi^{*}$ transitions should be out-of-plane polarized. The n-orbitals of the molecules of Figs. 3, 4, and 5 are symmetric to the molecular plane. The $\pi$-orbitals are antisymmetric to the molecular plane. An overlap integral between the $n$-orbitals and the $\pi$-orbitals over the whole space of the molecule will obviously be equal to zero, since the negative part of the overlap will cancel the positive part. Howerer, an electric dipole may be antisymmetric to the molecular plane: when a dipole operator acts on (multiples) an orbital distribution which is antisymmetric to the same plane, a symmetric clistribution function is obtained. Thus, an integral such as the transition moment integral (upper part of Fig. 11) will be non-vanishing if the electric vector of the light is polarized out-of-plane. The recent results Innes, et al. (14a), on rotational analysis of the furst ultraviolet absorption band of the diazines, confirm the polarization decluctions. 


\section{G. Low Transition Energy}

In many different types of heteromolecules it is observed that frequently the longest wavelength singlet-singlet transition is of $n \rightarrow \pi^{*}$ type. In the case of simple aldehydes and ketones the lowest ionization potential is for an n-orbital on the oxygen atom; consequently, longest wavelength $n \rightarrow \pi^{*}$ transitions might be expected. However, in the case of $\mathrm{N}$-heterocyclics the lowest ionization potential is almost certainly that for a $\pi$-electron, the $n$-orbital having an ionization potential which may be $2-3 \mathrm{ev}$. higher $(15,6)$. Nevertheless, as will be seen in the next section, the $n \rightarrow \pi^{*}$ transition is almost always lower in energy than the $\pi \rightarrow \pi^{*}$ transition in N-heterocyclics, although frequently very close to the lowest $\pi \rightarrow \pi^{*}$ transition. Probably the charge redistribution examined by Orgel (30) for $n \rightarrow \pi^{*}$ promotion greatly alters the relative orbital energies, reversing the expectation derived from the ionization potential concerning the relative excitation energy.

\section{H. Fluorescence Quenching}

The clue to the presence of lowest singlet-singlet $n \rightarrow \pi^{*}$ transitions in many diverse molecules was the absence of fluorescence iu such molecules (17). The interpretation of the intramolecular quenching depends especially on the greatly enhanced singlet-triplet mixing originally proposed on an empirical basis (17), but establisherl theoretically by Clementi and Kasha (5); the calculations of Sidman confirm this iclea (33a).

The detailed interpretation of the intramolecular quenching of lluorescence as a consequence of the existence of $n, \pi^{*}$ lowest excited singlet states has been given recently (15).

\section{Characteristic Vibrational Coupling}

One of the observations made on $n \rightarrow \pi^{*}$ transitions is the extremely characteristic vibrational-electronic coupling which occurs (15), permitting one to identify the heterogroup from which the $n \rightarrow \pi^{*}$ transition originates in many cases. Frequently, the appearance of a particular molecular vibration coupled with the electronic excitation is simply the result of the occurrence of a node between the atoms of a characteristic functional group in the excited state, leading to excitation of one to several quanta of a stretching frequency with the electronic transition.

However, in some cases, as in pyridine and the diazines, a particu- 
lar ring angular distortion frequency is excited, giving a very characteristic vibrational pattern, in the $n \rightarrow \pi^{*}$ transitions. This has been interpreted (10) as a consequence of promotion of a hybridized $n$-orbital electron, leading to changes in the ring bonding angles during excitation, since the orbitals forming the ring are also hybriclized. Mason (26) has also noted this point.

\section{J. Radical-Like Excited States}

The promotion of an (essentially atomic) n-orbital electron to a $\pi$-antibonding-orbital leaves one electron in the $n$-orbital. This highly localized single electron may impart radical-like behavior to the $n, \pi$ * excited state. The promoted electron is not likely to be completely localized, but may be found somewhat more probably at one atom of the molecule in certain cases. It is interesting that the photochemistry arising from the excited states of $n, \pi^{*}$ type requires radicallike, or diradical intermediates for its interpretation (16), whether singlet or triplet excited states are involved. (Burawoy's assumption of radical-like structures for the ground state of heteromolecules bears no relation to the present discussion.)

[Other characteristics of mainly spectroscopic interest have been cmumerated $(17,3.1)$ but ire omitted here.]

\section{SPECTRA OF N-HETEROCYCLICS}

\section{A. Pyridine}

The pyridine molecule is the first $\mathrm{N}$-heterocyclic which revealed the presence of $n \rightarrow \pi^{*}$ transitions (17). Fig. 13 shows the dramatic solvent effect observed. The curves shown were cletermined by a continuous recording Cary spectrophotometer and show features absent from curves obtained by manual spectrophotometers (38). The region 2700 to 2900 Angstroms shows structure in a hydrocarbon solvent in a region where the vapor spectrum shows very sharp lines. This structure is certainly due to an $n \rightarrow \pi^{*}$ transition since it strongly blue-shifts in ethanol as solvent, and is absent in the absorption spectrum of benzene itself. However, a more remarkable effect is the apparent sharpening of the main part of the band at $2500 \mathrm{~A}$ upon changing from isopentane solvent to ethanol (Fig. 13), which is of course the reverse of the usual blurring effect. This can only mean that there is significant $n \rightarrow \pi^{*}$ absorption even in the $\pi \rightarrow \pi^{*}$ region at $2500 \mathrm{~A}$. The increase of molar absorption coefficient from 1900 to 2500 with this solvent change facilitates the illusion of band 

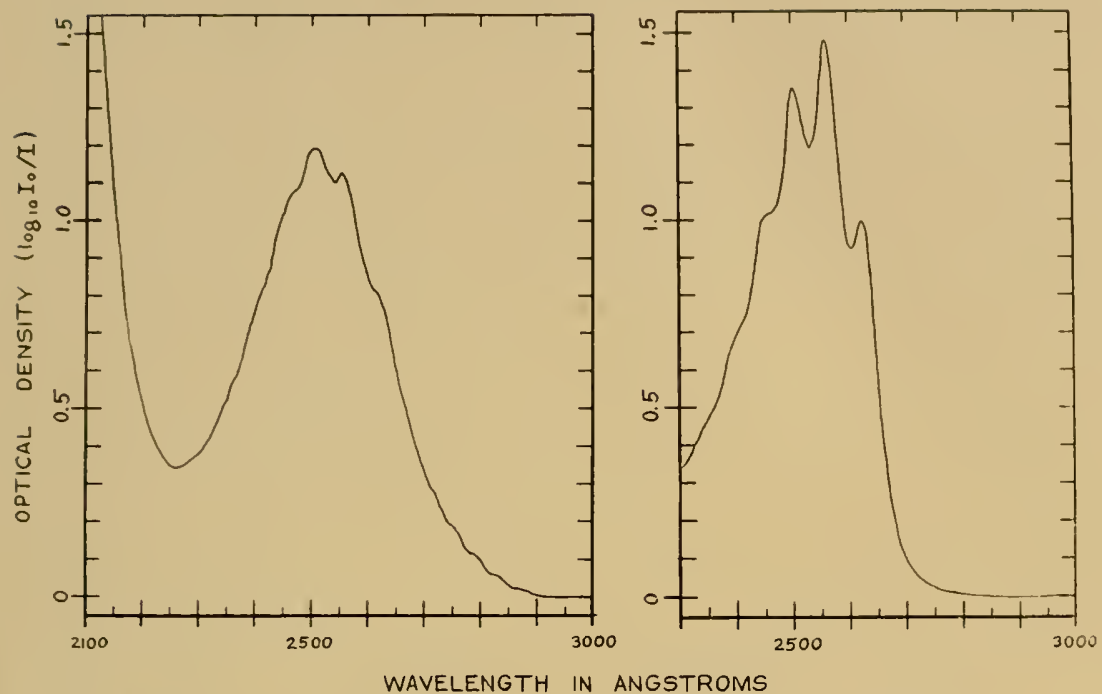

Fig. 13. Near ultraviolet absorption curves of pyridine at room temperature. Left, in isopentane; right, in absolute ethanol, after Kasha and Reid (18).

sharpening: the $\pi \rightarrow \pi^{*}$ transition is intensified in the solvated pyridine. Similar results were obtained by Stephenson (38). A fuller account of the spectra of this molecule is given in another place (18).

\section{B. The Dinzines}

The diazines are examples of N-heterocyclics in which the lowest $n \rightarrow \pi^{*}$ transition is completely separated from the lowest $\pi \rightarrow \pi^{*}$ transition. A partial reason (26) is the interaction between the lonepair orbitals (cf. Fig. 9). Pyridazine (12) has an $n \rightarrow \pi^{*}$ band at approx. $3500 \mathrm{~A}$ (cf. Figs. 12 and 14) which strongly blue-shifts upon changing solvent from hydrocarbon to water. The $\pi \rightarrow \pi^{*}$ absorption at approx. $2500 \mathrm{~A}$ in this case undergoes a small blue shift with the same solvent change.

Pyrazine (12) has an $n \rightarrow \pi^{*}$ band at approx. $3300 \mathrm{~A}$ (cf. Fig. 15), which undergoes a marked blue-shift with solvent change from hydrocarbon to water. The $\pi \rightarrow \pi^{*}$ band at approx. 2700 A undergoes a small red shift. The greater relative blurring of the $n \rightarrow \pi^{*}$. band with this solvent change is characteristic.

C. Molecules with $n \rightarrow \pi^{*}$ Long-Wavelength Shoulders and Tails

Quinoxaline (13) in hexane shows a highly structured $n \rightarrow \pi^{*}$ absorption region as a long-wavelength shoulder at $3500-3800 \mathrm{~A}$, which 
WAVENUMBERS, $C M^{-1}$

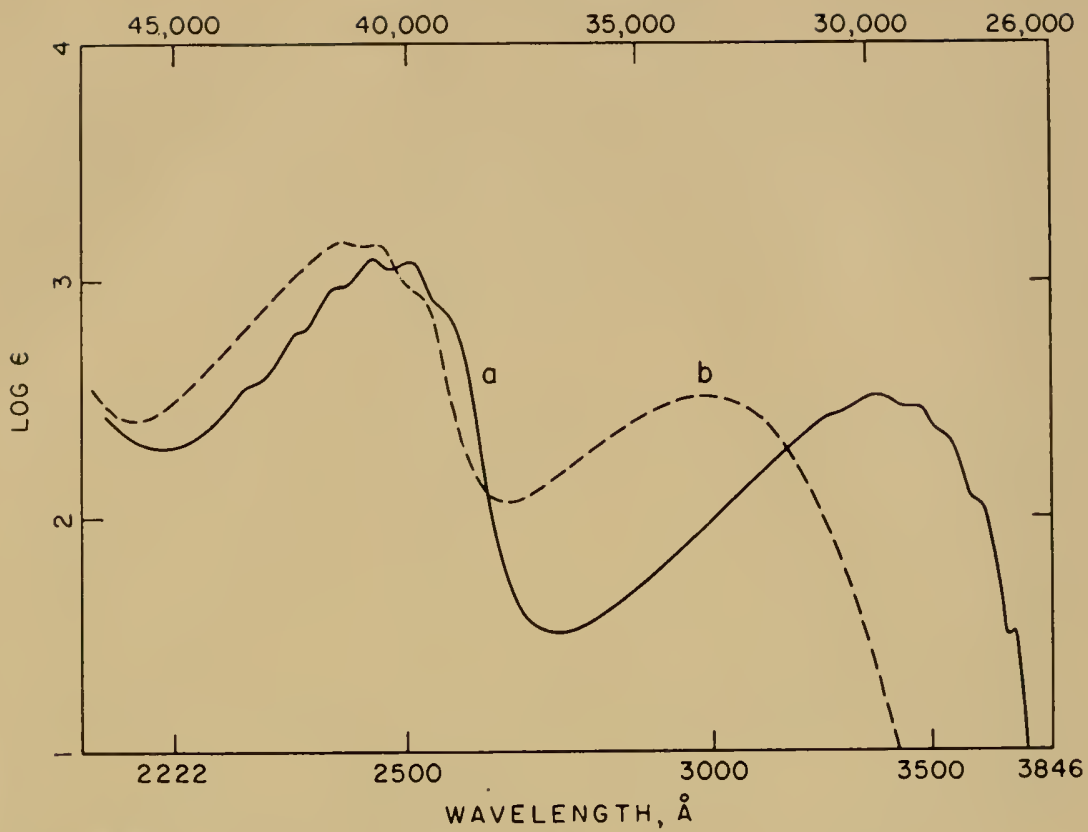

Fig. 14. Near ultraviolet absorption curves of pyridazine at room temperature, after Halverson and Hirt (12). Curve a, in cyclohexane; curve b, in water.

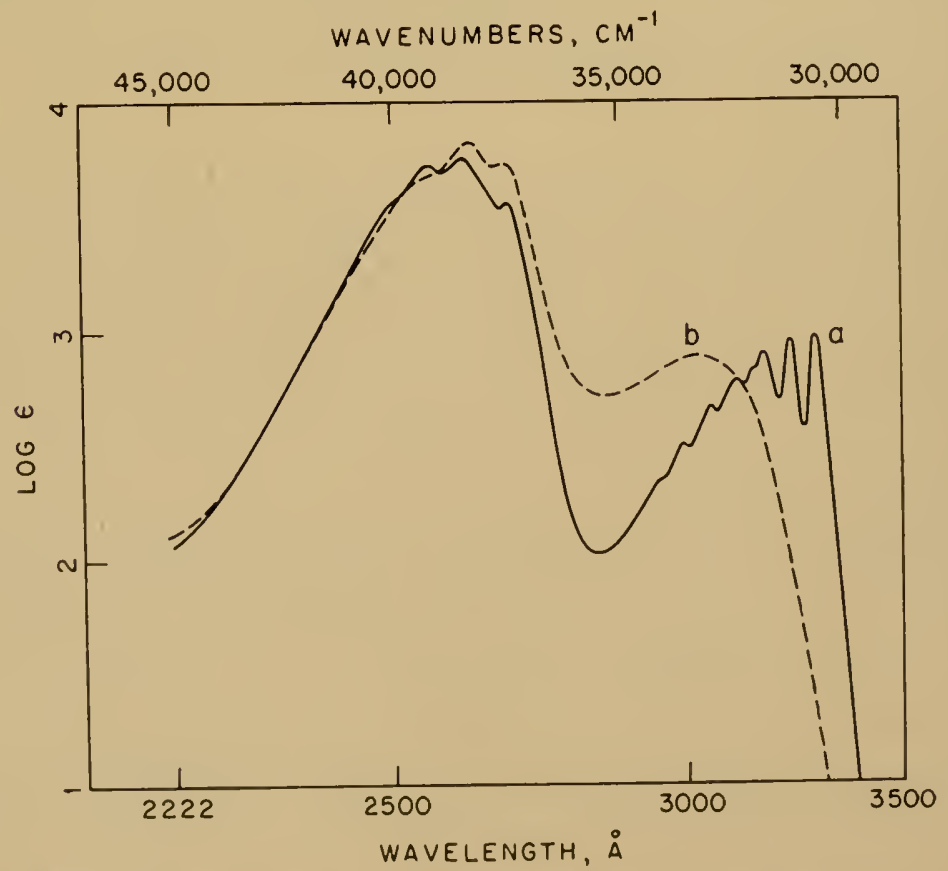

Fig. 15. Near ultratiolet absorption curves of pyrazine at rom temperature, after Halverson and Hirt (12). Curve a, in cyclobexane; curve b, in water. 
seems to disippear in water (Fig. 16). The $\pi \rightarrow \pi^{*}$ absorptions, which are naphthalene-like, are relatively unshifted with this solvent change.

Although in aqueous solution a resolved $n \rightarrow \pi^{*}$ shoulder is no longer present, the shape of the absorption curve of quinoxaline in water at about 3600 suggests that the $n \rightarrow \pi^{*}$ absorption region is still present, even though blue-shifted relative to hydrocarbon solvents. We shall find this a characteristic subtlety of N-heterocyclic spectroscopy.

Phenazine (12a) absorption spectra in hydrocarbon solvents show only a long-wavelength tail, which blue-shifts upon changing the solvent to ethanol, instead of a distinct resolved shoulder. This point was noted also by Badger and Walker (1). Since even in ethanol solvents the molecule is still non-fuorescent, having a strong phosphorescence only, the $n \rightarrow \pi^{*}$ transition is probably still the longest wavelength absorption (12a).

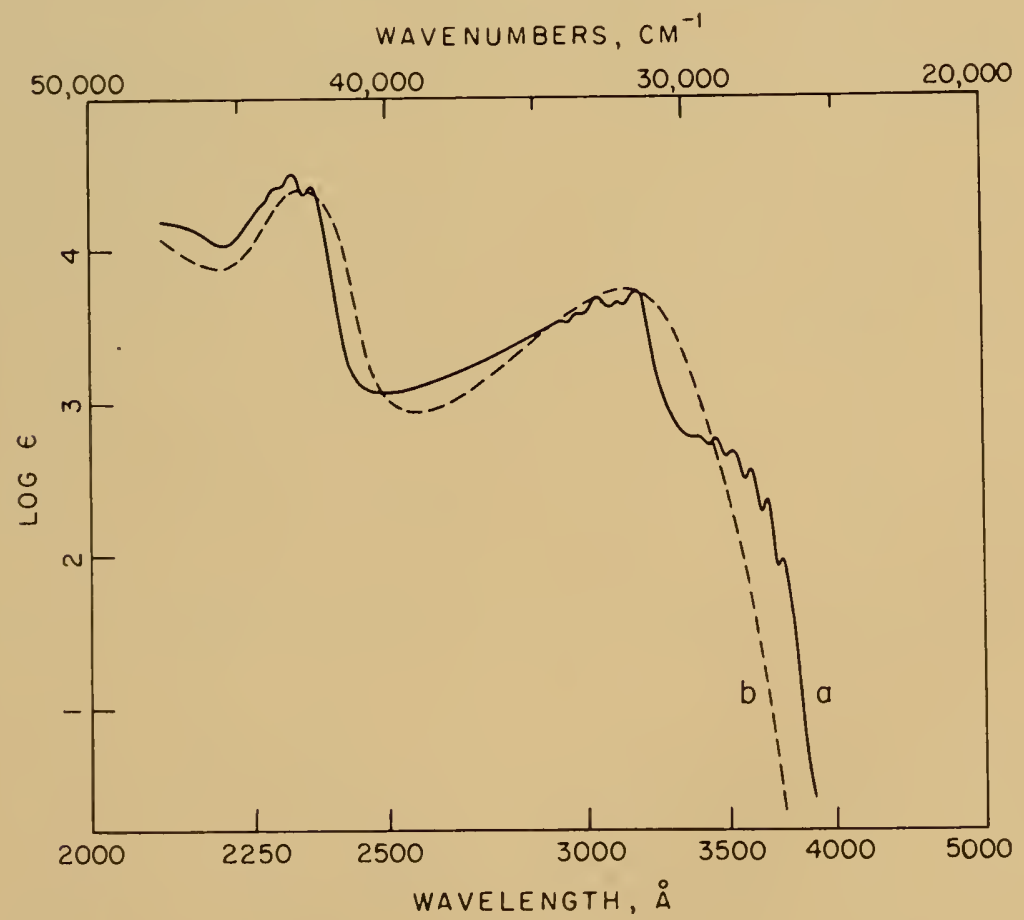

Fig. 16. Near ultraviolet absorption curses of quinoxaline at room temperature, after Hirt, King, and Cavagnol (13). Curve a, in cyclohevane: curve b, in water. 
Quinoline (6) represents a case in which a blue-shift of only a very small part of the long-wavelength absorption tail is observed as one changes from hydrocarbon to ethanol solvent. However, a dramatic change in the low-temperature emission spectra in rigid glass solvents is observed (6): in the hydrocarbon solvent, a strong phosphorescence is observed, whereas in an ethanol-ether-isopentane glass, fluorescence and phosphorescence are observed analogous to the emissions of naphthalene. Thus, in this case, an actual interchange of the electronic states of $n, \pi^{*}$ and $\pi, \pi^{*}$ type must occur as the solvent is changed.

\section{Pyrimidine and Purine}

The spectra of pyrimidine (26) are shown in Fig. 17. The lowest $n \rightarrow \pi^{*}$ transition appears as a distinct band in the region of approx.

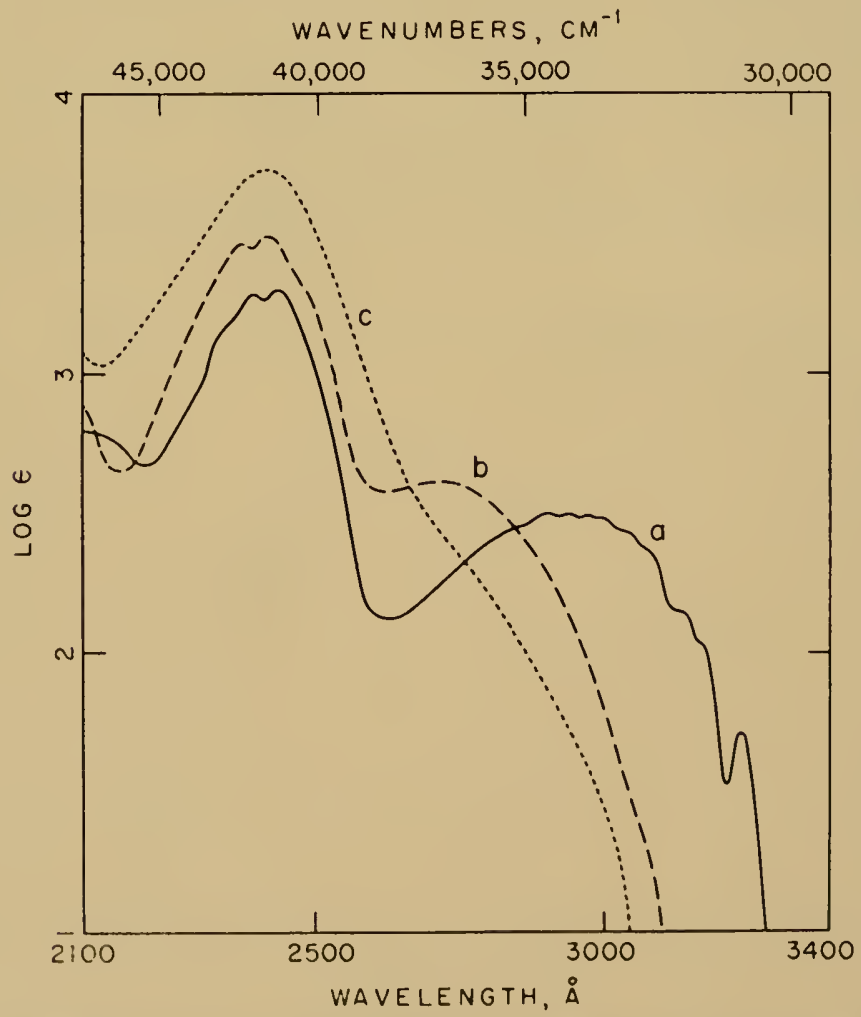

Fig. 17. Near ultratolet absorption curves of pyrimidine at room temperature. after Mason (26). Curve a, in crolohexane; curve b, in water (neutral); curve c. in $4 N$ sulfuric acid. 
3000 A in hydrocarbon solvent, and strongly blue-shifts in water. The $\pi \rightarrow \pi^{*}$ region at $2150 \mathrm{~A}$ is but slightly shifted. Even in $4 \mathrm{~N}$ sulfuric acid some $n \rightarrow \pi^{*}$ absorption remains as a shoulder.

Fig. 18 compares pyrimidine and 2,6-dichloropyrimidine (40), in which the substituents are in the positions corresponding to those of the biologically important pyrimidines. The effect of substitution is

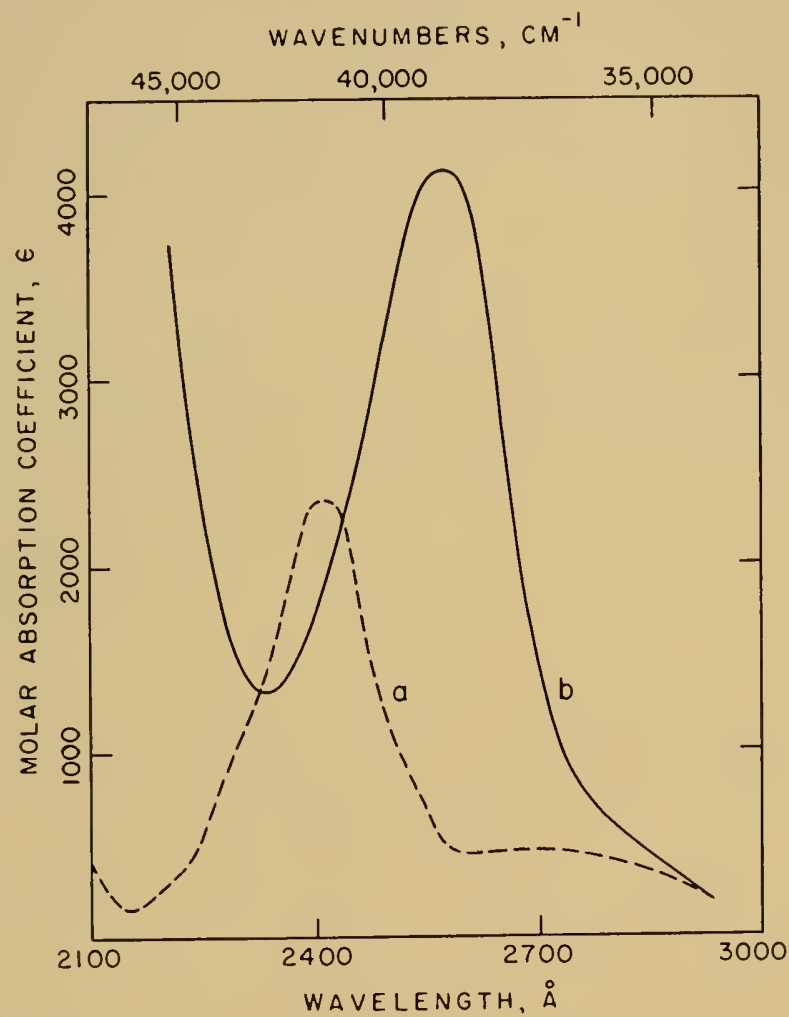

Fig. 18. Near ultraviolet absorption curves of pyrimidine in water (curve a), and 2, 6-dichloropyrimidine in methanol (curve b), at room temperalure. After Uber and Winters (40).

invariably to push together the $n \rightarrow \pi^{*}$ and $\pi \rightarrow \pi^{*}$ absorption regions of such a molecule. However, it is to be noted (curve b, Fig. 18) that even in the spectrum of the disubstituted pyrimidine in water, there is a long-wavelength shoulder at $2800-2900$ which must be due to the $n \rightarrow \pi^{*}$ absorption band.

An analogous situation is found for 9-methylpurine $(25,25 \mathrm{a})$, Fig. 19. Note that the $n \rightarrow \pi^{*}$ shoulder of this molecule in hydrocarbon 


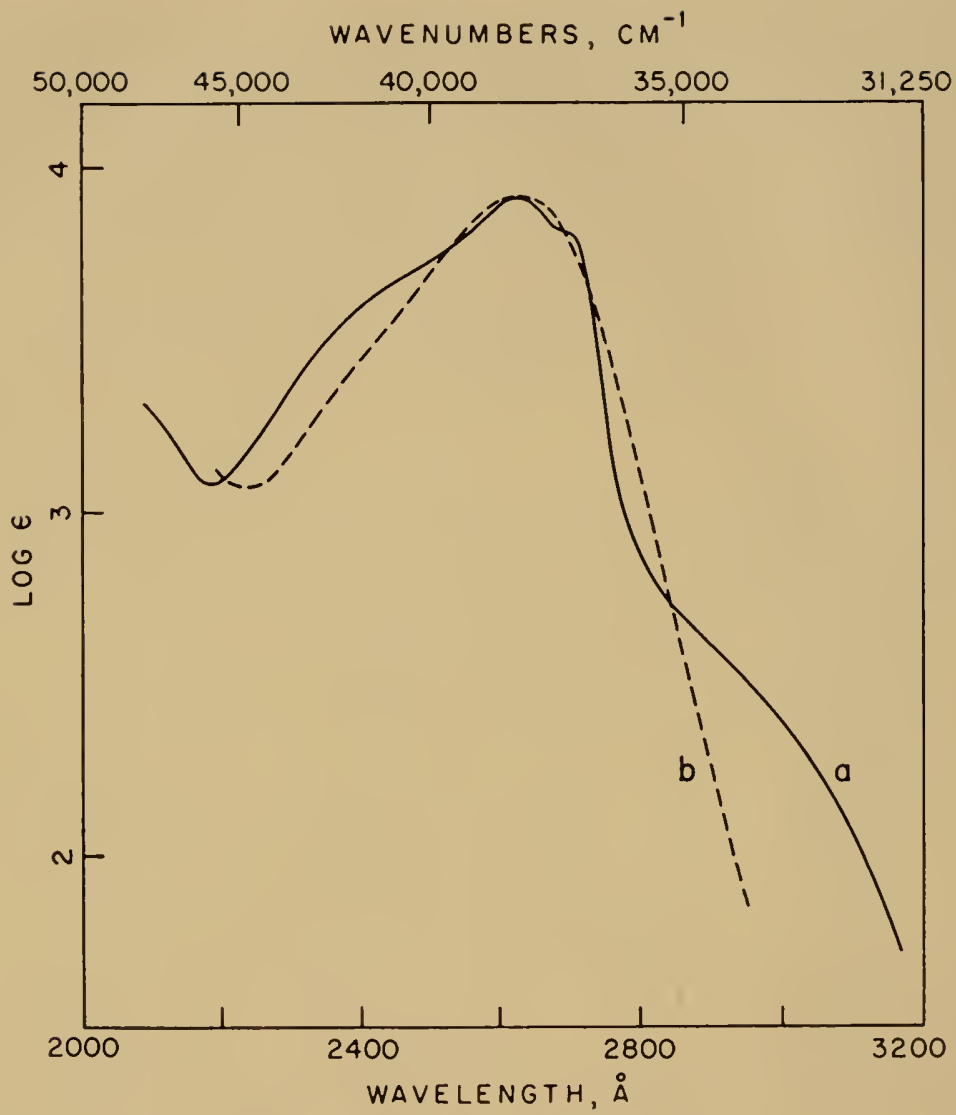

Fig. 19. Near ultraviolet abserption curves of 9-methylpurine at rom temperature, after Mason (25). Curve a, in cyclohexanc; curve b, in water; $p \mathbf{H} 5.05$.

(curve a) strongly blue-shifts, but is probably still present in the 2900 A region. Mason (25) has established by a study of the substitution of groups along different axes of the purime molecule that the absorption region from 2300 to 2750 consists of two $\pi \rightarrow \pi^{*}$ transitions, one polarized along the long axis (y) of the molecule, and one along the intermediate axis $(\mathrm{z})$.

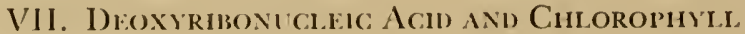

\section{A. Hypochromism in $D N A$}

It is well known that I)N $\Lambda$, "pon undergoing a randon-coil to helix transformation, exhibits a diminution of 30 per cent to 50 
per cent in its nolar absorption coefficient at $2600 \mathrm{~A}$. There is little spectral shilt or change in shape of the band. Tinoco (39) has applied the exciton model (16a) to this problem, taking into account the interaction between dipoles excited in the $\mathrm{N}$-heterocyclic base pairs. Since the base pairs have an essentially parallel stacking in native, or helical-coil, DN $\mathrm{I}$, the dipoles for $\pi \rightarrow \pi^{*}$ excitation will be essentially in a parallel side-by-side stacking, and hypochromism is anticipated by Tinoco's treatment, and was considered by him.

However, if there is an $n \rightarrow \pi^{*}$ long-wavelength tail in the purine and pyrimidine spectra, a head-to-tail stacking of the transition dipoles would be expected, since the $n \rightarrow \pi^{*}$ transitions in the N-heterocyclic bases will be polarized along the helical axis, or perpendicular to the planes of the base pairs. Consequently, a small long-wavelength hyperchromism would be expected in the random-coil to helix transformation according to Tinoco's interpretation. A. Rich and the author (33) have examined clenaturation curves of various synthetic polynucleotides and nucleic acids and have observed the expected hyperchromic effect for the helix-form.

\section{B. Rotatory Dispersion of $D N A$}

The presence of a long-wavelength $n \rightarrow \pi^{*}$ tail in the DNA absorption spectrum should be detectable by the observation of the rotatory dispersion curve. In particular, a Cotton effect might be anticipated corresponding to this band. Fresco and Gorn (9) have made a careful study of the optical rotatory dispersion of native calf thymus DNA and have found a Cotton effect centering on about $2900 \mathrm{~A}$, confirming a characteristic absorption band in this region. Murakami (27a) had previously suggested that pyrimidines might show this effect, but it appears from Mason's work (25) that purines should possess a long-wavelength $n \rightarrow \pi^{*}$ region as well, even in aqueous solution.

The fact that the exciton model seems to apply to the case of DNA implies that energy transfer down the DNA helix should be quite efficient. The application of this process is mainly to racliation effects, in which electronic excitation can result. In addition, the occurrence of $n \rightarrow \pi^{*}$ lowest transitions in the pyrimidines and purines suggests that photochemical processes in DNA will involve $n, \pi^{*}$ excited states with their characteristic features.

\section{The Chlorophylls}

Livingston, Watson, and McArcle (19) reported on the renarkable 
sensitivity of the optical properties of the chlorophylls to wet solvents. Fig. 20 shows their absorption curves for chlorophyll-b in anhydrous benzene and wet benzene. A long-wavelength shoulder at $6700 \mathrm{~A}$ is present in anhydrous benzene, but disappears when water or alcohols are added. Simultaneously, the fluorescence makes its appearance. Both of these phenomena are analogous to the behavior of some of the simpler heterocyclics.

Platt (31) interpreted these fluorescence activation experiments in terms of an interchange of electronic states of $n, \pi^{*}$ and $\pi, \pi^{*}$ type, but did not consider that the absorption spectral changes were clirectly related. This is possible, since the spectra of the porphyrins are rather complex in the region of the long-wavelength absorption band. Nevertheless, Becker and Kasha (2) advanced the same ex-

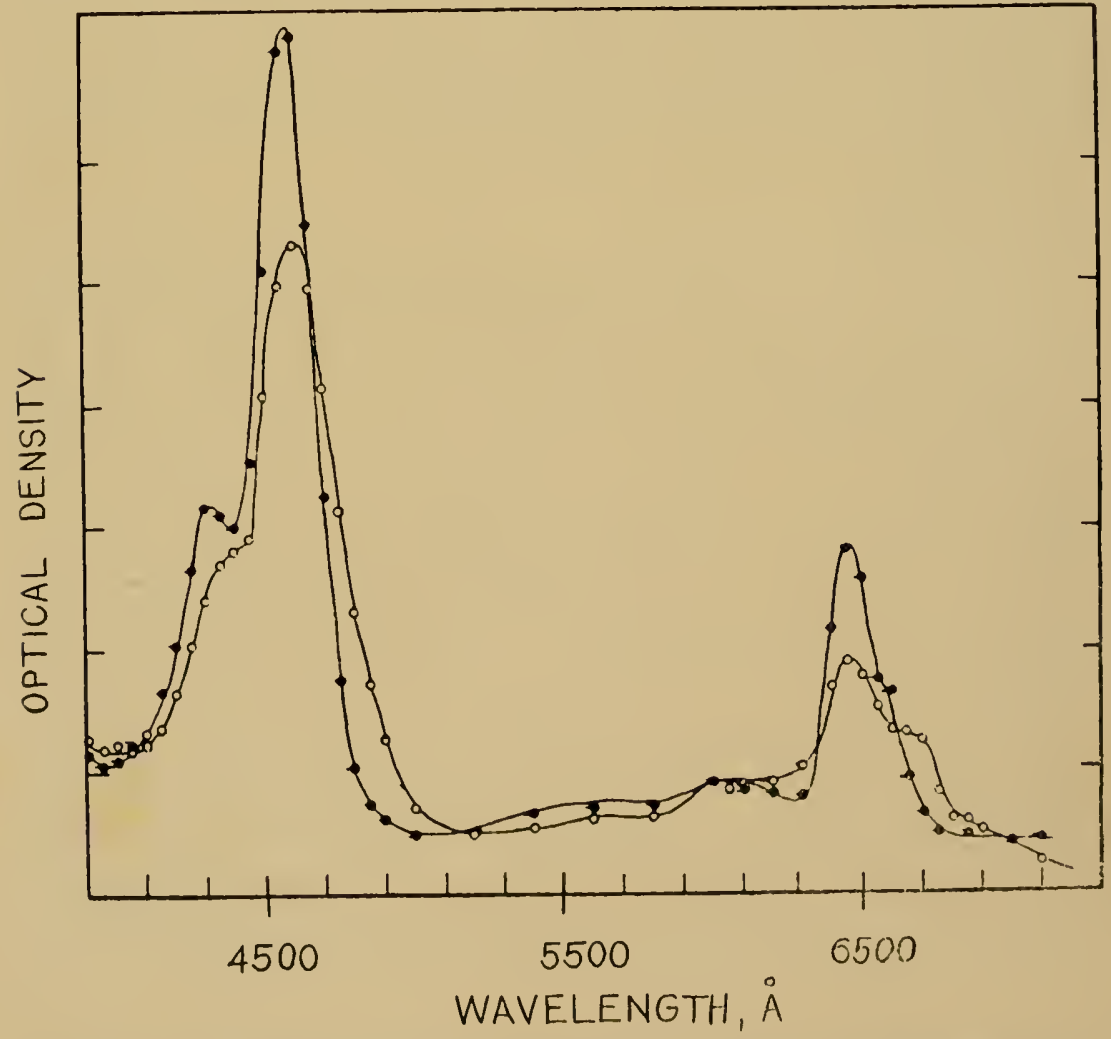

Fig. 20. Visible region absorption curves of chlorophyll-b at room temperature. Open circles in anhydrous benzene; black circles, in wet benzene. (After Livingston. Watson, and McArdle, 19). 
planation as Platt did, but were of the opinion that the long-wavelength shoulder was of $n \rightarrow \pi^{*}$ type, since the spectral shift required is well within the range of other $n \rightarrow \pi^{*}$ blue shifts. Fernandez and Becker (7a) have made further quantitative measurements on cholorphyll-a and clilorophyll-b, confirming the earlier results, and extending the observations to low temperature emission spectroscopy.

Becker's observations (7a) confurm the ideas of Franck (8) concerning the importance of lowest $n, \pi^{*}$ excited states in photosynthesis. In particular, they reinforced the possibility that two kinds of chlorophyll, wet chlorophyll in an aqueous phase, and dry chlorophyll, in a lipid phase, may each cary out complementary functions in the photosynthetic system.

The importance of $n \rightarrow \pi^{*}$ transitions in heteroaromatic systems should be evident, both from the point of view of their wide occurrence, as well as from the point of view of their characteristic features. New biological applications will doubtless be revealed. At the same time, further fundimental researches on the nature of the $n \rightarrow \pi^{*}$ transition are required belore the principal features of this process cail be thoroughly understood.

\section{REFERENCES}

1. Badger, G. M., and Walker, I. S., J. Chem. Soc., 122 (1956).

2. Becker, R. S., and Kasha, M., in Luminescence in Biological Systems (F. H. Johnson, ed.), p. 25. American Issociation for the Advancement of Science. Washinglon, D. C. (1955).

3. Brealey, G. J., and Kasha, M1., J. Am. Chem. Soc., 77, 4462 (19.55).

4. Burawor, A., J. Chem. Soc., 1177 (1939).

4a.Burawoy, A., J. Chem. Soc., 20 1941).

5. Clementi, E., and Kasha, M., J. Mol. Spectrossopy. 2, 297 (1958).

6. El Sayed, M. F. A., and Kasha, M., umpub.

7. Eyring, H., Walter, J., and Kimball, G. E., Quantum Chemistry, Chapters 8 and 12, John Wiley and Sons, New York (1941).

Fa.Fernandez, J., and Becker, R. S., J. Chem. Phys., 31, 467 (1959).

8. Franck, J., Daedalus, 86, I7 (1955); also in Research in Photosynthesis (11. Gaffron, ed.), pp. 19-30, 142-146, Interscience Publishers, New York (1957); Proc. Nat. Acad. Sci. L'.S., 44, 951 (1958).

9. Fresco, J. R., and Gorn, R., unpub.

9a.Goodman, L., and Harrelt, R. W., J. Chem. Phys., 30, II31 (1959).

10. Goodman, L., and Kasha, M., J. Mol. Spectroscopy, 2, 58 (1958).

lOa.Goodman, L., and Slıull, H., J. Chem. Phys., 22, II 38 (1954).

11. Halverson, F., and Hirt, R. C., J. Chem. Phys., 17, 1165 (1949).

12. Halverson, F., and Hirt, R. C., J. Chem. Phys., 19, 711 (1951).

12a.Harrell, R. W', and Kasha, M., Lmpub.

13. Hirt, R. C., King, F. T., and Cavagnol, J. C., J. Chem. Phys., 25, 574 (1956).

14. Hochstrasser, R. M., and Cullen, W. R., J. Mol. Spertroscopy (in press).

14a.Innes, K. K., Merril, J. A., Tincher, W. C., and Tilford, S. G., Nalure, 187. 500 (1960). 
15. Kasha, M., in Conference on Bioenergetics, Radiation Research, Supplement II $(1960)$.

16. Kasha, M., in Conference on Comparative Effects of Radiation (M. Burton, J. S. Kirby-Smith, and J. L. Magee, eds.), Wiley and Sons, New York (1960).

16a.Kasha, M., Rer. Modern Phys., 31, 162 (1959). Also, in Biophysical Science-A Study Program, (ed. by J. L. Oncley et al.) pp. 162-169, John Wiley and Sons, New York (1959).

17. Kasha, M., Discussions Faraday Soc., 9, 14 (1950).

18. Kasha, M., and Reid, C., unpub.

19. Livingston, R., Watson, W. F., and McArdle, J., J. Am. Chem. Soc., 71, 1542 (1949).

20. Maccoll, A., J. Chem. Soc., 670, (1946).

21. McConnell, H., J. Chem. Phys., 20, 700 (1952).

22. McMurry, H., J. Chem. Phys., 9, 231, 241 (1941).

23. McRae, E. G. and Goodman, L., J. Mol. Spectroscopy, 2, 464 (1958).

24. IclWeeny, R., and Peacock, T. E., Proc. Phys. Soc. (London), A70, 41 (1957).

25. Mason, S. F., J. Chem. Soc., 2071 (1954).

25a.Mason, S. F. in Recent Work on Naturally Occurring Nitrogen Heterocyclic Compounds, Special Publication No. 3, Chem. Soc. London, 139 (I955).

26. Mason, S. F., J. Chem. Soc., 1240 (1959).

27. Mulliken, R. S., and McMurry, H., Proc. Nat. Acad. Sci. U.S., 26, 312 (1940).

27a.Murakami, H., J. Chem. Plyys., 27, 1231 (1957).

28. Murrell, J., J. Chem. Phys., 26, 1738 (1957).

29. Nagakura, S., Bull. Chem. Soc. Jnpan, 25, 164 (1952). [in English.]

30. Orgel, L., J. Chem. Soc., 121 (1955).

31. Platt, J. R., in Radiation Biology (A. Hollaender, ed.), Vol. IlI, pp. 71-123, McGraw-Hill Book Company, New York (1956).

32. Pullman, B., and Pullman, A., Les Théories Electroniques de la Chimie Organique, Pp. 486-488, Masson et Cie., Paris (195\%).

33. Rich, A., and Kasha, M., umpub.

33a.Sidman, J., J. Mol. Spectroscopy, 2. 333 (1958).

34. Sidman, J., Chem. Rer.. 58, 689 (1958).

34a.Simpson, W. T., and Barnes, E., unpub.

35. Sponer, H., and Rush, J. H., J. Chem. Phys., 17, 587 (1919).

36. Sponer, H., and Rush, J. H., J. Chem. Phys., 20, 1817 (1952).

37. Sponer, H., and Stïcklen, H., J. Chem. Phys., I4, 101 (1916).

38. Stephenson, H. l'., J. Chem. Phy's., 22, 1077 (1954).

39. Tinoco, I., J. Am. Chem. Soc. (in press). The anthor is indebted to Prof. laul Doty for the opportunity of reading the manuscript of this paper before its publication.

40. Uber. F. M., and Winters, R., J. Am. Chem. Soc., 63, 137 (1911).

\section{DISCUSSION}

1)k. Brissmax: I would like to ask Dr. Kasha what is significant about the two and six positions of the substituted pyrimidines which make them miquely interesting.

1)R. KAsma: I was only suggesting that the Uber and Winter results on the 2,6-dichloropyrimidine have some bearing on thymine, which has keto groups in the 2 and 6 positions. On the other hand, I didn't have time to discuss tautomerism of the bases which is extremely important in terms of the origin of the $n \rightarrow \pi^{*}$ transitions. It is important because as one tautomerizes, 
one loses nitrogen base pairs and gains carbonyl base pairs and vice versa. The real problem, I think, is to find which tone pairs are the ones which are optically and photochemically active, and I think that substitution at the 2,6 positions only suggests that that conjugated substitution may cause spectral effects which might reflect themselves in the thymine spectrum.

DR. Kal.GAR: Since you brought up tautomerism and dipoles, I may refer to some recent optical studies in the infrared region by Todkl Miles* on nucleic acicts which. as I understand, might be quite pertinent to some of the problems you raised in connection with DNA.

Dr. Kasna: I know the work of Mason on the use of infrared spectroscopy to establish tautomeric forms of the bases. I don't know any work on DNA itself.

Dr. Kalckak: These studies were not done on DNA but on the enzymatically synthesized polynucleotide double strands of Warner and Ochoa which accorcling to Rich and Davis show some crystallographic resemblance with DNA. Todd Miles (Nature 183: 1814. I959) found striking spectral changes in the infrared region upon the formation of double helixes. These changes he ascribed for various reasons to changes in dielectric constants as well as to hyclrogen bonding.

DR. KASHA: I am unfamiliar with those infrared studies.

Dr. Calvis: I would like to ask Dr. Kasha for further elaboration on item 4 , that is, fluorescence versus phosphorescence comparing the $n \rightarrow \pi^{*}$ and $\pi \rightarrow \pi^{*}$ transitions. First of all, as I understand it, the excited $\pi$ state to which you go in both cases is the same and therefore the difference between the two spectra lies in the difference between the energy levels of the highest occupied $\pi$ state and the $n$ state from which it starts. Now what effect does that have on item 4 ?

DR. KASIA: I gave a paper at the Brookhaven Bioenergetics Conference on this point. Your statement is one that people commonly make but there are many subtleties behind this. Consider a system in which the first three $\pi$ orbitals are filled and the $\pi$ orbitals 4,5 and 6 are empty. You are then presuming that the final energy level is the same whether $\pi 4$ is reached through an $n \rightarrow \pi^{*}$ transition or a $\pi \rightarrow \pi^{*}$ transition. This is the simplest way of describing it in the LCAOMO molecular orbital description. But actually what happens is that the orbital order may not be the same because when you take an electron from a lone pair such as from the nitrogen in pyridine and put it into the ring. you leave a positive nitrogen. This has a tremendous affect on the $\pi$ orbital energy as has been pointed out by a number of people in the literature and it is even possible that the charge rearrangement may even upset the order of orbitals 4 and 5 .

Dr. Calvin: Can you conceive of a transition from the excited $\pi$ level to which you have gone by the $\pi \rightarrow \pi^{*}$ transition to the $\pi$ level to which you would normally go from $n$ ?

DR. KaSHa: They are formally and symmetry-wise alike, but energetically they can never be the same. 
Dr. CalviN: I don't mean that they are the same but can such a transition occur?

DR. Kasna: Oh, absolutely, and your other question was then why should the fluorescence and phosphorescence differ? Well, it turns out that when onc examines singlet-triplet mixing for $\pi$ electron states in a planar molecule, the singlet-triplet mixing vanishes for single-center interactions as McClure showed, and one has to consider very weak three-centered integrals in order to calculate any magnitude of single-triplet mixing. But in the $n \rightarrow \pi$ case you get one-center interaction, that is, the spin orbital coupling involves a single atomic center. So you get a large factor of gain in the rate constant for a single-triplet interaction.

DR. CaLviN: I wasn't thinking of another triplet. I was thinking of another singlet $\pi$ state which you achieve by an $n \rightarrow \pi^{*}$ transition which is different from a $\pi$ state which you achieve by a $\pi \rightarrow \pi^{*}$ transition. There are two different excited singlets, and you can transfer from one excited singlet $\pi^{*}$ to another. The one that you come to from the $n$ state is achievable through the excited $\pi \rightarrow \pi *$ transition.

DR. KAsna: Let me simply state that the major source of the difficulty in my understanding your question is that it is based on an orbital diagram. If we go to a state diagram, we get the following picture. If we go from the ground state to the singlet $\pi, \pi^{*}$ state, we can casily get to the singlet $n, \pi^{*}$ statc. Then we necessarily get down to the lowest triplet state, the $\pi, \pi^{*}$ state. If the singlet $n, \pi^{*}$ state were not lowest, the molecule could easily re-cmit from the singlet $\pi, \pi^{*}$ state. The reason for this behavior lies in the kinetics of excitation, and is largely due to enhanced singlet-triplet mixing in $n, \pi^{*}$ states.

I) R. Franck: May I say a word. I believe that I have some understanding of the difficulty that Dr. Calvin has. Namely, that the excited states that you arrive at by $\pi \rightarrow \pi^{*}$ transitions and by $n \rightarrow \pi^{*}$ transitions are different. In the one case you get a positive hole by removing an electron from the lone pair of the nitrogen atom, in the other case you get a positive hole in the highest occupied level of the $\pi$ orbital.

Dr. Calvin: But what I was asking is, can you get a transition between these excited states?

DR. Franck: Y'es, you can but you can't get it by radiative transitions, only by radiationless transitions, i.e., by internal conversion or by impacts.

DR. Wlibl: Dr. Kasha has spoken of some encrgy transfers in DNA, and I think it would be an appropriate time to describe an experinent that I have done. This is an actual experincnt showing that the singlet state can be transferred in DNA. In this experiment we studied the excitation fluorescence spectrum of solutions of DN.I to which auramin had been added. It lad been show' a number of years ago that atumin adsorbs on DNA with the appearance of fluorescence. Auramin itself does not fluoresce in water solutions. Therefore, in this experinent the fluorescence excitation spectrum 
is that of an auramin-1)NA complex. If we compare the fluorescence excitation spectrum of the auramin-DNA complex at wavelengths under $300 \mathrm{~m} \mu$ with that spectrum which one would expect if auramin would emit its own absorbed quanta, one observes that the complex has a higher fluorescence. The difference, of course, is due to the quanta absorbed by the DNA which are being transferred to and cmitted by the auramin. From the data one can calculate the number of purine and pyrimidine residues which are feeding into one auramin molecule. If one assumes a quantum efficiency of transfer of one, then the calculated number is 4 to 6 . The number, of coursc, would be higher if the quantum efficiency of trinsfer were less than onc. Therefore, it seems likely that we have proof from this experiment that the singlet state can be transferred from 1 pyrimicline or purine residue to the next, since it is unlikely that four pyrimidine or purine bases are so situated that they can transfer to auramin with the quantum efficiency of one.

Dr. Baxister: Dr. Kasha, is the activation of Huorescence of chlorophyll in hydrocarbon solvents to be given an explanation similar to the one you gave for quinolines?

Dr. Kasua: Yes, Platt has described this in his paper, in the book Radiation Biology, and Becker and I have discussed this as well (see text). There are three questions involved. (I) Are the solvent changes really associated with the $n \rightarrow \pi^{*}$ blue shift or not? They may be only partially associated with the $n \rightarrow \pi^{*}$ blue shift. There is a new peak and a tail. We think only the tail is associated with the $n \rightarrow \pi^{*}$ region. (2) A further problem which arises is this. When the non-fluorescent ary chlorophyll is studied, where is the phosphorescence? Fernandez and Becker, working in the University of Houston, have found that there is a unique emission in dry chlorophylls which is absolutely missing when the material is hydrated. The great curiosity, which I didn't have time to discuss, is why the intermediate triplet emits, not the lowest triplet. The lowest triplet emits in hydroxylic solvents, but in dry solvents the intermediate triplet emits. These are so close together, we normally would have expected a radiationless transition leading only to the lowest emission. However, I believe that the novel behavior comes because porphyrin is such a large molecule compared with the localized $n \rightarrow \pi^{*}$ excitation region, where, as a sort of contradictory answer to Calvin's question, here is a case where apparently the internal conversion process is restricted by the spatial characteristics of the orbitals with reference to the whole molecular system. If this observation is correct. I think it extremely important to study single lone pairs in other large molecules like formyl porphyrin and dyestuffs with one nitrogen lone pair, etc. to see whether it is characteristic that the rate constant for the radiationless process gets so limited that one cannot really get to the lowest $\pi . \pi^{*}$ triplet state from some upper $n \rightarrow \pi^{*}$ triplet. This will have some very important consequences on the photochemistry and is something which should be investigated. 
Dr. Abranamson: If I understand you correctly, you say that in chlorophyllclilorophyll b to be precise-one can obtain phosphorescence from the $n \rightarrow \pi^{*}$ as well as the $\pi \rightarrow \pi^{*}$ triplet.

DR. Kasina: Both, depending on your solvent. Fernandez and Becker have found it from the $n, \pi^{*}$ triplet in very dry solvents and Becker and Kasha have found phosphorescence from the lower $\pi, \pi^{*}$ triplet. So you can get cither one, curiously enough!

Dr. Abrahamson: It is generally true that $\pi \rightarrow \pi^{*}$ triplets lie at lower energies than $n \rightarrow \pi^{*}$ triplets?

Dr. KAsha: Not always. This is a very important thing. The $n, \pi^{*}$ singlet-triplet split is small in some types of $n \rightarrow \pi^{*}$ promotion, but not in heterocyclics. In heterocyclics this order can be inverted. You can have a small $n \rightarrow \pi^{*}$ singlet-triplet split or a large $\pi \rightarrow \pi^{*}$ singlet-triplet split. So we have every arrangement of singlet and triplet states of the two orbital types that you like. If you name it, we probably can give you an example, because the singlet-triplet splitting is variable and can be very large in $n \rightarrow \pi^{*}$, as much as 7,000 wave numbers. This is something which had not been considered possible before so that the order can interchange.

Dr. Franck: Flash experiments by Livingston, Linschitz, and others on chlorophyll dissolved in different organic liquids at room temperature showed that under these conditions only one metastable triplet was detectable by its absorption spectrum, supposed to be the lowest $\pi, \pi^{*}$ metastable triplet. It therefore seems that radiationless transition beween the triplet levels of $n, \pi^{*}$ and $\pi, \pi^{*}$ exist.

Dr. Kasha: I am not certain of that and I see Professor Porter is on the progran, and perhaps he can tell us. The flash experiments which people do in liquicl solutions would be, perhaps, the only way that one could distinguish between which lowest triplet is available. If there is a restriction on the conversion from the $n, \pi^{*}$ triplet to $\pi, \pi^{*}$ triplet, then one should get a different triplet-triplet absorption in the dry solvent compared with the wet.

DR. Lisscint: It has been found that one gets the same type of spectrum whether one works in polar or non-polar solvents.

Dr. Kasma: Well then, that is the answer, Dr. Franck. That establishes the radiationless transition in the presence of the licpuid solvent. 


\section{THE TRIPLET STATE IN FLUII) SOLUTION}

\section{GForge Porter}

Chemistry Department, l'nizersily' of Sheffield. England

In so filr as excited electronic states are of importance in photobiology two such states deserve primary consideration: the lowest excited singlet state and the lowest triplet state. The former determines the long wavelength absorption spectrum and is the first state reached by absorption of light in this region, the second is generally reached only indirectly by intersystem crossing from the singlet state but is the excited state of lowest energy and, being of different multiplicity from the ground state, it has a relatively long liletime and a consequently greater probability of entering into reaction.

There are three spectroscopic methods by means of which the lowest triplet state of a molecule may be studied.

(1) By direct absorption from the ground state. This requires long path-lengths owing to the forbidden nature of the transition, although the transition probability may be increased by perturbation techniques, e.g., by observation in a solution in equilibrium with a high pressure of oxygen (3). However, the only information obtained from such measurements is the energy separation between the lowest triplet and the ground state and also the extinction coefficients which are related to the transition probability and lifetime.

(2) Emission spectrum from the triplet to the ground state. This is known as phosphorescence and yields the energy of the lowest triplet level and its lifetime (5). Since the transition arises from the triplet state, phosphorescence may also be used, in principle, to study the lifetime of the triplet state as a function of conditions and hence to study its reactions. In practice this is not very useful, since phosphorescence is usually ouly observed in rigid glasses or in very viscous solutions, whereas as far as the reactions of the triplet state are concerned, we are normally interested in gases or in fluid solutions.

(3) Absorption from the triplet state resulting in excitation to a higher triplet level. This transition is spin-allowed and may therefore be very intense. It tells us nothing about the energy difference between the lowest triplet and the ground state, but this information is 
available from (1) and (2). It gives directly the energy of higher triplet levels. But much more important is the fact that it makes possible the clirect measurement of triplet concentration as a function of time and is equally applicable to gases, liquids, and solicls. In this symposimm the interest is principally in solutions and the remainder of this paper will deal only with this medium.

\section{Flash Photoly'sis}

Since the lifetime of the triplet state in fluid solution is normally not greater than one-thousandth of a second, special experimental techniques must be used. The technique of flash photolysis, originally developed for the study of free radicals ( 7$)$, is ideally suited to this purpose and is indeed the only general method at present available by which the triplet states of molecules can be observed and quantitatively studied in fluid solution.

A flash photolysis apparatus comprises a reaction vessel of some optically transparent material, an electronic flash which delivers a flash of light of at least several hundred joules energy and of a few microseconds duration, and some means of recording the transient absorption spectra of the reaction mixture as a function of time after the flash. This recording may be carried out either by means of a second electronic llash timed to photograph the absorption spectra at preset time intervals after the photolysis flash or by the continuous photoelectric recording of optical density at one wavelength. The former method is most suitable for the initial study of the transient spectra, and the latter method is more convenient and accurate for detailed kinetic studies once the spectrum is known.

The furst application of flash photolysis to the triplet state and the first detection of triplet state absorption spectra in solution were made by Porter and Windsor in 1952 (10). At this time it was not entirely clear whether the general absence of phosphorescence in gases and solutions was due to the fact that the conversion from upper singlet to triplet state occurred less readily than in solids or to the existence of radiationless processes in solution which were much more rapid than phosphorescence. Experiment showed that the latter was the case and that triplet states were readily populated in fluid solvents but nomally had a lifetime of the order of $10^{-4}$ seconds. This time was short enough to account for the absence of long-lived phosphorescence but long enough to be readily studied by llash photolysis techniques. Absorption spectra of a wide variety of molecules were recorded, and preliminary kinetic investigations were carried out by 
photographic methods (11). Subsequently more accurate kinetic studies have been carried out spectrophotometrically, and the results of them will now be briefly reviewerl.

\section{Kinltics of mile Triplet State in Solution}

The triplet state of a molecule is a species with its own structure and its own physical and chemical properties which may be very different from the properties of the same molecule in its ground state. These properties have not yet been studied in a wide enough range of molecules for secure generalization to be possible. Most detailed studies have been carried out on aromatic hydrocarbons and their derivatives and, in this series of compounds, certain modes of behavior appear to be rather general and probably applicable to most triplet states. Since most of these results have already been published in full, all that will be given here is an outline of the principal processes and references to the original papers.

\section{Ramiative and Radiationless Decay to Ground State}

In the absence of specific physical or chemical quenching processes, the triplet state disappears by a first-order conversion to the ground state. In rigid media the decay is usually radiative, and the lifetime, which is determined by the transition probability between the two states, is usually between $10^{-4}$ seconds and 10 seconds for molecules composed only of light atoms.

In fluid solution and in the vapor phase the lifetime is usually found to be between $10^{-5}$ and $10^{-3}$ seconds, and there is little relation between this lifetime and the radiative one. The radiationless decay is partly second order-brought about by collisions between two triplet molecules-and partly first order in triplet concentration. Both processes are viscosity-dependent and the nature of the firstorder process is still not fully understood. It is thought to be a true first-order radiationless conversion-at least in part-but the possibility that pseudo-first-order processes involving some unidentified quenching species may play a part is still under discussion.

\section{Quenching by Paramagnetic Substances}

The second-order decay of the triplet state mentioned above is only a special example of the rather general finding that all paramagnetic species quench the triplet state. The first quencher to be found $(10)$, and the most common, was oxygen, and quenching by this molecule is so efficient that very careful outgassing of solutions 
is necessary before triplet-state absorption can be observed. Both oxygen and nitric oxide quench with nearly unit encounter efficiency (11).

It was natural to enquire whether paramagnetic ions also quenched the triplet state, and the findings here were rather strange $(12,13)$. Whilst all paramagnetic ions were quenchers, and diamagnetic ions were not, the quenching efficiency of an ion showed no other correlation with magnetic susceptibility or number of unpaired electrons. Clearly the effect could not therefore be accounted for in terms of perturbations of the triplet state by the magnetic field of the quencher.

An explanation of this type of quenching has recently been given by Porter and Wright (13). The process

$$
\text { Triplet }+M \rightarrow \text { Singlet }+M \text {, }
$$

where $M$ is unchanged in multiplicity, is forbidden by spin selection rules if $M$ is a singlet but allowed if $M$ has any other multiplicity. Furthermore, from purely statistical considerations, the probability of the process is independent of the multiplicity of $M$ provided this is greater than singlet. The observed differences between different quenchers therefore arise from the varying degree of overlap of the orbitals of unpaired electrons in the triplet and in $M$ rather than from the magnetic field interactions.

\section{Quenching by a Molecule with a Lower Triplet State}

This is an energy-transfer process in which the quenching molecule becomes excited as follows:

$$
A_{T}+B_{S} \rightarrow A_{S}+B_{T}
$$

It is energetically possible whenever an encounter takes place with a molecule with a lower triplet level and, since spin is conserved in the overall process, it may occur with high probability. It has been demonstrated in rigid media by Terenin (14), in solution for the particular case of diacetyl by Backström (1), and in a range of systems in solution by Porter and Wilkinson (9). The maximum quenching constants observed in solution correspond to unit efficiency at normal collision diameters, but quencling constants as low as $10^{6} \mathrm{l} / \mathrm{mole} / \mathrm{sec}$ have also been measured. The present evidence indicates that exchange transfer rather than Förster type resonance uansfer is the preclominamt process. 


\section{Qunching by Chemeal Reaction}

Chemical reaction of the triplet state will clearly lead to its destruction. Photochemical reactions maly proceed from the lowest excited singlet or triplet states (and possibly in some cases from higher singlet states), but only in a very few cases has the state responsible for reaction been definitely identified. Examples of reactions involving the triplet state are liydrogen abstraction by benzophenone (9), clectron transfer between thionine dyes and ferrous salts (8), and addition of oxygen to anthracene (6). On the other hand similar reactions, such as hydrogen abstraction by duroquinone, appear to occur through the singlet state (2).

\section{Physico-Chemical Properties}

Properties such as redox potential and acidity are often entirely different in the excited state. An example of the difference in reclox potential between ground state and first triplet state is given by methylene blue, whose triplet state oxidizes ferrous to ferric, whilst the leuco form of the dye so formed is itself oxidized, in the ground state by ferric iron.

The acidity constants in excited states are particularly illuminating, since they provicle a rather direct measure of electron density distribution. Fluorescence measurements have shown that in molecules such as naphthols, naphthylamines, and acridine the first excited singlet state has an acidity constant differing from that of the ground state by as much as six $p K$ units (15). Recent measurements of the acidity constants of the corresponding triplet states have shown (4), rather surprisingly, that these differ very little from those of the ground state. It follows that the singlet transition in these molecules have considerably more charge transfer character than the transition to the triplet state.

The triplet state in solution is clearly a chemical species in its own right, with its own physical structure and chemical properties. Very little can be predicted about these properties from a knowledge of the ground state alone, but direct observation and measurement of its properties is now conveniently carried out by flash photolysis methods.

\section{REFERENCES}

1. Backström, L. J., and Sandros, K., Acta. Chem. Scand., 12, 823 (1958).

2. Bridge, N. K., and l'orter, G.. Proc. Roy. Soc., A244, 259, 276 (1958).

3. Evans, D. H., I. Chem. Soc., 1351, 3885 (19:5i). 
4. Jackson, G., and Porter, G., Discussions Faraday Soc., 27, 103 (1959).

5. Lewis, G. N., and Kasha, M., J. Am. Chem. Soc, 66, 2100 (1944).

6. Livingston, R., J. chim. Phys., 55, 887 (1958).

7. l'orter, G., Proc. Ro)'. Soc., A200, 284 (1950).

8. Porter, G., Nature, 182, 245 (1958).

9. Porter, G., and W'ilkinson, F., Discussions Faraday Soc., 27, 96 (1959); and unpub.

10. Porter, G., and W'indsor, M., J. Chem. Phys., 2I, 2088 (1953); Discussions Faraday Soc., 17, 178 (1954).

11. Porter, G., and Windsor, M., Proc. Roy. Soc., A245, 238 (1958).

12. Porter, G., and Wright, M. R., J. chim. Phys., 55, 705 (1958).

13. Porter, G., and Wright, M. R., Discussions Faraday Soc., 27, 18 (1959).

14. Terenin, A. N., and Ermolaev, V. L., Trans. Faraday Soc., 52, 1042 (1956).

15. Weller, A., Z. Physik. Chem., 17, 224 (1958); 18, 163 (1958).

\section{DISCUSSION}

Dr. Platt: Would you draw us an energy level diagram of duroquinone and benzophenone showing us the differences between the energy levels and which one is abstracting hydrogen?

Dr. Porter: I can't because I don't know the energy level of the triplet state of duroquinone. I don't mean that I just haven't read the literature, I just don't think it is known. It doesn't phosphoresce as far as I know. The triplet state for benzophenone is well known at $24,000 \mathrm{~cm}^{-1}$ and the singlet absorption for both compounds is about the same, that is, the longest wavelengh peak is at about 3500 angstroms. I don't know the triplet level of duroquinone. It hasn't been measured. We have looked for the phosphorescence but never observed it.

DR. PLATT: Would you summarize these results in about two sentences?

DR. PORTER: Y'es. In duroquinone the production of the semiquinone occurs from the singlet state, while in bentophenone that of ketyl radical occurs from the triplet state.

Dr. Franck: Do you regard it probable, that a reaction proceeding at the cost of the measurable triplet energy will not be promoted by the higher energy of the first excited singlet?

Dr. PORTer: In the case of benzophenone it could well be that the singlet was highly reactive, but the transition was very eflicient to the triplet. But in duroquinone the converse is not true. The triplet is there but does not react with anything like the efficiency of that of benzophenone.

DR. Franck: If the energy stored in the triplet is sufficient, then the reaction by the triplet is preferred because of its long lifetime but I am sure that the yield of the reaction per impact should be at least as high for the first excited state.

Dr. P'ORTER: This is very reasomable but energy is not the only consideration-electron density distribution in the two states is also important. I am rather loath to put forth explanations in terms of energy levels and lifetimes on so few examples. I did watnt to point ont, however, that this sort of 
thing must be done if we are crer to muderstand the photochemistry of this kind of system at all. A distinction must be made between the singlet and triplet. One must accumulate data and try to correlate this with whether the $n \rightarrow \pi^{*}$ or the $\pi \rightarrow \pi^{*}$ are the lowest state and this sort of thing.

Dr. Raвıомпncu: Since you brought up the reaction between a thiazine dye and ferrous ions, I thought it would be proper to mention some results recently obtained by Ainsworth in our laboratory. He concluded that, with thionine as oxidant, the reaction with ferrous ions goes partly through thionine molecules in the triplet state encountering ferrous ions, and partly through excitation and electron transfer in a complex of a ferrous ion and a dye molecule. Even in the latter case, however, there seems to be a delay between excitation and reaction. It looks as if in the complex, too, thionine, excited to the singlet state, can go down to the triplet state and stay there for at least $10^{-5}$ seconds, before accepting an electron from the ferrous ion. It seems a little peculiar that one can preserve an excited dye molecule next to an electron donor in a complex for this length of time.

Dr. Porter: Are you saying that even in the complex the singlet does uot react?

Dr. Rabinowitch: No, I didn't mean that. There must be a competition between singlet and triplet reaction but quenching observations suggest that the average time between excitation and the electron transfer is considerable, and that means that even within a complex, much of the reaction goes through the intermediary of a long-lived triplet state.

Dr. PORTER: Yes.

DR. KASHA: I was very impressed to see the progress in the plotochemistry of the triplet states. I am bothered by a few items here which I would like to bring up. One is the very high energy at which the triplet-triplet absorption comes. For example, in anthracene I calculate from your slide there are 38,000 wave numbers above the ground state in the first excited triplet that you find. You start at 14,000, and the first triplet $\rightarrow$ triplet is at 24,000 wave numbers, so you go 38,000 wave numbers up. That means you miss two triplets, which obviously require infra-red flash spectroscopy.

Dr. Porter: I missed two triplets, but the transitions to them are not allowed. As a matter of fact, the triplets which we clo observe correlate very beautifully with Pariseo's calculations. If one only takes his fully allowed transitions and plots those for the linear polyacenes they agree very nicely with yours for the ground state, with ours for the two principal excited states

Dr. KaSHa: But the forbidden ones would simply be weaker in intensity and should still be observable.

Dr. Platt: They should be down by a factor of 10 to 1,000 just the same as the weak bands in the aromatics.

Dr. PORTer: I thought it was even more than that, the calculated $f$ values were I think a good thousand down.

Dr. Platt: Yes, but they don't make a count of vibrations. Vibrations are equally allowed in the aromatics. There is only a factor of ten or so. 
Dr. Pokike: II it's only a factor of ten, I would think we might sec them, but I would not say that we do not see them. We do, in fact, if you look at the tracing of anthracene, give a longer wavelength transition than the one you see, but you have to make the principal one a complete blackout to see it. There are some weaker long wavelength transitions.

Dr. Kasıa: Secondly, on the phenanthrene transfer to naphthalene, I believe that the Focrster condition for resonance transfer is perfectly satisfied because the phenanthrene emission comes at about 4700 Angstroms which is well within the naphthalene singlet-triplet absorption region. I think a test of your contention would be to use phenanthrene-anthracene which would have a blank in the region of phenanthrene emission.

Dr. Porter: We have done that actually and a number of others as well. Transfer from phenanthrene to anthracene is very efficient. There is no correlation with the overlap calculated by the Foerster theory. Furthermore, you say that conditions are perfect, but they are not, because the Foerster mechanism preclicts a very low probability for triplet-singlet, singlet-triplet transfer.

Dr. Kasha: The Foerster spectral overlap conclitions are satisfied. As to the excited state coupling mechanism, one might anticipate a different r-dependence.

Dr. PORTER: Well, except that you have a condition of spin transfer. This makes the probability so low that the transfer distance becomes very small and I think exchange transfer will predominate.

Dr. Kaslla: I want to raise one more question. This is about the viscosity dependence of the radiationless process. I would tend to agree with Whiffen just because it would imply conservation of angular momentum as an important process in singlet-triplet intramolecular conversion. On the other hand, your ideas would suggest that the spectral features should show a viscosity dependence as well, I think. Or don't you think so? In other words, either the triplet-triplet or the triplet-singlet absorption emission spectra should then become strongly viscosity-dependent if you are saying that the potential function actually changes with viscosity.

Dr. PORTrR: I agree, but what I am not sure about is how much. There is a viscosity elependence in our triplet-triplet absorption spectra. In the first spectra of Windsor we pointed out that as one goes to paraffins and alcohols and so on, irrespective of the chemical constitution of these things, there is a gradual shift which correlates with viscosity rather than with anything else of the triplet-triplet absorption spectrum.

DR. KAsma: It is not so much the frequency which should change, or the spectral envelope.

DR. Portre: Well, of course, there is not a great cleal of detail in the absorption. It depends upon how much change is expected. There is a shift in the thing as a whole. I dont think the spectral data are precise enough to rule out a change in the potential energy diagram.

1) R. Insscant: In the quenching of the triplet state by various agents, I 
just want to point out that the spin of the quencher plays no role if the quenching process takes place by charge transfer from the triplet to the quencher or vice versi, because then the charge will be transferred in the complex with the same spin.

Dr. PORTf.r: If that is the mechanism.

DR. Lusscinc: But that is, in fict, the mechanism.

Dr. PORTER: You would then expect a correlation with redox potential or something like that, which you don't get.

Dr. Linschitz: But you do.

Dr. Porter: I don't. And you would expect diamagnetics to quench as well.

Dr. Lisschitz: Which you do find.

Dr. Porter: Which ones?

DR. LiNSChitz: Quinones and hydroquinones.

Dr. Porter: Ah, but other mechanisms become possible there.

Dr. SELIGER: Why, in the case of phenanthrene-naphthalene triplet-triplet quenching could it not be explained by a resonant energy transfer from the excited phenanthrene singlet to the naphthalene singlet and then to the naphthalene triplet?

Dr. Porter: You need an energy considerably greater than kt to go from the phenanthrene singlet to the naphthalene singlet. You just haven't got enough energy to make the transition and the system was chosen for that reason. This is an unequivocal case. There are others that we studied which were not unequivocal in this sense. 



\section{Part II}

EXCITED STATES OF MOLECULES OF BIOLOGICAL INTEREST 



\section{JaMes Franck \\ Department of Chemistry, Luiversily of Chicago (Fels Fund), Chicago, lllinois}

Apparently it is expected that the moderator makes a few general introductory remarks, comnected with the topics of the session. My own experience on excited states of molecules of biological interest concerns only those molecules which are important in the process of photosynthesis, i.e., the pigments of the chloroplasts, which, together with certain prosthetic groups, are adsorbed at proteins. According to views often expressed, the property of being a semi-conductor is ascribed to protein, and is assumed to be utilized in photosynthesis. Electrons liberated by photo-ionization of chlorophyll molecules are supposed to migrate through the protein to certain reaction centers. According to my own opinion, protein molecules are not semiconductors. All physical evidence available indicates that energetically low-lying conductivity bands are absent in protein. We may refer, e.g., to the survey on this subject matter written by Michael Kasha in the Review of Modern Physics, 1959. Observations on migration of energy through protein can be explained better as excitation energy transfer. Buecher observed that in the hemoglobin-CO complex, energy absorbed anywhere in the protein can be utilized for photochemical splitting of the complex. For many years this was regarded by biochemists as convincing proof for the occurrence of electron migration through protein. Actually, it has all the earmarks of migration of excitation energy. The two ways of excitation energy transfer, I) the phenomenon of sensitized fluorescence, and 2) that of exciton movement in crystalline and quasi-crystalline arrays of molecules, are known for nearly 40 , resp., nearly 30 years. It needed time and nany newer observations with complex organic molecules before the biochemists began to consider that these processes may be of importance for an understanding of certain biological problems. According to my view, their role in photosynthesis is still under-estimated. First, they provide the mechanism by which light energy absorbed by all plant pigments is transferred to the one whose absorption spectrum extends farthest to long wavelengths. Secondly, the same mechanism is utilized for the transfer in the particular dye itself, from the region of its own absorption act to the few places where the excitation energy is utilized for photochemical reactions. 


\title{
EXCITED STATES OF PROTEINS
}

\author{
G. WVEBER \\ Department of Biochemistry \\ Sheffield University, Sheffeld, England
}

In this survey the concept of the "excited state" is restricted to those states that result from the absorption, and are potentially capable of the emission, of radiation. Such states have a lifetime determined in principle by radiation theory and include both electronic and vibrational states. Knowledge of the vibrational excited states of proteins is at present virtually nil because of the experimental difficulties attaining their investigation. In turn, the electronic excited states may be expected to be either singlet states with a radiation lifetime of the order of millimicroseconds, or metastable states with lifetimes of milliseconds to seconds, states that after the original investigation of Lewis and Kasha (9) have been the object of considerable investigation by Livingstone (12), Linschitz and Sarkanen (10), and Porter (20). Interesting observations upon such metastable states of amino acids and proteins have been made by Debye and Edwards (4), Grossweiner (7), and Steele and Szent-Györgyi (21). Knowledge about them is, however, much more restricted than is that of the singlet states, which is already being used successfully in many problems of protein structure and function. In this summary, I shall restrict attention to those states that are brought about by absorption of light of $200 \mathrm{~m} \mu$ or longer wavelength. These excited states belong to one of three groups of absorbing species: (i) the aromatic amino acids; (ii) groups occurring naturally in certain proteins (heme, flavin mononucleotide, flavin adenine dinucleotide. carotenoids) or bound reversibly and specifically to certain proteins (DPNH and its analogues); and (iii) groups artificially attached to the protein by chemical linkage such as the anthracyl isocyanate conjugates (3) fluorescein isocyanates (2), or the dimethylaminonaphthalene conjugates (25).

Before attempting to discuss the phenomena observed in the proteins, it will be useful to discuss the propertics of the excited states of the components just mentioned. 


\section{Aromatic Amino Acins}

The absorption spectrum of each of the aromatic amino acids presents in the region of $20(0-300) m_{\mu}$ two absorption bands, a weak one at a longer wavelength and a stronger one at a shorter wavelength. These appear to correspond respectively to the two types of Platt's transitions: L (multipolar, weak) and B (dipolar, strong). The fluorescence of each compound consists in each case (23) of a single umresolved symmetric band which in water solution has a maximum at 282 $m \mu$ in phenylalanine, $303 \mathrm{~m} \mu$ in tyrosine, and $348 \mathrm{~m} \mu$ in tryptophan. Entirely similar bands are observed in the parent compounds benzene, phenol, and indole. The variations of the fluorescence spectrum with solvent composition are best studied with the parent compounds because of the insolubility of the dipolar molecules in non-polar media. In water, none of the spectra show any sign of vibrational structure; and this is true also of phenol and indole even when in hexane solution, in which the vibrational structure of the indole absorption spectrum is clearly discernible. While phenol shows virtually no change in the position of the fluorescence maximum in the different solvents, the fluorescence maximum of indole undergoes marked changes, appearing at $325 \mathrm{~m}_{\mu}$ in hexane and $340 \mathrm{~m}_{\mu}$ in methanol and other alcohols. These variations are due to dipole interaction between the solvent molecules and the excited molecule, and can be used with the help of a theory of Lippert (11) to determine the increase in dipole moment in the excited state over that of the ground state. The spectral shift is found to be dependent upon the dipole strength and upon the quantity

$$
\Delta f=\frac{D+1}{2 D+1}-\frac{n^{2}+1}{2 n^{2}+1}
$$

where $D$ is the dielectric constant and $n$ the refractive index of the medium, a quantity that determines the interaction energy between the excited molecular clipole and the induced or permanent dipoles of the solvent molecules, according to the theory of Onsager (14). Conversely, if the dipole strength in the excited state is known, by the determination of the fluorescence spectrum in a series of solvents the $\triangle f$ value of an unknown medimm can be determined. Thus in a protein the polarizability properties of the region surrounding the residues may be estimated from the fluorescent spectrum, and the same considerations apply to groups either chemically attached or physically adsorbed (e.g., Weber, 27). The quantum yield of the aronatic amino acids in water solution is 0.04 for phenylalanine, 0.21 for 
tyrosine, and 0.20 for tryptophan. White (30) has studied the influence of $p \mathrm{H}$ upon the fluorescence efficiency of tyrosine and tryptophan. From her studies it appears that protons can quench the fluorescence of both rings, and that the potential source of the quencher protons must be sought also in the $\mathrm{COOH}$ and $\mathrm{NH}_{3}{ }^{+}$ groups in the amino acid. Thus both tyrosine and tryptophan are

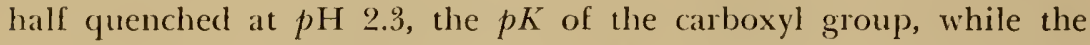
esters and the decarboxylated derivatives are quenched only at $p \mathbf{H}$

\section{ACID QUENCHING OF FLUORESCENCE OF TYROSINE DERIVATIVES}

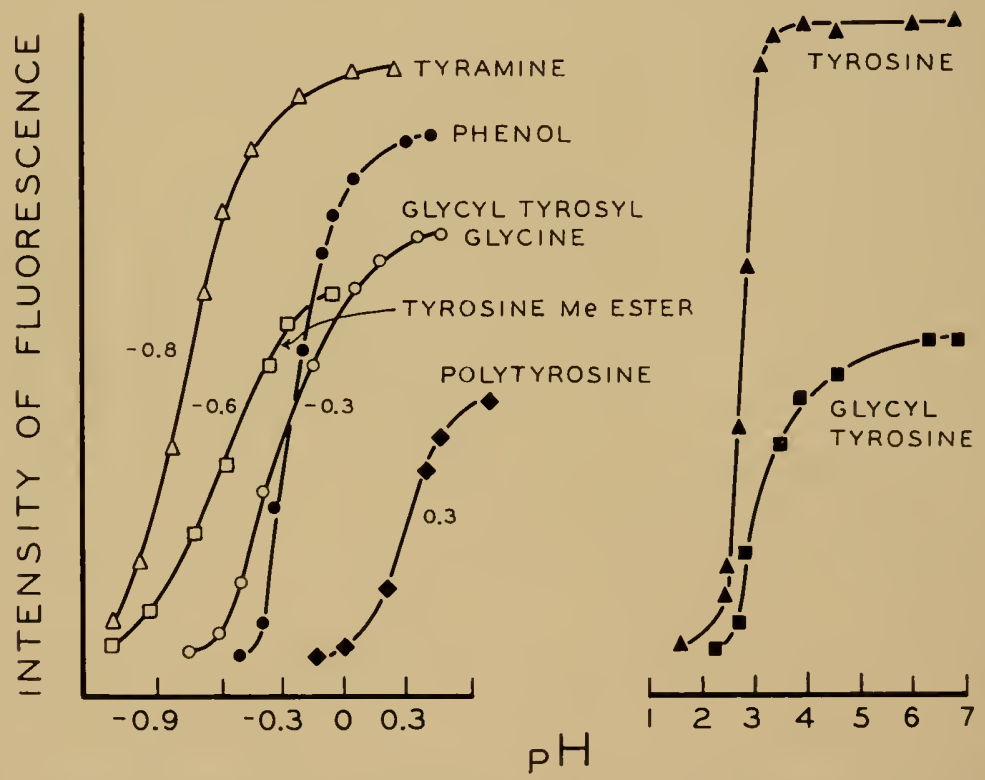

lig. 1. Effect of $/ 1$ upon the fluorescence of Iyrosine and derivalives (acid range).

-0.55 in the phenol series and at $p H 0.5$ in the indole series (Fig. I) In the alkaline range the phenolic derivatives lose all fluorescence upon dissociating to give the phenolate ion. Tryptophan and some tryptophan derivatives, though not indole, show a conspicuous increase in fluorescence in the $p \mathrm{H}$ region where the $\mathrm{NH}_{3}{ }^{+}$groups are discharged, and in the $\mathrm{NH}_{2}$ form can have absolute fluorescence efficiencies of the order of 0.6 (Fig. 2). Thus the excited indole ring is able to attract a proton from the $\mathrm{NH}_{3}{ }^{+}$in the side chain, in about 50 per cent of the excitations. In agreement with these ideas 
OCCURRENCE OF PEAKS IN

ALKALINE QUENCHING CURVES

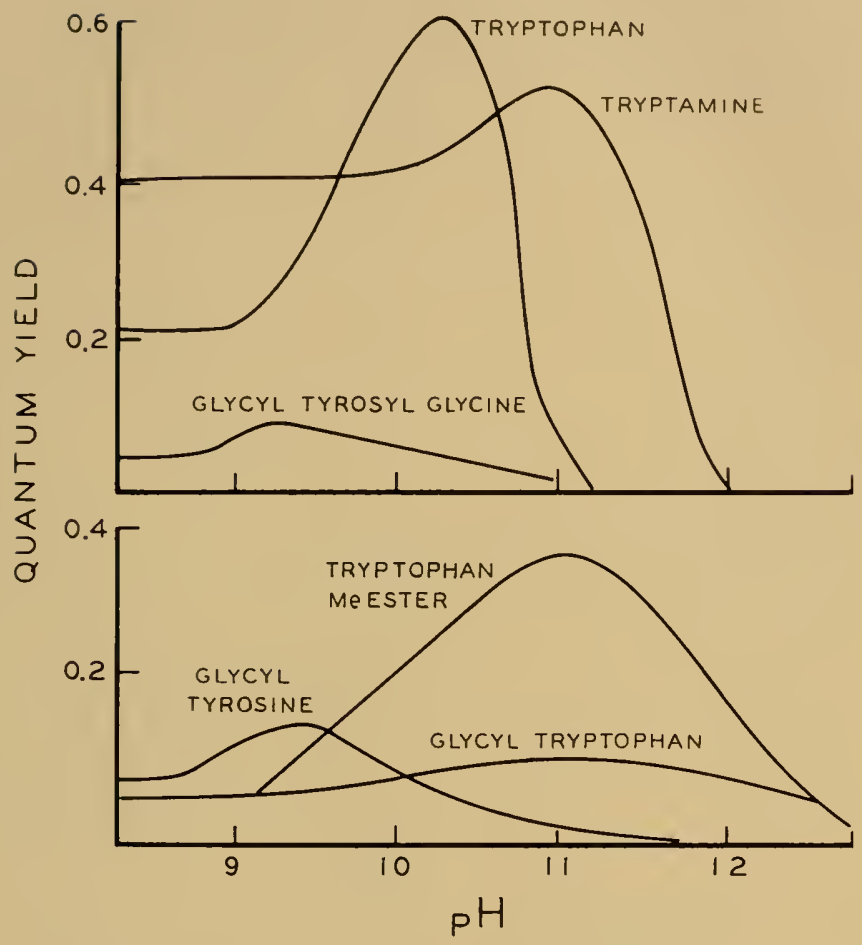

Fig. 2. Viffect of pH "1pon the fluorescence of indole and phenol derivatives (alkaline range).

it is possible to observe quenching of the fluorescence of indole and phenol by the addition of such simple substances as acetic acid and hydroxylamine (Fig. 3), and the interpretation of their quenching effects seems, in most cases at least, to be that of a straightforward proton transfer to the excited ring. Finally, tryptophan and indole show quenching of the fluorescence in alkaline solution $(\mathrm{NaOH}$, $0.01 M$ and higher). In accordance with ideas developed by Boaz and Rollefson ( 1 ) and by Förster (6), we have looked upon this as a reaction of the excited molecule with hydroxyl ion to yield the $\mathrm{N}$ compound, which undergoes a radiationless transition to the ground state before recombining with water to regenerate the uncharged indole and a hydroxyl ion. The postulated series of reactions is as follows: 
QUENCHING OF FLUORESCENCE BY HYDROXYLAMINE

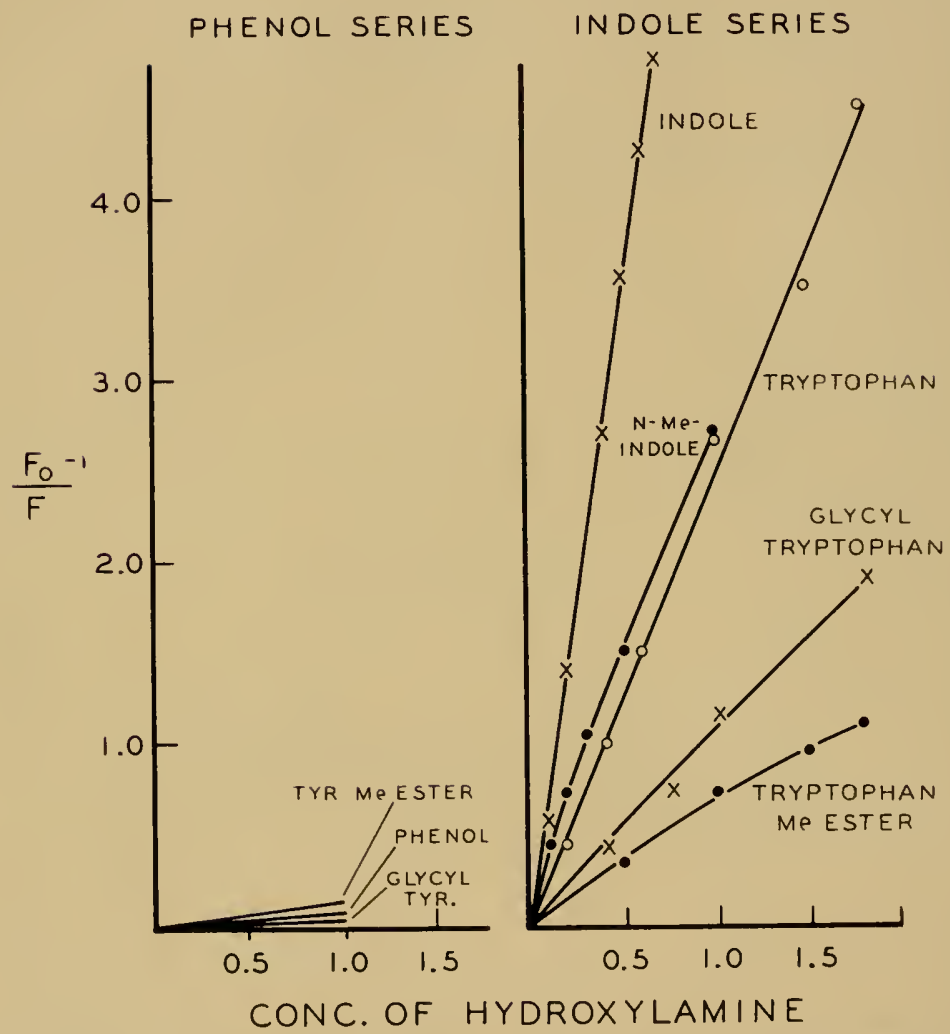

Fig. 3. Quenching of the fluorescence of phenol and indole derivatives by bydroxylamine. Abscissa, molarity of hydroxylamine. Ordinate: $F_{o}$, original fluorescence efficiency; F, fluorescence efficiency upon addition of hydroxylamine.

$$
\begin{aligned}
& R-\mathrm{NH}+h_{\nu} \rightarrow R \mathrm{NH}^{*} \\
& R \mathrm{NH}^{*}+\mathrm{OH}^{-} \rightarrow R \mathrm{~N}^{*-}+\mathrm{OH}_{2} \\
& R \mathrm{~N}^{*-} \rightarrow R \mathrm{~N}^{-} \text {(radiationless transition) } \\
& R \mathrm{~N}^{-}+\mathrm{OH}_{2} \rightarrow R \mathrm{NH}+\mathrm{OH}^{-}
\end{aligned}
$$

where $R \mathrm{NH}$ stands for the indole nucleus. The requirement of a mobile hydrogen for the reaction is demonstrated by the fact that $\mathrm{N}$-methyl indole is not quenched in alkaline solution. It may be pointed out that similas reactions with the hydroxyl ion have been postulated by Rollefson and by Förster for the naphthylamines, and 
that these molecules also exhibit, in common with indolc, large shifts in the fluorescence spectrum upon change of solvent. Thus in either case the contribution of ionic structures to the excited state is very important. It reaches nearly 50 per cent for some naphthylamine derivatives (Weber, unpublished) and is only slightly smaller in the case of indole. In these ionic structures the forms
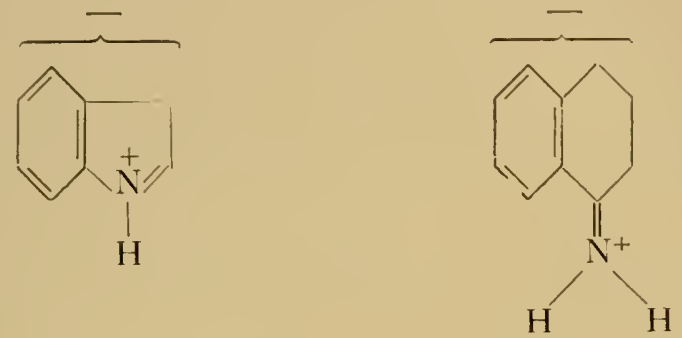

must make the greatest contribution in either case, and the reaction with the hydroxyl ion in the excited state receives a satisfactory explanation.

The interaction of the excited ring with proton carrier groups like COOH and $\mathrm{NH}_{3}{ }^{+}$receives also confirmation from experiments with glycyl peptides of both tyrosine and tryptophan. In these it would be expected that the short-range interaction between the relatively far away $\mathrm{COOH}$ or $\mathrm{NH}_{3}{ }^{+}$and the excited ring will require a rotational diffusion of the side-chain to bring the appropriate group into contact with the ring. Both glycyltryptophan and glycyltyrosine have low quantum yields (in the neighborhood of 0.04 ), which are increased to almost the normal value of 0.2 in a medium of high viscosity such as propylene glycol at low temperature (Teale, unpublished) .

\section{Fluorescence Excitation Spectrum}

This is coincident with the absorption spectrum over the range of wavelengths that has been investigated $(210-310 \mathrm{m \mu})$.

\section{LifETINE OF THE EXCITATION}

Direct measurements of this quantity are not yet available. By measurements of oscillator strength and application of the well-known radiation equation, the natural oscillator lifetime of tyrosine (and also of cresol and phenol) is found to be nearly $40 \mathrm{~m} \mu \mathrm{sec}$, while that of inclole tryptophan is $12 \mathrm{~m} \mu \mathrm{sec}$. With quantum yields of 20 per cent the respective lifetimes in water would be 8 and $2.5 \mathrm{~m} \mu \mathrm{sec}$ re- 
spectively. Measurements of the polarization of the fluorescence in viscous solvents, and the use of Perrin's equation (17), are in qualitative agreement with these figures; but since rotational relaxation times cannot be accurately assigned to the molecules in the viscous solvents used, the method cannot be made to yield precise values.

\section{Polakization of the Emitted Fluorescence}

Polarization measurements in viscous solvents can be used to obtain information on two important points: measurements of change in polarization with concentration at a fixed wavelength can be used to give information upon the transfer of electronic energy among like molecules $(16,18)$, while measurements at vanishing concentration and different exciting wavelengths can yield information about the relative directions of the transition moments of the different electronic bands to supplement and often clarily the absorption spectrum. To begin with the latter, this so-called polarization spectrum is best studied in rigid solutions at low temperature. We have used propylene glycol solutions at $-70^{\circ} \mathrm{C}$. Phenol solutions, and also those of tyrosine and cresol, show a polarization spectrum consisting of two regions, one positive at longer wavelength and one negative at shorter wavelength (Fig. 4). Calculation of the angle between the two transitions from the fluorescence data (28) with the aid of the equations of Jablonski, and on the assumption that the two main transitions are contained in the plane of the benzene ring, gives a value of

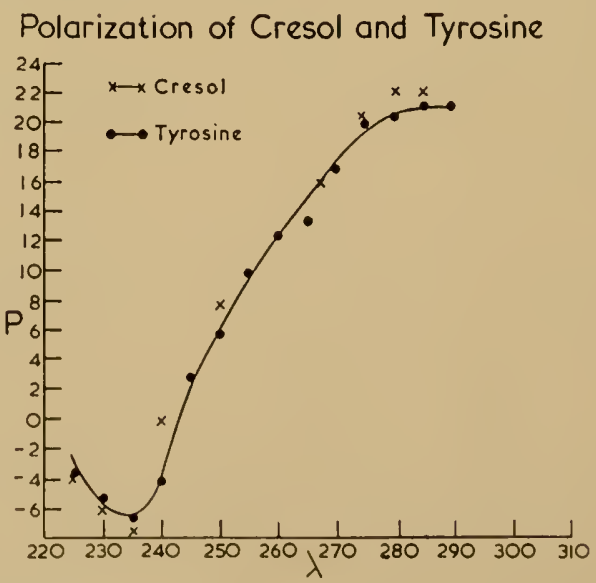

Fig. 4. The polariation spectra of tyrosine and cresol. P, polarization. $\lambda$. watelength. 
$88^{\circ}$. Thus the simple picture of two transitions at a right angle to each other, as suggested by the character of the transitions themselves (19), is experimentally confirmed. The maximum polarization (0.22) observed at $270 \mathrm{~m} \mu$ and longer wavelengths is still considerably below the theoretical maximum of 0.33 for the linear anisotropic oscillator, and it may be doubted whether this large difference can be entirely due to the depolarizing effect of torsional molecular vibrations, as Jabłonski (8) suggested.

The polarization spectrum of the fluorescence of indole and tryptophan (Fig. 5) is considerably more complex than that of tyrosine (28). No negative polarizations are observed at wavelengths of excitation of $220 \mathrm{~m}_{\mu}$ or longer. A region of positive polarization, with a flat maximum at $270 \mathrm{~m} \mu$, is followed by a sharp decrease in the polarization, with a minimum accurately corresponding to the spike (indole) or shoulder (tryptophan) observed in the absorption spec-

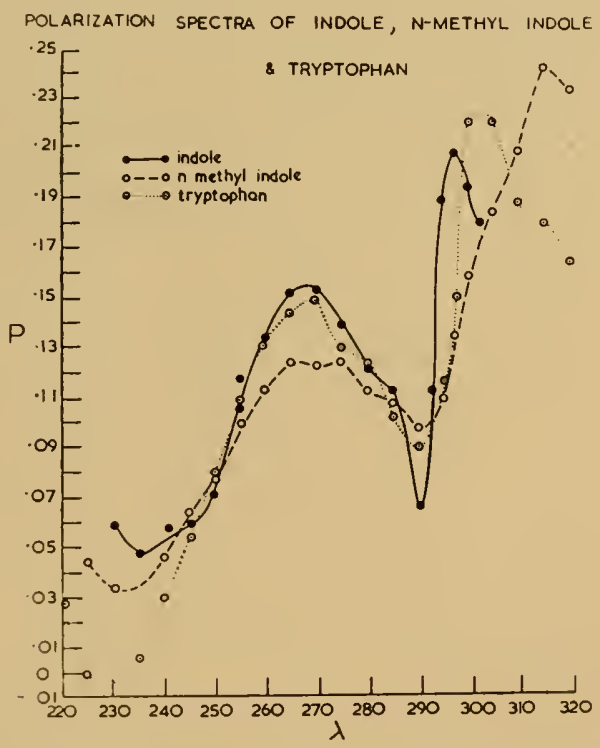

Iig. 5. Polarization spectra of indole, N-methyl indole, and tryptophan.

trum. The correspondence of the region of decreased polarization with the spike of indole shows that this is an independent electronic transition, with a transition moment at a large angle to the transition direction of the $270 \mathrm{~m} \mu$ band. In Platt's theory (19) of the electronic transitions of the aromatic hydrocarbons, the $L_{a}$ and $L_{b}$ 
transitions would be expected to have such a relation, and on the basis of the relative position, oscillator strength, and polarization, the $270 \mathrm{~m}_{\mu}$ and $295 \mathrm{~m}_{\mu}$ peaks can be provisionally identified with these two transitions.

At wavelengths longer than $300 \mathrm{~m} \mu$ the polarization rises very rapiclly, consiclerably surpassing the value for the $270 \mathrm{~m}_{\mu}$ peak. It is possible to account for this increase without assuming the existence of an independent electronic transition at this and longer wavelengths, as is shown by the Franck-Condon scheme of Fig. 6. G-S $S_{1}$ and $G-S_{2}$ are the two $L$ transitions already postulated. Wavelengths shorter than $270 \mathrm{~m}_{\mu}$ correspond to points above $V_{2}$ where excitation falls upon

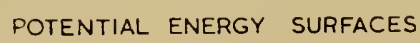

IN INDOLE

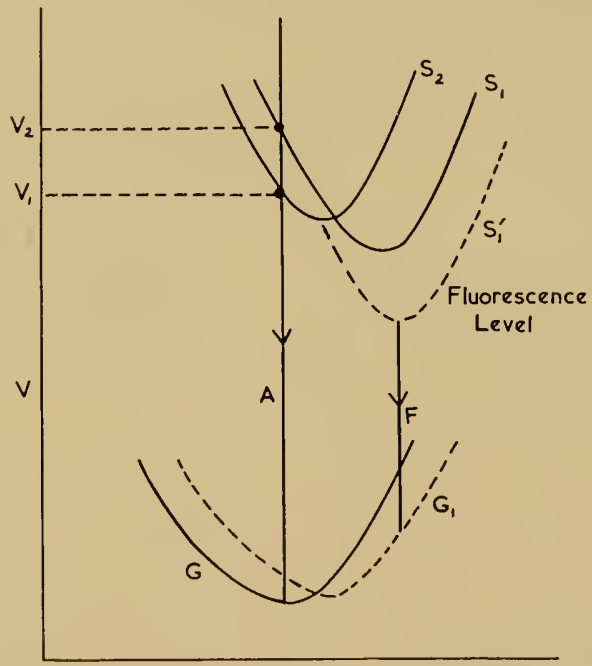

rig. 6. Potential energy surfaces in indole.

both bands but predominantly upon $G-S_{1}$, the main absorption band. Here the polarization increases monotonically. Between $V_{2}$ and $V_{1}$ the excitation to $G-S_{1}$ decreases, while that to $G-S_{2}$ reaches a maxi$m m m$ at $286 m_{\mu}$. Points below $V_{1}$ are those where excitation of $G-S_{2}$ is no longer possible, but excitation to $G-S_{1}$, although very weak, is still Franck-Condon allowed. The decrease observed in the polarization spectrum at still longer wavelengths than $305 \mathrm{~m} \mu$ has probably a different origin. Excitation here is probably restricted to molecules which are in levels above the zero vibrational level of the ground 
state. The molecular deformation caused by asymmetric vibrations results in a change in the direction of the oscillator of absorption as compared with the direction in a collection of molecules which are in the zero vibrational level. In emission the two types of molecules camnot be distinguished, since during the lifetime of the excitation, themul equilibration has an ample chance of occurring so that the enitting molecules are in both cases a collection of molecules predominantly in the zero level of the singlet excited state.

Tryptophan, indole, and $\mathrm{N}$-methyl indole show similar polarization spectra, with only minor differences between them. On the other hand, N-glycyl tryptophan shows an extra polarization band with a bandwidth of $10 m \mu$ and maximum at $290 m_{\mu}$ (Fig. 7). The

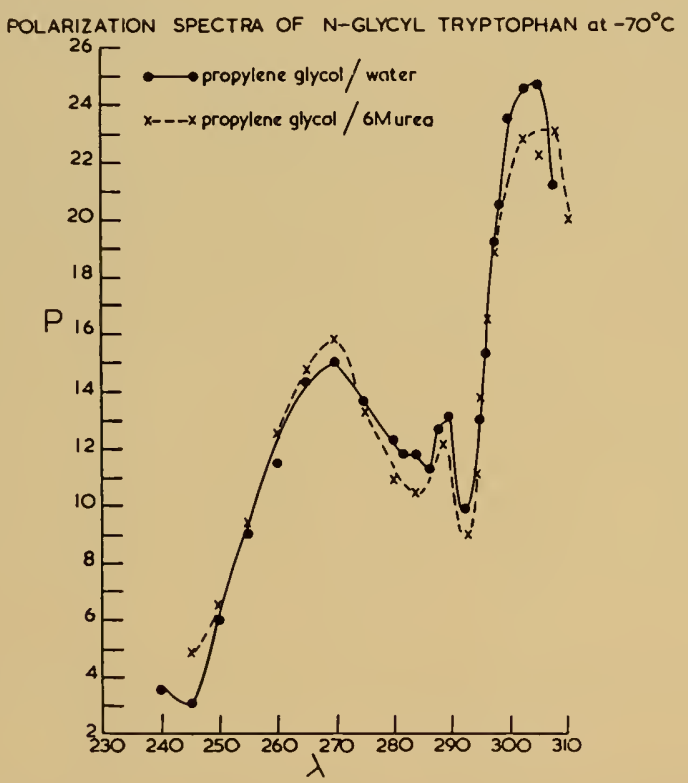

Fig. 7. Polarization spectra of $\mathrm{N}$-glycyl tryptophan at $-70^{\circ} \mathrm{C}$ in different solvents.

origin of this extra band is obscure. It is certain that it is not due to an impurity, since it is also present in most of the tryptophancontaining globular proteins so far examined.

The variations of fluorescence polarization with concentration have been studied in solutions of indole or phenol in propylene glycol at $-70^{\circ} \mathrm{C}$, examined in very thin layers (30 microns thickness). Examination of the concentrated solutions in these very thin layers 
is indispensable if the radiative transfer of the fluorescence is to be avoided, so that the depolarization observed may be unequivocally attributed to radiationless transfer of the excitation among the phenol molecules. Fig. 8 shows the different results obtained when thick and thin layers of phenol solutions are used. It has been found empirically (18) and it can be analytically derived (26) that the reciprocal of the linear polarization of the fluorescence is a linear function of the concentration. The figure shows that such a relation does hold

\section{CONCENTRATION DEPOLARIZATION OF PHENOL SOLUTIONS}

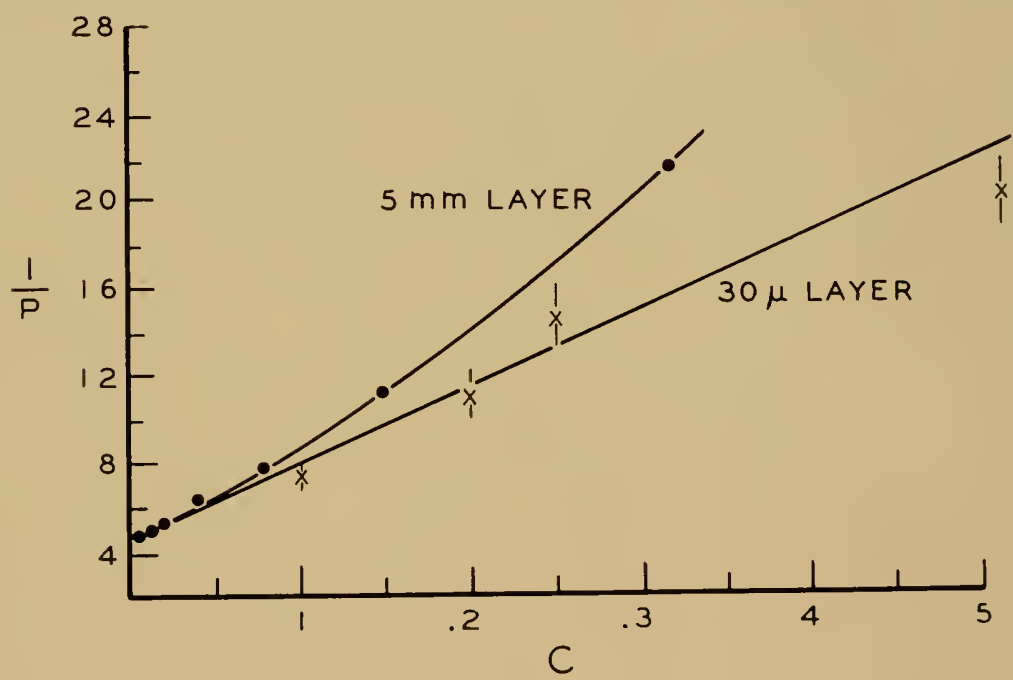

Fig. 8. Concentration depolarization of phenol solutions. Ordinate: 1/l', reciprocal of the linear polarization. Abscissa, molarity of indole.

for very thin layers, but that in thick layers an extra source of depolarization is found, one which can be attributed to radiative transfer, or, as it has been graphically described for the gas phase case, the imprisonment of the emitted radiation. The slope of the plot of the reciprocal of the polarization $(I / P)$ against molar concentration $(C)$ permits the calculation of the constant $R_{o}$ which is defined as the distance between two parallel molecules at which the excited state has a 50 per cent chance of migration from the excited to the unexcited onc. The distances thus derived may be compared with those predicted by the theory of Förster (5) on the 
basis of the overlap of emission and absorption bands and the oscillator strength of absorption of the acceptor molecule. An exact comparison of the experimental and theoretical values is made dilficult by the fact that Förster's theory utilizes the idealized model of two molecules isolated from the rest of the molecules in the solution, while in practice the value determined corresponds to a probability of repeated transfers not between isolated pairs of molecules but over a chain of molecules which camnot be broken up into independent pairs. Comparison of the values experimentally determined with those expected from Förster's theory shows that the overlap of absorption and emission (29) and the absolute oscillator strength of the absorption are indeed the parameters controlling the value of $R_{o}$, which has the magnitude predicted by the theory. Nevertheless, the theory predicts a spread of values between 10 and $100 \mathrm{~A}$, while in practice the observed values appear to cover a more restricted range, between 15 and $40 \mathrm{~A}$. For phenol, $R_{o}=17 \mathrm{~A}$, while the calculated value does not exceed 11 A. It must be stressed that these differences can in no case be attributed to experimental error, since $R_{0}$ is determined from the sixth root of the slope of the

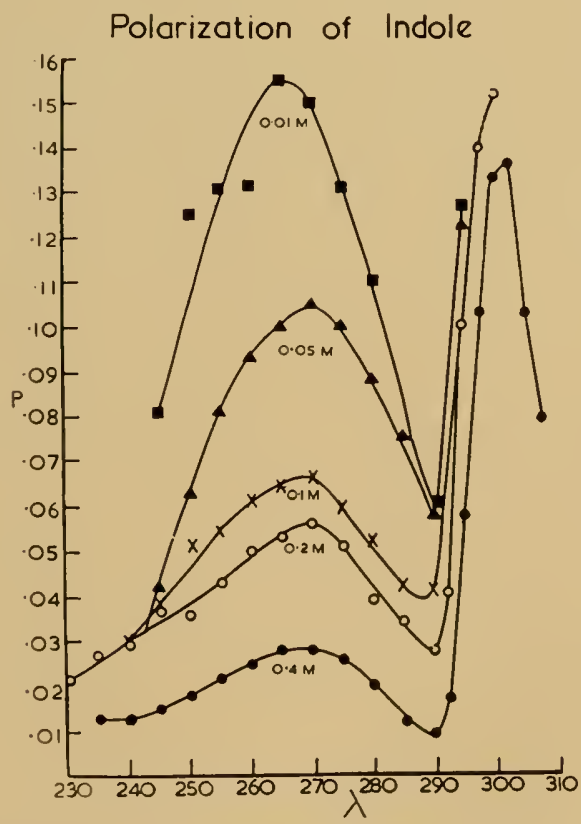

Fig. 9. Polarization spectra of indole at different concentrations. 
plot of the reciprocal of the polarization against the concentration and can therefore have significance to a fraction of an A unit.

Observation of the polarization spectrum at different concentrations indicates that the depolarization of the fluorescence is independent of the exciting wavelength in the case of phenol, though not in the case of inclole (Fig. 9). In the latter instance, excitation with wavelengths shorter than about $290 \mathrm{~m}_{\mu}$ results in transfer with $R_{o}=14 \mathrm{~A}$; but transfer is not conspicuously observed upon excitation with wavelengths longer than $305 \mathrm{~m} \mu$. From these measurcments, it appears that once the molecule has reached the zero level of the excited state of $S_{1}$, transfer to another indole molecule is improbable, and that it occurs only through the momentary thermal excitation to $S_{2}$ (improbable at $-70^{\circ} \mathrm{C}$ ) or during the thermal equilibration of the vibrational energy when the molecule is excited to $S_{2}$ or higher levels.

\section{Excited States Belonging to Spfecifically Bound Groups}

Since these are cliscussed in detail in the symposium by Dr. Velick, I will not attempt to cleal with them here.

\section{Excited States of Artificially Coupled Moieties}

Of these, the only one that has been studied from the points of view of interest to us are the conjugates of proteins with dimethylamino naphthalene sulfochloride (DNS) $(25,22)$. The lifetime of the excited state of DNS-conjugates with different proteins ranges from 12 to $15 \mathrm{~m} \mu \mathrm{sec}$. The fluorescence spectrum of simple DNS derivatives, e.g., the sulfonamide, shows very considerable displacement toward longer wavelengths in media of high dielectric constant. Fig. 10 shows this fact for mixtures of dioxane and water. A Lippert plot of the wave number difference between the maxima of absorption $v_{a}$, and fluorescence $v_{f}$ against the $\Delta f$ value shows the predicted straight-line relationship, from which it may be conclucled that the dipole moment in the excited state is higher than that of the ground state by $10-12 \mathrm{D}$ units and corresponds to a contribution of the ionic forms to the excited state of over 50 per cent. The absorption maximum of the last band is found in dioxane at $340 \mathrm{~m} \mu$ and in $80 \%$ water-20\% dioxanc at $3.92 \mathrm{~m} \mu$. Such changes can be correlated with the proton acceptor character of the $\mathrm{NH}_{2}$ in hyclrogen boncling, and the large contribution of the ionic form 


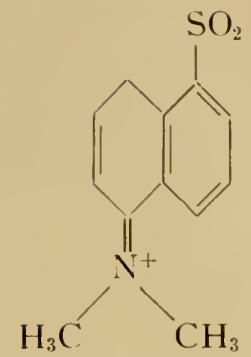

to the lowest excited state. The emission and absorption spectra can give information upon the properties of the enviromment in the protein-DNS conjugates.

\section{Excited States in the Protein}

Simple proteins: these can be divided into two groups: Class A, containing phenylalanine and tyrosine; and Class B, containing tryptophan as well. The contribution of phenylalanine to the total absorption is very small, and since its fluorescence yield appears to be also very small qualitatively, only tyrosine and tryptophan fluorescence is observed.

Class A proteins include insulin, tropomyosin, zein, and ribonuclease, besides several trypsin inhibitors. The first four proteins mentioned have quantum yields in the neighborhood of 0.03 , while the inhibitors have yields much smaller than 0.01 (24). The fluorescence spectrum of this class of proteins is indistinguishable from that of free tyrosine, with a maximum at $303 m \mu$. The polarization spectra, which were studied in $50-90 \%$ propylene glycol water mixtures, at $-70^{\circ} \mathrm{C}$ are essentially those of tyrosine, differing only by the fact that the absolute polarization values are smaller than those of tyrosine or phenol over the whole spectral region (Fig. 10). The conclusion is unavoidable that some transfer of the excited state among residues of tyrosine takes place. Since the distances between the tyrosine and their relative spatial orientation in these proteins is not known, an accurate estimation of the extent of the transfer cammot be given.

Class B proteins, which include the majority of globular proteins, show a fluorescence spectrum which is characteristic of tryptophan, and of tryptophan alone (24). The maxima of emission range from chymotrypsin $(332 \mathrm{~m} \mu)$ to pepsin $(346 \mathrm{~m} \mu)$ (Fig. 11). From these observations one could conclude that in pepsin the tryptophan residues of which the fluorescence is observed are in contact with the water or a medium of similar polarizability properties, while in 
Polarization Spectra of Tyrosine,

Ribonuclease, Insulin, and Zein

in Propylene Glycol at $-70^{\circ} \mathrm{C}$

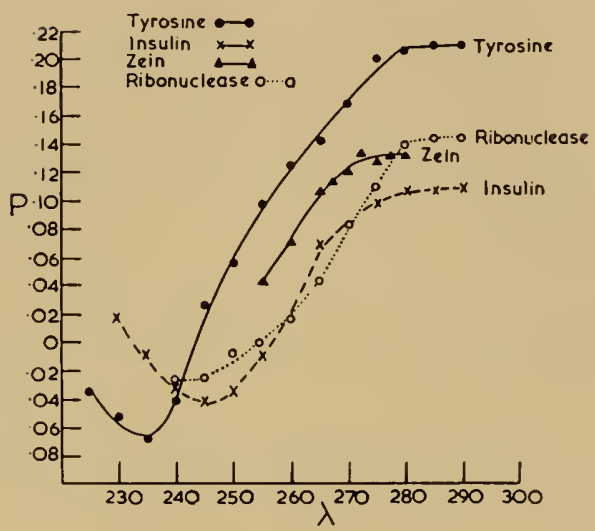

Fig. 10. Polarization spectra of tyrosine, ribonuclease, insulin, and zein in organtc solvent at $-70^{\circ} \mathrm{C}$.

\section{Excitation and Fluorescence Spectra} of Proteins

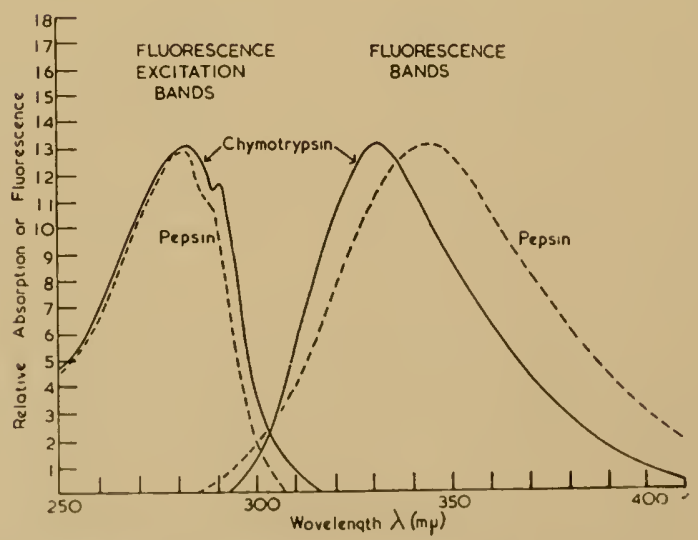

lig. II. Fuorescence excitation and emission spectra of pepsin and chymotrypsin. 
(hymotrypsin they are not in contact with water but with a medium of mainly non-polar character.

We have been unable to discem any contribution from tyrosine to the Class $\mathrm{B}$ proteins. Teale (Biochem. J., in press) has studied with particular care the albumins, human and bovine. Both of these proteins have the lowest (non-zero) relative content of tryptophan, which in human albumin accounts for 16 per cent of the absorption at $280 \mathrm{~m}_{\mu}$, while in bovine albumin it accounts for 31 per cent of it. Photooxidation of the tryptophan residue with methylene blue in visible light results in complete disappearance of the fluorescence without any restoration of tyrosine fluorescence. Urea and heat denaturation fail to induce any appearance of tyrosine emission. Direct evidence of the loss of the quanta absorbed by tyrosine is obtained by a measurement of the changes in absolute quantum yield of the protein with exciting wavelength (Fig. 12). At

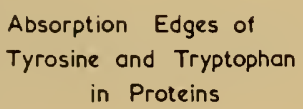

in Proteins

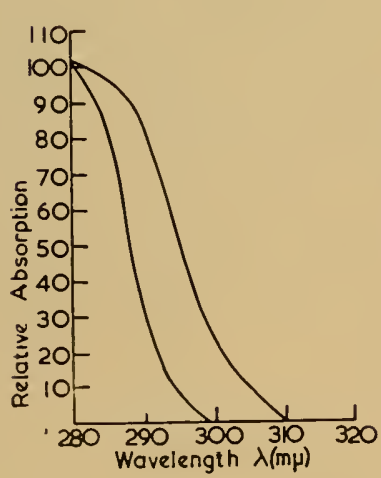

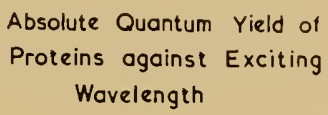

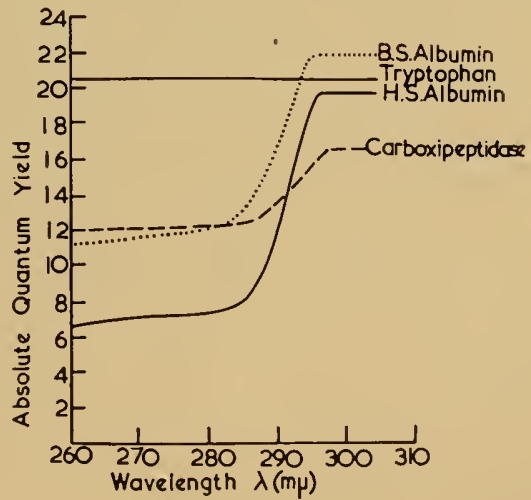

lig. 12. Absorption spectra of iyrosine and iryptophan in proteins, and absolute quantum yield of proteins at various exciting wavelengths.

wavelengths longer than $280 \mathrm{~m} \mu$ the absolute yield increases as the relative number of quanta absorbed by the tryptophan increases. At wavelengths longer than $300 \mathrm{~m} \mu$, where virtually all the absorption is due to tryptophan, the yield levels off, with similar values in both albumins.

Although the exact nature of the process by which the tyrosine 
fluorescence is quenched remains obscure, some important factors have been excluded:

(1) Methylation of the $\mathrm{OH}$ groups of tyrosine with dimethyl sulfate fails to restore the tyrosine fluorescence. Thus transfer of the $\mathrm{H}$ of the $\mathrm{OH}$ group to a hydrogen-bonding structure to yield transitorily the non-Iluorescent phenolate may be excluded.

(2) Recluction of the S-S groups with sodium borohydride followed by further stabilization with iodoacetate to give the S-carboxymethyl derivative does not change appreciably the fluorescence of the protein (Churchich, unpublished) .

The changes in quantum yield of several proteins with $p H$ have been studied by White (31). Particularly interesting is the comparison of bovine serum albumin, containing two tryptophan residues in the molecule, with human serum albumin, containing only one. In the latter, the quenching by $\mathrm{H}$ ions is half completed at $p H \quad 0.5$, while in the former the $p H$ fluorescence curve has two distinct steps, with midpoints at $p \mathrm{H} 3.5$ and $p \mathrm{H} 0.5$. Comparison with free tryptophan and its derivatives is immediate. Of the two residues of bovine albumin one must be located in the vicinity of a $\mathrm{COOH}$ group of aspartic or glutamic acid, while the other, as well as the unique resiclue in human albumin, must be located away from any such groups, so that they are quenched by the protons in the medium in much the same manner as the derivatives of tryptophan in which the COOH group is chemically engaged. Steps on the acid side of the $p \mathrm{H}$ fluorescence curve are also found in lysozyme, which shows steps on the alkaline side as well. These data indicate differences in the accessibility of the different tryptophan residues to protons and are difficult to reconcile with any theory which envisages the free migration of protons over the protein surface.

\section{Fluorliscence Liletime and Polarization}

An estimation of the lifetimes of the excited states in tryptophan in the proteins by methods already discussed gives values of 1-3 $m \mu \mathrm{sec}$. Since the rotational relaxation times of most globular proteins are 20 to 106 times longer (13, 25), a conspicious change in polariztion with temperature or viscosity may not be expected. Polariation meisurements should be useful in the determination of the rotational relaxation times of protein fragments of up to a few thousand in molecular weight.

The polarization spectrum of the fluorescence of proteins of Class B 
is essentially that of tryptophan and its simple derivatives, but with some interesting general differences, as well as characteristic differences among the proteins themselves.

In general, while the ratio of the polarizations observed upon excitation with $305 \mathrm{~m} \mu$ and $270 \mathrm{~m} \mu$ light $\left(p_{305} / p_{220}\right)$ has a value of 1.4-1.7 in tryptophan and the simple indole derivatives, the corresponding ratios in the proteins in cold propylene-glycol-water ranges from 2.2-3 and from 1.8-2.5 in water at room temperature.

The peak observed in $\mathrm{N}$-glycyl tryptophan at $295 \mathrm{~m} \mu$ is present in more than half of the globular proteins examined. The differences observed among the different globular proteins relate to (a) the ratio $p_{305} / p_{2 \pi 0}$, which is variable from one protein to the next; (b) the presence and magnitude of the small band at $295 \mathrm{~m}_{\mu}$, very noticeable in lysozyme, small in the albumins, and virtually absent in ovalbumin; and (c) the fall in polarization in the region of wavelengths longer than $310 \mathrm{~m} \mu$ (Figs. 13-16). In most cases a pronounced fall is observed, which is however less well marked in other albumins than in ovalbumin, where a flat peak at $310 \mathrm{~m}_{\mu}$ is observed. It is of significance that most of these details in the polariza-

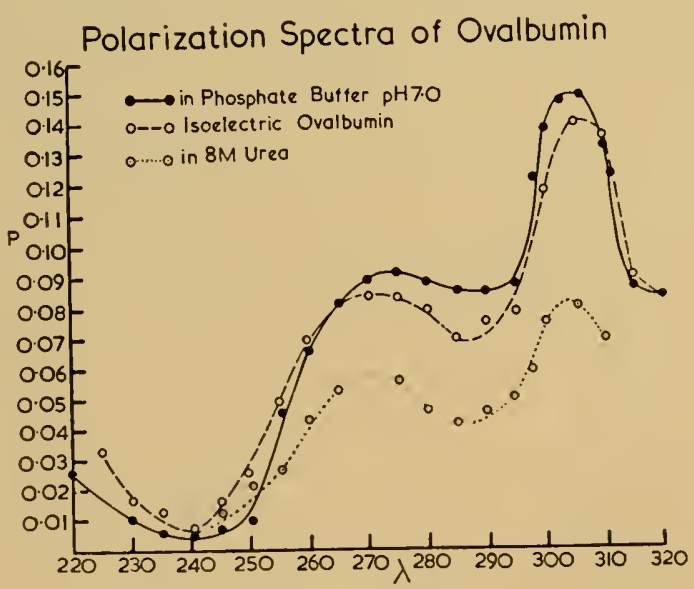

Fig. 13. Polarization spectra of ovallumin under various conditions.

tion spectrum are lost when the proteins are dissolved in urea. The details become much less conspicuous and the ratio $p_{305} / p_{2 \pi 0}$ reaches the values 1.3-1.6. Removal of the urea results in the case of some proteins in an almost complete restoration of the original spectrum (e.g., human and serum albumins), whereas in others, such as rabbit 


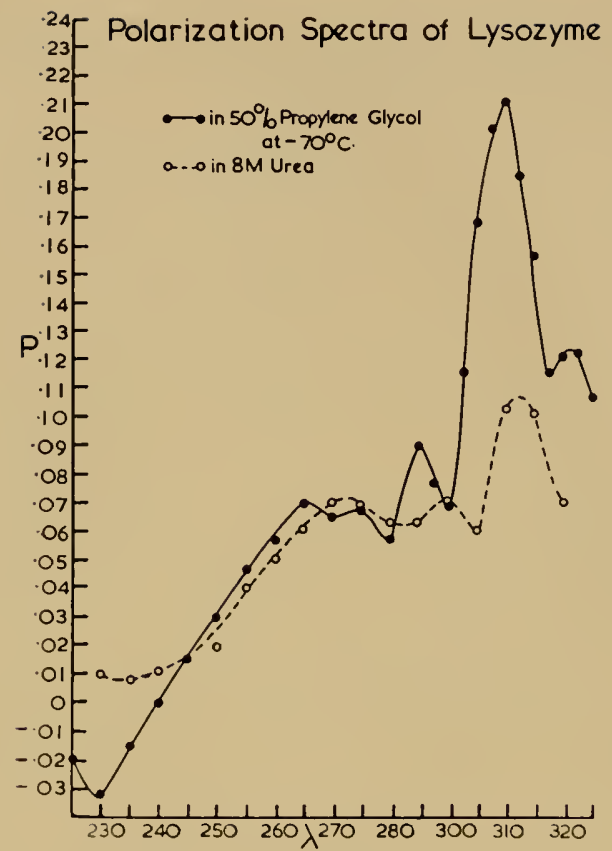

lig. 14. Polarization spectra of lysozyme in cold propylene glycol solient and in urea.

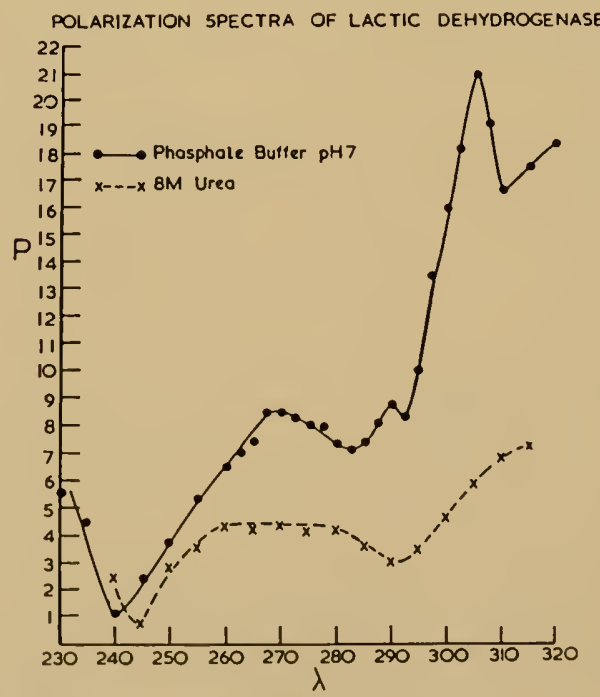

lig. 15. Polatiation spectra of lactic delydrogenase in nentral phosphate bulle: and in urea. 
muscle lactic dehydrogenase, it bears no great relation to that of the native protein. Perhaps the most interesting fact about the polarization spectrum of protein fluorescence is that a resolution of the order of $1 m_{\mu}$, as employed in these measurements, was necessary to reveal some of the details present, and that one gathers the impression that by further improrement in resolution and by the use of a contimuously recording method some more detail could be revealed. The interpretation of the polarization of the fluorescence of proteins from

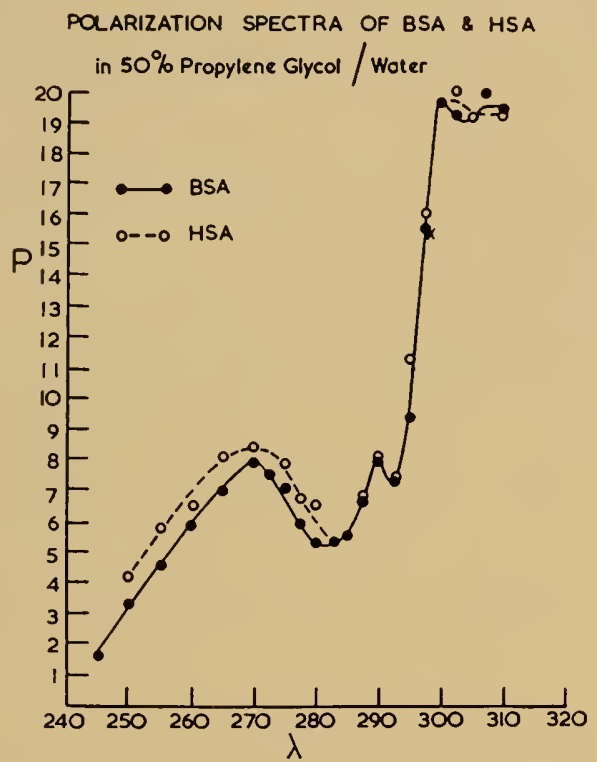

lig. 16. Polarization spectra of bovine serum albumin and of human serum albumin.

that of the simple derivatives is anything but straightforward. An increase in the ratio $p_{305} / p_{2 \pi 0}$ should not be taken as proof of an internal transfer of energy among the tryptophan residues, since a high ratio (I.8) is observable in human serum albunin, in which there is only one tryptophan. The effect of $p \mathrm{H}$ on bovine serum albumin, already mentioned as indicating that each tryptophan can be quenched independently, is strongly against the occurrence of any transfer between the two tryptophans of this protein; and the same catn be said of lysozyme. Nor does the transfer of energy from tryptophan to a uniquely attached substance, as in the case of DPNH and lactic dehydrogenase described by Velick (24a), constitute 
proof of an inter-tryptophan transfer, since each tryptophan resiche may be considered to lie within the distance from DPNH at which a considerable transler may be expected.

The large $p_{305} / p_{2 \pi 0}$ ratio, as well as its decrease in concentrated urea solutions, can be best explained as the result of interactions between the tryptophan residues and the protein backbone (28). In future work on this problem, one should aim at answering the question of the specificity of this interaction, and the participation of definable structural characteristics of the protein in the production of the details just mentioned. The importance of such studies for the knowledge of protein structure need not be stressed.

\section{Artificially Conjugated Proteins}

The studies carried out upon dimethylaminonaphthalene conjugates of proteins by Weber (25) and by Steiner and McAllister (22) have established the usefulness of fluorescence polarization in the determination of rotational relaxation times. It is very satisfactory that the values obtained for several globular proteins agree well with previous results of Oncley (13) by the dielectric dispersion method. Such an agreement can only by expected if the protein charges present at the isoelectric point can be considered virtually bound to the protein and incapable of showing a characteristic relaxation frequency of the order of magnitude of that of the protein itself $\left(10^{-7} \mathrm{sec}\right)$.

I will consider finally two applications to which these conjugates can be put, namely, the determination of the dielectric properties of the medium with which the fluorescent label is in contact and its variation under a variety of circumstances, and further, the determination of what may be called molecular expansion, by observations of the change in electronic energy transfer from the tryptophan residues to the attached DNS.

The determination of the dielectric properties of the enviromment by olsservations of the fluorescence spectrum is a simple application of the theory of Lippert already discussed. The fluorescence spectrum of conjugates of bovine serum albumin with dimethylamino naphthalene sulfochloride is found to have a maximum at $500 \mathrm{~m} \mu$, which after twenty hours' digestion with chymotrypsin shifts to $520-525 \mathrm{~m} \mu$. The shift towards longer wavelengths follows the replacement by water of the less polarizable protein residues found in the intact molecule in the vicinty of the attached DNS. Absorption spectra, and also Huorescence-excitation spectra, show a shift in the absorption maximum of some $10 \mathrm{~m} \mu$ toward the ultraviolet after proteolysis, as 
the dimethylamino gromp becomes a proton acceptor in a hydrogen bond with the water which has presently come into contact with it. Flnorescence excitation spectra of the DNS emission show also the transfer of electronic energy from the tryptophan residues to the DNS and the disappearance of the transfer alter twenty hours' digestion of the protein with chymotrypsin, which by breaking up the molecule into a series of independent pepticles causes the tryptophan and the naphthalene sulfonamide to be widely separated from each other (Fig. 17). A recent application of this energy transfer method to the detcrmination of protein expansion is shown in Fig. 18, where the action of methyl-ethyl carbinol at $3{ }^{\circ} \mathrm{C}$ and $38^{\circ} \mathrm{C}$ is shown. The physical

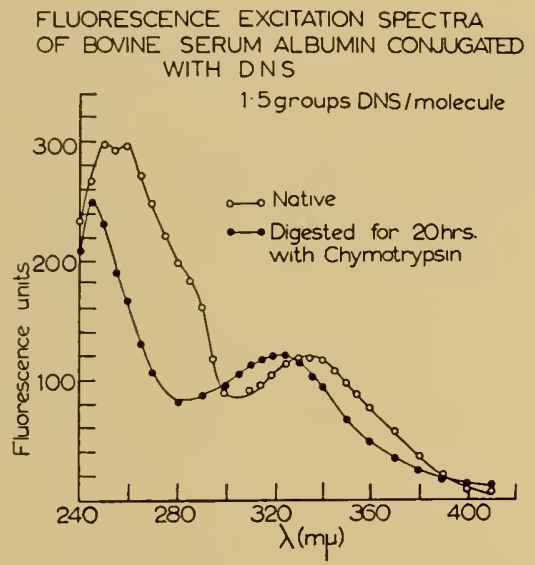

Fig. 17. The eftec of digestion with chymotrypsin upon the fluorescence excitation spectrum of bosine serum albumin conjugated with dimethylamino napthalene sulfochloride (DNS).

separation of parts of the protein produced by the penetration of the carbinol is followed by the disappearance of the energy transfer. These effects can be analyzed quantitatively by measurements of the protein (tryptophan) fluorescence respectively in the native species and in the DNS conjugate. If the two fluorescence efficiencies are respectively $F_{o}$ and $F_{c}$, application of the simple transfer theory recently described (28) gives

$$
\frac{F_{o}}{F_{c}}-1=(R / \bar{r})^{6}
$$

where $R$ is the characteristic distance for the transfer from trytophan to DNS and $r$ an effective average distance which separates both these 
residues in the protein conjugate. I have determined $R$ experimentally by a method similar to that employed in the study of transfer from phenol to indole (28), using $10 \mu$ layers of concentrated solutions of indole and $\mathrm{DNS}-\mathrm{NH}_{2}$ in a viscous medium. $R$ is found to be $24 \mathrm{~A}$,

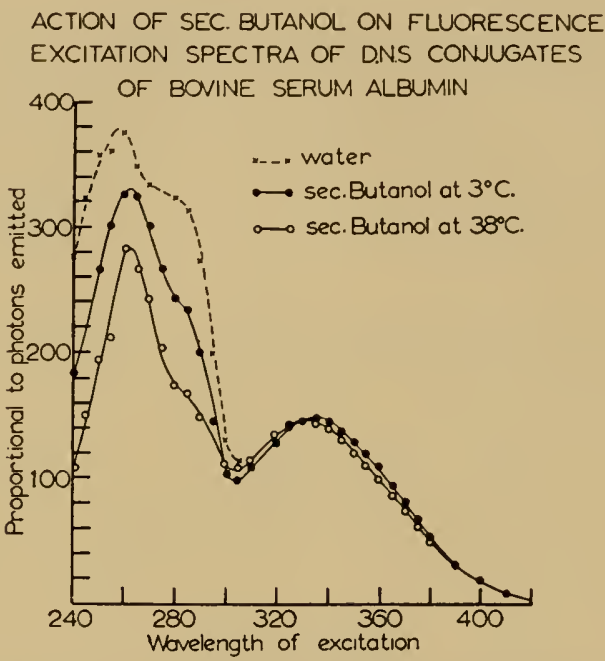

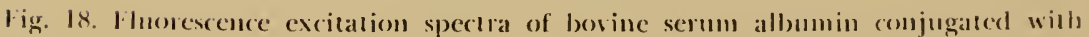
I).S. in methrledivf carbinol at Iwo temperatures, and in waler.

while the value calculated from Förster's theory is '26 $\mathrm{A}$.

The following table gives the values of $\bar{r}$ found for conjugates of bovine serum albumin, having 1.9 DNS conjugated groups per molecule, and the effect of the addition of $9.5 \mathrm{M}$ urea and of $30 \% \mathrm{v} / \mathrm{v}$ of methyl-ethyl carbinol upon $\bar{r}$.

Solvent

Water

$30 \%$ methyl-ethyl

carbinol

$9.5 M$ urea
$\bar{r}(\mathrm{~A})$

19.5

23.3

25.8 $\bar{r}-\bar{r}_{\text {water }}(\mathrm{A})$ Volume change

3.8

$50 \%$

6.3

The last column gives the effective increase in the molecular volume on the assumption that isotropic expansion is responsible for the observed increase in $\bar{r}$.

The examples quoted should suffice to give an idea of the type and range of application to which observations on the emission characteristics of native proteins and artificial conjugates can lend themselves. 


\section{REFERENCES}

I. Boaz, H., and Rollefson, G. K., J. Am. Chem. Soc., 72, 3435 (1950).

2. Coons, . 1. H., and Kaplan, M. H., J. Exptl. Med., 91, I (1950).

3. Crech, 11. J., and Jones, R. H., J. Am. Chem. Soc., 72, 1661, 1670, (1941).

4. 1)ebye, P., and Edwards. J. O., Science, 116, 143 (1952).

5. lörster, T., Ann. Physik. 2, 5.5 (1917).

ti. Förster, T., Z. Elektrochem., 54, 42 (1954).

7. Grossweincr, L. L., J. Chem. Phys., 24,|255 (1956).

8. Jablonski, A., Acta Phys. Polon., 10, 33, 193 (1950).

8a.Jablonski, A., Z. Physik, 96, 236 (1935).

9. Lewis, G. N., and Kasha, M., J. Am. Chem. Soc, 66, 2100 (1944).

10. Linschitz, H., and Sarkanen, K., J. Am. Chem. Soc., 80, 4826 (1958).

11. Lippert, E., J. Elektrochem., 61, 962 (1957).

12. Livingstone, R. A., J. Am. Chem. Soc., 77, 2179 (1955).

13. Oncley, J. L., Chem. Rer's. 30, 433 (1942).

14. Onsager, A., J. Am. Chem. Soc., 58, 1486 (1936).

15. Perrin, F., J. Phy's., 7, 390 (1926).

16. Perrin, F., Ann. Phy's., 12, 169 (1929).

17. Perrin, F., Amm. Plyss., 17. 283 (1932).

18. Pheofilov, P. P., and Sieshnikoff, B., J. Phy's. U.S.S.R., 3, 493 (1940).

19. Platt, J. R., J. Chem. Phy's., 17, 484 (1949).

20. Porter, G., Radiation Research Supplement 1, 479 (1959).

21. Steele, R., and Szent-Cyörgi, A., Proc. Nat. Acad. Sci. U. S., 44, 540 (1958).

22. Steiner, R. A., and Mc.Allister, A., J. Polymer Sri., 24, 205 (1957).

23. Teale, F. W. J., and Weber, G., Biochem. J., 65, 47t (1957).

24. Teale, F. W. J., and Weber, G., Proc. Biochem. Soc., in Biochem. J., 72, p. 15. (1959).

24a.Velick, S., J. Biol. Chem., 233, 1455 (1958).

25. Weber, G., Biochem. J., 51, 155 (1952).

26. Weber, G., Trans. Faraday Soc., 50, 554 (1954).

27. Weber, G., J. chim. phy's., 878 (1958).

28. Weber, G., Biochem. J., 73, 335 (1960).

29. Weber, G., and Teale, F. W. J., Discussions Faraday Soc., 27, 134 (1959).

30. White, A., Biochem. J., 71, 217 (196) 54

31. White, A., Doctoral Thesis, University of Sheffield, (1960).

\section{DISCUSSION}

Dr. Franck: Could you give us a résumé of the material you presented here and conclusions that you wanted to make.

DR. WEBER: There are many ways in which the properties of the excited state can be utilized to study points of ignorance of the structure and function of protein molecules.

DR. Franck: If you have polarization in protein molecules how do you line them up to the light?

DR. WEBER: The light picks out the molecules which are properly oriented.

Dr. Frescu: Did you say whether the urea influenced the intensity of fluorescence of proteins or not?

Dr. WEBER: Y'es, it does. There is no general rule. It influences very often in opposite directions. Some proteins on treatment with urea show a decrease 
in fluorescence, some show an increase, and some are not influenced at all.

Dr. Franck: What did you say about migration of the excited state?

Dr. Wris:R: In proteins which contain tyrosine alone, it is possible from the polariation measurements to see the excited state migrate from one tyrosine to another.

Dr. FRANCK: But do you have any quantitative ways of measuring this?

Dr. WEBER: No, because the only calculations which can be made assume a knowledge of the distance and orientation of the tyrosines in the molecule and this is unknown. One can make calculations assuming that the tyrosines are random, but this is certainly not true.

DR. FRANCK: Is the process by which it migrates the Foerster process?

DR. WEBr:R: I think there is no doubt that it is the Foerster process. I wanted to show an interesting example of the use of Foerster's theory by using conjugates in studying protein properties. In these conjugates one can show a transfer excitation from the tryptophan to an attached group, which is an aminonaphthalene group. When you put the protein into urea the relative distance between the two groups increases and the transfer becomes very much weaker or entirely disappears.

Dr. Franck: Did the proteins you used always contain a conjugated system of double bonds?

Dr. Weber: Yes, I have not used any special kind of protein but I think gelatin is the only one which does not have any absorbing tyrosine or tryptophan although it does have some phenylalanine.

Dr. Franck: Do they show no fluorescence?

Dr. Weber: They don't. except for impuritics. Polylysine of course I have used.

Dr. Seliger: I have two questions. Dr. Porter said that he used methylene blue and it was the triplet state of methylene blue which oxidized ferrous to ferric. He proposed that it would stay in a solution from which all oxygen had been removed. You, Dr. Weber, observed the same sort of thing and supposedly your solution was not oxygen-free.

DR. WliBLR: No, I was only attempting to photo-oxidize trypophan to excite it by oxygen. I did not care what the mechanism was. I was just measuring tryptophan disappearance.

Dr. Seliger: So you were not postulating the same mechanism. That is, that the triplet state is doing the oxidation.

Dr. WeBER: No, I was postulating nothing.

Dr. SliLIGI:R: Just one more question. Could you say something very briefly about the method used to obtain quantum yields and then could you postulate some mechanism whereby you conde explain the decrease in quantum yields with change in wavelength.

DR. Wribl:R: The method I used to measure quantum yichls was published in the Transactions of the Faraday Society in 1957. It consists in the comparison of the emission with a solution of glycogen in which the glycogen scatters light and sets a quantum yiekd standard of one, correcting for the 
geometry and spatial distribution of the glycogen scattering. Now, as to the question dealing with changes in quantum yield with wavelength in the case of a pure substance, one never observes these changes. But in the case of proteins in which there are complexities and where there are various kinds of resiclues absorbing you can have a change.

1)R. SrLigre: But this is not specifically the quantum yicld of the particular molecule that you are talking about.

DR. Wrbi:R: This is the quantum yicld defined as the number of quant. emitted divided by the number of quanta absorbed. I won't say anything lurther.

Dr. Ranisowitcu: This may be an unfair question, but one to which Professor Franck has referred at the beginning. What shall we think about the alleged existence of low excited levels of protein-levels supposed to be located only one electron volt above the ground state?

DR. WEBER: I don't know whether any experiment which I have done on fluorescence of proteins would be related to this question, to tell you the truth. I prefer not to cliscuss it.

Dr. PlatT: I would like to comment on Dr. Rabinowitch's question. These are awfully difficult experiments. There is no theoretical reason to expect that there should be any electronic excitation levels in proteins lower than those that Dr. Weber has talked about. I think theorists would strongly oppose the kind of results Healy has until they become confirmed by at host of other laboratories with the best semi-conductor technique, oxygen. free, free from false conductivity. from surface effects, etc. I clon't believe that one should believe in these low putative electronic states of proteins yet. I think we would do better for a while to ignore them until there is a lot better evidence.

DR. RABisowitch: I am grateful for your permission to ignore them.

Dr. Fraxick: Conclusions on the position of a conduction band and on the quantum yield of photo-ionization are, according to newer studies, mostly not reliable in so far as they refer to crystals or other ordered arrays of organic molecules, and are based on the usual observations of photoconductivity. The greatest part of the observed conductivity, and often all of it, is not caused by photo-ionization of the molecules in the crystal, rather it is caused by electron donation of the cathode to the crystal, respectively electron acception (i.e. hole production) at the anode. To avoid this effect, it is in crystals of aromatic compounds not sufficient to shicld the electrodes against direct irradiation, if excitation movement can carry excitation energy to the electrodes.

Dr. Cinavce: Mr. Patrick Taylor reported at the Faraday Society results on the possibility of conduction bands in cytochrome $c$ and these results make it even more unlikely that cytochrome c transfers electrons by means of conductor bands. 


\title{
SPECTRA AND STRUCTURE IN ENZYME COMPLEXES OF PYRIDINE AND FLAVIN NUCLEOTIDES
}

\author{
SIDNEY F. VeLicK \\ Department of Biological Chemistry, Washington \\ University, School of Medicine. St. Louis, Missouri
}

Of the many coenzymes that might profitably be discussed in a symposium on light and life I have selected two, the pyridine and the flavin mucleotides. The spectra and excited states of these compounds are neither simpler nor better understood than those of other coenzymes, but we are in a particularly good position to view them in relation to the substrates and enzymes with which they act. A coenzyme may be considered alternatively as a substrate or as a prosthetic group of an enzyme, depending upon the dissociability of the complex; and the distinction is often blurred. Once the position of a coenzyme in a metabolic pathway is established, it is the detailed relation of the coenzyme to the enzyme and substrate that is of interest to the biochemist. These are the relations that determine specificity and reaction mechanism and that are the focal points for metabolic integration and control. Absorption and emission spectra provide an approach to these problems and I shall discuss a few of the applications.

\section{Free Pyrimine Nucleotides}

We may begin by considering the three-dimensional structure of diphosphopyridine nucleotide (1)PNH) in solution. Fig. 1 illustrates two isomers, both of which are found in nature. The isomerism is at the glycosidic linkage between the nicotinamide ring and the ribose. Only the $\beta$ isomer is active with known enzymes. The $\alpha$ isomer, first isolated and characterized by Kaplan and coworkers (13), is a bit of a puzzle, and we will make use of it only by the contrasts which it presents. The lunctional part of the pyridine nucleotides is the substituted pyridine ring. Warburg and Christian (37) established the function as a reversible reduction and oxidation; and Vemesland, Westheimer, and coworkers (8) provided the evidence 

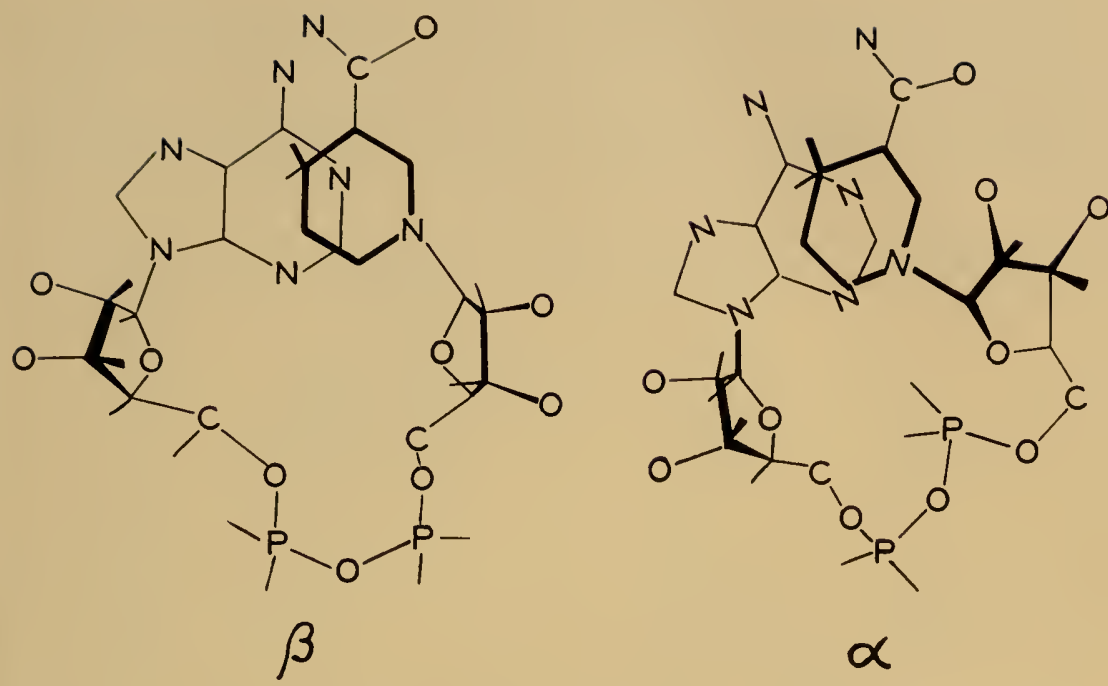

Fig. 1. Folded conformations of DPNH with $a$ and $\beta$ isomerism at the pyridine$\mathrm{N}$-riboside bond. These are projections of skeletal models in which the planes of the nitrogen rings are parallel to the plane of the paper, nicotinamide above and adenine below. Juxtaposition of the rings accounts for the excitation energy transfer, but in the absence of more detailed information the mutual orientations of the rings are arbitrary. Other bonds are out of plane and hence appear of rarying length in projection. Note the drastic effect of the $\alpha-\beta$ isomerism on the conformation of the ribose diphosphate moiety. It is to be observed also that the two hydrogens at pyridine-C-4 in a folded structure are not chemically equivalent.

that the reaction is a stereospecific transhydrogenation between substrate and pyridine ring, presumably involving the transfer of a hydride ion. There has been singularly little chemical study of reducible pyridine derivatives, and nearly twenty years elapsed between the work of Warburg and the observation by Pullman, San Pietro, and Colowick (18) that the enzymatic reduction occurs at the 1,4 and not at the 1,2 or 1,6 positions in the pyridine ring.

Substitution on the ring nitrogen is essential for the oxidation reduction behavior of the nicotinamide, and nature has selected for this purpose adenosine diphosphate ribose. Why so extended a structure is needed is not certain and is one of the problems that concerns us. The dinucleotide contains four ring structures connected by ten bonds around which free rotations may occur. There are, therefore, many possible spatial conformations. Only one of these conformations should occur in a given enzyme complex, and certain ones of them should be more stable than others in free solution. 
Spectral evidence for the folded conformation. The absorption spectrum of DPNH is shown in Fig. 2. At $260 m \mu$ there is a strong absorption band that arises chiefly from the adenine. At $340 \mathrm{~m}_{\mu}$ is the absorption band of the reluced nicotinamide ring. This band

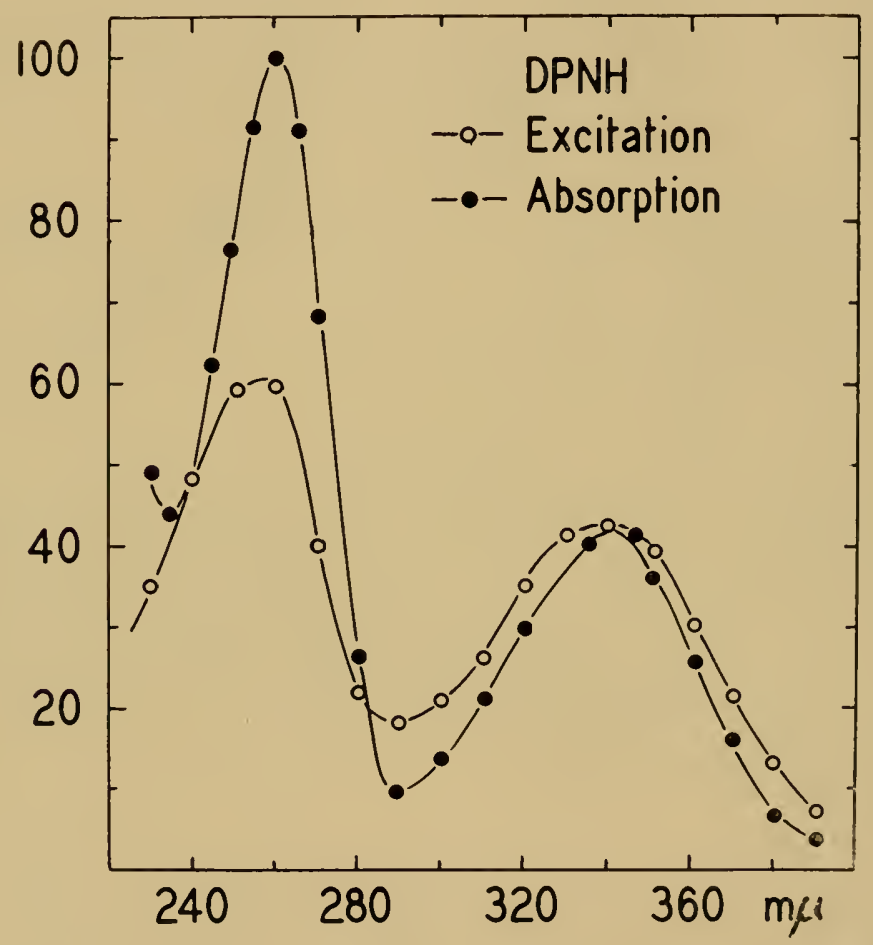

Fig. 2. The absorption and flnorescene excitation spectra of DPNH in water. emission was measured at $465 \mathrm{~m} \mu$ as a function of warelength of excitation. Correcions were applied at each excitation point to account for the spectrat energy distribution of the calibrated light source, a high pressure xenon are operated on D.C. The arbitrary units of the excitation spectrum were nomalized to coincide with the absorption spectrum at $310 \mathrm{~m} \mu$. Lack of perfect coincidence of the spectra in the $3.10 \mathrm{~m} \mu$ band are probably not real but the result of limitations in technique.

disappears when the coenzyme is reoxidized. It is restored by reduction and also by the non-enzymatic adclition of certain nucleophilic reagents, such as cyanide (5), which attack the reactive position at pyridline C-4. In the latter case the absorption band is shifted to the $325 m \mu$ region. The reduced pyridine ring is fluorescent with an emission maximum in water at $465 m_{\mu}$. The open circles in Fig. 2 
describe the excitation spectrmm for the fluorescence. A curve of this type was first obtained by Weber (39). The strong excitation band at $260 \mathrm{~m} \mu$, where more than 90 per cent of the light is absorbed by adenine, could only arise by the transfer of excitation energy from adenine to pyridine. The lifetime of the adenine excited state, a singlet state, is very short, since adenine fluorescence is not observed in neutral aqueous solutions at room temperature. Therefore, for

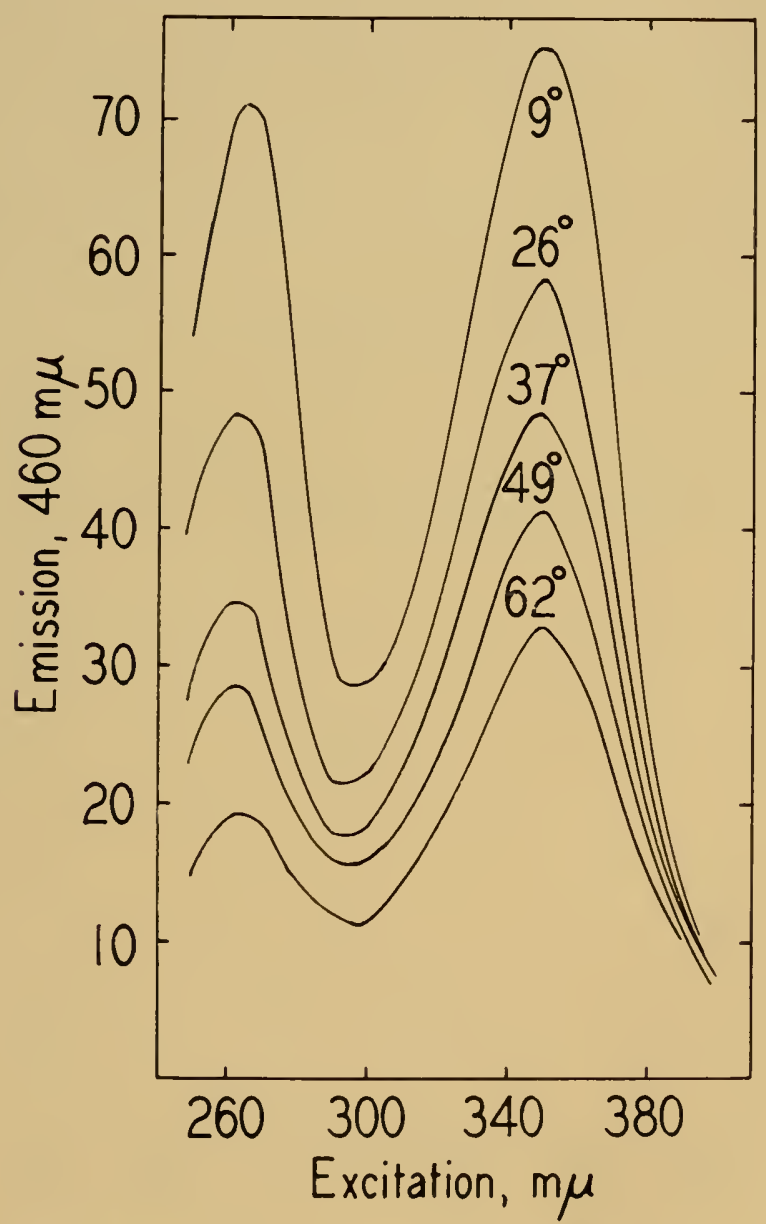

Fig. 3. The fluorescence excitation spectrum of DPNH in water as a function of temperature. Is in Fig. 2, the spectra are corrected to correspond to an excitation beain of constant intensity as a function of wavelength. The tempera. ture effects are completely retersiblc. 
the transfer to occur with the observed efficiency the adenine and pyridine rings must be in close juxtaposition. In order to observe the adenine excited state fluorometrically at room temperature it is necessary to work in strong acid solution. The emission band may be observed in neutral solutions at liquid nitrogen temperature. The adenine emission band overlaps the absorption band of the reduced pyridinium ring, and conditions are thus satisfied for excitation energy transfer by the coupled oscillator mechanism. However, the Förster equation (9) for interactions of this type applies to dipoles that are separated by several molecular diameters and must therefore be modified for interactions at short distances or within an inner complex.

Effect of temperature. If free DPNH occurs in solution as a folded inner complex the complex should be dissociable as a function of temperature, solvent, and structural modification. Fig. 3 shows the effect of temperature on the fluorescence excitation spectrum. You will notice that the relative quantum yield of excitation in the 340 $m_{\mu}$ band declines with increasing temperature as a result of increased

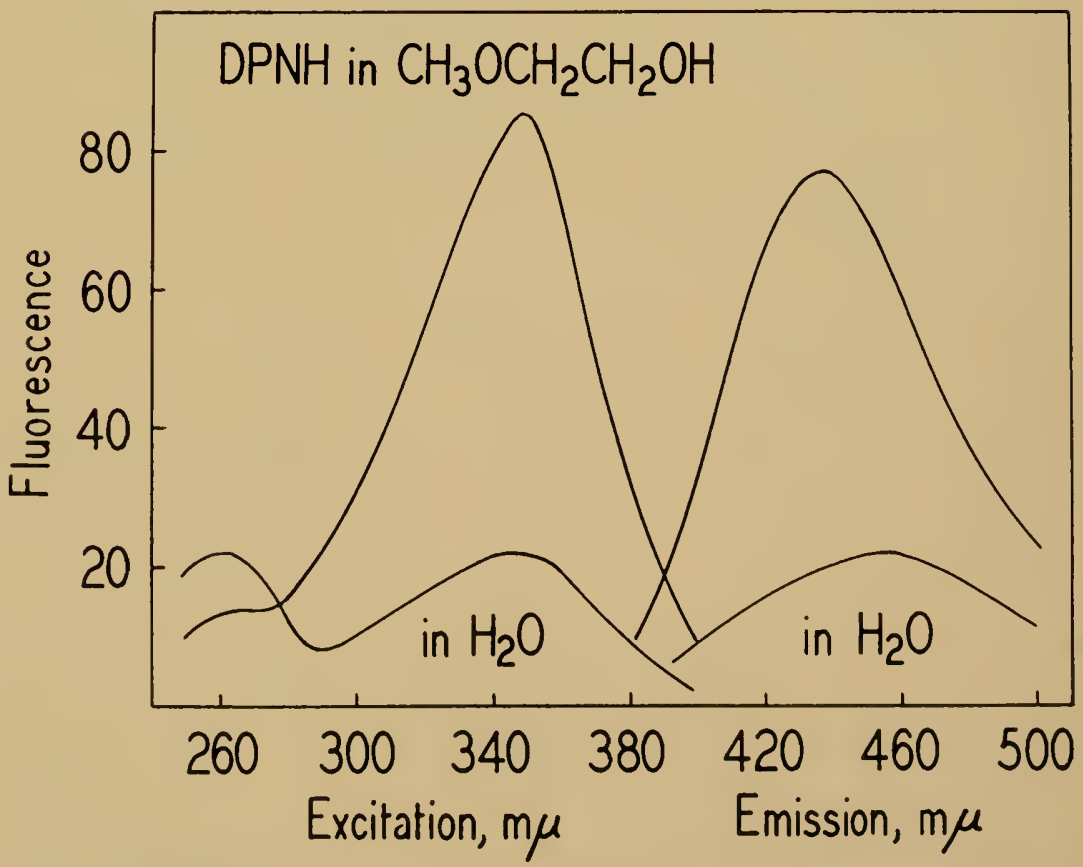

Fig. 1. Corrected fluorescence excitation and emission spectra of DPNH at the sane concentrations in water and in methyl carbitol. 
themal dissipation of the excitation energy. The efficiency of the $26011 \mu$ excitation declines even more rapidly because it involves two temperature-dependent processes, conversion to fluorescent light within the pyridine ring and energy transfer from adenine to pyridine. Weakening of the transfer interaction can arise both from increased thermal quenching of the adenine and from thermal clissociation of the immer complex. In so lar as dissociation is a factor, the amount of spatial separation or clisorientation need not be very large to produce a large effect on the transfer process.

Dissociation by change of solvent. The effect of solvent is much more pronounced than that of temperature (Fig. 4). The lower curve is the excitation spectrum of DPNH in water and the upper curve is the excitation spectrum of the same concentration of DPNH in ethylene glycol monomethyl ether. Notice that the emission maxi-

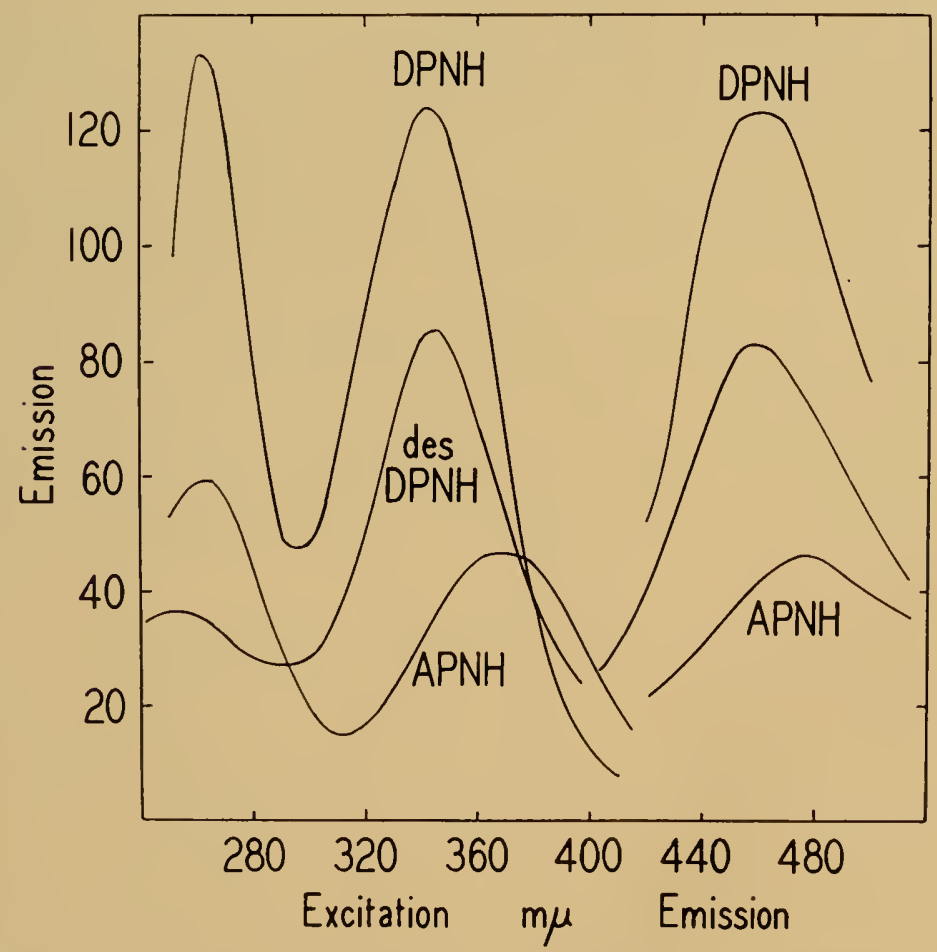

Fig. 5. Corrected fluorescence excitation and emission spectra of DPN desaminoD) PH (adenine replaced by hypoxanthine), and APNH (nicolinamide replaced by 3-acetyl pyridine). Measurements were made of dilute aqueous solutions the optical densities of which were equal at the respective long-wavelength absorption maxima. 
mum in the organic solvent is enhanced about fourfold and is shifted from $465 \mathrm{~m} \mu$ to about $440 \mathrm{~m} \mu$. There is a corresponding enhancement of the $340 \mathrm{~m}_{\mu}$ excitation band but the $260 \mathrm{~m}_{\mu}$ excitation band has nearly disappeared. The complex under these conditions, a moderate lowering of the dielectric constant of the solvent, has largely dissociated. These changes are precisely those that occur in the DPNH excitation and emission spectra when it is complexed to many but not all dehydrogenase proteins. The spectra of the complexes however involve some complications which will be taken up at a later point in the discussion.

The behavior of structural analogues. The fluorescence excitation and emission spectra of two structural analogues of DPNH are shown in Fig. 5. Replacement of the carboxamide group of the nicotinamide with acetyl yields the acetyl pyridine analogue, APNH. The emission band is weakened and shifted toward longer wavelengths, but the $260 \mathrm{~m} \mu$ excitation band is relatively stronger than before. The anide group is therefore not specifically involved in the formation of the inner complex. In the second analogue, desamino DPNH, the amino group of the adenine is replaced by hydroxyl. Note that the short wavelength excitation band has shifted to 250 $m \mu$, where hypoxanthine absorbs, and is rather weak, possibly because the amino group of the adenine is involved in the formation of the inner complex. Shifrin and Kaplan (21a) report that the $260 \mathrm{m \mu}$ excitation band of the $\alpha$ isomer of DPNH (Fig. 1) is absent. Although models of the $\alpha$ isomer may be folded to bring the pyridine and adenine rings in juxtaposition, the allowed conformations are different from those of the $\beta$ isomer. Either an inmer complex is not formed or one is formed in which the orientations are not favorable for the transfer of excitation energy.

\section{CONFORMATION AND THE STEREOCHEMISTRY OF OXIDATION AND Reduction}

Isomerism at pyridine C-t. When DPN is reduced enzymatically with a deuterimm-labelled substrate, deuterimm is transferred to the coenzyme to form DPNI. It is the deuterimm and not the hydrogen that is removed exclusively when the DPND is reoxidized in the reverse reaction catalyzed by the same enzyme. The enzyme thus distinguishes between the two bydrogen atoms at pyridine C-4, and this is not surprising in terms of what we know in general about enzyme specificity. However, it was observed by San Pietro and coworkers (19) that the wo hydrogen atoms at pyridine C-4 also behave dif- 
fercutially in non-enzymatic oxidations and reductions. The difcrences in reaction rates are significant after full cognizance is taken of the possible isotopic rate effects. It may be argued that the two hydrogen atoms at $\mathrm{C}-4$ are not exactly equivalent because of the asymmetric influence of the ribosidic bond, but in view of the distances involved such an effect would be expected to be rather weak. The probable explanation of the effect is based upon the propertics of the folded immer complex of the dinucleotide. It may be sccn in Fig. I that the two hydrogen atoms at pyridine-C-4 are in different chemical enviromments. As long as a specific folded conformation occurs, the chemical differcnce between the two reactive hydrogens is real even though the details of the inner complex may not be cxactly as depicted. The two steric positions at pyridine C-4 also react differentially when DPN is reduced by hydrosulfite. One may therefore infer that DPN, like DPNH, also occurs in a folded conformation, although fluorescence of DPN is not available to test the hypothesis.

Isomerism at the pyridine ring nitrogen. Conformations of the

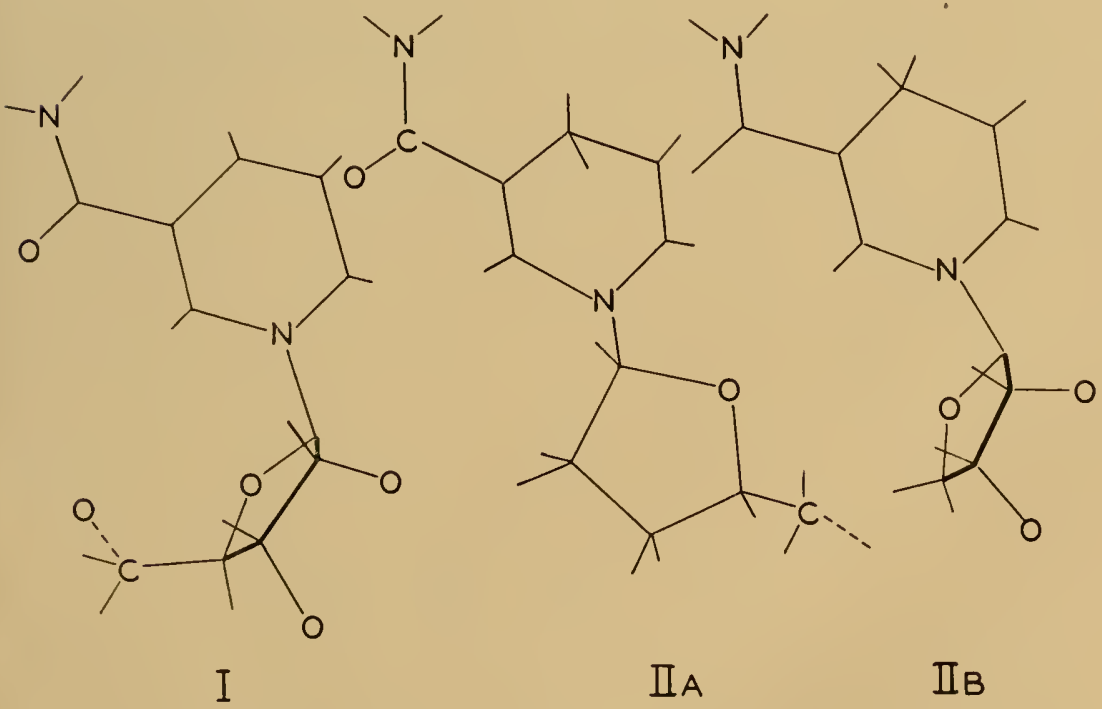

Fig. 6. Skeletal models of the nicotinamide riboside moiety of 1)PN (I) and 1)PNH (IIa and IIb). A pyrimidal ring nitrogen in IIa makes it possible for the pyridine and ribose rings to lie in nearly paral\}el planes. 1 planar ring nitrogen in IIb puts the $\mathrm{N}$-ribosidic bond in lise plane of the pyridine ring but makes its angles with the other two N-C bonds unequal. The actual stucture is probably closer to IIl) than to IIa. 
nicotinamide riboside portion of the pyridine nucleotides in the oxidized and reduced states are shown in Fig. 6. In DPN the pyridinium ring is aromatic and planar and the ribosidic bond lies in the plane of the ring. The ribose plane therefore lies somewhere between a right angle and a tetrahedral angle to the plane of the pyridine, depending on the conformation of the ribose. This is in accord with X-ray crystal structure analyses of simple ribosides $(11,4)$. Models of the reduced riboside are constructed in two ways: (a) with the trivalent ring nitrogen pyrimidal as in aliphatic amines; and (b) with the lone pair of the ring nitrogen involved in the conjuga-

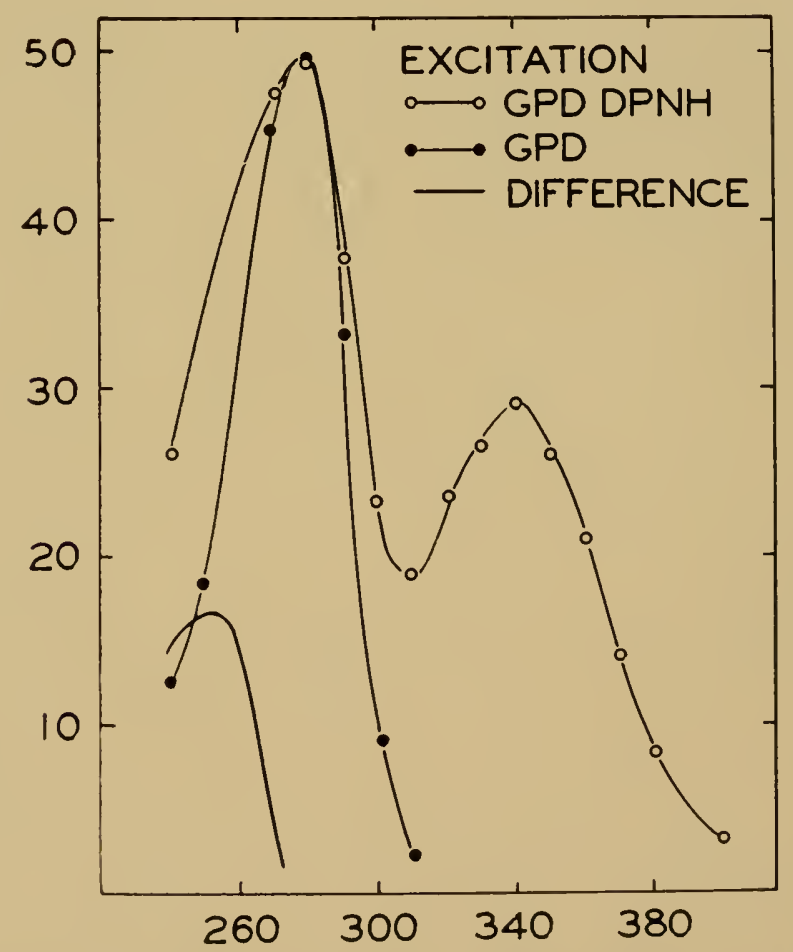

lig. 7. Flusescence excitalion spectrum, correcter, of DPNH (open circles) in

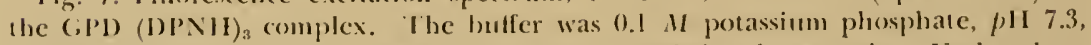
selected to minimize the quenching of DPNH emission by protein. Under these conditions the chief sign of complex formation is polariation and the excitation spectrum itself. DPXH emission was measmed at $185 \mathrm{~m} \mu$ to minimize the correction for the tailing protein emission when excitation was in the $280 \mathrm{~m} \mu$ band. superimposed is the excitation spectrmm of the protein fluorescence normalized at $280) \mathrm{m} \mu$ and measmed at $3.50 \mathrm{~m} \mu$. The cure on the lower lelt is the excitation difference spectrmm. 
tion. In model (a) the ribosidic bond is ont of the pyridine plane. In model (b), which is perhaps more probable, the ribosidic bond is in plane but does not form a $120^{\circ}$ angle with the bonds in the ring. The chiel point of these arguments is that in addition to the change in the charge on the ring nitrogen there is also a conformation change that accompanies oxidation and reduction. Both the oxidized and reduced forms of the coenzyme occupy the same binding sites in enzyme complexes. If the enzyme is constructed to fit one form closely, the fit will be less perfect and the binding interaction weaker for the other form. In most of the cases that have been examined by equilibrium methods, the enzyme interacts much more strongly with DPNH than with DPN. Specific instances are tabulated later, in Table I, where they are correlated with other properties of the complexes.

\section{Reduced Pyridine Nucleotide Enzyie Complexes}

Glyceraldehyde-3-phosphate dehydrogenase-DPNH. The persistence and the disappearance of a $260 m_{\mu}$ excitation band of DPNH fluorescence are criteria for closed and open conformations of the dinucleotide. In the complex of DPNH with glyceraldehyde-3phosphate dehydrogenase (GPD) the coenzyme fluorescence, contrary to its behavior in other knor'n enzyme complexes, is partially quenched (35) and the emission band shift, if significant at all, is slightly toward the red. The excitation spectrum is shown in Fig. 7. At $340 m \mu$ the excitation band is normal, but the short wave band is strong and maximal at 280 instead of $260 \mathrm{~m}_{\mu}$. The $280 \mathrm{~m}_{\mu}$ band occurs where protein absorption is maximal and nucleotide absorption weak, and corresponds to direct excitation of the protein and not of the coenzyme. However, emission was measured at $490 \mathrm{~m}_{\mu}$ and comes chiefly from the coenzyme. This effect, with uncorrected spectra, was described in an earlier report (35) and arises by the transfer of excitation energy from protein to bound coenzyme. If we superimpose on this excitation spectrum the excitation spectrum of the protein fluorescence, measured at $350 \mathrm{~m} \mu$, the two curves, normalized at $280 \mathrm{~m}_{\mu}$, are in agreement only near the maximum. On the long wavelength side there is divergence due to the rise of the $340 m_{\mu}$ absorption band of the coenzyme. On the short wavelength side there is divergence due to the persistence of the $260 \mathrm{~m} \mu$ excitation band of the coenzyme. The 260 $m \mu$ band is shown by the difference spectrum. The results thus indicate that the DPNH is bound in folded conformation to G.PD. Unlike the free coenzyme, the 


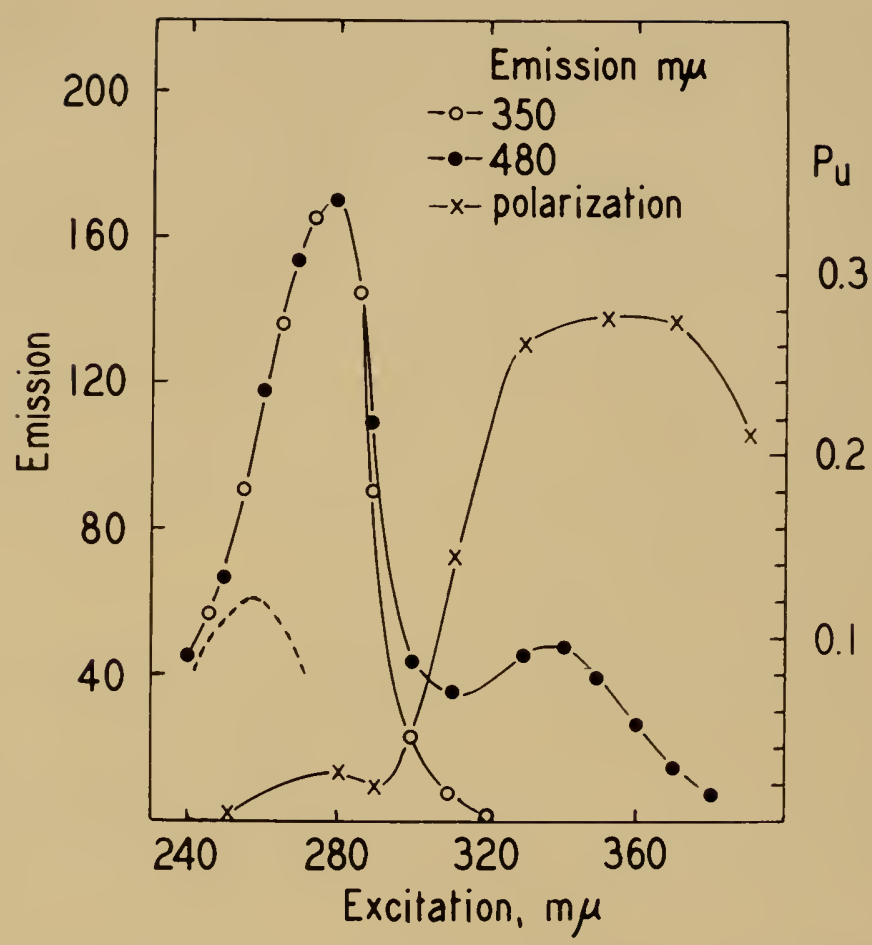

lig. 8. The fluosescence excitation spectum of DPNH in the LDH-(D) NH) complex in tris acetate, $0.1 \mathrm{M}, \mathrm{H} 7.3$. This buffer augments the enhancement of 1) P'xll emission by the protein. On the sate of the spectrum the contribution of protein cmission excited at $280 m \mu$ and measured at $180 m \mu$ is negligible. Ex(itation of protein cuission, measured at $350 \mathrm{~m} \mu$, is shown by the closed circles. The crosses show the changes in polarization of the emission of bound DPNH as a function of the wavelength of excitation with unpolarized incident light. If the $260 m \mu$ excitation band of the DPNH had becn retaincd in the complex its contribution would have been given by the dotted curve. Note that the polarization is not constant throughout the $340 \mathrm{~m}_{\mu}$ excitation band. This is the result of perturbation by the virbrational structure. The low polarization of DPNH fluoresconce excited in the $280 \mathrm{~m} \mu$ absorption band of the protein is discussed later.

ratio of the heights of the 260 and $340 \mathrm{~m} \mu$ excitation peaks remains constant as a function of temperature. This is to be expected, since the complex is catalytically active and the geometry of the bound (oensyme should therefore be rigorously fixed. Since the standard free cuergy change in complex formation is approximately 11,000 calories per mole and since the dissociation constant shows only a very small temperature dependence, a change in nucleotide conforma- 
tion as a function of temperature is not to be expected.

The lactic dehydrogenase-DPNH complex. The excitation of DPNH lluorescence in the lactic dehychrogenase (LDH) complex is shown in Fig. 8. As with GPD, there is a strong excitation band at 280 $m \mu$ but in this case the excitation of the coenzyme (solid line) and the cxcitation of the protein (open circles) show a point to point coincidence after normalization at $280 \mathrm{~m} \mu$. The dotted curve show's the $26011 \mu$ excitation that would have appeared if the coenzyme behaved as it does in the free form. Such a contribution could easily have been detected and was not observed. By this criterion the coenzyme is bound in an open conformation on LDH, as it is on all other dehydrogenases that have been examined with the exception of GPD. The extent to which the dinucleotide is unfolcled in the so-called open conformation is undetermined. Unfolding and disorientation of the inner complex need not be extensive in order to weaken greatly the interaction responsible for the energy transfer.

\section{COMPARISONS AND INTERPRETATIONS}

Each pyridine-nucleoticle-linked dehydrogenase has idiosyncrasies of its own which may or may not be expressed in the gross characteristics of the coenzyme complex. Some properties of a group of enzymes are listed in Table 1. In so far as the general pattern is concerned, most of the enzymes appear to be qualitatively similar; and we infer that the properties selected are not ones that are closely correlated with specificities of behavior. The aberrant case is GPD, and although the amount of cletailed interpretation that we can make is meager it is pertinent to consider a few of the properties briefly.

Fluorescence enhancement. This is a sign that the nucleotide in the complex is shielded and that vibrational modes, and hence chances for thermal deactivation, are restricted. Even in such a case, however, enhancement may not occur if polarities and conformation are not favorable. For example, Winer and Schwert observed that oxalate enhances and oxamate quenches the fluorescence of DPNH in the LDH complex (42). These ions are inhibitory substrate analogues and presumably interact with bound DPNH at the catalytic site. Groups with the same effects may in principle be integral parts of the protein structure. The DPNH in the GPD complex may be reell shielded, and the absence of enhancement may be the result of specific group interaction on the protein. That such an interaction occurs is indicated by the appearance of a $365 \mathrm{~m}_{\mu}$ absorption band in the GPD-DPN complex. 
TABLE 1

Some Properties of Pyridine Nucleotide Enzyme Complexes

Free DPNH in

Enzyme $\quad$ LDH $\quad \mathrm{ADH}^{6} \quad \mathrm{GDH}^{i} \quad \alpha \mathrm{GPDH} \quad \mathrm{GPD}$

Me.

Water Carbitol

\begin{tabular}{|c|c|c|c|c|c|c|c|}
\hline $\begin{array}{l}\text { Equivalent }{ }^{2} \\
\text { weight }(\mathrm{g} .)\end{array}$ & 37,000 & 36,000 & $(80,000)$ & 33,000 & 45,000 & & \\
\hline $\begin{array}{c}\text { Dissociation }{ }^{3} \\
K_{\mathrm{DPNH}}(\mu M) \\
\kappa_{\mathrm{DPN}}(\mu M)\end{array}$ & $\begin{array}{r}0.3 \\
>50.0\end{array}$ & $\begin{array}{r}0.4 \\
84.0\end{array}$ & $\begin{array}{r}3.0 \\
240.0\end{array}$ & 0.5 & $\begin{array}{l}0.2 \\
0.02\end{array}$ & & \\
\hline Stereospecificity ${ }^{4}$ & A & A & $\mathrm{B}$ & $\mathrm{B}$ & $\mathrm{B}$ & A & \\
\hline $\begin{array}{l}\text { Maxima }(\mathrm{m} \mu) \\
\text { Absorption } \\
\text { Emission }\end{array}$ & $\begin{array}{l}335 \\
440\end{array}$ & $\begin{array}{l}325 \\
450\end{array}$ & $\begin{array}{l}340 \\
445\end{array}$ & $\begin{array}{l}340 \\
447\end{array}$ & $\begin{array}{l}340 \\
465\end{array}$ & $\begin{array}{l}340 \\
465 \\
\end{array}$ & 440 \\
\hline $\begin{array}{l}\text { Fluorescence of } \\
\text { bound DPNH } \\
\text { Polarization } \\
\text { Enhancement } \\
\text { Quenching of } \\
\text { protein }(\%)\end{array}$ & $\begin{array}{l}0.47 \\
+\end{array}$ & $\begin{array}{l}0.42 \\
+\end{array}$ & $\begin{array}{l}0.44 \\
+\end{array}$ & $\begin{array}{l}0.43 \\
+\end{array}$ & $\begin{array}{l}0.28 \\
-\end{array}$ & 0.1 & + \\
\hline $\begin{array}{l}\text { Apparent } \\
\text { conformation }\end{array}$ & Open & Open & Open & Open & Closed & Closed & Open \\
\hline
\end{tabular}

1 The enzymes are respectively: lactic dehydrogenase of catte heart (LDH), alcohol dehydrogenase of horse liver $(\mathrm{NDH})$, glutamic dehydrogenase of catte tiver (GDH), a-glicerophosphate dehydrogenase of rablsit sheletal muscle ( $a$-GPDH), and ghiceraldehyde-3-phosphate dehydrogenase of rablit skeletal muscle (CPID).

- Gams of protein tha! combine with one mole of pyridine nucleotide.

${ }^{3}$ Intrinsic dissociation constants calculated for equivalent and independent comsbining sites.

1 and $B$ are arbitrary designations of the two reactive positions at pyridine-C-I.

"Polariation measured with plane-polarised incident light.

"Dissociation constants for (1) II from ref. 33.

(CDH is one of the few enrmes that works with both DPN and TPN. The dissociation constants for I)P'N and IPNH are approximately the same, (moublished fluorometric measurenents of D). Carl Frieden). I he value for the oxidized coensme was derived hineticalty for TPN. That for D) PN is also large.

Polarization. The polarization of DPNH in the LDH complex is near the theoretical maximum for solution in a rigid medium, but in the GPI) complex it is only half maximal. This suggests that there are elegrees of freedom in the (iPI) complex that are consistent with the absence of enhancenent, but such an explanation is hard to reconcile with the precise orientations that one would expect at a catalytic site. Such behavior may be related to the fact that the coenzyne on (iPI) is in some way involved in the phosphorylative as 
well as in the re-dox step and that orientation may shift as a function of reaction conditions. Orthophosphate ions produce some enhancement (35) but do not alter the observed fluorescence polarization.

Spectral shifts. In view of the similarity in the fluorescence spectra of IPNH in methyl carbitol and in the LDH complex, we can only state that in the complex the coenzyme behaves as though it were dissolved in a non-aqueous solvent of low or medium polarity. Specific effects are undoubtedly involved, but the emission spectrum tells us little about them. Absence of a shift in the GPD complex is again consistent with its other properties including the apparent binding in a closed conformation.

Relative affinities of $D P N$ and $D P N H$. The differences in free energy of complex formation of enzyme with reduced and oxidized forms of the coenzyme are determined by changes in conformation and charge distribution that accompany oxidation and reduction. In most cases the $\triangle F^{\circ}$ of complex formation is 2 to $3 \mathrm{kcal}$ more negative for DPNH than for DPN. The binding of DPN in several such cases is strongly promoted by inhibitory substrate analogues, and presumaably by substrate $(5,12,17)$, with the formation of an inhibitor-DPNHenzyme adduct. It was proposed previously that the inhibitor induces a conformation in DPN that resembles that of DPNH, for which the enzyme has a stronger affinity (35). This type of interaction should be at the core of the reaction mechanism, and the reader is referred to a recent example produced by Winer and Theorell (43). In this property, too, GPD is exceptional in that in the absence of substrate it binds DPN more strongly than DPNH and with a $\triangle F^{\circ} 3$ to $5 \mathrm{kcal}$ mole ${ }^{-1}$ more negative than is exhibited by other enzymes. To account for this we can invoke conformation and group interactions, but cannot specify the details.

Enzyme specificity at pyridine-C-t. It was pointed out earlier that the difference in the non-enzymatic reactivity of the two hydrogen atoms at pyridine-C-4 of DPNH could be accounted for by the fact that they have different chemical environments in the folded inner complex. This influence should, and does, disappear when the dinucleotide is bound to an enzyme in an open conformation. Individual enzymes of this group are seen to be specific for either side of the pyridine ring, the specificity being imposed entirely by the properties of the protein. The reactive hydrogen in the GPD coenzyme complex, on the other hand, might be expected to be the same as that of the free coenzyme in its folded conformation, but this is not the case. The opposite stereospecificity is observed. Ob- 
viously we need to know more about the details of the inner complex before we can interpret the above result. The selectivity in nonenzymatic reactions and the relative stability of the inner complex in water are easiest to account for if we postulate a face-to-face orientation of the adenine and pyridine rings. Energy transfer would also occur if the rings were aligned edge to edge in the same plane. This would be an unstable arrangement in free solution, even with the help of a hydrogen bond between the adenine amino group and the carbonyl oxygen of the nicotinamide, but such an orientation could be stabilized in the enzyme complex. In such a case, or in intermediate orientations, the substrate could have access to either sicle of the pyridine ring and the specificity would again be determined by the protein and the reaction that it catalyzes.

\section{Protein Fluorescence and the Transfer of Excitation Energy}

Protein fluorescence is described in detail in this symposium by Weber (40), and I will deal with the subject only in so far as it applies to the analysis of the enzyme-coenzyme complexes. The transfer of excitation energy from the aromatic amino acid residues of the proteins to the bound coenzyme is established by the excitation spectra of Figs. 7 and 8 . It will be profitable to consider the protein emission and the transfer process in more detail in order to see what can be learned about the properties of the proteins, the geometry of the complexes, and the mechanism of the excitation energy transfer. We will confine our attention chiefly but not entirely to LDH because the pertinent properties of LDH are extreme and most favorable for the analysis.

The number and nature of the emitting groups. LDH contains approximately 28 tryptophan and 56 tyrosine residues per molecule. Both tyrosine and tryptophan have absorption maxima near $280 \mathrm{m \mu}$ and the free amino acids emit respectively at 306 and $350111 \mu$ with quantum yields of 20 per cent. The LDH cmission maximum is near $350 \mathrm{~m} \mu$ and appears to be predominantly tryptophan emission. The cmission bands of LDH, free tryptophan, and G.PD obtained from solutions at the same optical density at the wavelength of excitation are shown in Fig. 9. The areas muder the curves obtained in this way represent relative quantum yields. It may be seen that the quantum yield of GPI) is low. However, the emission looks like tryptophan emission; and if we assume that about a third to onehalf of the light absorbed goes into tyrosine and is dissipated as heat, then the tryptophan emission from GPI) is approximately nomal. 


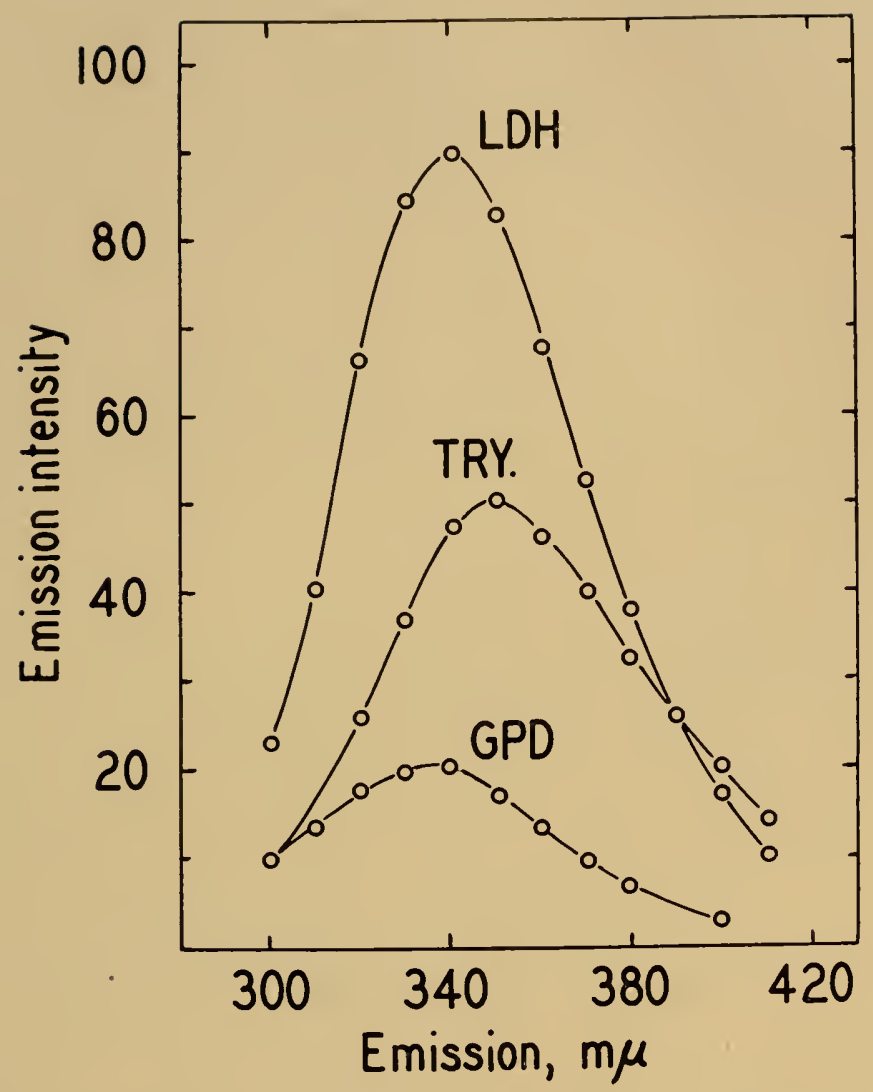

Fig. 9. Fluorescence emission bands of LDH, GPD, and free tryptophan excited at $280 \mathrm{~m} \mu$ from solutions of the same optical density at $280 m \mu$. The areas under the emission bands, under these conditions, are proportional to the respective fluorescence quantum vields.

On the other hand the quantum yield of the LDH emission is high, nearly 40 instead of the 10 per cent shown by GPD. If we assume that only light absorbed directly in the tryptophan of $\mathrm{LDH}$ is reemitted as fluorescent light, then the quantum yield of the tryptophan emission is of the order of 70 per cent. Since the tryptophan in the protein is in a protected environment, a high quantum yield introcluces no serious problem. That the high yield of tryptophan emission in $\mathrm{LDH}$ is a result of the special tertiary structure of the protein is shown in Fig. 10. The LDH is strongly quenched in $6 M$ urea, whereas free tryptophan is significantly enhanced. 


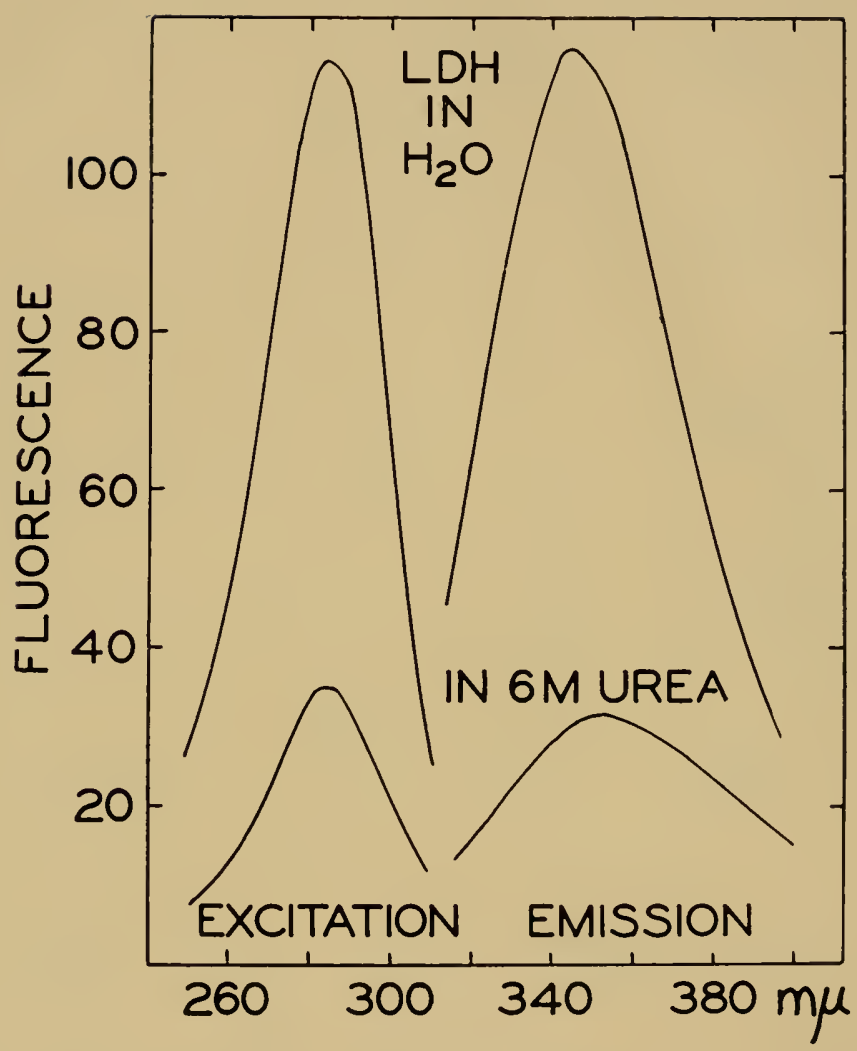

lig. 10. Fluorescence excitation and emission bands of equal concentrations of LDH in $0.1 \mathrm{M}$ phosphate buffer, and in $6.0 \mathrm{M}$ urea, showing the quenching effect of denaturation upon the protein emission. Free tryptophan emission in 6 M urea is enhanced about 40 per cent.

The polarization of the protein cmission, measured with planepolarized incident light, is about 25 percent for LDH, GPD, and other proteins of comparable size in dilute aqueous solution. As illustrated previously by the near maximal polarization of DPNH emission in the LDH complex, the rotational relaxation of proteins of this size is negligible during the lifetime of the excited state of DPNH. It is also small during the lifetime of the excited state of tryptophan. The partial depolarization of the tryptophan emission in the large proteins therefore arises either (a) from internal energy migrations, tyrosine to tryptophan or tryptophan to tryptophan, or (b) by vibrational perturbation or oscillatory motion of the indole 
side-chains. Explanation (b) is the more likely one, since free tryptophan in highly viscous media (35) and at low temperature (40) also exhibits $P$ values of about 25 per cent. In comparing polarizations, $P$, measured with plane-polarized incident light, and polarizations, $P_{u}$, measured with umpolarized incident light, the relation is

$$
P_{u}=\frac{P}{2-P}
$$

Since the theoretical maximum of $P$ is $1 / 2$ for right-angle emission, the maximal value of $P_{u}$ is $1 / 3$.

Energy transfer and quenching. When excitation energy is transferred from protein to bound coenzyme a new coenzyme excitation

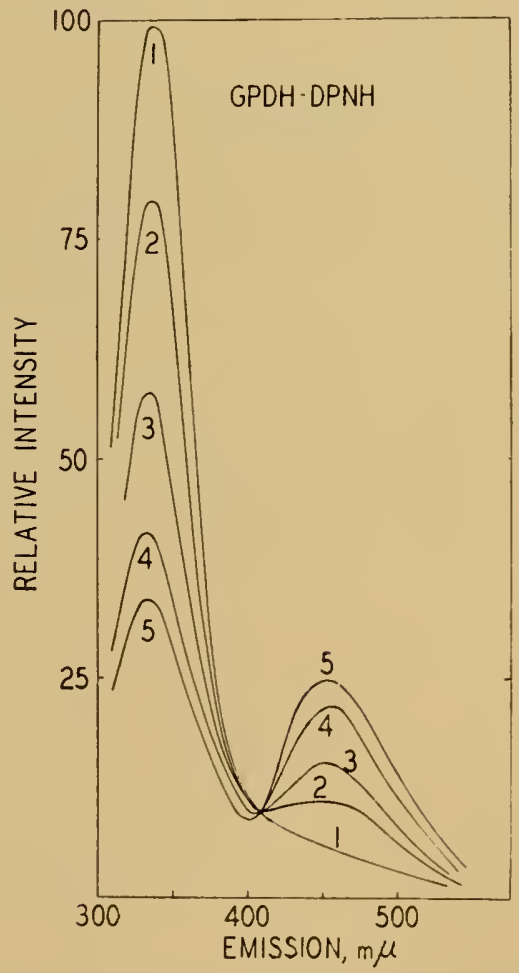

Fig. 11. The emission band of glycerophosphate dehvidrogenase, excited at 280 $111 \mu$, curve 1. The other curves show the effects on the emission spectrum of successive increments of DPNH. The bound cocmlyme quenches the protein emission by the capture of excitation energy and reemits a portion of it in its own emission band at $450 \mathrm{~m} \mu$. 
band appears (Figs. 7,8), and concomitantly the protein emission is partially quenched. Three examples of these effects are illustrated in Figs. 11 to 13. The amount of quenching that occurs when all binding sites are occupied on LDH is 67 per cent with DPNH and approximately $80 \mathrm{per}$ cent with APNH. This difference is correlated

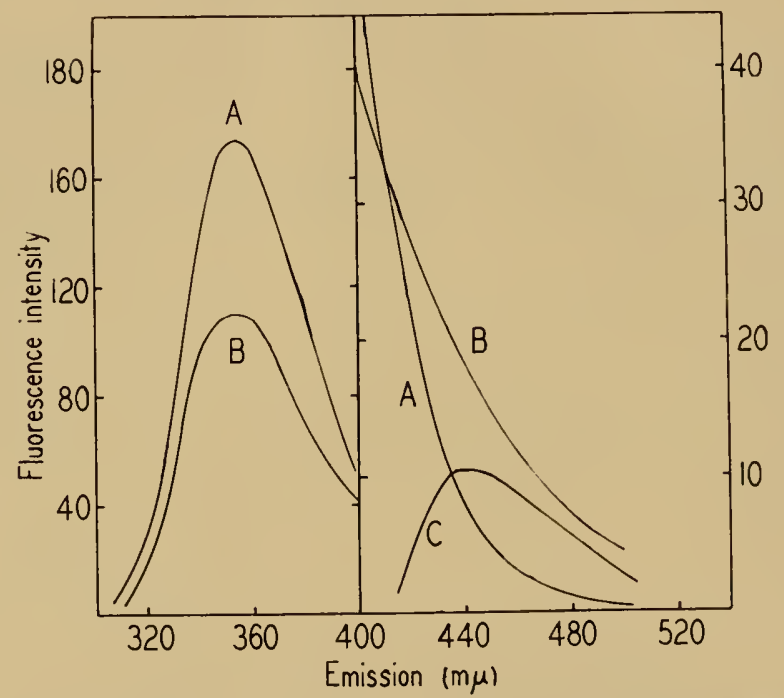

Fig. 12. The cmission of LDH (curve A) and of the LI)H-(I)PNH) complex $_{3}$ (curve B) cxcited at $290 \mathrm{~m} \mu$. Although the quenching of L.DH cmission by bound cocnzyme is appreciable, the high tryptophan content and high fluorescence quantum yield of $\mathrm{L1}) \mathrm{H}$ obscures the cocmeyme enission band, which is clearly rerealed only in the difference spectrum, curve C.

with the oscillator strengths of the coenzyme and its analogue, which exhibit molar extinction coefficients of 6200 and 8100 as their respective long wavelength absorption maxima. The polarization of APNH emission excited by transfer from $\mathrm{LDH}$ is also about 4 per cent higher, indicating a geometrical factor that might be in its favor.

It is useful to view the energy transfer in terms of normalized excitation spectra. In Fig. 14 the coenzyme excitation spectrum of the LDH-DPNH complex has been normalized to coincide with the absorption spectrum of the complex at $355 \mathrm{~m} \mu$. This is analogous to the proceclure employed with free DPNH (Fig. 2). The $280 \mathrm{~m} \mu$ excitation band is superimposed on two absorption curves, the absorption curve of the protein alter subtraction of the coenzyme contribution, and the absorption curve of the constituent tryptophan 


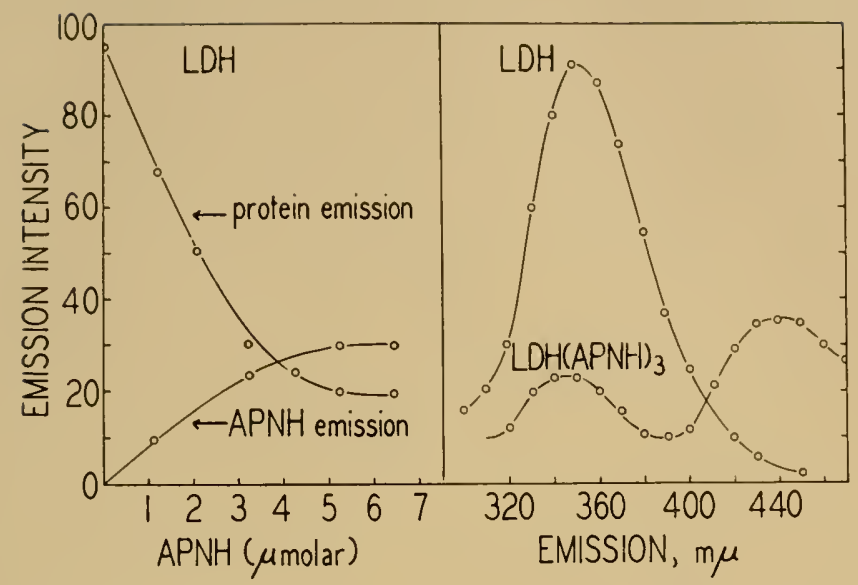

Fig. 13. The emission bands of LDH and of the LDH-(APNH) ${ }_{3}$ complex excited at $290 \mathrm{~m} \mu$. Note the extreme quenching of the protein emission band by the coenzyme analogue, and the relatively strong IPNH emission band excited by energy transfer from the protein. The curves on the left show the changes in intensity of protein and nucleoticle emission as the protein is titrated with APNH. Note that both curres level off as saturation is approached. Free $A P N H$, excited at $290 \mathrm{~m} \mu$. shows negligible emission at $460 \mathrm{~m} \mu$ on the scale of these curves.

calculated from the amino acid analysis and the assumption that the tryptophan in the protein absorbs like the free anino acid in alkaline solution. The area under the excitation band is about 40 per cent of that under the protein absorption curve, a relation which means that about 40 per cent of the light quanta absorbed in the protein are transferred to the coenzyme. On the other hand, the integrated excitation band is roughly 75 per cent of that of the absorption band of the constituent tryptophan. This corresponds approximately to the amount of quenching of the tryptophan emission. The results are only approxinnate because we do not know the exact position and shape of the tryptophan absorption band in the protein. The best fit between the observed protein absorption band and the contributions of its constituent tryptophan and tyrosine is obtained with the tryptophan band as shown and two types of tyrosine absorption, 69 per cent corresponding to the phenolic form and 31 per cent to the phenolate ion. The analysis of the curves in Fig. 14 is in accord with the idea (a) that the protein emission is chiefly tryptophan emission, (b) that tryptophan is the donor in the energy transfer to bound coenzyme, and (c) that the amount 
of intramolecular energy transfer between tyrosine and tryptophan is negligible.

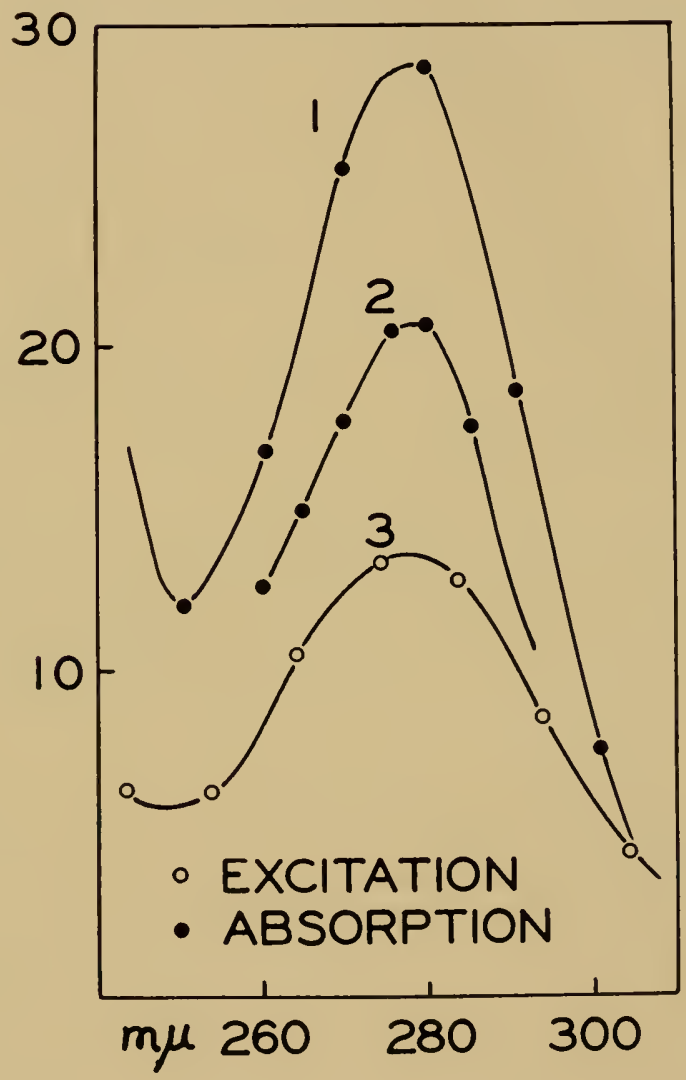

Fig. 14. Normalied excitation and absorption spectra of LDH and its DPNH complex. Curve I is the absorption band of LDH, and curve 2 the absorption band of its constitucnt tryptophan on the assumption that the peptide-bound tryptophan absorbs like the free amino acid in dilute alkali. Curve 3 is the excitation spectrum band of the bound DPNH nomalized to coincide with the absorption band of the complex at $335 \mathrm{~m} \mu$; the latter band and the point of normalization are not shown. This mormalization takes account of the quantum yield of the D) XH emission and puts the absorption and excitation bands on a comparable basis. The mits on the orclinate are arbitrary. The ratio of areas, curve 3 to curve 1 , is the quantum yield of energy transfer from protein to DlexH: the ratio of areas, curve 3 to curve 2, is the cuuntum yield of the energy transfer if the comstituent tyrosine is neglected and only the light absorbed directly in the tryptophan is transfered to the bound DPNH. It is the latter ratio that approximates the fractional guenching of the protein emission. 
Protein subunits or domains of interaction. When the fluorescence of a protein with multiple binding sites is quenched by addition of ligands there is no a priori reason for assmming that the amount of quenching should be linearly proportional to the number of ligands added. For eximple, the first DPNH molecule to become attached to a protein might in principle capture the major portion of the protein excitation energy. Subsequent molecules of DPNH might bind in statistically normal fashion but quench a disproportionately small amount of the protein emission. If one plotted a titration curve obtained under such conditions the apparent stoichiometry would be low and the binding sites would not appear to be equivalent and independent. In practice this has not happened (35). The titration curves of enzyme with DPNH, using either the quenching of protein fluorescence or the directly excited nucleotide emission as indicators, are strictly concordant with respect both to stoichiometry and to apparent intrinsic dissociation constants. It must be concluded therefore that each molecule of nucleotide quenches only the tryptophan within its own domain and that there is negligible overlap between domains. The proteins behave as though they possessed $n$-fold symmetry corresponding to $n$ binding sites, with $n$ equivalent subunits, real or virtual.

Although the proteins that have been examined in the present studies may indeed be specific aggregates of subunits, no direct evidence for subunits has been provided, and if they occur it is unlikely that they dissociate under the conditions of the fluorometric measurements. GPD, for example, shows no sign of dissociation by lightscattering measurements at concentrations down to $100 \mu \mathrm{g}$ per ml. Glutamic dehydrogenase is a rather large aggregate, of molecular weight 1,000,000, which dissociates at high dilution; and Frieden (10) has found that this dissociation is promoted by the binding of DPNH at specific inhibitory sites. The subunits in this case are, however, of large molecular weight (about 250,000) and still contain 3 to 4 coenzyme binding sites each. The occurrence of domains in GPD poses no serious problem because the maximal quenching of the protein by DPNH is only 40 per cent. There are about 15 tryptophans in GPD or 5 per binding site, and 40 per cent quenching corresponds to the capture of all the excitation energy from only two of them by a molecule of DPNH. The domains in this case could easily be determined by proximity of the tryptophans to the binding sites. The behavior of LDH-APNH is more unusual. In this complex the quenching is $80 \mathrm{per}$ cent. Each molecule of the bound coenzyme 
analogue captures, in effect, all of the excitation energy from 6 tryptophan resiches, or 80 per cent of the excitation from each of the 7 that occur within a domain.

What is the line of demarcation that prevents energy transfer between domains if the protein does not dissociate? Energy transfer of the type being considered is a quantum effect arising from an electrical dipole interaction. Förster has calculated the general expression for the rate constants of such transfers and has expressed the results in terms of a distance, $R_{0}$, between excited donor and acceptor at which the donor transfers the encrgy with the same probability that it emits a quantum of light.

$$
R_{o}^{6}=\frac{9(\ln 10)^{2} c \tau_{o} J_{\bar{\nu}}}{16 \pi^{4} n^{2} \Lambda^{\prime 2} \bar{\nu}_{0}{ }^{2}}
$$

where $c$ is the velocity of light; $n$, the refractive index of the medium (in this case of the protein); $\pi$, the lifetime of the excited state of the donor; $N^{\prime}$, Avogadro's number divided by $1000 ; v_{0}$, the frequency of the pure electronic transition; and $J v$, the overlap of the emission band of the donor and the absorption band of the acceptor. $J_{v}$ may be determined graphically as mentioned earlier, or analytically from the integral

$$
J_{\bar{\nu}}=\int_{0}^{\infty} \epsilon_{A}(\bar{\nu}) \epsilon_{D}\left(2 \nu_{0}-\bar{\nu}\right) \mathrm{d} \nu
$$

where $\epsilon_{A}(v)$ is the absorption coefficient of the acceptor as a function of wave number, and $\epsilon_{D}\left(2 v_{v_{1}}, v^{\prime}\right)$ is the emission band of the clonor set up as the mirror image of the absorption band around $v_{0}$. The factor $x^{2}$ is a mutual orientation factor and has the value

$$
x^{2}=(\cos \theta-3 \cos a \cos b)^{2}
$$

where $\theta$ is the angle between the two clipoles, and $a$ and $b$ are the angles cach makes with the line connecting their centers.

$R_{0}$, calculated from the above relations for tryptophan and DPNH in free solution, is $25 \Lambda(14)$. This is at best an approximation and is qualitatively in accord with experiment. From its hydrodynamic properties LDH maty be treated as a rod of axial ratio $2 / 1$ and dimensions of the order of $92 \times 46 \times 46 \AA$. The $R_{o}$ value for $A P N H$ and tryptoplam is a litte larger than $25 \Lambda$ but the dimensions of a quarter of the LDH molecule are large for the observed eighty per cent. transfer of excitation energy unless the average tryptophan residue 
is distributed closer to the binding site than it would be in a random or uniform distribution. The interacting tryptophan residues and the bound coenzyme are in fixed orientation in the protein molecule and a gain in transfer efficiency may occur through a lavorable value of $x^{2}$. The angle $\theta$ is related to the polarization as

$$
P_{o}=\frac{3 \cos ^{2} \theta-1}{\cos ^{2} \theta+3}
$$

In Fig. 8 it is seen that the DPNH emission excited at $340 \mathrm{~m} \mu$ with umpolarized light exhibits nearly the maximal theoretical polarization, whereas the polarization of the fluorescence excited by transfer from the tryptophan is quite low. This was to be expected since the energy reaches the coenzyme from seven different directions. The average value of $\theta$ of DPNH and tryptophan in an $\mathrm{LDH}$ domain is about $45^{\circ}$ but the $a$ and $b$ angles are unknown. $x^{2}$ is a sufficiently sensitive function of $a$ and $b$ to account for the demarcation of domains in cases where the transfer efficiency is high within a domain. It is to be expected that cases will arise, depending upon the structures of protein and ligand, in which there will be a strong overlap between domains. Because of this possibility, the quenching of protein fluorescence as a quantitative indicator of ligand attachment must be used with discretion. ${ }^{1}$

The triptophan emission of hemoglobin and myoglobin is quenched more than 99 per cent by the heme prosthetic group (41). In these cases each heme is associated with a protein subunit of about $18.000 \mathrm{~g}$ molecular weight, which is about half the size of a domain of interaction in the pyridine-nucleotide-enzyme c.mplexes. Extremely efficient quenching is also a consequence of the high oscillator strength of the heme. Titration of the chemically resolved apoprotein with heme (Furfine and Velick, unpublished), with quenching of protein fluorescence as indicator, shows normal stoichiometry. The quenching is about 85 per cent in the reconstituted system. The absence of strong overlap between domains in this casc is associated with the fact that the apoprotein, in the absence of heme, is a mixture of monomer and dimer subunits, chiefly dimer. One prosthetic group per dimer promotes aggregation to the tetramer, corresponding in molecular weight to the normal hemoglobin. Failure to obtain complete quenching and the apparent absence of quenching overlap between subunits is a sign that the reconstituted complex is not identical with the starting matcrial although it is quite similar in its other properties.

In unusual example of the quenching of protein fluorescence is provided by a pure antibody directed against 2,t-dinitrophenyl haptene groups. The antibody, of molecular weight approximately 180,000 , combines with two molecules of the haptene, 2,4-dinitrophenyl-L-lysine, with a dissociation constant of $10^{-0} \mathrm{M}$, remaskably low for an immunochemical system. There is a strong orerlap between the emission band of the protein and the absorption band of the haptene, and the quenching is about 75 per cent (Velick, Parker, and Eisen, Proc. Nat. Acad. sici. U. S., in press). 


\section{FLAVIN NUCLEOTIDES}

Flavin adenine dinucleotide conformation. The absorption spectra of the constituent mononucleotides of FAD are not additive in the dinucleotide, and the fluorescence quantum yield of the dinucleotide is about one-tenth that of the flavin monomucleotide. It was proposed, on these grounds, that FAD also occurs as a folded inner complex (38). One of the possible folded forms is illustrated in Fig. 15. The chiel points to be noticed are occurrence of an open chain ribitol instead of a ribose on the isoalloxazine and the congruent structures of the adenine and isoalloxazine which would favor a faceto-face contact.

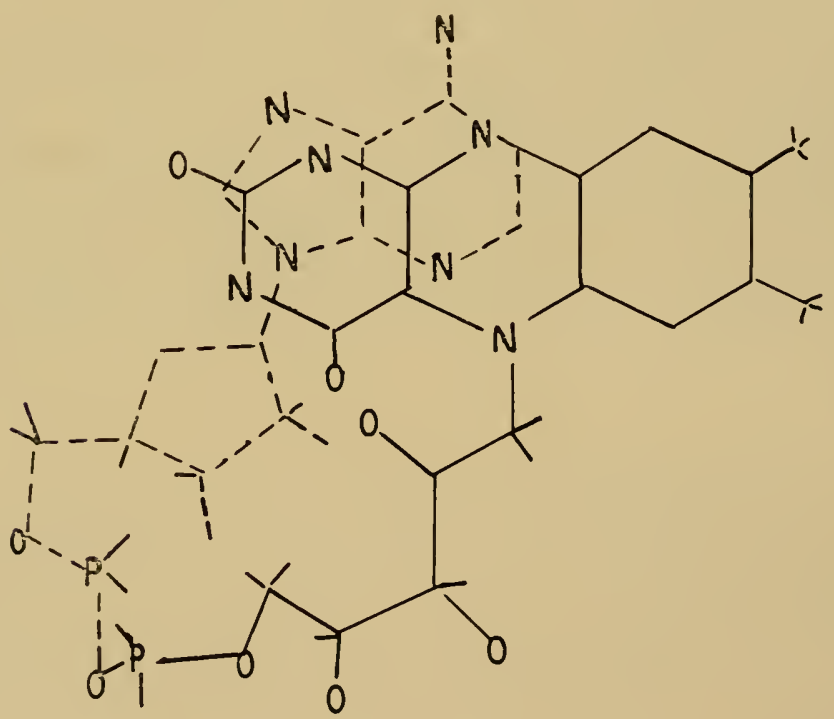

Fig. 15. A skeletal model of llavin adenine dinucleoticle in a folded conformation. I the dashed portion of the figure is the adenylic acid moiety of the dinucleotide and is located on a plane below that of the flavin mononucleotide.

Effects of temperature and solvent. The emissions of flavin mononucleotide (F.MN) and of FAD are maximal at about $520 m_{\mu}$ and extend out into the red. Fig. 16 shows the relative fluorescence quantum yields of the two nucleoticles as a function of temperature in two different solvents. The strong emission of F.MN, with a quantum yield of about 25 per cent, shows a typical negative temperature coefficient and is identical in water and in methyl carbitol. ln water, the emission of F.AD is virtually independent of temperature. 


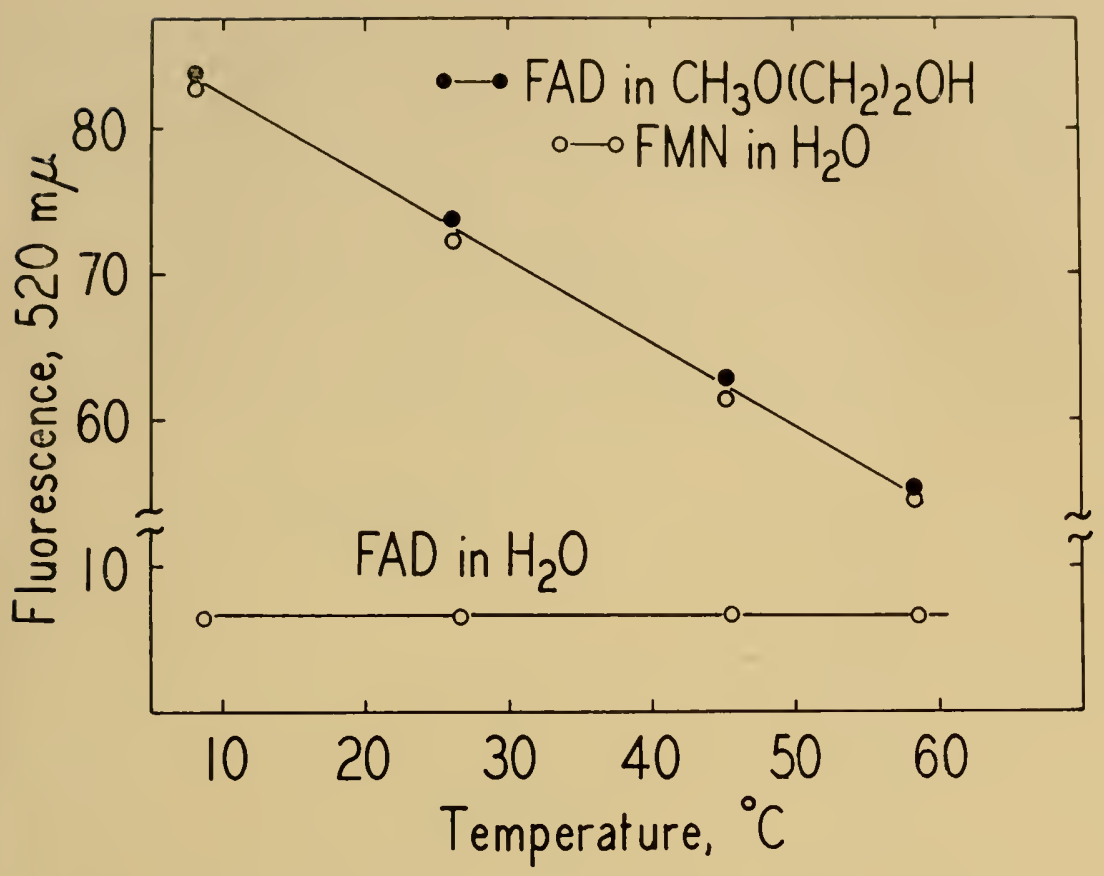

Fig. 16. The relative ffurescence quantum yields of flavin mononucleotide and dinucleotide as a function of lemperature in water and in methyl carbitol. The curve for FMN in methyl carbitol coincides with that in water and is not shown.

In methyl carbitol, the FAD emission is enhanced approximately tenfold and now coincides with that of FMN with respect both to quantum yield and to temperature coefficient. Thus the inmer complex of FAD behaves like that of DPNH. An explanation of the temperature-independence of $\mathrm{FAD}$ emission is provided by an examination of the excitation spectrum.

The excitation spectrum of FAD. Superimposed on the absorption spectrum of FAD in Fig. 17 is the fluorescence excitation spectrum. Within the limits of error of the light source calibration, the two curves coincide in the two long wavelength bands, but the quantum yield is lower when the excitation is in the ultraviolet. Unlike reduced nicotinamide, the contribution of the flavin to absorption in the $260 m \mu$ region is about twice that of the adenine. Consequently excitation in this region arises chiefly from direct absorption of photons in the isoalloxazine ring structure. There is in fact no fluorescence excitation by absorption in the adenine. The monomucleotide is excited more efficiently in this region than is the di- 
nucleotide. These results suggest that the dinucleotide does not emit at all and that the enission from FAD comes from about 10 per cent of the open form which is in thermal equilibrium with the inmer complex. The small or zero temperature coefficient of FAD fluorescence is thus probably a coincilence. Thermal quenching of the emission of the open conformation is compensated by the increased concentration of this form as a function of temperature.

spectral changes during oxidation and reduction. Unlike the pyri-

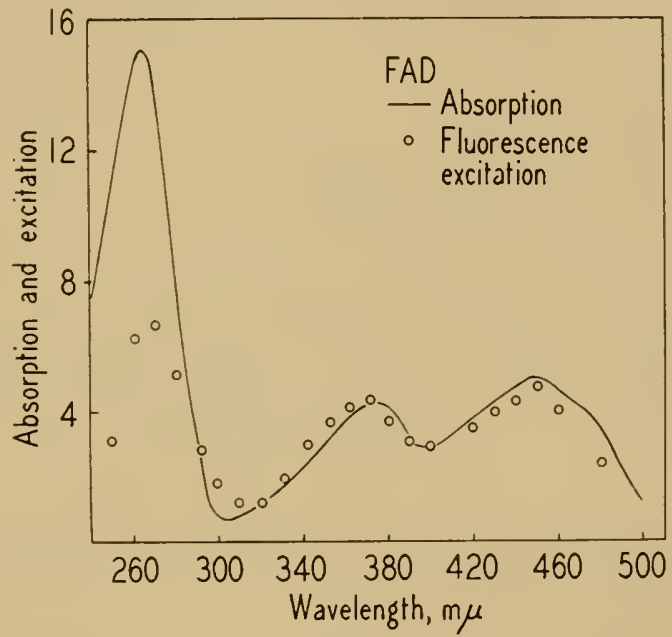

Fig. 17. The corrected fluorescence excitation spectum of FAD in water (open circles), normalised to coincide with the absorption spectrum (solid line), at about $370 \mathrm{~m} \mu$.

dine nucleotides, it is the oxidized forms of FMN and FAD which have strong absorption bands in the risible and near ultraviolet, and it is the oxidized and not the reduced forms that fluoresce. The spectral changes in FMN at various stages of reduction are shown in Fig. 18. Using a rapid scamning spectrophotometer, Beinert (2) observed two additional, very weak bands at longer wavelengths during the reduction of FMN by hychosulfite (Fig. 19). The band at 565 $1 m \mu$ rises to a maximum at 50 per cent reduction and then begins to disappear and is absent when reduction is complete. The band at $880 \mathrm{~m}_{\mu}$ exhibits a similar behavior but is strongly dependent upon concentration and temperature. Beinert therefore assigned the 560 $m_{\mu}$ band to a semiquinome or free radical intermediate and the 880 $m_{\mu}$ band to the semiquinone dimer. The occurrence of a semiquinone 
intemediate in the oxidation and reduction of flavins had previously been indicated by the potentionetric titrations of Michaclis (16), who postulated long ago that a semicuinone intermediate was obligate in the reduction of a univalent cytochrome by the divalent llavin in the pathways of biochemical electron transport.

\section{Flavin Nucleotide Enzyine Complexes}

Old yellow enzyme (OYE). Although its catalytic activity has not yet been thoroughly investigated, this flavoprotein was the first enzymecoenzyme complex to be purified, resolved, and reconstituted (30). In

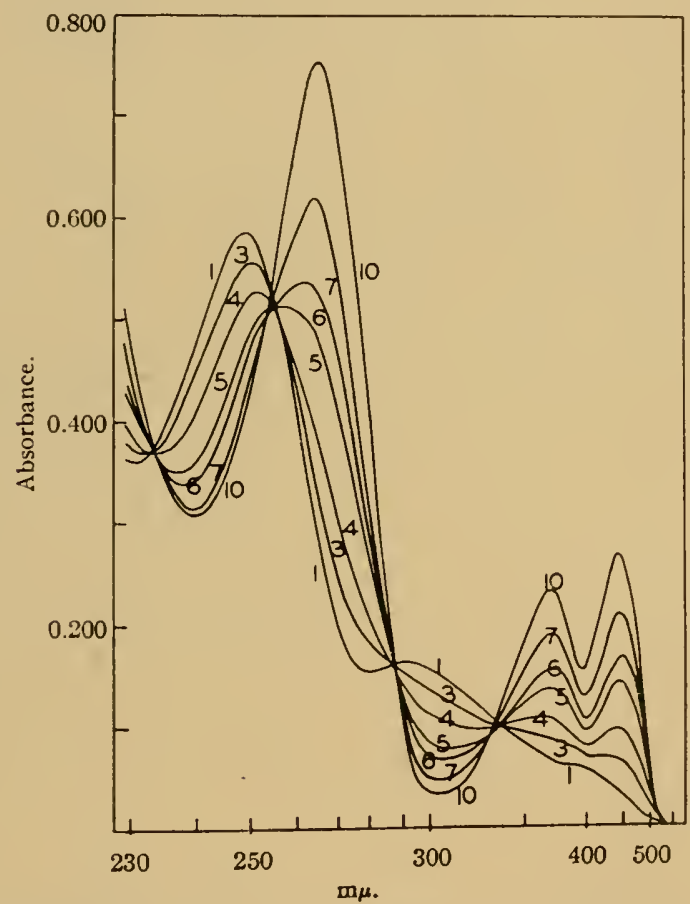

Fig. 18. The absorption spectrum of $0.25 M F M N$ in a $0.05 \mathrm{~cm}$ cell at various stages of reduction, $p \mathbf{H}$ 5.2. Curve 1, reduced; curve 10, oxidized. From Beinert (2.)

recent years Theorell and coworkers have completed the purification of the protein and have extended the early characterization of the complex $(31,32)$. The protein, of molecular weight 102,000 , carries two molecules of FMIN. In the complex the fluorescence of FMN is almost completely quenched. This effect has been utilized in the 


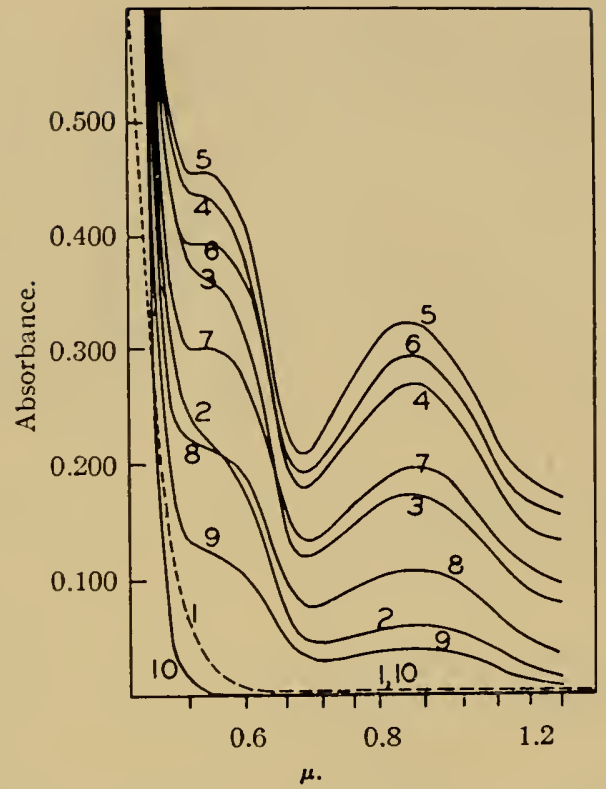

lig. 19. The absorption spectrum of the same solutions of FNI as in Fig. 18, but measured at longer wavelengths in a $10 \mathrm{~cm}$ cell. From Beincrt (2).

measurement of the second order rate constant for complex formation, $k_{1}=10.8 \times 10^{4} M^{-1} \mathrm{sec}^{-1}$, and the equilibrimm constant, $K=0.7 \times 10^{-8} M$. The first order rate constant for dissociation is accordingly $13.5 \times \mathrm{I}^{-4} \mathrm{sec}^{-1}$. Potentionetric titrations by Vestling (36) indicate a standard oxidation reduction potential, $E_{o}^{\prime}=0.190 \mathrm{v}$ for free F.IN, $-0.123 \mathrm{v}$ for the complex. If the positive potential shift is attributed to increased interaction of the protein with the reduced form of the nucleotide, then the dissociation constant of the protein and $\mathrm{FMNH}_{2}$ is $6 \times 10^{-11} \mathrm{M}$.

Ordinarily one would expect the rate constant for complex formation between enzyme and coenzyme to be too rapid to measure by simple direct methods. However, the OYE complex probably does not clissociate significantly under functional conditions. It is likely that when the complex is resolved chemically, conformational changes xccur in the protcin, the reversals of which are rate-limiting in the recombination reaction.

OYE catalyges the oxidation of TPNH by various acceptors, but its natural acceptor is unknown. When Ehrenberg and Ludwig (7) incubated the curye with excess TPNH in air the peculiar spectral 
shift shown in Fig. 20 was obtained. It was found that TPN had been formed and that one molecule of TPN per molecule of flavin hat become bound to OYE to form a stable complex in which the flavin also appears to be in the oxidized state. Upon incubation of the reaction mixture anaerobically, an electron spin resonance signal

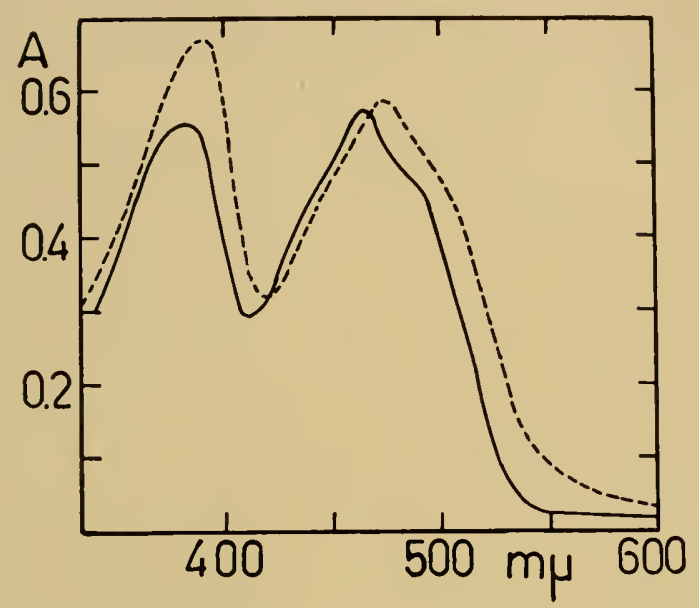

Fig. 20. The absorption spectrum of the old yellow en/yme, solid curre, and of its complex with TPN, produced as described in the text. From Ehrenberg and Ludwig (7).

corresponding in magnitude to 15 per cent of flavin semiquinone was detected. The signal disappeared upon admission of oxygen and returned when oxygen was removed, with no concomitant changes in the absorption spectrum. The location of the odd electron in the system is unknown. In order to remove the TPN from the complex by dialysis, it was necessary first to reduce both the pyridine and the flavin with hychrosulfite. How such a complex would dissociate in a catalytic situation, if it actually occurs as an intermediate, is not clear.

The acyl-coenzyme-A dehydrogenases. The reactions catalyzed by these FAD enzymes are reversible $\alpha-\beta$ dehydrogenations of the fatty acyl group in thio ester linkage with Co-A. Beinert (3) observed the development and decline of a semiquinone type of absorption band at $570 \mathrm{~m}_{\mu}$ during the reluction of the enzyme by substrate. This enzyme, too, forms a stable complex with substrate or product, but since the $570 \mathrm{~m}_{\mu}$ band can also be produced by non-specific reducing agents it is assigned directly to the bound flavin and not 
to a ternary complex. If a semiquinone intermediate is formed, as the spectrum indicates, there should also be an electron spin resonance signal, but none could be observed. A spin resonance absorption band could easily be missed if it were diffuse. Like many flavoproteins, the acyl-CoA dehydrogenase occurs in an organized subcellular particle. The particulate environment obscures details of function, but it is not known to what extent functions are modified or missed when such enzymes are examined in the isolated state.

Lipoic dehydrogenase. This enzyme, initially characterized by its catalysis of dye reduction by DPNH $(24,20)$ and termed diaphorase has recently been iclentified by Massey (15) as lipoic dehydrogenase. It is an FAD enzyme, noteworthy among flavoproteins in that the fluorescence of the flavin is not quenched in the enzyme complex but is enhanced about two-fold over that of free $\mathrm{FAD}$. The absorption bands of the FAD are bleached only about 15 per cent in the presence of excess DPNH, but one mole equivalent of DPNH in a dilute anaerobic system completely quenches the flavin fluorescence, suggesting the formation of a pyridine nucleotide complex. Inhibition with arsenite (15a) and the behavior of a $530 \mathrm{~m} \mu$ absorption band produced by reduced substrate $(15 b)$ are interpreted as evidence for vicinal dithiol groups which interact with the bound flavin to form free radical intermediates. Massey has also postulated the participation of a pyridine nucleotide free radical on the basis of the above observations and an unusual catalytic effect of DPN in the reduction of lipoic acid by DPNH.

Flavoproteins and molecular oxygen. Most flavoproteins in the reduced form are reoxidized slowly by molecular oxygen, with formation of hydrogen peroxide. A few special ones, such as xanthine oxidase (1), L-amino acid oxidase (22), and lactate oxidase (28), react rapidly and catalytically with molecular oxygen as the acceptor. The lactate oxidase, crystallized and characterized by Sutton, is of particular interest. Pyruvate and peroxide, produced in the initial reaction step, remain bound in some form to the enzyme and react in a second step to form acetate and carbon dioxide. No frec pyruvate or peroxide can be detected as intermediates or as products of the reaction. Concentrated solutions or slurries of this enzyme give a reak electron spin resonance signal, the intensity of which is dependent upon the concentration of lactate present (6).

The microsomal cytochrome reductase. This enzyme was isolated by Strittmatter and nuyself (27) from calf liver nicrosome particles. It catalyzes a rapid and specific reduction of the microsomal cyto- 
chrome-b- by DPNH. The red shift of the flavin absorption bands (Fig. 21) is typical of flavoproteins, but the enhancement of the shoulder around $485 m \mu$ is unusually pronounced. The FAD on this enzyme, of $40,000 \mathrm{~g}$ molecular weight per mole, is strongly bleached by DPNH, and a pyridine nucleotide complex is formed

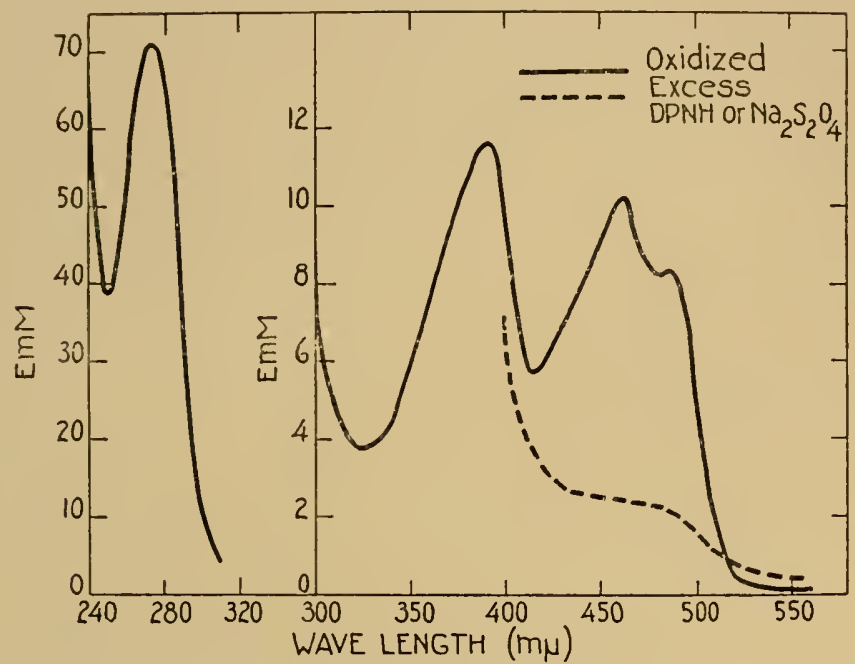

Fig. 21. The absorption spectra of the bound flavin in the microsomalcytochrome- $b_{5}$ reductase; solid line, oxidized; dashed line, reduced (27).

(25). The complex in this case is recognized by an absorption band at $315 \mathrm{~m}_{\mu}$, most clearly seen in difference spectra (Fig. 22). The pyridine nucleotide flavoprotein complex is only formed by admixture of cross conjugate pairs, DPNH and oxidized flavoprotein or DPN and reduced flaxoprotein. It dissociates when the complex is reoxidized by a variety of reagents, as it must do also in the rapid catalytic reaction with cytochrome- $b_{\overline{5}}$. The reductase contains a sulfhydryl group that is essential for the binding and oxidation of DPNH (27). Two additional sulfhydryl groups can be distinguished from this one by their chemical reactivity and do not seem to be directly involved in the catalysis (26). In the pyridine nucleotide enzyme complex the flavin is fully bleached and hence appears to be in the fully reduced form. If this is true, the pyridine nucleotide is in the oxidized state, and the $315 \mathrm{~m} \mu$ absorption band corresponds to the interactions that occur with DPN and inhibitor on LDH and other dehydrogenases. Such a band could arise, for example, from inter- 


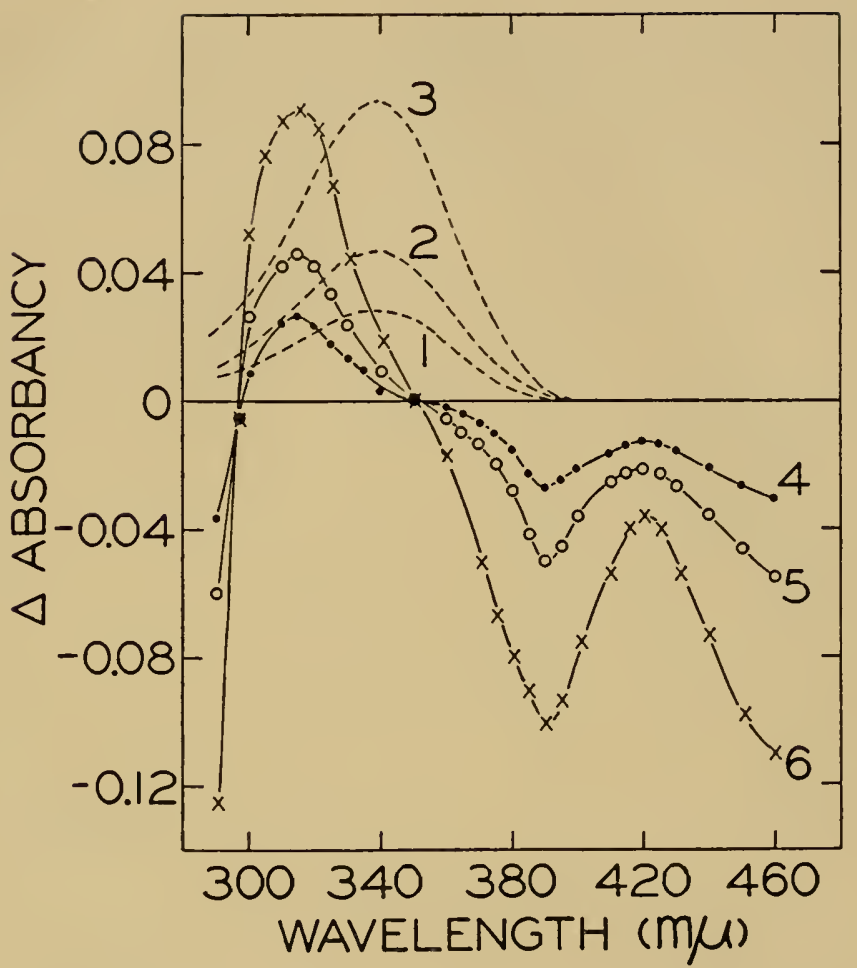

Fig. 22. The absorption spectrum of the pridine nucleotide complex of the microsomal cytochrome-b, reductase. Curves 1,2 , and 3 arc the absorption bands of $0.8,1.5$, and $3 \mu \mathrm{M} \mathrm{DPNH}$. Curves 4,5 , and 6 are difference spectra of 3 $\mu \mathrm{M}$ enzyme plus $0.8,1.5$, and $3 \mu \mathrm{M}$ DPN respectively, after reduction with $\mathrm{DDH}$ and ethanol minus the corresponding spectra before reduction. From Strittmatter (26).

action with the essential thiol group and would be like that produced in liver LDH-I)PN by sulfide or hydrosulfide ion (29). It does not resemble the $365 \mathrm{~m} \mu$ band of GPD-DPN. Since the bound flavin must be in the reduced form in the pyridine mucleotide complex, a reasonable alternative is that the interaction is not with thiol but with a nitrogen atom of the reduced flavin.

This work was supported in part br a research grant from the National Heart Institute, National Institutes of Ileath Some of the work on cocmyme confommtion in enzme complexes was intially described at the Symposimm on Plysical Methods in Biochenistry at the Iederation meetings, Itlantic City, Ipril, 1959. Experimental detals are in press elsewhere. 


\section{REFERENCES}

1. Wis, P. (,.. Bergel, F., and Bray, R. C., J. Chem, Soc., 1100 (195.5).

2. Beinert, H., J. Am. Chem. Soc., 78, 5323 (19.56).

3. Beinert, H., J. Biol. Chem., 225, 465 (1957).

I. Brown, D. M., Fasman, G. D., Magarth, D. I., Todll, A. R., Cochran, W., and Woolfson, M. M., Nature, 172. 1184 (1953).

5. Colowick, S. P.. Kaplan, N. O., and Ciotti, M., J. Biol. Chem., I9I, 477 (1951).

6. Commoner, B., Lippincoll, B., and Passoneat, J. V., Proc. Nat. Acarl. Sci. I. S., 44, 1099 (1958).

7. Ehrenberg, A., and Ludwig, G., Science, 127, 1157 (1958).

8. Fisher, H. F., Comn, E. E., Vennesland, B., and Westheimer, F., J. Biol. Chem., 202, 687 (1953).

9. Förster, Th., Fluorescenz organischer rerbindungen, Vandenhook and Rupprecht, Göttingen (1951).

10. Frieden, C., J. Biol. Chem,, 234, 809 (1959).

11. Furburg, S., Actu Cryst., 3, 325 (1950).

12. Kaplan, N. O., and Ciotti, M., J. Biol. Chem.. 211, 431 (1954).

13. Kaplan, N. O., Ciotti, N., Stolzenbach, F. E., ancl Bachur, N. R., I. Am. Chem. Soc., 77, 815 (1955).

14. Karreman, G., Steele, R. H., and Szent-Györgyi, A.. Proc. Nat. Acad. Sci. U. S., 44, 146 (1958).

15. Massey, V., Biochem. et Biophys. Actn, 37, 314 (1960).

15a.Massey, V., and Veeger, C., Biochem. et Biophys. Acta, 70, 184 (1960).

15̆b..Massey, V., and Gibson, Q. H., Federation Proc., 19, 27 (1960).

16. Michaelis, L., in The Enzymes (J. B. Summer and K. Myrbäck, eds.), Vol. II, part 1, Academic Press, New York (1951).

17. Pfleiderer, G., Jeckel, D., and Wieland, T., Biochem. Z., 328, 187 (1956).

18. Pullman, M., San Pietro, A., and Colowick, S. P., J. Biol. Chem., 206, 129 (1954).

19. San Pietro, X., Kaplan N. O., and Colowick, S. P'., J. Biol. Chem., 212, 911 $(1955)$.

20. Savage, N., Biochem. J., 67, 146 (195i).

21. Segal, H. L. and Boyer, P. 1)., J. Biol. Chem., 204, 265 (1953).

2la.Shifrin, S., and Kaplan, N. O., Nature. 183, 1529 (1959).

22. Singer, T. P'., and Kearney, E., Arch. Biochem., 29, 190 (1950).

23. Stockel, A., J. Biol. Chem., 234, 1286 (1959).

24. Straub, F. B., Biochem. J., 33, 787 (1939).

25. Strittmatter, P., J. Biol. Chem., 233. 748 (1958).

26. Strittmatter, P., J. Biol. Chem., 234, 2661 (1959).

27. Strittmatter, P., and Velick, S. F., J. Biol. Chem., 228, 78.5 (1957).

28. Suctou, W. B., J. Biol. Chem., 226, 395 (1957).

29. Terayama, H., and Vestling, C. S., Biochim. et Biophys. Actn, 20, 586 (1956).

30. Theorell, H., Biochem. Z., 978, 263 (1935).

31. Theorell, 11., and Ikesson, .1., Arh. Biochem. Biophys., 65, 439 (1956).

32. Theorell, H., and Nygaard, 1. P., Acta Chem. Scrund., 8, 877 (1954).

33. Theorell, H., and Winer, 1. J)., Aich. Bioctiem, and Biophys., 83, 291 (1959).

34. Vellick, S. F., in Mechanism of Enzyme Action (W. D. McElroy and B. Glass, eds.), The Johns Hopkins Press, Baltimore (1954).

3.). V'elick, S. F., J. Biol. Chem., 233, 145.5 (1958).

36. V'estling, C. S., Acta Chem. Scrand., 9, 1600 (195.5).

37. Warburg, O., and Christian, W., Biochem. \%., 287, 291 (1936).

38. Weber, G., Biochem. J., 47, $114(1950)$.

39. Weber, G., Nature, 180, 1409 (1958). 
10. Weber, G., this symposium.

11. Weber, G., and Teale, F. J. W., Discussions Faraday Sociely, 27, 134 (1959).

42. Winer, 1., and Schwert. G., J. Biol. Chem., 234, 115; (1959).

43. Winer, A., and Theorell, H., Acta. Chem. Scand., 13, 1038 (1959).

\section{DISCUSSION}

1) R. (Commonit: Since 1)r. Velick has brought up the matter of the evidence for frec radical formation in flavoprotein oxidation-reduction, it might be pertinent to mention the relevant data.

ESR signals due to free radicals in flavoprotein enzyme systems have been published by Commoner et al. (Science, 126, 57, 1957), Ehrenberg and Ludwig (Science, 127, 1177, 1958), Kubo et al. (Bull. Soc. Chem. Biol., 41, 981, 1958), and Bray et al. (Biochem. J., 73, 193, 1958). In these cases, kinetic data were lacking, so that no conclusions could be drawn regarding the precise origin of the free radical in the enzyme reaction mechanism. More recently it has been possible to carry out quantitative kinetic studies of the ESR signals observed in two flavoprotein enzyme systems, lactic oxidative decarboxylase (Commoner, Lippincott and Passomeau, Proc. Nat. Acad. Sci. U. S., 4t, 1099, 1958) and succinic dehydrogenase (Commoner and Hollocher, Proc. N'at. Acad. Sci. U. S., 46, 405, 1960; and Hollocher and Commoner, Proc. Nat. Acad. Sci. U. S., 46, 416, 1960). The latter investigations show unequirocally that the ESR signals arise from free radical intermediates in the oxidation-reduction of the flavoprotein.

Most detailed data are available in the case of succinic delydrogenase. ESR studies of mitochondrial particles from pig heart muscle reveal a signal which, from the effects of inhibitors, can be shown to arise from succinic dehydrogenase. The intensity of the signal bears simple relationships to temperature, enzyme concentration, the concentration of fumarate + succinate, the fumarate/succinate ratio, and the concentration of malonate.

These data provide a test of various possible reaction mechanisms. From these considerations it (an be concluded that the observed ESR signal is due to a free radical form of the enzune-substrate complex, which is in equilibrium with the nom-iree radical form of the complex. The free radical form of the complex occurs in the efuilibrium established among succinate, Ilavoprotein and funtratc. It does not onur when the reduced flavoprotein is reoxidized by eytochrounc $\mathrm{b}$.

The most recont investigations of this system (IIollocher and Commoner, Federation Proc. 19, 28, 1960) have been carried out with purified succinic dehydrogenase. The results are identical with those obtained from heare particle preparations and fead to quantitatice estimates of the various reaction constants. The equilibrimn constant relating the non-free radical form of the (encyme substrate complex to the frec ratical form is about .3.

It appears, therefore, that there is considerable evidence which shows that frec radical forms of flawoprotein enzymes play specific roles in the enzmatic reaction mechanisms. 
1)k. IRvox: I would lite to ask Dr. Velich if he would venture a guess on the reasons for the specificity of TPN-linked triose phosphate dehydrogenases in the photosynthetic reactions. Isolated chloroplasts reduce TPN bat not DPN in light and so the triose phosphate dehyetrogenase which is involved in the reduction of PG.S in light is alwalys the TPN-linked enzyme. If we take, however, another photosynthetic organism, Chromatium, where we can show that the pyridine nucleotide can be reduced with hydrogen in the dark, we find that the classical, DPN-linked triose phosphate dehyelrogenase is the effective enzyme in PGA reduction, as is, of course, the case in all other non-photosynthetic cells. There seems to be something peculiar about the link between light and the TPN-linked enzyme. Is the reason to be sought in the extrat phosphate of TPN as contrasted with DPN?

Dr. V'eluck: I don't think the specificity lies in that extra phosphate group. I think you will have to look for a teleological explanation.

DR. ARNox: Would you care to give it to me?

Dr. RaCkER: Dr. Velick, before you do this may I point out that there are several TPN-linked triose phosphate dehydrogenases that are found in nonphotosynthetic bacterial systems?

Dr. ARxox: But why does it always apply to the photosynthetic systems?

I)R. Arxox: Let us hear that last teleological comment before we adjourn, Mr. Chairman.

Dr. V'euck: 1 would say that in general the difference between TPNH and DPNH in intermediary metabolism is that TPNH is utilized in the reduction of organic substrates in animal metabolism and DPNH is in the oxidative pathway through flavins and cytochromes to oxygen. There is a demarcation of coentyme for functional convenience and this is the way it happens to come out in plants. 


\title{
INTERACTIONS IN PYRIDINE NUCLEOTIDES ${ }^{1}$
}

\author{
Sidney Shifrin and Nathan O. Kaplan \\ Graduate Department of Biochemistry \\ Brandeis University, Waltham, Massachusetts
}

The essentiality of specific functional groups in the diphosphopyridine nucleotide (DPN) molecule for biological activity has been studied by the use of coenzyme analogues in a large number of enzyme systems $(2,8,11,21,22)$. The usefulness of the coenzyme analogues in studying intramolecular interactions in the pyridine nucleotides will be presented in this report.

Weber $(24,25)$ has studied the interaction of the adenine and dihydronicotinamide moieties of DPNH by polarization fluorescence and by the ability of adenime to transfer excitation energy to the fluorescent dihydronicotinamicle moiety. From such studies, Weber (25) has suggested that there must be a strong force holding the two moieties of the dinucleotide in a position which facilitates interaction. The configurational requirements, as well as the nature of the forces facilitating intramolecular interactions, have been studied by the use of isomers as well as analogues of DPNH.

Kaplan et al. (12) reported the isolation of a compound from a "purified" DPN preparation which was identical with the biologically active coenzyme in its chemical analysis but differed from it in enzymatic and spectral properties. This nucleotide was identified as the alpha isomer of DPN; the compound is different from the enzymatically active coenzyme in the configuration at the nicotinamide ribose linkage. The enzymatically active DPN has a beta glycosidic linkage at this point, and both isomers contain the beta adenylic acid moiety. Table 1 compares the characteristics of DPN and the isolated material.

Examination of the chemically reduced $\alpha$ isomer for fluorescence properties revealed that the energy of emission was maximum at 465

\footnotetext{
This is publication No. fit of the Graluate Depamtment of Biochemistry, Brandeis Inicersity, Waltham, Massachusets. This work has becu supported by grants from the National Iustitutes of Health (NIH CY 36II) and the National frience Fomelation (NSF G.45I2).
} 
TABI.E 1

P'ROPPRTILS (I) $\beta$ AND \& DDPN

Properties

Enzymatic activity

Chemical reduction

Cyanide addition

Optical rotation

Optical rotation of corresponding

nicotinamide mononucleotides
$\beta-I) I N$

active

$\lambda_{\max } 340 \mathrm{~m} \mu$

$\lambda_{\max } 325 \mathrm{~m} \mu$

$-34.8$

$-38.3$
$\alpha-I) I^{\prime} N$

inactive

$\lambda_{\text {max }} 345 \mathrm{~m} \mu$

$\lambda_{\max } 332 \mathrm{~m} \mu$

$+14.3$

$+.58 .2$

$11 \mu$ upon excitation $345 m \mu$. However, when the reduced $\alpha$ isomer was excited at $260 \mathrm{~m}_{\mu}$, a wavelength at which 95 per cent of the light is absorbed by the adenine, the fluorescence intensity at $465 \mathrm{~m} \mu$ is only a fraction of that obtained from direct excitation of the dihydronicotinamide (Fig. 1) (20). These results are in contrast to those obtained with the beta, or enzymatically active, isomer of DPNH, in which $260 \mathrm{~m} \mu$ excitation results in the fluorescence of the dihydronicotinamide at $462 \mathrm{~m}_{\mu}$ with an intensity equal to that obtained from $340 \mathrm{~m} \mu$ excitation. The decreased efficiency of intramolecular energy transfer suggests that the two components of the $\alpha$-dinucleotide are not maintained in optimum conformations to allow for interaction.

Another variation in the structure of the DPNH molecule which may aid in elucidating the nature of the forces facilitating intramolecular interactions is the use of a thioamide group in place of the carboxamide of nicotinamide. Unlike DPN, which is transparent above 260-270 $\mathrm{m}_{\mu}$, the corresponding thionicotinamide analogue has a pronounced absorption maximum at $300 \mathrm{~m} \mu$, as shown by curve $\mathrm{A}$ in Fig. 2. The position of this maximum conforms to the suggestion made by Braude (5) that replacement of an oxygen by a sulfur auxochromic group in many chromophores results in a shift of the pertinent absorption maximum by $40-50 \mathrm{~m} \mu$ toward longer wavelengths, with an increase in the extinction coefficient. The generalization is further borne out by the spectrum of the enzymatically reduced analogue, shown in curve B. The absorption band has its maximum at $398 \mathrm{~m}_{\mu}\left(10^{-3} \epsilon=8.9\right)$ and the prominent band at $300 \mathrm{~m} \mu$ has become an inflection point with its maximum at 295 $m \mu$. The specific absorption band of the thiocarbonyl group permits an examination of the participation of this functional group in intramolecular interactions.

We postulate the structure of thionicotinamide-DPNH to be that shown in Fig. 3, in which the 6-anino group of adenine is hydrogen- 


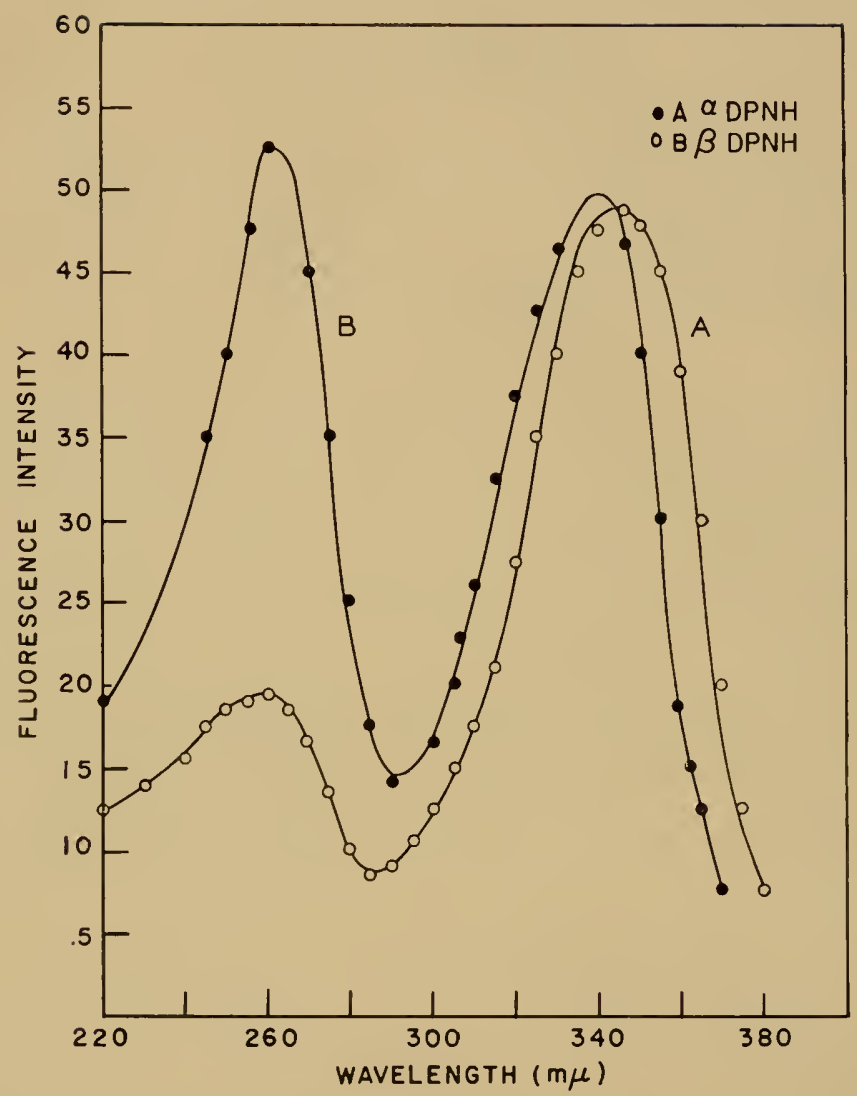

lig. 1. Fluorescence excitation spectra of alpha and beta DeNH. Curve $A$, fluotescence excitation spectrum of beta 1$) l^{\mathrm{NH}} 1.5 \times 10^{-4} \mathrm{M}$, in water. Fluorescence maximum $=462 \mathrm{~m} \mu$. Curre B, fluorescence excitation spectum of alpha DPNH, $1.5 \times 10^{-4} \mathrm{M}$, in water. The spectra were studied at $5^{\circ} \mathrm{C}$ and correcions are made for the variation in intensity of the exciting light.

bonded to the thiocarbonyl function. That the same kind of interaction occurs in the DPNH molecule is suggested from the fact that replacement of the amino group by a hydroxyl in deamino-DPNH reduces the efficiency of intramolecular excitition energy transfer (20). Modification of the adenine moicty by reacting DPN with ethylene oxide was carried out by Dr. Windmueller of our laboratory. The structure of the reduced compound under the conditions for studying excitation energy tansler is given in Fig. 4. While the $E_{260} / E_{340}$ ratio for this compound is the same as that found in DPNH, the modified material shows no transfer of energy from 260 $111 \mu$ excitation to the dihydronicotinamide moiety. 


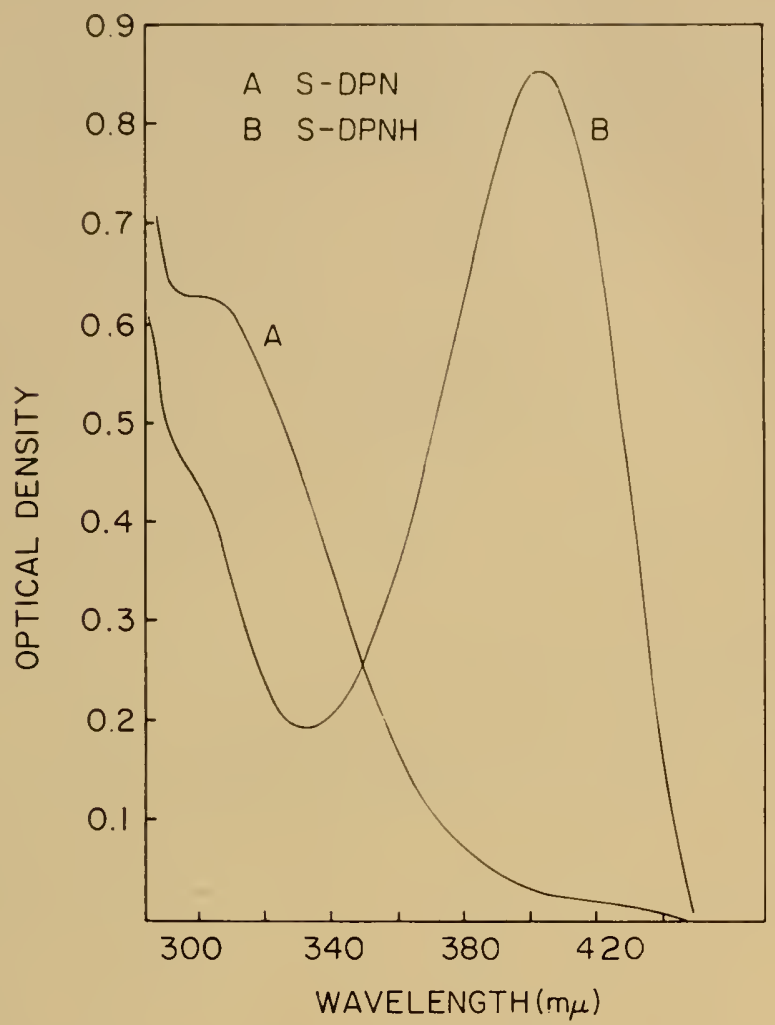

Fig. 2. Comparison of the absorption spectra of oxidized and reduced thionicotinamide DI'N. Curve A. absorption spectrum of $9.3 \times 10^{-5} \mathrm{M}$ thionicotinamide-DPN) (S-DPN) in $0.1 M$ phosphate buffer $p H$ 9.2. Curve B, absorption spectrum of $9.3 \times 10^{-5} \mathrm{M}$ thionicotinamide $\mathrm{DPNH}$ (S-DPNH) obtained by enzynatic reduction of the oxidized analoguc, using $1 \times 10^{-5} M$ horse liver alcohol dehylrogenase and $2 \times 10^{-5} M$ ethanol in $0.1 M$ phosphate buffer, $p \mathbf{H}$ 9.2.

Although the thionicotinamide analogue of DPNH is very weakly fluorescent and thereby does not permit studies of intramolecular energy transfer, spectrophotonetric analysis has allowed a determination of the interacting groups within the molecule. A comparison of the absorption spectrum of the thionicotinamide analogue of DPNH in water and propylene glycol is shown in Fig. 5. It can readily be observed that the inflection at $295 \mathrm{~m} \mu$ in water has been shifted to $300 \mathrm{~m}_{\mu}$ and is more prominent in propylene glycol.

From a study of the position of the absorption naximum of thionicotinamide as a function of the dielectric constant of the solvent, it appears that the $300 \mathrm{~m} \mu$ band results from an $n \rightarrow \pi^{*}$ transition, as suggested by Kasha (13). A schematic diagram of the 


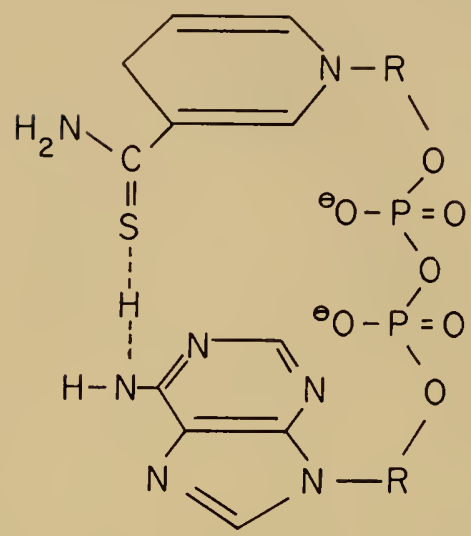

Fig. 3. Suggested structure of thionicotinamide I)PNH. R, ribose.

effect of hydrogen-bonding on the $n \rightarrow \pi^{*}$ transition is shown in Fig. 6. The excitation of one of the $n$ (non-bonding, lone pair) electrons to the antibonding $\pi$ orbital requires a certain amount of energy, $h_{v}$. If the $n$ electrons participate in hydrogen-bonding, as shown by the equation at the bottom of the figure, the energy $\left(h_{v}{ }^{1}\right)$ required to promote the electron to the same excited state will be greater than $h_{v}$, and will give an absorption maximum at shorter wavelengths. Brealey and Kasha (6) have convincingly demonstrated that in hydroxylic solvents the blue shift of $n \rightarrow \pi^{*}$ transitions is due primarily to hydrogen-bonding of the non-bonding electrons by the hydroxylic solvent, and in these cases specific hydrogen-bonding is more important than any general effect which depends on the dielectric constant.

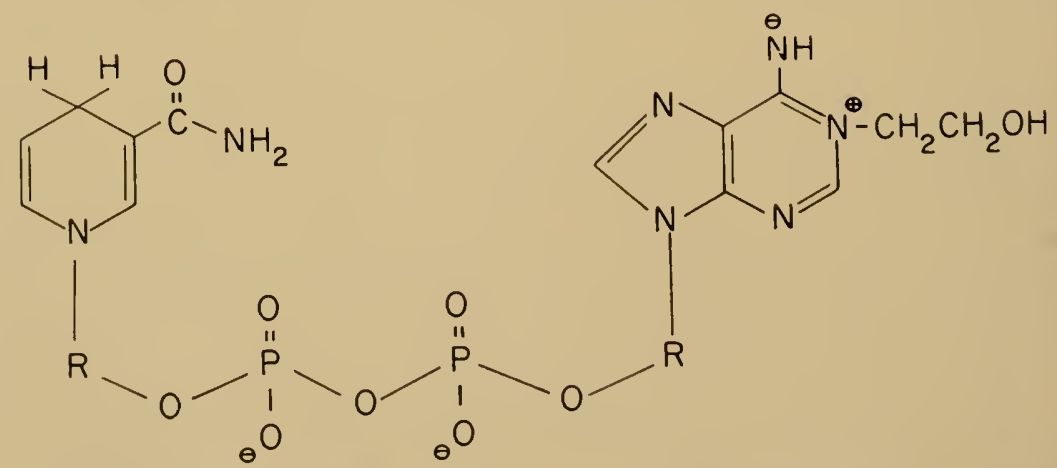

lig. 4. Structure of the reducel ethylene oxide analoguce of D)I. R, ribose. 
Recent stuclies by Sheinker et al. (19), using 2-mercaptothiazole, which exists in the thione form, have demonstrated intermolecular hychogen bonding from the amino to the thione group:
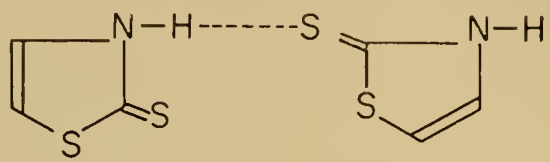

An interesting reaction of the thionicotinamide-DPNH was observed upon exposure of a solution of the reduced coenzyme analogue to strong light. The $398 m \mu$ maximum is almost completely lost under such conclitions, and a new band appears with a maximum at $341 \mathrm{~m} \mu$, as shown in Fig. 7. In addition, the absorption in the 295-300 $m_{\mu}$ region is no longer present, a lact suggesting that the thione func-

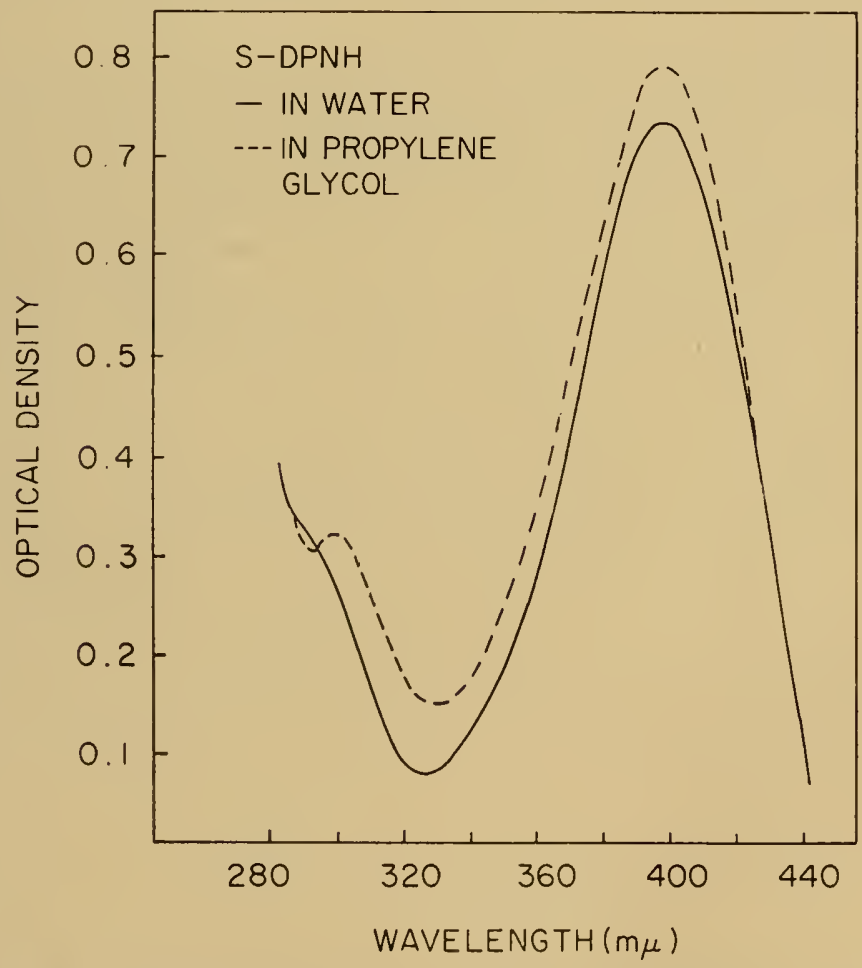

Fig. 5. Comparison of the spectra of thionicotinamide DPNH in water and propylene glycol. Solid line, absorption spectrum of $9.0 \times 10^{-5} \mathrm{M}$ thionicotinamide-DPNH in water. Broken line, absorption spectrum of the same concentration of the reduced analogue in propylene glycol. 


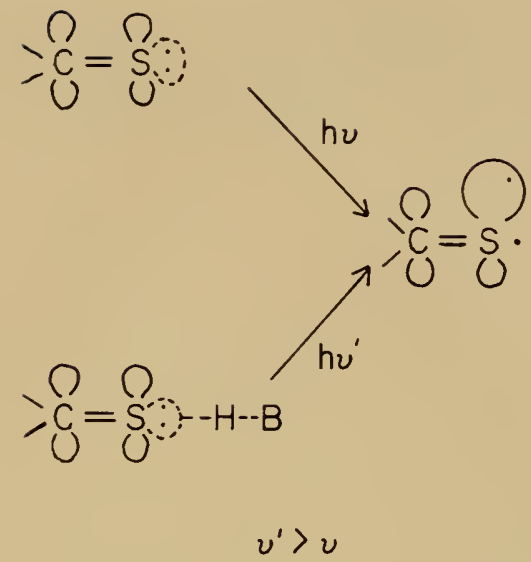

Fig. 6. The effect of hydrogen bonding on the thione absorption band. $B$, an anion.

tion has disappeared. The formation of carbonyl compounds resulting from illumination of the corresponding sulfur analogues in the presence of oxygen ( 17 ) suggested that DPNH may have been formed from illumination of its thionicotinamide analogue. However, the spectral changes resulting from exposure of the reduced coenzyme analogue to illumination are also observed when the analogue is contained in an anaerobic curette flushed with nitrogen. The following properties of the illumination product were also observed:

(a) absence of enzymatic activity;

(b) regeneration of the material absorbing at $398 m \mu$ after the solution was allowed to remain in the dark; and

(c) enhancement of fluorescent intensity upon addition to beef heart lactic dehydrogenase.

The reversibility of the reaction suggested that illumination results in the shift toward one side of an equilibrium. The thioamides may exist in an equilibrium involving the following tautomers (4, 14):<smiles>[X]C(=N)S</smiles>

If illumination caused a shift toward the thiol form, complexation of the thione grouping should prevent formation of the illuminated product. One of the earliest reactions noted with thiones was the formation of complex addition compounds with the salts of heavy 


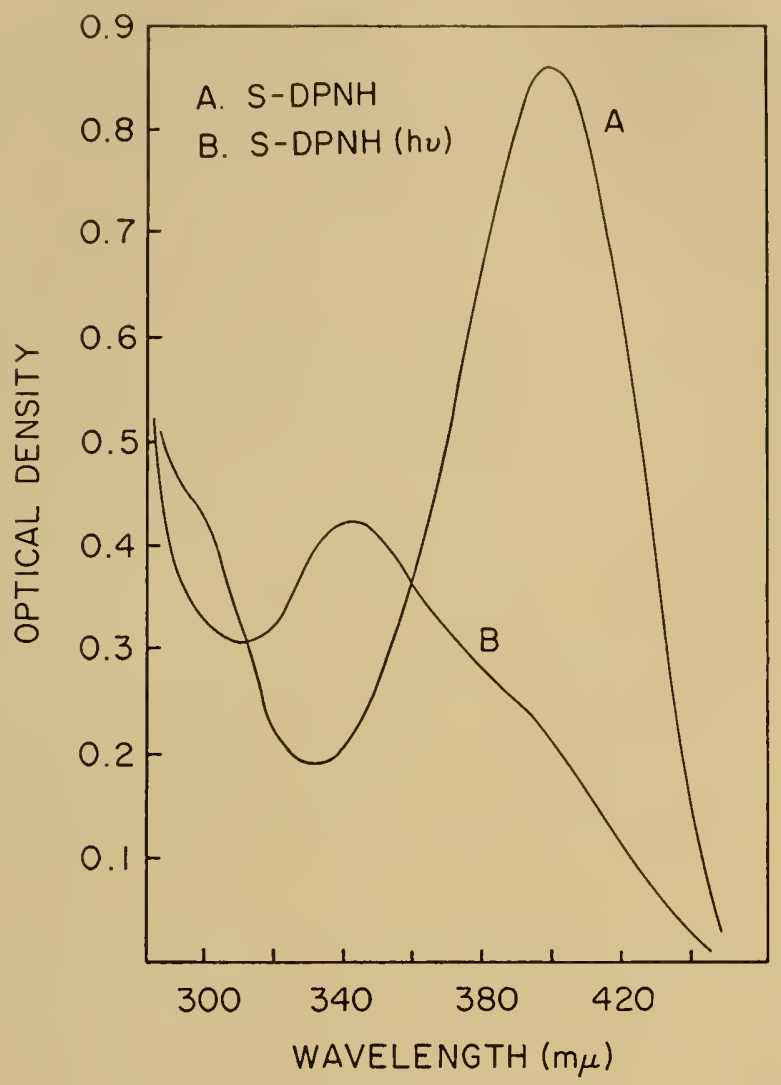

Fig. 7. Spectral changes of thionicotinamide DPNH after illumination. Curve A, absorption specirum of $9.3 \times 10^{-5} M$ thionicotinamide-DPNH in $0.1 M$ phosphate buffer, $p \mathrm{H}$ 9.2. Curve $\mathrm{B}$, absorpion spectrum of same concentration of reduced analogue after a 5 -minute exposure to the infrared source of the Cary recording spectrophotometer kept at $400 \mathrm{~m} \mu$.

metals, a formation accompanied by distinct color changes (15). The addition of equimolar amounts of p-chloromercuribenzoate to solutions of thionicotinamide-DPNH resulted in an intensification of the yellow color of the analogue and a shift of the absorption maximum toward the red. The rate of bleaching of the complex was only one-tenth that observed with the untreated coenzyme analogue.

Almost all known enolizable thiocarbonyl groups are alkylated in such a way as to suggest that the molecule exists in large part as the thiol form. When the thionicotinamicle analogue of DPNH was treated with dimethyl sulfate in sodium bicarbonate buffer, at $p \mathrm{H}$ 


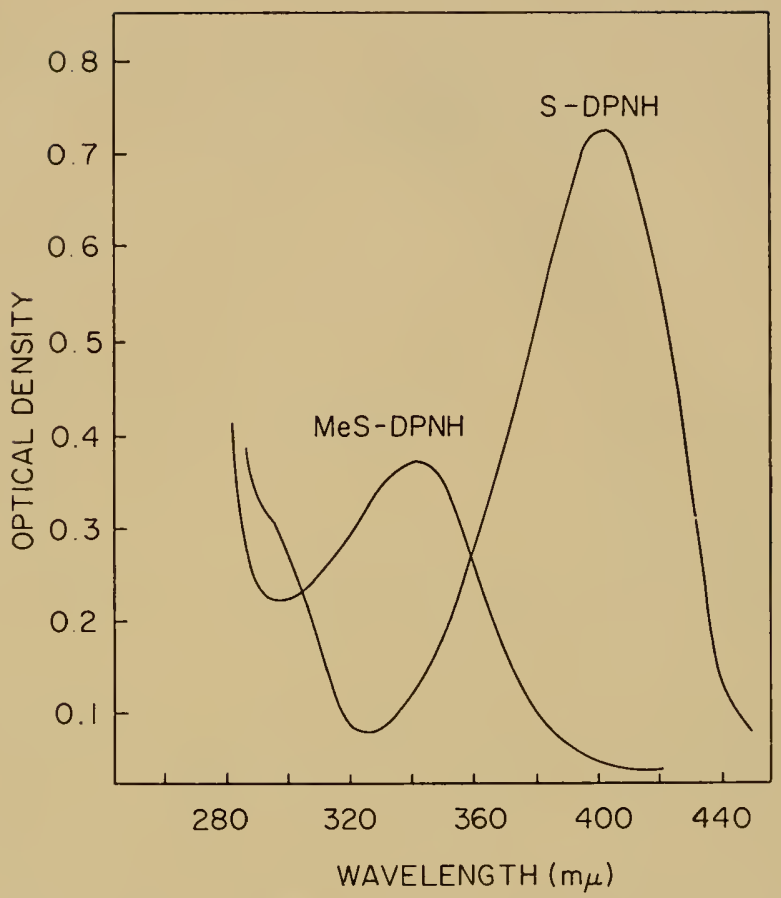

Fig. 8. Spectrum of the methylated product of thionicotinamide analogue of DPNH. S-DPNH, $9.0 \times 10^{-5} M$ thionicotinamide-1)PNH in $0.1 M$ soditm bicarbonate buffer, $p H$ 9.0. MeS-DPNH, $9.0 \times 10^{-5} M$ thionicotinamide-DPNH reated with $2 \times 10^{-4} M$ dimethyl sulfate at room temperature in $0.1 M$ soclium bicarbonate buffer. The absorption spectrum was taken one hour after the reactants were mixed. Precautions were taken to prevent exposure of the reduced analogue to light during this period.

TABLE 2

Absorption Spectra of Thiol Pyridine Derivatives

\begin{tabular}{lcc}
\multicolumn{1}{c}{ Compound } & $\lambda_{\max }(\mathrm{m} \mu)$ & $10^{-3} \epsilon$ \\
\hline S-DPNH & 398 & 8.9 \\
S-DPNH $(h \nu)^{\oplus}$ & 342 & 4.4 \\
Pyrid-2-thione & 340 & 8.7 \\
2-Benzylthiopyridine & 290 & 5.5
\end{tabular}

9.0 and at room temperature, the spectral changes shown in Fig. 8 are obtained. While actenine has been shown to react with dimethyl sulfate $(7)$, the portion of the spectrum which is examined represents absorption by the dihydrothionicotinamide moiety. The reaction of methyl sulfate with the reduced thionicotinamicle may be represented by the following equation: 


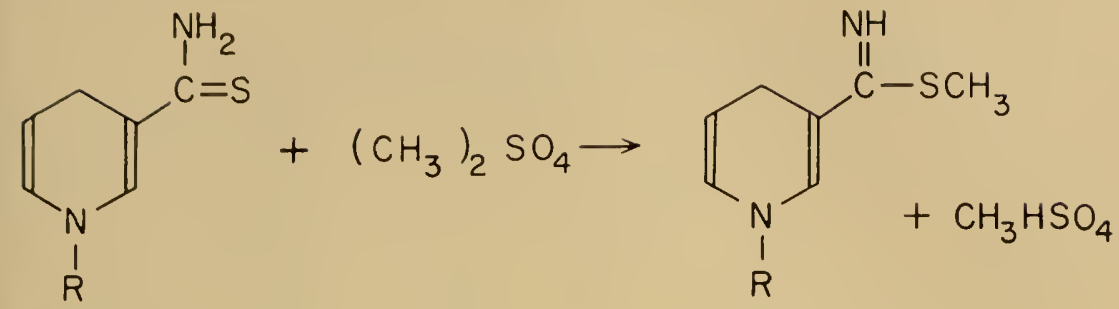

The absorption of the methylated coenzyme analogue is maximal at $341 \mathrm{~m} \mu$ and has a millimolar extinction coefficient of 4.8. These values are comparable to those observed for the illumination product of the reduced thionicotinamide analogue $\left(\lambda_{\max } 342 \mathrm{~m} \mu, 10^{-3} \epsilon 4.4\right)$.

The virtual transparency of a methyl group when attached to nitrogen, oxygen, or sulfur has permitted a study of tautomerism in the mercapto derivatives of some heterocyclic compounds $(19,16,10,1)$. A comparison of the absorption data of the thionicotinamide analogue of DPNH, of its illumination product, of pyrid-2-thione, and of 2benzylthiopyridine are given in Table 2.

The similarities in the spectrophotometric changes accompanying illumination and alkylation suggest that the light-induced product $\mathrm{SH}$ 1

has the structure of a thiolimide, $-\mathrm{C}=\mathrm{NH}$. Although there are reports of a great many photochemical rearrangements (3) and reactions of non-enolizable ketones in sunlight (18), there are very few well-described examples of photo-induced tautomerism (9). It has not been possible to isolate the product of the illumination reaction because of its rapid equilibration with the thione form. The structural evidence is based entirely on its spectral properties, which are compared with those of known thioamide systems.

While evidence for interaction between the components of pyridine dinucleotides in the reduced form has been presented, there has thus far been little indication that adenine interacts with the pyridinium portion of DPN. The 3-aminopyridine analogue of DPN has been prepared (2), and its ultraviolet absorption spectrum has been shown to have a maximum at $332 \mathrm{~m} \mu$ in water. The spectral shifts observed upon changing the solvent to propylene glycol are compared for the analogue and 3-aminopyridine methiodide in Table 3.

The "blue shift" which is observed on increasing the dielectric constant of the solvent is in accord with the suggestion made by Kasha (13) that the absorption results from an $n \rightarrow \pi^{*}$ transition. 
TABLE 3

Effect of Solvent on Absorption Properties of 3-Aminopyidine Derivatives

\begin{tabular}{lrrrrr} 
& \multicolumn{2}{c}{ Water } & \multicolumn{2}{c}{ Propylene Glycol } \\
\hline & $\lambda_{\max }$ & $\epsilon$ & $\lambda_{\max }$ & $\epsilon$ \\
\cline { 2 - 6 } & & & 336 & 3440 \\
3-Aminopyridine methiodide & 323 & 3440 & 331 & 5930 \\
3-Aminopyridine analogue of DPN & 332 & 5350 & 341 &
\end{tabular}

Although there are very distinct differences between the absorption properties of the analogue and the methiodide, the most dramatic changes are observed in the fluorescence properties of the analogue. When an aqueous solution of the analogue is excited at its absorption maximum, fluorescence is observed maximally at $420 \mathrm{~m} \mu$, with an intensity which is one-twenty-fifth that of an equimolar solution of aminopyridine methiodide. On the other hand, the fluorescence intensity of the analogue is only one-half that of methiodide in propylene glycol as shown in Table 4. A comparison of the fluorescence spectra of the analogue in water and propylene glycol is shown in Fig. 9.

The ten per cent greater molar absorption of the analogue in propylene glycol as compared with that in water cannot account for the fluorescence changes observed in the two solvents. It appears that adenine-quenching of the aminopyrictine fluorescence is comparable to the quenching of the isoalloxazine moiety of flavin adenine dinucleotide (FAD) by the aminopurine base, as reported by Weber (23) .

It should be pointed out that we do not mean to imply that the aminopyridine analogue serves as a model for oxidized DPN. The amino group of aminopyridine is an electron-donating group, while the carboxamide of DPN is an efficient electron withdrawing group. 'These have opposite effects on the electron density of the pyridinium ring and should also affect the nature of adenine-pyricline interactions.

TABLE 4

EFFect of a SOLVEnt on FluOResclence Intensities of 3-Aminopyridine Derivatives

\begin{tabular}{lrrrrr} 
& \multicolumn{2}{c}{ Water } & \multicolumn{2}{c}{ Propylene Glycol } \\
\hline & $\lambda_{\text {fluor }}$ & Intensity & $\lambda_{\text {fluor. }}$ & Intensity \\
\cline { 2 - 5 } & 400 & 210.0 & 400 & 210 \\
3-Aminopyridine methiodide & 420 & 8.0 & 420 & 90 \\
3-Aminopyridine-DPN & & & &
\end{tabular}


FLUORESCENCE OF 3-AMINOPYRIDINE -DPN

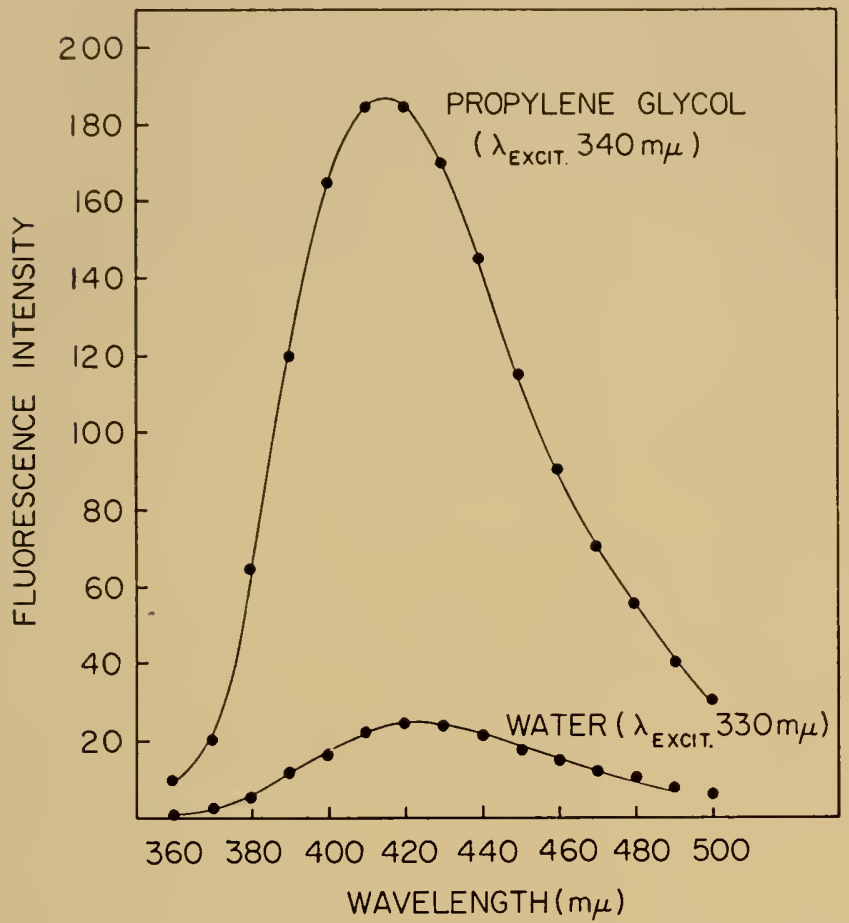

Fig. 9. Fluorescence spectra of 3-aminopyridine-DPN in $\mathrm{H}_{2} \mathrm{O}$ and in propylene glycol. The fluorescence spectrum of aminopyridine-DPN, $4 \times 10^{-4} M$, in water and in propylene glycol, shown using the wavelengths of maximum excitation. These spectra were taken at room temperature.

Nevertheless, by comparing properties of various analogues it may be possible to learn more about intramolecular interactions in pyridine nucleotides and intermolecular forces with enzymes.

\section{REFERENCES}

1. Albert, A., and Barlin, G., J. Chem. Soc., 2384 (1959).

2. Anderson, B. M., and Kaplan, N. O., J. Biol. Chem., 234, I226 (1959).

3. Barton, D. H. R., Helt. Chim. Acta, 42, 2604 (1959).

4. Bernthsen, A., Ann. Chern., Liebigs, 192, 39 (1878).

5. Braude, E. A., Ann. Repts. Chem. Soc., 42, 105 (1945).

6. Brealey, G. J., and Kasha, M., J. Am. Chem. Soc., 77, 4462 (1955).

7. Brookes, P., and Lawley, P. D., J. Chem. Soc., 539 (1960).

8. Dolin, M. I., J. Biol. Chem., 235, 544 (1960).

9. Iredale, T., Nature, 150, 579 (1942).

10. Jones, R. A., and Katritzky, A. R., J. Chem. Soc., 3610 (1958). 
11. Kaplan, N. O., Ciotti, M. M., and Stolzenbach, F. E., J. Biol. Chem., 221, 833 (1956); Arch. Biochem. and Biophys., 69, 441 (1957).

12. Kaplan, N. O., Ciotti, M. M., Stolzenbach, F. E., and Bachur, N. R., J. Am. Chem. Soc., 77, 815 (1955).

13. Kasha, M., Discussions Faraday Soc., 9, 14 (1950).

14. Laar, C., Chem. Ber., 19, 730 (1886).

15. Schonberg, A., Chem. Ber, 62B, 195 (1929).

16. Schonberg, A., and Aster, W., J. Chem. Soc., 198 (1945).

17. Schonberg, A., and Mustafa, A., J. Chem. Soc., 275 (1943).

18. Schonberg. A., and Mustafa, A., Chem. Revs., 40, 181 (1947).

19. Sheinker, Yu. N., Postorskii, I. Ya., and Voronina. N. M., Zhur. Fiz. Khim., 33, 302 (1959); Chem. Abstr., 54, 4147 (1960).

20. Shifrin, S., and Kaplan, N. O., Nature, 183, 1529 (1959).

21. Stockell, A., J. Biol. Chem., 234, 1293 (1959).

22. Strittmatter, P., J. Biol. Chem., 234, 2665 (1959).

23. Weber, G., Biochem. J., 47, 114 (1950).

24. Weber, G., Nature, 180, 1409 (1957).

25. Weber, G., J. chim. phy's., 878 (1958). 
ON THE MECHANISM OF THE DIHYDROTHIOCTYL DEHYDROGENASE REACTION

\author{
Robert L. Searls and D. R. Sanadi \\ Gerontology Branch, National Heart Institute, National Institules of Health, \\ and the Ballimore City Hospilals, Baltimore, Maryland
}

The dihydrothioctyl dehydrogenase purified from $\alpha$-ketoglutaric dehydrogenase complex of $\log$ heart muscle catalyzes the reversible oxidation of DPNH by thioctamide $\left(\mathrm{TS}_{2}\right.$ ) or thioctate (lipoate) (Equation 1 ) as well as the oxidation of DPNH by 2,6-dichlorophenol indophenol and ferricyanide (diaphorase reaction, Equation 2) (1).

$$
\begin{gathered}
\mathrm{DPNH}+\mathrm{H}^{+}+\mathrm{TS}_{2} \rightleftarrows \mathrm{DPN}^{+}+\mathrm{T}(\mathrm{SH})_{2} \\
\mathrm{DPNH}+\mathrm{H}^{+}+\text {Dye } \rightarrow \mathrm{DPN}^{+}+\mathrm{Dye}^{-} \mathrm{H}_{2}
\end{gathered}
$$

Recent work $(6,10,12,13)$ has shown that this flavoprotein is identical with Straub's diaphorase (14). The reduction of thioctate is inhibited by reagents which are known to inhibit vicinal sulfhydryl groups (arsenite and cadmium ions), while the diaphorase reaction is activated several fold under identical conditions $(9,11)$. These results indicate that the enzyme reaction involves, besides flavin adenine dinucleotide (FAD), a closely juxtaposed (vicinal) disulfhydryl group and that this disulfhydryl is functional in the oxidationreduction with thioctate but is not required for the diaphorase reaction. The disulfhydryl on the flavoprotein is not a derivative of thioctic acid $(10,13)$.

Fig. I shows that the $530-540 \mathrm{~m} \mu$ peak $(8,10,13)$ of the flavoprotein reduced with DPNH (curve B) disappears instantaneously upon addition of $4 \times 10^{-4} M$ arsenite (curve C), along with further bleaching of the flavin $\left(400-500 \mathrm{~m}_{\mu}\right)$ spectrum. When the enzyme is reduced by $\alpha$-ketoglutarate through the thioctate bound to the $\alpha$-ketoglutaric dehydrogenase complex (Fig. 2, curve B), according to equation 3 ,

$$
\begin{array}{r}
R-\mathrm{CO}-\mathrm{COOH}+\mathrm{CoA}-\mathrm{SH}+\text { Flavoprotein } \rightarrow \\
R-\mathrm{CO}-\mathrm{S}-\mathrm{CoA}+\text { Flavoprotein- } \mathrm{H}_{2}+\mathrm{CO}_{2}
\end{array}
$$

again the $535 \mathrm{~m} \mu$ peak is eliminated by the addition of arsenite. At the same time, the flavin spectrum returns to the original oxidized state (curve $\mathrm{C}$ ). Similar results were obtained in both experiments by replacing arsenite with $3 \times 10^{-5} \mathrm{M} \mathrm{Cd}^{++}$. 


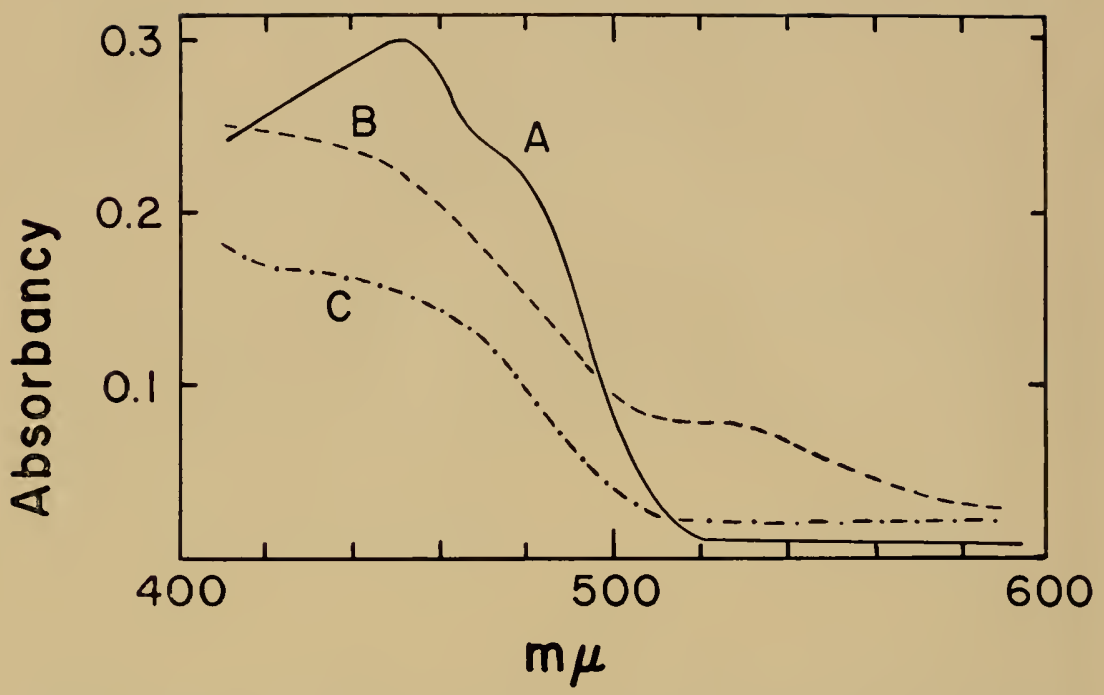

Fig. 1. Effect of arsenite on the spectrum of dihydrothioctyl dehydrogenase reduced with DPNH. Curve A, $1.8 \mathrm{mg}$ flavoprotein in $0.69 \mathrm{ml}$ phosphate buffer, $p H$ 7.3. Curve B, same plus $1.2 \mu$ moles DPNH. Curve C, reduced flasoprotein plus $0.2 \mu$ mole sodium arsenite. Experimental conditions are described in ref. $10,13$.

These and previous results suggest the following type of mechanism for the enzyme reaction.

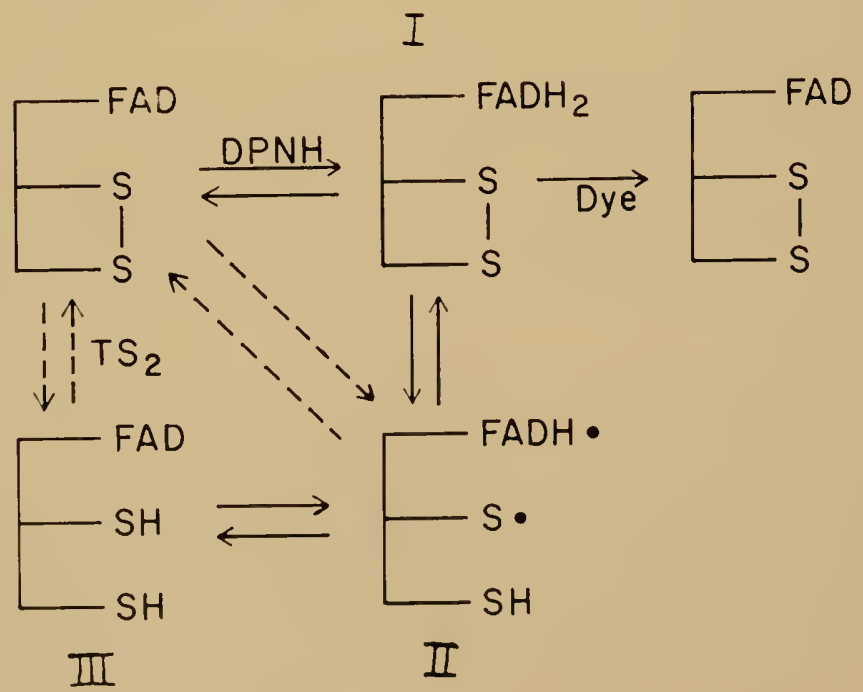




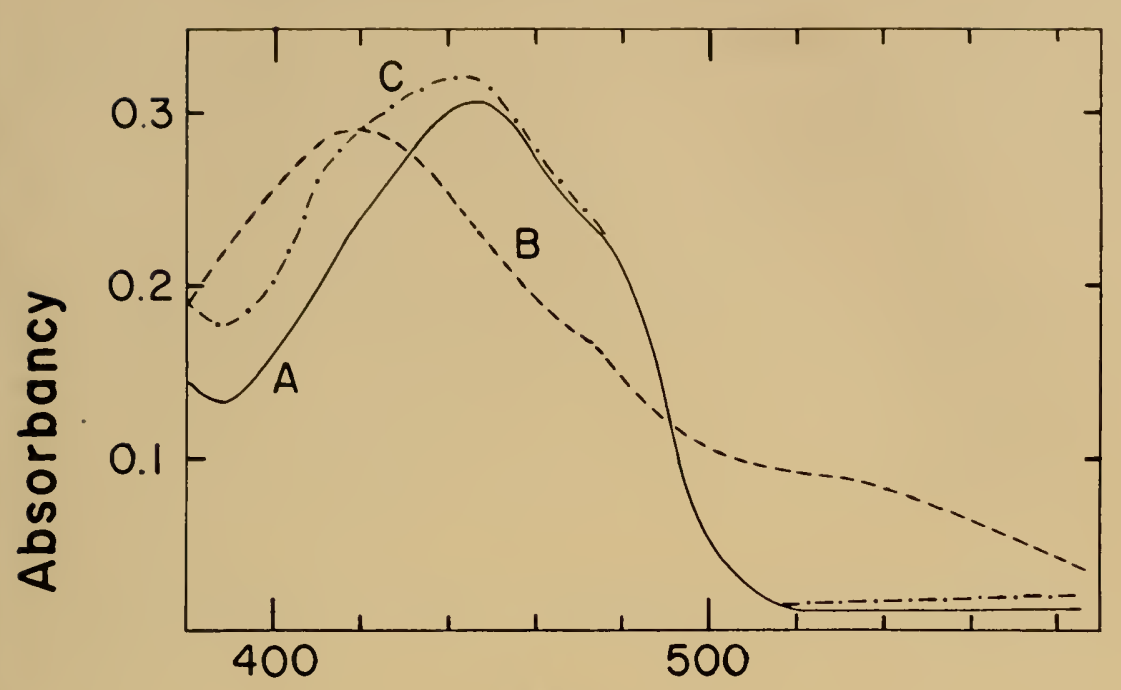

$m \mu$

Fig 2. Effect of arsenite on the spectrum of dihydrothioctyl dehydrogenase reduced with $a$-ketoglutaric dehydrogenase. Curve A, $2.0 \mathrm{mg}$ flavoprotein, $0.02 \mathrm{mg}$ Co., $3 \mu$ moles cysteine, and $0.2 \mathrm{mg} \alpha$-ketoglutaric dehydrogenase in $0.71 \mathrm{ml}$ phosphate buffer, $p \mathrm{H}$ 7.3. Curve $\mathrm{B}$, same plus $2 \mu$ moles $a$-ketoglutarate. Curve $\mathrm{C}$, reduced flavoprotein plus $0.4 \mu$ mole arsenite.

It is possible, by analogy with the recent work on other flavoproteins $(2,3)$, that the $535 m \mu$ peak associated with the reduced enzyme is due to a semiquinoid free radical represented by Intermediate II or something very closely related to it. Addition of arsenite or $\mathrm{Cd}^{++}$ would eliminate Intermediate II by shifting the equilibrium to Intermediate III, in which the flavin is in the oxidized state. When DPNH is present in excess, the FAD would be reduced again (spectrum C, Fig. 1). When dihydrothioctate (or $\alpha$-ketoglutarate) is the reductant, the FAD would remain oxidized (spectrum C, Fig. 2) since arsenite blocks the clisulficle group essential for this reaction.

A flavin semiquinone stabilized by an - $\mathrm{SH}$ group has been proposed as an intermediate based on inhibition studies with $p$-chloromercuribenzoate and $\mathrm{Cu}^{++}(15,7)$. However, these results do not necessarily implicate a vicinal disulfhydryl group.

A model reaction between a disulfhydryl compound and riboflavin5 -phosphate (FMN) leading to formation of a product with an absorption maximum at $535 \mathrm{~m}_{\mu}$, is shown in Fig. 3. The absorption spectrum of a mixture of $1.8 \times 10^{-3} M$ dihydrothioctate and $15 \times$ 


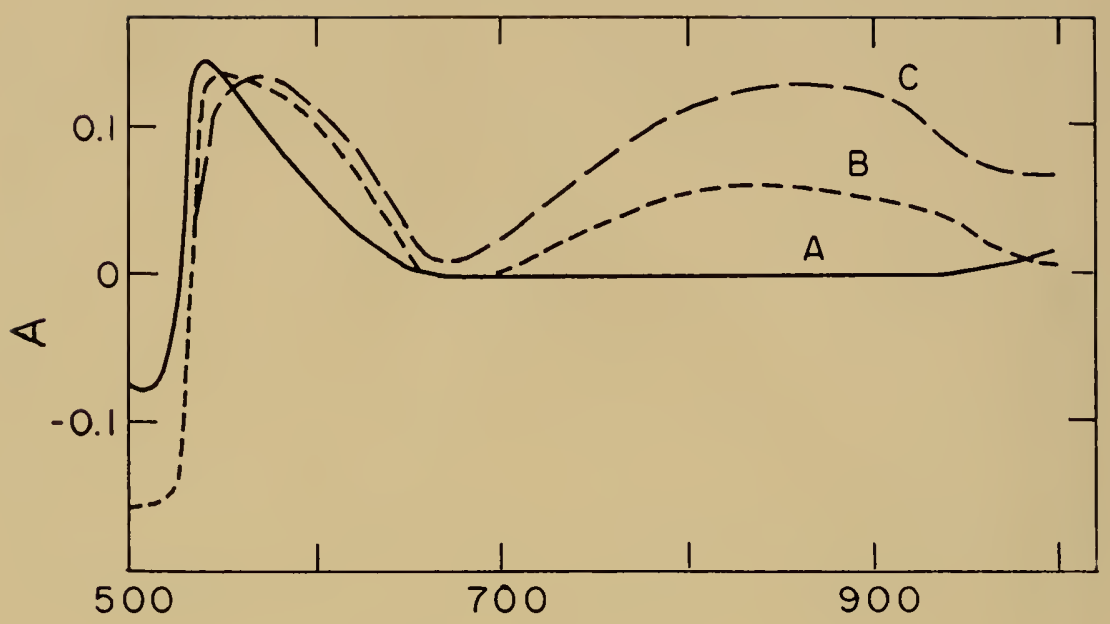

$\mathrm{m} \mu$

Fig. 3. Spectrum of complex between dihydrothioctate and flavin mononucleotide. Curve A, 5 minutes after mixing. Curve B, 20 minutes after mixing. Curve C, 200 minutes after mixing.

$10^{-3} M$ FMN at $p \mathrm{H} 6.4$ was measured against the same concentration of FMN at intervals. A peak at $535 \mathrm{~m} \mu$ appeared immediately after mixing; visually it is seen as an intensification of the orange color of FMN. On standing at room temperature, two new broad peaks centered around $565 \mathrm{~m} \mu$ and $880 \mathrm{~m} \mu$ appeared gradually and the solution turned olive green. The 565 and $880 \mathrm{~m}_{\mu}$ peaks correspond respectively to the peaks of the flavin semiquinone and its dimer (1). It is likely that the $535 \mathrm{~m} \mu$ peak in the model reaction corresponds to the $530-540 m \mu$ peak of the reduced dihydrothioctyl dehydrogenase, although the possibility that the $565 \mathrm{~m} \mu$ peak in the model system shifts to lower wavelengths as a result of binding to the enzyme protein cannot be ruled out.

Isenberg and Szent-Györgyi have obtained spectral evidence for the formation of a complex between FMN and tryptophan and several other electron acceptors $(4,5)$. They derived the kinetic equation describing the complex formation and found that, as expected, a straight line resulted from a plot of the reciprocal of the absorbancy of the complex against the concentration of one of the reactants while holding the second reactant constant. In the following equations, which are similar to those above, $\mathrm{T}(\mathrm{SH})_{2}$ is the reduced thioctate, 
$A_{C}$ is the absorbancy of the complex $(C)$ and $K$ is the dissociation constant.

$$
\begin{gathered}
\mathrm{FMN}+\mathrm{T}(\mathrm{SH})_{2} \rightleftarrows C \\
\frac{1}{(\mathrm{FMN})}=\frac{\left(\mathrm{T}(\mathrm{SH})_{2}\right) \underline{K}}{A_{C}}-K
\end{gathered}
$$

Fig. 4 shows the dependence of the absorbancy of the $535 \mathrm{~m} \mu$ peak, obtained within 5 minutes after mixing, upon the concentration of FMN in the mixture (which is varied from $5 \times 10^{-3} \mathrm{M}$ to $15 \times 10^{-3}$ M) at a fixed concentration $\left(1.8 \times 10^{-3} M\right)$ of reduced thioctate. The relationship, as plotted, is linear and meets the criterion expected for a complex.

Electron spin resonance (ESR) measurements, carried out in the laboratory of Dr. B. Chance, showed no signal immediately after mixing equal volumes of dihydrothioctate $\left(2.4 \times 10^{-2} M\right)$ and FMN

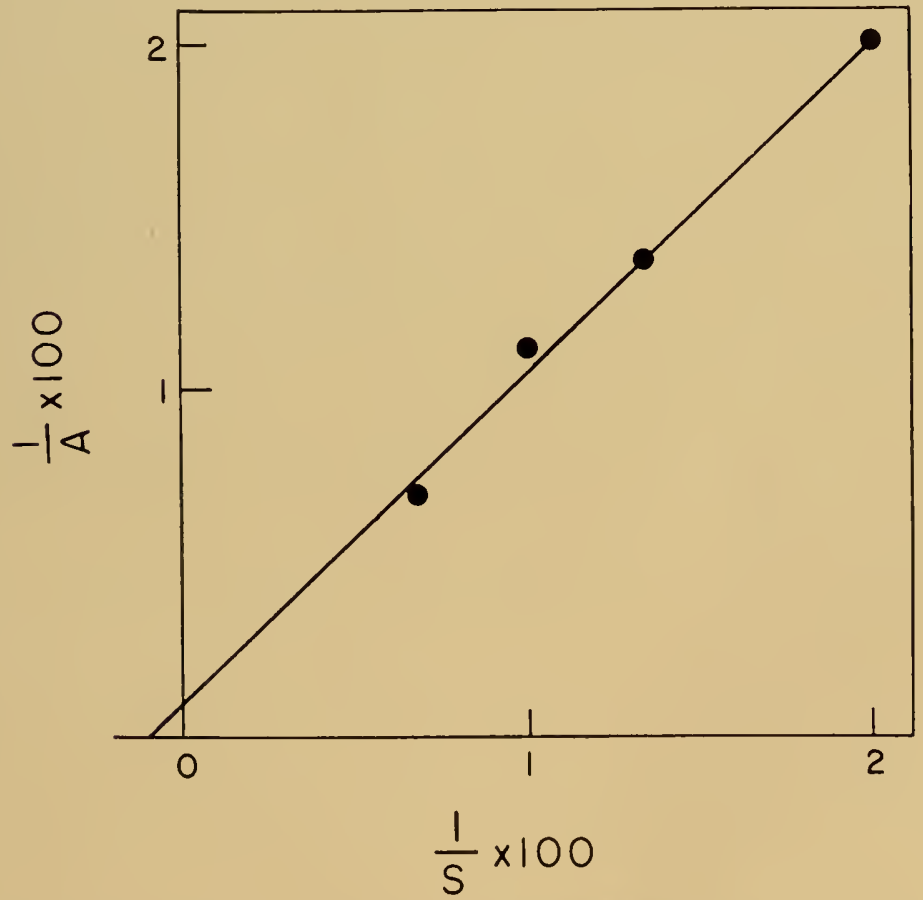

Fig 4. Effect of reactant concentration on the FMN-T(SH) $)_{2}$ complex. $A$ is the height of the peaks obtained 5 minutes after mixing: $S$ is the FMN concentration; the $\mathrm{T}(\mathrm{SH})_{2}$ concentration was $1.8 \times 10^{-3} \mathrm{M}$. 
$\left(3 \times 10^{-2} M\right)$. The solution turned green and a free-radical signal ( $g=2.006$, signal width $=14$ gauss) appeared after the solution stood at room temperature for 20 minutes. On the other hand, dihydrothioctyl dehydrogenase (approximately $10^{-5} M$ ) showed no signal alone or with $6 \times 10^{-3} M \mathrm{DPNH}$. The concentrated enzyme turned green on reduction because of the combined effect of the $a b$. sorption in the region above $500 \mathrm{~m}_{\mu}$ and the residual flavin color. Since there is no evidence for unpairing of electrons, the $535 \mathrm{~m} \mu$ peak in the reduced flavoprotein may be due to a charge transfer complex. If free radicals do exist (as represented tentatively in Intermediate II), there is sufficiently strong interaction between them that the ESR spectrum does not detect it. These results may be the first demonstration of a charge transfer complex in a biological system, although further work is necessary to establish the nature of the $\mathrm{T}(\mathrm{SH})_{2}$-FMN complex.

This work was supported in part by a grant-in-aid from the American Heart Association, Inc. The able assistance of Miss Patricia Knell in the preparation of the enzyme is acknowledged with appreciation.

\section{REFERENCES}

1. Beinert, H., J. Amer. Chem. Soc., 78, 5323 (1956).

2. Beinert, H., J. Biol. Chem., 225, 465 (1957).

3. Ehrenberg, A., and Ludwig, G. D., Science, 127, 1177 (1958).

4. Isenberg, 1., and Szent-Györgyi, A., Proc. Nat. Acad. Sci. U. S., 44, 857 (1958).

5. Isenberg, I., and Szent-Györgyi, A., Proc. Nat. Acad. Sci. L'. S., 45, 1229 (1959).

6. Massey, V., Biochim. et Biophys. Acia, 37, 314 (1960).

7. Massey, V., and Cibson, Q. H., Federation Proc., 19, 27 (1960).

8. Savage, N., Biochem. J., 67, 146 (1957).

9. Searls, R. L., Federation Proc., 19, 36 (1960).

10. Searls, R. L., and Sanadi, D. R., Proc. Nat. Acad. Sci. U. S., 45, 697 (1959).

11. Searls, R. L., and Sanadi, D. R., Biochem. Biophys. Res. Comm., 2, 189 (1960).

12. Searls, R. L., and Sanadi, D. R., J. Biol. Chem., 235, PC32 (1960).

13. Searls, R. L., and Sanadi, D. R., J. Biol. Che'm., 235, 2485 (1960).

14. Straub, F., Biochem. J., 33, 787 (1939).

15. Veeger, C., and Massey, V., Biochim. et Biophys. Acta, 37, 181 (1960). 


\title{
SOME COMMENTS ON FLAVIN AND FLAVOPROTEIN COMPLEXES AND SEMIQUINONES*
}

\author{
Helmut BeinerT \\ Institute for Enzy'me Research \\ University of Wisconsin \\ Madison, Wisconsin
}

\section{INTERACTION OF Light with Flavins}

The flavins belong to the most "colorful" of the coenzymes-whether we use this word in its true sense or in a metaphoric one. Not only do they appear in forms of almost all colors of the spectrum, but they also undergo a variety of reactions and are involved in a great number of biological processes. The light energy absorbed by flavins is dissipated in various ways: Flavins may fluoresce or phosphoresce under proper conditions, they may be photolysed, photoreduced, or may act as photosensitizers of other compounds. Their chemiluminescence has also been observed.

Although these light reactions of flavins appear to be particularly germane to the main topic of this symposium, I shall refrain from discussing them here, as I have not made any contribution to this subject myself and also very recently have reviewed this topic elsewhere (2). Furthermore, it is not clear whether the light reactions of the flavin compounds are of major biological significance per se, or whether they are merely an expression of the great reactivity of this class of compounds.

I would therefore like to extend here Dr. Velick's discussion on the observation of excited states of coenzymes as a means of recognizing their state, their environment, or structure. I shall summarize some recent work on various forms of flavins and flavoproteins which can be studied by their specific light absorption. Although our knowledge in this area rests on purely empirical ground thus far, it appears that a rational approach to the interpretation of the spectra and spectral peculiarities of flavins may not be in the too distant future (cf. 10).

* Recent experimental work of the author's laboratory, which is discussed here, was supported by the National Institutes of Health (Grant $\Lambda-2512$ ). 


\section{Changes in the Absorition Spectra of Flavins and Flavolroteins} Due to SPECIFIC INTERACTIONS

Fig. I shows schematically a flavin or flavoprotein spectrum (dotted line) and the various solid lines, appearing in different regions of the spectrum, indicate the approximate shapes and positions of new absorption bands which have been observed under specific conditions or with certain flavin compounds. The intensities of these bands relative to the main flavin band at about $450 \mathrm{~m} \mu$ are approximately correct, except for the bands at 560 and $900 \mathrm{~m} \mu$, which are of much

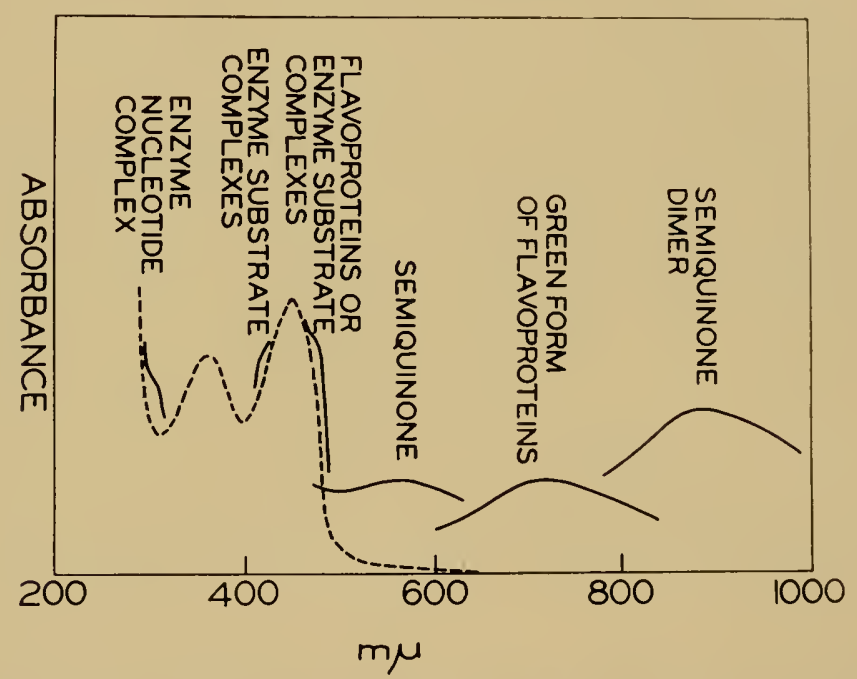

Fig. 1. Schematic representation of flavin or flavoprotein spectrum (dotted line) and spectral changes which occur on specific interactions or with certain flavin compounds (solid lines).

lower intensity with free flavin compounds (1). The bands observed in the region between 500 and $1000 m_{\mu}$ are all rather broad, extending over $100-200 \mathrm{~m} \mu$, whereas those shown at shorter wavelengths are more narrow and fall within about $20 \mathrm{~m} \mu$. Broad bands in the latter region camnot be readily recognized because of the absorption of the flavin itself.

\section{The $400-500 m_{\mu}$ Region}

It has long been known that a spectral shift toward longer wavelengths and a slight decrease of absorption occurs when flavin is attached to adenine, as it is in flavin adenine dinucleotide (FAD) 
(22). These changes, as well as the quenching of fluorescence, have been attributed to formation of an intramolecular complex between the isoalloxazine moiety and the adenine moiety of FAD $(22,21)$. Similar spectral changes are found in a variety of flavin complexes. As examples we may mention those with phenol (23), benzoate, or naphthoate (9). In addition to a shift toward longer wavelengths and a decrease in absorption, such complexes often show a pronounced shoulder in the $460-490 \mathrm{~m} \mu$ region. It is of interest here that a number of pure flavoproteins show a shoulder in this region of the spectrum $(16,8,18,15)$ or even an additional peak $(19,5)$, which may indicate an intranolecular complex of the protein moiety with the prosthetic llavin. Other flavoproteins, which show a smooth Havin spectrum initially, may develop a shoulder in this region when substrate or an inhibitor is bound to them $(17,24)$. The spectral changes demonstrated by Yagi (24), which occur in going from FAD to D-amino acid apooxidase-FAD and then to the D-amino acid apooxidase-FAD-benzate complex are typical here. Recently Harbury et al. succeeded in producing a shoulder and even an additional peak in the 460-490 $m \mu$ region of the spectrum of 3-methyl humiflavin when they dissolved this compound in solvents of decreasing polarity (10). They observed the simultaneous emergence of a shoulder at 400-430 $m_{\mu}$, which has also been lound to be associated with the $460-490$ $m_{\mu}$ shoulder in flavoproteins (17).

It is often assumed that a shoulder at $400-430 m \mu$ in a pure flavoprotein indicates contamination with heme compounds. The lact, however, that this shoulder can be produced by changing solvents (10) or adding substrate (17) shows that this interpretation is not always correct. Observation of the spectral changes on reduction may permit one to reach a decision here, as heme compounds should then show a distinct peak.

It is not clear whether the complex formation of the old yellow enzyme with TPNH $(7,6)$ bears more relation to those interactions which lead to a band in the $460-490 \mathrm{~m} \mu$ region or to those which produce spectral changes at longer wavelengths, such as oxidoreductive interactions. It appears from the spectrum $(7,4)$ that both components may be represented. This complex represents one of the first instances in which a flavoprotein substrate complex was clearly demonstrated by a spectral shift (7). Interpretation is, however, complicated by the fact that a certain oxidoreductive interaction between the complexing partmers is bound to occur. The actual nature of the complex is still unknown $(6,4)$. 


\section{The 300-400 $m_{\mu}$ Region}

Harbury et al. (10) observed that the ability of a solvent to form hydrogen bonds with 3-methyl lumiflavin has the most pronounced influence on the position of the secondary flavin band in the near ultraviolet. Hydrogen-bonding solvents tend to shift this band toward $370 \mathrm{~m} \mu$, whereas solvents which are unable to form hydrogen bonds shift it toward $330 \mathrm{~m}_{\mu}$. It is of particular interest here that a flavinpeptide fragment which Kearney (11) obtained from digests of succinic dehydrogenase also shows a shift of the secondary flavin peak from 375 to $350 \mathrm{~m} \mu$.

Strittmatter (20) showed that the complex of DPNH cytochrome reductase of liver microsomes with its substrate DPNH has a distinct band at $315 \mathrm{~m}_{\mu}$. Although Strittmatter attributes this band mainly to an interaction of the nicotinamide ring and the isoalloxazine ring system of the reactants, he has also presented evidence that the adenine moiety of the substrate is involved in enzyme-substrate binding. It is therefore of interest that a band in the same spectral region is found (3) when crotonyl CoA is added to the yellow form (17) of butyryl dehydrogenase. As mentioned above, the butyryl dehydrogenase-crotonyl CoA complex is also characterized by bands at about $420 m_{\mu}$ and at $470 m_{\mu}$. When, however, the analog of crotonyl CoA, crotonyl thioethylamine, which lacks the nucleotide moiety of CoA, is adcled to the same enzyme, only the bands at 420 and $470-480 \mathrm{~m}_{\mu}$ appear, and not that at $315 \mathrm{~m} \mu$. In the case of the yellow form of butyryl dehydrogenase, at least, we may therefore assign the $315 m \mu$ band to an interaction of the isoalloxazine ring system with a nucleotide, and the $420 \mathrm{~m} \mu$ and $470-480 \mathrm{~m}_{\mu}$ bands to the interaction of flavin with the non-nucleotide moiety of the substrate. The double bond contained in the crotonyl moiety of the substrate may be necessary for the appearance of this latter band.

\section{The 500-1000 $m_{\mu}$ Region}

A new absorption band in the $600-800 \mathrm{~m} \mu$ region has only been found with two flavoproteins thus far. These proteins are of deep green color. Butyryl dehydrogenase, as isolated from beef and pig liver or beef heart, shows this peculiarity $(17,3,13)$; and the old yellow enzyme of yeast could be transformed into a green-colored protein on treatment with ammonium sulfate at $p \mathrm{H} 10$ (14). This transformation of the old yellow enzyme appears to be reversible, whereas the green form of butyryl dehydrogenase could never be produced artificially. The green butyryl dehydrogenase can be converted, how- 
ever, into a yellow form by reducing agents, substrate, and some other substances. The long-wavelength band of this enzyme had previously been attributed to the presence of a heavy metal, but recent work (17) has made it likely that it is clue to the interaction of a component of the enzyme protein itself with its own prosthetic flavin. No analogies are available from interactions of simple flavin compounds which would assist in clarifying the kind of reaction involved.

The characteristics of the appearance and disappearance of the bands at about $560 \mathrm{~m} \mu$ and $900 \mathrm{~m} \mu$ indicate that they belong to intermediates in the oxidation-reduction of flavin compounds (1). Because of its concentration and temperature dependence the band at $900 \mathrm{~m}_{\mu}$ has been attributed to a dimer of the semiquinone form of flavins. This band has never been seen with flavoproteins. It is of considerable interest that such quinhydrone-type dimers show in the relatively unexplored near-infrared region.

The band at $560 m \mu$ is thought to indicate the monomeric semiquinone at neutral $p \mathrm{H}$. Recent work by paramagnetic resonance (EPR) spectroscopy has confirmed this view (4). A broad band with maximum absorption at wavelengths from 530 to $570 m \mu$ also appears temporarily during oxidation-reduction of several flavoproteins by dithionite or by substrate. In the cases which were studied, EPR has again provided confirmatory evidence that semiquinones are formed under these conditions. There are, however, several flavoproteins which show a persistent, and not a transient, absorption band of very similar shape and intensity when substrate is added.

Although one is inclined to infer from spectral analogy that this also indicates semiquinone formation, no semiquinone was detectable by EPR in the only case which has so far been thoroughly investigated (4) .

\section{Semiquinone Formation of Flatins and Flavoproteins}

The evidence for semiquinone formation of flavins and flavoproteins obtained by optical methods and by EPR methods, and the correlation of the results obtained by both methods, was discussed at a recent symposium by Beinert and Sands (4). It may therefore suffice to state here the conclusions they reached. The appearance of an absorption band with a maximum from $530-570 \mathrm{~m}_{\mu}$ in many cases coincides with the appearance of EPR signals indicative of free-radical formation, particularly when the absorption band makes a transient appearance. The appearance of a persistent absorption band in this region, however, does not necessarily coincide with formation of 
free radicals which are measurable by EPR. It is therefore clear that no simple monomeric semiquinone is formed under such conditions. A more complicated situation obtains. In those cases in which a persistent band is observed, a very strong binding of the substrate to the enzyme occurs (17). Interaction with neighboring substrate radicals in such a complex may make semiquinones undetectable by EPR. Since many flavoproteins contain two prosthetic flavins, flavin biradical formation may also occur in these complexes. EPR signals would not be expected in this case. The spectral band, however, which is due to transitions of a considerably higher energy level would not necessarily be influenced by such interactions and would thus still be visible.

It is not easy to prove that interactions of the type mentioned are indeed responsible for the failure of EPR to detect lree radicals when certain flavoproteins react with substrate and when the spectral band appears which was found characteristic of semiquinone formation in other cases. One may have to consider other possibilities of explaining the observations. It appears extremely unlikely that the strong complexes formed between what was initially oxidized flavoprotein and reduced form of substrate would not include some kind of oxidoreductive interaction. It has been suggested that this interaction may be of a charge transler type. This contention again is hard to prove experimentally. Absence of EPR signals is certainly in accord with it, but the fact that the characteristic absorption band is found in almost the same position with a variety of flavoproteins and substrates is not in agreement with observations on charge transfer bands (12). The occurrence of a charge transfer absorption also implies that charge transfer only takes place significantly in the excited state. One may therefore wonder whether an enzymatic oxidoreduction reaction could proceed efficiently between a substrate and an enzyme which require energy of the magnitude of light energy for electron transfer.

It is possible that present concepts and terminology are not able to describe satisfactorily the kind of intermediate and of interaction which we are actually dealing with in these complexes.

Although I have tried in the foregoing remarks to arrive at a few general conclusions from a vast and diverse body of experimental material, I would at the same time like to express my view that generalizations are hazardous and that at this early stage of our knowledge of the phenomena under discussion we have little choice but to consider every case individually and to study it on its own merits. 


\section{REFERENCES}

1. Beinert, 11., J. Am. Chem. Soc., 78, 5323 (1956).

2. Beinert, 11., in The Enzymes (P. D. Boyer, H. A. Lardy, and K. Myıbäck, eds.), pp. 339-416, Academic Press, New York (1960).

3. Beinert, H., umpub.

1. Beinert, H., and Sands, R. H., in Symposium on Free Radicals in Biological Systems, Academic P'ress, N. Y. (1960, in press).

.. Crane, F. L., and Beinert, 11., J. Biol. Chem., 218, 717 (1956).

6. Ehrenberg, A., and Ludwig. G. D., Science, 127, 1177 (1958).

7. Haas, E., Biochem. Z., 290, 291 (1937).

8. Haas, E., Biochem. Z., 298, 378 (1938).

9. Harbury, H. I., and Foley, K. A., Proc. Nall. Acad. Sci. U. S., 44, 662 (1958).

10. Harbury, H. I., La Noue, K. F., Loach, P. I., and Imick, R. M., Proc. Natl. Acad. Sci. U. S., 45, $17(1) 8$ (1959).

11. Kearney, E. B., J. Biol. Chem., 235, 865 (1960).

12. Kosower, E. M., in The Enzymes (P. D. Boyer, H. A. Lardy, and K. Myrbäck, eds.), pp. 171-194, Academic Press, New York (1960).

13. Mahler, H. R., J. Biol. Chem., 206, 13 (1954).

14. Rutter, W. J., and Rolander, B., Acta Chem. Scand., 11, 1663 (1957).

15. Savage, N., Biochem. J., 67, 146 (1957).

16. Singer, T. 1'., and Kearney, E. B., Arch. Biochem., 27, 348 (1950).

17. Steyn-Parvé, E. P., and Beinert, H., J. Biol. Chem., 233, 853 (1958).

18. Straub, F. B., Biochem. J., 33, 787 (1939).

19. Strittmatter, P., and Velick, S. F., J. Biol. Chem., 228, 785 (1957).

20. Strittmatter, P., J. Biol. Chem., 233, 748 (1958); 234, 2665 (19:9).

21. Weber, G., Biochem. J., 47, 114 (1950).

22. Whitby, L. G., Biochem. J., 54, 437 (1953).

23. Yagi, K., and Matsuoka, Y., Biochem. Z., 328, 138 (1956).

24. Yagi, K., Ozawa, T., and Harada, M., Nature, 184, 1938 (1959). 



\section{Part III}

LIGHT EMISSION BY CHEMICAL REACTIONS 



\title{
CHEMILUMINESCENGE IN PORPHYRIN-CATALYZED
}

\section{DECOMPOSITION OF PEROXIDES}

\author{
HENRY LINSCHITZ \\ Department of Chemistry \\ Brandeis University \\ IValtham, Massachusetts
}

The thermal decomposition of peroxides, catalyzed by heavy metal ions (12), is a reaction of great chemical interest and biochemical relevance. Via appropriate enzymes, this reaction of course plays a key biochemical role in peroxide and oxygen metabolism. In the case of certain metallo-porphyrins in vitro, the reaction is of further interest in that the catalysis is often accompanied by chemiluminescence. A stucly of the process may therefore be helpful in establishing possible mechanisms of biolnminescence.

The chemiluminescence of porphyrins in the presence of peroxides was discovered by Helberger $(5,6)$, who observed a red emission when magnesium phthalocyanine was added to hot tetralin. Further work showed that tetralin hydroperoxide (THP), present as impurity in the solvent, is required for the luminescence, and that the emission is accompanied by the breakdown of both the peroxide and the metallo-porphyrin. In our own work, we have established that the chemiluminescence spectrum corresponds to the fluorescence of the original metallo-porphyrin. Thus, some of the energy released in the exergonic peroxide breakdown may be used for electronic excitation of the catalyst.

Among the metallo-porphyrins and related compounds which show the reaction is chlorophyll itself (11). The process thus becomes still more intriguing in connection with its possible bearing on the photosynthesis problem. The catalyzed breakdown of THP results mainly

${ }^{1}$ This work was supported by grants from the U. S. Alomic Energy Commission (0) Siracuse University (Contract No. AT(30-1)-820) and 10) Brandeis Iniversio (Confract . Yo. AT (30-I)-2003). The experimental studies stmmarized briefly in this paper were carried out in collaboration with Drs. E. W. Abrahamson, D. R. Cross, and Mrs. M. K. Schumm. It is a pleasure to acknowledge their contributions. A detailed joint report will appear elsewhere. 
in $\alpha$-tetralone and water $(6,3)$. We may therefore write ${ }^{*}$ signifies an electronically excited molecule) :<smiles>O=C1CCCC2CCCCC12</smiles>
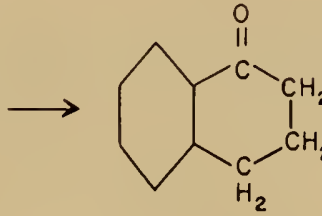

$$
+\mathrm{H}_{2} \mathrm{O}+[\text { Porphyrin }]
$$

If this (strongly exergonic) reaction is run backward, water is split and a hydroperoxide is obtained. In purely formal terms, at least, this corresponds to a reaction of the general type that might be looked for in plant photosynthesis, although it is emphasized that the equilibrium point of the process written above must lie far to the right. In this paper we wish simply to point out such possible relationships. Our chief concern at this time is with the mechanism whereby the porphyrin becomes electronically excited.

A preliminary kinetic study of the chemiluminescent decomposition of THP, catalyzed by zinc tetraphenylporphine (ZnTPP), was reported by Linschitz and Abrahamson (9). We briefly summarize here the results of further work on the reaction in our laboratory.

\section{EXPERIMENTAL}

Kinetic and luminescence measurements were made on solutions of THP and ZnTPP in $t$-butyl benzene. Reagents and solvents were highly purified. Samples were degassed on the vacuum-line and the reaction run in sets of sealed-off tubes, at temperatures between $135^{\circ}$ and $155^{\circ} \mathrm{C}$. At suitable intervals, a tube was withdrawn from the thermostat, quenched, and its contents analyzed for peroxide (iodimetrically) and for porphyrin (spectrophotometrically). During the course of the reaction, the decay of the chemiluminescence was followed by a photomultiplier photometer, in which the luminescence intensity was compared with the light output from a standard lamp.

\section{Results}

The general leatures of the reaction and luminescence kinetics are given in Figs. 1 and 2, which show respectively the time course of peroxide and porphyrin concentration, and of the chemihuminescence for a typical run. For comparison, an uncatalyzed peroxide decomposition is also shown in Fig. 1. Table 1 presents smoothed concentra- 


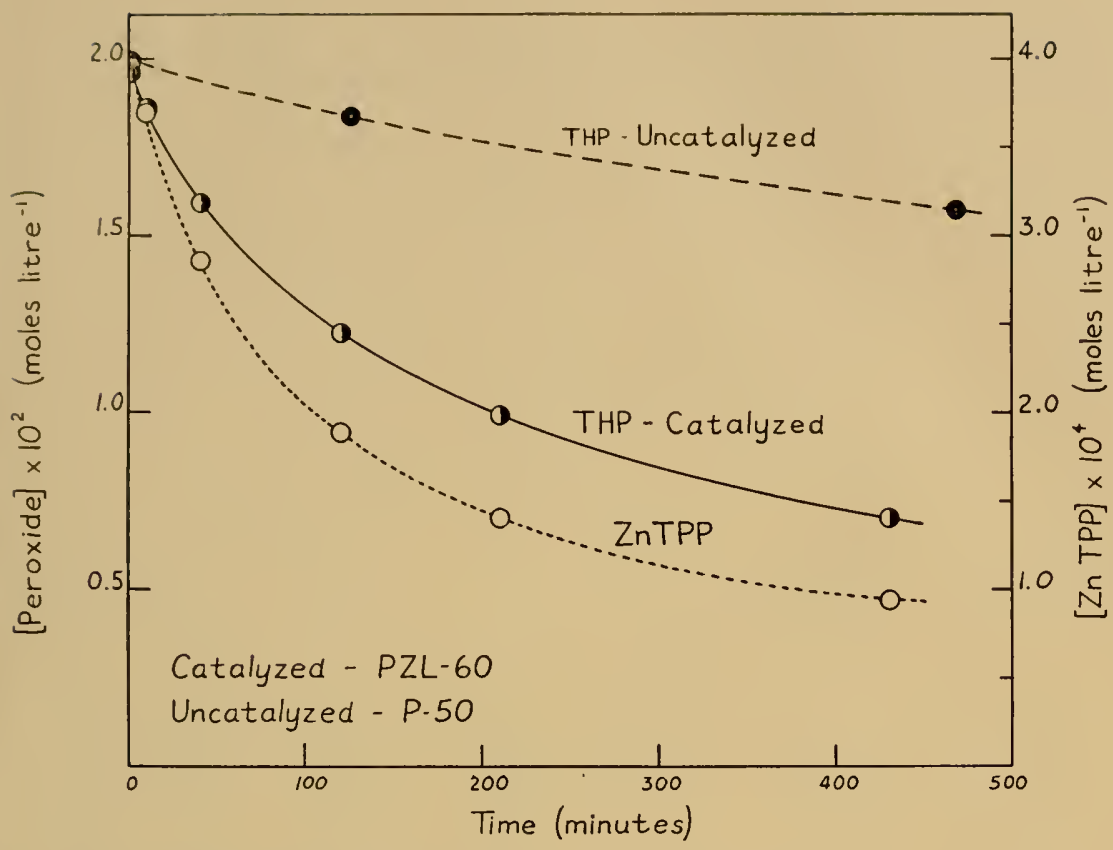

Fig. 1. Decomposition of tetralin hydroperoxide (uncatalyzed and catalyzed) and inc tetraphenyloporphine, in $t$-butyl benzene, at $148^{\circ} \mathrm{C}$.

tion and luminescence-time data, read from these curves, as well as specific rate constants for the various decays. These constants were obtained by first establishing values of the actual rates at various times by drawing tangents to the curves of Fig. 1, and then dividing these rates by suitable powers of the corresponding reagent concentrations. In Table 1 , the intensity of luminescence is measured by the photometer galvanometer. This intensity is itself a rate (of photon emission in photometer units) and thus may be compared directly with the chemical rates. Correction is made in Table $\mathbf{l}$ for the uncatalyzed decomposition of peroxide, by making the dubious assumption that the uncatalyzed rate applies also in the presence of active porphyrin. In the case presented, this correction is small and introduces no essential change in the kinetic characteristics.

1. Rate laws. It is evident from Table 1 that the peroxide decomposition rate accurately parallels the luminescence intensity $(I)$. Both rates show good second-order kinetics (first order in both peroxide and porphyrin) over most of the course of reaction, and both 


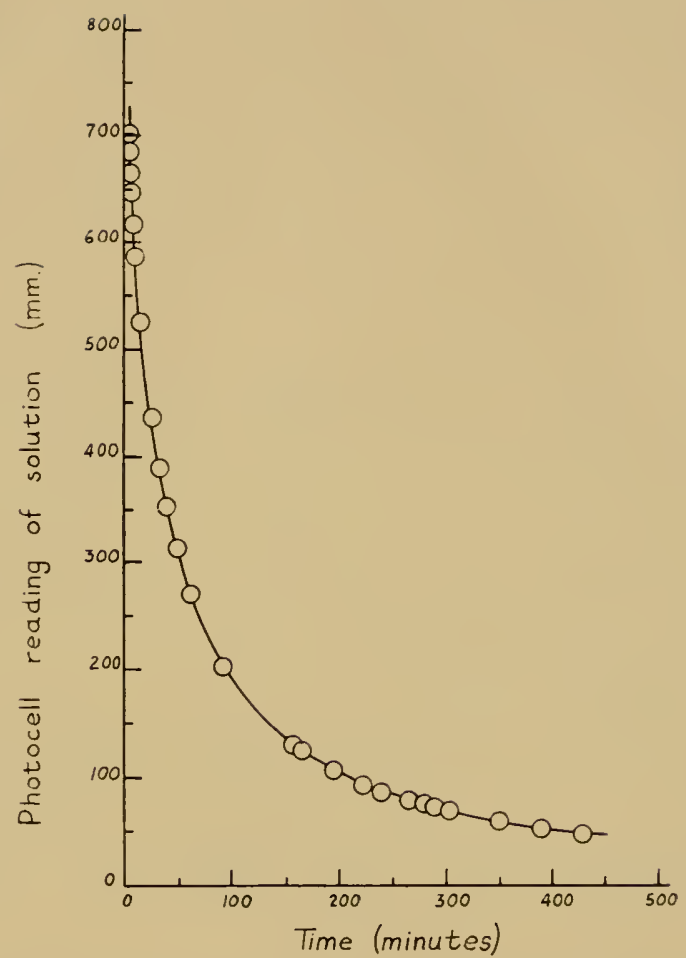

Fig. 2. Decas of chemiluminescence of tinc tetraphenglporphinc in same run illuslated in Fig. I.

show an initial somewhat rapid phase as well. Further, the ratio of initial rate to steady rate for both peroxide decomposition and luminescence intensity is the same, within experimental error. At lower initial concentration ratios of porphyrin to peroxide the apparent $k_{1}$ 's and $k_{i}$ 's both increase. The rate law for the irreversible porphyrin decomposition is somewhat more complex. Within a given run, the rate appears to be second order in peroxide and first order in porphyrin (Table 1). However, variation of the initial peroxide concentration $[\mathrm{THP}]_{0}$, indicates that a better fit is obtained by a rate proportional to the product $[\mathrm{THP}]^{2}\left[\mathrm{THP}_{1}{ }^{-1}[\mathrm{ZnTPP}]\right.$.

2. Ratio of peroxide to porphyriu decomposed. Over about the first (peroxide) hall-life, about thirty molecules of peroxide are decomposed for every mole of dye which disappears. Despite the kinetic complications, this ratio is remarkably constant over the temperature 
TABLE I (3)

Kinetics of Cheniluminescent Zn-Porphivin-Catalyzed Decomposition of Tetralin Hydroperoxide

Run: PZL-60 Temp: $148.0^{\circ} \mathrm{C}$.

$S=$ Photometer Sensitivity Correction $(\sim 0.98)$

$$
\begin{aligned}
& k_{1}(\text { corr. })=\left(\frac{\mathrm{d}[\mathrm{THP}]}{\mathrm{d} t}\right)_{\text {eat. }} \frac{1}{[\mathrm{THP}]} \frac{1}{[\mathrm{ZnTPP}]} \\
& k_{Z}=\frac{\mathrm{d}[\mathrm{ZnTPP}]}{\mathrm{d} t} \frac{1}{[\mathrm{THP}]^{2}} \frac{1}{[\mathrm{ZnTPP}]} \\
& k_{I}=\frac{I}{S} \frac{1}{[\mathrm{THP}]} \frac{1}{[\mathrm{ZnTPP}]}
\end{aligned}
$$

\begin{tabular}{|c|c|c|c|c|c|c|}
\hline \multirow[b]{2}{*}{$\begin{array}{l}\text { Time } \\
(\mathrm{min})\end{array}$} & & \multirow[b]{2}{*}{$I(\mathrm{~mm})$} & \multirow{2}{*}{$\begin{array}{c}\left.h_{1} \text { (corr. }\right) \\
1 \mathrm{~mol}^{-1} \\
\min ^{-1}\end{array}$} & \multirow{2}{*}{$\begin{array}{c}h_{Z} \\
\mathrm{I}^{2} \mathrm{~mol}^{-2} \\
\min ^{-1}\end{array}$} & \multirow[b]{2}{*}{$\begin{array}{c}k_{I} \times 10^{7} \\
\left(\mathrm{~mm} \mathrm{l}^{2} \mathrm{~mol}^{-2}\right.\end{array}$} \\
\hline & $\underset{10^{2}}{[\mathrm{THP}]} \times$ & $\underset{10^{4}}{[\mathrm{ZnTPP}]} \times$ & & & & \\
\hline 0 & 1.975 & 4.08 & & 17.1 & 27.5 & \\
\hline 3 & 1.935 & 3.925 & 702. & & & 9.41 \\
\hline 5 & 1.910 & 3.855 & 667. & 15.3 & & 9.21 \\
\hline 10 & 1.853 & 3.685 & 588 . & 14.7 & 26.6 & 8.73 \\
\hline 20 & 1.750 & 3.362 & 476 & 14.5 & & 8.23 \\
\hline 50 & 1.525 & 2.670 & 306 . & 12.9 & 28.1 & 7.65 \\
\hline 80 & 1.373 & 2.250 & 226.0 & 12.5 & & 7.41 \\
\hline 100 & 1.291 & 2.042 & 190.8 & 12.4 & 27.1 & 7.37 \\
\hline 150 & 1.129 & 1.675 & 135.7 & 12. & 27.1 & 7.25 \\
\hline 200 & 1.009 & 1.436 & 103.5 & 12.3 & 28.0 & 7.28 \\
\hline 250 & 0.912 & 1.260 & 83.9 & & 28.4 & 7.47 \\
\hline 300 & 0.836 & 1.136 & 70.6 & & 25.9 & 7.66 \\
\hline 350 & 0.775 & 1.047 & 59.2 & & 25.2 & 7.44 \\
\hline 400 & 0.724 & 0.975 & 50.0 & & 25.5 & 7.28 \\
\hline
\end{tabular}

Conc. $(\mathrm{Mol} / \mathrm{l})$

Concentration and luminescence is. time, smoothed data taken from curves of Figs. 1 and 2.

range studied, and is relatively independent of variations in the initial concentration ratio of peroxide and catalyst $(3,9)$.

3. Spectra. Fig. 3 gives a comparison between the fluorescence spectrum of $\mathrm{ZnTPP}$, as excited at $148^{\circ} \mathrm{C}$ in $t$-butyl benzene by 436 $\mathrm{m}_{\mu}$ mercury radiation, and the chemiluminescence spectrum at the same temperature (Abrahamson). It is seen that both emissions have their maximum at $6506 \mathrm{~A}$ (neon spectrum). A small high-energy shoulder is also found in the fluorescence spectrum, which may be due to a trace of impurity. A similar close correspondence between fluorescence and chemiluminescence emission was found by Knorr and Albers, using chlorophyll as catalyst (8) .

4. Metal Requirement. Free tetraphenylporphine (TPP) has no accelerating effect on the rate of THP breakdown, and shows no 


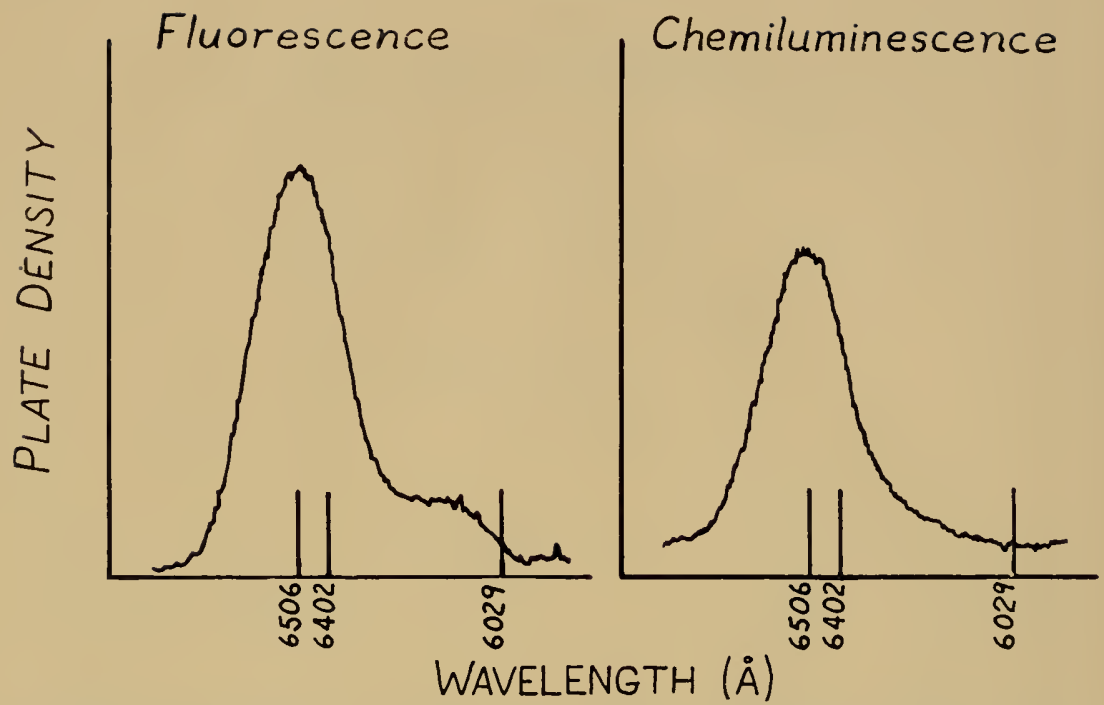

Fig. 3. Comparison of fluorescence and chemiluminescence spectra of zinc tetraphenylporphine at $148^{\circ} \mathrm{C}$. (The indicated wavelengths are neon calibration lines).

luminescence in hot peroxide solutions (Kalberman, 7). If anything, addition of TPP slightly inhibits peroxide decomposition. The presence of the complexed metal is therefore a strict requirement, in agreement with the findings of Cook in hydrogen peroxide systems (2). It is difficult to compare the effectiveness of the various TPP metal complexes which were tested, because of the widely different stabilities of the metallo-porphyrins. However, the order is approximately $\mathrm{Cu}>\mathrm{Pb}>\mathrm{Zn}>\mathrm{Sn}$. Of these, the copper salt is by far the strongest catalyst, as shown by Kalberman (7).

\section{Discussion}

The first question to be clecided is whether we are observing a true catalysis or merely a stoichiometric reaction between peroxide and porphyrin. There is certainly no doubt that a certain fraction of the total peroxide decomposed is used to break down the dye. On the basis of other studies $(5,6)$ establishing formation of pthalimide and ammonia from metallo-phthalocyanines in the peroxide reaction, we might expect that succinimide and possibly benzoic acids would result from ZnTPP breakclown. It is not likely, however, that each ZNTPP molecule would require as many as thirty molecules of peroxide, which is the observed ratio. If we confine ourselves to the 
question of the luminescence alone, the evidence of Fig. 3 that the luminescent molecule is the excited unchanged porphyrin itsell also indicates that the dye is excited in the course of its catalytic function, rather than its breakdown. The proposed mechanisms are based on this reasonable assmmption.

The metal requirement suggests that the first step in the reaction is complexing of the peroxide at the central metal ion. By analogy with the classic mechanism of Haber and Weiss $(4,1)$, we then assume an initial oxidation of the metallo-porphyrin. This may occur via hydrogen atom or electron transfer (9). In tetraphenyl porphyrins and phthalocyanines, it is evidently much more likely that electron transfer is involved. We therefore write $(M=$ metallo-porphyrin):

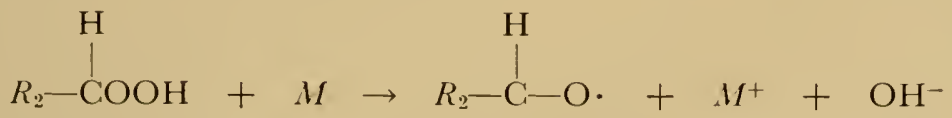

Particularly in non-polar solvents, the driving force of this reaction will be increased by binding $\mathrm{OH}^{-}$to the central metal ion, giving a type of charge-transfer complex:

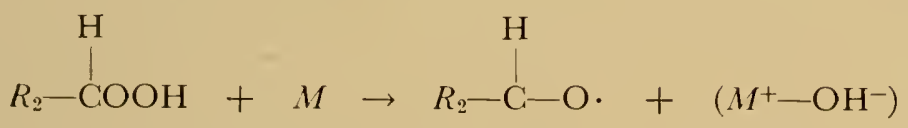

Various reactions may now ensue. However, in all of these it is proposed that electronic excitation of the metallo-porphyrin results from removal of $\cdot \mathrm{OH}$ from the charge-transfer complex to form. $\mathrm{H}_{2} \mathrm{O}$, and the return of the electron into the $\pi$-system of the porphyrin.

(A) Excitation in bimolecular reaction. Following reaction (1), we may have $\left(M^{*}=\right.$ excited metallo-porphyrin):

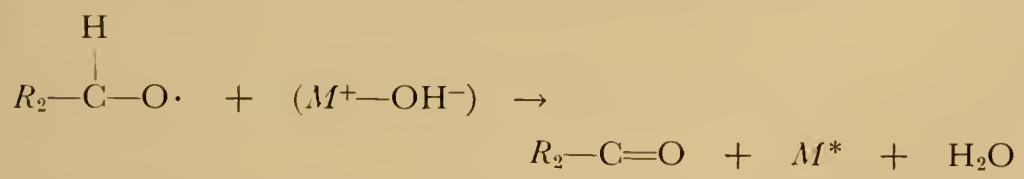

In this case, reactions ( $I$ ) and (2) may be considered to occur without separation of the intermediate products. However, the kinetic complications of this reaction and of peroxide chemistry in general (12) indicate that chain processes are involved. Reasonable mechanisms may be written in which the excitation process may occur in chain-starting, propagating, or ending steps, as follows: 
(B). Excitation in chain-ending step. Reaction (1) would be followed by the chain:<smiles></smiles>

$$
R_{2}-\mathrm{C}=\mathrm{O}+R_{2}-\stackrel{\mathrm{CO}}{\mathrm{H}}+\mathrm{H}_{2} \mathrm{O},
$$

and finally by reaction (2).

(C). Excitation in chain-starting step. Reaction (I) would be followed by:

$$
\begin{gathered}
\left(\mathrm{M}^{+}-\mathrm{OH}^{-}\right)+R_{2}-\stackrel{\mathrm{COOH}}{R_{2}-\dot{\mathrm{COOH}}} \rightarrow M^{*}+\mathrm{H}_{2} \mathrm{O} \\
R_{2}-\dot{\mathrm{COOH}} \rightarrow R_{2}-\mathrm{C}=\mathrm{O}+\cdot \mathrm{OH} \\
\cdot \mathrm{OH}+R_{2}-\mathrm{COOH} \rightarrow \text { chain }
\end{gathered}
$$

(D). Excitation in chain-propagating step. Depending on the relative reactivity of peroxide and porphyrin toward hydroxyl radicals, we may have, after reaction (5),

$$
\mathrm{M}+\cdot \mathrm{OH} \rightarrow\left(\mathrm{M}^{+}-\mathrm{OH}^{-}\right)
$$

followed again by reaction $(f)$. Hydroxyl radicals in this mechanism may also arise originally from the simple thermal cleavage of the peroxide.

Other possible sequences may evidently be written.

The close parallel between intensity of luminescence and rate of peroxide decomposition is consistent with all these mechanisms, as is the overall second-order character of the respective constants. In mechanism B, for example, any other competing chain-ending step (due perhaps to intermediates in dye breakdown) rould diminish both the peroxide decomposition rate and the luminescence. Mechantisms for excitation involving reactions of type $(f)$, in which the products include both free radicals and excited molecules, are energetically leasible, but would seem to be intrinsically less probable 
than those resulting in non-radical products only. The ultimate breakdown of the dye may result from molecules of $\left(\mathrm{M}^{+}-\mathrm{OH}^{-}\right)$which are cliverted from the reaction sequence of Mechanism $\Lambda$, or from the simple chain mechanisms. Alternatively, the dye breakdown may proceed by free-radical reactions not directly related to those which result in excited catalyst molecules.

The excitation process itsclf may perhays be understood more simply by saying that the electron, transferring from $\mathrm{OH}_{-}^{-}$to $\mathrm{M}^{+}$, drops back into an excited orbital instead of the gromol state. In classical terms, we may consider that the electronic oscillator (the harp-string of the porphyrin!) is set into vibration by the electron displacement and return. In lact, if sufficient energy is available, one might expect that quite generally the returning electron will fall into another orbital, since the reaction is accompanied by movements of the heavy nuclei, and the electronic energies of the various orbitals are coupled to the nuclear configuration. Charge-transfer will thus be accompanied by a change in molecular shape, and provide the opportunity for transition into a new electronic state during the backtransfer.

The frequent association of peroxide-breakdown reactions with chemiluminescence of complex organic molecules suggests that the mechanism in all such cases may be essentially the same as that proposed here for the porphyrin reaction-that is, the molecule being excited, or its precursor, functions alwalys as a catalyst for peroxide decomposition, the excited state being reached following charge-transfer processes involved in this catalysis. However, the general chargetransfer mechanism for excitation need not, of course, specifically require peroxides.

Recent work on quenching of excited (triplet) states of aromatic molecules (including porphyrins) by heavy metal ions indicates that charge-transfer intermediates also are involved here (10). Thus, if $R^{*}$ is an excited molecule and $I$ is the quenching ion, we would have (direction of charge-transfer is arbitrary) formation of the complex

$$
R^{*}+I \rightarrow\left(R^{+}-I^{-}\right) \rightarrow R+I+\text { heat }
$$

followed by radiationless transition to the ground state. It thus appears that ionic intermediates play an important role in catalyzing transitions among the electronic states of the separated donor or acceptor. This is not to say that charge-transfer processes are the only ones which lead to chemiluminescence. In cases where atom rather than charge transfer may appear to be the reaction mechanism, we may, of course, utilize the coupling of clectronic and vibrational 
energies to permit radiationless transitions leading to excited states of the product (13). However, a reaction mechanism becomes a less probable pathway toward chemiluminescence to the extent that it entails conversion into vibration of any part of the released chemical energy, particularly in condensed phase.

\section{REFERENCES}

1. Bark, W. G., Baxendale, J. H., George, ''., and Hargrave, K. R. Trans. Faraday Soc., 47, 462, 59 I (1951).

2. Cook, A. H., J. Chem. Soc., 1761, 1774 (1938).

3. Cross, D. R., Ph.D. Thesis, Syracuse University (1960).

4. Haber, F., and Weiss, J., Proc. Roy. Soc. London, A 147, 233 (1939).

5. Helberger, J. H., Naturwiss. 26, 316 (1938).

6. Helberger, J. H., and Hever, D. B., Chem. Ber., 72B, 11 (1939).

7. Kalberman, M., M.A. Thesis, Syracuse University (1955).

8. Knorr, H. V., and Albers, V. M., Phys. Rev. 61, 730 (1942).

9. Linschitz, H., and Abrahamson, E. W., Nature 172, 909 (1953).

10. Linschitz, H., and Pekkarinen, L., J. Am. Chem. Soc. 82, 2411 (1960).

11. Rothemund, P., J. Am. Chem. Soc., 60, 2005 (1938).

12. Tobolsky, A. V., and Mesrobian. R. B., "Organic Peroxides", Interscience Publishers, New York (1954).

13. Compare, for example: Weiss. J., Trans, Faraday Sor., 35, 219 (1939), and Evans, M. G.. and Polanyi, M., ibid., 35, 178 (1939). 


\title{
THE CHEMILUMINESCENCE OF LUMINOL ${ }^{1}$
}

\author{
EMIL H. WhIT: \\ Department of Chemistry, The Johns Hopkins Unitersity, \\ Ballimore, Maryland
}

Visible light is emitted from a moderate number of chemical reactions at room temperature (2). Only two chemiluminescent reactions, the oxidation of luminol (5-anjino-2,3-dihydro-1,4-phthalalinedione, Fig. 1, I) (1) and the oxidation of lucigenin (10, I0'-

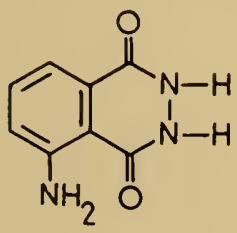

I

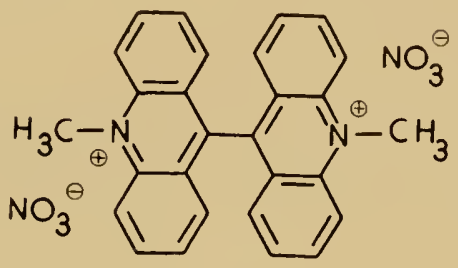

II

Fig. I. I, Structural formula of luminol. II, Structural formula of lucigenin.

dimethyl-9,9'-biacridinium nitrate, Fig. I, II) (14), are reasonably efficient in this respect, however. The brilliant chemiluminescence of the latter compounds has attracted the attention of a large number of investigators, some interested in the mechanism of the reaction $(\stackrel{2}{)}$, and others in practical applications to the detection and analysis of hydrogen peroxide (19), free radicals (4), blood (24), etc. (13) . Despite this interest and effort, it must be concluded that very little is known about chemiluminescence today. ldeally, a study of chemiluminescence should lead to an understanding of the chemical processes involved as well as to an understanding of the important problem of the interconversion of chemical energy and light energy. An approach to such a study of the chemiluminescence of luminol and a critical review of previous work in this field are the subjects of this paper.

this investigation was stpported in part b) a research grant from the Public Health Service. 


\section{Structure and Chemiluminfascence}

The chemiluminescence of luminol was first reported in 1928 by Albrecht (1). Since that time, a large number of hydrazides have been tested for their chemiluminescence activity but none has been found to surpass luminol in this respect. A knowleclge of relationships between structure and activity has resulted from these investigations, however (Fig. 2). The substituted luminol III, for example, proved<smiles>CNc1cccc2c(=O)[nH][nH]c(=O)c12</smiles>

III<smiles>Nc1cccc2c1C(=O)N(N)C2=O</smiles>

VI<smiles>[R]NNC(=O)c1ccccc1</smiles><smiles>[R]O[OH+]</smiles><smiles>Nc1c2ccccc2cc2c(=O)[nH][nH]c(=O)c12</smiles>

$X I I$<smiles>Cn1[nH]c(=O)c2c(N)cccc2c1=O</smiles>

IV<smiles>Nc1cccc2[nH]c(=O)c(=O)[nH]c12</smiles>

VII<smiles>O=c1[nH][nH]c(=O)c2cnccc12</smiles>

$\mathrm{X}$<smiles></smiles>

XIII<smiles>COc1n[nH]c(=O)c2c(N)cccc12</smiles>

V<smiles>O=C1C=CC=CC1=O</smiles>

VIII<smiles>O=c1[nH][nH]c(=O)c2nc3ccccc3nc12</smiles>

$X I$<smiles>[R]c1cccc2c(=O)[nH][nH]c(=O)c12</smiles> 
to be as efficient as luminol (10), whereas the $\mathrm{N}$-methyl and the O-methyl analogs (IV and V) with blocked hydrazide rings were not chemiluminescent (7). Two isomers of luminol, VI with a fivemembered ring (15) and VII with exchanged nitrogen and carbonyl groups (17), were also not chemiluminescent. Maleic hydrazide (VIII) and the linear hydrazides (IX) proved to be chemiluminescent, but only weakly so. In general, therefore, a diacylhydrazine bearing (wo hydrogen atoms (insuring the ready formation of nitrogen) is required in the heterocyclic ring, and for efficient chemiluminescence this system should be attached to an aromatic ring (presumably to increase the efficiency of the light emission). Although a few hydrazides have been prepared in which this attached ring is heterocyclic ( $\mathrm{X}$ and XI; both only weakly chemiluminescent) (31) or naphthalenic (XII; almost as efficient as luminol) (9), most of the cyclic hydrazides that have been synthesized correspond in type to general formulas XIII and XIV.

Two generalizations correlating structure and efficiency of chemiluminescence have been made by Drew (10). He noted that the 5 -substituted phthalazinediones (XIV) were superior to the 6-substituted isomers (XIII), and that within each group the chemiluminescence efficiency was related to the ability of the substituent $R$ to release electrons into the conjugated system. Selected compounds illustrating these conclusions are given in Table 1. The increase in light emission caused by the halogens, the relative order of the halogens, and the effect of the nitro group suggest that the resonance effect, not the inductive effect, of the substituents is involved (Fig. 3). The same
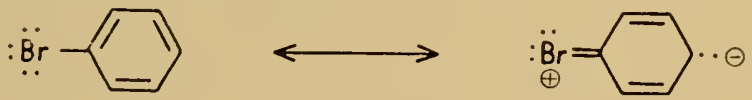

Fig. 3.

order of substituents has been observed for organic reactions in which the substituent is called upon to stabilize a partial positive charge in the transition state (3). The generalization is too simple to account for the effect of all of the substituents, however, since the order of the 5 -methyl and the 6,7-dichloro derivatives is anomalous.

Whatever the detailed explanation for the substituent effect in chemiluminescence may be, it is significant that a similar effect has been found for the fluorescence of these compounds (23) as well as for the fluorescence of the corresponding phthalic acids. This observation strongly suggests that the effect of the substituent is exerted 
T.ABLE I

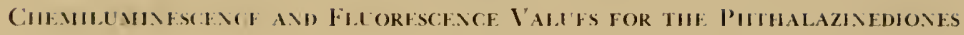<smiles></smiles>

\begin{tabular}{|c|c|c|c|c|}
\hline \multirow{2}{*}{ SUBSTITUENT } & \multicolumn{2}{|c|}{ CHEMILUN INESCENCE } & \multicolumn{2}{|c|}{ FLUORESCENCE (in acid) } \\
\hline & RELATIVE INTENSITY & $\lambda, m \mu$ & YIELDS $\quad(\%)$ & $\lambda, \mathrm{m} \mu$ \\
\hline $5-\mathrm{NH}_{2}$ & 100 & 424 & 10 & 424 \\
\hline $5-\mathrm{NHCH}_{3}$ & 60 & 451 & 10 & 449 \\
\hline $5-\mathrm{OH}$ & 20 & 416 & 2 & \\
\hline $6-\mathrm{NH}_{2}$ & 4 & 418 & 2 & 423 \\
\hline 5-NHCONHCO & 2 & 463 & 0.1 & 424 \\
\hline $5-B r$ & 1 & 417 & 2 & 424 \\
\hline $5-\mathrm{NHCOCH}_{3}$ & 1 & 424 & 7 & 425 \\
\hline $5-\mathrm{NHNHSO}_{3}^{\ominus} \mathrm{Na}^{\oplus}$ & 0.2 & 424 & 0.02 & 424 \\
\hline None & 0.02 & 413 & & \\
\hline $5-\mathrm{NO}_{2}$ & 0.01 & 402 & 0.02 & 425 \\
\hline
\end{tabular}

IDDITIONAL RELATIVE CHEMILUMINESCENI INIENSTTHE (9, 10)

$$
\begin{aligned}
& 5-\mathrm{Br}>6-\mathrm{Br}>6-\mathrm{I}>\text { none }>5-\mathrm{CH}_{3} \\
& 5,8-\mathrm{di}\left(\mathrm{NHCOCH}_{3}\right)>5-\mathrm{NHCOCH}_{3}>5-\mathrm{Cl}>\text { none }>6,7-\mathrm{di}(\mathrm{Cl})
\end{aligned}
$$

largely at the light-emission stage and that the efficiency of the various hydrazides in chemiluminescence is also determined at this point. Consistent with this view is the fact that the rates of oxidation of a number of hydraricles that differ markedly in their light output are the same (32).

\section{The Chemiluminescent Reaction in IVater}

The chemiluminescence of luminol and related hycharicles has 
been examined in the past almost exclusively in a system composed of water, sodium or potassium hydroxide, hydrogen peroxide, the hydravide, and an "activating" agent (2). The latter reagent usually consists of either an oxidizing agent such as sodium hypochlorite or potassium ferricyanicle, or a transition metal. A chelating agent is used with the metal to prevent the precipitation of the metal oxide or hydroxide in the basic solutions which are required for chemiluminescence. Typical metal "activators" are iron in the form of hemin (2) or the polyamine or Versene complexes (30), and copper in the form of its ammonia complex $(30)$. The role of the metal is a double one; in its reaction with hydrogen peroxide, two necessary reactants, oxygen gas and hydroxy or hydroperoxy radicals are formed (16). In a few instances hydrogen-peroxide-free systems have been used, but under these conditions oxygen must be supplied and an oxidizing agent such as potassium ferricyanide must be used to get any appreciable amount of light.

The quantum yield has been measured for the luminol reaction under a number of conditions with various combinations of reagents; a convenient summary of the results has been given by Bremer (6a). To date, the highest quantum yield measured for a water system has been found to be 0.003 .

Mechanism of the Reaction. Very few facts are available on which to base a mechanism. No product other than nitrogen (26) has been isolated from a chemiluminescent reaction of luminol in water. The chemiluminescent reaction of the unsubstituted phthalic hydrazide has yielded phthalic acid $(8,32)$ but this is largely a product of the "dark" reaction. With a quantum yield of 0.003 for the chemiluminescence in water of the most efficient hydrazide, the possibility of isolating the products of the "light" reaction is, at best, slim.

In view of the nitrogen formed in the reaction and in view of the nature of the reaction in organic solvents (vide infra), it is rather certain that luminol is not a "catalyst" in the chemiluminescence; it is consumed in the reactions leading to the excited state of some emitter. The belief that luminol is not consumed in the chemihminescence stems from the work of two investigators in particular. Drew showed that in a sodium hypochlorite-hydrogen peroxide system, ca. 70 moles of hydrogen peroxide were consumed per mole of luminol (8). Actually, large amounts of oxygen are evolved, and the excess of hydrogen peroxide is merely lost in this side reaction which has nothing to do with luminol. Oxygen evolution from the hydrogen-peroxide-based systems occurs whenever a strong oxidizing 
agent or a transition metal is present. The second observation which seemed to many authors to demand a "regeneration" of luminol was first made by Albrecht (1). Albrecht, and later workers (18, 27), showed that the ftuorescence spectrum of luminol (in acidic solutions) was a rather good match for the chemiluminescence spectrum (in basic solutions!). This match of the spectra does not warrant identification of luminol as the light emitter, however. The correspondence which was observed holds only when the fluorescence is measured in neutral or acidic solutions. In the basic solutions used for chemiluminescence, luminol exists only as the mono anion, and the fluorescence efficiency of this species is essentially zero $(22,23)$. That is, there is no luminol as such in the chemiluminescent systems. A far better case can be made for the 3-aminophthalate ion as the light emitter. There is not only a close match between the fluorescence spectrum of this compound and the chemiluminescence spectrum of luminol, but in addition, the $p H$-fluorescence efficiency profile is essentially the same as the $p \mathrm{H}$-chemiluminescence profile (22) .

A number of detailed mechanisms have been proposed for the luminol chemiluminescence by various workers $(1,5,8,18,26)$. In general, the mechanisms are variations of two types, based on the key internsediates XV and XVII (Fig. 4). The evidence for the peroxide XV consisted solely of an elementary analysis (8). It has been suggested that $\mathrm{XV}$ is in reality a salt of luminol in which the metal ion is solvated by hydrogen peroxide (6b) (i.e., structure XVI). Our evidence is in agreement with this view, since the infrared spectrum of this salt ( $M=$ barium), prepared according to the directions of Drew (8), showed the normal carbonyl frequencies of the simple salts of luminol. If structure $\mathrm{XV}$ were correct, no absorption would have occurred in this region of the spectrum. Also, the addition of peroxide ion or oxygen to form compounds such as XV has little or no analogy in organic chemistry.

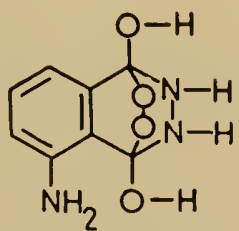

XV

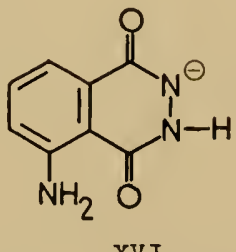

$\mathrm{XVI}$

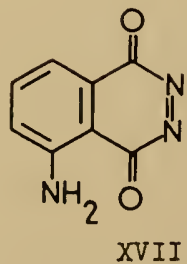

XVII

Fig. 4. 
There is, furthemore, no evidence for the formation of the azo compound XVIl in the luminol chemiluminescence. Albrecht and Kautsky (18) proposed that XVIl was a reaction intermediate, and both of them attempted to prepare XVII from luminol. They were mable to isolate it because of its instability, and their tests were run on the crude material. Albrecht's observations can be accounted for by the presence of luminol in the crude samples which he used, and this may also be the explanation for Kautsky's results. We have examined the relatively stable azo compound XVIII (Fig. 5)

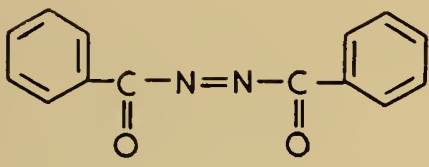

XVIII

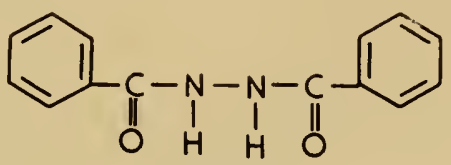

XIX

Fig. 5 .

and compared it with dibenzoylhydrazine (Fig. 5, XIX). The hyIrazide is chemiluminescent, but the azo compound is not. Furthermore, the products obtained from the two reactions are different. Only benzoic acid was obtained from the hydrazide XIX, whereas approximately equimolar amounts of benzoic acid and benzaldehyde were obtained from XVIII (Fig. 6).

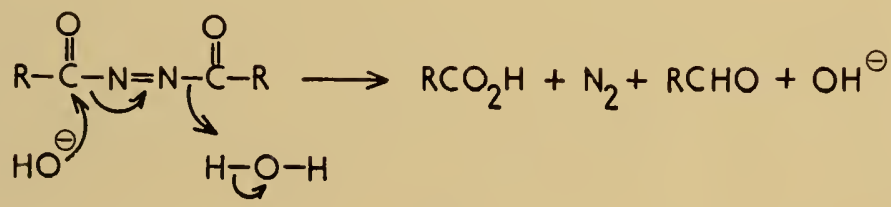

Fig. 6.

The above discussion is intended to show that there is actually no unambiguous experimental evidence supporting any of the mechanisms that have been proposed for the chemiluminescence of Luminol. Practically the only lacts available for a discussion of the mechanism are the following: (1) oxygen is required (6); (2) the anion of luminol is the reactant $\left(K_{a}\right.$ for Iuminol $\left.=10^{-6}\right)(26)$; (3) free radicals are involved; and (4) nitrogen is a product of the reaction. The requirement for radical internediates is based largely on the observation that radical chain stoppers inhibit the chemiluminescence $(5 b, 23,26)$, and on experiments such as the following. A basic 
aqueous solution of luminol $(p \mathrm{H}$ ca. 11) is dark both at room temperature and at $75^{\circ} \mathrm{C}$. When a small amount of a free-radicalforming compound such as azoisobutyronitrile, or better, benzoyl peroxide is added to the hot solution, light is emitted. The emission maximum is at the same wave length $\left(430 \mathrm{~m}_{\mu}\right)$ as the emission from the hydrogen-peroxide-based chemiluminescence. Oxygen gas is required for light production, and this system is therefore very similar to the oxygen-ferricyanide system mentioned earlier. Our rorking hypothesis for the chemiluminescence in the water systems, based on these lacts, is given in Fig. 7 .

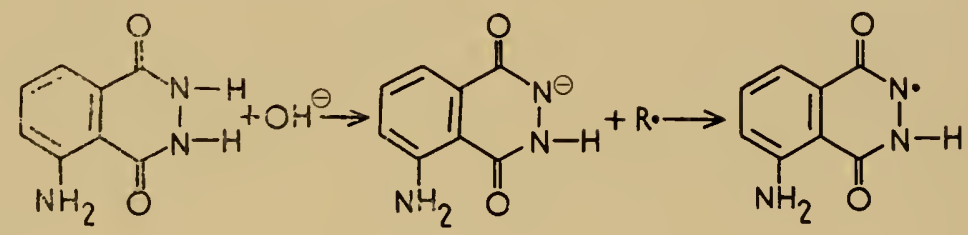

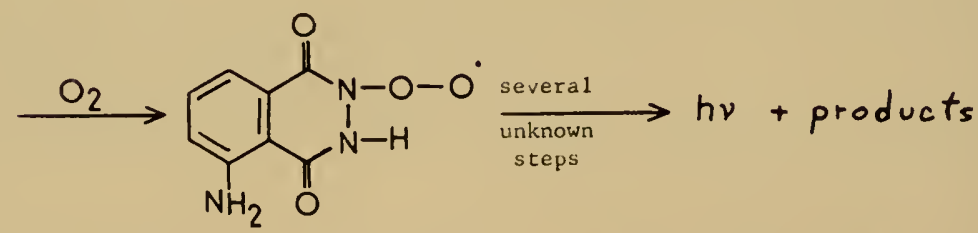

Fig. 7 . Reaction scheme for the chemiluminesence of luminol in water.

A rather different approach to the study of chemiluminescence has been made by several investigators interested in sensitized chemiluminescence (21). This phenomenon involves the transfer of energy from an excited molecule to a "foreign" molecule which subsequently emits a photon. There seems to be no evidence, however, that this can occur with the excited species in the luminol reaction (29).

It is apparent that the study of chemiluminescence in water systems has not been very successful. Part of the difficulty can be attributed to the use of powerful oxidizing agents that degrade the products which one might normally want to isolate. A system which is free of hydrogen peroxide and oxidizing agents consists of luminol, hot $30 \%$ potassium hydroxide, and oxygen. The efficiency of this system is, however, very low. To date, we have been able to carry out efficient chemiluminescent reactions under mild conditions only in organic solvents. 


\section{The Chemiluminescent Reaction in Organic Solvents}

The hydrogen-peroxide-luminol systems discussed in the previous section can be carried out without much change in solvents such as acetone and ethanol. An interesting mixture from which a deep blue light is obtained consists of luminol, hydrogen peroxide, ammonia, a trace of copper, and glycerin as the solvent. When organic solvents are used, alkyl hydroperoxides can be substituted for hydrogen percxide if iron cattalysts such as the iron-polyanine complexes (11) are used: copper is not a catalyst under these conditions. These systems are actually not much different than the corresponding ones in water.

A major change in the chemiluminescent reaction occurs, however, when certain weakly acidic, highly polar organic solvents are used; only luminol, oxygen, and a base are required, and the light emission is superior to that observed in water. Dimethyl sulfoxide is the best of the solvents that we have tested and dimethyl formamide is the second best. The advantage of using solvents of this type is that bases stronger than hydroxide ion can be used. We normally use t-butoxicle ion (prepared from the alcohol and potassium metal); with this base, the dianion of luminol is formed and, as will be shown later, this ion can react directly with oxygen.

\section{Luminol $+\mathrm{O}_{2} \stackrel{\text { Base }}{\longrightarrow}$ Light}

The reaction is thus similar in general outline to a number of other chemiluminescent reactions (Fig. 8) $(12,14,20)$.

These equations illustrate the interesting fact that in most, if not all, chemiluminescent and bioluminescent reactions at room temperature, oxygen is a reactant. (See McElroy and Seliger, this symposium). The ground state of oxygen is a triplet state, and the significance of the oxygen requirement may be that, in these cases, adducts of oxygen and the organic molecules are formed with no reversal of an electron spin; i.e., the adducts may be formed directly in a triplet excited state.

The products of the luminol chemiluminescence in dimethyl sulfoxide are nitrogen and 3-aminophthalate ion (Fig. 9). The nitrogen was identified and measured by gas-liquid chromatographic techniques. The aminophthalate ion was determined as dimethyl 3aminophthalate; this ester was obtained in 89 per cent yield from the successive treatment of the reaction mixture with hydrochloric acid and diazomethane. Since the quantum yield for the reaction in dimethyl sulfoxide was found to be 0.1 and since it is unlikely that 


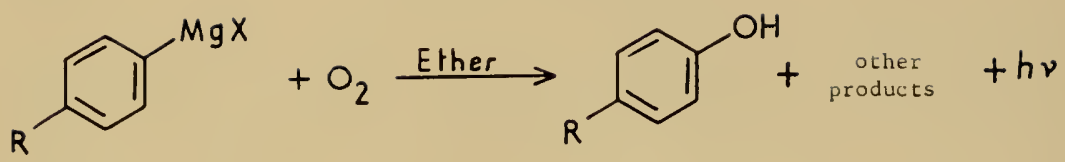

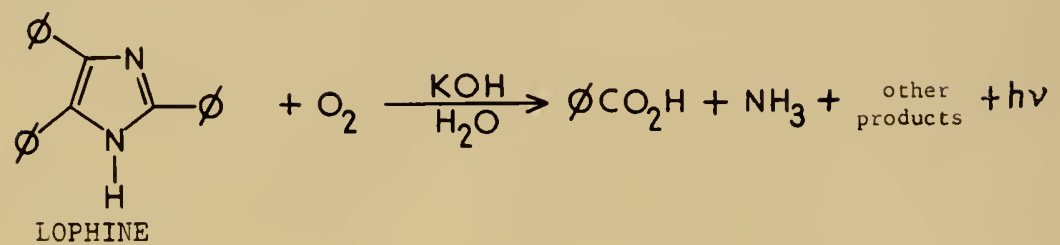<smiles></smiles>

lïg. 8. Chemiluminescent reactions.

our product recovery was quantitative, we feel that the aminophthalate ion is indeed a product of the light-emission reaction. Further work is in progress to improve the product isolation and, if possible, the quantum yield.

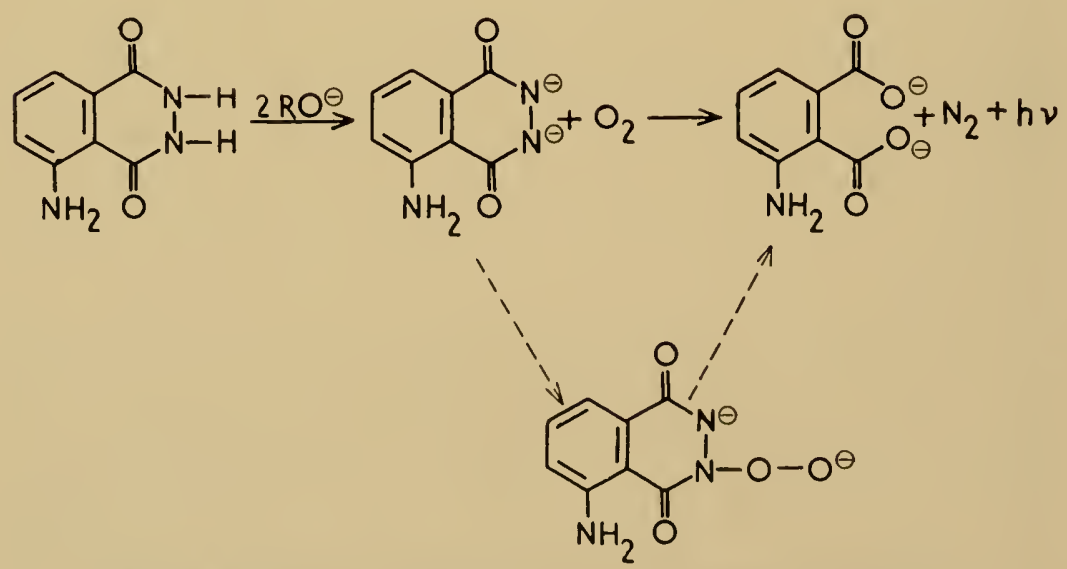

Fig. 9. The chemiluminescence of huminol in dimethyl sulfoxide. 
The stoichionetry of the reaction is as shown in Fig. 9. One molecule of the dianion reacts with one molecule of oxygen to form one molecule of the dianion of aminophthalic acid and one molecule of nitrogen.

The first step in the reaction must involve the dianion and oxygen, since no light is observed when the monoanion is exposed to oxygen, and the dianion undergoes no reaction in the absence of oxygen. A possible intermediate is the hyperoxide ion shown in Fig. 9. A closely related reaction of Grignard reagents has been reported recently (28).

$$
R^{-} \mathrm{Mg} \mathrm{I}^{+}+\mathrm{O}_{2} \rightarrow R-\mathrm{O}-\mathrm{O}^{-} \mathrm{Mg}_{\lambda^{+}}
$$

Unlike the reaction in water, the chemiluminescent reaction in dimethyl sulfoxide is not noticeably affected by cyanide ion or by the transition metals. A radical decomposition path for the hyperoxide is, therefore, unlikely. An intramolecular decomposition is probably involved, since it has been found that peroxide ion is not an intermediate; that is, the oxygen is eventually reduced by four electrons without passing through $\mathrm{O}_{2}=$. Very little more can be said about the intramolecular reaction at the present time.

The luminol chemiluminescence in climethyl sulfoxide has a peak emission at $480 m \mu$, a wavelength corresponding to about $60 \mathrm{kcal}$. per einstein. Approximate calculations of the energy available from the<smiles>Nc1cccc2c(=O)[n-]n(O[O-])c(=O)c12</smiles>
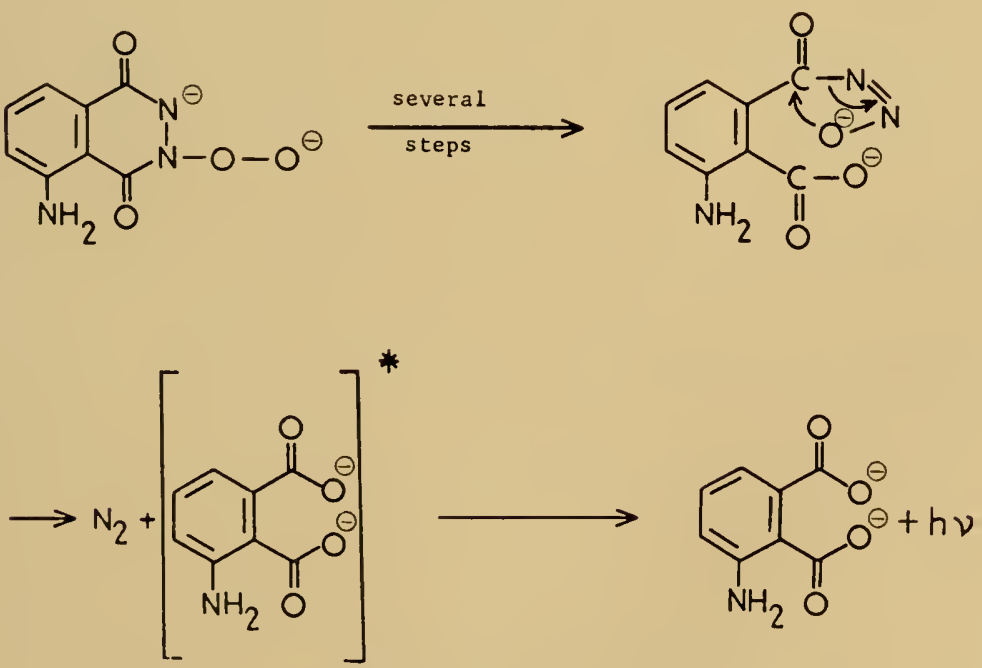

lig. 10. $A$ possible path for the formation of nitrogen. 
reaction (Fig. 9), based on bond energy values, indicate that ca. $90 \mathrm{kcal}$ are available and that most of this energy is associated, in a sense, with the formation of the nitrogen molecule. The latter possibility suggests that the emitting species is excited during the ejection of the nitrogen molecule and that the phthalate ion might be the emitter (Fig. 10). Indeed, the eviclence for this choice seems rather good for the water systems (previous section). The evidence is not

TABLE 2

Culmulumingchen: and Fluorescence Maxina in Various Solvents

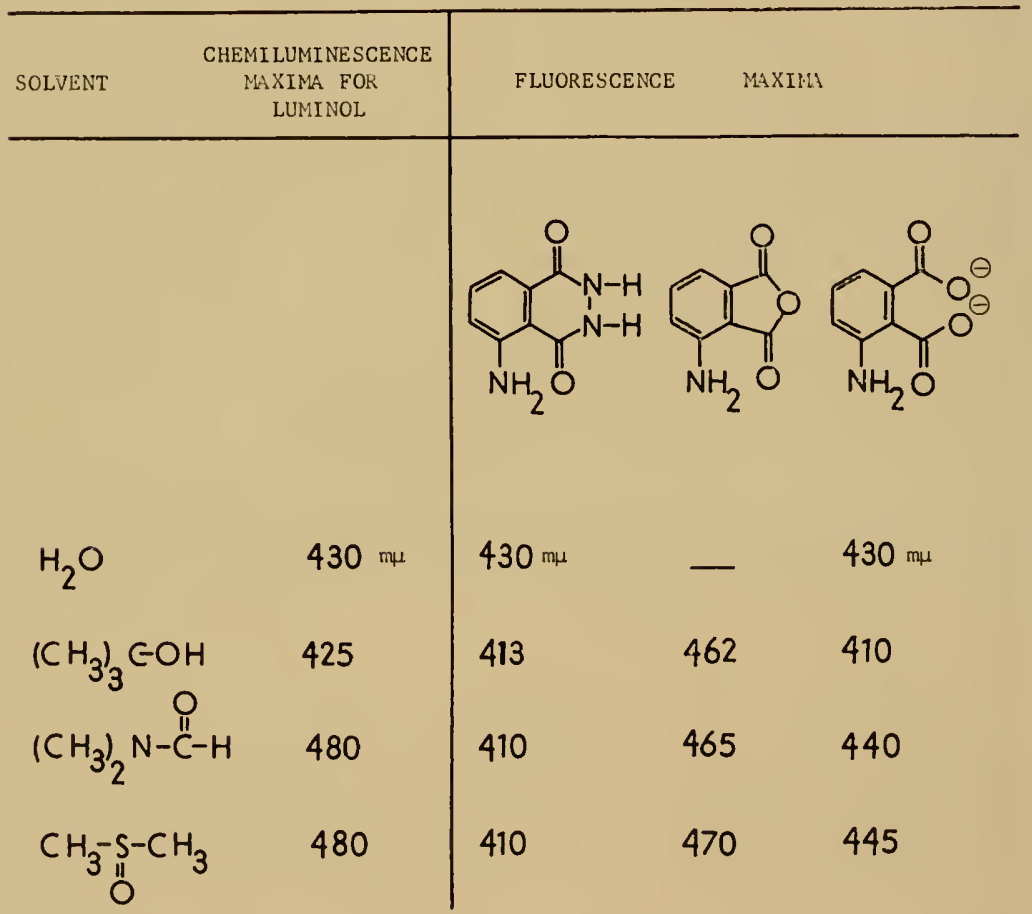

compelling for the reaction in organic solvents, however. We have compared the chemiluminescence spectra in lour solvents with the fluorescence spectrat of various possible emitters (Table 2). Nlthough a case might be made for the aminophthalate ion in water, and for the anhydride in dimethyl formamide and in dimethyl sulfoxide, no completely satisfying general correspondence has been found. It has been recently observed, however, that immediately after the chemiluminescence is over in dimethyl sulloxide, the solution fluoresces at $480 \mathrm{~m} \mu$; attempts to isolate and identily the emitter are in progress. 
It is obvious that much more must be known before the phenomenon of chemiluminescence can be outlined in any detail.

\section{ACKNOWLEDGMENTS}

The author wishes to thank Dr. Howard H. Seliger for the quantum yield measturements, and Drs. John H. M. Hill and Eugene Galantay for some of the experiments :eported in this paper.

\section{REFERENCES}

1. Albrecht, H. O., \%. physik. Chem., 136, 321 (1928).

2. (a) Audubert, R.. Trans. Faraday Soc. 35, 197 (1939); Harey, E. N., A History of Luminescence, The American Philosophical Society, Philadelphia, Pa. (1957). (b) Extensive reviews of chemiluminescence have been published by Bernanose, A., Bull. soc. chim. France, 567 (1950); 39D (1952); ref. 5. Anclerson, R. S., Ann. N. Y. Acad. Sci., 49, 337 (1948); and Vaughan, W., Chem. Revs., 43, 447 (1918).

3. Bennct, G. M., and Jones, B., J. Chem. Soc., 1815 (1935).

4. Bergstemann, J., Strahlentheropie, 98, 474 (1955).

5. (a) Bernanose, A.. Bull. soc. chim. France, 329 (1951); (b) Weber, K., Chem. Ber., 75, 565 (1942).

6. (a) Bremer, T., Bull. soc. chim. Belges., 62, 569 (1953); (b) Wilhelmsen, P. C., Lumry, R., and Eyring, H., The Luminescence of Biological Systems, p. 77, Anerican Association for the Advancement of Science, Washington, D. C. (1955).

7. Drew, H. D. K., and Garwood, R. F., J. Chem. Soc., 1841 (1937).

8. Drew, H. D. K., and Garwood, R. F., J. Chem. Soc., 791 (1938).

9. Drew, H. D. K., and Garwood, R. F., J. Chem. Soc., 836 (1939).

10. Drew, H. D. K., and Pearman, F. H., J. Chem. Soc., 586 (1937).

11. Embree, W. H., Spolsky, R., and Williams, H. L., Ind. Eng. Chem., 43, 2553 (1951), have used similar systems as polymerization initiators.

12. Evans, W. V., and Diepenhorst, E. M., J. Am. Chem. Soc., 48, 715 (1926); Duf. ford, R. T., Nightingale, D., and Calıert, S., J. Am. Chem. Soc., 47, 95 (1925).

13. Friedman, A. S., J. Appl. Plys., 27, 417 (1956).

14. Gleu, K., and Petsch, Wr., Anger'. Chem., 48, 57 (1935).

15. Gleu, K., and l'fannstiel, K., J. prakt. Chem., 146, 137 (1936); and also ref. 8.

16. Haber, F., and Weiss, J., Proc. Roy. Soc. London, A, I47, 332 (1934).

17. Humtress, E. H., and Gladding, J. V. K., J. Am. Chem. Soc., 64, 2644 (1942).

18. Kautsky, H., and Kaiser, K. H., Z. Naturforsch., 5b, 353 (1950).

19. Langenbeck, W., and Ruge, U., Chem. Ber., 70, 367 (1937).

20. Radziszewski, 13i., Chem. Ber., 10, 70 (1877).

2I. Schales, O., Chem. Ber., 72, 1155 (1939); Iamamushi, 13., Naturwiss., 28, 722 $(1940)$.

22. Seliger, H. H., this symposium.

23. Spruit-Van Der Burg, A., Rec. trar. chim., 69, 1.536 (1950).

24. Steigman, A., Chem. Ind. (London), 60, 889 (1941).

25. Stollé, R., Chem. Ber., 45, 273 (1912).

26. Stross, F. H., and Branch, G. E. K., J. Org. Chem., 3, 38.5 (1938).

27. Si ešnikov, Acta Physicochim. U.S.S.R., 8, 441 (1938).

28. Walling, C., and Buckler, S. A., J. Am. Chem. Soc., 77, 6032 (1955).

29. Weber, K., and Ochsenfeld, W., Z. physik. Chem., B. 51.63 (1912).

30. White, E. H., J. Chem. Educ., 34, 275 (195i). 
31. Yale, et al., J. Am. Chem. Soc., 75, 1933 (1953), prepared compound X, and XI was prepared and tested by Wegler, J. prakt. Chem., 148, 135 (1937).

32. Zellner, C. N., and Dougherty, G., J. Am. Chem. Soc., 59, 2580 (1937). These authors isolated benzil-2,2'-dicarboxylic acid from the reaction of phthalic hydrazide with sodium hypochlorite. The chemiluminescence under these conditions is extremely weak.

\section{DISCUSSION}

Dr. Kisha: I would like to direct a question to Dr. Linschitz. I am impressed by what a mute harp the porphyrin ring makes when the chemiluminescence arises as "one twangs the porphyrin ring." It is the low quantum yield to which I am referring, which makes a very interesting comparison to the second reaction of yours. This suggests that in both cases the emitting molecule is in a different environment. The point is that I think that the porphyrin spectra characteristically show vibrational structure and yet I sec you attribute the second band in the porphyrin base to some impurity. I think that this may not be correct. But, if not, the question is why does the second band disappear in the chemiluminescent reaction?

DR. LiNschitz: Professor Dorough has also measured the fluorescence of the same prophyrin at low temperatures. His spectrum does not have the sicle band in it, and his spectrum checks exactly with our luminescence spectrum. To be sure we measured our fluorescence spectrum at the same elevated temperatures as our chemiluminescent reaction, and I think our explanation makes sense for this reason. As far as overall chemiluminescence quantum yields are concerned, it is hard to say. They are probably very, very low. If you calculate the number of molecules which are going through this system and if the yields were $100 \%$, you would expect an output of sontething like $10^{15}$ to $10^{16}$ photons per second. I would expect our yields to be below $0.1 \%$. 'This represents, of course, the primary yield of excited molecules produced in the reaction, multiplied by the fluorescence yield. The point is that the chemiluminescence corresponds to the fluorescence of the original dyc. Thus, it is hard to see how this can be excited in any breakdown processes involving the dye. The point is that the one unambiguous fact which 1 am really sure of is that the chemiluminescence corresponds to the fluorescence of the original dye. Thus, it is hard to see how this can be excited in the breakilown processes.

Dr. Cinvin: 1 have a question for Dr. Linschitz. Is there any evidence for the kind of complexes with which you start the reaction if you examine the spectrum carefully of porphyrins at low temperatures in the presence of peroxides?

D). Lixs(antz: No, we have not been able to find any evidence for that.

1)R. Cisvix: It's a rather sharp spectrum.

DR. Inscintr: I would think so, and we lowe looked for it.

I)R. M(iliko: I would like to ask a question on the inhibitor product 
you are sugesesting which might change your rate constant in the first fow seconds. Why doesu't it continue en change your constant?

DR. Lexsents: The only way that I call make scuse out of that is to assume that there is a steady state concentration of the inhibitor which is maintaned more or less constant during the course of the reaction. The best source of this would probably be some intermediate breakdown product of the dye, which is extensively decomposed. If you start, for example, with phthalocyaninc, you obtain phthalimide as the end product of the dye breakdown. The final absorption spectrum of our reacted solutions shows no traces of any long wavelength absorption components. You really shatter the dye. The breakdown of the dye itself apparently needs metal. If you run this reaction in the presence of uncomplexed porphyrin. then the dye rides right through and there is apparently little attack on the dye. That is rather surprising because presumably the uncatalyzed normal breakdown of peroxide must go through some chain process involving hydroxyl. And yet the porphyrin structure seems to come through hydroxyl radicals unchanged.

DR. Platt: How great a range of metals have you tried?

DR. Linscintz: Not too great; zinc, magnesium, lead, tin and copper. I know that this is rather a bizarre choice of metals. It was made for spectroscopic as well as chemical reasons.

Dr. PLatT: Would the disodium complex work as well?

DR. CALvis: I do not think that this compound would last through the peroxide treatment. Would copper really work as well as the other metals? The other metals are all diamagnetic.

Dr. Linscintz: Well, I have a feeling that the magnetism per se does not play any important role in the catalysis. Copper is extremely active.

Dr. Calvis: But can you get chemiluminescence?

DR. Lisschitz: You do not get the luminescence, but then the copper complex does not fluoresce.

Dr. CalviN: But that is just the point.

Dr. LinscuITz: Y'es, that's the point. It may very possibly go through some excited state, but you never see it, because the copper porphyrin immediately falls over quite likely to the triplet, and then very quickly to the ground state. It is a very active catalyst for the peroxide breakdown, much more active than the other metals.

Dr. Weber: I wanted to make some remarks on the general energetics of luminescent reactions. It has been said many times that these reactions are strongly exothermic. What has not been used to my knowledge is the balance of the bonds broken and formed during the reactions to calculate $\Delta H$ which is approximately equal to $\Delta F$. This should give us some idea of how much energy we should get in the reaction. For example, in the peroxidation of an aldehyde there is one carbon-hydrogen bond broken which requires 85 kilocalories and one oxygen-oxygen bond broken which requires 40 kilocalories. One carbon-oxygen bond is formed releasing 75 kilocalories, and one oxygen-hydrogen bond formed releasing 110 kilocalories. Thus, the 
total reaction is exothermic with the release of 60 kilocalories. This will be the energy available for the formation of the excited state. So the important point is we are breaking bonds which are strong. The only labile bond which secms to be really useful in these circumstances seems to be the oxygen-oxygen bond. I believe it is very interesting that all these reactions involve peroxides, either hydrogen peroxide or the transitory formation of another peroxide which, of course, all have this oxygen-oxygen bond. Moreover, if one produces a scheme of reactions in which an excited state is created, it would appear to me logical that one should create this excited state at the moment at which this bond disappears.

Dr. Hastings: I would like to ask Dr. White if any of these nonchemiluminescent, substituted derivatives are fluorescent?

Dr. WhIte: The answer is, in general, no. The chemiluminescence effciencies of the various phthalic hydrazides are roughly proportional to their fluorescence efficiencies. This relation seems to hold for the phthalic acids also.

Dr. Hastings: If one could find one which was fluorescent but nonchemiluminescent, and whose fluorescent emission spectrum differed from the emission spectrum of the chemiluminescent compound, then one could look for an energy transfer to the non-chemiluminescent molecule in the reaction mixture.

Dr. White: So far, we have assumed the simpler picture of chemiluminescence; namely, that an energy transfer is not involved and that one of the reaction intermediates is the light emitter.

Dr. Linschitz: We do find that at low temperatures (these things are degassed in a racuum line) some reversible changes take place which may correspond to semiquinone formation. These changes are being looked into in more detail, but for studying the chemiluminescent reaction we took pains to aroid any possibility of having a photochemical reaction.

I would like to ask Dr. White a question. You make a comparison of a chemiluminescence spectrum at high $p H$ and a fluorescence spectrum measured at a different $p \mathrm{H}$. Now, if they do correspond, one may I think legitimately argue that the luminescence is coming from the same state, despite the difference in $p \mathrm{H}$, since it is the acid strength of the excited state that counts. Thus, it is possible that the same excited ionic form may be present in both the chemihminescence and fluorescence experiment. In fact. the spectrosepic data seem to me to be rather convincing on this point.

DR. WHite: This possibility has been eliminated (assming that no special interactions occur in the chemiluminescence) by the fincling that basic solutions of luminol do not fluoresce. It seems unlikely, therefore, that the emission occurs from the singlet state of either luminol or the luminol anion.

Dr. STREntik: Firstly, with respect to the effect of light on porphyrin catalyzed luminescence, the following may be of interest. A few years ago we observed that chloroplyyl in basic methanol in the presence of aldehyde gives off a continuous luminescence for a number of hours. After this 


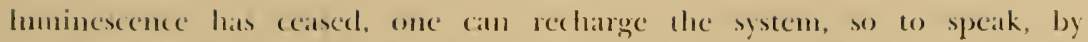
illuminating with visible light which causces a reappearance of luminescence.

Secondly, I would like to ank Dr. White to what he ascribes the barimm salts of peroxide derivatives which Drew isolated. Are they consistent with any of the schemes you made? Would you also comment on the $p \mathrm{H}$ dependence of the fluorescence and the fluorescence yields of the various phthallic acid derivatives you believe might be the emitting molecule?

DR. Wmin:: The salt that Drew isolated wats just a barimm salt of luminol in which the barium ion was solvated by hydrogen peroxide. I don't molerstand your second question. Which derivatives do you mean?

DR. STR:Allik: You mentioned the possibility of the phthallic acid derivative being the emitting molecules. What is its Iluorescence yield at the $p \mathrm{H}$ at which the reaction takes place? Is it as low as that of the luminol itsell? Or is it quite a bit higher?

Dk. Winte: The fluorescence yield is about one-third that of luminol.

DR. STRFHLF: And that yield is 0.2 ?

Dr. W'mT: Y'es, 0.2 is the maximum value. This wals measured at the $p H$ of maximum chemiluminescence. 


\title{
SOME ASPECTS OF THE LUMINOL LIGHT REACTION
}

\author{
H. H. Seliger \\ McCollum-Pratt Institute and Department of Biology \\ The Johns Hopkins University, Baltimore, Maryland
}

\section{INTRODUCTION}

With the exception of bioluminescence, the oxidation of luminol is the most efficient chemiluminescent reaction known and has been the subject of comprehensive chemical investigations, the most recent by White and coworkers (7). With material purified in the latter's laboratory the luminol light reaction is being investigated by quantitative spectroscopic techniques and the following is a summary of some of the results obtained to date.

\section{EXPERINENTS AND DISCUSSION}

The primary object of the present investigation is to identify the molecule whose excited state gives rise to the cheniluminescence. This in itself requires two assumptions relative to the oxidation reaction, and the validity of any conclusions reached rests on how closely these assumptions hold within the general framework of the mechanism of chemiluminescence. Briefly, one assumes that (I) light emission occurs from the excited state of a relatively stable molecule, either the original molecule or an oxidized product; and (II) the ionic environment in which the excited molecule finds itself immediately after the oxidation process is not dissimilar from the general ionic environment.

In order to eliminate any possible effects of resonance self-absorption, the chemiluminescence emission spectrum of a highly purified $10^{-5} M$ luminol solution in .1 $N$ NaOH was measured photoelectrically by a charge-nomblization technique already described (5), and is shown in Fig. 1 together with the fluorescence spectrum of the neutral luminol molecule in acicl solution. These data are similar to the results of Spruit and Spruit van der Burg (6), who suggest that, by virtue of the coincidence of the spectrat, the neutral huminol molecule is the emitting species in the light reaction. This picture seems to be generally accepted (1), and the further assumption is 


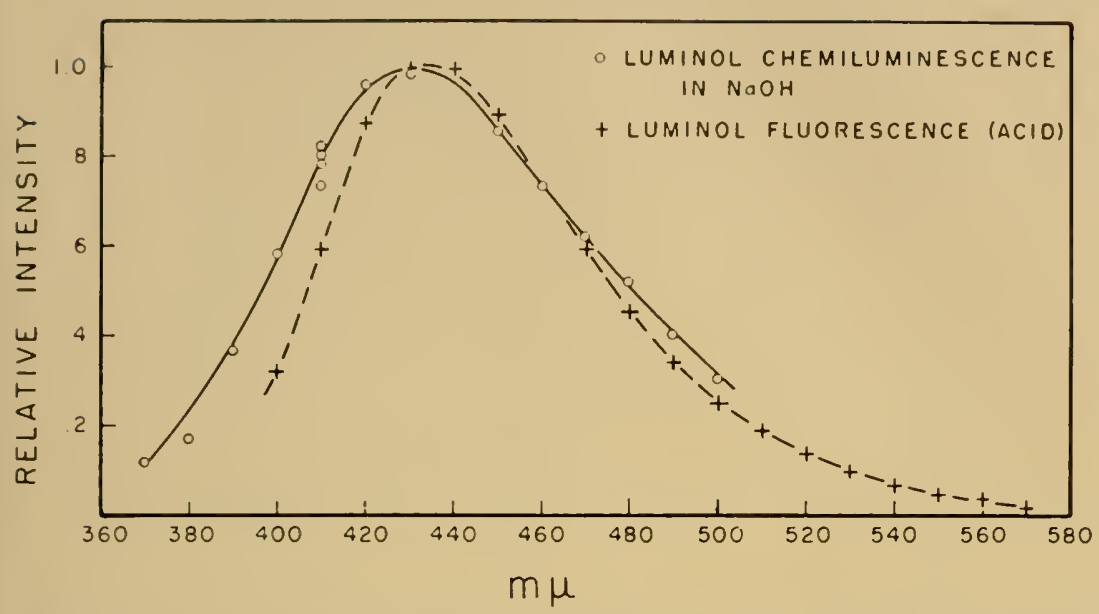

Fig. 1. Chemiluminescence emission spectrum of luminol in $0.1 \times \mathrm{NaOH}$ initiated with $\mathrm{H}_{2} \mathrm{O}_{2}+\mathrm{NaOCl}_{\text {, and }} \mathrm{Nompared}$ with fluorescence of the neural luminol molecule in acid solution, $p \mathbf{H} 4$.

made that in alkaline solutions the chemical excitation of luminol leaves it in the acid-stable form (3).

It is proposed that perhaps this picture is an unnecessarily complicated one and that the enitting species in the luminol chemiluminescence is an excited state of the oxidized product, with a structure not too different from the original luminol molecule. Since White (7) has shown a concurrent evolution of $\mathrm{N}_{2}$ for luminol consumed in the oxidation process, the assmuption that light emission is due to the neutral luminol molecule implies an energy transfer from the excited oxidation product to a neutral huminol molecule. This energy transfer further implies a concentration dependence of the chemiluminescence quantum yield. This has been found not to be the case. The relative chemiluminescence quantum yield for luminol was measured over the concentration range from $10^{-7} M$ to $10^{-4} M$ in $0.1 \mathrm{~N} \mathrm{NaOH}$ and was found to be constant, and independent of concentration. A further argument against the supposition that the luminol molecule is the emitting species, based on assumption II above, is given by the data of Fig. 2 in which the experimentally determined fluorescence quantum yield of the luminol molecule is plotted as a function of $p \mathrm{H}$. At precisely those $p \mathrm{H}$ values where chemiluminescence occurs and is most efficient the fluorescence quantum yield of the $430 m_{\mu}$ band decreases to zero.

However, following the line of proposed intermediates suggested 


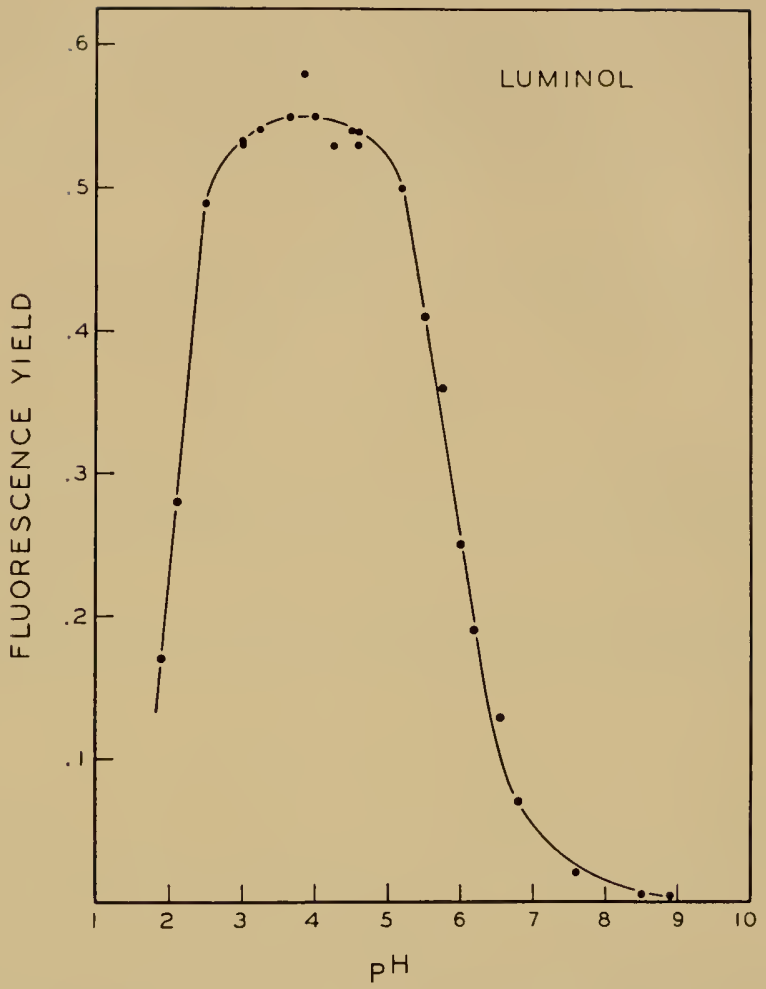

lig. 2. Fluorescence quantum yield of luminol as a function of $p \mathrm{H}$.

by White, the fluorescence quantum yield of a crystallized sample of the HCil salt of aminophthalic acid was measured as a function of $p \mathrm{H}$. These data are shown in Fig. 3. Fig. 4 shows the comparison between the luminol chemiluminescence enission in 0 .I $N \mathrm{NaOH}$ and the fluorescence emission of aminopluthalic acid, also at $0.1 \mathrm{~N}$ $\mathrm{NaOH}$. If anything, the spectra appear to coincide even more closely than in the case of luminol shown in Fig. 1. If the oxidized product molecule is the light-emitting species, it would certainly simplify the picture by obviating the necessity for imposing energy transfer.

From Fig. 3 it is seen that the fluorescence quantum yicld of aminophthalic acid decreases rapidly below $p \mathrm{H}$ ll. The luminol light reaction initiated with $\mathrm{NaOCl}$ has been reported to show an intensity maximmm at $p H \mathrm{H} / \mathrm{l}$ (1). Although light intensity is primarily a measure of the rate of a reaction rather than the efficiency, these facts might suggest a direct relation between the chemiluminesrence quantum yield and the fluorescence efficiency of excited amino- 


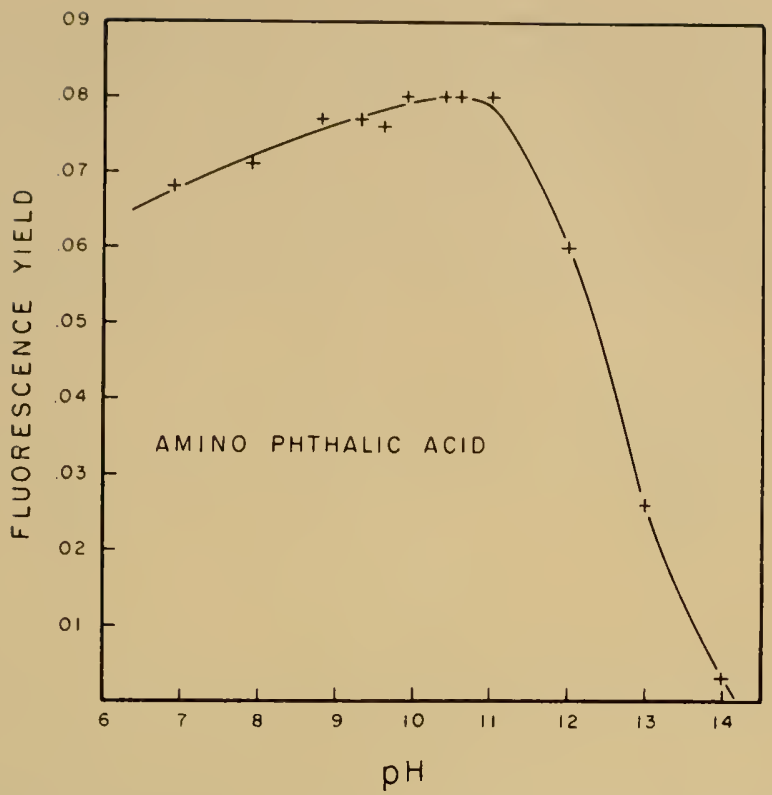

Fig. 3. Fluorescence quantum yield of aminophthalic acid as a function of $p \mathbf{H}$.

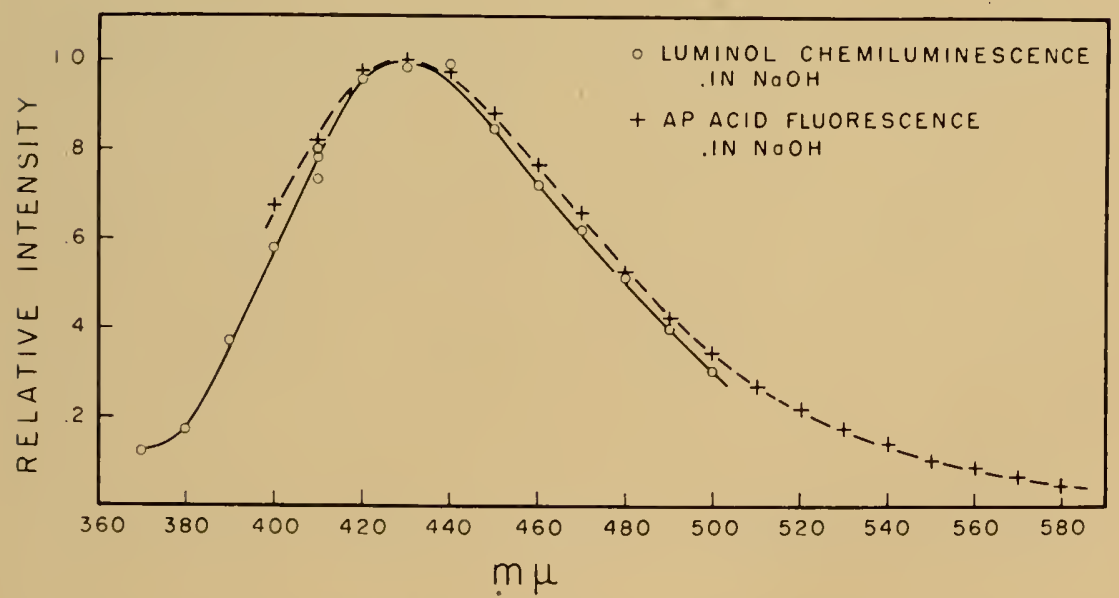

Fig. 4. Chemiluminescence emission spectrum of luminol in $0.1 \mathrm{~N} \mathrm{NaOH}$ initiated with $\mathrm{H}_{2} \mathrm{O}_{2}+\mathrm{NaOCl}$, and compared with the thorescence of aminophthalis. acid, also at $0.1 \mathrm{~N} \mathrm{NaOH}$.

phthalic acid. In order to check this point and to eliminate any possible $p H$-dependent rate reactions, the chemiluminescence quantum 
yield of luminol in aqueous solution was measured in bicarbonate buffer at $p \mathrm{H} 9.6$, in $0.001 \mathrm{~N} \mathrm{NaOH}(p \mathrm{H} 11), 0.01 \mathrm{~N} \mathrm{NaOH}(p \mathrm{H} 12)$, $0.1 N \mathrm{NaOH}(p \mathrm{H} 13), 1 N \mathrm{NaOH}(p \mathrm{H} 14)$, and $10 N \mathrm{NaOH}$. These results are shown in Table 1.

TABLE 1

Chemiluminescence Quantum Yield of Luminol for Various $p$ H Values

\begin{tabular}{lcc} 
Solution & $p \mathrm{H}$ & $\begin{array}{c}\text { Chemiluminescence } \\
\text { Quantum Yield }\end{array}$ \\
Bicarbonate Buffer & 9.6 & .01 \\
$.001 \mathrm{~N} \mathrm{NaOH}$ & 11 & .02 \\
$.01 \mathrm{~N} \mathrm{NaOH}$ & 12 & .01 \\
$.1 \mathrm{~N} \mathrm{NaOH}$ & 13 & .006 \\
$1 \mathrm{~N} \mathrm{NaOH}$ & 14 & .002 \\
$10 \mathrm{~N} \mathrm{NaOH}$ & 15 & .0004 \\
\hline
\end{tabular}

\section{Conclusions}

The data of Table 1 are significant in two respects. Firstly, they indicate that with properly purified material and other proper experimental conditions a minimum of one light quantum is emitted per approximately 50 luminol molecules oxidized, a much higher efficiency than has been reported previously (2). Secondly, the rapid decrease in the chemiluminescence quantum yield with increasing $p \mathrm{H}$ roughly parallels the decrease in the fluorescence quantum yield of aminophthalic acid, as shown in Fig. 3. This, together with the lack of concentration dependence of the chemiluminescence quantum yield, the fact that aminophthalic acid is fluorescent in alkaline $p \mathrm{H}$ whereas luminol is not, and the correspondence of the fluorescence emission spectrum of aninophthalic acid with the chemiluminescence emission spectrum of luminol would favor the former as the emitting species over the neutral luminol molecule. It would be extremely valuable to be able to correlate the chemical yield of aminophthalic acid with the chemiluminescence quantum yielk. However, owing to the low quantum yiclds observed in aqueous solution and also owing to the possibility of further dark reactions caused by the strong oxidants used to initiate the chemiluminescence, the unambiguous identification of the excited oxidation product as the light-emitting species camnot be made at this time, although it is strongly inclicated.

White (7) has lomnd that the chemiluminescence of luminol in organic solvents such as dimethyl sulfoxide appears to be even more efficient. The cmission spectrum of this chemiluminescence is shifted, peaking aromed $180 \mathrm{~m} \mu$. Preliminary measurenents of the chemi- 
luminescence quantum yield of fuminol in dimethyl sulfoxide, initiated with tertiary butoxide, give values of the order of 0.1 . This begins to look promising, in that if conditions can be found under which the quantun yiclds in organic solutions can be increased by a factor of only" or 3 it may be possible to correlate the chemical analysis directly with the chemiluminescence quantum yield and to establish once and for all the nature of the excited molecule in chemiluminescence.

\section{ACKNOWLEDGMENTS}

This worh has been supported in part by the Atomic Energy Commission, the National Institutes of Health and the National Science Foundation. Dr. F. White has kintly supplicd the purified reagents used in the measurements.

\section{REFERENCES}

1. Bernanose, A., and Simon, J., Bull. Soc. Pharm. Nancy, 24, I (March, 1955).

2. Bowen, E. J., in Chemical Aspects of Light, p. 270, Oxford University Press (I949).

3. Johnson, F. H., Eyring, H., and I'olissar, M. J., in The Kinetic liasis of Molecular Biology, p. 162, John Wiley and Sons, New York, 1954.

4. Pringsheim, P., in Luminescence, p. 33, Interscience Publishers, New York (1946).

5. Seliger, H. H., Anal. Biochem. I, 21 (1960).

6. Spruit, C. J. P., and Spruil, van der Burg, A., in The Luminescence of Biological Systemis (F. H. Johnson, ed.), p. 99, American Association for the Adrancement of Science, Washington, D. C. (1955).

7. White, E., this symposium. 


\title{
THE LUCIFERIN-LUCIFERASE REACTION ${ }^{1}$
}

\author{
Frank H. Johnson and Edward H.-C. Sie \\ Department of Biology, Princeton University, Princeton, New Jersey \\ AND Y. HANEDA \\ Yokosuka City Museum and Tokyo Jikeikai Medical School, Japan
}

\section{ORIGIN AND DEFINITION}

Nearly half a century ago, a book by a French general physiologist, Raphaël Dubois (11), was published under the title La Vie et la Lumière. In subject matter as well as title it was similar to the present symposium, for it clealt not only with the phenomenon of bioluminescence, which was Dubois' major interest and took up almost half the book, but also with problems of photosynthesis, with the biological action of a wide spectrum of radiations, from $\mathrm{X}$-rays to Hertzian, and with other topics pertinent to the general subject. Most of the topics that were included bear to some extent on the comfort or well-being of man. Extensive, accelerating research since that time has brought tremendous advances in knowledge of these subjects. Bioluminescence, however, is of no obvious importance to man, or even to the great majority of the organisms which possess it, and advances in this area have been less spectacular.

Dubois was a singularly appropriate author for a book emphasizing bioluminescence as at least a lascinating aspect of "Light and Life." He was responsible, in 1885, for the first really substantial breakthrough from a biochemical point of view (9), and had spent much of the cusuing years pursuing further investigations in this area. His initial, now classic, experiment consisted simply in (1) plunging a brightly luminescing photogenic organ of the West Indian elaterid beetle Pyrophorus into boiling water, which extinguished the light,

\footnotetext{
'Some of the woth reported in this paper has been aided in part by Office of Varal Research contract Nom 13.3.3(00), by National Science fomblation grants (; 1578 and (, 6106, by the Eugene Higgins Fund allocated to Princeton Universily, and by the Japanese National Commisson for UNLSCO. Reproduction, Uanslation, publication, use, and disposal in whole or in patt by or for the Inited states foremment is permilled.
} 
then cooling (quickly, (2) triturating a sinilar organ in a little water at room temperature until the light disappeared, and (3) pouring the two preparations together in the clark, whereupon light appeared at once.

Because the quantity of photogenic substances in Pyrophorus scemed to be small and the specimens were hard to procure, he sought more lavorable material and found it in the luminous clam, Pholas dactylus, which yielded analogous extracts that were active in light emission in response to essentially the same procedure $(10)$. With Pholas, the hot water extracts were prepared by heating to 7()$^{\circ} \mathrm{C}$, rather than boiling, in order to conserve the relatively, but not lully, heat-stable component. The relatively heat-labile component was rapidly eliminated at $60^{\circ} \mathrm{C}$. For the active principle in the hot water extract he introduced the name "luciférin," and for the one in the cold water extract the name "luciférase" $(10)$. Inrestigation of the properties of these components led him to conclude that the former was a natural albumin whose oxidation by molecular oxygen was catalyzed by the latter, a specific enzyme, with the production of light. This conclusion was correct except for the chemical nature of the luciferin, which is not an albumin.

In terms of the above procedure, the expression "luciferin-luciferase reaction" can be clearly defined, namely, light emission on mixing a cooled, hot water extract, whose luminescence was destroyed by heating, with triturated photogenic cells or tissue, whose luminescence has disappeared by other causes. In terms of the information that such a procedure yields, however, the expression can have as many meanings as such widlely encompassing terms as "protein denaturation." For reasons that are now understandable, a negative result, i.e., failure to obtain light on mixing crude extracts, does not prove the absence of luciferin or luciferase or even of a reaction between the two. Conversely, positive results do not prove that the observed light has originated in the manner in which it seemed to, i.e., from a reaction between the presumed components. Testing for a luciferinluciferase reaction in extracts of various types of organisms is nevertheless an important preliminary step, since the separation of components that will give a luminescent reaction in vitro is a sine qua non in progress toward biochemical elucidation of the system.

Shortly before Dubois' book appeared, the first paper in a long, comprehensive series of studies on bioluminescence by the late Professor E. Newton Harvey (17) was published. No few words of tribute could possibly do justice to the magnitude of his work, and the 
limitations of space prevent a detailed discussion of his contributions here. Further information and references to the literature in general can be conveniently found in his several books $(21,26,27,29)$. With particular reference to the luciferin-luciferase reaction, however, certain facts should be noted, as follows.

\section{The Known Examples}

Harvey made exhaustive tests for a luciferin-luciferase reaction in extracts from virtually every type of luminescent organism he could obtain. To the list of two examples found by DuBois, he added the American (Photimus and Photuris) and Japanese (Luciola) fireflies $(18,20)$, the ostracod crustacea Cypridina (19) and Pyrocypris (22), the marine fireworm Odontosyllis (25), and the deep sea decapod shrimp Systellaspis (25). Apart from a few falsely positive results in the early studies, a luciferin-luciferase reaction could not be demonstrated in any of various other groups of luminous organisms, including bacteria, fungi, sponges, radiolaria, flagellates, medusae, pennatulids, ctenophores, ophiuroids, tomopterids, chaetopterids, terebellids, polynoeids, earthworms, schizopods, myriapods, cephalopods, ascidians, balanoglossids, or fish (26).

The list of examples of definitely positive reactions among different types of organisms was not extended until 1950, when Bowden (5) succecled in clemonstrating the luciferin-luciferase reaction in extracts of a Iresh-water limpet, Latia. At the moment, the only additional examples are the luminous bacteria Achromobacter and Photobacterium $(38,39,43,44,46)$, a dinoflagellate, Gonyaulax (31), two kinds of fish, Parapriacanthus (15) and Apogon (16), the luminous fungi Collybia and Armillaria (1), the sea pansy Renilla (7), and a second decapod shrimp, Heterocarpus (13). It is noteworthy that most of the established examples of a lucifcrin-luciferase reaction have been discovered by Harvey and his students, his students' students, or collaborators within this group, the only exceptions being the examples found by Dubois $(9,10)$ and Bowden $(5)$, respectively.

Despite the many failures to demonstrate a huciferin-luciferase reaction in crude extracts of various organisms, there is little reason to doubt that such a system is involved in light emission in essentially all instances of bioluminescence. The failures can result from various possible causes, some of which are indicated in paragraphs that follow. Obrious possibilities for failures with crude extracts include dilution of reactints, which may be normally held together in gramules of some sort, or the presence of inhibitors, which seems to 
be the case with fompunlax (31), of to instability of either the lucilerim, such as that of lumgi (1). of the lucilerase, such as that of cratin fircllies $(37)$.

\section{Luminescence of Dried Materials}

The fact, however, that a number of instances have been found in which the whole organism, or its photogenic organ or cells, can be dried and can then luminesce again on moistening, shows that the necessary reactants are not hopelessly unstable except in a living environment. Examples of this phenomenon, anong organisms which thus far have not yielded active extracts of luciferin and luciferase, inclucle the following: jellyfish, sea pens, ctenophores (Beröe), nemerteans, polychaetes (Chaetopterus), earthworms, nudibranchs, cephalopods, copepods, myriapods, ophinrids, and tunicates (27).

Indirect evidence for an enzyme-substrate system, where these two components have not been successfully separated, can sometimes be lound in the effects of factors which influence enzyme action or states of proteins in general. For example, if urethane is added during the decay of luminescence in the slime secreted by Chaetopterus, the intensity of the light drops at once, but if sea water is then added to reduce the concentration of the drug, the intensity immediately increases, thus indicating a reversible inhibition (33). Nore recently2 it has been shown that desiccated photogenic tissue of jelly fish (Aquorea) or sea pens (Leioptilus) can be ground to a powder and taken up in concentrated solutions of urea, anmonium sulfate, sodium chloride, etc., which partially suppress the light normally emitted on moistening, but a bright flash of light will subsequently occur on dilution with distilled water (Fig. l). In $6 M$ urea, the system gradually deteriorates at room temperature, and in $10 M$ urea it undergoes rapid destruction. Similar results are obtained with homogenates prepared by squeezing whole jellyfish, or excised ring canals with their associated photogenic tissue, through several layers of cheese cloth. On adding ammonium sulfate to 60 per cent saturation, a precipitate forms which is highly active on dilution with distilled water, and retains its activity for days. The ring canals can be dehyclrated with acetone at $-10^{\circ} \mathrm{C}$, or colder, and on adding water to the dry powder prepared therefrom, a bright flash of light occurs. No additional light could then be elicited by addition of

2 The work on Aquorea and Lefoptilus reported here was carried out in the summer of 1959 at the . Iarine Laboratory of the Lniversity of Washington. Friday Harbor, Washingion. 
certain cofactors that function in the luminescence of other systems (vide infar), viz, adenosine triphosphate $(\Lambda T P)$ ), flavin mononucleotide (FMN), diphosphopyricline mucleotide in the oxidized (DPN) or reduced state (DPNH), or decaldehyde, separately or in combination. The same factors had no definite effect on the luminescence of fresh homogenates, except for decaldehyde, which caused

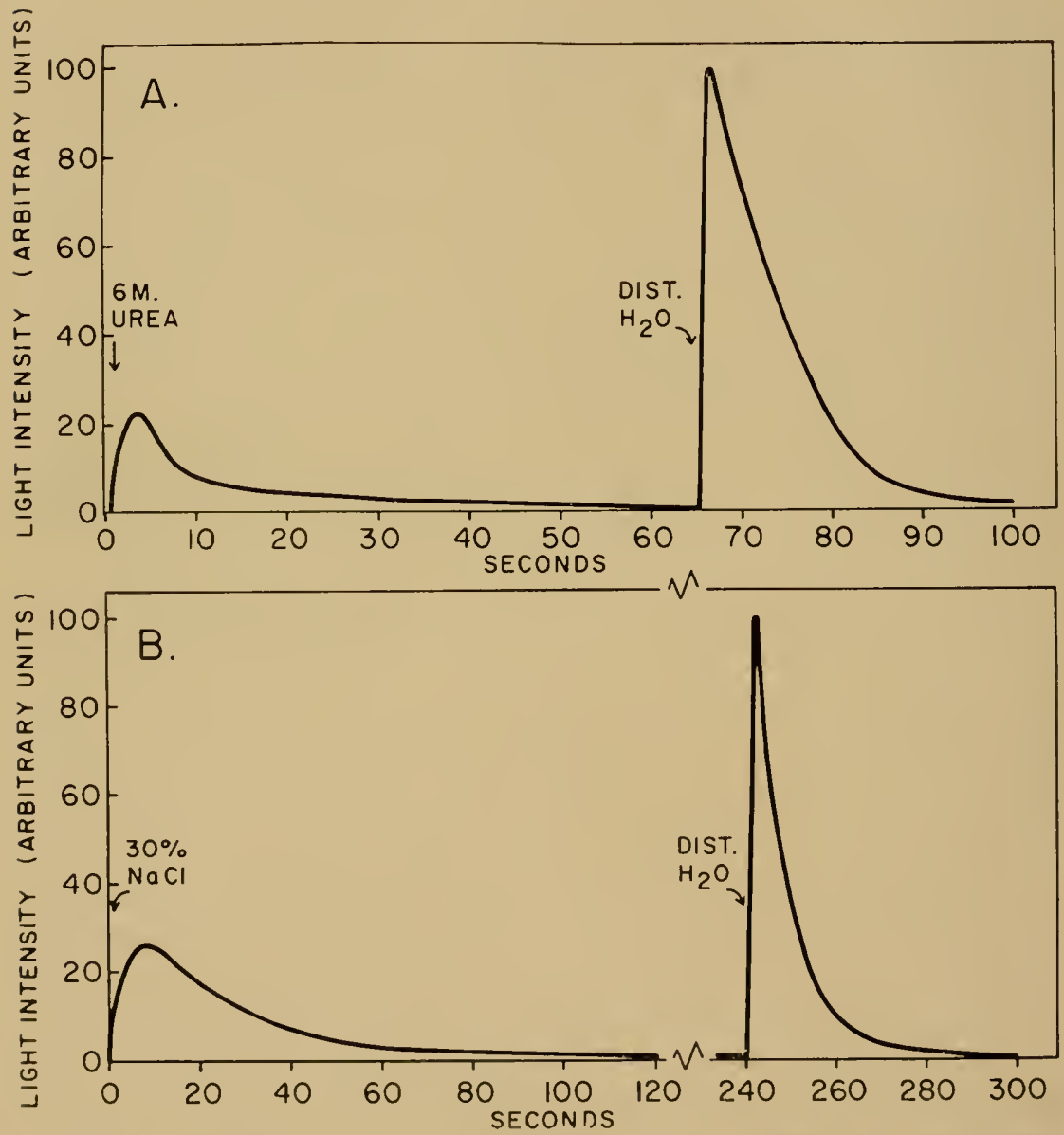

lïg. 1.1. Course of light imensity after adding $0.2 \mathrm{ml}$ of $6 \mathrm{M}$ wea 40 desiccated, powelered ring canals of Afuoren, and then adcling $2.8 \mathrm{ml}$ of distilleci water, at fines indicated by the respective arrows in the figure. Data ploted from EsterlineIngus antomatic recordings of amplitied ouput from RC.I I I'2I plostomultiplier (ube used to measure instantancous intensity of luminescence.

lig. IB. Similat $16 \times 1$, but $0.5 \mathrm{ml}$ of $30 \% \mathrm{~K} \mathrm{NaCl}$ solution was adcled instead of urea, followed by $3 \mathrm{ml}$ of clistilled water. 
a quick flash. I similat quick tlash could be caused, however, by nonpecilic cytolytir agents, such as octyl alcohol or soclimm taurocholate. Attempts were made to prepare an active heilerase from dry powelers, or from freshly excised rings ground with aluminum oxide and then centrifuged in the cold at high speeds, or in other ways. Tests lor their activity were macle by adding the residue of acetone extracts, methyl alcohol extracts, cooled hot water extracts, and others, but no luminescence resulted that could not be accounted for in terms of some remaining traces of photogenic tissue. Since the luminescent system of jellyfish is one of the few examples wherein light emission can take place in the complete absence of dissolved oxygen (24), it seems mlikely that instability of the luciferin in presence of oxygen is responsible for these negative results.

\section{The Three Systems Best Known at Present}

At the present time, the three biochemically best known systems are those of Cypridina, of the firefly, and of luminous bacteria. The over-all reactions leading to light emission in aqueous solutions with minimal components can be represented by the following schemes wherein $C$ stands for Cypridina, $F$ for firefly, $B$ for bacteria, $L$ for luciferin, Ase for luciferase, $R$-CHO for a long chain, aliphatic aldehycle, the $k$ 's for rate constants, and the cofactor symbols have the usual meaning:

\section{Cypridina}

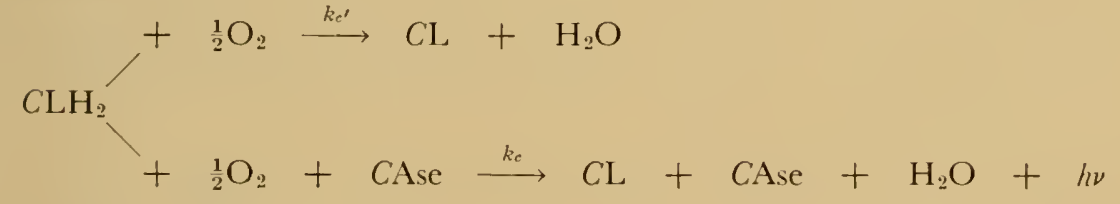

Firefly

$$
\begin{aligned}
\mathrm{FLH}_{2}+ & F \text { Ase }+\mathrm{ATP}+\mathrm{Mg}^{++}+\frac{1}{2} \mathrm{O}_{2} \stackrel{k s}{\longrightarrow} \\
& \text { FL.FAse.AMP }+\mathrm{POP}+\mathrm{Mg}^{++}+\mathrm{H}_{2} \mathrm{O}+h \nu
\end{aligned}
$$

Bacteria

$\mathrm{F}_{\mathrm{NNH}}$

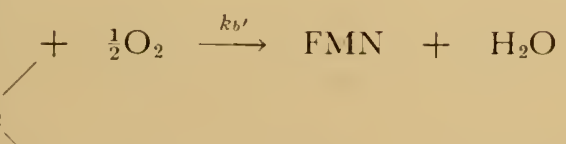

$$
\begin{aligned}
& +\frac{1}{2} \mathrm{O}_{2}+B \text { Ase }+ \text { R.CHO } \stackrel{k_{b}}{\longrightarrow} \\
& \text { FMN }+ \text { BAse }+ \text { products }+\mathrm{H}_{2} \mathrm{O}+h \nu
\end{aligned}
$$


The Cypridina system appears to be the simplest of the three, involving only an oxidizable substrate and enzyme catalyst, in addition to water and molecular oxygen. Anderson (3) demonstrated the non-luminescent auto-oxidation designated above by $k_{c}$, and found that it could be induced by ferricyanide and certain other agents. The product is inactive in light emission, and decomposes on standing, but can be initially reduced to active luciferin $\left(C \mathrm{LH}_{2}\right)$ by $\mathrm{Na}_{2} \mathrm{~S}_{2} \mathrm{O}_{4}$ and certain other reductants. The changes in absorption spectrum of fairly well-purified luciferin (48) indicate that the product of reactions $k_{c^{\prime}}$, and $k_{c}$ is the same, although no one has succeeded in regenerating, by the action of reducing agents or other means, any very considerable amounts of active luciferin after it has undergone a luminescent oxidation $\left(k_{c}\right)$ in presence of luciferase. The similarity in absorption spectra of the products of oxidation is therefore somewhat puzzling, and is possibly indicative of impurities in the luciferin solution, or else the seeming irreversibility of the luminescent reaction is to be attributed to catalysts other than luciferase in the relatively crucle enzyme solution.

The kinetics of light emission may be profoundly influenced by the proportion of $C \mathrm{LH}_{2}$ and $C \mathrm{~L}$ in equilibrium with each other at the start of the reaction with luciferase. Moreover, false "crossreactions" may occur with enzymes of other systems when the luciferase solution contains appreciable amounts of reversibly oxidized luciferin which undergoes reduction and then a light-emitting oxidation by its own specific enzyme. This circumstance is the most likely explanation for the fact that, in cross-reactions between the systems of different fireflies or different kinds of Cypridina which have different emission spectra (23), the color of the light is associated with the enzyme preparation.

Crystalline preparations of Cypridina luciferin, with a light-emitting potency some 40,000 times that of the starting material (whole chied organisms), have been obtained $(42)$ and their properties studied. The active luciferin is yellow in color, and gives a yellow fluorescence in ultraviolet light. On standing in solution exposed to air, the color changes to red, and three substances can be isolated by chromatography, namely, an inatctive red substance, plus two colorless, bluc-fluorescent substances which have been designated oxyluciferin A and B, respectively. The former of these two substances separates from ammoniacal solution in the form of yellow crystals, but the latter has not been crystallized. The empirical formula adduced for luciferin (32) is $\mathrm{C}_{21} \mathrm{H}_{25} \mathrm{O}_{22} \mathrm{~N}_{1} ; 2 \mathrm{HCl}$, and on the basis of various lines 
of evidence, the following provisional structure has been suggested:

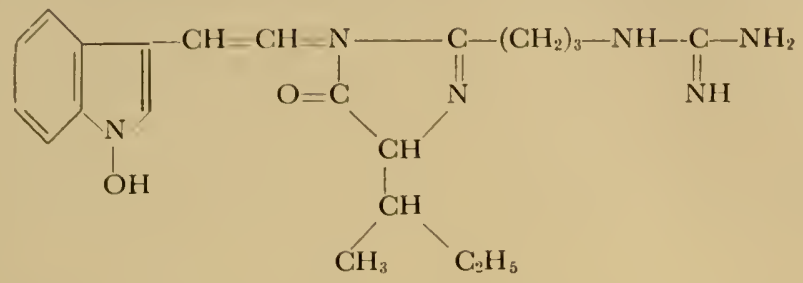

With relerence to the firefly, the luciferin, in both oxidized and reduced forms, as well as the lucilerase have been crystallized and their properties studied $(2,4,12,38)$. The reduced form of luciferin, active in light emission, is slightly yellow in alkaline solution, and has a greenish yellow fluorescence. It has the empirical formula $\mathrm{C}_{13} \mathrm{H}_{12} \mathrm{~N}_{22} \mathrm{~S}_{2} \mathrm{O}_{3}$, and is converted into the oxidized form with the loss of two hydrogens. The latter form is a potent inhibitor of luminescence through the formation of a relatively stable, intermediate complex with the luciferase, from which it can be removed by the addition of pyrophosphate or coenzyme A. Extensive research by McElroy and his associates has clarified the role of ATP and various factors that influence the amount and intensity of light emitted by the firefly system. Although the details can scarcely be touched upon in the present paper, it is interesting to note that this system has the extraordinarily high quantum efficiency of one, or close to one (4l). The important point with respect to luciferin-luciferase reactions is the requirement of ATP (35), a cofactor required for certain reactions involved in energy transfer within virtually every living cell.

Finally, the luminescent system of bacteria likewise requires a type of cofactor that has a widespread importance in cellular oxidations, namely, a flavin $(39,44)$. The precise role of the FMN is uncertain; like Cypridina luciferin, flavin mononucleotide is yellowcolored and fluoresces yellow, whereas the bio- or chemi-luminescence is much more in the blue: Although an exact correspondence between fluorescent and chemiluminescent spectra of a substance would not necessarily be expected, such correspondence is sometimes quite close, e.g., among the 2,3-dihydrophthalazine-1,4-diones (6). Marked differences between the emission spectrum of fluorescence and that of chemiluminescence suggest that the same molecule undergoes considerable modification in the chemical reaction before it emits. In bacterial Imminescence the aldehycle can scarcely be the emitting molecule inasmuch as it has no absorption in the visible. The 
aldehyde increases the efficiency of the luminescent oxidation. The evidence indicates that it becomes bound to the luciferase along with two molecules of flavin phosphate, and possibly furnishes part of the energy of excitation by undergoing peroxidation $(8,36,44$, $46,47)$. With the essential reactants in limiting concentrations, the total amount of light emitted is proportional to the amount of aldehycle added, with an efficiency of about 20 molecules of aldehyde used per quantum of light emitted, while the flavin is not used up (8). In the sustained luminescence which results when DPNH is ackded to the above components, the efficiency with respect to DPNH is only about 1 quantum of light per 2,800 molecules, a result in keeping with the influence of variations in temperature and hydrostatic pressure on the system, to the effect that most of the hyclrogen transfer from DPNH is via non-hminescent pathways (45).

\section{Specificities and Relationships of Different Systems}

The Cypridina, firefly, and bacterial systems clearly differ from one another biochemically. Because of the known differences in these systems, together with wide variations in the emission spectrum among different types of luminescent organisms, (or even in the same animal, as witnessed by the red and green luminescence of the "railroad worm Phrixothrix (27)), as well as the random occurrence of huminescence on the evolutionary scale of organisms, and finally, the failure of inmumerable attempts to obtain luminescence in "crossreactions" between the huciferin contained in extracts of one type with the huciferase in extracts of a different type of organism, the more or less prevalent view has arisen that essentially all luminescent systems are chenically unlike $(27,28)$.

Other considerations, however, do not lend support to this view. In the first place, almost no information is available concerning the actual chemical structure of different luciferins, and generalizations based on indirect evidence are not strictly reliable. Moreover, only slight modification of a chemiluminescent or fluorescent molecule is sometimes sulficient to effect wide differences in emission spectra, e.g., the wavelength of maximum chemiluminescene intensity of 3nitrophthalhyclravide is 4,020, while that of 3-acetyl carbaminophthalhydrazide is $4,625 \mathrm{~A}$, and the fluorescence peak of 3-aminophthalhydrazide occurs at slightly over $4,000 \mathrm{~A}$ in $0.01 \mathrm{~N} \mathrm{KOH}$, but at over $5,000 \wedge$ in $0.1 \times \mathrm{KOH}$ (6). Thus, it is unnecessary to postulate totally different types of compounds to account for variations in emission spectra. The random distribution of the property of bio- 
Imminescence on the phylogenetic tree could conceivably represent dilferences primarily in the efficiency of light emission, due to diflerences in internal cellular enviromments, such as the presence of quenching agents, or absence of a lactor such as the aldehycle (e.g., in aldehyde-less mutants $(40))$ of the bacterial system, which is somewhat non-specific in the sense that palmitic aldehyde, effective in promoting light emission of this system, is of some general occurrence in cells. Finally, the failure to obtain "cross-reactions," or even a lucilerase-luciferin reaction, in crucle extracts of different organisms could result in considerable measure from the presence of inhibitors, or to dilution, as mentioned earlier. Success in clemonstrating a luciferin-Inciferase reaction in bacterial extracts depended in part on the elimination of inhibitors and on concentrating the reactants $(39,43,44)$; the same is true with respect to extracts of higher fungi (1). Demonstrating the effects of ATP, ADP, and AMP on the luminescence of Renilla extracts also necessitated purification of the extracts (7). It is impossible to say just how many other systems, of the many tested with crude extracts $(14,27,30)$, will reveal a luciferin-luciferase reaction or a function of ATP in light emission, until suitably purified preparations become available. It is reasonable to expect that new types of organic cofactors, in addition to adenosine phosphates and flavin nucleoticles, will be ultimately discovered, and the word "luciferin" will take on a definite meaning, such as the substance or complex that actually emits, in the systems that are still biochemically obscure.

At the moment three general types of luminescent systems, illustrated by those cliscussed above, can be recognized, namely, (i) the simple enzyme-substrate system, as in the crustacean Cypridina, the protozoan Gomyaulax (31), and possibly the fungi Collybia and Armillaria (1), even though the last of these three types appears to require DPNH; (ii) systems that require in addition to a luciferin and luciferase, one or another of the adenosine phosphates, as in the firefly (35) and the seat pansy Renilla (7) ; and (iii) systems that involve an enzyme plus a flavin and an aldehycle, as in luminous bacteria $(39,43,44)$. The easily auto-oxidizable flavin system needs a continual source of hydrogen transfer from a more reduced compound, such as DPNH, for sustained luminescence. The role of DPNH in fungal luminescence is perhaps somewhat analogous.

Despite differences in detail, the fundamental types of luminescent systems among different types of organisms may ultimately turn out to be relatively few. Suggestive evidence in favor of this view re- 
sides in the recent discovery of a luminescent cross-reaction between the luciferin and lucilerase of a fish, Apogon, and the crustacean Cypridina $(16,34)$. In this instance, the cross-reaction has been studied both quantitatively (Fig. 2) and with purified preparations of each system. The absorption spectra of chromatographically puri-

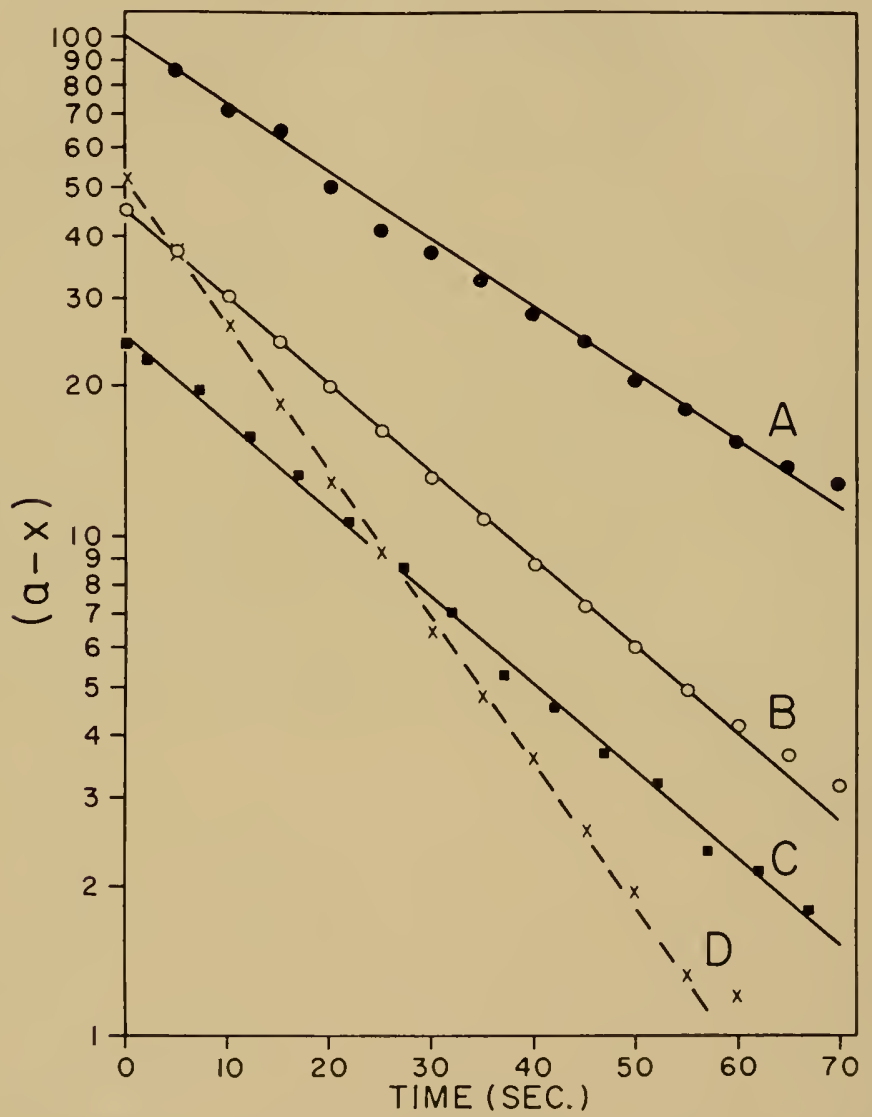

fig 2. 1.uninescent crosi-teaction of partially purified, highly diluted luciferase of

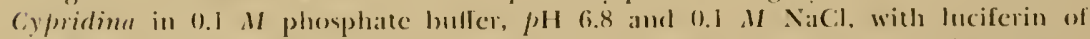
llic: posterior photogenic organs of d/ogon obtaincal by boiling the dried organs momentarily in distilled water and then cooling rapidly. The data are plotted in the manner of a reation that is first order with respect to luciferin concentration, "reptescuting total light in alstutary units at the completion of the reaction. and $x$ representing the amomt of light emitted up to time t. Concentration of Inciferase nsed for 1) was double that which was used for $\triangle$. B, and C. Initial luciferin concentrations were in the proportion of 1 (curve 1 ), 0.5 (curves $B$ and IJ), and 0.25 (curse $($ ). In abstract based in part on these data has been published (16i). 
fied luciferin of Cypridina and Apogon have points in common but are not identical. It stands to reason that the lucilerases of two such widely different types of organisms are not identical, though their activity towards the substrate of the other organism is indicative of some basic similarity. Other propertics of the Apogon system have been studied, and the details will be published in due course.

Within the past few years important advances in the biochemistry of luminescent systems have been achieved. Y'ct the actual number of different systems about which any detailed biochemical information now exists is still only a small fraction of the multitude known to biology. As a fascinating, intellectually challenging ficld of research, bioluminescence offers vast possibilities.

\section{REFERENCES}

I. Jirlh, R. L., and McElroy, W. 1)., J. Bacteriol., 77, 249-250 (1959).

2. Airth, R. L., Rhodes, W. C., and McElroy, W. D., Biochim. et Biopliys. Acta, 27, 519-532 (19:38).

3. Inderson, R. S., J. Cellular Comp. Physiol., 8, 261-276 (1936).

4. Bitler, B., and McElroy, W. D., Arch. Biochem. and Biophys., 72, 358-368 (1957).

5. Bowden, B. J., Biol. Bull., 99, 373-380 (1950).

6. van der Burg, A., Spektrale onderzoekingen over chemo- en bioluminescentie. Thesis, Unechl (1913).

7. Cormier, M. J., J. Am. Chem. Soc., 81, 2592 (1959).

8. Cormier, M. J., and Toller, I. R., Biochim. et Biophys. Acta, 25, 229-237 $(1957)$.

9. Dubois, R., Compt. rend. soc. biol., 37, 559-562 (1885).

10. Dubois, R., Compt. rend. soc. biol., 39, 564-566 (1887).

11. Dubois, R., La Vie et la Lumière, Librairie Félix Mlcan, Paris (1914).

12. Green, $\Lambda$., and McElroy, W. D., Biochim. et Biophys. Acta, 20, 170-176 (1956).

13. Haneda, Y., in The Luminescence of Biological Systems, (F. H. Johnson, ed.), pp. 335-385, American Association for the Advancement of Science, Washington, D. C. (1955).

14. Haneda, Y., and Harrey, E. N., Arch. Biochem. and Biophys., 48, 237-238 (1954).

15. Haneda, Y., and Johnson, F. H., Proc. Natl. Acad. Sci. U. S., 44, 127-129 (1958).

16. Haneda, Y., Johnson, F. H., and Sie, H.-C., Biol. Bull., 115, 336 (1958).

17. Harvey, E. N., Biochem. Bull., 2, 456-457 (1913).

18. Harrey, E. N., Science, 44, 208-209 (1916).

19. Harrey, E. N., Am. J. Physiol., 42, 318-324 (1917).

20. Harvey, E. N., Am. I. Plysiol., 42, 342-348 (1917).

21. Harvey, E. N., The Nature of Animal Light, Lippincolt, Philadelphia (1920).

22. Harvey, E. N., I. Cien. Physiol., 4, 285-295 (1921-22).

23. Harvey, E. N., Am. J. Plisiol., 70, 619-623 (1924).

24. Harvey, E. N., Biol. Bull., 51, 89.97 (1926).

2.). Harrey, E. N., Zoologica, 12, 71-it (1931).

26. Harvey, E. N.. Living I.ight, l'rinceton Unis. P'ress (1940).

27. Harvey, E. N., Biolmminescenrr. Icarlemic Press, Now York (1952).

28. Harves, l.. V., Federation Proc., 12, $597-606$ (1953). 
29. Harrey, E. N., A History of Luminescence, American Philosophical Socicty, Philadelphia (1957).

30. Harvey, E. N., and Haneda, Y., Arch. Biochem. and Biophys., 35, 470-471 (1952).

31. Hastings, J. W., and Sweeney, B. M., J. Cellular Comp. Physiol., 49, 209-225 $(195 \overline{7})$.

32. Hirata, Y., Shimomura, O., and Eguchi, S., Tetrahedron Letiers, No. 5, 4-9 (1959).

39. Jolinson, F. H., J. Cellular Comp. Physiol., 53, 259-278 (1959).

34. Johnson, F. H., Haneda, Y., and Sie, H.-C., Science, 132, 422-423 (1960).

35. McElroy, W. D., Proc. Natl. Acad. Sci. U. S.. 33, 342-315 (1947).

36. McElroy, W. D., and Green, A., Arch. Biochem. and Biophys., 56. 240-255 (1955).

37. McElroy, W. D., and Harvey, E. N., J. Cellular Comp. Physiol., 37, 83-90 (1951).

38. McElroy, W. D., and Hastings, J. W., in The Luminescence of Biological Systems (F. H. Johnson, ed.), pp. 161-198, A.A.A.S., Washington, D. C. (1955).

39. Micklroy, W. D., Hastings, J. W., Sonnenfield, D., and Coulombre, J., Science, 118, 385-386 (1953).

40. Rogers, P., and McElroy, W. D., Proc. Natl. Acad. Sci. L'. S., 41, 67-70) (1955).

41. Seliger, H. H., and McElroy, W. D., Aich. Biochem. and Biophys. (in press).

42. Shimomura, O., Goto, '. ., and Hirata, Y., Bull. Chem. Sor. Japan, 30, 929-933 (1957).

43. Strehler, B. L., J. Am. Chem. Soc., 75, 1264 (1953).

44. Strehler, B. L., in The Luminescence of Biological Systems (F. H. Johnson, ed.), pp. 209-25.5, 1.1.1.S., Washington, D. C. (1955).

45. Strehler, B. L., and Johnson, F. H., Proc. Natl. Acad. Sci. U. S., 40, 606-617 (1954).

46. Terpstra, W., Biochim. et Biophys. Acta, 28, 159-168 (1958).

47. Totter, J. R., anri Commier, NI. J., J. Biol. Chem. 216, $801-811$ (1955).

18. Tsuji, F. I., Arch. Biochem. and Biophys., 59. 452-46t (1955). 


\title{
MECHANISMS OF BIOLUMINESCENT REACTIONS
}

\author{
W. D. McElroy and H. H. Seliger \\ Department of Biology and McCollum-Pratt Institute \\ Johns Hopkins University, Baltimore, Maryland
}

\section{INTROIUCTION}

Bioluminescence is an enzyme-catalyzed chemiluminescence in which oxygen acts as an electron acceptor. The enzyme effectively channels the oxidative events so that light is emitted with a high efficiency as compared with ordinary chemiluminescence (8). So far as we can determine, the requirements for chemiluminescence and bioluminescence are an ionizable substrate which can form a peroxide addition, molecular oxygen, and presumably a fluorescent product molecule. These requirements appear to be fulfilled for both the firefly and the bacterial systems. In the examination of these light-emitting processes we have been concerned with the isolation and purification of the components of the reactions, the identification of end-products and possible intermediates, and the mechanism of the conversion of the chemical energy in to light energy.

In chemiluminescence in aqueous media, hydrogen peroxide or the formation of a peroxide appears to be a necessary factor for the production of light. On this basis a number of workers have suggested that an organic peroxide radical is the important intermediate in bioluminescence, particularly since oxygen is essential for light emission (19).

The enzyme molecule must therefore be able to bind the substrate molecule in such a manner that a catalyzed peroxide addition can occur at the proper ionizable site, and further must be able to protect the excited state from non-luminescent quenching interactions. In this respect the configuration of the substrate on the enzyme molecule appears to be extremely important for intramolecular energy transfer which will determine the efficiency of light emission, and in some cases, the color.

In the present paper we shall summarize the data available on the chemical and physical properties of the firefly and the bacterial bio= 
luminescence systems. Mechanisms are proposed for the light-producing reactions, but must be strongly qualified as being only tentative because of our lack of detailed knowledge of the exact mechanism of the more general case of chemiluminescence.

\section{FIREFLY LUMINESCENCE}

The factors required for light production in extracts from fireflies have now been prepared in a highly purified crystalline state $(2,7)$. The crystallization of firefly luciferase and luciferin has allowed for the first time an extensive quantitative study of the interaction of these substances during the process of light emission. Luciferase, purified by repeated crystallization, is homogeneous as judged by electrophoresis and ultracentrifugation, both run at various $p \mathrm{H}$ values. This method of purification, which eliminates the contaminating enzyme pyrophosphatase, is an important factor in the interpretation of the kinetics of the reaction. The isoelectric point of luciferase is between $p \mathrm{H} 6.2$ and 6.3 , and the sedimentation constant at $25^{\circ} \mathrm{C}$ is 5.6, a value consistent with a molecular weight of about 100,000 (7). The properties of purified luciferin will be described later. However, the evidence available suggests that it has the empirical formula $\mathrm{C}_{13} \mathrm{H}_{12} \mathrm{~N}_{2} \mathrm{~S}_{2} \mathrm{O}_{3}$ and, as the following addendum describes, it is probably a benzthiazole derivative (2). Additional evidence indicates that a carboxyl group of luciferin reacts with adenosine triphosphate (ATP) to form luciferyl adenylate and inorganic pyrophosphate $(14,12)$. Magnesimm ion is required for this activation step. As indicated in Fig. 1, the luciferase-catalyzed reaction

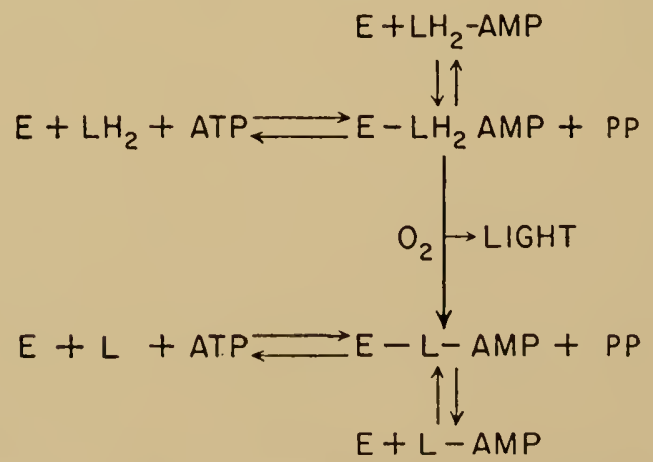

Fig. 1. Summany of the reaclions concerned with light emission in firefly extracts. $\mathrm{LH}_{2}$, luciferin; ATP, adenosine triphosplote; PI, pyrophosphate; E, lnciferase; L, oxyluciferin; LH.-AMP and L-AMIP, luciferyl and oxyluciferyl adenylic acid, respectively. 
between luciferin and adenosine triphosphate leads to the formation of an intermediate enzyme-lucileryl-alenylate complex, E- $\mathrm{LH}_{2}-\mathrm{AMP}$, and pyrophosphate (PP). Light is produced when, in the presence of oxygen, E-LH.-AMP is oxidized to the enzync-oxyluciferyl-adenylic acid complex (E-L-AMP) (20, 21). This last-11aned compound can also be produced enzymatically by the reaction of ATP with oxyluciferin. We shall review the experinental evidence for these various reactions and describe some of the effects of coenzyme $A$ and pyrophosphate on the light reaction, prior to discussing the mechanism of light emission.

\section{The Production of Light from ATP and $\mathrm{LH}_{2}$}

In the study of the kinetics of the light reaction the reactants are delivered to a buffer solution in a $10 \mathrm{~mm} \times 75 \mathrm{~mm}$ test-tube with a fixed geometry relative to a phototube. The reaction is then initiated by injecting with a hypodermic syringe quickly, and in a reproducible manner, approximately $0.1 \mathrm{ml}$ of an ATP solution. The light intensity observed as a function of time is shown in Fig. 2 by the solid circles. There is at first a rapid rise in light intensity which is followed by a relatively rapid decrease for the first few seconds and then a much slower decay that may last for several minutes or even hours. This typical flash height curve can be separated into three parts. The initial rise is due to the finite mixing time of the reactants, as can be shown by separate fluorescence measurements in which a fluorescent solution is injected into a tube under similar geonetrical conditions. The rapid decrease is caused by the initial inhibition of the enzyme because of the rapid formation of E-L-AMP complexes. Thus the flash-height peak, which is the resultant of the above two effects, is strongly dependent upon the method of injection. However, with sufficient practice in injection procedure this flash-height peak is directly proportional to the reaction rate and therefore is valid for Michaelis-Menten analysis. It is possible to separate the clecay portion into two exponential decays. For the concentrations of reactants used for Fig. 2 the initial decay has a half-value time of 0.25 seconds and the slow decay has a half-value time of 13 seconds. Extrapolation of the first-order plot of the initial decay to zero time suggests that the initial flash ideally should be more than twice that oloserved. The slower decay has been shown to be due in part to the fact that the production of pyrophosphate in the initial flash is sufficient to partially reverse the enzyme inhibition due to the product E-L-AMP. 


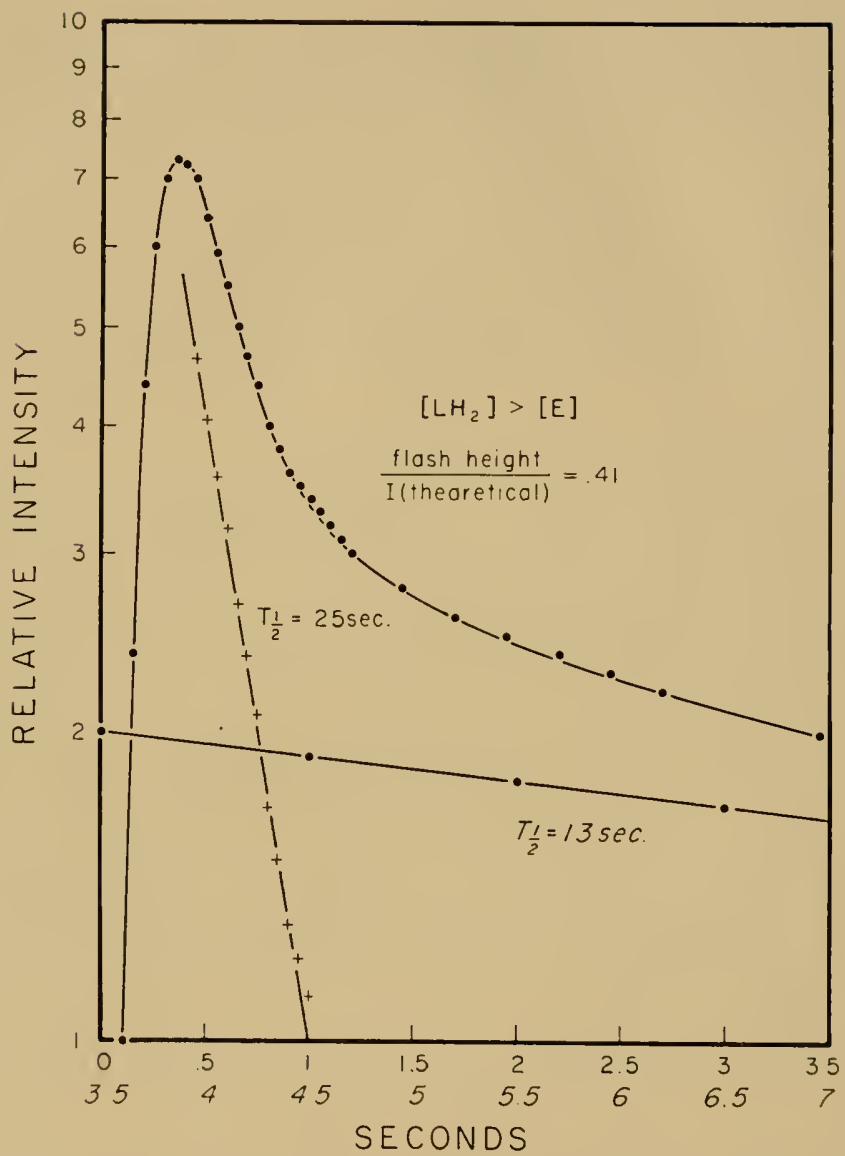

Fig 2. Light emission response to ATP. The initial rapid decay with a halftime of 0.25 seconds represents product inhibition. The slower decay after the first few seconds, with a half-life of 13 seconds, represents a partial reversal of the inhibition by pyrophosphate and ATP. See texı for details.

The importance of pyrophosphate in maintaining this stustained but inhibited level of luminescence was first observed during the carly purification of luciferase (II). The evidence indicated that in the presence of inorganic pyrophosphatase the light response to ATP decreased in a few seconds to a very low level (Fig. 3). An extensive series of investigations has shown that the nature of this response definitely depends upon the quantity of inorganic pyrophosphatase present in the luciferase preparation $(15,16)$. If highly purified inorganic pyrophosphatase is added to the luciferin-luciferase 


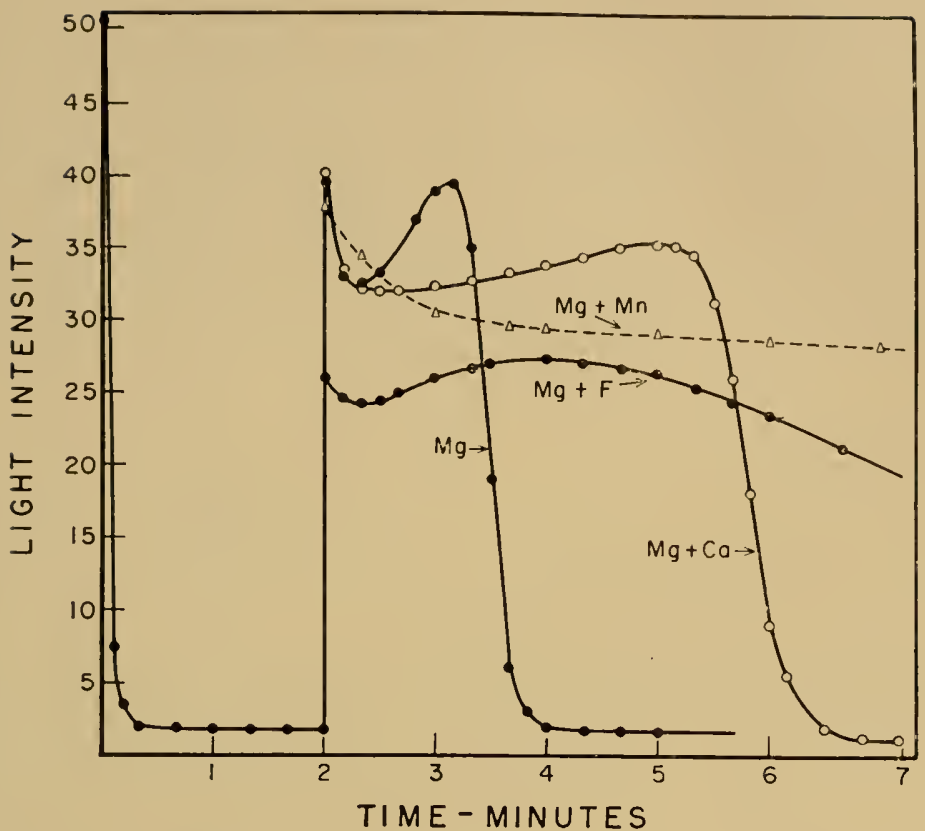

Fig. 3. Effect of inorganic pyrophosphatase and pyrophosphate on light emission. The initial rapid decline of light intensity after the addition of ATP is due to product inllibition and the remoral of pyrophosphate which favors the removal of L-AMP from the enzyme, as described in Fig. 1. The addition of pyrophosphate at two minutes stimulates light emission until it is removed by hydrolysis. Inorganic pyrophosphatase is present to hydrolyze the pyrophosphate produced in the activation reaction.

reaction, the light intensity curve seen in Fig. 2 is altered to give the initial response indicated in Fig. 3. After the initial flash has decreased to the low level hminescence a secondary addition of pyrophosphate results in a stimulation of light emission. The luminescence remains at this high level until all of the pyrophosphate has been hydrolyzed. Ions such as fluoride, which are known to inhibit pyrophosphatase activity, greatly influence the response to added pyrophosphate.

It is significant that pyrophosphate, even in low concentration, is a potent inhibitor of the liminescent reaction if added prior to the ATP. Apparently it can compete with the latter to form an inactive intermediate with the luciferin-luciferase system. A large amount of data, some of which will be presented later, indicates quite clearly that the oxidized lucileryl-adenylic acid derivative is tightly complexed to the enzyme and effectively removes the latter from catalytic activity. Low concentrations of pyrophosphate react with the enzyme- 
oxyluciferyl-adenylic acid complex to give free enzyme, АTP, and free oxyluciferin. The free enzyme in the presence of ATP is now available to catalyze the oxidation of a second luciferin molecule. We believe that the stimulation by pyrophosphate in the manner discussed above is the mechanism of control of the firefly flash $(9,15)$. We have discussed previously the existence of such inactive complexes within enzyme-catalyzed reactions and have considered their importance as controlling mechanisms not only in the firefly flash but in other enzyme-catalyzed processes (12).

Response to $\mathrm{LH}_{2}-A M P$

Probably the best eviclence for the proposed scheme in Fig. 1 is the demonstration that $\mathrm{LH}_{2}$-AMP, formed chemically by the condensation of $L_{2}$ and $A M P$, can act as an active substrate in the presence of the enzyme and without ATP or magnesium ion to produce light. Starting with $\mathrm{LH}_{2}$-AMP and crystalline luciferase in solution, the only other requirement for light emission is oxygen. Free oxyluciferin has no inhibitory effect on the rate of light emission. However, ATP gives a slight inhibition of the rate of light emission which becomes much more pronounced in the presence of magnesium ion.

The product of the enzymatic light reaction initiated with $\mathrm{LH}_{2}$ AMP has been identified as the oxyluciferyl-adenylate derivative. The inhibitory effect of oxyluciferyl-aclenylate on the flash height is even more pronounced in the absence of ATP and free $\mathrm{LH}_{2}$ when starting with $\mathrm{LH}_{2}-\mathrm{AMP}$ as the reactant. This is shown in Fig. 4, where light production from $\mathrm{LH}_{2}$-AMP is compared to that initiated with ATP and an equal quantity of luciferin. The continuous production of light from $\mathrm{ATP}$ and luciferin suggests that either ATP or luciferin, or both, are capable of removing the L-AMP inhibitor from the enzyme surface. This is not the case with $L_{2}-A M P$. The fact that the secondary addition of luciferase to the reaction mixture showed a burst of light indicates that excess $\mathrm{LH}_{2}$-AMP was present. The production of pyrophosphate in the presence of ATP and $\mathrm{LH}_{2}$ because of the initial activating reaction is undoubtedly important in sustaining a low but continuous light emission. As the results in Fig. 5 indicate, the addition of pyrophosphate along with $\mathrm{LH}_{2}$ - $\mathrm{M}$ MP to the lucilerase solution decreases the initial rate. However, the total light production has been greatly increased due to the release of enzyme from the E-L-AMP inhibited complex. It should be noted that if both magnesium ions and pyrophosphate are added, there is 


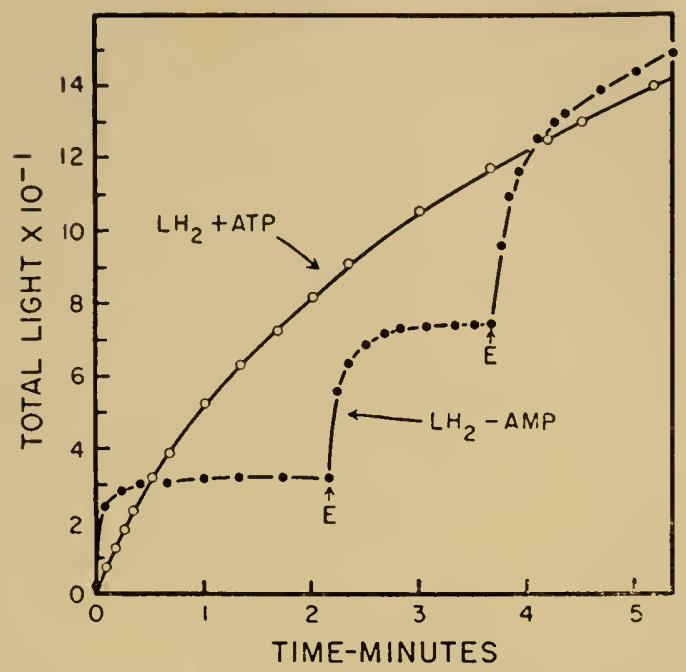

Fig. 4. Light from $\mathrm{LH}_{2}-\mathrm{AMP}$ compared with light from luciferin and ATP. $\mathbf{L H}_{2}-\mathrm{A} . \mathrm{MP}$ is in excess of enzyme. Note extreme product inhibition. Second addition of enzyme is indicated by arrows.

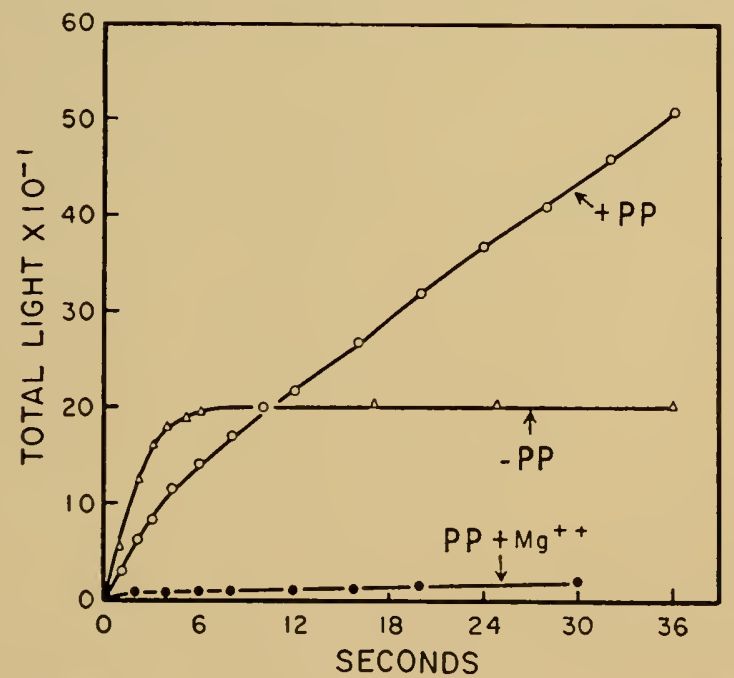

Fig. 5. Effect of pyrophosphate on light emjssion from $\mathrm{LH}_{2}-\mathrm{AMP}$.

no appreciable light production; magnesium alone has no effect. These results can be interpreted in terms of the reaction of pyrophosphate with E- $\mathrm{LH}_{2}$-AMP and E-L-AMP to produce free luciferin and free oxyluciferin respectively, and ATP; that is, the reversal of the 
activating reactions (see Fig. I). Competition for E- $\mathrm{LH}_{2}-\mathrm{AMP}$ by pyrophosphate and by oxygen in the light-producing reaction results in a decrease in the initial rate. Howerer, the reaction of pyrophosphate with E-L-AMP removes the inhibitor from the enzyme surface; and the result is an increase in the turnover of the enzyme and a consequent increase in total light production. Magnesium ion increases the rate of the reaction between PP and E- $\mathrm{LH}_{2}-\mathrm{AMP}$ and thereby affords much greater competition with the light reaction (20, 21).

The reversal of these two activating reactions by pyrophosphate has actually been shown by demonstrating a net synthesis of ATP as well as the incorporation of P $^{32}$-labeled pyrophosphate into ATP. That L-AMP is the inhibitor of luciferase is clearly shown when chenically synthesized L-AMP is added to a light-producing reaction, which is initiated with either $\mathrm{LH}_{2}$ and ATP or $\mathrm{LH}_{2}$-AMP. Because of the very tight binding of the oxyluciferyl adenylate to the enzyme, equimolar amounts of L-AMP and enzyme essentially produce no light

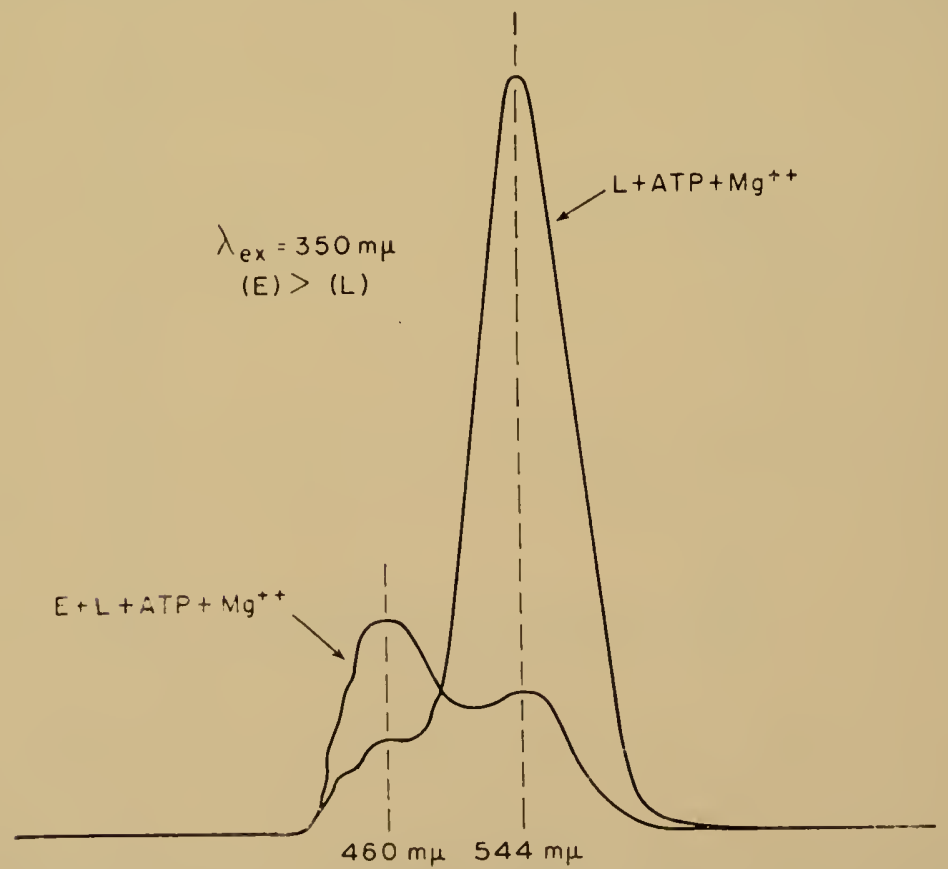

Fig. 6. Fuorescence of L, and E-L-. IMI'. Relative fluorescence is plotted along the ordinate and the watelength for emission along the abcissa. When exress enzyme is ardeded, llac intense fluorescence at $5.11 \mathrm{~m} \mu$ decreases rapidly. 
in the presence of $\mathrm{XTP}$ and luciferin. It is of interest in this connection that the chemically synthesized inosinic acid derivative of oxylucilerin. L-IMP, is also an extremely potent inhibitor, while the IMP derivative of $\mathrm{LH}_{2}$ is not effective for light production (21).

\section{Fluorescence of Oxyluciferin and Oxyluciferyl-Adenylate}

One of the interesting changes in the properties of oxylucilerin in the enzymatic reaction with ATP is the tremendous decrease in the intensity of fluorescence. Normally the fluorescence of oxyluciferin gives a strong emission peak at 514 millimicrons (Fig. 6). However, when ATP and enzyme are added to such a reaction mixture the fluorescence intensity of E-L-AMP at this wave length decreases dramatically. Other fluorescence properties of L-AMP will be discussed later. Knowing these fluorescence properties of L-AMP, it has been possible to study a number of interesting reactions of the enzymeL-AMP complex (1). For example, an enzyme has been found in the firefly which will catalyze the hydrolysis of both $\mathrm{L}-\mathrm{AMP}$ and $\mathrm{LH}_{2}$ AMP. We have followed the hydrolysis of the L-AMP by observing the increase in the fluorescence upon the formation of the free oxyluciferin. This hydrolytic enzyme will not attack L-AMP when it is tightly bound to the luciferase. However, when the hydrolase is present in the solution of luciferase-L-AMP there is a very slow but definite recovery of enzymatic activity. This can be explained in terms of the equilibrium between the enzyme-L-AMP complex and free enzyme and free L-AMP.

Another interesting aspect of the enzyme-L-AMP complex has been studied by using fluorescence as an indicator of free oxyluciferin. A light-producing reaction mixture containing luciferin, ATP, luciferase, and magnesium continues to produce pyrophosphate long after the enzyme is inhibited for light production, provided pyrophosphatase is present to pull the reaction. The same property of the system is observed if luciferin is replaced by oxyluciferin (21). The only reasonable conclusion, therefore, is that the enzyme-L-AMP complex is capable of causing the hydrolysis of ATP to adenylic acid and pyrophosphate. There are several possible mechanisms for the interaction between ATP and the E-L-AMP complex. Luciferase-LAMP could act as a unit to catalyze the hydrolysis of ATP. If this were true, the AMP moiety of L-AMP would not be replaced by ATP. Another possibility is that ATP in some way causes the hydrolysis of luciferase-bound L-AMP, after which oxyluciferin reacts with ATP to release pyrophosphate. The evidence presented in Fig. 
7 suggests that the latter mechanism is more nearly correct. The results in the figure indicate that in a mixture of luciferase and L-AMP in which the latter is at a concentration greater than that of the enzyme there is a fluorescence increase upon the addition of ATP. The fluorescence increase corresponds to the hydrolysis of the amount of L-AMP which is in excess of luciferase. A similar experiment carried out with L-AMP concentration less than the enzyme indicates very little effect of ATP on fluorescence. These results can be interpreted most readily in terms of the second mechanism described above and on the basis of the high affinity of the L-AMP for the luciferase. If ATP in some way causes the labilization of the luciferasebound L-AMP, with subsequent hydrolysis, this would leave free oxyluciferin on the enzyme surface. If there is no free L-AMP in solution, the oxyluciferin molecule would react immediately with ATP to produce another molecule of E-L-AMP and pyrophosphate. However, this would not always be the case when free L-AMP is present. Because of the extremely high affinity of L-AMP for luciferase, L-AMP could readily replace oxyluciferin on the enzyme surface and subsequently be hydrolyzed. The other observation in sup-

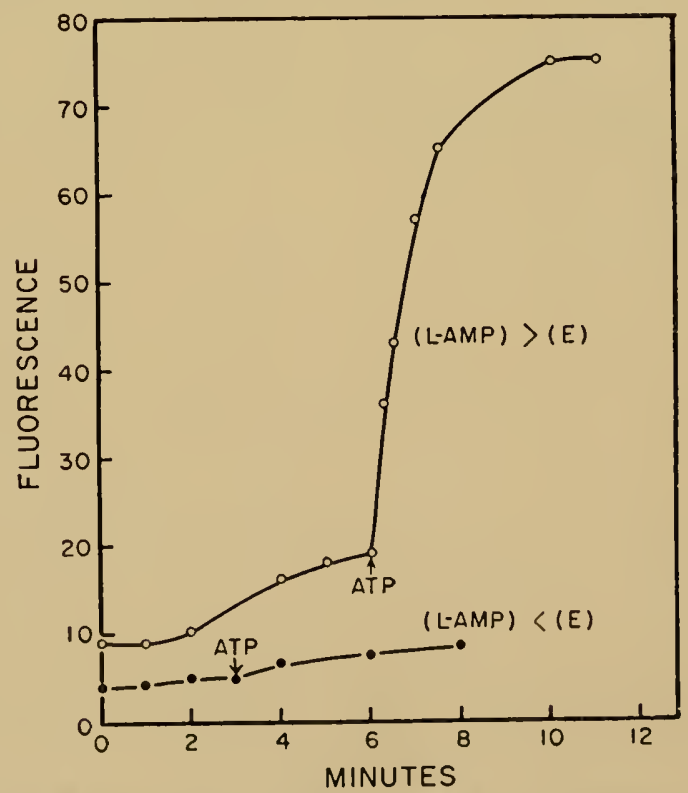

Fig. 7. Effect of ATP on the hydrolysis of $L-A M P$. Fhorescence increase is due (6) the hydrotysis of L-A.MP to $\mathrm{L}$ and AMP. Excitation at $350 \mathrm{~m} \mu$; emission measured at $510 \mathrm{~m} \mu$. Sec text. 
port of this idea was mentioned previously, namely, that when ATP and lnciferin are present in an enzyme-catalyzed reaction there is always a prolonged light production, in contrast to starting with the $\mathrm{LH}_{2}-\mathrm{A}$.MP. Thus in some way the ATP is decreasing the inhibitory effect of L-AMP and making the enzyme available to luciferin.

\section{Dissociation Comstant of E-L-AMP}

The dissociation constant of the E-L-AMP complex may be defined as follows:

$$
K=\frac{[\mathrm{E}]_{\text {free }}[\mathrm{L}-\mathrm{AMP}]_{\text {free }}}{[\mathrm{E}-\mathrm{L}-\mathrm{AMP}]}
$$

This dissociation constant has been determined by incubating mixtures of lucilerase and oxylucileryl-adenylate and determining at equilibrim the concentration of the free enzyme. The ratio of the flash height in the presence of L-AMP to the flash height in the absence of L-AMP is equal to the ratio of free enzyme to total enzyme. This ratio has been defined as $f$. Various concentrations of L-AMP

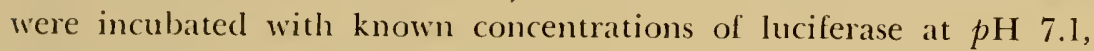
and the light intensities for each mixture were measured by the addition of equal amounts of $\mathrm{LH}_{2}$-AMP. The total luciferase was determined from protein concentration and an estimated molecular

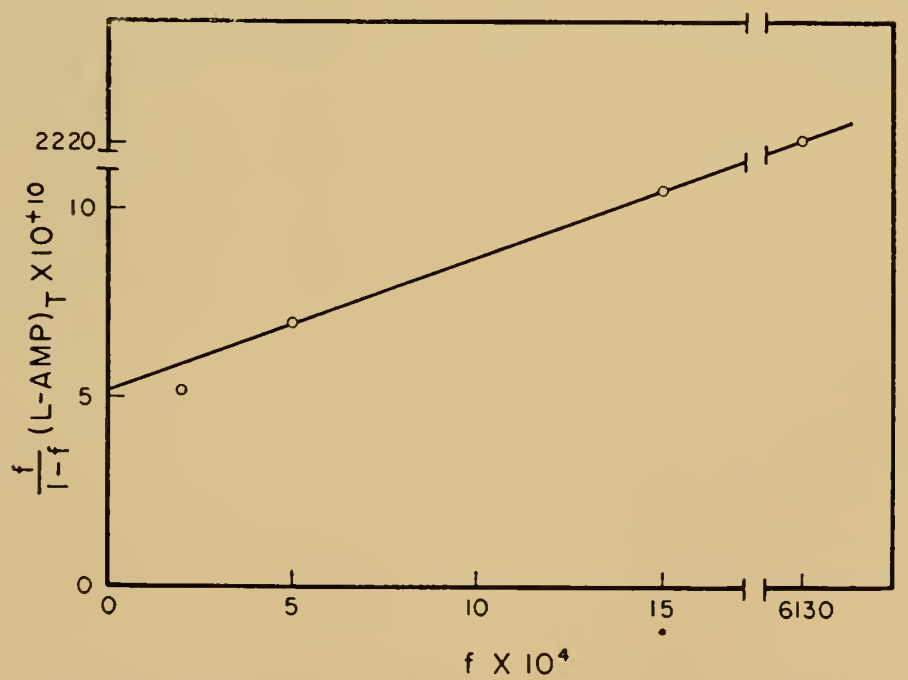

Fig. 8. Dissociation of luciferase-L-AMP. $f$ is the ratio of free enzyme to total enzyme. The slope is the equivalent concentration of total enzyme, whereas the intercept is $K$. 
weight of $10 \%$. From such experiments we conclude that $K$ is equal to approximately $5 \times 10^{-10}$ moles per liter. This relationship is shown graphically in Fig. 8. Such a plot prockuces a curve that has a constant slope of $3.6 \times 10^{-7}$ molar, a value which is in reasonable agreement with the calculated enzyme concentration of $3 \times 10^{-7} \mathrm{M}$. The fact that one obtains a straight line from such a plot suggests that luciferase has one active site for light production and that one molecule of L-AMP combines with this site. Also the value of $K$ obtained by extrapolation of $f$ to 0 (infinite L-AMP concentration) is in good agreement with the calculated $K$ values (21).

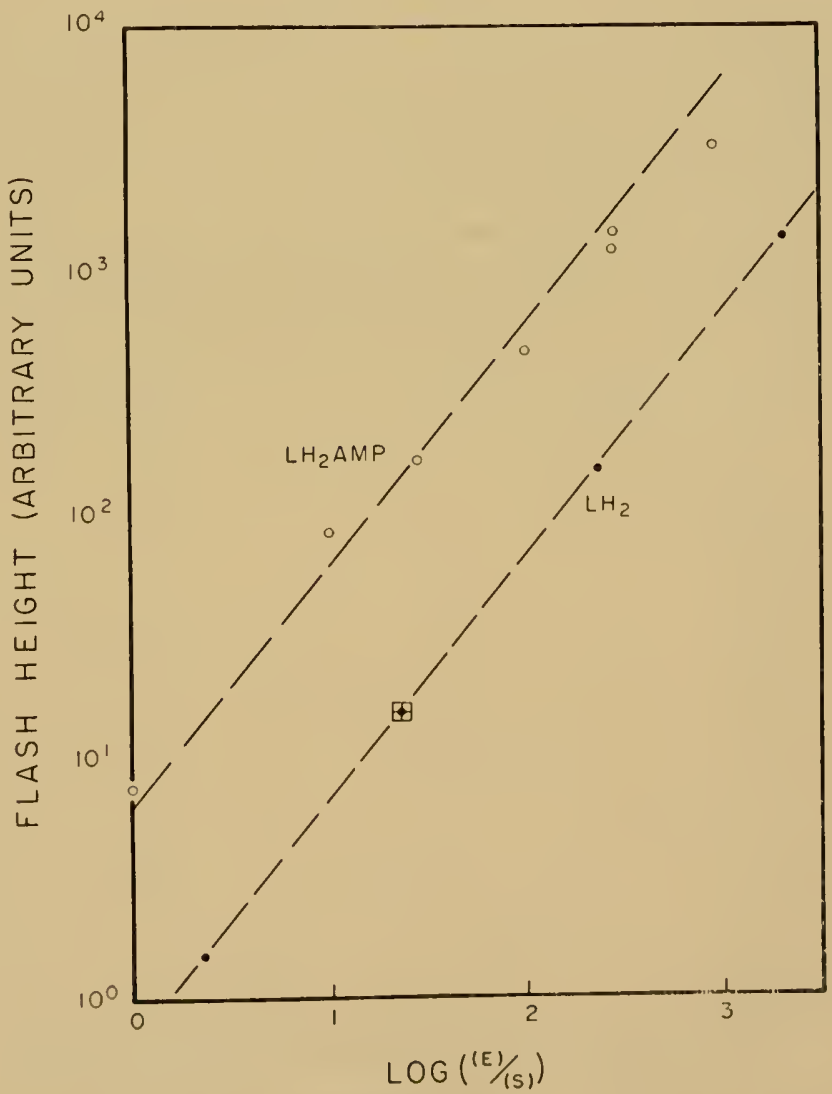

Jig. 9. Initial llash height with valying amoum of enzyme. The upper curve is the flash response due to the addition of LH. $H_{2}$. WIIP. The lower cure is the

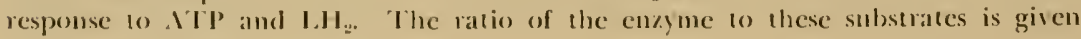
along the abcissa. 
Aelditional evidence that only one active site is involved in light production has been obtained by studying the flash height in the presence of $\mathrm{L}_{2} \mathrm{H}_{2} \mathrm{AMI}$, and $\mathrm{L}_{2} \mathrm{H}_{2}$ and $\mathrm{ATP}$ with large variations in the en/yme concentuation. The results of such an experiment are presented in Fig. 9. II more than one luciferin or $\mathrm{LH}_{\mathrm{w}} \mathrm{AM} \mathrm{MP}$ were required for light emission one might expect an inhibition of the flash height with excess enzyme. However, the evidence indicates that a 1,000-fold cxcess of en\%yme concentration relative to the substrate concentration has no effect on the flash height. We conclude from such evidence that there is one active $\mathrm{LH}_{\underline{w}-\mathrm{A}} \mathrm{MP}$ formed on the enzyme surface, and that this interacts with oxygen in some way to produce the excited state.

\section{The Reaction of Coenzyme A with Enzyme-L-AMP Complex}

When coenzyme $A$ is ackled to a reaction mixture in which $\left[\mathrm{L.H}_{2}\right]$ $>$ [E] and which has been allowed to proceed to the region of slow light decay, there is an immediate rise in the light intensity, a rise clirectly proportional to the coenzyme A concentration. After the initial rise due to the coenzyme $A$ addition the luminescence will continue at this high level of light emission and finally return to the normal slow decay level. The duration of this stimulated light emission is also directly proportional to the initial CoA concentration. If CoA is adcled initially to the reaction mixture there is no effect on the initial light intensity, but the reaction mixture containing CoA always remains at a higher value than an identical mixture without CoA (1).

CoA is very specific in its stimulatory effect. Neither dephosphoCoA, nor phosphopantothine, nor cysteine is effective in this respect. The inhibitory effect of oxyluciferin or oxyluciferyl-adenylate can be removed by the addition of CoA. A considerable amount of evidence now indicates that $\mathrm{CoA}$ stimulates light production by reacting with the E-L-AMP to produce AMP, oxyluciferyl-CoA, and free enzyme. The results diagrammed in Fig. 10 indicate that $\mathrm{L}$-CoA can be produced from oxyluciferin if ATP, lucilerase, and magnesium ion are present in the reaction mixture. Spectrophotofluorometry provides a good means of measuring the production of L.CoA since its fluorescence intensity is approximately 2 per cent of that of oxyluciferin. The reaction producing L-Cod has been shown to be enzymatically reversible by obtaining a net synthesis of $\mathrm{ATP}$ from L-Coג, AMP, and pyrophosphate; by measuring a coenzyme- $\mathrm{A}$-clependent incorporation of $\mathrm{C}^{1+}$-labeled $\mathrm{AMP}$ into $\mathrm{ATP}$; and by measuring the formation 


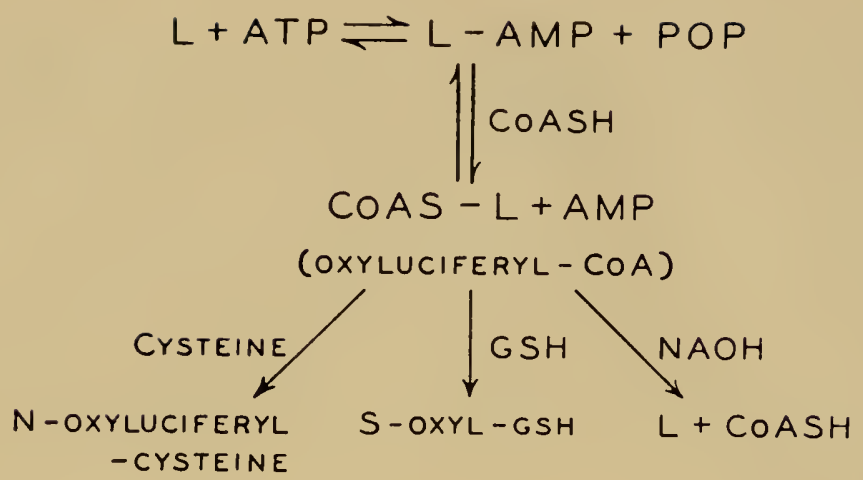

Fig. 10. The activation of oxyluciferin and the reaction of coenzyme 1 .

of oxyluciferin by fluorescent increase in a mixture of L-CoA, AMP and pyrophosphate.

The results presented in Fig. II represent a spectrophotofluorometric study of part of the reaction described in Fig. 10. In this experiment the initial rapid decrease in fluorescence is due to the formation of the CoA derivative of oxyluciferin in the presence of ATP, magnesium, and luciferase. The L-CoA derivative has been isolated and identified by using paper chromatography and enzymatic procedures. Cysteine is capable of reacting with the L-CoA to produce oxyluciferyl-cysteine. The fluorescence increase on the addition of cysteine at 12 minutes (Fig. 11) is due to the formation of the cysteine clerivative, which has a fluorescence which is only about 50 per cent less than that of free oxyluciferin. The cysteine oxyluciferin derivative has also been identified by chromatographic procedures. The enymatic activation of oxyluciferin by XTP in the presence of coenzyme $A$ leads to the formation of pyrophosphate and free adenylic acid as well as oxyluciferyl-coenzyme $A$. The results (Fig. 12) indicate that this reaction does occur. Oxyluciferyl-CoA was produced in a reaction mixture consisting of oxyluciferin, luciferase, coenzyme A, and ATP. This is shown on the graph by the initial decrease in fluorescence. Upon the addition of adenylic acid after 11 minutes there was a small increase in fluorescence. When high concentrations of pyrophospluate were added there was a large increase in Huorescence because of the formation of free oxyluciferin. ATP was also formed in this reaction and was measured by the firefly system in a separate experinent. The high concentration of pyrophosphate required to obtain an increase in fluorescence indicates that the equilibrium is in favor of oxyluciferyl-CoA formation. 


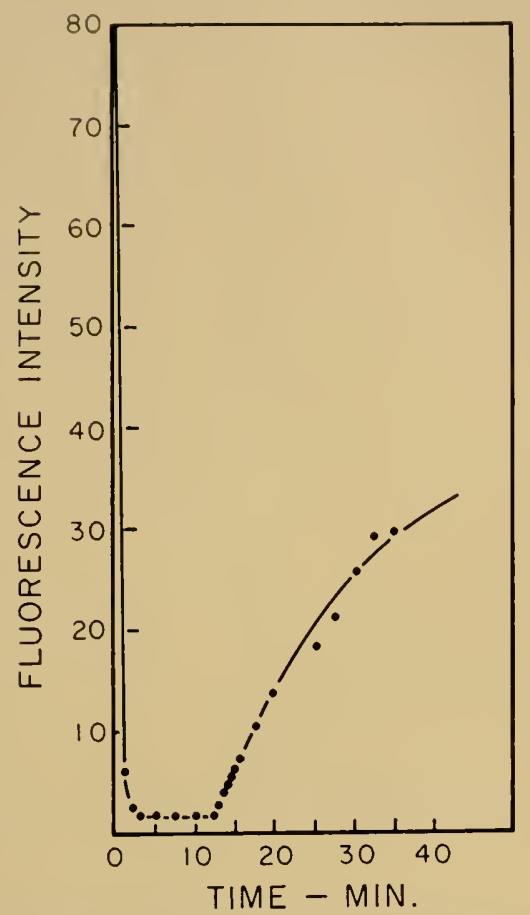

Fig. 11. Effect of cysteine on oxyluciferyl-CoA fluorescence. The formation of L-CO.A is indicated by the initial decrease in fluorescence. Cysteine was added at 12 minutes.

The evidence presented above indicates that in the firefly system, luciferase catalyzes the interaction of luciferin and ATP to form the enzyme-luciferyl-adenylate complex. The luciferyl-adenylateenzyme complex in the presence of oxygen leads to the formation of the enzyme-oxyluciferyl-adenylate complex and the emission of light. The dissociation constant for the luciferase-oxyluciferyladenylate complex indicates that the latter is very stable, thus accounting for the rapid product inhibition of luminescence. The knowledge of the dissociation constant for the luciferase-oxyluciferyladenylate complex, together with the equilibrium constant for the activation of oxyluciferin, has made it possible to calculate the standard free energy of hydrolysis of oxyluciferyl-adenylate as being -13.1 kilocalories per mole. The stimulation of luminescence by pyrophosphate can be explained by its reaction with the complex to produce free luciferase, ATP, and oxyluciferin. The luciferase then reacts again with luciferin and $A T P$ to give more light production. 


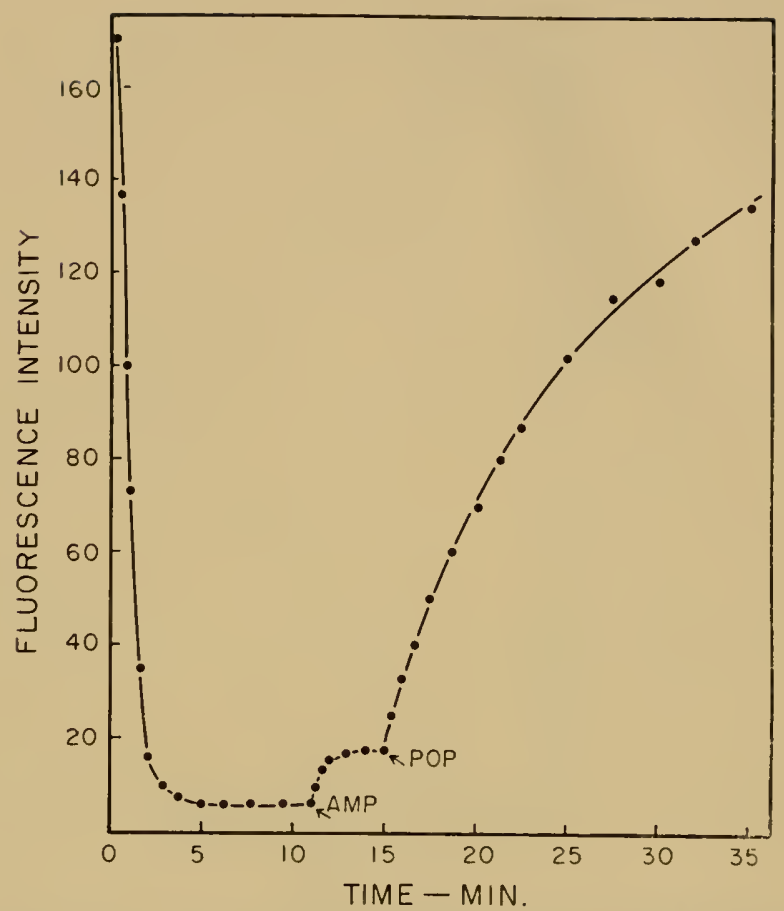

Fig. 12. Effect of aclentic acid and pyrophosphate on oxyluciferyl-CoA decomposition.

\section{Other Factors Affecting the Light Reaction}

The results shown in Fig. 13 indicate that the $p H$ optimum for the flash height in the presence of glycine or glycyl-glycine buffer is approximately 7.8 (11). In phosphate buffer the light intensity ap. pears to be greatly reduced and, as will be discussed later, the actual emission spectrum is altered. The effect of temperature on the flash height is shown in Fig. I4. The experimental energy of activation for the reaction is calculated to be approximately 18 kilocalories per mole. The decrease in the light intensity above the optimum of $25^{\circ} \mathrm{C}$ is due to the reversible inactivation of the lucilerase.

The results presented in Fig. 15 indicate that oxygen is an absolute requirement for the light-emitting reaction (10). Maximum luminescence is observed at an oxygen concentration greater than approximately $0.5 \%$ and really significant decreases in the light intensities are not observed until the oxygen concentration is less than $0.1 \%$. The results presented in Fig. 16 were obtained by de-aerating the enzyme solution prior to the mixing of the reactants. The reaction 


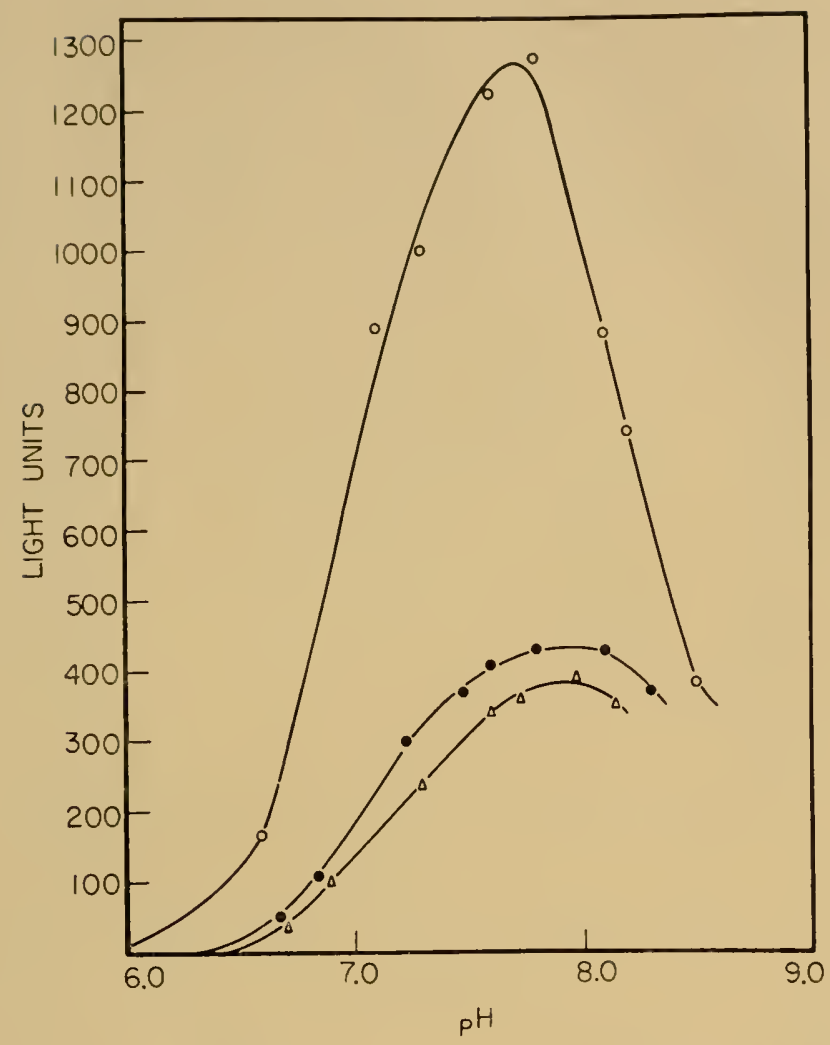

lig. 13. Effert of $p H$ aud buffers on light emission. The upper curve is for glycine buffer and the lower curves for phosphate buflers $\left(\mathrm{Na}^{+}\right.$and $\mathrm{K}^{+}$).

mixtures were degassed, and oxygen was added to the concentration indicated in the table. The particular gas mixture was bubbled continuously, and the light intensity was measured after the enzyme was adcled to start the reaction. The intensities observed at these various oxygen concentrations and at various time intervals are indicated in the figure. After the reaction had run for approxinately $21 / 2$ minutes, oxygen was added to the reaction mixture and the resulting flash was recorded. The peaks of these flashes are indicated in the table. These results are taken to indicate that oxygen plays an important role in the reaction leading to light emission. In addition, it can be demonstrated that under anacrobic conclitions some active intermediate accumulates which then rapidly reacts with oxygen when the gas is readmitted to the reaction vessel. A large amount of ex- 


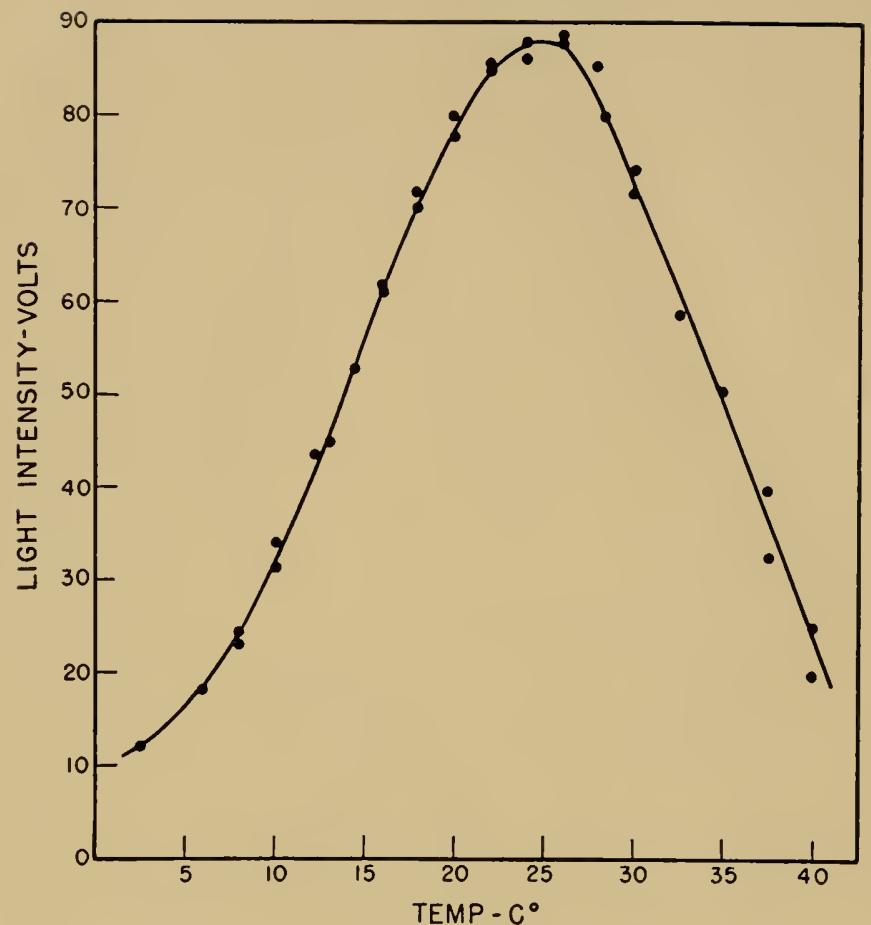

Fig. 14. Effect of temperature on light intensity.

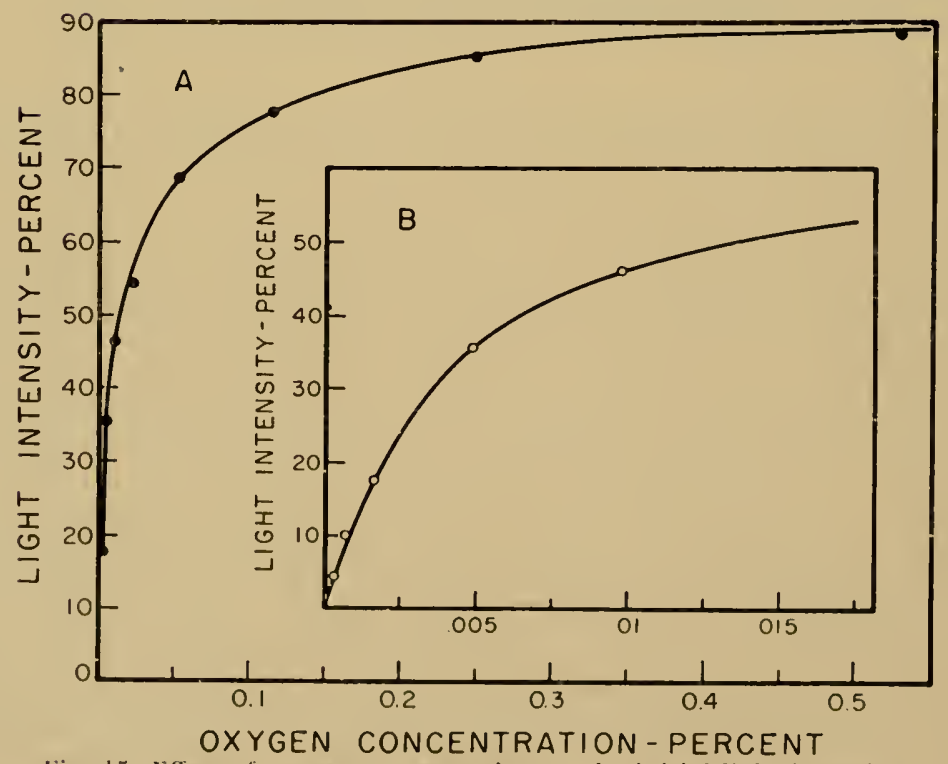

Fig. 15. Effect of oxygen concentration on the intial light intensity. 
perimental evidence indicates that the material accumulated under anacrobic conditions is the emyme-luciferyl-adenylic acid complex.

A rapid recording of the oxygen flash indicates that the shape of the curve is very similar to the pseudo-flash of the intact firefly which has been placed under anaerobic conditions. Although the oxygen flash provides a model mechanism for normal flashing, the

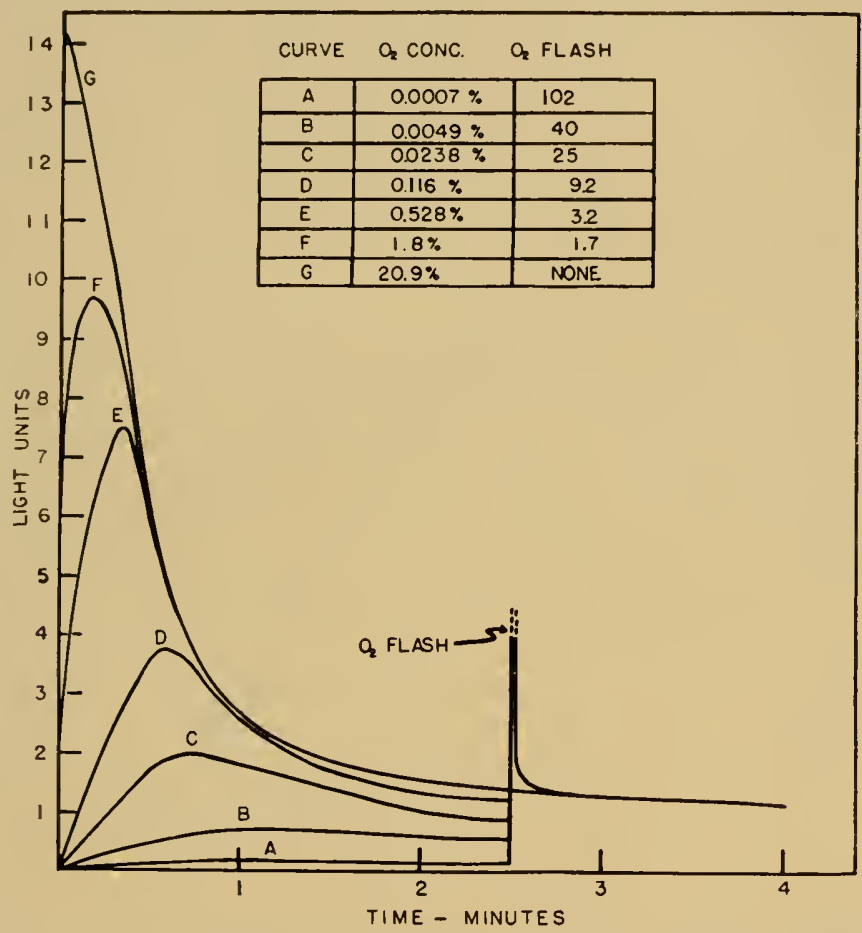

Fig. 16. The effect of oxygen concentration on the time course of luminescence. Oxygen was added to the reaclion mixture at $2 \frac{1}{2}$ minutes, and the resulting flash height was recorded as indicatert in the table.

duration of the oxygen flash is much longer than the normal firefly flash. By varying the luciferin, luciferase, magnesium, ATP, or oxygen concentration to obtain the flash indicated in Fig. 16 we have not been able to obtain one which is typical of the normal firefly flash. For this and other reasons we prefer the idea that the flash in the intact firefly is che to a reversal of the inhibition of the enzyme by pyrophosphate. 


\section{Emission Spectra}

Fig. 17 shows the true emission spectrum of the firefly light reaction in vitro in glycyl-glycine buffer at $p H 7.6$ (24). The relative spectral photon sensitivity of the phototube used is also included in the figure, and the observed spectrum was corrected for phototube sensitivity. The peak emission for the bioluminescence is 562 millimicrons, with the band ranging from 500 millimicrons to 630 millimicrons. Certainly, as an absolute minimum, the energy requirement for the bioluminescence should therefore exceed $57 \mathrm{kcal} / \mathrm{mole}$.

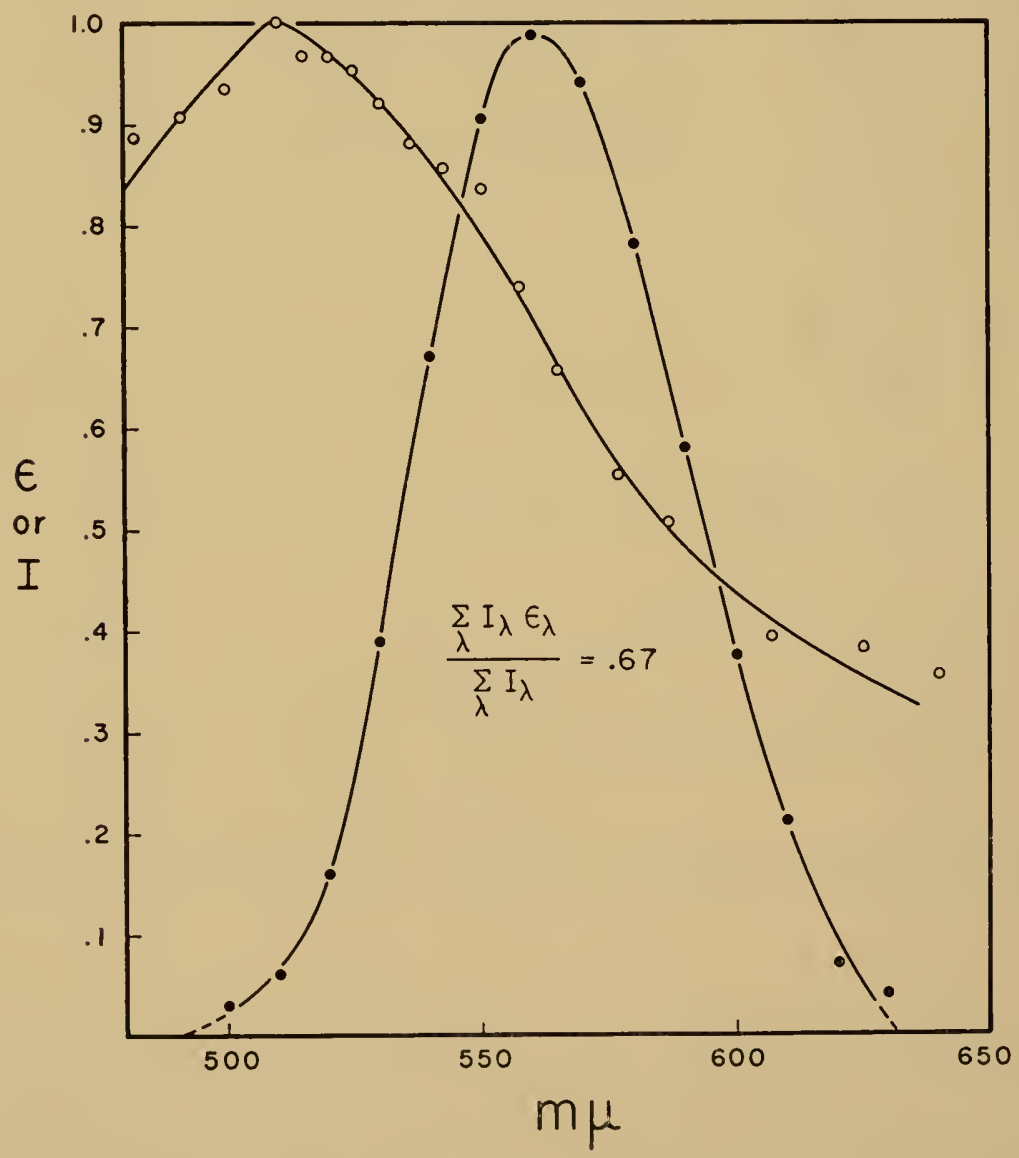

Fig. 17. Emission spectrmm of firefly light in vitro. Bioluminescence (solid cir(les) in glycyl-glycine bulfer at $p$ H 7.6. The peak and emission is at $562 \mathrm{~m} \mu$. Superimposed upon this emission spectrum is the normalized photon spectral efficiency of the photocathode of the phototube used in the quantum yield measurements. 
One might expect that some product would occur in the lightcmitting step which would have fluorescence properties similar to the bioluminescence enission and which could possibly be identified as the light-emitting species.

For this reason careful measurements were made of the absorption and emission properties of a number of luciferin and oxyluciferin derivatives. The results, presented in Fig. 18, show normalized ultraviolet absorption spectra of luciferin and oxyluciferin in acid and base. At neutral $p \mathrm{H}$ or below, Iuciferin shows an absorption peak at 327 millimicrons which corresponds to the activation peak for its fluorescence. When the $p H$ is made higher than 9, the absorption peak shifts from 327 to 381 millimicrons. The small absorption in the short ultraviolet also shifts to a longer wavelength. The molar absorbance at 327 millimicrons is proportional to concentration. The $327 / 263$ ratio is used as a criterion of purity, and for crystalline luciferin is approximately 4.5 .

The absorption spectrum of oxyluciferin under the corresponding conclitions is also shown in Fig. 18. The absorption spectrum of oxyluciferin show's the same general shape and shift with $p \mathrm{H}$ as that of luciferin. The shift of approximately 20 millimicrons to longer wavelength as we go from the dihycho form to the oxidized form might be just what would be expected from the addition of a double bond to the aromatic structure. The retention of both absorption

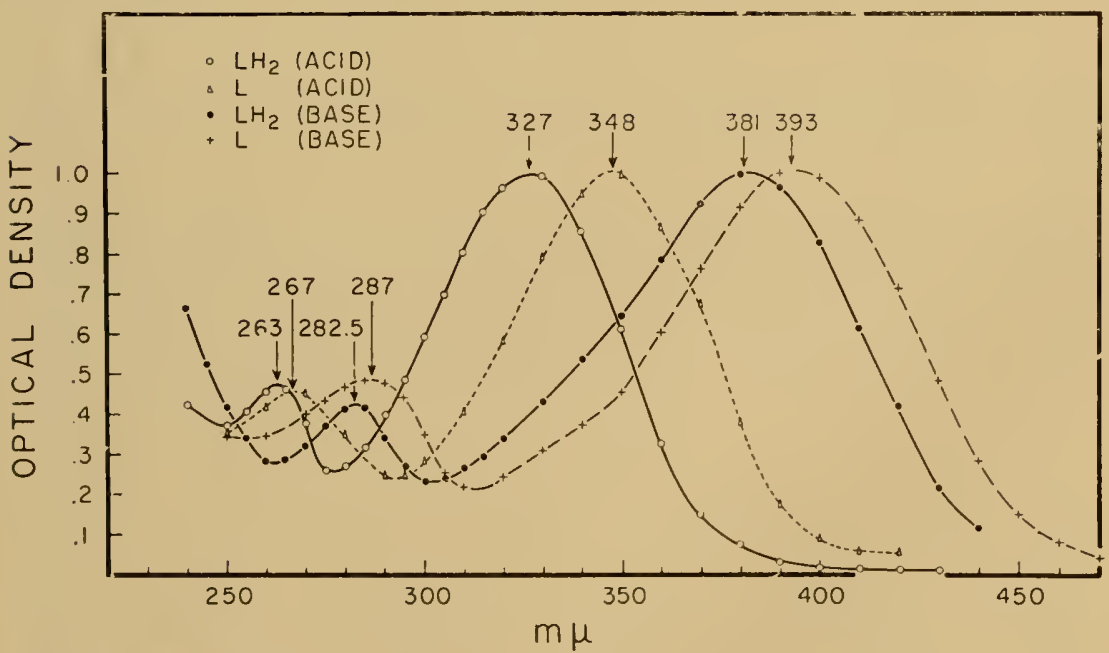

Fig. 18. Normalized absorbance spectra of luciferin and oxyluciferin. The points of crossing of the acid and base curves are also isobestic points. 
bands with the same relative absorbances would also indicate that the only difference between the reduced and oxidized forms is the loss of two protons.

The fluorescence emission spectra of luciferin and oxyluciferin are shown in Fig. 19. The fluorescence emission peak of luciferin is at 535, and for oxyluciferin, at 544 millimicrons. There appears to be a very slight shoulder of blue fluorescence for oxyluciferin at around $450-460$ millimicrons at acid $p H$. This is shown schematically

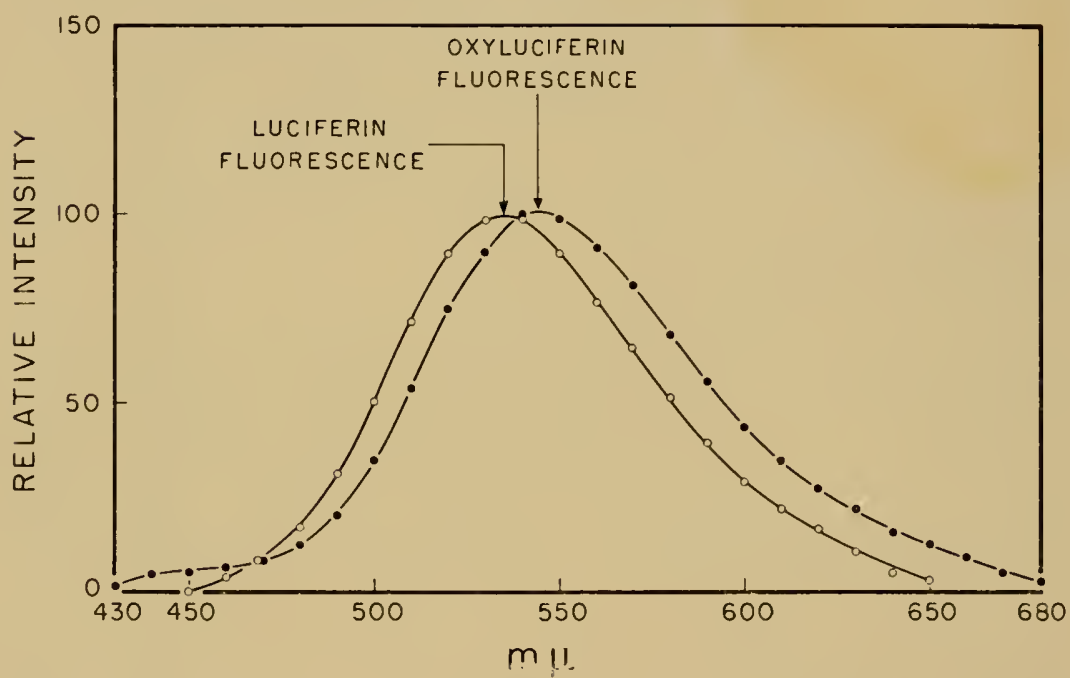

Fig. 19. Fluorescence spectra of Juciferin and oxyluciferin. The peaks ate at 535 and $34 t$ millimicrons, respectively. These data have been corrected for phototube spectral efficiency.

in Fig. 2(), in which the peak energy levels for both absorption and lluorescence are shown in analogy with an energy-level diagram for atomic spectra. At the right of the diagram the absorption and fluorescence bands are shown to inclicate the degree of overlap among them. The 460 millimicron fluorescence remains relatively constant with $p H$. The reason why it is not seen at alkaline $p H$ values is that the 5 . If millinicrom band becomes very intense and overshadows the lowintensity blue emission with the spectrophotofluorometer used for the measurements. It is of interest that although the absorption peak shifts ralically with changes in pH from acid to base, the fluorescence emission remains at 5.14 millimicrons although altering in intensity. This would indicate that a separate portion of the molecule is re- 


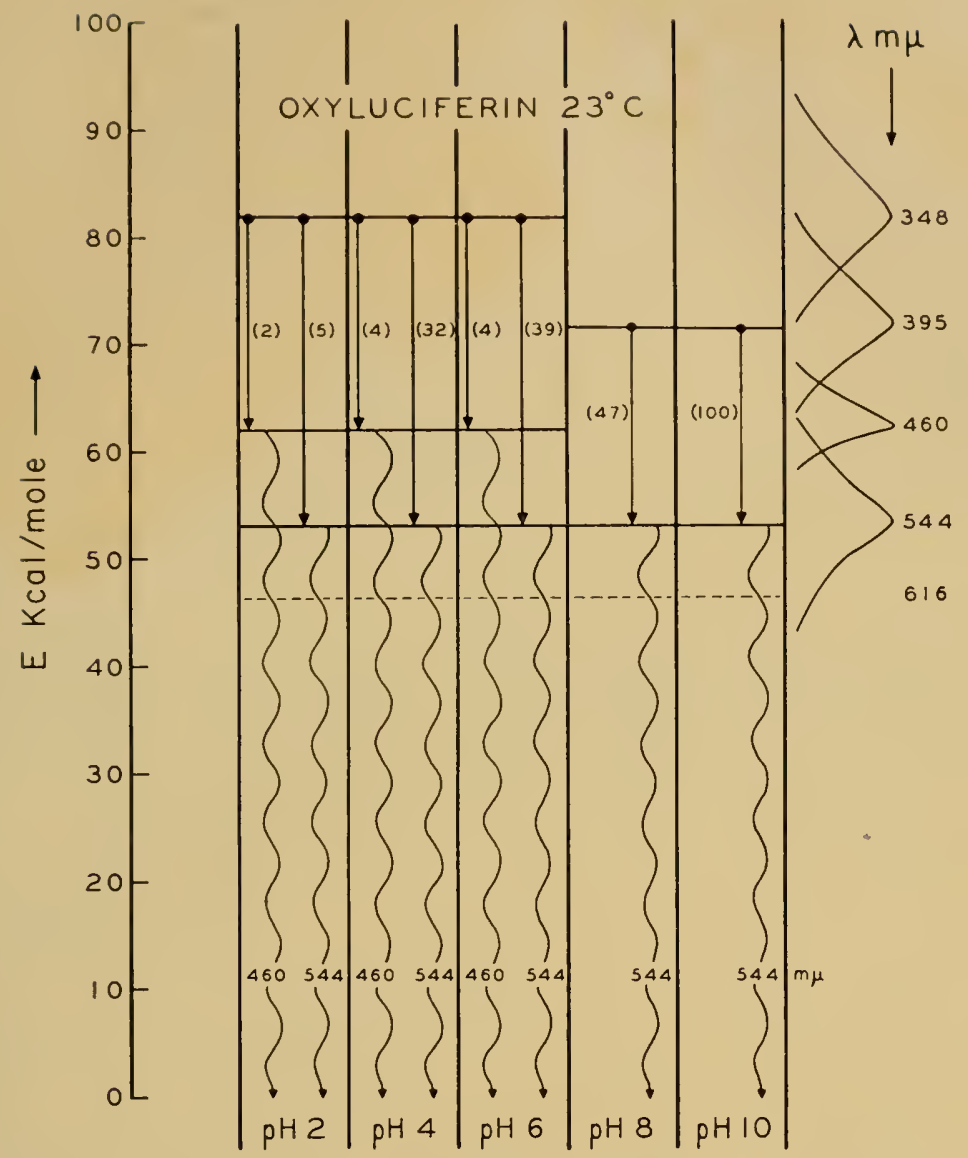

Fig. 20. Energy-level diagram of oxyluciferin absorption and fluorescence. The solid dots represent peak absorption levels, the straight arrows represent internal transitions, and the wavy arrows the emission of light whose wavelength in millimicrons is given in the break of the arrow. The energies in $\mathrm{kcal} / \mathrm{mole}$ corresponding to these absorptions and emissions are given on the left ordinate. To show the degree of overlap among the various levels, the approximate spectral distributions are plotted at the right of the figure.

sponsible for the emission as contrasted with absorption. Thus the intramolecular energy transfer is dependent upon the ionic species present, which in turn is a function of $p H$ or, in the bioluminescence case, upon the type of hydrogen-binding to the enzyme surface. This intramolecular transfer efficiency is reflected in the fluorescence yield of the luciferin and oxyluciferin molecules (Fig. 21). These data were obtained using the combination of a spectrophotofluorometer 


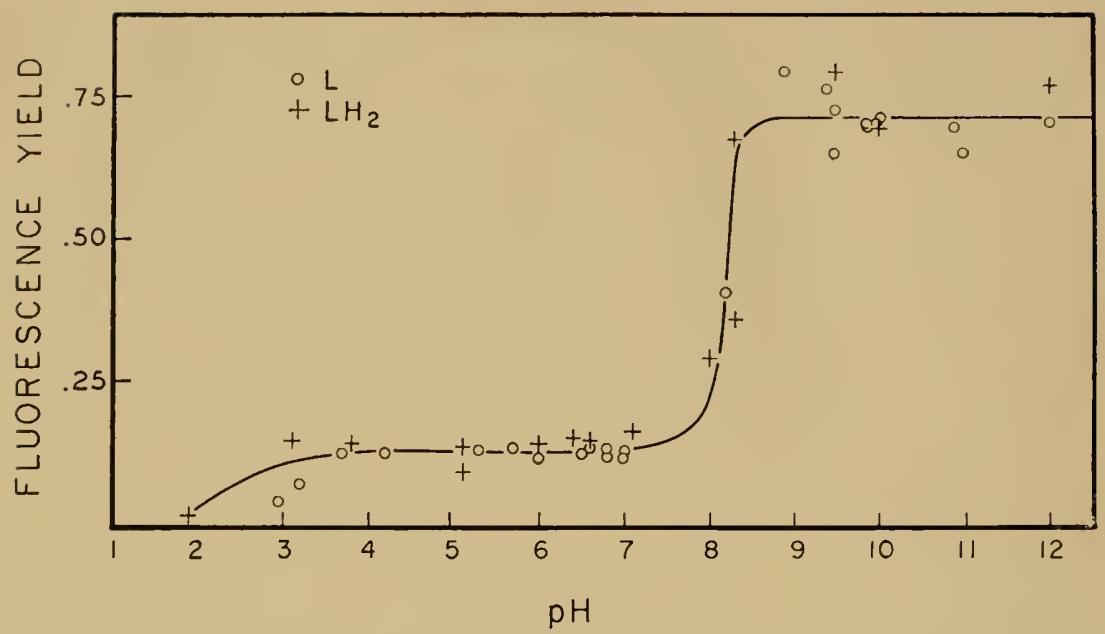

Fig. 21. Fluorescence yields of luciferin and oxyluciferin as functions of $p H$. The measurements were complicated by the absorption shifts, shown in Fig. 18. The quality of the emission, however, was unchanged by $p \mathrm{H}$. Measurements were made relative to fluorescein in alkaline solution. The rather sharp $p K$ at pH 8.4 corresponds to the ionization of the hydroxyl group of the molecule, and it is apparently the ionized specics which emits light in bioluminescence.

and a spectrophotometer, taking account of the spectral absorption shifts shown in Fig. 18. The relative data were then compared directly with fluoresccin in basic solution, since the spectral emission of this compound is very near to that of luciferin and its absolute Huorescence yield is well known. The curve indicates that both luciferin and oxyluciferin have the same ionizable groups, a carboxyl group showing a $p K$ between $p H 3$ and 4 and a phenolic OH group with a $p K$ at $p H$ 8.4. The carboxyl group has been confirmed on the basis of infra-red spectra and other chemical tests. The fluorescence yield exhibits the most dramatic change depending upon the ionization of the hydroxyl group and it is most likely this group which is prevented effectively from ionizing when oxyluciferin is bound on the enzyme surface, since the fluorescence of L-AMP relative to $L$ shows the same relative decrease in intensity.

The absorption and fluorescence levels of oxyluciferin at three diflerent $p$ H's and at different temperatures are shown in Fig. 22. At liquid nitrogen temperatures regardless of $p \mathrm{H}$ there is only one major emission, and this is at 480 millimicrons. At minus 70 degrees and pH 10 there is a 180 millimicron peak which changes gradually to 540 millimicrons as the temperature is raised. A similar change in 


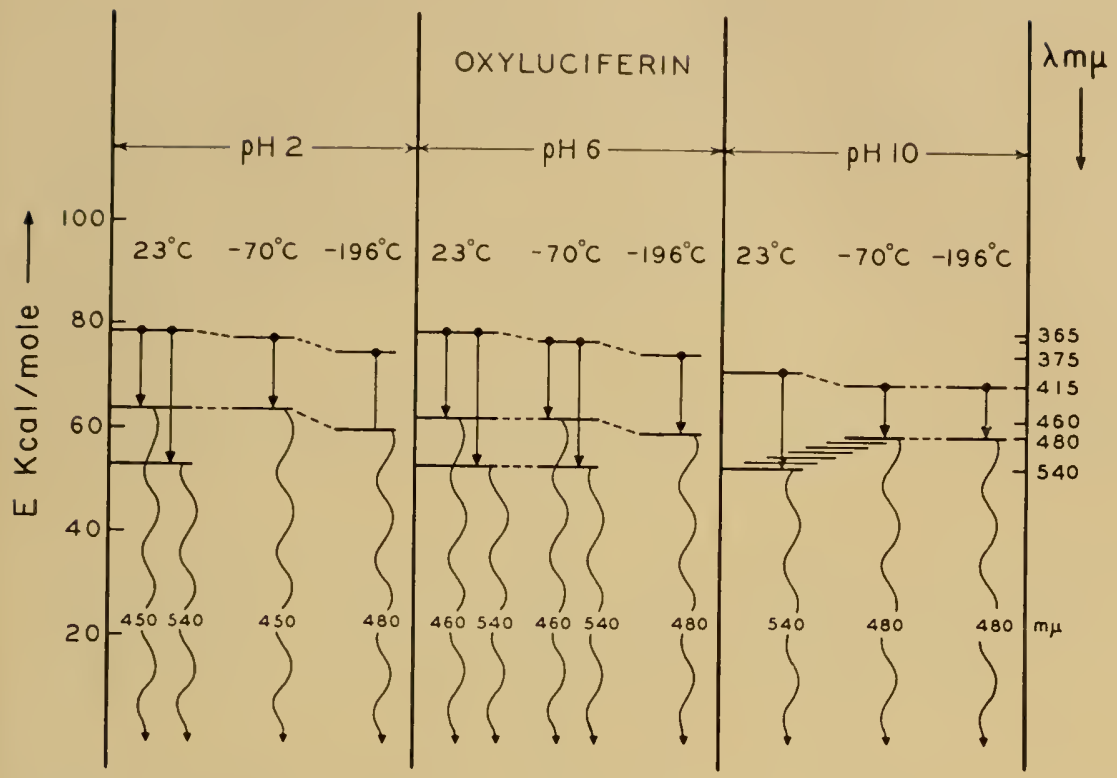

Fig 22. Absorption and fluorescence energy level diagram for oxyluciferin. The measurements were made at various $p \mathrm{H}$ values and at various temperatures, as indicated. The blue shift at low temperatures appears to be a highly efficien fluorescent state.

fluorescence and in intensity with temperature is observed with luciferin. In Fig. 23 the fluorescence emission of luciferin at $-196^{\circ} \mathrm{C}$ and at neutral $p \mathrm{H}$ is shown in Curve 1. As the temperature is warmed gradually going from curves 1 through 8 , one observes the slow disappearance of the $460 \mathrm{~m} \mu$ peak and a gradual appearance of the $535 \mathrm{~m} \mu$ peak.

In none of these fluorescence measurements of luciferin and oxyluciferin under the various conditions described have we observed a fluorescence emission which is comparable to the bioluminescence emission spectrum. However, as the results in Fig. 24 indicate, the adenylic acid derivatives of luciferin and oxyluciferin radically change the emission peaks. $\mathrm{LH}_{2}$-AMP acid fluorescence has an emission peak at 570 millimicrons which corresponds closely with the bioluminescence emission curve. On the other hand, oxyluciferyl-adenylate fluoresces with a major peak around $460 \mathrm{~m} \mu$ and a secondary minor peak at around $625 \mathrm{~m} \mu$. We shall discuss these data in connection with the mechanism of the light-emitting reaction in a later section. 


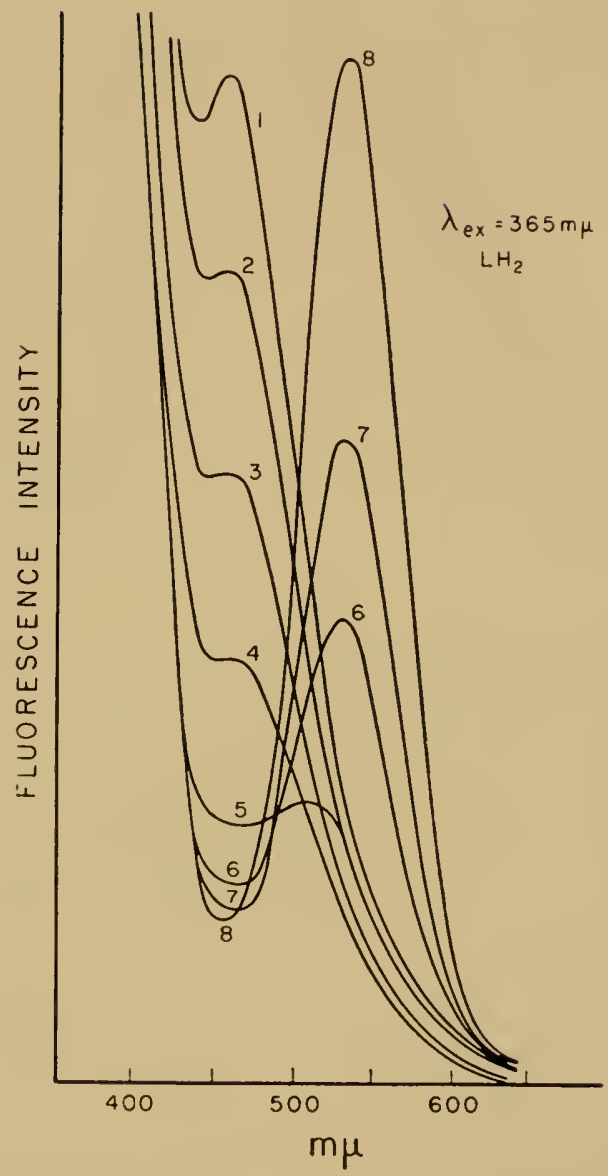

Fig. 23. Relative fluorescence spectra of luciferin. The measurements were made at various temperatures, beginning at $-195^{\circ} \mathrm{C}$ for curve 1 and increasing to room temperature for curve 8. The large apparem peak around $400 \mathrm{~m} \mu$ is due to the scatter of the exciting light by the crystalline ice at the low temperatures.

\section{Quantum Yield of Firefly Bioluminescence}

In considering the mechanism of the firefly light-emitting system it was essential that we have some understanding of the number of light quanta emitted per luciferin molecule used.

The absolute spectral sensitivity of a phototube was determined using an NBS standard of radiation intensity and a sensitive thermopile in combination with a guark prism monochromator. With this phototube it was possible to determine quantitatively the total num- 


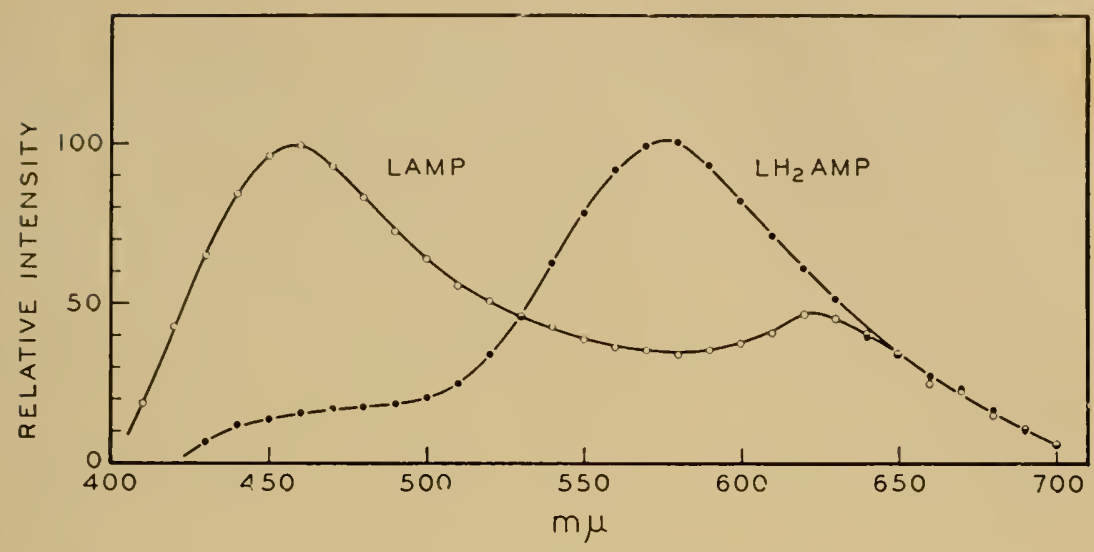

Fig 24. Fluorescence spectra of chemically svinthesized L-.M.MP and $\mathrm{LH}_{2}-\mathrm{AMIP}$ in acid solution. The acid condition was required, since both compounds hydrolve rapidly in neutral and basic solutions, giving the Huotescence of free $\mathbf{L}$ and $\mathbf{L H}_{2}$ respectively. The position of the peak fluorescence of $\mathbf{L H}_{2}-$ IMP appears to be a function of $p \mathrm{H}$, and in more acidic solutions there is a red fluorescence.

ber of photons emitted in a bioluminescent reaction. The total light emitted during the enzymatic oxidation of luciferin over the range $10^{-12}$ mole to $10^{-10}$ mole was measured, and from these data we conclude that one light quantum is emitted per luciferin molecule oxidized $(24,23)$.

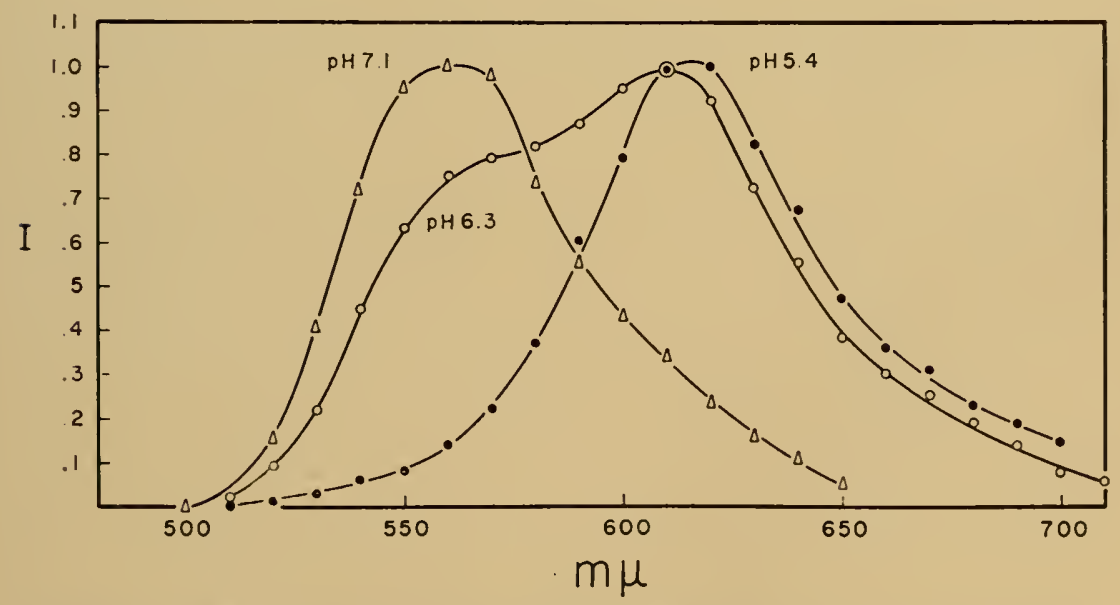

Fig. 25. Emission spectra of firefly bioluminescence for various $p H$ values in glycylglycine buffer solution. These spectra were measured with an RC.I 7326 phototube sensitive out to $8000 \mathrm{~A}$. 
This 100 per cent efficiency for light emission is indicative of the high degree of protection afforded to the activated substrate molecule by the enzyme. That the tight binding of the $\mathrm{LH}_{2}$-AMP or L-AMP to the enzyme is an important factor in determining the quantum yield is also suggested from temperature measurements. The quantum yield has been determined at $3{ }^{\circ} \mathrm{C}$ and at $27^{\circ} \mathrm{C}$, with no observed change in efficiency over this temperature range. It is considered significant that the fluorescence of luciferase (peak at $350 \mathrm{~m} \mu$ ) overlaps the broad absorption band of luciferin and oxyluciferin (peak at approximately $395 \mathrm{~m}_{\mu}$ ) at neutral or alkaline $p \mathrm{H}$ where the quantum yield is unity. It may be possible that the enzyme-substrate complex forms a resonant molecule which is able to transfer energy via tryptophan until it is trapped at an emitting site, in analogy

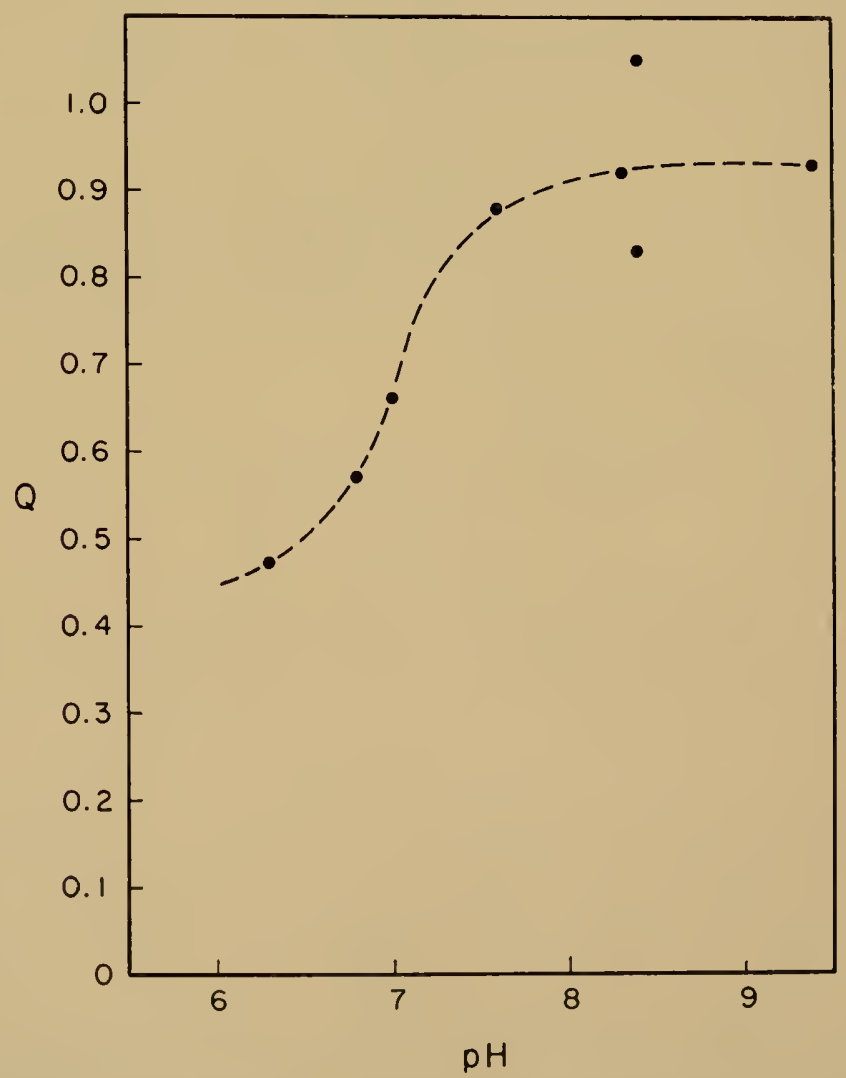

lig. 26. Quantum yicld (Q) of furefly bioluminescence as a function of $p$ II. 
with a trapping center in a crystal lattice or possibly with a wavelength shifter in an organic liquid scintillator.

As the $p H$ of the firefly solution is reduced torvard acid it can be observed that the yellow-green light intensity decreases tremendously, leaving a dull brick orange glow that can be seen in a darkened room. This variation in bioluminescence emission with $p H$ is shown in Fig. 25 for three different $p \mathrm{H}$ values. As can be seen, at neutral (and alkaline) $p \mathrm{H}$ there is a single emission band in the yellowgreen region. At intermediate $p \mathrm{H}$ a red emission band appears at

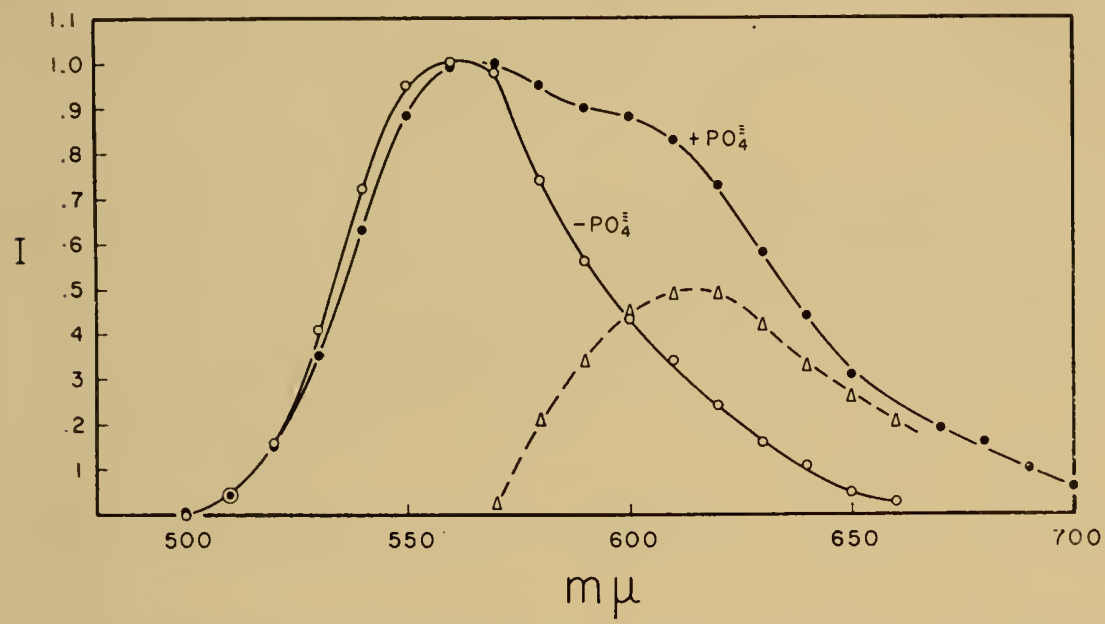

Fig. 27. Emission spectra of firefly bioluminescence at neutral $p \mathrm{H}$ with and without phosphate buffer. The effect of phosphate ion is similar to a lowering of the $p \mathrm{H}$ by approximately 1 unit.

$616 \mathrm{~m} \mu$ and at $p \mathrm{H}$ values below $5.5 \mathrm{~m} \mu$ the yellow-green emission is completely suppressed, and only the red band is evident. The quantum yield has also been measured for various $p H$ values (Fig 26). At acid $p H$ the number of light quanta emitted per luciferin molecule oxidized is markedly lower than 1 , and indicates a predominantly dark reaction. However, at alkaline $p \mathrm{H}$, although the rate of light emission is reduced to a small fraction of the rate at $p \mathrm{H} 7.6$, the quantum yield is essentially unity. The change of quantum yield with $p \mathrm{H}$ corresponds rather closely in form to the fluorescence yield of luciferin and oxyluciferin at various $p H$ 's, as shown in Fig. 21, except for the fact that the $p K \alpha$ has been essentially shifted one whole $p \mathrm{H}$ unit to the acid range for the bioluminescence quantum yield. This may represent the interaction of the enzyme with the phenolic 
$\mathrm{OH}$ group, altering in effect the fluorescence or chemiluminescence properties of the lucileryl-adenylate compound.

One other factor has been shown to modify the emission spectrum of the bioluminescence reaction in vitro. We have indicated previously that inorganic phosphate is an inhibitor of light emission; the results shown in Fig. 27 indicate that phosphate produces a condition even at neutral or alkaline $p \mathrm{H}$ which is essentially similar to a reduction of the $p H$ of the solution, that is, the appearance of a red emission band. The shift from the $562 \mathrm{~m}_{\mu}$ peak to the 616 $\mathrm{n}_{\mu}$ peak in the presence of phosphate or under acid $p \mathrm{H}$, and the sharp decrease in quantum yield in acid $p \mathrm{H}$, are additional evidence that the enzyme is important in influencing the excited state (23).

\section{Discussion of MECHANISM}

It would be appropriate at this time to summarize what is known of the mechanism of the bioluminescence and to point out some of the questions still unresolved. We know that during the enzymatic oxidation of luciferin, oxyluciferin is formed. On the basis of quantitative analysis on ashing, the chemical formula of oxyluciferin differs from luciferin by only two hydrogen atoms. That oxyluciferin differs only slightly from luciferin is also evidenced by the striking similarity of their absorption, fluorescence, and fluorescence yield curves and their respective variations with $p H$. This is then different than in the case of the chemiluminescence of luminol, where the release of $\mathrm{N}_{2}$ from the phthalhydrazide molecule is sufficiently exergonic to leave the product phthalic acid molecule in an excited state from which blue light can be emitted. We have yet to resolve the steps by which the $\mathrm{LH}_{2}$-AMP active intermediate, bound on the enzyme molecule, reacts with dissolved molecular oxygen to produce L-AMP and a light quantum. From the kinetic data on the fluorescence of $\mathrm{E}-\mathrm{L}-\mathrm{AMP}+\mathrm{PP} \leftrightarrows \mathrm{E}+\mathrm{L}+\mathrm{ATP}$ and from the flash height data as a function of enzyme concentration, we can safely say that there is only one site for light emission on the cnzyme surface. The quantum yicld of unity also precludes the utilization of two luciferin radicals on an enzyme molecule.

The fact that the acid fluorescence of synthetically formed $\mathbf{L H}_{2}$ AMP is close to the bioluminescence emission would imply an energy transfer from an excited product to the $\mathrm{LH}_{2}-\mathrm{AMP}$ molecule. This picture is further strengthened by the fact that the acid fluorescence of L-AMP show's no yellow-green peak but does have a red fluorescence. However, (a) $\mathrm{LH}_{2}$ - AMP can also be shown at more strongly 
acid $p \mathrm{H}$ to exhibit a red fluorescence; (b) the bioluminescence, and most chemiluminescences, occur at neutral or basic $p \mathrm{H}$ values, so that acid $p \mathrm{H}$ may have little relation to the actual cuvironment during chemiluminescence: and (c) energy transfer should be concentrationdependent and the results of the flash height versus enzyme concentration would therefore rule out this type of transfer.

These data eliminate the possibility that two LH.-AMP molecules would interact with oxygen in a reaction analogous to the one proposed by Linschitz, in which one reduced molecule (in our casc $\mathrm{LH}_{2}-\mathrm{AMP}$ ) is regenerated. It was conceivable that the exergonic step was creating the excited state of $\mathrm{LH}_{2}$-AMP, whose emission would correspond to the observed fluorescence of this compound. Energy transfer to the oxidized product (L-AMP) would under these conditions give rise to the red emission.

Thus we must account for a minimum of approximately $60 \mathrm{kcal} /$ mole in the oxidation of a single $\mathrm{LH}_{2}$-AMP molecule, an amount which is more than is available from the production of a peroxide, obtained from a simple balancing of the equation $\mathrm{LH}_{2}$-AMP $+\mathrm{O}_{2} \rightarrow$ L-AMP $+\mathrm{H}_{2} \mathrm{O}_{2}$. We have not yet been able to isolate unambiguously the excited state of the product molecule from which the light quantum is emitted, although we infer that its fluorescence yield must be practically 100 per cent. It is possible that the oxygen activation leading to the formation of hydrogen peroxide could furnish sufficient energy. The formation of a double bond in the ring structure of luciferin could contribute as much as $30 \mathrm{kcal}$ of resonance energy. The difference in bincling energy between L-AMP and $\mathrm{LH}_{2}$-AMP may contribute, as well as energy from the formation of hydrogen peroxide. Further experimental evidence, particularly on the possible formation of $\mathrm{H}_{2} \mathrm{O}_{2}$, is necessary before a detailed description of mechanism can be given. It is possible that knowledge of the structure of the luciferin molecule will furnish a better idea as to the chemistry involved, and this is being pursued at the present time.

\section{BACTLRIAL LUMINESCENCE}

Early attempts to extract the luminescent system from bacteria were almost uniformly unsuccessful, although Gerretsen in 1920 succeeded in obtaining a reak light-emitting cell-frce extract. This observation was repeated by Shoup and Strehler in 1951. In 1953 Strehler demonstrated that DPN and DPNH would substitute for the hot water extract to restore light emission in a cold water bacterial extract (25, 26). Later experiments by Strehler indicated that DPNH was the 
limiting factor for light emission. Simultaneous studies by McElroy, Hastings and associates demonstrated that FMN and an additional factor, subsequently identified by Strehler and Cormier as a longchain aldehyde, were required for light emission $(17,18)$. These and other observations indicated that the following over-all reaction was the basic one concerned with light emission:

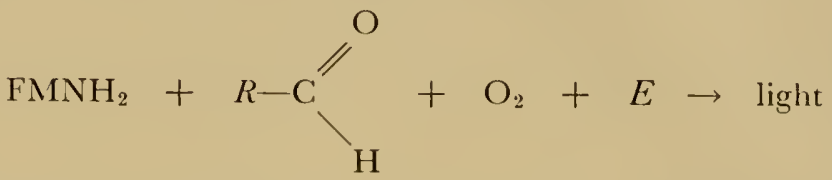

Studies by Cormier and Totter (5) and by McElroy and Green (13) indicated that at least two enzymes were required to obtain light emission from DPNH. By dialysis at low $p \mathrm{H}$ Cormier et al.

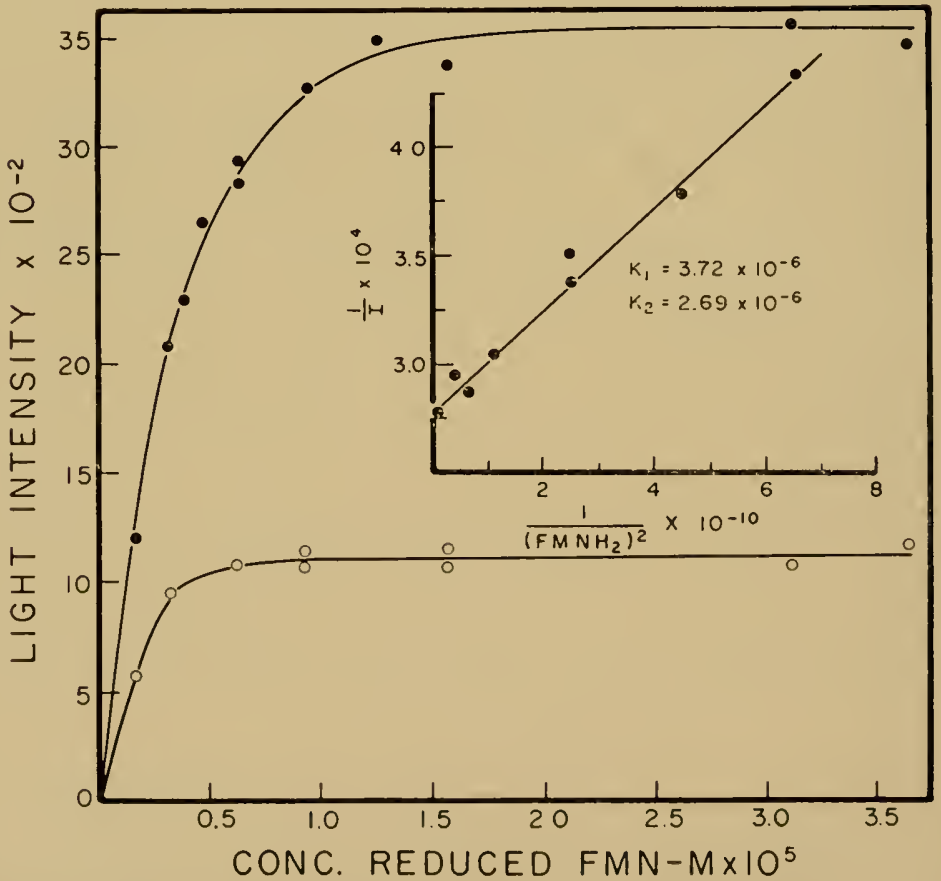

lig. 28. Relationship between light intensity and reduced FMN. Initial light intensity, in arbitary units, of a constant batcterial extract solution as a function of the amome of reduced filN injected into the sulution. The clesed circles eppesent a duplicate run statting with a higher ensume concentsation. In the "pper righe portion of the figure is a Michaclis-Menten plot of the data, indicating a lwo-site cmsmatic oxidation of FMNH. 
(6) were able to inactivate the light-entitting system which depended on DPNH. The system was able to emit light, however, in the presence of reduced FMIN. Subsequently Green and McElroy were able to separate by calcium phosphate gel columms the DPNH-FMN oxidase system from the luciferase preparation. Ammonimm sulfate fractionation of this preparation yields an active enzyme which is homogeneous in the ultracentrifuge (mol. wt. ca. 85,000) but which contains three components as judged by electrophoresis. The active fraction contains over 70 per cent of the total protein and cmits light only when reduced FMN, aldehyde, and oxygen are present. No emission is observed with reduced DPN.

The biochemical and physiological aspects of bacterial luminescence have been reviewed recently, and only that evidence which seems to be essential for a discussion of the mechanism will be presented here.

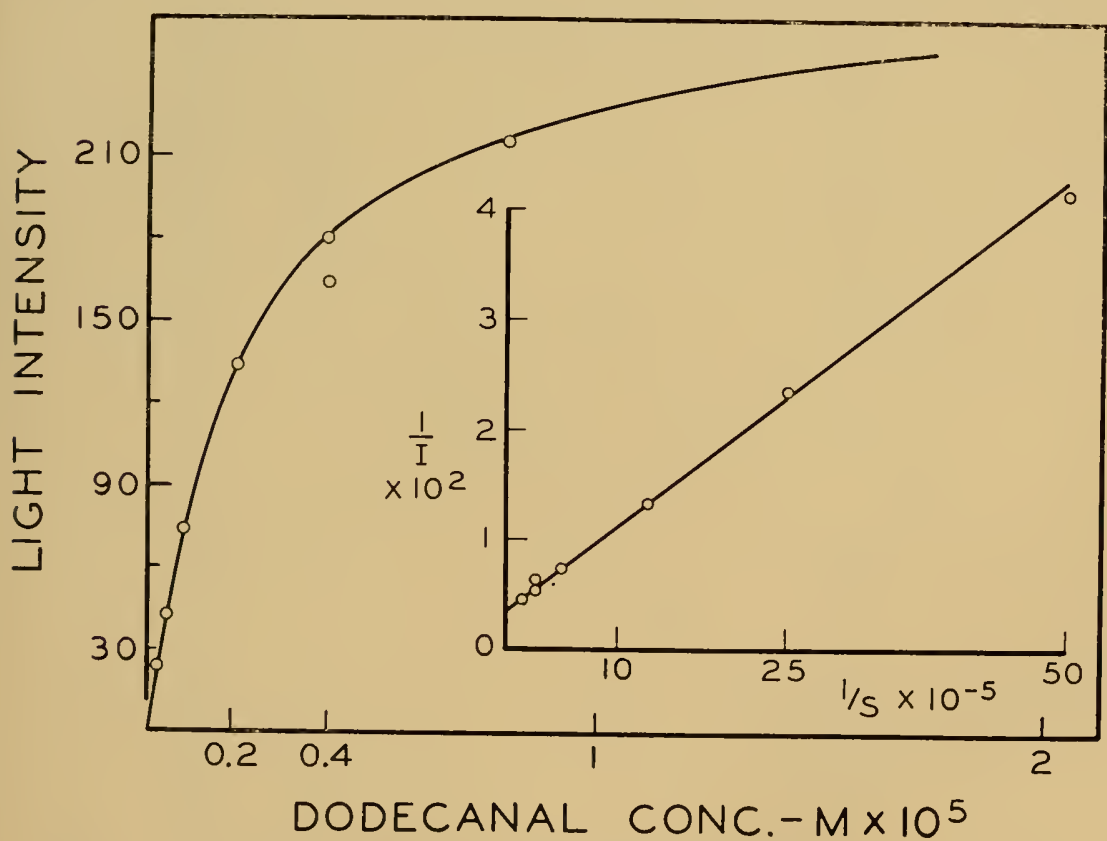

Fig 29. Relationship between light intensity and aldehyde. Initial light intensity, in arbitrary units, of a bacterial extract solution initiated by injection of a constant amount of reduced DPN, but as a function of the concentration of dodecanal in the solution. The insert is a Michaclis-Menten plot of the dita, indicating a simple relationship between aldehyle and initial rate of the right reaclion. 


\section{Reduced FMN Requirement}

$\Lambda$ detailed analysis of the reduced FMN requirement for light emission (Fig. 28) led McElroy and Green (1.3) to conclude that two FMNH. molecules were required. Subsequent studies by Totter, Connier, and Rostorler (6) suggest that there is one tightly bound flavin and one which freely dissociates.

\section{Aldehyde Requirement}

Similar observations with regard to the aldehyde required indicate that one molecule per enzyme molecule is required for light emission. The results obtained for dodecanal are shown in Fig. 29.

Using a highly purified enzyme preparation, McElroy and Green were able to demonstrate that the total light output was directly dependent on the amount of aldehyde added (Fig. 30). These results suggested that aldehyde utilization was essential for light emission.

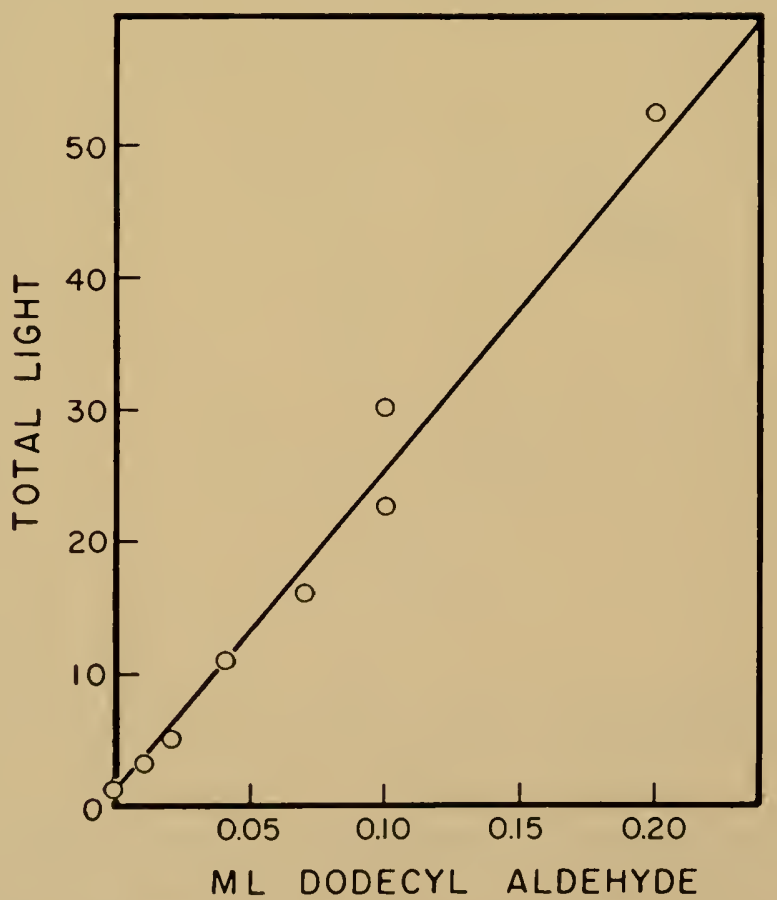

lig. 30. Total light versus aldehyde concentration. Elfect of varying amounts of dodecyl aldehide added 10 a bacterial empune extract 1 pon the total light emitted during the reaction. 
Subsequent studies by Totter and Cormier indicated that one light quantum is emitted for each twenty aldehyde molecules used.

Using a partially purified enzyme, Strehler and Cormier demonstrated that a number of straight-chain, saturated aldehydes would support light emission. The results obtained by Rogers and McElroy (22) indicated an interesting difference in light intensity with alde-

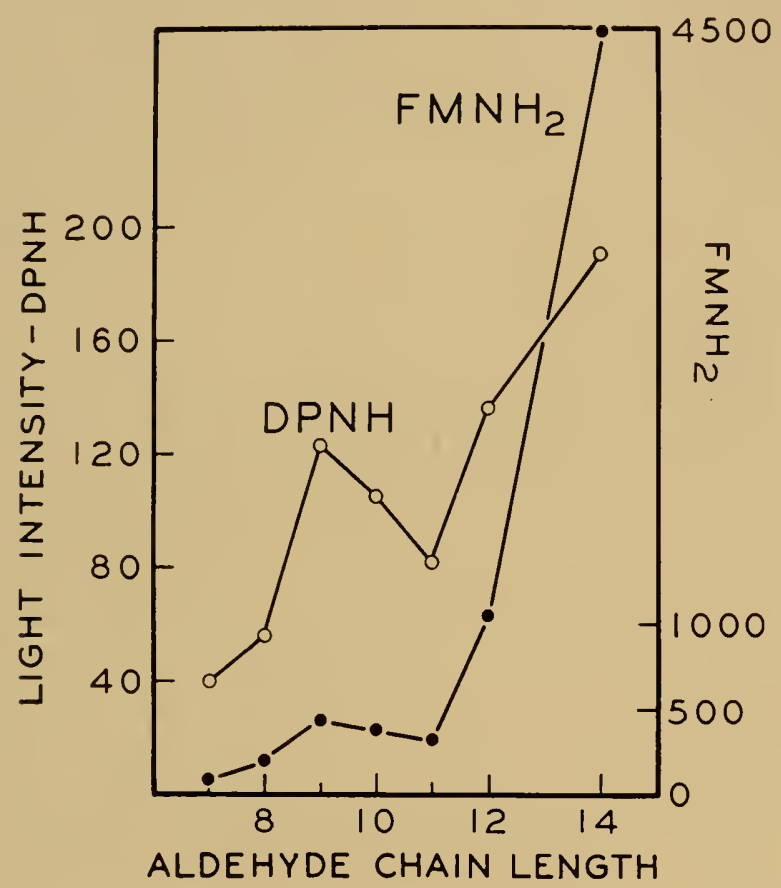

Fig. 31. Effect of aldehyde chain length on the initial light intensity. The light reaction in the bacterial extracts was initiated upon addition of reduced DPN and reduced F.IN respectively.

hydes of various chain lengths. The results given in Fig. 31 suggest that the effectiveness of the aldehydes containing an even number of carbon atoms increases with chain length, while the response to the aldehydes containing an odd number of carbon atoms reaches maximum effectiveness with $\mathrm{C}_{9}$.

\section{MFGHANISM OF Light EMISSION}

Before discussing the possible chemical and physical mechanisms of bacterial light emission, it is well to summarize the following points which any proposal must satisfy. (1) The peak of the emission spec- 
trum of bacterial light (Achromobacter fischeri) is $495 \mathrm{~m} \mu$. This is considerably removed from the yellow-green fluorescence $(530 \mathrm{~m} \mu)$ of riboflavin or FMN. (2) Kinetic data indicate that two reduced FMN molecules may be required. (3) The data demonstrate that total light emission is dependent on the amount of aldehyde added. The utilization of aldehyde occurs rapidly only under conditions which favor light emission. (4) Thermodynamically at least 58 kilocalories per mole are required to obtain an excited state which will give the peak emission. Since light quanta at the wavelength of $120 \mathrm{~m} \mu$ are also observed, the energy requirement must be as high as $68 \mathrm{kcal} /$ mole. (5) Hydrogen peroxide addled to the complete enzyme system does not stimulate, nor does catalase inhibit.

From such consiclerations McElroy and Green (13) suggested the possibility that a peroxidation of an aldehyde molecule was the source of the excitation energy. They suggested that one reduced FMN molecule combined with the aldehyde and that the second FMNH.2 molecule combined with oxygen to form a peroxide similar to that suggested by Drew for aminophthalic hydrazide. The presumption was that the organic peroxide acted as an oxidant for the aldehydeFMNH., compound to give a highly excited molecule which would emit light. Although this proposal is in keeping with the observed facts, there is no direct chemical evidence to support it. It is possible, as Strehler (25) argues, that the utilization of aldehyde represents dark reactions and that such dark reactions occur only under those conditions which support luminescence. Unfortunately, until one can obtain a system which gives a quantum yield of one, as in the case of the firefly, this hypothesis is difficult if not impossible to eliminate.

\section{ADDENDUM: The Stricture of Firefly Liciferin}

\section{Frank McCapra and Emil $H$. White}

The following is a brief indication of the probable constitution of luciferin, as deduced from preliminary investigations.

Luciferin, $\mathrm{C}_{13} \mathrm{H}_{10-12} \mathrm{O}_{3} \mathrm{~N}_{2} \mathrm{~S}_{2}$, obtainable from the clried light organ of the firefly, is an optically active crystalline solid, highly fluorescent in solution. Earlier evidence has suggested the presence of a carboxylic acid grouping and a phenolic hydroxyl group. Tests for basic nitrogen, thioketone, or sulfhydryl groups were all negative. 
Recently it was found that reduction of lucilerin with Raney nickel gave an aminophenol, with an ultraviolet spectrum in acid solution identical to the acid spectrum of p-anninophenol.

Vigorous acid hydrolysis of luciferin and sublimation of the product gave a componnd which proved to be the hydroxybenzothiazole (III), by comparison with a synthetic sample. Cysteine was also produced in this hydrolysis, and in view of the negative tests referred to earlier, and the properties of the oxidation product of luciferin, the latter is probably present as a thiazoline. Oxyluciferin, which appears to differ from luciferin simply by two hydrogen atoms, is inferred to have a thiazole as part structure, in view of its much greater overall stability, especially to hot strong acid, and the changes in its spectra.

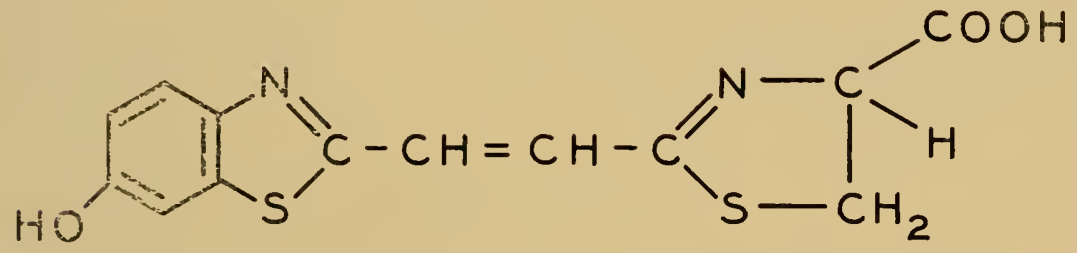

I

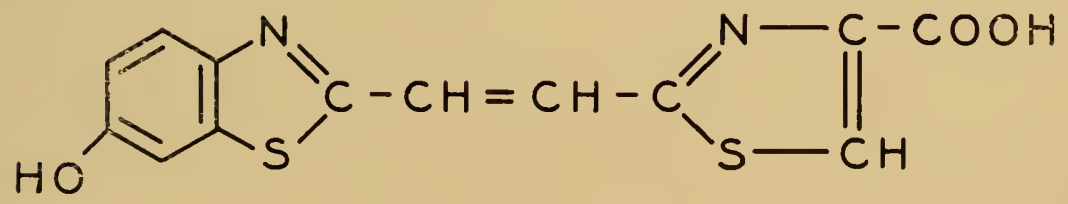

II

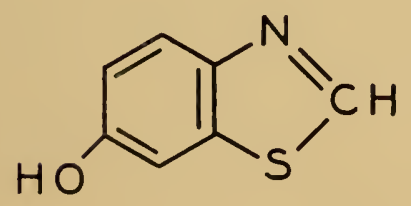


Hydrogenation of luciferin over palladium on charcoal results ir the uptake of one mole of hydrogen, and a compound with an almost identical ultraviolet spectrum to the very distinctive one of the hydroxybenzothiazole (III). Catalytic reduction of a thiazoline is unlikely, and hydrolysis of the reduction product by acid produces no change in the spectrum.

These facts, together with excellent agreement between the properties of luciferin and those of certain model compounds, lead to the probable structures for luciferin (I) and for oxyluciferin (II) .

A more complete report, with further confirmatory evidence, will be published elsewhere.

\section{ACknowledgments}

The investigations summarized in this paper were supported in part by the Atomic Energy Commission, National Institutes of Health, National Science Foundation, and the Office of Naval Research.

\section{REFERENCES}

1. Airth, R. L., Rhodes, W. C., and McEIroy, W. D., Biochim. el Biophy's. Acla, 27, 519 (1958).

2. Bitler, B., and McElroy, W. D., Arch. Biochem. Biophys., 72, 358 (1957).

3. Cormier, M. J., and Strehler, B. L., J. Am. Chem. Soc., 75, 4864 (19.53).

4. Cormier, M. J., and Strehler, B. L., J. Cellular Comp. Physiol., 44, 277 (1954).

5. Cormier, M. J., and Totter, J. R., Biochim. el Biophys. Acta, 25, 229 (1957).

6. Cormier, M. J., Totter, J. R., and Rostorfer, H. R., Arrh. Biochem. Biophy's., 63, $414(1956)$.

7. Green, A. A., and McElroy, W. D., Biochim. et Biophys. Acta, 20, 170 (1956).

8. Harvey, E. N., Bioluminescence, Academic l'ress, New York (1952).

9. Hastings, J. Woodland and John Buck, Biol. Bull., 111, 101 (1956).

10. Hastings, J. Woodland, McElroy, W. D., aud Coulombre, Jane, J. Cellular Comip. Physiol., 42, 137-150 (1953).

11. McElroy, W. D., J. Biol. Chem., 191, 547 (1951).

12. McEtroy, W. D., in The Harrey Leclures, Acadamic Press, Inc. (1957).

13. McElooy, W. D., and Green, A., Arch. Biochem. and Biophys., 56, 240 (1955).

14. Mcklroy, W. 1), and Green, A., Arch. Biochem. and Biophys., 64, 257 (1956).

15. M(Elroy, W. D., and Hastings, J. W., in The Luminescence of Biological Sys. lems (F. H. Johnson, ed.), pp. 161-198, American Issociation for the Ar. vancement of Science, Washington (19.5).

16. McElroy, W. 1)., Hastings, J. W.. Coulombre, Jane, and Somnenfeld, Valerie, Arrh. Biochem. and Biophys., 46, 399-416 (1953).

17. McElroy, W. 1)., Hastings, J. W., Sonnenfeld, V., and Coulombre, J., Science, $118,385,(1953)$.

18. Mclilroy, W'. 1)., llastings, J. W.. Sonncufeld, V., and Coulombre, J., J. Bacteriol., 67, 102 (1951).

19. McEIroy, W. D., and Strehler, B. L., Barteriol, Rev。, 18.177 (1951).

20. Rhodes, W. C., and McElroy, W. 1)., Science, 128, 253 (1958).

21. Rhorkes, W. C., and McElroy, W. D., J. Biol. Chem., 233, 1.528 (1958).

22. Rogers, 1'., and McElroy, W. 1)., Arch. Biochem. and Biophys., 75, 87 (19.58). 
23. Scliger, H. 11., and McElroy, W. D., Biochem. Biophys. Research Commun., 1, 21 (19.59).

24. Seliger, H. H., and Mclilroy. W. D., Arch. Biochem. and Biophys., 88, 136 (1960).

25. Sec Strehler, 13. 1.., "Luminescence of Biological Systems", Proc. Conf. on Luminescence, Asiomar, Calif., 1954 (1955).

26. Strehler, B. L., and Cormier, M. J., Arch. Biochem. and Biophys., 47, 16 (1953); J. Am. Chem. Soc., 75, 1264 (1953).

27. Totter. J. R., and Commier, M. J., J. Biol. Chem., 216, 801 (1955). 


\title{
CYPRIDINA LUCIFERIN OXIDATION AS A FUNCTION OF $p \mathrm{H}$, AND REDUCTION OF LUCIFERIN BY ASCORBIC ACID ${ }^{1}$
}

\author{
A. M. Chase, J. H. Ball, C. E. Cornelius, and R. J. Lederman
}

Physiological Laboratory, Princeton University, Princeton, New Jersey

Studies, in vitro, of the kinetics of the luminescent reaction of Iuciferin and luciferase from Cypridina have long been plagued by a complication clue to the presence of reversibly oxidized luciferin (1). The curve relating total light emitted to time (the integral curve) becomes distorted when more than a small amount of reversibly oxidized luciferin is present, and this often makes it difficult or impossible to analyze data in terms of effects of experimental variables upon the activity of the enzyme. Also, procedures for purifying Cypridina luciferin are complicated by the fact that this luciferin is so unstable in the presence of clissolved oxygen.

We have extended earlier studies on the effects of temperature upon the non-luminescent oxidation of Cypridina luciferin (2) to inclucle measurements of the rate of this process as a function of $p \mathrm{H}$, and have found that this luciferin is least stable at about $p \mathrm{H} 9$ and becomes considerably more resistant to oxidation at $p H$ 's more acid or more alkaline than this.

The experimental procedure and materials used were essentially the same as in the carlier work involving temperature (2). Luciferin was allowed to stand in $0.1 M$ phosphate buffers of the clesired $p \mathrm{H}$ at $26^{\circ} \mathrm{C}$. Samples from the reaction mixtures were periodically mixed with luciferase, and the amount of light still remaining was measured with a recording photoelectric light integrator. The firstorder reaction-rate constant for the disappearance of the luciferin by non-luminescent oxidation was then calculated for each $p \mathrm{H}$.

Fig. I shows the results of these experiments. It is eviclent that non-luminescent oxidation is most pronounced at about $p \mathrm{H} \mathrm{9}$, being nearly fifty times as fast there as at $p \mathrm{H} 5$ and about three times as fast as at $p \mathrm{H} 13$ or 14 . Anderson stated some years ago (1) that this luciferin was relatively stable in clilute acid or alkali but gave no details.

\footnotetext{
${ }^{1}$ Supported by a National Science foumdation grant.
} 


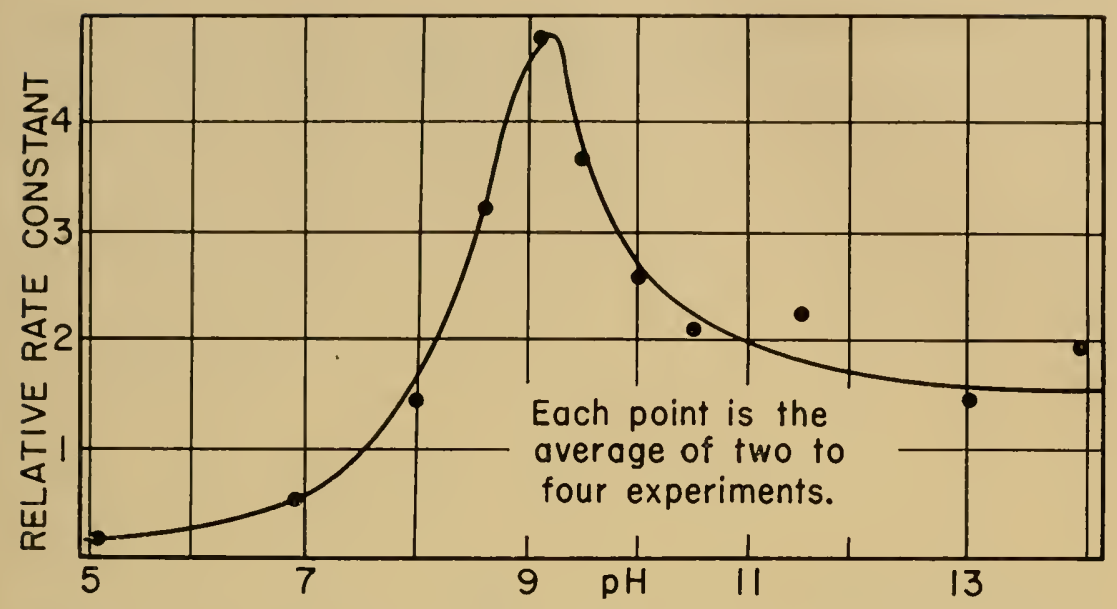

Fig 1. The relative first-order reaction-rate constant for the non-luminescent oxidation of Cypridina luciferin during exposure, at $26^{\circ} \mathrm{C}$, to the $p \mathrm{H}$ 's indicated.

Ascorbic acid was found to be a most effective inhibitor of this non-luminescent oxidation of luciferin. Selecting $p \mathrm{H} \mathrm{8.6,} \mathrm{where} \mathrm{the}$ luciferin is very unstable, experiments were performed in the presence of (a) $0.01 M$ ascorbic acid, (b) $0.001 M$ ascorbic acid, and (c) no ascorbic acid. The lower half of Fig. 2 shows typical results. It is eviclent that $0.01 M$ ascorbic acid almost completely prevents the loss of luciferin by non-luminescent oxidation, and $0.001 \mathrm{M}$ ascorbic

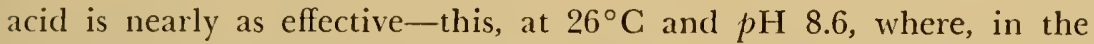
absence of the ascorbic acid, half the luciferin is lost through oxidation in less than ten minutes.

The addition of a trace of ascorbic acid to a reaction mixture of Cypridina luciferin and luciferase completely abolishes the dim light emission which has been attributed to reversibly oxidized luciferin (1), and a reaction mixture so treated shows only the regular bright luminescence characteristic of luciferin which has not undergone any oxidation. Evidently any reversibly oxidized luciferin in such a mixture is recluced by the ascorbic acid.

This effect of ascorbic acid on the course of light emission when luciferase is added to partially oxidized luciferin is illustrated very strikingly in the upper part of Fig. 2, which is traced from the chart of the recording photoelectric light integrator. The compound nature of the reaction in the absence of ascorbic acid, and the delayed dim light emission due to reversibly oxidized luciferin (1) are very evident. This luminescence curve cannot be described by 

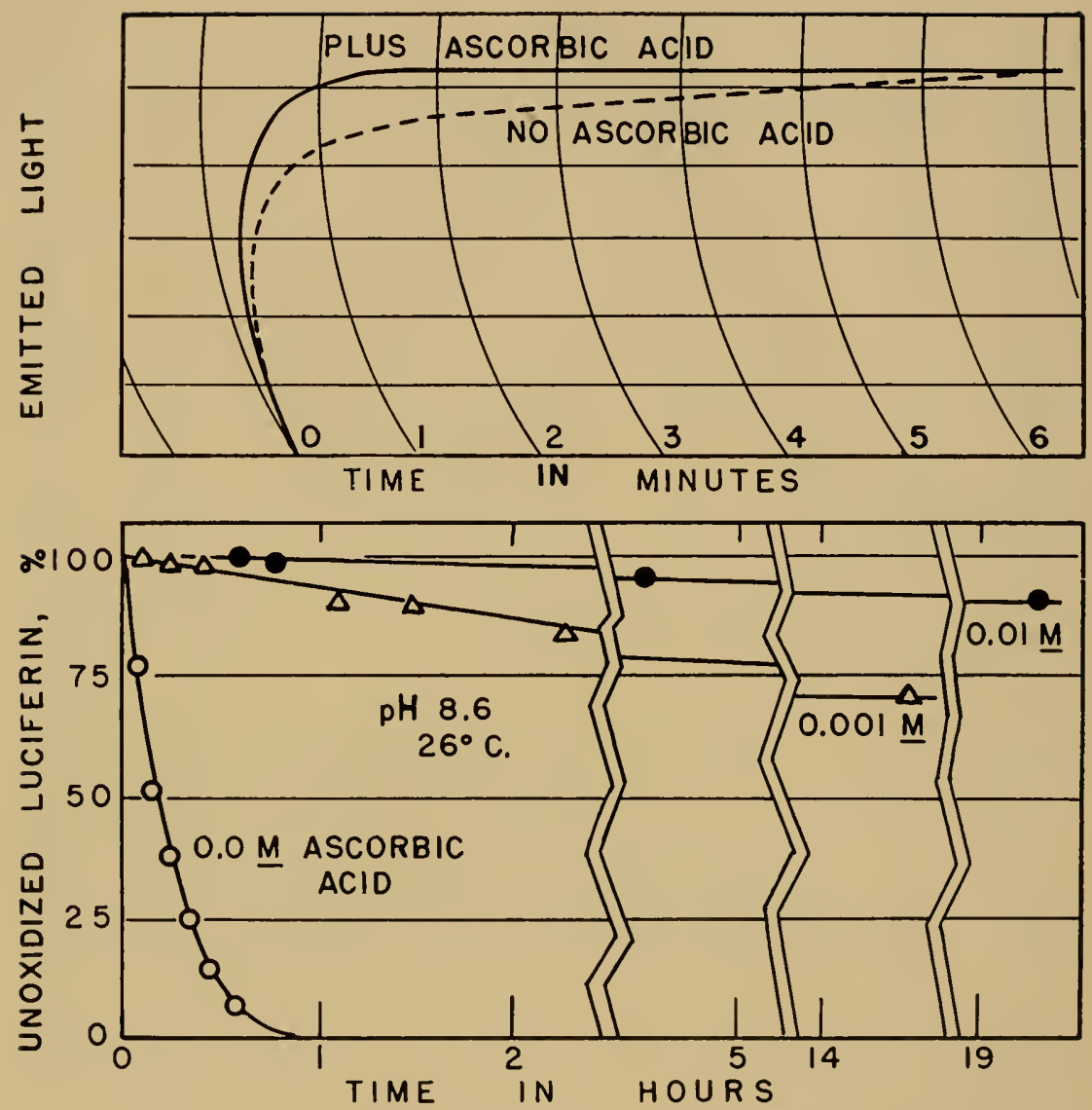

Fig. 2. Upper: Tracings of automatically recorded curves showing the luminescent reaction of luciferase and partially oxidized luciferin in the presence and absence of ascorbic acid. The latter evidently reduces reversibly oxidized luciferin, and thereby prevents the delayed dim luminescence which may otherwise complicate the luminescent reaction.

Lower: Inoxidized fuciferin remaining after various times of incubation, at $266^{\circ} \mathrm{C}$ and $p H 8.6$, in the presence of (a) $0.01 \mathrm{M}$ ascorbic acid, (b) $0.001 \mathrm{M}$ ascorbic acid, and (c) no ascorbic aciol. It is clear that ascorbic acid effectively protects lnciferin against non-luminescent oxidation.

the equation for a simple first-order reaction, although the equation will fit the other luminescence curve exactly.

The use of such a reductant as ascorbic acid should do much to simplify measurements of the kinetics of the Cypridina luciferinluciferase reaction, by abolishing the complicating effects often produced by the presence of reversibly oxidized luciferin. In addition, 
it should be useful in retarding oxidation of luciferin during isolation and purification procedures (3).

\section{REFERENGES}

I. Inderson. R. S., J. Cellular Comp. Physiol., 8, 261 (1936).

2. Chase, 1. M., Hurst. F. S., and Zeft. H. J.. J. Cellular Comp. Pligsiol., 54. $115(1959)$.

3. Dr. Peter Marfey (pers. commun.) used ascorbic acul in unpublished experiments done in 1957, while studying methods for purifying Cypridina luciferin. 


\title{
CHARACTERISTICS OF CELL-FREE FUNGAL BIOLUMINESCENCE ${ }^{1}$
}

\author{
R. L. AIRTH \\ Department of Botany \\ University of Texas, Austin, Texas
}

The emission of light by certain fungal species has been observed by many investigators, one of the early notations being made by Pliny. Indeed, one of the first "practical applications" of this luminous form was to serve as a light source on a submarine, the Turtle, built for the United States Navy and used during the Revolutionary War (5). There have been many technological advances in the propelling force of submarines since the Turtle, but very little attention has been given to the original light source. While not at the atomic level, the obvious final objectives, a study of luminous molds at the molecular level, has been initiated.

Harvey (6) has made note of his own unsuccessful attempts and the failure of several other investigators to obtain the classical luciferin-luciferase reaction in a cell-free preparation of luminous fungi. Recently, however, this was accomplished by mixing a cold water extract containing the enzyme luciferase, a hot water extract containing the substrate luciferin $\left(\mathrm{LH}_{2}\right)$, and a reduced pyridine nucleoticle (1). The pyridine nucleotide (PN) requirement is nonspecific in that either reduced triphosphopyridine nucleotide (TPNH) or diphosphopyridine nucleotide (DPNH) can act as the hydrogen donor, the former being about 2.5 times more reactive both as to the initial rate and the maximum light intensity. A "typical" light emission curve from such a reaction mixture is presented in Fig. 1. All reactions in vitro were carried out with Collybia velutipes as an enzyne source and Armillaria mellea as the source of substrate.

Light emission is not obtained until the reaction mixture is conplete. Though a fresh luciferase preparation will enit a small amount

\footnotetext{
The incestigations in this paper were supported by research grants from the National lustitutes of Health, the National Science Foundation, and The Uniersity of Texas Research Instiute.
} 


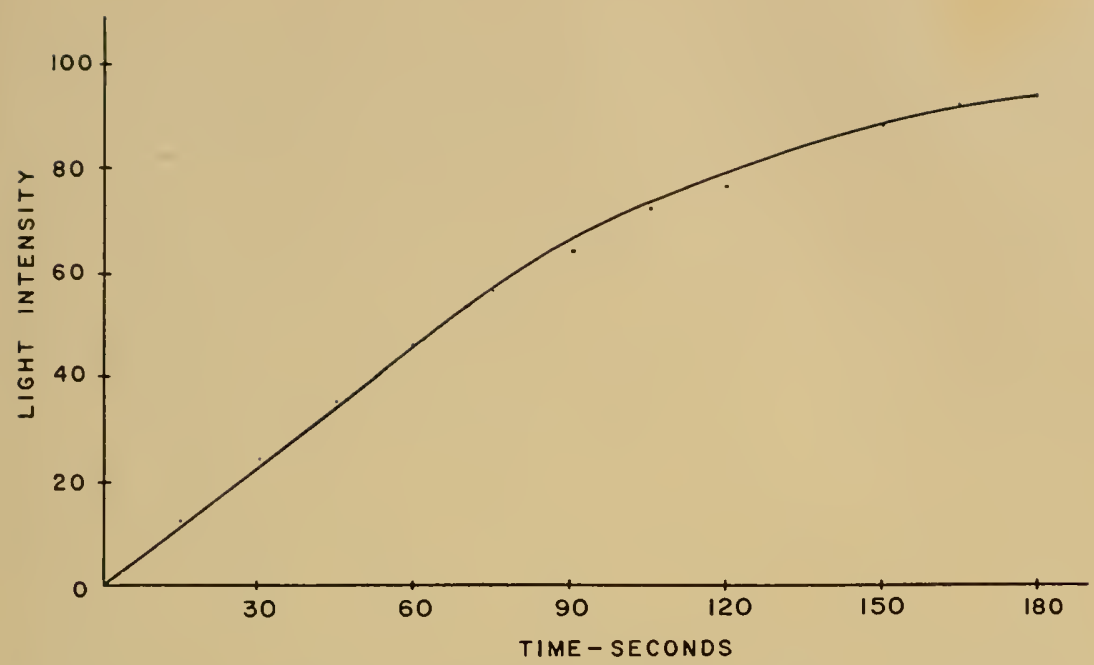

Fig. I. In vitro fungal bioluminescence reaction. Lucilerase (homogenate-see text) $2.7 \mathrm{mg}$; luciferin, $0.20 \mathrm{ml}$; 'TPNH, $0.65 \mu$ moles; $0.05 M \mathrm{~K}-\mathrm{l} \mathrm{O}_{4}$ bulfer, $p \mathrm{H} 7.4$, containing $10-3 \mathrm{M}$ Versene brought to a final volume of $3.0 \mathrm{ml}$.

of light with no further additions, this capacity is quickly lost. The explanation for the rather slow increase in light intensity during the course of the reaction is open to several interpretations.

When first isolated, fungal luciferase was reported as quite stable; it could be repeatedly frozen and thawed with very little loss of activity (1). It is now known that this is the case only when very crude homogenates are used and that on further purification the enzyme appears to become quite unstable. The enzyme is now prepared by making a homogenate of an air-dried, surface-grown mycelia mat with $0.05 M$ potassium phosphate buffer, $p \mathrm{H} 7.45$, the buffer containing $0.001 M$ Versene and $0.001 M$ mercaptoethanol. It is of interest that liquid cultures grown with any form of agitation do not yield luminous mycelia. Attempts to demonstrate a deficiency of enzyme or substrate under such conditions have been inconclusive to date. The homogenate is treated with protamine sulfate and subsequently fractionated with $\left(\mathrm{NH}_{4}\right)_{2} \mathrm{SO}_{4}$. This procedure has resulted in approximately a 5 -fold purification in the $30 \%$ to $50 \%$ $\left(\mathrm{NH}_{4}\right)_{2} \mathrm{SO}_{4}$ fraction. This increase in activity refers to both the initial rate of the light-emitting reaction and the maximum value finally attained. This preparation loses approximately $75 \%$ of its activity on freezing and thawing once. Storage at room temperature or at $35^{\circ} \mathrm{C}$ results in complete loss of activity in 12 hours. 
Luciferase, isolated and purified in this fashion, is probably not soluble. The addition of Treen 80 to a final concentration of $0.03 \%$ in the reaction mixture has been found to stimulate both the initial rate of the reaction and the maximum light intensity attained. This addition now is made routinely during the assay. The homogenate is not made up in a buffer containing Tween 80 , as this causes a more rapid loss in activity. Treatment of the $30-50 \% \quad\left(\mathrm{NH}_{4}\right)_{2} \mathrm{SO}_{4}$ fraction with wheat germ lipase or snake venom has not resulted in increased activity or in reduction of the stimulatory effect of the Tween 80 addition. If the mold luciferase is particulate in nature, then the particle must be of comparatively small dimensions, as activity is still restricted to the supematant fraction after centrifugation of the homogenate at $29,000 \times g$ for 30 minutes. In view of these findings, and since facilities for routine high speed centrifugation are not available, we have proceeded with the objective of learning as much as possible about the system in this state. The results set forth in Fig. I could be attributed to an increase in the amount of active enzyme during the course of the reaction. Throughout the initial phases of this work it was noticed that enzyme preparations frequently were completely inactive. Precedent to this possibility is given by the fact that the initial stages of luciferase purification from both the firefly and Photobacterium fischeri also have been complicated by the fact that the enzyme is readily inhibited, a lact leading one to believe that the preparation is very unstable (9) . Treatments of the inactive fungal cnzyme by dialysis against $p H 7.0$ phosphate buffer, precipitation with cold ethyl alcohol or acetone, and additions of Versene or sulfhydryl reagents, or both, have been unsuccessful in liberating the presumed inhibitor. However, the addition of crystalline bovine plasma albumin (Armour and Company) to the reaction mixture stimulates the reaction. Such an addition has subsequently been found not only to "reactivate" the inactive enzyme but also to stimulate the response from an active preparation. In Fig. 2, albumin is seen to give about a 5 -fold increase in the maximum amount of light emitted and a corresponding increase in the rate. This effect is specific for bovine plasma albumin, bovine serum albumin, or crystalline human albumin; neither crystalline egg albumin, bovine albumin-fraction V, nor gelatin will clicit the same response. The possibility that the crystalline bovine plasma albumin protects the luciferase, due to its low concentration, from denaturation is unlikely since the en/yme preparation at this stage of purification contains from 20 to 30 milligrams of protein per 


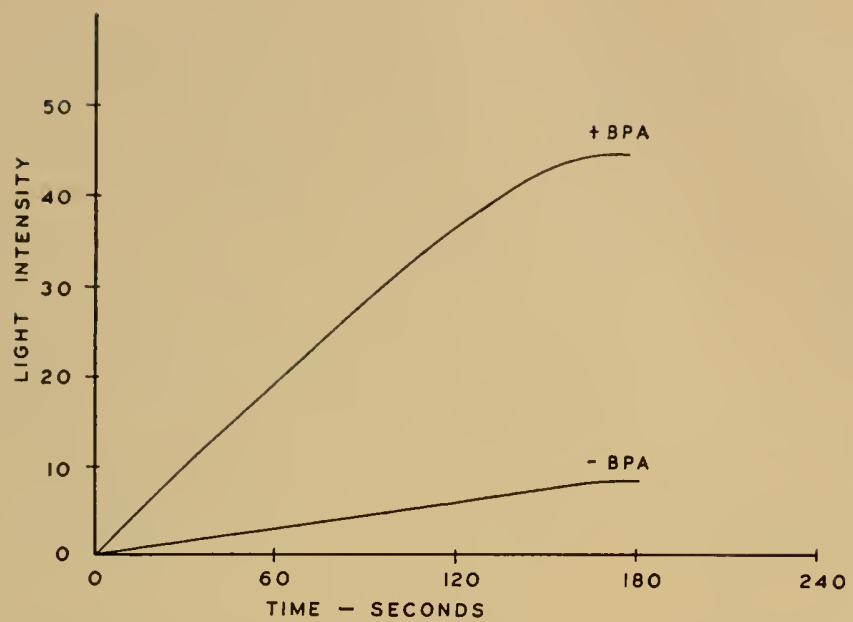

Fig 2. Effect of crystalline bovine plasma addition on fungal bioluminescence in vitro. Luciferase $\left(0-75 \% \quad\left(\mathrm{NH}_{4}\right)_{2} \mathrm{SO}\right.$, fraction) $3.5 \mathrm{mg}$; luciferin, $0.20 \mathrm{ml}$; DPNH,

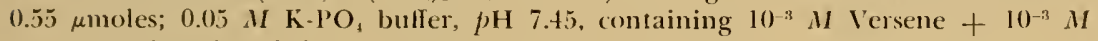
mercaptoethatol to bring the linal volume to $3.0 \mathrm{ml}$.

milliliter. Fig. 3 indicates that luciferase can be titrated with crystalline bovine plasma albumin. The luciferase preparation was completely inactive until the albumin was added. Both the maximum light intensity and the change in the initial rate have been plotted, and, on the whole, these two characteristics of the system behave in a similar fashion. It has been found, in regard to both light emission and the rate of increase in light emission, that an albumin to enzyme-protein ratio of about 5 gives optimal activity. Ratios greater than 5 tend to inhibit the reaction. The above could be ex. plained on the basis of adsorption by the plasma albumin of an inhibitor from the reaction mixture.

The source of this inhibitor could be the hot water extract or the enzyme preparation or both. Attempts to verify such an interpretation of the data and the source of the presumed inhibitor have been indecisive. Heating the albumin to $100^{\circ} \mathrm{C}$ for 3 minutes before use completely eliminates its stimulatory effect. Preincubation with the luciferin has no effect, and subsequent boiling of this preincubation mixture necessitates further addition of the albumin for stimulation. This latter case is inconclusive, however, for, if a protein inhibitor complex is formed during preincubation, it may be broken down during subsequent heating. Likewise, preincubation of the albumin and huciferase gives no data indicative of the adsorption 


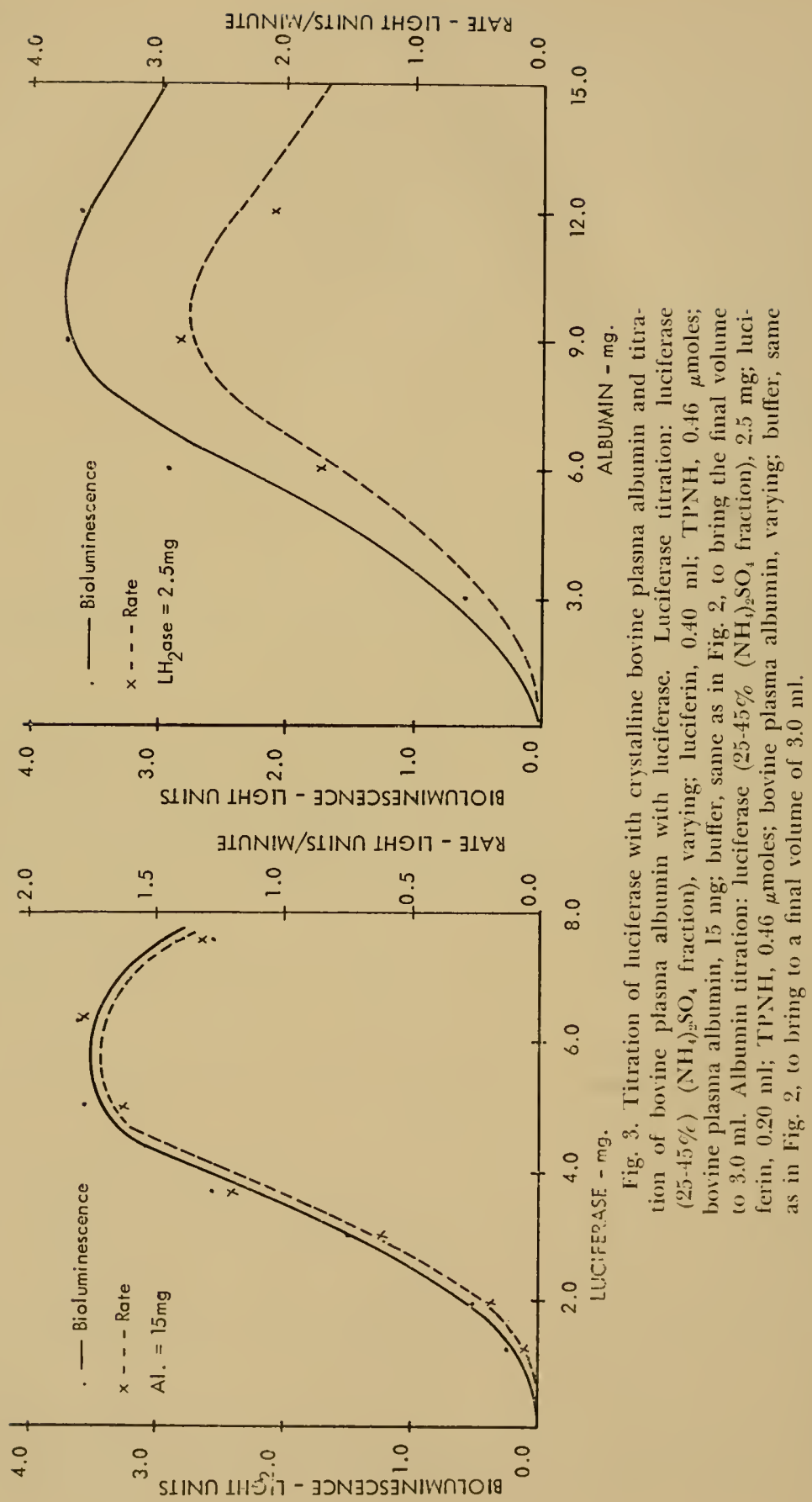


of an inhibitor. During the course of this type of experiment, secondary additions of bovine plasma albumin also were tested. Results of one of these experiments are presented in Fig. 4.

An enzyme preparation that had some activity was assayed with albumin which was added either before the reaction was initiated with DPNH or after 4 minutes. Upon secondary addition of plasma albumin (curve $A$ ), there was an immediate increase in light intensity to a value approximating that of curve $B$-in which case the albumin was added before the reaction was started. The gradual increase in light intensity with time in the absence of albumin (Fig. 1) may be explained on the basis of (a) increase in the amount of substrate-luciferin, (b) increase in the amount of active enzyme, (c) production of some component (s) - other than luciferin-essen-

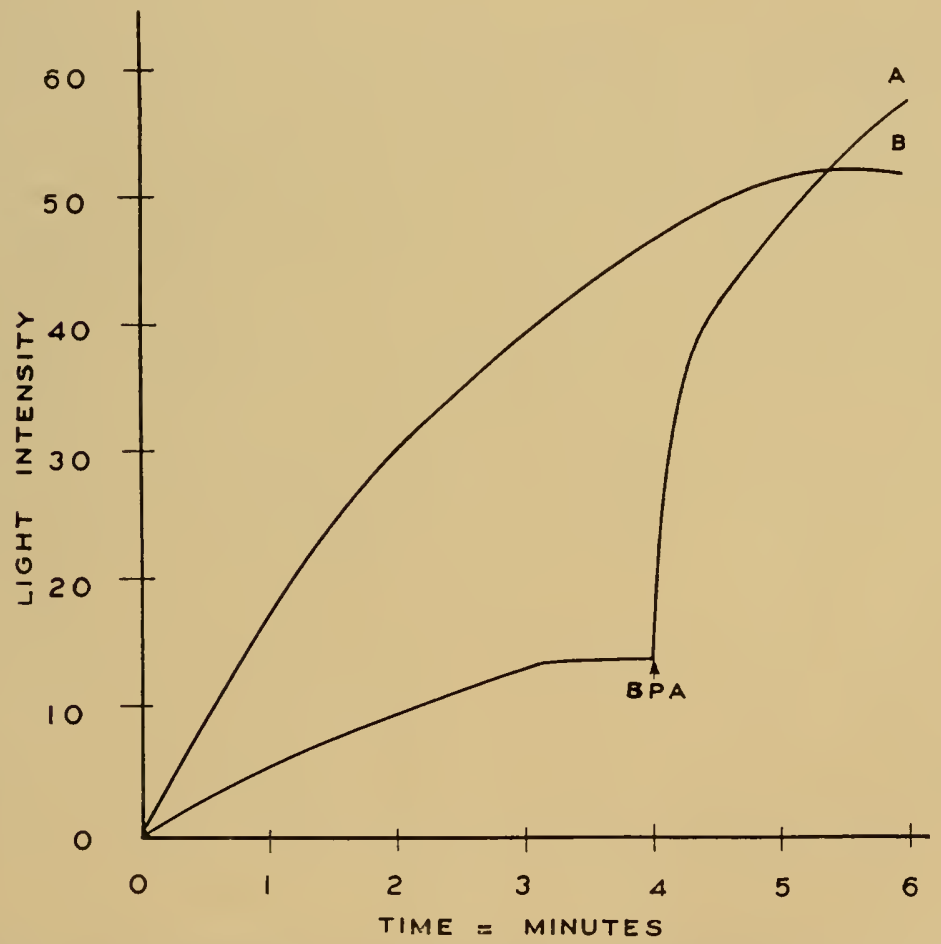

Fig. 4. Effect of secondary crystalline bovine plasma adclition on fungal bioluminescence in vitro. Reaction conditions: $B$ : bovine plasma albumin, 12 ng; luciferase $\left(0-75 \% \quad\left(\mathrm{NH}_{4}\right)_{2} \mathrm{SO}_{4}\right.$ fraction), $3.5 \mathrm{mg}$; luciferin, $0.10 \mathrm{ml}$; DPNH, 0.46 umoles; buffer, same as in Fig. 2, to bring to a final volume of $3.0 \mathrm{ml}$. $A$ : Same as $B$, except that the albumin was added 4 minutes after the reaction was initiated with DPNH. 
tial for light emission, or (d) a combination of the above three. There is no a priori reason for assuming that the hot water extract contains luciferin. For example, this fraction could be oxyluciferin and the function of the reduced pyridine nucleotide to reduce it to luciferin. On the other hand, the pyricline nucleotide requirement may be for the recluction of some component other than oxyluciferin. Experimental evidence for selecting between these two possibilities is lacking. The experiments with albumin strongly suggest that not only is possibility (a) or (c) taking place but also that the enzyme preparation is inhibited also. The results presented in Fig. 4 may be explained in the following manner. When bovine plasma albumin is present during the entire course of the reaction, the intensity of light emission is determined by some factor other than the amount of active luciferase. The results of curve $A$ conform to the following interpretation. The reactions leading to components necessary for light emission are not affected by albumin, and they proceed at the same rate either in its presence or absence. However, the reaction catalyzed by luciferase and leading to light emission is ratelimiting due to enzyme inhibition, and hence the unknown components accumulate. The secondary addition of bovine plasma albumin effectively increases the concentration of the luciferase by reacting with the presumed inhibitor and consequently there is an immediate increase in light intensity. If this suggestion is valid, curve $A$ inclicates that the time necessary for the adsorption of the inhibitor is comparatively short. In subsequent experiments similar results have been obtained upon the secondary addition of the enzyme preparation. These results are amenable to the same interpretation. In this case more luciferase is being added to the reaction mixture. Postulation of rate limitation in the presence of albumin to unknown reactions previous to light emission is preferred since the velocity constants of all known luciferin-luciferase reactions are extremely rapid, thus accounting for the "flash" of light seen on mixing these reactants. If enzymes other than luciferase are involved in light emission from these preparations, they are not separable by the purification procedure outlined above.

The nature of the albumin response is somewhat comparable to that found in the reduction of cytochrome $\mathrm{c}$ by cytochrome c reductase. Nason et al. (10) found that the addition of $\alpha$-tocopherol stimulated cytochrome c reduction in a manner similar to crystalline bovine serum albumin. Acklition of bovine plasma albumin, isooctane-extracted in a mammer similar to that described by Nason $(10)$, to the 
mold bioluminescent reaction did not result in loss of the albumin effect. Atso, neither $\alpha$-tocopherol, $\alpha$-tocopherol aretate, nor menadione were found to stimulate light entission. Addition of antimycin $A$, at a final concentration of 5 micrograms per milliliter, had no effect on a reaction $\mathrm{rmm}$ in the presence of allumin. Dialysis of the bovine plasma albumin at $p H$ 7.0, previous to use, had no effect on its stimulatory activity. Indeed, if the light reaction is carried out with the albumin contained in clialyzing tubing, there is no stimulation. The actual function of the bovine albumin is unknown. Similar stimulatory effects have bcen found in other systems upon addition of amino acids, chelating agents, calcium phosphate gel, or protein $(2,3,4,7)$. Such effects could be, and have been variously ascribed to (a) adsorption of an inhibitor, (b) non-specific effects on the steric relationship of the reactants, or (c) addition of a component other than protein acting as the stimulating agent.

As noted, both the biolmminescent systems of the bacteria and firefly have presented the problem of luciferase inhibition during the intial stages of isolation. McElroy et al. (8) found, during the purification of the bacterial system, two effects of irradiating various enzyme fractions with ultraviolet light: (1) photo-destruction of an inhibitor-presumably flavin mononucleotide (FMN), and (2) production of an essential factor in the 20-30\% $\left(\mathrm{NH}_{4}\right)_{2} \mathrm{SO}_{4}$ enzyme fraction. In view of these results, work was initiated concerning the response of the luminous fungi to ultraviolet light.

In Fig. 5, the response of a slant of the luminous mold Armillaria mellea to ultraviolet light (Black Ray 366OA Lamp) is presented. This experiment was carried out with the slants in a $22^{\circ} \mathrm{C}$ water bath and with adequate controls to assure that the effects measured were light, and not themal, effects. During exposure the luminescence decreased markedly. When the ultraviolet light source was removed, there was a rapid increase in light emission, finally reaching a value approximately 70 per cent greater than the original steady state. The results suggest that there may be present in the luminous system in vivo: (a) a photo-labile component which is essential for light emission; and (b) a component, also photo-labile, that normally inhibits the emission of light from this organism. A similar experiment was carried out in which both the hot water extract (luciferin) and a $0-75 \% \quad\left(\mathrm{NH}_{4}\right)_{2} \mathrm{SO}_{4}$ enzyme preparation were exposed to ultraviolet light for varying periods of time. The solutions were irradiated at $5{ }^{\circ} \mathrm{C}$. In the dark controls, the enzyme and the hot water extract were placed in separate beakers that had been 


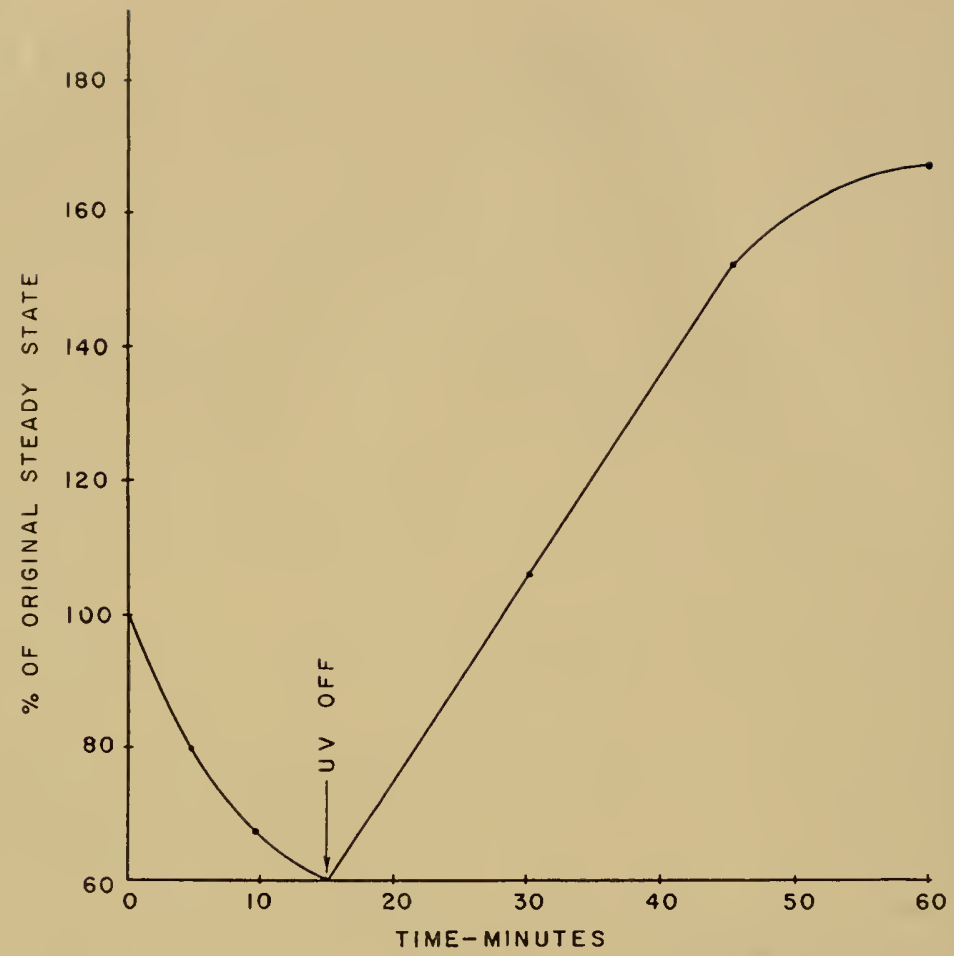

Fig. 5. Effect of ultraviolet light $(3660 \mathrm{~A})$ on a slant of Armillaria mellea. Culture was grown on $5 \%$ mycophil agar $+0.5 \%$ reast extract at $p \mathrm{H} 6.0$ for 9 days at $18^{\circ} \mathrm{C}$. Light source 4 inches from slant.

wrapped with aluminum foil. The assays were conducted in the following mammer. When irradiated luciferase was used as the enzyme, the clark control luciferin was used as the substrate; when irradiated luciferin was the substrate, dark control enzyme was used as the catalytic source. In one set of reactions both the irradiated enzyme and substrate were used in the assay. Results of these assays are presented in Table 1.

With the reactants exposed for approximately 80 minutes, the values obtained for the 60-minute dark control have been used as the basis for comparative calculations. Where the reactants were exposed to ultraviolet light for approximately 145 minutes, the 128-minute dark control values have been used as a base for comparative calculations. The values given are the maximum light intensities attained in terms of light units ( $\mathrm{LU}$ ) per milligram of enzyme protein $\left(\mathrm{I}_{\mathrm{mnx}}\right)$. Also presented are the values for the increase in the initial rate of 
TABLE 1

The EfFect of Ultraviolet Ligut (3660A) on fungal Luciferin AND LUCIFLRASE:

Reaction conditions: luciferin, $0.20 \mathrm{ml}$; luciferase $\left(0-75 \%\left(\mathrm{NH}_{4}\right)_{2} \mathrm{SO}_{4}\right.$ fraction) $2.2 \mathrm{mg}$; crystalline bovine plasma albumin, $10 \mathrm{mg}$; DPNH, $1.0 \mu$ mole; $1 \%$ Tween 80 , $0.1 \mathrm{ml}$; buffer, same as Fig. 2, to bring to a final volume of $3.0 \mathrm{ml}$.

\begin{tabular}{|c|c|c|c|c|c|}
\hline $\begin{array}{l}\text { Length of } \\
\text { UV cxposure } \\
\text { minutes }\end{array}$ & Reactant exposed & $\begin{array}{c}\mathbf{I}_{\max } \\
\mathrm{LU} / \mathrm{mg} \\
\text { protein }\end{array}$ & $\begin{array}{c}1 \% \\
\text { change }\end{array}$ & $\begin{array}{c}\text { Rate } \\
\mathrm{LU} / \mathrm{min} . / \mathrm{mg} \\
\text { protein }\end{array}$ & $\begin{array}{c}\% \\
\text { change }\end{array}$ \\
\hline 60 & dark control & 8.95 & 0 & 2.48 & 0 \\
\hline 128 & dark control & 9.70 & 0 & 2.60 & 0 \\
\hline 77 & luciferase & 12.3 & +34 & 3.72 & +50 \\
\hline 147 & luciferase & 12.0 & +27 & 3.36 & +29 \\
\hline 70 & luciferin & 2.98 & -75 & 1.49 & -60 \\
\hline 130 & luciferin & 2.94 & -77 & 0.60 & -77 \\
\hline 87 & luciferin and luciferase & 2.24 & -81 & 0.36 & -90 \\
\hline 155 & luciferin and luciferase & 1.49 & -81 & 0.41 & -89 \\
\hline
\end{tabular}

the reaction in terms of increase in light units per minute per milligram of enzyme protein. Although the percentage changes in these values are not identical, they do suggest a common trend.

The lability, on exposure to ultraviolet light, of the active component in the hot water extract-whether oxyluciferin or luciferinis quite apparent from these data. It has been found that exposures as short as 15 minutes will reduce the activity by approximately the same amount. Experiments have been conducted to determine whether irradiated hot water extracts inhibit a reaction carried out with non-irradiated substrate and enzyme. This does not appear to be so.

The data obtained with irradiated luciferase are not as convincing as those discussed above. There is certainly a suggestion of stimulation, and this aspect is being studied further. The response of the mold to ultraviolet light is quite similar to that obtained with the bacterial system. The similarity is even more striking when the results presented in Table 2 are considered. Both systems are inhibited by flavin derivatives. In fungi this is not a matter of inhibition due to the limiting hydrogen donor-DPNH. Contrary to the bacterial system, however, the fungal system is not stimulated by the addition of either recluced flavin mononucleotide $\left(\mathrm{FMNH}_{2}\right)$ or flavin adenine dinucleotide $\left(\mathrm{FADH}_{2}\right)$. The flavins were reduced by the hydrogen paladium asbestos method. Here, inhibition could be due to the presence of oxidized flavin. The bacterial and mold systems also differ in that the latter in vitro reaction is not stimulated by n-dodecylaldehyde. 
Inhibition of the fungal luminous system in vitro by such compounds as cyanide, 8-hydroxyquinoline, and ortho-phenanthroline suggests a metal requirement, and this matter is being investigated.

In conclusion, results so far confirm the fact that under certain conditions it is possible to conduct in vitro light reactions of the classical luciferin-Iuciferase type for the fungi. The hot water extract used in these reactions may represent oxyluciferin, and this could be reduced-presumably by reduced pyridine nucleotide-to luciferin, the latter reacting with luciferase for emission of light. The number of enzymes involved in this reaction sequence is un-

TABLE 2

I.MHIBTORS OF FUNGAL BIOLEMINISCFNCF IN VITRO

\begin{tabular}{lcc}
\hline \multicolumn{1}{c}{ Inhibitor } & $\begin{array}{c}\text { Concentration } \\
\text { (Molar) }\end{array}$ & $\begin{array}{c}\%_{\text {inhibition }} \\
\text { of } \mathbf{I}_{\max }\end{array}$ \\
$\mathrm{KCN}$ & $3 \times 10^{-4}$ & 56 \\
& $1 \times 10^{-3}$ & 70 \\
ortho-phenanthroline & $2 \times 10^{-3}$ & 76 \\
8-hydroxyquinoline & $1 \times 10^{-3}$ & 49 \\
$\mathrm{FMN}$ & $1 \times 10^{-3}$ & 88 \\
$\mathrm{FAD}$ & $7 \times 10^{-6}$ & 26 \\
$\mathrm{FMNH}_{2}$ & $5 \times 10^{-5}$ & 25 \\
$\mathrm{FADH}_{2}$ & $3 \times 10^{-5}$ & 51 \\
$\mathrm{NaN}_{3}$ & $5 \times 10^{-5}$ & 37 \\
$\mathrm{NaAsO}_{2}$ & $1 \times 10^{-3}$ & 0 \\
$\mathrm{Na}_{2} \mathrm{HAsO}_{4}$ & $1 \times 10^{-3}$ & 0 \\
& $1 \times 10^{-3}$ & 0 \\
\hline
\end{tabular}

known. The results obtained on the secondary addition of bovine plasma albumin suggest that there are at least two. This matter should be resolved by further purification of the enzyme system. Along the same line, there is no indication of a pyridine nucleotide specificity, but the presence of a transhydrogenase is quite conceivable. In several respects the mold system is not comparable with the bacterial system: (a) addition of reduced flavin mononucleotide does not stimulate the fungal bioluminescent system; (b) long-chain aldehydes are not required; and (c) the emission maximum of the luminous mold in vivo is at $530 \mathrm{~m} \mu$ while that of Photobacterium fischeri is at approximately $480 \mathrm{~m} \mu$. The excitation energy requirement for the fungal bioluminescence would be approximately 55 kilocalories per mole quanta rather than the 60 kilocalories required by the luminous bacteria. 


\section{REFERENCES}

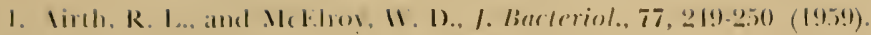

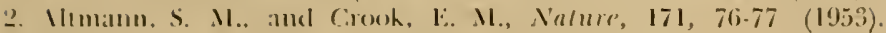

3. Ball, E. C., and Cooper, Detalia, J. Biol. Che'll., 180, 113-124 (1919).

4. Bommer, W. D., Nalure. 165, 757-758 (1950).

i. Cross, Wr, Challengers of the Derep. William Sloan Issociates, New York (1959).

6. Harvey, E. N., Bioluminescence, Icademic l'ress, New York (1952).

7. Keilin, D., and Hartree, l.. F., Biochem. J., 44, 205-218 (1919).

‥ McEloy, W. D., Hastings, J. W., Sommenteld, Valeric, and coulombre, Jane. J. Bacteriol., 67, 402-408 (1954).

9. McElroy, W. J., pers. commun.

10. Nason, A., Atuerbach, Bella C., and Terrell, Imn J., Biochim. el Biophys. Acla, 19, 395-397 (1956). 


\title{
LUMINESCENCE ${ }^{1}$
}

\author{
Milton J. Cormier \\ Department of Chemistry, University of Georgia, Athens, Georgia, \\ and the University of Georgia Marine Institute, \\ Sapelo Island, Georgia
}

\section{INTRODUCTION}

During the last ten years, our knowledge of the biochemical factors involved in bioluminescent reactions and their mechanisms has increased considerably. From the classical work of E. N. Harvey and his associates (7) on Cypridina luminescence, a description of the factors involved in firefly (11) and bacterial luminescence (4, 5, $8,15)$, and their mechanisms, has arisen. More recently, one of the components involved in fungal luminescence has been described (1).

Aside from the four systems mentioned above, no detailed report has appeared on the biochemistry of any other luminescent system. The author therefore feels that it may be of general interest to describe some recent work on the luminescent system of the sea pansy, Renilla reniformis.

Luminous extracts of the sea pansy have been prepared, and certain factors involved in this system have been identified. The system is an interesting one since it differs biochemically from other known bioluminescent reactions.

In fact, one of the striking features about these luminescent systems is that, in general, they differ biochemically. This is true for the components involved, and for the mechanisms of the reactions. It would be more unusual, it seems, to find luminescent systems that are biochemically identical, rather than different, among the various luminous species. Thus biochentical evidence is beginning to mount to support the general observation that luminous forms have appeared sporadically during evolution and have not evolved along the "same line."

Some of the data given below are based on a preliminary report (2) and a more detailed manuscript now in press (3).

${ }^{1}$ This work was supported in part by the National Science Foundation and is contribution No. 25 from the University of Georgia Marime Institute, Sapelo Island, Gcorgia. 
P'RIPARATION OF EXTRACTS ANU OF CRUDE LUCHFERIN

\section{Extracts}

Freshly collected sea pansies were placed in a dark, ruming seawater aquarium for 4 to 8 hours. Since these animals have a diurnal rhythm of luminescence $(7)$, this procedure was necessary to rejurenate the luminescence system. Approximately 50 animals at a time were destemmed and dropped into a cold mortar containing $10 \mathrm{ml}$ of saturated ammonium sulfate, $p \mathrm{H} \mathrm{7.5}$. They were cut into small pieces with scissors and finally ground with sand for 30 minutes. A total of $200 \mathrm{ml}$ of saturated ammonium sulfate was gradually added with stirring, and the suspension was centrifuged at $12,000 \times g$ for 10 minutes. The precipitate was carefully drained to remove excess ammonium sulfate, and then extracted by grinding in a mortar with

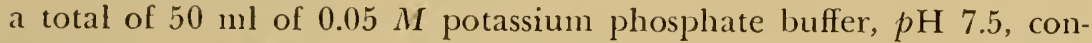
taining $0.001 M$ glutathione (phosphate-GSH buffer). The extract was centrifuged for 10 minutes at $12,000 \times g$, and the resulting supernatant centrifuged for 30 minutes at $140,000 \times g$. The latter centrifugation yields a clear, amber-colored supernatant solution which contains the activity and will be referred to as the crude extract. The activity of the extract was preserved by storing at $-20^{\circ} \mathrm{C}$. The grinding, centrifugation, and extraction procedures were done at 0 to $3^{\circ} \mathrm{C}$.

For the experiments reported here, unless otherwise indicated, the crude extract was precipitated once with saturated ammonium sulfate, $p H 7.5$ (to 75 per cent saturation), and the precipitated protein was redissolved in phosphate-GSH buffer.

Treatment of the animals with ammonium sulfate is an essential feature of the extraction process. Grinding rejuvenated organisms with sand in the presence of phosphate-GSH buffer without ammonium sulfate treatment does not yield an active extract. Ammonium sulfate produces at least two observable effects: first, it allow's a greater amount of protein to be extracted; and second, it acts as an anesthetic and thus prevents an excessive luminescent response during grinding, such as would result in oxidation of Renilla luciferin.

\section{Luciferin}

The once-extracted Renilla residue was reextracted with phosphateGSH buffer in the manner described for preparation of the crucle extract, except that centrifugation at $140,000 \times g$ was not required. 
This extract was heated at $100^{\circ} \mathrm{C}$ for $1 \frac{1 / 2}{2}$ minutes, cooled to room temperature, and the denatured protein then removed by centrifugation. The supernatant solution contained Renilla luciferin, which can be stored at $-15^{\circ} \mathrm{C}$ for weeks without significant loss of activity.

\section{Assay SYSTEM}

Unless otherwise specified, the luminescence assay system consisted of the following additions: potassium phosphate buffer, $p \mathrm{H} 7.5$ (50 $\mu$ moles) ; GSH (2 $\mu$ moles) ; ATP, ADP, or AMP ( $5 \mu$ moles); Renilla luciferin $(0.2 \mathrm{ml})$; enzyme $(0.5 \mathrm{mg}$ protein); and water to $1.5 \mathrm{ml}$. In the experiments reported, ADP was used in preference to ATP, since the latter compound functions indirectly in this system. All reactions were carried out at $30^{\circ} \mathrm{C}$ unless otherwise stated.

\section{Properties and Characteristics of the System}

\section{Requirement for Adenine-Containing Nucleotides}

Crude extracts from $R$. reniformis give a relatively weak luminescence that is stimulated approximately 10- to 30 -fold (depending upon the extract) upon addition of ATP, ADP, or AMP. These results are presented in Table 1. Enzyme preparations can be obtained, by repeated precipitations with saturated ammonium sulfate, that are stimulated 100 -fold upon the addition of adenine-containing mucleotides. Adenosine is inactive in this system, as well as the mono-

TABLE 1

Effect of Nucleotides on the Luminescence of $R$. reniformis Extracts

\begin{tabular}{ccc}
\multicolumn{1}{c}{ Experiment } & \multicolumn{1}{c}{ Addition } & Relative Light Intensity \\
\hline 1 & None & 16 \\
ATP & 445 \\
ADP & 390 \\
ANP & 440 \\
Adenosinc & 16 \\
GTP, CTP, or UTP & $16 \pm 1$ \\
& GMP, CMP, or UMP & $16 \pm 1$ \\
& None & 20 \\
& ADP & 450 \\
GDP & 23 \\
& GDP & 36 \\
& UDP & 122
\end{tabular}

Conditions: Same as described under assay system. $5 \mu$ moles of each nucleotide or nucleoside were added as indicated. ATP, ADP, AMP, adenosine triphosphate, diphosphate, and monophosphate, respectively. GT ', CTP, UTP, guanosine, cytidine,

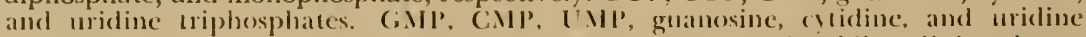

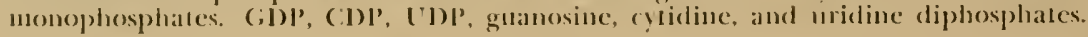


phosphates and triphosphates of guanosine, cytidine, and uridine. The diphosphates of gumosine and cyticline are also inactive, although some activity was exhibited by uridine diphosphate. Whether the latter activity is clue to contumination with adenine-containing nucleotides is not certain, although on the basis of concentration curves (see Fig. 1), a 1 per cent contamination with any one, or a combination, of the adeninc-containing nucleotides would have been suffcient to account for the light observed. Furthermore, since UTP and U.MP are inactive, it appears unlikely that uridine-containing nucleoticles are active in this system. Thus, luminescence in extracts of $R$. reniformis is dependent upon the presence of adenine-containing nucleotides and is specific for the adenine moiety, at least in the initial step of the reaction.

Since light emission in the firefly requires a primary activation by ATP (10), and since the Renilla system responds to either ATP, ADP, or AMP, there appeared to be a good possibility that the two systems were similar with respect to nucleotide requirements. The purity of the ADP and AMP was therefore checked for contamination with ATP. The AMP used in these experiments did not contain ATP as determined by ATP analysis using the firefly technique (13, 17). The sensitive light-detecting apparatus used in these experiments readily allowed the detection of $10^{-3} \mu \mathrm{g} A T P / m l$. Likewise, insufficient amounts of ATP were found in the ADP preparations to account for the light observed. Furthermore, various coinmercial sources of ADP and AMP were found to be equally effective. In addition, the AMP used was not contaminated with ADP, which was measured by coupling adenylate kinase to the firefly system. The activities exhibited by AMP and ADP, therefore, were not due to contamination with ATP.

The possibility existed, however, that the AMP and ADP activities could be attributed to phosphorylations in the crude extract leading to the formation of ATP. This possibility can also be ruled out, since AMP and ADP are fully effective in a phosphate-free system, as well as in the presence of 2,4-dinitrophenol (10-2 $M$, final concentration). The method of Fiske and Subbarow (6) was used to determine the absence of inorganic phosphate in these experiments. In addition, adenylate kinase could not be demonstrated in the crude extracts since, under anaerobic or aerobic conditions, no ATP formation could be detected from ADP using the firefly assay. Thus, the AMP and ADP activities are not due to contamination with ATP or to their conversion to ATP. 
At this point it is significant to note that crude extracts contain a phosphatase (s) that causes the liberation of inorganic phosphate from ATP and ADP. In one experiment, for example, it was found that a preparation containing $1.5 \mathrm{mg}$ protein $/ \mathrm{ml}$ caused the release of 0.5 and $1.0 \mu$ mole of $P_{i}$ per 10 minutes from ADP and ATP, respectively, under the experimental conditions used. Under conditions that support luminescence, then, these extracts apparently convert $\mathrm{ATP}$ to $\mathrm{ADP}$, whereas $\mathrm{ADP}$ is apparently converted to AMP. That these products are actually formed via phosphatase action was confirmed by separation and identification of the nucleotides on formate-charged Dowex-1 columns, as described by Hurlbert et al. (9). The fact that ATP can be broken down to ADP and AMP in the crude extracts, whereas AMP or ADP are not converted to ATP, suggests that ATP functions as a generating system for ADP and AMP and that one (or both) of the two latter nucleotides is directly responsible for driving the light reaction. This suggestion is now known to be the correct interpretation, and the supporting data are given later.

The effect of nucleoticle concentration on the luminescence of Renilla extracts is illustrated in Fig. 1. The data plotted in Fig. I show that ATP is more effective than ADP or AMP at the lower con-

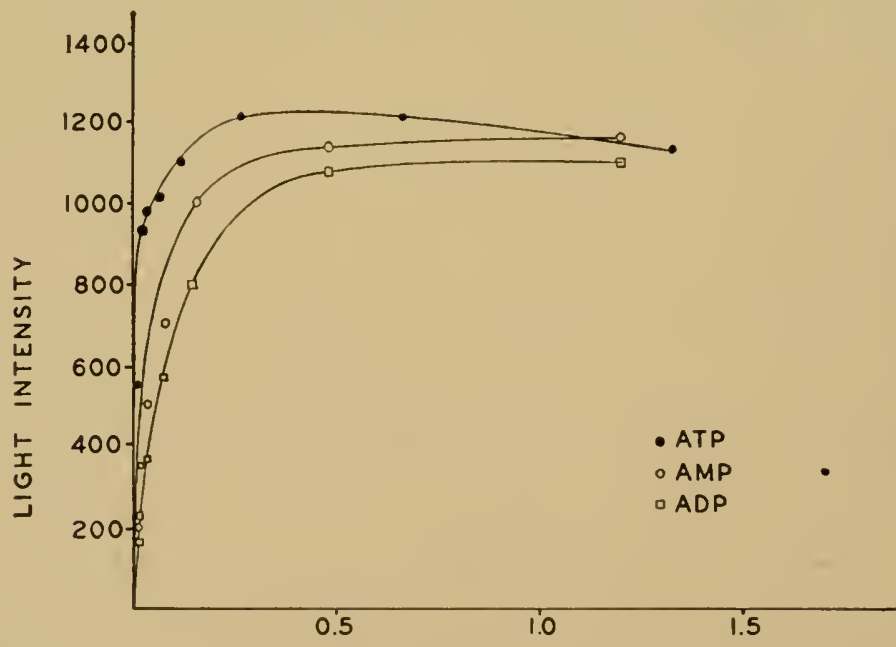

NUCLEOTIDE CONCENTRATION ( $\mu$ molos/ml)

Fig. 1. Effect of nucleoticle concentration on the luminescence of Renilla reniformis extracts. 
centrations, and that the response obtained with ADP is somewhat less than that with AMP. The higher efficiency of ATP at lower concentrations is also shown by calculation of the apparent Michaclis constant, which, for the ATP reaction, is approximately one-fifth that of the ADP- or AMP-stimulated reactions. Since generating systens are frequently more efficient than substrate levels of a reactant, the higher efficiency of ATP could be explained on the basis that it acts as a generating system for ADP and AMP.

Requirement for Luciferin and Oxygen

Since other bioluminescence systems require an oxidizable substrate, termed luciferin, for luminescence, an attempt was made to demonstrate the necessity for luciferin in this system. The requirement for an additional factor became apparent by studying luminescence decay as a function of time. Fig. 2 shows that, at the end of approximately 70 minutes, the luminescence rate decays to a negligible value that is not affected by the addition of nucleotides. Although ADP was added in this experiment, ATP and AMP are also without effect. Nucleotides are required, however, to bring about the luminescence decay. For example, an extract incubated 70 minutes

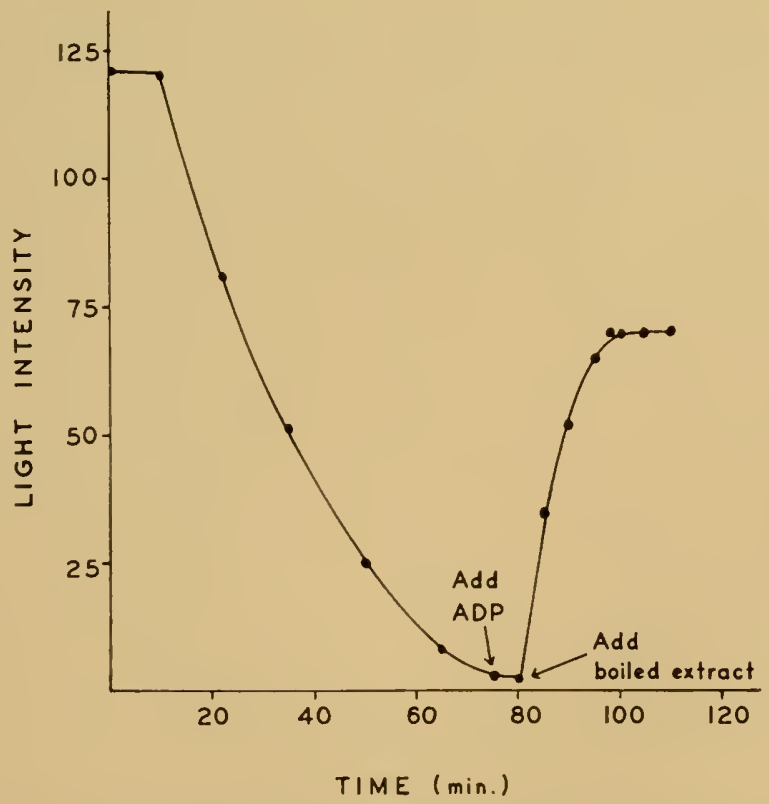

Fig. 2. Effect of boilerl extract on luminescence after luminescence decay. 
in the absence of nucleotides responds to nucleotide addition in a manner analogous to non-incubated extracts. The addition of boiled extract promotes a rapid return to a steady-state value, which depends upon the amount of boiled extract added. Thus, the decay of luminescence is brought about as the result of a nucleotide-dependent utilization of some unknown factor required for luminescence. The factor in the boiled extract, which has been found to be dialyzable, will be referred to as Renilla luciferin by analogy to other bioluminescence systems.

Oxygen, as well as nucleotides, is required for luciferin utilization, since a decrease in luminescence activity does not occur by incubation of the complete system under anaerobic conditions. The effect of oxygen on the luminescence of Renilla extracts is illustrated in Fig. 3. Apparently, normal oxygen tension is not sufficient to saturate this system, since flushing with oxygen causes an increase in the steady-state luminescence rate, whereas flushing with air does not. The light is essentially abolished in two minutes by flushing with nitrogen, and the signal is returned by subsequently flushing with oxygen. The somewhat lower steady-state value following the second oxygen flushing is due to some enzyme inactivation as a result of agitation during the flushing process.

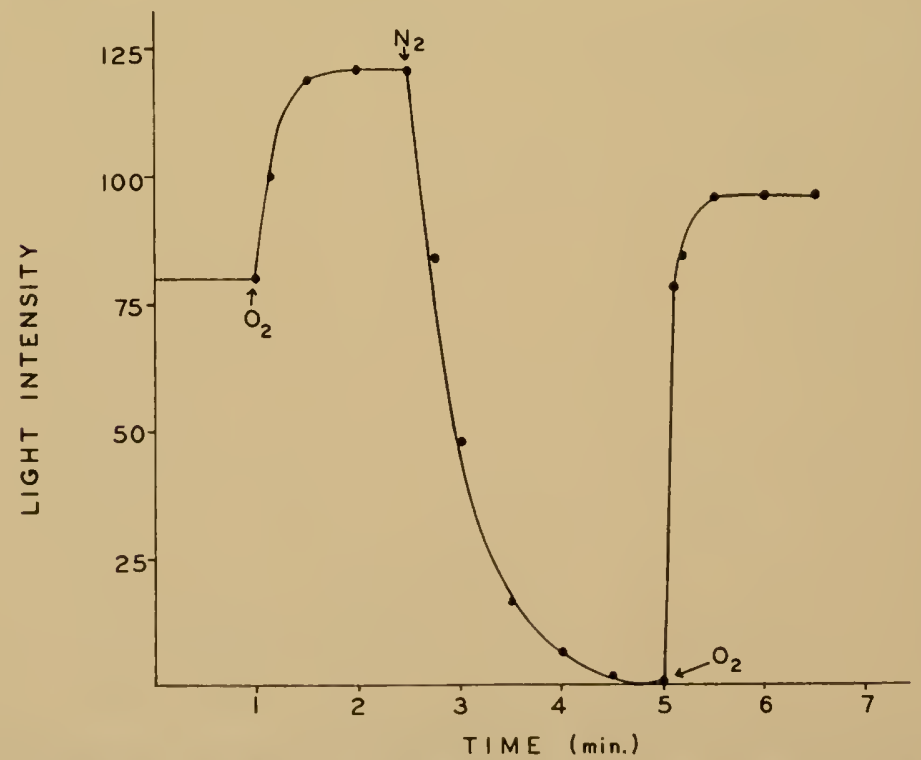

Fig. 3. Elfect of oxygen on luminescence. The system was flushed alternately with oxygen and nithegen, as indicated. 
Thus the luminescent reaction involves a nucleotide-dependent oxidation of luciferin. Indeed, some preliminary observations on the properties of luciferin indicate that it is oxidizable under certain conditions. For example, luciferin activity is destroyed by heating at $100^{\circ} \mathrm{C}$ for 1 minute in the presence of $0.06 \mathrm{~N} \mathrm{HCI}$ and oxygen, but is stable at this temperature and $\mathrm{HCl}$ concentration providing the system is kept anaerobic.

An enzyme system whose luminescence has been allowed to decay to a negligible level in the presence of nucleotide can be treated with ammonium sulfate to precipitate the enzyme, and the latter (resolved enzyme) can be used as an assay for the unknown factor. When this is done, the effect of increasing concentrations of boiled extract on the light intensity can be studied. The results (Fig. 4) show that 80-fold stimulations of luminescence were observed upon the addition of sufficient amounts of crude luciferin. No increase above background is observed in the absence of added nucleotide. The apparent saturation at higher levels may be due to inhibitors of luminescence in the boiled extract. Luciferin camnot be replaced by divalent cations such as magnesium or manganese and many others that were tested. No luciferin activity could be demonstrated upon the addition of long-chain aldehydes which are involved in bacterial

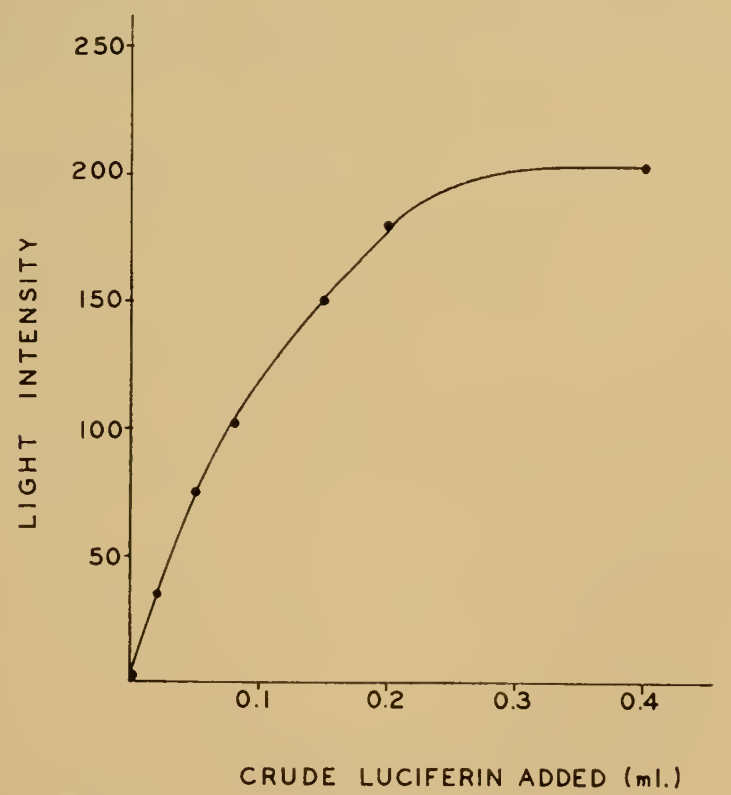

Fig. 4. Effect of luciferin concentration on luminescence. 
luminescence, yeast concentrates, or of crude extracts from beef or rat liver, heart, kidney, or spleen. After each of these additions, the luminescence system would still respond to the addition of boiled Renilla extract. Thus Renilla luciferin cannot be demonstrated in a variety of naturally occurring materials. Similar observations have also been made in this laboratory for fungal and firefly luciferins.

\section{Specificity of Renilla Luciferin and Luciferase}

Luciferin preparations from a number of bioluminescent systems were tested for activity in the Renilla luminescence system. Table 2 shows that firefly, Cypridina, bacterial, or fungal luciferin will

\section{TABLE 2}

Effect of Various Luciferin Preparations on the

Luminescence of Renilla Extracts

Source of Luciferin

Preparation Added

Light Intensity

$\begin{array}{lr}\text { None } & 0 \\ \text { Renilla } & 60 \\ \text { Firefly } & 0 \\ \text { Cypridina } & 0 \\ \text { Bacterial }\left(\mathrm{FMNH}_{2}\right) & 0 \\ \text { Fungal } & 0\end{array}$

Conditions: Same as described under assay system except that the source of luciferin was that listed in the table, and the enzyme preparation was a resolved enzyme of the type used to obtain the data plotted in Fig. 4.

not replace Renilla luciferin. All luciferin preparations were shown to be active in their respective luminescence systems prior to testing them with Renilla luciferase. Likewise, Renilla luciferin will not react in any of the above-mentioned luminescence systems. Renilla luciferin is specific, therefore, for Renilla luciferase.

Effect of Enzyme Concentration, $p H$, and Temperature

Fig. 5 illustrates the effect of luciferase concentration on luminescence. A typical straight-line response is obtained with increasing enzyme concentration.

The response of this luminescence system to $p \mathrm{H}$ is shown in Fig. 6. The activity declines on the acid side of neutrality, but shows considerable activity over a relatively wide range on the alkaline side. with an optimum at $p H 7.5$.

Fig. 7 illustrates the effect of temperature on luminescence. Under these experimental conclitions, the optimum for this reaction is $32^{\circ} \mathrm{C}$. 


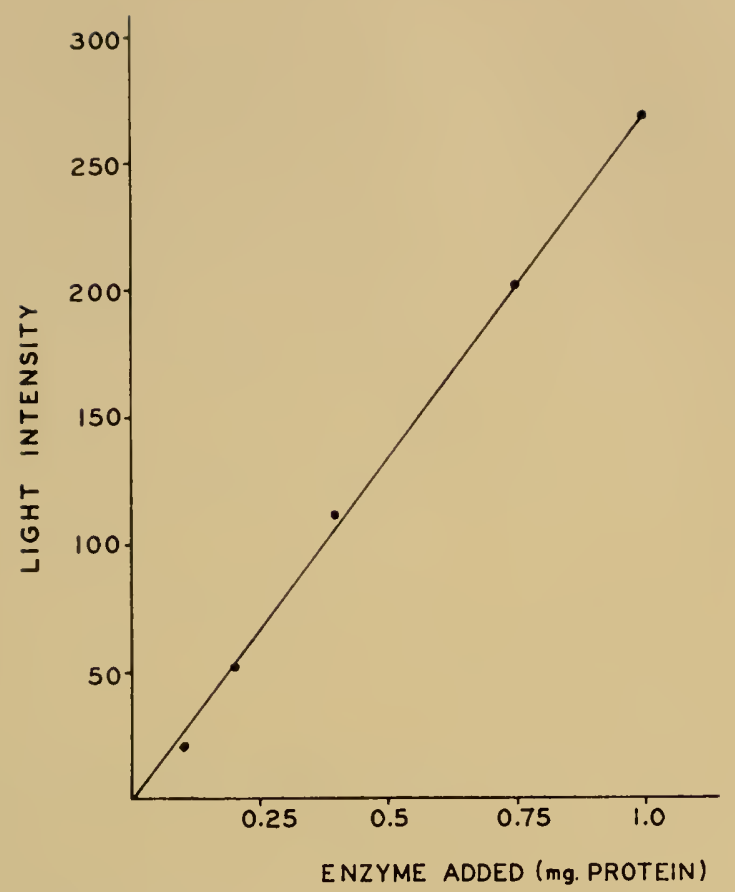

Fig. 5. Effect of enzyme concentration on luminescence.

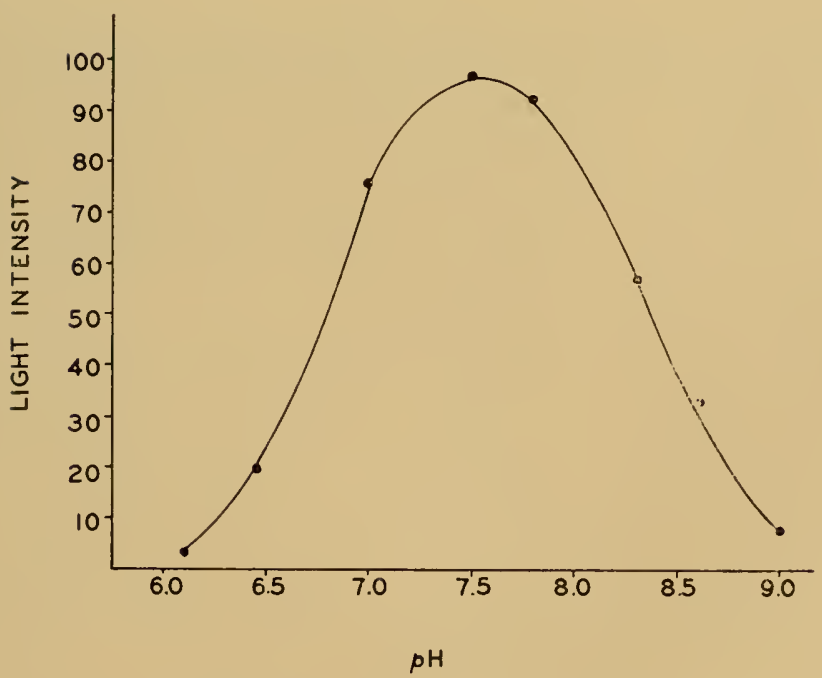

Fig. 6. Effect of $p H$ on luminescence. 


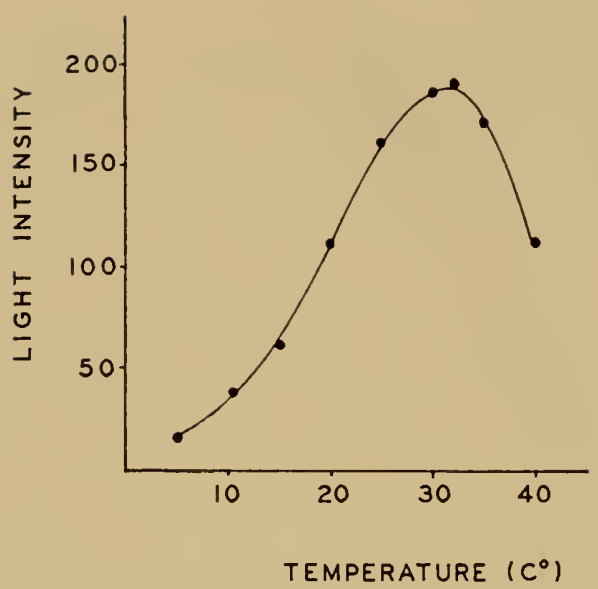

Fig. 7. Effect of temperature on luminescence.

Spectral Energy Distribution

Although the spectral energy distribution of the light from this luminescent reaction has not been determined, the luminescence is visible to the 5-minute dark-adapted eye, and some visual observations have been made. The greenish-blue color of the light in extracts appears to the eye to be the same color as that emitted from the intact animal.

\section{Inhibitor Studies}

A number of well-known inhibitors of other bioluminescent systems, as well as classical enzyme inhibitors, were tested for their inhibitory action on Renilla luminescence. Table 3 show's that potent inhibitors of bacterial luminescence, such as menadione and cytochrome c $(5,13)$, do not inhibit the luminescence of Renilla extracts, and indicates that these compounds do not oxidize Renilla luciferin as they do bacterial huciferin $\left(\mathrm{FMNH}_{2}\right)$.

Fluoride is a partial inhibitor at high concentrations, but only when ATP is added. For example, when ADP is used, fluoride does not inhibit luminescence, as shown in Table 3. Similar results were ubtained with AMP. In the presence of ATP, however, a 33 per cent inhibition was observed. Under the same conclitions, a 34 per cent inhibition of ATPase activity by fluoricle was observed. These observations support the suggestion that $\Lambda T P$ is involved indirectly in this system via an $A T$ Pase reaction.

The respiratory inhibitors cyanicle and azide are noninhibitory at 
the concentrations tested. FMN and FAll) are slightly inhibitory at $10^{-5} \mathrm{M}$ whereas arsenate does not inhibit.

It has been shown, in the case of firefly luminescence, that inorganic pyrophosphatase can adversely affect the steady-state lumincscence (12). Likewise, inorganic pyrophosphate can either inhibit or stimulate luminescence, depending upon whether it is added prior to or after ATP. These results have been explained in terms of a primary ATPdependent activation reaction that results in the formation of luciferyl adenylate and pyrophosphate (14). As seen from Table 3, inorganic pyrophosphate has little or no effect on Renilla luminescence. This is true regardless of the order in which pyrophosphate is added. Furthermore, the addition of inorganic pyrophosphatase (300-600 units) has no effect on luminescence of this system. These observations are in clirect contrast to those made by McElroy et al. (12) on firefly luminescence, and indicates a difference in the mechanism of ATP action in the two systems. In addition, it would be difficult to explain the stimulation of Renilla luminescence by AMP on the basis of identical mechanisms.

A requirement for free sulfhydryl groups in the luminescent system is indicated by the inhibitory action of parachloromercuribenzoate (PCMB), N-ethylmaleimide, and copper and silver salts, as shown in Table 3. The inhilition produced by PCMB can be partially reversed (to $60 \%$ of the initial response) by the addition of $4 \mu$ moles

TABLE 3

EFfect of INHibitors on Renilla LuMinescence

\begin{tabular}{|c|c|c|}
\hline Addition & $\begin{array}{l}\text { Final Molar } \\
\text { Concentration }\end{array}$ & $\begin{array}{l}\text { Per Cent } \\
\text { Inhibition }\end{array}$ \\
\hline $\mathrm{NaF}$ & $10^{-1}$ & 0 \\
\hline Menadione & $10^{-4}$ & 0 \\
\hline Cytochrome c & $10^{-5}$ & 0 \\
\hline $\mathrm{KCN}$ & $10^{-3}$ & 0 \\
\hline $\mathrm{NaN}_{3}$ & $10^{-3}$ & 0 \\
\hline FMN & $10^{-5}$ & 17 \\
\hline FAD & $10^{-5}$ & 8 \\
\hline $\mathrm{Na}_{3} \mathrm{AsO}_{4}$ & $10^{-3}$ & 0 \\
\hline \multirow[t]{2}{*}{ Versene } & $10^{-3}$ & 58 \\
\hline & $10^{-2}$ & 63 \\
\hline \multirow[t]{2}{*}{ Sodium Pyrophosphate } & $10^{-3}$ & 0 \\
\hline & $3 \times 10^{-2}$ & 10 \\
\hline PCMB & $10^{-4}$ & 93 \\
\hline N-Ethylmaleimide & $10^{-4}$ & 31 \\
\hline \multirow[t]{2}{*}{$\mathrm{CuSO}_{4}$} & $10^{-4}$ & 100 \\
\hline & $10^{-5}$ & 85 \\
\hline \multirow[t]{2}{*}{$\mathrm{AgNO}_{3}$} & $10^{-4}$ & 100 \\
\hline & $10^{-5}$ & 63 \\
\hline
\end{tabular}


of glutathione (GSH). In addition, GSH has been found to help maintain luciferase activity during initial purification steps.

Regardless of the nucleotide used in the assay system, a large number of metal ions have no effect on the activity of the crude extract at $10^{-3} M$ concentrations. These include magnesium, manganese, nickel, calcium, cobalt, zinc, molybdenum, and iron. Although metal ions have no effect on the crude extract, chelating agents such as Versene are potent inhibitors (Table 3). Regardless of the nucleotide used in the test system, the inhibitory action of Versene requires approximately 15 minutes incubation with the test system to reach a maximum level. The addition of magnesium ions, in amounts in excess of that of Versene, cloes not reverse the inhibition. Thus, Versene inhibition cannot be explained simply in terms of binding one of these metal ions.

In the absence of phosphate, zinc ion is a potent inhibitor of luminescence. Under these conditions, for example, luminescence is completely inhibited at $2 \times 10^{-3} M$ zinc ion. The lack of zinc inhibition in phosphate buffer is due to the fact that an insoluble zinc phosphate forms at the $p \mathrm{H}$ used in the assay system.

Although the luminous bacteria and sea pansies discussed here are both marine organisms, the properties of the enzymes involved in the two luminescent systems differ considerably in their response

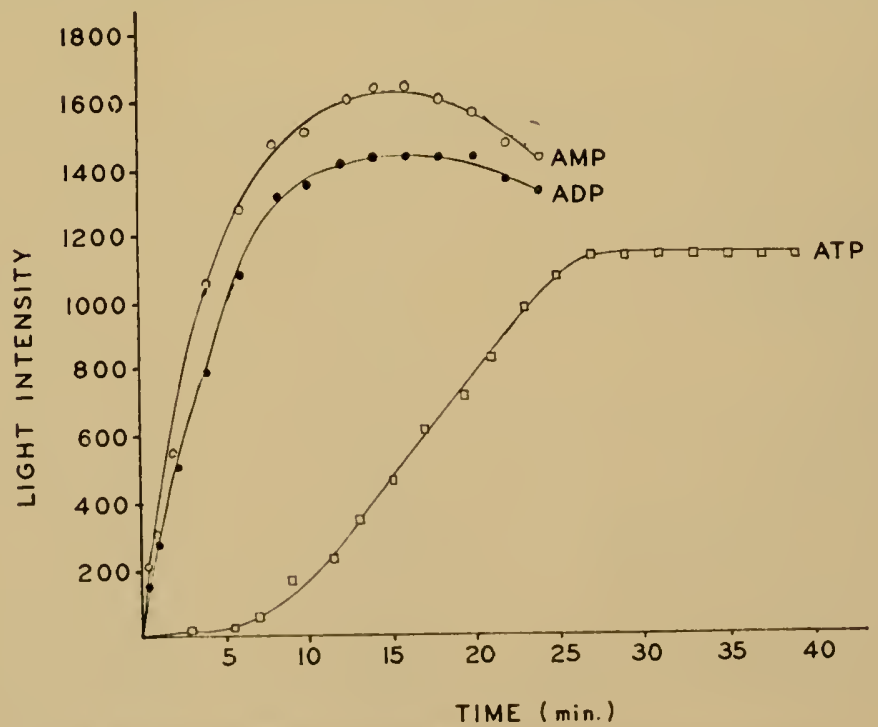

lig. 8. Comparison of the kinetics using different mucleotides. 
to salt. For example, salts such as $\mathrm{NaCl}$ and $\mathrm{KCl}$ inhibit the luminescence system of Renilla. The inhilsition increases linearly with inreasing salt concentration, 50 per cent inhibition being reached at approximately $2 \times 10^{-1} M$ salt. The luminescence of extracts of luminous bacteria, on the other hand, is stimulated by increasing salt concentrations, reaching a maximum steady state at approximately $1.7 \times 10^{-1} M$ salt $(16)$.

\section{Kinetic Data}

Although freshly prepared crude extracts usually do not show significant differences in kinetics between AMP, ADP, and ATP, differences in these kinetics do present themselves when extracts are used that have been repeatedly precipitated two to three times with ammonium sulfate. The latter enzyme preparation, for example, shows large differences in ATP versus ADP or AMP kinetics. Fig. 8 illustrates the results of such an experiment. ADP and AMP kinetics are essentially identical, under these conditions, requiring 3.3 minutes to reach half maximum velocity, whereas the response to ATP is much slower, shows a lag period, and requires approximately 17 minutes to reach half maximum velocity.

In addition to observing kinetic differences with the nucleotides tested, this enzyme preparation also requires a metallic ion activator for ATP, but not for ADP or AMP, activity. Ions of magnesium and manganese are among the best activators, but nickel, cobalt, and calcium ions also show considerable activity. The kinetic experiments described above were therefore done in the presence of magnesium ion. None of these metal ions has any effect on ADP. or AMP-stimulated luminescent reactions. These results, using magnesium ion as an activator, are presented in Table 4. It is of in-

TABLE 4

EFfect of Magnesium Ion on Luminescence in the Presence of Various Nucleotides

\begin{tabular}{lc}
\multicolumn{1}{c}{ Additions } & Relative Light Intensity \\
\hline None & 69 \\
ATP & 34 \\
ATP + magnesium & 320 \\
ADP + magnesium & 360 \\
ADP + & 360 \\
AMP + magnesium & 380 \\
AMP + magn & 380 \\
\hline
\end{tabular}

Conditions: Same as described under assay system, except that $5 \mu$ moles of $\mathrm{MgCl}_{2}$ were added where indicated and the enzyme used was that described in the text. 
terest that the ATPase and ADPase activities are also dependent upon the same metal ions for activity in this enzyme preparation.

The kinetic data, as rell as differences in metal activator requirements for the active nucleotides, lend strong support to data presented earlier in the text which suggested that ATP functions indirectly in this system by acting as a generating system for ADP and AMP via an ATPase reaction. That this is, in fact, the case is clearly shown by experiments using partially purified fractions of Renilla luciferase, the results of which are presented in the following sections.

\section{Preparation of DEAE-cellulose Fractions}

The $0-75 \%$ ammonium sulfate fraction of the crude extract was dialyzed against $0.002 M$ potassium phosphate buffer, $p \mathrm{H} 7.5$, containing $0.001 M \mathrm{GSH}$, for four hours at $0-3{ }^{\circ} \mathrm{C}$. The dialyzed fraction was then absorbed on a $\mathrm{NaOH}$-charged DEAE-cellulose column and eluted off the column with $0.05 M$ potassium phosphate, $p \mathrm{H}$ 7.5. The resulting column fractions showed a 0 to 5 -fold purification based on specific activity measurements using ADP in the assay system.

\section{Properties of DEAE-Fractionated Luciferase}

\section{Phosphatase Content and Nucleotide Requirement}

The data in Table 5 show that the phosphatase activity for both ATP and ADP decreases approximately 10 -fold in the DEAE fractions as compared to the crude extract. Furthermore, when phosphatase activity is lost, the ability to produce light with ATP is

\section{TABLE 5}

Pilospinatase and Nucleotide-Stimulated Luminescence Activities of Crude and DEAE-fractionated Remilla Luciferase.

\begin{tabular}{|c|c|c|c|c|c|c|}
\hline \multirow{3}{*}{$\begin{array}{l}\text { Enzyme } \\
\text { Added }\end{array}$} & \multicolumn{2}{|c|}{ Phosphatase Activity } & \multicolumn{4}{|c|}{ Luminescence } \\
\hline & \multirow{2}{*}{$\underset{\text { ATP }}{(\mu \text { moles }}$} & \multirow{2}{*}{$\begin{array}{c}\left.\mathrm{P}_{\mathrm{i}} / 20 \min \right) \\
\mathrm{ADP}\end{array}$} & \multicolumn{4}{|c|}{ (Relative Light Intensity) } \\
\hline & & & None & $A M P$ & $\mathrm{ADP}$ & ATP \\
\hline $\begin{array}{l}\text { Crude } \\
\text { DEAE- }\end{array}$ & 1.60 & 0.87 & 40 & 400 & 330 & 215 \\
\hline fractionated & 0.13 & 0.09 & 30 & 380 & 350 & 35 \\
\hline
\end{tabular}

Conditions: Same as described under assay system except that the enzyme added in a'l cases represents $0.25 \mathrm{mg}$ protein. When $A T P$ was used for the luminescence assay, $5 \mu$ moles of $\mathrm{MgCl}_{2}$ were added. $\mathrm{MgCl}_{2}$ was also incorporated into the phosphatase assay al equimolar concentrations with that of the nucleotides. 
also lost. On the other hand, a loss of phosphatase activity has little or no effect on the ability to stimulate light production by ADP or AMP. The stimulation of luminescence by ATP can be restored in the DEAE fractions by the addition of commercial preparations of alkaline phosphatase. These results are presented in Table 6.

Thus, the activity observed with ATP in the crude extracts can be attributed to phosplatase action which results in the generation of ADP and AMP from ATP. The functional nucleotide, therefore, in the luminescence of the sea pansy is either ADP or AMP, or both. It has not been possible, on the basis of kinetic data, and using partially purified preparations, to distinguish between the activities of the latter two nucleotides.

TABLE 6

Effect of Alkaline Phosphatase on the ATP Response in DEAE Fractions

System

Relative Light Intensity

Complete

minus ATP

234

minus phosphatase

16

minus phosphatase (ADP replacing ATP)

(ADP replacing ATP)

224

211

Conditions: Same as under assay system except that $1 \mathrm{mg}$ of crude alkaline phosphatase and $5 \mu$ moles of $\mathrm{MgCl}_{2}$ were added.

\section{Mechanisir Studies}

\section{Evidence for Formation of an Intermediate}

If crude Renilla luciferase is added to a stanclard assay system under anaerobic conditions, and preincubated under anaerobic conditions for varying lengths of time prior to flushing with oxygen, one obtains kinetic data of the type illustrated in Fig. 9. The system is flushed with oxygen for 20 seconds beginning at zero time (after 20 minutes anaerobic preincubation) in the case of Figure $9 \mathrm{~A}$, and after 40 minutes anaerobic preincubation in the case of Figure 9B. Absolutely no light can be detected unless oxygen is introduced. The fact that the time required to reach half the steady-state value is approximately 2 seconds as compared to 3.3 minutes for ADP kinetics (see Fig. 8) suggests the formation of an intermediate during anaerobic preincubation. Although the decay of luminescence from the steady state is relatively rapid, as shown in Figure 9B, 10 to 15 minutes is required to return to a level near the normal steady-state value where 
the decay rate is not nearly so rapid. This observation and the fact that, under these conditions, a doubling of the preincubation time essentially doubles the steady state value suggest that an intermediate is not only formed, but is also slowly accumulated.

The intermediate is relatively heat-stable. If, for example, a standard assay system is preincubated for 30 minutes under anaerobic conditions, heated at $100^{\circ} \mathrm{C}$ for $1 \frac{1 / 2}{2}$ minutes (without prior flushing with oxygen), and then cooled to room temperature, an intermediate

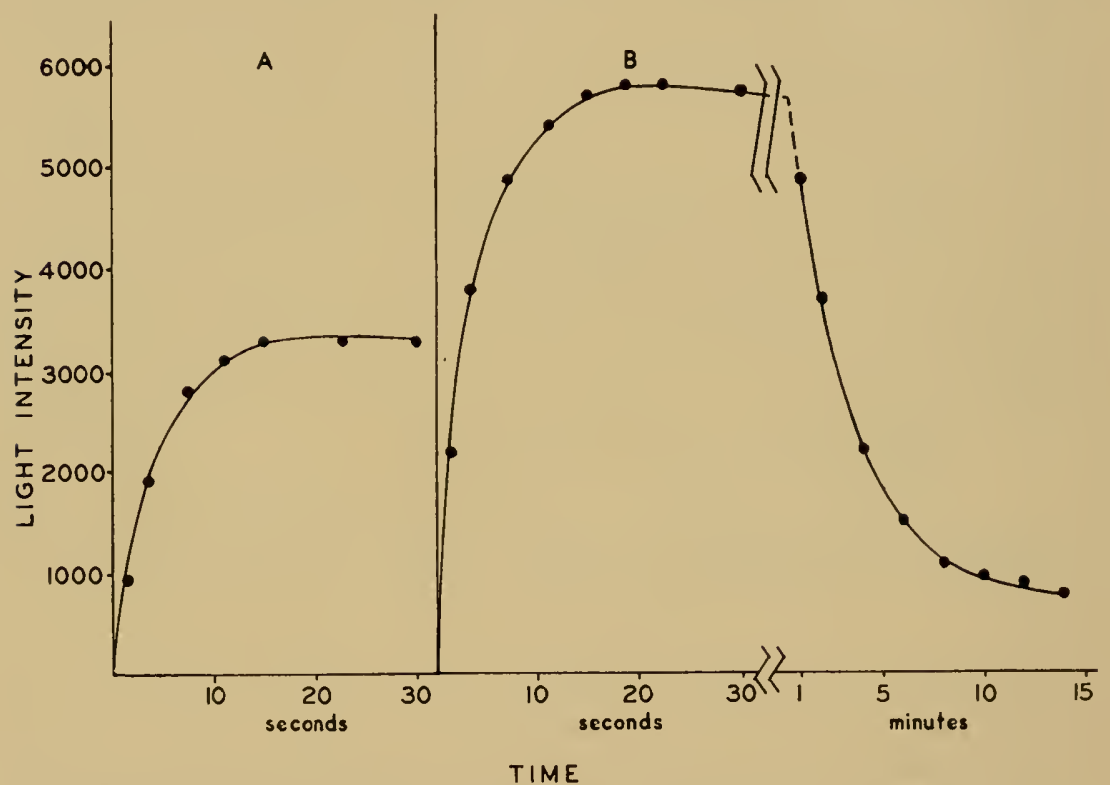

Fig. 9. Eflect of anaerobic preincubation on the kinetics of the reaction.

can be demonstrated in the heated extract. Fig. 10 shows that the addition of a portion of such a heated extract to a system containing only crude lucilerase, GSH, and phosphate buffer results in an almost instantaneous rise to a steady state. The kinetics of this reaction is too fast to follow with the recorder used (relaxation time $=1$ second full scale). Thus the linitations of the recorder make kinetic measurements uncertain for time periods of less than 1 second. No light is obtained above background by flushing the preincubated and heated extract with oxygen. Apparently the somewhat slower kinetics observed in Fig. 9 are due to the limiting rate at which oxygen is dissolved in water under these conditions. 


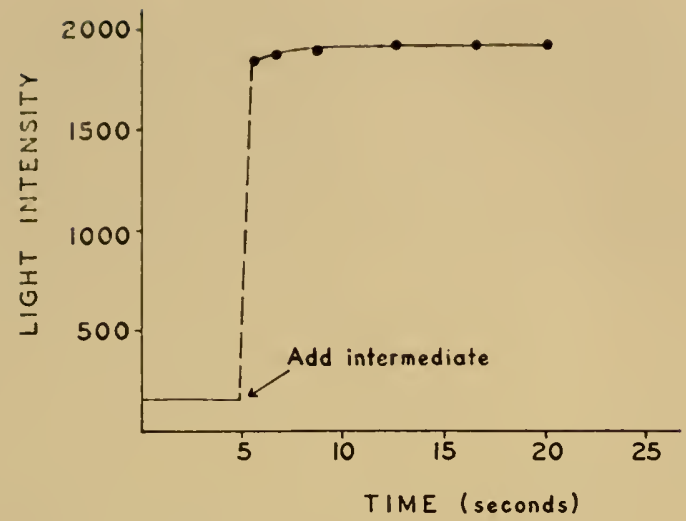

Fig. 10. Kinetics of the reaction upon addition of preformed intermediate.

Requirements for Formation of the Intermediate

A study of the requirements for the formation of the intermediate was possible using a DEAE-fractionated enzyme of the type used to obtain the data in Table 5. This enzyme preparation contains a negligible amount of adenine-containing nucleotides, on the basis of luminescence determinations, and is essentially devoid of luciferin. As seen from Table 7 , the formation of the intermediate requires the presence of both ADP and luciferin. The relatively small light signal obtained when the complete system minus ADP is used is presumably due to low levels of contaminating nucleotides in the crude luciferin. Although the data are not shown, AMP is about equally active under these conditions, whereas ATP plus magnesium ion is totally inactive.

In addition to ADP and luciferin, the enzyme preparation is also required for intermediate formation. The intermediate is not formed, for example, if ADP and luciferin are preincubated under anaerobic conditions in the absence of enzyme.

The above data suggest that a heat-stable intermediate is formed by an enzyme-catalyzed reaction involving luciferin and either ADP

TABLE 7

Requirements for INTERMediate Formation

Test System

Complete

minus luciferin

minus ADP

minus ADP and luciferin

minus enzyme
Relative Light Intensity

162
6
36
3
8


or AMP. Since the DEAE fractions do not contain significant ADPase activity, however, it follows that ADP is functional in this system without first being converted to AMP. Furthermore, it seems unlikely that luciferase would non-specifically utilize either AMP or ADP, but not ATP, for light production. Thus AMP may be involved in this system via a cyclic phenomenon that results in the formation of ADP. In order to satisfy the kinetic data, however, one would have to assume that the equilibrium of the reaction leading to the formation of ADP from AMP lies far in the direction of ADP.

An example of a cyclic mechanism of the type discussed would be one involving the intermediate formation of luciferyl phosphate from ADP and luciferin. The product of this reaction, AMP, might then react, after the light reaction, with oxyluciferyl phosphate to form ADP and oxyluciferin. This mechanism, of course, is purely speculative and simply provides a rorking hypothesis for future investigations.

The data do not rule out a nucleotide-dependent enzymic release of bound luciferin. It is difficult with such a mechanism, however, to visualize a cyclic scheme that would explain the activity of both nucleotides in this reaction.

\section{ACKNOWLEDGMENTS}

The author wishes to acknowledge the competent technical assistance of Mrs. R. J. Eckroade during the course of these investigations. In addition, these studies would not have been possible without the aid and close cooperation of Drs. J. M. Teal and L. R. Pomeroy of the University of Georgia Narine Institute, Sapelo Island, Georgia, during the collection of the animals used in these experiments. The author also wishes to express his appreciation to Dr. R. A. McRorie for his helpful criticisms and suggestions during the preparation of the manuscript. The author wishes to express his appreciation for the generous gifts of firefly and Cypridina luciferins by Drs. W. D. McElroy and E. N. Harvey, respectively.

\section{REFERENCES}

I. Mirth, R. I., and McElioy, W7. D., J. Bacteriol.. 77. 249-2.50 (1959).

2. Cormier, M. J., J. Am. Chem. Soc., 81, 2592 (1959).

3. Cormier, M. J., Biochim. et Biophys. Acta (in press).

4. Cormier, M. J. and Totter. J. R.. Biochim. el Biophys. Acla, 25, 2299-237 (1957).

5. Cormies, M. J., Totter, J. R., and Rostorler, II. H., Arh. Biochem. and Biopliys., 63, 41.1-426 (1956).

6. Jiske, C. HI., and Sublbarow, Y., I. Biol. Chem., 66, 375-386 (1925).

7. Harvey, I. N., Bioluminescence, Icarlemic Press, New York (19;2).

8. Hastings, J. W., and MrLilrov, W. D., in The L.uminescence of Biological Systems (F. H. Johnson, ed.), pp. 257-269. American Issociation for the Alvancement of Science, Washinglon, D. C. (19.5.5).

9. Huslbert, R. B., Schmit, K., Brumm, I. F., and Potser, V. R., J. Bio?. Chem., 209.221, 23 (1954). 
10. Malios. W. W., and Green, A., Arch. Biochem. and Biophys., 64, 257 (1956).

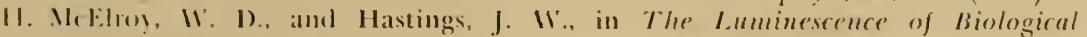
systems (1. 11. Johnson, ed.), pp. J61-198, Imerican Issociation for the Adrancement of feience, Washington, D). C. (I955).

12. Mclitroy, W. 1)., Hastings, J. W.. Coulombre, J., and Somnenfeld, I'., Areh. Biochem. and Biopleys., 46. 399-116 (1953).

13. Nalidos, W. 1)., and Strehler, 13. 1.., Bacteriol. Re7., 18, 177-194 (1954).

1. Rhodes, W. (.., and Mclidroy, W. 1)., J. Biol. Chem., 233, 1528-1537 (1958).

1.5. Strehler, 13. L.. in The Luminescence of Biological Systems (1. 11. Johnson, ed.), pp. 2(19-25.5. Anerican Association for the Mdvancement of Science, liahington, 1). C.. (1955).

16. Strehter, B. I.. and Comier, M. J., Arch. Biochem. and Biophys., 47, 16-33 (19.53).

17. Strchler, B. 1.. and 'outer, J. R., Arch. Biochem. and Biophys., 40, 28-41 (1952).

18. Isuji, F. L., Chase, I. M., and Harvey, E. N., in The Luminescence of Biological systems (ed. F. H. Johnson), pp. 127-159, Imerican Association for the Idrancencnt of Science, Washington, D C. (1955). 


\title{
IONIC EFFECTS UPON BIOLUMINESCENCE IN GONYAULAX EXTRACTS
}

\author{
J. Woodland Hastings and Vernon C. Bode ${ }^{1}$
}

Division of Biochemistry, Department of Chemistry and Chemical Engineering, University of Illinois, Urbana, Illinois

The beautiful and sometimes spectacular "phosphorescence" of the seas is due in many, though not all instances, to the bioluminescence of unicellular organisms, the dinoflagellates (3). This same group of organisms is also responsible for the well-known phenomenon of red tides. The sea water at such times appears blood-red in color because of an excessively large dinoflagellate population, and sometimes only one species is involved. The conditions which give rise to such an excessive growth are presumably nutritional, but they are not yet fully understood (12).

B. M. Sweeney has isolated and cultured in the laboratory a number of marine dinoflagellates. One of these, Gonyaulax polyedra, was found to be brilliantly luminescent in culture (6). Light emission in cell-free extracts from these organisms was found to require, in addition to oxygen, at least three factors: a heat-labile enzyme, a factor in the boiled extract, and salt in a ligh concentration (5). The mechanism of the reaction appeared similar in certain respects to the luciferin-luciferase systems described for other organisms $(2,3$, $8,10,13,15)$, but it was found that there was no cross-reaction between Gonyaulax luciferin or luciferase and those of the firefly, bacteria, or Cypridina. We can report, however, that Gonyaulax polyedra luciferin and luciferase preparations will cross-react with preparations from another species, Gonyaulax monilata.

Gonyaulax is an obligate photoauxotroph, and is grown in large quantities in Fernbach flasks, utilizing fluorescent bulbs as the light source. Either of two culture media are usecl: a supplemented seawater medium modified from an earlier one (14), or an artificial sea-water medium, modified from the ASP medium of Provasoli,

\footnotetext{
${ }^{1}$ Predoctoral Fellow, National Cancer Institute, l’ublic Health Service.
} 


\section{J. WOODLANII HASTINGS AND VERNON C. BODE 295}

McLaughlin, and Droop (9). Details of these media as well as information concerning the growth of the organism will be published subsequently.

At densities of 10,000 to 20,000 cells per milliliter cells are harvested from the growth medium by filtration on a Büchner funnel, washed twice with distilled water, and scraped into a container. Water extracts may be made immediately or, alternatively, the cells maly be dried for future use. There is little loss of activity in dried cells.

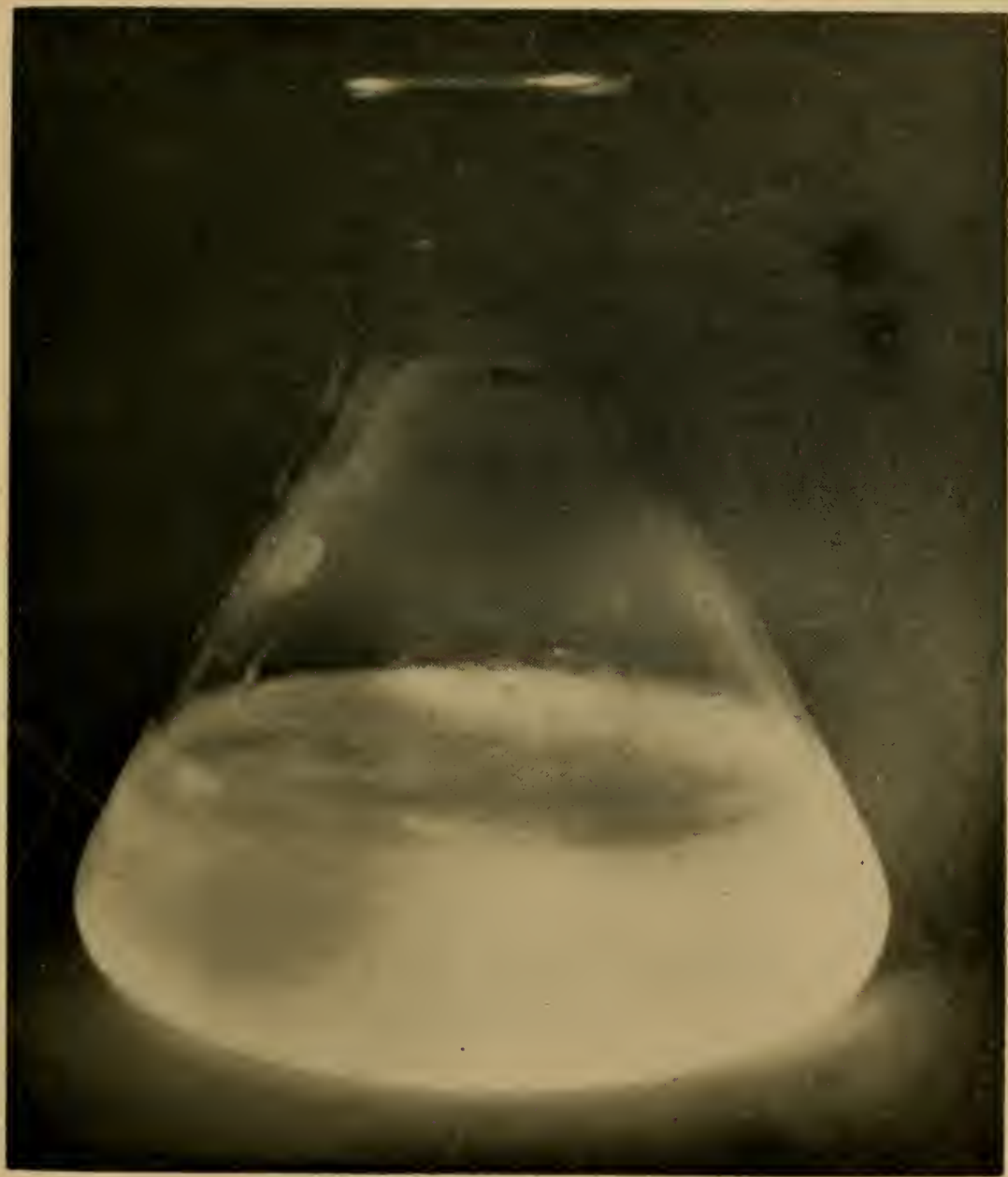

Fig. 1. Photograph of a flask of Conyaulax polyedra cells, taken with the light emitted by the cells as they were stimulated. Exposure, about 5 seconds. 
A Gonyaulax cell is about 40 microns in diameter and emits bright flashes of light which last for less than one-tenth of a second $(4,5)$. This flashing occurs when the cell is agitated or stimulated. The process of harvesting in itself, therefore, causes a large quantity of light to be emitted, meaning that a considerable amount of luciferin has been utilized and presumably lost. Chemical agents also stimulate flashing, and attempts to block luminescence during harvesting by

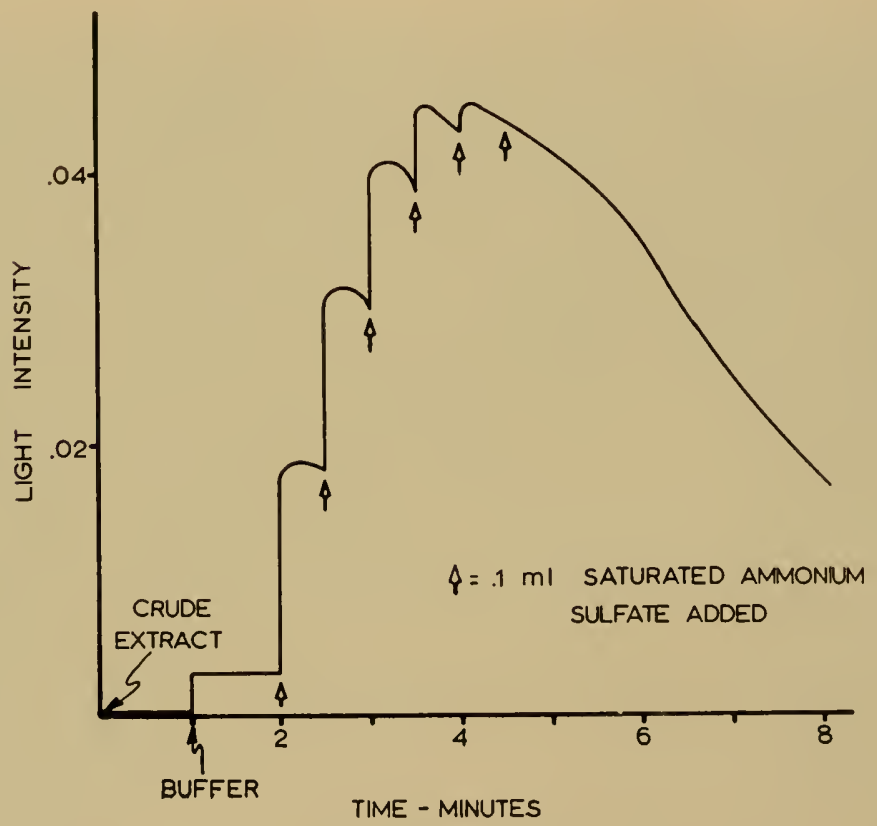

Fig. 2. This illustrates the effect of added salt on the luminescence of a cellfree extract. At zero time $0.1 \mathrm{ml}$ of a crude extract was aclded to $0.7 \mathrm{ml}$ of water. This was followed at the times indicated by $0.5 \mathrm{ml}$ of $0.1 \mathrm{M}$ Tris-maleate-NaOH buf. fer, and $0.1 \mathrm{ml}$ quantities of saturated ammonium sulfate.

adding inhibitors have resulted only in stimulation of this sort. A photograph of the luminescence of Gonyaulax taken with its own light emission is shown in Fig. 1.

A cell-free cold water extract of Gonyaulax cells contains both the luciferin and the luciferase, but does not emit light unless salt is added (Fig. 2). A variety of salts are effective in stimulating light emission, and for each of them a relatively high concentration is required for optimum activity of the luminescent system (see below). 
In addition to the cell extract and salt, the luminescent reaction requires molecular oxygen (Fig. 3).

The cxtract may be fractionated with ammonimm sulfate to yield an enzyme purified by abont 20 -fold, as shown in Table 1 . Dried or fresh cells are extracted three times with a total of $100 \mathrm{ml}$ of clistilled water per gram of cells, dry weight. The dark brownish-green extract is then fractionated with ammonium sulfate to give an active fraction which precipitates between 35 and $65 \%$ saturation of am-

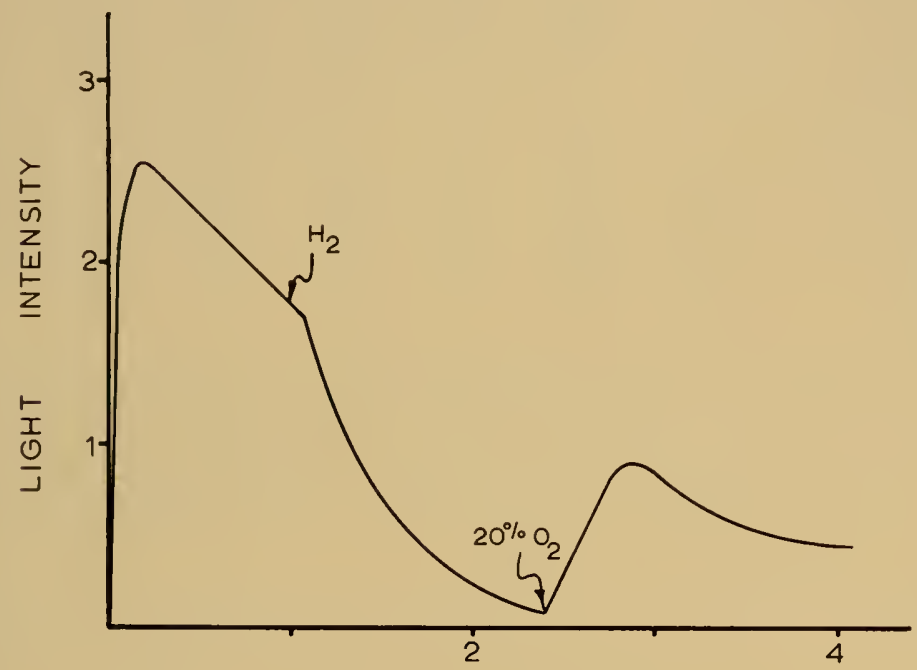

TIME - MINUTES

Fig. 3. This illustrates the requirement for oxygen in the Gonyaulax luminescent reaction. A reaction was initiated with air bubbling through $i t$ and about one mintte later the gas flow was changed to hydrogen. The light intensity decreased rapidly, bu became higher when the gas tlow was changed back to air $\left(20 \% \mathrm{O}_{2}\right)$.

monium sulfate. At low ammonium sulfate concentrations a deep green protein precipitates, and at higher concentrations a pink-red protein is precipitated. Upon subsequent purification it is found that the activity of the luciferase does not accompany the colored fractions. A second ammonium sulfate fractionation results in further purification. Enzyme stored at $-10^{\circ} \mathrm{C}$ is stable for at least a year.

This partially purified enzyme contains no measurable amounts of substrate, i.e., luciferin. Luciferin may be prepared by extracting cells for two minutes with water at $90^{\circ} \mathrm{C}$. Luciferase activity is thereby destroyed, and when the luciferin is added to a partially 
TABLE 1

Purification of Gonyaulax Luciferase

\begin{tabular}{|c|c|c|c|c|c|c|c|}
\hline \multirow[t]{2}{*}{ Fraction } & \multirow{2}{*}{$\begin{array}{c}\text { Volume } \\
\mathrm{ml}\end{array}$} & \multicolumn{2}{|c|}{ Light Units } & \multicolumn{2}{|c|}{ Protein, mg } & \multicolumn{2}{|c|}{$\begin{array}{l}\text { Specific Activity } \\
\text { Activity Recovery }\end{array}$} \\
\hline & & per ml & total & per $\mathrm{ml}$ & total & $\begin{array}{l}\text { light } \\
\text { units/ } \\
\text { mg } \\
\text { protein }\end{array}$ & $\begin{array}{l}\text { per } \\
\text { cent }\end{array}$ \\
\hline $\begin{array}{l}\text { combined crude } \\
\text { first }\left(\mathrm{NH}_{4}\right)_{2} \mathrm{SO}_{4}\end{array}$ & 780 & 1.5 & 1170 & 3.3 & 2570 & 0.45 & - \\
\hline $0-35 \%$ & 38 & 0.5 & 19 & 6.8 & 260 & 0.08 & 2 \\
\hline $35.65 \%$ & 30 & 32.0 & 960 & 21.6 & 650 & 1.5 & 82 \\
\hline $\begin{array}{l}\text { 65-85\% } \\
\text { second }\left(\mathrm{NH}_{4}\right)_{2} \mathrm{SO}_{4} \\
35-65 \% \text { (from }\end{array}$ & 28 & 0.5 & 14 & 5.4 & 150 & .09 & 1 \\
\hline above) & 10 & 32.0 & 320 & 21.6 & 216 & 1.5 & - \\
\hline after dialysis & 10 & 32.0 & 320 & 20.1 & 201 & 1.6 & 100 \\
\hline $0-35 \%$ & 3.5 & 1.0 & 4 & 40.0 & 140 & .03 & 1 \\
\hline $35-42 \%$ & 2.3 & 14.0 & 32 & 6.6 & 15 & 2.1 & 10 \\
\hline $42-52 \%$ & 3.5 & 56.0 & 196 & 7.2 & 25 & 7.8 & 61 \\
\hline $52-60 \%$ & 2.0 & 20.0 & 40 & 3.9 & 8 & 5.1 & 13 \\
\hline $60-85 \%$ & 2.0 & 1.1 & 2 & 4.2 & 8 & 0.3 & 1 \\
\hline
\end{tabular}

purified luciferase preparation in the presence of salt, light emission occurs. The light intensity is a measure of the velocity of the reaction. Light intensity increases linearly with increasing enzyme concentration, whereas luciferin shows a typical substrate saturation curve with increasing concentration (5).

These facts make it possible to represent the reaction by using a modification of Harvey's (3) scheme for bioluminescent reactions (Fig. 4). A reduced luciferin molecule $\left(\mathrm{LH}_{2}\right)$ is oxidized enzymatically to yield an excited molecule, $L^{*}$, which falls to the ground state with the emission of a quantum of light.

The side reactions shown are based upon several observations. Hot water extracts rapiclly lose luciferin activity. After 24 hours the activity remaining is usually less than 10 per cent of the original. First of all, the loss of activity can be prevented by the exclusion of oxygen. Thus, a nonenzymatic nonluminescent oxidation is indicated. Sccond, treatment of nonenzymatically inactivated preparations with reducing agents, such as cysteinc, or hydrogen in the presence of platinized asbestos, will partially restore activity. This is not observed with enzymatically oxidized luciferin. Third, the total light yicld is much greater at higher enzyme concentrations. This may be explained in terms of a competition for reduced huciferin. At higher enzyme concentrations a greater percentage of the $\mathrm{LH}_{2}$ is channeled through the enzymatic pathway. Fourth, the addition of crystalline 


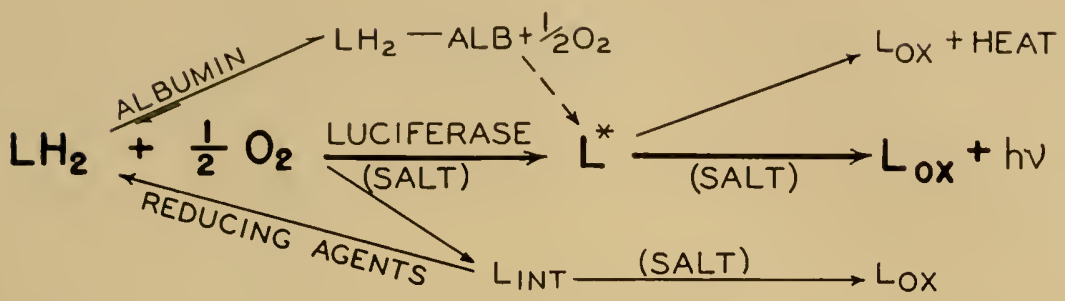

lig. 4. This figure illustrates the proposed pathways for the breakdown of luciferin. The reduced starting material, or luciferin, is designated as $\mathrm{LH}_{2}$. Its oxidation may proceed ria a nonenzymatic pathway (bottom) which may be reversed by the use of reducing agents. 'This reversal is possible from an intermediate $\left(L_{\xi n t}\right)$ but not from the final product $\left(L_{o x}\right)$. Alternatively, the enzymatic oxidation results in the production of an excited molecule, $L^{*}$, which may decay to the ground state either with the emission of light, or via a radiationless pathway with the production of heat. $L_{o x}$ is the oxidized luciferin. We have also represented the formation of an albumin-luciferin complex (top), which is less sensitive to nonenzymatic oxidation, and may presumably also be capable of enzymatic oxidation.

TABLE 2

The Effect of Salts Upon Luminescence

$\begin{array}{ccc}\text { Compound } & \text { Final Molar } & \\ \text { Concentration } & \text { Relative Intensity } \\ \text { Tris-maleate-NaOH, } p \mathrm{H} 6.5 & .12 & 15^{\mathrm{a}}\end{array}$

$\mathrm{Na}_{2} \mathrm{SO}_{4}$

$\left(\mathrm{NH}_{4}\right)_{2} \mathrm{SO}_{4}$

$\mathrm{K}_{2} \mathrm{SO}_{4}$

$\mathrm{Li}_{2} \mathrm{SO}_{4}$

$\mathrm{MgSO}_{4}$

$\mathrm{Na}_{2} \mathrm{~S}_{2} \mathrm{O}_{3}$

\section{$\mathrm{KCl}$}

$\mathrm{NaCl}$

$\mathrm{NH}_{4} \mathrm{Cl}$

$\mathrm{LiCl}$

$\mathrm{MgCl}_{2}$

$\begin{array}{lr}1.0 & 120 \\ 1.0 \quad & 100 \\ .5 \text { (solubility } & 90 \\ \text { limit) } & \\ .8 & 70 \\ .6 & 30 \\ 1.2 & 150\end{array}$

1.2

.8

1.0

.15

.05
100

70

15 (no effect)

18

Inhibits

\begin{tabular}{lcc}
\hline $\mathrm{NaF}$ & .8 & 50 \\
$\mathrm{KBr}$ & .1 & 15 (no effect) \\
$\mathrm{KI}$ & .1 & Inhibits
\end{tabular}

Ammonium acetate

Potassium acetate

Sodium acetate

Lithium acetate

.6
1.0
1.0
.1

a Tris-maleate buffer was present in all assays except two, as noted. A stimulation of light intensity can be ascribed to added salt, therefore, only when the relative intensity is greater than the value of 15 which is attributable to the buffer. 
TABLE 2-continued

Sodium formate

Compound

Sodium citrate

Potassium citrate

Potassium oxalate

Potassium sodium tartrate

Sodium succinate

Potassium hydrogen phthalate

$\mathrm{K}_{x} \mathrm{H}_{y} \mathrm{PO}_{4}$ (no buffer)

Sodium pyrophosphate

$\mathrm{MnSO}_{4}$

Guanidine sulfate

$\mathrm{NiSO}_{4}$

$\mathrm{AlNH}_{4} \mathrm{SO}_{4}$

$\mathrm{Cr}_{2}\left(\mathrm{SO}_{4}\right)_{3}$

$\mathrm{ZnSO}_{4}$

$\mathrm{CuSO}_{4}$

$\mathrm{CsCl}$

$\mathrm{SrCl}_{2}$

$\mathrm{CaCl}_{2}$

$\mathrm{CoCl}_{2}$

$\mathrm{AlCl}_{3}$

$\mathrm{FeCl}_{3}$

$\mathrm{NaNO}_{3}$

$\mathrm{KNO}_{3}$

$\mathrm{AgNO}_{3}$

$\mathrm{NaSO}_{3}$

$\mathrm{KIO}_{3}$

$\mathrm{KCNS}$

$\mathrm{K}_{3} \mathrm{Fe}(\mathrm{CN})_{6}$

$\mathrm{Na}_{2} \mathrm{WO}_{4}$

$\mathrm{Na}_{2} \mathrm{HAsO}_{4}$

$\mathrm{BaCl}_{2}$ (no buffer)

Urea

Sucrose
Final Molar

Concentration Relative Intensity

$\begin{array}{rr}2.4 & \text { Relative In } \\ .5 & 120 \\ .5 & 120 \\ .6 & 80 \\ .7 & 110 \\ .5 & 100 \\ .1 & 80\end{array}$

.1 Inhibits

$.5 \quad 70$

$.2 \quad 50$

$\begin{array}{ll}.5 & 15 \text { (no effect) } \\ .5 & 15 \text { (no effect) }\end{array}$

.1 Inhibits

.05 Inhibits

$10^{-3} \quad$ Inhibits

$10^{-4} \quad$ Inhibits

$10^{-5} \quad$ Inhibits

.15

.05

.05

.05

$5 \times 10^{-3}$

$5 \times 10^{-4}$

Inhibits

Inhibits

Inhibits

Inhibits

30

Inhibits

Inhibits

Inhibits

Inhibits

Inhibits

Inhibits

80 (rapidly inactivates)

60 (rapidly inactivates)

10 (rapidly inactivates)

.1

.05

15 (no effect)

15 (no effcct)

bovine serum albumin has a marked stimulatory effect upon both the intensity of luminescence and the total light obtained (Fig. 5). With high concentrations of albumin, the light intensity (rate) is decreased, whereas the total light (yield) from the reaction remains maximal. This effect of albumin has been shown to be due to the formation of an albumin-luciferin complex which does not so readily undergo nonenzymatic dark oxidation.

The effect of concentration of several salts upon the light intensity of the reaction is shown in Fig. 6. Remarkably high concentrations are required for maximum activity. Most of the curves do not intersect the origin, since buffer was present (see Fig. 1). With no resi- 
dual buffer adcled, however, as in the case of phosphate, the curve goes through the origin. This indicates the absolute nature of the salt requirement.

Table 2 lists other compounds which have been tested for their ability to fulfill the salt requirement. Several properties of each of

\section{THE EFFECT OF ADDED ALBUMIN}

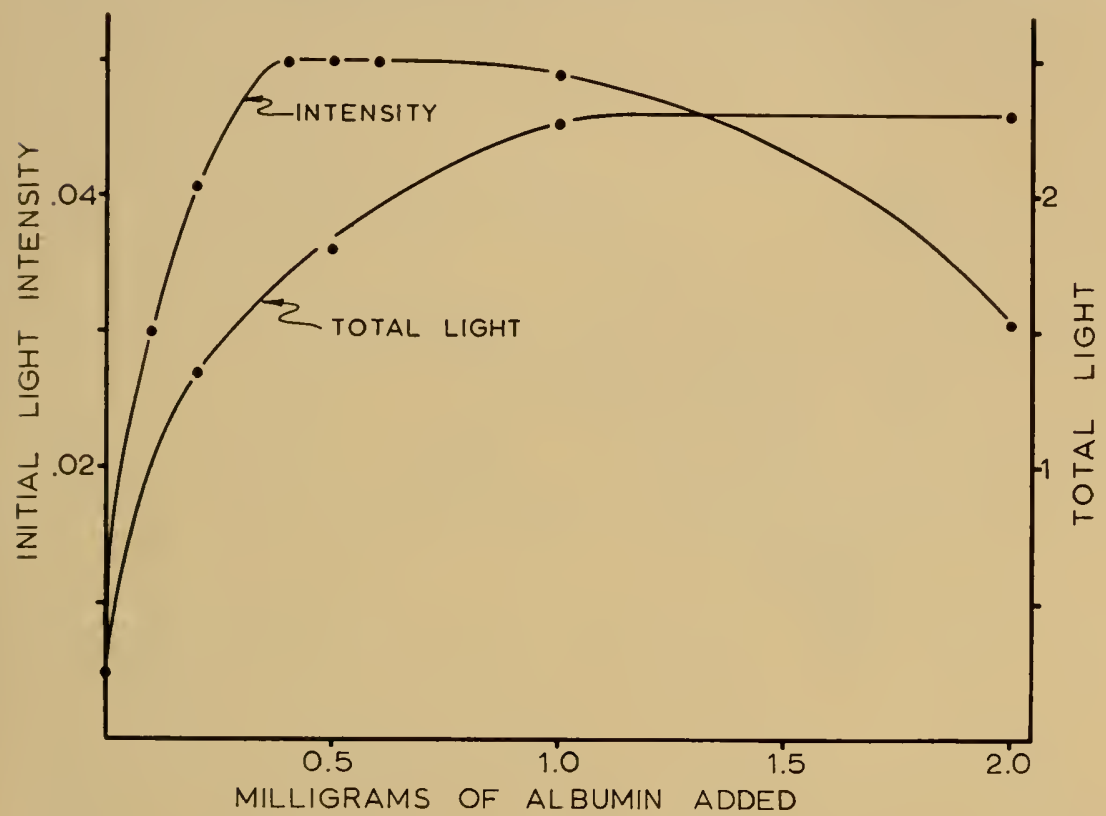

Fig. 5. This figure illustrates the effect(s) of cristalline bovine serum albumin ipon the luminescent reaction. Each point is from a separate experiment. The reaction mixture in each case was composed of $0.45 \mathrm{ml}$ saturated ammonium sulfale, $0.55 \mathrm{ml}$ of $0.5 \mathrm{M}$ Tris-maleate buller. $p \mathrm{H} 6.5,0.1 \mathrm{ml}$ of partially purified elisme (0.1 $\mathrm{mg}$ protein), $0.1 \mathrm{~m}$ luciferin (hot water extract of freshly harvested (ells), crystalline bovine serum albumin as indicated on the abscissa, and water to a total volume of $2.0 \mathrm{ml}$. The reactions were initiated by adding the luciferin last. It may be seen that an increased quantity of albumin results in an increased vield of light from the luminescent reaction. The rate of the reaction (intensity) is also greater.

the salts must be considered in an evaluation of their relative effectiveness. A few of these are: ionic strength, molecular size, solubility (see, for example, $\mathrm{K}_{2} \mathrm{SO}_{4}$ in Fig. 6), the potential for oxidizing or reducing (e.g. $\mathrm{K}_{3} \mathrm{Fe}(\mathrm{CN})_{1 ;}: \mathrm{Na}_{2} \mathrm{~S}_{2} \mathrm{O}_{3}$ ), and effectiveness as protein precipitants. The possible multiplicity of effects, along with the 
fact that the luminescent system is not highly purified, makes it difficult to ascribe a specific role or importance to each of the above factors.

Attempts to replace the salt requirement by the addition of trace amounts of various inorganic ions were unsuccessful. Moreover, with extracts centrifuged for 2 hours at $105,000 \times g$ in the Spinco preparative ultracentrifuge, the activity was found exclusively in the supernatant. Thus, the salt effect is not apparently related to an effect upon a particulate system.

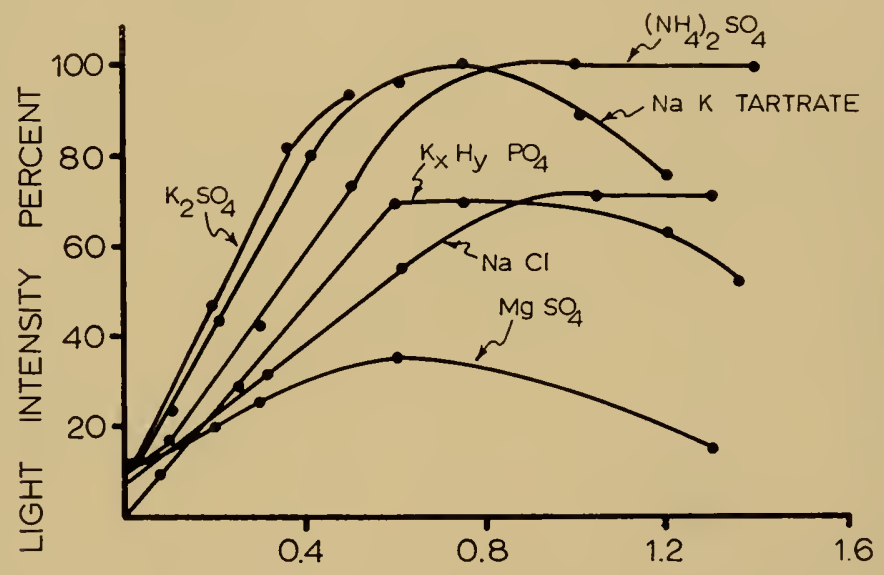

FINAL CONCENTRATION - MOLAR

Fig. 6 . The etfect of various salts upon the initial light intensity in the luminescent reaction. Light intensity is expressed in arbitrary units relative to $\left(\mathrm{NH}_{4}\right)_{2} \mathrm{SO}_{4}$, which was taken as 100 at its optimum concentration. The reaction mixture was composed of $0.45 \mathrm{ml}$ of $0.1 \mathrm{if}$ Tris-maleate buffer, $p \mathrm{H} 6.5,0.1 \mathrm{ml}$ of $1 \%$ crystalline bovine serum albunim, $0.1 \mathrm{ml}$ cold water (ionyaulax extract, salt, and water to a total volume of $2 \mathrm{ml}$. The $p H$ of the salt solutions were adjusted to pH 6.5 where necessary. Similar results were obtained using more concentrated buffer, as well as with partially purifierl enzume (Table 1).

There are at least three possible ways in which salt may be effective in promoting the lumincscent reaction. Nonc of these alternatives have been excluded at the present time. First, the salt may act directly upon the enzyme. Alternatively, the luciferin molecule may be modified to an active form in the presence of salt. A third possibility is that the quantum yield from the reaction might be increased in the presence of salt, thus implying an effect upon the excited state, $L^{*}$. 


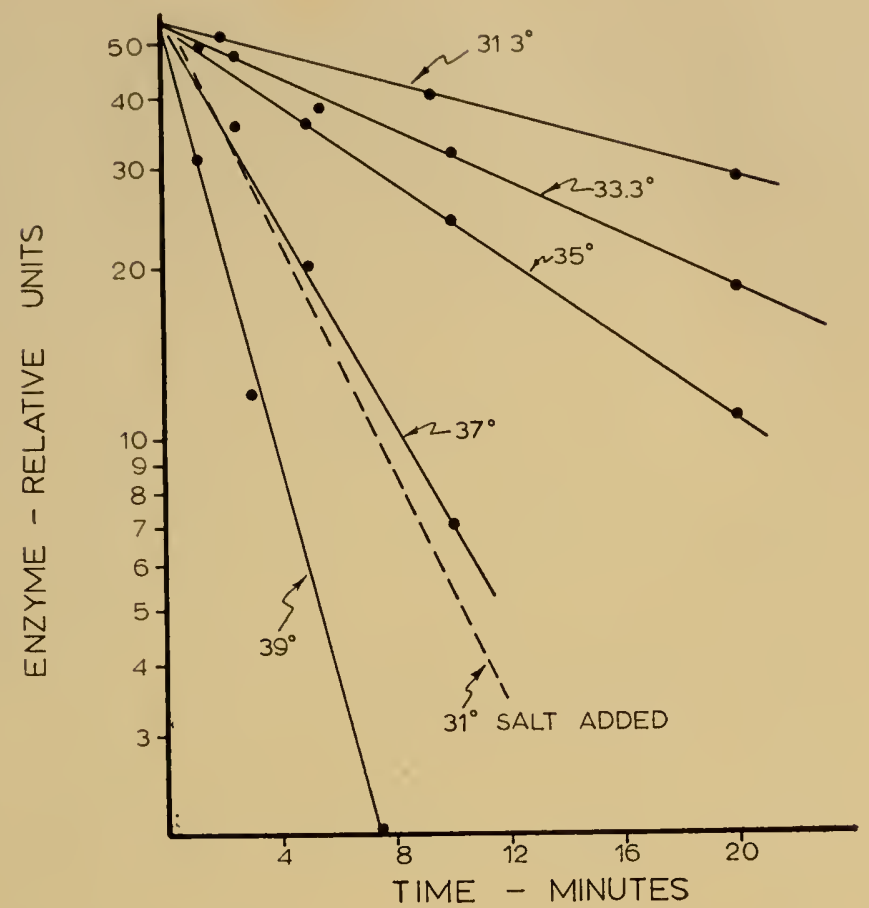

Fig. 7. This figure shows the rates of inactivation of Gomyulax luciferase at several different temperatures. The enzyme used was the active fraction from a lirst ammonium sulfate precipitation (sce Table 1). In all curres except onc, cnzyme alone was present during the incubation. In one (dotted line), the enzyme was incubated in the presence of $2 \mathrm{M} \mathrm{NaCl}$. Aliquots were remover from the incubation mixtures at the times indicated and assayed for enzyme by measuring the initial intcnsit! of luminescence in a reaction mixture with added luciferin.

An effect of added salt upon the enzyme is evident from the fact that enzyme is more unstable in the presence of salt. This is illustrated in Fig. 7, where the curves show the rate of denaturation at different temperatures. All curves are for enzyme alone except the dotted line, which is enzyme in the presence of salt, at $31{ }^{\circ} \mathrm{C}$. The velocity constant for heat denaturation of the enzyme is much greater with added salt. In addition, the rate of denaturation is greater at higher salt concentrations.

An Arrhenius plot of these data (Fig. 8) reveals that the slope (which defines the enthalpy of activation) is the same in the presence and in the absence of the salt. But the temperature range in which denaturation occurs is different, and indicates an increase in the entropy of activation with adled salt. 
From this it may be inferred that an appreciable change in the configuration of the protein occurs in the presence of salt at ambient temperatures, and it is possible that this change might be related to the concomitant increase in enzymatic activity. Such an effect upon the catalytic activity might be mediated either via general changes in

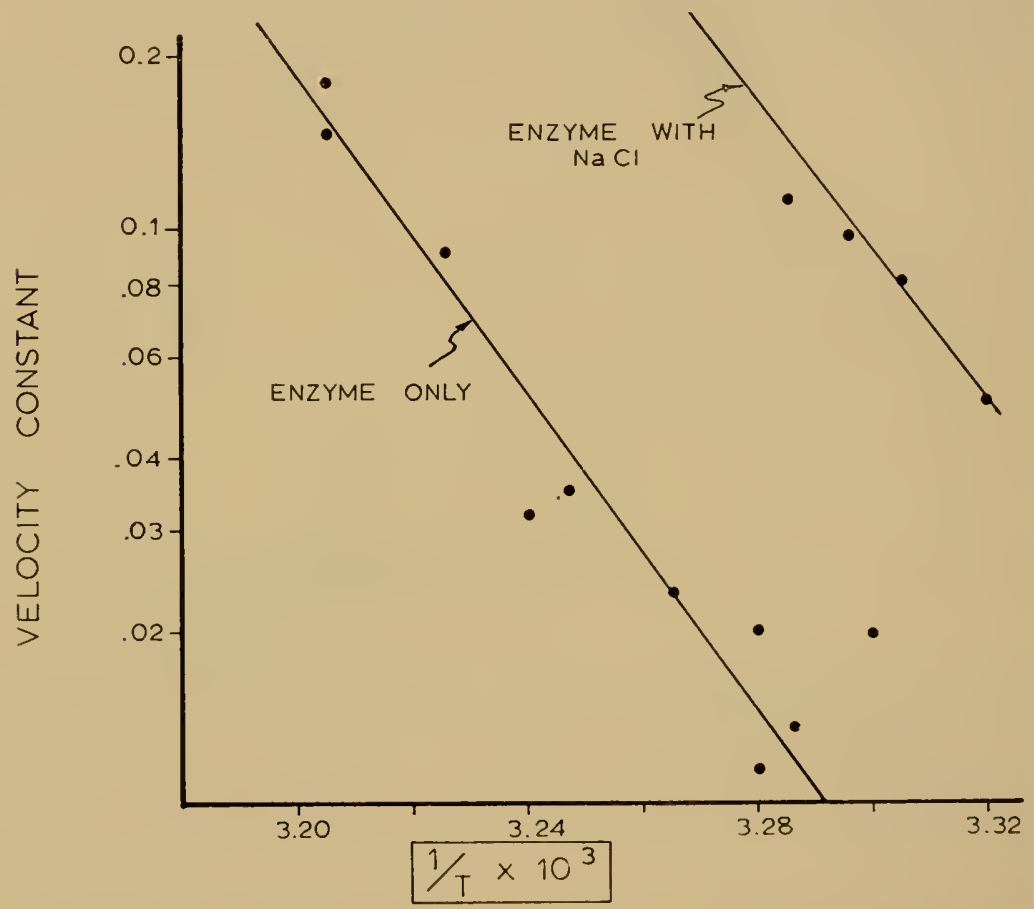

lig 8. Data for heat inactivation of lucilerase plotted according to the Arrhenius equation. On the ordinate are plotted on a log scale the velocity constants for enzyme inactivation, and on the abcissa the reciprocal of absolute temperature. From the slope of the line, the cnthalpy of activation may be calculated to be about 62,000 calorics, both for enzyme in the presence of $\mathrm{NaCl}$ and for enzyme in the absence of salt.

the conformation or tertiary structure of the protein, or via effects upon the dissociation of groups at, or near, the active center ( $l$, 7 , II). The relatively nonspecific ion activation is consistent with effects of this nature.

It can also be shown that lucilerin itself, in the absence of enzyme, undergoes the nonenzymatic oxidation more rapidly in the presence of salt (Fig. 9). This effect is concentration- and temperature- 
dependent, and protection against the destruction may be obtained by adding serum albumin to bind the luciferin.

The albumin effect suggests that enzymatic and nonenzymatic oxidations may be similar, and that the major difference may lie in the participation of the protein, one of whose functions might be to trap and conserve the energy from the reaction. The reversibility of the nonenzymatically oxidized luciferin may be from an intermediate.

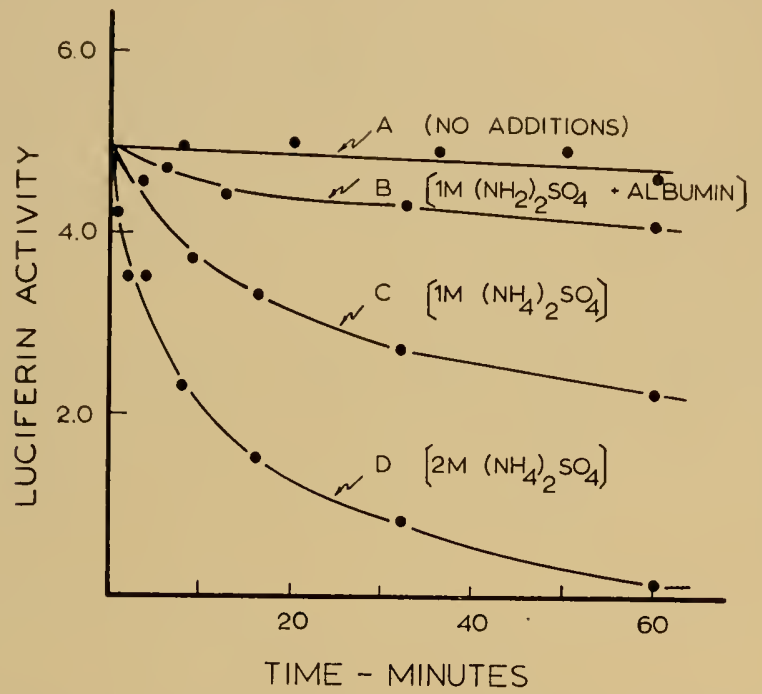

Fig. 9. This figure illustrates the nonenzymatic loss of luciferin under several conditions. Idded salt increases the rate of the loss of Ituciferin activity, anci albumin protects against this loss. The solutions were incubated at $22^{\circ} \mathrm{C}$ in a water bath. and aliquots were removed at the times indicated and assayed for tuciferin. The $p \mathrm{H}$ of all incubation mixtures was adjusted to 7.4 .

A final consideration relates to the possibility that salt has an effect upon the quantum yield of the reaction. Any effect which decreases the energy losses via competitive radiationless pathways should increase the lifetime of the excited state, and therefore the quantum yield.

In the case of bioluminescence, a quantum yield increase might result from an altered or more effective combination of luciferin with the luciferase. In fact, the role of albumin might be related to a quantum yield effect. To test these questions experimentally it will be of importance to learn more concerning the nature of the excited complex which enits. In particular, it will be important to 
understand more concerning the fluorescence of luciferin, and the effect of ionic enviromment and other factors upon the fluorescence.

\section{ACKNOWLEDGMENTS}

This research has been supported in part by grants from The National Science Foundation and the Graduate Research Board, University of Illinois. We are grateful to Dr. L. Provasoli, of the Haskins Laboratory, for the culture of Gonyaulax monilata, and for helpful suggestions in the preparation of artificial sea-water media.

\section{REFERENCES}

1. Alberty, R. A., J. Am. Chem. Soc. 76, 2494-2498 (1954).

2. Cormier, M. J., and Totter, J. R., Biochim. et Biophy's. Acta, 25, 229.237 (1957).

3. Harrey, E. N., Bioluminescence, Academic Press, N. Y. (1952).

4. Hastings, J. W., in Proc. Nat. Biophysics Conf. (Henry (Quastler, edl.), pp. 427434, Yale University Press, New Haven, Conn. (1959).

5. Hastings, J. W., and Sweeney, B. M., J. Cellular Comp. Physiol., 49, 209-226 (1957).

6. IIaxo, F. T., and Swecney, B. M. in The Luminescence of Biological Systems (F. H. Jolinson, ed.), pp. 415-420, American Association for the Advancement of Science, Washington, D. C. (1955).

7. Massey, Y'., and Nlberty. R. A., Biorhem. et Biophy.s. Acta, 13, 35t-3.59 (1954).

8. McElroy, W. D., and Green. A. I., Arch. Biochem. and Biophys., 56, 240-255 (1955).

9. Provasoli, 1., McLaughlin, J.J.A., and Droop, M. R., Arch. Mikrobiol., 25, $392-428$ (1957).

10. Rhodes, W. C., and McElroy, W. D., J. Biol. Chem., 223, 1528-1537 (1958).

II. Rutter, W. J., Acta. Chem. Scand., 11, 1576-1.586 (1957).

12. Ryther, J. H., in The Lumimescence of Biological Systems (F. H. Johnson, ed.), pp. 387-414, Imerican Association for the Advancement of Science, Washington, D. C. (1955).

13. Strehler, B. L., in The Luminescence of Biological Systems (F. H. Johnson, edl.), pp. 209-2.55, American Association for the Advancement of Scicnce, Washington, D. C. (1955).

14. Sweeney, B. M., and Hastings, J. W., J. Cellular Comp. Physiol., 49, 115-128 (1957).

15. Tsuiji, F. I., Chase, I. M., and Harvey, E. N., in The Luminescence of Biological Systems (F. H. Johnson, ed.). Pp. 127-159, Amcrican Association for the Advancement of Science, Washington, D. C. (1955).

\section{DISCUSSION}

1)R. SIRlallik: We agree in most particulats with the picture Dr. MeElroy has outlined in his presentation. There are, however, three points I should like to make. The first is that molecular oxygen does not appear to be a universal requirement for bioluminescences. Even though most light-producing forms require oxygen, several species have been studied by the late Dr. E. N. Harvey which yield bright luminescence even under the most strictly anacrobic conditions for several lours. I refer particularly to his studies 
of the ctenophore, Mnemiopsis. These studies either mean that oxygen docs not participate at all, or that it is bound in some form for long periods of tine and is inaccessible to the normal respiratory catalysts.

Secondly, the possible reversibility of $\mathrm{ATP}$ utilization in the hminescence of fireflies, which Dr. McElroy alluded to, while not rigorously comparable to the findings reported in our manuscript, bears a resemblance to them in principle. The "cofactors" required to reverse the process are, one might saly, particulates from green plants called chloroplasts.

Thirdly, whereas it may well be that the scheme for the terminal steps in bacterial luminescence will turn out to be that outlined by Dr. McElroy, I do not believe the present evidence can, as yet, rule out the alternative scheme we presented a number of years ago.

Our hypothesis is based upon the following observations and concepts: (1) We suggested (on energetic grounds) that two flavin molecules should be required for luminescence. This prediction is consistent with evidence obtained by Dr. McElroy and by Drs. Cormier and Totter. (2) The rate of Havin oxidation, as well as the enzyme affnity for oxygen, are increased by the addition of aldehyde. (3) The quantum yield for flavin is greater than 1 per molecule, whereas the aldehyde yield is quite low (about 1 quantum per 20-30 molecules). Since so large a percentage $(95-98 \%)$ of the aldehycle is oxidized without light emission, it appears to be quite possible that the observed oxidation of aldehyde is incidental to the luminescent reaction (i.e., a side or alternate pathway) and that aldehyde is not used in the actual reactions producing light, but only in certain reactions where light is not evolved.

The scheme which we still prefer is shown in Figure A.

Whether the aldehyde operates catalytically could be most rigorously tested by measuring the number of additional moles of $\mathrm{O}_{2}$ consumed per mole of alchehyde added in the presence of an excess and regenerating supply of reduced FMN.

DR. RACKER: Before we let Dr. McElroy get away without asking him any questions, I have one question which maybe either Dr. McElroy or Dr. Strehler can answer. Have you tried to reverse the oxidation of the aldehyde to the acids by putting light in? Dr. NcElroy has a lot of time. Have you tried it?

Dr. McElroy: Well, I would like to add one thing first. We have clone experiments over a period of one hour with aldehydes in our preparation where the alclehycle is not used if you do not add reduced FMN. The only time you use aldehyde in the system, with any combination you want to use, is when light is emitted. The correlation with the light emission and the aldehyde utilization is perfect as far as we can see. It is true that the quantum yield so far has been quite low, but I think that there is no question that this can be improved. The only thing is that under the conditions in which Dr. Cormier and Dr. Totter did the experiment (using crude enzyme preparations) aldehyde utilization takes place much faster than the observed 


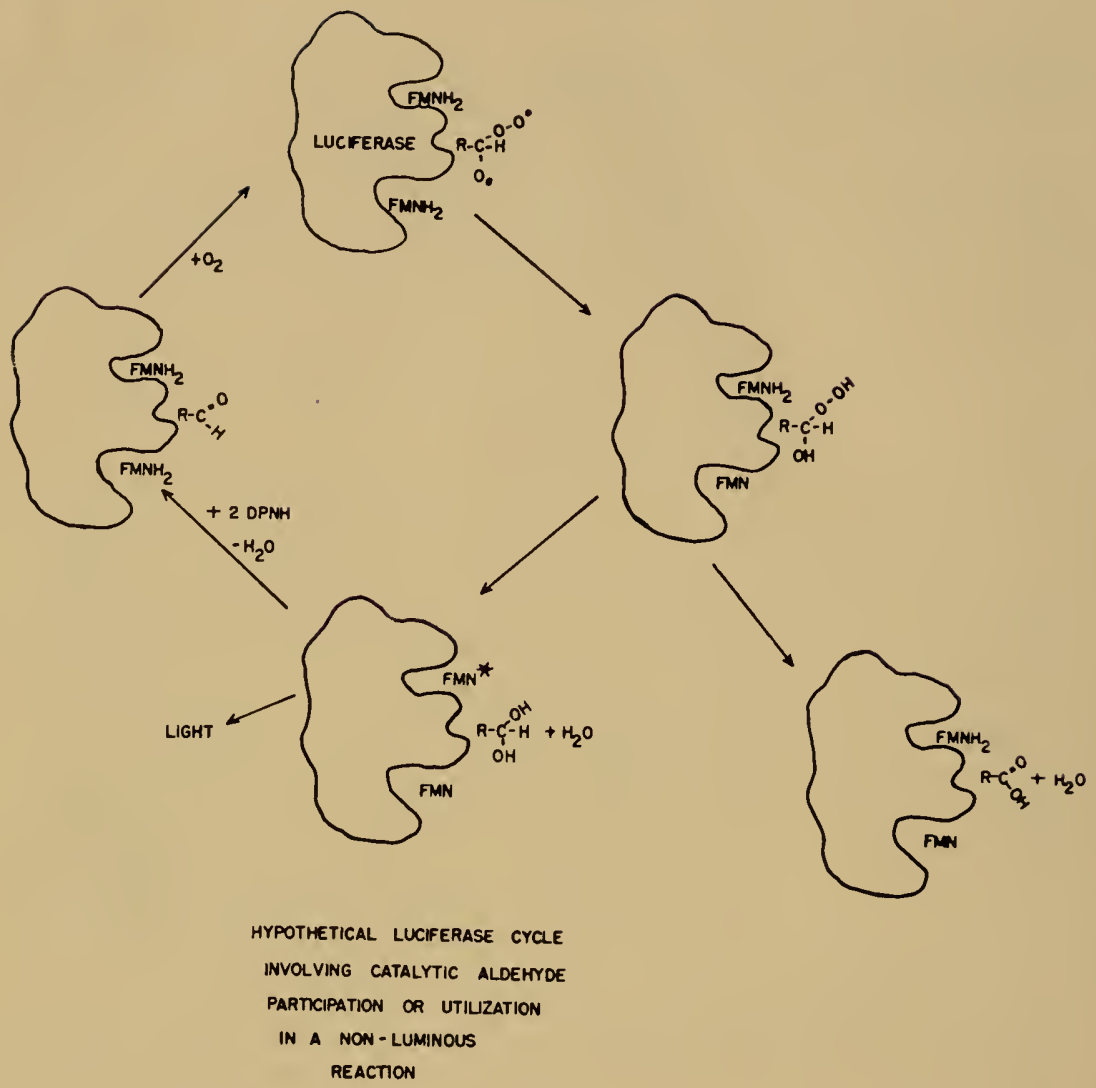

Fig. A

aldehyde oxidase activity. But when you use purified enzyne, which I insist on, the only time we see rapid aldehyde utilization is when you get light emission. For that reason, we suggested that aldehyde is used in the process of excitation.

DR. RACKER: How about the reversal?

Dr. McElroy: The reversal-you mean starting with the acid? You cannot reverse it, even in the presence of light. You can do a chemical reduction. as a matter of fact, but that is an artificial system.

1) . Stranter: Apropos to the utilization of aldehyde under conditions in which you get luminescence, it is well known that reduced flavin autooxidizes quite readily to produce peroxide. Might it not be that peroxide gives by-products in a non-enymatic or even in an ensmatic reaction which isn't directly connected with luminescence.

1)R. M(cileros: No, that is not quite right. You can actually run at control for this type of experiment. You can start non-enzymatically with reduced 
Fin phus aldeligde plus oxygen and the aldehyde concentration drops lincarly and slowly with time. If you put the cnyme in this preparation, the aldehyde concentration drops very rapidly and then slows up. So there is no question that the rate of aldehyde utiliation is directly correlated with the rate of luminescence. In all cases, the total light emitted is proportional (o) the amount of aldehycle used.

1)R. STREHLer: You would expect such a result with many mechanisms you might propose.

Dr. MeElroy: Well, then you would have to explain to me why this cnyme is acting like an aldehyde oxidase. That is all I am saying, it's an alclehyde oxidase.

Dr. POrter: I would just like to make a suggestion of how some of these effects of buffers which Dr. McElroy mentioned on spectra and yields might occur. In fact, there is a precedent already from fluorescence. If the $p \mathbf{H}$ of the excited state is different from that of the ground state, then the luminescence we get comes from another species provided equilibrium is established. Now, equilibrium very often is not established. It has to compete with the radiation process which goes in $10^{-8}$ seconds. The equilibrium is speeded up by the addition of bulfers. And one can, therefore, by adding salts or buffers change the species which is emitting from the original one to the one which is in equilibrium with it at that $p H$. Therefore, you can change the fluorescence spectrum, and of course, the yield if one of the species is not fluorescent. You can change the yield also by adding buffer.

1)r. McElroy: I hope I did not bring the salt eflect up because, as a matter of fact, in the firefly system there is no salt effect in the highly purified enzyme except in this conversion to the red peak. That is very specific for inorganic phosphate. In the case of the bacterial system, once you have it purified then you do not have any salt effect there as far as we can observe. In other words, you can go up to $0.01 \mathrm{M}$ sodium chloride or any other salt and you do not change the emission peak or, as far as we can tell, the total light. This is the same for the firefly system.

DR. PORTER: What about bicarbonate?

Dr. McElroY: It has no effect. As long as you maintain the $p \mathrm{H}$ constant.

Dr. PORTER: What about arsenate?

Dr. McElroy: Arsenate is an inhibitor, but not on the quantum yield.

DR. Ar.sox: I just have a very brief comment on Dr. McElroy's paper. I think that very often when biochenical evidence is given for photochemical events our colleagues, the physicists and photochemists, subject it to severe and rigid scrutiny and analysis on the basis of yield. They say that certain proposed mechanisms are not probable because the yield is too low. I was particularly impressed that in Dr. NcElroy's work a simple thing such as $p \mathrm{H}$ changed yelds almost $100 \%$. I think this is another beautiful exanple of the innate perversity of biohgical materials which I would like to bring to the notice of our colleagues here from the physical sciences.

DR. UDENFRExid: The importance of oxygen here has been stressed previ- 
ously. Dr. Chase has said that he has been able to run this in the presence of huge amounts of ascorbic acid, which we know is an autoxidizable substance utilizing oxygen, without any decrease in the rate of luminescence or having any influence at all on the rate of luminescence. I wonder if this should not have some influence if this is an absolute oxygen-requiring system.

Dr. Chase: Actually, the ascorbic acid seems only to be causing the reduction of oxidized luciferin so that it will give bright light when luciferase is added. I don't believe the autoxidation of ascorbic acid in the absence of the oxidase would be fast enough at $26^{\circ} \mathrm{C}$ and $p \mathrm{H} 8$ to reduce the concentration of dissolved oxygen enough to affect luminescence in this system. Would you agree with that, Dr. McElroy?

Dr. MCELroy: That's right. As a matter of fact, you get maximal luminescence in the firefly system with approximately $0.05 \%$ of oxygen and the same way in the Cypridina system.

Dr. Hastings: It isn't quite the same as Cypridina.

Dr. MCELroy: No, in Cypridina the luminescence is clecreased at higher oxygen concentrations than in the firefly.

Dr. Hastings: That's correct. If you decrease the oxygen concentration below that of air-which is $20 \%$-even by just a little, the luminescence of Cypridina decreases. Luminescence intensity is a continuous function with oxygen concentration from zero to $20 \%$. I think, Dr. Udenfriend, that the answer to your question lies in the fact that Dr. Chase probably does his experiments by incubating luciferin in ascorbic acid and then taking out aliquots for assay-which would dilute the ascorbate and it would then have a negligible effect.

Dr. Chase: No, I don't think that's it. The dilution was only one in three, which wouldn't reduce the ascorbic acid concentration enough to make much difference.

Dr. Hastings: Does it highly autoxidize rapidly or is it fairly slow?

Dr. Chasf: Well, I don't think it autoxidizes much under these particular conditions. I think that what oxidation occurs is from the reduction of the luciferin. The oxygen concentration shouldn't be aflected.

Dr. Veitıck: People often refer to effects produced by tris buffer, obscuring the possibility that the anion of the buffer may be the culprit. For example the fluorescence quantum yield of DPNH in the glyceraldehyde-3-phosphate dehydrogenase complex is diminished in tris acetate and enhanced lightly in tris phosphate. In the LDH complex the DPNH emission is enhanced by acetate.

Dr. Hastings: The anion in our tris buffer was maleate.

Dr. Cormitr: Just a couple of comments. I wouldn't want us all to go away thinking that salts are required for all marine bioluminescence, because this is certainly not the case with the sea pansy. As you approach distilled water you are much better off. The other comment I would like to make 
is that with respect to the mechanism in the bitcterial luminescent system, I don't have any evidence whether one molecule of FNN is reduced or whether two molecules of FMN are reduced on the enzyme surface. I thiuk you can write reaction medhanisms for either one or the other. l'erhaps Dr. Mcklroy has some evidence that there are two. but if he does, I would like to know.

Dr. Malinor: The only evidence we have is purely kinetics, i.e., we must plot the square of the FMNH $\mathrm{F}_{2}$ concentration in the Lineweater-Burk plot in order to obtain a straight line.

DR. Conmer: The evidence that we had of course for two flavins was th: fact that you could isolate an enzyme in which FMN was bound to the enzyme and that this did not produce light until one added DPNH, and aldehyde, FMIN, and DPNH oxidase. You had to add exogenous FMIN to do it. However, I never could find out whether it was necessary to have only one or both molecules of FMN reduced prior to light emission.

Dr. Chase: May I ask if there is anything in the structure of the Cypridina luciferin which would react with cyanide? Cyanide combined apparently very readily and irreversibly with luciferin so that you can't get light.

DR. WHIE: Does it combine with luciferin or with the enzyme?

DR. MCCAPRA: This is with the luciferin, not with the enzyme. Well, I don't think that under neutral conditions cyanide adds in any specific way to luciferin. I mean it has to be catalyzed some way.

Dr. MCELroy: There is an important point here, though, with regard to the Apogon system. It seems like a possibility from Dr. Johnson's data that the enzyme can lose a metal quite readily. If the metal isn't an absolute requirement for luminescence then cyanide would have no inhibitory effect. There would be an inhibitory effect only when you add the metal. This is one difference between the Apogon luciferase and the Cypridina luciferase.

Dr. Chase: I have learned through a personal communication from Dr. Hirata that cyanide has no effect at all on their crystalline Cypridina luciferin. He didn't give any details. This is exactly contrary to the very definite results that Dr. Giese and I got with cyanide.

DR. Chase: Richard J. Lederman, a student at Princeton, and I have been studying the effects of certain factors on the activity of Cypridina luciferase, measuring the luminescent reaction with a photoelectric recording light integrator, and taking the first order reaction rate constant as representing the activity of the enzyme.

In the course of this work it was found that the concentration of the phosphate buffer which was used to stabilize the $p \mathrm{H}$ of the reaction mixture had a considerable effect upon the enzyme activity. Ordinarily one uses a phosphate concentration of about $0.05 . / 1$ for the luminescent reaction of Cypridina luciferase and luciferin. We found that if the phosphate buffer concentration was increased to $0.3 \mathrm{M}$ the rate constant was reduced to about one-half, whereas when phosphate concentration was lowered to about $0.01 \mathrm{M}$, 
the rate constant (compared with that at $0.05 M$ concentration) was increased by more than 50\% (With concentrations of less than $0.01 \mathrm{M}$ the rate constant decreased again.

Recalling the complications that phosphate may cause in certain enzyme systems, we ran another series of experiments using Tris buffer and found e'ssentially the same results. As a further check we tried barbital buffer but. for solubility reasons, could work only at relatively low concentrations. Here, again, a striking change was observed in the activity of the enzyme, depending on the concentration of the buffer.

These results are summarized in Figure $\mathbf{B}$, which is self-explanatory. The differences in activity of the luciferase are in no way due to changes in $p H$ of the buffers on dilution, for this was especially looked into.

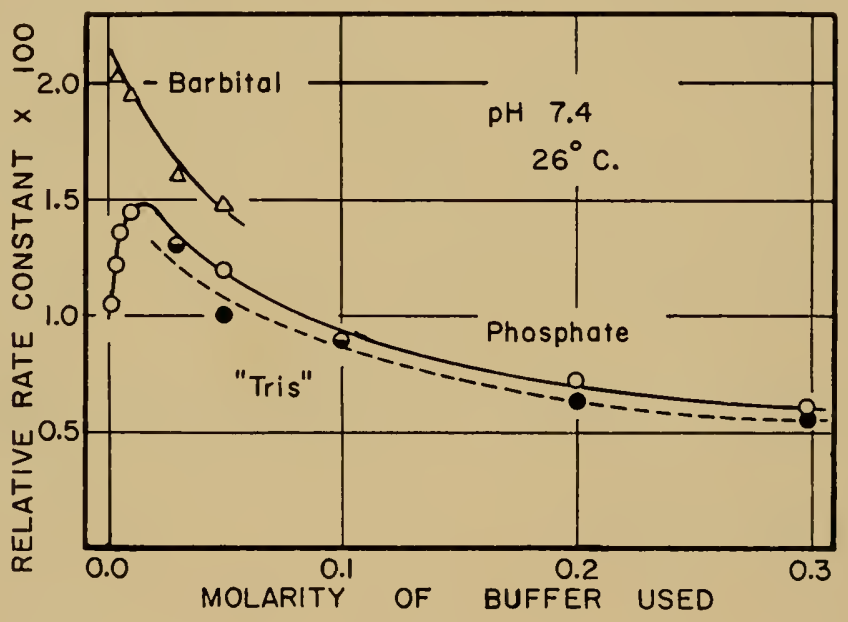

Fig. B

It is very evident from these results that not only the kind of buffer used, but its concentration as well, must be carefully controlled when studying the kinctios of this luminescent reaction. 


\section{Part IV}

\section{PHOTOSYNTHESIS}





\title{
INTRODUCTORY COMMENTS
}

\author{
C. B. VAN NIEL \\ Hopkins Marine Station of Stanford University, \\ Pacific Grove, California
}

On introducing the session on photosynthesis in this symposium, I may be permitted a few introductory remarks. It is not uncommon to find that scientists, like artists, have a habit of defending what they like to do best as the best possible approach to it. There is considerable justification for this because it implies a certain amount of pleasure in one's work. On the other hand, it has also been defended in a beautiful manner in that little treatise of Bronowski on "Science and Human Values", where he paraphrases Coleridge who spoke of poetry, by saying that science is the search for unity in the wild variety of our experiences. Now this emphasizes two different things. It emphasizes unity and it also emphasizes variety. While there is every reason to emphasize unity perhaps more, this is not a good justification for eliminating variety. For that reason, I would like to state that in my opinion the notion that we can understand life if and when we understand energy transfer is perhaps too restricted. Energy transfer is no doubt one of the fundamental aspects, but so are other things. Life is characterized perhaps more than anything else by organization. Organization in its very simplest form is the organization of elementary particles into molecules. It is this aspect that has for several years been in the hands of biochemists who have studied enzyme-controlled reactions, and it should be admitted that on the basis of that approach a great deal has been learned. The importance of that aspect for photosynthesis can perhaps be introduced by saying that about a decade ago the first attempts were made to reconstitute the enzymatic mechanism of the photosynthetic process, approached independently by Vishniac and Ochoa, by Tolmach, and by Arnon and Whatley. From this starting point Calvin, Benson, Bassham and their collaborators have taken off and in a period of ten years have amassed a quantity of beautiful evidence that has given us a very considerable insight into the details of the manner in which carbon dioxide is converted 
into organic molecules. It is a pleasure to pay tribute to the magnificent work of Calvin and co-workers. But, in a way, all that already belongs to the past; it is known. And science has the habit of continuing from what is known to the unknown. From here it has become evident that one of the fundamental, as yet unsolved, aspects of the photosynthetic mechanism is the first step, the conversion of light into chemical energy. From what $I$ have seen of the program of Session IV, it seems that this is largely what the session will be occupied with. It is a pleasure to announce that the first paper in this session will be given by Dr. Melvin Calvin. 


\title{
SOME PHOTOCHEMICAL AND PHOTOPHYSICAL REACTIONS OF CHLOROPHYLL AND ITS RELATIVES ${ }^{1}$
}

\author{
Melvin Calvin \\ Department of Chemistry and Laurence Radiation Laboratory \\ University of Califormia, Berkeley, California
}

\section{INTRODUCTION}

This is a discussion of some of the photochemistry and photophysics of porphyrins which has accumulated in the course of the last fifteen to trenty years and which has some bearing on the problem in which we are primarily engaged, namely, the conversion of electromagnetic into chemical energy as it occurs in photosynthetic organisms. The history of the photochemical and similar properties of chlorophyll and its related materials is an old one. From the very beginning of the recognition of chlorophyll as a prime light-absorber and converter in green plants (26), there has been a steady flow of model experiments with chlorophyll and related materials, intended to discover in simplified systems the types of transformations which conceivably might be taking place in the living organism in its operations for the conversion of electromagnetic into chemical energy.

From very early times, even before the chemical structure of chlorophyll and its relatives was known $(32,76)$, a number of photochemical properties of the chlorophyll molecules in solution had been observed. These can be most easily described in two classifications. The first is a photosensitized oxidation reaction with molecular oxygen in which the chlorophyll is the photosensitizer, that is, the chlorophyll absorbs the light and causes, in some way, the oxidation of some other substrate with molecular oxygen; some of the pigment may be destroyed, depending on the conditions of the reaction, but conditions can be found in which the chlorophyll itself is relatively stable and acts as a photosensitizing dyestuff which will cause the

\footnotetext{
${ }^{1}$ The preparation of this paper was sponsored by the U.S. Aomic Fnergy Commission.
} 
oxidation of a good many substrates. Recent examples of this type of reaction are the studies of G. Schenck in Germany (57), in which he has studied the photosensitized oxidation of a whole variety of materials. One of the very early and more quantitative studies was that of Gaffron (29), in which he used chlorophyll as a photosensitizer for the oxidation of allylthiourea and, in fact, studied it thoroughly enough so that it could be used as an actinometer-that is, as a means of measuring actual light intensity in a beam, particularly in the red. The quantum yield for this reaction, that is, the number of allylthiourea molecules oxidized per quantum absorbed by chlorophyll, is approximately one.

The other type of photochemical reaction in solution which chlorophyll is known to sensitize is a hydrogen transfer from some reducing agent to some oxidized substance. The classic example is the reduction of an azo dye, such as methyl red, by a reducing agent (hydrogen donor) such as ascorbic acid, and chlorophyll has long been known to sensitize the transfer of hydrogen from the reducing agent to the acceptor.

Both these types of cases, for the most part, are photosensitized reactions in which the thermodynamics favors the reaction itself, and the light largely serves the function of overcoming activation energy for the reaction. In general, then, there is not, in any of these reactions, a conversion of electromagnetic into chemical energy.

\section{Photochemistry of Chlorophyll Models}

Relationships between Chlorophyll, Bacteriochlorophyll, and Protochlorophyll

In 1937, I first became acquainted with the chemistry of porphyrins and recognized the relationship of porphin to chlorin. In order to see the type of reasoning involved, I think it is best to look at the structural formulas of the principal energy-capturing molecules in the photosynthesizing organism. Fig. I shows the structural formula of chlorophyll as we now believe it to be, and you will notice that it is a porphyrin with an isocyclic ring and an "extra" pair of hydrogens on one of the pyrrole rings, making the chlorin a dihydroporphyrin. In the last few years, Linstead and his coworkers have proved that these two hydrogens are trans to each other (50).

It is interesting to see the relationship of the chlorophyll molecule to two others, one of which is bacteriochlorophyll (the lightcapturing pigment in the photosynthetic bacteria), and that relation- 
ship can also be seen in Fig. 1. There are two more "extra" hydro. gens in bacteriochlorophyll, presumably on a pyrrole ring on a diagonal from the hydrogen-bearing one in chlorophyll; and on the No. 1 pyrrole ring the vinyl group has been transformed into an acetyl group. The protochlorophyll (Fig. 1), which is the material formed in plants when they are grown in the absence of light, can be recognized as the dehydrochlorophyll (a porphin). The No. 4 pyrrole ring in protochlorophyll has a double bond in it, and it has been shown by a number of workers that the first thing that happens in etiolated plants (which have no chlorophyll in them) when the light is turned on, is the addition of the two hydrogens to the double bond in ring No. 4 to generate chlorophyll.

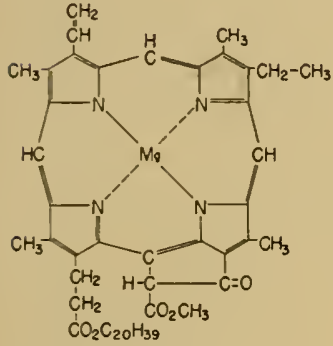

PROTOCHLOROPHYLL

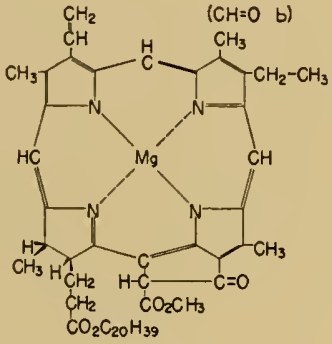

CHLOROPHYLL a (b)

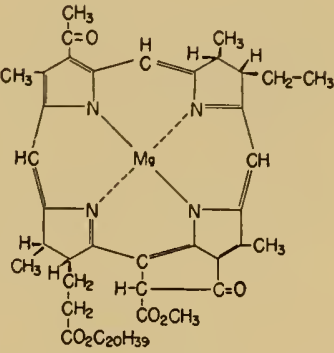

BACTERIOCHLOROPHYLL

Fig. 1. Structural formulas of chlorophyll, bacteriochlorophyll, and protochlorophyll.

An examination of these three formulas shows this relationship of chlorophyll to protochlorophyll and bacteriochlorophyll very neatly. It shows that the macrocycle of chlorophyll lies midway in the oxidation level between that of protochlorophyll and that of bacteriochlorophyll. With the diffusion of the idea of Van Niel (72) that the primary photochemical reaction of green plants involved the fission, or splitting, of the water molecule to give hydrogen and oxygen, or to give a reducing agent and an oxidizing agent, and that this reducing agent was used to recluce carbon dioxide while the oxidizing agent actually generated oxygen, it occurred to me that the function of the chlorophyll might be as a hydrogen carrier from the water toward the ultimate reducing agent which is used to reduce carbon dioxide. Today we believe one of these ultimate reducing agents to be pyricline nucleotide. It seemed likely that the chlorophyll might be functioning between the stage of chlorophyll and protochlorophyll, 
that is, between the stage of dihydroporphyrin and porphyrin, as a transferring agent of the hydrogen from water to something else.

This was a very early notion, and the earliest experiments which were devised to test it, such as doing photosynthesis in deuterated water to see if the two hydrogens that were picked up on the chlorophyll macrocycle were deuterium, failed of positive results. The first experiment of this kind was done by Ruben back before the war (53) and a second one was done in our own laboratory (6), using tritium in the hope we could detect smaller amounts of photosensitized exchange; this also failed to show a tritium incorporation into chlorophyll very much greater than that of new synthesis of the entire molecule.

These unsuccessful results then led to the next notion, namely, that the chlorophyll might be functioning not between the level of photochlorophyll and chlorophyll (between the level of porphyrin and dihydroporphyrin) but between the level of dihydroporphyrin and tetrahydroporphyrin, as represented by bacteriochlorophyll. In that case, any study of deuterium exchange in chlorophyll would fail. If the hydrogen transfer involved first the photochemical reduction of the chlorophyll (dihydroporphyrin) to tetrahydroporphyrin, and if this, then, was transferring its hydrogen to the acceptor which ultimately reduced $\mathrm{CO}_{2}$, and we analyzed only for the dihydroporphyrin, we would not, of course, find any isotope in the dihydroform. It would have been passed on to the ultimate reducing agent.

This would require that in the green plant there should be traces of dihydrochlorophyll (tetrahydroporphyrin), although the steady state, or equilibrium, amount of dihydrochlorophyll might be minutely small and hard to discover. We have yet to perform an experiment in which we seek to find dihydrochlorophyll (or something close to it) in the green plant and to determine whether or not it undergoes a photosensitized isotope exchange. A similar experiment might very well be done in photosynthetic bacteria in which, presumably, the steady state, or equilibrium, amount of tetrahydroporphyrin (or dihydrochlorin) is large and the dihydroporphyrin (or chlorin) is small. This should show, if this type of transformation is the way in which the reaction is proceeding, a large and easily detectable photosensitized deuterium, or tritium, exchange. As far as I know, this experiment has not yet been done.

There is, however, one type of photosensitized deuterium exchange experiment which has been successful, and this is the experiment of Vishniac (75) in which he has shown what appears 
to be the exchange of a labile proton, presumably on chlorophyll, which is photocatalyzed. He believed it to be the relatively labile isocyclic hydrogen which is exchangeable, since it is enolizable. Vishniac also belicves to have shown a photosensitized, or plotoaccelerated, excliange of some proton, on other compounds not identical with chlorophyll. Perhaps some of this could possibly be the dihydrochlorophyll mentioned earlier. Some of it might also be in the form of the next hydrogen carriers (see later). It remains to be seen what the exact nature of this exchange reaction is and whether it has any connection with the photosynthetic process.

\section{Photochemical Hydrogen Transfer-Model Systems}

With this background it seems worthwhile to examine some model systems for photochemical hydrogen transfer. The model systems chosen (long before the aforementioned exchange experiments with chlorophyll were (lone) were those which did not have the sidechains on them which made the compound considerably more labile with respect to incidental transformations. Chlorophyll itself was relatively difficult to obtain in completely pure form, so we undertook to synthesize a model substance which would not be subject to the above-mentioned difficulties and which would have only the porphyrin nucleus and the dihydro- and tetrahydroporphyrin possibilities. Such a molecule is the simple tetraphenylporphyrin whose structure is shown in Fig. 2 and in which the four phenyl groups are on the bridging carbon atom and which contains a simple porphyrin nucleus. This material is relatively easy to synthesize. It is made simply by heating benzaldehyde with pyrrole in the presence of zinc
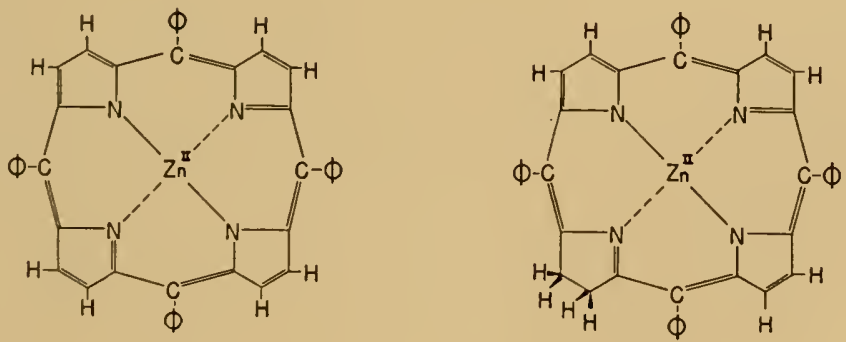

Zn TETRAPHENYLPORPHIN

Zn TETRAPHENYLCHLORIN

Fig. 2. Structural formula of zinc tetraphenylporphin and zinc tetraphenylchlorin. 
ion to obtain a 10-15 per cent yield of the zinc porphyrin, with traces of zinc chlorin in which one of the double bonds is reduced. These substances can be separated by chromatography and fractional crystallization, their properties determined independently and unequivocally, and their photochemistry studied. Fig. 2 shows also the structure of the zinc chlorin in which one of the pyrrole rings is in the dihydro form, and Fig. 3 shows the absorption spectra of these two forms. It is very easy to distinguish between the dihydroporphyrin, that is, the chlorin, and the porphyrin, and the spectral difference between the two substances has been used to study the kinetics of the photochemical transformation of one to the other (21).

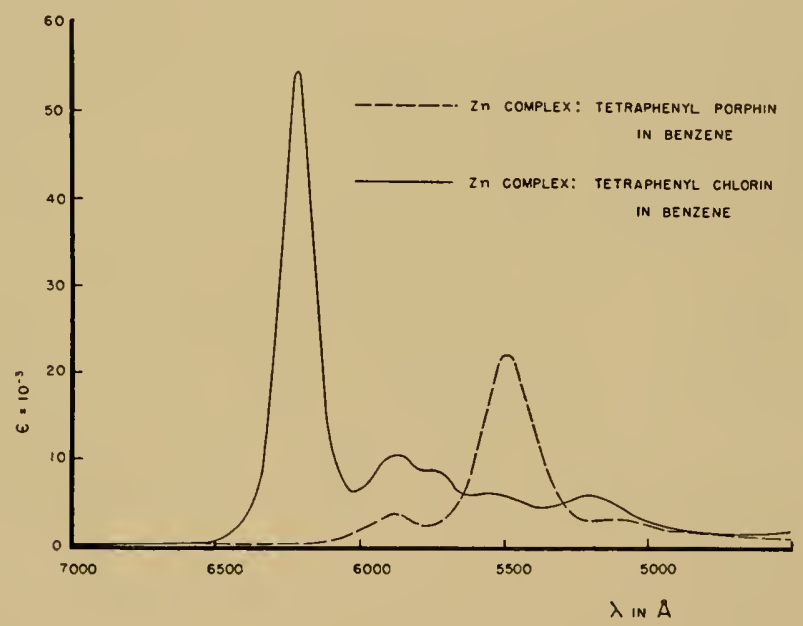

Fig. 3. Absorption spectra of tinc tetraphenylporphin and zinc tetraphenylchlorin.

The first of these transformations (and the easiest to study) was the photoinduced conversion of zinc dihydroporphyrin (chlorin) into zinc porphyrin, using some hydrogen acceptor. A whole series of hydrogen acceptors were used, most of them being quinones or molecular oxygen (33). It was easy to demonstrate a very clean photochenical conversion of dihydroporphyrin into porphyrin and nothing else. Fig. 4 shows the relative rates (quantum yields) of the transformations, and the relationship is clear between the rate of hydrogen transfer from chlorin to quinone and the ability of the quinone to hold the hydrogen, that is, the oxidation potential of the quinone. The greater the oxidation potential of the quinone, the faster is the transfer. Two series of experiments were done, one 
with para- and one with ortho-quinones; oxygen behaves like an ortho-quinone in the transformation, and there is a very nice relation between the potential and the photochemical yield.

Unfortunately, none of these transfers of hydrogen from chlorin to these oxidation agents (hydrogen acceptors) involved the storage of chemical energy. In every case, the thermodynamics is such as to favor the system porphyrin + hydroquinone (over chlorin + quinone), and the light is simply overcoming the activation energy. The kinetics of these reactions were studied in some detail (Dorough and Calvin, 21), and it was easy to demonstrate that a long-lived

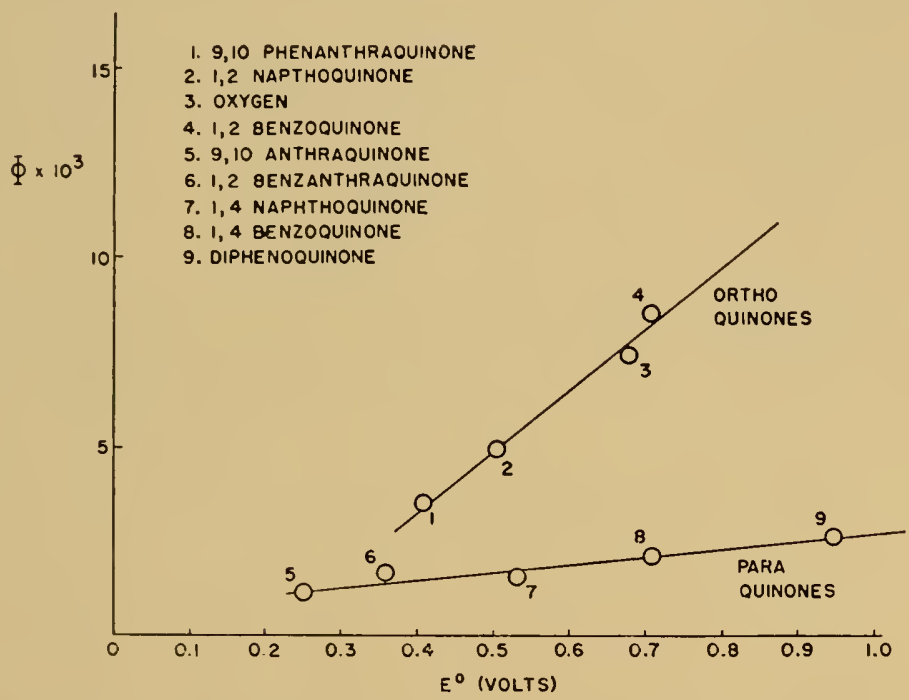

Fig. 4. Relation of quantum yield in photooxidation of zinc tetraphenylchlorin to the oxidation potential of hydrogen acceptor.

excited state of the chlorin was involved, since the rate of the reaction did not depend upon the concentration of the hydrogen acceptor at all, down to very low concentrations. This led to the suggestion that the excited state was the triplet state of the chlorin which has been found in a whole variety of chlorins, including chlorophyll $a(7,21)$.

Fig. 5 shows the kinetic analysis of this experiment. It is evident that the quantum yield is dependent upon the ratio of $k_{3}$ to $k_{3}+k_{\bar{n}}$, and what we suggest is that the ratio depends upon the quinone. You will notice that the rate law does not contain a factor for the concentration of quinone, but the quantum yield does contain a factor which is dependent upon which quinone you use. In this way we 
have accounted for the kinetic results. In this case the hydrogen acceptor is a very good oxidizing agent and the transfer does not entail any energy storage. If the transfer of hydrogen could be clemonstrated from such a dihydroporphyrin to a more powerful reducing agent, that is, a molecule (viz., pyridine nucleotide) which in its reduced form was more nearly like molecular hydrogen, perhaps something of more direct interest to photosynthesis could be shown.

The next question to be answered involved the possibility of doing the reverse reaction, i.e., a transfer of hydrogen from something which

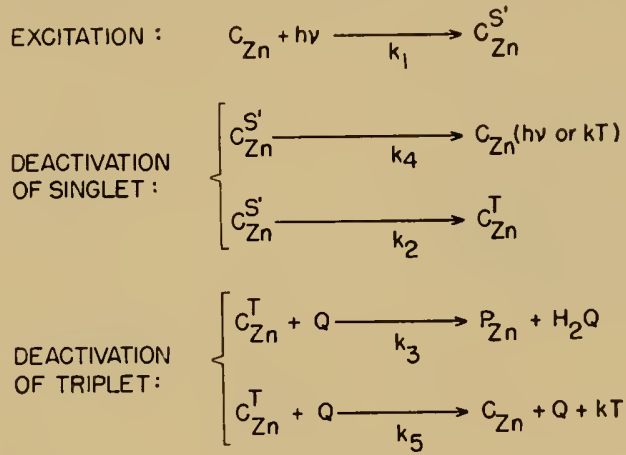

RATE LAW RESULTING FROM THESE STEPS:

$$
\begin{aligned}
& \left(C_{Z n}\right)+\frac{1}{\sum d} \log \left(1-10^{-\sum\left(C_{Z n}\right) d}\right)=r k a 10 t+\pi \\
& \text { WHERE QUANTUIM YIELD, } r \text {, EQUALS: } \frac{k_{2}}{k_{2}+k_{4}} \cdot \frac{k_{3}}{k_{3}+k_{5}}
\end{aligned}
$$

\section{KINETIC ANALYSIS OF PHOTOXIDATION OF Zn TETRAPHENYLCHLORIN}

lïg. 5. Kinclic analysis of plotooxidation of zinc teraphenylchlorin.

clearly was not as good a reducing agent as the dihydroporphyrin to the porphyrin to make the dihydroporphyrin; this actually would involve a storage of energy (58). Fig. 6 shows the results of such an experiment. Here zinc tetraphenylporphyrin is being reduced by benzoin, which is an ene-diol resembling ascorbic acid in some respect. The solid line in Fig. 6, upper, is the spectrum of the porphyrin, and after 7 minutes of illumination the porphyrin is dropping and the chlorin is coming in. Alter one and one-half hours of illumination, most of the dye is in the form of the chlorin and most of the porphyrin is gone. The fuantum yield of this reaction was extrenely small, much 
smaller than that for the transformation in the other direction, but it involved, I believe, a storage of chemical energy.

The reation does not generally stop at the dihydro-stage but goes on into the tetrahydro- and hexahydroporphyrin stages. The various relationships of these porphyrins are shown in Fig. 7, which illus-

Photoreduction of 21 ne Tetraphenylporphin

by Benzoln In Bonzone

Run $X I$ of Table VIII
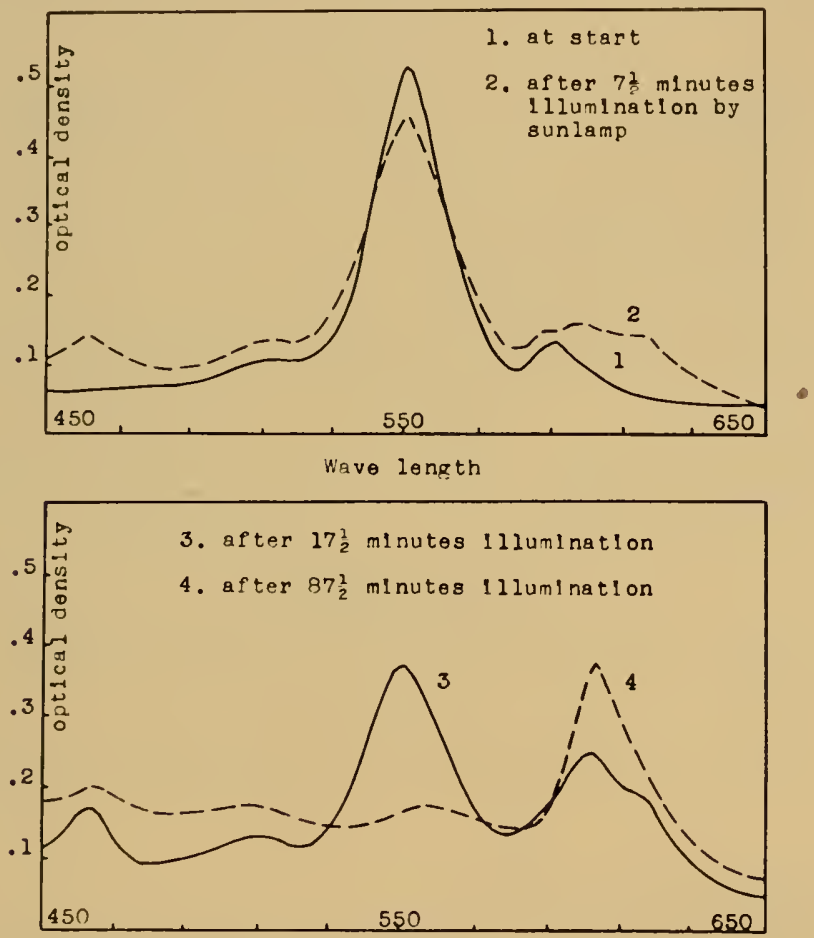

Fig. 6. Spectra showing photoreduction of zinc tetraphenylporphin by benzoin.

trates the hydrogen transfer reactions that have been demonstrated for this particular porphyrin. The first of these reactions was the transformation of chlorin by light into porphyrin using quinones as the acceptor. The reverse reaction, that is, the transformation of porphyrin into chlorin using ene-diols, is a variable one, depending on the ene-diol and also on the conditions of the reaction. The chlorin 
can then go further, into a tetrahydroporphyrin, which is a very good reaction compared to the first one. This result was in keeping with the notion that perhaps chlorophyll in the green plant was functioning not between chlorophyll and protochlorophyll but between the chlorophyll and dihydro- or bacteriochlorophyll. The latter reaction does not stop at the tetrahydroporphyrin but under vigorous conditions it can be pushed to the hexahydroporphyrin.

The reverse reaction, namely, tetrahydroporphyrin and quinone, goes directly to porphin. The dihydro-form is not observed in between. The reaction of chlorin is much faster, so that the accumula-

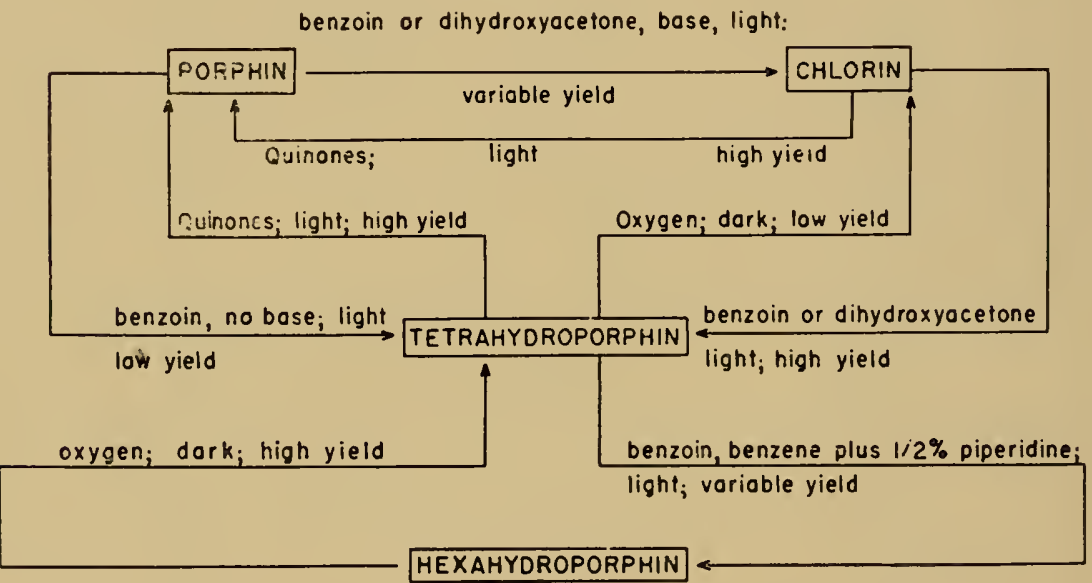

Fig. 7. Redox relations among the zinc tetraphenylporplyrins.

tion of chlorin is not seen. The hexahydro-form will auto-oxidize, even in the dark, with oxygen to give the tetrahydroporphyrin very readily.

\section{Thermodynamic Relationships}

The question arises as to what indeed are the various energy levels of these porphyrins with respect to water, pyridine nucleotide, oxygen, etc., the various molecules which are involved in the process of photosynthesis itself. There is no direct and unequivocal information about the energy of these various transformations, primarily because the energy of hydrogenation of porphyrin to chlorin, or chlorin to dihydrochlorin, is not known. Only indirect information is available about this, and one must deduce, by indirection, what these energies might be. It is interesting to note what is evolved if one makes the 
best estimates one can about the energies involved in these transformations.

In the conversion of a porphyrin to a chlorin, a double bond is hydrogenated to give the dihydro compound. In doing so, the conjugated macrocycle is not destroyed. One such double bond can be removed without destroying the conjugated macrocycle, and in bacteriochlorophyll, two such clouble bonds can be removed and a conjugated macrocycle still exists. Therefore, as a first approximation, I would suggest that we use, in attempting the thermodynamic estimate, a $\triangle H$ for this reaction of about $-30 \mathrm{kcal}$, which is approximately that of a substituted olefin. ${ }^{2}$

$$
\text { porphin }+\mathrm{H}_{2} \rightarrow \text { chlorin } \Delta H \simeq-30 \mathrm{kcal}
$$

In order to estimate the energy for the hydrogenation of the pyridine nucleotide, one must remember that the free energy of this reaction is very nearly zero, that is, the reduction potential of TPN is very nearly the same as that of molecular hydrogen. If the free energy of this reaction is near zero, the heat will be equal to the entropy loss, which is 9 kcal.

pyridine nucleotide $+\mathrm{H}_{2} \rightarrow \cdot \mathrm{PNH}_{2} \quad \Delta H \simeq-9 \mathrm{kcal}$

The reason for this figure being so small is that a very large aromatic resonance is destroyed, and that is why there is $20 \mathrm{kcal}$ less energy evolved in the hydrogenation of this material (TPN) than in the hydrogenation of an ordinary olefin.

Combining these two reactions, one can write for the hydrogen transfer from chlorin to pyridine nucleotide, to give reduced pyridine nucleotide:

chlorin $+\mathrm{TPN} \rightarrow$

or

(dihydrochlorin)

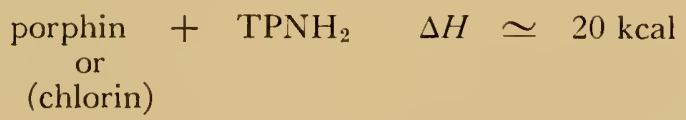

This is an "uphill" reaction of the order of $20 \mathrm{kcal}$. To complete the cycle, the dihydroporphyrin has to be recovered. What is available now is a porphyrin (protochlorophyll, if the reaction is running

"The relation of protochlorophyll to chlorophyll might very well be materially different due to the steric requirements of the isocyclic $\left(\mathrm{C}_{5}\right)$ ring. Here one might expect somewhat less steric interference in the chlorophyll and thus the $\Delta H$ might he more negative. 
between chlorophyll and protochlorophyll) which has to be returned to the chlorin stage in order for the reaction to continue. The hydrogen for this retum must ultimately come from water, knowing what we clo about the stoichiometry of the photosynthetic reaction. The energy of getting hydrogen from water is $+56 \mathrm{kcal}$. This reaction can now be combined with the hydrogenation of the porphyrin to the dihydroporphyrin:

$$
\mathrm{H}_{2} \mathrm{O} \rightarrow \mathrm{H}_{2}+1 / 2 \mathrm{O}_{2} \quad \Delta H \simeq+56 \mathrm{kcal}
$$

porphin $+\mathrm{H}_{2} \mathrm{O} \rightarrow$

$$
\text { chlorin }+1 / 2 \mathrm{O}_{2} \quad \Delta H \simeq+26 \mathrm{kcal}
$$

The photolysis of water has now been divided into two steps, (5) and (3), one of which is the transfer of hydrogen to the porphyrin, if the reaction is ruming between porphyrin and chlorin (5). The calculations for the two reactions between chlorophyll and bacteriochlorophyll would be exactly the same as far as our precision is concerned. The total energy required for the reaction of two electrons - the generation of one-half mole of oxygen-has now been divided into two approximately equal parts, with one reaction "uphill" approximately $20 \mathrm{kcal}$ and the second one approximately $26 \mathrm{kcal}$.

In actual fact, this half mole of oxygen probably does not come off directly as molecular oxygen but goes through some oxygen acceptor species, which then goes on to molecular oxygen. Reaction (5) would thus be broken into several steps, the size of which would depend on the nature of the unknown oxygen carrier.

Our model reactions have indeed shown that hydrogen can be transferred from dihydroporphyrin to an acceptor. Unfortunately, this particular hydrogen acceptor-quinone-is a very good one (not as poor a hydrogen acceptor as pyridine nucleotide), so it does not really correspond to such a transformation as reaction (3) with a $+\triangle H$. The fact remains, however, that light will produce an excited state which induces the transfer of these hydrogen atoms to an acceptor. The reduction of the doulsle bond has been achieved only with much better hydrogen donors than water. They have always been relatively good reducing agents, such as ascorbic acid and a varicty of other cne-diols or hydravines which are the common materials used for this kind of transformation.

In fact, the plotochemistry of chlorophyll models, and chlorophyll itself for that matter, has not yet produced in solution a model reaction in which such reactions as those described above, which involve relatively large encrgy storage acts, have been accomplished. 


\section{Chloromitil Photosensitized Transformations}

\section{Hydrogen Transfer Reactions Catalyzed by Chlorophyll}

Let us retum to the sensitised hydrogen transfer reaction, which is what one of the overall reactions of photosynthesis is presumed to be (hydrogen transfer from water to pyridine nucleotide), and determine which ones may be catalyzed by chlorophyll. The classic examples are the hydrogen transfers from materials such as ascorbic acid and hydrazine to dyestuff-acceptors such as azo dyes (methyl red). These reactions have been known for some time, and in the last 15 years they have been studied a great deal, particularly in the Soviet Union. One such reaction is called the Krasnovskii reaction after the man who has spent a great deal of time studying it (42, 43, 44, 46, 47). Krasnovskii used chlorophyll and porphyrin model substances as sensitizers to transfer hydrogen from a variety of donors (ascorbic acid, particularly) to methyl red and other azo dyes. He did it under such conditions that he was able to show two steps as separate events, that is, the transfer of hydrogen from the hydrogen donor to chlorophyll to give some intermediate, followed by the transfer of hydrogen from this intermediate to the hydrogen acceptor, giving back again the initial chlorophyll. By cooling the reaction mixture, and performing the experiment in a basic solvent such as pyridine, Krasnowskii was able to show that chlorophyll plus ascorbic acid, without the addition of a hydrogen acceptor, would go from a green color to a pink color. This pink color was presumed to be some intermediate, not necessarily bacteriochlorophyll, since the spectrum did not correspond. The reaction reverses in the dark, and the "pink" intermediate is not a radical (49).

The general result of all of these studies is shown in Fig. 8 (27, 42, $43,44,46,47)$, in which the whole series of transformations is systematized. The chlorophyll absorbs the light, the excited chlorophyll (probably in a triplet state, as the kinetics indicate that such is the case) removes either a hydrogen atom, or an electron from the donor $\left(A \mathrm{H}_{2}\right)$ to give what Krasnovskii believes to be a radical, or a radical ion $\left(\dot{C} h^{-}\right)$, (at low temperatures, he believes he has caught this radical ion (45)), leaving behind a positive radical $\left(A \mathrm{H}_{2}{ }^{+}\right)$which then dissociates to give a proton. The free radical or ion $\left(\dot{A} \mathrm{H}\right.$ or $\left.\dot{A} \mathrm{H}_{2}{ }^{+}\right)$can then go ahead and reduce another chlorophyll, and the free radical ion of chlorophyll $\left(\dot{C} h^{-}\right)$can hand on the hydrogen atom, or electron (or both) to the clyestuff $\left(D_{y}\right)$ to give back the chlorophyll starting material and the partly reduced semi- 
quinone of the dye. This, then, finishes its reduction, either by combination with a radical or by taking a proton directly off the hydrogen donor itself, to give the colorless dyestuff and the dehydroascorbic acid or other dehydro compound. This is a generalized scheme which appears to apply for a whole variety of hydrogen donors, hydrogen acceptors, and sensitizing dyes. The reaction will work, for example, with acridine orange as the sensitizing dye, for allylthiourea as the hydrogen donor, and for oxygen as the hydrogen acceptor.

In general, these reactions do not involve the storage of any energy, that is, the reaction hydrogen donor + dyestuff $\rightarrow$ reduced dye + dihydro hydrogen donor (ascorbic acid, etc.) is thermodynamically

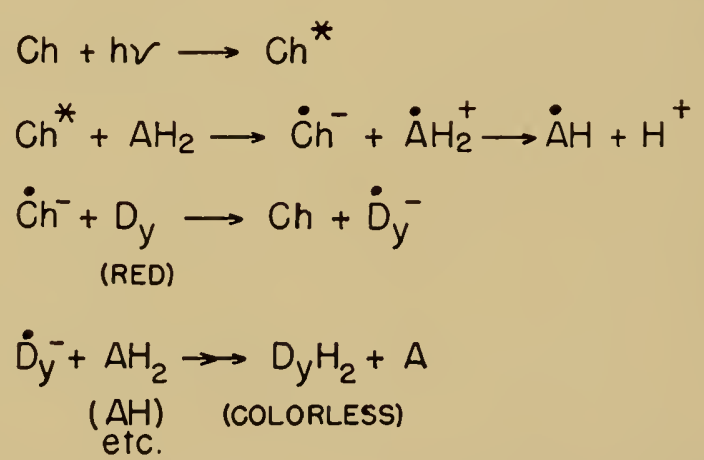

Fig. 8. Chlorophyll-sensitized reduction of dye by ascorbic acid (Krasnorskii,

Erstigneev). Ch, chlorophyll; D, dye; A, hydrogen doner.

positive; the energy is "downhill" in that direction. However, there are a few cases in which the reaction seems to be reversible, that is, when the light is turned off there follows the reappearance of oxidized dyestuff. Whether this indeed represents a small degree of energy storage (a few kcal) or whether it represents a trace of oxygen in the reaction mixture which, in general, will oxidize most of these reduced materials, remains to be demonstrated. In any case, there is no great storage of free energy in this system; most frequently these reactions are "downhill" thermodynamically and will not go backwards. When they do go backwards, it seems as though these dihydro dyestuffs are auto-oxidizable, and traces of oxygen in the reaction mixture may carry them back.

There are a number of photochemical electron transfer reactions involving dyestuffs unrelated to chlorophyll and which apparently do 
constitute some energy storage. One of these is the reaction $(1,10$, $54)$

$$
\text { thionine }+\mathrm{Fe}^{++} \underset{\text { dark }}{\stackrel{h v}{\rightleftarrows}} \text { leucothionine }+\mathrm{Fe}^{+++}
$$

Here also the energy storage is small ( $\sim 5 \mathrm{kcal}$ per quantum) at best.

\section{Photophysical EFFects in Monel Systems}

\section{Energy Transfer in Solid Systems}

The failure of all of these various types of solution model reactions to provide a case where energy of the order of 20 to $40 \mathrm{kcal}$ per quantum is being stored is in itself significant. I think it may be demonstrated that this is not the direction in which to look for the energy-storing reaction in photosynthesis. Some years back, as the structure of the chloroplast became somewhat clearer to us (primarily through electron microscopy) $(28,66)$ and as our knowledge of the photochemical or photophysical behavior of ordered systems developed in the form of a body of theory and information on the photoresponse of atomic crystals $(31,70)$, the notion that the photosynthetic apparatus might not be functioning as ordinary molecules in solution, but rather as something approaching molecular crystal behavior, became popular $(13,67)$.

We undertook to seek possible models for such systems in the laboratory. In addition to the electron microscopy on the chloroplasts, there was, of course, the very well-known fact that the absorption spectrum of chlorophyll in the living organism is not identical with the absorption spectrum of chlorophyll in solution. This, together with the ordered structure that was seen in the electron microscope, suggested that the chlorophyll in the living organism might be in a physical form quite different from a true solution. The difference in the spectra is quite obvious. The solution spectrum of chlorophyll has a peak at about $6600 \mathrm{~A}$ and in the living organism chlorophyll has its peak somewhere near $6800 \mathrm{~A}$. This shift toward a longer wavelength, from 6600 to $6800 \mathrm{~A}$, is exactly the kind of shift observed in the spectra of all sorts of pi-molecules when they are packed in crystals. When the pi-clourls of large, conjugated systems are brought close together, there is an interaction which shifts the energy of the excited state (or the difference between the ground and the excited state). This is quite common in all pi-molecules, and in chlorophyll it has been examined by Rabinowitch and by 
Trurnit $(35,71)$. Fig. 9 shows that the so-called amorphous solid layers of chlorophyll have absorption in the $6800 \mathrm{~A}$ region, and when the chlorophyll layers are allowed to "crystallize" the absorption spectrum moves out almost to $7200 \mathrm{~A}$. Intermediate spectra of chlorophyll can be obtained, depending on the nature of the monolayers, in which the peaks lie between the $6600 \mathrm{~A}$ peak of the true chlorophyll solution and the 7200 A peak of the crystalline chlorophyll.

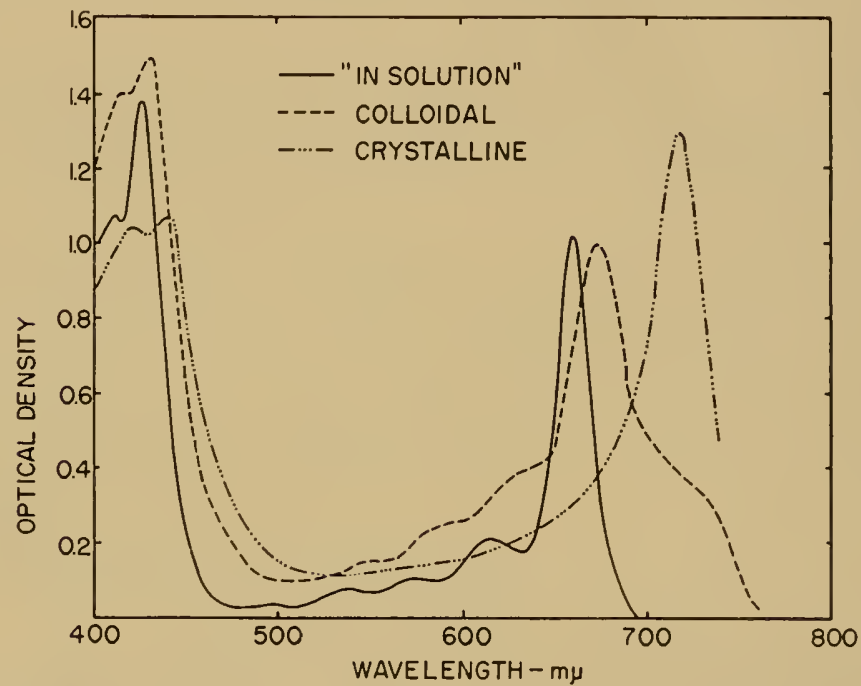

Fig. 9. Ibsorption spectra of chlorophyll in various states (Rabinowitch).

\section{Phthalocyanine as a Model for Chlorophyll Energy Transfer}

The ordered structure of the chloroplasts, as observed in the electron microscope, together with the difference in the chlorophyll spectra in solution and in the crystalline state, were some of the things which induced us to think in terms of solid lattices as a possible way in which the energy, which is absorbed in the chlorophyll molecule, might be handled in the chloroplast, and in which the oxidizing and reducing power might be separated. Again, we sought models of various kinds so we could experimentally develop some concepts for such a separation, and here we turned to the experiments which were begun in the Soviet Union in 1919 by Vartanyan $(73,74)$ and which wore extended by Eley in England $(22,23,24)$. Eley examined the electronic properties of crystals of a very stable molecule related to chlorophyll, namely, phthalocyanine, the structure of which is shown in Fig. 10. One can again recognize the tetrapyrrolic structure 
of the pigment, and this molecule has some resemblance to the porphyrin structure previously discussed. There are major differences, however, which must be kept in mind: the bridging atoms in phthalocyanine are nitrogen atoms instead of carbon atoms, and fused onto each of the pyrrole rings is a benzene ring which, of course, changes the nature of the compound considerably. It happens that I became familiar with this molecule in 1937, shortly after its discovery by Linstead, and participated in the demonstration of some of its catalytic abilities at the same time that Eley was working with it $(16,17)$. Eley has gone on to examine the electrical properties of phthalocyanine, and in recent years we turned to this also.

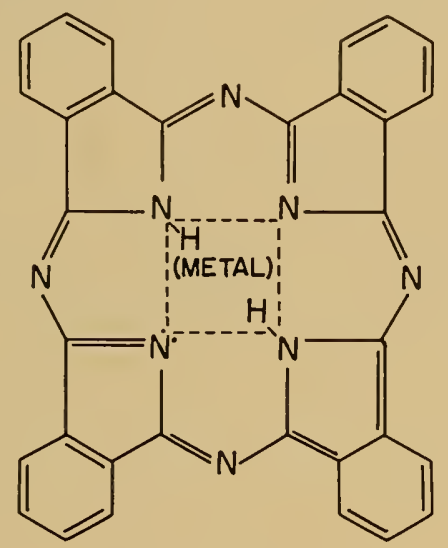

\section{PHTHALOCYANINE}

Fig. 10. Structural formula of phthalocyanine.

Phthalocyanine is a very stable substance, easy to prepare and not easy to destroy (compared to chlorophyll), and we have used it as a model in our photophysical measurements. The first experiments undertaken were to demonstrate the effect of electron acceptors, or electron donors, added to crystalline phthalocyanine, on its conductivity and its photoinduced conductivity. These experiments were one step beyond what Vartanyan and Eley had done. They studied primarily the behavior of what they believed to be the pure phthalocyanine.

We made layers of phthalocyanine on a conductivity cell and then adcled electron donors or acceptors to it to see what effect these would have on the electrical conductivity in the dark and on the photo- 
"SURFACE" CELL SHOWING ARRANGEMENT OF ELECTRODES

(A) Negative

Electrode spocing

Aquodog

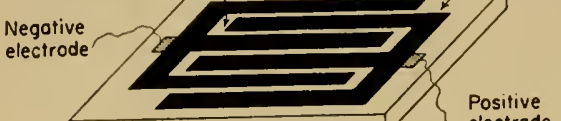

$\xi n v$

(B)

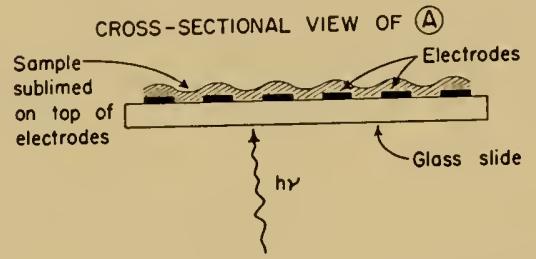

Fig. 11. Diagram of sample cells (conductivity).

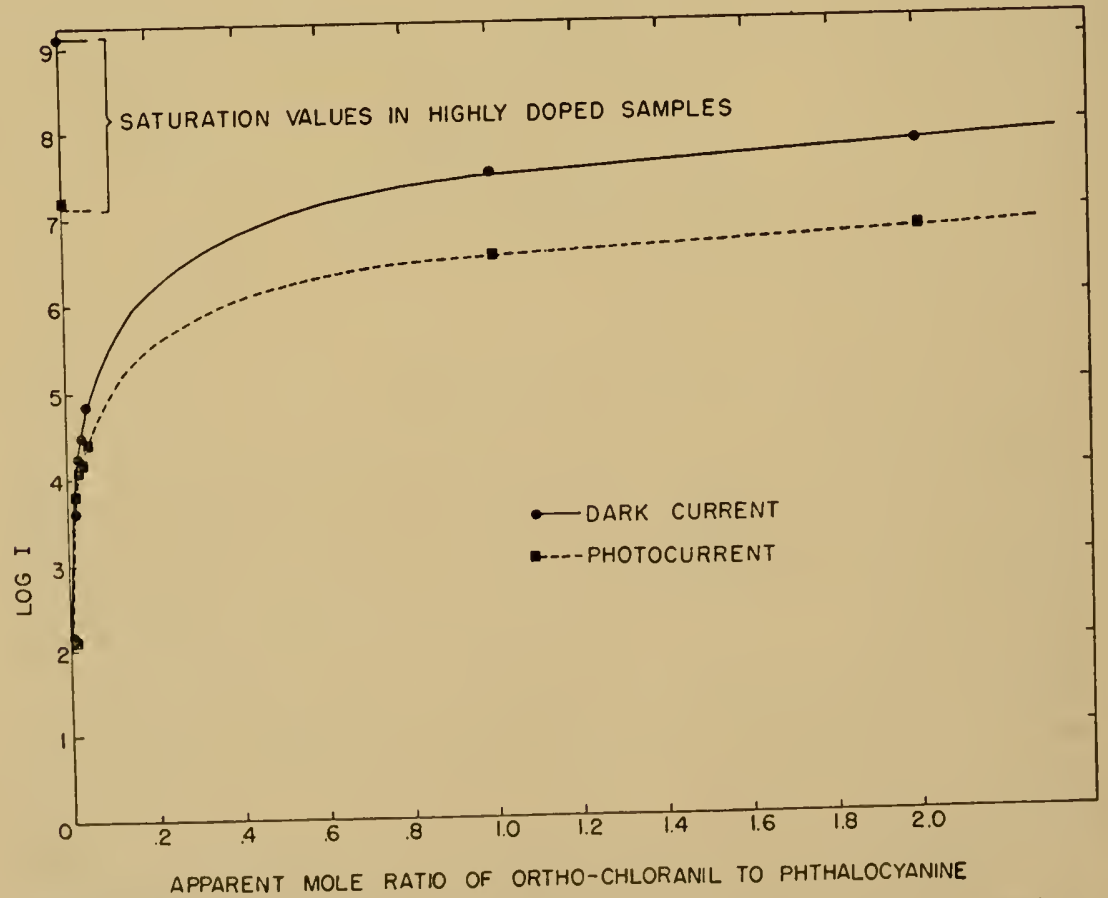

lig. 12. Variation of dark conductivity and photoconductivity of phthalocyanine witl amomm of $o$-chloranil added. 
induced conductivity $(38,39,40)$. Fig. II shows such a conductivity cell with the electrodes, on top of which is placed a layer of phthalocyanine. On top of that is laid the layer of electron acceptor. The results of experiments using such a conductivity cell are shown in Fig. 12, where the solicl line gives the effect of added electron acceptor on the dark current. The dark current conductivity of such a sample, if electron acceptor is added, goes up as much as seven powers of ten. The same type of thing is true of the photoinduced conductivity, which goes up by as much as five powers of ten as electron acceptor is added on top of the layer of the phthalocyanine. I shall not try to review all of the kinetic and spectral studies which have been performed on this phthalocyanine system, but I shall show only a few of the highlights and then present to you what we believe to be the behavior of this molecular crystal in electronic terms.

When these electron acceptors were added to the phthalocyanine layer, it was found that electron transfer took place, from the phthalocyanine to the electron acceptor, even in the dark, as evidenced by the presence of free-radical-like signals, determined by electron spin resonance (ESR), in such a "doped" or treated phthalocyanine sample (40). This is shown in Fig. 13, and the interesting fact is that by treating (doping) the phthalocyanine with electron acceptor (o-chloranil) we increase the dark current and also increase the lightinduced conductivity. When the light is turned on such a sample as this, the number of unpaired electrons is decreased, as indicated by the electron spin signal. Fig. 14 shows how this system behaves.

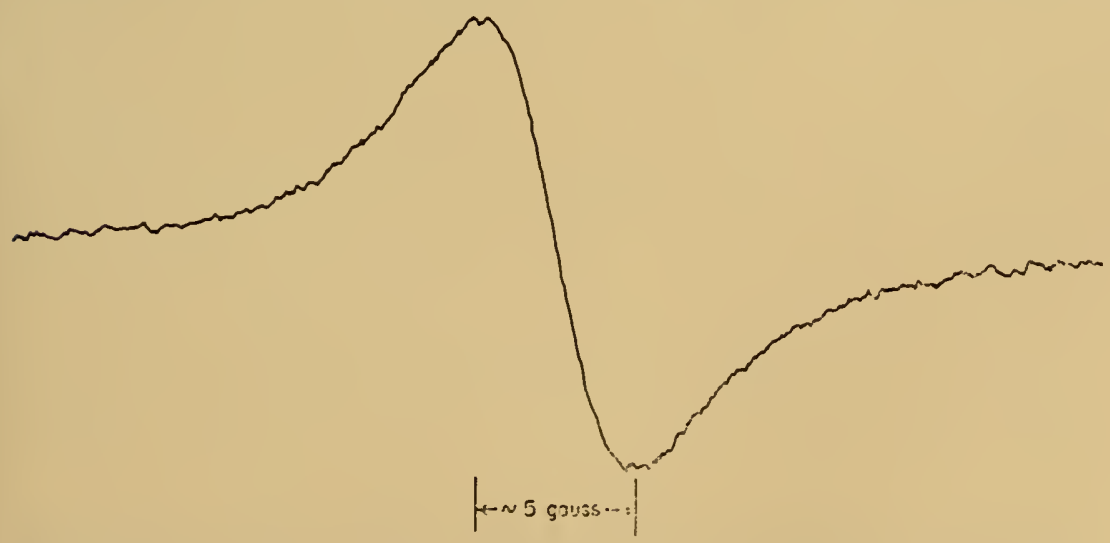

Fig. 13. Electron spin resonance (ESR) spectrum of o-chloranil-"doped" phthalocyanine. The curve represents the first derivative of the absorption. 


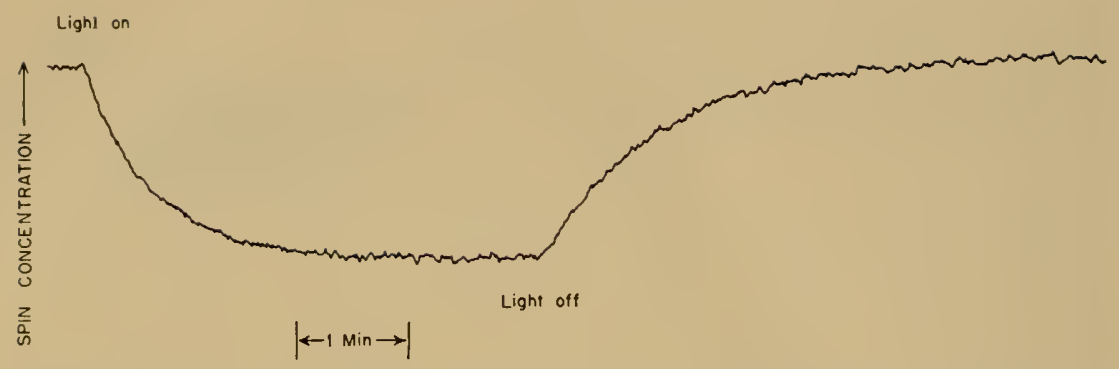

Fig. 14. Effect of illumination on the electron spin resonance signal of $o$-chloranil "doped" metal-free phthalocyanine. Curve represents unpaired spin concentration is. time.

The conductivity, of course, goes in the reverse direction; when the light is turned on, the conductivity increases and when the light is turned off, the conductivity decreases, with rates corresponding to these spin signal changes.

Fig. 15 shows the relationship between the kinetics of the spin signal behavior and the several other associated phenomena in the same lattice, such as conductivity, etc. Fig. 16 shows the interpretation of these phenomena. They are interpreted in terms of electron transfer from phthalocyanine molecules, in the lattice, to the $o$ chloranil to give positive ion radicals of phthalocyanine which are in a crystal lattice, and these are responsible for the conductivity (Fig. 16-I). The electrons on the o-chloranil negative ion radical are not mobile, and presumably they are the things which the ESR equipment sees. When the light is turned on, it is absorbed by the phthalocyanine, and the exciton can migrate around in the phthalocyanine until it is ionized (Fig. 16-2). This ionization may take place either at some unknown center, or the exciton may come directly in contact with the $o$-chloranil negative ion, transferring a second electron to the o-chloranil negative ion, and thus reducing the number of unpaired spins but increasing the conductivity. In the dark the reverse reaction occurs. When light at $4000 \mathrm{~A}$ is used, the reverse effect is observed, that is, there is a transfer of the electrons from the o-chloranil negative ion radical into an unoccupied orbital of the phthalocyanine crystal (Fig. 16-3). This is also an casily movable electron rather than a trapped one, and it will immediately and rapidly recombine with the conducting holes (positive ion centers) originally present in the lattice, and thus result in a decrease in conductivity but an increase in electron spin signal (Fig. 16-4) . 


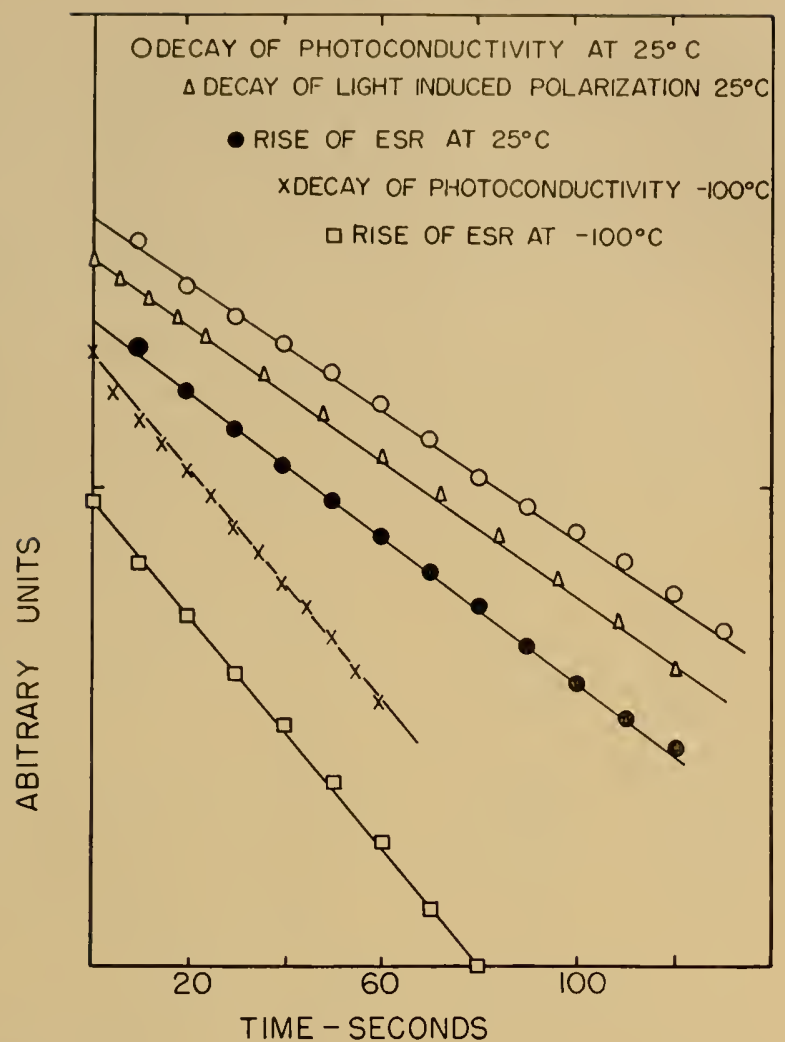

Fig. 15. Semilog plot of time dependence of photoconductivity, light-induced electron spin resonance (ESR) and light-induced polarization in doped phthalocyanine.

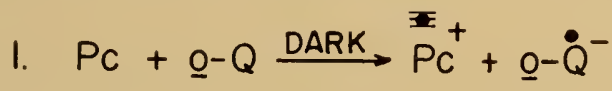

$$
\begin{aligned}
& \text { 2. } P C+\underline{O}-\dot{Q}^{-} \underset{\text { DARK }}{\stackrel{h \nu(7000 \AA)}{\rightleftarrows}} P^{+}+\underline{0}-Q^{=}
\end{aligned}
$$

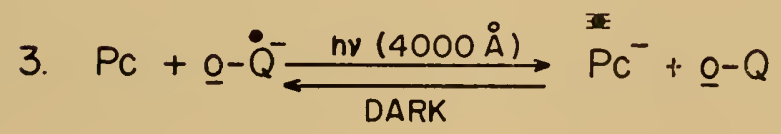

$$
\begin{aligned}
& \text { 4. } \mathrm{PC}^{-}+\mathrm{PC}^{+} \stackrel{\text { DARK }}{\longrightarrow} 2 \mathrm{PC}
\end{aligned}
$$

Fig. 16. Reactions of a solid matrix of P'c with a film of o-Q. Pc, phthalocyanine; $o$-Q, o-chloranil. 
Fig. 17 shows a representation of the phthalocyanine negative ion, phthalocyanine itself, phthalocyanine positive ion radical, o-quinone, $o$-quinone negative ion radical, and o-quinone double negative ion, in molecular orbital terms, to call to mind the way in which we have been thinking about the $\pi$-energy levels of these conjugated molecules.

The actual processes which were seen in Fig. 16 can now be illustrated in terms of molecular energy levels, and Fig. 18 shows that interpretation. Reaction (1) is a transfer, in the clark, of an electron from the highest occupied phthalocyanine orbital to the lowest unoccupied o-quinone orbital, leading to the formation of phthalocyanine positive ions. This occurs in the crystal lattice so these are conductivity holes, and the trapped electrons are in the o-quinone

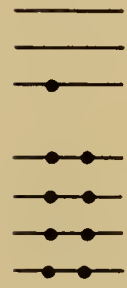

$\stackrel{\mathrm{PC}^{-}}{ }$

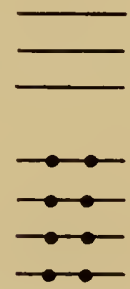

Pc
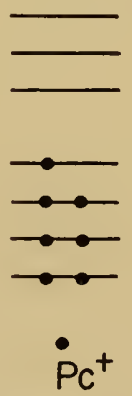
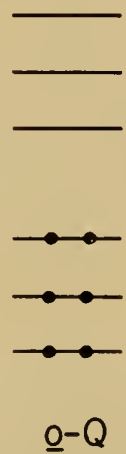
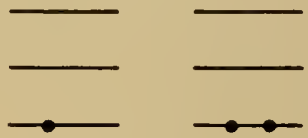

Fig. 17. Schematic representation of the electronic energy levels of rarious l'c and $o \cdot Q$ species (the various species should not be compared). P'c, phihalocyanine: $o-Q, o$-chloranil.

negative ion. The lowest moccupied quinone level is shown below the highest phthalocyanine occupied level, and this reaction takes place quite spontaneously in the dark. The photochemical transformation, reaction (2), involves, first the excitation of the phthalocyanine itself, which could be represented by the raising of an electron from the highest occupied $\pi$-orbital (or from a $N-n$ orbital) to the lowest unoccupied $\pi$-orbital, which must lie very nearly at the same level as the singly-unoccupied orbital of the o-quinone negative ion. The reason for this shift of relative levels is that, whereas in the first instance transfer is occurring from one neutral molecule to another, here the transfer is from a neutral molecule to a negatively-charged, already singly-occupied orbital.

The third process, in Fig. 18, the one represented by the decrease in photoconductivity by illumination at $4000 \mathrm{~A}$, involves the excita- 
tion of the electrons in the o-quinone negative ion up to an excited orbital which can then be mansferred into the lowest unoccupied orbital of the phthalocyanine crystal (a conduction orbital). This, then, is a relatively mobile electron which can rapidly find and neutralize the conductivity holes in the phthalocyanine lattice, leading to a decrease in conductivity. These represent the principal processes shown in Fig. 16.
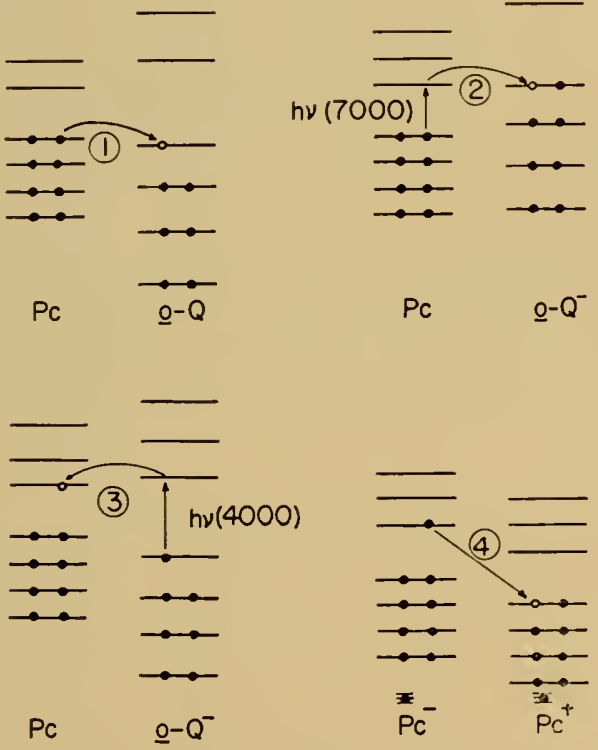

Fig. 18. Diagrammatic molecular orbital representation of a solid matrix of phthalocyanine with $0-\mathrm{Q}$.

Fig. 19 shows the actual separation of charge that can be accomplished in this model system if it is constructed properly. Here is shown a matrix of phthalocyanine, on the surface of which lies an o-quinone layer. There will be some negative charge trapped in the o-quinone (acceptor) layer, and the positive charge will remain in the phthalocyanine (donor) layer. This will induce a polarization in the pair of electrodes between which the double layer is placed. The polarization will be increased by shining light absorbed by phthalocyanine on the clouble layer, in which case there will be an additional accumulation of negative charge in the quinone and an 


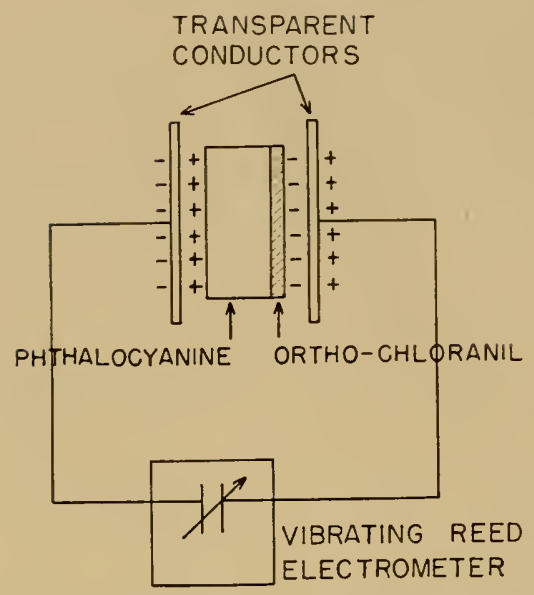

Fig. 19. Schematic diagram of polariation apparatus.

additional accumulation of positive charge in the phthalocyanine. This is exactly what happened (40), and it is a photochemically induced separation of oxidizing power (positive holes) and reducing power (quinone double-negative ions). Presumably, this sort of thing could occur in the individual layers which can actually be seen in the chloroplasts.

\section{The Relation to the Photosynthetic Apparatus}

What bearing does this information have on the photosynthetic apparatus itself? The obvious relationship is that the phthalocyanine might be considered as a model for the chlorophyll layer itself. The electron acceptor, here listed as o-chloranil, might indeed be some electron acceptor in the chloroplasts such as Cocnzyme Q.25s (Plastoquinone $)(8,20,11,18,60)$, which conceivably could have a function similar to the function that the $o$-chloranil has in the model system, but with certain differences.

\section{Electron Spin Resonance in Chloroplast Materials}

If this is a model for the actual chloroplast behavior, then we should see some of these electronic properties in the chloroplast itself. First of all, the change of absorption spectrum in the chloroplasts from that of a true solution has been mentioned earlier. Unfortunately, we camnot place electrodes on cither side of the lamellar layers of the chloroplasts as we have been able to do in the phthalocyanine system, but there are a number of properties which can be 
observed. One of these is the generation and disappearance of the unshared electrons which we have seen manipulated in the model system. Fig. 20) shows the photoproduction of unshared electron pairs in whole spinach chloroplasts at room temperature and at $-150^{\circ} \mathrm{C}$ (61). The fict that unshared pairs of electrons can be produced by
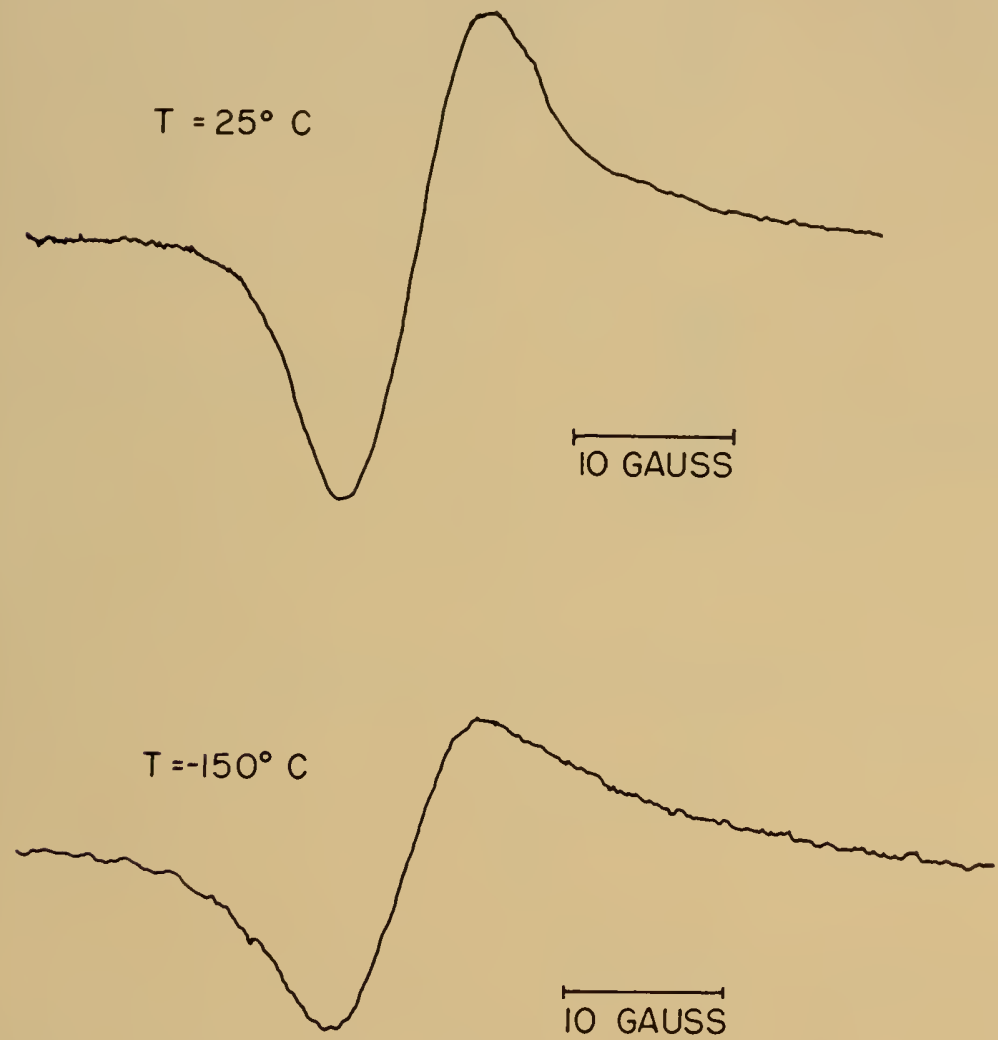

Fig. 20. Light signals from whole spinach chloroplasts.

red light is in itself some indication that rather profound changes are occurring in the chloroplast. These cannot be clue to triplet states because the interaction of the two electrons in a single triplet molecule in randomly arranged chloroplasts would broaden the signal so as to make it unobservable (34). The direct photochemical fission of a chemical bond (by some sort of predissociation process) scems entirely unlikely by a quantum supplying no more than $40 \mathrm{kcal}$ of energy at.most. 
As in the model systems, these signals must be due to the generation of unpaired electrons somewhere in the system. The fact that they can be induced by red light, and incluced just as rapidly at $-150^{\circ} \mathrm{C}$ as they are at $+25^{\circ} \mathrm{C}$, suggests that this is not due to an ordinary chemical reaction which requires any kind of activation energy. If that were the case, the formation reactions at $-150^{\circ} \mathrm{C}$ should have very different rates than the reactions at $+25^{\circ} \mathrm{C}$, and they do not. In Fig. 21 you can see the growth and decay of these signals, as far as they have been measured. At room temperature the signal rises
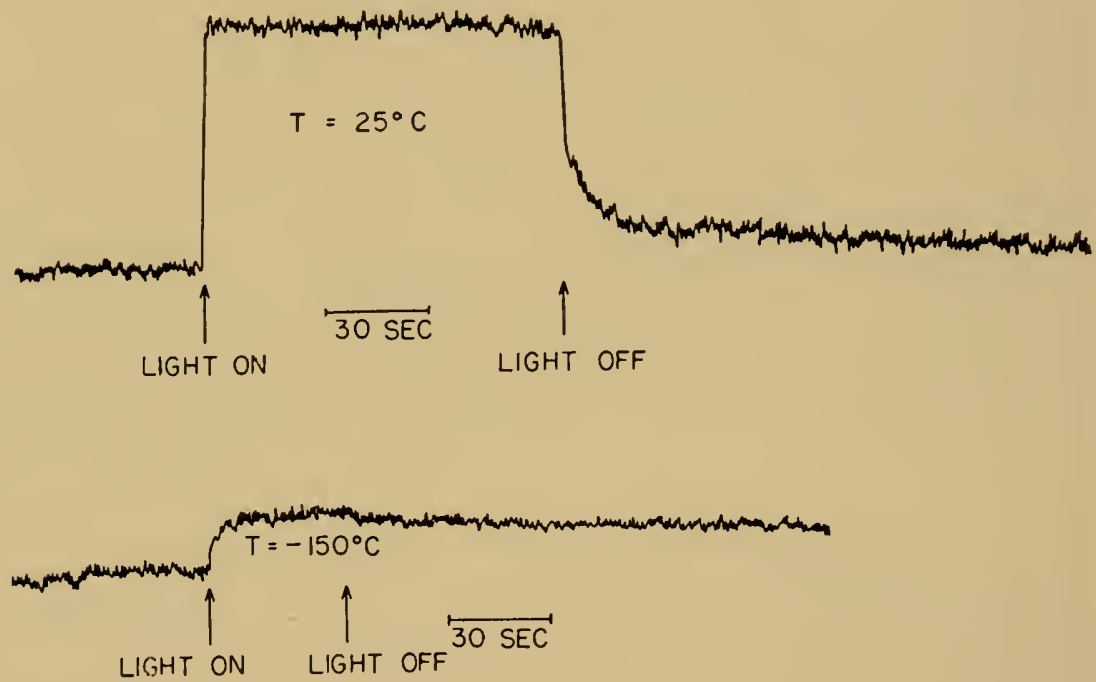

WHOLE SPINACH CHLOROPLASTS

Fig. 21. Growth and decay curves of whole spinach chloroplasts.

just as fast as the instrument can measure it and part of the signal falls very rapidly when the light is turned off, thus indicating the presence of at least two different kinds of unpaired electrons. At $-150^{\circ} \mathrm{C}$ the rise of the signal is just as fast as the instrument can follow it, but in the case of the chloroplasts it does not fall at all at low temperatures, indicating that the back-reactions in this case do indeed have a temperature coefficient.

Fig. 22, opposite. Left, lSR signals from Rhodospirillum rubrum, 5 minutes continuous illumination. Right, rise and decay of ESR signals from Rhodospirillum rubrum. 

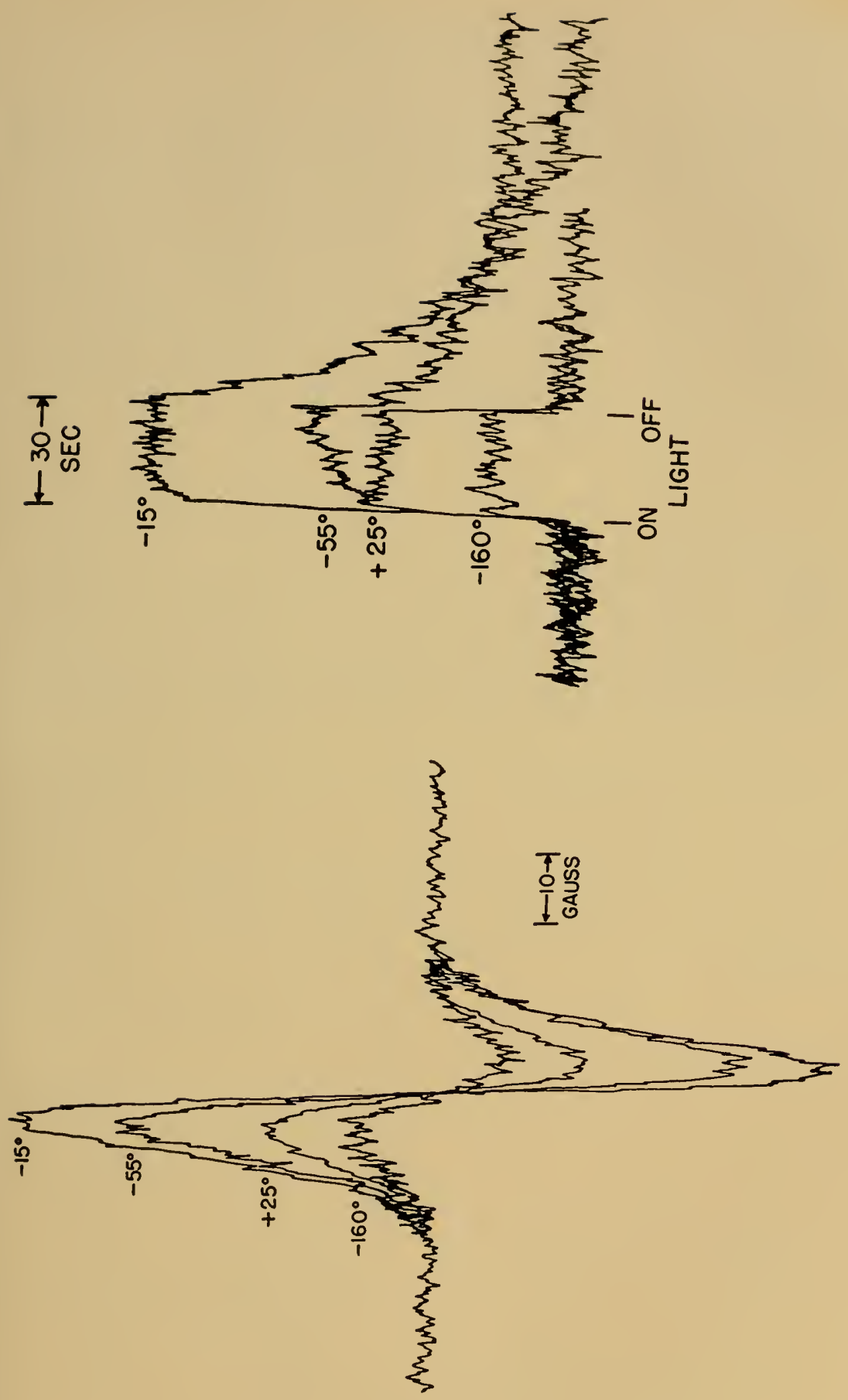
In the case of Rhodospirillum rubrum the reaction is also complex; some of it has a temperature coefficient and some of it does not. Fig. 22 shows the Rhodospirillum signal growth and decay (14). At both $+25^{\circ} \mathrm{C}$ and $-150^{\circ} \mathrm{C}$ the growth and clecay were as fast as the instrument could follow. In the intermediate temperature regions, some fraction of the decay was slow, indicating that this is a complex signal made up of several different kinds of unpaired electrons, probably formed in sequence. At low temperature there are no slowly formed, unpaired electrons; they are formed extremely rapidly and they decay extremely rapidly.

\section{The Structural Requirement}

One could ask the question: Is the chloroplast needed to produce such unpaired electrons? Could not such unpaired electrons be produced photochemically, just using the chlorophyll and its associated pigments? $(12,55)$. This experiment has been performed by several workers (61), including some in our own laboratory (2). Fig. 23 shows the production of such unpaired electrons by the pigments which are extracted by methanol from chloroplasts. The signals so

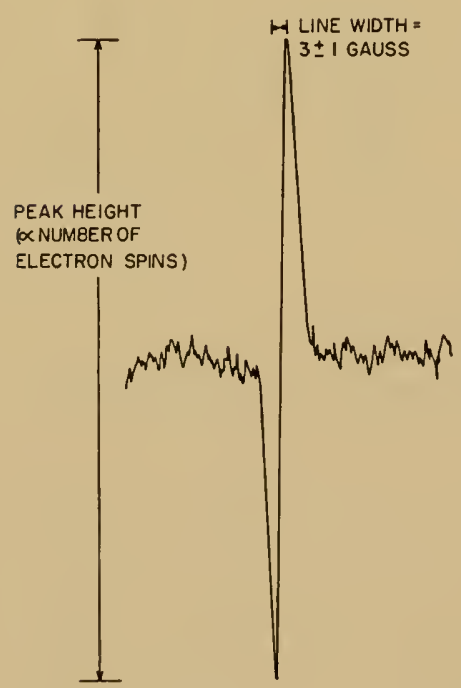

SIGNAL

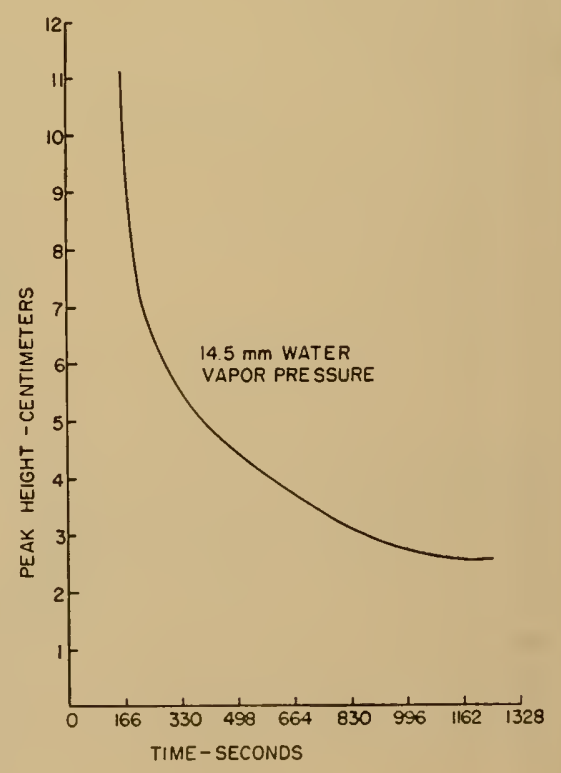

DECAY

lig. 23. Pholo-induced lESR of methanol extract of Chlorella. 
produced at $25^{\circ} \mathrm{C}$ are quite different from the signals produced in the whole chloroplasts, either at room temperature or at very low temperatures (see Fig. 23). The signals in the chloroplasts were 10 to 20 gauss wide and the signals in the chloroplast extracts are only 3 gauss wide. Furthermore, their decay at room temperature is slow compared to the decay of signals in the chloroplasts. It is clearly possible, therefore, to produce such signals in methanolic extracts, and if this methanolic extract is thoroughly dried, there is no signal. Furthermore, if the methanolic extracts are fractionated by petroleum ether so a cleaner chlorophyll is obtained, then the signals that are produced are broader and smaller.

\section{Carotenoid Requirement}

There is one other type of experiment which has been performed in an attempt to determine the point at which these signals originate in the chloroplast and what factors determine this point, and that is the experiment done with Rhodopseudomonas, of which we have two types: the will type, which contains carotenoid; and the mutant (65), which does not contain the conjugated carotenoid. An attempt was made to see if the conjugated carotenoid was involved in the photoproduction of the spin signal $(4,5)$. Very nearly the same characteristics in the signal are obtained (Fig. 24) whether the wild

\begin{tabular}{|c|c|c|c|}
\hline & $\begin{array}{l}\text { RHODOPSE UDOMONAS } \\
\text { SPHEROIDES (WILD) }\end{array}$ & $\begin{array}{l}\text { RHODOPSEUDOMONAS } \\
\text { SPHEROIOES (MUTANT) }\end{array}$ & $\begin{array}{l}\text { RHODOSPRILLUM } \\
\text { RUBRUM }\end{array}$ \\
\hline$g$ & $<2$ BY $\sim 1 \%$ & $<2$ BY 1\% & 2 \\
\hline LINE WIDTH & $20 \mathrm{~g}$ & $20 \mathrm{~g}$ & $10 \mathrm{~g}$ \\
\hline $\begin{array}{l}\text { CHLOROPHYLL } \\
\text { CONTENT }\end{array}$ & $0.4 \mathrm{mg} / 100 \mathrm{ml}$ sol. & $0.2 \mathrm{mg} / 100 \mathrm{ml} \mathrm{sol}$ & - \\
\hline $\begin{array}{l}\text { SIGNAL } \\
\text { AMPLITUDE }\end{array}$ & $A_{0}(S M A L L)$ & $A_{0}$ & $\geqslant 5 A_{0}$ \\
\hline $\begin{array}{l}\text { INITIAL } \\
\text { DECAY }\end{array}$ & $\begin{array}{l}\text { SECONDS } \\
\text { TEMPERATURE } \\
\text { INDEPENDENT }\end{array}$ & $\begin{array}{l}\text { SECONDS } \\
\text { TEMPERATURE } \\
\text { INDEPENDENT }\end{array}$ & $\begin{array}{l}\text { < SECONDS } \\
\text { TEMPERATURE } \\
\text { INDEPENDENT }\end{array}$ \\
\hline $\begin{array}{l}\text { FINAL } \\
\text { DECAY }\end{array}$ & $\begin{array}{l}\text { HOURS } \\
\text { TEMPERATURE } \\
\text { DEPENDENT }\end{array}$ & $\begin{array}{c}\text { HOURS } \\
\text { TEMPERATURE } \\
\text { DEPENDENT }\end{array}$ & $\begin{array}{c}\text { MINUTES } \\
\text { TEMPERATURE } \\
\text { DEPENDENT }\end{array}$ \\
\hline
\end{tabular}

Fig. 24. Properties of the photo-spin signals in purple bacteria. 
type or the mutant type (which does not contain the carotenoid) of Rhodopseudomonas is used (3). The signal is characterized by various physical methorls such as the signal growth rate, decay rate, band width, etc.

\section{Apparent Spectral Efficiency}

Finally, an apparent action spectrum for the production of these signals (63), both in the green plant material (chloroplasts) (Fig. 25)

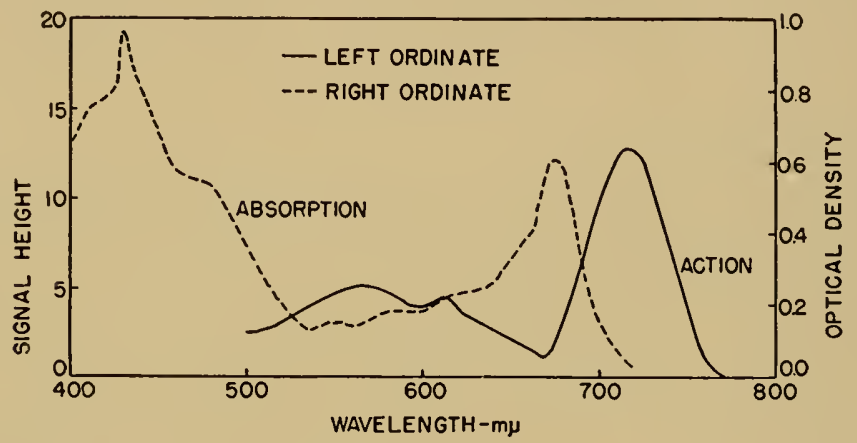

Fig. 25. Ibsorption and action spectra of chloroplasts (Sogo) $\mathbf{I}_{\theta}=10^{15}$ quanta/sec. Band width $=100.1$.

and in the red bacteria (Fig. 26), is shown. This apparent action spectrum is a very curious one and has a peak at about $7200 \mathrm{~A}$, with a minimum approximately where the peak of absorption of the chlorophyll in the living organism is, namely, at 6800 A (59). A similar behavior for the apparent action spectrum of the signal pro-

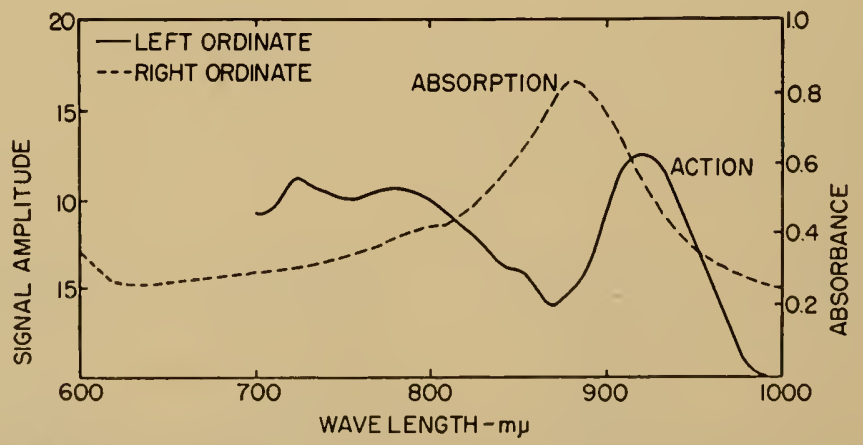

lig. 26. Absorptim spectrmm (Shibata, Benson) and action spectrmm (Sogo) of Rhodospirillum rubrum. $\mathbf{1}_{0}=5 \times 10^{11}$ quantal/sec. Band width $=60 \mathrm{\Lambda}$. 
duction in the Rhodospirillum appears, namely, one in which the maximum for the production of the signal is at, somewhat longer wavelength $(9100(\lambda)$ than the maximum for the pigment absorption (8800 A) (59).

lart of this shift of the wavelength for most efficient spin signal production is certainly due to the way in which the experiment was performed. The samples were totally-absorbing and relatively thick $(\sim 0.1 \mathrm{~mm})$. This resulted in the total absorption of the maximally absorbed light $(6800 \mathrm{~A}$ for the chloroplasts and $8800 \mathrm{~A}$ for the Rhodospirillum) in a very thin layer near the surface of the sample. This situation resulted in the production of unpaired spins at a very much higher real concentration than would be the case if the light were absorbed throughout the sample. This latter situation would be approached by light of wavelengths not so strongly absorbing, such as wavelengths on either side of the absorption maximum in both cases $(6800 \mathrm{~A}$ for the chloroplasts and $8800 \mathrm{~A}$ for the Rhodospirillum).

Since there is an indication that the decay rate of the spin signals is greater the higher their concentration, it is easy to see that any attempt to measure the number of spin signals produced for a constant incident number of quanta will be in error on the side of too few electrons per quantum absorbed, the greater is the concentration of the unpaired spins produced. This would depress the apparent number of spin signals produced at the very point of maximum light absorption, as indeed is the case. This, however, is not enough to account for the fact that the efficiency of spin signal production is actually higher on the longer side of the maximum light absorption than it is on the short side of the maximum. In order to account for this, another process must be invoked.

Something else besides the simple absorption of light by the chlorophyll into its ordinary excited state at $6800 \mathrm{~A}$ is involved in the production of the spin signal. Presumably there is another state, or another substance, which leads to the maximum at longer wavelengths. In the crystal spectra of the monolayers of chlorophyll (see Fig. 9) there was indeed in the crystalline layers a peak at $7180 \mathrm{~A}$. This, together with the fact that our spin signal occurred at $\sim 7200$ A, prompted us to seek some evidence for another excited state in the living organism, somewhere around $7200 \mathrm{~A}$.

In looking back over our earlier studies on the luminescence of living organisms $(68,69)$, we did indeed find emission at $7200 \mathrm{~A}$. In Fig. 27 is shown the emission spectrum of Chlorella as it has been 


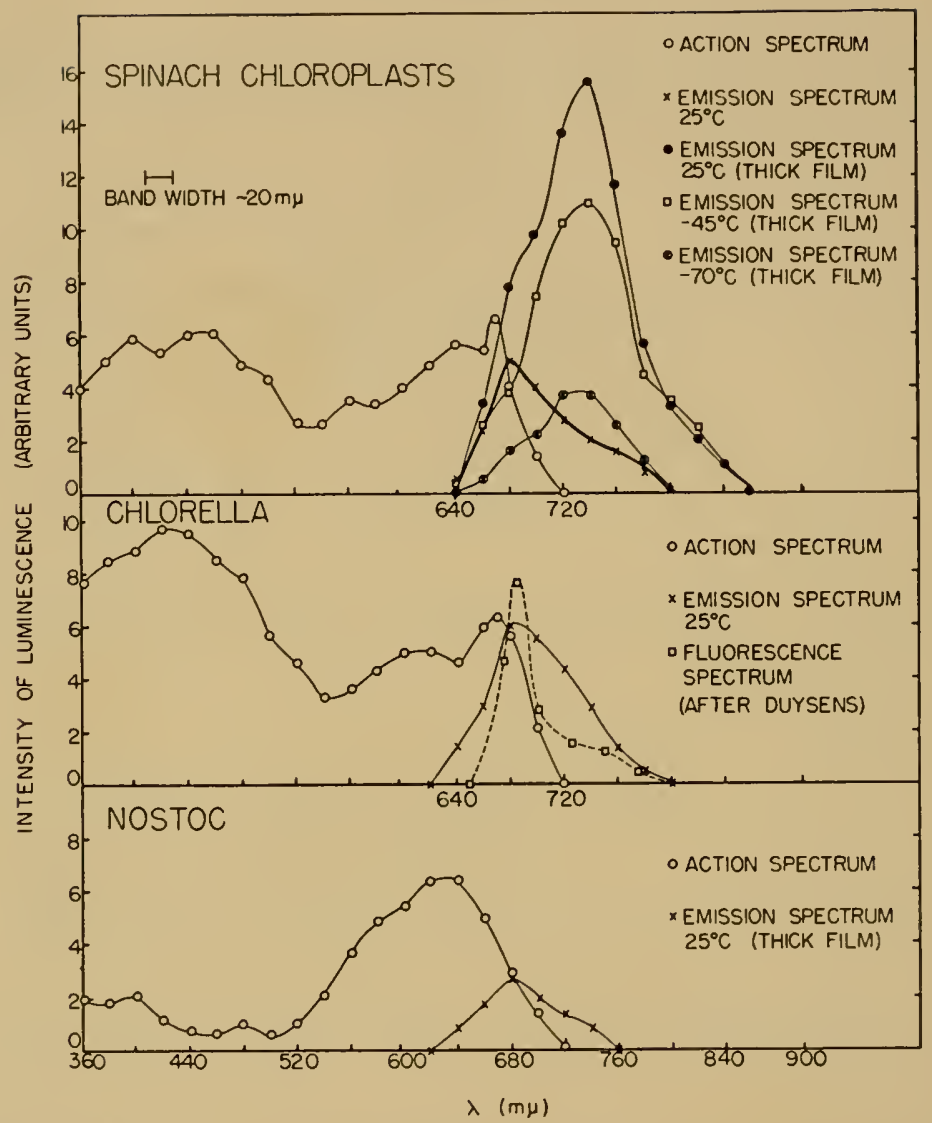

Fig. 27. Action, emission, and fluorescence spectra of spinach chloroplasts, Chlorella, and Nostoc.

observed for long-lived emission. The peak is at $6800 \mathrm{~A}$, but there is a very prominent shoulder at $7200 \mathrm{~A}$. This was originally interpreted as due to self-absorption of the ordinary fluorescence. However, the fact that this is such an asymmetric curve now scems to suggest that there may be another emission band somewhere beyond $7000 \mathrm{~A}$.

A better experiment was performed by Brody (11), in which he found exactly that: a very pronounced enission with a peak at $7180 A$ (Fig. 28). The ordinary fluorescence spectrum of Chlorella has a peak at around $6900 \mathrm{~A}$, but if one cools the Chlorella to $-190^{\circ} \mathrm{C}$ a strong emission at $7180 \mathrm{~A}$ appears. This cannot be a self-absorption effect, because if it were, there would not be a minimum between 


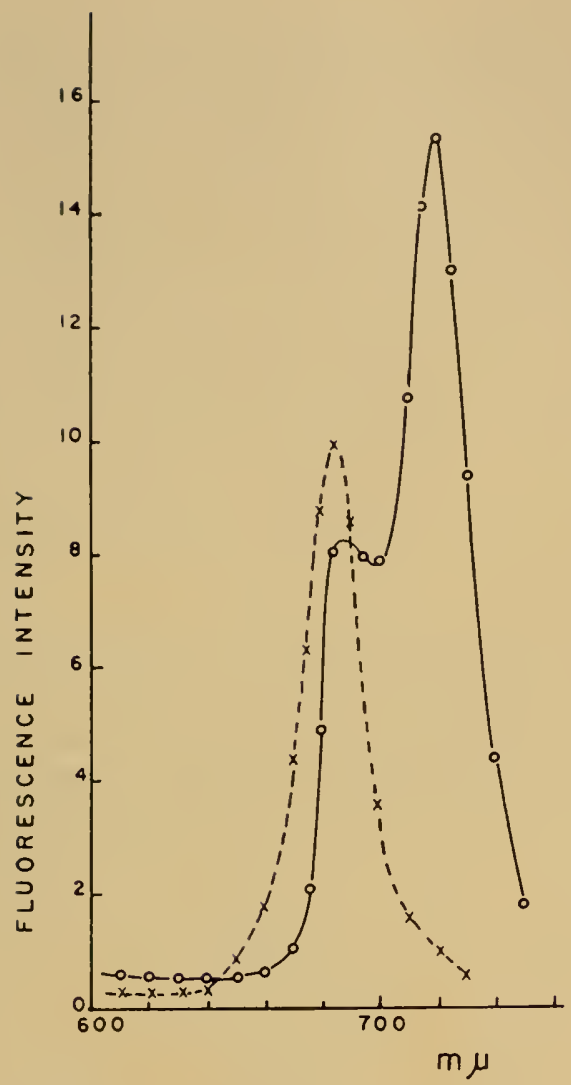

Fig. 28. Fluorescence spectra of Chlorella at room temperature (crosses) and $-193^{\circ} \mathrm{C}$ (open circles). The fluorescence intensities indicated are the same for both curres. The decrease in fluorescence yield at $690 \mathrm{~m} \mu$ is probably due to the increased scattering of the exciting and fluorescent light.

the two absorption peaks. This means that there is a new state emitting, and quite different from the one producing fluorescence emission at $6900 \mathrm{~A}$. The triplet emission is out still further, at around $7600 \mathrm{~A}$, and this has been observed in pure chlorophyll samples. Brody has seen the $7180 \mathrm{~A}$ peak both in whole Chlorella as well as in concentrated chlorophyll solutions, and he believes this to be the emission of a state of aggregated chlorophyll and quite different from the triplet emission but corresponding to something which exists in the living organism. Another possibility is that it may be the lowest $n, \pi^{*}$ state as distinct from the more readily reached $\pi, \pi^{*}$ state. One can presume, now, that excitation to such a state as this is 
required in order to produce the mpaired electron, and that this may occur either by resonance transfer of exciton energy amongst the chlorophyll molecules until it comes to molecules so situated that this state may be excited, or by direct excitation of this state by absorption, as presumably we have done when we examined the action spectrum for the production of unpaired spins. Failure to observe a distinct absorption peak at this point would have to be accounted for. What the nature of this emitting state is remains to be seen. It could be, of course, that this state is one from which an electron transfer to a certain low-lying acceptor occurs. Energy absorbed in the $6800 \mathrm{~A}$ state might be degraded to this emitting state to produce the same electron transfer, or might be used directly from the higher energy state to transfer an electron to a somewhat higherlying acceptor.

It should be noted that this would, in fact, correspond to two different types of primary quantum conversion processes. Such an idea has already appeared in the work of Emerson (25), which has since been explored further by French and Myers $(9,57)$. Emerson had observed that the apparent long-wave limit for photosynthesis was shifted to still longer wavelengths (somewhat beyond $7000 \mathrm{~A}$ ) if light of shorter wavelength (around $6500 \mathrm{~A}$ ) was also present. A further examination of this effect by Myers and French seemed to confirm the suggestion that the quantum yield of an increment of $7000 \mathrm{~A}$ light is greater when light of shorter wavelength (around $6500 \mathrm{~A})$ is also impinging than when it is not. In addition to this, Myers, following lBlinks (9), observed a number of transients in changing from one wavelength of light to another, transients best interpreted in terms of the requirement for the collaboration of two different products resulting from two different quantum conversions, one in the region of $6500 \mathrm{~A}$ and another in the region of $7000 \mathrm{~A}$.

It is tempting to suggest, following the analogy of the phthalocyanine model experiments described earlier, that corresponding to the two model pigments, we have present in the chloroplast both chlorophyll and plastoquinone $(8,20,48)$. The plastoquinone in this case would not have a sufficiently low-lying orbital to act as acceptor to the chlorophyll in its ground state, but could accept an electron from chlorophyll brought to an excited state by illumination, corresponding to the $7200 \mathrm{~A}$ emission. The transfer of a second electron to the quinone negative ion radical thus produced would require the excitation of the chlorophyll to a somewhat higher state, which could result from absorption at $6800 \mathrm{~A}$ or shorter wavelength. 
The quinone double negative ion thus produced would then be a sufficiently powerful reducing agent in its lipid medium to reduce such enzymatic colactors as lipoic acid or pyridine mucleotide (18).

The remaining positive ion in the chlorophyll matrix would have to fiud its waly to some donor, ultimately accepting electrons from witer. These donors might very well be other metal ions such as iron, which is very common in the chloroplast and which is associated with chlorophyll. In fact, a low temperature $\left(70^{\circ} \mathrm{K}\right)$ lightinduced electron abstraction from a ferrocytochrome in a bacterium has been reported (19). Room temperature photo-oxidation of the ferrocytochromes of photosynthetic bacteria has been known for some time $(36,37)$.

The system might structurally then bear some resemblance to the model (see Fig. 19) which we have used, the chlorophyll layer having associated with it on one side the electron acceptor, quinone, in a lipid environment, and on the other side electron donor materials, such as the cytochromes, in an aqueous environment. Following the absorption of a quantum in chlorophyll (Fig. 29, equation 1), the energy will migrate by resonance transfer to a suitable site near the quinone, at which point electron transfer to the quinone will take
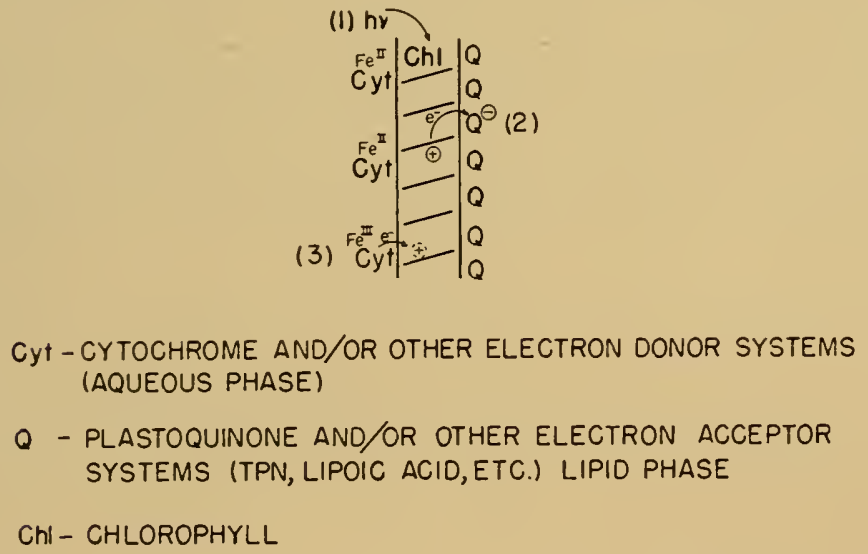

lig. 29. Schematic arrangement of chlorophyll and possible donor and acceptor molectules in the chloroplast. 
place (Fig. 29, equation 2). The resulting vacancy, or chlorophyll positive ion, can then migrate by hole diffusion, that is, electron transfer from normal chlorophyll, into the vacant orbital of the neighboring chlorophyll positive ion. This process is the one in the entire sequence which most nearly resembles the properties of a semiconductor and permits the oxidizing point (the chlorophyll positive ion) to separate from the reducing point (the electrons in the quinone orbitals) by a process which is very nearly temperature-independent. The oxidizing point will make itself apparent as a chemical change, finally, when it captures an electron from a suitable reducing agent, in this case shown ats a ferrocytochrome, thus producing a ferricytochrome and regenerating chlorophyll (Fig. 29, equation 3).

It is conceivable that in order for the reduction of pyridine nucleoticle to occur, possibly through lipoic acid $(51,56)$, the quinone must be in the form of a cli-anion, in which case a second electron transfer from an excited chlorophyll to a quinone negative ion radical, produced in equation 2 (Fig. 29) will take place. This clearly will require somewhat greater energy than the first reaction, if only to overcome the electrostatic repulsion of the pre-existing negative charge. It is interesting to view these two steps as a possible means of understanding the collaborational requirement of light of tro wavelengths $(7000 \mathrm{~A}$ and $6500 \mathrm{~A})$ which was mentioned earlier (52).

Another alternative would involve the transfer of the electron from the donor (ferrocytochrome) to the excited chlorophyll as the first act. This would lead to a chlorophyll negative ion radical in which an electron has been placed in the lowest $\pi$-orbital of the chlorophyll. The migration would then have to occur in this form until the acceptor site (quinone) is arrived at.

We prefer the first formulation described above, since every effort we have made to find either clark- or photo-induced electron transfer from a clonor to the neutral phthalocyanine in our phthalocyanine model has failed. Beyond this, in practically every case in which it has been determined, the charge migration in an organic molecular crystal takes place via hole migration rather than via electron migration $(30)$.

\section{Conclusion}

In summary, then, we can see that while the solid state model (phthalocyanine) allows an approach from at somewhat different point of view, the net result is the same as what was sought, but so far not found, when we looked at the solution chemistry of chloro- 
phyll (and chlorophyll model substances), namely, the transfer of an electron, or hydrogen atom, from the excited porphyrin to an electron acceptor at a high reduction level which can be used to reduce the ultimate carbon dioxide reducers, followed by the donation of an electron, ultimately from water, to the remaining radical ion, or lattice, which produces the net result of the transfer of hydrogen from water to carbon dioxide.

How much of the solid state picture will be required to fully understand this separation of oxidant and reductant I think is yet to be cletermined. However, I believe it is quite clear that we are coming to the same kind of conclusion from both ends, that is, from both the pure solution chemistry which involves electron transfer from donor to acceptor, and from the solid state experiments which involve the same kind of electron transfer from donors to acceptors. The difference lies in the types of lattices involved. The back-reaction in the solid state experiments is demonstrably slower than one can visualize for the solution electron transfer reaction in which no provision is made for the rapid, relatively temperature-independent separation of the products, electron (reducing agent) and hole (oxidizing agent).

\section{REFERENCES}

I. Ainsworth. S., and Rabinowitch, E., Science, 131, 303 (1960).

2. Inderson, A. F. H., unpub.

3. Indrocs, C.. Singleton, I., and P'ark, R. B., unpub.

4. Irnold, W., and Maclay, H. K., Brookhaven Symposia in Biol., I1, 1-9 $(19 ; 8)$.

5. Irnold, W., and Sherwood, H. K., Proc. Natl. Acad. Sci. U. S., 43, 105-114 $(19 ; \%)$.

6. Ironolf, S., and Dorough, G. D., unpub.

7. Becker, R. S., and Kasha, M., J. Am. Chem. Soc., 77, 3669-3670 (1955).

8. Bishop, N. I., Proc. Natl. Acad. Sri. U. S., 45, $1696-1702$ (1959).

9. Blinks, L. R., Proc. Natl. Acad. Sci. U. S., 46, $327-332$ (1960).

10. Bowen, E. J., Chemical Aspects of Light, p. 163, Oxford University Press. Oxford, Eugland $(19+6)$.

11. Brody, S. S., Science, 128. $838-839$ (1958).

12. Brody, S. S., and Rabinowitch, E., Science, 125, 5:55 (1957).

13. Calvin, M., Brookhaven Symposia in Biol., 11, 160-180 (1958).

14. Calıin. .1., Rey. Modern Phvicic, 31, 147.156 (1959).

1.7. Calvin, M., Rev. Modem Physics, 31, 157-161 (1959).

1(i. Calvin, M., Cockbain, E. C., and Polanyi. M., Trans. Faraday Soc., 23, 1436 (1936).

17. Calvin, M., Eley, D. D., and Polanyi, M., Trans. Faraday Soc., 32, 1433 (1936).

18. Chance, B., Federation Proc., 19, Part I, 38 (1960).

19. Chance, B., and Nishimura, M., Proc. Natl. Acad. Sci. U. S., 46, 19-25 (1960).

20. Crane, F. C., Plant Physiol., 34, 128-131 (19.59). 
21. Dorough, G. D., and Calvin, M., Science, 105, 433 (1947); J. Am. Chem. Soc., 70, 699-708 (1948).

22. Eley, D. D., Nature, 162, 819 (1948).

23. Elcy, D. D., and l'arfitt, G. D., Trans. Faraday Soc., 51, 1529 (1955).

24. Eley, D. D., Parfitt, G. D., Perry, M., and Taysum, D. H., Trans. Faraday: Soc., 49, $79-86 \quad(1953)$.

25. Emerson, R., Chalmers, R., and Ceclerstrand, C., Proc. Natl. Acad. Sci. U. S., 43, 133-143 (1957).

26. Engelmann, Th. W., Botan. Z., 42, 81, 97 (1884); or Botan. Z., 45, 393, 409, 425. 441,457 (1887).

27. Evstigneev, V. B., and Gavrilova, V. A., Doklady Akad. Nauk. SSSR, 95, 841-844 (1954); Doklady Akad. Nauk. SSSR, 98, $1017-1020$ (1954); Biofizika, 4, 641 (1959).

28. Frey-Wyssling. 1., Macromolecules in Cell Structure, Chapter 4, Harvard University Press, Cambridge, Mass. (19.57).

29. Gaffron, H., Biochem. Z., 264, 251 (1933).

30. Garrett, C. G. B. in Semiconductors (N. B. Hannay, ed.), Chapter 15, Rein. hold Publishing Co., New York, N. Y. (1959).

31. Hannay, N. B. (ed.), Semiconductors, Reinhold Publishing Co., New York. N. Y. (1959).

32. Hill, R., and Whittingham, C. P., Photosynthesis, p. 23, Methuen (1955).

33. Huennekens, F. M., and Calvin, M., J. Am. Chem. Soc., 71, 4024-4031 (1949); 71, 4031-4035 (1949).

31. Hutchinson, C. A., Jr., and Mangum, B. W., J. Chem. Phys, 29, 952-953 (1958).

35. Jacobs, E. E.. Holt, A. S., Kromhout, R., and Rabinowitch, E., Arch. Bicchem. and Biophys., 72, 495 (1957).

36. Kamen, M. D. in Entymes: Units of Biological Structure and Function, p. 483, Academic Press, New York, N. Y. (1956).

37. Kamen, M., and Vernon, L. P., J. Biol. Chem., 211, 663-675 (1954).

38. Kcarns, David R., Thesis, "Elcctrical Properties of Organic Solids", University of California, Bcrkeley (June, 1960).

39. Kearns, 1. R., and Calvin, M., J. Chem. Phys., 29, 950-951 (1958).

40. Kearns, D. R., Tollin, G., and Calvin, M., J. Chem. Phys., 32, 1013-1019 (1960); 32, I020-102: $(1960)$.

4. Kofler, M., Langemann, A., Rucgg, R., Gloor, U., Scluwieter, U., W'ursch, J., Wiss, O., and Isler, O., Helv. Chim. Acta, 42, 22.52-22.54 (1959).

42. Krasnorskii, . A. A., Doklady Akad. Nauk. SSSR, 60, 421 (1948); 61, 91 (1948).

43. Krasnovskii, A. A., and Briu, G. P., Doklady Akad. Nauk. SSSR, 89, 527-530 (1953).

H. Krasnovskii, A. A., and Gavriolova, V. A., Doklady Akad. Nauk. SSSR, 81, I 105 (19.5I).

4.) Krasnowskii. A. A., and Jmrihkina, A. V., Biofizika, 3, $547-557$ (1958).

46. Krasnorskii. A. A., and V'oinorskaya, K. K., Doklady Akad. Nauk. SSSR, 66. 663-660 (1949).

17. Krasnovskii, A. A.. Brin, G., and Voinorsksya, K., Doklady Akad. Nauk. SSSR, 69. 393-396 (1919).

18. Lester, R. 1.., and Crane, F. L., J. Biol. Chem., 234, 2169-2175 (1959).

49. Linscluity, 11., and Weissman, S. I., Arch. Biochem. and Biophys., 67, 491-492 $(19: 7)$.

50). Linstcat, R. I'., Eisner, U., Ficken, G E., and Johns, R. B., Chemical Sociely special Publication 3, 83-96 (1955).

5. Massey, V.., aud Cibsou, Q. H., Federotion Proc., 19. Part I, $2 \bar{i}$ (1960).

52 Mvers, J., and French, C. S., Ann. Rep. Dircctor Dept. Plant Biol., Carnegie 
Institute of W'ashington, Ycarbook 58, Pp. 318-323, July 1, 1958-June 30, 1959.

53. Norris, 'T. H., Ruben, S., and Allen, M. B., J. Am. Chem. Soc, 64, 30373040 (I942).

54. Rabinowitch, E.., Proc. 6th Summer Conf. Spectroscopy, pp. 1-17-148, John Wiley and Sons, New York, N. Y. (1939).

5.5. Rabinowitch, E.., and Brody, S. S., J. chim. phys., 55, 927-933 (1958).

56. Sanadi, 1). R., pers. commun.; Sanadi, D. R., Langley, M., and White, F., Biochim et Biophy's. Acta. 29, 218-219 (1958).

57. Schenck, G. O., Angew. Chem., 69, 579-599 (1957); Proc. 2nd. Intern. Cong. on Photobiology, Turin, Iraly (1957).

58. Sceley, G.. and Calvin, .Y., J. Chcm. Phys., 23, 1068-1078 (1955).

59. Shibata, K., Benson, A. A., and Calvin, M., Biochim. et Biophys. Acta, 15, $461-470 \quad$ (1954).

60. Shunk, C. H., Erickson, R. E., Wong, E. L., and Folkers, K., J. $A m$. Chem. Soc., 81, 4999-5000 (1959).

61. Smaller, 13., Symposium, Free Radicals in Biological Systems, Stanford University, March, 1960 (in press).

62. Smith, J. H. C., and Coomber, J., Ann. Rep. Director Dept. Plant Biol., Carnegie Institute of Washington, Yearbook 58, p. 331, July I, 1958-June $30,1959$.

63. Sogo, P. B., Carter, L. A., and Calvin, M., Proc. symposium on Free Radicals in Biological Systems, Stanforrl University, March, 1900 (in press).

64. Sogo, 1. 13., Pon, X. G., and Calvin, M., Proc. Natl. Acad. Sci. U. S., 43, 387. 393 (1957).

65. Stanier, R. Y., Brookhazen Symposia in Biol., 11, 43-53 (1958).

66. Steinmann, E., and Sjostrand, F. J., Exptl. Cell Research, 8, 15-23 (1955).

67. Terenin, A. N., Putzeiko, E., Akimov, A., "Energy Transfer in Systems of Connected Organic Molecules," Discussions Faraday Soc., 27, 83-93 (1959).

68. Tollin, G., and Calvin, M., Proc. Natl. Acad. Sci. U. S., 43, 897-908 (1957).

69. Tollin, G., Fujimori, E., and Calvin, M., Nature, 181, 1266-1267 (1958); Proc. Natl. Acad. Sci. U. S., 44, 1035-1047 (1958).

70. Tollin, G., Sogo, P. B., and Calvin, M., Aun. N. Y. Acad. Sci., 74, 310-328 (1958); J. chim. phys., 55, 919-926 (1958).

71. Trumit, H. J., and Colmano, G., Biochim. et Biophy's. Acta, 31, 434-447 (1959).

72. van Niel, C. B., in Plotosynthesis in Plants (J. Franck and W. E. Loomis, eds.), pp. 437-495, lowa State College Press, Ames, Iowa (1949).

73. Vartanyan, A. T., Thur. Fiz. Khim., 20, I065 (1946); 24, 1361 (1950).

74. Vartanyan, A. T., and Karpovitch, I. A., Doklady Akad. Nauk. SSSR, 111, 561 (1956).

75. Vishniac, W., and Rose, I. A., Nature, 182, 1089-1091 (1958).

76. Willstätter, R.. and Stoll, A., Cntersuchungen über Chlorophyll. Springer, Berlin (1913). 


\title{
ELECTRON SPIN RESONANCE STUDIES OF PHOTOSYNTHETIC SYSTEMS 1
}

\author{
BARRy COMMIONER
}

The Henry Shaw School of Botany

and

The Adolphus Busch III Laboratory of Molecular Biology

W'ashington Unitersity

St. Louis, Missouri

\section{INTRODUCTION}

There are good theoretical grounds for expecting free radicals to occur as intermediates in photobiological processes, and reference to almost any recent discussion of the mechanism of photosynthesis reveals the considerable frequency with which hypothetical free radical intermediates are proposed. These expectations result from the necessity of uncoupling paired electrons during one or more phases of the photochemical or oxidation-reduction processes that participate in photosynthesis. We may regard an organic molecule which contains one or more umpaired electrons as a free radical.

Until about ten years ago investigations of possible free radical intermediates in dilute conditions such as those found in most biochemical and biological processes were hampered by the lack of suitably sensitive detection methods. The discovery by Zavoisky in 1945 of electron spin resonance (ESR) absorption led to the possibility of constructing spectrometers which were at least potentially capable of detecting exceedingly low concentrations of unpaired electrons, including those associated with free radicals.

Begimning in 1949 a group of investigators in the departments of physics, chemistry, and botany at Washington University developed a concerted effort to apply the new technique to a variety of relevant problems including, among others, an analysis of the mechanism of photosynthesis and other photobiological processes. The present paper is a brief review of the development of this work as it re-

This work was supporterl in part by research grant C-3983 from the National Cancer Inslitule, United States Public Health Service, and by Contract Nont$816(10)$ (NR 101.227) between the Otfice of Naval Reseatch, Department of the Nary, and Washington Universily. 
lates to photosynthesis, and it summarizes the present status of our knowledge about the participation of lree radicals and similar components in photosynthesis.

The ESR phenomenon depends on an inherent property of the electron-the magnetic moment arising from its spin, and ESR data are capable of describing, at least directly, only this property of the electron. The basic effect ariscs when an unpaired electron is placed in an external magnetic field. The electron's magnetic moment may assume one of two oricntations to the external field, one of which represents a higher energy state than the other. The separation between these energy levels is a function of the strength of the external field $(H)$, so that onc may impose on the system an energy gap of any chosen value by selecting the appropriate external magnetic field. The energy levels imposed on the system by the external magnetic field may result in a capacity for the resonance absorption of incident radiation of a particular frequency $(v)$, according to the relationship:

$$
\nu h=g \beta H,
$$

where $h$ is Planck's constant, $\beta$ is a constant (the Bohr magneton) and the value of $g$ describes the interaction between the inherent magnetic moment due to the electron's spin and the external magnetic field. For the free electron the value of $g$ is 2.0023, but the $g$ value exhibited by a localized unpaired electron may depart from this figure as a result of the interaction between the magnetic moment due to the spin and that due to the electron's orbital angular momentum. The magnetic moment of an unpaired electron also interacts with the magnetic moments of atomic nuclei with which it is associated. As a result, the single energy gap characteristic of an isolated electron becomes split, so that a series of evenly spaced absorptions occur. The most important nuclei normally encountered in biological material that possess magnetic moments and are therefore capable of inducing such hyperfine structure in the ESR signal are $\mathrm{H}^{1}$ and $\mathrm{N}^{14}$. $\mathrm{C}^{13}$ has a magnetic moment, but $\mathrm{C}^{12}$ does not. The magnetic moments of $\mathrm{H}^{2}$ and $\mathrm{N}^{15}$ are different from those of the normal isotopes. The hyperfine structure is often distinctively related to the molecular structure of the component and may be employed to describe the locus of the electron in the molecule. In this situation isotope substitution experiments can be carried out which yield detailed information regarding the location of the unpaired electron in the molecule. 
In most ESR spectrometers, the sample is placed within a cavity designed to establish a standing wave of microwave radiation. The cavity itself lies between the poles of a variable magnet. To detect an ESR signal, the absorption of microwave energy is monitored as the strength of the external magnetic field $(H)$ is gradually varied. At a value of $H$ which is given by the above relationship and by the particular value of $g$ characteristic of the unpaired electrons present in the sample, an absorption of microwave energy will be noted. Since most spectrometers impose a modulation of variable amplitude of the external field, the ESR signal is usually received as the derivative of the absorption curve, i.e., as the rate of change of the microwave absorption, with respect to field strength. By integrating such a signal, an actual absorption curve can be obtained. The relationship between these two types of presentation of the ESR signal is shown in Fig. 2. The microwave frequency employed in most present ESR spectrometers is 9000 megacycles/sec; when $g$ is about 2, an unpaired electron induces a resonance absorption at an external magnetic field of about 3300 gauss.

Various types of components that are encountered in biological systems may possess umpaired electrons capable of yielding an ESR signal. These are summarized briefly below.

\section{a. Paramagnetic Ions}

A number of metals of the transition series contain unpaired electrons in their normal ground state and these will give rise to an ESR signal. Typically these signals are complex, and the hyperfine structure exhibits spacing which is characteristic and is usually very much wider than the hyperfine splitting in organic free radicals. Manganous ion is an interesting example which is observed fairly often in biological material. This gives rise to an ESR signal consisting of six hyperfine peaks each separated by 96 gauss. Concentrations of manganous ion as low as $10^{-5} M$ are easily detectable in a suitable spectrometer. We have detected ESR signals due to manganous ions in living bacteria, algae, and the green gland of the crayfish, which contains an unaccountably high manganous ion concentration.

\section{b. Free Radicals}

In the context of this paper we shall regard a free radical as a covalent-bonded molecule which contains an umpaired electron. A biradical is a molecule which contains two unpaired electrons. One 
type of biradical is represented by the triplet state. The $g$ value of an aromatic free raclical may vary between 2.002 and 2.007 (14). A particular free radical is characterized, but not uniquely, by a given $g$ value, so that in practice it is often difficult to distinguish between ESR signals due to different free radicals solely by this means. The width and shape of the ESR signal obtained from organic free radicals is affected by a relatively large number of factors and varies markedly from one free radical to the next. It is also affected by the structural organization of the substance (12). As already indicated, an organic free radical may yield an ESR signal which is characterized by a precise pattem of hyperfine splitting if the unpaired electron is localized near nuclei which themseives possess magnetic moments. From quantitative determinations of the $g$ value, line width, and the spacing and intensities of hyperfine peaks exhibited by an ESR signal, one can extract considerable information about the molecular structure and kinetic behavior of a free radical. The methods and principles employed in such an analysis of ESR signals are described in a recent sumnary by Weissman (13).

Despite the considerable interest in the possibility of ESR studies of triplets, which is in part engendered by their supposed importance in biological systems, this has proved to be one of the most difficult matters in ESR technique. The difficulties arise from the complex interactions which are possible between two unpaired electrons that are present in the same molecule. In general, if the two electrons are umpaired and separated by only $4 \mathrm{~A}$ or less, their interaction is sufficiently strong to cause a broadening of the ESR signal so extreme as to result in undetectability. This effect may be overcome if the free radical is in the form of a single crystal and is observed in a specific orientation. Hutchinson has observed a light-induced triplet in these special conditions (8). Triplet molecules that are randomly distributed in space do not exhibit a detectable ESR signal at $g=2$. There is some very recent evidence (11) that in random glassy solutions triplet molecules may give rise to a characteristic signal at $g=4$ (i.e., a signal which occurs at a magnetic field strength which is $1 / 2$ of that required to induce a signal at $g=2$ ). In general, there is no expectation of detecting an ESR signal due to triplet molecules in biological systems at the ordinary $g$ value of 2, unless they occur in a macroscopically large anisotropic structure-a very unlikely circumstance. No ESR signal that can be attributed to a triplet state has been observed, as yet, in any biochemical or biological system. 
In a biradical in which two umpaired electrons occur at distances greater than $4 \mathrm{~A}$, the electrons may be regarded as essentially independent and each of them will give rise to a separate ESR signal. If the two unpaired electrons occur in similar atomic environments (with respect to paramagnetic nuclei), they may yield identical ESR signals which superimpose; such a signal is indistinguishable from that of an ordinary free radical containing only one unpaired electron. If the two electrons are located in enviromments which include different paramagnetic nuclei, they may give rise to ESR signals which are distinguishable in $g$ value, and possibly with respect to hyperfine structure, but which are of equal intensity.

\section{c. Semiconductors}

In physical systems that are capable of conducting electrons (i.e., metallic conductors and semiconductors of various types), the mobile electrons are unpaired and therefore give rise to an ESR signal. It is characteristic of these systems that the conduction electrons are essentially uncompled to any other paramagnetic constituents and therefore hyperfine structure is absent. Unfortunately, apart from this negative characteristic (which is of course not unique) ESR signals due to semiconductors cannot be distinguished, on their face, from signals resulting from a free radical. Localized regions of the semiconductor structure (electron traps) may tend to bind an unpaired electron at an energy level slightly below that of the conduction electron. Such an impaired electron may become closely associated with magnetic atomic nuclei so that the resultant ESR signal exhibits hyperfine structure. Hence an ESR signal due to a trapped unpaired electron may be distinguished from the signal due to a conduction electron by the occurrence of hyperfine structure. However, this distinction is not unique, since ordinary free radicals also exhibit both types of signal.

It has been suggested by Calvin (3) that the light-induced ESR signal observed in chloroplasts at temperatures that are so low as to preclude ordinary chemical reactions must be due to semiconductors. This conclusion is not required by such data. Lewis and Lipkin (9) have shown that in glassy solutions illuminated at liquid nitrogen temperatures, the absorption of light may eject an umpaired electron from an organic molecule, which is then in a free radical form as long as the ejected electron remains trapped in the structure of the surrounding medium. Such a system is not necessarily a semiconductor but would give rise to an ESR signal when illuminated at liquid nitrogen temperatures. 


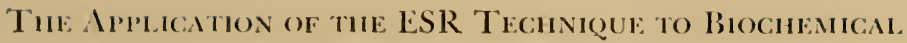

ANi) Biological Sistems

Several special problems arise in the application of the ESR technique to systems of interest to the biologist. Because the technique is relatively new, there have as yet been relatively few efforts to develop specific modifications of the basic instrument which are suited to the needs of the biochenist or the biologist, rather than to the needs of the physical chemist or physicist.

Fortunately, since its inception, the program at Washington University has been designed to correlate the interests and needs of investigators in physics, chemistry, and biology. One of our earliest decisions was to undertake the development of a series of ESR spectrometers designed specifically to accommodate biochemical and biological experiments. These developments, which have been carried out by Professor Townsend, have been absolutely essential to the experimental work which is described below.

The crucial problem which must be solved in applying ESR spectroscopy to biochemistry and biology is the matter of sensitivity. It was at first apparent on theoretical grounds, and it is now evident from our own results, that the concentration of free radicals expected in such systems is of the order of $10^{-7}$ to $10^{-5} M$, and this fact alone requires a spectrometer of high intrinsic sensitivity. Unfortunately, two additional factors, inherently associated with the systems of interest to the biologist, happen to militate against the realization of a spectrometer's full sensitivity. The first of these is the ubiquitous presence of liquid water in functional biochemical and biological systems. The microwaves employed in ESR spectrometers are heavily absorbed by liquid water, thereby resulting in a significant loss in sensitivity. To some extent this problem can be counteracted by drying the material or by examining it in the frozen condition, since ice does not have a high absorbancy for microwaves. However, under these conditions only static data are possible and one loses a great deal of information which could be obtained from functional systems. The first spectrometer developed at Washington University in 1950 was not sufficiently sensitive to accommodate wet samples, and we were forced to study frozen-dried materials rather than active systems. A second problem is due to the relatively fast rates of processes of interest to the biologist. This precludes the use of an ESR spectrometer in which a slow scanning speed (which averages out noise and thereby increases the effective signal:noise ratio) is used to attain an enhanced sensitivity.

When it became apparent from our earliest studies of frozen-dried 
materials (7) that free radicals do indeed occur in biological systems, Professor Townsend set himself the task of constructing a spectrometer of maxinum sensitivity which would tolerate samples containing liquid water and yield ESR signals at scanning speeds of the order of 30 to 90 seconds. All of the experiments described below were carried out with an instrument of this type. Our present instrument will accommodate a liquid aqueous sample of about 0.1 $\mathrm{ml}$ in volume and can detect free radicals at a minimal concentration of about $10^{-7} M$ with scanning speeds of the order of 60 seconds (instrumental time constant, $0.1 \mathrm{sec}$ ) when the ESR signal has a half-width of 10 gauss. We believe that the sensitivity achieved by this instrument for aqueous systems is the greatest reported to date. To illustrate the crucial importance of this level of sensitivity we may note that ESR signals from living animal tissues are only about 3 to 10 times the noise level in this spectrometer, so that they are completely undetectable if any appreciable loss of sensitivity occurs.

The crucial importance of high sensitivity is also evident from consideration of the matter of resolving power. The sensitivity of most ESR spectrometers depends on the use of a modulated variation in the external magnetic field. As the magnetic field is gradually increased the instrument sweeps back and forth across the mean field strength at a fixed frequency (which in our present instrument is 1000 kilocycles per second) but with an adjustable amplitude. It is useful to draw an analogy between the amplitucle of this modulation of the external magnetic field and the slit-width in an ordinary optical spectrophotometer. A large modulation amplitude corresponds to a wide slit; sensitivity is enhanced at the expense of resolving power. Thus, the inherent sensitivity of the spectrometer places a serious limit on the degree to which ESR signals can be resolved. To illustrate the importance of this problem, Figure 1 show's what happens to the intensity of the ESR signal yielded by a preparation of living Chlorella when the modulation amplitude is reduced from 24 gauss (analogous to a wide slit-width) to 3 gauss. An appreciable signal observed with 24 gauss modulation becomes almost imperceptible when the modulation is reduced to 3 gauss. As will be noted from Figs. 1, 2 and 3, the resolution of the complex ESR signals observed in plotosynthetic systems requires a low modulation amplitude, so that without an inherent instrument sensitivity sufficient to permit operation at such a low modulation, one can only obtain unresolved signals which represents a mixture of the signals derived from two quite distinct components. 


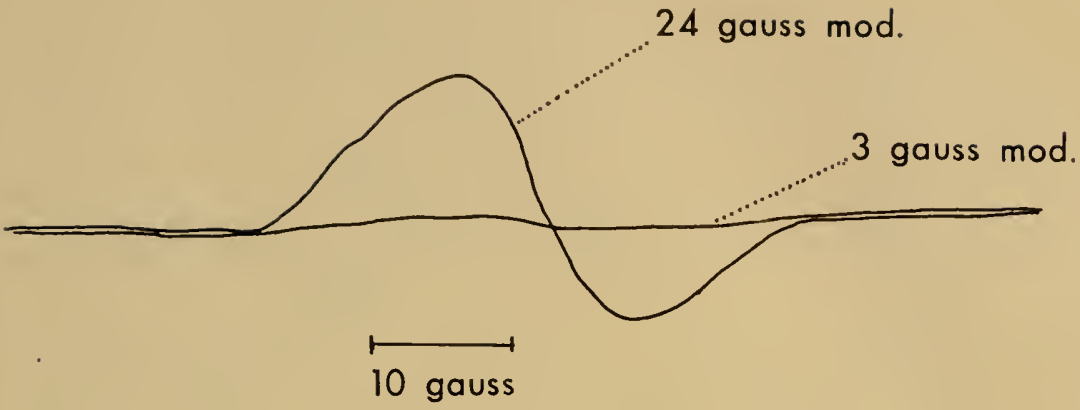

Fig. 1. ESR signals from a sample of living Chlorella under two different instrument conditions (24 gauss modulation amplitude and 3 gauss modulation amplitude) to show the effect of high resolution conditions (3 gauss modulation) in reducing instrument sensitivity. See legend to Fig. 2 for other details.

This account suggests the crucial relation between the characteristics of the ESR spectrometer, and the level of information which it yields regarding biochemical and biological systems. With sufficient sensitivity the spectrometer can detect free radicals at very low concentrations in functional systems. High sensitivity also permits the resolution of separable components, and an estimate, from the intensities of the separate signals, of their concentrations. If such sensitivity can be achieved at rapid scanning speeds, it also becomes possible to obtain quantitative kinetic data regarding the free radical concentration in a functioning system. Such data lend themselves to analysis with respect to reaction mechanism. But in their absence, the spectrometer tells us little more than that some component which contains one or more unpaired electrons is present.

\section{ESR Studies of Photosynthesis}

Our first observations of ESR signals in frozen-dried preparations of biological material, which began in 1952, included investigations of green leaves. These relatively crude observations showed that the concentration of unpaired electrons in etiolated leaves is rather low but increases markedly after they are exposed to light and become green (7). It was also found that the ESR signal in a green leaf frozen-dried after exposure to light is significantly greater than the signal yielded by preparations made previous to illumination (4).

Following the completion of a spectrometer of improved sensitivity in 1956, it was possible to study aqueous suspensions of chloroplasts for the first time. An ESR signal was observed which became considerably intensified on illumination (6). The signal was apparently 

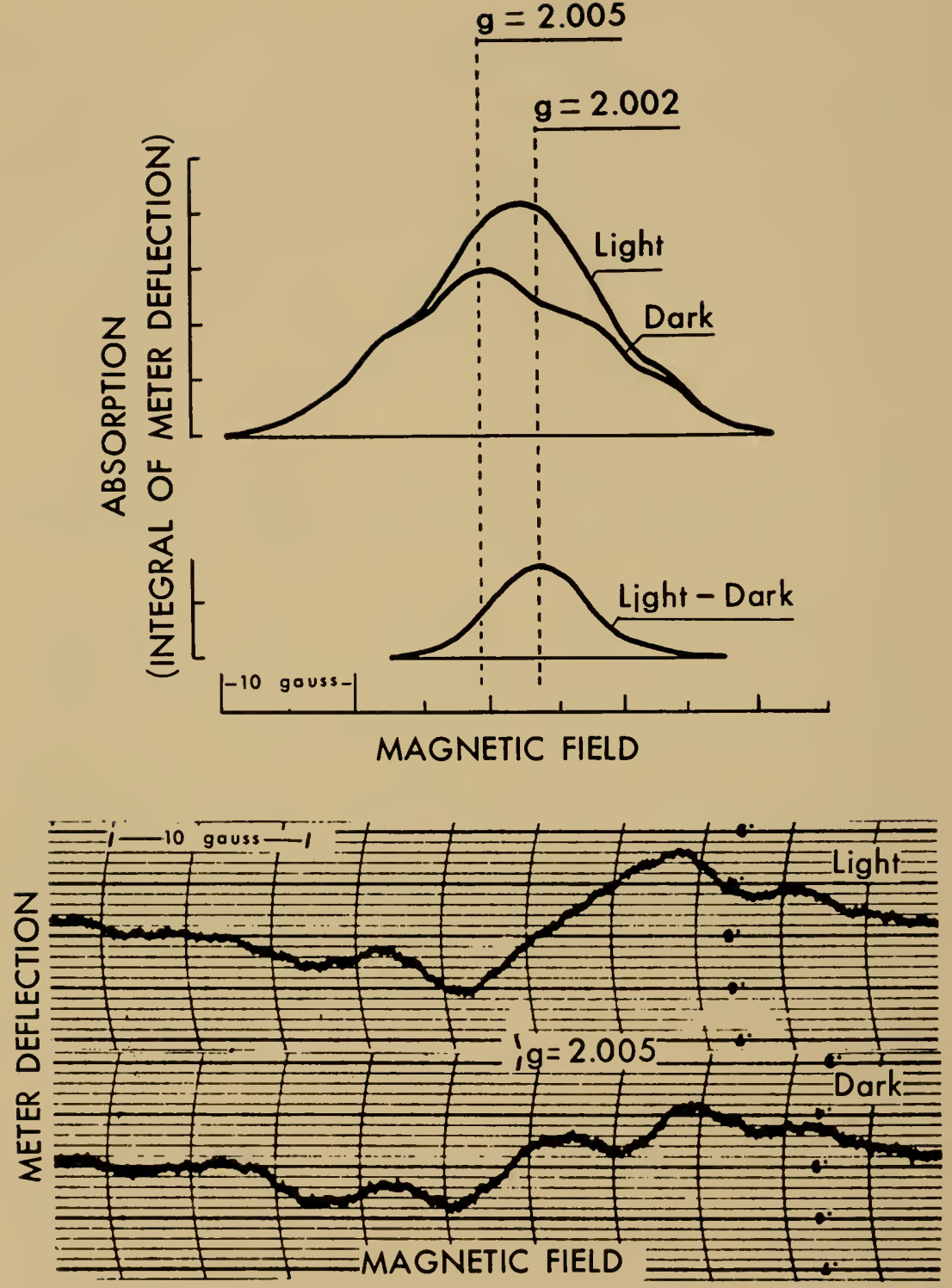

Fig. 2. ESR signal from spinach chloroplasts (washed twice with $0.5 \mathrm{M}$ sucrose) in the dark and during illumination with white light. The Iower curres represent the original ISSR siguals; magnetic field incicases toward the right along the abscissa, and the ordinate represents the rate of change with respect to ficld strength of microwave energy $(9000 \mathrm{Mc} / \mathrm{sec})$ absorbed by the sample. The uppermost curves represent machine integrals of the lower curve; below them is the absorp)tion due to the effect of light alone which is obtained by subtracting the dark integral curve form the light integral curce. Modulation amplitude: 8 gauss. rigure from reference (5). 
simple and lacking in hyperfme structure. Howerer, the signal was poorly resolved (the spectrometer sensitivity being too low), and precluded any effective quantitative dati, or conclusions regarding the true shape of the signal. Similar results were later obtained by Calvin et al. in 1957, who showed also that the light response was exhibited to a certain degree at liquid nitrogen temperatures and that signals resembling those of chloroplasts conld be observed in samples of Rhodospirillum rubrum (10).

In the meantime Professor Townsend managed to provide us with a further increase in sensitivity, and we were finally in a position to obtain highly resolved signals from photosynthetic systems at 30 to 90 -second scamning speeds.

When the chloroplast system was reinvestigated with the new spectrometer, it was discovered that what had previously been observed in illuminated chloroplasts as a simple ESR signal was in reality due to two components (5). Chloroplasts observed in the dark under conditions of high resolution revealed for the first time an ESR signal with distinctive hyperfine splitting. This signal was centered at $g=2.005$, had a half-width of about 19 gauss, and 5 hyperfine peaks evenly spaced at 6 gauss intervals. When chloroplasts prepared by ordinary methods were illuminated the signal became more intense, but its shape changed, and it exhibited a somewhat lower $g$ value. When the light and dark signals were integrated and subtracted from each other, it became apparent that on illumination a new ESR signal was superimposed on the signal observed in the dark. The signal due to light was centered at $g=2.002$, had a half-width of about 9 gauss, and exhibited no hyperfine structure. These results are shown in Fig. 2. In what follows, the ESR signal at $g=2.002$, and the component which gives rise to it will be designated as I; while II will refer to the signal centered at $g=2.005$, and the free radical to which it is due.

Further investigations (5) suggested that the two ESR signals arose from components that probably represented sequential steps in a light-induced electron transfer process. It was observed that signal I appeared only in chloroplasts that were thoroughly washed or dialyzed. When chloroplasts prepared with a minimum of washing were illuminated, they exhibited only an increased intensity of the signal (II), which was also present in the dark. Kinetic studies showed that signal I was associated with a relatively rapid rate of onset and decay (5- to 10-second half-time), while the onset of signal II followed a time constant of 40 seconds for onset and 100 seconds 
for decay. In well-washed chloroplasts, the onset of signal I became more rapid (time constant about 2 seconds) while the rate of decay was reduced (time constant about 26 seconds).

These observations suggested that signal II arises from a substance produced in association with the decay of the component that causes signal I. Since signal II is also present in the dark, and because its hyperfine structure is characteristic of an organic free radical, it appeared probable that it represented a free radical intermediate of a dark oxidation-reduction process. Thus in the more nearly intact chloroplast, the rate of decay of component $I$ is so fast as to preclude the accumulation of a detectable steady-state concentration. In this circumstance, the light-induced unpaired electrons appear to be transferred to component II, which by virtue of its relatively low decay rate, then tends to accumulate to a degree.

The component which yields ESR signal II has a hyperfine structure which is characteristic of an organic free radical, and further evidence of its structure will be cited below. The foregoing data offer no further characterization of the component which is the source of the signal I. Its possible relation to a free radical form of chlorophyll will be discussed below.

More recently we have turned our attention toward living Chlorella in order to study free radical processes in a functionally complete photosynthetic system. The algae are harvested, washed, and either packed into an ESR cell, or examined while flowing through the cavity in a circulation system which includes a gas-exchanger. Typical ESR signals from living Chlorella under various intensities of illumination are shown in Fig. 3. The ESR signals are quite similar to those observed in isolated chloroplasts. In the dark, Chlorella exhibits an ESR signal centered at $g=2.005$, with a half-width of about 20 gauss, with 5 hyperfine peaks at 6 gauss intervals. On illumination the intensity of this signal increases, but in addition (at appropriate temperatures) a second signal is observed which enhances the absorption at the low-g side of the signal. By subtraction of suitable signals it can be shown that the second signal is centered at $g=$ about 2.002, has a half-width of about 9 gauss, and no observable hyperfine structure.

Thus, the two ESR components I and II observed in spinach and tobacco chloroplasts also occur in living Chlorella. That these components are closely related to the photosynthetic processes in green algae is shown by the results shown in Fig. 4. The upper curve shows the ESR signals exhibited by the normal green strain of Euglena, 


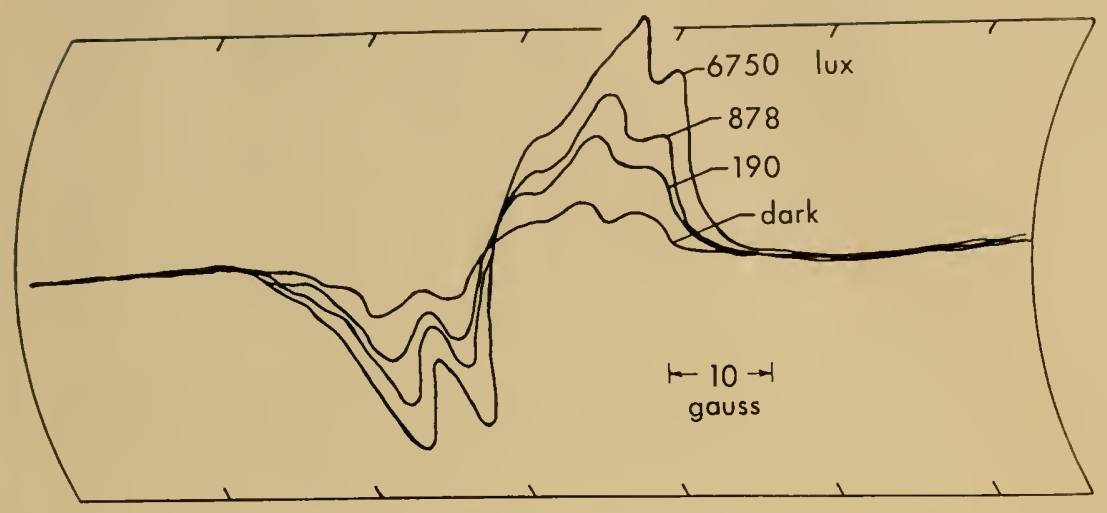

\section{MAGNETIC FIELD}

Fig. 3. ESR signals from living Chlorella in the dark and various intensities of white light. Modulation amplitude: 3 gauss.

and by a colorless mutant. The signals obtained from the green strain are essentially identical to those observed in Chlorella, both with respect to $g$ values, hyperfine structure, and the effect of light. In contrast, the colorless strain yields a single ESR signal at about $g=2.006$, which shows no hyperfine structure, and does not respond to illumination. Fig. 4 also shows that when $R$. rubrum is examined under instrument conditions which will resolve the hyperfine structure in the signals from green algae, a single signal at about $g=$ 2.003 and a half-width of 7 gauss is observed. Although this signal somewhat resembles component I of green algae, and is responsive to light, a considerably more detailed study is required for any further comparisons.

Fig. 5 show's that the presence of $\mathrm{CO}_{2}$ has a characteristic effect on these signals. In the dark, when only component II is present, its intensity is reduced by the presence of $5 \% \mathrm{CO}_{2}$ in air. When this gas mixture is present, and the cells are illuminated, the intensity of signal II is reduced but that of signal I is enhanced.

The effects of various intensities of light on the two ESR signals exhibited by Chlorella provides further evidence of their relationship to photosynthetic processes. From data of the type shown in Fig. 3 , it is possible to estimate, separately, the effects of light intensity on the two ESR components. The meter deflection at the first hyperfine peak at the low magnetic field side of the overall ESR signal is due only to signal II (see Fig. 1). On the other hand, the 

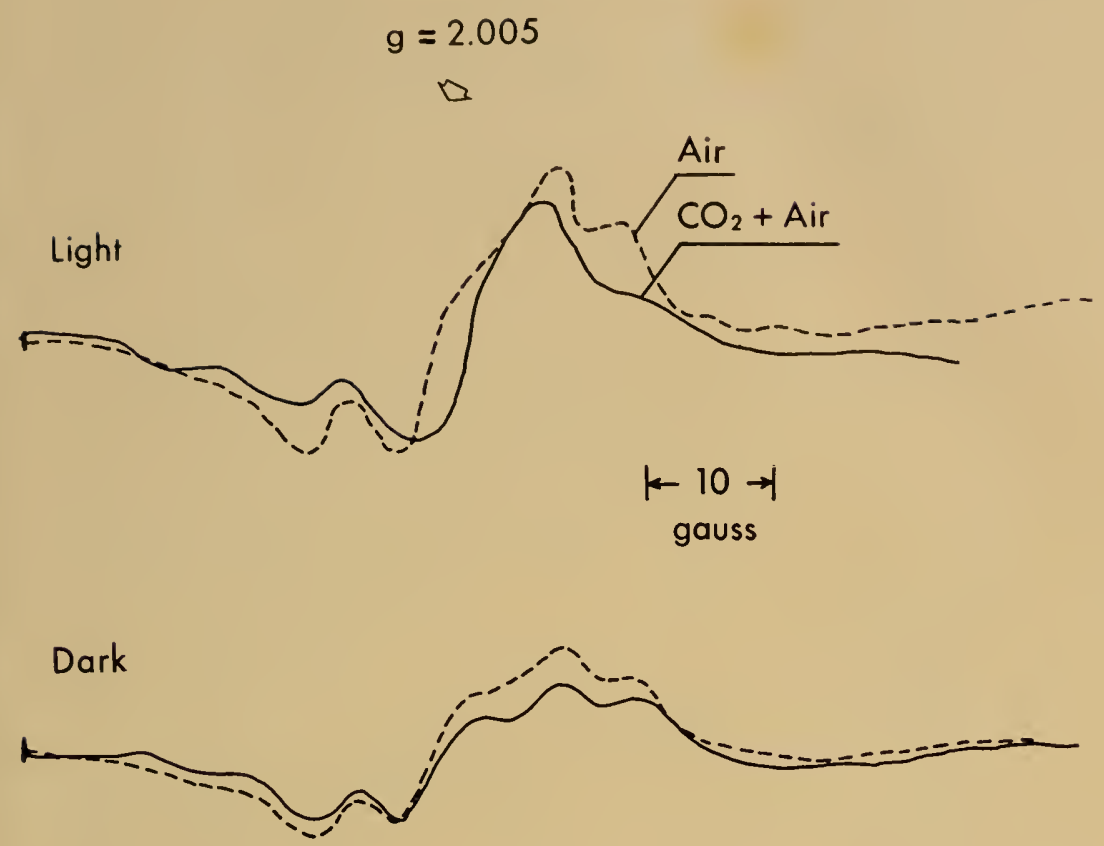

Fig. 5. ESR signals from living Chlorella after equilibration with air and $5 \%$ $\mathrm{CO}_{2}$ in air. Modulation amplitude: 3 gauss.

meter deflection at which the pure signal II crosses the baseline (as observed in the (lark) can be due only to signal I. From these two determinations, one can estimate the relative intensities of the two components in the overall ESR signal.

The effects of various light intensities on the two components at two different temperatures are shown in Fig. 6. At $29: 5^{\circ} \mathrm{C}$ signal I is not observed under any conditions. Signal II exhibits a lightsaturation curve typically observed in photosynthesis which reaches a maximum at 20,000 lux. At $12.8^{\circ} \mathrm{C}$, component II shows a similar effect of light intensity, except that saturation occurs at about 10,000 lux. However, at the lower temperature, illumination also induces the appearance of the component I, which increases with light intensity without exhibiting a tendency to saturate until an intensity of 60,000 lux is attained.

From kinetic determinations of the type discussed earlier in connection with chloroplast studies (see ref. 5 for details), we find in Chlorella a single time constant after onset of illumination (about 10 seconds), and under certain circumstances 2 time constants (about 


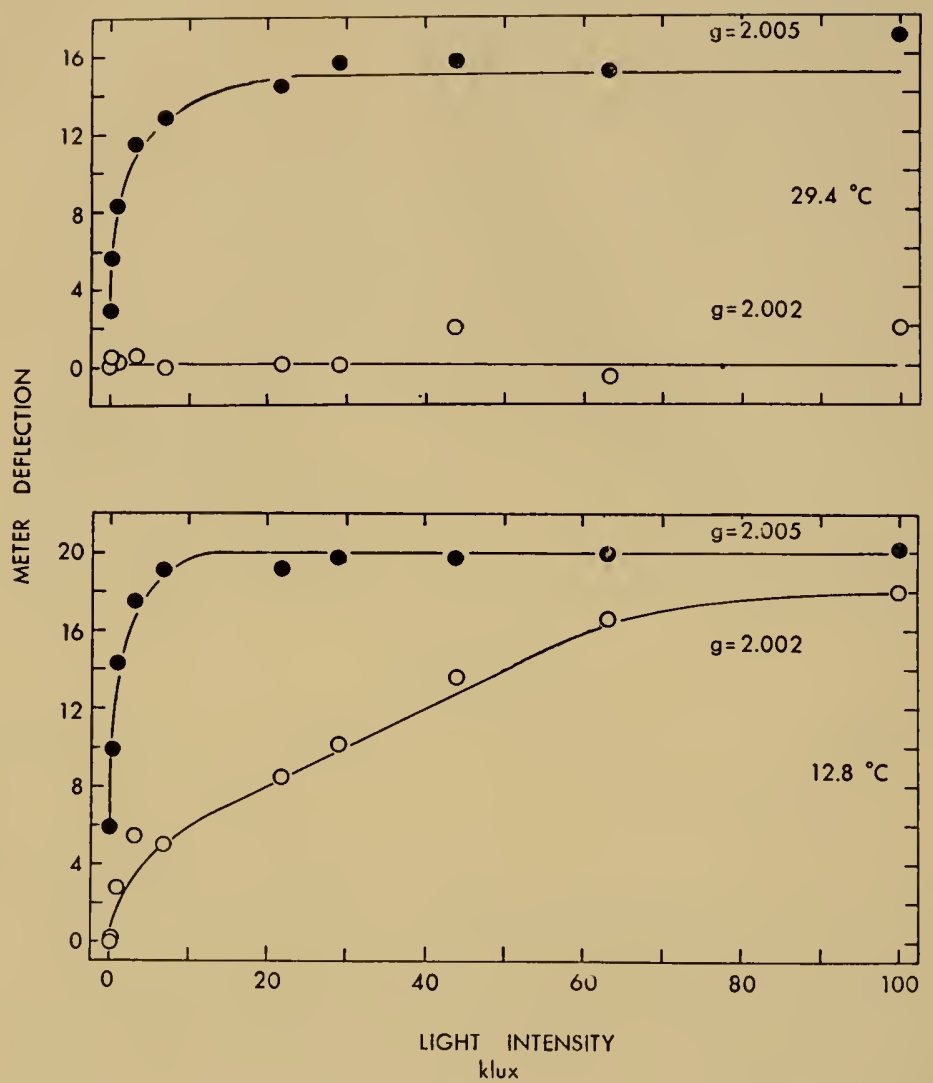

Fig. 6. Relative contribution of the ESR signals at $g=2.002$ (open circles) and at $g=2.005$ (closed circles) to the total signal intensity of Chlorella cells at various light intensities, at temperatures of $29.5^{\circ} \mathrm{C}$ and $12.8^{\circ} \mathrm{C}$. See text for details of mcasurement.

11 seconds and 20 seconds respectively) during decay in the dark. By methods which have been described elsewhere (5), it is possible to estimate the relative contribution of the two processes to the total ESR signal. Results of such an analysis of the ESR signal exhibited by Chlorella at various temperatures are shown in Fig. 7. The upper curve shows that at all temperatures the signal appears after onset of light with a single, relatively long time constant. The signal intensity is at a minimum at $18-28^{\circ} \mathrm{C}$, and is maximal at low temperatures. When illumination stops, the decay process exhibits two time constants. The fast component makes almost no contribution to the decaly process at temperatures of $18-28^{\circ} \mathrm{C}$, but represents an increas- 


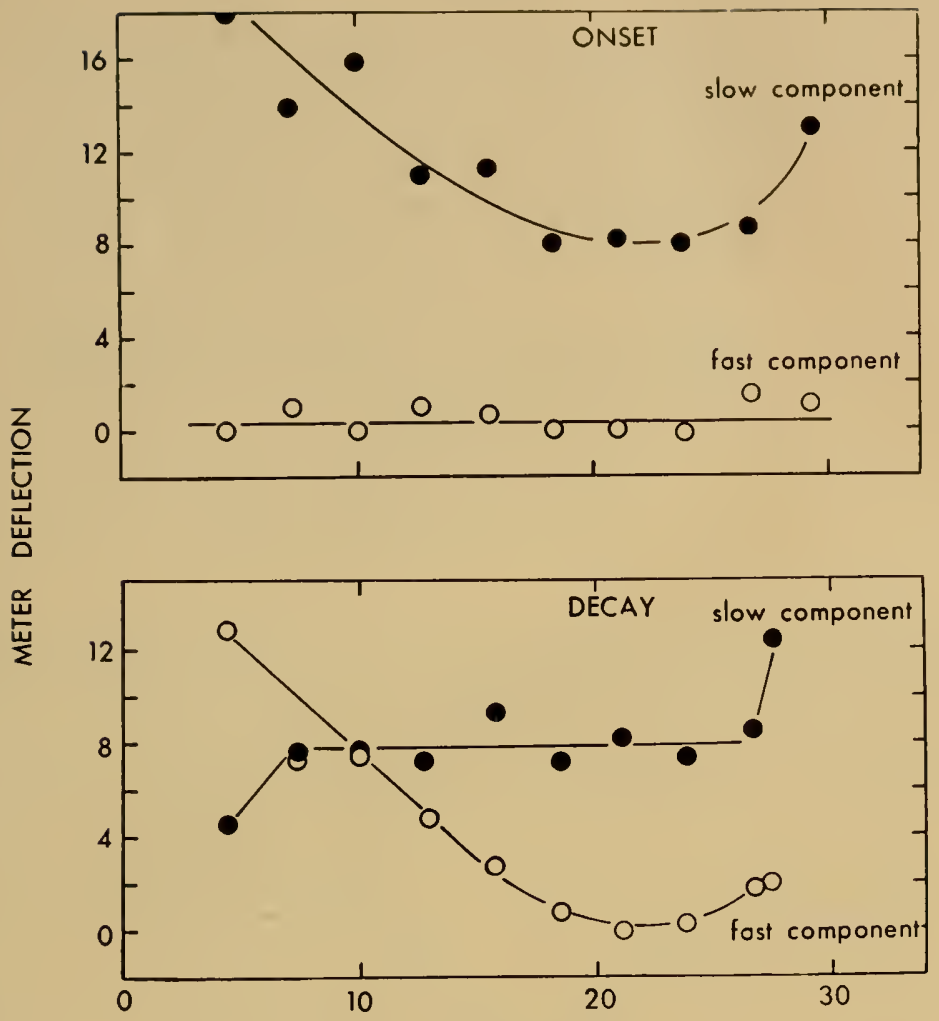

TEMPERATURE $C^{\circ}$

Fig. 7. Analysis of the rate of onset and decay of the ESR signals yielded by Chlorella at various temperatures. The values indicated are given in arbitrary scals units and represent the relative signal intensity accounted for by onset and decay processes which follow a fast time-constant (10 to $11 \mathrm{sec}$.) and slow time-constant (20 sec.) respectively, as explained in text.

ingly larger part of the overall loss in signal intensity as the temperature is reduced. In contrast, over nearly the entire range of temperatures the slow component contributes a constant part of the decay process-about 8 units on the scale of meter deflection.

The relationship between the slow onset and fast decay processes apparent from these data is shown more explicitly in Fig. 8. This figure shows that when the signal exceeds 8 meter units, the amount of the fast decay is about equal to the excess, over 8 units, in the signal intensity. 


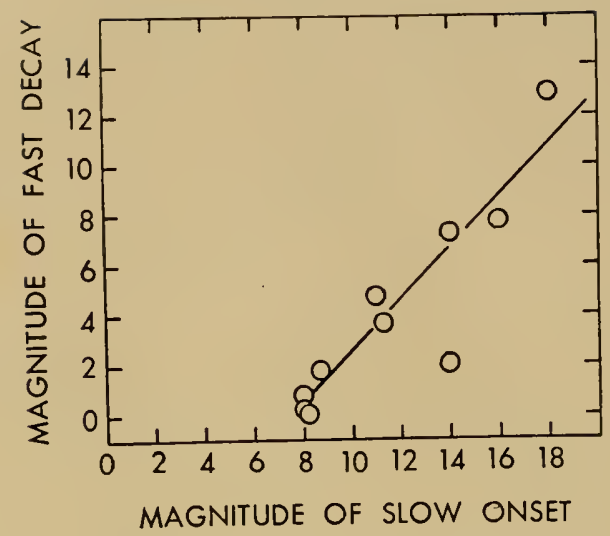

Fig. 8. Data replotted from Fig. 7 .

When these data are compared with the evidence regarding the relative responses of the two ESR signals to light intensity and temperature, the following relationships emerge. In the temperature range which is optimum for photosynthesis $\left(18-30^{\circ} \mathrm{C}\right)$, ESR signal II, which is present in the dark, increases in the light at a relatively slow rate, to attain a total signal intensity of about 8 units. When the light ceases, the signal decays with a single, relatively slow rate constant.

At lower temperatures matters are different. The total ESR signal intensity in the light now exceeds 8 units and is due to two superimposed effects: (a) signal II increases from a dark value of about 3 units to a maximum of 8 units, and (b) signal I rises from a clark value of zero to a level which increases with decreasing temperature. On illumination, both components increase in intensity at the same rate, so that at all temperatures a single rate constant is observed for onset. At low temperatures the decay process occurs with two time constants. Signal II decays slowly from its maximum value of 8 units. Signal I decays more rapidly.

Direct evidence of these relationships can be scen in the upper part of Fig. 9, which represents the change in meter deflection during a brief decay of the light-induced signal at $12.5^{\circ} \mathrm{C}$. This figure shows a remarkable "undershoot" effect, in that the signal intensity exhibited by a briefly darkened sample continues to decline for a fraction of a second after being again illuminated. This result is to be expected from the fact that the rate of onset for both ESR components that contribute to the overall meter deflection is relatively 

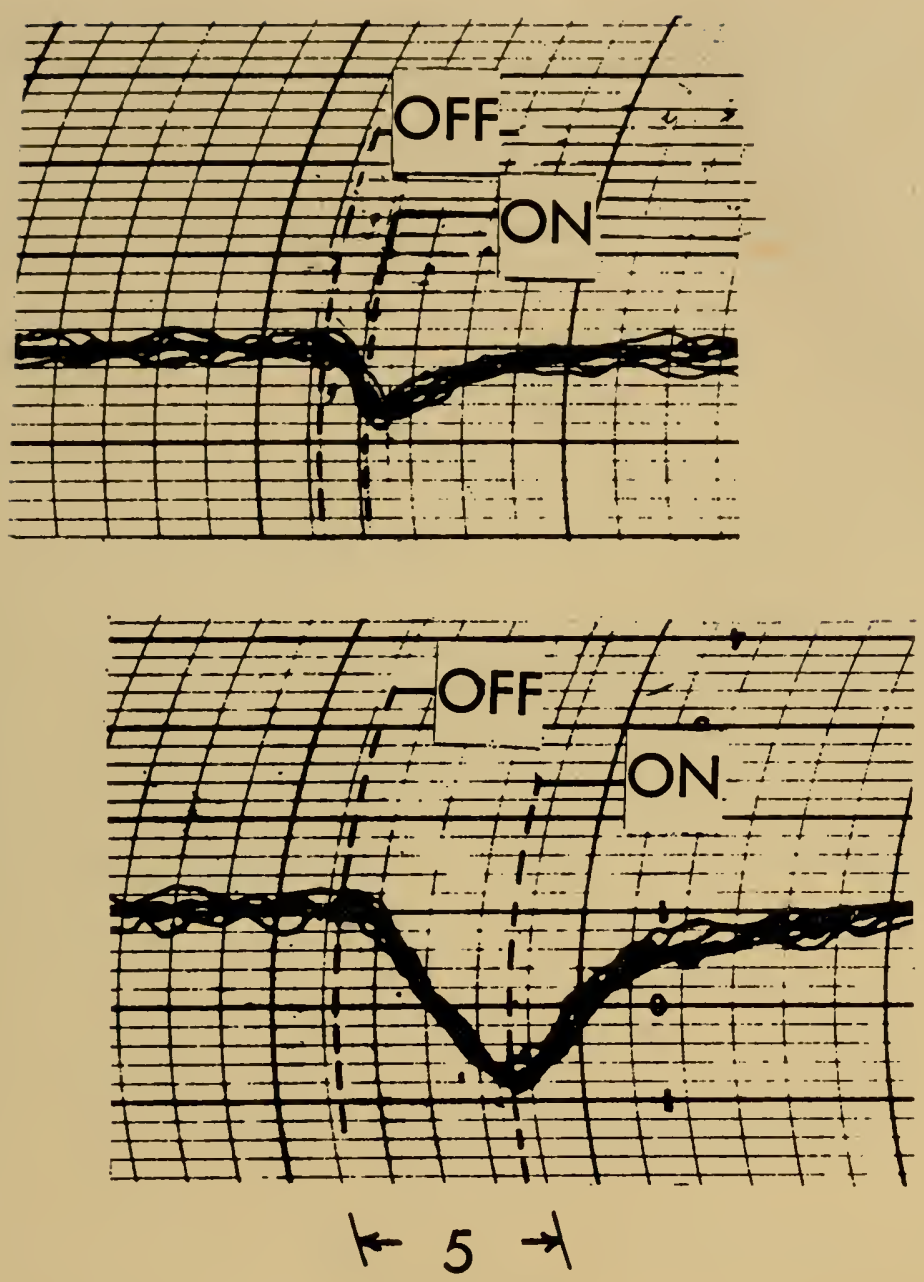

\section{seconds}

Fig. 9. Variation in ESR signal intensity, with time after cessation and onset of illumination. In this experiment the ESR spectrometer was maintained at a fixed magnetic field and the meter deflection at that field was monitored as a function of time. Temperature, $12.8^{\circ} \mathrm{C}$. The upper curve shows evidence of an "undershoot" effect.

slow, while the initial rate of decay of component $I$ is rapid. As a result, for a brief period after onset of illumination, the decay process overbalances the increasing signal due to illumination, and the "undershoot" is observed. 
These observations, like the earlier data on chloroplast preparations, suggest that the light-induced ESR effects involve the sequential formation of two components which contain unpaired electrons. Tentatively, it would appear that of the two, component $I$ is the more proximal to the initial photochemical event. This component cannot represent an actual light-induced excited state, because its decay rate, though rapid compared to that of the second component, is still small compared to that exhibited by light-excited states. There is evidence that component $\mathrm{I}$ is not ordinarily observed (i.e., at optimal photosynthetic temperatures) because it rapidly decays, perhaps by means of an electron transfer process which in turn gives rise to component II. However, since component II is always present in the dark it is also capable of being produced as an intermediate in a dark oxidation-reduction process. The data of Fig. 7 and 8 suggest that the steady-state concentration which can be attained by component II in the light is limited, and that component I makes its appearance when this limit is reached. These relationships suggest that electron transfer from component I to component II normally results in a zero steady-state concentration of the former, which is overcome only when the transfer process is inhibited by the failure of component II to decay sufficiently rapidly (as at low temperatures).

This description of the ESR-yield components of the photosynthetic system has been presented, intentionally, in purely operational terms, because the data available at the present time do not warrant any other approach. It is, of course, obvious that the foregoing relationships can be fitted into any one of a number of schemes of photosynthesis that have already been proposed. However, if this correlation is to be meaningful, both of the ESR components which we have observed must be identified with specific known constituents of the photosynthetic apparatus. The data available at present do not permit such an identification, except in the most general terms.

All that can now be said in this matter is the following: The ESR component at $g=2.005$ (II) is probably an organic free radical capable of being produced in the dark. It is uniquely present in green plant photosynthesis. This component may be a free radical form of any one of a number of reversibly oxidizable components that occur in this system. Conceivably, it represents umpaired electrons bound by a specific electron trap in a semiconductor. If so, the presumed electron trap is unique to green plants, and is for example, not present in $R$. rubrum. Less definite conclusions can be drawn with regard to the component at $g=2.002$ (I). We have observed relatively 
narrow, unstructured ESR signals at about this $g$ value in a preparation of purified chlorophyll. The signal is present in the dark, increases On illumination, and decalys very slowly when illumination ceases. But there is no clear evidence that the ESR signal is due to a free radical form of chlorophyll. The number of umpaired electrons observed is less than $0.1 \%$ of the chlorophyll present, and it is possible that the signal arises from an impurity. Similar results have been reported by Brody, Newell, and Castuer (1). ESR studies of the Krasnorsky reaction are not very helpful either. We have found that on illumination of the Krasnovsky preparation (chlorophyll, aqueous pyridine, and ascorbate) an intense ESR signal is observed-but it is clearly due to an ascorbate free radical. A similar observation has been reported by Krasnovsky himself (2). There is therefore only the most slender basis at this time for relating the signal at $g=2.002$ to chlorophyll.

Recent experiments with deuterated Chlorella provide additional data on the identities of the ESR signals observed in photosynthetic systems, and offer a hope that complete identification will be achieved tefore long. Fig. 10, which is based on studies made in collaboration with Dr. Joseph J. Katz of the Argonne National Laboratory, shows the ESR signal from Chlorella cultured in $99.9 \% \quad \mathrm{D}_{2} \mathrm{O}$. As indicated carlier, the hyperfine splitting due to $\mathrm{H}^{1}$ and deuterium are distinc-

$$
g=2.005
$$

CHLORELLA

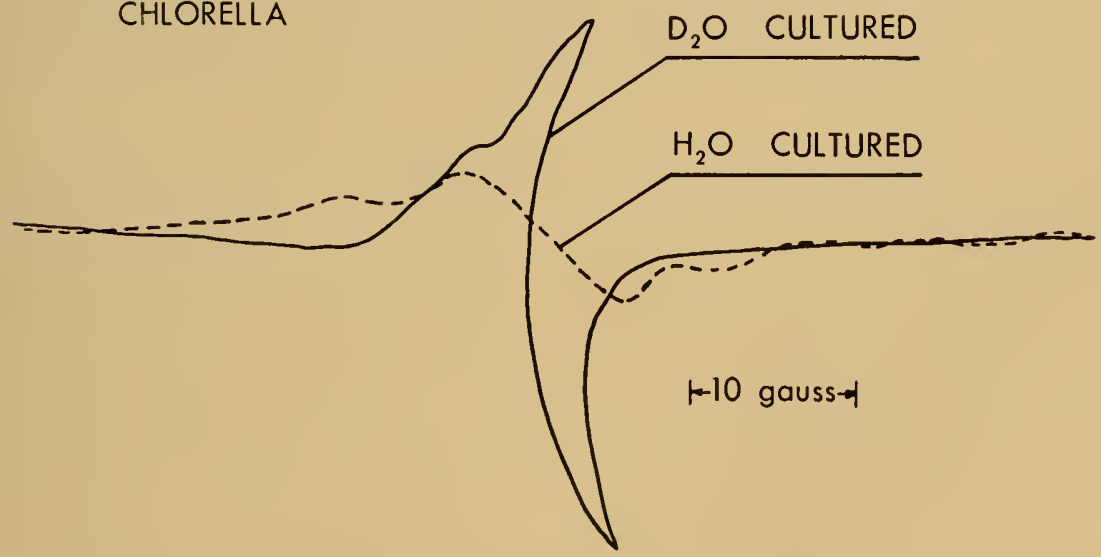

Fig. 10. ESR signals, under identical instrument conditions from $\mathrm{H}_{2} \mathrm{O}$-cultured Chlorella and Chlorella cultured in $99.9 \% \quad \mathrm{D}_{2} \mathrm{O}$. Modulation amplitude: 3 gauss. 
tive, and one can predict that an ESR signal affected by protons will become very much narrowed if cleuterons are substituted. Fig. 10 show's that the D.O-cultured algae yield an ESR signal which resembles that of ordinary Chlorelln-except that it is very much compressecl. Signal 11, which in normal algae has a half-width of 19 gauss, exhibits a half-width of about 5.5 gauss in the $\mathrm{D}_{2} \mathrm{O}$ material. The corresponding half-widths for signal I are 9 gauss and about 3 gauss respectively.

In the case of component II the deuterium effect is so marked as to indicate that the umpaired electron is affected exclusively by association with hydrogen atoms. Once this conclusion is reached, it follows that the 5-line hyperfine structure observed in the ordinary signal from component II is due to protons. This being the case, it can be concluded, on the basis of rules developed from studies of known free radicals (see Weissman, 12 and 13), that this component is an organic free radical in which the umpaired electron is associated with a resonance system composed of four chemically equivalent carbons, to each of which a proton is attached. We are now studying the effects of adding known substances to the $\mathrm{D}_{2} \mathrm{O}$-cultured algae, and expect, in this way, to make a positive identification of this free radical. The $\mathrm{D}_{2} \mathrm{O}$ data also show that the umpaired electron of component $\mathrm{I}$ is associated with hychogen atoms.

The association of both components with protons precludes the possibility that the unpaired electrons that give rise to the two ESR signals are concluction electrons in a semiconductor. Thus, the isotope clita inclicate that no part of the ESR signal resulting from illumination can be attributed to concluction electrons, and provide a useful partial description of the free raclical that is responsible for one of the observed signals.

In presenting this summary of our ESR studies of photosynthetic systems, an effort has been made to clescribe some of the experimental data, the principles on which the measurements are based, and the imitations that these principles place on possible interpretations of the results. It is evident that the physical principles which govern ESR measurements and the relationships between the observed signals and molecular structure are essential as guides in these investigations. However, our studies also show that biological principles are equally important experimental guides. A frequent comment about the earliest efforts to apply the ESR technique to biological investigations was that the presence of numerous types of free radicals in living cells would preclude precise clata relevant to a specific 
biochemical process. This problem is familiar to biologists, and the solution-the use of highly specialized cells as objects of study-is equally well known. The discovery of the highly specific ESR signal due to a photosynthetic free radical component (II) which is readily identifiable in isolated chloroplasts and in whole living algae is additional evidence of the usefulness of this biological principle.

The dependence of ESR studies of biological systems on principles of physics, chemistry, and biology is also exemplified by the great debt which this work owes to the efforts of my colleagues Professor Jonathan Townsend of the Department of Physics and Professor S. I. Weissman of the Department of Chemistry.

\section{REFERENCES}

I. Brody, S. S., Newell, G., and Castner, T., J. Phys. Chem., 64, 554 (1960).

2. Bubnov, V. N., Krasnorsky, A. A., Umrichina, A. V.. Tsepalor, V. F., and Shlyapintokh, V. Y., Biofizika, 5, 12I (1960).

3. Calvin, M. in Brookhaven Symposia in Biol., 11, 160 (1958).

4. Commoner, B., paper presented at Gatlinburg Conf. on Photosynthesis, 1952.

5. Commoner, B., Heise, J. J., Lippincott, B. B., Norbert, R. E., Passonneau, J. V.. and Townsend, J.. Science, 126, 57 (1957).

6. Commoner, 13.. Heise, J. J., and Townsend, J., Proc. Natl. Acad. Sci. L. S.. 42, 710 (1956).

7. Commoner, B., Townsend, J., and Pake, G. E., Nature, 174, 689 (1954).

8. Hutchison, C., and Mangum, B., J. Chem. Phy's., 29, 952 (I958).

9. Lewis, G. N., and Lipkin, D., J. Am. Chem. Soc., 63, 3005 (1941).

10. Sogo, P. B., Pon, N. G., and Calvin, Ml., Proc. Natl. Acad. Sci. U. S., 43, 387 (1957).

11. Van der Waals, J. H., and DeGroot, M. S., Molecular Physics, 2, 333 (1959).

12. Weissman, S. I., J. Chem. Phys., 22, 1378 (1954).

13. Weissman, S. I., Chapter on "Electronic Paramagnetic Resonance" in Comprehensive Biochemistry, Elsevier Publishing Co. (in press).

14. Wertz, J. E., Chem. Revs., 55, 829 (1955). 


\title{
DIFFERENT FORMS OF CHLOROPHYLL $A$ IN VIVO
} AND THEIR PHOTOCHEMICAL FUNCTION

\author{
Eugene Rabinowitch and Govindjee
}

Photosynthesis Research Laboratory

Department of Botany

University of Illinois, Urbana, Illinois

\section{INTRODUCTION}

From the study of the action spectra of chlorophyll fluorescence in algae containing different pigment complements, it has been concluded that quanta absorbed by pigments other than chlorophyll a are transferred by resonance to the latter pigment, with an efficiency between $<20 \%$ (for some carotenoids) and $>90 \%$ (for chlorophyll $b$ and phycocyanin). Comparison with the action spectra of photosynthesis suggested that these quanta contribute to photosynthesis in proportion to the efficiency of their transfer-in other words, that ultimately photosynthesis is sensitized only by excited molecules of chlorophyll $a$, whether their excitation be due to direct absorption or to resonance transfer. This simple and eminently plausible picture is, however, unable to explain two phenomena: (I) The quantum yield of photosynthesis declines, in green algae (Chlorella) above $680 \mathrm{~m}_{\mu}(12,13)$ (Fig. 1), and in red algae (Porphyridium cruentum) above $650 \mathrm{~m}_{\mu}$ (1) (Fig. 2)-i.e., in the region where light is absorbed only by chlorophyll $a$. The action spectrum of chlorophyll $a$ fluorescence shows a similar "red drop," and it secms that it, too, occurs at considerably shorter wavelengths in red algac than in green algae (8). (II) The quantum yield of photosynthesis in the "red drop" region can be brought up, often to the normal level of about 10 quanta per molecule $\mathrm{O}_{2 .}$, by simultancous absorption of light of shorter wavelengths $(9-11,14)$. The action spectrum of this so-called "second Emerson effect" follows the absorption curves of the main accessory pigments (chlorophyll $b$ in green algae, fucoxanthol in brown algae, the phycobilins in red and bluc-green algae); or, more precisely, the curves showing the proportion of incident light absorbed by these pigments. This suggests that excitation of chlorophyll $a$, far from being the only mechanism of sensitization of photosynthesis, 


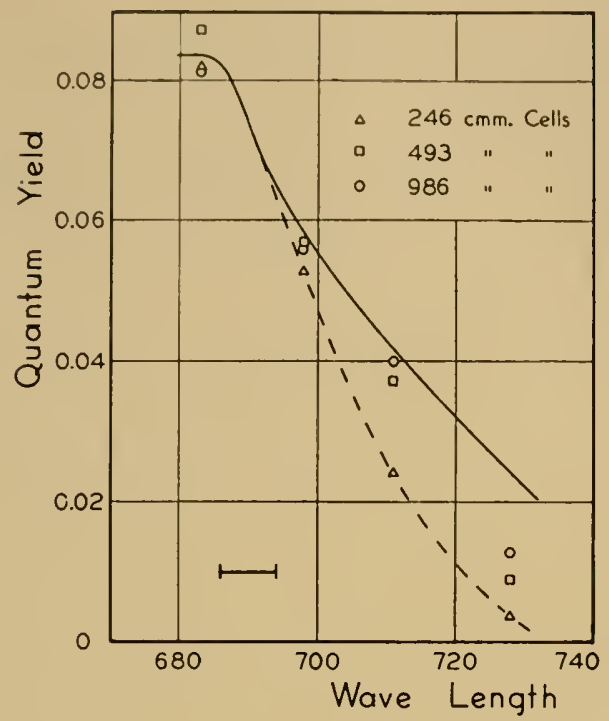

Fig. 1. Decline of quantum yield, $\phi$, in the far red in Chlorella. Dashed curve, incomplete absorption; solid curve, approach to complete absorption (showing that red drop is not simply the consequence of incomplete absorption). (After Emerson and Lewis, 13).

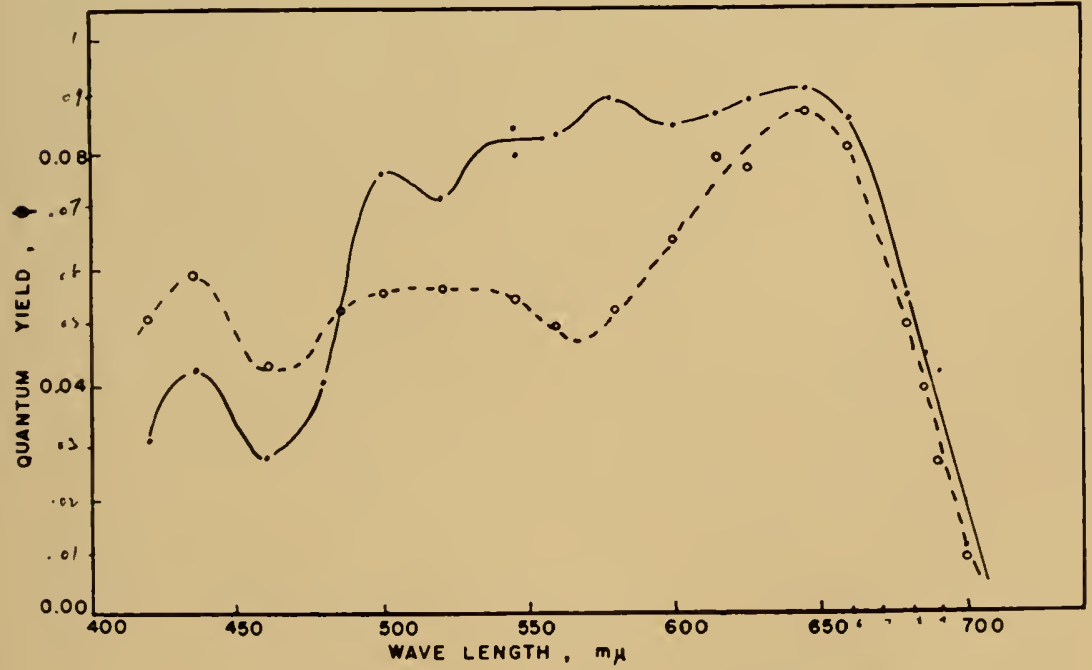

Fig. 2. Decline of quantum yield, $\phi$, in the red in Porphyridium. Solid curve, cells adapted to green light; dotted curve, cells adapted to blue light. (After II. Brody and R. Emerson, I.) 
is in itself insufficient to produce photosynthesis, and needs supplementation by the absorption of quanta by one of the accessory pigments.

This picture, too, seems consistent and plausible-but it fails to explain the above-mentioned parallelism of the action spectra of photosynthesis and of chlorophyll a fluorescence. Why should excitation of chlorophyll a need supplementation by excitation of an accessory pigment, if the latter excitation is transferred practically quantitatively to chlorophyll $a$ ?

Prior to Emerson's experiments on the red drop, Duysens (8) suggested, as explanation of the apparent low photochemical efficiency of chlorophyll $a$ in red algae, the presence in these organisms of two forms of chlorophyll $a$, one of which is preferentially coupled to the accessory pigments. This form is fuorescent and photochemically active, while the rest of chlorophyll $a$ is non-fluorescent and photochemically inactive. To account for the "red drop," it could be further assumed that the two forms of chlorophyll have somewhat displaced absorption bands. Specifically, it could be suggested that the main red bands of both Chlorella and Porphyridium consist of two superimposed component bands-one with a peak at about 670 $\mathrm{m}_{\mu}$ ("Chl a 670"), which belongs to the photochemically active form of chlorophyll $a$, and another with a peak at $690-700 \mathrm{~m}_{\mu}$ ("Chl a $690^{\prime \prime}$, which belongs to the photochemically inactive form of the same pigment. To explain the action spectrum of chlorophyll fluorescence, one would naturally suggest that the Chl a 670 form is fluorescent and the Chl a 690 form, non-fluorescent. (However, there is evidence (2) of a fluorescent band at $710-715 \mathrm{~m} \mu$ probably associated with the $695 m \mu$ absorption band.) It was suggested by Brody (2, 3) that the difference in the location of the red drop in Chlorella (at 680 $\mathrm{m}_{\mu}$ ) and in Porphyridium (at $650 \mathrm{~m}_{\mu}$ ) can be due simply to a much larger proportion of the inactive form, Chl a 690 , in the latter. However, this interpretation probably is insufficient. The shape of the band is, in every case, suggestive of the presence of a third, and per-

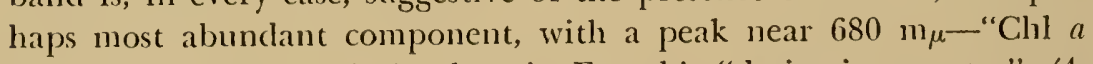
680." This is particularly clear in French's "derivative spectra" (4, 16). (He assigns positions at 673,683 , and $695 \mathrm{~m}_{\mu}$ to the three component bands in Chlorella). The question of the nature, the photochemical role, and the fluorescence of Chl a 680 naturally poses itself and the answer to it is far from clear. We will return to this subject in discussing the new experimental results presented in the following section. 


\section{NEW OBSERVATIONS}

Action Spectra of the Emerson Effect

The hypothesis that wo (or more) lorms of chlorophyll a, with different photochenical properties, are present in vivo was confirmed by new and more precise measurements of the action spectrum of the Emerson effect (Figs. 3. A, B). Fig. 3A applies to the green alga, Chlorella. It show's the extent to which the quantum yield of photosynthesis produced by the absorption of light in a "far red" band $(685-700 \mathrm{~m} \mu)$ is enhanced by monochromatic supplementary light of different wavelengths. The curve has two sharp maxima. The one at $650 \mathrm{~m}_{\mu}$ (already observed by Emerson) must belong to chlorophyll $b$; the other, at $670 \mathrm{~m}_{\mu}$, not noted by Emerson, indicates the existence of a chlorophyll $a$ form having an absorption peak in this region-a form whose excitation can supplement that in the farred band as effectively as does the excitation of chlorophyll $b$.

Fig. 3B refers to the diatom Navicula. The action spectrum of the Emerson effect in this diatom shows three peaks, at $535 \mathrm{~m}_{\mu}$ (attributalble to fucoxanthol-this is not the peak of the fucoxanthol band, but the region of maximum participation of fucoxanthol in total absorp-

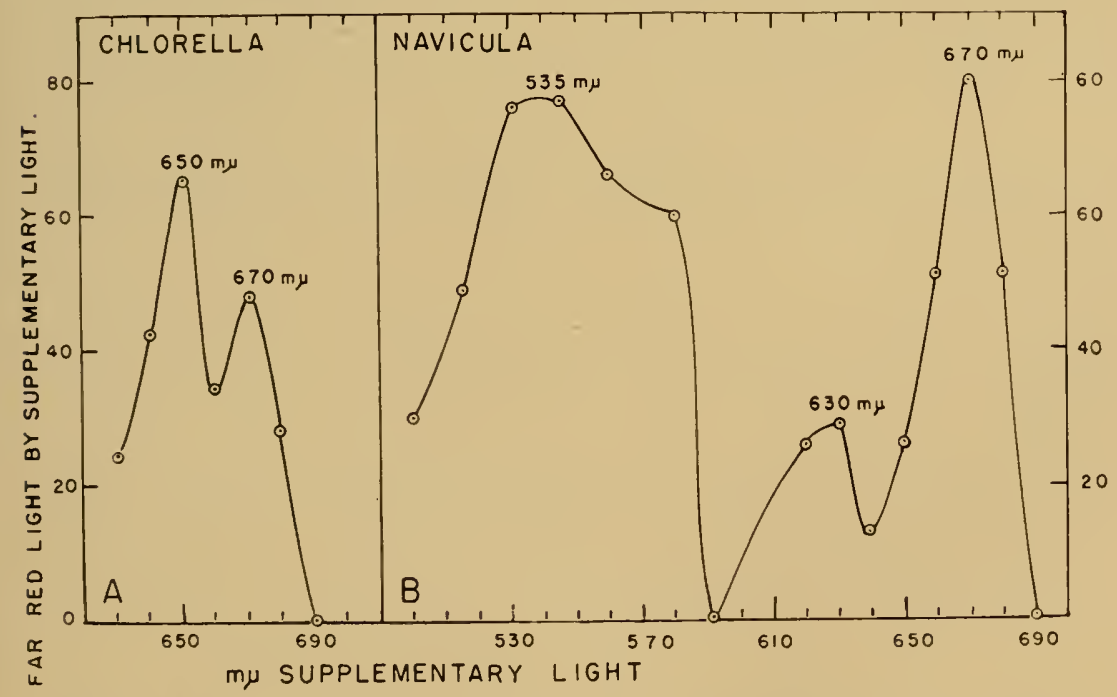

Fig. 3. A, Action spectrum of Emerson effect in Chlorella (after Covindjec and Rabinowitch, 17). B, Action spectrum of Enerson effect in Navicula (after Govindjee and Rabinowitch, 17). 


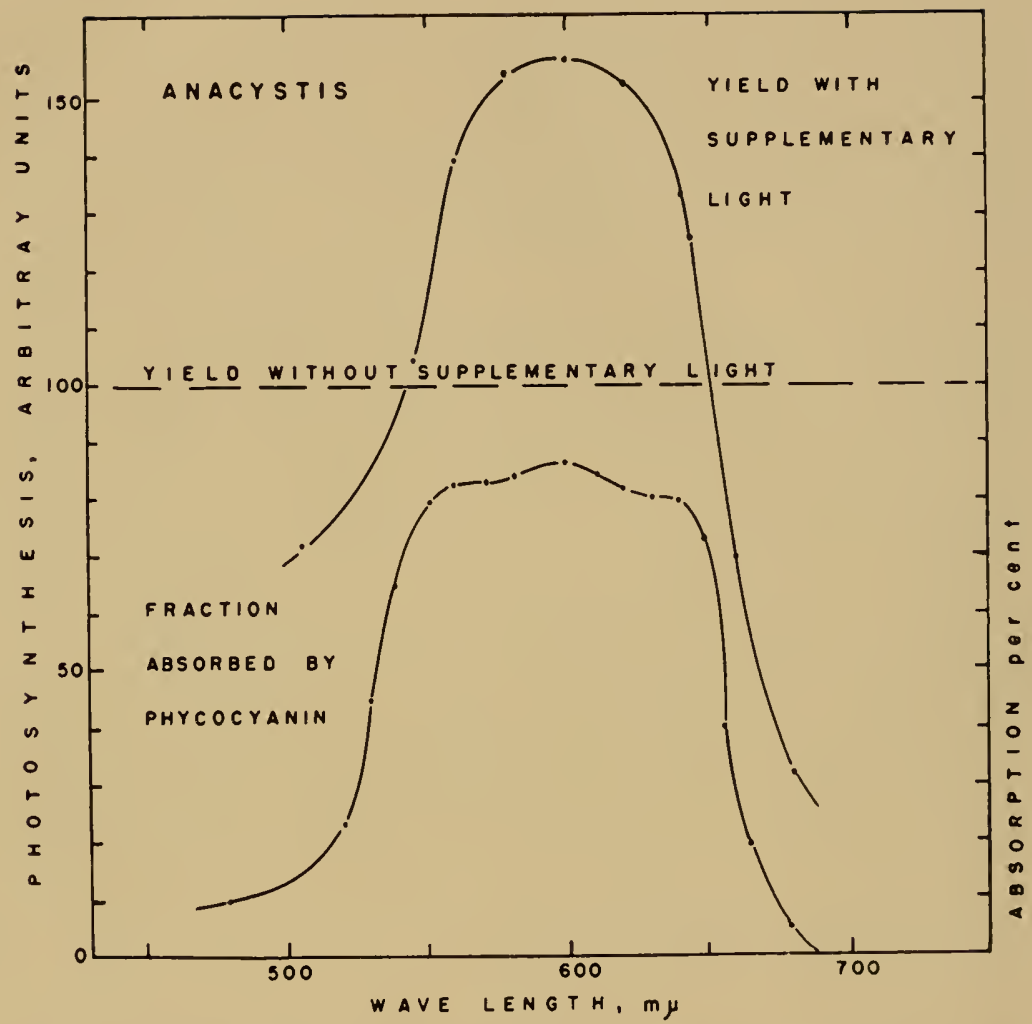

Fig. 4. Ipper curve, action spectrum of Emerson ellect in Anarystis. Lower curn, fraction of absorbed light absorbed by plycocyanin. (Ifter Emerson and Rabino. witch, 14).

tion, cf. Tanada, 19), at $630 \mathrm{~m}_{\mu}$ (chlorophyll c), and at $670 \mathrm{~m}_{\mu}$. There is no chlorophyll $b$ in these algae and therefore no peak at $650 \mathrm{~m}_{\mu}$.

These two curves seem to suggest that the form Chl a 680 does not supplement effectively the photochemical action of Chl $a 690$.

So far, so good; but now we are up for trouble. Fig. 4 was obtained by Emerson with the blue-green alga Anacystis. It shows only one peak, at $600 \mathrm{~m}_{\mu}$, clearly attributable to phycocyanin; but when we move to longer waves, not only is there no clear peak at $670 \mathrm{~m} \mu$, but there is a negative region. This means that combination of farred light $(685-700 \mathrm{~m} \mu)$ with monochromatic light in the region 670$700 \mathrm{~m} \mu$ produces, in Anacystis, less photosynthesis than the two light beams separately! Emerson's measurements cf. 14 showed negative 
effects not only in the action spectrum of Anacystis, but also in those of Noricula and Porphyridium.

The negative effect in the region $670-700 \mathrm{~m}_{\mu}$ in Anacystis suggests that this alga contains relatively little, if any, of the "activating" form, Chl a 670, and that the main chlorophyll a component in it, Chl a 680 , not only does not complement the photochemical action of Chl a 690, but counteracts it! (The existence of such photochemical countereffects is well known in various photobiological phenomena.)

Another approach to the interpretation of the negative effects is through the study of light saturation in monochromatic light. So far, it has been presumed, on theoretical grounds-and confirmed by observations in filtered colored light-that light saturation of photosynthesis is independent of the spectral nature of the absorbed light, being imposed by the limited amount of some enzyme, and a consequent "ceiling" on the overall rate of photosynthesis. Some preliminary obscrvations suggest, however, that the saturation rate may be much lower at 699 and $700 \mathrm{~m}_{\mu}$ than at the shorter wavelengths. If this is true, then the superposition of two beams, both belonging to the far red region, may produce a negative effect simply by bringing the process into the light-saturation range, even though one would not expect this to happen from the known extension of the "linear range" in white light. Such low saturation level may be itself conceivably due to some kind of a "photochemical counter effect."

\section{Difference Spectra}

The different fates of several chlorophyll a components in photosynthesis are suggested also by the shape of the "difference spectrum" of Chlorella (cf. Fig. 5, based on observations by Coleman in our laboratory; 5). The reversible bleaching of chlorophyll in strongly illuminated Chlorella cells is shown by this curve to affect Chl $a$ 680 more strongly than Chl $a 670$ or Chl $a 690$; in fact, it seems possible that a reversible transformation of Chl a 680 into $\mathrm{Chl} a 670$ and Chl a 690 takes place in light. Since, however, the whole difference spectrum remains negative above $600 \mathrm{~m}_{\mu}$, this conversion must be superimposed on the transformation of Chl $a$ into a compound with no red absorption band at all. (This compound may absorb in the $520 \mathrm{~m} \mu$ band, and may perhaps be identical with Krasnovsky's "pink" reduced chlorophyll, for which the name "eosinophyll" was suggested (14).)

Krasnorsky $(18,20,21)$ has observed preferential bleaching of Chl a 670 in light; but this was not confirmed by Brown and French (4), 


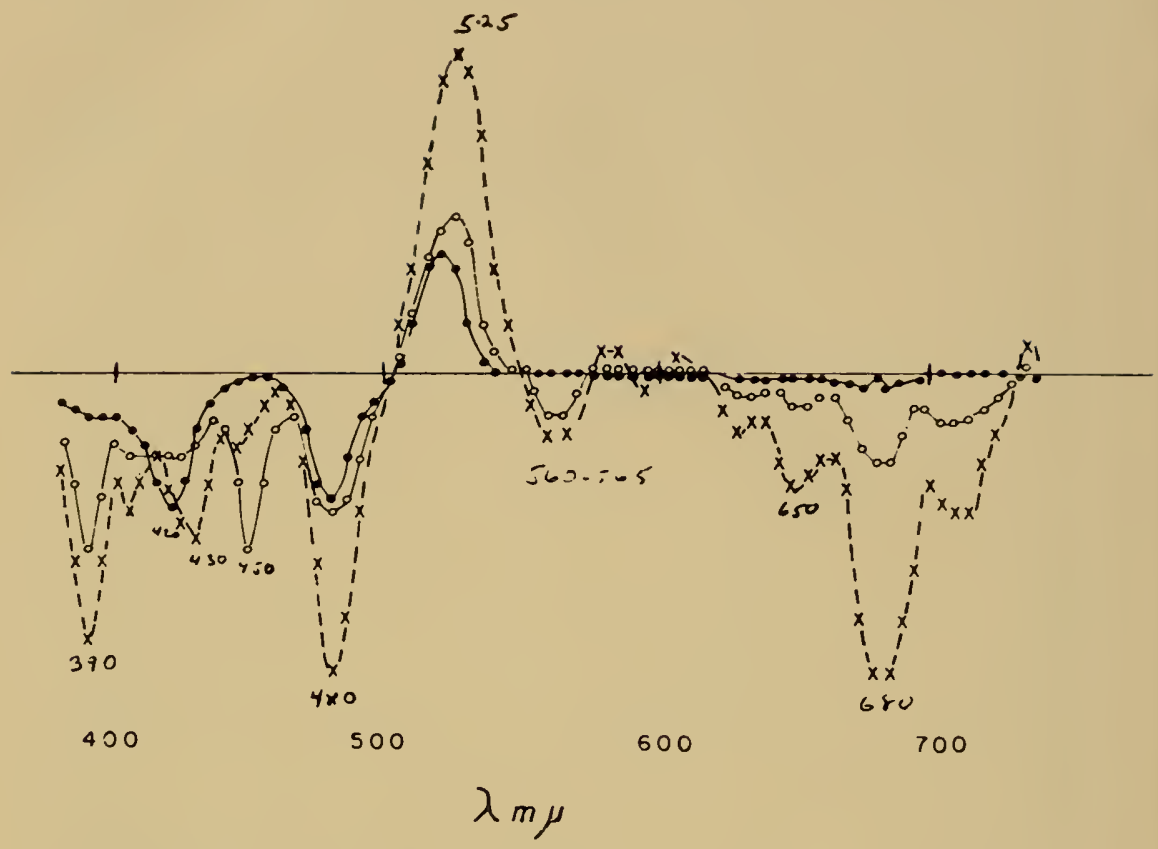

Fig. 5. Difference spectrum of Chlorella (after Coleman and Rabinowitch, 5). The three curres correspond to three light intensities $\left(3.4,12.0\right.$, and $31.4 \times 10^{14}$ $h_{\nu} /\left(\mathrm{sec} \times \mathrm{cm}^{2}\right)$. Reversible bleaching in the red chlorophyll a band is observed only at the higher intensities. It is strongest at $680 \mathrm{~m} \mu$, weaker at $670 \mathrm{~m} \mu$ and $690 \mathrm{~m} \mu$. Maximum eflects are of the order of $0.2 \%$.

who found that the bleaching affects most strongly the $690 \mathrm{~m} \mu$ form, next the $680 \mathrm{~m}_{\mu}$ form and least of all the $670 \mathrm{~m}_{\mu}$ form. In any case, these observations applied to more extensive and not immediately reversible bleaching, rather than to the small $(\sim 0.1 \%)$ instantaneous and fully reversible effect shown in Fig. 5.

\section{Conclusions}

All these results suggest that, in addition to the "one-dimensional" action spectra of photosynthesis, one should investigate systematically also "two-dimensional action spectrat," i.e., the effect on the yield of photosynthesis of combinations of two monochromatic light beams of systematically varied wavelengths. That certain combinations of light colors have specific effects on photosynthesis has been asserted in the past, e.g., by Danilov $(6,7)$; but conclusions were unconvincing because of the primitive techniques used. It seems that these 
studies are worth being repeated using more precise photochemical methods.

The cuhancement of the quantum yield in the far red by axiliary light suggests that the "inactive" form, Chl a 690, is not entirely inactive, but requires a balanced co-excitation of an "active" form to contribute fully to photosynthesis. It secms natural to think in this connection of the possibility that the primary photochemical process in photosynthesis might consist of two steps. Excited Chl $a$ 690 may be able to bring about only one of these steps, while excited Chl a 670 may be able to sensitize both of them. More specifically, Franck (15) suggested that the Chl a 690 excitation leads practically entirely to the triplet state (because it occurs in chlorophyll associated only with lipides, in which the singlet-triplet conversion is accelerated by the existence of an intermediate $n, \pi$ state); while the Chl a 670 excitation leads to a singlet excited state longlived enough to emit fluorescence (because it occurs in chlorophyll $a$ associated with water, in which the $n, \pi$ state lies above the singlet excited $\pi, \pi^{*}$ state). Why the cooperation of a chlorophyll $a$ molecule, in an excited singlet state, with a chlorophyll $a$ molecule in the triplet state is needed to bring about photosynthesis, is a separate question.

Franck's hypothesis is, of course, not the only possible explanation of the different photochemical capacities of Chl $a 670$ and Chl $a 690$ and of their cooperation in photosynthesis. Another alternative would be, for example, to postulate a limited photochemical capacity of a dimeric or polymeric form of chlorophyll $a$, and more versatile capacities of the monomeric form.

\section{REFERENCES}

1. Brody, M., and Emerson, R., J. Gen. Physiol., 43, 251 (1959).

2. Brody, S. S., Science, 128, 838 (1958).

3. Brody, S. S., pers. commun.

4. Brown, J. S., and French, C. S., Plant Physiol., 34, 305 (1959).

5. Coleman, J. W., and Rabinowitch, E., J. Phys. Chem., 63, 30 (1959).

6. Danilov, A. N., Soret. Botan., No. 4, 3 (1935).

7. Danilov, A. N., Eksp. Botanika, 2, 5 (1936).

8. Duysens, L. N. M., Ph.1). Thesis, Univ. of Utrecht (1952).

9. Emerson, R., Science, 125, 746 (1957).

10. Emcrson, R., Science, 127, 1059 (1958).

11. Emerson, R., and Chalmers, R. V., Phycol. Soc. Am. News Bull., 11, 51 (1958).

12. Emerson, R., Chalmers, R. V., and Cederstrand, C., Proc. Natl. Acad. Sci., U. S., 43, 133 (1957).

13. Emerson, R., and Lewis, C. M., Am. J. Bot., 30, 16.) (1913).

14. Emerson, R., and Rabinowitch, E., Plant Physiol., 35, 477 (1960).

15. Franck, J., Proc. Nat. Acad. Sci. U. S., 44, $94 \mathrm{i}$ (19.58).

16. French, C. S., Brookhaven Symposia in Biol., 11, 65 (1959). 
17. Govindjee, and Rabinowitch, E., Science, 132, 355 (1960).

18. Krasnowsky, A. A., and Kosobutskaya, L. M., Doklady Akad. Nauk. SSSR, 104, 440 (1955).

19. Tanada, 'T.. Am. J. Botany, 38, 276 (1951).

20. Vorobyova, L. M., and Krasnovsky, A. A., Biokhimiya, 2I, 126 (1956).

21. Vorobyova, L. M., and Krasnovsky, A. A., Biokhimiya, 23, 760 (1958).

\section{DISCUSSION}

DR. Franck: I am grateful for the opportunity to compare the basic differences of Dr. Calvin's concept of the photochemical steps of photosynthesis with mine. That I accept his biochemical results on the pathway of the carbon in photosynthesis, is self-evident. Onr main difference is that Dr. Calvin, as practically all biochemists, takes it for granted that the reduction of the final photosynthetic oxidant proceeds by dark reactions with a photochemically produced reducing agent. On the other hand, according to my view, physical evidence indicates direct photochemical reduction acts with direct contact between the oxidants and the excited chlorophyll molecules. In the last years Dr. Calvin emphasized the hypothesis that the only light reaction of photosynthesis consists in a photoionization of chlorophyll which by migration of electrons and positive holes, produces a charge of a 'solar battery' sufficient for electrolysis of water. The original model of this battery is, according to my opinion, based on very improbable assumptions; the new one presented today avoids some difficulties, but others remain. I enumerate the main ones briefly:

1) I still have grave doubts whether the quantum yield of photoionization can be high enough to match the high yield of photosynthesis. That a limited photoionization occurs, is shown by the behavior of the afterglow of chlorophyll, found by Strchler and Arnold, and explained as recombination luminosity of positive chlorophyll ions with electrons, by Armold. That this hypothesis fits all observations of afterglow, has been discussed in a paper of Brugger and Franck.

2) The assumption that light energy is fully utilized for photoionization has failed to explain the very characteristic relations between chlorophyll funorescence intensity and utilization of light energy for photosynthesis. All factors which lower the photosynthetic utiliation of excitation energy. ats low temperature, absence of $\mathrm{CO}_{2}$, induction phenomena, adclition of ensymatic poisons, ete., enhance the fluorescence, but not more than by a factor of ca. 2, uncler conditions where photosynthetic rates are zero cluring illumination. A detailed up-to-date description of such observations and their interpretation based on the concept of direct contact between oxidants and excited chlorophyll, will be found in an article on chlorophyll afterglow in the Handbuch fïr Pfanzenbiologie, Vol. V. (The article was originally written several years ago and is fually (he to appear this spring).

3) Another point not considered so far in Dr. Calvin's concept is an 
explanation of the Emerson effect. Emerson found that illumination with two light sources of different wavelengths, if applied together, gave a higher photosynthetic rate than the sum of the two independent irradiations. Of special interest is Jack Myer's new result that this is still the case if the irradiations are offered in succession, separated by dark periods of several seconds. It secms difficult to reconcile these data with the assumption that light is solely used for photoionization; the indications are rather, that the first irradiation produces a photochenical intermediate stable enough to survive a dark period and to undergo a second photochenical change by the following irradiation with light of shorter wavelengths.

The examples of clifficulties encountered by the assumption that light is solely used in photosynthesis for photoionization of chlorophyll make it equally difficult to accept any other concept which ascribes specificity to the photochemical acts of photosynthesis. Photochemistry is unspecific and permits all those reactions of substance which come in contact with the excited dye molecule for which the energy stored in the excited state is sufficient. The hypothesis that light energy in photosynthesis is specifically used for reduction of TPN inplies that the excited chlorophyll encounters TPN as the only photosensitive species. Oxygen present in the cells under aerobic condition in higher concentration is known to make during impacts efficient use of cxcited singlet energy, and of the energy of the metastable triplet state. Why does it in the region of light linitation not interfere with photosynthesis?

Our conclusion is that the photosynthetic oxidants must be present in unbstrate concentration rather than in catalytic ones. The final photosynthetic oxidants PGA, and probably the short-lived acid formed by addition of $\mathrm{CO}_{3}$ to ribulose diphosphate, are present during the steady state of photosynthesis in substrate concentration, and, as we want to mention as last point of this discussion, we have reason to assume that they are able to undergo photochemical changes at the cost of the lowest excited state of chlorophyll- $a$, its metastable triplet state.

Our interpretation of the photochemical acts of photosynthesis may be visualized by a diagram represented by a lantern slide.

1)
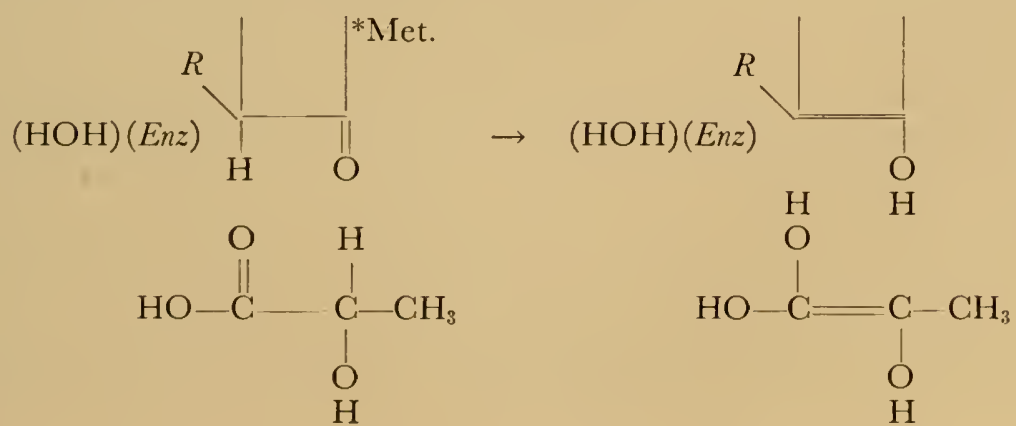


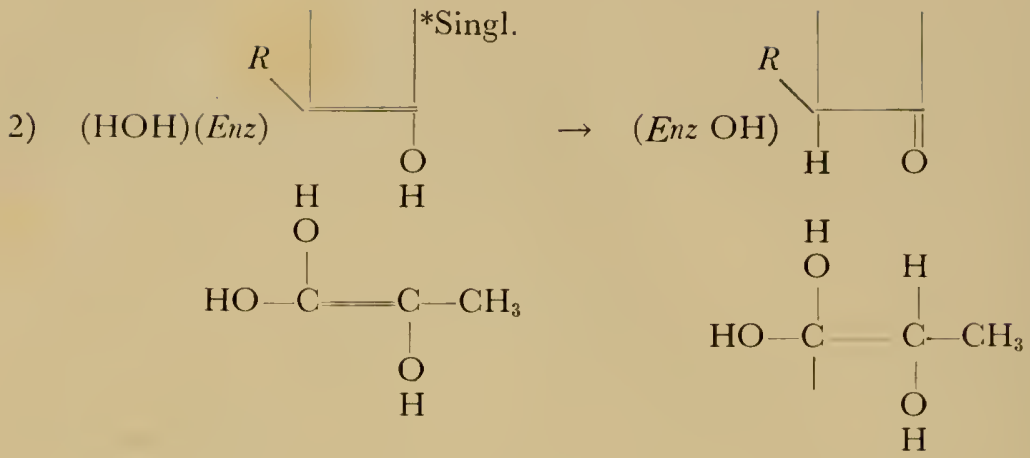

The purpose of the first photochemical reaction (represented by the chemical symbols of the upper half of the figure) is to store temporarily chemicat energy at the cost of the metastable state of chlorophyll- $a$. The reaction is supposed to be a simultaneous keto-enol transition in the fifth ring of chlorophyll-a and in the photosynthetic oxiclant. In the diagram, PGA is chosen, but other keto acids would show the same isomer transition. A complex of chlorophyll with keto acids should easily be formed by hydrogen bonds. The energy of the metastable excitation of chlorophyll should be sufficient to promote by an exchange of two hydrogen atoms the two enolization processes. The shift of the hydrogen atoms during the transfer is small. Thus, losses lyy heat of activation should be small and enough energy would be available to store about $20 \mathrm{kcal}$. If onc uses the conventional bond energies for the keto and enol forms, each of the latter should be about $10 \mathrm{kcal}$. above the energy levels of the keto forms. The symbol Enz. in the diagram stands for cytochromes which in our theory are direct neighbors of each water-exposed chlorophyll. Neither will the cytochrome be changed chemically during this reaction, nor will water be split. However, one water molecule can be formed by dehydration of the two hydroxyl groups attached to the same carbon of this acid. The sccond step is initiated by arrival of singlet excitation at the complex. Its energy, together with the $20 \mathrm{kcal}$. stored in the enol states and relcased again during the second photochemical step, represents ample cnergy for the splitting of water, connected with a transfer of an H-atom to the PGA, and of an OH to the cytochrome. Two of the radicals formed by addition of one hydrogen atom to PGA, are supposed to dismutate into one phosphotriose molccule and one PGA. The dismutation reaction is exothemic to an amount of ca. 40 kcal. The energy liberated can be used for $\Lambda \mathrm{TP}$ formation from $\Lambda \mathrm{DP}$ and phosphate, if the dismutation occurs on a surface of the enzymatic system promoting phosphorylation. This type of phosphorylation is probably the one which Dr. Arnon calls primary phosphorylation comnceted with the reduction process. This picture fits in with all the observations on fluorescence and quencling and so on. I believe it is also not in contradiction to any 
biochemical results gained with the method of radioactive tagging. I have the highest regard for Dr. Calvin's elucidation of the pathway of carbon in photosynthesis. but physical evidence seems to me not in accordance with this hypothesis that light is used in photosynthesis only for photoionization. Even the problem of the quanum yield of photoionization is by 10 means solved.

Dr. Calvix: May I answer l'rofessor Franck? The quantum yield on this for carrier production is one.

Dr. Franck: How do you know?

Dr. CALvin: I measured it.

Dr. Franck: How?

Dr. Calvin: By electron spin and by conductivity, both. The two things together.

Dr. Franck: In the photosynthetic apparatus of the chloroplasts?

Dr. CalviN: No. I am not talking about chloroplasts. I am talking about this system.

Dr. Franck: Well, I must say this is a much better system than the less ordered chlorophyll layers of the chloroplasts. How great in the chloroplasts is the ratio of the concentrations of quinone and chlorophylls?

Dr. Calvix: Dr. Franck, may I paraphrase your remarks? Dr. Franck doesn't believe at the moment there is enough quinone, that is, enough electronic acceptor present in the chloroplast. I don't know the exact number. Do you, Dr. Bishop?

DR. Bishop: Yes, approximately $12 \%$ of the chlorophyll on a molar basis, and that's a minimum.

Dr. Franck: That's disagreeable.

Dr. CalviN: That's a disagreeable minimum?

Dr. Franck: Yes.

Dr. Calvin: No, that's quite all right as far as I am concerned. You don't need very many, you know. There will be one quinone for a number of chlorophylls. Some of these chlorophylls, as you yourself have said, are not in active position.

DR. FRANCK: But if certain chlorophyll molecules are not in contact with quinone, how will the quantum absorbed by them be used?

Dr. Calvin: Resonance transfer to the place where they are.

DR. FRANcK: . Mh, resonance transfer. Once you accept it, I see no need to introduce the concept of electron transfer in photosynthesis.

Dr. Calvin: That is precisely what we do need. Eventually there must be an electron transfer to the quinones to produce a reducing agent. I believe that a charge migration with little or no temperature coefficient is the best way to separate the high-energy products of the quantum conversion and reduce non-productive back reaction to a minimum.

I sincerely regret that Dr. Franck has had to offer his criticism of this proposal without having had the opportunity to hear it presented with the evidence. Fortunately the manuscript, which presumably will appear in 
connection with this discussion, will have in it the last third of the talk which this audience had no opportunity to hear. Perhaps the readers will thus be better equipped to judge the importance and the value of the notion that a charge migration through a pi-system of electrons involving several if not many molecules is the essential feature required for the separation of the high-energy products of the primary quantum conversion act in photosynthesis. In this sense, and in this sense only, do we invoke some of the notions of solid state electronic (hole) conductivity, a phenomenon which is now well established for the type of organic molecules available in the chloroplasts.

Dr. Rabinowitch: Dr. Calvin is right when he objects to the treatment of photosynthesis as a homogeneous reaction; the structure is important as he and many others have pointed out for a long time. (I have called photosynthesis a "topochemical reaction" since 1940.) I think that one should be careful in going to the other extreme and saying that since photosynthesis is not a reaction in a homogeneous solution, it must be a reaction in a solidmeaning a crystalline solid, because the only really solid systems are crystals. One can, of course, interpret any intermediate situation by starting from the one or from the other end; and it is useful to explore how far one can get in a case like that of photosynthesis, by starting from the phenomena in the crystalline state. However, I think that if one looks at what we know about the real structure of chloroplasts and the distribution of chlorophyll in it, one is inclined to see it as rather far from a true crystal. The monomolecular layers of chlorophyll supposed to exist in the chloroplasts, areas witnessed by their absorption spectra-not two-dimensional crystals. In two-dimensional crystalline chlorophyll monolayers, the absorption band is shifted to $740 \mathrm{~m} \mu$ as against $675-680 \mathrm{~m} \mu$ in the chloroplasts. (It lies at $670 \mathrm{~m} \mu$ in non-crystalline colloids.) If a solicl state were realized, and excitation were to throw electrons out of a molecule either into the crystalline interspace, or outside, where they can join some electron acceptor, the absorption spectrum of chlorophyll in vivo would differ from that of chlorophyll in molecular dispersion much more strongly than it actually does. The band of chlorophyll in the living state is rather similar to the absorption band of chlorophyll in molecular solution. The arrangement of chlorophyll molecules in the chloroplasts is dense enough to permit migration of energy because this migration requires closeness only on the scale of the wavelength of visible light-which is large compared to the scale of molecular clianeters. It is not close cnough, I believe, to permit free movenent of electrons because this requires overlipping of eigenfunctions. However interesting it may be to explore the solicl state picture I think one has no reason to be too optimistic about explaining with its help what is going on in the chloroplasts.

Dr. Kastua: May l make one comment between these three comments of Calvin and Rabinowitch. Nlthough it is true that there may be electron transport between the pigments, electronic overlap would be required. On 
the other hand, the energy transfer phenomenon which insolves exciton interaction depends on three different parameters. One is common to the chlorophylls in any situation, and it is that the intensity of the alsorption band for individual molecules determines the energy of the band clisplacement in the aggregate ats well als the rate of transfer of the energy. But on the other hand, there are wo other factors, the distance between the molecules and their mutual orientation. It is possible to have in a helical arrangement rero exciton interaction (unfarorable geometry) yet with very good electron overlap. The fact that the bands shift very little is no indication that there camot be good electron overiap beciuse if the mutual transition moments were unfavorably arranged as one runs clown the aggregate, there would be zero displacement of the spectrum. Therefore, I think the question is to try to find out by some experimental method whether it is the clistance between chlorophylls or whether it is the orientation of adjacent chlorophylls which is giving the relatively small spectral shift in the chloroplast itself. 


\title{
LIPID FUNCTION IN THE PHOTOSYNTHETIC STRUC'TURE
}

\author{
A. A. Benson \\ Department of Agricultural and Biological Chemistry \\ The Pennsylvania State University \\ University Park, Pennsylvania
}

Lipids constitute about 30 per cent of the dry weight of the chloroplast. Their high degree of orientation has been revealed by determination (6) of birefringence by dye molecules absorbed in the lipid regions. Chlorin orientation as a result of absorption of the phytol rest of the chlorophyll molecule in lipid media is generally accepted. Most concepts of chloroplast structure and function embrace these considerations.

The major fraction of the lipids within the chloroplast are surfactant molecules concentrated within the grana. Rather little neutral triglycerides are observed. The two galactolipids (Fig. I) predominate in the chloroplasts $(2,12)$ and in the chromatophores of photosynthetic bacteria. They differ from most lipids in that they lave but one fatty acid. The most important of the ionic surfactants are the phosphatidyl glycerols (Fig. 2) and sulfodeoxyglycosyl monoglyceride (Fig. 1), the plant sulfolipid. It is proposed that these neutral and anionic surfactants stabilize the large interfacial areas between pigmented and protein phases of the lamellae. Their adsorption at the interfaces would provide the physical medium necessary to account for the observed structural and optical properties. In addition, laminae of cationic or anionic functional groups would allow a variety of suggestions for their function in the electrontransfer reactions of photosynthesis.

\section{Light-Dependent Lipid Metabolism}

\section{The Phospholipids}

Phosphatidyl glycerol is the predominant phosphatide in the photosynthetic structure and is by far the most actively metabolized (8). Observations by Miller in this laboratory revealed the active meta- 


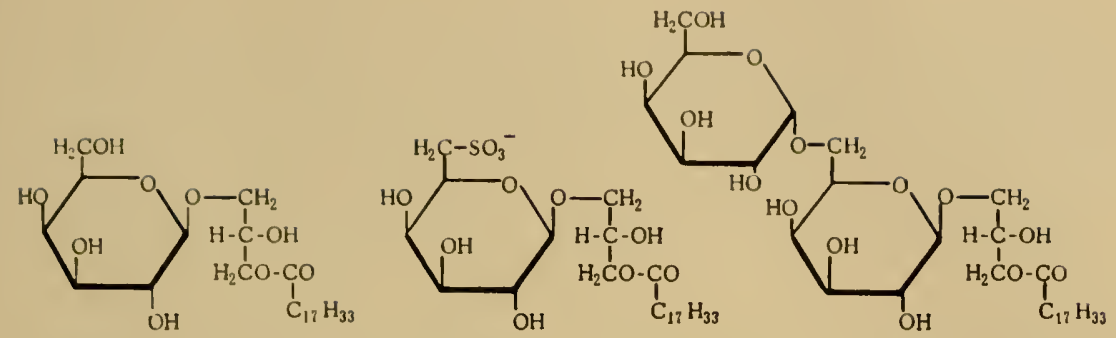

l:ig. 1. The chloroplast glycolipids, $\beta$-1)-galactopyranosyl monoglyceride, sulfodeoxyhexopyranosyl monoglyceride (structure not completely established), and $\alpha$-1)-galactopyranosy $(1 \rightarrow 6) \beta$-b-galactopyranosyl monoglyceride.

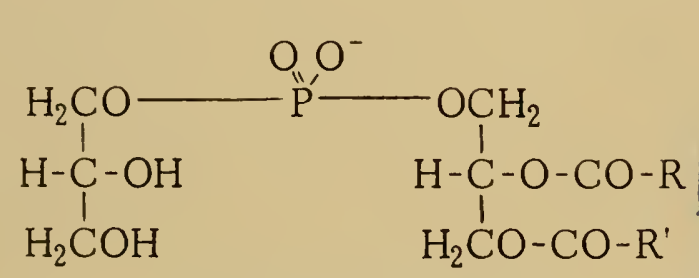

Fig. 2. Phosphatidyl glycerol.
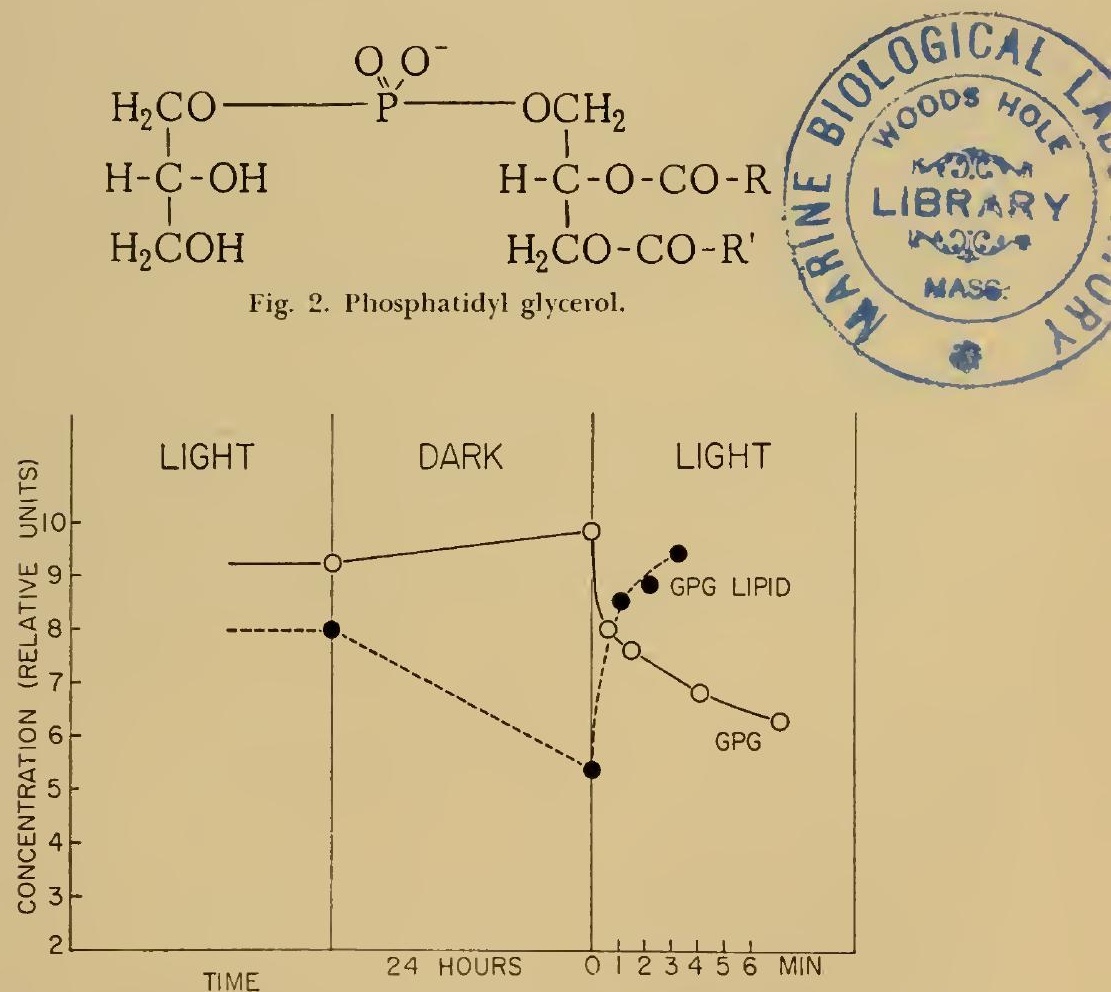

Fig. 3. Effect of illumination upon concentrations of diglycerophosphate (GP'G) and phosphatidyl glycerol (GPG lipid) in Chlorella.

bolism of diglycerophosphate in Chlorella, even before its chemical identification. Algae grown and harvested at high light intensity contained small amounts of the diester, while cells cultured at low in- 
tensities had concentrations exceeding those of other phosphorylated metabolites by a factor of ten. More recent observations revealed an inverse relationship between free diglycerophosphate and its esterified derivative, phosphatidyl glycerol. The concentration changes shown in Fig. 3 occur within two or three minutes of illumination.

The total concentration of glycerophosphate derivatives in Chlorella approaches $10^{-2}$ molar. This is largely within the chloroplast, and the concentration there may be even higher. Metabolism on this scale at this speed suggests that the nature of the lipid phase within the lamellae may be changing upon illumination. Since glycerophosphate oxidation can provide one mole of DPNH and one of triose phosphate, one may interpret the observed "reducing power" accumulation upon preillumination (10) and consequent enhanced $\mathrm{C}^{14} \mathrm{O}_{2}$ fixation in the dark. The reservoirs of glycerophosphate could well provide this capacity, and further, the kinetics of the changes described by Fig. 3 are of the same order as those observed for decay of "reducing power" in Chlorella. Our observation that the concentration of DPN exceeds that of TPN in the chloroplasts may be associated with this metabolic transformation.

\section{The Glycolipids}

The galactolipicls comprise about two-thirds of chloroplast lipids. They are even more rapidly labeled than phosphatidyl glycerol during photosynthesis in $\mathrm{C}^{14} \mathrm{O}_{2}(1,2,4)$. The galactose moieties possessed most of the newly incorporated $\mathrm{C}^{14}$ while the activity in the monoglyceride accumulated slowly. It is an obvious possibility, therefore, that the galactolipids are localized at the phase bounclaries adjacent to the transglycosylating enzymes of carbohydrate synthesis. Their galactose is in surprisingly rapid equilibrium with the nucleotide diphosphogalactose pool observed by Buchanan et al. (3).

\section{Plant Mitochondria}

The observed predominance of the galactolipicts in photosynthetic tissues and Wintermans' detailed study of chloroplast lipids (12) suggested that these compounds are concentrated in the photosynthetic structure. The lipids of corn coleoptile mitochondria were examined for comparison and found quite devoid of galactosyl monoglycerides. The predominant surfactant lipid was phosphatidyl glycerol (ca. $80 \%$ in this group). The mitochondria of animal tissues, on the other hand, contain phosphatidyl esters of choline, ethanolamine, and inositol with 10-15\% of diphosphaticlyl glycerol. 


\section{Location of Pigments in THE Lamellae}

Since the galactosyl monoglycerides ${ }^{1}$ are situated within the lamellar structure, their interfacial orientation would place the galactose moieties in contact with the stroma and the fatty acids in a lipid layer adjacent to the protein-rich phase visualized by Hodge, McLean, and Mercer $(\bar{T})$. There must be a vigorous competition between the chlorophyll and the surfactant lipids for adsorption at the interface. Then, either the chlorin groups are situated in the same layer as the galactose or in an "inner" interface of the granum which is not in contact with the stroma. The close packing of the chlorophyll, as cstimated by Rabinowitch (9) and others (II), would hardly allow appreciable space for interfacial adsorption of surfactant lipids which far exceed its concentration. It would, therefore, appear reasonable to anticipate two types of lipid laminae, an outer one dominated by galactolipids in the region of carbohydrate synthesis and an inner one dominated by chlorophyll and electron transport systems in the region of oxygen production. Gas diffusion from the inner region of the granum would be more plausible than would be transport of carbohydrate. Such a picture would be in accord with the "semiconductor" concept of granum function.

Recent observations by French (5) indicate the existence of several forms of chlorophyll $a$ in plants. Franck, earlier, suggested that the clifferences in observed fluorescence properties of chlorophyll $a$ were due to variations in the medium surrounding the pigment. It now appears reasonable to consider that heterogeneous distribution of surfactants may provide the pigment molecules with media of widely differing polarity.

\section{SUMMARY}

The high lipid content of the chloroplast is associated with the lamellar pigmented structures. The major chloroplast lipids are the galactosyl monoglycerides and the phosphatidyl glycerols, both of which are actively involved in the carbohydrate metabolism of photosynthesis. The rapid turnover of the galactose and glycerophosphate moieties suggests that these surfactant lipids are oriented in the

\footnotetext{
${ }^{1}$ New evidence indicates that the glycolipids are, in fact, glycosyl diglycerides rather than glycosyl monoglycerides as reported. C"-labeling of both fatty acids is slower than anticipated and only about 18 units of $\mathrm{C}^{14}$ are readily incorporated into these moieties. "Lyso"-glycolipids result from controlled enzymatic degradation, thereby indicating that each glycerol bears two fatty acid ester groups as in the glycerol phosphatides.
} 
external surfaces of the grana and are in contact with the stroma of the chloroplast containing the enzyme systems of carbohydrate metabolism. Being surfactant and in high concentration, these lipids may leave insufficient interfacial area for uniform chlorophyll adsorption. Competition for interface adsorption could lead to domains of varied polarity surrounding the groups of associated chlorophyll molecules.

\section{Acknowledganents}

The author is inclebted to the efforts of his collaborators, Drs. J. F. G. M. Wintermans, J. A. Miller, and R. A. Ferrari for information presented in this paper. Corn coleoptile mitochondria were kindly provided by Dr. H. W. Siegelman. This work was supported by the Atomic Energy Commission, the National Science Foundation, and the Pennsylvania Agricultural Experiment Station.

\section{REFERENCES}

1. Benson, A. A., Wintermans, J.F.G.M., and Wiser, R., Plant Physiol., 34, 315-317 (1959).

2. Benson, A. A., Wiser, R., Ferrari, R. A., and Miller, J. A., J. Am. Chem. Soc., 80, 4740 (1958).

3. Buchanan, J. G., Lynch, V. H., Benson, A. A., Bradley, D. F., and Calvin, M., J. Biol. Chem., 203, 935 (1953).

4. Ferrari, R. A., and Benson, A. A., unpub.

5. French, C. S., Brookhaven Symposia in Biol., 1I, 305-309 (1959).

6. Goedheer, J. C., "Optical properties and in vivo orientation of photosynthetic pigments." Dissertation, Utrecht (1957).

7. Hodge, A. J., McLean, J. D., and Mercer, F. V., J. Biophys. Biochem. Cytol., 6 , 605-614 (1955).

8. Miller, J. A., and Benson, A. A., unpub.

9. Rabinowitch, E., Discussions Faraday Soc., 27, 161-172 (1959).

10. Tamiya, H., Miyachi, S., and Hirokawa, T., "Some New Preillumination Experiments with Carbon-14," in Research in Photosynthesis (H. Gaffron, ed.), p. 213-223. Interscience Publishers, New York (1957).

11. Thomas, J. B., and Nuboer, J. F. W., J. Phys. Chem., 63, 39-44 (1959).

12. Wintermans, J.F.G.M., unpub. 


\title{
SPECTRAL CHANGES IN PHOTOSYNTHESIS ${ }^{1}$
}

\author{
Bessel KoK ANd George Hoci \\ Photosynthesis Group, \\ Research Institute for Advanced Studies \\ Baltimore, Maryland
}

\section{Energy Transfer in Photosynthesis}

It is well known that in photosynthesis the number of sensitizing pigment molecules is far in excess of the number of catalytic chains which process the photochemical products. In order to absorb most of the light falling onto it, the plant requires a high pigment concentration. But even in full sunlight each chlorophyll molecule receives a quantum only a few times per second-a rate which is several fold lower than the turnover rate of most metabolic reactions. However, a mechanism is provided whereby all quanta received by a large. number of pigment molecules are fumneled into a single reaction center. Flashing light measurements $(7,12)$ indicate that this number (often called the photosynthetic unit) amounts to between 300 and 500 pigment molecules.

In an array of closely spaced pigment molecules, such as we find in the chloroplast, the quanta received by the various absorbers have been shown to flow towards the longest wavelength absorption band: in aerobic photosynthetic organisms this is the red $(675 \mathrm{~m} \mu)$ peak of chlorophyll $a$. In turn, a very small concentration of a pigment absorbing slightly beyond $675 \mathrm{~m}_{\mu}$ would suffice to trap efficiently all energy conveyed to its neighboring chlorophyll a molecules. It therefore could function as the focal point of the light-collecting step, and be the link between photochemistry and dark metabolism. The first evidence for the existence of such a pigment was obtained in fluorescence studies with red and blue-green algae by Duysens (3). He observed that the fluorescence of chlorophyll $a$ was excited to a larger extent via accessory pigments (phycobilins) than by chlorophyll $a$ itself. This was explained by assuming two fractions of

${ }^{1}$ The reported work was supported in part by grant No. 6692 of the National Institute of Health. 
chlorophyll $a$ : one intimately connected to accessory pigment, activating photosynthesis (cf. next section), and free to fluoresce; the other connected to a quenching pigment, the concentration of which is so small that its presence is indicated only by a weak fluorescence at longer wavelengths than that of chlorophyll $a$ emission.

In summary, kinetic and fluorescence data indicate that the key intermediate between the light and the dark steps in photosynthesis might be a pigment possessing an absorption band in the far red.

\section{Sensitization of Photosynthesis}

The ubiquitous presence of chlorophyll $a$ in aerobic photosynthesis has designated it as the primary pigment in this process. Occurring with chlorophyll $a$ are always accessory pigments, such as carotenoids, chlorophyll $b$ in green plants, and phycobilins in other plants. The occurrence of two pigments with such similar absorption spectra as chlorophyll $a$ and $b$ in the green plant has long been a mystery. Since the red absorption band of chlorophyll $b$ lies at shorter wavelengths than the corresponding band of chlorophyll $a$, energy transfer from $b$ to $a$ is possible (and has been shown to occur). Because of a lack of evidence to the contrary, the accessory pigments have been assigned the passive role of gathering light energy and transferring it to chlorophyll $a$. In this picture they have no specific function in energy conversion reactions.

However, in recent years abundant evidence has come to light to indicate that sensitization of aerobic photosynthesis is more complex. Haxo and Blinks (9) found that in red algae the phycobilins were more efficient sensitizers than chlorophyll $a$. This phenomenon can be correlated with the earlier observation of Emerson and Lewis (8) that the quantum yield of green plant photosynthesis starts to decline beyond $680 \mathrm{~m} \mu$, in the middle of the chlorophyll $a$ absorption band (and at the long wavelength limit of absorption by chlorophyll $b$, the accessory pigment of green plants).

Emerson and his coworkers (6) later observed that the long wavelength limit of photosynthesis can be extended (i.e., the apparent efficiency of chlorophyll $a$ can be increased) by simultaneous illumination absorbed by an accessory pigment. It was shown that this so-called enhancement effect was sensitized by chlorophyll $b$ in green plants and a small fraction of the chlorophyll $a$ (cf. also Blinks (I) and Myers (18)), by fucoxanthol in brown algae, and by phycobilins in blue and red algae.

Various workers seem to agree that the rate of photosynthesis 
sensitized by $700 m_{\mu}$ light may be increased about twice by addition of supplemental light. This maximum enhancement requires an intensity of supplemental light which by itself gives about twice the rate of photosynthesis produced by the $700 \mathrm{~m} m$ light alone. Dr. French will discuss these matters in more detail.

Figs. 1 and 2 show some clata we have obtained with the bluegreen alga Anacystis nidulans and may serve to illustrate the enhancement. The action spectrum (Fig. I) clearly shows the relative inefficiency in oxygen evolution and $\mathrm{CO}_{2}$, uptake of chlorophyll $a$ if it alone is excited. The rate of oxygen production mediated by chlorophyll a $(700 \mathrm{~m} \mu)$ is increased by a simultaneous excitation of phycocyanin $(638 m \mu)$. Fig. 2 plots the magnitude of the enhancement versus the ratio of rates induced by the two beams separately.

Thus, together with the observations discussed in the previous section, these data lead to a rather embarrassing picture. Chlorophyll a-the most abundant and the only universally occurring pigmentis unable to sensitize photosynthesis efficiently (and to fluoresce) un-

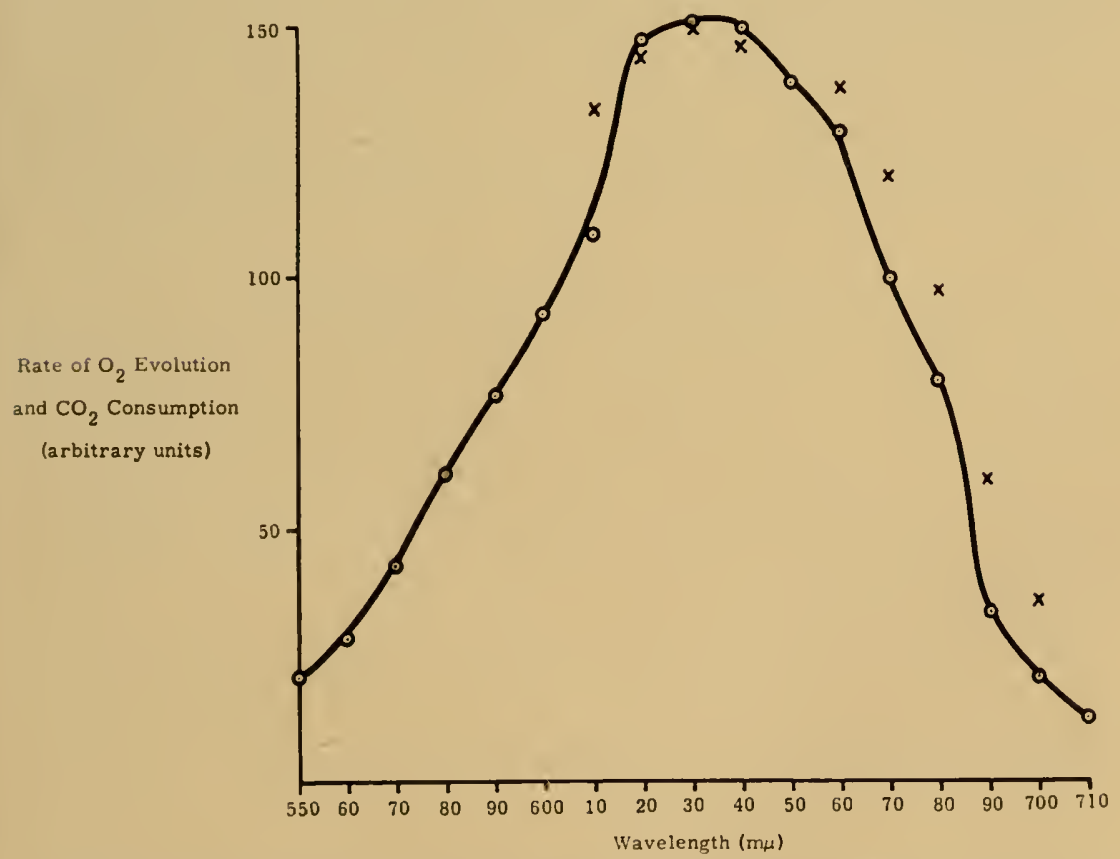

Fig. I. Aclion spectrum of photosynthesis by Anacystis nidulans. Rate of gas uptake or evolution measured with a mass spectrometer. Circles, oxygen evolulion crosses; $\mathrm{CO}_{2}$ consumption. 


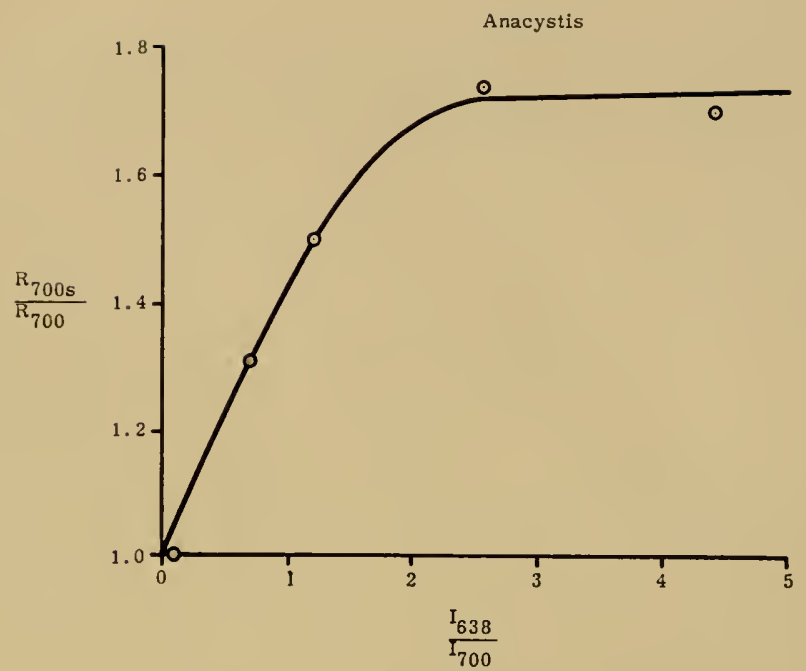

Fig. 2. "Enhancement effect" in Anacystis nidulans. Calculated from rates of oxygen evolution. Abcissa: ratio of rates induced by the individual beams $\left(I_{700}\right.$ constant throughout). Ordinate: $R_{\text {roms }}$ equals total rate with both beams concurrently minus the rate in $I_{638}$ separately. $R_{\text {roo }}$ equals the rate in $I_{700}$ alone.

less it is excited via transfer from some accessory pigment, or is assisted in some indirect way as in the enhancement experiment.

\section{Light Minus Dark Difference Spectra}

A powerful approach toward understanding the effects brought about by illumination of photosynthetic material-first applied by Duysens (3) -is the application of difference spectroscopy.

A rather obvious question to ask is the following: do one or more of the sensitizing dyes or the pigment-electron carriers undergo a change of absorption spectrum as a direct or indirect consequence of the photochemical act?

Although, in algae and chloroplasts, chlorophyll may amount to 4 per cent of the total dry weight, it turns out that illumination yields only minuscule changes of absorption (as long as we disregard the nots-specific photooxidative blcaching in excessive light). Additional difficulties are encomntered in these measurements, due to the strong pigmentation of the matcrial and the fluorescence of chlorophyll, excited or altered by the actinic illumination. Various rather special techniques to study photosynthetic difference spectra have been de- 
veloped, all based on the cross-illumination of a sample with a monitoring light $\left(I_{l}\right)$ and an actinic light $\left(I_{A}\right)$.

To survey all the phenomena observed in light mimus dark spectra, especially those occurring at wavelengths shorter than $650 \mathrm{~m} \mu$, would be an undertaking in itself. Changes in nearly all known pignents: cytochromes, pyridine nucleotides, carotenoids (the latter only in green plants, brown algae, and purple sulfur bacteria) and chlorophylls, have been observed and reviewed on earlier occasions (5, 14, 20). Dr. Hill will more specifically review the possible role of cytochromes (cf. also Fig. 3). We therefore will limit our present consideration to aerobic photosynthesis and to a discussion of phenomena occurring in the far red part of the spectrum. Our own work has been largely concentrated on these, since they seemed to offer the most direct approach to the primary energy conversion step or steps, as was explained in the previous sections.

It would be too time-consuming to give a detailed account of our measurement technique $(15,16)$ or to describe the difficulties which are often confronted in obtaining very accurate data. Among the bewildering number of phenomena observable with these sensitive techniques, often a rather subjective choice must be made as to what effects are the most significant. The experiments we will present are described, or will be described, in more detail elsewhere.

\section{The Direct Photochemical Bleaching of an Absorption at $700 \mathrm{M} \mu$}

The difference spectra shown in Fig. 3 (14) were obtained by comparing (at each wavelength of the detecting beam) the absorbance of a sample before and after a flash of bright white light. The specimens studied are representatives of four classes of aerobic photosynthesizers: green (Scenedesmus and spinach), brown (Nitzschia), red (Porphyra), and blue-green (Nostoc) organisms.

Except for a broad but weak band of increased absorbance at wavelengths beyond $725 \mathrm{~m}_{\mu}$ (which is found universally), all changes are negative. One can correlate the decrease at $650 \mathrm{~m} \mu$, found in green cells only (cf. also Strehler and Lynch, 21, with the disappearance of chlorophyll $b$. The small decreases around $680 \mathrm{~m}_{\mu}$ correspond to a minute bleaching of chlorophyll a (Coleman et al., 2). The most striking shift, which is found universally, occurs slightly beyond 700 $m \mu$. It appears quite prominent in blue-green and red algae, as is illustrated for the former in the difference spectrum of Fig. 4. In this experiment the only other pronounced effect occurs in the blue, where an absorption band at about $420 \mathrm{~m}_{\mu}$ shifts towards 410 


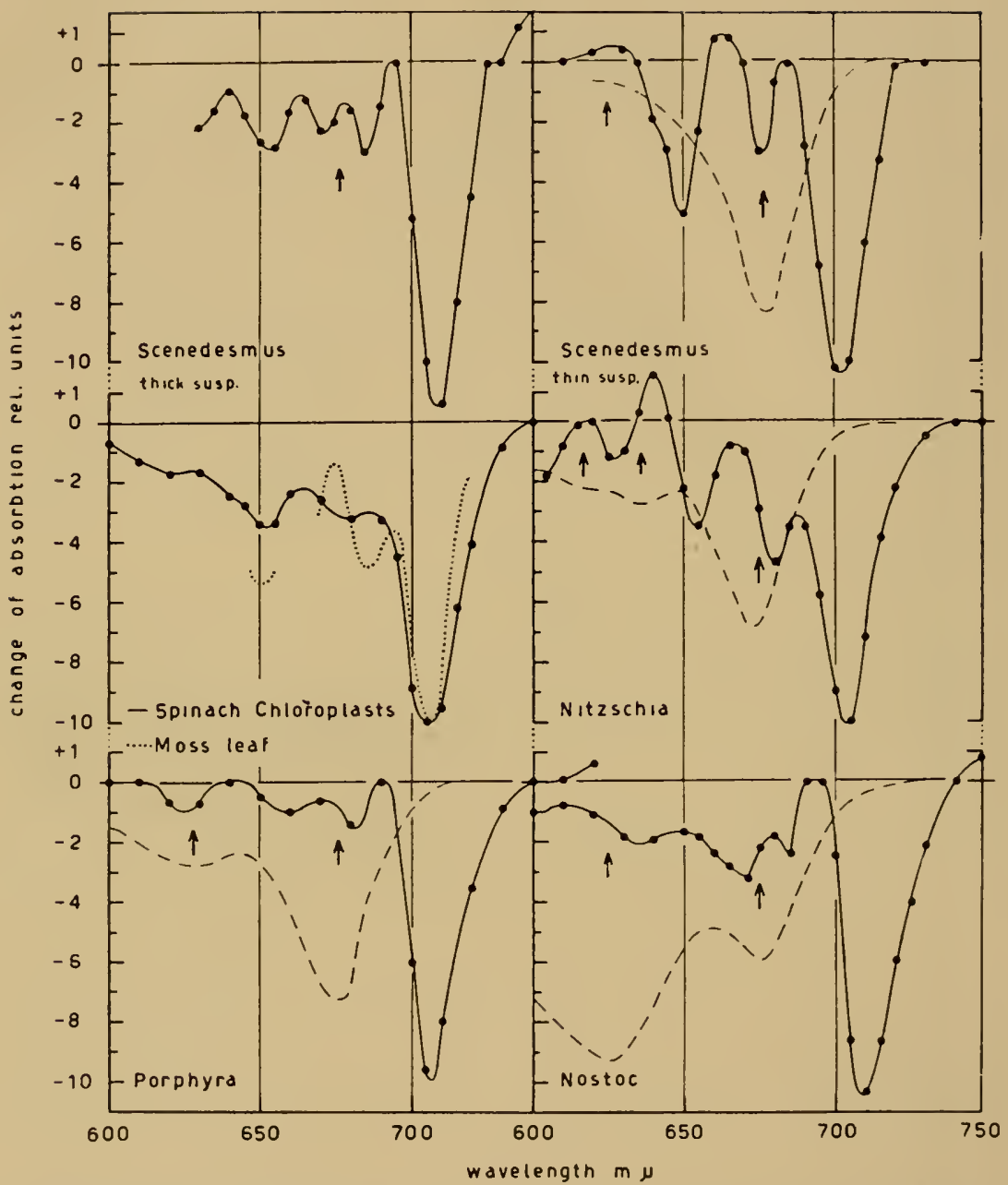

Fig. 3. Liglit-induced changes of absorption as observed in the long wavelength region with four types of acrobic plants. (Fast, reversible effects induced by flashes of white light.) For the various spectra the shifts at $705 \mathrm{~m} \mu$ are brought to about equal height. Arrows indicate absorption maxima in the absorption spectra plotsed as broken curves. (From Kok, 1.t.)

$\mathrm{m}_{\mu}$. Together with the smaller negative change at $555 \mathrm{~m} \mu$, one could interpret this as the oxidation of cytochrome $f$ by the light (14). (A similar effect was found by Duysens (4) in red algae). We have shown earlier (12) that this shift is a fast phenomenon induced by brief flashes, like the negative $700 m_{\mu}$ shift. 


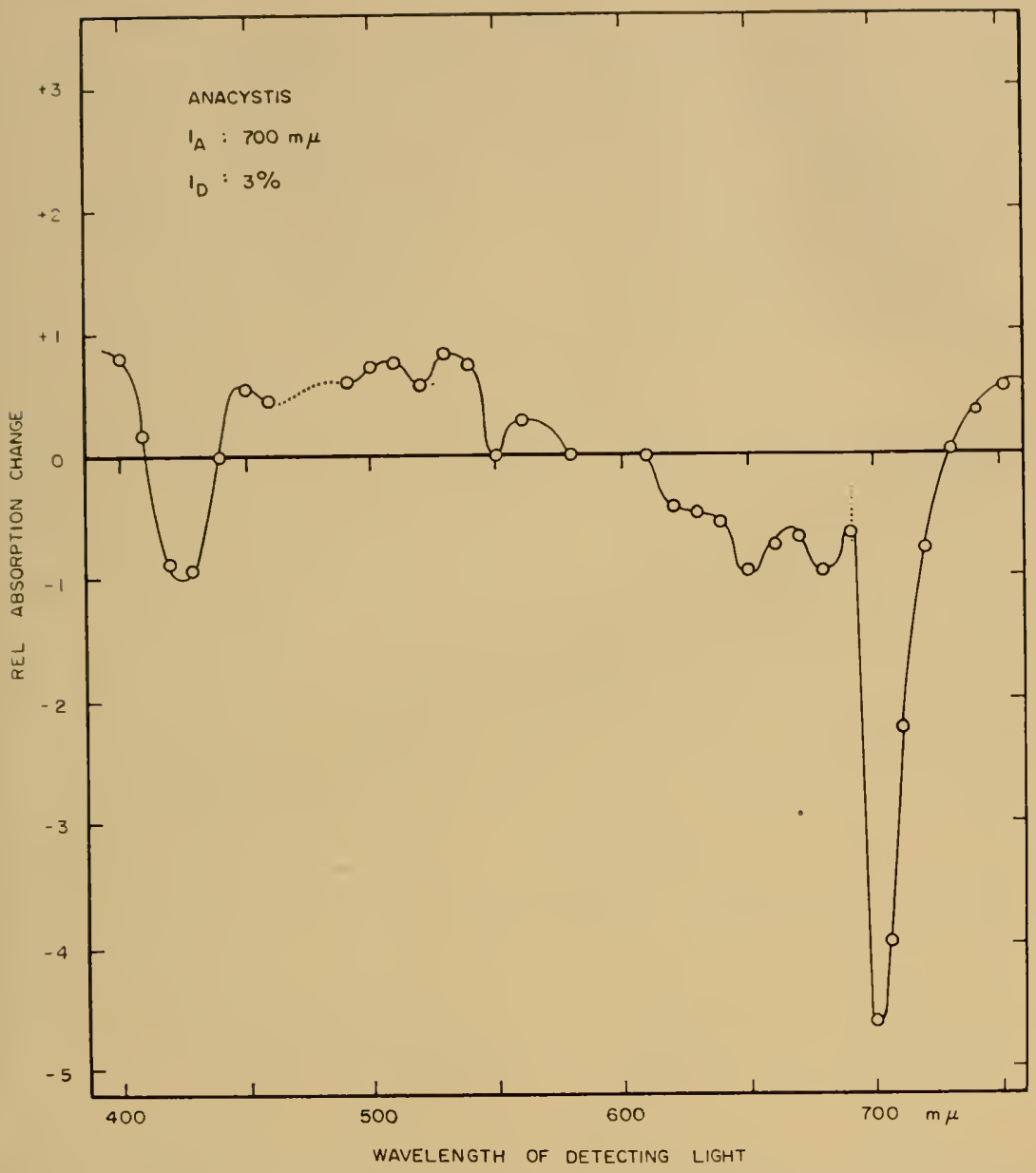

Fig. 4. Changes of absorption induced in a suspension of the blue-green alga Anacystis nidulans by far-red flashing light $(650-700 m \mu)$. Dark-time between flashes $0.01 \mathrm{sec}$ (from 17).

Most of the remainder of this discussion will be restricted to a number of observations of a pigment ("P 700 ") which is presumably responsible for the $700 \mathrm{~m} \mu$ shift. Table 1 summarizes the characteristics of the fast absorption decrease at $700 \mathrm{~m} \mu$.

An often observed asymmetry of the band (13) could be due to screening by chlorophyll $a$, but might also indicate that we are dealing with a band shift (in addition to a decreased extinction coefficient).

The fact that with even the shortest flashes we could obtain $(3 \times$ 
TABLE I

Characteristics of Negative Absorption Shift at $700 \mathrm{~m} \mu$

Wave length maximum effect Band shape

Magnitude

i.e. Ratio $\mathrm{P}_{700}$ : Chlorophyll $a$

Velocity of bleaching

Return reaction

Quantum yield of bleaching

Bleaching sensitized by $\sim 703 \mathrm{~m} \mu$

Often asymmetric

$\Delta \sim 0.01$ OD if $\mathrm{OD} 675 \simeq 4$

$\sim 1: 400$

Instantaneous: Photochemical

In dark, $\mathrm{t}_{1 / 2} \geq 5 \mathrm{msec}$

Order of unity

Chlorophyll $a$, carotenoid

$\left.10^{-t} \mathrm{sec}\right)$ bleaching was induced, as well as other evidence, indicates a direct photochemical event. During dark periods of suitable length, a restoration of the absorption occurs. As will be discussed later, the rate of this back reaction is influenced by various agents. In steady-state flashing light (dark time $\sim 60 \mathrm{msec}$.) we measured (in whole algae) a half restoration time of some 7 milliseconds.

In such a steady-state sequence of flashes and dark periods one can determine: (a) the size of the cyclic absorbance shift; (b) the number of flashes per unit time; and (c) the amount of absorbed actinic light per unit time. If we now assune that the involved pigment has a molar extinction like chlorophyll $\left(10^{5} M^{-1} \mathrm{~cm}^{-1}\right)$ then the rate of pigment turnover and the quantum yield can be calculated. Although with our present apparatus we were able to make only rough estimates (with Anacystis and relatively weak actinic light of 670 $m \mu$ ), a quantum yield of the order of unity was inclicated. This indicates that at least a substantial fraction of the light quanta absorbed by chlorophyll a contribute to the bleaching of "P 700."

Finally, we tried the effect of varying the wavelength of the activating light, that is, we determined an activation spectrum of this negative shift. It appeared that under normal conditions the effect was sensitized mainly (but hardly ever exclusively) by chlorophyll a and probably also by carotenoids (cf. Fig. 6). However, in aged or poisoned preparations, accessory pigment can also contribute considerably to this sensitization (see below).

In conclusion, these data fit the picture that "Pigment 700 " is an efficient trap, collecting excitations from surrounding chlorophyll a molecules, and undergoing a temporary bleaching each time.

\section{TuF Photoghemigally Indugen Fordation of "p 700"}

In the previous section we have dealt only with instantaneously induced, quickly reversible phenomena ("fast effect"). However, we 
have strong indications that not all available " $P$ " 700 " is necessarily involved in these cyclic changes (at least in weak intensity flashes). They represent only "wiggles" of concentration and our measurements revealed that the backgromnd level, on which they are superimposed, is not constant. This background level of "P 700" (the envelope of the wiggling curve) progressively (lecreases if a steady sequence of fashes absorbed by chlorophyll $a$ is applied. This is easily understood. If each flash bleaches more pigment than the back reaction can restore during the intermediate dark period, all available "P 700 " will disappear in the course of a few seconds.

Surprisingly, however, if the activating light excites accessory pigment, the background level of "P 700" increases (Fig. 5). This hap-

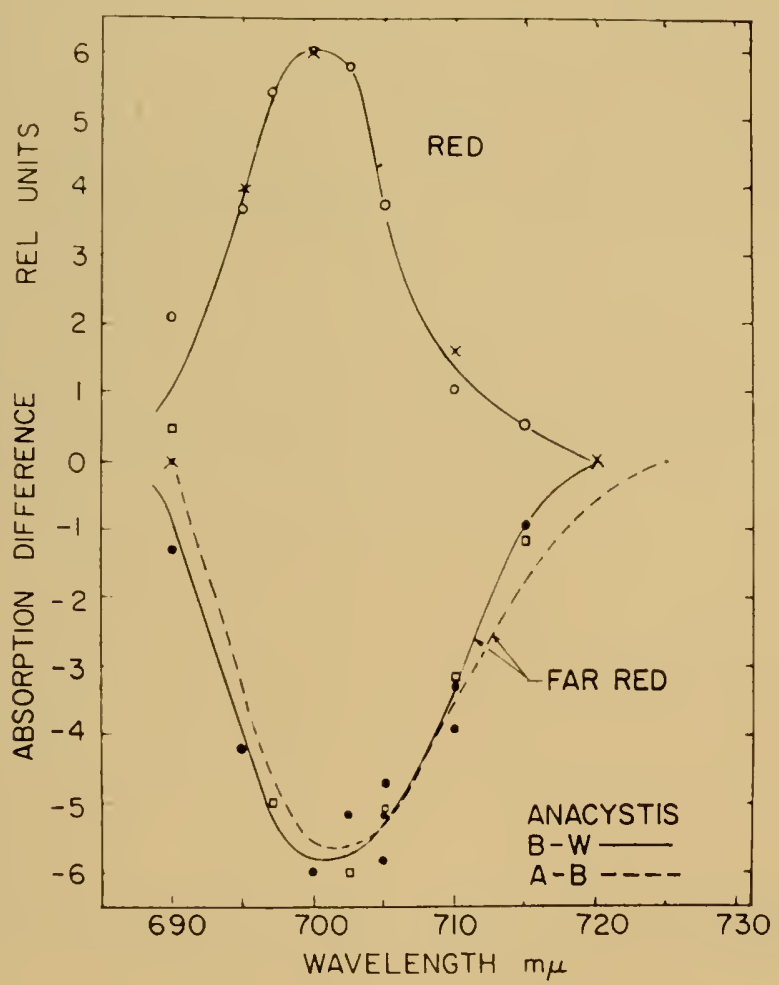

Fig. 5. Change of absorption around $700 \mathrm{~m} \mu$ incluced in Anacystis cells by either red light $(\lambda 580-6.50 \mathrm{~m} \mu$ crosses, circles) or far-red light $(\lambda>6.50 \mathrm{~m} \mu$, solid circles. squares). Both curves represent the background concentration of "P 700." The dashed curve shows the fast reversible change having negative sign only (from 16). A-B: fast photochemical bleaching. B-W: change of background absorption level. 
pens even if chlorophyll $a$ is excited simultaneously and the flashes of mixed wavelengths induced considerable direct photochemical bleaching.

The obvious conclusion is that formation of "P 700" (during the dark periods) is stimulated by light absorbed by accessory pigment, and that there occur two light reactions with opposing effects.

The experiment described in Fig. 6, and made with whole Anacystis cells, demonstrates the above phenomena. The dotted curve shows the activation spectrum of the fast absorption decrease (discussed in

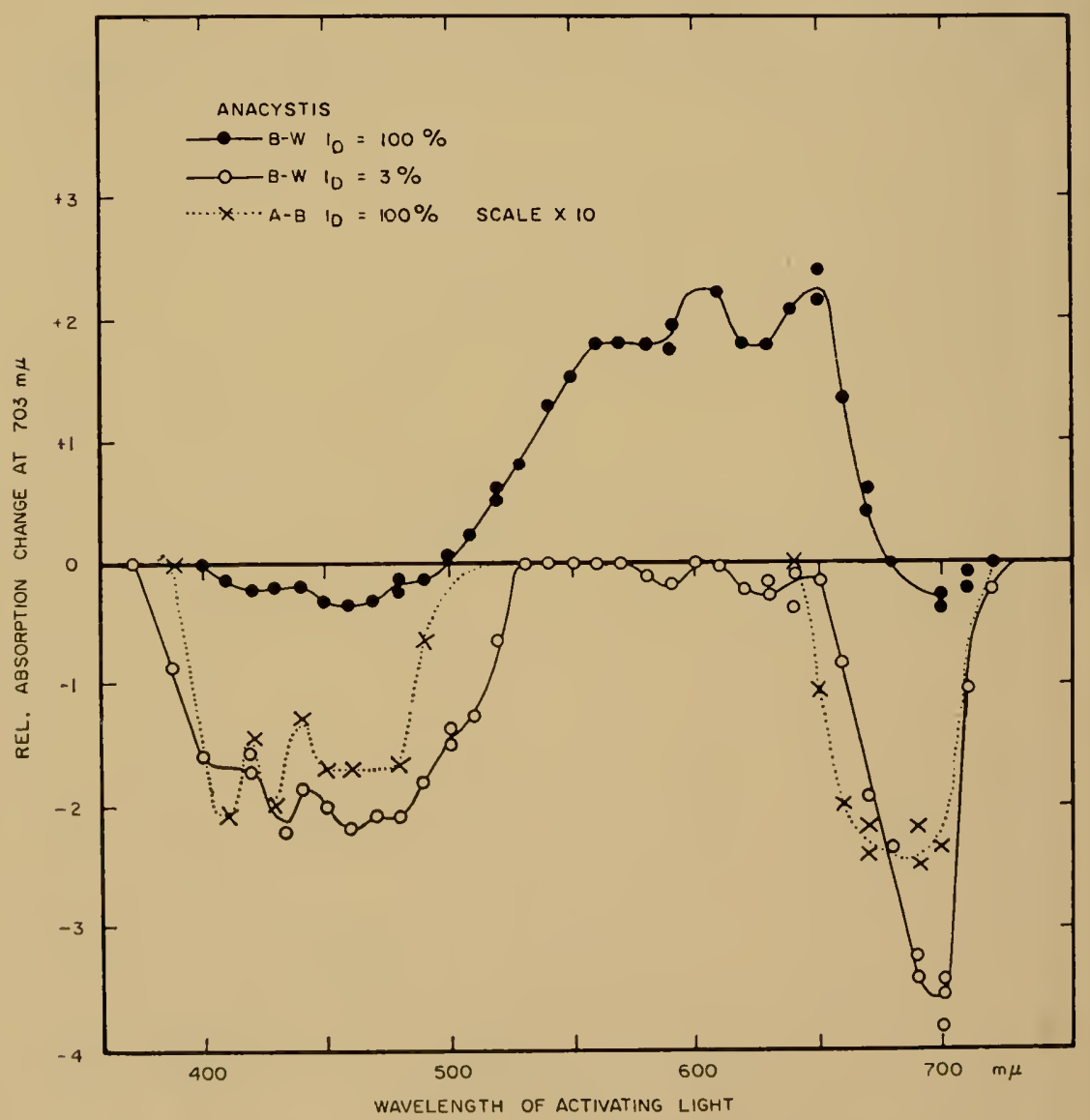

Fig. 6. Activation spectra of the $700 \mathrm{~m} \mu$ absorption shifts measured with the long datk-time arrangement and whole Anacystis cells. Open circles, background signal $I_{D} 3 \%$; solid circles, background signal $I_{D} 100 \%$; crosses, fast reversible change I, $100 \%$. A.B: fast photochemical bleaching. B-W: change of background absorption level (from 17). 
the previous section). The full curves indicate the background concentration of "p 700 " as dependent upon the wavelength of the actinic beam. The spectrum measured with strong detecting light shows an increase of "P 700 " absorption sensitized by phycocyanin (the accessory pigment), and indicates that a decrease of "P 700" concentration is induced by chlorophyll a and carotenoid. The overlapping absorption spectrum of the two pigments probably accounts for the peculiar shape of the activation spectrum around $650 m_{\mu}$.

Noteworthy in Fig. 6 is that a 30 -fold decrease of the intensity of the detecting beam results in complete disappearance of the positive phycocyanin-scnsitized effect and a strong enhancement of the negative (background) change induced by chlorophyll a. (In contrast, the fast reversible bleaching is less influenced by the intensity $I_{D}$ ) .

There is an obvious explanation for the fact that the positive effect, in order to occur at all, requires a considerable intensity of the detecting beam. This latter beam has to be of a wavelength around $700 \mathrm{~m} \mu$ (absorbed by chlorophyll $a$ and by "P 700 " directly), and therefore will itself bleach "P 700 " and thus lower its steadystate concentration. Only if this occurs to a sufficiently large extent, will the $630 \mathrm{~m} \mu$ beam find enough bleached material to convert and cause a detectable reformation of "P 700."

Conversely, the establishment of a low steady-state "P 700" concentration by a strong detecting beam will decrease the negative effect of the actinic light, since both compete for the same substrate. Fig. 6 show's that the positive change seems to be largely sensitized by phycocyanin in Anacystis. In green cells chlorophyll $b$ is the main sensitizer, but there is a defunite involvement of part of the chlorophyll $a$ (18).

\section{Is Photosynthesis Driven by Two Light Reactions?}

The observations discussed in the above sections strongly indicate the occurrence of two different light-induced reactions: the first sensitized by chlorophyll $a$ and a direct photochemical bleaching of " $P$ 700 "; the second, sensitized by accessory pigment, acting indirectly via the mediation of dark steps, and restoring "P 700."

So far we have no way to approach the quantum yield of the second step, but, as cliscussed in the beginning, photosynthetic action spectra invariably indicate a high conversion efficiency of sensitization by accessory pigment.

We also should recall that fluorescence data, if significant, indicate that accessory pigments transfer their energy efficiently to chlorophyll 
a (at least to a fraction of it). This could mean that perhaps ultimately both steps are sensitized via the two Iractions of chlorophyll $a$, but we can for the present only speculate about the possible operation of such a mechanism.

It is hard to suppose that the most abundant and the only universally occuring sensitizer-chlorophyll a-could be largely nonfunctional in photosynthesis. Therefore, rather than conceiving of it as a useless drain of light quanta, we prefer to ascribe functional significance also to the negative effect sensitized by chlorophyll $a$. An obvious solution is offered by the assumption that both steps are essential, and clo cooperate in a cyclic fashion.

Dr. Ruth Aranow derived some kinetic consequences of such a cyclic mechanism, which is depicted in Fig. 7. Light received by the absorbing state of " $\mathrm{P} 700$ " (indicated as $\mathrm{P}_{\text {red }}$-see below) leads to an excited state $\left(\mathrm{P}^{*}\right)$ and from there to the bleached form $\left(\mathrm{P}_{\mathrm{ox}}\right)$. $k_{1}$ and $k_{2}$ are rate constants for the first light step as it is activated by the detecting and the activating beam. $k_{3}$ and $k_{4}$ bear a similar relationship for the production of $\mathrm{P}_{\text {red }}$ by the second light step; $k_{f}$ and $k_{b}$ are rate constants for eventual dark reactions. These constants were inserted for complete generality and could in special cases have zero value. The derived formula show's the dependency of $\Delta I$ (the

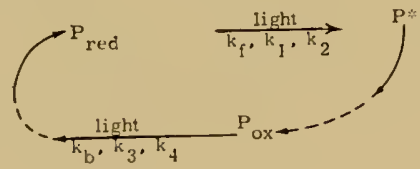

$$
\begin{aligned}
& P_{\text {red }}+P^{*}+P_{\text {ox }}=P_{\text {total }} \\
& \frac{d\left[P_{r e d}\right]}{d t}=-\left[k_{f}+k_{1} I_{D}+k_{2} A_{A}\right] \quad\left[P_{\text {red }}\right]+\left[k_{b}+k_{3} I_{D}+k_{4}{ }^{1} A\right]\left[P_{o x}\right] \\
& =0 \text { steady state } \\
& \text { 1f: } \mathrm{P}_{\text {total }} \cdots \mathrm{s}_{\mathrm{i}} \mathrm{P}_{\mathrm{i}}^{*} \text {, then: } \\
& {\left[P^{\prime}, d\right]=\frac{\left(k_{b}+k_{3}{ }^{1} D+k_{4}{ }^{1} A\right) P_{t o t}}{\left(k_{f}+k_{b}\right)+\left(k_{1}+k_{3}\right) I D+\left(k_{2}+k_{4}\right) I_{A}}} \\
& \Delta I=I_{D} k P_{\text {total }}\left[\frac{k_{b}+k_{3} I_{D}+k_{4} A_{A}}{\left(k_{f}+k_{b}\right)+\left(k_{1}+k_{3}\right) I_{D}+\left(k_{2}+k_{4}\right)_{A}^{-}} \quad-\frac{k_{b}+k_{3} I_{D}}{\left(k_{f}+k_{b}\right)+\left(k_{1}+k_{3}\right) I_{D}}\right]
\end{aligned}
$$

Fig. 7. Kinetics of a cyclic system. 
observed signal) on rate constants, light intensities, total pigment concentration $\left(\boldsymbol{P}_{\text {tot }}\right)$ and $k$, a proportionality factor correlated with Becr's law. Fig. 8, which is based on this formula, predicts the pattern of obscrvations for a variety of intensities of detecting beam (700 $m \mu$ ) and actinic beam (absorbed by accessory pigment). Actual experimental findings agree with this pattern (17). The most significant aspect is that at a certain value of the detecting intensity the activating light induces no effects.

The implication is that two light steps, and at least one dark step, must be inrolved in the behavior of "P 700."

\section{Effects of Various Agents on the Two Light Effects}

One, of course, would like to obtain more knowledge about the chemical nature of the $700 \mathrm{~m} \mu$ pigment and the reactions involved in its turnover. Table 2 show's the influence of various agents and treatments on the two absorption shifts as observed with the difference apparatus.

The negative light effect, i.e., the photochemical bleaching and spontaneous dark regeneration, appears very stable and is only slightly affected by any of the inhibitors employed. In cell-free Anacystis

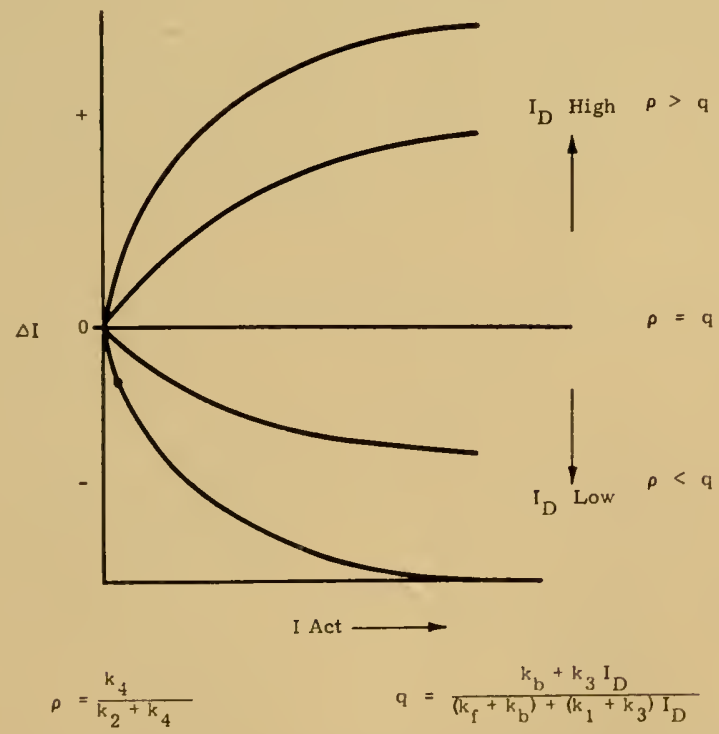

Fig 8. Expected dependence of sign and magnitude of the background absorption changes at $700 \mathrm{~m} \mu$ as a function of detecting intensities $I_{D}(700 \mathrm{~m} \mu)$ and actinic intensity $I_{A}(600 \mathrm{~m} \mu)$ in Anncystis. (Experimental data in 17). 
preparations it survives prolonged sonication, lyophilization, cold hexane extraction, probably complete removal of phycocyanin, and a partial removal of chlorophyll a-in short, any treatment which leaves a particulate chlorophyll protein complex intact. However, it slowly tends to disappear with aging and disappears much faster during dialysis. We observed that such inactive preparations could be revived by the addition of $10^{-3}$ to $10^{-4} M$ phenazine methosulfate (PMS) (and with some reducing agents, such as ferrocyanide, and palladium-hydrogen; PMS is probably reduced by endogenous substrate). Noteworthy also in table 2 is that ferricyanide, flavin adenine mononucleotide (FMN), and various dyes, often used as Hill reagents, markedly inhibit the negative shift in the concentration range $10^{-4}$ to $10^{-2} M$.

The positive light effect, on the other hand, is extremely susceptible to aging and heating and requires all the usual precautions to retain activity in chloroplast preparations.

The extreme sensitivity of the positive shift to 3 (3,4-dichlorophenyl)-l,I-dimethylurea (DCMU), the most potent known inhibitor of photosynthetic oxygen evolution (22), is illustrated in Fig. 9. The effect is reduced to zero at a concentration of $10^{-7} M$. Note that higher concentrations induce phycocyanin to sensitize the negative shift, i.e., to act exactly like chlorophyll $a$.

10-5 $M$ hydroxylamine causes complete inhibition, but no "flipover" effect is induced by higher concentrations of this poison. We may conclude from these inhibitor data that the positive shift must be intimately connected with the oxygen evolution reaction.

TABLE II

Effect of Various Agents on $700 \mathrm{~m} \mu$ Shift

\begin{tabular}{lll}
\hline & \multicolumn{1}{c}{ Positive Shift } & \multicolumn{1}{c}{ Negative Shift } \\
\hline Iodoacetate & No effect $<10^{-1} M$ & No effect $<10^{-1} M$ \\
Azide & No effect $<10^{-2} M$ & No effect $<10^{-2} M$ \\
KCN & Slight inhibition $<10^{-2} M$ & Slight stimulation $<10^{-2} M$ \\
PCMB & No cffect $<10^{-3} M$ & No cffect $<10^{-3} M$ \\
Dinitrophenol & Inhibits $\mathrm{C}_{2}^{1}=10^{-4} M$ & $\mathrm{C}>10^{-3} M$ retards back reaction \\
o-Phenanthroline & Inhibits $\mathrm{C}_{2}^{1}=10^{-4} M$ & $\mathrm{C}>10^{-3} M$ retards back reaction \\
Hydroxylamine & Inhibits $\mathrm{C}_{2}^{1}=3 \times 10^{-4} M$ & \\
DCMU & Inhibits $\mathrm{C}_{2}^{1}=10^{-7} M$ & $\mathrm{C}=10^{-4} M$ Inhibits to $50 \%$ \\
Fe $(\mathrm{CN})_{6}++$ & Inhibits & Stimulates \\
Fc $(\mathrm{CN})_{6}{ }^{+++}$ & Stimulates & Inhibits \\
PMS & Inhibits & Stimulates \\
FMN & & Inhibits \\
Dyes & Complete inhibition in 10 sec. & Inhibit \\
$100^{\circ} \mathrm{C}$ & &
\end{tabular}


We finally should draw attention to the remarkable phenomenon that in chloroplasts and broken duacystis cells PMS proved to be an inhibitor of the positive shift (as much as it stimulated the negative change) and that conversely lerricyanicle and some other Hill reagents (inhibitors of the negative change) act as stimulators.

\section{Sensitization of Photophosinorýlation in the Presence of PMS}

The striking effect that PMS exerts on the absorption shifts and the peculiar type of DCMU - insensitive "closed" photophosphorylation which is cattalyzed by PMS $(11,10)$, made us wonder what type of sensitization is involved in the latter process.

Fig. 10 show's two rate (inorganic phosphate esterified per unit time) vs. intensity curves measured with totally absorbing chloroplast suspensions. Two monochromatic lightbeams of different wavelengths were used: one at $670 \mathrm{~m} \mu$, where chlorophyll $b$ and chlorophyll $a$ both absorb, one at $710 \mathrm{~m} \mu$, where almost all incident light is absorbed by chlorophyll $a$.

These data show a better rate at the medium and high intensities,

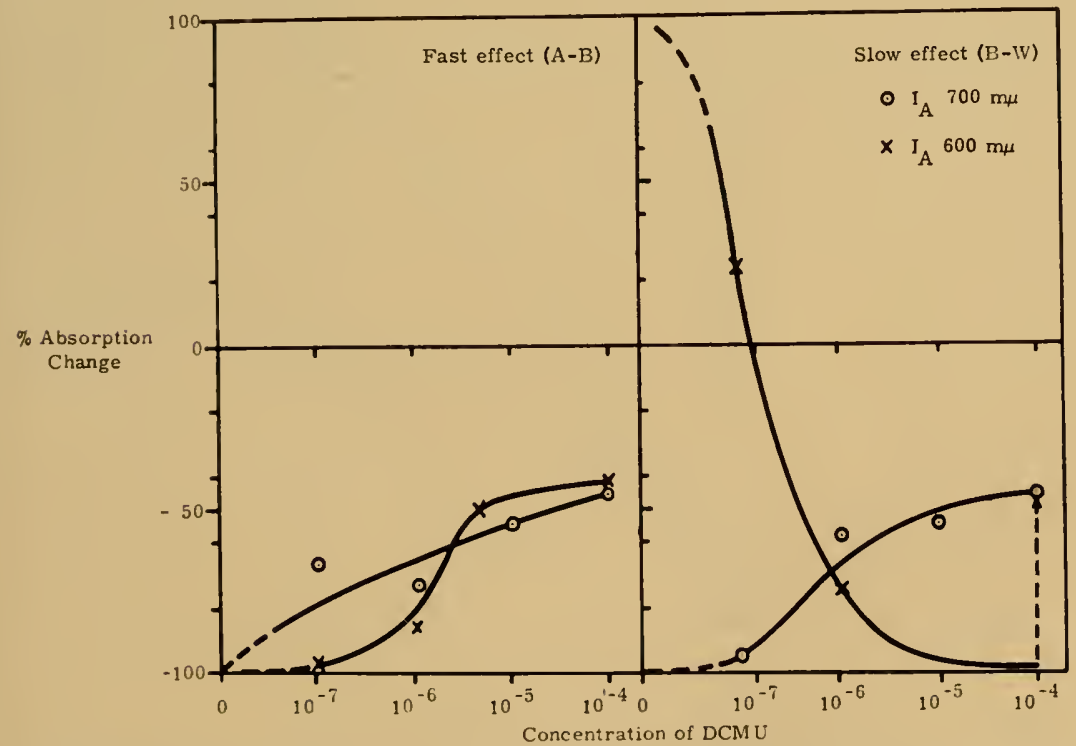

Fig. 9. Effect of elichlorophenyl dimethylurea (DCMU) upon the $700 m \mu$ absorption changes in Anacystis. Dashed arrow in lower corner indicates that in $10^{-4} M$ DC.IfU the (now negative) background change induced with $600 \mathrm{~m} \mu$ light equals the one induced by $700 \mathrm{~m} \mu$ light. 
possibly a better quantum yield at very weak ones, and different kinetics at $710 \mathrm{~m}_{\mu}$ compared to shorter wavelengths.

As shown in Fig. 11, the rates of PMS photophosphorylation, which were measured at the full intensity of Fig. 9, show a pronounced maximum at $710 \mathrm{~m}_{\mu}$. This effect is the opposite of the well-known drop of the quantum yield beyond $680 \mathrm{~m}_{\mu}$ that occurs in complete photosynthesis. Whereas in the previous section evidence was presented that the light step leading to formation of "P 700" was connected with oxygen evolution, the similar sensitization of the negative absorption shift at $700 \mathrm{~m} \mu$ and cyclic photophosphorylation indicates a correlation between these phenomena.

In this connection an initial report by Petrack (19) with cell-free Anacystis preparations is of interest. Preparations largely devoid

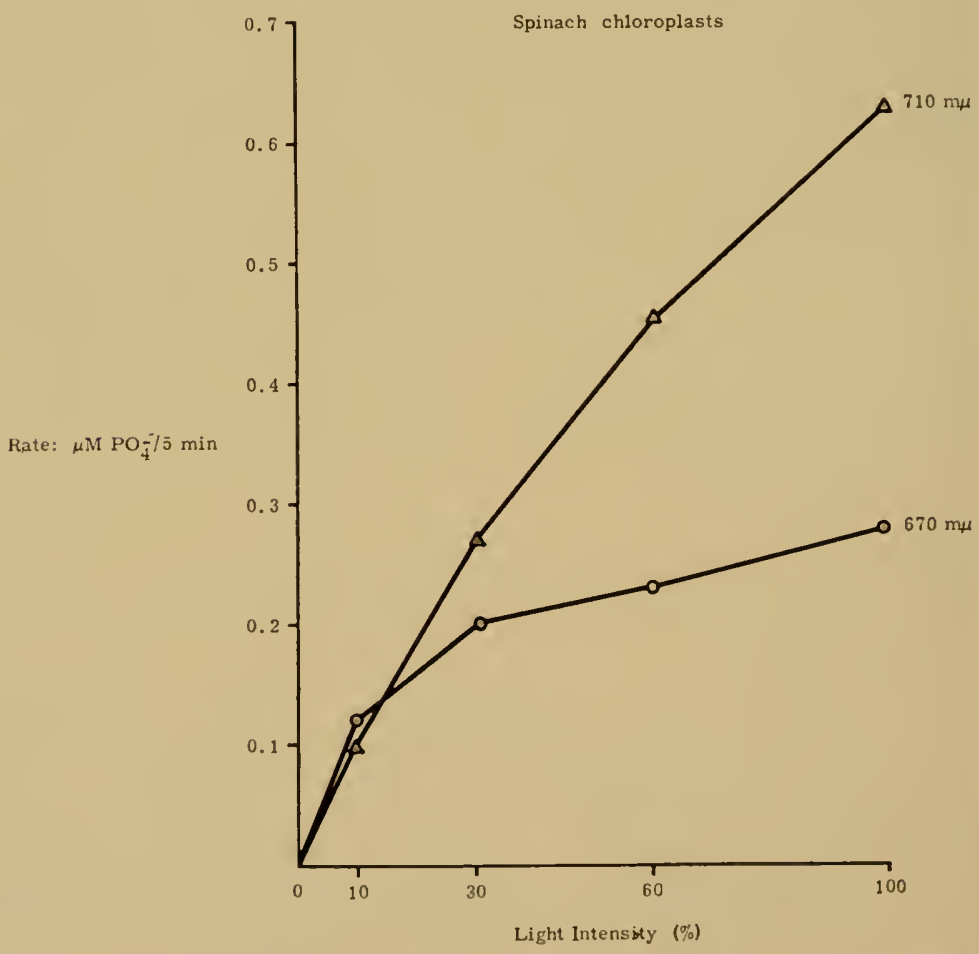

Fig. 10. Rate of photosynthetic phosphorylation is. light intensity. Flask contents: I micromole inorganic phosphate; 2 micromoles adenosine diphosphate; I micromoles $\mathrm{MgCl}_{2}$; spinach chloroplasts containing $250 \mu \mathrm{g}$ chlorophylt: 0.1 micromoles phenazine methosulphate; 5 micomoles "tris" bulTer, $p H$ 7.4 ; in a total volume of $0.3 \mathrm{ml}$. Fir c-minute reaction time; temperature, $18^{\circ} \mathrm{C}$. 
of phycocyanin were found capable of cyclic photophosphorylation. This indicates that chlorophyll a can sensitize this process by itself.

\section{Simulation of the Light Effects with Chemical Reagents}

We have tried to obtain more insight into the character of the "P 700 " shifts by attempting to reproduce the light-induced changes by chemical means. Two curettes, each containing a sample of the same chloroplast suspension, were placed in the paths of a specially constructed double beam spectrophotometer. If the two monochromatic beams and the two samples are exactly alike, a zero difference sigual will be recorded at all wavelengths. Addition of various agents to one of the curettes indeed yielded spectral shifts similar to the light-induced ones. As an example, the experiment shown in Fig. 12 reveals that under proper conditions addition of PMS results in formation of "P 700," whereas this effect can be reversed by a subsequent addition of ferricyanicle. The pigment seems to be oxidizable in the clark only by fairly strong anionic oxidants; quinone, for

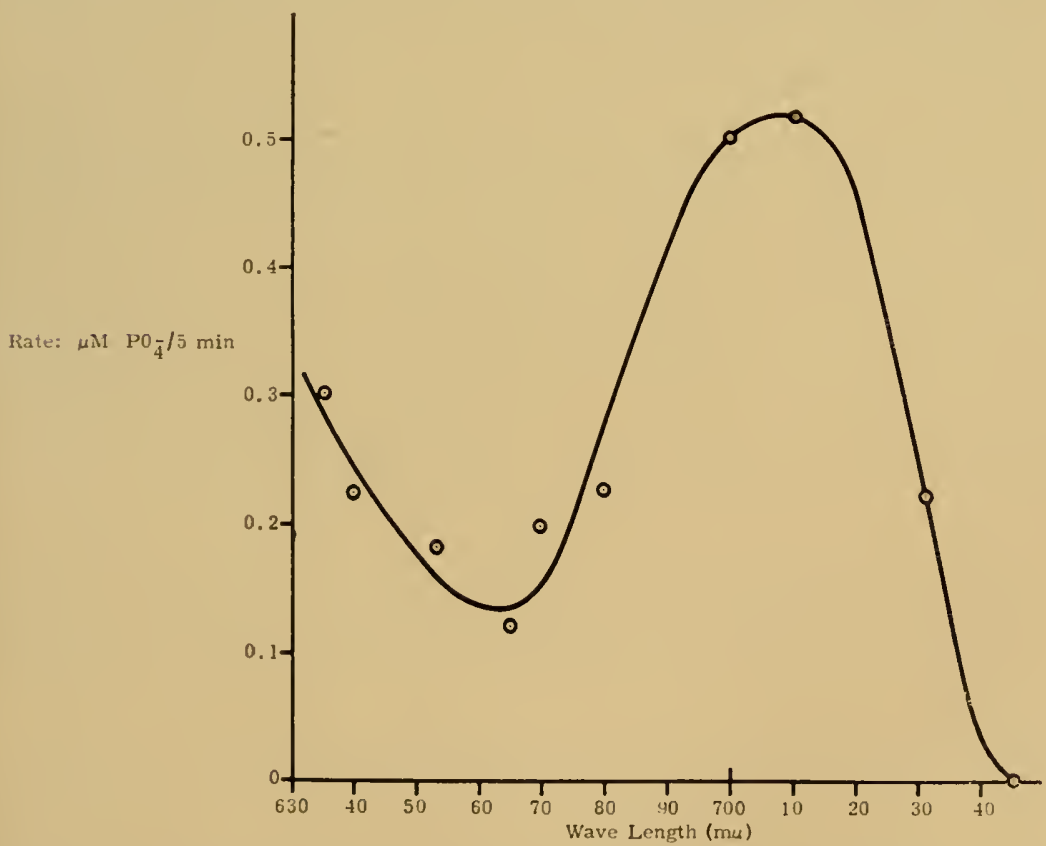

Fig. 11. Photosynthetic phosphorylation as a function of wavelength. Same experimental conditions as in Fig. 9. Lamp output equal to $100 \%$ as shown in Fig. 9. 


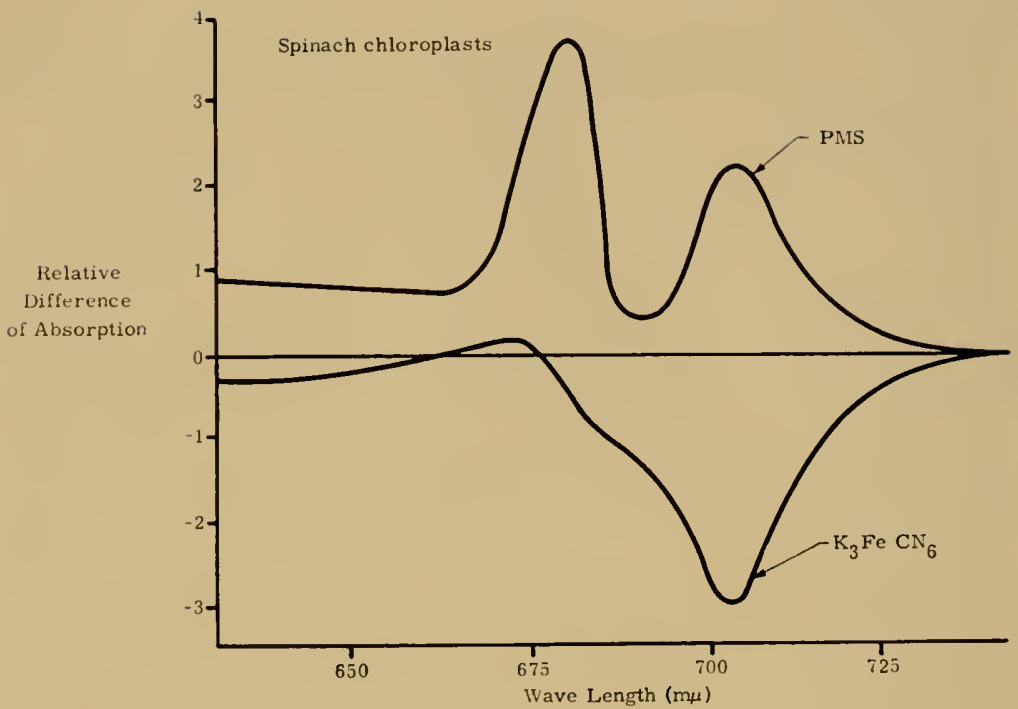

Fig. 12. Changes of absorption induced in a suspension of spinach chloroplasts by addition of either phenazine methosulfate or potassium ferricyanide. The chemically induced differences at $700 \mathrm{~m} \mu$ are of the same order of magnitude as the lightinduced changes shown in other figures.

example, was ineffective. In a few preliminary measurements of this type (using Anacystis preparations and ferrocyanide instead of PMS), it appeared that in mixtures of ferri-/ferro-cyanide the $700 \mathrm{~m} \mu$ peak is half maximal at a ratio $\mathrm{Fe}^{+++} / \mathrm{Fe}^{++} \sim 3$. Such data indicate "P 700" to be a redox system with an $E_{o}^{\prime}$ value around +0.46 volt, the colored state being the reduced one. These latter results are quite tentative, however. If confirmed, they might elucidate that the fast oxidation of cytochrome $f\left(E^{\prime}{ }_{0}+0.36\right.$ volt) by light (Fig. 4) is mediated by the light-induced oxidized form of "P 700. ."

\section{Conclusions}

The phenomena discussed in the last three sections can be satisfactorily combined so as to expand the scheme of Fig. 7 into that of Fig. 13, a working hypothesis for pigment interaction in photosynthesis.

Each of the two light steps supposedly drives an oxidoreduction reaction. In the step sensitized by chlorophyll $a$, the natural photosynthetic oxidant $X$ becomes reduced and "P 700" is oxidized. By means of the second, and more complex, light-induced step, "P 700" is regenerated and oxygen evolved. Several types of evidence indicate 


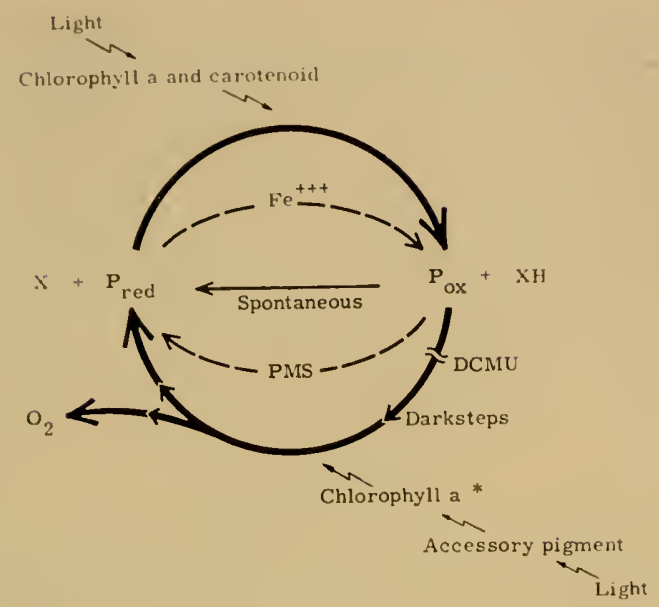

Fig. 13. Schematic summary of discussed observations.

that the sensitization of this step, although mainly by accessory pigment, is still mediated by a small fraction of the total chlorophyll $a$.

As shown in the previous section, ferricyanide may spontaneously carry out the first step, in other words, it can short-circuit the chlorophyll $a$-sensitized light reaction (and the negative absorption change induced by it). But once all " $\mathrm{P} 700$ " is converted (and a correspondingly small amount of $\mathrm{Fe}^{+++}$is reduced to $\mathrm{Fe}^{++}$), the reaction comes to a halt. If now light of proper wavelength (accessory pigment sensitization) is given, oxygen will be evolved, "P 700 " is again reduced and will in turn recluce another batch of $\mathrm{Fe}^{+++}$, so that a continuous cycle occurs. This concept-or should we say oversimplification-thus states that the Hill reaction with ferricyanicle is only half of the photosynthetic process.

A similar reasoning can be based upon the second observation: that PMS spontaneously converts $\mathrm{P}_{\mathrm{ox}} \rightarrow \mathrm{P}_{\text {red }}$. Obviously this entails a short-circuit of the second (accessory-pigment-sensitized) light reaction. Oxygen evolution is bypassed and, with all "P 700 " in the reduced state, no positive light-induced absorption change will occur. To drive a cyclic process in which ATP is generated, light is required, but only the oxidative (chlorophyll- $a$-sensitized) step suffices. This prediction grossly fits the observed sensitization data of Fig. 10. No guess can be made about the site in the above scheme where coupling with phosphorylation (either in the $\mathrm{Fe}^{+++}$or PMS system) is most likely to be. An important question remains to be answered. In 
complete photosynthesis (consuming 2 quanta per electron), how are the absorbed quanta distributed over the two pathways in the proper ratio? In other words, how can, as in green plants, the quantum yield remain virtually constant over a spectral region in which the ratio Chl. $a /$ Chl. $b$ absorbance varies widely. Several ad hoc hypotheses can be advanced, some of which are open to experimental test.

It must be reemphasized that these thoughts about the two-quanta interaction in the sensitization of photosynthesis are speculative and are presented as a basis for discussion. However, evidence gathered to date indicates a mechanism of this general type.

\section{REFERENCES}

1. Blinks, L. R., Proc. Nat. Acad. Sci. U. S., 46, 327 (1960).

2. Coleman, J. W., Holt, A. S., and Rabinowitch, E. 1., in Research in Photosynthesis (H. Gathon, ed.), Interscience Publishers, న̀. Y. (1957).

3. Duysens, L. N. M., "Transfer of excitation energy in photosynthesis," Thesis. Utreclit (1952).

4. Duysens, L. N. M., Srience, 120, 353 (1954).

5. Diysens, L. N. M., Ann. Rev. Plant Physiol., 7, 25 (1956).

6. Emerson, R., Ann. Rev. Plant Physiol., 9, 1 (1958).

7. Emerson, R., and Arnold, W., J. Gen. Physiol., I5, 391 (1932).

8. Emerson, R., and Lewis, C. M., Am. J. Botan., 30, 165 (1943).

9. Haxo, F., and Blinks, L. R., J. Gen. Physiol., 33, 389 (1950).

10. Jagendorf, A., in "The Photochemical Apparatus, its Structure and Function," Brookharen Symposia in Biol., 2, 237 (1959).

11. Jagendorf, A., and Avron, M., J. Biol. Chem., 231, 277 (1958).

12. Kok, B., Biochim. et Biophys. Acta., 2I, 245 (1956).

13. Kok, B., Biochim. et Biophys. Acta, 22, 399 (1956).

14. Kok, B., Proc. Intern. Congr. Photobiol., 2nd Congr., p. 369, Turin, Italy (195i).

15. Kok, B., Acta Botan. Neer., 6, 316 (1957).

16. Kok., B., Plant Physiol., 34, 184 (1959).

17. Kok, B., and Gott, J. W., Plant Physiol. (in press).

18. Mvers, J., and French, C. S., J. Cen. Physiol., 43 (in press, 1960).

19. Petrack, 13., Federation Proc., 18, 302 (1959).

20. Smith, L. and Chance, B., Ann. Rev. Plant Physiol., 9, 449 (1958).

21. Strehler, B. L., and Lynch, V. H., Arch. Biochem. Biophys., 70, $52 \bar{i}$ (1957).

22. Wessels,, J. S. C., and van der Veen, R., Biochim. et Biophys. Acta, 19. 548 (1956).

\section{DISCUSSION}

DR. Arvos: I have several questions. I will not take the time to ask them all, but will stirt with the last point first. Your concluding sentence was that the yield of photophosphorylation was very low, and that the quantum yield was also so low that it is questionable whether it should be taken seriously. Yet earlier you stated that the cliloroplast material that you had used was kept for long periods in the refrigerator. Now, it happens that the photophosphorylation yield is very much dependent upon the con- 
dition of the chloroplasts. It is very hard to get good yields with poor chloroplasts. 1) you see any possible relation between the condition of your chloroplasts and the yields which you obtained?

Dr. Kok: No. For phosphorylation measurements all the care that you described in your papers for treating the leaves was taken. The quantum yelds always come out very, very low $(\sim 0.02)$. Have you measured it?

DR. ARxos: We have nothing to report on quantum yiclds at this time. But I would like to ask another question. I am not quite clear what you are measuring with PMS. Do you measure photophosphorylation? Spectral changes? Have you compared phenazine methosulfate with other catalysts for photophosphorylation and were there any differences?

Dr. KoK: I want to emphasize the fact that PMS is the only one and very typically the only one that will stimulate the light-induced bleaching of P700 in cell-free preparations. FMIN, dye, whatever you use, and there are a number of others, always tend to have the opposite effect on our pigment system. They do not act like PMS at all.

DR. JAGENDORF: I am surprised that you call PMS a reducing agent. It should be oxidized when you add it unless you have ascorbate there also.

Dr. Kok: I do not know what it means, but it stimulated the shift reduced dye no matter how you put it in, as pyocyanine or as PMS. We tricd all sorts of things and it seems to establish itself in illuminated chloroplasts.

Dr. Lip.laxi: If you have a chloroplast to which you give some substrate, you get a reduction of PMS. But one cannot call PMS a reducing agent because it gets reduced.

Dr. Kok: That's right, but we can produce the same effect with reducing agents like ferrocyanide or molecular or atomic hydrogen. This similarity of action makes us think that PMS carries out a reducing step some way or other.

Dr. Calvix: I just wanted to add a remark at the end of the talk earlier today. I just didn't get a chance to. The wavelength for maximum quantum yield of spins is around $7 \mathrm{I} 5 \mathrm{~m} \mu$ for chloroplasts.

Dr. Commoner: It seems to me that in the absence of a fully resolved EST signal (which is the case in Dr. Calvin's results) quantitative estimates of spin numbers should be treated with considerable caution.

DR. CALviN: I said the wavelength for maximum of the total number of unpaired spins produced in chloroplasts is $715 \mathrm{~m} \mu$, the total number of spins. They are unpaired electrons.

Dr. Rabisowitcr: l'd like to ask Dr. Kok how it is from his point of view that monochromatic light absorbed entirely by phycoerythrin can give complete photosynthesis with a high yield.

Dr. Kok: If you look at the spectrum for activation of the positive return step in chloroplasts, it looks exactly like enhancement activation. It has a shoulder at $670 \mathrm{m \mu}$, a peak at $650 \mathrm{~m} \mu$ (and then it gets lost in the blue. Something strange is going on there). This means that the plus shift does 
not involve only chlorophyll $b$. Chlorophyll $a$ is also involved. Among several hypotheses one can conceive the presence of two pigment groups: (1) chlorophyll $a$ and (2) accessory pigment combined with a small fraction of the total chlorophyll $a$. The first pigment group cannot perform complete photosynthesis by itself since it can only transfer light to P700. In doing so it generates the reaction center for the second pigment group. The second group will preferentially transfer to this latter center, but if not there it can pass the light on to the first group (whose chlorophyll a should therefore absorb at slightly longer wavelengths) and so reconvert P700-the other trapping center. Via such a mechanism, light received by the second pigment group would not only be automatically distributed over the two paths but assist the conversion of radiation falling in the first group (enhancement).

Dr. ARNoN: Do I understand you have measured phosphorylation in monochromatic light at $705 \mathrm{~m} \mu$ with a chloroplast system that was catalyzed by PMS. Is that correct?

Dr. KoK: Yes.

Dr. ArNon: Would you tell us something about the magnitude of this phosphorylation. How does it compare with phosphorylation in white light?

Dr. KoK: Would you care to answer that question, Dr. Hoch?

Dr. Hoch: It doesn't make much sense to put it on a chlorophyll basis, because we are totally absorbing the light.

Dr. ArNon: On any basis.

DR. HocH: Well, for instance, we measure in $0.3 \mathrm{ml}$ about 0.8 micromoles of phosphate disappearing in five minutes. Y'et we have run experiments in white light and gotten up to four to five hundred micromoles of phosphate per milligram of chlorophyll per hour, and we have never tried to get the maximum rate.

DR. ARvoN: Did I understand that the comparison was between 400 and 0.8 ?

DR. Hoch: There's about one micromole in five minutes.

Dr. ARNox: Let's put it on the same basis.

Dr. Hocn: You can't put it on the same basis.

Dr. Arvon: My question is, is there any basis on which we can compare them?

Dr. Hocn: All I can say is that under the conditions that we run the monochromatic light, we have also run white light experiments in which the rate compared-that is, with datit available in the literature.

DR. AR.Nox: You saly they do compare?

Dr. Hoch: That is, on a milligram chlorophyll basis. That is, with white light of high intensity.

Dr. Arvox: Well, if there are no figures, may I ask for an opinion as to the order of magnitude of phosphorylation in red light as compared to white light?

DR. Hocn: I camnot give an opinion because I cannot find any basis for 
comparison. It does not make sense to put it on a per milligram chlorophyll basis.

DR. JAGNDorr: If you apply monochromatic light or white light at equal energies under the same experimental conditions, you would have a common basis.

Dr. Hocи: W' liave not done that experincut.

DR. Arvox: May I ask this question? Do you think that the phosphorylation you get at $705 m \mu$ represents a substantial proportion of the total phosphorylation you get in white light? Is this a fair question?

Dr. Kок: Let's run an experiment tomorrow.

Dr. STRE:Inlik: I recall that Dr. Duysens' findings, some later work that Lynch and I did (in which we used circulating Chlorella rather than measurement during illumination) and certain experiments which Chance and I reported all gave essentially negative results for the region 660 to $750 \mathrm{~m} \mu$. Certainly, there were not changes of nearly the order of magnitude of those occurring in the regions of 480 to $515 \mathrm{~m} \mu$. In view of these other negative findings. I want to ask a question concerning the technique of measurement. It seems to me that if you have extremely dense chloroplasts of Chlorella as you have in your chamber and you pass light through it so that only 0.0001 of the total light comes through at the maximum of the chlorophyll peak, then such fluorescence excitation by the measuring beam as occurs at the side of the curette nearest the photomultiplier will be much more pronounced at wavelengths where chlorophyll does not absorb so strongly, i.e., out at the long wavelength end of the spectrum. Now, if the exciting beam, but not the measuring beam, produces a change in the fluorescence yield of the chlorophyll at the far end of the tube, one would expect to find changes in the fluorescence intensity produced by the measuring beam on the red edge of the band which would not be apparent when you are measuring at the peak of the chlorophyll (where most of the fluorescence would be reabsorbed). I am sure you have some experiments which would rule out this possibility.

Dr. Kok: No, I haven't. All we have done is our best to go down to the weakest suspensions we can use. 0.01 optical density is a big shift for us. You can use very dilute suspensions and still get this shift. This also holds for the chemically induced shifts, which one can even observe with a standard Cary instrument. However, the signal to noise ratio may be very poor.

Note added in proof: After the meetings we made a few experiments specifically designed to meet Dr. Strehler's doubts (with his consultation). It appeared that fluorescence induced by the detecting beam, although under conditions contributing significantly to the total photosignal, could not account for the light-induced changes of this signal.

Dr. Strenll:R: I was quite convinced by the phosphate esterification action spectrum, until Dr. Hoch mentioned you are absorbing all the light. The explanation there might be somewhat similar: namely, that in the region 
where chlorophyll absorbs quite strongly you are already above saturation. This would occur in the upper layers of the suspension that are exposed to the light whereas when you go out further toward the red, the light is transmitted down deeper into the suspensions, giving a higher rate.

Dr. Kок: Your worries are our worries. To help light penetration we stir as fast as we can. We checked this point again just before the meeting. This work is done with interference filters-to obtain measurable rates we have to use this rather impure monochromatic light. Now, we placed a Schott RG8 glass filter in front of the 700 or 710 interference disc so that all radiation below $690 \mathrm{~m} \mu$ is cut off. This cuts the total transmitted light by a factor of two or more. Still the rate was higher than that with the full transmission of a 670 interference filter.

Note added in proof: Perhaps the most convincing evidence [against Dr. Strehler's hypothesis] is that the phosphorylation associated with ferrocyanide reduction and that mediated by riboflavin 5-phosphate or vitamin $\mathrm{K}_{3}$ (measured in the same set up under identical conditions to PMS) does not show the maximum at $700-710 \mathrm{~m} \mu$.

Dr. STREHLER: I would like to suggest that you could absolutely rule out this possibility if you were to measure the fluorescence yield under your various conditions of illumination. If the fluorescence yields changed in the opposite direction from your transmission measurements or if they didn't change at all, then you would certainly rule them out.

DR. KoK: No, that fluorescent measurement wouldn't work. This problem doesn't bother us, because whatever fluorescence is caused by the activating beam the photocell could only see a change in fluorescence induced by the detecting beam and occurring after the flash is orer. That's all that is plausible. You do not see fluorescence. Right? That's what you are worried about. Fluorescence after the flash?

Dr. Streuler: You pick up fluorescence from the measuring beam which should be more intense at the far end of the chlorophyll spectrum.

Dr. Kок: No, we do not see activating beam fluorescence. We have a phosphoroscope arrangement. Only if the actinic beam should produce an after-effect in the fluorescence of the detecting beam would we see it. But then this fluorescence would be seen by the photocell regardless of the wavelength setting of the monochromatic beam (cf. above).

Dr. Raninowitch: May I come back to our old observations on the difference spectrum in Chlorella. Although these results differ somewhat from the ones described by Dr. Kok, our experiments made were under different conditions-in constant light-and therefore do not invalidate each other. I want to answer Dr. Strehler's skeptical question: why other people did not find any effects in the red part of the spectrum. If one measures the difference spectrum of Chlorella in light of clifferent intensities, one finds that even weak light produces a positive effect at $520 \mathrm{~m} \mu$, as well as a negative effect at $480 \mathrm{~m} \mu$, (and also the cytochrome effects). On the other hand, in 
weak light, you sec practically nothing in the red. However, if you increase the light intensit? and bring it into the saturation region of photosynthesis, the negative ellect at $680 \mathrm{~m} \mu$ develops, and other negative bands appear, e.g. at 650 and 700$) m \mu$. In the strongest light we used, the cytochrome effects were practically gone. The strongest remaining effects were those at 180 and $520 \mathrm{n} \mu$ (which Dr. Chance, and we, too, ascribed to a transformation of carotenoids): and the negative effects at 680 and $430 \mathrm{~m} \mu$, which are most likely due to the bleaching (reduction?) of chlorophyll, I would like to note here that the bleaching of the red chlorophyll band does not occur uniformly over its width. The several components of chlorophyll a appear to respond differently. However, the important point at the moment is that bleaching of the red chlorophyll band is not a linear function of light intensity, but follows a sigmoid curve. There is no significant change as long as you are in the linear part of the photosynthesis light curve. Only when you get into the saturation region, something begins to happen to chlorophyll.

DR. Fraick: I was very interested to hear that changes in the absorption spectrum of chlorophyll become strong as soon as the irradiation intensity surpasses saturation. Here photo-oxidation of chlorophyll might be responsible for the bleaching. If the spectral region around $7000 \mathrm{~A}$ is, as we are inclined to believe, the region of light absorption by water-exposed chlorophylls, the bleaching in this region is supposed to be the strongest.

Dr. Kok: I am sorry but on this point I cannot agree. The cliscussed effects don't show sigmoid light curves. Other things besides activating intensity determine their sign and magnitude. With the short darktime arrangement the effects in the red began only to make sense after we found that the wavelength of activating light mattered. Still, green cells are confusing, maybe because their pigments overlap so closely and (or) photosynthetic sensitization is so well balanced over the entire spectrum until $680 \mathrm{~m} \mu$.

Possibly we notice the light-induced shifts at $700 \mathrm{~m} \mu$ only if the system is out of balance: in continuous illumination at saturating intensities, as Dr. Rabinowitch showed, or in our long darktime flashing light ("fast effect"). In the latter case photosynthesis still runs at normal integrated rate and efficiency, but the involved intermediates can be easier observed since they undergo cyclic changes. We also found earlier (I2), that the (fast) shifts at $520 m \mu$ saturated earlier than the red and blue shifts. This does not necessarily imply that photooxidation underlies the latter ones. As I said, it might be explained on the basis that the oxygen evolution step (correlated with the slow effect) saturates earlier and that not until then does a negative effect become noticeable. The situation might be analogous in the case of cytochrome $f$ oxiclation. In green material the $555 \mathrm{~m} \mu$ shift is not perceptible with continuous actinic light, but we found it repeatedly with long darktime flashing light.

In Anacystis, where the quantum yield is normally poor in the far red, the system might always be off balance and the $700 \mathrm{~m} \mu$ shifts (as well as 
the one at $555 \mathrm{~m} \mu$ ) show up in flashing as well as in weak continuous ilfumination. Itso an interpretation of the effects on the basis of induction phenomena, as Dr. Franck suggests, I cannot quite see. The fast effect with its very short lifetime we measured only by integration over many cycles. Time response of the instrument was of the order of a second. Only the positive effect may show incluction and overshoot but both effects reach a steady state in the light.

Dr. Commoner: I would like 10 make a very brief extension of the earlier remarks on electron spin resonance. Dr. Franck's remark may be very pertinent. From our clata, it would appear that the signal at $g=2.005$ saturates at very low light intensities, whereas the signal at $g=2.002$ saturates hardly at all. It is probable if any signal is associated with chlorophyll, it will turn out to be the signal at $g=2.002$. If so, Dr. Franck may be right in concluding that chlorophyll is converted, photochemically, into a free radical form. The chlorophyll free radical, which in Chlorella is observed only at high light intensities, may have little to do with the photosynthetic process which is already saturated with respect to light intensity. Considerably more kinetic data are needed before this question can be resolved.

DR. RABiNowitcil: I am not inclined to agree that this eflect has nothing to do with photosynthesis. I believe it is related to the light saturation of photosynthesis, and is not something extraneous like photooxidation. It is instantancously reversible. I quite agree with Dr. Kok that these experiments should be done with monochromatic actinic light; we are trying to do it now. Our first experiments with colored glatss filters did not show much variation in the difference spectrum, whether obtained with red or green or bhe light. However, it should be done with only monochromatic light. To return to results obtained on white light: now that we have a much improved instrument, we get the following picture. At a given wavelength of the monitor beam, there is a steady signal in the dark; when actinic light is admitted, there is a practically instantaneous effect (in less that one second.) If the light is left on for one minute, a second effect appears, a "slow" effect, which can hate the same sign as the fast effect, or an opposite sign. In the latter case, the total effect maly go through zero and reverse its sign. Strehler has observed this behavior. If after a mimute you switch the light off, you get an instantancous reversal of the instantaneous effect; then the slow effect is also reversed, but slowly-in a couple of minutes. One thus sees the induction plenomena-first the instantaneous inchuction (ewo seconds induction), then the slow induction (two minutes incluction)-both well known from fluorescence measurentent; then the reversal of the instantaneous induction, and the resersal of the slow induction, somcthing you camnot observe in fluorescence. Sometimes the cycle encls on a somewhat different level, indicating an irreversible eflect. From the induction phenomena of the fluorescence, we know that induction involves chloropliyll; but in fluorescence, you ouly sec the dark-to-light part; in absorption spectra you 
also sece the lightedo-dark part, which is something new. W'e also see that both the slow and the fast induction effects are due to changes in the chlorophyll complex.

Dk. Kok: I would like to add that at least in our later experiments with monochromatic actinic light the intensities-even using wide slits-were extremely weak. We certainly could not approach photosynthetic saturation, let alone photoinhibitory phenomena.

DR. JAGNDORF: I would like to comment on the physiological significance of high light intensity in respect to phosphorylation with pyocyaninc. I)r. Arron finds that he doesn't reach saturation of photophosphorylation until 15.000 foot candles, using Swiss chard chloroplasts.

DR. Strenlr:R: Apropos of Dr. Commoner's and Dr. Rabinowitch's remarks about the induction period, I rould like to point out that the luminescence of green plants (following their illumination) also shows a diphasic decay phenomenon which has somewhat different time constants, however, from those Dr. Conmoner has observed. We have simultaneously measured the luminescence intensity from plants and absorption spectrum changes at $515 \mathrm{~m} \mu$ and have also measured the degree to which the alsorption phenomenon is suppressed in the dark alter illumination and have found close kinetic correspondences between the albsorption spectrum changes and the luminescence. It would be interesting to tie these three phenomena, the absorption spectrum, the luminescence of the chloroplasts. and the electron spin resonance changes, together.

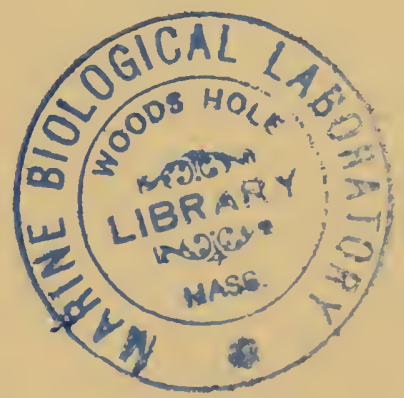




\title{
THE NATURE AND POSSIBLE FUNCTION OF CHLOROPLAST CYTOCHROMES
}

\author{
Robert Hill and Walter D. Bonner, Jr. \\ Department of Biochemistry, University of Cambridge, \\ England, and Johnson Research Foundation, \\ L'niversity of Pennsyltania, Philadelphia, Pennsylvania
}

This discussion will be limited exclusively to the cytochromes of higher plant chloroplasts. It was in higher plants that a cytochrome, localized in the photosynthetic apparatus, was first cliscovered.

Hill and Scarisbrick (9) showed that a cytochrome, named by them cytochrome $f$, could be observed in acetone powiders of green leaves. This cytochrome was readily extractable from green tissues and was obtained in a highly purified form. Later work by Davenport and Hill (4) localized cytochrome $f$ to green leaves and described an improved method for extraction and purification of cytochrome $f$. In his work relating to the localization of cytochrome $f$ within the chloroplast, Davenport (5) observed a second cytochrome. The spectroscopic properties of this second chloroplast cytochrome were studied by Hill $(7)$, who named this component cytochrome $b_{6}$. Some of the characteristics of cytochromes $f$ and $b_{6}$ are given in Table 1.

Cytochrome $f$ is the only chloroplast cytochrome which is readily brought into solution and to a high state of purity. It is nonautoxidizable, is reduced by ascorbate, DPNH, and TPNH, and is oxidized by ferricyanide.

The absorption maxima of cytochrome $f$ are slightly shifted toward the red, when compared to cytochrome $c$; and the former has a very asymmetric $\alpha$-band which at low temperature is resolved into very sharp $\alpha_{1}$ and $\alpha_{2}$ peaks. Fig. 1 illustrates the low temperature difference spectrum (oxidized-reduced) of highly purified cytochrome $f$ compared to its room temperature absolute spectrum.

The spectroscopic observation of cytochrome of freshly prepared chloroplasts is complicated, because of the large concentration of chlorophyll which is present. Through the use of a low dispersion spectroscope it is possible, however, to observe the appearance of a broad absorption band, in the region $550-560 \mathrm{~m}_{\mu}$, following hydro- 
ROBERT HILL AND WALTER D. BONNER, JR.

TABLE 1

Some Cimaracteristics of Crtochiromes $\int$ and $b_{6}$

\begin{tabular}{cccc} 
Cytochrome & $\begin{array}{c}\text { Absorption Maxima } \\
\left(25^{\circ} \mathrm{C}\right)\end{array}$ & $\begin{array}{c}\text { Absorption Maxima } \\
\left(-190^{\circ} \mathrm{C}\right)\end{array}$ & $\begin{array}{c}E^{\prime}{ }_{0} \\
(\text { Volts })\end{array}$ \\
\hline$f$ & $\alpha-554.5, \beta-524, \gamma-422$ & $\begin{array}{l}\alpha_{1}-552, \beta_{1}{ }^{*}-529, \gamma-420.8 \\
\alpha_{2}-548, \beta_{2}-524\end{array}$ & +0.365 \\
$b_{6}$ & $\alpha-563, \beta-534, \gamma-429$ & $\alpha-557, \beta-530, \gamma-427$ & -0.030
\end{tabular}

* 9 B-bands can be observed at low temperature.

sulfite reduction of whole chloroplasts. Since no absorption in the region $550-560 \mathrm{~m} \mu$ can be observed in the absence of hydrosulfite, it may be concluded that in the light the readily discernible chloroplast cytochromes are oxidized.

It has been shown by Hill and his associates (see Hill, 7) that the two cytochrome components of chloroplasts are not denatured by

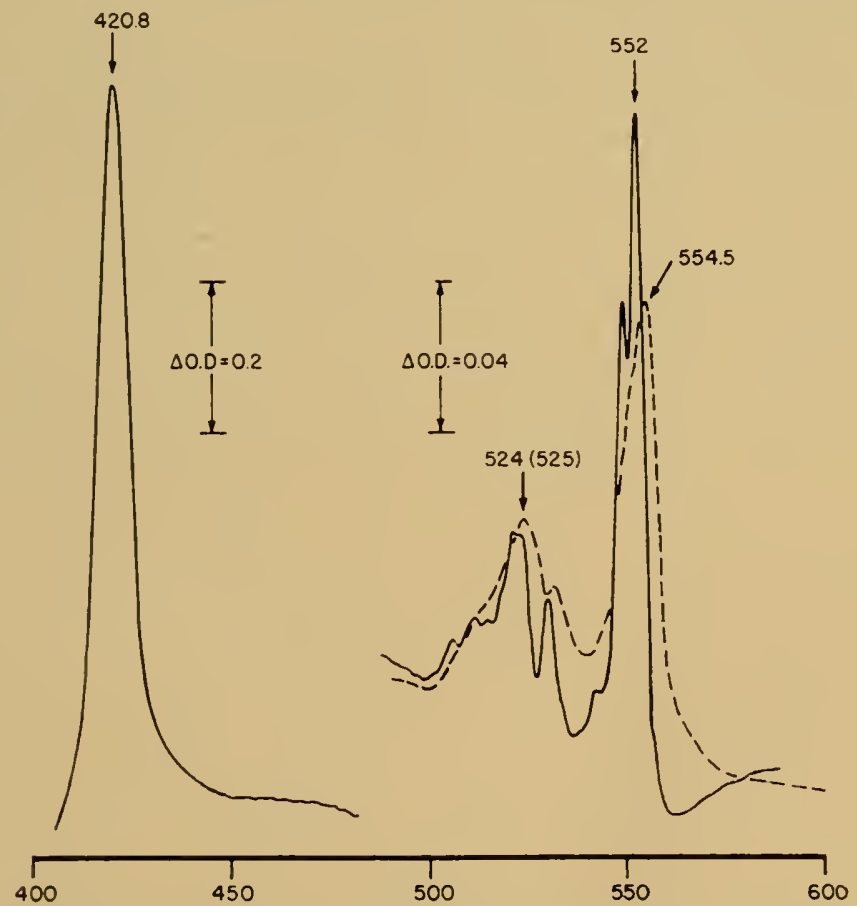

Fig. 1. Spectra of highly purified cytochrome $f$. Solid line, a low temperature $\left(-190^{\circ} \mathrm{C}\right)$ oxidized-reduced difference spectrum. Dashed line, a room temperature absolute spectrum. This latter spectrum was replotted from recent unpublished data of H. E. Davenport. 
cold 80 per cent acetone, a behavior that is in marked contrast to that of the mitochondrial cytochromes. The extraction of freshly prepared chloroplasts by cold 80 per cent acetone removes chlorophyll and allows quantitative spectroscopic and spectrophotometric observation of the residue. Such an extraction of chloroplasts changes the oxidation state of the cytochrome components, and cytochrome $f$ becomes fully reduced while the highly autoxidizable $b_{6}$ remains oxidized.

A spectrum showing the difference in absorption between 80 per cent acetone-extracted chloroplasts and similar chloroplasts reduced with hydrosulfite is shown in Fig. 2. This spectrum clearly shows a single cytochrome component, a component whose low-temperature absorption maxima do not correspond to any of the plant mitochonchial or microsomal cytochromes (cf. Bonner, 2). The spectrum shown in Fig. 2 is that of cytochrome $b_{6}$. No spectral changes have been observed with hyclrosulfite-reduced $b_{6}$ preparations in the presence of cyanide, azide, carbon monoxide, or antimycin $A$. The lack of any effect of carbon monoxide on the spectrum indicates a minimum of denaturation during the acetone treatment. Frequently, however, the $\alpha$-band of cytochrome $b_{6}$ in the acetone-extracted prepara-

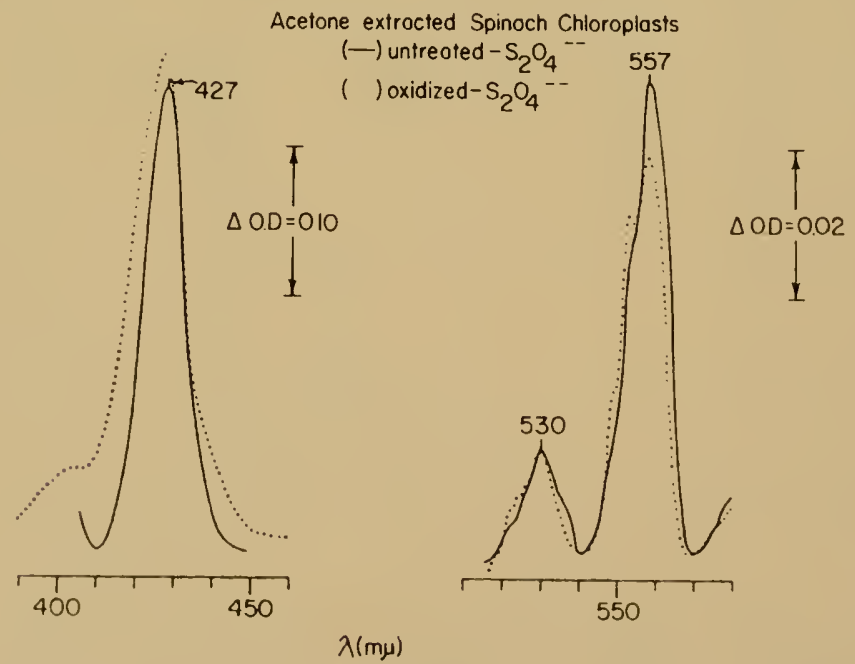

Fig. 2. Low-temperature difference spectra of 80 per cent acetone-extracted spinach chloroplasts. The solid line, intreated - hydrosulfite-reduced chloroplast residue, shows the spectrum of cytochrome $b_{8}$ only. The dotted line, oxidized (chlorox) - bydrosulfite-reduced chloroplast residue, shows, in addition to $b_{b}$, the peak, at $551 \mathrm{~m} \mu$, of cytochrome $f$. 
tions shows a marked asymmetry on the short-wavelength side. This sante figure (Fig. 2) also shows an oxidized - reduced spectrum of 80 per cent acetone-extracted chloroplasts in which the two cytochromes, $f$ and $b_{6}$, are clearly illustrated. The ratio, $b_{6} / f$, over several experiments, was 1.3 .

The spectrum of cytochrome $f$, present in 80 per cent acetoneextracted chloroplasts, is given in Fig. 3. This same figure has for comparison a spectrum of cytochrome $c$. Both of these spectra are difference spectra, oxidized - reduced.

It is very clear from the investigations presented above that the chloroplasts of higher plants contain at least two cytochrome components, and that these components are present in relatively high concentration. Since acetone extraction destroys the reactions that are characteristic of isolated chloroplasts and changes the oxidation state of the chloroplast cytochromes, it becomes necessary to refine the techniques to the point where cytochrome components in whole

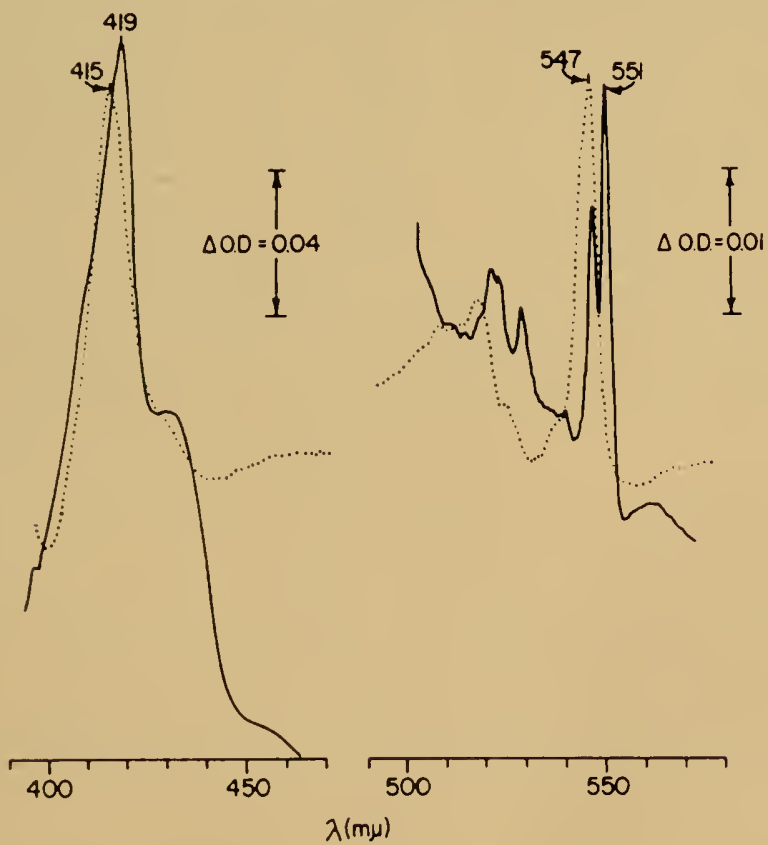

Fig. 3. Low-temperature difference spectrum of 80 per cent acetone-extracted spinach chloroplasts, oxidized (chlorox) - Intreated, showing the characteristic. spectrum of cytochrome $f$. For comparison, an oxidized - redifcel difference spectrum of cytochrome $c$ (dotted line) is included. 
chloroplasts can be observed optically. By providing a suitable optical path length it is possible to obtain low-temperature spectra of the cytochromes present in freshly prepared whole chloroplasts. Such a spectrum, showing the difference in absorption between illuminated and hydrosulfite-reduced chloroplasts, is shown in Fig. 4. Inspection of this figure shows that the cytochrome spectrum of whole chloroplasts only superficially resembles that obtained from 80 per cent acetone-extracted chloroplasts. With the whole chloroplast, the peaks of absorption are shifted to slightly longer wavelengths as compared to the acetone-extracted chloroplasts, and there are several indications that there is present, in whole chloroplasts, a third component which absorbs at a wavelength intermediate between $f$ and $b_{6}$. It is concluded here that treatment of green plant chloroplasts with cold 80 per cent acetone does more than remove the chlorophylls and carotenoids.

The study of cytochromes in chloroplasts has been extended by many workers to algae and bacteria. These studies have been critically reviewed by Smith and Chance (15). In general, the results of such
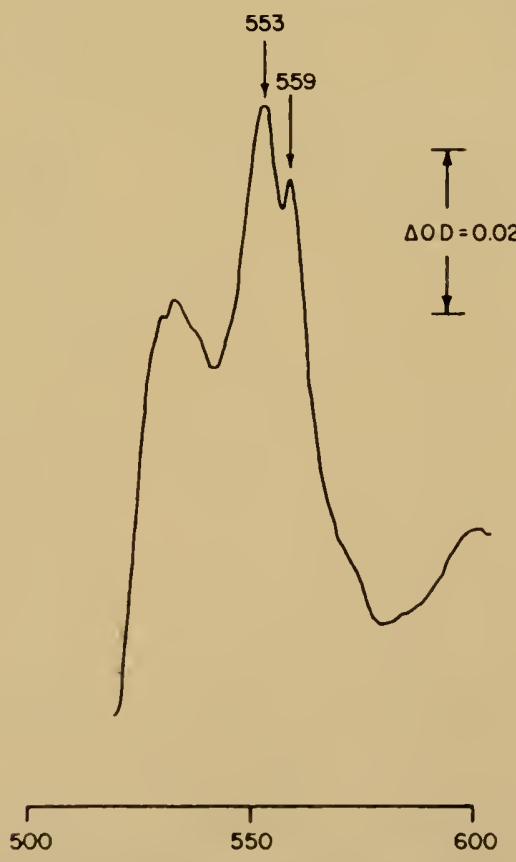

Fig. 4. Iow-temperature difference spectrum of whole spinach chloroplasts; untreated - higdrosulfic-reduced. 
investigations show that photosynthetic structures or organisms contain an f, or f-type, cytochrome and a $b$ cytochronce. The direct participation of these cytochromes in the photosynthetic process remains to be denonstrated.

Before discussing a possible role of chloroplast cytochromes in photosynthesis, it is advisable to consider some general properties of electron transport systems and how such properties could apply to the photosynthetic process.

In respiration, the utilization of oxygen for reactions depending on hydrogen transfer is represented by the following equation:

$$
4(\mathrm{H})+\mathrm{O}_{2} \rightarrow 2 \mathrm{H}_{2} \mathrm{O}
$$

Keilin's classical work (12) showed that the transfer of $(\mathrm{H})$ to oxygen is a stepwise process which is carried out through the cytochrome components; the reduction and oxidation of the cytochrome components is a univalent or one-electron process and can be considered to be a series of thermochemical reactions which take place in the clark. The gradient of these reactions is toward oxygen and its recluction.

Mitochondrial preparations can catalyze the oxidation of a variety of hydrogen donors according to the following equation:

$$
R \mathrm{H}_{2}+1 / 2 \mathrm{O}_{2} \rightarrow R+\mathrm{H}_{2} \mathrm{O}
$$

Illuminated chloroplasts have been found to carry out the reverse of Equation 2. The production of oxygen can occur with a variety of $(\mathrm{H})$-acceptors; they can be $2(\mathrm{H})$ acceptors, such as quinones or dyes, or one-electron acceptors such as ferricyanide. The latter case is illustrated in Equation 3:

$$
\begin{aligned}
4 \mathrm{Fe}(\mathrm{CN})^{3-}=+2 \mathrm{H}_{2} \mathrm{O} & \rightarrow 4 \mathrm{Fe}(\mathrm{CN})^{4-} \\
& =+4 \mathrm{H}^{+}+\mathrm{O}_{2}
\end{aligned}
$$

The light-dependent production of oxygen in the presence of various $(\mathrm{H})$ acceptors is called the chloroplast reaction, because the active production of molecular oxygen, from the chloroplasts, in the process of photosynthesis has been known for nearly 80 years (6). Fig. 5 is a reproduction of an old illustration of Engelmann's experiments. These experiments, published in 1884, clearly showed that oxygen production was confined to illuminated chloroplasts. The chloroplast reaction is limited to green cells; photosynthetic bacteria do not produce the oxygen molecule. 

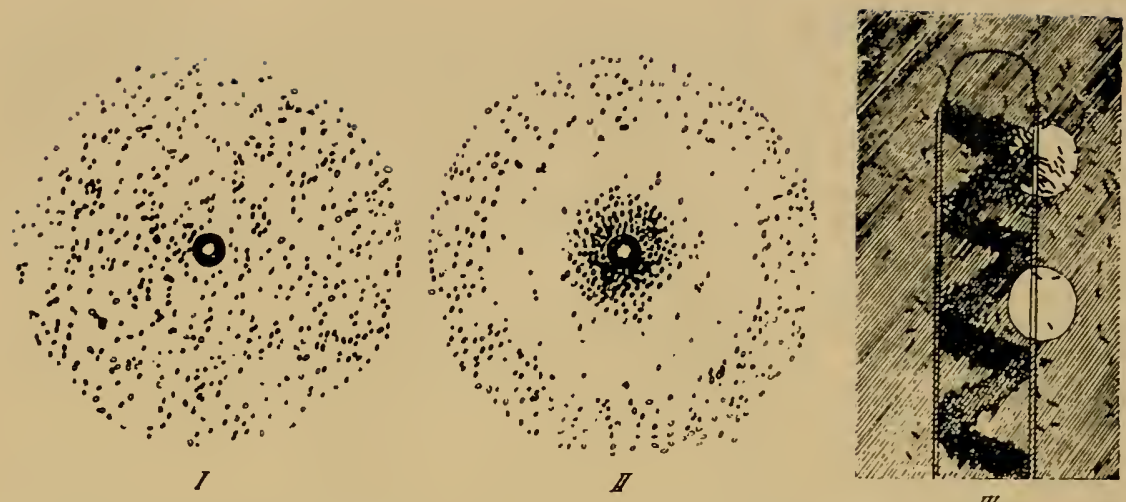

III

Fig. 5. Engelmann's experiment which showed that oxygen production is confined to the illuminated chloroplast.

I, Green algae placed in the middle of a suspension of motile bacteria, as observed following a period in the dark. Since the bacteria require minute traces of oxygen for motility they are immobilized and randomly distributed in the dark.

II, Same preparation as in I following brief illumination.

III, A Spirogyra cell illuminated in two different locations. The bacteria congregate only where the chlorophyll is illuminated.

[From Plant Physiology, by W. Benecke, and L. Josi, Volume I, p. 175. Gusta: Fischer, Jena, 1924.]

Keilin, in his study of the function of the cytochrome system in respiration, found that the oxidation of cytochrome $c$ and the reduction of oxygen was inhibited by cyanicle, while the reduction of cytochrome $c$ was inhibited by urethane. The production of oxygen in the chloroplast reaction is not affected by cyanicle but is very sensitive to urethane. This latter fact indicates the importance of (H) transfer in photosynthesis, a point first emphasized many years ago by van Niel (14). He used the concept of $(\mathrm{H})$ transfer to give a general explanation for all photosynthetic processes; the production of the oxygen molecule in photosynthesis was regarded as a special case of light-induced $(\mathrm{H})$ transfer.

The light-induced $(H)$ transfer in photosynthesis, which is generally represented by Equation f, may be regarded as being opposite to the thermochemical gradient, a suggestion that may be illustrated by a very simple experiment. Basically this experiment consists of mixing together chloroplasts, mitochondria, a crude green-leaf juice, and air. Observation of such a mixture with a low dispersion spectroscope shows that cytochrome $c$ becomes reduced in the light and oxidized in the dark. The light-dark reduction and oxidation of cytochrome $c$ 
can be repeated any number of times. The dark oxidation is due to endogenous respiration, mediated by the mitochondrial oxidase. The reduction of cytochrome $c$ in this experiment may be viewed as a light-induced $(\mathrm{H})$ transfer against the thermochemical gradient, in other words, an exogenous reaction.

$$
\mathrm{H}_{2} \mathrm{O}+\mathrm{Y} \rightarrow \mathrm{XH}+\mathrm{YOH}
$$

The suggestion that $(\mathrm{H})$ transport in the respiratory chain is in the opposite direction to that in the "photosynthetic chain" invites further comparison between these two systems. Fig. 6 shows some

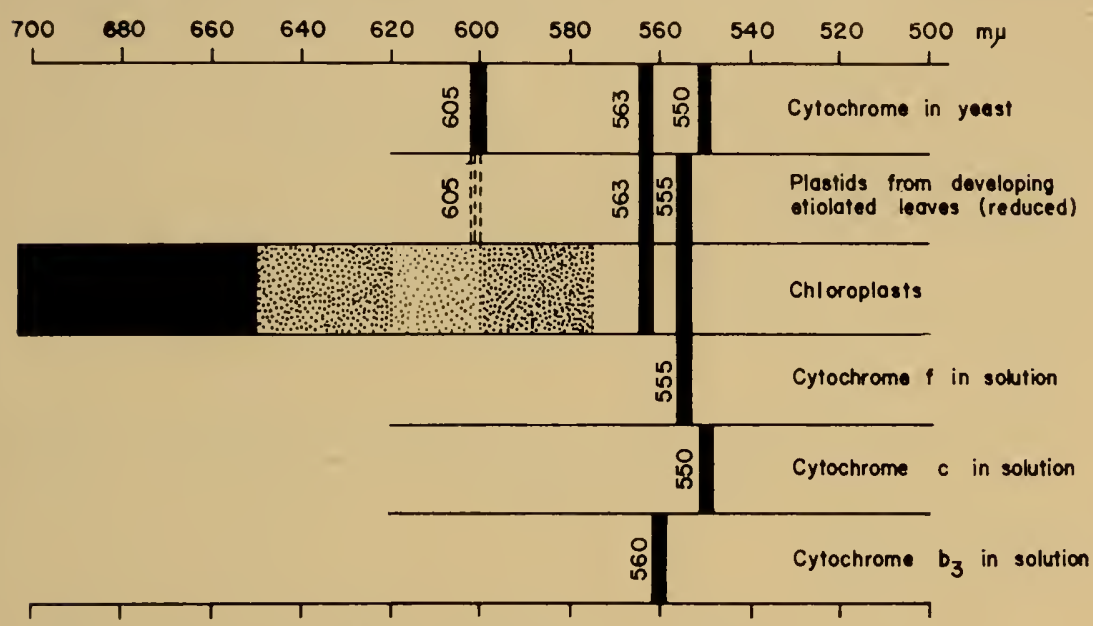

Fig. 6. Spectra, as observed in a low-dispersion spectroscope of chloroplasts and of some hematin compounds extracted from leaves, compared to the cytochrome spectrum of yeast. Only the $a$-bands are represented.

spectra of the hematin compounds present in leaves compared to those present in yeast. Also shown is a chloroplast spectrum, but it should be emphasized that this spectrum is a combined one, derived from our knowledge concerning plastids from etiolated plants or 80 per cent acetone-extracted chloroplasts (for cytochromes) and from green chloroplasts (for chlorophyll). Fig. 7 compares the oxidationreduction potentials of some hematin components in leaves and in yeast.

Further comparisons between mitochondria and chloroplasts may be made as follows:

(1) Mitochondria perform oxygen reduction. Illuminated chloroplasts perform oxygen production. 


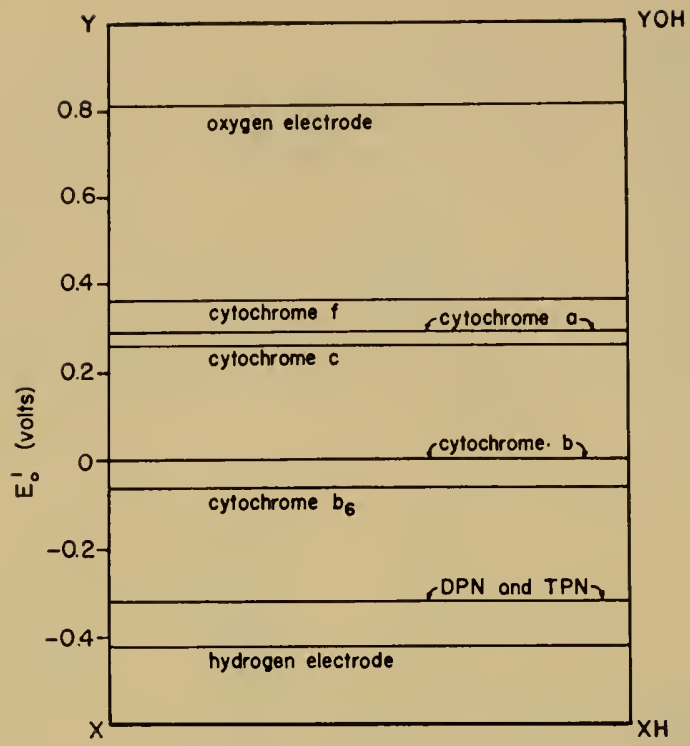

Fig. 7 . Oxidation-reduction potentials of some chloroplast and mitochondrial cytochromes.

(2) Mitochondria oxidize hydrogen donors. Illuminated chloroplasts reduce hydrogen acceptors.

(3) Mitochondria oxidize DPNH. Illuminated chloroplasts reduce TPN.

(4) Mitochondria carry out oxidative phosphorylation. Illuminated chloroplasts carry out "reductive" phosphorylation (10). 1

In the mitochondria the cytochromes $a+a_{3}$ catalyze the reduction of oxygen. In the chloroplast the illuminated chlorophyll could be regarded as supplying both the oxidase, as $Y O H$, and the $(H)$ acceptors, as $\mathrm{XH}$. Hence, using such assumptions, one would expect that the chloroplast cytochromes might function in a manner similar to those of mitochondria. This suggestion is illustrated in Equation 5.

$$
\mathrm{YOH} \rightarrow f \rightarrow b_{6} \rightarrow \mathrm{XH}
$$

In other words, in the illuminated chloroplasts $Y O H$ and $\mathrm{XH}$ might react through the $(\mathrm{H})$ transport system, $f$, and $b_{6}$ to give $\mathrm{H}_{2} \mathrm{O}$ and the concomitant phosphorylation of ADP. In such a hypothesis one would expect that addition of inorganic phosphate and $\triangle D P$ to

"The use of the term "reductive" here is controversial, but it describes the process as observed experimentally. 
the system would result in decreased rates of $(\mathrm{H})$ acceptor recluction and of oxygen production, il phosphorylation was obligatory, or no change if phosphorylation was not necessary. However, such additions result in greatly increased rates of both reactions (1), and the above simple hypothesis has to be abandoned. It was inclicated above that such a series of reactions was unlikely in chloroplasts where some of the reactions of $(\mathrm{H})$ transport must be against the thermochemical gradient.

A second hypothesis would involve three light-driven reactions, and ones that proceed against the thermochemical gradient as first ontlined by Davenport and Hill (4). This hypothesis is illustrated diagrammatically in the left-hand portion of Fig. 8.

Recently Hill and F. Bendall (8) have suggested that most of the experimental observations relating to ferricyanide reduction, oxygen evolution, etc., are best explained in a sequence of reactions illustrated diagrammatically in the right-hand portion of Fig. 8. Involved in this suggestion are two light-driven reactions, against the thermochemical gradient. The two light-driven reactions are considered to
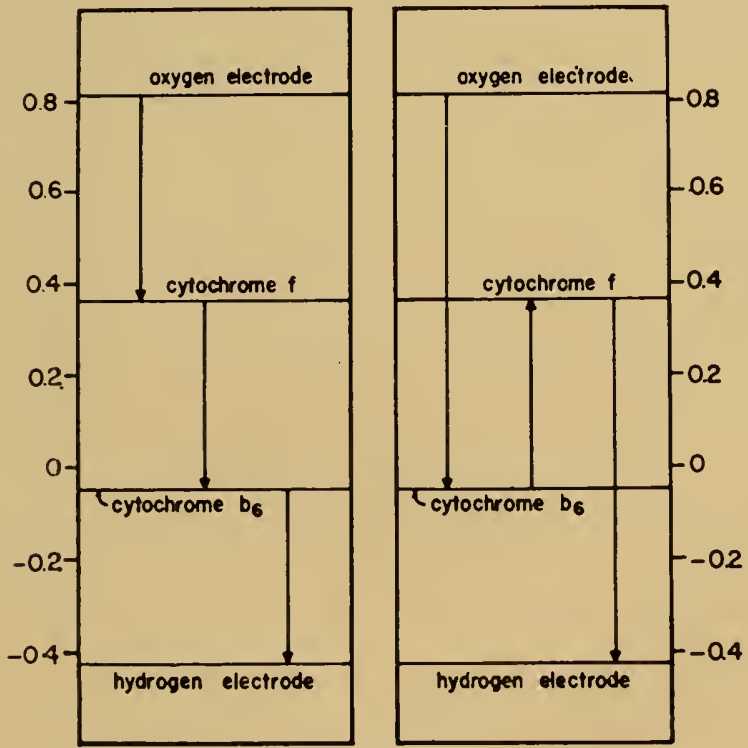

Fig. 8. Hypothetical sequences in the chloroplast reaction. Downward pointing arrows indicate endergonic reactions, against the themochemical gradient; upwat pointing arrows indicate exergonic reactions. On the left, a sequence involving three light-dricen reactions; on the right, wo light-driven reactions connected by an exergonic reaction between cytochromes $f$ and $b_{0}$. 
be comnected into a complete sequence by a chemical reaction between cytochromes $f$ and $b_{6 j}$.

The only evidence for the above hypothesis, in higher plants, is the observation that illumination of pale yellow-green leaves causes an oxidation of $f$ and the reduction of $b_{6}$. As yet, this sequence of reactions has not been observed, in our laboratories, with normal green leaves or chloroplasts.

There are a few observations in the literature that are pertinent to this discussion. One of these observations is that of Lundegårdh (13), who reported that in Chlorella, following illumination, a reduced peak disappeared and an oxidized peak appeared in a spectral region that was ascribed to cytochrome $f$. Similarly, James and Leech (11) reported spectral changes following illumination of chloroplasts derived from Caulerpa prolifera and Vicia faba. Interestingly enough, the light-induced spectral changes reported by James and Leech correspond exactly to those suggested here, viz., disappearance of $555 \mathrm{~m} \mu$ absorption followed by appearance of $563 \mathrm{~m} \mu$ absorption. The time relations of the above two observations leave much to be desired; an interpretation of such results would be facilitated greatly if the observations could be made over very short time intervals. The importance of the time interval between observations, as well as other possible sources of error, in experiments of the kind just described has been discussed by Smith and Chance (15).

In algae also there has been no clear-cut demonstration of changes in cytochrome absorption following illumination of normal cells. Interestingly enough, Chance and Sager (3) have shown the oxidation of cytochrome $f$ following illumination of a pale-green mutant of Chlamydomonas, a mutant that contains unusually low concentrations of chlorophylls and carotenoids and yet retains efficient photosynthesis. The situation in the Chlamydomonas mutant is exactly analogous to that in the pale yellow-green leaves mentioned above. An unequivocal demonstration of a light-induced cytochrome absorption change in normal green cells or chloroplasts must be made before the role of cytochromes in the photosynthetic process can be assessed properly.

It is our hope that future investigations will be able to elucidate more fully the role of cytochromes in the photosynthetic process of green plants. The suggestions given here are made only as a working hypothesis and to serve as models for further experimentation. It is hoped that the spectral properties of the chloroplast cytochromes which are described clearly here will help in such experimentation. 


\section{REFERENCES}

1. Amon, D. 1., Whatley, I. R., and Allen, M. B., Science, 127, 1026 (1958).

2. Bonner. W. 1).. Jr., in Proc. Haematin Enzyme Symposium. Canberra (J. L. lalk. M. R. Lemberg, and R. K. Norton, eds.). Pergamon Press, London (in press).

3. Chance, B., and Sager, R., Plant Physiol., 32, 548 (1957).

1. Davenport, H. L..., and IIill, R.. Proc. Roy. Soc. Lomdon, R, 139, 327 (19.52).

5. Davenport, H. E., Nature, 170, 1112 (1952).

6. Engelmanı, 'T. W., Arch. ges. Physiol. (Pflügers), 57, 375 (1891).

7. Hill, R., Nature, 174, 501 (1954).

8. Hill, R., and Bemdall, F., Nature, 186,136 (1960).

9. Hill, R., and Scarisbrick, R., New Phytologist, 50, 98 (1951).

10. Hill, R., and Walker, D. A., Plant Physiol., 34, 240 (1959).

11. James, W. O., and Leech, R. M., Nature, 182, 1684 (1958).

12. Keilin, D., Proc. Roy. Soc. London, B, 98, 312 (1925).

13. Lundergardh, H., Physiol. Plantarum, 7, 375 (1954).

14. van Niel, C. B., Arch. Microbiol., 3. 1 (1931).

15. Smith, L., and Chance, B., Ann. Rev. Plant Physiol., 9, 449 (1958). 


\title{
REACTIONS OF GYTOCHROME PIGMENTS IN PHOTOSYNTHETIC BACTERIA
}

\author{
LuCILE SMITH \\ Department of Biochemistry \\ Dartmouth Medical School \\ Hanover, New Hampshire
}

We have made some observations of changes in the absorption spectra of pigments in photosynthetic bacteria upon illumination or upon reaction with oxygen. So far, it has proved to be easier to observe the reactions of cytochromes in these bacteria than in green plant tissues.

The photosynthetic bacteria investigated were Rhodospirillum rubrum and Rhodopseudomonas spheroides, grown uncler illumination. Measurements of changes in the absorption spectrum on illumination or oxygenation of anaerobic suspension of intact bacteria or of cell-free extracts were made in spectrophotometers specially designed to measure small changes in optical density of the pigments present, with minimal interference from non-spectral changes in signal (1, $15,2)$.

Illumination of anaerobic suspensions of $R$. rubrum or $R$. spheroides with light absorbed by the bacteriochlorophyll (light of wavelength greater than $720 \mathrm{~m} \mu$ ) results in changes of absorption spectrum of two kinds of pigments in the wavelength region between 400 and $630 \mathrm{~m}_{\mu}$ : (1) there is oxidation of cytochrome pigments; and (2) there are changes in the absorption spectrum of carotenoid pigments $(2,11)$. These changes are illustrated in Figs. 1 and 2, for R. rubrum and $R$. spheroides, respectively. Curve $b$ in Fig. 1 and the dashed curve of Fig. 2 plot the difference in absorption spectrum between illuminated and dark anaerobic cells.

The absorption peaks at 550 and $515 \mathrm{~m} \mu$ in the $R$. rubrum difference spectrum and at 508, 47., and $445 m \mu$ in that with $R$. spheroides result from a decrease of the absorption peaks of the carotenoid pignents of these organisms on illumination, while the troughs at $575 \mathrm{~m} \mu$ in $R$. rubrum and at 525, 490, and $460 m_{\mu}$ in $R$. spheroides result from the appearance of absorption peaks at 

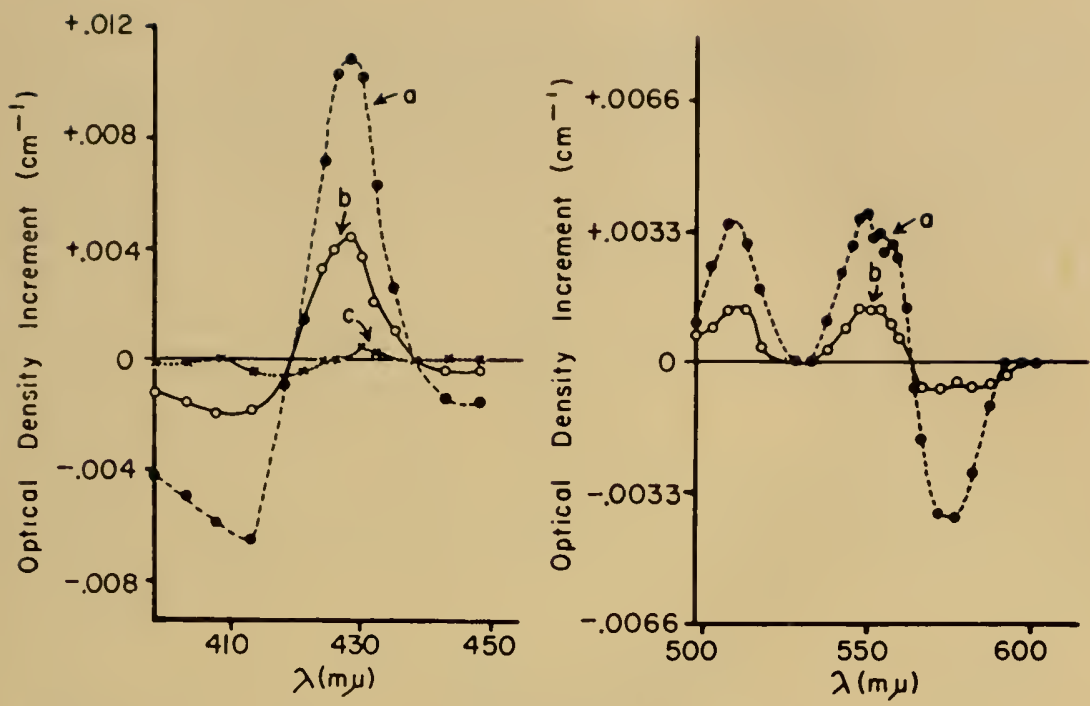

Fig. 1. Difference spectra of Rhodospirillum rubrum grown under illumination. Measurements were made in the double-beam spectrophotometer at the wavelengths indicated. $a$, Anaerobic minus aerobic bacteria in the dark. $b$, Dark minus illuminated anaerobic bacteria. $c$, Dark minus illuminated aerobic bacteria. Different samples of bacteria were used in the two wavelength regions.

longer wavelengths (11). Figs. 1 and 2 also plot the difference in absorption spectrum between anaerobic and aerobic cells in the dark. These difference spectra show that the same carotenoid difference spectra result from either illumination of the anaerobic cells or from addition of oxygen in the clark. Since we are discussing here the reactions of cytochromes, the changes of absorption spectrum related to the carotenoid pigments will be only briefly summarized (12). The changes of carotenoid absorption spectrum take place very rapidly on illumination, more rapidly than the oxidation of the cytochromes. (2) The carotenoid changes can be seen, under some conditions, when there are very small or no cytochrome changes. (3) The rapid shift in carotenoid absorption bands to longer wavelengths appears to follow any procedure which initiates electron transport processes in the bacteria, either in light or dark.

The changes in absorption spectrum in the region between 400 and $450 \mathrm{~m} \mu$ shown in the difference spectra of Figs. 1 and 2 result from the oxidation of one or more cytochromes. This is the region of the absorption spectrum where the large "Soret" or $\gamma$-absorption bands of the cytochromes are seen. In $R$. rubrum the changes in the $\alpha$ and 


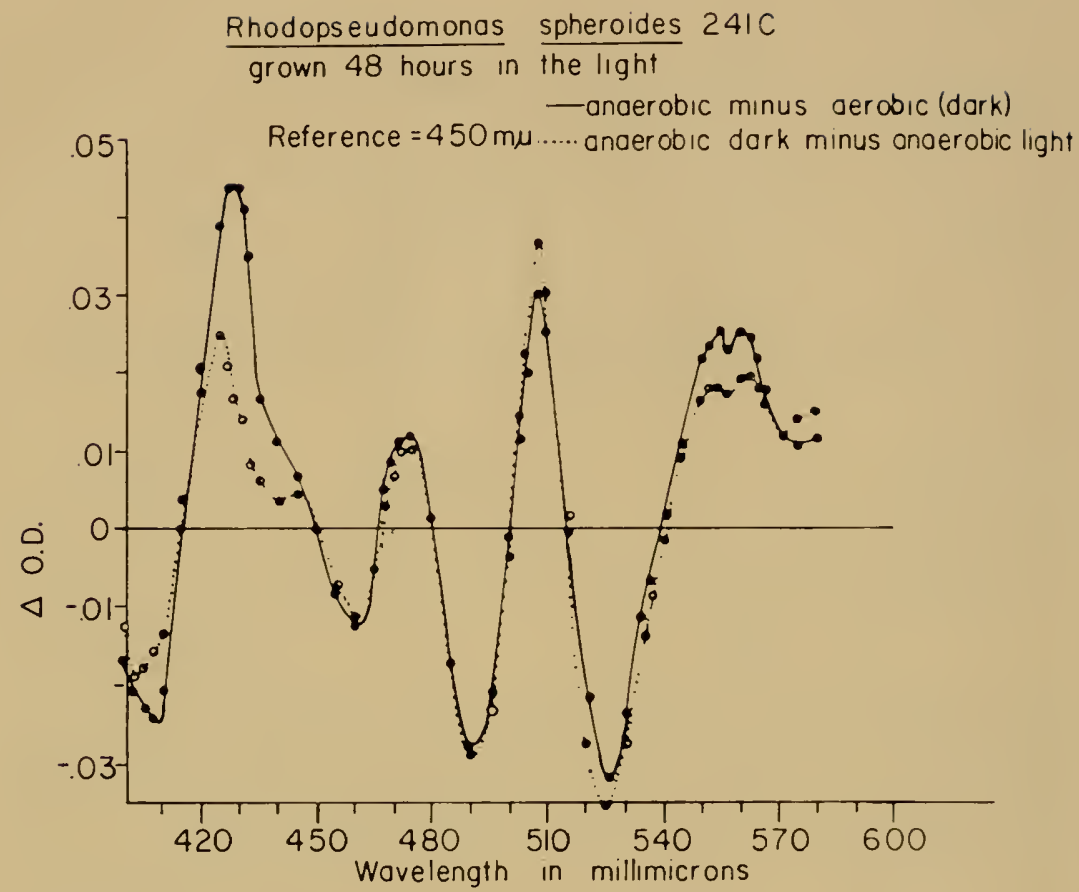

Fig. 2. Difference spectra of $R$. spheroides grown nuder illumination. The measurements were made in the double-beam spectropholometer at the wavelengths indicated; the reference wavelength was $450 \mathrm{~m} \mu$. The bacteria were used clirectly from the culture bottle and contained $1.3 \mathrm{mg}$ prolein per $\mathrm{ml}$.

$\beta$ cytochrome absorption bands are obscured by the large changes in the carotenoid absorption spectrmm. Small changes in the $\alpha$ absorption bands of the cytochromes can be seen in the difference spectra of R. spheroides (Fig. 2) around $550-560 \mathrm{~m} \mu$, where they are superimposed upon the carotenoid changes. Thus the cytochrome reactions can be seen most clearly in the region of the Soret bands. The difference spectral of Fig. 2 show that different cytochrome pigments are oxidized on illumination, as compared with those oxidized on addition of oxygen in the dark. This relationship is shown more clearly in Fig. 3, which plots the difference spectra of $R$. nubrum in the region of the Soret cytochrome peaks under conditions where the intensity of illumination was greater than that used in Fig. I (12). The cytochromes oxidized on acration of bacteria in the dark would be those of the "respiratory chain," over which substrates are oxidized by molecular oxygen. The bacteria can grow either under illumina- 
tion or in the dark with acration, and the same substrates can be oxidized in the two conditions (13). The difference spectra of Fig. 3 show that illumination results in the oxidation of the same cytochrome system which is oxidized in the dark with oxygen, and in addition at least one more cytochrome is also oxidized on illumination. The latter cytochrome has a peak in the difference spectrum around $420 \mathrm{~m} \mu$, which would correspond to the cytochrome $c_{2}$ of the bacteria, which has been isolated from these organisms by Vernon $(14,4)$. Isolated cytochrome $c_{2}$ does not interact rapidly with the dark respiratory chain of the bacteria (8).

Further studies were made with cell-free extracts of $R$. nuburn, which can respire in the lark (8) and, as shown by Frenkel (5), can promote rapid phosphorylation of adenosine diphosphate (ADP) on illumination. Fig. 4 shows that illumination of cell-free extracts

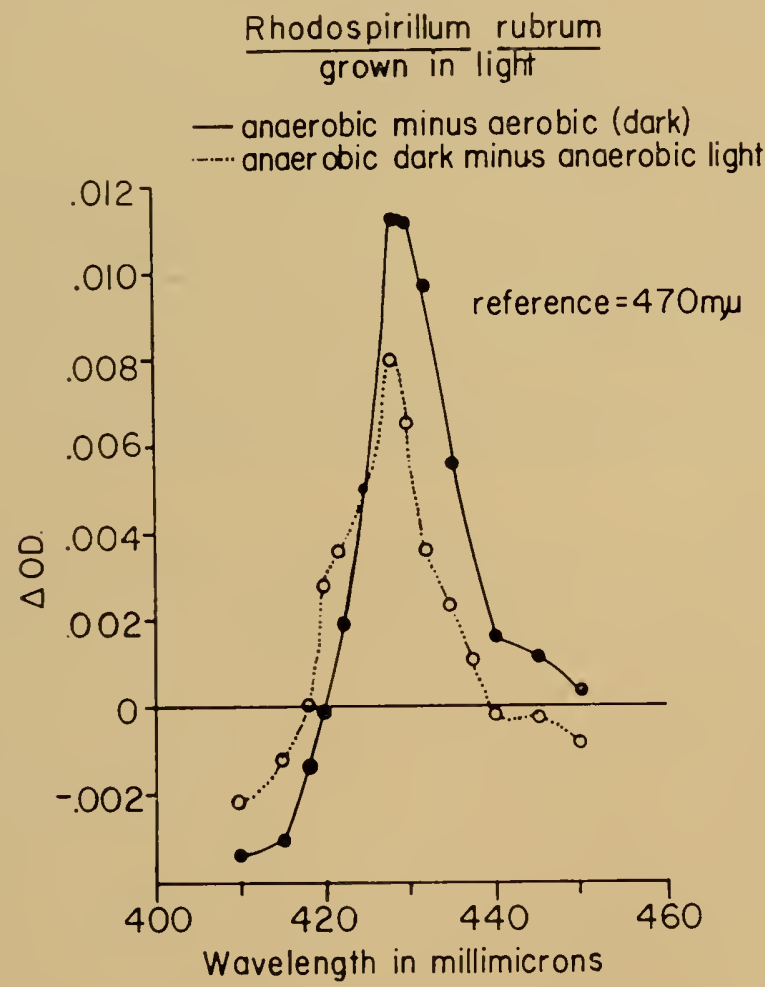

Fig. 3. Difference spectra of $R$. rubrum grown under illumination. The data were obtained with the double-beam instrument at the wavelengths indicated; the reference wavelength is $470 \mathrm{~m} \mu$. 


\section{Rhodospirillum rubrum extract \\ Anaerobic extract \\ Difference spectra of \\ illuminated minus dark extract}

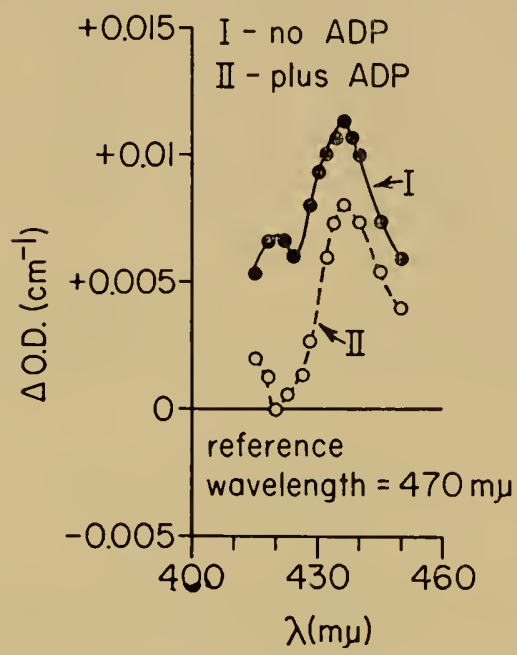

Fig 4. Changes in absorption spectrum of cell-free extract of $R$. rubrum in the presence or absence of ADP. The extract was diluted with buffer $(0.2 M$ glycylglycine, $p \mathrm{H} \mathrm{7.4)}$ containing $0.01 M \mathrm{Mg}^{++}$and $\mathrm{F}^{-}$ions and $0.0067 \mathrm{M}$ inorganic phosphate and $0.015 M$ succinate. Curve I shows the difference in absorption spectrum between illuminated and dark extract in the anaerobic state. Then ADP was added to the same mixture to a concentration of $0.0034 M$ and curve II was obtained, which represents the difference between illuminated and dark extracts in the anaerobic state when light-induced phosphorylation was taking place.

diluted in buffer containing magnesium and phosphate ions results in no oxidation of cytochrome pigments (9). A broad absorption band appears around $434 m \mu$ on illumination. The compound responsible for this absorption band is unknown, but it probably is not involved in normal light-induced reactions $(10)$. When the same particulate suspension is illuminated after addition of a phosphate acceptor $(\mathrm{ADP})$, a trough is seen in the illuminated minus dark difference spectrum at $420 m \mu$, indicating that the cytochrome $c_{2}$ becomes more oxidized on illumination under conditions where lightinduced phosphorylation is proceeding. This implicates the cytochrome $c_{2}$ in an electron-transfer system which reacts during illumination to promote the phosphorylation of $\mathrm{ADP}$. Frenkel has shown that substrates are not oxidized during this reaction (6). There seems to be obligate coupling of the oxidation-reduction reactions involving 
cytochrome $c_{2}$ and the phosphorylation systent. Krogman et al. have also shown evidence of obligate coupling of phosphorylation and electron transport in chloroplasts $(7)$.

There is additional evidence (9) for the existence of two electrontransport chains in $R$. rubrum, one being the respiratory chain system which can oxidize substrates with oxygen and a second chain which is involved in phosphorylation of ADP on illumination. The two chains of oxidative enzymes show different reactivities on addition of substrates or inhibitors; these effects are summarized in Tables 1 and 2.

The scheme presented in Fig. 5 pictures the simplest systems which would explain our observations on the photosynthetic bacteria (9). It includes two cytochrome chains, one of which is the respiratory

\section{TABLE 1}

Respiration and Light-induced Phosphorylation in Extracts of Rhodospirillum rubrum

\begin{tabular}{lcccc}
$\begin{array}{c}\text { Substrate } \\
\text { added }\end{array}$ & $\begin{array}{c}\text { Dark respiration } 29.7^{\circ} \mathrm{C} \\
\mu \mathrm{l} \mathrm{O}_{2} / \\
\mathrm{hr} \times \mathrm{D}_{800}\end{array}$ & $\begin{array}{c}\text { Light-induced } \\
\mathrm{hr} \times \mathrm{D}_{800}\end{array}$ & $\begin{array}{c}\text { Oxidative } \\
\text { phosphorylation } \\
28^{\circ} \mathrm{C}\end{array}$ & $\begin{array}{c}\text { in dark } \\
\text { inhorylation }\end{array}$ \\
\hline None & 12 & 0.5 & 40 & very low or none \\
\hline $\begin{array}{l}\text { Succinate } \\
20 \mathrm{mM}\end{array}$ & 83 & 3.6 & 42 & very low or none \\
& 12 & & &
\end{tabular}

TABLE 2

EFfect of Inhibitors on Respiration and Light-induced Phosphorylation IN Extracts of Rhodospirillum rubrum

\begin{tabular}{|c|c|c|c|c|c|}
\hline \multirow{2}{*}{ Inhibitor } & \multirow{2}{*}{$\begin{array}{l}\text { Concentration } \\
\text { of inhibitor }\end{array}$} & \multicolumn{2}{|c|}{$\begin{array}{l}\text { Respiration in dark } \\
\text { per cent inhibition }\end{array}$} & \multicolumn{2}{|c|}{$\begin{array}{l}\text { Light-induced } \\
\text { phosphorylation } \\
\text { per cent inhibition }\end{array}$} \\
\hline & & $\begin{array}{l}\text { no added } \\
\text { substrate }\end{array}$ & $\begin{array}{c}\text { succinate } \\
20 \mathrm{mM}\end{array}$ & $\begin{array}{l}\text { no added } \\
\text { substrate }\end{array}$ & $\begin{array}{l}\text { succinate } \\
20 \mathrm{mM}\end{array}$ \\
\hline
\end{tabular}

3-Hydroxyl-

1-heptyl quinoline

$10^{-6} M$

0

0

100

100

Ni-oxime

Phenylmercuric

$10^{-4} M$

33

45

acetate

\begin{tabular}{lccccc}
\hline Cyanide $^{*}$ & $10^{-4} M$ & 44 & 0 & 0 \\
\hline Malonate & $0.1 M$ & 59 & 69 & 17 & 35
\end{tabular}

* Data on cyanide inhibition of respiration are crratic. Unless acetate is also present with the succinate, sometimes $10^{-4} \mathrm{M}$ cyanide is more inhibitory than $10^{-3} \mathrm{M}$. Possibly keto acids, formed in the absence of acetate, will bind cyanide. 


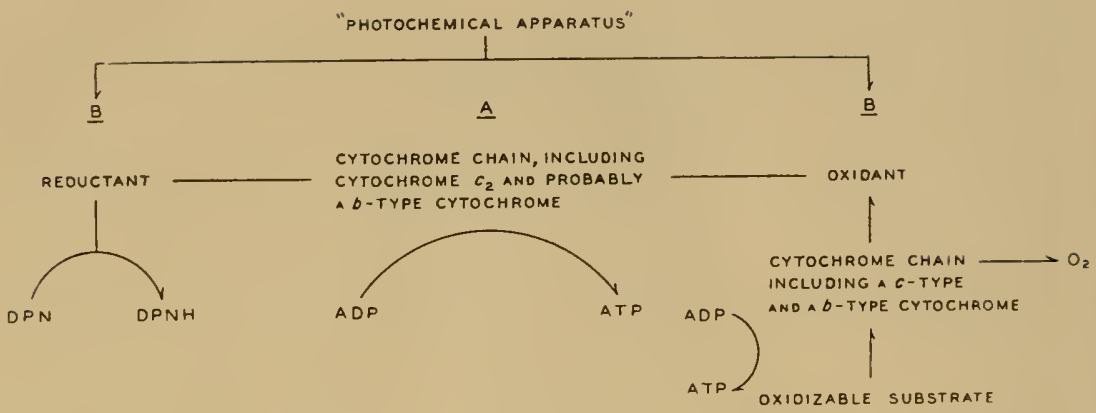

Fig. 5. Schematic representation of the postulated electron transport chains in photosynthetic bacteria.

chain which can oxidize substrates with oxygen in the dark or can pass electrons to the oxidant formed upon illumination of the bacteria. In the latter reaction, an equivalent amount of photoreductant brings about the reduction of DPN. The reduction of DPN upon illumination of $R$. rubrum has been demonstrated by Duysens and Sweep (3) . The photosynthetic bacteria do not evolve oxygen, but instead oxiclize a substrate (13). In the system pictured, ATP is formed from ADP on oxiclation of substrate either by chain $B$ reacting with oxygen or the photo-oxidant. In addition a second electron-transport chain (chain $A$ ) exists, over which the photoreductant and the photooxidant can interact with the phosphorylation of ADP. All of our observations, particularly those on the effects of inhibitors (9), indicate that the latter electron-transport chain is similar in properties to the extensively studied enzyme system which mediates oxiclation of substrates by molecular oxygen with accompanying phosphorylation. This agrees with the idea that similar pigments, such as $b$-and $c$-type cytochromes, are components of the chain, and that the light-induced phosphorylation may have a similar mechanism to that of "oxidative phosphorylation." This is all in agreement with the suggestions of Dr. Hill on the role of the cytochromes in the oxidation-reduction reactions of photosynthesis.

\section{REFERENCES}

1. Chance, B., Rev. Sci. Instr., 22, 619 (1951).

2. Chance, 13., and Smith, L., Nature, 175, 803 (1955).

3. Duysens, L. N. M., and Swecp, G., Biochim. el Biophys. Acta, 25, 13 (1957).

4. Elsden, S. R., Kamen, M. 1., and Vernon, L. P., J. Am. Chem. Soc., 75, 6347 (1953).

5. Frenkel, A., J. Am. Chem. Soc., 76, 5568 (1951). 
6. Frenhel, A., J. Biol. Chem., 222, 823 (1956).

7. Krogman, D). W., Jagendorf, A. T., and Avron, M., Plant Physiol., 34, 272 (1959).

8. Smith, L., J. Biol. Chem., 234, 1571 (1959).

9. Smith. L., and Baltscheffsky, MI., J. Biol. Chem., 234, 1575 (1959).

10. Smith, L., Baltscheffsky, M., and Olson, J. M., J. Biol. Chem., 235, 213 (1960).

11. Smith, L., and Ramires, J., Arch. Biochem. Biophy's., 79, 233 (1959).

12. Smith, L.. and Ramíres, J.. J. Biol. Chem., 235. 219 (1960).

13. Van Niel., C. B., Adr, Enzymol., 1, 263 (1941).

14. Vernon, L. 1'., Arch. Biochem. Biophys., 43, 492 (1953).

1.5. Yang, C. C., and Legallais, V., Rev. Sci. Instr., 25, 801 (1954).

\section{DISCUSSION}

Dr. Lipuani: I think Dr. Smith, in summarizing, has said about what I want to sily, namely, that mitochondrial phosphorylation and photophosphorylation are very similar. I do not think that the term reductive phosphorylation should be used for photophosphorylation. Dr. Hill has given us earlier in his talk a scheme in which the $Y O$ is about at the potential of oxygen, that is, +0.81 and the $\mathrm{XH}$ is at about the potential of hydrogen, that is, -0.41 , and all that light does is make $Y O$ and $X H$. I am a little less prejudiced with oxygen because when Dr. Frenkel found Rhodospirillum photophosphorylation in my laboratory, it was quite obvious that it had no relation to oxygen evolution. It was fortunate, I believe, that we got our first glimpse at it in this bacterial system. It became very soon evident that no substrate was involved or needed in this system of photophosphorylation; the light in this system was furnishing both oxidant and reductant. Photophosphorylation was coupled, as far as we could see, to the recombination of the oxidant and the reductant producing the same gradient in potential differences as is set up in the mitochondrial system by oxygen against substrate. There certainly are differences between photophosphorylation and oxidative phosphorylation. One of them is that photophosphorylation is not as sensitive to the classical uncouplers such as dinitrophenol. In his early experiments, Dr. Frenkel thought that nitrophenol was entirely inactive. However, Geller later could show that nitrophenol in higher concentration interrupts photophosphorylation. Dicunerol, which is a good uncoupler in oxidative phosphorylation, uncouples in many systems of photophosphorylation; and a substance which we have been using as an uncoupler and which is related to thyroxin (the compound is the butyl ester of diiodo-para-hydroxybenzoic acid) uncouples photophosphorylation in all cases where we have tried it in very similar concentrations as it uncouples oxidative phosphorylation. So I agree with Dr. Smith that photophosphorylation and mitochondrial phosphorylation are really very sinilar, although some of the components, e.g., the cytochromes, are of a different type.

Dr. ArNox: 1 would like to say a word about this matter of nomenclature. This may seem at first unimportant, but really is important, because it adds to clarity of thinking. I cannot agree more with what Dr. Lipmann said, that 
insofar ats the recombination of the oxidized and reduced moieties is concerned, we see in photosynthetic phosphorylation a phenomenon very much analogous to oxidative phosphorylation. In other worls, we see high energy phosphate bonds made at the expense of energy released during electron transport. There are, however, fundamental clifferences. In photosynthetic phosphorylation, we do not use or do not consume an external hydrogen donor, and we do not consume external oxygen. I will have more to say about this this afternoon. So, in one case we have to pay for it very dearly by providing a substrate for getting the energy and by providing oxygen. In the other case, the only substrate which is being used is light. Light makes both the oxidant and the photoreductant. For this reason, we introduced the term photosynthetic phosphorylation to distinguish it from oxidative phosphorylation while fully maintaining the concept of electron flow between oxidized and reduced moieties.

Dr. McElroy: I would say I need clarification on that because it seems to me what you are saying is that you are merely using light energy to create your substrate and your oxidant. That is the same as what Dr. Lipmann just said.

Dr. ARNon: I find it clarifying to think that light and cytochrome constitute the oxidant in photosynthetic phosphorylation. This is what we replace oxygen with. At the reducing end, we release an electron, which instead of combining with oxygen as it does in oxidative phosphorylation, it recombines with chlorophyll via cofactors and cytochrome. I think it is very important to keep in mind the distinction between oxidative and photosynthetic phosplorylation. I do not think this is just a play on words. It is rather important whether we consume sugar or succinate or whether we can get ATP without it. That is a rather fundamental point.

Dr. KAMEN: I was somewhat informally deputized to discuss Dr. Hill's paper. However, before begimning my informal remarks, I wish to express my feeling that these symposia are not places to rehash at length things that are in the literature. They should be a forum for presentation of ideas, whether half-baked or wholly baked, which are worthy of consideration even though the work on which they should be based has not been clone. Now many of the ideas that you will hear about cytochrome function in phosphorylation are already in the literature. They are available and I clon't think we should take up our time with them unless there is something to discuss in connection with them which is new. Otherwise, we will never get to things which I think might also be interesting. Now, as I mention in the paper I am submitting, it is a privilege to appear as a discussant in the first place following Dr. Hill inasmuch as it was an attempt to create his reaction in bacteria which led Dr. Vermon and me to our research some 10 years ago on cytochrome. I venture to say that the result of this has been a remarkalble number of investigations. Unwittingly, we opened a Pandora's box. Dr. Vernon and I a long time ago proposed that there would be back- 
oxidation to the cytochrome chain, and in fact we expected something like phosphorylation (which we managed to prevent ourselves from finding by clever reasoning!!). I wish to give Dr. Vemon some credit for the fact that he introduced me to cytochromes. I do not know whether this was a good idea or not. As he has not been mentioned very much in this symposium I feel it imperative to saly that it was his work with Alberty and Malhler on cytochrome $c$ reductase which began all this. Now until recently, Dr. Hill and I were the only ones that talked about cytochromes in photosynthetic systems, so it is very gratifying to see the tremendous amount of interest which is now developing in these pigments. It is not so gratifying to see what little work is being done on them. I must emphasize that we will have no conception of what these are doing in photosynthesis unless we isolate them and determine their physico-chemical properties. And in this connection, what Dr. Arnon said about nomenclature comes up. The term cytochrome has been battered about in a rather glib way. It should be emphasized that the cytochromes we are talking about in the photosynthetic chain are not the cytochromes that are present in mitochondria. In at least three or four instances in which we have gone to the trouble of isolating and crystallizing and determining something about the structure of these proteins, we find we do not have anything like the kind of cytochrome in the respiratory chain.

DR. WALD: How can a thing be reduced by molecular hydrogen without combining with it?

DR. KAMEN: I did not mean to imply no combination. I meant that there is no combination without reduction.

Dr. WALD: Is not that a pretty unusual reaction you have in your scheme with molecular hydrogen?

DR. KAMEN: There are hydrogenases present.

DR. WALD: Isn't RHP one of the hydrogenases?

DR. KAMEN: No. RHP is not a hydrogenase. There is a special hydrogenase enzyme in Rhodospirillum rubrum which can be extracted and purified and which appears to be the mechanism for the introduction of hydrogen as a hydrogen donor in the photosynthetic process.

Dr. Rabinowitch: I am glad that someone goes beyond the "ordinary" cytochromes, and "ordinary" TPN and thinks of "super-cytochromes" with an oxidation potential permitting them to oxidize water, and of "superTPN" with a potential permitting it to recluce TPN. But if so, how do you know that the oxidant is an iron complex, and not something altogether different?

Dr. KamEx: Why, indeed? I would like to make a plea that people stop for the moment and work hard on the molecular analysis of chloroplasts and cliromatophores. There are many crucial problems in photosynthesis which have not been solved yet. One is, what is in the chloroplasts? and what is in the chromataphore? Except for the state of knowledge back in 
the early 1900's that there was carbon, oxygen, nitrogen and sulfur, and in 1935 that there might be fat, proteins, and carbohydrates, and now that we have cytochromes, flavins, quinones, and some metals and a few enzymes, there are still many gaps. We cannot make a molecular balance sheet for these particles. I feel that it will be very hard to explain what these particles do without a molecular balance sheet. The same is true for other biological structures of course. In this connection one asks what is the minimum number of things that you need to make a phosphorylation system. Here a biological approach is required. The problem is, how do you construct a structure biologically which will begin to do something at a certain point in its evolution? Nobody has done this yet. What has been clone is take organisms which are alrearly established, chop them to pieces, and then dig out the pieces and find the smallest piece and say this is the minimal structure. The biochemical criterion is that if you get to a point where nothing happens with some of these structures and you can add something back to get back to activity, you have the minimal structure. This really does not mean that you have the minimal amount. The point is how few chlorophylls do you need, how few proteins, how few flavins, etc. Until this question is settled, it will be very difficult indeed to decide about the metal requirement, be it iron or copper or manganese. Copper and manganese are certainly some of the possibilities, among many.

Dk. Rabinowitch: I think that it makes the game less interesting to think that everything is either pyridine nucleoticle or cytochrome.

Dr. Kamen: Well, at least we are making progress; up to now we were restricted to $x$ and $y$.

Dr. Linschitz: In connection with Dr. Kamen's remarks on possible interactions between excited chlorophyll and cytochromes. l'd like to mention some studies we have made on the quenching of triplet states by heary metal ions. The mechanism we have been forced to assume for this quenching process on the basis of evidence which I do not want to take time to go into, but which is in a paper in press at the moment, involves a charge-transfer interaction between the excited states, call it the triplet state, of authracene or porphyrin, and the metal ion. This is followed by a rearrangement of charge inside the complex leading back to the ground state. One gets the same kind of mechanism for quenching of anthracene or porphyrins. It is now of interest to look at the eflect of complexing of the metal ions on the rate of this process. One finds indeed that the quenching is very sensitive to the presence of complexing groups which block oll the metal ions, but if you use porphyrin complexes the rate continues at a high value. In fact, we are now extending this work to the quenching of chlorophyll triplets by other porphyrins. The only data I can give now is on the quenching of anthracene triplet by copper, copper chloride uncomplexed, and copper porphyrin complex. The rates in each case are very high. That is to say, the blocking off of the metal by this very large group in no way impecles the formation of the charge-transfer complex. 


\title{
LIGHT, PIGNENTS, AND PHOTOSYNTHESIS
}

\author{
C. S. French \\ Department of Plant Biology, Carnegie Institution of Washington \\ Stanford, Califormia
}

\section{INTRODUCTION}

Chlorophyll has long been known to be the major light-absorbing pigment in plants. Somehow it converts its captured energy into chemical form. About 1880, Engelmann lound that the red and blue phycobilin pigments of some algae also used their absorbed light for photosynthesis. Furthermore, the light absorbed by some carotenoids is photosynthetically active. The name "accessory pigments" has become associated with all photosynthetically useful pigments other than chlorophyll. The nature of these accessory pigments and the evidence for their participation in photosynthesis has been discussed by Blinks (5), in an article with a full bibliography. Some of the properties of accessory pigments and some recent experiments concerning the way in which they are used in the process of photosynthesis will be reviewed here.

The general idea has been that accessory pigments help the plant to catch more of the incident energy because they can take up green light, which is poorly absorbed by chlorophyll. The accessory pigments can transfer their absorbed energy to chlorophyll a. This transfer process is detected through the emission of chlorophyll fluorescence by plants exposed to light of wavelengths that are absorbed almost entirely by the accessory pigments $(12,13,29,59)$. Energy transfer has been considered the mechanism by which the accessory pigments function. in photosynthesis. Thus the carotenoids, the phycobilins-phycoerythrin and phycocyanin-and chlorophyll $b$ have been thought of as accessory only in the sense of extra light-gatherers that funnel the light into the usual channel of photosynthesis via chlorophyll $a$.

Several experiments of the last few years lead to a different idea of the function of accessory pigments. The accessory pigments now seem to have a much greater importance than had previously been realized. Apparently they are responsible for another chemical reac- 
tion different from and accessory to that performed by chlorophyll $a$.

The accessory function looks like a specific chemical effect rather than a simple increase in the light-absorbing capacity of plants. Furthermore, it appears that chlorophyll $b$, which is in all ordinary land plants, plays the same accessory role as do the more spectacular coloring materials of the blue-green, red, and brown algae.

The two experiments forcing a revision in the concept of the function of accessory pigments are the chromatic transient experiments and the enhancement of photosynthesis by two wavelengths given together. Furthermore, the comparative inactivity of chlorophyll in red algae $(33,61)$, as well as the expancling realization of the simultaneous occurrence of several different forms of chlorophyll $a$ in plants, has contributed to the present picture of the interrelated function of plant pigments in photosynthesis.

We will first review the various photosynthetic pigments, together with the typical spectra by which they may be identified in living plants. Then we will consicler the action spectrum measurements that in comparison with absorption spectra have shown these accessory pigments to be active in photosynthesis. An improved method for measuring action spectra and its use in studying the enhancement spectra will be mentioned. An unusual new fact, the variation of photosynthesis rate with wavelength at saturating intensity, discovered by McLeod (47), will be clescribed. In conclusion, some relations between several other effects related to the separate function of individual photosynthetic pigments will be postulated.

All of these phenomena are believed to be due to the interaction of different photochemical products formed by the action of light on different groups of pigments. In short, it appears that there are qualitative differences in the chemistry of photosynthesis dependent upon the pigments by which incoming light quanta are collected and channeled.

\section{Photosy nthetic Pignents in Plants}

\section{Chlorophyll a}

The main red absorption band at $670-680 \mathrm{~m}_{\mu}$ of all photosynthetic plants except the photosynthetic bacteria is due to chlorophyll $a$. In ether solution, chlorophyll a has absorption bands at 662, 615, $578,534,430$ and $+10 \mathrm{~m} \mu$ as well as other minor bands (55). (The major bands are italicized.) In absorption spectra of live plants the chlorophyll a bands may be identified but are found at longer wave- 
lengths than in ether solution. The blue absorption bands of chlorophyll are largely obscured by carotenoid absorption and the 615, 578, and 535 bands are small. The spectroscopic study of chlorophyll $a$ in live plints is therefore mainly restricted to the major red absorption band. The shape of this band has been investigated in many different algate and found to be a composite of several individual components (26).

The purple bacteria show various forms of the complexed bacteriochlorophyll with great clarity, since the bands are more widely spaced than in green plants $(13,19,36,57,58)$. Duysens (13) designates the forms of bacteriochlorophyll in vivo as B800, B860, $\mathrm{B} 870$, B890, etc., according to the wavelength of the main absorption peak of each type. IVe use this notation to distinguish the various forms of chlorophyll $a$ and $b$ and protochlorophyll in plants as $\mathrm{C}_{\mathrm{b}} 650, \mathrm{C}_{\mathrm{a}} 673, \mathrm{C}_{\mathrm{a}} 695, \mathrm{P}_{635}, \mathrm{P}_{6: 0}$, etc.

The chemical nature of the natural chlorophyll complex (holochrome) is not known. In green plants chlorophyll $a$ is present in several different forms, like the bacteriochlorophyll in photosynthetic bacteria, but the bands in green plants are much closer together. Chlorophyll $a$, instantly after its formation in leaves from protochlorophyll, absorbs at $682 \mathrm{~m} \mu .(53,54)$, as seen in Fig. 1 (fluorescence peak $691 \mathrm{~m}_{\mu}$, Latimer, 44). After a few minutes of illumina-

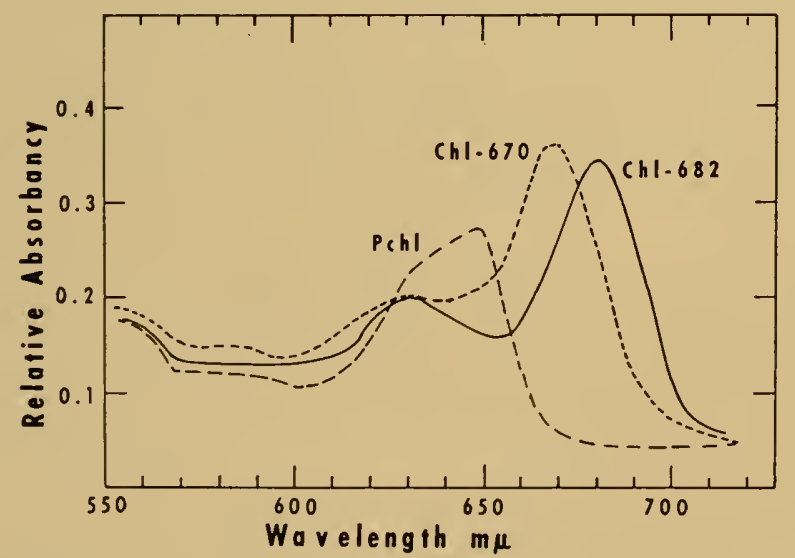

Fig. 1. Two kincls of chlorophyll $a$ in a bean leaf. The dashed curve, Pchl, is the absorption spectrim of protochlorophyll in a bean leaf grown in the dark. The full line, Chl 682, is the absorption of the freshly formed chlorophyll $a$ iminediately after exposure to light. The dotted curve. Chl 670, shows the spectrum after the same leat had been $30 \mathrm{~min}$. in the dark following illumination. From J. H. C. Smith (54). 
tion, or in the dark, the peak position of the newly formed $C_{a} 682$ shifts to $670 \mathrm{~m} \mu$. Mature green plants and algae usually contain both $\mathrm{C}_{\mathrm{a}} 682$ and $\mathrm{C}_{\mathrm{a}} 670$ in roughly equal amounts ( $1 \mathrm{l}$ ), giving an absorption peak at $675-680 \mathrm{~m} \mu$.

The chemical nature of the different forms is not known, but their simultaneous presence can be recognized from detailed analyses of the shape of the red absorption band which, in live plants, is the sum of several overlapping components. The forms of chlorophyll $a$ at present recognizable in the plant or in the water extracts of chloroplast particles have their red absorption peak at about 670 , 682 , and $695 m \mu(3,10,11)$, but all these substances revert to ordinary chlorophyll a on extraction with organic solvents. These various forms of the natural holochrome are therefore different kinds of a complex containing a single extractable chlorophyll. The diversity of the spectra of live plants is due to the simultaneous presence of these forms in cliffering proportions in vivo. The exact wavelength of these forms may vary a few $\mathrm{m}_{\mu}$; a fine distinction of the peak positions of overlapping components better than $\pm 2 \mathrm{~m}_{\mu}$ is not yet possible.

Because these absorption bands overlap extensively, their presence is hard to detect in absorption spectra as ordinarily measured. We therefore measure the first derivative of absorbance with respect to wavelength. These derivative spectra show the presence of the individual components by shoulders or extra peaks on the derivative curves, as in Figs. 2 and 3. To get an approximate idea of the peak positions and relative quantities of the components represented by derivative spectra, we have attempted to match the observed spectra by adding together derivatives of normal probability curres, as illustrated in Fig. 4.

The forms $\mathrm{C}_{\mathrm{a}} 684$ and $\mathrm{C}_{n} 673$ are those whose presence has long been suspected, and which have been frequently used to explain various phenomena $(37,41,42,43,60)$. Their separate identity is clearly shown by the characteristic shoulder near the peak absorption wavelength in the derivative spectra of Chlorella and other green algac, Fig. $2(25,26) . \mathrm{C}_{\mathrm{i}} 684$ is probably the major functional entity classically known as chlorophyll $a$ in vivo. $\mathrm{C}_{n} 673$ may do the same job as $\mathrm{C}_{\mathrm{n}} 684$ and also is suspected to function in part as does $\mathrm{C}_{\mathrm{b}} 650$, as we will see later. There is also evidence for less effective light utilization by $\mathrm{C}_{21} 684$ than by $\mathrm{C}_{\mathrm{a}} 673$ in red algae $(30,33,61)$.

The $\mathrm{C}_{\mathrm{a}} 695$ form found in weak-light cultures of Euglena (25) also appears in small amounts in the detailed analysis of many plant 


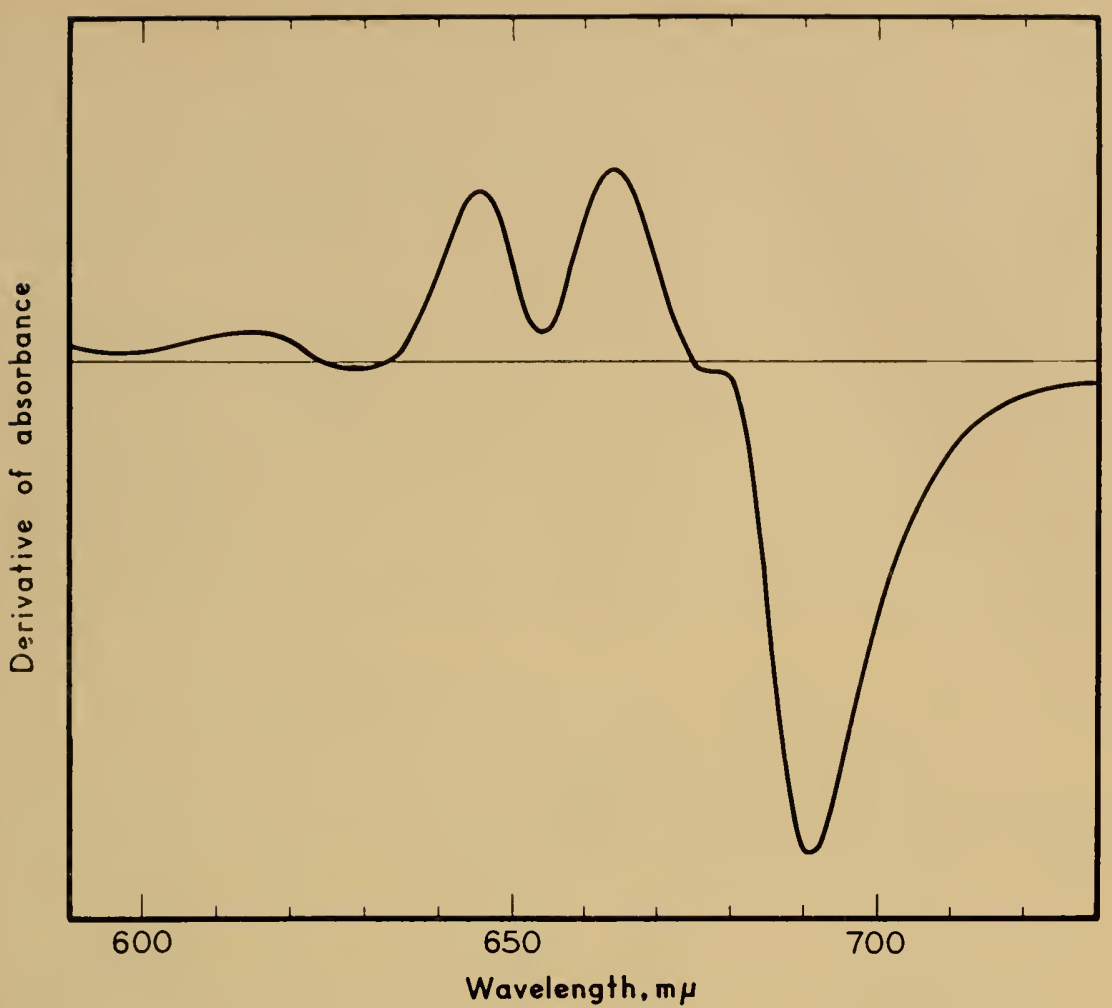

Fig. 2. The derivative absorption spectrum of living Chlamydomonas moewusii, Starr No. 96, which is typical of green algae. The peak and dip at 640-660 $\mathrm{m} \mu$ is due to chlorophyll $b$. The shoulder at $675-680 \mathrm{~m} \mu$ indicates the presence of two components of chlorophyll $a$.

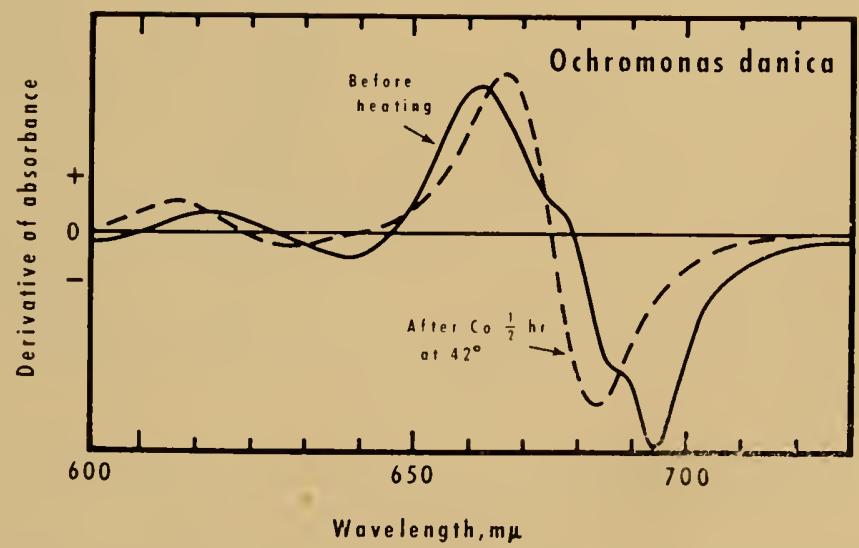

Fig. 3. The derivative absorption of Ochromonas danica, showing three forms of chlorophyll a. Warming destroys the longer wavelength components. From (3). 
spectra $(10,11)$. This $\mathrm{C}_{\mathrm{a}} 695$ pigment is shown below to be the form of chlorophyll a which, when activated together with chlorophyll $b$ in green algae or with phycobilins in red algae, is responsible for the Emerson enhancement effect.

$\mathrm{C}_{\mathrm{a}} 707$, shown in Fig. 5, has occasionally appeared in abundance in both Ochromonas danica (3) and Euglena gracilis (10). The conditions promoting its formation in large amounts are not at all understood, but, like $\mathrm{C}_{\mathrm{a}} 695$, its presence in smaller quantities is indicated in some leaves and algae by analysis of their derivative absorption spectra. Recent unpublished work of J. S. Brown has shown a correlation of $707 \mathrm{~m} \mu$ absorption with the pheophytin content of the cells. It may be that $707 \mathrm{~m} \mu$ absorption is due to a combined form of

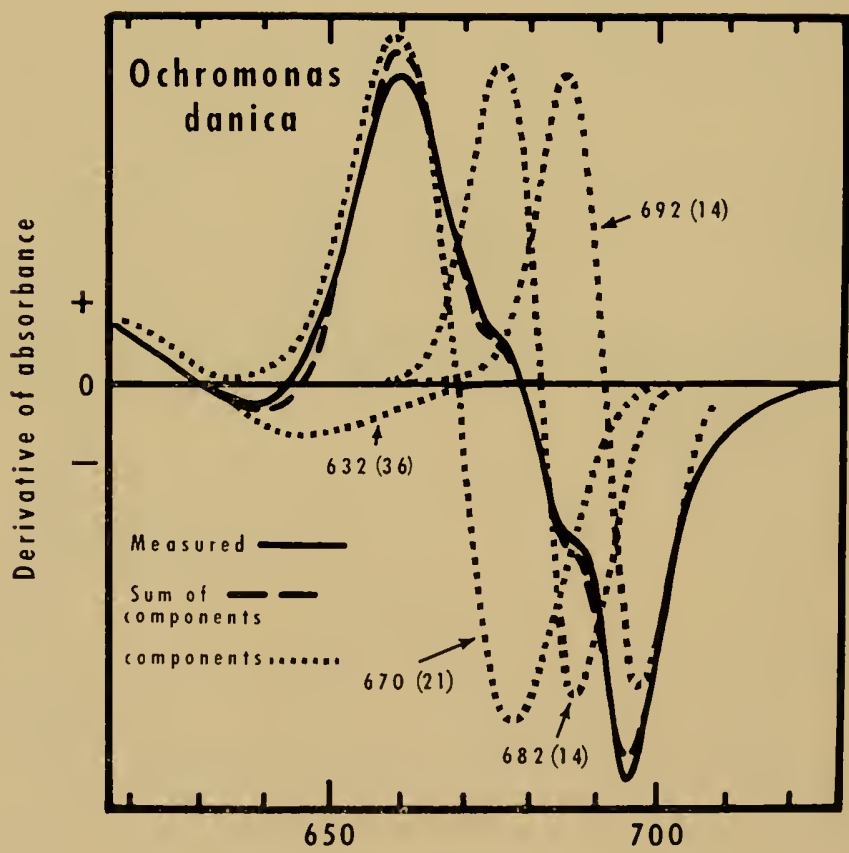

Wavelength, $m \mu$

Fig. 1. The analysis of a derivative absorption spectrum of Ochromonas danica. the individual components are derivatives of normal probability curves. The sum of these componcms is shown as the dashed curce that very nearly matches the meastued spectrmm. The half widths of the components are given in parentheses. from (3). 


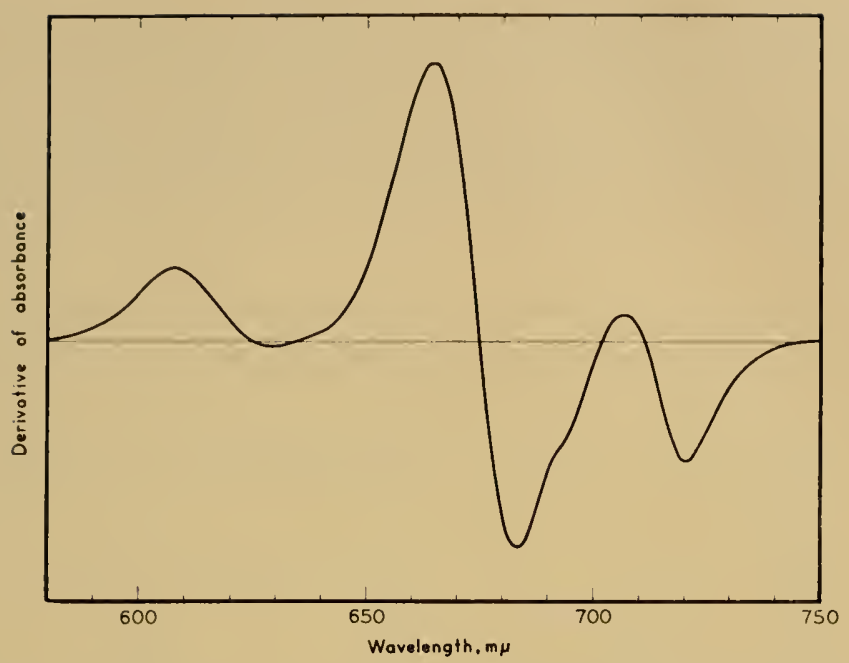

Fig. 5. The derivative absorption spectrum of a culture of Ochromonas danica having a large amount of a chlorophyll $a$ form with an absorption peak at about $712 \mathrm{~m}_{\mu}$. (Unpublished measurements of M. B. Allen and J. S. Brown).

pheophytin rather than of chlorophyll. Kok (38, 39) found reversible changes of absorption at $705 \mathrm{~m} \mu$ on illumination of cells.

Whether these different forms of chlorophyll may have their own specific functions in photosynthesis is a problem of major interest to our group at the present time.

\section{Chlorophyll b}

In ether, chloroplyyll $b$ has absorption bands at $674,595,549,455$, and $730 m \mu$ (55). The "644" band is recognizable in plants at about $650 \mathrm{~m} \mu$. The absorption spectra of plants usually have a shoulder at $480 \mathrm{~m}_{\mu}$ which is generally attributed to carotenoids. Barer (4) has suggested that the $480 \mathrm{~m} \mu$ band in vivo may be primarily due to the chlorophyll $b$ band found in ether solution at $455 \mathrm{~m} \mu$. This idea may be possible in spite of the umsually large wavelength shift. The other chlorophyll $b$ bands are not generally recognizable in spectra of whole plants. Even the $650 \mathrm{~m} \mu$ band is only a shoulder on the side of the red chlorophyll a absorption. In Ulva, but in no other plant studied, there are two forms of chlorophyll $b$, or else there is some other umrecognized pigment absorbing near $650 \mathrm{~m}_{\mu}$ in this species $(22,23)$. 
Action spectra for enhancement in brown algae and dinoflagellates reflect some active chlorophyll $c$ absorption, though certainly nothing to indicate a role different from chlorophyll $b$ (Haxo, pers. commun. and Blinks, 9). Since there have been no indications of a photosynthetic function of chlorophyll $d$, it is omitted from this discussion.

\section{Carotenoids}

There is strong absorption by a mixture of carotenoids from about $500 \mathrm{~m}_{\mu}$ to the near ultraviolet in all photosynthetic plants except for

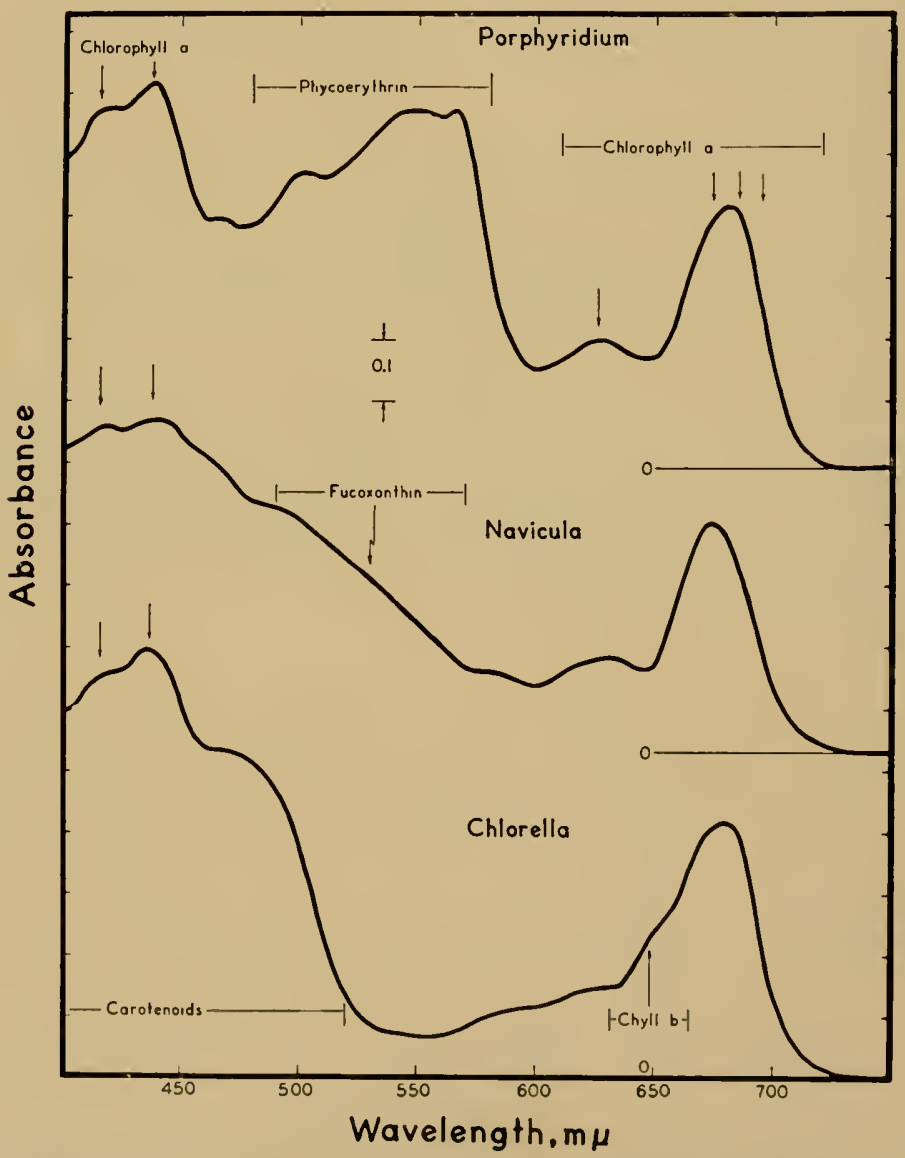

Fig. 6. Absorption spectra of a red alga. Porphyridium cruentum, a diatom, Navicula minima (Starr No. 656), and a green alga. Chlorella rulgaris (Starr No. 398 ), to show the absorption bands of various photosynthetic pigments. (With G. C. McLeod). 
certain green bacteria. Not only are the individual carotenoids inpossible to identify in spectra of intact plants, but they seriously obscure the shorter wavelength chlorophyll bands. Some brown algae and diatoms, however, have broad absorption bands in the 480-550 $m \mu$ region attributable to the carotenoid fucoxanthin in combination with a protein.

\section{Phycobilins}

Unlike clilorophylls and carotenoids, the phycobilins-red phycoerythrin and blue phycocyanin-occur as water-soluble pigment protein complexes. The spectra of the extracted and purified substances may be compared directly, without wavelength shifts, with spectra of intact plants. Both phycoerythrins and phycobilins come in several different types characterized by their spectra. References 1, 2, 5, 28, $30,31,32,34,35,40,50,51$ discuss the properties of some phycobilin pigments studied since the work of Lemberg and Legge (45). Evidence for the participation of the phycobilins in photosynthesis has been reviewed $(5,15,30)$.

Since the phycobilins are highly fluorescent, their fluorescence spectra may also be used for identification in intact plants, if the distortion of the spectrum by reabsorption within the sample is avoided by using very thin layers $(20)$. Phycoerythrin has a fluorescence peak at $578 \mathrm{~m} \mu$ with a broad shoulder around $625 \mathrm{~m} \mu$, phycocyanin fluoresces at $637 \mathrm{~m} \mu$, and allophycocyanin at $663 \mathrm{~m} \mu$ (28). The main absorption bands by which the common photosynthetic pigments may be identified in live plants are shown in Fig. 6.

\section{Action Spectra of Photosynthesis}

\section{The Participation of Accessory Pigments Shown by}

Action Spectra of Photosynthesis

The fact that chlorophyll is the primary light absorber for photosynthesis was guessed long ago from the observation that only green parts of plants produce oxygen. The only clear evidence for this function of chlorophyll is, however, the qualitative agreement of the absorption spectrum of chlorophyll in vivo with the action spectrum for photosynthesis.

A large number of action spectra for photosynthesis have been measured for many species, with techniques varying from some of remarkable ingenuity and high precision to some of ridiculous inadequacy. Comparatively crude action spectra showing the partici- 
pation of accessory pigments were made long ago by Engelmamn. The precise work of Emerson and Lewis $(17,18)$, of Haxo and Blinks (33), of Tanada (56), and of Yocum and Blinks (61), in which the absorption was compared with the action spectra, has substantiated Engelmann's conclusions and resulted in the remarkable discovery of inactive chlorophyll in the red algae. Most of this work is too well known to require further discussion here, but Fig. 7 (from Haxo and Blinks) is given to show an example of action and ab-

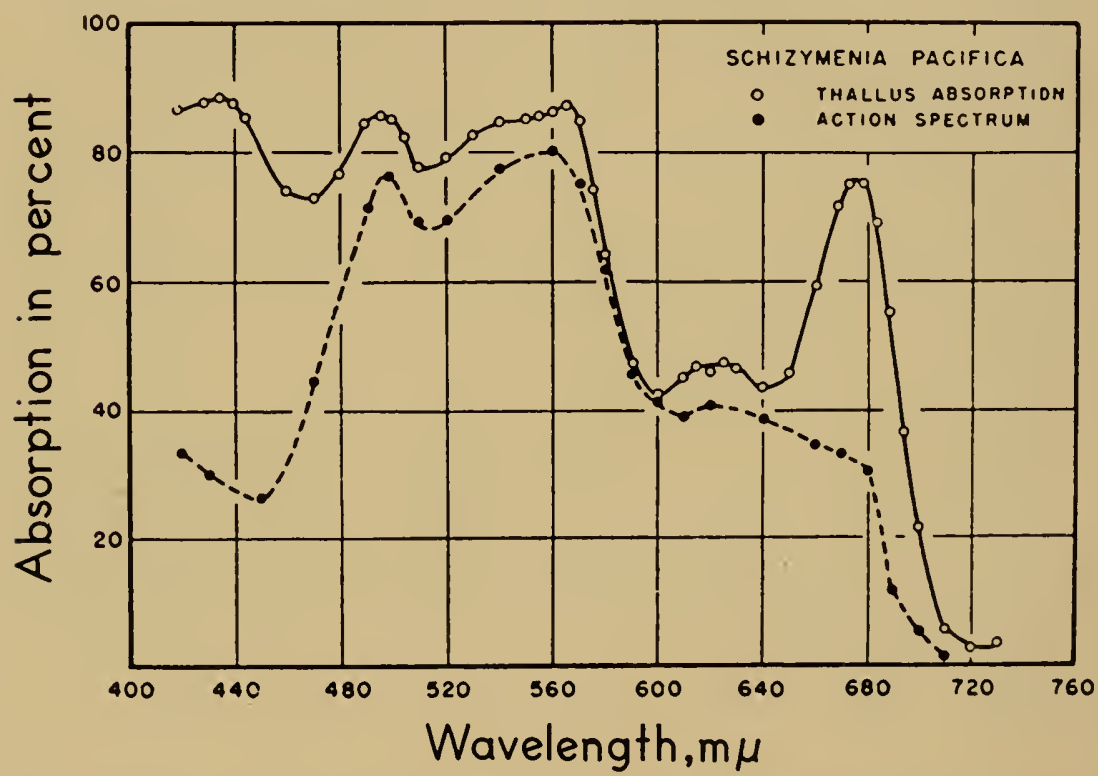

Fig. 7. The action and absorption spectra of a red alga showing more photosynthesis by phycoerythrin than by chlorophyll. From Haxo and Blinks (33).

sorption measurements on the same sample. Some of the principles and techniques useful in such measurements have been reviewed $(21,29,30)$.

\section{Automatic Recording of Action Spectra}

After the $695 \mathrm{~m} \mu$ form of chlorophyll had been found in Euglena, the question arose as to its possible participation in photosynthesis with energy acquired either by direct absorption or through energy transfer. Largely for this reason we thought the time had come to build a device that had been in mind for some time. This is a recording spectrophotometer to plot action spectra with somewhat 
the same convenience that commercial instruments record absorption spectrat. $\Lambda$ block diagram is given in Fig. 8.

The principle of the action spectrophotometer is to use nonochromatic light slowly changing in wavelength and having its intensity continuously adjusted to keep the rate of photosynthesis at a constant value as the spectrum is traversed. This intensity adjustment is done automatically by a servo-driven optical wedge con-

\section{Photosynthesis Action Spectrophotometer \\ Operating principles}

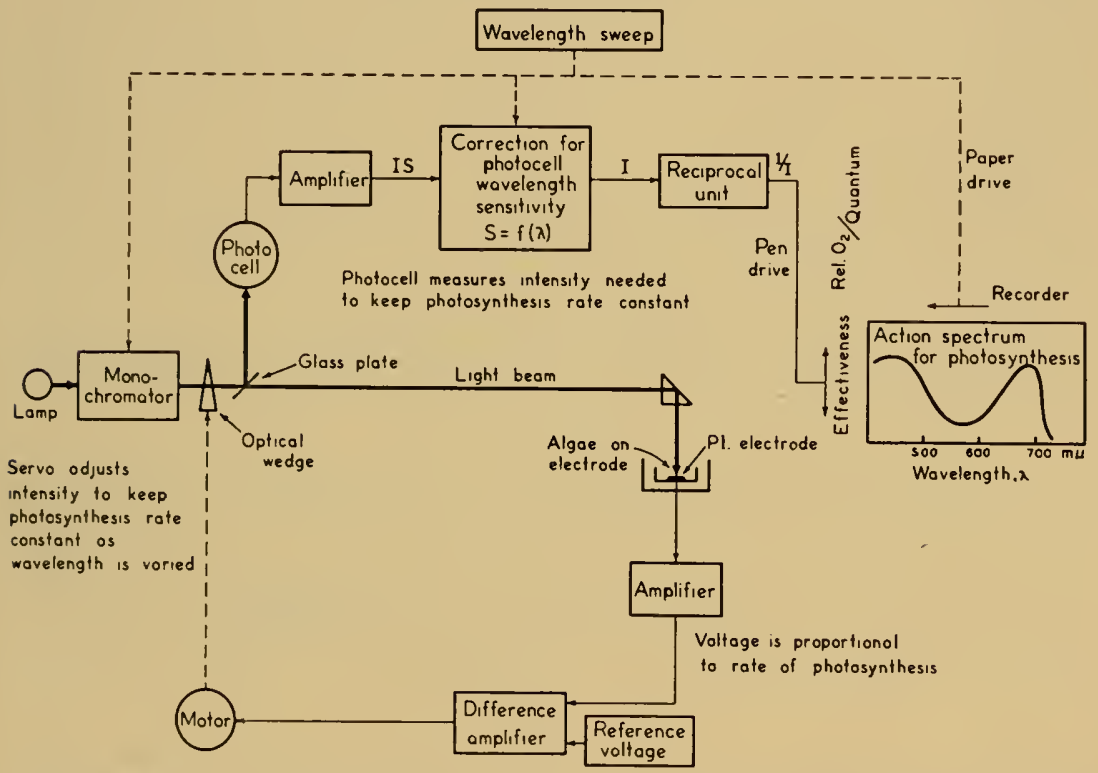

Fig. 8. A block diagram of the recording action spectrophotometer (From 27).

trolled by the rate of photosynthesis itself. A platinum electrode (Haxo and Blinks, 33; Haxo and Fork, 34) gives a current proportional to the photosynthesis rate and is used to control the light intensity. A fraction of the light beam is continuously measured by a photocell, and a value proportional to its reciprocal (expressed in relative numbers of incident quanta and corrected for the wavelength sensitivity of the photocell) is plotted against wavelength on a recording potentiometer. Thus the action spectrum is plotted directly without calculation. The system works reasonably well for very thin layers of algae that do not swim. A record is shown in Fig. 9. 


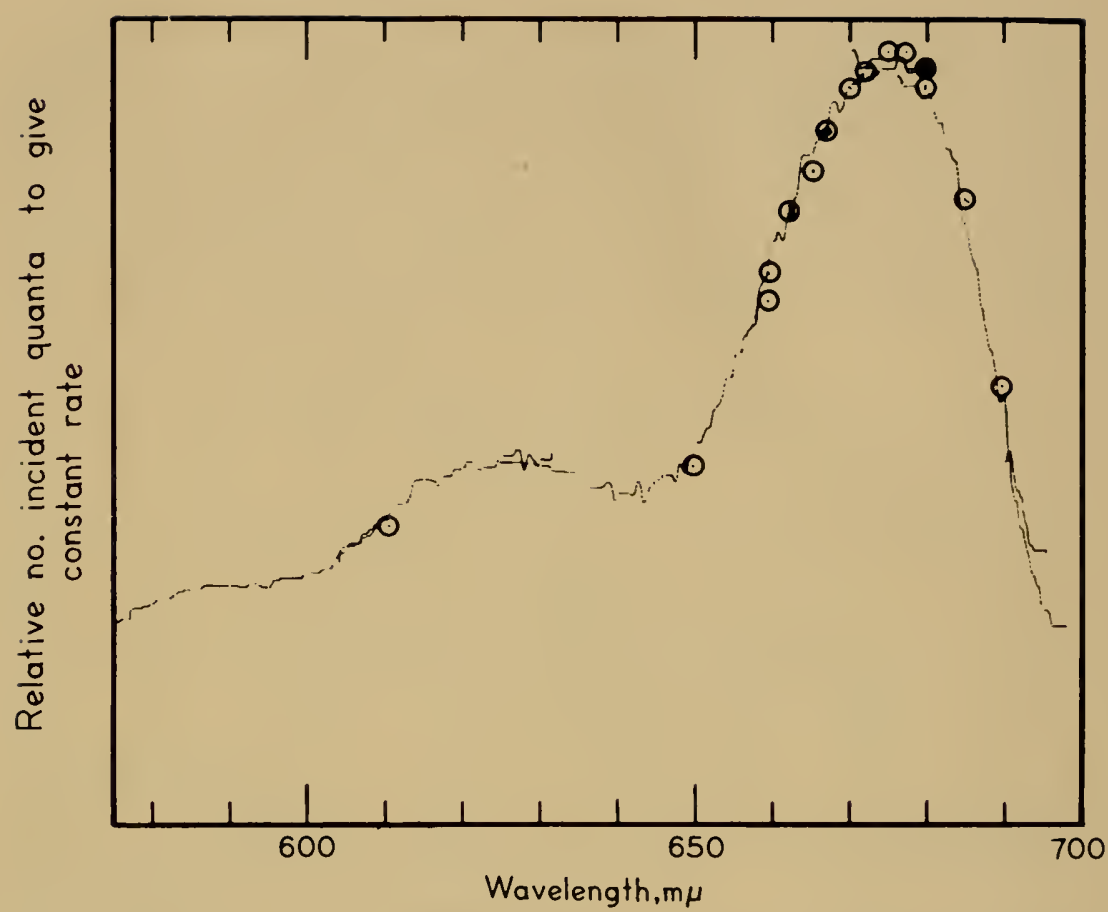

Fig. 9. An automatically recorded action spectrum for photosynthesis in Botrydiopsis alpina, Starr No. 295. The wavelength was varied at about $10 \mathrm{~m} \mu / \mathrm{min}$; after each segment of about $20 \mathrm{~m} \mu$ the wavelength was returned to $680 \mathrm{~m} \mu$ ant the zero point readjusted if necessary. The points were plotted by changing rapidly from $680 \mathrm{~m} \mu$ to the indicated wavelength and then waiting about 1 min for the steady rate to be reached. The lower point at $680 \mathrm{~m} \mu$ show's the drift in twice the time used to plot a point. (With G. C. McLeod).

The device is well adapted for studies of the Emerson enhancement effect of photosynthesis by added background light of controlled wavelength and intensity.

\section{Action Spectra for Enhancement and for Chromatic Transients}

Emerson and Lewis (18) found the efficiency of photosynthesis to decline much more steeply than does the absorption itself on the long-wave side of the red chlorophyll absorption band. Many years later, Emerson, Chalmers, and Cederstrand (16) found that the low efficiency of light in this $680-710 m \mu$ region could be greatly increased by adding light of shorter wavelengths during the measurements. This Emerson enhancement effect is illustrated in Fig. 10, where it is seen that $\lambda 650$ and $\lambda 700$, when given together, give more photo- 


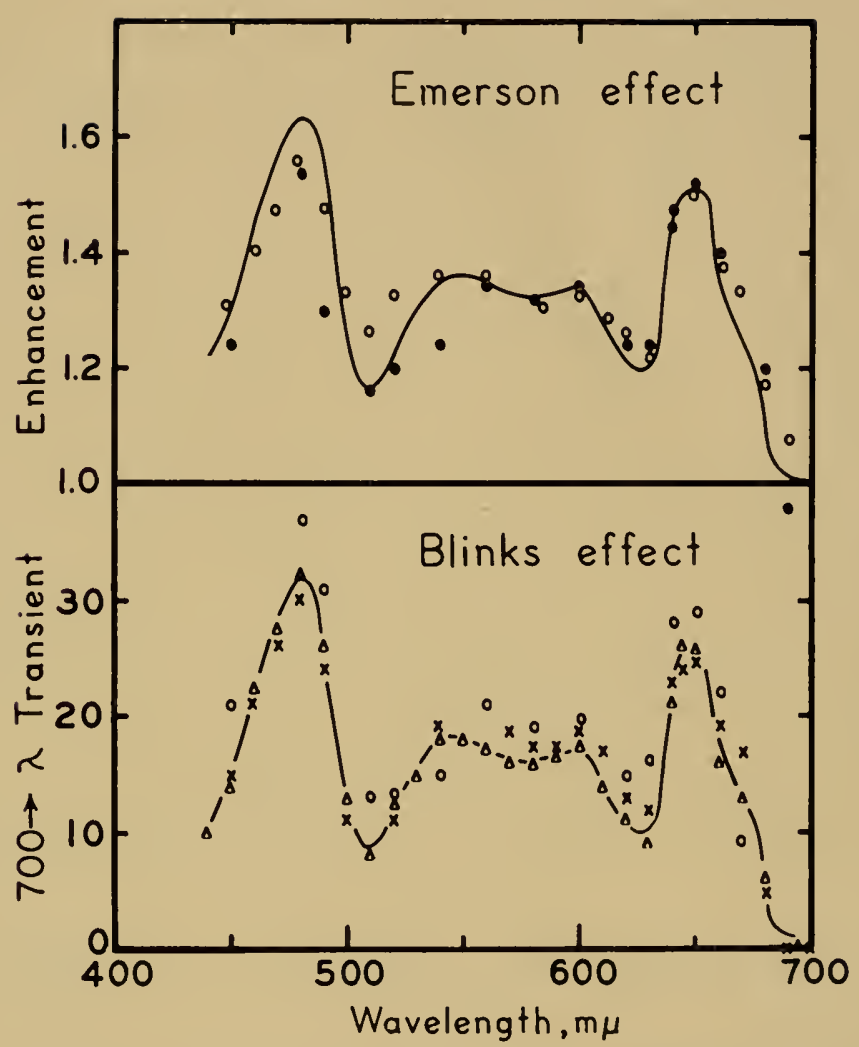

Fig. 11. The action spectra for chromatic transients and for enhancement are identical in Chlorella. Lower: The transient as percentage of the sleady rate in changing from $700 \mathrm{~m} \mu$ to $\lambda$. I'pper: The enhancement of photosynthesis by $700 \mathrm{~m} \mu$ light cansed by adding wavelength $\lambda$. Each wavelength, $\lambda$, was adjusted in intensity to give a rate equal to that from $700 \mathrm{~m}_{\mu}$ alone. (From 48).

are involved. The enhancement may be looked at in two ways. Traclitionally the effects of short wavelengths on the enhancement of photosynthesis by light of about $700 \mathrm{~m} \mu$ has been measured. The experiment can be performed in the reverse way. The enhancement of photosynthesis at $650 \mathrm{~m} \mu$ may be studied by adding various wavelengths in the $700 m_{\mu}$ region.

The enbancement spectrat for the two pignents may also be determined by measuring the action spectrum of photosynthesis with a background light of about $650 m_{\mu}$ and again with a $700 m_{\mu}$ background. The curve with the $650 \mathrm{~m} \mu$ background is enluanced in the $700 \mathrm{~m}_{\mu}$ region, and the curve with the $700 \mathrm{~m}_{\mu}$ background is enhanced 
in $650 \mathrm{~m} \mu$ region. Such a pair of curves, attomatically recorded for Chlorella, is given in Fig. 12. The difference between the curves in the shoter wavelength region gives enhancement spectra similar to those of Eunerson suggestive of chlorophyll $b$. The difference between the curves in the long wavelength region gives the action spectrum of the long wavelength partner. The difference curve for one of the active components has its peak at $695 \mathrm{~m} \mu$. This active pigment is believed to be the chlorophyll $a$ form, $\mathrm{C}_{\mathrm{a}} 695$, found in the absorption spectrum of Euglena and in smaller amounts also in Chlorella (11). This part of the spectrum was checked by individual point-bypoint measurements (Fig. 13). It thus appears that enhancement of photosynthesis by light absorbed by chlorophyll $b$ is somehow due to the simultaneous light activation of $\mathrm{C}_{b} 650$ and of $\mathrm{C}_{\mathrm{a}} 695$. Chlorophyll $a$ 695 and $C_{1}, 650$ act as a mutually enhancing pair. Similar meastrements by McLeod and by Fork (as reported by Haxo, 3) have shown enhancement of chlorophyll a photosynthesis by the accessory phycobilin pigments, and vice versa (27).

Enhancement thus looks like a two-way balanced affair. We see no reason to suppose that either of the two pigments involved plays a primary role and the other a secondary or "accessory" role.

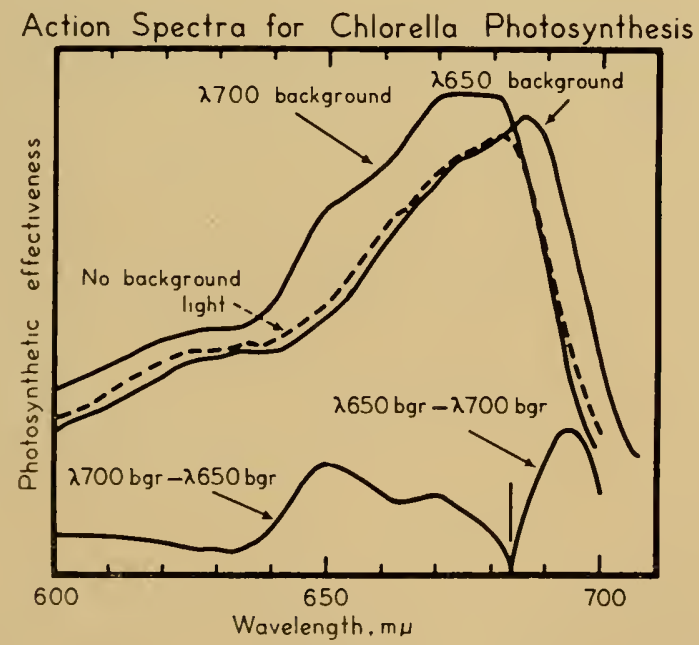

Fig. 12. Action spectra for photosynthesis by Chlorella pyrenoidosa. Dashed curve: no extra light. Other two upper curves with background light as indicated. Lower curve: difference between 700 and $650 \mathrm{~m} \mu$ background curves. The part of this curve below $683 \mathrm{~m} \mu$ represents the action spectrum for chlorophyll $b$, one of the mutually enhancing pair of pigments, while the part at longer wavelengths than $683 \mathrm{~m}_{\mu}$ is the action spectrum for the other partner, $\mathrm{C}_{\mathrm{a}} 695$. (From 27). 


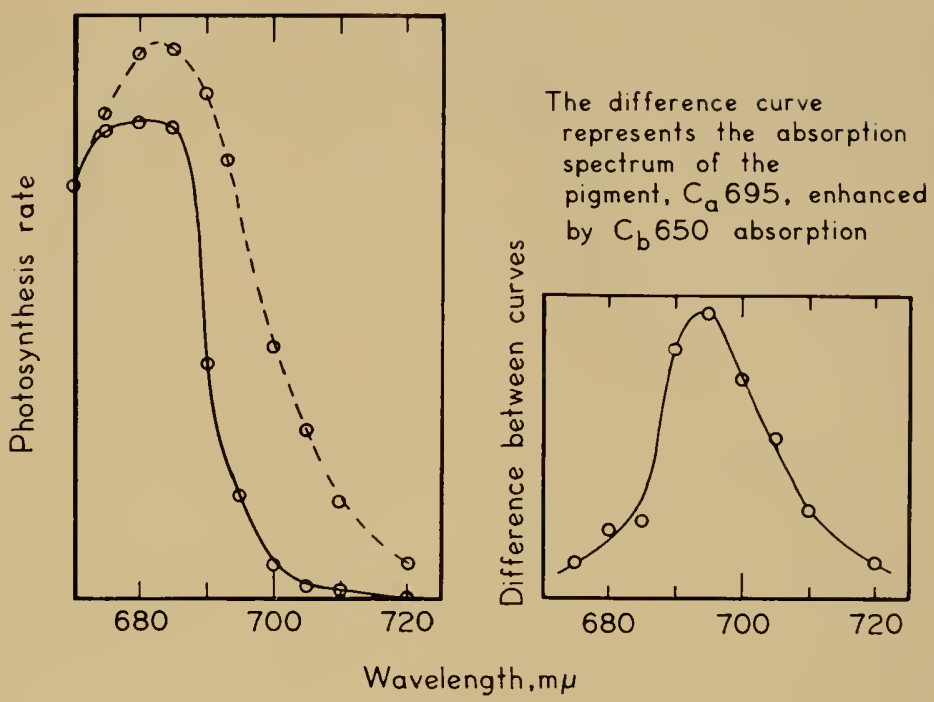

Fig. 13. An action spectrum for Chlorella photosynthesis with (dotted line) and without (solid line) background light of $650 \mathrm{~m} \mu$. The difference between the curves is shown at the right.

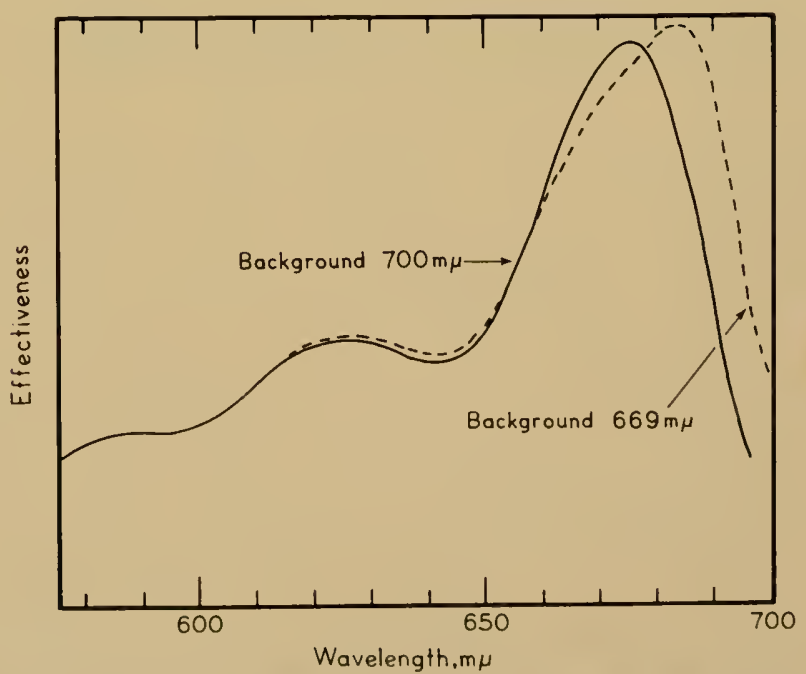

Fig. 14. Action spectra of photosynthesis in Bolrydiopsis alpina, Starr No. 295, with two different background colors. This alga does not contain chlorophyll $b$. The cuhancement appears to be caused by the simultaucons illumination of $\mathrm{C}_{\mathrm{a}} 670$ and $\mathrm{C}_{\mathrm{a}} 695$, indicating a different chemical function for the two forms of chlorophyll a. 
The enhancement spectrum giving the chlorophyll $b$ peak at 650 $m_{\mu}$ to our surprise consistently shows a shoulder at about $670111 \mu$ in the data of Myers and French (48), of Blinks (9, Figs. 2 and 8), and also here in Fig. 12. We therefore speculate that the $673 \mathrm{~m}_{\mu}$ form of chlorophyll a may, at least in part, be functionally equivalent to chlorophyll b. Enhancement experiments were therefore made with Botrydiopsis, which contains several forms of chlorophyll $a$ but lacks chlorophyll $b, c$, and $d$, as well as the phycobilins. The data of Fig. It show that in this alga having only chlorophyll $a$ the "accessory" function can be performed by the $670 \mathrm{~m} \mu$ form of chlorophyll a. However, with amother chlorophyll a alga, Ochromonas danica, no enhancement was found between 669 and $694 \mathrm{~m} \mu$.

At the moment it seems that "accessory" pigments work mainly by activating $\mathrm{C}_{\mathrm{a}} 695$. If so, should $\mathrm{C}_{\mathrm{a}} 695$ also be called an accessory pigment? In time it will be more clearly obvious what chemical reactions are activated by the different pigment systems, and when that time comes the vague term of accessory pigments will presumably be replaced by a more specific name based on physiological function.

\section{Action Spectra of Photosynthesis at Saturating Light Intensity}

Myers (unpublished) found that Chlorella exposed to bright $700 \mathrm{~m} \mu$ light gave a photosynthetic rate at light saturation far below the saturation rate measured at shorter wavelengths. This incidental observation was the starting-point of a thorough study of the rate of photosynthesis at saturating intensities of different wavelengths by McLeod $(47)$.

As we all know from one of the long-accepted basic facts of photosynthesis, illustrated in Fig. 15, the rate of photosynthesis rises at low intensity in direct proportion to the intensity, and then more slowly up to a constant rate. Above this region of intensity further increases of light give no greater rates of photosynthesis.

At low light intensity the rate is dependent on the color as well as the intensity and is very nearly independent of the temperature. The rate is limited by the photochemical first step of the reaction. On the other hand, at high intensity, the rate is limited by a dark reaction, and the intensity or color of the light should have no effect whatever on the measured rate of photosynthesis.

With this clear and simple picture in mind, all action spectra have been made at low intensity, where the light reaction determines the measured rate. Any attempt to measure an action spectrum of photosynthesis with saturating intensities of light would at first sight seem to be absurd. 


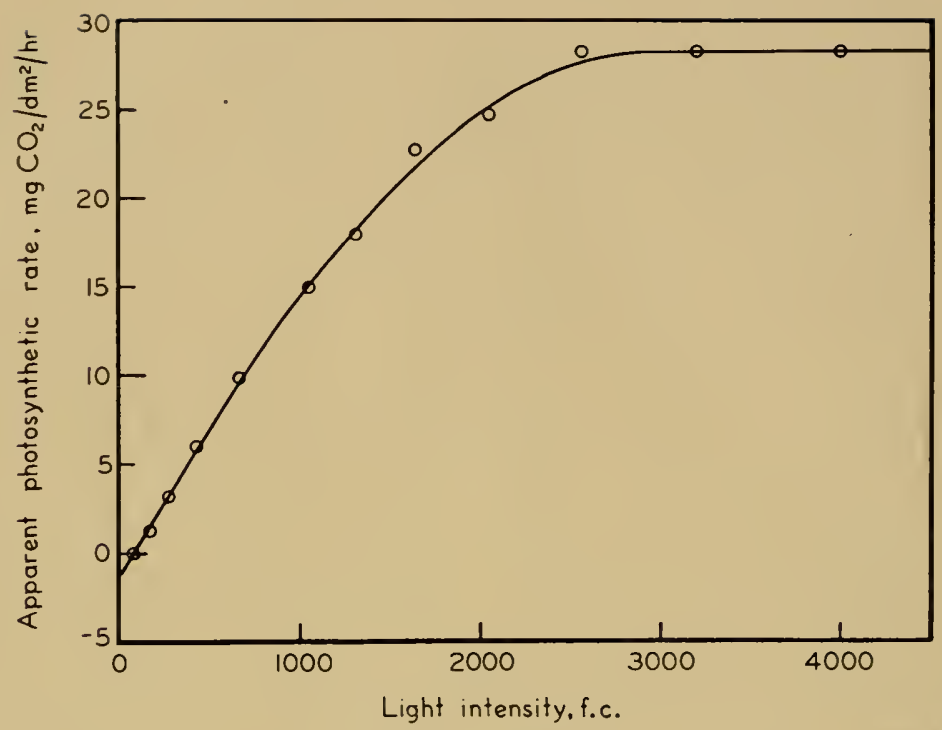

Fig. 15. A typical intensily curve for photosynthesis. From data of H. W. Milner for Mimulus cardinalis $7119-1$ (Baja Calif.). CO.2 uplake measured at $30^{\circ} \mathrm{C}$.

McLeod (47) has recently measured the action spectrum of photosynthesis using high intensities that, at each wavelength, were shown to be saturating. The results of such an experiment, when plotted as rate against wavelength, should give a perfectly straight and horizontal line over the wavelength region effective in photosynthesis. However, it turned out that the beatifully simple concept of a single saturation rate independent of wavelength is inadequate. The results obtained were not at all like the expected horizontal line but gave a curve with significant peaks and valleys. These data themselves show that there is not one photochemical first step, but two different ones that are promoted by different pigments. Fig. 16 compares the low intensity conventional action spectrum of Chlorella photosynthesis with the action spectrum measured at light saturation for the red part of the spectrum. The low intensity spectrum shows a peak at $680 \mathrm{~m}_{\mu}$, (chlorophyll $a$ ), while the high intensity spectrum has a peak at $650 \mathrm{~m} \mu$ where chlorophyll $b$ also absorbs light.

We believe the shape of the high intensity action spectrum is in some way caused by the Emerson enhancement effect. At $650 \mathrm{~m} \mu$ both chlorophyll $a$ and $b$ are activated, at $680 m \mu$ the absorption is almost entirely by chlorophyll $a$. Some difference in the relation of intensity to rate for the $\mathrm{C}_{\mathrm{a}} 680$-promoted photochemical reaction and 


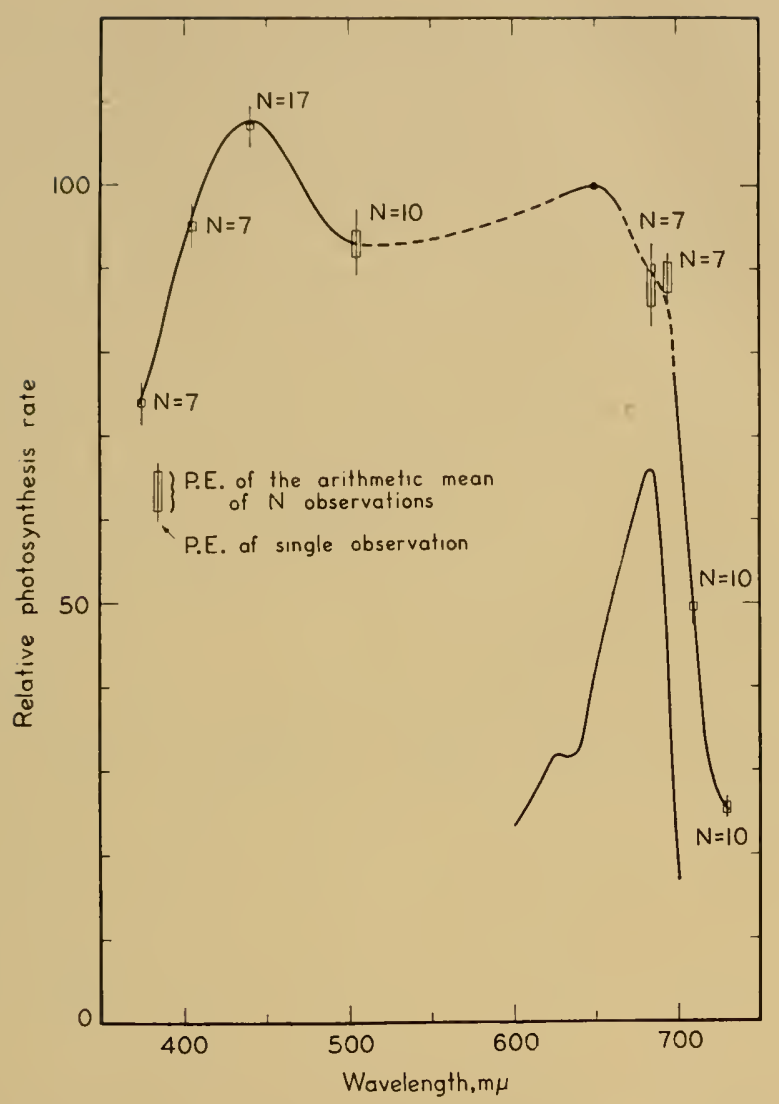

Fig. 16. The action spectrum for photosynthesis by Chlorella at low and at saturating light intensity. The low intensity curve is the reciprocal of the relative quantum intensity needed to give a constant rate of photosynthesis at various wavelengths.

The high intensity curve is the rneasured rate at saturating intensity of each wavelength. The rates for $N$ experiments at each point were normalized at 650 $m_{\mu}$. According to the usual concepts of photosynthesis this should be a horizontal line, with no structure. The photosynthesis rate at which the low intensity spectrum was measured was $0.006 \times$ that for a typical measurement at light saturation at $650 \mathrm{~m} \mu$. The scales for the two curves are not comparable. From MIcLeod (47).

the "accessory pigment reaction" promoted by $\mathrm{C}_{1}, 650$ and $\mathrm{C}_{2} 695$ together may well be involved in a way that is not yet understandable. Certainly the relative photochemical activity of the less absorbing pigments in proportion to the total activity would increase with intensity at levels above the saturation range of the major absorber. 
The reason for greater effectiveness of $\lambda 650$ than of $\lambda 680$ at high intensity seems to be that at $680 \mathrm{~m} \mu$ the fraction of light absorbed by $\mathrm{C}_{\mathrm{b}} 650$ and $\mathrm{C}_{a} 695$, is negligibly small. Therefore the maximum attainable rate at $680 \mathrm{~m} \mu$ is essentially that of unenhanced photosynthesis. At $650 \mathrm{~m} \mu$, however, the enhancement appears to be added to the basic rate. This happens because there is significant absorption by $\mathrm{C}_{\mathrm{il}} 695$ at $650 \mathrm{~m} \mu$, but very little absorption by $\mathrm{C}_{1}, 650$ at $680 \mathrm{~m}_{\mu}$.

\section{The Variation of the Course of Photosynthesis with Wavelengths}

An effect underlying the chromatic transients, and probably itself also a consequence of the enhancement effect, is the variation of photosynthesis rate during the induction period with wavelength. This effect is shown in Fig. 17 for Chlorella, a repetition of the experiment of Fig. 1 in Blinks (6) done with Porphyra. With appropriate choice of conditions, such curves have been used to calculate the chromatic transient curves (49).

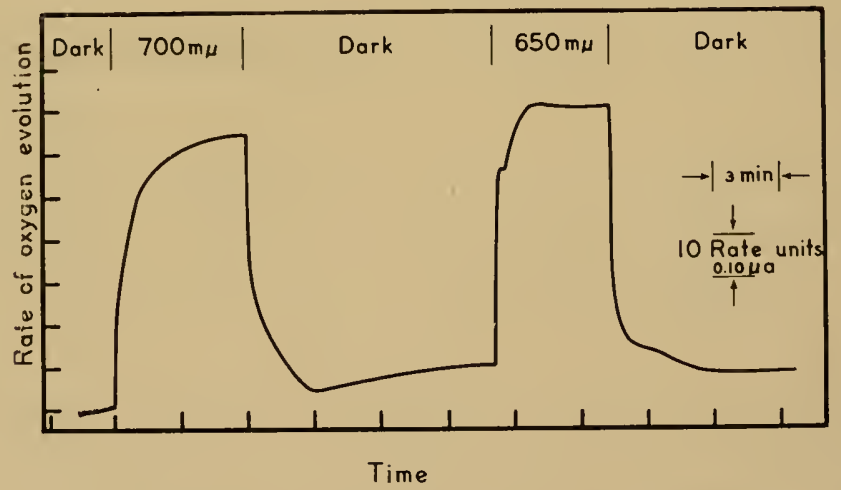

Fig. 17. The time cousse of $\mathrm{O}_{2}$ exchange rate of Chlorella pyenoidose for two different wavelengths. (From +8$)$.

\section{Discussion}

The history of research on photosyuthesis covers a fascinating maze of well-founded experimental facts of general significance, mixed with quantities of trivial observations often of cloubtful validity. There is a pressing nced for clear underlying concepts, expressed in terms of chemical kinetics, and compatible with the physical and photochemical knowledge about the bchavior of colored molecules simpler than chlorophyll. Ideas from fields such as classical organic chemistry, dye photochemistry, enzyme biochemistry, and solid state 
physics have been used to explain different aspects of the photosynthetic process. So far we have no muiversally acceptable concept as to how the photochemical part of the reaction takes place, but we have no lack at all of proposed theories.

The basic difference between well-known photochemical reactions and photosynthesis is that what seems to be a single step-the splitting of a water molecule-takes more energy than is contained in one quantum. Ordinary photochemical reactions use one quantum at a time to do a job within their capacity; photosynthesis seems to go by means of some mechanism for collecting the energy of several quanta for use at one time. The present discussion will avoid this more basic problem and will be limited to the question of how the accessory pigments may come into the picture.

The phenomena presumably attributable to the action of accessory pigments are: chromatic transients, enhancement, the wavelength dependence of the time course, and the action spectrum at saturating light intensity. All of these effects appear to be related, and are probably most easily discussed as enhancement, which may perhaps give rise to the other phenomena. That the $670 \mathrm{~m} \mu$ form of chlorophyll $a$ appears to act as an accessory pigment, and that the activation of the accessory pigments makes $\mathrm{C}_{\mathrm{a}} 695$ more effective, may refer the whole accessory pigment question back to that of understanding the mode of action of the various forms of chlorophyll $a$.

Without facing the fundamental problem of collecting the energy of several quanta, we may nevertheless speculate about the means by which some extra photosynthesis is produced by the action of accessory pigments to give the phenomena discussed above.

Possible theories to explain enhancement fall into two classes: (a) theories that account for the interaction within the pigment system itself; and (b) theories that have various pigments produce different photochemical products and therefore locate the interaction in the products rather than in the pigment system.

The latter concept is favored by the possible separation in time of the two light beams giving enhancement and by the long time constants involved (see Fig. 10). It is supported even more strongly by the different time course of respiration after illumination by 650 $\mathrm{m}_{\mu}$ and by $700 \mathrm{~m}_{\mu}$. How can cells remember the color of light they have previously been exposed to, except by storing different chemical products?

Theories of interaction within the pigment system can no doubt be made in terms of solid state physics and may even account for the 
possible separation in time of the mutually enhancing beams. The theory of different products from specific pigments and the interaction of these products to give a greater rate when both are formed together leads back to a biochemical search for the products themselves. If the investigators of the photochemical products of photosynthesis will stop using white light and will define their experimental wavelengths with interference filters, I am sure we will see progress in this field.

It looks as though the effects we have been reviewing were superimposed on the main path of photosynthesis. A tentative scheme for the accessory pigment function is illustrated in Fig. 18. In this picture most of the photosynthesis is presumed to be done by the major forms of chlorophyll $a$, either by their own absorption or by energy transfer through the accessory pigments. A second mechanism leads to extra oxygen evolution by what is labelled the enhancement pathway. To drive this enhancement mechanism, light must be absorbed both by $\mathrm{C}_{\mathrm{a}} 695$ and by an accessory pigment. Absorption by an accessory pigment produces a photochemical product called $A$, while absorption by $\mathrm{C}_{\mathrm{a}} 695$ forms the photoproduct $B . A$ and $B$ then react to give oxygen.

The diagram also states that $A$ and $B$ spontaneously decompose to inactive substances, $P$. The half lives of $A$ and of $B$ are not identical but are both between 1 and 10 seconds.

Qualitatively the cliagram appears to provide for transients, enhancement, wavelength dependence of time course during and after

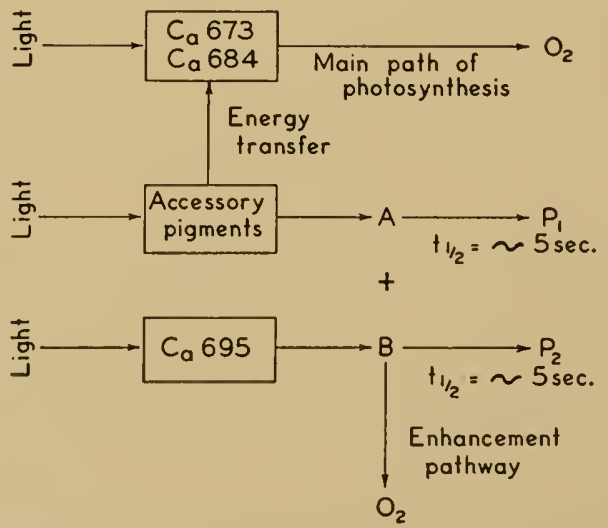

Fig. 18. A scheme to show the possible functional relations between scveral forms of chlorophyll $a$ and the accessory pigments of photosynthesis. 
illumination, and perhaps somehow for the variation of rate with wavelength at light saturation.

One reason for the current interest in the long wavelength forms of chlorophyll $\mathrm{C}_{n} 695$ and $\mathrm{C}_{\mathrm{n}} 707$ is the expectation that they might be the final pigments in the energy transfer chain. It is evident from the enhancing effect of $695 \mathrm{~m} \mu$ light on photosynthesis from $650 \mathrm{~m} \mu$ light that such transfer to $\mathrm{C}_{\mathrm{a}} 695$ is not taking place. This step is therefore intentionally absent in the diagram. This scheme is presented only as a starting-point for discussion and will no doubt be modified or abandoned as new experiments and better understanding clarify the role of accessory pigments in photosynthesis.

\section{REFERENCES}

1. Airth, R. L., and Blinks, L. R., J. Gen. Physiol., 4I, 77-90 (1957).

2. Allen, M. B., Dougherty, E. C., and McLaughlin, J. J. A., Nature, I84, 1047 1049 (1959).

3. Allen, M. B., French, C. S., and Brown, J. S., in Comparative Biochemistry of Photoreactive Systems (M. B. Allen, ed.), Academic Press, New York (in press, $1960)$.

4. Barer, R., Science, 121, 709-715 (1955).

5. Blinks, L. R., in Symposium on Autotrophic Microorganisms, Soc. of General Microbiology, pp. 224-246, Cambridge University Press (1954).

6. Blinks, L. R.. in Research in Pholosynthesis (pp. 444-449), Interscience Publishers. New York (1957).

7. Blinks, L. R., Plant Physiol., 34, 200-203 (1959).

8. Blinks, L. R., in Comparative Biochemistry of Photoreactive Pigments (M. B. Allen, ed.), Academic Press, New York (in press, 1960).

9. Blinks, L. R., Proc. Nat. Acad. Sci. U. S., 46, 327-333 (1960).

10. Brown, J. S., unpub.

11. Brown, J. S., and French, C. S., Plant Physiol., 34, 305-309 (1959).

12. Dutton, H. J., Manning, W. M., and Duggar, B. M., J. Phys. Chem., 47, 308-3 I3 (I943).

13. Duysens, L. N. M., Thesis, Utrecht (1952).

14. Emerson, R., Science, 127, 1059-1060 (1958).

15. Emerson, R. and Chalmers, R. F., Phycol. Soc. Am. News Bull., 11, 5 I-56 (1958).

16. Emerson, R., Chalmers, R. F., and Cederstrand, C., Proc. Nat. Acad. Sci. U. S., 43, $133-143$ (1957).

17. Emerson, R., and Lewis, C. M., J. Gen. Physiol., 25, 579-595 (1942).

18. Emerson, R., and Lewis, C. M., Am. J. Bot., 30, I65-I78 (1943).

19. French, C. S., J. Gen. Physiol., 23, $483-494$ (1940).

20. French, C. S., in The Luminescence of Biological Systems (F. H. Johnson, ed.), pp. 5I-74, American Association for the Advancentent of Science, Washing. ton, D. C. (195.5).

21. French, C. S., in Photoperiodism and Related Phenomena in Plants and Animals (R. B. Withrow, ed.), pp. 51-74, American Association for the Advancement of Science, Washington, D. C. (1959).

22. French, C. S., in The Photochemical Apparatus, Pp. 65-73, Brookhaven Symposia in Biol. (1959). 
23. French, C. S., in Photobiology-Nineternth Ammual Biology Colloquium, Corz'allis, Oregon, pp. 52-64 (1958).

24. Frcuch, C. S., in Encyclopedia of Plant Physiology, Part II, Vol. 5 (W. Ruhland, e(1.). pp. 252-297, Springer, Berlin (1960).

25. French, C. S., and Elliott, R. F., Carnegie Inst. Wash. Y'car Book, 57, 278-286 (1958).

26. French, C. S., and Helen S. Huang, Comegic Inst. H'ash. Year Book, 56, 266-268 (1957).

27. French, C. S., Myers, J., and McLeod, G. C., in Comparative Biochemistry of Photoreactive Systems (M. B. Allen, ed.), Academic Press, New York (1960).

28. French, C. S., Smith, J. H. C., Virgin, H. I., and Airth, R. L., Plant Physiol., 31, 369-3T4 (1956).

29. French, C. S., and Young, V. K., I. Cien. Physiol., 35. 873-890 (1952).

30. Frencl, C. S., and Yoming, V. K., in Radiation Biology (A. Hollaender, ed.), Vol. 3, pp. 343-391, McGraw-Hill, New York (1956).

31. Hattori, A., and Fujita, Y., J. Biochem. (Japan), 46, 633-644 (1959).

32. Hattori, A., and Fujita, Y., J. Biochem. (Iapan), 46, 903-909 (19.59).

33. Haxo, F. T., and Blinks, L. R., J. Gen. Physiol., 33, 389-422 (1950).

34. Haxo, F. T., and Fork, D. C., Nature, 184, $1051-1052$ (1959).

35. Haxo, F. T., O'hEocha, C., and Norris, P., Arch. Biochem. Biophys., 54, $162-$ 173 (1955).

36. Katz, E., and W'assink, E. C., Enzymologia, 7, 97-112 (1939).

37. Kaıtsky, H., Appel, E., and Amann, H., Biochem. Z., 332, 277-292 (1960).

38. Kok, B., Biochim. et Biophys. Acta, 22, 399-40I (1956).

39. Kok, B., in 2nd Intern. Congr. Photobiology, pp. 370-384, Turin (195i).

40. Krasnowskii, A. A., Evstigncev, V. B., Brin, G. P., and Gavrilova, V. A., Doklady Akad. Nauk. SSSR, 82, 947-950 (1952).

41. Krasnowsky, I. A., and Kosobutskaya, L. M., Doklady Akad. Nank. SsSR, 91, 343-346 (1953).

12. Krasnovsky, A. A., and Kosobutskaya, L. M., Doklady Akad. Na1lk. SSSR, 104, $440-443$ (1955).

43. Krasnorsky, A. A., Vorob'cra, L. M., and Pakashina, E. V., Fiziol. Rastinii, Akad. Nank S.S.S.R., 4, 123-133 (1957).

44. Latimer, P'. (pers. (ommum.).

45. Lemberg, R., and Legge, J. W.. Hematin Componnds and Bile Pigments, Interscience Publishers, New York (1949).

46. Li, Tsi-Tung, Anm. Bot., 43, 587-601 (1929).

47. McLcod, G. C. (in press).

48. Myers, J., and French, C. S., J. Gen. Physiol., 43, 723-736 (1960).

49. Myers, J., and French, C. S., Plant Physiol., (in press, 1960).

50. O'hEocha, C., Arch. Biochem. Biophys., 73, 207-219 (1958).

51. O'bEocha, C., and Raferty, M., Nature, 184, 1019-1051 (1959).

52. Rabinowitch, E., Proc. Intern. Botan. Congr., 9th Congr. Montreal (1959).

53. Slibata, K., J. Biochem. (Japan), 44, 147-173 (1957).

51. Smith, J. H. C.., in Intern. Congr. Photobiology, Curin. Italy (1957).

55. Sunith. J. H. C., and Benite\%, X., in Modern Methods of Plant Analysis, Vol. 1, pp. 143-196, Springer, Berlin (195.).

56. Tanada, T., Am. J. Bot., 38, 276-283 (1951).

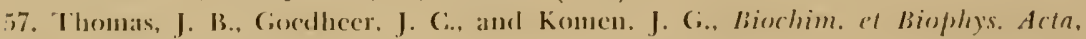
22, $1-8(19.56)$.

58. Wassink, I., C... Kat/, F., and 1)orrestein, R., Enzymologia, 7, 113-129 (1939).

59. Wassink, E. C., and Kersten, J. A. H., Enzymologia, 12, 3-32 (1916). 
60. Witt, H. T., Moraw, R., Miiller, A., and Rumberg, B., Z. physik. Chem. N. F. 23. $133.138(1960)$.

(il. Yocum, C.. S.. and Blinhs, L. R., I. (ien. Physiol., 41, 1113-1117 (1958).

\section{1)ISCUSSION}

Dr. Frixcin: I would like to saly that it is nice to measure the action spectrum for the other one of the pair of the mutually enhancing pigments by doing it the other way around, set on $670 \mathrm{~m} \mu$ and then vary the long wavelength beam.

Dr. Rabixowrich: I think you are too modest when you say don't know which yield is alfected. On the one side you have a wavelength which by itself gives normal high yield, on the other side a wavelength which gives a subnormal yield, with a quantum requirement of 20 or more. I don't think there is any doubt that what is happening upon combination of the two wavelengths is improvenent of activity in the far red.

Dr. French: I still like to look at it as a balanced affair. There is no real reason for this opinion.

Dr. Fraick: The new results on the Emerson effect presented by Drs. French and Rabinowitch are very interesting. So far as I can tell without more careful studies, they are in perfect agreement with an interpretation of the Emerson effect given by me in a paper (Proc. Natl. Acad. Sci. U.S. H, $941,1958)$. It astonished me that no attempt was reported of comparing these results with the theory mentioned. In case our explanation is unacceptable to the speakers, they should have mentioned their objections. The whole purpose of this meeting is airing of differences of opinions and attempts to straighten them out. Since I have neither the intention, nor the time to discuss the theory in any detail, I mention only its basis and refer those who are interested to the published paper and to a supplement I might write to include new results on chlorophyll in vitro and vivo. A comparison of the spectroscopic results on chlorophyll in vitro with those in vivo, is the fundament of our interpretation of the Emerson effect. Chlorophyll a possesses two sets of electronic transitions which lie close together: the so-called $\pi \rightarrow \pi^{*}$ transitions and $n \rightarrow \pi^{*}$ transitions. The relative energetic position of the two sets of levels depends on presence or absence of water, as absorption spectra, fluorescence olsservations, etc., reveal. There can be no doubt that both types of electronic transitions appear in chlorophyll $a$ in vivo, too. The structure of the chloroplas!s is responsible for certain differences. One of them is that in vivo the bulk of chlorophyll $a$, sandwiched between lipid and protein layers, is protected against fluid water and its solutes, and has, dependent upon the cell material, to a limited and varying degree access to single water molecules. Only a small percentage is exposed to liquid water and its solutes. These exposed chlorophylls receive their excitation energy from the protected ones by resonance transfer, or by transfer from excited accessory pigments. It depends upon the wavelengths of the irradiated light. 
which of the two transfers occurs. In those cells in which a strong lack of water exists for the protected chlorophylls, the unprotected receive a surplus metastable triplet excitation from them, while the accessory pigments transfer singlet excitation. As mentioned in the cliscussion after Dr. Calvin's talk, the metastable state can carry out only one of the two photochemical steps connecterl with one splitting of water; singlet excitation is needed for the second step. Thus the cooperation between the products of two absorption acts of clifferent wavelengths, i.e., the Emerson effect, is understandable.

Dr. French: I am sure I have had that same experience, Professor Franck, in having people not pay attention to what I think important. The only thing that I have against this is that it is hard to think of a part of the insicle of the chloroplasts as being protected from water, water being such a small molecule.

Dr. FrANCK: If I speak of protection from water, I mean only protection against water as a lluicl. Certainly, protein contains water, but if for each chlorophyll at the lipid layer one water molecule should be available, its concentration should be high, as high as that of chlorophyll. Furthermore, I expect only small water concentrations at the position where the chlorophyll is adsorbed; it is itself not water-soluble, and the fatty acid sicle of the lipid will repel water, too. The probability of finding water at the phosphate group is supposed to be much greater.

Another point 1 want to mention is that your scheme does not contain any explanation why (according to Duysen's and your remarks), the Iluorescence intensity of chlorophyll $a$ in red algate is higher if the light is absorbed by phycobilins than if chlorophyll a absorbs it.

DR. French: I am sure a lot of things clon't fit in that little scheme. I don't take it very seriously except as a means of correlating the facts we have discussecl.

Dr. Rabinowitcin: I just wanted to answer Dr. Franck. My manuscript contains a cliscussion of your interpretation. I didn't luave cnough time to mention it in my talk, and 1 thought you were going to bring it up anyhow so I did not discuss it. It was just a question of time. The other thing that I would say is that your interpretation suggests something about the fluorescent capacity of the dillerent forms of chlorophyll.

DR. Wrist: I would like to ask Prolessor Franck how he proposes to reconcile the olsserved half-life of five seconds of the two effects with metastable states which have a half-life of only a fraction of a second. The two illuminations enhance each other even when onc comes five seconds after the other. How is this compatible with metastable states which last only a fraction of a second?

DR. Fravik: You speak of my older ideal of a cooperation between a metastable triplet state and a singlet state, to reach a higher excited state of chloropliyll. This idea, which I called 'physical cooperation,' I supplemented by the picture of a 'chemical cooperation,' and finally gave "up the 
first one for the reason you mentioned. In the chemical picture the metastable state is supposed to produce a chemically changed substance (the enol complex, see, c.g., the discussion after Calvin's paper), and it can live for a number of seconds before it vanishes by back reactions.

DR. PLATT: I would like to make an extension of some of the thoughts we have all had. When we get physical chemists together with biologists, we should not talk entirely about theory or interpretation. We should also talk tools, because it is entircly possible that physicists and physical chemists may be able to suggest certain tooling effects which might be useful to biologists and vice versa. It is possible that biologists may be able to suggest certain types of measurements they would like to make which they cammot make with existing tools. At the Brookhaven Conference last October we had almost a half day on the discussion of possible tools. I would like to mention two possible tools which it seems to me would be of great advantage in distinguishing one pigment from another in a cell, or one complex from another in a cell without taking the cell apart. Now, this means that we have to find a physical method which we can apply externally to the cell with mininal damage. The ones that I would like to mention that we can put into the cell from outside without wrecking it perlaps are light, changes of temperature, and changes in pressure. In the case of light, of course, we have been cliscussing some of the changes that are observed in the form of bleaching. Let me mention one thing that has not been mentioned and which might be illuminating-and I don't mean that as a pun-for some of these studies, that is, to try polarized light in the photo-bleaching experiments. The reason is that the bleaching should be polarized if the incoming light is polarized, if the bleaching is in the same molecule that is absorbing the light. So one would be able to correlate one peak with another peak in the same spectrum by finding a concomitant polarization and say that these are probably the same molecule or closely associated molecules. Peaks which don't have polarized bleaching, then, might be further away or more randomly arranged. This might be an additional cheap piece of information. In the case of temperature we have seen a number of high temperature and low temperature spectra. Let me emphasize one feature when we go from a high temperature spectrum to a low temperature spectrum. If we have several vibrational peaks in the same molecule which we will call $0-0,0-1,0-2$ one would not expect to change much the spacing between these peaks for a given molecule with temperature. However, if we have two different electronic transitions which lie in the same region and which have a different electronic structure, or come from a different molecule, they will shift by different amounts with temperature. So there will be relative shifts of one set of peaks with respect to the other, which proves that the set which shifts together is one electronic transition, or one pigment, or one molecule, or one complex and the other set which has a different amount of shift belongs to another, very probably. The same argument maly be made for pressure, 
and let me suggest that small pressure apparatuses are now very cheap. One can make a little hand pump machine with a little bomb which you can set on your laboratory table and get something of the order of a thousand atmospheres. Now, it is always something of a surprise to a physicist that biological creatures survive at the bottom of the sea where the pressures are so high. It is clear that biological systems are less sensitive to pressure than you might suppose. Perhaps you require mutation or perhaps you require adaptation, I don't know. Nevertheless, it seems to me possible that for a great number of systems that you work with, especially the smaller ones, one-celled systems, chromatophores, it might be possible by pressure to push them into configurations which would change somewhat the distribution of products light performance. In particular, it will shift absorption spectra in the same way that temperature will shift absorption spectra. I think sometimes the little bombs with sapphire windows on them to look through may be simpler in fact than a low-temperature apparatus. I would like to urge the use of more high pressure experiments, and the examination of spectra for these interactions between pigments and for different pigment effects to try to isolate different electronic transitions and different pigments, let me say, particularly in these cytochrome problems. 


\title{
ON THE LONG-WAVE DECLINE OF THE QUANTUM YIELD OF PHOTOSYNTHESIS IN THE RED ALGA PORPHYRIDIUM CRUENTUM
}

\author{
J. B. Thomas and Govindjee. \\ Photosynthesis Research Laboratory \\ Department of Botany \\ Unirersity of Illinois, Urbana, Illinois
}

\section{INTRODUCTION}

The quantum yield of photosynthesis, $\Phi$ in the far red, instead of remaining constant until a region is reached where chlorophyll $a$ no longer absorbs at all, declines with increasing wavelength $(8,9)$. Emerson and coworkers (cf. 3) found that the wavelength at which this decline starts varies with the pignent composition of the photosynthetic system. They also observed that irradiation with light of shorter wavelengths can restore the reduced quantum yield to its full value $(6,7)$. This "Emerson effect" was interpreted as evidence of a need for cooperation in photosynthesis of an excited auxiliary pigment with an excited chlorophyll $a$. It was noted (5) that the amount of activation by auxiliary light depends on the intensity ratio of auxiliary light and far red light. No effect occurred unless this ratio exceeded a certain minimum.

The published data do not prove whether, in the case of exclusive excitation of chlorophyll $a$, the rate of photosynthesis becomes zero, or has a certain small finite value. (The smallest value measured by M. Brody and R. Emerson (2) with Porphyridium was $\phi=0.01$ in a narrow band centered at $700 \mathrm{~m} \mu$ ). The present study was intended to answer this question.

\section{METHODS}

We wanted to be able to vary the composition of the incident light so that the proportion of energy absorbed by auxiliary pigments could change in small steps. To this purpose, an organism had to be used in which the auxiliary pigments absorbed well outside the red chlorophyll $a$ band (which has a peak between 670 and $680 \mathrm{~m} \mu$ ). 
This requirement is met by red algae, which contain chlorophyll $a$, but not chlorophyll $b$. The main auxiliary pigments are the phycocyanin, absorbing around $625 \mathrm{~m} \mu$, and the phycoerythrin, absorbing from 565 to $500 \mathrm{~m} \mu$. The red alga, Porphyridium cruentum, was grown over fluorescent white light tubes in the medium described by M. Brody and Emerson (1). Gradual removal of light absorbed by the phycobilins was obtained by filtering the incident white light through cuvettes containing aqueous phycobilin extracts of increasing concentration. As a light source, a 225-watt ribbon-filament, incandescent lamp was used. Care was taken to remove, as far as possible, the infrared radiation (not absorbed by the algae), by using a 6-inch water layer and two American Optical IR-absorbing glass filters. A correction was applied to account for the remainder of the radiation beyond $720 \mathrm{~m}_{\mu}$.

The phycobilin extracts were prepared by storing thalli of Porphyra perforata in clistilled water in the dark at room temperature (about $23^{\circ} \mathrm{C}$ ) for at least five days. In this time the cells died and the phycobilins, but not the chlorophyll and the carotenoids, were released. The extract was partly purified by filtering through a Seitz filter, centrifuging, and repeated precipitation with ammonium sulfate. The final preparation was highly concentrated, but clear, showing only very little light-scattering. Our thanks are due to Dr. L. R. Blinks for providing us with the algal material, and to Mr. C. Cederstrand for measuring true (scattering-free) absorption of the Porphyridium suspensions in an integrating sphere set-up.

Photosynthetic oxygen production was measured manometrically, while the incident energy was determined with a bolometer, as described by Emerson and Chalmers (4).

\section{Results and Discussion}

The results of six consecutive experiments are shown in Fig. I, in which quantum yield in percentages of that in full light is plotted against the fraction of total incident light absorbed by the phycobilin filter. The curve consists of three sections. In the first section, the quantum yield does not change as the absorption of the phycobilin filter is raised from 0 to $20 \%$. Further increase of the filter density up to $80 \%$ absorption leads to a lincar decline of the quantum yicld. When the concentration is increased still further, the quantum yield declines more stecply, until, at about $95 \%$ absorption, photosynthesis ceases to be measurable $(-0.24 \pm 0.25 \mu \mathrm{l} \mathrm{O} /$ hour $)$. However, the Emerson effect can still be observed: if a background of orange light 


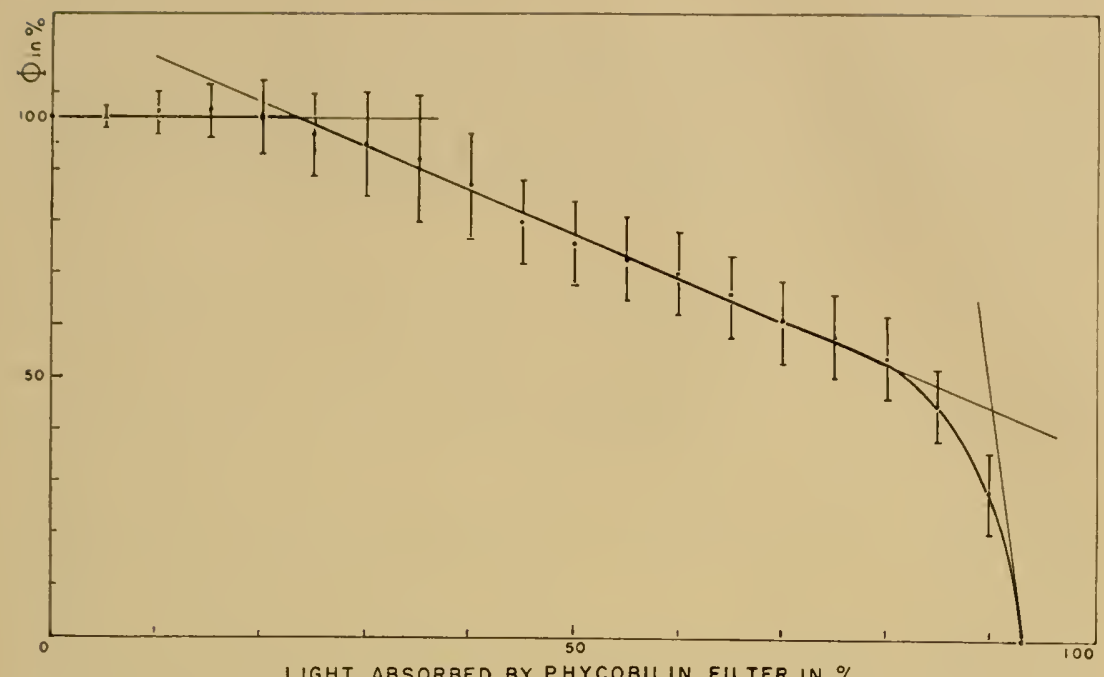

Fig. 1. Relation between quantum yield and light absorption by phycobilin filters.

is provided, the light transmitted by the $95 \%$ filter clearly shows a measurable photosynthetic effect $(+1.66 \pm 0.30 \mu \mathrm{l} \mathrm{O}$ /hour). This show's that the disappearance of photosynthetic activity in light filtered through the $95 \%$ filter was not due to the residual light being practically unabsorbable by chlorophyll $a$. The same conclusion can also be derived by calculation, using the energy distribution curve of the incident light, the absorption curve of the filter, and the absorption curve of the cell suspension (determined in an integrating sphere and therefore free from scattering effects). This calculation shows that much of the transmitted light falls into the region $680-700 \mathrm{~m}_{\mu}$, and is largely absorbed by the cell suspension.

The effect of removal of light predominantly absorbed by phycobilins, shown in Fig. 1, may be explained in terms of the dependence of $\Phi$ on the intensity ratio of auxiliary light to far red light, as noted by Emerson and coworkers. As long as the phycobilin filter absorbs less than $20 \%$ of the incident light, this ratio may remain above the critical value, and the maximal quantum yield is maintained. If more than $20 \%$ but less than $80 \%$ of light is absorbed in the filter, the quantum yield becomes dependent on the intensity ratio; i.e., light energy absorbed by chlorophyll $a$ produces photosynthesis only in proportion to the energy absorbed by the auxiliary pigment. When the intensity ratio declines below another critical value, no enhancement of the quantum yield in the far red by light of shorter wave 
is possible. One would then expect the yield either to become constant, or to decline to zero, depending on whether the light absorbed only by chlorophyll $a$ (or, more precisely, the form of chlorophyll $a$ responsible for the absorption in this region) does or does not produce by itself a finite yield of photosynthesis. The curve in Fig. 1 suggests that the latter is the case.

However, according to a suggestion made by Dr. E. I. Rabinowitch, the situation may be more complex. The measurements by Brody and Emerson (2) (and confirmed by Govindjee) suggest that $\phi$ remains measurable in the narrow spectral bands centered at $695 \mathrm{~m} \mu$ and even $700 \mathrm{~m}_{\mu}$. Why, then, should there be no photosynthesis at all in the " $5 \%$ band" which extends from $680 \mathrm{~m} \mu$ to longer waves? Perhaps there exists in photosynthesis, as in many other photobiological phenomena, a "counter-effect" caused by extreme red light, which deactivates some of the intermediates in photosynthesis. The amount of this light in the " $5 \%$ band" may be sufficient to reduce the photosynthesis to zero. Further experiments are in progress to check this tentative suggestion.

\section{SUMMARY}

The relation between quantum yield and white light from which the wavelengths predominantly absorbed by both phycobilins were removed was established for the red alga Porphyridium cruentum. It proved possible to obtain incident light of such a spectral composition that no photosynthesis could be measured in it at all, although a significant part of it was absorbed, and while the Emerson effect still occurred.

\section{REFERENCES}

1. Brody, M., and Emerson, R., Am. J. Botany, 46, 433-440 (1959).

2. Brody, M., and Emerson, R., J. Gen. Physiol., 43, 25l-26t (1959).

3. Emerson, R., Ann. Rev. Plant Physiol., 9, 1-24 (1958).

4. Emerson, R., and Chalmers, R., Plant Physiol., 30, 504-529 (195.5).

5. Emerson, R., and Chalmers, R. V., Plycol. Sor. Am. Neu's Bull., 11, $51-56$ (1958).

6. Emersoln, R., Chatmers, R., and Cederstrand, C., Pror. Nat. Acad. Sri. U. S., $43,113-143(1957)$.

7. Emerson, R., Chalmers, R., Cederstrand, C., and Brody, M., Srience, 123, $673(1956)$.

8. Emerson, R., and Lewis, C. M., Am. J. Bolany, 30, 165.178 (1943).

9. Haxo, F., and Blinks, L. R., J. Gen. Physiol., 33. 389-422 (1950). 
EVIDENCE FOR PIGMENTS ABSORBING AT 705-710 $11 \mu$ IN PHOTOSYNTHETIC ORGANISMS

\author{
Mary Belle Allen \\ Kaiser Foundation Research Institute \\ Laboratory of Comparative Biology \\ Richmond, California
}

The recent results of $\operatorname{Kok}(5,6)$ indicating changes in absorption in the 705-710 $\mathrm{m} \mu$ region during photosynthesis of the blue-green alga Anacystis nidulans have given impetus to a search for pigments absorbing in this region of the spectrum. The first suggestion that light of wavelengths greater than $700 \mathrm{~m}_{\mu}$ might be effective for oxygenproducing photosynthetic organisms came from the work of Dangeard (2), who in 1927 found that a motile Oscillatoria accumulated in the $710-730 \mathrm{~m} \mu$ region of a projected spectrum, and that a strain of Phormidium would grow in light of these wave lengths. Dangeard's experiments have been criticized on the ground of an insufficient spectral purity of his light, and, possibly for this reason, these experiments seem never to have been pursued further.

The next suggestion of pigments with absorption at longer wavelengths than that of chlorophyll came from Duysens $(3,4)$, who obtained fluorescence spectra for the red algae Porphyra lacineata and Porphyridium cruentum and the blue-green alga Oscillatoria. These spectra suggested the presence of an unknown pigment with a fluorescence maximum at $725 \mathrm{~m}_{\mu}$. Duysens first attributed this fluorescence to chlorophyll $d$. Since this chlorophyll is not known to occur in the Bangiaceae, and is certainly absent from Porphyridium and Oscillatoria, the fluorescence observed must be either an artifact of measurement or due to a previously unknown pigment.

In a survey of the in-vivo spectra of chlorophyll in a number of algae, carried out in collaboration with Dr. C. S. French, the chrysomonad Ochromonas danica was observed at times to contain a pigment absorbing around $710 \mathrm{~m}_{\mu}$, as shown in Fig. 1. Brown and French (pers. commun.) have obtained similar spectra from Euglena gracilis. The $710 \mathrm{~m}_{\mu}$ pigment in $O$. danica is somewhat evanescent; studies on the conditions for its appearance are in progress. 


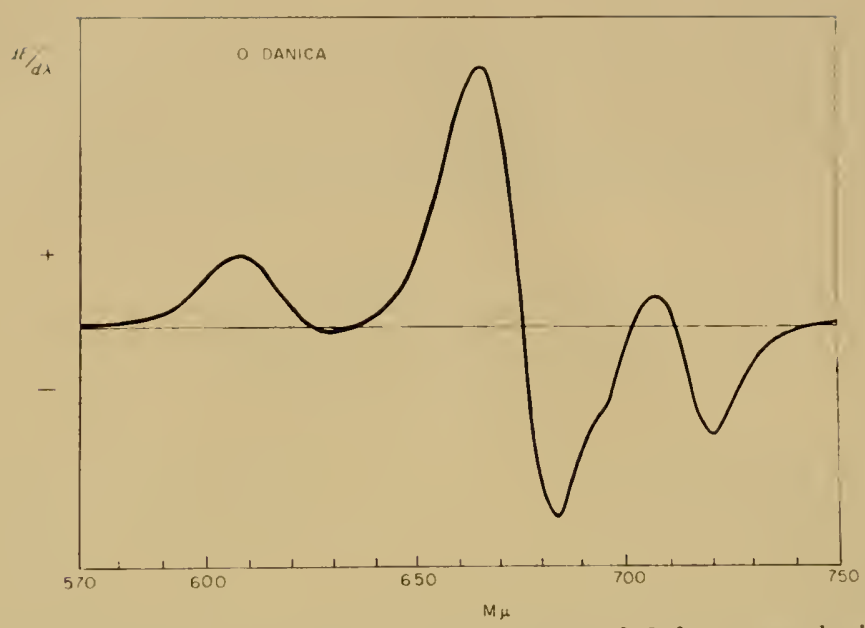

Fig. 1. Derivative absorption spectrum of a culture of Ochromonas danica showing absorption at around $710 \mathrm{~m} \mu$. Obtained through the collaboration of Dr. C. $S$. French.

Ochromonas danica is an organism notable for the complexity of its absorption spectrum of chlorophyll $a$ in vivo and for the presence in young healthy cells of a consiclerable proportion of a form of chlorophyll $a$ absorbing at $695 \mathrm{~m} \mu$ (1). Integral absorption spectra have at times been obtained showing a shoulder at $710 \mathrm{~m} \mu$; in other spectra this peak may not have been resolved from the $695 \mathrm{~m}_{\mu}$ absorption. Fluorescence clata support the conclusion that a pigment absorbing at wavelengths longer than that of chlorophyll is present in this organism. As seen in Table 1, the fluorescence at $725 \mathrm{~m}_{\mu}$ is much higher in relation to that at the chlorophyll absorption maximum in $O$. danica than in Chlorella or in chlorophyll a solutions $(O$. danica contains no chlorophyll other than chlorophyll $a$ ). Moreover, there is no constancy of ratio between the absorption at $725 \mathrm{m \mu}$ and at the chlorophyll maximum, and the $725 \mathrm{~m} \mu$ fluorescence is high in samples in which absorption at $710 \mathrm{~m} \mu$ is clearly shown. Since there is no correlation between the amount of fluorescing pigment present and the ratio of fluorescence at $725 \mathrm{~m}_{\mu}$ and the chlorophyll maximum, it scems unlikely that these results are due to reabsorption of fluorescent radiation.

An absorption peak at around $710 \mathrm{~m} \mu$ has also been observed in certain mutants of Chlorella pyrenoidosa (Fig. 2). The mutants in which this has been observed are all of the type known as "apple green," which contain chlorophyll $a$, in a quantity considerably less 
TABLE 1

Fluorescence of Ochromonas danica Cultures ${ }^{5}$

$\begin{array}{ccc}\text { Fluorescence at } & \text { R chl. } \\ \lambda \text { chl. } & \text { chl. max. 725 in } \mu & \text { max./ }\end{array}$

$\max ^{2} \quad$ (arbitrary units) $725 \mathrm{~m} \mu$

Culture Conditions

\begin{tabular}{|c|c|c|c|c|c|c|}
\hline $690 \mathrm{~m} \mu$ & 13.1 & 3.1 & 0.236 & \multicolumn{3}{|c|}{1 day dark, medium $\mathrm{O}^{3}$} \\
\hline 691 & 16.3 & 3.8 & 0.233 & 2 days & & \\
\hline 692 & 12.1 & 3.9 & 0.322 & $3 " \#$ & $"$ & $"$ \\
\hline 681 & 14.5 & 6.5 & 0.448 & 25 " light, & $"$ & $"$ \\
\hline 693 & 14.4 & 4.2 & 0.282 & 1 day " & $"$ & $"$ \\
\hline 692 & 19.8 & 5.5 & 0.277 & 2 days " & & $"$ \\
\hline 690 & 17.8 & 5.7 & 0.320 & $3 " \prime$ & $"$ & $"$ \\
\hline 691 & 16.6 & 4.2 & 0.253 & $3 "$ & $"$ & $"$ \\
\hline 682 & 14.0 & 4.9 & 0.350 & $4 "$ & $"$ & $"$ \\
\hline 682 & 18.2 & 7.7 & $0.423^{1}$ & $5 "$ & $"$ & $"$ \\
\hline 692 & 21.0 & 6.5 & 0.310 & $6 "$ & $"$ & $"$ \\
\hline 678 & 17.7 & 6.8 & $0.385^{1}$ & $7 "$ & $"$ & $"$ \\
\hline 678 & 19.8 & 6.7 & 0.338 & $3 "$ & dium & $\mathrm{N}^{4}$, broken cells \\
\hline 690 & 16.4 & 4.6 & 0.280 & $" \quad "$ & $"$ & $"$, intact cells \\
\hline 691 & 13.4 & 4.5 & 0.336 & 3 days dark, & & \\
\hline 691 & 15.9 & 5.1 & 0.321 & $" \Rightarrow "$ & & $"$, after 4.5 hr. illum \\
\hline 680 & 16.5 & 8.8 & 0.531 & 8 days light, & totrop & phic \\
\hline 688 & 12.8 & 5.6 & 0.437 & $5 " \#$ & $"$ & \\
\hline 690 & 14.6 & 5.4 & 0.370 & $2 "$ & $"$ & \\
\hline 678 & 16.0 & 7.0 & 0.437 & 5 and 8 days & ht, $\mathrm{m}$ & gedium $N$ \\
\hline 678 & 16.8 & 7.7 & 0.458 & 21 days light, & totrop & ohic \\
\hline 688 & 16.8 & 6.5 & 0.386 & $2 " \prime$, & dium & $\mathrm{N}$ \\
\hline 677 & 10.5 & 4.5 & 0.428 & 24 days light, & dium & $\mathrm{O}$ \\
\hline 678 & 13.7 & 6.5 & 0.473 & $10 " \|$ & $"$ & $"$ \\
\hline 678 & 15.2 & 6.7 & 0.440 & $8 "$ & $"$ & $"$ \\
\hline 688 & 20.7 & 7.8 & 0.377 & $5 "$ & $"$ & $"$ \\
\hline 691 & 15.6 & 4.9 & 0.314 & $2 "$ & $"$ & $"$ \\
\hline $\begin{array}{l}\text { chl. a } \\
\text { (ether) }\end{array}$ & 一 & - & $\begin{array}{l}0.167 \\
0.125\end{array}$ & $\begin{array}{l}\text { Zscheile \& Ha } \\
\text { Duysens (4) }\end{array}$ & s (7) & \\
\hline Chlorella & - & - & $\begin{array}{l}0.192 \\
0.135\end{array}$ & $\begin{array}{l}420 \mathrm{~m} \mu \text { exciti } \\
480 \mathrm{~m} \mu\end{array}$ & light $\}$ & Duysens (4) \\
\hline
\end{tabular}

${ }^{1} 710 \mathrm{~m}_{\mu}$ absorption peak clearly shown.

${ }^{2}$ The position of the fluorescence maximum of chlorophyll in $O$. danica depends on the proportion of the different in-vivo components of the chlorophyll $a$ spectrum (1).

${ }^{3}$ Complex organic medium.

'Synthetic medium.

${ }^{5}$ Fluorescence measurements made in collaboration with Dr. C. S. French.

than that of the wild type, as their only green pigment, and which lack colored carotenoids. It is possible that the $710 \mathrm{~m} \mu$ pigment is more evident here because of the reduced quantity of the betterknown photosynthetic pigments.

It is premature to hazard any guesses as to the nature of the 710 $m \mu$ pigment. It may be another in-vivo spectroscopic form of chlorophyll or another pigment of unknown nature. Work on its iclentifica- 


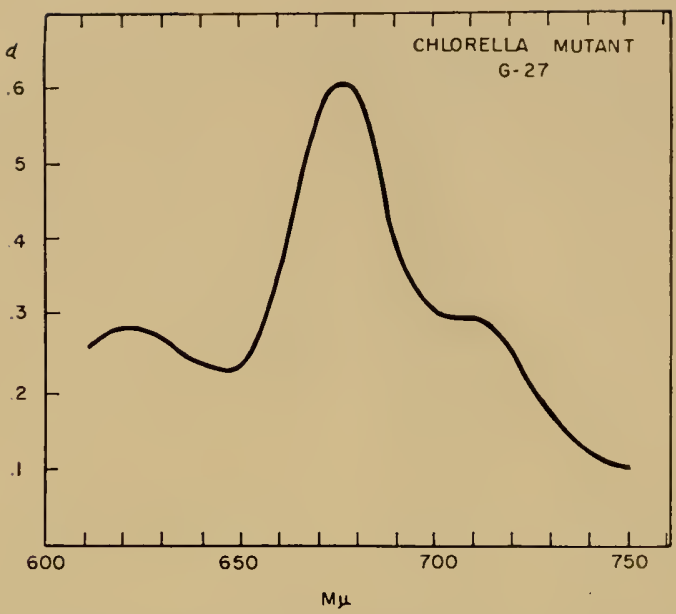

lig. 2. Absorption spectrum of an "apple green" mutant of Chlorella pyrenoidosa, showing absorption at $710 \mathrm{~m} \mu$. The spectrum was measured with a Cary recording spectrops tometer with opal glass to minimize the effects of scattering.

tion is in progress. Whatever its nature, if it is, as Kok suggests the ultimate energy converter in photosynthesis, it must be of great interest to students of this subject.

\section{REFERENCES}

1. Allen, M. B., French, C. S., and Brown, J. S., in Comparative Biochemistry of Photoreactive Systems (M. B. Allen, ed), Academic J'ress, New York, 1960.

2. Dangeard, P.-..., Le Botaniste, 19, 200-208 (1927).

3. Duysens, L. N. M., Nature, 168, 548-549 (1951).

4. Duysens, L. N. M., Dissertation, Utrecht (1952).

5. Kok, B., Plant Physiol., 34, 184-192 (1959).

6. Kok, B., this symposimm.

7. Zscheile, l. P., and Harris, 1). G., J. Phys. Chem., 47, 623-637 (1943). 


\title{
COMMENTS ON THE FUNCTION OF HAEM PROTEINS AS RELATED TO PRIMARY PHOTOGHEMICAL PROCESSES IN PHOTOSYNTHESIS1
}

\author{
MARTIN D. KAMEN \\ Graduate Department of Biochemistry \\ Brandeis University \\ IJaltham, Massachusetts
}

It is a privilege to appear as a discussant following the presentation by our distinguished colleague, Professor Robin Hill. Much of our own work in recent years was prefigured in his classic memoir of 1939 on the chloroplast reaction, wherein he offered the suggestion that a respiratory pigment might be intimately linked in a chemosynthetic system coupled to the initial quantum absorption process in photosynthesis (12). Since then, data have been accumulating and are now sufficient to indicate that in the early phases of photosynthesis there is a close relation between tetrapyrrolic chelates of iron present as haem proteins on the one hand, and of magnesium, present as chlorophylls, on the other. There is neither time nor space to enter into a detailed discussion of these data which for the most part are adequately documented in the literature $(13,14)$. I wish only to call attention to a possible mechanism for chemical coupling to the initial quantum absorption process which may be inferred, if it is granted that chelates of iron and magnesium exist in close contact in the photosynthetic apparatus, whether chloroplast or bacterial chromatophores.

As we know from the voluminous literature on electron transfer processes in systems containing conjugates or chelates of metal ions, the presence of a macrocyclic resonating system may induce rapid electron exchange between ions otherwise shielded by solvent (3, $8,19,7)$. In all photosynthetic systems a situation exists in which an efficient resonating macrocyclic system-porphyrin or a derivative

1*This is publication No. 66 of the Graduate Department of Biochemistry, Brandeis University, Waltham, Massachusetts. Much of the work on which this report is based has been supported by grants from the National Science Foundation, the National Instituses of Heals and the C. F. Kettering Foundation. 
recluced porphyrin ring-is chelated to magnesium or iron as the central metal ion. Let us suppose that a magnesium chlorin (as in chlorophyll) lies close to a haem iron (as in haem protein). If the magnesium chlorin is excited by absorption of a photon in the characteristic red band, it will reach a singlet (or triplet) excited state with an energy equivalent $\sim 1.8$ e.v. above the ground state. We can expect that de-excitation can proceed by electron transfer from the neighboring haem. In this process there will be produced a partially reduced chlorophyll, which can be represented as a "semichlorinogen," and a partially oxidized haem. If the iron complex is originally one in which the metal is formally in the (III) state of oxidation, the process can be written as in Fig. 1, in which only the essential portions of the ring structure are shown.

This process most likely yields both products in their ground states. The energy difference can be expected to be close to the original energy of excitation of the chlorophyll. Hence, in this system, a potential difference can be considered to exist in which the oxidized form of haem is $\sim 1.8$ e.v. more oxidizing than the semichlorinogen radical. However, stabilization of the products sufficient to prevent rapid back-reactions may require $0.2-0.3$ e.v., so that perhaps $\sim 1.5$ e.v. is the maximum spread in potential.

For the present, it is possible to infer consequences of such a process only by analogy because nothing is known directly about the solution chemistry of the higher oxidation states of iron, either as the hydrated ion or as a clielate. George and Irvine (10) have shown that metmyoglobin treated by a variety of oxidizing agents (peroxide,

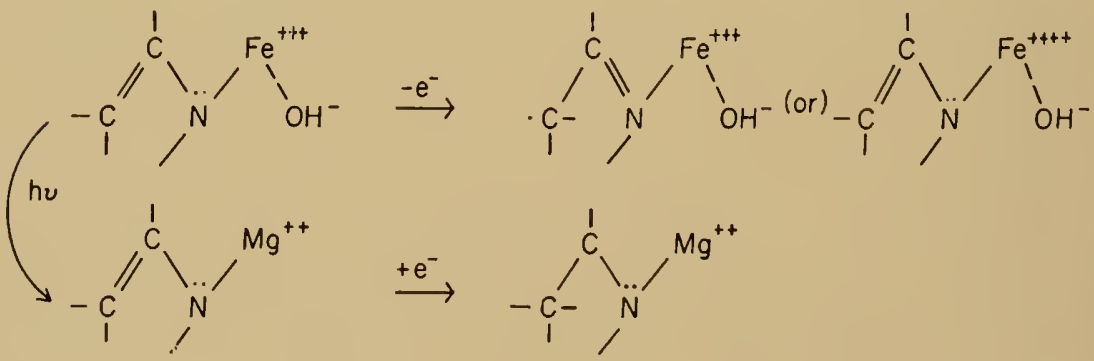

SCHEME: $\mathrm{Chl}+\mathrm{Fe}_{\mathrm{hp}}^{+++} \stackrel{\mathrm{hu}}{\longrightarrow} \mathrm{Chl}^{-}+\mathrm{Fe}_{\mathrm{hp}}^{++++}$

lig. 1. Sclseme for the reaction of chlorophyll with a hacm protein. Mlthough $\mathrm{OH}^{-}$is slown as ligand, it is more likely that water or protein supplies the co-ordinating gromp). 
permanganate, (hloriridate) gives at product spectroscopically idential with the internediate "complex II" formed when metmyoglobin acts as a peroxidase. They have established the $\mathrm{E}^{\prime}{ }_{0}$ as $\sim+0.9 \mathrm{v}$. This value is $\sim 0.1 \mathrm{v}$. more oxidizing than that for the standard oxygen electrode. Hence, the oxidizing equivalents present in this complex can extract an electron from $\mathrm{H}_{2} \mathrm{O}$. If it is assumed that the hacm chelate-protein complex acquires a similar $\mathrm{E}_{0}^{\prime}$, then the $\mathrm{E}^{\prime}$ 。 for the semichlorinogen formed would be $\sim-0.6$ to -0.7 e.v., assuming $1.5 \mathrm{e.v}$. as the value of $\Delta E_{o}^{\prime}$ between the reducing and oxidizing components. Such a strong reducing potential would be more than sufficient to provide an electron transfer step to pyridine mucleotide $\left(E_{o}^{\prime}=-0.3\right)$ which could be coupled to formation of ATP from ADP and inorganic phosphate ("photophosphorylation") $(1,9)$. On this basis, the "photoreductase" of San Pietro and Lang (18) would have as its substrate the semichlorinogen which is identified as the photoreductant generated by the light reaction.

The reactions initiated by the presence of the $\mathrm{Fe}^{\text {IV }}$-haem complex depend on the environment presented. A simple combination of chlorophyll and haem protein would have only the possibility of back-reaction, or reversal of the process shown in Fig. I. However, if an enzymic pathway (such as through the photoreductase to pyridine nucleoticle) is available to remove electrons from the semichlorinogen, then it can be expected there will be a preferential flow of electrons to the enzyme substrate. If a source of electrons is present in the haem complex, either in the protein or as a simple ligand (water), then reduction of the $\mathrm{Fe}^{\mathrm{Ir}}$-haem to its original state would occur with the production of a free radical, probably in the protein moiety.

The evidence available from paramagnetic spin resonance studies of the metmyoglobin oxidation complex, while somewhat ambiguous, appears to be consistent with this postulated sequence of events. Gibson, Ingram, and Nichols (Il) have shown that the complex, studied by George and Irvine and produced by peroxidation of metmyoglobin, exhibits an ESR signal with a $g$-value close to that for the free electron. The precise value for $g$ is somewhat smaller than expected for a $\pi$-electron localized at a methine bridge carbon. It is more consistent with the presence of a delocalized electron in an orbital spread over the whole macrocyclic structure, or of a substrate free radical, such as $\mathrm{OH}$. At the same time, the signal at $g=6$, corresponding to the unpaired electrons at the Fe site, is quenched, indicating a change in the bonding at the metal ion site. 
George and Irvine (10) have presented evidence for this change as a production of "ferryl iron" $\left(\mathrm{FeO}^{++}\right)$or, alternatively, as in the formulation of Fig. 1. At present it is not necessary to postulate production of a ferryl complex, which requires movement of two protons off the ligand water. This is indicated in Fig. 1 by leaving the iron in a formal valence state equivalent to $\mathrm{Fe}^{4+}$, without alteration of the chemical nature of the ligand.

An alternative reaction scheme, which has been discussed by Calvin (4), begins with loss of an electron from excited chlorophyll, concomitant with generation of a positive hole in the chlorophyll complex. This postulate necessitates a delayed oxidation of the cytochrome, or at least reduction of some acceptor, such as pyridine nucleotide, before oxidation of haem iron occurs. There is no conclusive evidence at present to refute this notion, although the lowtemperature measurements of Chance and Nishimura (5) on the photo-induced oxidation of the Chromatium cytochrome system, together with the quantum yield data of Olson and Chance $(17,6)$, seem to favor prior oxidation of haem iron as a primary reaction following quantum absorption.

A variety of interesting problems comes to mind when predictions are attempted for the chemical behavior of a higher oxidation state such as that postulated in Fig. 1. Fe $\mathrm{Fe}^{4+}$, which is isoelectronic with $\mathrm{Mn}^{3+}$, would contain four unpaired electrons distributed in the five $3 d$ orbitals of the metal ion. Upon combination with the ligand groups, at least two could pair, leaving two unpaired electrons and the two free $3 d$ orbitals, so that the $\mathrm{Fe}^{4+}$ orbitals could hybridize as usual to give the octahedral complexes found for $\mathrm{Fe}^{3+}$ and $\mathrm{Fe}^{2+}$. There is evidence from the studies on magnetic susceptibility of metmyoglobin-peroxide complexes that this occurs $(20,2)$. If all the electrons paired, then seven orbitals rather than six would be available, with the $\mathrm{Fe}^{++}$in a diamagnetic state. A ligand such as $\mathrm{OH}^{-}$, but not $\mathrm{H}_{2} \mathrm{O}$, would favor such an arrangement, if analogy with the lower valence forms holds.

The stabilizing effect of both the porphyrin ring, and possibly the protein moiety, in a higher valence form can be inferred from many well-known exanples such as the metal porphyrin complexes of silver, bismuth, cobalt, etc. Winfield and King have emphasized this possibility (15). Dwyer (7) has discussed similar situations, especially the case of the nitroprusside ion, and it is from his discussion that the suggestion of a possible dianagnetic complex structure is Irawn.

One point which should be made is that until direct data can be 
obtained on the chemistry of iron haen chelates, when in a state of oxidation formally higher than $\mathrm{Fe}^{3+}$, it is unsafe to assume that a molecule such as $\mathrm{CO}$ is specific lor the $\mathrm{Fe}^{++}$state. The criterion of a light-sensitive CO-binding has been used universally to establish the presence of lerrous iron, but the possibility that $\mathrm{Fe}^{4+}$ could bind $\mathrm{CO}$ in a similar fashion is not excluded.

Returning to the photochemical system, it may be inagined that in the green plant chloroplast, chlorophyll molecules are arranged in an oriented, or aggregated fashion, so that exciton transfer, or a transfer of energy by inductive resonance, is possible. Upon excitation of a given chlorophyll, the quantum energy is transferred rapidly to successive chlorophylls $\left(\tau=\sim 10^{-12}\right.$ to $\left.10^{-9} \mathrm{sec}\right)$. When a chlorophyll adjacent to a haem protein is involved $(\sim 1$ in $100-500)$ the quenching process shown in Fig. I could occur. If enough components of an electron-transfer chain were present (and they seem to be from molecular analyses of the active photophosphorylating systems studied so far-see, for example, 16), a reaction chain would result involving reduction of the pyridine nucleotides and thence flavin, quinone, hacm protein, etc., including the coupled phosphorylation observed in illuminated chloroplasts. If substrate amounts of pyridine nucleotide are present and fumnelled off to $\mathrm{CO}_{2}$ fixation, the $\mathrm{Fe}^{4+}$-haem component could initiate electron withdrawal from a moiety, such as protein, leading to eventual formation of oxygen. It can be supposed that reversal of an analogue of an oxiclase system is involved, depending on the protein moiety linked to the haem. In bacterial systems, the protein moiety may be one which allows only peroxidation reactions, so that oxygen is not evolved, but rather substrate must be supplied to be oxidized. Another possibility is that there is insufficient energy in the effective quantum for bacterial photosynthesis $(\sim 1.3$ e.v. $)$ to allow the formation of the strongly oxidizing $\mathrm{Fe}^{4+} \cdot \mathrm{Fe}^{3+}$ couple.

Implicit in this formulation is the assumption that no cytochrome oxidase is present in the oxidation chain. All experimental evidence at present supports this assumption. A detailed discussion of this evidence is not within the scope of this paper and in any case is available in the literature (13).

Many more consequences can be pictured, none of which are at variance with what is known about plant and bacterial photosynthesis, but these can be omitted in the interests of brevity. What is of immediate interest is the experimentation indicated. It is evident that chemical interaction initiated by light between model systems com- 
prising first chlorophyll-haem adducts, and then more complex systems, should be studied using the techniques developed recently for following fast reactions, such as those based on relaxation methodology, flash spectrophotometry, etc. The reactions involved which occur in times less than milliseconds fill the present gap between the fast physical processes initiated by quantum absorption in the condensed organized aggregates of photosynthetic systems and the relatively slow biochemical and physiological reactions leading to cell growth.

\section{REFERENCES}

1. Amon, 1). I., Whatley, F. R., and Allen, M. B., J. Am. Chem. Soc., 76, 6324 (1954).

2. Brill, A. S., Ehrenberg, A., and den Hartog, H., Biochim. et Biophys. Arti (in press, 1960$)$.

3. Cahill, A. E., and Taube, H., J. Am. Chem. Soc., 73, 2847 (1951).

4. Calvin, M., this symposium.

5. Chance, B., and Nishimura, M., Proc. Nat. Acad. Sci. L'. S., 46, 19 (1960).

6. Chance, B., and Olson. J. M., Arch. Biochem. Biophys. (in press, 1960).

7. Dwyer, F. P'., Symposium on Haematin Enzymes, Canberra, Australia (1959), Pergamon Press, London (in press).

8. Dwyer, F. P.., and Gyarfas, E. C., Nature, 166, I181 (1950).

9. Frenkel, A. W., J. Am. Chem. Soc., 76. 5568 (19.54).

10. George, P'., and lrvine, D. H., Biochem. J., 58, 188 (1954).

11. Gibson, J. F., Ingram, 1). J. E., and Nichols, P., Nature, 181, 1398 (1958).

12. Hill, R., Proc. Roy. Sor. London B, 127, 192 (I939).

13. Hill, R., Proc. 3rd Intern. Cong. Biochem., Brussels, p. 205, Academic I'ress New York (1958).

14. Kamen, M. D., in Enzymes: Lnits of Biological Structure and Function, (O. H. Gaebler, e(1.). p. 483, Academic Press, New York (19.56).

15. King, N. K., and Winfield, M. E., Austral. J. Chem., 12, 47 (1959).

16. Newton, J. W., and Newton, G. A., Arch. Biochem. Biophys., 71, 250 (1957).

17. Olson, J. M., and Chance, B., Biochim. et Biophys. Acta, 28, 27 (1958).

18. San Pietro, A., and Lang. H. M., J. Biol. Chem., 231, 211 (19.58).

19. Taube, H., Adunces in Inorg. Chem. (and) Radiochem., 1, 1 (1959).

20. Theorell, H., and Ehrenberg, A., Aich. Biochem. Biophys., 41, 442 (1952). 


\title{
CELL-FREE PHOTOSYNTHESIS AND THE ENERGY CONVERSION PROCESS
}

\author{
DANIEL I. ARNON 1 \\ Laboratory of Cell Physiology \\ Unizersity of California, Berkeley, California
}

"The most fundamental character of the living state is the occurrence in the parts of the cell of a continuous and clirected movement of electrons." A. J. Kluyver, Microbial metabolism; evidence for life's unity (83).

\section{INTRODUCTION}

Life on our planet depends on the key event in photosynthesis, the conversion of light into chemical energy. This was first expressed with great clarity by two founders of thermodynamics, J. R. Mayer in 1845 (106), and Ludwig Boltzmann in 1886 (29). Mayer, who three years earlier enunciated the law of the conservation of energy, now stressed its applicability to plant life: "Plants are able only to convert energy not to create it." They accomplish this because during their lifetime they "absorb sunlight and use its force to add continuously to a sum of chemical difference" (106).

Boltzmann, whose name is linked with the Second Law of Thermodynamics, expressed the cosmic significance of the photosynthetic energy conversion process in these words:

"There exists between the sun and the earth a colossal difference in temperature; ... the equalization of temperature between these two bodies, a process which must occur because it is based on the law of probability, will, because of the enormous distance and magnitude involved, last millions of years. The energy of the sun may, before reaching the temperature of the earth, assume improbable transition forms. It thus becomes possible to utilize the temperature drop between the sun and the earth for performing work, as is the case with the temperature drop between steam and water.

${ }^{1}$ Aided by grants from the National Institutes of Health and the Office of Naval Research. 
"The universal struggle for existence in the living world is therefore not a struggle lor raw materials-all organisms can find these in abundance in air, water and soil . . but a struggle for entropy that becomes available in the energy transition from the hot sun to the cold earth. To make the most use of this transition, green plants spread the enormous surface of their leaves and, in a still unknown way, force the energy of the sun to carry out chemical syntheses, before it cools down to the temperature level of the earth's surface. These chemical syntheses are to us in our laboratories complete mysteries. The products of this chemical kitchen form the prize for which the animal world struggles."

To investigators of photosynthesis the core of the energy conversion problem is the identification, by experiment rather than by surmise, of these "improbable energy transition forms" before they become consumed in the "chemical kitchen." Different investigators have followed different experimental approaches in studying this problem. The approach emphasized in my laboratory has been the investigation of photosynthesis in cell-free systems. The main purpose of this article is to summarize some of our findings and to interpret them from the standpoint of a unified but still tentative theory. No comprehensive coverage of the pertinent literature will be attempted here, since much of it has recently been reviewed elsewhere $(10,74)$.

\section{Photosynthesis as an Isolated Process}

In the intact cell the energy conversion in photosynthesis occurs simultaneously with non-photosynthetic energy conversion processes, notably respiration, that are essential to the maintenance of cellular structure and function. The products of photosynthesis become the substrates of respiration; and conversely, the products of respiration become the substrates of photosynthesis. This intertwining of the two processes extends to their intermediate products and to the enzymes and coenzymes that are concerned in the relevant syntheses and degradations.

Given the structural and functional complexity of an intact cell, it was apparent that the study of the energy conversion process of photosynthesis would be greatly simplified if it could be separated from the degradative energy conversion process of respiration. The most hopeful possibility for isolating photosynthesis from other physiological processes secmed to lic in chloroplasts. Isolated chloroplasts, freed from other cytoplasmic impurities, do not respire-a 
conclusion $(6,7,8)$ which has recently reccived support from the work of James and Das (79) and Warburg et al. (165).

Chloroplasts were once widely believed to be the site of complete photosynthesis, but this view was not supported by critical cxperimental cvidence $(131,121)$ and was later abandoned because of much evidence to the contrary $(70,33,26,125,98)$. In 1954, Lumry ct al. (98) summed up the many investigations with isolated chloroplasts as pointing to the conclusion that the chloroplast was a "system much simpler than that required for photosynthesis" and was the sitc of only "the light-absorbing and watersplitting reactions of the overall photosynthetic process" (Hill reaction). It seemed likely, in the words of Rabinowitch (124, p. 355), that "the inability of isolated-even if apparently intact-chloroplasts to carry out photosynthesis is an indication that this process requires the cooperation of the cytoplasm."

The possibility has always remained that the observed failure of isolated chloroplasts to fix $\mathrm{CO}_{2}$ was merely a consequence of inappropriate experimental mechods that were used in different laboratories, including our own (5). Further work with isolated chloroplasts has shown that the difficulties were indeed methodological. In 1954, another phase of chloroplast investigations began with the discovery that, under suitable experimental conditions, isolated whole chloroplasts can assimilate carbon clioxide (11). Photosynthesis, as it is usually defined (124, p. 61), was found to be independent of the organization of the living cell. Reduction of carbon dioxide to carbohydrates accompanied by oxidation of water to oxygen was carried out, at physiological temperatures and with no energy supply except visible light, by isolated chloroplasts unaided by other cellular particles or enzyme systems $(11,6,1)$.

$\mathrm{CO}_{2}$ fixation by isolated whole chloroplasts was strictly light-dependent and proceeded at an almost constant rate for at least an hour. There was approximate correspondence between the oxygen evolved and the $\mathrm{CO}_{2}$ fixed, as would be expected from the well-known photosynthetic quotient in green plants $\left[\frac{\mathrm{O}_{2}}{\mathrm{CO}_{2}}\right]=1$. Both soluble and insoluble products resulted from the fixation of radiocarbon by chloroplasts. The insoluble product of $\mathrm{CO}_{2}$ fixation was identified as starch. Among the soluble products the following were identified: phosphate esters of fructose, glucose, ribulose, secloheptulose, dihydroxyacetone, and glyceric acid; glycolic, malic, and aspartic acids: alanine, glycine, and free dihydroxyacetone and glucose (1). 
The ability of isolated whole chloroplasts to carry out the synthesis of starch from $\mathrm{CO}_{2}$ and water, without the aid of external enzyme systems and substrates, provided the final link in the chain of evidence for the conclusion that chloroplasts are autonomous cytoplasmic bodies containing the complete cellular apparatus needed for photosynthesis (cf. also 157, 73, 147, 66, 148, 65, 136, 137).

In later experiments, $\mathrm{CO}_{2}$ fixation was investigated not with whole chloroplasts but with disrupted chloroplasts (13, 173, 153, 151, 96, 152). The disruption of chloroplast structure impeded starch synthesis, but was very useful in the study of the soluble products of $\mathrm{CO}_{2}$ assimilation. Under suitable experimental conditions the same soluble compounds were formed in light by chloroplasts isolated from a number of different species; spinach, sugar beet, sunflower, Phytolacca americana, and Tetragonia expansa $(174,175)$. The soluble products of $\mathrm{CO}_{2}$ assimilation by broken sugar beet chloroplasts are shown in Fig. 1.

The close similarity of photosynthetic products of intact cells and of chloroplasts isolated from different species left little doubt that chloroplasts are the sites of the complete photosynthetic process in green plants. It seemed legitimate therefore to explore the pattern of energy conversion in photosynthesis, using isolated chloroplasts with the expectation that it would also be relevant to the experimentally much more complex situation in whole cells.

\section{The Role of Light in CO.2 Assmilation}

It is an interesting aspect of the history of photosynthesis that the energy conversion process appeared settled in broad outline for well over a hundred years. Few hypotheses in biology seemed as secure with the passage of time, and had a more tenacious hold on the thinking of biologists for over a century, as the one that the function of light in photosynthesis is the splitting of $\mathrm{CO}_{2}$ with a resultant liberation of $\mathrm{O}_{2}$ and the retention of "C" for forming plant substance. From the time it was first proposed by Ingenhousz in 1796 that plants absorb from "carbonic acid ... in the sunshine, the carbon, throwing ont at that time the oxygen alone, and keeping the carbon to itself, as nourishment" (72), this hypothesis grew in the 19th century to the stature of a fixed principle which served to divide all living organisms into two main groups: green plants that lived by using radiant energy for the assimilation of $\mathrm{CO}_{2}$ and organisms without chlorophyll that were incapable of using radiant energy for $\mathrm{CO}_{2}$ 


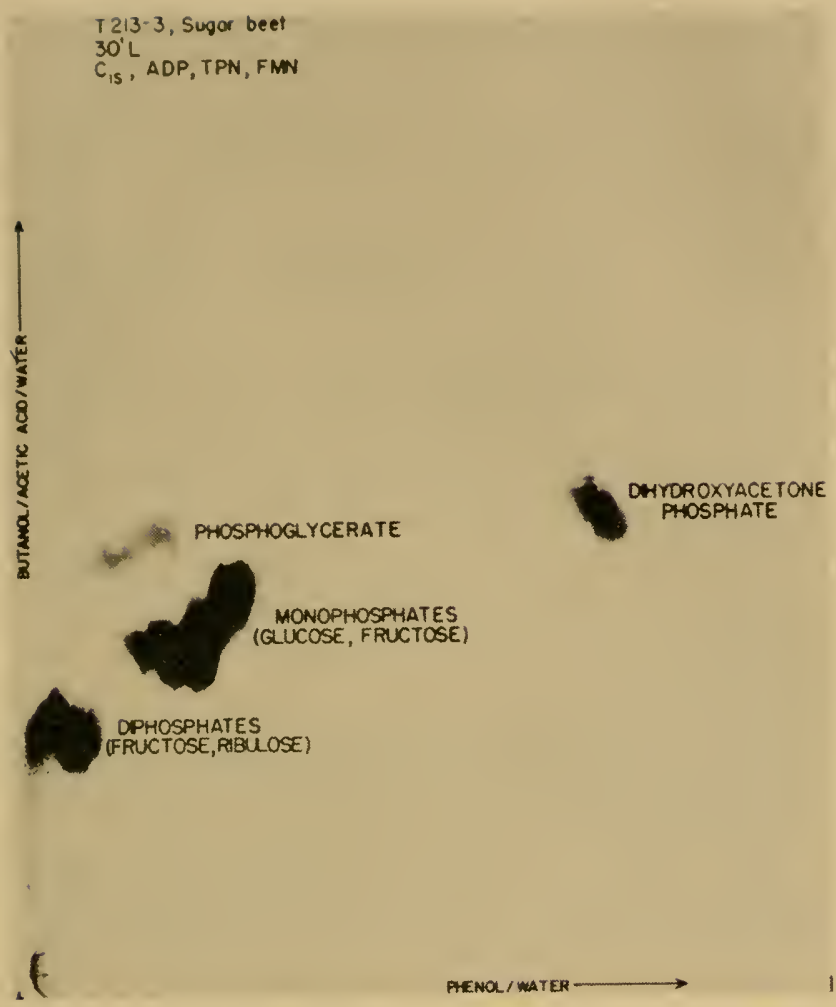

Fig. 1. Products of $\mathrm{CO}_{2}$ assimilation by illuminated chloroplasts isolated from sugar beet leaves. (Whatley, Allen, Trebst and Arnon, 175).

assimilation and could therefore subsist only by consuming the organic substances formed by the photosynthetic group.

So firm was the conviction that only green plants could form organic substances by assimilating $\mathrm{CO}_{2}$ that in the 19th century the term "assimilation" became established in several European languages to describe what is now called photosynthesis. Assimilation, i.e., the formation of organic substances from $\mathrm{CO}_{2}$, was believed to be peculiar to green plants, whereas metabolism, i.e. the utilization of organic substances formed by plants, occurred in both plants and animals (67).

This seemingly logical and orderly division of the living world was destroyed by two discoveries in the 1880 's. Winogradsky (178, 179) discovered chemosynthetic bacteria, i.e., cells without chlorophyll that could form their cellular substance by assimilating $\mathrm{CO}_{2}$ in the clark, 
and Engelmam $(46,47)$ discovered the purple bacteria that carried on a kind of photosynthesis which apparently liberated no oxygen. Neither discovery was fully appreciated in its day; their effect was not that of an explosive charge, but rather that of a time-bomb that dicl its devastation later.

The far-reaching consequences of Winogradsky's discovery were ignored by most of his contemporaries, who persisted in their belief in what Lebedev, one of Winogradsky's few adherents, called, with remarkable insight, a "methodological error" (89). Lebedev pointed out that since the discovery of chemosynthetic bacteria revealed that $\mathrm{CO}_{2}$ assimilation can also occur in the dark, it was no longer permissible to consider $\mathrm{CO}_{2}$ assimilation as peculiar to photosynthetic cells. What was peculiar to these cells was their ability to use light as a source of energy for that process, whereas chemosynthetic organisms obtained the needed energy by oxidation of chemical substrates. $\mathrm{CO}_{2}$ assimilation itself was in both cases an endergonic process, independent of light.

But Lebedev's views were too advanced to gain wide acceptance. This was delayed for over forty years. Only after the discovery of the Wood-Werkman reaction and the use of tracer isotopes by many investigators in the past twenty years, did it become generally recognized that all living cells, photosynthetic and non-photosynthetic, autotrophic and heterotrophic, assimilate $\mathrm{CO}_{2}$, but differ as to the sources of energy that must be expended during this process.

The full impact of the discovery of photosynthetic bacteria was also delayed for about forty years. There was at first no compelling evidence for, and great resistance to, the notion that photosynthesis could proceed without oxygen evolution. The view that $\mathrm{CO}_{2}$ assimilation always involved a liberation of oxygen was so firmly cntrenched that it was even extended to the dark $\mathrm{CO}_{2}$ assimilation by chemosynthetic bacteria. For example, Lebedev's (87-89) early conclusion about the fundamental similarity of $\mathrm{CO}_{2}$ assimilation in chemosynthetic and photosynthetic organisms rested on his belief that hydrogen bacteria use molecular oxygen, released by the decomposition of $\mathrm{CO}_{2}$ for the oxidation of hydrogen gas. The idlea, therefore, of photosynthesis without oxygen evolution seemed a contradiction of terms until the basic facts of bacterial photosynthesis were experimentally unravelled and clearly interpreted by the incisive work of van Niel $(158,159)$. It then became evident that bacterial photosynthesis proceeds anacrobically without oxygen evolution, and the 
hypothesis that lighe functioned in photosynthesis by decomposing $\mathrm{CO}_{2}$ into " $\mathrm{C}$ " and $\mathrm{O}_{2}$ could no longer be maintained ${ }^{2}$.

\section{Renucing, Power Versus Assmillatory Power}

If the function of light was not the photodecomposition of $\mathrm{CO}_{2}$, where was the common role for light to be found in bacterial and plant photosynthesis? This question was answered by van Niel's hypothesis (159) that envisaged a basic similarity between bacterial and plant photosynthesis in a common photochemical origin of the reductant for $\mathrm{CO}_{2}$ assimilation. Van Niel proposed that the light reaction in both groups of organisms is a photolysis of water which results in the formation of a reductant $(\mathrm{H})$ and an oxidant $(\mathrm{OH})$, the latter being the precursor of molecular oxygen.

To account for the lack of oxygen evolution in bacterial photosynthesis, van Niel pointed to the requirement of this process for an external hydrogen donor. The external hydrogen donor was, in his postulation, required for reducing the $(\mathrm{OH})$ back to water, and in this manner keeping the photosynthetic apparatus in bacteria active. Only in green plants did $(\mathrm{OH})$ give rise to molecular oxygen, and in this capacity for oxygen evolution lay the difference between bacterial and plant photosynthesis.

The postulated photolysis of water by radiation at an energy level of visible light was a reaction unknown in chemistry. There were therefore no experimental restrictions on the surmises as to the nature of the $\mathrm{CO}_{2}$ reductant, $(\mathrm{H})$. One postulation which has figured in numerous hypotheses [see for example $(143,56)]$, considered $(\mathrm{H})$ to be a "reducing power" of a potential sufficiently strong to reduce $\mathrm{CO}_{2}$ to the level of carbohydrates by some mechanism that is peculiar to photosynthesis and clifferent from the known biochemical reactions for the dark assimilation of $\mathrm{CO}_{2}$. This hypothesis has thus far remained speculative.

The alternative view regarded $\mathrm{CO}_{2}$ assimilation in photosynthesis as being fundamentally a reversal of the degradative reactions of carbohydrates, i.e., a dark chemosynthetic process which depends on only two products formed by light reactions, reduced pyridine nucleotide and ATP. This hypothesis was formulated on theoretical grounds in 1943 by Ruben (130) and incorporated earlier suggestions

${ }^{2}$ This generalization would apply only to the overall process and would not necessarily exclude the catalytic participation of $\mathrm{CO}_{2}$ in the mechanism of oxygen eiolution, as has recently been proposed by Warburg et al. (165). 
made by Thimann (145) about $\mathrm{CO}_{2}$ fixation and by Lipmann about a possible role of ATP in photosynthesis-the latter being, so far as I know, the first suggestion of this kind ever made (92, p. 148).

Ruben's proposal clistinguished two phases of sugar synthesis in the dark: a carboxylative phase, dependent on ATP only, in which $\mathrm{CO}_{2}$ enters cellular metabolism by carboxylating an acceptor molecule, and a reductive phase, in which a carboxyl group is reduced by pyridine nucleotide with the aid of ATP.

In the ensuing fifteen years Ruben's scheme received experimental support from several directions: (a) Calvin, Benson, Horecker, Ochoa, Racker, and their associates (123, 166, 78, 127) have identified the ATP-dependent carboxylative phase in $\mathrm{CO}_{2}$ assimilation. They have shown that the entry of $\mathrm{CO}_{2}$ into the metabolism of photosynthetic cells depends on the phosphorylation of ribulose monophosphate by ATP to ribulose diphosphate, which is then carboxylated by $\mathrm{CO}_{2}$ and cleaved to give 2 molecules of phosphoglyceric acid (PGA). (b) The kinetic studies of Calvin's group (35) suggested that the reductive phase of $\mathrm{CO}_{2}$ assimilation is the reduction of PGA to triose phosphate by a reversal of the well-known glycolytic reactions-a mechanism that was known to require reduced pyridine mucleotide and ATP. (c) In addition to a carboxylative and a reductive phase, the finding of components of the pentose cycle in photosynthetic tissue (mainly in Horecker's and Racker's laboratories, of. review, 161) afforded a mechanism for the regeneration of the $\mathrm{CO}_{2}$ acceptor in photosynthesis, in what might be designated as a third, regenerative phase of $\mathrm{CO}_{2}$ assimilation. (d) Racker (126) obtained in the dark a syuthesis, driven by $\mathrm{DPNH}_{2}$ and exogenous $\mathrm{ATP}$, of hexose phosphate from $\mathrm{CO}_{2,}$, in a model multi-enzyme system consisting of glycolytic cnzymes from rabbit muscle and yeast (128) and pentose cycle enzymes from spinach leaves.

The carboxylative, reductive, and regenerative phases constitute a cyclic sequence of dark reactions which jointly might be termed a reductive carbolnydrate cycle (cf. 127). The fact that this reductive carbohydrate cycle, comprising exclusivcly dark reactions, was not peculiar to photosynthetic cells was demonstrated when the most distinctive enzyme of the cycle, ribulose cliphosphate carboxylase, was found in such heterotrophs as Escherichia coli $(161,53)$, and when the cycle in its entirety was found in the non-photosynthetic sulfur bacterim Thiobacillus denitrificans $(155,20)$. It thus became clear, as was already earlier anticipated by Benson and Calvin, that "it is not to be expected that this type of work (investigation of the carbon 
(yile) will lead directly $t o$ a solution of the unique problems of photosynthesis, namely, the knowledge of the act or acts by which clectromagnetic energy is tamsformed into chemical energy" (26, p. 10).

The investigation of $\mathrm{CO}_{2}$ assimilation by isolated chloroplasts has shown that the energy requirements for this process were satisfied jointly by $A T P$ and $\mathrm{TPNH}_{2}(153,151,96,152)$. When experimental conditions were so arranged that only $\mathrm{TPNH}_{2}$ was formed in the light, no formation of sugars occurred. In the absence of ATP the photosynthetic "reducing power," TPNH., was powerless to reduce $\mathrm{CO}_{2}(96)$. Work with reconstituted chloroplast systems and inhibitors demonstrated $(96,152)$ that both ATP and $\mathrm{TPNH}_{2}$ must be supplied for photosynthetic $\mathrm{CO}_{2}$ assimilation. ATP and $\mathrm{TPNH}_{2}$ have therefore been jointly termed "assimilatory power" (18) . A general scheme for $\mathrm{CO}_{2}$ assimilation by isolated chloroplasts is summarized in Fig. 2.

To recapitulate, the work with isolated chloroplasts provided no evidence for postulating a photochemical formation of a special re-

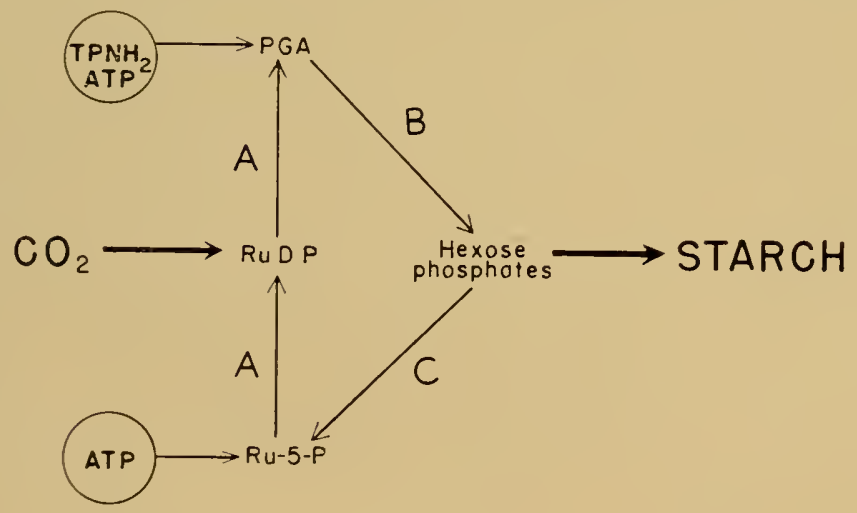

Carbohydrote synthesis by isoloted chloroplasts.

Fig. 2. Diagram of the reductive carbohydrate cycle in chloroplasts. The cycle consists of three phases. In the carboxylative phase $(A)$, ribulose- 5 -phosphate (Ru-5-P) is phosphorylated to ribulose diphosphate (RuDP), which then accepts a molecule of $\mathrm{CO}_{2}$ and is cleaved to 2 molecules of phosphoglyceric acid (PGA); in the reductive phase (B) PGA is reduced and converted to hexose phosphates; in the regenerative phase $(C)$ hexose phosphate is converted into storage carbohydrates (starch) and into the pentose monophosphate needed for the carboxylative phase. All the reactions of the cycle occur in the dark. The reactions of the carboxylative and reductive phases are drisen by $\mathrm{\Lambda TP}$ and $\mathrm{TPNH}_{2}$ formed in the light. 
ductant capable of direct reduction of $\mathrm{CO}_{2}$ to the level of carbohychate by some unknown reaction(s) peculiar to photosynthesis. The investigation of $\mathrm{CO}_{2}$ assimilation by isolated chloroplasts revealed that here also carbohydrate formation occurs by dark reactions that are driven by reduced pyridine nucleotides and ATP.

The "photosynthetic" carbohydrate cycle in green plants differed from the reductive carbohydrate cycle in non-chlorophyllous cells in having a TPN-dependent rather than the conventional DPN-clependent triose phosphate dehydrogenase (96). But this special feature of photosynthetic $\mathrm{CO}_{2}$ assimilation did not alter the reducing potential level of the needed reductant, which is the same for both $\mathrm{TPNH}_{2}$ and $\mathrm{DPNH}_{2}$.

The investigation of $\mathrm{CO}_{2}$ assimilation by isolated chloroplasts has thus completed the chain of evidence for the view that carbohydrate synthesis is removed from the domain of photosynthetic reactions proper in which light is converted into chemical energy. In green plants the first stable, chemically defined products of this energy conversion are not intermediates of $\mathrm{CO}_{2}$ assimilation but are the two components of assimilatory power, $\mathrm{TPNH}_{2}$ and $\mathrm{ATP}(9,153)$.

\section{Photosythetic Phosphory lation}

The experiments with isolated chloroplasts have thus narrowed down the problem of energy conversion in photosynthesis to the identification of those reactions in which $\mathrm{TPNH}_{2}$ and $\mathrm{ATP}$ are formed by light. With respect to $\mathrm{TPNH}_{2}$ it had already been shown independently by Vishniac and Ochoa (162), Tolmach (149), and Arnon (5) that isolated chloroplasts can reduce pyridine mucleotides and simultaneously evolve oxygen, if this photochemical reaction is coupled with a "pulling reaction," i.e., an enzyme system capable of utilizing at once the newly formed reduced pyridine nucleotide.

A direct reduction of pyridine mucleotide by illuminated chloroplasts was first demonstrated by San Pietro and Lang (132), who measured the accumulation of the reduced nucleotides, but not oxygen evolution. In their experiments DPN was reduced in preference to TPN and the reduction of pyridine nucleotides occurred only in the presence of appreciable concentrations of chloroplast material. However, they have also noted that the reaction proceeded at lower .oncentrations of chlorophyll if a soluble extract from chloroplasts was added.

The observations of San Pietro and Lang were confirmed in our inboratory except that under our conditions TPN, instead of IPN, 
was preferentially reduced (17). A water-soluble factor (F), readily extractable from chloroplasts, was required for TPN reduction and was found to be a protein in nature (17). In the presence of the soluble protcin, catalytic amounts of chlorophyll (0.1 micromole) reduced substrate amounts of TPN (4 micromoles). The accumulation of $\mathrm{TPNH}_{2}$ was found to be (17) accompanied by an evolution of oxygen (Table 1), in accordance with reaction $l$ :

$$
\begin{array}{r}
2 \mathrm{TPN}+4 \mathrm{H}_{2} \mathrm{O} \frac{\text { light }}{\text { chloroplasts }+\mathrm{F}} \\
2 \mathrm{TPNH}_{2}+\mathrm{O}_{2}+2 \mathrm{H}_{2} \mathrm{O}
\end{array}
$$

The TPN-reducing factor (F) has subsequently been purified by San Pietro and Lang, who called it the photosynthetic pyridine nucleotide reductase (133). They found the purified enzyme to be more active in reducing TPN than DPN, as was observed with the chloroplast extract by the Berkeley group (17).

It is clear, therefore, that chloroplasts have an enzymatic apparatus necessary for the photochemical reduction of TPN that is coupled with oxygen evolution. What remains to be determined is the source of ATP in photosynthesis, or more specifically, the cellular site and the mechanism by which ATP is formed during photosynthesis. From the standpoint of cellular physiology, the important question is whether the ATP used in photosynthesis is formed by some lightdriven assimilation of inorganic phosphate, catalyzed by enzymes

TABLE 1

Equivalence of TPN Reduction and Oxygen Evolution by Illuminated

Chloroplast Particles in the Presence of

Excess Chloroplast Extract (CE)

(Arnon, Whatley, and Allen, 18)

$\mu$ moles TPN added

0
1.0
2.0
3.0
4.0

$\mu$ moles TPN reduced

$$
\begin{aligned}
& 0.1 \\
& 0.9 \\
& 1.9 \\
& 2.9 \\
& 4.1
\end{aligned}
$$

$\mu$ atoms oxygen evolved
0.2
1.2
2.2
3.1
4.2

The reaction mixture included, (in addition to TPN) in a final volume of $3 \mathrm{ml}$, "broken" chloroplasts $\left(\mathrm{P}_{\mathrm{is}}\right)$ containing $0.1 \mathrm{mg}$ chlorophyll; chlorophyll-free chloroplast extract made from chloroplasts containing $2 \mathrm{mg}$ of chlorophyll; tris-buffer, $p \mathrm{H} 8.3$, $80 \mu$ moles; and $\mathrm{NaCl}, 35 \mu \mathrm{m}$. The reaction was run to completion at $20^{\circ} \mathrm{C}$ under nitrogen, at an illumination of about $35,000 \mathrm{Lux}$, with $\mathrm{KOH}$ in the center well of the manometer vessel. After centrifuging off the particles, reduced TPN in the supernatant fluid was measured spectrophotometrically at $340 \mathrm{~m} \mu$. $\mathrm{O}_{2}$ evolution was measured. manometrically. 
closely bound to the chlorophyll system, or whether ATP is formed by mitochondria, by the process of oxiclative phosphorylation.

The only cytoplasmic particles known to form ATP were mitochondria, and oxidative phosphorylation by them has usually been invoked in explaining the source of ATP used in photosynthesis [see, for example, ref. 24, Fig. 7; also review, ref. 7]. In early models of ATP formation in photosynthesis it was proposed that the reduction of pyridine nucleotide was carried out by illuminated chloroplasts and the resulting reduced pyricline nucleotide was re-oxidized with molecular oxygen by mitochondria (163). This coupled chloroplast-mitochondrial system differed from conventional oxidative phosphorylation only in the source of the reduced pyridine nucleotide. In one case the pyridine nucleotide was reduced by light and in the other by a respiratory substrate. The phosphorylation reactions proper leading to the synthesis of ATP were in both cases supposed to be clependent on enzymes localized in mitochondria.

This model for the generation of ATP in photosynthesis posed a serious difficulty. The most specialized photosynthetic tissue, the mesophyll of leaves, is noted for its paucity of mitochondria. Within the mesophyll cells, especially in the palisade parenchyma, chloroplasts are the dominant cytoplasmic bodies; mitochondria are few $(48,79)$. It was difficult, therefore, to visualize how oxidative phosphorylation by mitochondria could generate enough ATP in tissues noted for their vigorous photosynthetic activity.

The difficulty was removed by the discovery in 1954 that isolated chloroplasts are capable of synthesizing ATP in light without the aid of mitochondria (11). When conditions were so arranged that $\mathrm{CO}_{2}$ assimilation was excluded, isolated chloroplasts used light energy for the esterification of inorganic phosphate in accord with the over-all reaction:

$$
n \cdot \mathrm{P}+n \cdot \mathrm{ADP} \stackrel{\text { light }}{\longrightarrow} n \cdot \mathrm{ATP}
$$

Light-induced ATP formation in chloroplasts raised at once the question whether this process is analogous to oxidative phosphorylation by mitochondria. Two fundamental differences were apparent. ATP formation by illuminated chloroplasts occurred without the consumption of molecular oxygen and without the addition of a (hemical substrate to supply free cnergy needed for the formation of the pyrophosphate bonds of $\mathrm{ATP}$. The term photosynthetic phosphorylation $(11,6)$ was therefore given to the light-induced ATP 
formation by chloroplasts to distinguish it from oxidative (respiratory) phosphorylation by mitochondria and the anacrobic phosphorylations at substrate level that occur in glycolysis. In both of these processes ATP formation occurs at the expense of energy liberated by the oxidation of a chemical substrate, whereas the only "substrate" which is being consumed in photosynthetic phosphorylation is light.

\section{Photosythetic Phosphorylation and Molfcular Oxygen}

Although there was no net consumption of molecular oxygen in photosynthetic phosphorylation (as measured by manometric pressure change), the process, when first discovered, proceeded at a sustained rate only in the presence of oxygen (11, Fig. 2b). We noted at the time that "the overall reaction of photosynthetic phosphorylation differs from the conventional Hill reaction in that there is no net $\mathrm{O}_{2}$ evolution"; and that "photosynthetic phosphorylation also differs from oxidative phosphorylation of the type investigated by Lehninger. In oxidative phosphorylation oxygen is consumed, while in photosynthetic phosphorylation there is no evidence of utilization of atmospheric oxygen" (11). Oxygen thus acted as a catalyst in photosynthetic phosphorylation, not as a substrate, as it does in oxidative phosphorylation. A decisive difference between photosynthetic and oxidative phosphorylation was the inability of chloroplasts to form ATP in the dark by oxidizing hydrogen donors of oxidative phosphorylation with molecular oxygen (12).

Further investigation of photosynthetic phosphorylation by spinach chloroplasts soon resulted in the identification of flavin mononucleotide $(\mathrm{FMN})$ and vitamin $\mathrm{K}$ as catalysts in the process (15, p. $6326 ; 16,170)$. At optimal (but still catalytic) concentrations of either FMN (170) or vitamin K (Fig. 3), photosynthetic phosphorylation became independent of external oxygen and proceeded vigorously in an atmosphere of nitrogen or argon. At a much lower, "microcatalytic," concentration of the added cofactors, photosynthetic phosphorylation remained dependent on oxygen, although still showing no net oxygen consumption.

The effects of added FMN and vitamin $\mathrm{K}$ were thus compatible with two hypotheses that were advanced earlier to account for the absence of a net oxygen consumption in photosynthetic phosphorylation. When no cofactors were added (11) or at low concentrations of FMN and vitamin $\mathrm{K}$ (Fig. 3), the dependence on external oxygen was consistent with the hypothesis that under these conditions oxygen was acting catalytically and sustaining photosynthetic phosphorylation by 
INTENSE LIGHT and HIGH CHLOROPHYLL

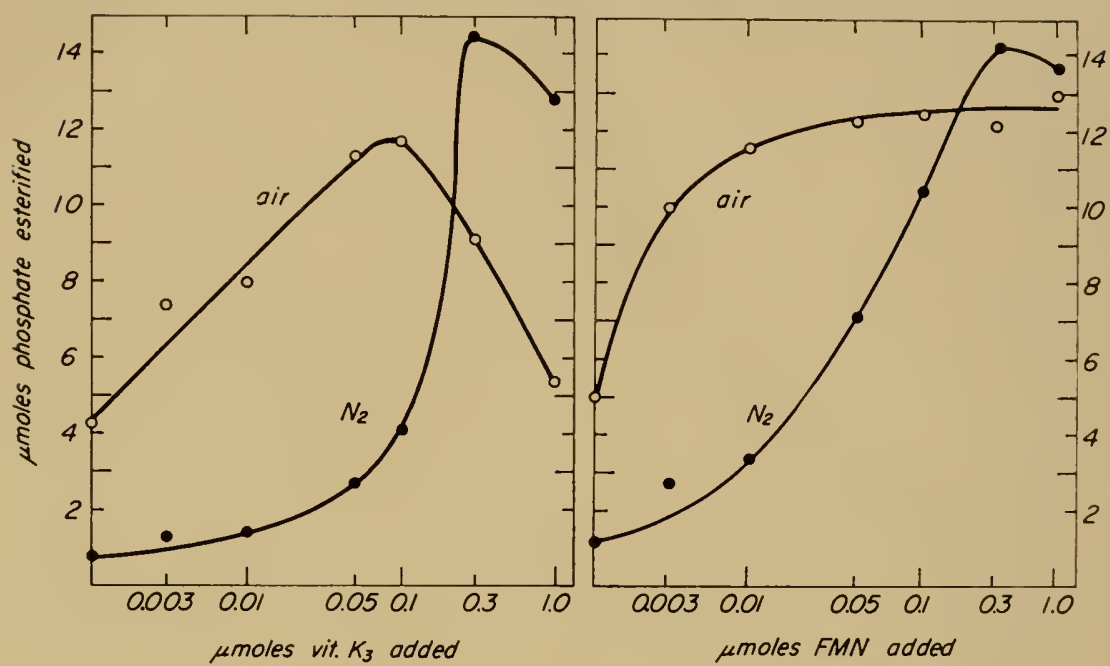

Fig. 3. Effect of vitamin $\mathrm{K}_{3}$ (2-methyl-1,t-naphthoquinone) and FMN concentration on cyclic photophosphorylation by spinach chloroplasts in nitrogen and air at a high light intensity. The reaction mixture (3 $\mathrm{ml}$ final volume) included chloroplast fragments $\left(\mathrm{C}_{1 \mathrm{~s}}\right)$ containing $1.5 \mathrm{mg}$ chlorophyll; and in micromoles: Tris buffer $p \mathrm{H} 8.3 .80 ; \mathrm{K}_{2} \mathrm{H}^{32} \mathrm{PO}_{1}, 20 ; \mathrm{ADP}, 20$; $\mathrm{IgSO}_{1}, 5$; and TPN, 0.3 (only in the FMN series). FIIN and vitamin $K_{3}$ were added as indicated. The reaction was run for 5 minutes at an illumination of 50.000 Lux. (Tsujimoto, Hall, and Irmon, 156; Arnon, Whatley, and Allen, unpublished data, 1954.)

reoxidizing the requisite catalysts-when these are present in limiting concentrations. At the higher concentrations of added cofactors, photosynthetic phosphorylation could proceed in the total absence of oxygen (see Section 16).

This interpretation is in agreement with the recent results of Wessels (169), Jagendorf and Avron (77), and Nakamoto, Krogmann, and Vennesland (111), that photosynthetic phosphorylation with suboptimal amounts of cofactors was oxygen-dependent but became oxygen-independent at higher concentrations of cofactors.

In charting their subsequent investigation, the Berkeley group laid special stress on the anaerobic photosynthetic phosphorylation which proceeds in isolated chloroplasts at optimal catalytic concentrations of FMN and vitamin $\mathrm{K}$. They considered this type more fundamental to photosynthesis in general than the oxygen-catalyzed type because it would also apply to bacterial photosynthesis, which is an anaerobic process. 
Soon after the discovery of photosynthetic phosphorylation in isolated chloroplasts, Frenkel (19) reported a similar phenomenon in the photosynthetic bacterium Rhodospirillum rubrum. Although Frenkel suggested that the light-induced ATP formation in bacterial preparations was similar to that in chloroplasts, the similarity seemed uncertain at first, because Frenkcl's photophosphorylation system, which was a sonic macerate of $R$. rubrum cells, differed in several respects from its comnterpart in isolated chloroplasts (11). Frenkel's preparations became substrate-dependent after washing; the rate of phosphorylation was doubled on adding $\alpha$-ketoglutarate (49). But in later expcriments he ruled out the dependence on an added chemical substrate $(50)$ and the equivalence of chloroplast and bacterial photophosphorylation could no longer be cloubted.

Frenkel's findings were followed by those of Williams (177), who demonstrated photosynthetic phosphorylation in cell-free preparations of the obligately anaerobic photosynthetic bacteria, Chromatium and Chlorobium. It thus became clear that a common anaerobic mechanism for a light-induced phosphorylation, that does not depend on an exogenous chemical substrate or on oxygen consumption, is shared by both green plants and photosynthetic bacteria. The energy conversion process proper seemed to be fundamentally independent of oxygen, although it was still possible that details of mechanisms were different in green plants and photosynthetic bacteria.

The discovery of photosynthetic phosphorylation in chloroplasts by Arnon et al. (I I) and in bacterial particles by Frenkel (49) was confirmed and extended in a number of laboratories ${ }^{3}$. Photosynthetic phosphorylation in isolated chloroplasts was observed by Avron and Jagendorf $(21,22)$, Wessels $(168)$, and Vemnesland and her associates (37, 111); in algae by Thomas and Haans (146) and Petrack (120); and in photosynthetic bacteria by Geller and Gregory (60), Geller (59), Kamen and Newton (81), and Anderson and Fuller (3). In later experiments Whatley et al. $(174,175)$ have shown that photosynthetic phosphorylation by chloroplasts, which had previously been almost entirely limited to observations on chloroplasts isolated from one species, viz., spinach, is also demonstrable with chloroplasts

${ }^{3}$ Other accounts of the discovery of $\mathrm{CO}_{2}$ assimilation and photosynthetic phosphorylation by isolated chloroplasts are given by Calvin. In 1956 he ascribed (25, p. 31) the discovery of $\mathrm{CO}_{2}$ assimilation by isolated chloroplasts to Boychenko and Baranor (31) and in 1959 he ascribed the discovery of both $\mathrm{CO}_{2}$ assimilation by isolated chloroplasts and photosynthetic phosphorylation to his own laboratory (36, p. 152). 
isolated from several other species of higher plants. It now seems well established, therefore, that the cytoplasmic particles, which in all photosynthetic organisms contain the chlorophyll pigments, also contain a closely bound phosphorylation system.

Soon after the demonstration of photosynthetic phosphorylation in isolated chloroplasts, attempts were made to compare its rate with that of $\mathrm{CO}_{2}$ assimilation by illuminated whole cells. Since, as with most newly discovered cell-free reactions ${ }^{4}$, the rates of photosynthetic phosphorylation were rather low, there was little inclination at first to accord this process quantitative importance $(57$, p. 292, 345) as a mechanism for converting light into chemical energy.

With further improvement in experimental methods we obtained rates of photosynthetic phosphorylation up to 170 times higher (2) than those originally described (11), and even these high rates were exceeded by Jagendorf and Avron (76). The improved rates of photosynthetic phosphorylation were equal to or greater than, the maximum known rates of carbon assimilation in intact leaves. It appeared, therefore, that isolated chloroplasts retain, without substantial loss, the enzymatic apparatus for photosynthetic phosphorylationa conclusion which is in harmony with evidence that the phosphorylating system is tightly bound in the water-insoluble grana portion of the chloroplasts (see Section 11).

Unlike the phosphorylation system, the enzymes catalyzing $\mathrm{CO}_{2}$ assimilation are water-soluble $(13,173,153)$ and are therefore readily lost in the isolation of chloroplasts. This results in lower rates of $\mathrm{CO}_{2}$ assimilation by isolated chloroplasts than by the intact parent leaves. The difference between the rate of $\mathrm{CO}_{2}$ assimilation by isolated chloroplasts and that of intact leaves may be made to appear greater, though less relevant, if the comparison is made not between isolated chloroplasts and their parent leaf tissue, but between isolated chloroplasts and maximum rates of $\mathrm{CO}_{2}$ assimilation observed in unrelated leaf material and under different experimental conditions. Nevertheless, the now known rates of $\mathrm{CO}_{2}$ assimilation in isolated chloroplasts [ 10 to 20 per cent of that in parent leaf tissue $(136,96)$ ] are substantial enough to strengthen the conclusion that photosynthesis by isolated chloroplasts mirrors that in the intact leaf. This conclusion is fortified by the identity of the photosynthetic products found in both cases.

The most recent instance of this kind is the cell-free synthesis of DNA investigated by Komberg. "The first positive results represented the conversion of only a very small fraction of the acid-soluble substrate into an acid-insoluble fraction (50 or so counts out of a million added)" (85). 


\section{Catalssts of Photosythetic Phosphorilation}

The most significant conclusion drawn from the recent investigations with isolated chloroplasts was that photosynthetic phosphorylation represents a major mechanism for converting light into useful chemical energy independently of $\mathrm{CO}_{2}$ assimilation. We shall now disctuss what has been learned about the mechanism and the physiological significance of this direct conversion of light into pyrophosphate bond cnergy.

The first factors which were found to stimulate cyclic photophosphorylation without themselves being consumed in the reaction were magnesium ions and ascorbate $(11,15)$; the next to be recognized were FMN and vitamin $\mathrm{K}$ compounds $(170,16)$.

In searching for factors of photosynthetic phosphorylation by isolated chloroplasts special attention was given to normal constituents of chloroplasts and green leaves. Magnesium and ascorbate have long been known to be present in chloroplasts (124). FMN is widely distributed in green leaves (134). Ohta and Losada in our laboratory (unpub.) have found FMN to be a regular constituent of chloroplasts. Of unusual interest, however, was the antihemorrhagic factor, vitamin $\mathrm{K}$, which occupied, since its discovery in plants, a unique position among other vitamins in being specifically associated with chloroplasts $^{5}(40)$. Moreover, Martius and others have recently assigned a role to vitamin $\mathrm{K}$ in oxidative phosphorylation (103, 104; cf. 10 , review).

The working hypothesis first formulated to account for the role of cofactors in photosynthetic phosphorylation visualized the light reaction as resulting in the simultaneous generation, through a photolysis of water, of a reducing agent $(\mathrm{H})$ and an oxidizing agent $(\mathrm{OH})$ at opposite ends of an clectron transport chain; vitamin $\mathrm{K}, \mathrm{FMN}$, ascorbate, and cytochromes were tentatively proposed as members of

${ }^{5}$ Bishop, who earlier presented evidence that vitamin $\mathrm{K}$ is an essential factor for the photochemical activity of isolated chloroplasts (27), has reported in a more recent publication (28) that spinach chloroplasts do not contain the naphthoquinones of the vitamin $K$ type but contain instead the benzoquinone $0-255$ ("plastoquinone"), which Crane (39) and Folkers and his associates (15t) found in green tissues and which Crane also found to be specifically concentrated in chloroplasts (39). Bishop (28) has reported that Q-255 activates the Hill reaction. The role of $\mathrm{Q} .25 \mathrm{~J}$ in photosynthetic phosphorylation is still unknown, but it should be noted that from the standpoint of the mechanism of photosynthetic plosphorylation (see next section), either a naphthoquinone of the vitamin $\mathrm{K}$ type or a benzoquinone would appear suitable as an electron carrier in the process. A clarification of the disagreement between the earlicr reports of vitamin $\mathrm{K}$ distribution in chloroplasts (40) and the recent reports on $Q-255$ will be awaited. 
an electron transport chain which catalyzed a stepwise recombination of the products of photodecomposition of water (6). It was suggested that the energy liberated in this stepwise reconstitution of water became available for ATP formation. The role of the first electron or hydrogen acceptor was tentatively assigned to either FMN (6) or, as proposed by Wessels (167), to vitamin $\mathrm{K}(6,16,12)$.

Further studies of photosynthetic phosphorylation made it apparent that the initial hypothesis required revision. TPN, not included in earlier formulations, was identified as a catalyst of photosynthetic phosphorylation (17, cf. 101). Evidence was also obtained that, under modified experimental conditions, FMN and vitamin $\mathrm{K}$ may be active in catalyzing separate pathways of photosynthetic phosphorylation (171, 172). Maximal rates of phosphorylation occurred with either FMN or vitamin $\mathrm{K}$ as catalysts. At a saturating concentration of one catalyst, the addition of the other gave no further increase in photosynthetic phosphorylation, but little phosphorylation occurred unless one of them was added.

The distinction between the FMN and the vitamin K pathway of photosynthetic phosphorylation was made on the basis of their diflerential dependence on TPN and differential sensitivity to clinitrophenol and o-phenanthroline. A similar conclusion that FMN and vitamin $\mathrm{K}$ catalyze alternative pathways for electron transport in photosynthetic phosphorylation was independently reached by Wessels (168), but on the basis of findings which in some respects did not agree with our own (171, 172).

Apart from the catalytic effect of FMN and vitamin $K$, photosynthetic phosphorylation may also be increased by the addition of nonphysiological cofactors (76). Among these, of particular interest is phenazine methosulfate, since this dye is known to be a strong reducing agent for cytochromes (105). Phenazine methosulfate was found to stimulate photosynthetic phosphorylation in bacterial preparations by Geller (59) and Kamen and Newton (81) and in spinach chloroplasts by Jagendorf and Arron (76).

With respect to inhibitors, the phenazine methosulfate system resembled the vitamin $\mathrm{K}$ pathway in its resistance to dinitrophenol and ()-phenanthroline (172). But in other respects phenazine methosulfate differed from either the FMN or the vitamin $\mathrm{K}$ pathways. The use of phenavine methosulfate in photophosphorylation seemed to dispense with the requirement for other cofactors, such as ascorbate, and also gave higher rates of photophosphorylation than were obtained with either FMN or vitamin K (76). 


\section{Cofactors of Bacterial Photophosphorylation}

Of the cofactors of photosynthetic phosphorylation discussed so far, ascorbate and phenazine methosulfate, but not FMN, were found to be effective in photosynthetic phosphorylation by cell-free preparations from Rhodospirillum rubrum (59) and Chromatium (81). In addition, Geller (59) has also found a stimulatory effect of vitamin $\mathrm{K}_{3}$.

Under our experimental conditions photosynthetic phosphorylation by cell-free preparations of Chromatium showed no response to added cofactors when the particles were freshly prepared under anaerobic conditions. On aging, however, an effect of added vitamin $\mathrm{K}$ and phenazine methosulfate was observed (Table 2); the joint

\section{TABLE 2}

Effect of Vitamin $K_{5}$ and Phenazine Methosulfate (PMS) on Photophosphorylation by Aged Cell-free Preparations of Chromalium [Chromatophores (P) and Supernatant Fluid (S)]

(Ogata, Nozaki, and Arnon, 115)

\section{$\mu \mathrm{moles} \mathrm{P}$ esterified/mg chlorophyll/hr.}

\begin{tabular}{|c|c|c|c|c|c|}
\hline \multirow[b]{2}{*}{ Treatment $^{1}$} & \multicolumn{5}{|c|}{ Aging time (days) } \\
\hline & 0 & 1 & 2 & 4 & 8 \\
\hline 1. $\mathrm{P}+\mathrm{S}$ & 83 & 55 & 42 & 40 & 26 \\
\hline 2. $\mathbf{P}+\mathrm{S}$, vit. $\mathrm{K}_{5}, \mathrm{PMS}$ & 99 & 98 & 91 & 80 & 66 \\
\hline 3. PS & 87 & 30 & 27 & 21 & 15 \\
\hline 4. PS, vit. $\mathrm{K}_{5}, \mathrm{PMS}$ & 96 & 106 & 75 & 65 & 45 \\
\hline
\end{tabular}

${ }^{1} \mathrm{P}$ and $\mathrm{S}$ were stored separately in Treatments 1 and 2 and together in Treatments 3 and 4.

Each vessel contained, in a final volume of $3.0 \mathrm{ml}$, cell-free preparation containing

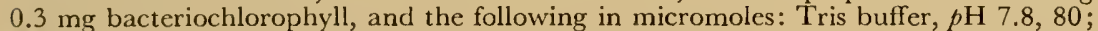
$\mathrm{MgCl}_{2}, 5 ; \mathrm{K}_{2} \mathrm{H}^{32} \mathrm{PO}_{4}, 10$; and ADP, $10.0 .1 \mu$ mole each of vitamin $\mathrm{K}_{5}$ (2-methyl-4amino-1-naphthol hydrochloride) and PMS were added as indicated. Gas phase was argon. The reaction was carried out at $20^{\circ} \mathrm{C}$ for 30 minutes and stopped by adding $0.3 \mathrm{ml}$ of 20 per cent trichloracetic acid TCA to each ressel. Illumination 35,000 Lux.

addition of these two cofactors gave a greater increase of phosphorylation than when they were added singly. The addition of FMN gave no increase $(59,81)$, and in fact, under our experimental conditions, inhibited photosynthetic phosphorylation by Ghromatium particles.

For the purposes of experimental analysis, bacterial photophosphorylation thus appeared to be of the vitamin $\mathrm{K}$ or phenazine methosulfate type rather than of the FMN type (172). This conclusion was confirmed by the response of Chromatium photophosphorylation to inhibitors, as shown in Table 3. Photophosphoryla- 
TABLE 3

Effect of Inhibitors on Photophosphorýlation by Chromatium Particles (OGATa, Nozaki, AND ARnON, 115)

Treatment

$\mu$ moles P esterified $\mathrm{mg}$ chlorophyll/hr.

$\begin{array}{lr}\text { Control } & 75 \\ 10^{-3} M \text { dinitrophenol } & 52 \\ 5 \times 10^{-5} M o \text {-phenanthroline } & 50 \\ \text { Control } & 126 \\ \text { Antimycin A, } 10 \mu \mathrm{g} & 119 \\ \text { Gramicidin, } 40 \mu \mathrm{g} & 119 \\ 10^{-4} M \text { methylene blue } & 100 \\ 10^{-5} M p \text {-chloromercuribenzoate } & 14\end{array}$

Each vessel contained, in a final volume of $3.0 \mathrm{ml}$, cell-free preparation (PS) containing $0.2 \mathrm{mg}$ bacteriochlorophyll and the following, in micromoles: Tris buffer, $p \mathrm{H} 7.8,80 ; \mathrm{MgCl}_{2}, 5 ; \mathrm{K}_{2} \mathrm{H}^{32} \mathrm{PO}_{4}, 15 ; \mathrm{ADP}, 15$; vitamin $\mathrm{K}_{3}$ (2-methyl-1,4-naphthoquinone) 0.3 ; and phenazine methosulfate, 0.1 . Inhibitors were added as indicated. Other conditions were the same as those described for Table?.

tion by Chromatium particles was resistant to inhibition by dinitrophenol and o-phenanthroline, as was also the case with vitamin $\mathrm{K}$ and phenazine methosulfate type photophosphorylation in chloroplasts. These inhibitor effects for Chromatium are similar to those reported for $R$. nubrum $(49,59)$.

Table 3 shows that photosynthetic phosphorylation by Chromatium particles also resembled that of chloroplasts in its resistance to gramicidin and antimycin A [when phenazine methosulfate was present in the reaction mixture (cf. 59) ] and its sensitivity to $p$-chloromercuribenzoate (cf. 172). However, unlike chloroplasts (12, 172), photophosphorylation by Chromatium particles was, uncler our experimental conditions, resistant to inhibition by methylene blue (cf. 59) .

\section{The Electron Flow Mechanism of Photosinthetic PIIOSPHORYLATION}

Photosynthetic phosphorylation has provided direct experimental evidence for the view that the key event in photosynthesis, the conversion of light into chemical energy, is independent of the classical manifestations of this process in green plants: oxygen evolution and $\mathrm{CO}_{2}$ recluction. If it is accepted that photosynthetic phosphorylation represents the simplest common denominator of photosynthesis in green plants and bacteria, then a mechanism for this process would be expected to provide a basic pattern for the conversion of light into chemical energy. The salient facts which must be explained are that 
a "high-chergy" pyrophosphate bond is formed at the expense of absorbed light energy. There is no nced, a priori, to connect this reaction either with photolysis of water or with reduction of $\mathrm{CO}_{2}$. Photosynthetic phosphorylation catalyzed by phenazine methosulfate or vitamin $\mathrm{K}$ produces ncither a reductant for $\mathrm{CO}_{2}$ assimilation nor molecular oxygen; the sole product is ATP.

The simplest hypothesis to account for the formation of ATP in photosynthetic phosphorylation is to assume that, as in the dark phosphorylations of glycolysis and respiration, the formation of a pyrophosphate bond is also coupled with a release of free energy which occurs during electron transport, i.e., when an electron drops from the higher energy level (that it has when it resides in the electron donor molecule) to the lower energy level that it assumes on joining the electron acceptor molecule. But a mechanism for photosynthetic phosphorylation must also account for its unique features: ATP is formed without the consumption of an exogenous electron donor and electron acceptor. Unlike oxidative phosphorylation, photosynthetic phosphorylation consumes neither exogenous substrate nor molecular oxygen, only light energy.

A mechanism for photosynthetic phosphorylation must, therefore, provide for the generation of both an electron donor and an electron acceptor in the primary photochemical act when radiant energy is absorbed by chlorophyll. Investigations of photosynthesis at the cellular level, in which the main preoccupation has usually been with $\mathrm{CO}_{2}$ assimilation and oxygen evolution, led to no cogent theory of the primary act of photosynthesis that would fit the experimental facts of photosynthetic phosphorylation. As summed up recently by Livingston, "physiologists and biochemists appear to believe that this question (the primary act of photosynthesis) was answered long ago by physicists while physicists find the problem distressingly complicated and therefore uninteresting" (93).

The mechanism of photosynthetic phosphorylation that we have proposed (9) regards the photosynthetic particle, chloroplast or bacterial chromatophore, as a "closed" catalytic system. We have suggested that during the primary photochemical act one component of the "closed" system, chlorophyll (bound to protein), becomes excited on absorbing a photon and "expels" one of its electrons that has been raised to a higher energy level. The excited chlorophyll thus becomes the electron donor. On losing an electron, chlorophyll assumes an oxidized state, and in this way also becomes the electron acceptor in photosynthetic phosphorylation. 
The "expelled" electron returns in a stepwise mamner to the oxidized chlorophyll molecule, which thereupon resumes its normal ground state. On its return "downhill" path, the expelled electron releases free energy as it passes through several electron carriers. These intermediate electron carriers are coupled with enzyme systems that catalyze the phosphorylation process during which electron energy is converted into pyrophosphate bond energy. After returning to chlorophyll, the cyclic journey of the electrons begins once more as chlorophyll molecules acquire fresh excitation energy by recurrent absorption of photons. The stepwise interaction of the "expelled" electron with the intermediate electron acceptors constitutes the energy conversion process in photosynthetic phosphorylation. Because of the cyclic path travelled by electrons that are activated by light, this type of photosynthetic phosphorylation has been called cyclic photophosphorylation $(18,9)$.

Chlorophyll can, of course, also be restored to the ground state when the excited electron returns directly without going through the enzymatic "energy transformer stations," but in that case electron energy has not been converted into chemical energy and hence photosynthesis has not occurred. Instead, a light quantum is emitted and the characteristic fluorescence of chlorophyll is observed.

The primary photochemical reaction in which an absorbed light quantum "excites" a chlorophyll molecule and "expels" an electron, is represented by Equations 3 and $3 a$. The symbol $[\mathrm{Chl}]+$ is intended to denote that the chlorophyll molecule as it loses an electron becomes oxidized or forms a "hole" ["odd ion", (91)] that is ready to accept another electron and to return in this way to its normal ground state.

$$
\begin{aligned}
& \mathrm{Chl}+h_{v} \rightarrow \mathrm{Chl}^{*} \\
& \mathrm{Chl}^{*}=[\mathrm{Chl}]^{+}+\mathrm{e}^{-}
\end{aligned}
$$

In the proposed mechanism of cyclic photophosphorylation it was suggested (9) that $\left[\mathrm{Chl}_{1}\right]^{+}$is restored to its ground state by accepting an electron from a cytochrome present in the photosynthetic particle. This "terminal" cytochrome component, i.e., a cytochrome that is adjacent to, and interacts with, the excited chlorophyll molecule, becomes oxidized after donating an electron to chlorophyll. We have visualized (9) that phosphorylation is coupled with the oxidation of the terminal cytochrome, in a manner analogous to the phosphorylations which accompany the oxidation of cytochromes by oxygen in 
mitochondria (90). Thus, chlorophyll with the aid of light is the ultimate oxidant in photosynthetic phosphorylation and plays a part which corresponds to that of molecular oxygen in oxidative phosphorylation (cf. 71$)$. The terminal phosphorylation reaction is represented by Equation $t$.

$$
\begin{array}{r}
2[\mathrm{Chl}]++2 \mathrm{Fe}^{2+} \mathrm{cyt}+\mathrm{ADP}+\mathrm{H}_{3} \mathrm{PO}_{4} \rightarrow \\
\mathrm{ATP}+2 \mathrm{Chl}+2 \mathrm{Fe}^{3+} \mathrm{cyt}
\end{array}
$$

The photophosphorylation reaction would leave the cytochrome in the oxidized state. Since cytochromes are present in catalytic amounts, cyclic photophosphorylation would soon cease unless the cytochrome could become reduced again. Our theory provides that in cyclic photophosphorylation the reduction of cytochrome occurs by the return of the electron originally "expelled" from chlorophyll in the primary photochemical reaction (Equations 3 and $3 a$ ).

In isolated chloroplasts, the reduction of cytochrome by the electron expelled from chlorophyll requires an added catalyst, i.e., an intermediary electron carrier. In the simplest case, as shown in the scheme in Fig. 4, the part of the electron carrier is played by a nonphysiological catalyst, phenazine methosulfate. Phenazine methosulfate is known to be a very effective electron carrier in reactions inrolving cytochromes. For example, Massey (105) has found that

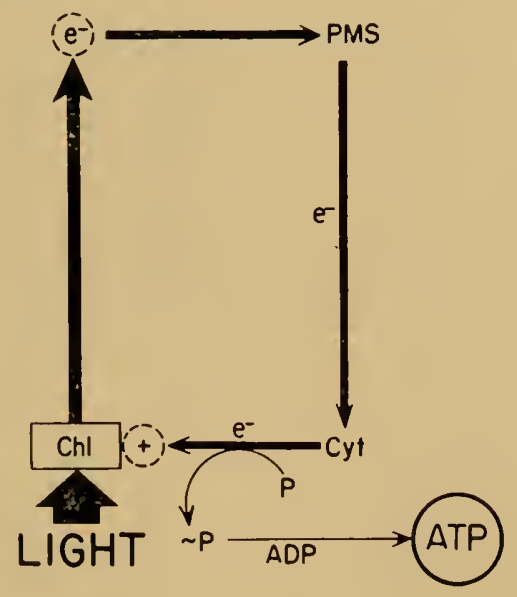

Cyclic photophosphorylation (PMS type)

Fig. 4. Scheme for anaerobic cyclic photophosphorylation catalyzed by phenazine methosulfate (PMS). Details in the text. 
cytochrome $c$ is rapidly reduced in a non-enzymic reaction with reduced phenazine methosulfate. In Fig. 5, the intermediary electron carrier is vitamin $\mathrm{K}$ or FMN.

The cyclic electron flow diagrams, illustrated by Figs. 4 and 5, are components of the scheme presented earlier (9). The key reaction in the proposed mechanism, the photooxidation of chlorophyll by the loss of an electron, is based on a type of reaction in photochemistry that was experimentally documented by Lewis and Lipkin (91). They found, by illuminating a variety of substances in rigid media, "that one of the commonest photochemical processes is the mere loss of an electron by an activated molecule" (91). The evidence for the then (in 1942) "new and somewhat surprising phenomen" (91) was, for example, "that chemical oxidation at room temperature and photooxidation at liquid air temperature (of tri$p$-tolylanine) have given the same substance, namely, the positive ion left, $\left(p-\mathrm{CH}_{3}-\mathrm{C}_{6} \mathrm{H}_{4}\right)_{3} \mathrm{~N}^{+}$, when one electron has been removed" (91).

In the reactions studied by Lewis and Lipkin the fate of the ejected electron was uncertain but, as they pointed out, "the electron must lie in a potential hole which is cleep enough so that the large electrostatic field of the ion is unable to dislodge it" for a considerable period of time. This was indicated by the fact that "the [blue] color (of the 'odd ion' formed) persists at liquid air temperature for several

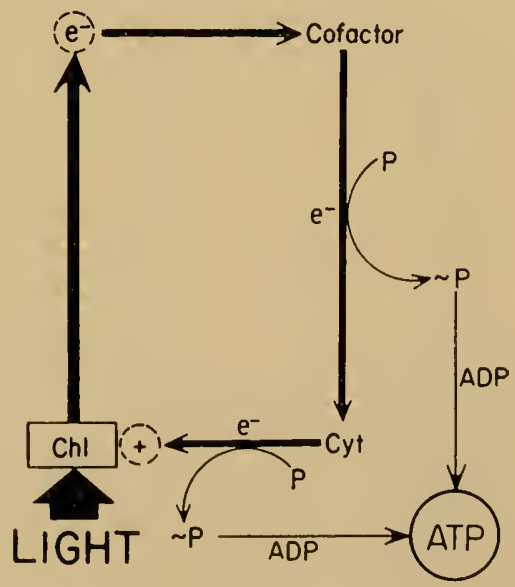

Anoerobic cyclic photophosphorylation

Fig. 5. Schemc for anacrobic cyclic photophosphorylation catalyzed by vitamin $\mathbf{K}_{3}$ or FMN. Details in the text. 
days, but at only slightly higher temperatures the color disappears. Then presumably the electron has returned to the ion" (91). In cyclic photophosphorylation the electron expelled from chlorophyll is visualized as reaching the first intermediary acceptor in the electron transport chain either by resonance transfer or by a semi-conductor mechanism (122a), and thus initiating the clectron transfer process that makes cyclic photophosphorylation possible (9).

To summarize, then, the simplest experimentally demonstrable case of conversion of light energy into chemical energy, a case that is common to all chlorophyll-containing particles, is cyclic phosphorylation. In cyclic photophosphorylation electrons flow from chlorophyll to a cofactor (Figs. 4 and 5), from the cofactor to cytochromes, and from cytochromes back to chlorophyll. During this cyclic flow of electrons the cytochromes present in the photosynthetic particles undergo oxidation-reductions which are believed to be coupled to phosphorylation reactions that produce ATP.

The proposed mechanism for this process may be divided into three phases: (a) the light-induced generation of a high-energy electron and the ultimate electron acceptor [Chl]+; (b) electron transport; and (c) phosphorylation reactions coupled to electron transport. Phases (b) and (c) are analogous and possibly identical in some respects with their counterparts in oxidative phosphorylation, whereas phase (a) is peculiar to photosynthetic phosphorylation.

\section{Evidence for Electron Flow Mechanism in Cyclic}

\section{PHOTOPHOSPHORYLATION}

The validity of the proposed mechanisms for cyclic photophosphorylation is supported by several lines of evidence. These include recent experiments on the effect of chloride and ferricyanide on photosynthetic phosphorylation in isolated chloroplasts and chromatophores, and experiments on the effect of light and vitamin $\mathrm{K}$ on cytochromes of chlorophyllous particles. This evidence will now be discussed in more detail.

\section{Effect of Chloride}

The role of chloride in photosynthesis was discovered by Warburg (164), who found that chloride, replaceable by bromide but not by other anions, was essential for oxygen evolution by isolated chloroplasts. This discovery was fully confirmed by Arnon and Whatley (14), but they were disinclined to accept, at that time, Warburg's conclusion that chloride is a coenzyme of photosynthesis, because this 
conclusion would have conferred on chloride the then unwarranted status of an essential element for green plants. However, they envisaged the possibility, which has since been documented by Broyer and associates (34) and Martin and Lavollay (102), that chloride may prove to be an essential micromutrient for green plants.

A reinvestigation by Bové et al. (30) of the role of chloride in the photochemical reactions of chloroplasts confirmed Warburg's conclusion that chloricle is essential for those photosynthetic reactions in which oxygen is liberated. Chloride was not required, however, for the anaerobic cyclic photophosphorylation that is shared by bacterial particles and chloroplasts. Thus, in the absence of chloride, chloroplasts behaved like bacterial chromatophores. They were able to carry out the anaerobic cyclic photophosphorylation but were $\mathrm{m}$ able to evolve oxygen.

\section{Effect of Ferricyanide}

The key premise in the proposed mechanisms for cyclic photophosphorylation is that the electron expelled from the chlorophyll molecule in the primary photochemical act is not removed from the "closed circuit" within which it travels before it returns to the chlorophyll. If this basic postulation is correct it follows that cyclic photophosphorylation should be abolished if the electrons are prevented from completing the cycle because of capture by an external electron acceptor. To be convincing, such an experiment should be carried out with an electron acceptor which would be free from the suspicion that it prevented phosphorylation by acting as an uncoupler, or in some toxic manner.

An electron acceptor that fulfills these requirements is ferricyanicle. As shown by Jagendorf (74), and confurmed in this laboratory, ferricyanide has a great affunity for trapping electrons during photophosphorylation. Thus by adding ferricyanide, in the absence of chloride, cyclic photophosphorylation in both chloroplasts and chromatophores should be inhibited, if the proposed hypothesis is correct. The cyclic flow of electrons in the closed circuit would be interrupted when the electrons are trapped by, and used in, the reduction of ferricyanide.

Table 4 shows that this theoretical prediction has been experimentally verified. The addition of ferricyanide abolished cyclic photophosphorylation both in chloroplasts and in chromatophores. Adding this ion in its reduced form, as ferrocyanide, was withont effect. The reduction of ferricyanicle with ascorbate either prior to, or during, illumination of the photosynthetic particles restored in full their 
TABLE 4

INHAHNG OF FFRICYANIDE (IN THE ABSENCT, OF CIILORIBH) ON Ciclic Photophosphorylation by Spinach Chloroplasts and Bacterial. Cilromatophores (Chromatium)

(Micromoles Phosphate Esterified in 30 Minutes)

(Bové, Bové, Whatley, and Arnon, 30)

Treatment

Control

Ferricyanide, $1 \mu \mathrm{m}$

Ferricyanide, $2 \mu \mathrm{m}$

Ferricyanide, $3 \mu \mathrm{m}$

Ferricyanide, $5 \mu \mathrm{m}$, reduced by ascorbate ${ }^{1}$

Ferrocyanide, $5 \mu \mathrm{m}$
Chloroplasts

$\begin{array}{ll}9.2 & 4.9 \\ 0.5 & 0.4 \\ 0.5 & 0.5 \\ 0.5 & 0.4 \\ 7.2 & 6.2 \\ 9.4 & 5.4\end{array}$

${ }^{1}$ Sodium ascorbate $(5 \mu \mathrm{m})$ was tipped in from a sidearm 15 minutes after the beginning of the experiment, and illumination (35,000 Lux) was then continued for 30 minutes.

capacity for cyclic photophosphorylation. The conclusion seemed justified therefore that the inhibitory effect of ferricyanide resulted from the capture by this ion (in its oxidized form) of electrons which would have normally travelled the cyclic electron transport route (Fig. 5). This conclusion was strengthened by the finding that the inhibition was produced by very low concentrations of ferricyanicle. This would be expected if, as demanded by the hypothesis, the quantity of ferricyanide needed to capture electrons from the cyclic system needs only to be sufficient to leave the catalytic components of the system in an oxidized form.

\section{Light-induced Oxidations of Cytochromes}

Our theory assigns to cytochromes the role of electron carriers in photosynthetic phosphorylation. The initial suggestion that cytochromes oxidized by light may act as electron carriers in the electron transport chain of photosynthetic phosphorylation was based on the observation of Lundegardh (99) that the cytochrome peculiar to chloroplasts, cytochrome $f(71)$, is oxidized upon illumination. The oxidation of cytochromes upon illumination has also been observed in intact algae and photosynthetic bacteria by Duysens $(43,44)$ and by Olsen and Chance (116). Of special relevance to our theory (9) is the recent finding of Chance and Nishimura (36a) that, in whole Chromatium cells, a light-induced oxidation of cytochrome $c_{2}$ is independent of temperature. This accords with the main postulate of our theory (9) that the primary photochemical act in photosynthesis consists of electronic excitation and is thus independent of a thermal reaction. 
In illuminated cell-free preparations of $R$. rubrum, Smith and Ramirez (139) and Smith and Baltscheffsky (138) have observed changes in the absorption spectrum of cytochrome $c_{2}$ that were associated with phosphorylation. Their conclusions (138), that in the facultative anaerobe $R$. rubrum the "photosynthetic" cytochrome $c_{2}$ is not a part of the respiratory chain, and that "two different enzyme systems mediate the oxidation of substrates by oxygen and the phosphorylation of ADP on illumination," are concordant with our distinction between photosynthetic and oxidative phosphorylation in green cells $(6-8,12)$.

A reversible light-induced oxidation of cytochrome $c_{2}$ in cell-free preparations of Chromatium-a cytochrome that has been isolated and purified by Bartsch and Kamen (23) - was measured by Nozaki,

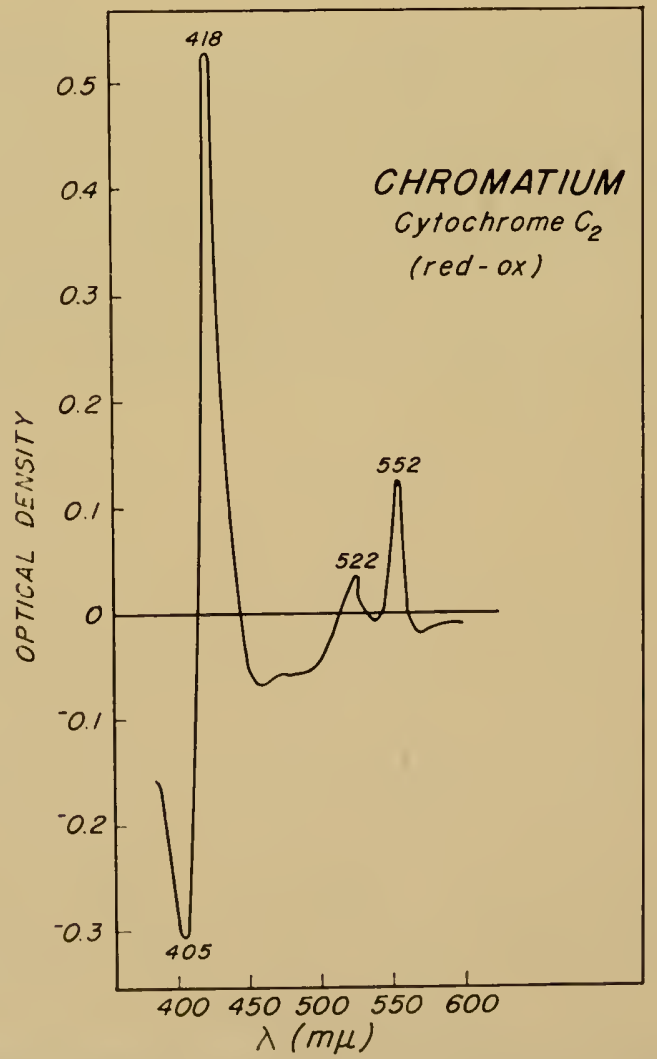

Fig. 6. Diflerence spectrum (reduced minus oxidized) of purified cytochrome $c_{2}$ of Chromatium (Nozaki, Ogata, and Arnon, 114). 
Ogata, and Arnon (114). The absorption spectrum of the reduced form of a purified cytochrome $c_{2}$ from our preparations (Fig. 6) is the same as that described by bartsch and Kamen (23).

The effect of light on the absorption spectrum (difference spectrum, light minus dark) of cytochromes in cell-free preparations of Chromatium is shown in Fig. 7. On illumination, the absorption spectrum of cytochrones shows oxidation followed by a reduction in the dark and reoxidation upon repeated illumination. Under the experimental conditions in which the Chromatium cell-free system was investigated, the light-dark reversible oxidation-reduction reactions

Reversible oxidotion of $\frac{\text { Chromatium cytochromes by light }}{(P+S)}$

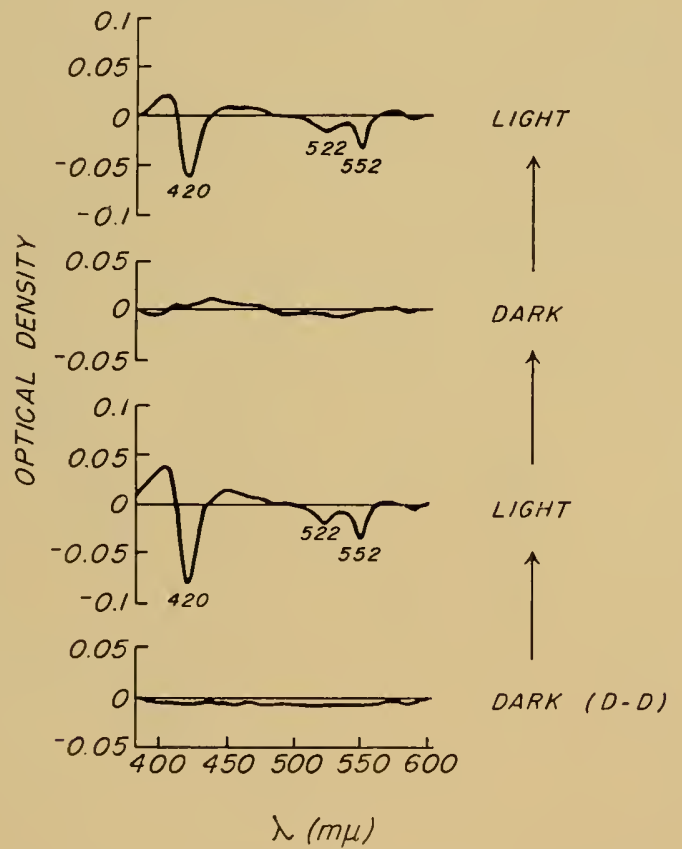

Fig. 7. Successive oxidation by light and reduction in the dark of cytochromes in cell-free preparations of Chromatium. The reaction mixture included in a final volume of $3.0 \mathrm{ml}$, chromatophores $(P)$ containing $0.06 \mathrm{mg}$ bacteriochlorophyll and supernatant fluid $(S)$ corresponding to $0.5 \mathrm{mg}$ bacteriochlorophyll. A small amount of $\mathrm{Na}_{2} \mathrm{~S}_{2} \mathrm{O}_{4}$ was previously added to $S$, which was then dialyzed against $0.2 \mathrm{M}$ tris buffer, $p \mathrm{H} 7.8$, prior to use. The reaction was carried out at room temperature. Gas phase, argon. Difference spectra, using the dark treatment as control, were made in Thunberg type curettes, with a Cary recording spectrophotometer. Illumination was by a tungsten lamp (35,000 Lux). (Nozaki, Ogata, and Arnon, 114). 
were sufficiently decelerated to be conveniently measurable with a recording spectrophotometer at room temperature.

\section{Effect of Vitamin $K$}

In fresh preparations of chromatophores the reduction of oxidized cytochrome in the dark was not influenced by the addition of added cofactors (compare Table 2). However, as shown in Fig. 8, in aged preparations the reduction of the oxidized cytochromes was greatly accelerated by the addition of vitamin $\mathrm{K}$, either in the oxidized form (vitamin $\mathrm{K}_{3}$ ) or in the reduced form (vitamin $\mathrm{K}_{5}$ ). The effect of vitamin $\mathrm{K}$, as an electron carrier, in accelerating the reduction of oxidized cytochrome depended on the presence of chromatophores. Without chromatophores, using a purified cytochrome $c_{2}$, a hundred-fold greater concentration of reduced vitamin $\mathrm{K}$ was required to reduce the oxidized cytochrome.

The observed effects of vitamin $\mathrm{K}$ in catalyzing the reduction of
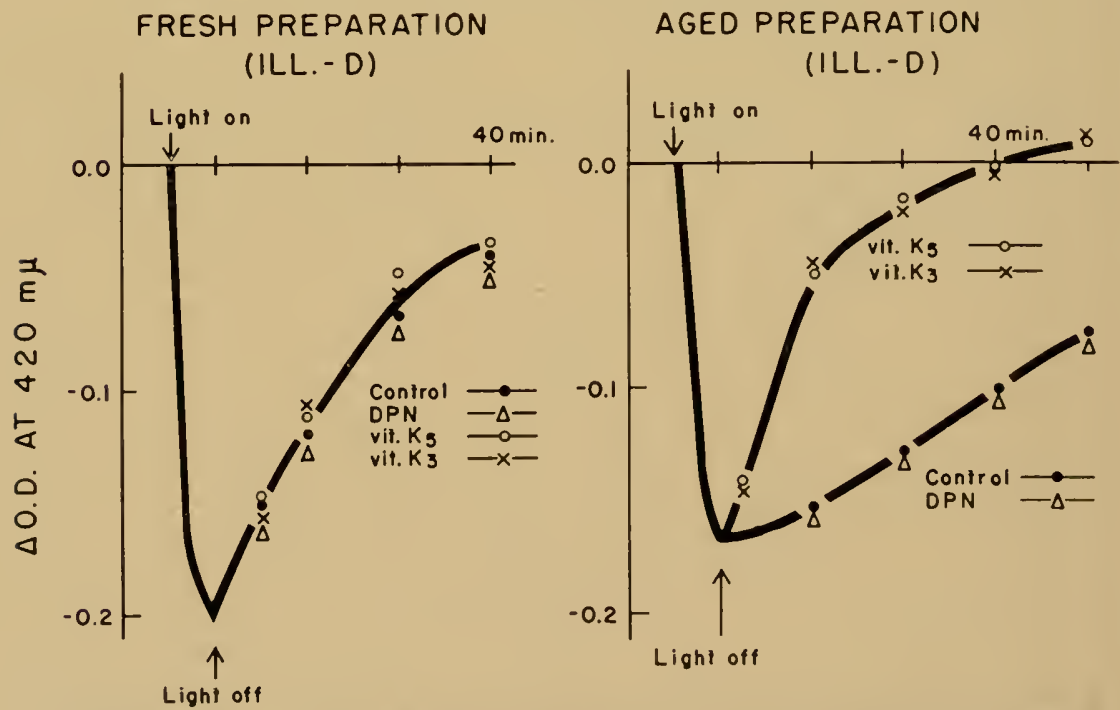

Fig. 8. Elfect of vitamin $k$ and other cofactors on the reduction cytodhome $c_{2}$ in cell-free preparations of Chromatium. The cylochrome was oxidized by previous ilfumination ( $c$. Fig. 7 ). The reaction mixture included in a final volume of $3.0 \mathrm{ml}$, dialyzed cell-free strspension (PS) containing $0.06 \mathrm{mg}$ bacteriochlorophyll, $0.02 \mu$ moles of purifica cytochrome $c_{2}$ and $0.03 \mu$ moles of the respective cofactors. Dilference in optical deusity was measured on a Bechman 1) $\mathrm{C}$ spectrophotometer with an attached photomultiplier, and using as controls culettes, hept in the dark, with the respective colactor omitted (Nozaki, Ogata, and 1mon, 114). 
cytochromes which had been oxidized in light support the electron flow theory (9) for cyclic photophosphorylation. This theory assigris to vitamin $\mathrm{K}$, or some analogons quinonc, the role of an intermediate electron carrier in the clectron transport chain associated with photophosphorylation ${ }^{6}$.

\section{Multiple Sites in Cyclic Photophosphorylation}

As already discussed, cyclic photophosphorylation is catalyzed by vitamin $\mathrm{K}$ and FMIN and also by non-physiological factors such as phenazine methosulfate. The latter fact has given rise to questions whether vitamin $\mathrm{K}$ and $\mathrm{FMN}$, or equivalent quinone and flavin constituents of photosynthetic tissues, are to be considered the physiological catalysts of cyclic photophosphorylation or whether they are to be regarcled as non-specific agents in no way distinguishable from non-physiological catalysts. The question was of special interest in connection with phenazine methosulfate because, as already mentioned, this dye has given rates of photosynthetic phosphorylation higher than either vitamin $\mathrm{K}$ or FMN (cf. 76).

The marked effectiveness of phenazine methosulfate could be explained by its acting as an electron carrier that bypasses a ratelimiting step in photosynthetic phosphorylation. This is suggested by the observation of Geller (59) that the severe inhibition of photophosphorylation in $R$. rubrum by antimycin A does not occur in the presence of phenazine methosulfate. On this hypothesis, phenazine methosulfate might give higher rates of phosphorylation at high light intensity when there is a rapid flux of electrons from excited chlorophyll. Assuming, however, several sites of phosphorylation in the cyclic pathway, the advantage of phenazine methosulfate might disappear at low light intensity when the overall rate of the process is limited by the electron flux. Under such conditions the highest rate of photophosphorylation would be observed in a system in which none of the phosphorylation sites was bypassed. Thus, a comparison of photosynthetic phosphorylation, catalyzed by vitamin $\mathrm{K}$ or phenazine methosulfate under conditions of limiting light, seemed desirable.

The results of such a comparison are shown in Fig. 9. At low light intensity photophosphorylation catalyzed by vitamin $\mathrm{K}_{3}$ was markedly greater than that catalyzed by phenazine methosulfate or

'Added nole: Ifter this Symposium, new experimental evidence for the electron flow theory has come from the work of Arnold and Clayton (4), who, on illuminating bacterial chromatophores, observed temperature-independent $\left(1^{\circ} \mathrm{K}\right.$ to $300^{\circ} \mathrm{K}$ ) reversible spectral changes near the absorption bands of bacteriochlorophyll. 


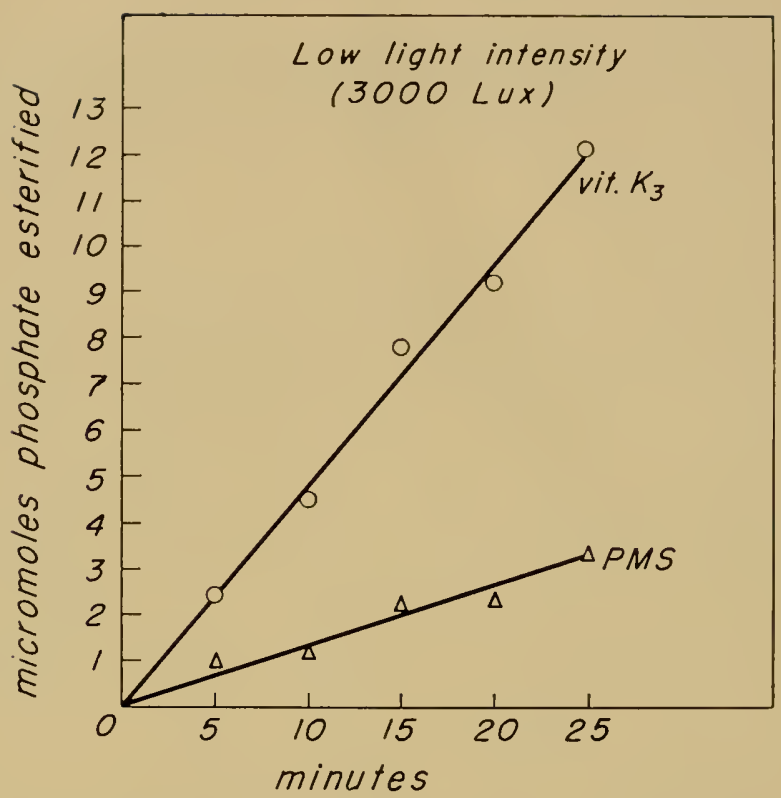

Fig. 9. Effect of vitamin $\mathrm{K}_{3}$ and phenazine methosulfatc (PMS) on anacrobic cyclic photophosphorylation in spinach chloroplasts at a limiting light intensity (3000 Lux). Reaction mixture included chloroplast fragments $\left(\mathrm{C}_{1 \mathrm{~s}}\right)$ containing 1 mg chlorophyll and in micromoles: Tris buffer, $p \mathrm{H} 8.3,80 ; \mathrm{K}_{2} \mathrm{H}^{32} \mathrm{PO}_{4}, 15 ; \mathrm{MgSO}_{4}$, 5; ADP, 15; vitamin $\mathrm{K}_{3}$ or PMS, 0.3; gas phase nitrogen (Tsujimoto, Hall, and Arnon, 156).

pyocyanin. This difference was persistent and gave a straight-line relationship for a considerable period of time.

These experiments were extended by comparing the rates of photosynthetic phosphorylation at different light intensities. The results shown in Fig. 10 confirm and extend those illustrated in Fig. 9. At low light intensity, cyclic photophosphorylation catalyzed by either vitamin $\mathrm{K}_{3}$ or FMN proceeded at a much higher rate than that catalyzed by phenazine methosulfate. However, at higher light intensities the phenazine methosulfate system gave much greater rates of phosphorylation (156).

These results suggest that at high light intensity the vitamin $\mathrm{K}$ and FMN systems became limited by enzymatic reactions which were unable to keep pace with the rapid electron flux. The increasing rates of phosphorylation obtained at high light intensity with the phenazine methosulfate system are consistent with the explanation that this agent docs indeed serve as a bypass around some rate- 


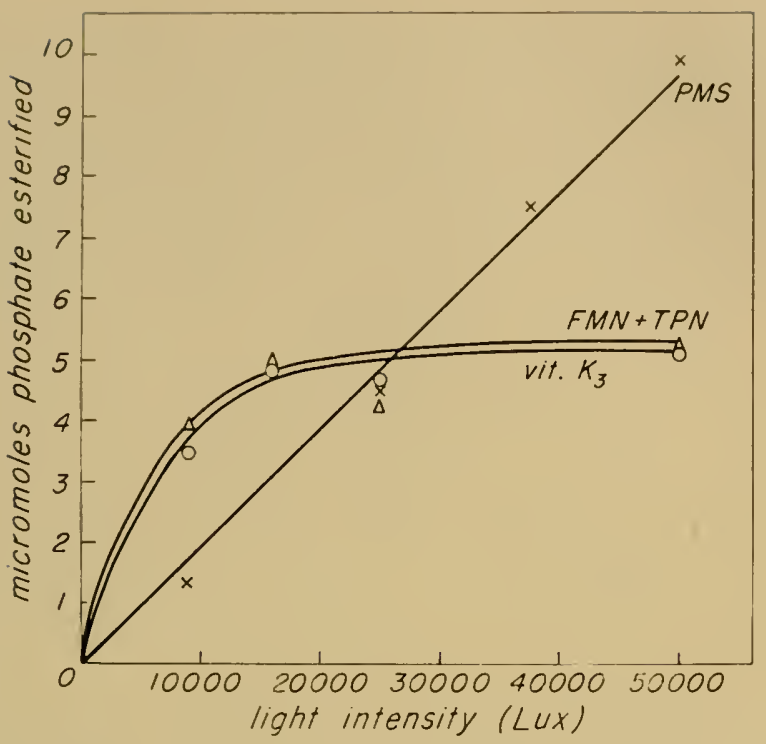

Fig. 10. Effect of light intensity on anaerobic cyclic photophosphorylation. Gas phase. nitrogen. Illumination period, 30 minutes. The reaction mixture included chloroplast fragments $\left(\mathrm{C}_{1 \mathrm{~s}}\right)$ containing $0.1 \mathrm{mg}$ chlorophyll and chloroplast extract equivalent to $1 \mathrm{mg}$ chlorophyll. $0.3 \mu$ moles TPN and $0.3 \mu$ moles FMN were included in the FMN system. Other conditions as given for Fig. 9.

limiting step. These findings provide the first indication of enzymatic steps that may limit cyclic photophosphorylation at high light intensities, when physiological catalysts such as vitamin $\mathrm{K}$ or FMN (or their analogues) are involved.

The higher rates of photophosphorylation catalyzed by vitamin $\mathrm{K}$ and FMN than those catalyzed by phenazine methosulfate, when light is limiting (Fig. 10), suggest the involvement of at least two phosphorylation sites in the vitamin $\mathrm{K}$ and FMN pathways. A diagrammatic representation of this mechanism is given in Fig. 5 .

A primary phosphorylation reaction, common to all pathways of cyclic photophosphorylation, is envisaged as being coupled with the oxidation of the terminal cytochrome in the photosynthetic particle, i.e., the cytochrome that reacts with the excited chlorophyll molecule (compare Figs. 4 and 5). A second site of phosphorylation in the vitamin $\mathrm{K}$ pathway is likely to occur upon oxidation of reduced vitamin $\mathrm{K}$ (or its analogue) by cytochromes, as was suggested by the model reactions proposed by Wessels (167), Harrison (68), and Clark, Kirby, and Todd (38). In the FMIN pathway, an additional phos- 
phorylating site can be readily envisaged in the span between TPN and cytochromes (9).

Further evidence, derived from fractionating chloroplasts, will be given in the next section for the conclusion that cyclic photophosphorylation catalyzed by phenazine methosulfate probably proceeds by way of a "bypass" and is less dependent on enzymatic chloroplast constituents than photosynthetic phosphorylation catalyzed by either vitamin $\mathrm{K}$ or FMN.

\section{Structural Association of Chorophyll with the \\ Photophosphorylating System}

In photosynthetic bacteria the photophosphorylating system is structurally bound to chlorophyll in the smallest particles that function as units in the absorption of light energy, the chromatophores. Their analogues in green plants are the grana. It was of interest, therefore, to determine whether in chloroplasts photophosphorylation is indeed localized in the grana.

Photosynthetic phosphorylation was first observed in intact chloroplasts (Fig. 11), but experiments with disrupted chloroplasts soon demonstrated that structural integrity was not essential for this process. When whole chloroplasts were broken, active photophosphorylation systems were reconstituted by a recombination of chloroplast fractions and added cofactors $(13,173)$. This technique proved effective in investigating the mechanism of photophosphorylation but provided no rigorous evidence that the site of photophosphorylation is in the grana.

Direct evidence for the localization of photosynthetic phosphorylation in grana, freed from other chloroplast fractions, was obtained by Müller, Steere, and Arnon (110), who prepared purified grana by sonication of isolated whole chloroplasts followed by a density gradient centrifugation technique. The purity of the grana obtained by these methods was determined by examination of electron micrographs of freeze-dried and air-dried grana preparations (Figs. 12 and 13). The freeze-drying technique avoids artilacts resulting from chemical fixation and retains the natural shape of the particles (142). Cyclic photophosphorylation by purified grana is shown in Table 5.

Table 5 shows that, at the high light intensity at which cyclic photophosphorylation by purified grana was measured, the highest rates were obtained in the system catalyzed by phenazine methosulfate. Photophosphorylation in this system was not increased by the addition of an aqueous chloroplast extract. By contrast, photophosphory- 


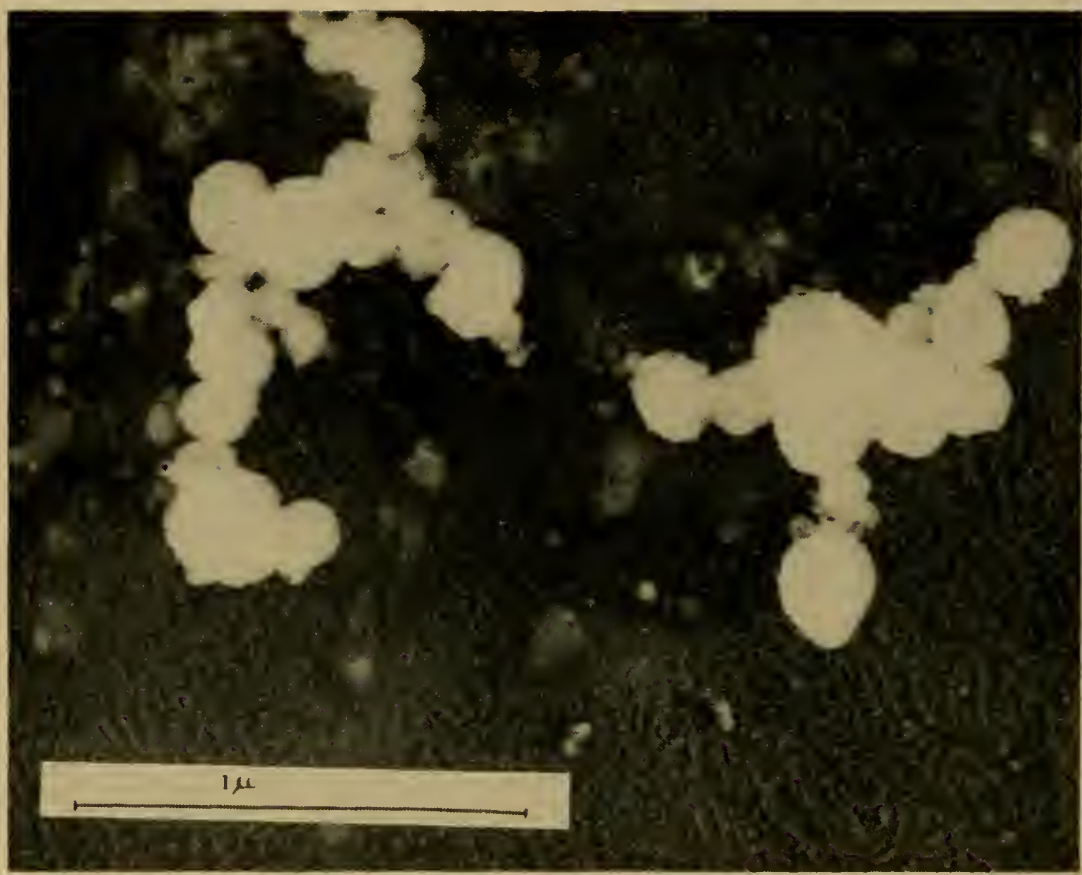

Fig. 12. Electron micrograph of isolated spinach grana prepared for electron microscopy by a freeze-drying technique. [One spinach chloroplast contains about 1000 grana (147a). I $\mathrm{cm}^{2}$ of leaf surface is estimated to have $50 \mathrm{million}$ chloroplasts.] Whole chloroplasts were disrupted by a sonic vibration treatment for 10 sec. The grana were isolated in sucrose by a density gradient centrifugation technique, and the sucrose was removed by washing with $2 \% \mathrm{NaCl}$. The grana were used in electron microscopy involving a modificl frecze-drying technique (1+2) that avoids possible artifacts resulting from chemical fixation and retains the natural shape of particles. Magnification: 59,000 X. (Mïller, Steerc, and Irnon, 110).

cytochrome and is shown in Fig. 4 as being catalyzed by phenazine methosulfate. [Significant in this connection are the recent findings of James and Leach that, like the bacterial cytochrome in chromatophores, the chloroplast cytochromes, $f$ and $b$, are "entirely confined to the grana" (80) ]. At high light intensity, the "bypass" pathway catalyzed by phenazine methosulfate gives high rates of photophosphorylation because it is not limited by the absence of chloroplast constituents that hie ontsicle the grana. The beneficial effect of adcled chloroplast extract on the FMN and vitamin $\mathrm{K}$ systems (Table 5) suggests that the extrace contains some chloroplast constituents that are involved in these pathways but not in the pathway catalyzed by phenarine methosulfate. 


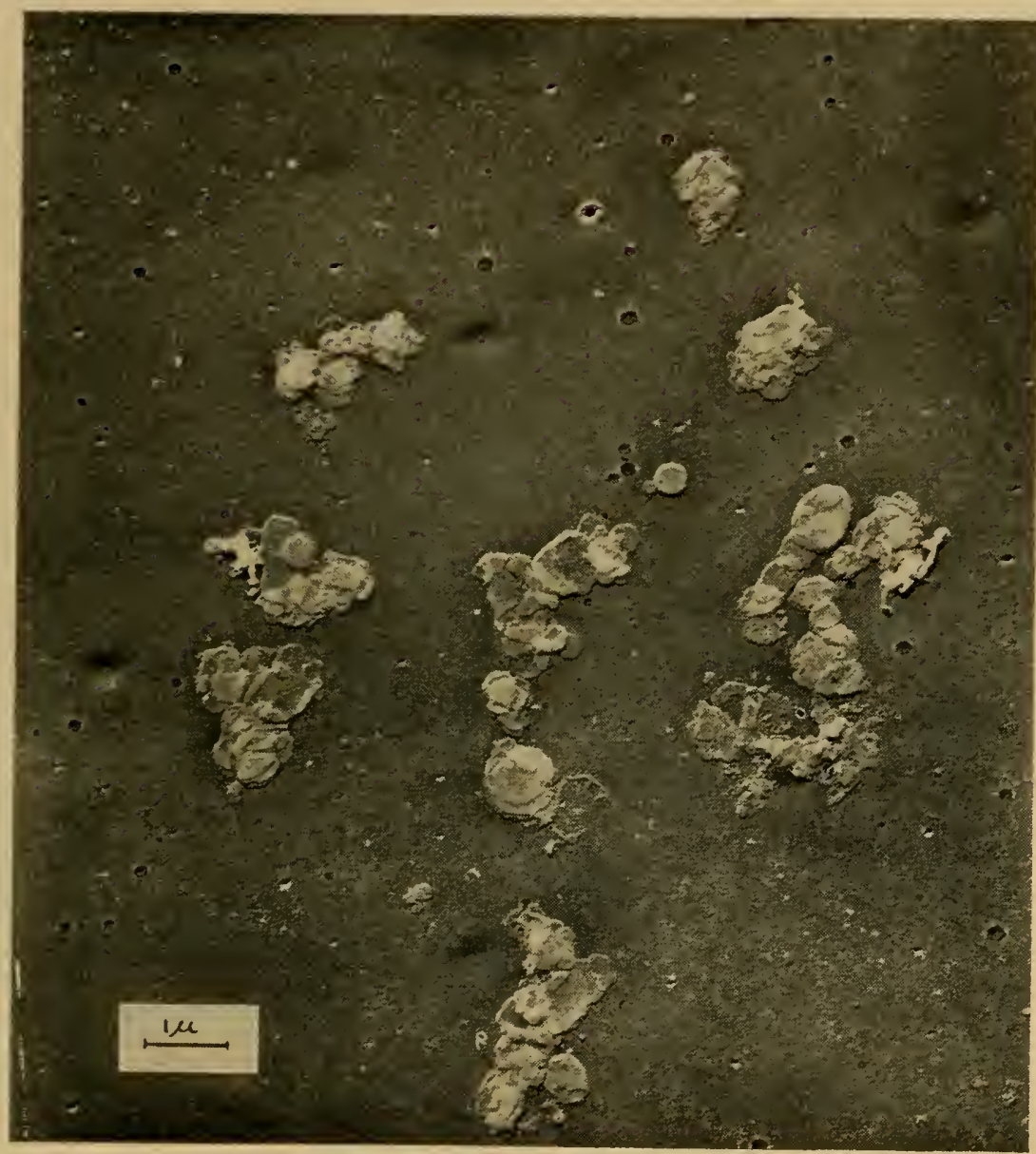

Fig. 13. Electron micrograph of isolated spinach grana fixed in formaldehyde. Whole chloroplasts were disrupted by sonic vibration treatment for 10 sec. The grana were isolated by differential centrifugation and filtration through a double layer of Whatman No. 2 filter paper. The isolated grana were fixed for $2 \mathrm{hr}$ in $6 \%$ formaldehyde at $p \mathrm{H} 6.5$. A comparison with similar particles prepared by the freeze-drying technique (Fig. 12) shows that the grana have collapsed in the formaldehyde treatment. The backgroms in the formaldehyde treatment is free from salt (compare with lig. 12). Magnifications: 10,000 X Miiller, Steere, and Arnot, 110).

The close structural association, in both chloroplasts and bacterial chromatophores, of phosphorylating activity with the chlorophyll pigments suggests that the harnessing of light energy in photosynthesis is more closely associated with ATP formation than with $\mathrm{CO}_{2}$ assimi- 
TABLE 5

Cíclic Photophosphorvlation by Purified Grana withi and without Added Chloroplast Extract (Illumination 35,000 Lux.)

(Müller, Steere, and Arnon, 110)

\begin{tabular}{lc}
\multicolumn{1}{c}{ Treatment } & $\mathrm{Q}_{\mathrm{p}}^{\mathrm{Cbl} *}$ \\
Phenazine methosulfate & 157 \\
Phenazine methosulfate + chloroplast extract & 145 \\
Vitamin $\mathbf{K}_{3}$ & 39 \\
Vitamin $\mathbf{K}_{3}+$ chloroplast extract & 78 \\
FMN & 46 \\
FMN + chloroplast extract & 69 \\
\hline
\end{tabular}

* Micromoles orthophosphate esterified per mg chlorophyll per hour.

lation. The enzymes responsible for $\mathrm{CO}_{2}$ assimilation are easily dissociable from grana in the case of chloroplasts $(13,173,153)$, and are not even structurally joined together in the case of bacterial chromatophores $(3,97)$. It seems reasonable to conclude, therefore, that in the course of biochemical evolution, photosynthesis first emerged as a process for converting light energy into ATP and this "primitive" photosynthesis only later became a process linked to $\mathrm{CO}_{2}$ reduction.

\section{Cyclic Photophosphorylation as Primtive Photosynthesis}

In the conventional view of photosynthesis, the chemical energy formed by the conversion of absorbed light is always used for the reduction of $\mathrm{CO}_{2}$. The case of cyclic photophosphorylation as being a "primitive" photosynthesis, in the evolutionary sense, would therefore be strengthened if an example could be found today of a photosynthetic organism in which the only contribution of light to carbon assimilation was the formation of ATP.

Two such cases of photosynthesis in Chromatium have recently been described by Losada et al. (97). In one case the sole source of carbon was acetate, and in the other, $\mathrm{CO}_{2}$. The photoassimilation of acetate occurred in the absence of an external hydrogen donor, whereas in the photoassimilation of $\mathrm{CO}_{2}$ the reductant was exogenous hydrogen gas. The sole contribution of light in both cases was the formation of ATP.

In the photoassimilation of $\mathrm{CO}_{2}$, ATP was required for the formation of an activated internediate (ribulose diphosphate, phosphoenol. pyruvate, or 1,3-diphosphoglycerate) for a subsequent carboxylation or reduction, whereas in the photoassimilation of acetate, ATP was required for the activation of the carbon source itself, by forming 
acetyl-CoA from acetate and coensyme $A$. The activated compounds then become ready for participation in the synthetic reactions that are catalyzed by specific enzyme systems, all of which function in the dark. A summary of those reactions of ATP that have now been experimentally documented in the carbon metabolism of Chromatium is given in Fig. 14.

Evidence that the sole contribution of light in these reactions is the formation of ATP was obtained by replacing light with a supply of exogenous ATP and finding that carbon assimilation would then proceed in the same manner in the dark as in the light (97). Other evidence for the equivalence of light and ATP is given in Table 6. Here assimilation occurred either in the dark with added ATP or in the light when ATP was allowed to form photosynthetically. If, however, the ATP which was formed in light was trapped by an added hexokinase-glucose system, than acetate assimilation ceased. The addition of hexokinase alone, without glucose as the ATP acceptor, was not inhibitory (Table 6).

The experimental substitution of ATP for light was considered

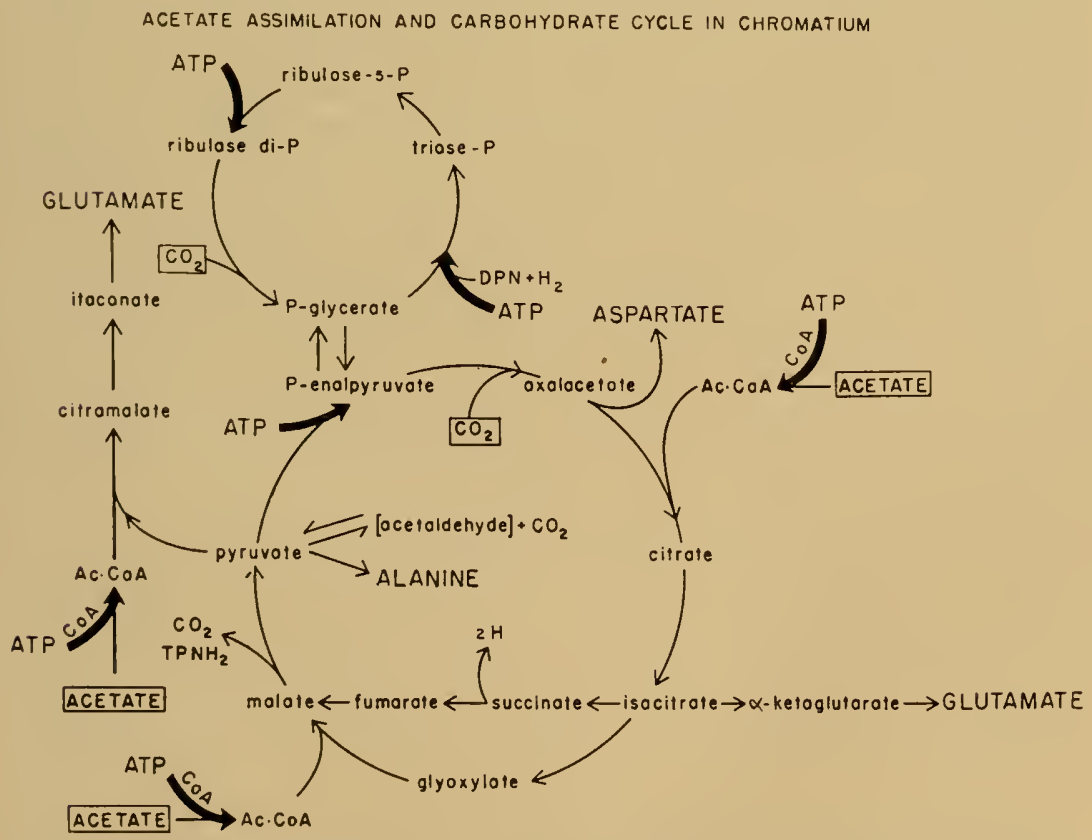

Fig. 14. Reactions of carbon assimilation in Chromatium. Further details are given by Losada, Trebst, Ogata, and Arnon (97). 
TABLE 6

Equivalence of ATP and Ligut in the Assimilation of $\mathrm{G}^{14}$-acetate by Cell-free Preparations of Chromalium (Losada, Trebst, Ogata, and Arnon, 97)

\section{Treatment}

1. Dark, control

2. Dark, ATP

3. Dark, ATP, hexokinase

4. Dark, ATP, hexokinase, glucose

5. Light, control

6. Light, hexokinase

7. Light, hexokinase, glucose
Carbon ${ }^{14}$ fixed in soluble compounds (Thousands of counts/min)

Each vessel included, in a final volume of $1.5 \mathrm{ml}$, cell-free extract, containing $0.3 \mathrm{mg}$

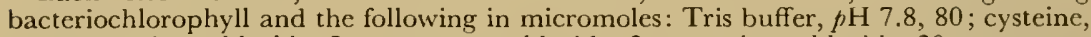
20; magnesium chloride, 5; manganese chloride, 2; potassium chloride, 20; coenzyme A, 0.3; oxaloacetate, 10; carboxyl-labelled $\mathrm{C}^{11}$-acetate, 3 . I.5 mg hexokinase, type III (Sigma Chemical Co.), 10 micromoles glucose, and 4 micromoles ATP were added as indicated. In treatments 5, 6, and 7, no addition of ADP and phosphate was necessary to supplement the catalytic amounts present in the cell-free extracts.

particularly significant because it was found in such photosynthetic bacteria as Chromatium, that are unique in the living world in being strict phototrophs. Chromatium, unlike, for example, Chlorella or photosynthetic bacteria of the genus Rhodospirillum, cannot replace its light-dependent mode of life by a heterotrophic, aerobic metabolism in the clark $(158,109,159)$. Chromatium grows only in the light (158, 109), and being an obligate anaerobe, does not possess an alternative way for forming ATP by the mechanism of oxidative phosphorylation.

As regards the photoassimilation of acetate in another photosynthetic bacterium, the facultative anacrobe $R$. rubrum, a similar view that the contribution of light is limited to cyclic photophosphorylation was recently expressed, on the basis of independent evidence, by Stanier, Doudoroff, Kunisawa, and Contopoulou (141).

In certain circumstances, ATP formation may be the sole contribution of the photosynthetic process, not only in bacteria but also in higher plants. We have suggested elsewhere (18) that in green plants cyclic photophosphorylation may continue forming ATP when $\mathrm{CO}_{2}$ assimilation is, for one reason or another, reduced or even stopped altogether. This might arise during the well-known midday closure of stomata in leaves of higher plants $(140,69)$, a closure which restricts the supply of $\mathrm{CO}$. . The closure of stomata often coincides with an abundance of starch and an incipient water deficit in the photosynthesizing cells. Under these conditions cyclic photophos- 
phorylation, which consumes neither CO. nor witter, would be a uselul device for generating $\triangle T P$ to drive the many $A T P$-dependent reactions, notably the syuthesis of polysaccharides, proteins, and fats.

These theoretical deductions for higher plants have recently received experimental support from the work of Maclachlan and Porter (100). They reported the first known instance of utilization of light energy in leal tissue for the synthesis of starch from labelled glucose, under conditions when $\mathrm{CO}_{2}$ assimilation was excluded but cyclic photophosphorylation conld proceed.

\section{P'ridine Nucleotide Reduction by Hydrogfasase in the Dark}

In the examples of photosynthesis in which the contribution of light was limited to ATP lormation, no reductant was needed in the conversion of glucose to starch. In the assimilation of acetate, hydrogen is released for metabolic purposes and no addition of hydrogen donor is required (97). But the assimilation of $\mathrm{CO}_{2}$ requires, in addition to ATP, a supply of a reductant, viz., reduced pyridine nucleotide. It was stated earlier that in photosynthesis of green plants both of these components of assimilatory power are formed at the expense of light energy. It is necessary, therefore, to trace the transition from a primitive photosynthesis, in which light is used only for the formation of ATP, to the "advanced" type of photosynthesis, observed in green plants, in which light energy is used not only for ATP formation but also for the reduction of pyridine nucleotide and the accompanying evolution of oxygen.

In the photoassimilation of $\mathrm{CO}_{2}$ by Chromatium the added reductant was hydrogen gas (129). This is the simplest reductant usable by living cells. Cell-free hydrogenases from non-photosynthetic bacteria are known to reduce pyridine nucleotides with molecular hyclogen $(84,118$; cf. 126). From the standpoint of photosynthesis, it was important to know if the hydrogenases of photosynthetic bacteria could also reduce pyridine nucleotide with molecular hydrogen in the dark, since this would provide a mechanism, independent of light, for the formation of the reductant for $\mathrm{CO}_{2}$ assimilation. In photosynthetic bacteria, the only cell-free hydrogenase tested in this respect, that of $R$. nubrum, was reported to be unable to reduce acceptors with potentials less than 0 volts $(82)$, which would thus exclude pyridine nucleotides $\left(E_{0}^{\prime}=-0.32 \mathrm{v}\right)$.

The subject was reinvestigated by Ogata, Nozaki, and Arnon (115), using the cell-free hydrogenase of Chromatium. As in R. rubrum (82). the hydrogenase of Chromatium was found to be localized in the 
chromatophores. The Chromatium hydrogenase reduced DPN and TPN with molecular hydrogen in the dark in the presence of benzyl viologen. The enzyme was more active toward DPN than TPN (Table 7) .

These results indicated that in the presence of hydrogen gas Chromatium cells do not require light for the reduction of pyridine nucleotides. The role of light is, then, limited to ATP formation, without which $\mathrm{CO}_{2}$ assimilation cannot occur (97). Photosynthesis by Chromatium in the presence of molecular hydrogen may, therefore, be summarized as follows:

LIGHT PHASE

Cyclic photophosphorylation:

$n \cdot \mathrm{ADP}+n \cdot \mathrm{P} \rightarrow n \cdot \mathrm{ATP}$

DARK PHASE

DPN reduction: $\quad 2 \mathrm{DPN}+2 \mathrm{H}_{2} \rightarrow 2 \mathrm{DPNH}_{2}$

$\mathrm{CO}_{2}$ assimilation: $\mathrm{CO}_{2}+2 \mathrm{DPNH}_{2}+n \cdot \mathrm{ATP} \rightarrow$

$\left(\mathrm{CH}_{2} \mathrm{O}\right)+\mathrm{H}_{2} \mathrm{O}+2 \mathrm{DPN}+n \cdot \mathrm{ADP}+n \cdot \mathrm{P}$

$$
\mathrm{SUM}: \mathrm{CO}_{2}+2 \mathrm{H}_{2} \stackrel{\text { light }}{\longrightarrow}\left(\mathrm{CH}_{2} \mathrm{O}\right)+\mathrm{H}_{2} \mathrm{O}
$$

Several algal species are known to contain hydrogenases and to acquire, after adaptation to hydrogen, a capacity to photoassimilate $\mathrm{CO}_{2}$ with the aid of molecular hydrogen $(54,55,52)$. This process,

\section{TABLE 7}

Pyridine Nucleotide Reduction with Molecular Hydrogen by

Cell-free Preparations of Chromatium

(Ogata, Nozaki, and Arnon, 115)

\begin{tabular}{|c|c|c|c|c|}
\hline \multirow[b]{2}{*}{ Treatment } & \multicolumn{2}{|c|}{ DPN Series } & \multicolumn{2}{|c|}{ TPN Series } \\
\hline & Light & Dark & Light & Dark \\
\hline $\begin{array}{l}\text { Pyridine nucleotide (PN) } \\
\text { Benzyl viologen (BV) } \\
\text { PN + BV }\end{array}$ & $\begin{array}{r}0.08 \\
-0.08 \\
0.80\end{array}$ & $\begin{array}{r}0.09 \\
-0.08 \\
0.86\end{array}$ & $\begin{array}{r}0.07 \\
-0.08 \\
0.33\end{array}$ & $\begin{array}{r}0.07 \\
-0.08 \\
0.42\end{array}$ \\
\hline \multicolumn{5}{|c|}{ 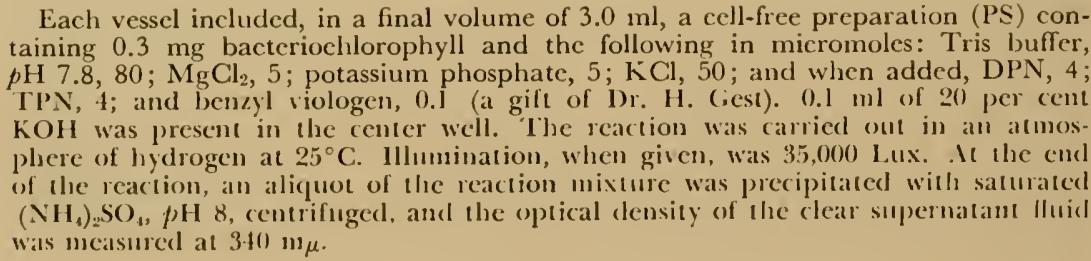 } \\
\hline
\end{tabular}


which Gallron named photoreduction (55), appears to be the same type of photosynthesis as that in Chromatium when it is supplied with hydrogen gats. It secms likely that photoreduction by algae is a reversion to a prinitive photosynthesis in which the $\mathrm{CO}_{2}$ reductant was hydrogen gas present in the environment, and the sole contribution of light was the formation of ATP by cyclic photophosphorylation.

\section{Photopronuction of Hyidrogen Gas ani Its Significance to Photosinthesis}

Chromatium, like other photosynthetic bacteria, grows photosynthetically with hydrogen donors more oxidized than molecular hydrogen $(158,109)$. Is the role of light in these cases also limited to ATP formation? Or is light energy used for the recluction of pyridine nucleotide, as is the case in green plants?

Among the hydrogen donors used by Chromatium for growth are some which are known to reduce pyridine nucleotides enzymatically in the dark. In these cases, assuming the requisite enzymes are present, there is no need to invoke the participation of light in that step. A case in point is malate, an excellent hydrogen clonor for Chromatium cells, which are known to contain the malic enzyme that catalyzes the reduction of TPN. But Chromatium can also grow photosynthetically with an inorganic electron donor, such as thiosulfate, or with succinate $(158,109)$, a substrate that donates electrons at the level of flavin and is therefore unable to reduce pyridine nucleotides. How, in terms of the electron flow theory (9), can pyrichine nucleotides be reduced in bacterial photosynthesis with thiosulfate or succinate as hydrogen donors?

An attractive hypothesis would be to consider photoproduction of hydrogen gas as resulting from an electron flow mechanism that generates, with the aid of light energy, a photosynthetic reductant at the level of molecular hydrogen, from various electron donors having a potential less reducing than hydrogen. Photoproduction of hydrogen would be limited to those photosynthetic cells that are either known to have or to be able to form hydrogenase adaptively, namely, certain species of algae $(58,48 \mathrm{a})$ and photosynthetic bacteria $(63,64,135$, $32,113,62,61)$. The source of the evolved hydrogen gas would be either an endogenous electron donor, as seems to be the case in adapted algae (58), or an exogenous electron donor, organic or inorganic, that is always required for the growth of photosynthetic bacteria $(158,109)$.

A similar hypothesis that the origin of the photoproduced hydrogen 
gas in algae is a photodecomposition of an endogenous hydrogen donor was considered by Gaffron and Rubin but rejected by them in favor of photodecomposition of water (58). In their view, the hydrogen came from water and the role of the endogenous hydrogen donors was to reduce the remaining oxidized moiety (OH) of water. Likewise, in the case of photosynthetic bacteria, neither current interpretations nor existing evidence supported the photochemical electron flow theory as a mechanism for evolving hydrogen from inorganic and organic electron clonors.

With R. rubrum, Gest, Kamen, and Bregoff (64) found that the most active substrates for photoproduction of hydrogen gas were malate, oxaloacetate, and fumarate. Siegel and Kamen concluded that, in general, "the presence of a certain steady-state concentration of $\mathrm{CO}_{2}$ appears to be a major requirement for $\mathrm{H}_{2}$ evolution" (135). Thus, they found succinate, which had earlier been reported as not giving a photoproduction of hydrogen (64), could evolve hydrogen gas in the presence of $\mathrm{CO}_{2}$.

The dependence of photoproduction of hydrogen gas on $\mathrm{CO}_{2}$ suggested that the evolved hydrogen may be a product of interconversions of certain organic hydrogen donors. However, Siegel and Kamen recognized the possibility that "essentially all readily metabolized substrates are capable of inducing $\mathrm{H}_{2}$ evolution under appropriate conditions" (135). A decisive test of this possibility would have been evidence of hydrogen evolution from inorganic hydrogen donors such as hydrogen sulfide or thiosulfate. But when this was experimentally tested by Bregoff and Kanen with Chromatium, they found that "using only inorganic media, no gas production could be observed even over periods as prolonged as 2 to 3 weeks. Whenever malate media were used, good gas production, as well as growth, was observed in 2 to 3 clays" (32).

Photoproduction of hydrogen gas in Chromatium wats also observed by Newton and Wilson (113). In their experiments the photoproduction of hydrogen was strictly dependent on the presence of $\mathrm{CO}_{2}$ and was inhibited by $\mathrm{N}_{2}$ and $\mathrm{NH}_{4}+$ - an inhibition that was first noted by Gest and Kamen (63) .

These experimental facts have been variously interpreted. Newton and Wilson (113) regarded the photoproduction of hydrogen by Chromatium as resulting from a condensation of a C-4 compound with $\mathrm{CO}_{2}$ to form $\alpha$-ketoglutarate. They suggested that when a shortage of nitrogen prevents the subsequent formation of glutanate, its precursor, $\alpha$-ketoglutarate, is decomposed to give molecular hydrogen. 
I more general hypothesis of the photoproduction of hydrogen was recently proposed by Gest (6I), namely, that "the source of the $\mathrm{H}_{\text {? }}$ produced in these light-dependent reactions is evidently the ' $H$ ' atom pool gencrated by photolysis of water" and that "when the metabolism of the 'accessory donor' provides a steady stream of reducing power (reduced pyridine nucleotide etc.) the ' $\mathrm{H}$ ' atoms from water photodecomposition may be diverted through the appropriate carriers to hydrogenase, thus yielding $\mathrm{H}_{2}$ gas."

l'hotoproduction of hydrogen by Chromatium has recently been inrestigated in our laboratory with special reference to succinate and thiosulfate as hydrogen donors. The results with thiosulfate, which are presented in the companion paper by Losada, Nozaki, and Arnon (95), provided the first evidence of a light-dependent hydrogen evolution by photosynthetic bacteria from an inorganic electron donor. There was thus an experimental basis for considering the photoproduction of hydrogen by photosynthetic bacteria as being primarily related to a light-induced electron transport process that is fundamentally independent of interconversions of organic compounds.

This conclusion was strengthened by the results of Ogata et al. with succinate $(115)$. A vigorous production of hydrogen from succinate occurred only in light (Fig. 15) and was independent of $\mathrm{CO}_{2}$. The identity of hydrogen was established by absorbing the gas on palladium asbestos (150). Little hydrogen evolution occurred in the dark, or in the light without the addition of succinate. The photoproduction of hydrogen was strongly inhibited by carbon monoxide. These results are summarized in Table 8.

Photoproduction of hydrogen gas indicates that photosynthetic bacteria should be capable of reducing DPN or TPN with light, in the presence of succinate or some other electron donor that is less reduced than pyridine nucleotide because they are able to form photochemically a reductant at the level of molecular hydrogen. A photochemical reduction of pyridine nucleotide with succinate was indeed observed by Frenkel (51) and Vernon and Ash (160) and confirmed by Ogata et al. (115).

As to the mechanism of this reaction it is not possible, on present evidence, to decide whether molecular hydrogen is produced first and then used to reduce pyridine nucleotide or whether the reduction is accomplished by electrons generated in the photochemical act before they are combined with protons to give molecular hydrogen. The elucidation of the mechanisms involved in these reactions is likely to come from work on cell-free systems. 
PHOTOPRODUCTION OF $\mathrm{H}_{2}$ FROM SUCCINATE

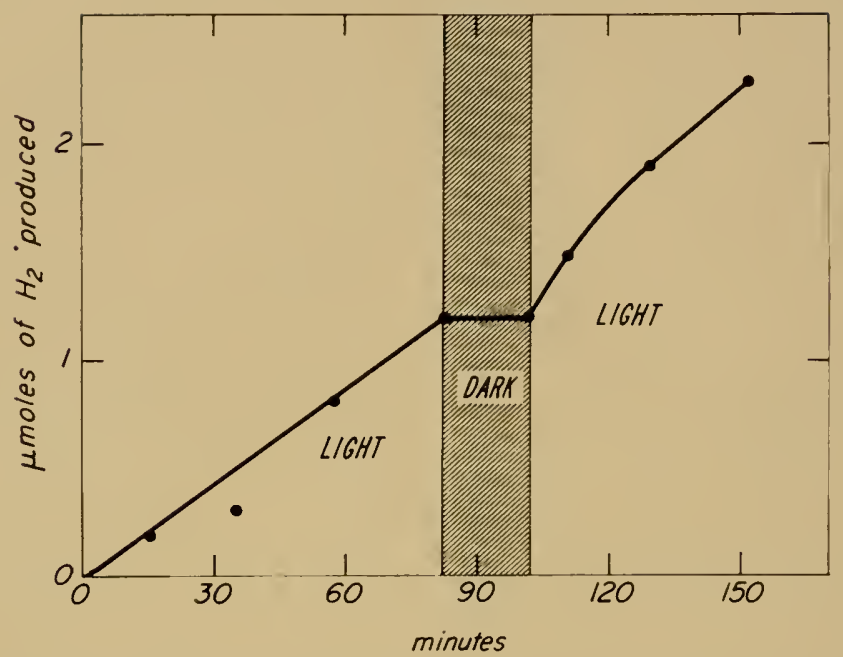

Fig. 15. Photoproduction of hydrogen gas from succinate by Chromatium cells. Experimental conditions as described in Table 8. (Ogata, Nozaki, and Arnon, $115)$.

Investigations of cell-free hydrogenase preparations from photosynthetic bacteria have begun only recently. The hydrogenase of $R$. rubrum, in a cell-free state, reduced certain dyes such as methylene blue with $\mathrm{H}_{2}$, but the enzyme was incapable of reducing one-electron

TABLE 8

Photoproduction of Hydrogen from Succinate by Chromatium (Ogata, Nozaki, and Arnon, 115)

\section{Treatment}

1. Complete

2. Complete, $\mathrm{KOH}$ omitted

3. Complete, dark

4. Complete, succinate omitted

1. Complete

2. Complete, succinate omitted

3. Complete, plus palladium asbestos

4. Complete, plus carbon monoxide $\mu$ moles $\mathrm{H}_{2}$ evolved

8.6
8.2
1.5
0.6
5.9
0.7
0.3
-0.7

The complete system contained, in a final volume of $3.0 \mathrm{ml}, 100 \mathrm{mg}$ wet cells that were suspended in a modified nutrient solution with nitrogen omitted, and the following in micromoles: Tris buffer, $p \mathrm{H} \mathrm{7.2,80}, \mathrm{MgCl}_{2}, 5$; succinate, $20.0 .1 \mathrm{ml}$ of $20 \% \mathrm{KOH}$ was present in the center well. The reaction was carried out for 2 hours at $30^{\circ} \mathrm{C}$ in argon. Illumination 35,000 Lux. 
viologen dyes of low redox potential or of producing $\mathrm{H}_{2}$ from reduced methyl viologen (82). Both of these dark reactions are known to be catalyzed by cell-free hydrogenases from heterotrophic anaerobes $(141,119)$.

By contrast, the hydrogenase from Chromatium, in a cell-free state, was found in a recent investigation to be capable of reducing viologen dyes with $\mathrm{H}_{2}$ (115) and of evolving hydrogen in the dark from reduced methyl viologen (Table 9). Experiments on the photoevolution of hydrogen from cell-free preparations of Chromatium are currently under way in this laboratory.

In other experiments with cell-free preparations of Chromatium, we found that chromatophores contained succinic dehydrogenase.

TABLE 9

Hydrogen Evolution from Reduced Methyl Viologen by

Cell-free Hydrogenase from Chromatium

(Ogata, Nozaki, and Arnon, 115)

$\mu$ moles $\mathrm{H}_{2}$ evolved $/ 10 \mathrm{~min} / \mathrm{mg} \mathrm{chl}$.

Complete system

Methyl viologen omitted

$\mathrm{Na}_{2} \mathrm{~S}_{2} \mathrm{O}_{4}$ omitted

Hydrogenase omitted
12.8

none

none

none

Complete system contained, in a final volume of $3.0 \mathrm{ml}$, cell-free suspension (PS)

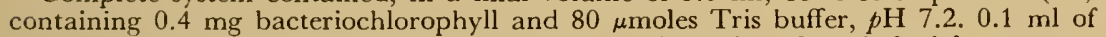
$20^{\circ} \mathrm{KOH}$ was present in the center well and $16 \mu$ moles of methyl viologen were added to the sidearm. Methyl viologen was reduced by adding $\mathrm{Na}_{2} \mathrm{~S}_{2} \mathrm{O}_{4}$ to the same sidearm while gassing with argon. The reaction was carried out at $30^{\circ} \mathrm{C}$ in the dark.

As shown in Fig. 16, the addition of succinate reduced the Chromatium cytochromes. The reduction by succinate was slow at first but became accelerated with time (114).

Since Chromatium cytochromes are oxidized by light (Fig. 7) and reduced by succinate (Fig. 16) or thiosulfate (95) in the dark, it seems permissible to regard succinate, thiosulfate, and other substrates in bacterial photosynthesis as electron donors for the cytochrome-chlorophyll system of the chromatophores. Following the absorption of photons by chlorophyll the electrons donated by those substrates are raised to a high reducing potential, at least equal to that of molecular hydrogen. It follows from the electron flow theory, that when these electrons do not return to the cytochrome-chlorophyll complex by the cyclic route (Figs. 4 and 5 ), but are instead transferred by hydrogenase to protons, that hydrogen gas will be produced. A non-cyclic, light-clependent electron path of this sort, capable of 


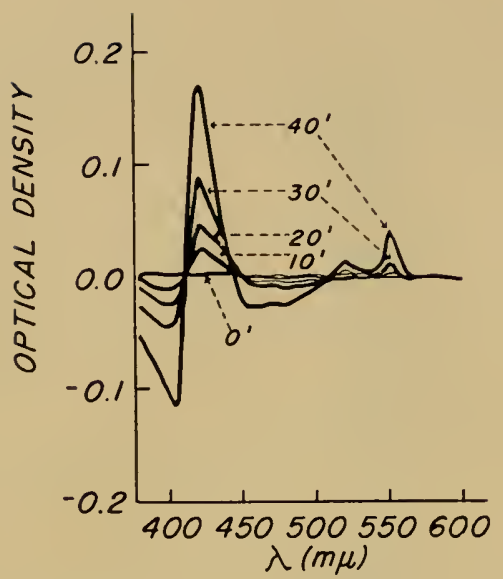

Fig. 16. Reduction of Chromatium cytochromes by succinate in a cell-free system. Reaction mixture included, in a final volume of $3.0 \mathrm{ml}$ of $0.2 M$ Tris buffer, pH 7.8, chromatophores $(P)$ containing $0.06 \mathrm{mg}$ bacteriochlorophyll and supernatant fluid $(S)$ corresponding to $0.3 \mathrm{mg}$ bacteriochlorophyll. $10 \mu$ moles of succinate were added to one of a pair of curettes, and the resulting difference spectrum was measured in a Cary spectrophotometer at the indicated time intervals (Nozaki, Ogata, and Arnon, 114).

evolving hydrogen gas, is represented by Fig. 17. HA represents an inorganic or organic electron donor which gives low-energy electrons to the cytochrome-chlorophyll complex, where with, the aicl of absorbed light, they are raised to the energy level of electrons in molecular hydrogen.

With succinate as the electron donor, the proposed sequence of reactions for the non-cyclic electron flow which results in the photoproduction of hydrogen (Fig. 17), is represented as follows:

$$
\begin{aligned}
& \text { Succinate }+2 \mathrm{Fe}^{3+} \mathrm{cyt} \rightarrow \text { fumarate }+2 \mathrm{Fe}^{2+} \mathrm{cyt}+2 \mathrm{H}+ \\
& 2 \mathrm{Fe}^{2+} \mathrm{cyt}+2[\mathrm{Chl}]^{+} \rightarrow 2 \mathrm{Chl}+2 \mathrm{Fe}^{3+} \mathrm{cyt} \\
& 2 \mathrm{Chl} \stackrel{\text { light }}{\longrightarrow} 2[\mathrm{Chl}]++2 \mathrm{e}^{-} \\
& 2 \mathrm{e}^{-}+2 \mathrm{H}+\stackrel{\text { hydrogenase }}{\longrightarrow} \mathrm{H}_{2}
\end{aligned}
$$

Sum: succinate $\stackrel{\text { light }}{\longrightarrow}$ fumarate $+\mathrm{H}_{2}$

If the electron donor $(\mathrm{HA})$ in Fig. 17 is thiosulfate, an analogous sequence of reactions would, as is discussed in the companion paper 


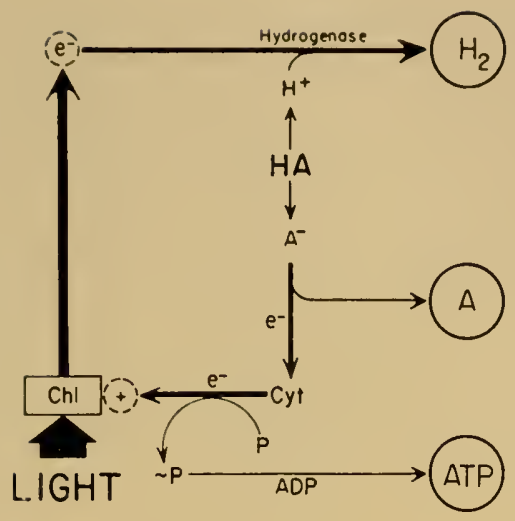

Non-cyclic photophosphorylation (bacteria)

Fig. 17. Scheme for non-cyclic photophosphorylation in Chromatium. Details in the text.

(95), account for the photoproduction of hydrogen from thiosulfate in accordance with the overall reaction:

$$
\mathrm{S}_{2} \mathrm{O}_{3}=+5 \mathrm{H}_{2} \mathrm{O} \stackrel{\text { light }}{\longrightarrow} 4 \mathrm{H}_{2}+2 \mathrm{SO}_{4}^{=}+2 \mathrm{H}^{+}
$$

To recapitulate, then, the scheme shown in Fig. 17 is supported by experimental evidence for the photoproduction of hydrogen from succinate (Fig. 15 and Table 8) or thiosulfate (95) and for the reduction of Chromatium cytochromes by succinate (Fig. 16) or thiosulfate (95). By analogy with non-cyclic photophosphorylation in chloroplasts (9), the non-cylic electron flow mechanism in bacteria should also result in a phosphorylation, as is indicated by preliminary experiments (94). We further conclude, that hydrogen gas is evolved in light when the "reducing" electrons are not consumed in metabolic reactions, as, for example, in the reduction of $\mathrm{CO}_{2}$ via pyridine nucleotide (114).

\section{Photoproduction of Reductant in Plants: Non-cyclic PHOTOPHOSPHORYLATION}

Photosynthetic bacteria can reduce pyridine nucleotide in photosynthesis, either with molecular hydrogen in the clark or with a less reduced electron donor, organic or inorganic, in the light. Green plants that do not contain hydrogenase depend unconditionally on light for reducing pyridine nucleotide in photosynthesis, the more so since they use water as the electron donor. The reduction of pyri- 
dine nucleotides with electrons donated by water requires a considerable input of energy which in photosynthesis is supplied by light.

We have seen in Section 5 that isolated chloroplasts can reduce TPN in light with an accompanying stoichiometric evolution of oxygen, in a reaction that is catalyzed by a specific TPN reductase, an enzyme that is localized in chloroplasts (Equation 1). Recently, the relation of photosynthetic phosphorylation to the photoreduction of TPN, and indeed to the photoreduction of non-physiological Hill reagents, was found to be very direct (18). In the presence of ADP and orthophosphate $(\mathrm{P})$, the photoreduction of TPN and oxygen evolution was coupled with the formation of ATP in accordance with Equation 5.

$$
\begin{array}{r}
2 \mathrm{TPN}+2 \mathrm{ADP}+2 \mathrm{P}+2 \mathrm{H}_{2} \mathrm{O} \rightarrow 2 \mathrm{TPNH}_{2} \\
+\mathrm{O}_{2}+2 \mathrm{ATP}
\end{array}
$$

Under appropriate experimental conditions (18a), the evolution of one mole of oxygen was accompanied by the reduction of two moles of TPN, and the esterification of 2 moles of orthophosphate (Fig. 18). The stoichiometry of this reaction was the same when TPN was replaced by ferricyanide. With either TPN (4l) or ferricyanide $(18,74)$, the rate of oxygen evolution is greatly increased when it is coupled with phosphorylation. The conventional Hill reaction could thus be viewed as an uncoupled photophosphorylation, i.e., a photochemical electron transport that is proceeding without its normally associated phosphorylation reaction? ${ }^{7}$.

The novel features of reaction 5 were, first, that its ATP formation, unlike any other known phosphorylation, was coupled with an evolution of oxygen. Second, contrary to an analogy with oxidative phosphorylation, ATP formation resulted not in the ultimate oxidation but in the reduction of pyridine nucleotide.

The mechanism of reaction 5 was also different, notably in its dependence on chloride (30), from that of the anaerobic cyclic photophosphorylation that is common to chloroplasts and chromatophores (Section 9). In the presence of chloride, ferricyanide was not an

"It may be of interest to record in passing that the finding of Reaction 5 in chloroplasts was as umexpected to its discoverers as it was to other investigators in this field. The extent to which it was mexpected was expressed by Jagendorf in lhese words: "When it was announced at lhe .1IBS meetings in August 1957 that the 'Hill reaction' is really due to uncoupled phosphorylation, i.e., that ferricyanide reduction was more rapicl if phosphorylation occurred simultaneously, we were as surprised and skeplicat as anyone clse. However, by now we have been able to confirm this rery amply" (74, p. 215). 


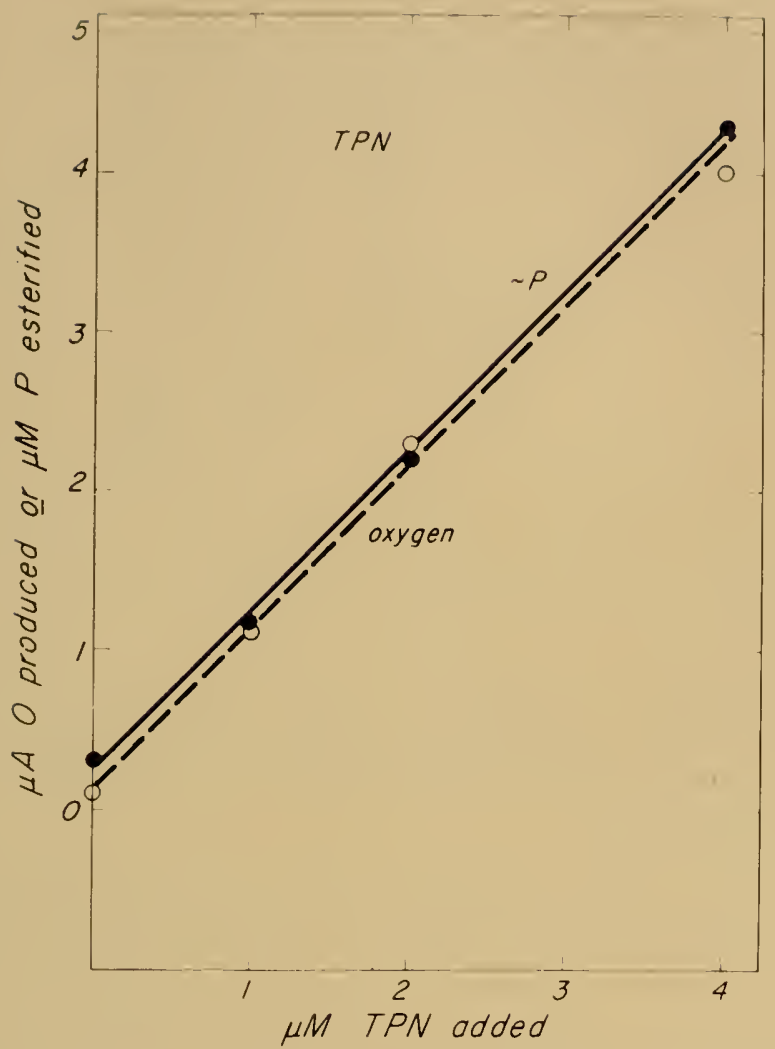

Fig. 18. Stoichiometry of oxygen evolution and ATP formation resulting from the photochemical reduction of TPN (Annon, Whatley, and Allen, 18, 18a). $\mu_{\mathrm{A}}$. microatoms.

inhibitor as it was in cyclic photophosphorylation (Table 4), but an electron acceptor which promoted ATP formation. The results are shown in Table 10.

The mechanism of reaction 5 is visualized as the non-cyclic electron flow mechanism in photosynthetic bacteria (Fig. 17) but with water as the electron donor $(\mathrm{H} A)$. Fig. 19 gives a diagram of the noncyclic photophosphorylation of green plants, represented here as a part of the general electron flow mechanism in photosynthesis that was shown elsewhere (9).

We visualize non-cyclic photophosphorylation of green plants (Fig. 19) as having in common with other photophosphorylations the same primary photochemical act (Equations 3 and $3 a$ ) and the same pri- 
TABLE 10

Effect of Chloride on Non-cyclic Photophosphorylation By Isolated Chloroplasts

(Bové, Bovḱ, Whatley, and Arnon, 30)

\begin{tabular}{ccccc} 
& & \multicolumn{2}{c}{ Micromoles ATP formed } \\
Experiment & Electron acceptor & - chloride & + chloride \\
A & Ferricyanide & 0.1 & 3.3 \\
B & Ferricyanide & 0.3 & 3.7 \\
C & TPN & 0.7 & 3.6 \\
D & TPN & 0.7 & 4.2
\end{tabular}

mary phosphorylation reaction at the site of the cytochrome adjacent to the excited chlorophyll molecule (Equation 4). Chloroplasts have no hydrogenase and hence can give no hydrogen evolution. The electron "expelled" in the primary photochemical act is accepted by TPN and used (along with an accompanying proton) for the production of $\mathrm{TPNH}_{2}$. $\mathrm{TPNH}_{2}$ can either accumulate under special conditions, as in the experiments with isolated chloroplasts (Table 10), or be used jointly with ATP for the formation of carbohydrates during normal photosynthesis.

In a non-physiological variant of reaction 5, TPN can be replaced by ferricyanide. In that case the electrons from chlorophyll are accepted by ferricyanide which becomes reduced and accumulates as ferrocyanide. The protons also accumulate, causing the acidity to increase, as has been found to be the case with potassium ferric oxalate by Holt and French (7la).

To keep the chlorophyll system functional the electrons removed by non-cyclic transport must be continuously replenished. We suggested that this is accomplished in a reaction between hydroxyl ions (or water) and a cytochrome peculiar to the photosynthetic apparatus of green plants but absent in photosynthetic bacteria (9). This reaction is visualized as being possibly an "anocle"-type, clark reaction, in which $\mathrm{OH}^{-}$ions yield molecular oxygen and donate electrons, via a cytochrome chain, to chlorophyll, where they are raised at the expense of absorbed light energy to the reducing potential required for TPN reduction. A corollary of the scheme is that water is still the source of the oxygen evolved in photosynthesis by green plants (9).

In the electron transfer sequences visualized in non-cyclic photophosphorylation (Fig. 19), we have ruled out the participation of cytochromes in the transfer of electrons from chlorophyll to TPN because the reclox potential of TPN $\left(E^{\prime}{ }_{o}=-0.32 \mathrm{v}\right)$ is altogether 


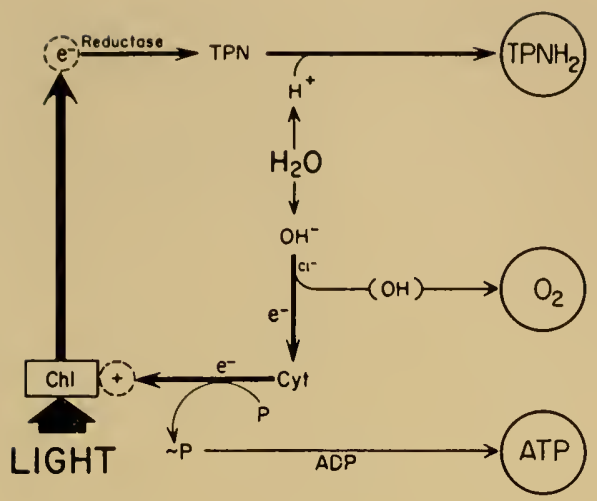

Non-cyclic photophosphorylation (chloroplasts)

Fig. 19. Scheme for non-cyclic photophosphorylation in chloroplasts.

Details in the text.

too negative for cytochromes to serve here as electron carriers. This leaves the electron transfer from water (hydroxyl ions) to chlorophyll as the sequence of reactions in which cytochromes can serve as electron carriers.

The proposed mechanism, which assigns to cytochromes a role in the electron transport from $\mathrm{OH}^{-}$ions (or water) to chlorophyll, implies that the chlorophyll-cytochrome complex must generate a sufficient oxidizing potential to drive the reaction $2 \mathrm{H}_{2} \mathrm{O} \rightarrow \mathrm{O}_{2}+$ $4 \mathrm{H}^{+}+4 \mathrm{e}^{-}$, which at $25^{\circ} \mathrm{C}$ and at $p \mathrm{H} 7$, has $E^{\prime}{ }_{o}=+0.815 \mathrm{v}$.

It might be argued that cytochrome $f$, the most oxidizing cytochrome now known to occur in chloroplasts, has a reclox potential lower than oxygen (42), i.e., $E_{o}^{\prime}=+0.365$ v. However, it would be premature to conclude that our knowledge of redox potentials of cytochromes in chloroplasts is now complete.

The proposed reactions leading to oxygen evolution appear to be thermodynamically feasible. The energy contribution of one einstein of red light, about $43 \mathrm{kcal}$, is equivalent to a potential of $1.9 \mathrm{v}$ per faraday, and is sufficiently large, after making allowances for TPN reduction and ATP formation, to endow a chlorophyll-linked cytochrome with a redox potential more oxidizing than $0.815 \mathrm{v}$, as is needed for oxygen evolution.

We wish to reiterate that, in our present state of knowledge, the proposed mechanism for oxygen evolution must remain tentative. The possibility is not excluded that the transfer of electrons from $\mathrm{OH}^{-}$to cytochromes may require an additional input of energy. 


\section{Oxygen-Catalyzen Cyclic Photophosphorylation}

The mechanisms of photosynthetic phosphorylation in chloroplasts discussed thus far include anaerobic cyclic photophosphorylation (Figs. 4 and 5) and non-cyclic photophosphorylation (Fig. 19). Recent work by Tsujimoto, Hall, and Arnon (156) suggests the operation in chloroplasts of a third mechanism, an oxygen-catalyzed cyclic photophosphorylation.

As already discussed in Section 6, a catalytic role for oxygen was envisaged in explaining the first experiments on photosynthetic phosphorylation, in which the presence of oxygen was required but no oxygen consumption was observed (11). Interest in the role of oxygen was heightened when several laboratories reported that at low, "microcatalytic" concentrations of FMN or vitamin K (Fig. 3), photophosphorylation remained dependent on oxygen $(169,77,111)$.

From the standpoint of cellular physiology, it was interesting to contrast the role of oxygen in ATP formation in photosynthesis with that in respiration. The participation of oxygen as the terminal electron acceptor in oxidative phosphorylation has conferred a marked superiority on respiration over fermentation, in the efficiency of converting the free energy of the substrate into the energy of the pyrophosphate bonds of ATP. Was the efficiency of conversion of light energy into ATP also increased by the presence of oxygen?

To answer this question, photophosphorylation by chloroplasts was investigated in air and in nitrogen, at different concentrations of FMN or vitamin $\mathrm{K}$, and particularly, at a limiting light intensity, when the efficiency of the energy conversion process could be best observed (compare Section 10). The results are shown in Fig. 20.

In limiting light, the highest rate of photophosphorylation was obtained in nitrogen at a concentration of approximately $10^{-4} \mathrm{M}$ of either FMN or vitamin K. No photophosphorylation occurred in nitrogen without added cofactors but when these were added at an optimal concentration, the anaerobic system was about twice as effcient in converting light energy into ATP as the acrobic system.

The experiments represented by Fig. 20 were carried out with relatively high concentrations of chloroplast material. Under these conditions the aerobic system showed little increase in photophosphorylation from adding FMN or vitamin $\mathrm{K}$. Howerer, high concentrations of chloroplast material were found to be necessary to insure the effective operation of the anaerobic FMN system. The anaerobic vitamin $\mathrm{K}$ system functioned optimally at lower concentrations of chloroplast material, suggesting that it required less or fewer 
CIMITING LIGHT and HIGH CHLOROPHYLL

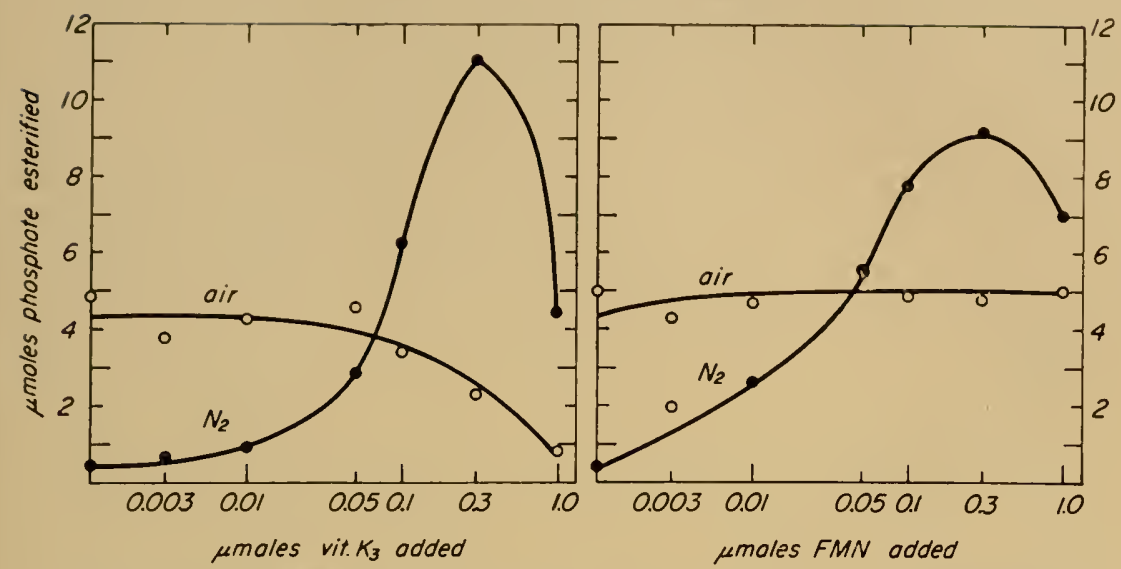

Fig. 20. Effect of FMN and vitamin $\mathrm{K}_{3}$ concentration on cyclic photophosphorylation by spinach chloroplasts in nitrogen and air, at a low light intensity. The reaction mixture included, in a final volume of $3.0 \mathrm{ml}$, chloroplast fragments

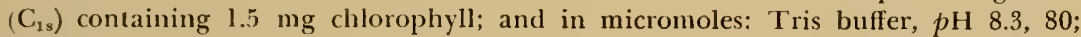
$\mathrm{MgSO}_{4}, 5: \mathrm{K}_{2} \mathrm{HP}^{\prime: 2} \mathrm{O}_{4}, 15$; ADP, $15 \mathrm{TPN}, 0.3$ (only in the FMN series). FMN or vitamin $K_{3}$ was added as indicated. The reaction was run for 30 minutes at an illumination of 2.000 Lux (T'sujimoto, Hall, and .Imon, 156).

of the chloroplast factor (s) than were required for the anaerobic FMN system. These chloroplast factors for the FMN system appeared to be bound in the grana fraction and were not supplied by an aqueous extract of chloroplasts.

On comparing the aerobic and anaerobic systems under conditions when they responded optimally to the addition of cofactors, a marked difference was observed, depending on the presence or absence of oxygen, in the effect of two inhibitors, o-phenanthroline and C.MU [p-chlorophenyl dimethylurea] (a gift of Dr. C. E. Hoffman). The results are shown in Table 11 .

In agreement with findings of Wessels (169), Jagendorf and Avron (7i), and Nakamoto et al. (111), o-phenanthroline and CMU, in the presence of air, inhibited photophosphorylation in the FMN and vitamin $\mathrm{K}$ systems. Relatively little inhibition by these two inhibitors was observed in an atmosphere of nitrogen. In other experiments, not reported here, phenazine methosulfate was found to differ from FMN and vitamin $\mathrm{K}$ in that its pathway was resistant to inhibition by o-phenanthroline and CMU, both in air and in nitrogen.

$o$-Phenanthroline and CMU are powerful inhibitors of oxygen evolution by illuminated chloroplasts (cf. 169, 77, 111). It seems 
TABLE 11

EFECT OF O-PUENANIIROLINE AND CMU ON CrCLIC P'HOTOPHOSPHORYLATHON in Nitrogen or Air

(Tsujimoto, Hall, And Arnon, 156)

\begin{tabular}{|c|c|c|c|c|}
\hline \multirow[b]{3}{*}{ Treatment } & \multicolumn{4}{|c|}{ Inhibitor added } \\
\hline & \multicolumn{2}{|c|}{$\mathrm{CMU}$} & \multicolumn{2}{|c|}{$o$-Phenanthroline } \\
\hline & $\begin{array}{l}\mu \text { moles P } \\
\text { esterified }\end{array}$ & $\begin{array}{l}\text { Percent } \\
\text { Inhibition }\end{array}$ & $\begin{array}{l}\mu \text { moles P } \\
\text { esterified }\end{array}$ & $\begin{array}{l}\text { Percent } \\
\text { Inhibition }\end{array}$ \\
\hline \multicolumn{5}{|l|}{ Nitrogen series } \\
\hline Control & 0.7 & - & 0.7 & 一 \\
\hline FMN added & 8.1 & - & 9.7 & - \\
\hline FMN and inhibitor added & 6.1 & 25 & 7.8 & 20 \\
\hline Vitamin $\mathrm{K}_{3}$ added & 9.3 & - & 9.4 & - \\
\hline Vitamin $\mathrm{K}_{3}$ and inhibitor added & 7.5 & 19 & 6.7 & 27 \\
\hline \multicolumn{5}{|l|}{ Air series } \\
\hline Control & 1.9 & - & 2.6 & - \\
\hline Inhibitor added & 1.6 & - & 2.3 & - \\
\hline FMN added & 5.8 & - & 9.5 & - \\
\hline FMN and inhibitor added & 1.7 & 97 & 3.9 & 77 \\
\hline Vitamin $\mathrm{K}_{3}$ added & 5.8 & - & 9.2 & - \\
\hline Vitamin $\mathrm{K}_{3}$ and inhibitor added & 2.2 & 85 & 4.7 & 64 \\
\hline
\end{tabular}

In the nitrogen series the illumination was $2000 \mathrm{Lux}$ for $30 \mathrm{~min}$ and the reaction mixture included, in a final volume of $3 \mathrm{ml}$, chloroplast fragments $\left(\mathrm{C}_{1 \mathrm{~s}}\right)$ containing 1.5 mg chlorophyll and $0.3 \mu$ moles of $F M N$ or vit. $K_{3}$. In the air series the illumination was 50,000 Lux for $5 \mathrm{~min}$ and the reaction mixture included chloroplast fragments $\left(\mathrm{C}_{\mathrm{s}}\right)$ containing $1 \mathrm{mg}$ chlorophyll and $0.003 \mu$ moles of FMN or vitamin $K_{3}$. The final inhibitor concentrations were, $3 \times 10^{-5} M /$ for 0 -phenanthroline and $2 \times 10^{-6} M I$ for CMU. Other common components of the reaction mixtme were, in micromoles: Tris butfer, $p \mathrm{H} 8.3,80 ; \mathrm{K}_{2} \mathrm{H}^{32} \mathrm{PO}_{4}, 15$; $\mathrm{ADP}, 15$; and $\mathrm{MgSO}_{4}, 5$. In the air series, the photophosphorylation in the control and "inhibitor added" treatments was subtracted from that in the other treatments when calculating the per cent inhibition.

TABLE 12

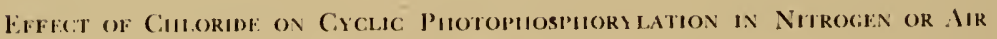
(Tstijimoto, Hall and Armon, 156)

pmoles P' esterified

Treatment mimus chloride

plus chloride

Nitrogen, liNN

Nitrogen, vit. $K_{3}$

Air, IMN

Air, vit. $\mathrm{K}_{3}$

5.1
9.7
0.5
0.4

6.1

Experimental conclitions as in Table 11 , except that chloroplasts were prepared in $0.5 \mathrm{M}$ sucrose and chloride-frce reagents were used. $0.2 \mathrm{mg}$ chlorophyll was used in the air series, and $2.5 \mathrm{mg}$ chlorophyll per tessel was used in the nitrogen series. 'I he reaction was run for 30 minutes. 
likely, therefore, that as was concluded earlicr by Wessels (169) and Nakamoto et al. (111) oxygen evolution is a component step in the "acrobic" photophosphorylation catalyzed by FMN or vitamin $\mathrm{K}$, and that molecular oxygen, when present, acts as an electron acceptor in photosynthetic phosphorylation. This conclusion is supported by the observed effect of chloride on cyclic photophosphorylation with vitamin $\mathrm{K}$ and FMN in air and in nitrogen (Table 12). (Chloride, it will be recalled, is required for the photochemical production of oxygen.) The omission of chloride had scarcely an effect on photophosphorylation in nitrogen, but it inhibited severely photophosphorylation in air.

The similarity in the effects of chloride, o-phenanthroline, and CMUU, either in air or in nitrogen, on the FMN and vitamin $\mathrm{K}$ pathways, under the modified experimental conditions which we now use, has blurred the distinction between the two pathways that was made on the basis of earlier inhibitor experiments (172). Apart perhaps, from the greater dependence of the FMN pathway on TPN (172) (a dependence that has not yet been reinvestigated under the new experimental conditions), what seems now to distinguish the two anaerobic pathways is the greater requirement, in the case of FMN, for a higher concentration of chloroplast material.

The participation of oxygen in cyclic photophosphorylation may increase the overall rate of ATP formation but only when light is abundant and phosphorylation is limited by a low concentration of cofactors. However, present evidence indicates (Fig. 20) that, in contrast to oxidative phosphorylation, the intervention of molecular oxygen in photosynthetic phosphorylation is an energy-wasteful step that lowers the efficiency of the anaerobic cyclic photophosphorylation process when light is limiting.

On the basis of evidence now available, the participation of oxygen as a catalyst in cyclic photophosphorylation may be represented by the diagram in Fig. 21. Here the electron flow mechanism (marked by a heavy line) is composed of two parts. The first part is completed when electrons from excited chlorophyll are accepted by $\mathrm{O}_{2}$ and, in combination with protons, form water. In the second part, these electrons are replaced by those donated by $\mathrm{OH}$, with a concomitant evolution of oxygen, as was described for the non-cyclic electron flow pathway for chloroplasts (Fig. 19). The proposed mechanism, in which oxygen acts catalytically, provides for an exchange between molecular oxygen and the oxygen of water and is 


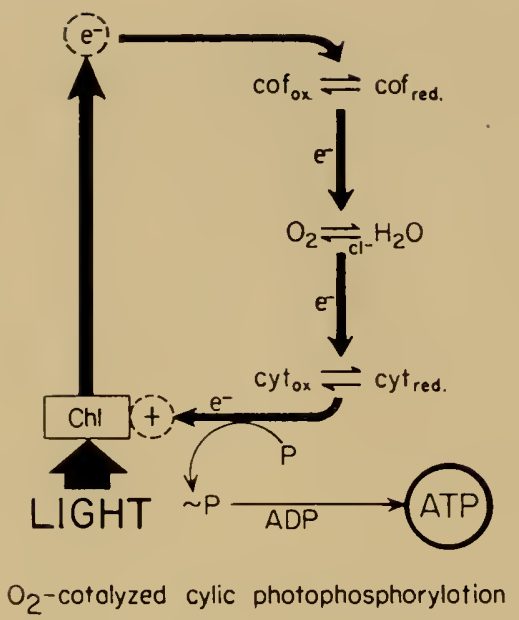

Fig. 21. Scheme for oxygen-catalyzed cyclic photophosphorylation in chloroplasts. Details in text.

in agreement with the $\mathrm{O}^{18}$ exchange data recently reported by Nakamoto and Vennesland (112) and Jagendorf (75).

In summary, then, FMN and vitamin $\mathrm{K}$ seem to catalyze two pathways of cyclic photophosphorylation, one anaerobic and one catalyzed by molecular oxygen (cf. 168). The anaerobic pathway, when investigated in an atmosphere of nitrogen, requires appreciable, although still catalytic, concentrations of cofactors and, particularly in the case of FMN, high concentrations of chloroplast material that evidently supply the additional lactor (s) needed for the efficient conversion of light energy into ATP under anaerobic conditions. The oxygen-catalyzed pathway for FMN or vitamin $\mathrm{K}$ is catalyzed by very low, "microcatalytic" concentrations of these cofactors and is much less dependent on additional chloroplast material than the anaerobic pathway.

These findings are interpreted to mean that oxygen, when present in a system catalyed by either FMN or vitamin $\mathrm{K}$, is able to conpete effectively for the electrons of cyclic photophosphorylation. Once the electrons are accepted by oxygen and form water, the cyclic pathway can be maintained only by a release of electrons in the oxygenforming reaction of non-cyclic photophosphorylation in chloroplasts (Section 15). By contrast, phenazine methosulfate catalyzes the transler of electrons to cytochrome so effectively (105) that it is able to prevent their "escape" to oxygen, and hence the phenazine metho- 
sulfate pathway remains an "anacrobic" one even when molecular oxygen is present.

As lar as efficiency of conversion of light encrgy into ATP is concerned, it appears from experiments at liniting light intensities that the anaerobic cyclic photophosphorylation with FMN or vitamin $\mathrm{K}$ is more efficient that with phenazine methosulfate (Figs. 9 and 10). Also, the anacrobic FMN and vitamin $\mathrm{K}$ cyclic pathways are more efficient than their oxygen-catalyzed ${ }^{8}$ counterparts (Fig. 20). These fundings suggest the participation of more than one phosphorylation site in the anacrobic FMN and vitamin $\mathrm{K}$ pathways (compare Fig. 5 with Figs. 4 and 21).

\section{Rflation of Cyclic to Non-cýglc Photophosphorylation in Chloroplasts}

The ability of isolated chloroplasts to carry out both cyclic (Fig. 5) and non-cyclic photophosphorylation (Fig. 19) raises the question of the mutual relation of these two processes. Specifically, what effect would the addition of one of the cofactors of cyclic photophosphorylation have on the reduction of TPN and evolution of oxygen which accompany ATP formation in non-cyclic photophosphorylation?

As shown in Figs 22 and 23, the addition of either FMN or vitamin $\mathrm{K}$ altered non-cyclic photophosphorylation profoundly. ATP formation was sharply increased, whereas oxygen evolution and the accumulation of reduced TPN were abolished. It appears, therefore, that cyclic photophosphorylation is a more "tightly coupled" mechanism for converting light energy into ATP than non-cyclic photophosphorylation. In the presence of the requisite cofactors, cyclic photophosphorylation is capable of diverting all of the absorbed light energy for the formation of $A T P$, and suppressing TPN reduction and $\mathrm{O}_{2}$

- Distinct from the oxygen-catalyzed cyclic photophosphorylation discussed here is the "oxidative photosynthetic phosphorylation" (86) by chloroplasts in which oxygen consumption was induced by a joint use of a dye (trichlorophenol indo. phenol), an inhibitor (o-phenanthroline or C.MU), and $\mathrm{DP}^{\mathrm{N}} \mathrm{NH}_{2}$. The correspondence of the term "oxidative photosynthetic phosphorylation" to oxidative phosphorylation by mitochondria appears to be fortutous. The role of $\mathrm{DPNH}_{2}$ in this chloroplast system was not that of a physiological electron donor but that of a nonspecific reducing agent for the dye, as one of several reducing agents that were effective. That the consumption of oxygen was artificially induced and was onlv a feature of the special system used, is made clcar by the authors' observations that "there was ample energy released by the dark oxidation of the DPNH to form the high energy phosphate bonds. Nevertheless, the reaction gave no phosphorylation unless the system was illuminated, even though the light caused no increase in the rate of oxygen consumption" (86). 


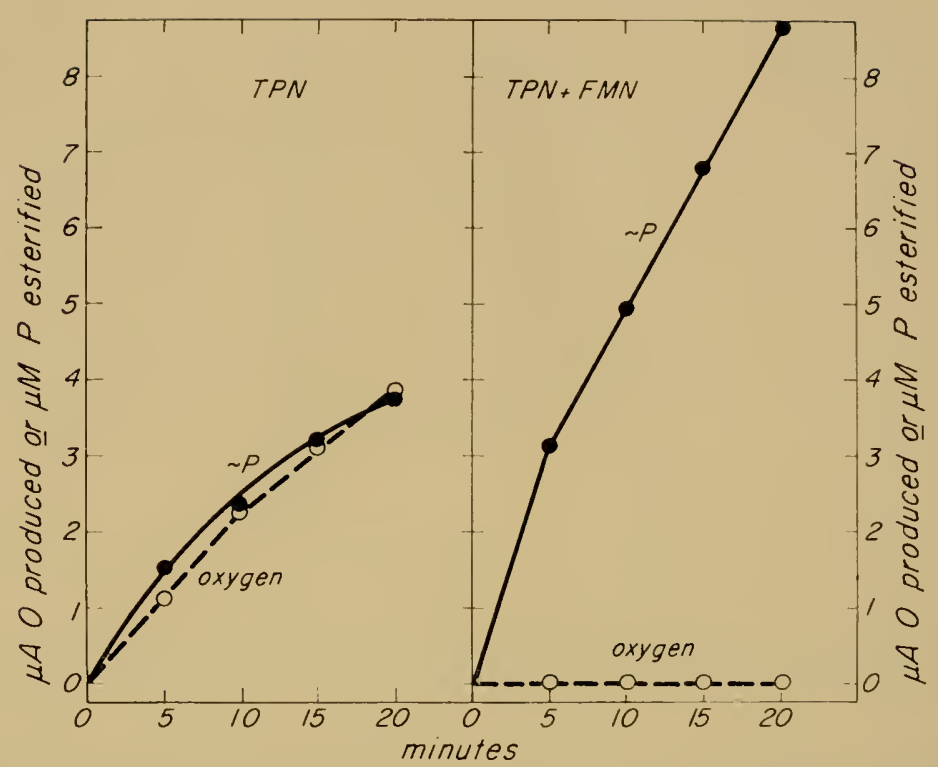

Fig. 22. Photophosphorylation and oxygen evolution by isolated chloroplasts in the presence and absence of FMN (Arnon, Whatley, and Allen, 18, 18a). $\mu \mathrm{A}$, microatoms.

evolution. It is assumed that the intact cell has suitable regulatory mechanisms for keeping cyclic and non-cyclic photophosphorylation in balance.

The marked increase in phosphorylation accompanied by a total abolition of oxygen evolution and $\mathrm{TPNH}_{2}$ accumulation, shown in Figs. 22 and 23, occurred on adcling 0.1 micromoles of FMN or 0.2 micromoles of vitamin $\mathrm{K}$ (in a final volume of $3 \mathrm{ml}$ ). However, the adclition of even extremely minute amounts of either FMN or vitamin $K$ had a measurable effect on reaction 4 . Table 13 shows that the addition of as little as 0.0002 to 0.0005 micromoles of FMN or vitamin $\mathrm{K}$ increased ATP formation without appreciably depressing oxygen evolution (and the corresponding $\mathrm{TPNH}_{2}$ accumulation). Similar effects were observed on adding small amomts of phenazine methosulfate (Table 14).

As will be discussed more fully later, the addition of small amounts of one of these catalysts of photosynthetic phosphorylation had an influence on carbon assimilation. Concentrations of FMN and vitamin $\mathrm{K}$ which suppressed oxygen evolution (and $\mathrm{TPNH}_{2}$ accumulation) also suppressed reductive $\mathrm{CO}_{2}$ fixation, but the minute concentrations 


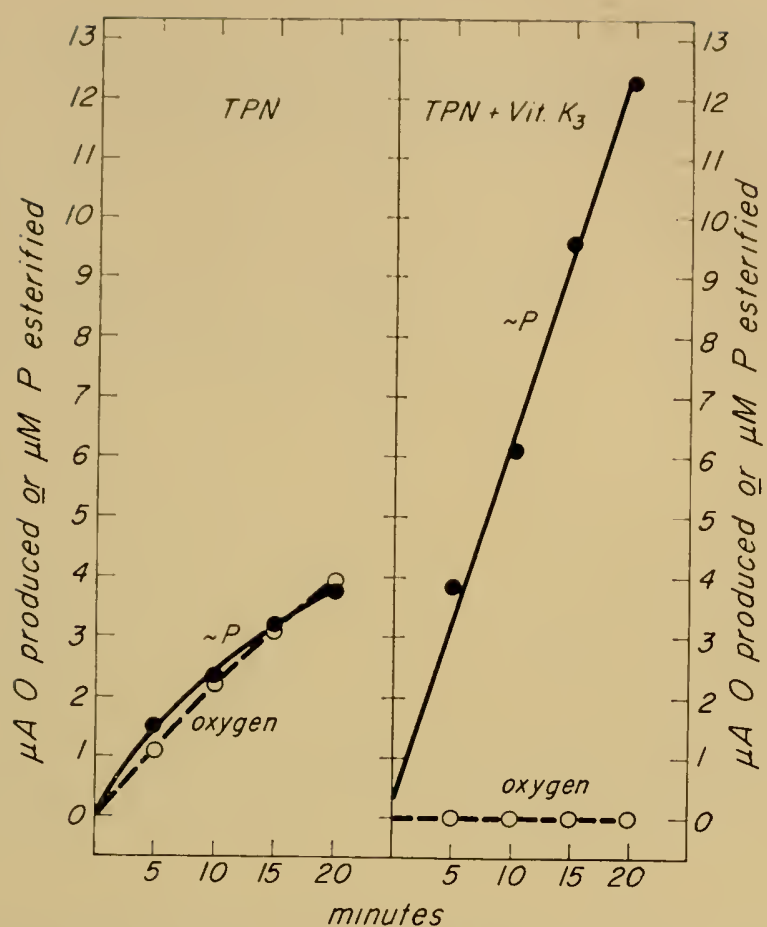

Fig. 23. Photophosphorylation and oxygen evolution by isolated chloroplasts in the presence and absence of vitamin $\mathrm{K}_{3}$ (Arnon, Whatley, and Allen, 18, 18a). $\mu \mathrm{A}$, microatoms.

of these catalysts which increased ATP formation without markedly depressing oxygen evolution also increased reductive $\mathrm{CO}_{2}$ fixation (see Section 18).

\section{Sfparation of Light and Dark Phases of Photosynthesis in Isolated Chloroplasts}

A special contribution of cell-free photosynthesis to the study of the mechanism of the process has been the experimental separation of the light-dependent phase, in which photosynthetic events proper occur, from the dark phase during which $\mathrm{CO}_{2}$ is assimilated. This separation has already been discussed for bacterial photosynthesis in Section 12. It will now be reviewed for green plants as it has been observed in chloroplasts. The literature on similar investigations with whole cells has been reviewed elsewhere (153).

A physical separation of the light and dark phases of photosyn- 
TABLE 13

EfFect of FMN and Vitamin $\mathrm{K}_{3}$ on Photophosphorylation and Oxygen Evolution Linked to TPN Reduction

(Arnon, Whatiley, and Allen, 18a)

\begin{tabular}{|c|c|c|c|c|}
\hline \multirow{2}{*}{$\begin{array}{c}\text { FMN or } \\
\text { vitamin } \mathrm{K}_{3} \\
\text { added } \\
\text { (micromoles) }\end{array}$} & \multicolumn{2}{|c|}{ FMN system } & \multicolumn{2}{|c|}{ Vitamin $K_{3}$ system } \\
\hline & $\begin{array}{l}\text { P esterified } \\
(\mu \text { moles })\end{array}$ & $\begin{array}{c}\mathrm{O}_{2} \text { evolved } \\
\text { ( } \mu \text { atoms) }\end{array}$ & $\begin{array}{c}\text { P esterified } \\
\text { ( } \mu \text { moles })\end{array}$ & $\begin{array}{c}\mathrm{O}_{2} \text { evolved } \\
\text { ( } \mu \text { atoms) }\end{array}$ \\
\hline none & 5.6 & 3.6 & 5.6 & 3.6 \\
\hline 0.0002 & 6.5 & 4.2 & 6.1 & 3.6 \\
\hline 0.0005 & 7.4 & 3.8 & 7.5 & 2.9 \\
\hline 0.001 & 7.9 & 3.3 & 8.0 & 2.3 \\
\hline 0.003 & 8.4 & 1.2 & 9.6 & 0.9 \\
\hline 0.01 & 9.0 & 0.4 & 10.0 & 0.9 \\
\hline
\end{tabular}

thesis by fractionating isolated chloroplasts has recently been described by Trebst, Tsujimoto, and Amon (153). The light phase was completed first by the complete chloroplast system, in the absence of $\mathrm{CO}_{2}$, and resulted in an evolution of oxygen accompanied by an accumulation of substrate amounts of $\mathrm{TPNH}_{2}$ and $\mathrm{ATP}$ in the reaction mixture. The green portion of the chloroplasts (grana) was then discarded and $\mathrm{CO}_{2}$ was next supplied to the remaining non-green portion of the chloroplasts in the dark.

As shown in Table 15, in the presence of assimilatory power, i.e., $\mathrm{TPNH}_{2}$ and ATP, the chlorophyll-free extract was able to fix $\mathrm{C}^{14} \mathrm{O}_{2}$ in the dark. Only leeble $\mathrm{C}^{14} \mathrm{O}_{2}$ fixation occurred in the dark without the components of assimilatory power (Treatments 1 and 2). The dark fixation of $\mathrm{CO}_{2}$ by the chlorophyll-free extract supplemented with assimilatory power was comparable with fixation in the light by the complete chloroplast system, to which $\mathrm{C}^{14} \mathrm{O}_{2}$ was supplied at the beginning of the illumination period. Here assimilatory power was formed continuously in the light and used at once for $\mathrm{CO}_{2}$ fixation (Treatment 3). Very little $\mathrm{CO}_{2}$ fixation occurred in the complete chloroplast system in the dark (Treatment 4).

\section{TABLE 14}

Efiect of Phenazine Methosulfate (PMS) on Phospiorýlation and Oxygen Evolution Linked to TPN Reduction

(Arnon, Whatley, and Allen, 18a)

\begin{tabular}{ccc}
$\begin{array}{c}\text { PMS added } \\
(\mu \text { moles })\end{array}$ & $\begin{array}{c}\text { P esterified } \\
(\mu \text { moles })\end{array}$ & $\begin{array}{c}\mathrm{O}_{2} \text { evolved } \\
(\mu \text { moles })\end{array}$ \\
none & 4.5 & 4.0 \\
0.003 & 6.1 & 4.0 \\
0.01 & 9.0 & 3.2 \\
0.1 & 10.0 & 0.8 \\
\hline
\end{tabular}


T. IBI.E 1.5

CO2 hixation by a Chlorophyll-frle Extract and a Compiete

Chlloroplast System from Spinacil

(Trebst, TsujImoto, And Arnon, 153)

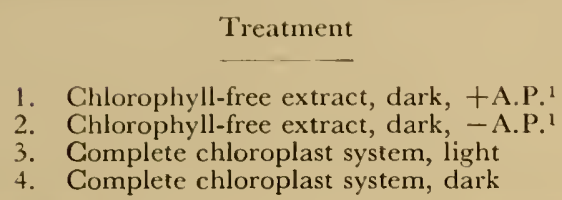

Treatment

$\mathrm{C}^{14} \mathrm{O}_{2}$ fixed counts/minute
134,000
9,000
200,000
20,000

${ }^{1}$ A.P. = Assimilatory power, i.e., $\mathrm{TPNH}_{2}$ and $\mathrm{ATP}$, formed in the light by chloroplasts.

The complete chloroplast system contained in a final volume of $2.5 \mathrm{ml}$, "broken" chloroplasts $\left(\mathrm{C}_{1 \mathrm{~s}}\right)$ containing $0.5 \mathrm{mg}$ chlorophyll, chlorophyll-free extract $(C E)$ equiv-

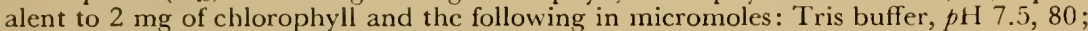
$\mathrm{IgCl}_{2}, 5 ; \mathrm{MnCl}_{2}, 2 ; \mathrm{Na}$ ascorbate, 10; glucose-1-phosphate, potassium salt, 0.3 ; sodium phosphate, $p \mathrm{H} \mathrm{7.5,5;ADP,2}$; and TPN, 2. The reaction was carried out at $20^{\circ} \mathrm{C}$ for 30 mintutes; gas pliase, argon. In Treatments 3 and $t, 10$ micromoles $\mathrm{NaHCO}_{3}$, containing $\mathrm{C}_{1}^{14} \mathrm{O}_{2}$, were injected at the beginning and the reaction vessels were illuminated or left in the dark, respectively. In Treatment 1, the reaction mixture was first illuminated without the addition of bicarbonate. After 30 minutes, the chlorophyllcontaining particles were removed by centrifugation, and the chlorophyll-free supernatant fluid was incubated in the dark for 30 minutes with 1.5 micromoles cysteine and 10 micromoles of $\mathrm{NaHCO}_{3}$, containing $\mathrm{C}^{14} \mathrm{O}_{2}$. The same procedure was followed in Treatment 2 as in Treatment 1, except that ADP and TPN were omitted.

The products of $\mathrm{CO}_{2}$ assimilation were found to be the same whether $\mathrm{CO}_{2}$ assimilation occurred during continuous illumination (Fig. 24) or in the dark, at the expense of assimilatory power generated during a preceding light period (Fig. 25). The products included hexose and pentose mono- and di-phosphates, phosphoglyceric acid, dihydroxyacetone phosphate, and small amounts of phosphoenolpyruvate and malate.

Similar products of $\mathrm{CO}_{2}$ assimilation by chloroplast extracts, including the formation of phosphorylated sugars, were obtained in a total dark chemosynthesis where $\mathrm{TPNH}_{2}$ and $\mathrm{ATP}$ were not formed photochemically but were prepared either chemically or enzymatically, or were derived from animal material (153).

An important link in the chain of evidence connecting the photosynthetic events with the dark assimilation of $\mathrm{CO}_{2}$ in chloroplasts was provided by experiments showing that both cyclic and non-cyclic photophosphorylation were essential. The ATP formed in non-cyclic photophosphorylation alone (Equation 5) is insufficient for the assimilation of $\mathrm{CO}_{2}$, to the level of carbohyclrates (151).

Experimental evidence for the participation of cyclic photophosphorylation in $\mathrm{CO}_{22}$ assimilation by chloroplasts had to be sought in 


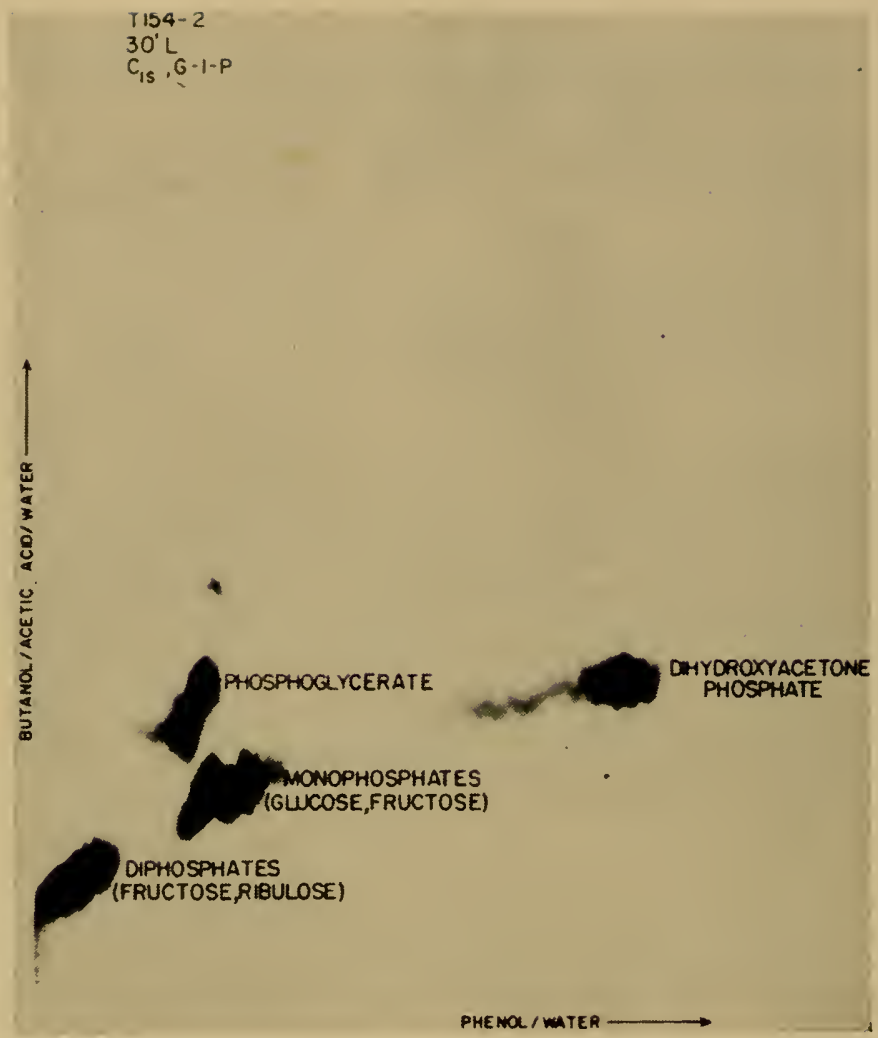

Fig. 24. Radioautograph of a chromatogram showing products of photosynthetic $\mathrm{C}^{14} \mathrm{O}_{2}$ assimilation by illuminated spinach chloroplasts. Expcrimental conditions as in Treatment 3 of Table 15 (Trebst, Tsujimoto, and Arnon, 153).

a system different from the one described above, wherein $\mathrm{TPNH}_{2}$ and ATP were first generated in substrate amounts in light (by Reaction 5) and then used later for $\mathrm{CO}_{2}$ assimilation in the dark. Here $\mathrm{TPNH}_{2}$ and ATP were already supplied in excess and a second reaction for generating ATP would be superfluous. However, evidence for the participation of cyclic phosphorylation in $\mathrm{CO}_{2}$ assimilation by isolated chloroplasts was recently obtained by Trebst et al. (151) in a catalytic system, i.e., one in which, as in an intact cell, $\mathrm{TPNH}_{2}$ and ATP were present in catalytic amounts and $\mathrm{CO}_{2}$ fixation was therefore possible only in the light while $\mathrm{TPNH}_{2}$ and ATP were being continuously regenerated at the expense of absorbed light energy.

$\mathrm{CO}_{2}$ assimilation in this catalytic system was investigated, under 


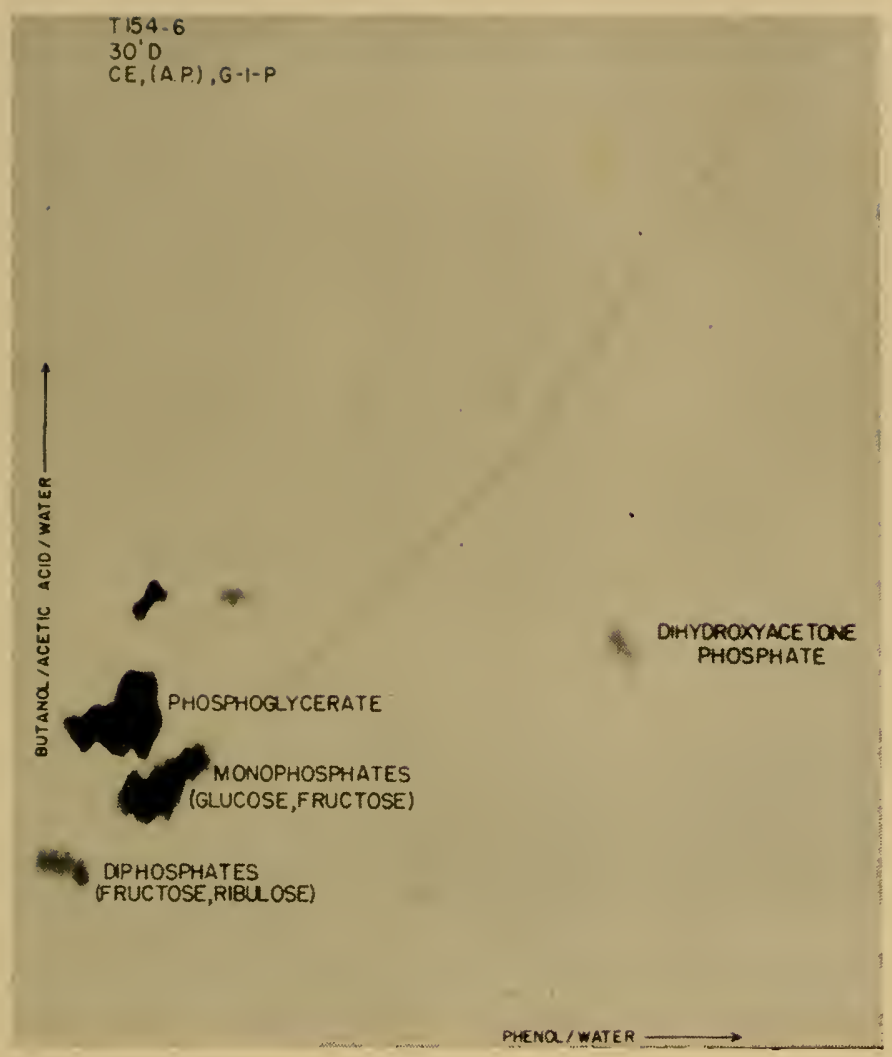

Fig. 25. Radioautograph of a chromatogram showing products of dark $\mathrm{C}^{14} \mathrm{O}_{2}$ assimilation by a chlorophyll-free extract of chloroplasts. Experimental conditions as in Treatment 1 of Table 15 (Trebst, Tsujimoto, and Arnon, 153).

three conditions: (a) when the photochemical phase was limited to non-cyclic photophosphorylation (reaction 5), (b) when the photochemical phase was limited to cyclic photophosphorylation (Fig. 5), and (c) when the photochemical phase included both (a) and (b).

Figs. 26 and 27 show that under conditions (a) and (b) $\mathrm{CO}_{2}$ assimilation was limited almost entirely to the formation of phosphoglycerate. As shown in Fig. 28, the formation of sugar phosphates, which is taken as a measure of a recluctive (photosynthetic) $\mathrm{CO}_{2}$ assimilation in this reconstituted chloroplast system, was observed only in case (c), when a proper balance was established between cyclic and non-cyclic photophosphorylation.

In the experiments illustrated by Figs. 26, 27, and 28, the balance 


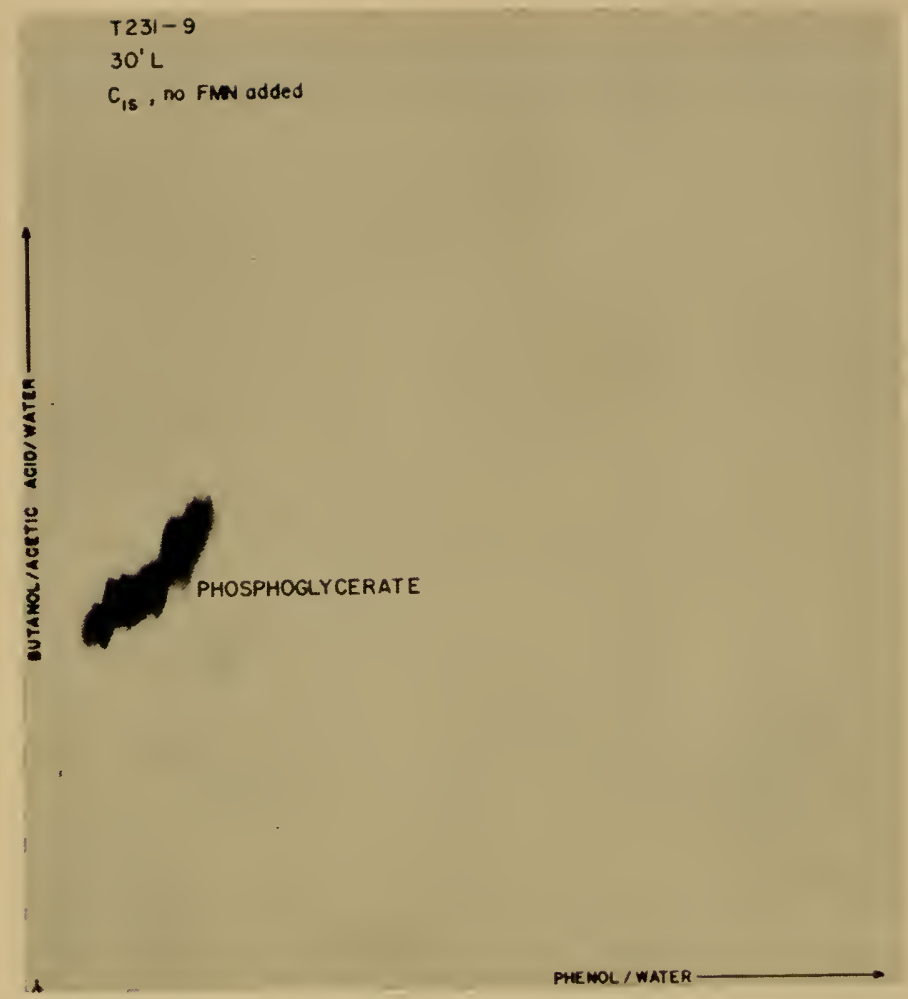

Fig. 26. Radioautograph of a chromatogram showing products of photosynthetic $\mathrm{C}^{14} \mathrm{O}_{2}$ assimilation by illuminated chloroplasts in the absence of added FMN (Trebst, Losada, and Arnon, 151).

between the non-cyclic and cyclic photophosphorylation was maintained by adding clifferent amounts of one of the catalysts of cyclic photophosphorylation, FMN, vitamin $\mathrm{K}$, or phenazine methosulfate (cf. Tables 13 and 14). Concordant results were also obtained by Trebst et al. (152) with inhibitor experiments. For example, using the uncoupling effect of ammonia $(74,152)$ on both cyclic and noncyclic photophosphorylation, it was possible to suppress the formation of ATP by illuminated chloroplasts without inhibiting the reduction of TPN. Under these conditions, $\mathrm{CO}_{2}$ fixation was completcly abolished except when the "acceptor" substance for $\mathrm{CO}_{2}$ was ribulose diphosphate. In that case a single product, phosphoglyceric acid, was formed by the carboxylase reaction which does not depend on added ATP (cf. review 161). Howerer, no sugar formation occurred because the acid cotld not be reduced by $\mathrm{TPNH}_{2}$ in the absence of ATP. 


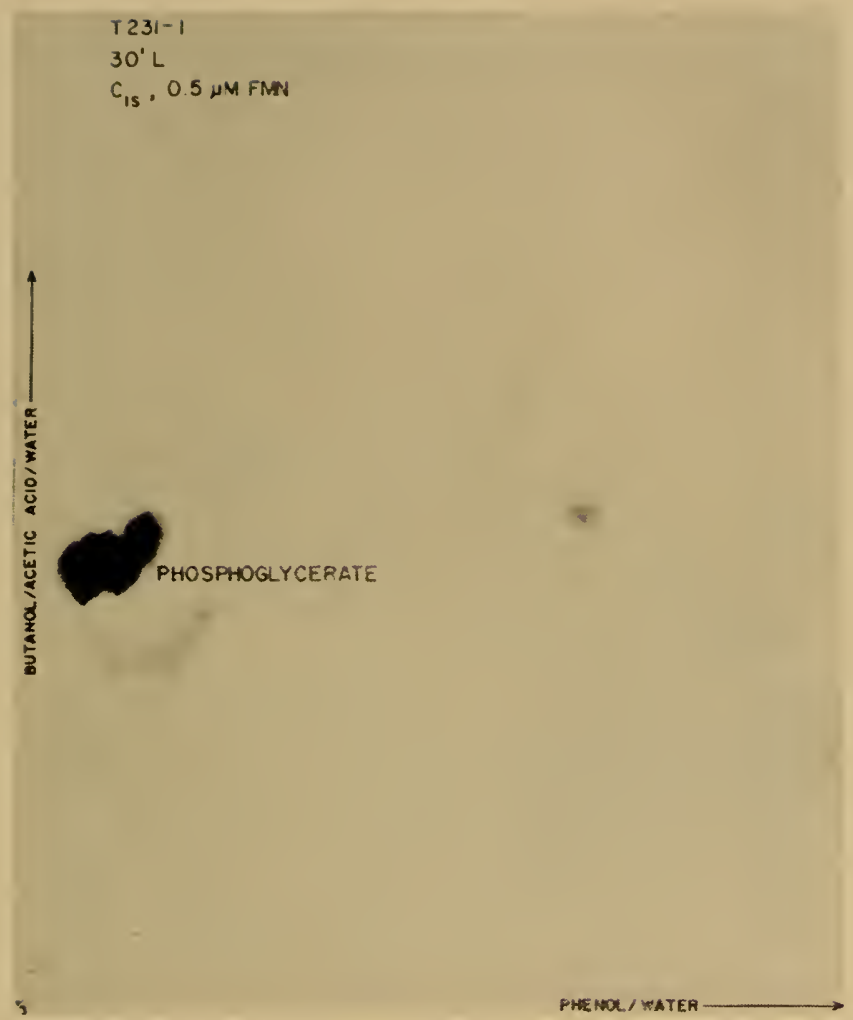

Fig. 27. Radioautograph of a chromatogram showing products of photosynthetic $\mathrm{C}^{14} \mathrm{O}_{2}$ assimilation by illuminated chloroplasts supplied with 0.5 micromoles FMN (Trebst, Losada, and Arnon, 151).

Parallel experiments of Losada et al. (96) on specific enzyme systems in chloroplasts fortified these lines of evidence and supported the conclusion that in a reconstituted "catalytic" chloroplast system (in which $\mathrm{CO}_{2}$ assimilation can occur only in the light), noncyclic photophosphorylation alone does not provide sufficient ATP for a reductive assimilation of $\mathrm{CO}_{2}$ to the level of carbohydrate. Additional ATP must be supplied by cyclic photophosphorylation.

\section{Photosynthesis and Biochemical Evolution}

The insight into the mechanism of photosynthesis gained from cellfree experiments with chloroplasts and chromatophores permits us to interpret, with somewhat enhanced confidence, certain aspects of biochemical evolution which we have already discussed elsewhere $(18,97)$. 


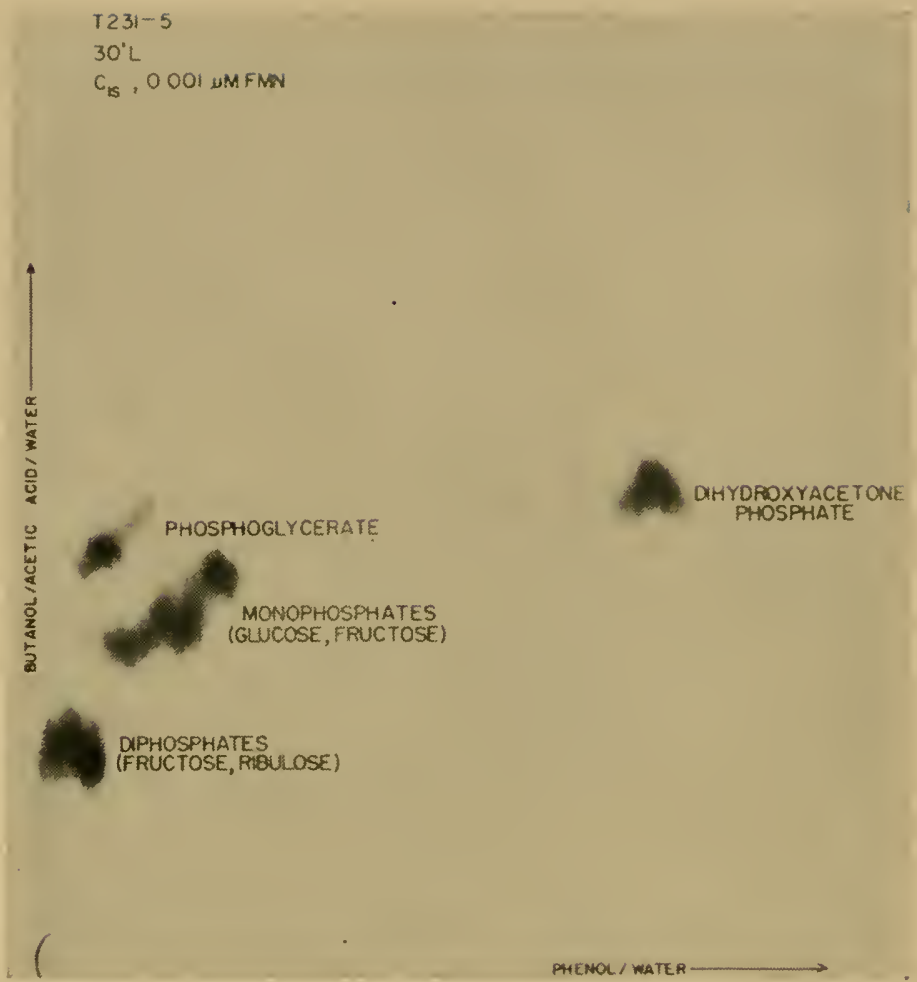

liig. 28. Radioatutograph of a chromatogram showing products of photosynthetic $\mathrm{C}^{14} \mathrm{O}_{2}$ assimilation by illuminated chloroplasts supplied with 0.001 micromoles FMN (Trebst, Losada, and Arnon, 151).

If, in the early periods of evolution of life forms, organic compounds were abundant and the earth's atmosphere was a reducing one (117, 107 ), then the beginning of photosynthesis may be viewed as an emergence of a porphyrin that gave rise to chlorophyll and permitted the cell to use for metabolic purposes the energy in the visible spectrum of sunlight. This primitive photosynthesis consisted only of anaerobic cyclic photophosphorylation. No oxygen was evolved and no reduction of $\mathrm{CO}$. was essential, since our premise is that the cnvironment contained preformed carbon compounds. This primitive type of photosynthesis is excmplified today by the already discussed photoassimilation of acetate by the obligate phototrophic anacrobe, Chromalium (97).

The harnessing of light energy for the synthesis of XTP was an event of supreme importance to the cell. It provided the cell, in an 
anaerobic ensironment, with a much more efficient mechanism than fermentation for the formation of ATP, which is the unversal cellular "energy currency" needed in the transformation of existing carbon compounds into fats, carbohydrates, proteins, etc. Cyclic photophosphorylation gave the anaerobic photosynthetic cell a mechanism which in efficiency of ATP formation is comparable with the process of oxidative phosphorylation in aerobic cells, that followed it later in the evolutionary scale.

From the point of view of biochemical evolution, one of the most interesting findings in the study of cell-free photosynthesis was that higher aerobic plants have retained to this day the anaerobic cyclic photophosphorylation as a mechanism for making ATP, while sharing with other organisms the acquisition of the process of oxidative phosphorylation by mitochondria.

We can next envisage that the photoassimilation of organic compouncls was gradually replaced by the assimilation of $\mathrm{CO}_{2}$, which gained in importance as the supply of organic compounds in the environment diminished. Here the main contribution of light was still the formation of ATP by cyclic photophosphorylation. The reductant, it is reasonable to suppose, was at first the molecular hydrogen of the surrounding atmosphere. Chromatium, for example, is still capable of using molecular hydrogen for reducing (in the dark) the pyridine nucleotide that is needed for $\mathrm{CO}_{2}$ assimilation.

Next, the photosynthetic cell acquired the enzymatic apparatus for photochemically generating electrons with a reducing potential equal to molecular hydrogen, from more oxidized electron donors, such as succinate or thiosulfate. In organisms which contain, or can adaptively form, hydrogenase (photosynthetic bacteria and algae), this phase of photosynthesis can also be observed today as a photoproduction of molecular hydrogen. Light energy now served a dual purpose. It supplied ATP by cyclic photophosphorylation and it provided electrons lor reducing pyridine nucleotides (Fig. 17).

Finally, in the most advanced type of photosynthesis, found in green plants, $\mathrm{CO}_{2}$ became the sole carbon source and water became the electron donor. Here the function of light was not only to provide ATP, but also to raise the electrons from water to an energy level high enough for the reduction of TPN. Only in the last case, when water became the electron donor, did oxygen evolution form an inseparable part of photosynthesis, as is indicated in the proposed mechanism for non-cyclic photophosphorylation in chloroplasts (Fig. 19) . 
Non-cyclic photophosphorylation enabled green plants to form a $\mathrm{CO}_{2}$ reductant at the expense of light energy with the aid of an ubiquitous substance, water, and in this way to invade and live autotrophically in areas devoid of reduced sulfur compounds or of other electron donors of restricted distribution. The resultant proliferation of plant growth was responsible for releasing to the atmosphere the oxygen, locked in the water molecule, by the only known mechanism capable of accomplishing this, the photosynthesis of green plants $(117,107)$.

Once molecular oxygen became available, the way was open for biochemical evolution to progress toward aerobic metabolism. The oxygen-independent cyclic photophosphorylation by chlorophyll-containing particles could now be paralleled by an efficient biological utilization of the energy of chemical substrates through the mechanism of oxidative phosphorylation of mitochondria. Plotosynthesis of green plants now provicled both the substrates and oxygen to make oxidative phosphorylation and aerobic life on this planet possible.

An interesting aspect of the relation between photosynthetic and oxidative phosphorylation in biochemical evolution is the common phylogenetic relationship between proplastids and mitochondria, as it was recently reported by Mühlethaler and Frey-Wyssling (108). Their observations on proplastid development in embryonic cells suggest that mitochondria followed rather than preceded chloroplasts as functional organclles in cellular metabolism. This is in harmony with the biochemical eviclence, since photosynthetic phosphorylation by chlorophyll-containing particles, being independent of molecular oxygen, could occur before oxidative phosphorylation by mitochondria, which requires molecular oxygen.

\section{Concluning Remakks}

The concept of photosynthesis to which we were led in the six years since the process was first completely localized in isolated chloroplasts $(11,6,1)$ differs from the conventional view of photosynthesis that it is mainly a process of $\mathrm{CO}_{2}$ assimilation. Photosynthesis appears to be first and foremost a process for converting sunlight into chemical energy and this conversion is more directly associated with phosphorus than with carbon assimilation. The trapped light energy is frist converted into ATP and reduced pyridine nucleotides, and then may be used by the cell for assimilation of carbon, nitrogen, phosphorus, or sulfur compounds, or for any other encrgy-requiring cellular activity. 
The photoassimilation of acetate by Chromatium is a case of photosynthesis without either oxygen evolution or $\mathrm{CO}_{2}$ reduction (97). So is the light-dependent conversion of glucose into starch (100). In the conventional view of photosynthesis this state of affairs would be a contradiction of terms, but according to our present concept these examples represent photosynthesis because they involve biochemical syntheses that are being driven by light cnergy after light has been converted by the cell into ATP.

In this view of photosynthesis, $\mathrm{CO}_{2}$ assimilation, although quantitatively the dominant form of photosynthesis on our planet, is fundamentally only a special case of the use and storage of light energy. $\mathrm{CO}_{2}$ assimilation proper, in both green plants and photosynthetic bacteria, consists of exclusively dark reactions that are not peculiar to photosynthesis. The familiar accumulation of carbon compounds as carbohydrates during photosynthesis in green plants constitutes storage of trapped light energy. The first products of photosynthesis in green plants $(9,96), \mathrm{ATP}$ and $\mathrm{TPNH}_{2}$, are present in the cell only in catalytic amounts and cannot be stored to any appreciable degree for future use, whereas carbohydrates or fats can.

The proposal that ATP formation is the main event in photosynthesis has been made earlier, notably in 1944 by Emerson, Stauffer, and Umbreit (45), who suggested that the "sole function of light energy in photosynthesis is the formation of 'energy-rich' phosphate bonds." They included in this generalization both green plants and photosynthetic bacteria, but made no provision for a photochemical generation of a reductant for $\mathrm{CO}_{2}$ assimilation by green plants. The main reason, however, why their proposal met with little acceptance in the ensuing years, and had little influence on subsequent research in photosynthesis, was the lack of substantial experimental eviclence for the participation of phosphorus in their experiments with intact Chlorella cells (cf. 124, p. 228; 176). The theoretical proposals of Emerson et al. could not be adequately defended against the theoretical objections levelled against them [as for example by Rabinowitch (124, p. 229) ], particularly since the first experiments with $\mathrm{P}^{32}$ to test the occurrence of light-induced phosphorylation in cell-free systems led to negative results. Aronoff and Calvin, who made these experiments with spinach grana, reported that "there is no direct connection between light and the gross formation of organic phosphorus compounds" (19).

As the history of photosynthesis approaches the end of its second century, one finds fresh relevance in the admonition of Priestley, 
whose discoveries marked the beginning of this field of inquiry. $\mathrm{He}$ wrote in 1777: "This is a new and a wide field of experiment and speculation, and a premature attachment to hypothesis is the greatest obstruction we are likely to meet with in our progress through it . . . Speculation is a cheap commodity. New and important facts are most wanted, and therefore of most value" (122).

The new facts which led to the concept of photosynthesis presented here are the following: (a) Isolated chloroplasts use the energy of visible light for converting $\mathrm{CO}_{2}$ to sugars and starch with a simultaneous evolution of oxygen. (b) Isolated chloroplasts use light energy for a simultaneous reduction of TPN, formation of ATP, and evolution of oxygen (non-cyclic photophosphorylation). (c) Isolated chloroplasts and bacterial chromatophores use light energy for an anaerobic formation of ATP as the sole product of the light reaction (cyclic photophosphorylation). (d) By fractionating chloroplasts and Chromatium cells, the light-dependent phase of photosynthesis has been separated, in time and space, from the dark phase that is concerned with carbon assimilation. Variants of these reactions and the supporting evidence have been discussed above.

In closing, I wish to borrow these words from Priestley, which accurately express the spirit in which this survey was undertaken. "I have been most particularly careful to distinguish facts from hypothesis; and whenever I have occasionally attempted anything of this latter kind, it has been with diffidence, and in such a manner as it would give me no pain to relinquish my own opinions, and adopt those of any other person that should appear to me more naturally to arise from the lacts" (122).

\section{REFERENCES}

I. Allen, M. B., Arnon, D. I., Capindale, J. B., Whatley, F. R., and Durham, L. J., J. Am. Chem. Soc., 77, 4149-4155 (1955).

2. Allen, M. B., Whatley, F. R., and Arnou, D. I., Biochim. et Biophys. Acta, 27, $16.23(1958)$.

3. Auderson, 1. C., and Fuller, R. C., Arrh. Biochem. Biophys., 76, 168-179 (1958).

4. Anold, W., and Clayion, R. K., Proc. Nat. Acod. Sci. Ü. S., 46, 769-776 (1960).

5. Aruon, D. I., Nature, 167, 1008-1010 (1951).

6. Arnon, D. I., paper presented at the Cell Symposum, American Association for the Advancement of Science, Berkeley Meeting (1954); Science, 122 , $9-16$ (1955).

7. Arnon, D. I., Ann. Rev. Plant Physiol., 7, 325-354 (1956).

8. Aruon, D. I., in Enzymes: Units of Biological Structure and Function. (O. H. Gacbler, ed.), pp. 279-313, Acarlemic Press. New York (1956).

9. Arnon, D. I., Nature, 184, 10-21 (1959).

10. Arnon, D. I., in Handbuch der Pflanzenphysiologie, Vol. 5, (A. Pirson, ed.). pp. 773-829, Springer, Heidelberg (1960). 
11. Imon, I). I., Alien, M. B., and Whatley, I. R., Noture, 174, 394-396 (1951).

12. Amon, 1). 1., Allen, M. B., and Whatley, I. R., Buchim. et Biophys. Acta, 20, $119-461(1956)$.

13. Arnon, 1). 1., Nllen, M. B., Whatley, I. R., Capindale, J. B., and Rosen. berg, L. L., Proc. Inlern. Congr. Biochem., 3rd Congr., (Brussels), 227-232, Academic l'ress, N. Y. (195.)).

14. Arnon, D. 1.. and Whatley, I. R., Science, 110, 5.54-556 (1949).

15. Arnon, D. 1., Whatley, F. R., and Allen, M. B., J. Am. Chem. Soc., 76, 6.324$6329(1954)$.

16. Arnon, 1). 1., Whatley, F. R., and Allen, M. B., Biochim. et Biophys. Acta, $16,607-608$ (1955).

17. Amon, D. I., Whatley, F. R., and Allen, M. B., Nature, 180, 182-185 (1957).

18. Amon, 1). I. Whatley, I. R., and Allen, M. B., Science, 127, 1026-[034 (1958).

I8a. Imon, 1). I., Whatley, F. R., and Allen, M. B., Biochim. el Biophys. Acta, 32, $47-57$ (1959).

19. Aronoff, S., and Calvin, M., Plant Physiol., 23, 351-358 (1948).

20. Aubert, J. P., Milhaud, G., and Millet, J., Ann. inst. Pasteur, 92, 515-528 (1957).

2I. Aron, M., and Jagendorf, A. T., Nature, 179, 428-429 (1957).

22. Arron, M., Jagendorf, A. T., and Evans, M., Biochim. et Biophys. Acta, 26, $262-269(1957)$.

23. Bartsch, R. G., and Kamen, M. D., J. Biol. Chem., 235, 825-831 (1960).

24. Bassham, J. A., Benson, A. A., Kay, L. D., Harris, A. Z.. Wilson, A. T., and Calvin, M., J. Am. Chem. Soc., 76, 1760-1770 (1954).

25. Bassham, J. A., and Calvin, M., Photosynthesis, in Currents in Biochemical Research (1). E. Green, ed.), Interscience Publishers, New York (1956).

26. Benson, A. A., and Calvin, M., Ann. Rev. Plant Physiol., 1, 25-42 (1950).

27. Bishop, N. I., Proc. Nat. Acad. Sci. U. S., 44, 501-504 (1958).

28. Bishop, N. I., Proc. Nat. Acad. Sci. U. S., 45, 1696-1702 (1959).

29. Boltzmann, L., "Der zweite Hauptsatz der mechanischen Wärmetheorie" (1886), Essay in Populäre Schriften, p. 39, Barth, Leipzig (I905).

30. Bové, J., Bové, C., Whatley, F. R., and Arnon, D. 1., presented by D. I. Arnon at Intern. Bot. Congr., 9th Congr., Montreal, Canada (Angust, 1959).

31. Boychenko, E. A., and Baranov, V. I., Doklady Akad. Nauk S.S.S.R., 95, 10251027 (1954); Chem. Abstr., 48, 8881 (1954).

32. Bregoff, H. M., and Kamen, M. D., J. Bacteriol., 63, 147-149 (1952).

33. Brown, A. H., and Franck, J., Arch. Biochem., 16, 55-60 (1948).

34. Broyer, T. C., Carlton, A. B., Johnson, C. M., and Stout, P. R., Plant Physiol., 29, 526-532 (1954).

35. Calvin, M., Proc. Intern. Congr. Biochem., 3rd Congr. Brussels, p. 211-225, Academic P'ress, Inc., New York (1956).

36. Calvin, M., Ret. Modem Phys., 31, 147-156 (1959); also in Biophysical Science -A Study Program (J. L. Oncley, ed.), 147-156, John Wiley and Sons, New York (1959).

36a. Chance, B., and Nishimura, M., Proc. Nat. Acad. Sci. U. S., 46, 19-24 (1960).

37. Chow, C. T., and Vennesland, B., Plant Physiol., 32 (Supp.), iv (1957).

38. Clark, V. M., Kirby, G. W., and Todd, A., Nature, 181, 1650-1652 (1958).

39. Crane, F. L., Plant Physiol., 34, 128-13I (1959).

40. Dam, H., Hjorth, E., and Kruse, 1., Physiol. Plantarum, 1, 379-381 (1948).

41. Davenport, H. E., Biochem. J., 73, $45 \mathrm{P}$ (1959).

42. Darenport, H. E., and Hill, R., Proc. Roy. Soc. (London) B, 139, 327-345 (1952).

43. Duysens, L. N. M., Nature, 173, 692 (1954).

44. Duysens, L. N. M., Science, 121, 210-211 (1955).

45. Fmerson, R. L., Stanffer, J. F., and Umbreit, W. W., Am. J. Botany, 31, 107. 120 (1944). 
46. Engelmann, Th. W., Arch. ges. Physiol. (Pflüger's), 30, 95-124 (1883).

47. Engelmann, Th. W., Bolan. Zig., 46, $661 \mathrm{ff}$ (1888).

48. Esau, K., Plant Anatomy, J. W'iley and Soms, New York (1953).

48a Frenkel, A. W., Arch. Biochem. Biophys., 38, 219-230 (1952).

49. Frenkel, A. W., J. Am. Chem. Soc., 76, 5568-5569 (1954).

50. Frenkel, A. W., J. Biol. Chem., 222, 823-834 (19:6).

51. Frenkel, A. W., Brookhaven Symposia in Biol., 11. 276-288 (19:58).

52. Frenkel, A. W., and Lewin, R.. Am. J. Bot., 41, 586-589 (1954).

53. Fuller, R. C., and Gibbs, M., Plant Physiol., 34. $324-329$ (19:9).

54. Gaffron, H., Nature, 143, 204-205 (1939).

55. Gaffron, H., Biol. Revs., 19, 1-20 (1944).

56. Gaffron, H., in Autotrophic Microorganisms (B. A. Fry and J. L. l'eel, eds.), p. 163, Cambriclge University Press (1954).

57. Gaffron, H. (cd.), Research in Photosynthesis, Interscience Publishers, Ncw York (1957).

58. Gaffron, H., and Rubin, J., J. Gen. Physiol., 26, 219-240 (1942).

59. Geller, D. M., Doctoral Diss., Div. Med. Sci., Harvard University (1957).

60. Geller, D. M., and Gregory, J. S., Federation Proc., 15, 260 (1956).

61. Gest, H., Proc. Intem. Symposium Enz. Chem., Tokyo and Kyoto, 250-256 (1957).

62. Crest, H., Judis, J., and Peck, H. D., in Inorganic Nitrogen Metabolism (W. D. McElroy and B. Glass, eds.), p. 310, The Johns Hopkins University Press, Baltimore (1956).

63. Gest, H., and Kamen, M. D., J. Bacteriol., 58, 239-245 (1949).

64. Gest, H., Kamen, M. D., and Bregoff, H. M., J. Biol. Chem., 182, 153-170 (1950).

65. Gibbs, M., and Calo, N., Plant Pltysiol., 34, 318-323 (1959).

66. Gibbs, M., and Cynkin, M. A., Nature, 182. 1241-1242 (1958).

67. Hansen, A., Alb. Botan. Inst. Hïrzburg, 2, 537-626 (1882).

68. Harrison, K., Nature, 181, 1131 (1958).

69. Heath, O. V. S., and Orchard, B., Nature, 180, 180-181 (1957).

70. Hill, R., Symposia Soc. Exptl. Biol., 5, 223-231 (1951).

71. Hill, R., Proc. Intern. Congr. Biochem., 3rd Congr., Brussels, 1955, pp. 22:)227, Academic Press, New York (1956).

7la. Holt, A. S., and lirench, C. S., Arch. Binchem., 9, 25-43 (1946).

72. Ingenhousz, J., Essay on the food of plants and the renovation of soils, London (1796).

73. Irmak, L. R., Rev. fac. sci. univ. Istanbul, Sir. 13, 20, 237-243 (1955).

74. Jagendorf, A. T., Brookhaven Symposia in Biol., 11, $236-258$ (1958).

75. Jagendorf, A. T., pers. commun.

76. Jagendorf, A. T., and Arron, M., J. Biol. Chem., 231, 277-290 (1958).

77. Jagendorf, A. T., and Avron, M., Arch. Biochem. Biophys., 80, $246-257$ (1959).

78. Jakolsy, E. B., Brummond, D. O., and Ochoa, S., J. Biol. Chem., 218, 811 $822(1956)$.

79. James, W. O., and Das, V. S., Neu Phylologist, 56. 325-313 (19:37).

80. James, W. O.. and Leach, M. R., Endenzour, 19, 108-11+ (1960).

81. Kamen, M., and Newton, J. W., Biochim. et biophys. Acla. 25, 462.474 (1957).

82. Karunairatnam, M. C.. and Gest, I1., Abstracts, Th Intern. Congr. Microbiol. pp. 74-75, Nmquist and Wiksells, Uppsala (1958).

83. Kluyver, A. J., and van Niel, C. B., in The Microbe's Conlribution to Biology, p. 71, Harvard University P'ress, Cambridge, Mass. (1956).

81. Korkes, S., J. Biol. Chem., 216. $737-748$ (195.5).

85. Kornberg. A., Srience, 131, 1503-1508 (1960).

86. Kugmuan, 1). W', and Vennesland, B.. J. Biol. Chem., 234, 2205-2210 (1960).

87. I.eluerlev, A., Biochem. \%., 7, 1-10 (1907). 


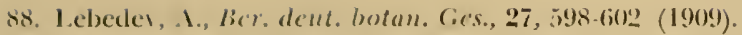

89. Lebedev, A. 1... Izz'est. Donskoz'o Gosudarst. ('niv'., 3, 25.55 (1921); Iranslated in Am. Rer. Soviet Medicine, 5, 15-27 (1918).

90. Lehninger, A. L., in The Harrey Lectures, 49, $176-216$ (1955).

91. Lewis, G. .1., and Lipkin, D., J. Am. Chem. Soc., 64, 2801-2808 (1912).

92. Lipmann, F., Adv. Lnzymol., 1, 99-162 (19.11).

93. Livingston, R., in Handbuch der P/lanzenphysiologie, Vol. 5, (A. Pirson, ed.), p. 832. Springer, Heidelberg (1960).

91. Losada, M., Nozaki, M., and Mrnon, D. 1. (unpub.).

95. Losada, M., Nozaki, M., and Arnon, D. I. (this symposium).

96. Losada, M., Trebst, . I. V'., and Arnon, D. I., J. Biol. Chem., 235, 832-839 (1960).

97. Losada, MI., Trebst, A. I., Ogata, S., and Arnon, D. I., Nature, 186, 753-760 (1960).

98. Lumry, R., Spikes, J. D., and Eyring, H., Anm. Rev. Plant Plyysiol., 5, 271-340 (1954).

99. Lundegardh, H., Physiol. Plantarum, 7, 375-382 (1954).

100. Naclachlan, G. A., and Porter, H. K., Proc. Roy. Soc. (London) B, 150, 460473 (1959).

101. Marrè, K., and Servettaz, O., Arch. Biochem. Biophys., 75, 309 (1958).

102. Martin, G., and Lavollay, J., Experientia (Basel), 14, 333 (1958).

103. Martius, C., Biochem. Z., 326, 26-27 (1954).

104. Martius, C., Proc. Intern. Congr. Biochem., 3rd Congr. Brussels, 1-9, Academic Press, N. Y. (1955).

105. Massey, V., Biochim. et Biophys. Acta, 34, 255-256 (1959).

106. Mayer, J. R., Die Organische Bewegung (1845), Essay in Mechanik der Wärme, p. 54, J. G. Cotta, Stuttgart (1874).

107. Miller, S. L., and Urey, H. C., Science, 130. 245-251 (1959).

108. Mühlethaler, K., and Frey-Wyssling, A. J., Biophys. Biochem. Cytol., 6, 507512 (1959).

109. Nüller, F. M., Ark. Mikrobiol., 4, 131-166 (1933).

110. Müller, H. R., Steere, R. L., and Arnon, D. I. (unpub.).

111. Nakamoto, T., Krogmann, D. W., and Vennesland, B., J. Biol. Chem., 234, 2783-2788 (1959).

112. Nakamoto, T., and V'ennesland, B., Federation Proc., 19, 329 (1960).

113. Newton, J. W., and Wilson, P. W., Antonie van Leeuwenhoek, J. Microbiol. Serol., 19, 71-77 (1953).

114. Nozaki, M., Ogata, M., and Amon, D. L., reported by D. I. Arnon at Syn1posium on Comparative Biochemistry of Photoreactive l'igments, Pacific Grove, Calif., Sept. 1959.

115. Ogata, S., Nozaki, M., and Arnon, D. L., reported by D. I. Arnon at Symposium on Comparative Biochemistry of Photoreactive Pigments, Pacific Grove, Calif., Sept. 1959.

116. Olson, J. M., and Chance, B., Biochim. et Biophys. Acta, 28, 227-228 (1959).

117. Oparin, A. 1., The Origin of Life on The Earth, 3rd Ed., Academic Press, New York (195i).

118. Peck, H. D., and Gest, H., Biochim. et Bioply's. Acta, 15, 587-588 (1954).

119. Peck, H. D., and Gest, H., J. Bacteriol., 71, 70-80 (1956).

120. Petrack, B., Federation Proc., 18, 302 (1959).

121. Pfeffer, W., Pliysiology of Plants, Clarendon Press, Oxford (1900).

122. Priestley, J., Experiments and Observations on Different Kinds of Air, Vol. III, J. Johnson, London (17\% $i$ ).

122a.Prince, M. B. J., Appl. Physics, 26, 534-540 (1955).

123. Quayle, J. R., Fuller. R. G., Benson, A. A., and Calvin, M., J. Ain. Chem. Soc., 76,3610 (1954). 
124. Rabinowitch, E. 1., Photosynthesis and Related Processes, Vol. 1, Interscience Publishers, New York (1945).

125. Rabinowitch, E. I., Anu. Rer. Plant Physiol., 3. 229-264 (1952).

126. Racker, E., Nature, 175, 249-251 (1955).

127. Racker, E., Arch. Biochem. and Biophys., 69, 300-310 (1957).

128. Racker, E. (pers. commun.).

129. Roelfsen, P. A., Proc. Koninkl. Ned. Akad. Welenschap., 37, 660-669 (1934).

130. Ruben, S., J. Am. Chem. Soc., 65, 279-282 (1943).

131. Sachs, J., Lectures on the physiology of plants, Clarendon Press. Oxford (1887).

132. San Pietro, A., and Lang, H. M., Science, 124, 118-119 (1956).

133. San Pietro, A., and Lang, H. M., J. Biol. Chem., 231, 211-229 (1958).

134. Sebrell, W. H., and Harris, R. S., The V'ilamins, 3 vols., Academic Press, New York (1954).

135. Siegel, J. M., and Kamen, M. D., J. Bacteriol., 61, 215-228 (1951).

136. Smillie, R. M., and Fuller, R. C., Plant Physiol., 34, 651-656 (1959).

137. Smillie, R. M., and Krotkov, G., Canad. J. Bot., 37, 1217-1225 (1959).

138. Smith, L., and Baltscheflsky, M., J. Biol. Chem., 234, 1575-1579 (1959).

139. Smith, L., and Ramirez, J., Brookhaten Symposia in Biol., 11, 310-315 (1958).

140. Stalfelt, M. G., Physiol. Plantarum, 8, 572-593 (1955).

141. Stanier, R. Y., Doudoroff, M., Kunisawa, R., and Contopoulou, R., Proc. Natl. Acad. Sci. U.S., 45, 1246-I260 (1959).

142. Steere, R. L., J. Biophys. Biochem. Cytol., 3, 45-60 (1957).

143. Strehler, B. L., Arch. Biochem. and Biophys., 43, 67-79 (1953).

144. Tamiya, N. Kondo, Y., Kameyama, T., and Akabori, S., J. Biochem., (Japan). 42, 613-614 (1955).

145. Thimann, K. V., Srience, 88, 506-507 (1938).

146. Thomas, J. B., and Haans, A. M. J., Biochim. et Biophys. Acta, 18, 286-288 (1955).

147. Thomas, J. B., Haans, A. J. M., and Van der Leun, A. A., Biochim. et Biophys. Acta, 25, 453-462 (1957).

I47a. Thomas, J. B., Minnaert, K., and Elbers, P. F., Acta Botan. Neer., 5, 315-321 (1956).

148. Tolbert, N. E., Brookhaven Symposia in Biol., 11, 271-275 (19.78).

149. Tolmach, L. J., Nature, 167, 946-948 (1951).

150. Treadwell, F. P., and Hall, W. T., Analytical Chemistry, Vol. II, p. 665, 7th ed., John Wiley and Sons, New York (1930).

151. Trebst, A. V., Iosadla, M., and Amon, D. I., J. Biol. Chem., 234. 3055-3058 (1959).

152. Trebst, A. V., Losada, M., and Arnon, D. 1., J. Biol. Chem., 235, 840-844 (1960).

153. Trebst, A. V., Tsujimoto, H. Y., and Arnon, 1). I., Nature, 182, 351-355 (1958).

134. Trenner, N. R., Arison, B. H., Erickson, R. R., Shunk, C. H., Wolf, D. E., and Folkers, K., J. Am. Chem. Soc., 81, 2026 (1959).

155. Trudinger, 1'. A., Biochem. J., 64, 274-286 (1956).

156. Tsujimoto, H. Y., Hall, D. O., and Arnon, D. I. (impub.).

157. Ueda, R., Botan. Mag. (Tokyo), 62, 731-732 (1919).

158. Van Niel, C. B., Arch. Microbiol., 3, 1-112 (1931).

159. Van Niel, C. B., in Photosynthesis in Plants (J. Franck and Wr. E. Loomis. eds.), pp. 437-495. lowa State College Press (1949).

160. Vernon, L. l'., and Ish, O. K., J. Biol. Chem., 234, 1878-1882 (1959).

161. Vishniac, W'., Horecker, 13. I... and Ochoa, S.. Adv. in Enzymol., 19. 1-77 (1957).

162. Vishniac. W., and Ochoa. S.. Nature, 167. 768-769 (19.51).

163. Vishniac, W., and Ochoa, S., J. Biol. Chem.. 198, $501-506$ (1952). 
164. Waburg, (O., in Heary Metal Prosthetic Ciroups and Enzyme Action, pp. 213 214, Clarendon Press, Oxford (1949).

16.5. Warburg, O., Krippahl, G., Gewitz, 11. S., and V'olker, W. Z., Naturforsch., 14b, $712.724(1959)$.

166. Weissbach, A., Horecker, B. L., and Hurwitz, J., J. Biol. Chem., 218, 795-810 (1956).

167. Wessels, J. S. C., Rec. trat. chim., 73, 529-536 (1954).

168. Wesscls, J. S. C., Biochim. et Biophys. Acta, 25, 97-100 (1957).

169. Wessels, J. S. C., Biochim. et Biophiys. Actu, 29, $113-123$ (1958).

170. Whatley, F. R., Allen, M. B., and Arnon, D. I., Biochim. et Biophys. Acta, 16, 605-606 (1955).

171. Whatley, F. R., Allen, M. B., and Arnon, D. I., Plant Physiol., 32, (Supp.) iii $(1957)$.

172. Whatley, F. R., Allen, M. B., and Amon, D. I., Biochim. et Biophys. Acta, 32. $32-46(1959)$.

173. Whatley, F. R., Allen, M. B., Rosenberg, L. L., Capindale, J. B., and Arnon, D. I., Biochim. et Biophys. Acta, 20, $462-468$ (1956).

174. Whatley, F. R., Allen, M. B., Trebst, A. V., and Arnon, D. 1., Plant Physiol., 33, (Supp.) xxvii-xxviii (1958).

175. Whatley, F. R., Allen, M. B., Trebst, A. V., and Arnon, D. I., Plant Physiol., $35,188-193$ (1960).

176. Whittingham, C. P., Biol. Rev, 30, 40-64 (1955).

177. Williams, A. M., Biochim. et Biophys. Acta, 19, 570 (1956).

178. Winogradsky, S., Botan. Ztg., 45, 489 ff. (1887).

179. Winogradsky, S., Zur Morphologie und Physiologie der Schwefelbacterien, Arthur Felix, Leipzig (1888).

\section{DISCUSSION}

Dr. FRENKEL: If Chromatium can produce hydrogen in the light, its cellfree extracts should be capable of photoreducing pyridine nucleotides comparable to isolated chloroplasts or to chromatophores from Rhodospirillum rubrum. I cannot see that the mechanism for the production of photoreductant and photo-oxidant is necessarily different in bacteria and in higher plants.

Dr. ARNoN: First of all, let me say that we have observed in cell-free Chromatium preparations reduction of pyridine nucleotides with succinate and light, similar to the one you have reported with Rhodospirillum rubrum. The difference between plants and photosynthetic bacteria is this. Bacteria, for example, can use succinate to reduce pyridine nucleotide and as a result fumarate is formed. However, green plants use water and form reduced pyridine nucleotide and oxygen. That is the difference. Bacteria, in our view, do not use water as a reductant.

DR. FRENKEL: Yes, but in your earlier statement you implied that in higher plants you have photolysis of water, whereas in bacteria you have some kind of mysterious process in which high energy phosphate is formed which does not involve photolysis. You stated that this is the big difference. You stated definitely that photolysis is not occurring in bacteria.

Dr. ArvoN: I might plead guilty to an even greater heresy and say that photolysis of water does not even apply to green plants. I think photolysis 
of water has been one of the most fruitful theories in biology for a very important reason: it has led to experiments. It has guided the work on photosynthesis for the last 20 years, including specifically our own research up to the last year. Now we have gotten awaty from this hypothesis. Because of our results with Chromatium and with green plants, we now visualize the primary photochemical act not as a photolysis of water, where we split water to an $\mathrm{H}$ and $\mathrm{OH}$ radical, but rather as the formation of an oxidized cytochrome. Instead of photolysis we have an ionization of water as we have described in our scheme published in Nature (5). We use the proton from the water, not the hydrogen atom, and we use the $\mathrm{OH}^{-}$. By a reaction which is really an analog of the anode reaction, we take the electron from the $\mathrm{OH}^{-}$, to a cytochrome to the chlorophyll to the pyridine nucleotide, with a concomitant release of oxygen.

Dr. ForTI: I think that Dr. Vishniac reported recently that the hydrogen is incorporated from water into the chlorophyll molecules. I wonder if Dr. Arnon or Dr. Vishniac would comment on that. How would this fit with Dr. Arnon's ideas about splitting of water?

Dr. ArNon: I would like to defer this question to Dr. Vishniac.

Dr. Vishniac: Would you repeit that question, please?

DR. Forti: Well, I know that you reported very recently that tritium is incorporated from tritiated water into chlorophyll. It seems to me that this would really imply a splitting of water.

Dr. Visiniac: No, I don't think so. All I can say is that in the light we get incorporation of tritium from water into cllorophyll $a$. This need not be a direct incorporation of tritium, it could be a secondary elfect of electron transport.

DR. FORT1: What about the subsequent transfer of tritium from chlorophyll, also reportecl.

Dr. Visminac: This is more complicated than it might appear. I would rather not cliscuss it right now.

Dr. Jagindorr: 1 was a little bit confused about the ratio between the anacrobic and oxygen-requiring phosphorylation, which you find as 2.2. Can't you alter that ratio quite at will by just varying the concentration of cofactors?

1)R. ArNon: The concentrations of cofactors in these experiments were selected so that they were optimum for each type of reaction. That is, the optimum concentration for the anaerobic type and the oxygen-catalyzed type that Dr. Vennesland and you have studied.

DR. JAGENDORF: How do you define the borderline between the two?

Dr. BiSHOP: 1 have found no vitamin $\mathrm{K}$ at all in chloroplasts of spinach, sugar bects or in Swiss chard. My techniques are perhaps not as definitive as those of Dr. Zill and he also has not detected vitamin $K$ in chloroplasts. Also in photosynthetic bacteria, including Chromatium, I have not observed 
bitumin $\mathrm{K}$ but rather an appreciable amount of Coemryme Q272. Now, would you connent on this?

Dr. ArNon: Yes, als I said at the beginning, what we need there is a quinone. Now we satid that it was vitamin $K$, because we accepted the work of Dr. Dim. He funds vitamin $K$ specifically concentrated in the chloroplasts. If it turns out that plastoquinone gives the same effect as vitamin $K$ we will accept that result without altering our scheme in any essential respect.

DR. Thomas: I would like to apologize to Dr. Arnon with regard to measurement of oxygen evolution in isolated chloroplasts. In a paper I mentioned that Dr. Arnon measured photosynthetic oxygen evolution as a Hill reaction. This has been done in many cases and I must confess that I dicl not read the section on methods too well. For in the second paper, I think, in the section on methods he measured oxygen evolution in photosynthesis. The point I made is that Dr. Arnon stated that oxygen evolution in the Hill reaction is the same as oxygen evolution in photosynthesis. Now in some work as we did in Utrecht we tried to find oxygen evolution by isolated chloroplasts in photosynthesis, not in the Hill reaction. In the paper describing this study, we claimed to be the first to find a true photosynthetic oxygen evolution by chloroplast suspensions. As the misinterpretation of Dr. Arnon's results was brought to our attention after publication of our paper, I am glad to find an opportunity here to state that, contrary to our previous remark, Dr. Arnon and coworkers were the first to obtain photosynthetic oxygen evolution with isolated chloroplasts.

DR. Кок: I an rather impressed by this idea of Chromatium making only ATP in the light, using hydrogen as the reductant. But I was so brought up in van Niel's thinking that it is hard to digest. If you only need light to form ATP and not to form the reductant, why do you need so many quanta? Why is this process so inefficient? You would think that one quantum per $\mathrm{CO}_{2}$ would be enough to work.

Dr. ARNoN: I am not prepared to discuss quantum efficiency in photosynthesis from our own data. But if I an asked to speculate I would say that the minimum that we would need, according to our scheme, for green plants, bașed on our work with cell-free systems, is about 4 and a fraction quanta. Let me make another point that 1 forgot to mention. This Chromatium photosynthesis with hydrogen is, 1 believe, nothing else than Dr. Gaffron's photoreduction in hydrogen-adipted algae, where he simply forced the algae to take a march back in evolution $\mathrm{X}$ million years, and in this way they have learned again to live as they used to in the old days, reducing pyridine mucleoticle in the clark and using light only for ATP formation.

Now as far as TPN reduction in chloroplasts is concerned we need at least two quanta to reduce 1 TPN. If we get the electron from $\mathrm{OH}^{-}$to the cytochrome-chlorophyll complex and then to TPN, we would need two of 
these electrons to reduce 1 TPN. Since we need four hydrogens to reduce one molecule of $\mathrm{CO}_{2}$, we would therefore need 4 quanta to get 2 TPNH. Thus we arrive at a minimum of 4 quanta. In Chromatium the problem doesn't arise, if one uses hydrogen gas. What remains then is to use light energy only for making ATP.

Dr. VAN Niel: But I thought that the point which Dr. Kok wanted to make is exactly that it makes it very unintelligible.

Dr. Arnon: Then I don't understand the question.

Dr. VAN Niel: If you make ATP with light energy, a single quantum will produce enough ATP to carry on a lot of photosynthesis.

Dr. ArNon: In the non-cyclic photophosphorylation in chloroplasts we get one phosphorylation per two electrons transferred. This is the stoichiometry which we observe with ferricyanide. Now two einsteins at a wave length of red light give roughly about 80 kilocalories. So from 80 kilocalories we can theoretically get 6 ATP's using 12 kilocalories as the value for making 1 ATP at a physiological $p \mathrm{H}$. This figure of 12 seems to go up and down like the stock market. According to Dr. H. A. Krebs, under physiological conditions 12 kilocalories is still the figure to use. In mitochondrial systems one gets 3 ATP's formed in the transfer of two electrons from DPNH to oxygen. So, if an analogy to oxidlative phosphorylation is allowed, with 2 quanta of red light we would also expect to get only 3 ATP's. Photosynthetic bacteria use quanta of even longer wavelengths than green plants so that they receive even less energy, and therefore the efficiency would come out even better. If one gets 3 ATP's recuiring 36 kilocalories from two quanta of light supplying 80 kilocalories, the efficiency is over $50 \%$. Using light at longer wavelengths, the efficiency is even better. As a historical sidelight, let us not forget that when oxidative phosphorylation was first discovered, experimentally, only slightly better than 1 ATP per two electrons was observed and it took about 10 or 20 years to get up to 3 .

Dr. LipmanN: I would like to say that no one knows the quantum yield of phosphorylation. I think these chloroplast preparations vary in their photophosphorylation capacity, because they may be damaged. Therefore one cannot get the true quantum yield from photophosphorylation from chloroplasts. Many of the reactions can go on in chloroplasts which are not capable of giving good photophosphorylation. Therefore, I do not think it is a good idea to juclge quantum yields now.

Dr. KoK: One should recall the work of Wassink et al. (Wassink, E. C., E. Katz and R. Dorrestein, Enzymologia 10: 285, 1942) with Chromatium, which showed equal quantum requirements $\left(10 \mathrm{~h} / \mathrm{CO}_{z}\right)$, with either hydrogen or thiosulfate as the reductant (this was later confirmed by Larsen [Larsen, H., C. S. Yocum and C. B. van Niel, J. Gen. Physiol. 36: 361, 1952] with Chlorobium). P'ossibly even more striking was that the bacteria showed no preference for hydrogen compared to thiosulfate when both were fed simultancously. Hardly any light energy is required to reduce $\mathrm{CO}_{2}$ 
with molecular hydrogen. But thiosulfate puts a demand of some $70 \mathrm{kcal}$ per mole $\mathrm{CO}$, fixed, a figure pretty close to the $112 \mathrm{kcal}$ required if water is the reductant as in icrobic photosynthesis. Chromatium therefore must have a photosynthetic machinery about as potent as the one of green plants. In the peculiar case of feeding with hydrogen or other "easy" donors, this big motor must be idling or short circuited, since only the ATP generated by, let's say, the fuel pump is required for the job.

Dr. ArNoN: Well, I think we can only answer this question by discussing the $\mathrm{CO}_{2}$ fixation, which we cannot do here now. Let me summarize in a nut shell the reason why we thought that if in Chromatium all that light does is make ATP, it should then be possible to replace light by exogenous ATP in the dark. Unlike Rhodospirillum rubrum we have no alternatives here. Rhodospirillum rubrum but not Chromatium can also have oxidative metabolism. The experimental evidence is that in a cell-free Chromatium system light is replaceable by ATP. The products of $\mathrm{CO}_{2}$ or acetate assimilation are exactly the same in both cases.

Dr. Kok: You agree, though, that this does not prove that this is all the light is doing.

Dr. ARNoN: I an now at the point where I feel I do not wish to go beyond these data which we have. I will leave everything else to future evidence.

Dr. FRENKEL: I believe this question can be handled experimentally in the Rhodospirillum system where you can reduce DPN in the breakdown of succinate. If Dr. Arnon's mechanism is correct, you should be able to start with labeled succinate, and you might be able to get the incorporation of hydrogen into DPN. If the data are positive, it would strongly suggest the Krasnowsky reaction of sensitized reduction. If it is negative, you can't tell anything.

Dr. Strehler: I also have the impression that you are retreating a little from the idea you expressed-that the only function of light in the photosynthetic bacteria is to produce ATP-since you later said that possibly the hydrogen from succinate went down to chlorophyll and then was kicked up to TPN. Wouldn't it to more parsimonious to assume (as has been suggested earlier) that the ATP that you generate in the light is used to drive the electrons from the potential of succinate up to that of TPN?

Dr. McElroy: Dr. Petrack, have you looked at the fluorescence?

Dr. Petrack: No. 


\title{
PHOTOPRODUCTION OF MOLECULAR HYDROGEN FROM THIOSULFATE BY CHROMATIUM CELLS
}

\author{
M. Losada, M. Nozaki, and Daniel I. Arion \\ Laboratory of Cell Physiology \\ University of California, Berkeley, California
}

Photoproduction of molecular hydrogen by photosynthetic cells was first observed by Gaffron and Rubin in the green alga Scenedesmus (5) and by Gest and Kamen in photosynthetic bacteria $(7,8)$. These findings were confirmed and extended in other laboratories $(4,9)$.

The evolution of hydrogen was attributed either to a decomposition of a dicarboxylic acid (9) or to a photolysis of water into $(\mathrm{H})$ and $(\mathrm{OH})(5,6)$. In the latter proposal, it was suggested that $(\mathrm{H})$ is eventually liberated, with the aid of hydrogenase, as molecular hydrogen, whereas the $(\mathrm{OH})$ is reduced back to water, by reacting in the case of algae with endogenous lydrogen donors (5), and in the case of photosynthetic bacteria with exogenous hydrogen clonors (6). Photosynthetic bacteria evolve hydrogen in light only in the presence of an exogenous hydrogen donor $(7,8,10)$.

The endogenous electron donors for algae were not chemically identified (5). The exogenous electron donors for photosynthetic bacteria were organic acids, mainly malate $(7,8,9,10)$. There was no evidence that thiosulfate or any other physiologically suitable inorganic electron clonor was compatible with photoproduction of hydrogen (3) .

From the standpoint of the electron flow theory of photosynthesis, which exchudes a photolysis of water $(1,2)$, the photoproduction of hydrogen could be viewed as a reduction of protons by a hydrogenase with the aid of electrons, "expelled" by excited chlorophyll molecules. The electrons come to the excited chlorophyll via cytochromes from the external electron clonors and are raised to the reducing potential of molecular hydrogen during the primary photochemical act (1, 2).

If this interpretation was correct, then photoproduction of hydrogen by photosynthetic bacteria should occur not only at the expense of organic electron donors but also at the expense of physiologically 
suitable inorganic electron clonors, and the latter should be capable of reducing the bacterial cytochromes that are oxidized by light in the course of photosynthesis (2). An investigation of photoproduction of hydrogen from thiosulfate by Chromatium was undertaken to test this prediction.

As shown in Fig. 1, in the light but not in the dark, gas was evolved by Chromatimu cells in the presence of thiosulfate. No $\mathrm{CO}_{2}$ source was supplied and $\mathrm{KOH}$ was present in the reaction vessel. Gas evolution ceased when light was turned off and resumed when light was tumed on again. The evolved gas was identified as hyclrogen (Table 1) by adsorption on palladium asbestos (11).

The evolution of hydrogen by illuminated Chromatium cells showed a marked $p \mathrm{H}$ dependence (Fig. 2). The reaction was most vigorous at the more acid $p \mathrm{H}$. Little hydrogen was evolved at $p \mathrm{H}$ 8.0.

Similar to the inhibition of hydrogen evolution in the presence of organic hychrogen clonors $(7,9)$, photoproduction of hydrogen from

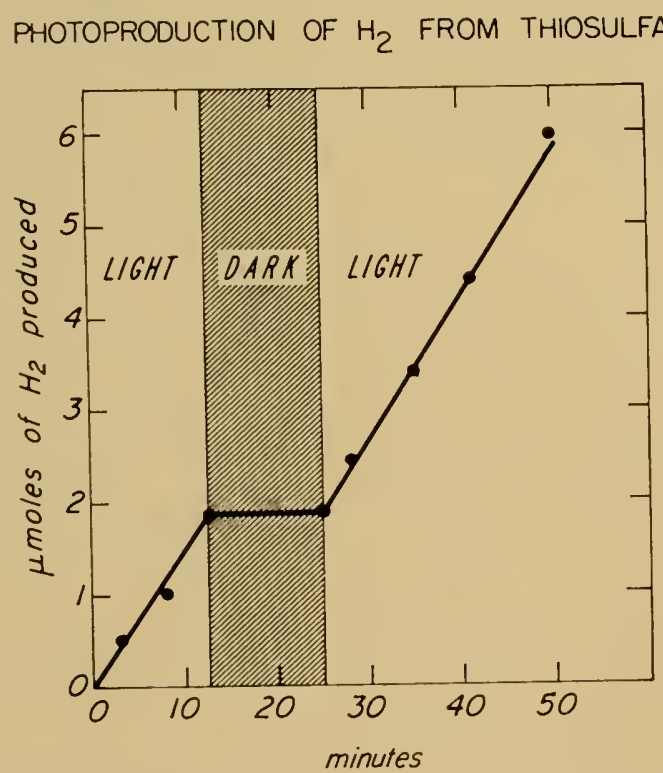

Fig. 1. Light-dependent evolution of hydrogen from thiosulfate by Chromatium cells. The reaction mixture included $0.1 \mathrm{~g}$ of washed cells, suspended in $2.6 \mathrm{ml}$ of a modified nutrient solution from which nitrogen was omitted, $0.3 \mathrm{ml}$ of 0.5 $M$ Tris buffer, $p \mathrm{H} 7.2$, and $0.1 \mathrm{ml}$ of $0.2 M$ sodium thiosulfate. $0.1 \mathrm{ml}$ of $20 \%$ $\mathrm{KOH}$ was placed in the center well of the Warburg manometer flasks. The reaction was run at $30^{\circ} \mathrm{C}$. Gas phase, argon. Illumination, 50,000 Lux. 
TABLE I

Adsorpition on Palladium Asbestos of Hidrogen, Photochemically Evolved BY Chromatium Cells From THIOSUlfate

$\mu$ moles $\mathrm{H}_{2}$ evolved

\section{Control \\ Palladium asbestos added}

7.2

0.6

Experimental conditions as in Fig. 1 except that no Tris buffer was added and $50 \mu$ moles thiosulfate were used. $100 \mathrm{mg}$ palladium asbestos (11) were placed in the sidearm of the Warburg manometer flask. Reaction time, 2 hours.

thiosulfate was also inhibited by molecular nitrogen and ammonium ions. The results are shown in Fig. 3.

The photoproduction of hydrogen was dependent on the concentration of thiosulfate and was abolished by heating the cells (Fig. 4). Growing Chromatium cells were found, by analysis, to have oxidized in four days 27 millimoles of added thiosulfate into 54 millimoles of sulfate. During this period the appearance of the

\section{PHOTOPRODUCTION OF $\mathrm{H}_{2}$ FROM THIOSULFATE}

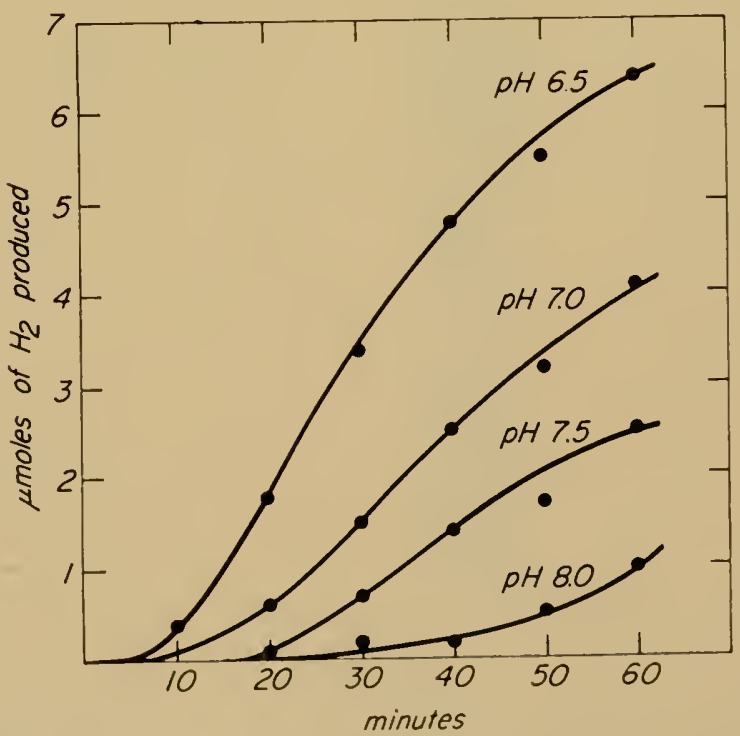

Fig. 2. Effect of $p H$ on photoproduction of hydrogen from thiosulfate by Chromatium cells. Phosphate buffer was used to adjust $p H$ to 7.0 and below, and Tris buffer to adjust $p H$ to 7.5 and above. Other experimental conditions as described for Fig. 1. 


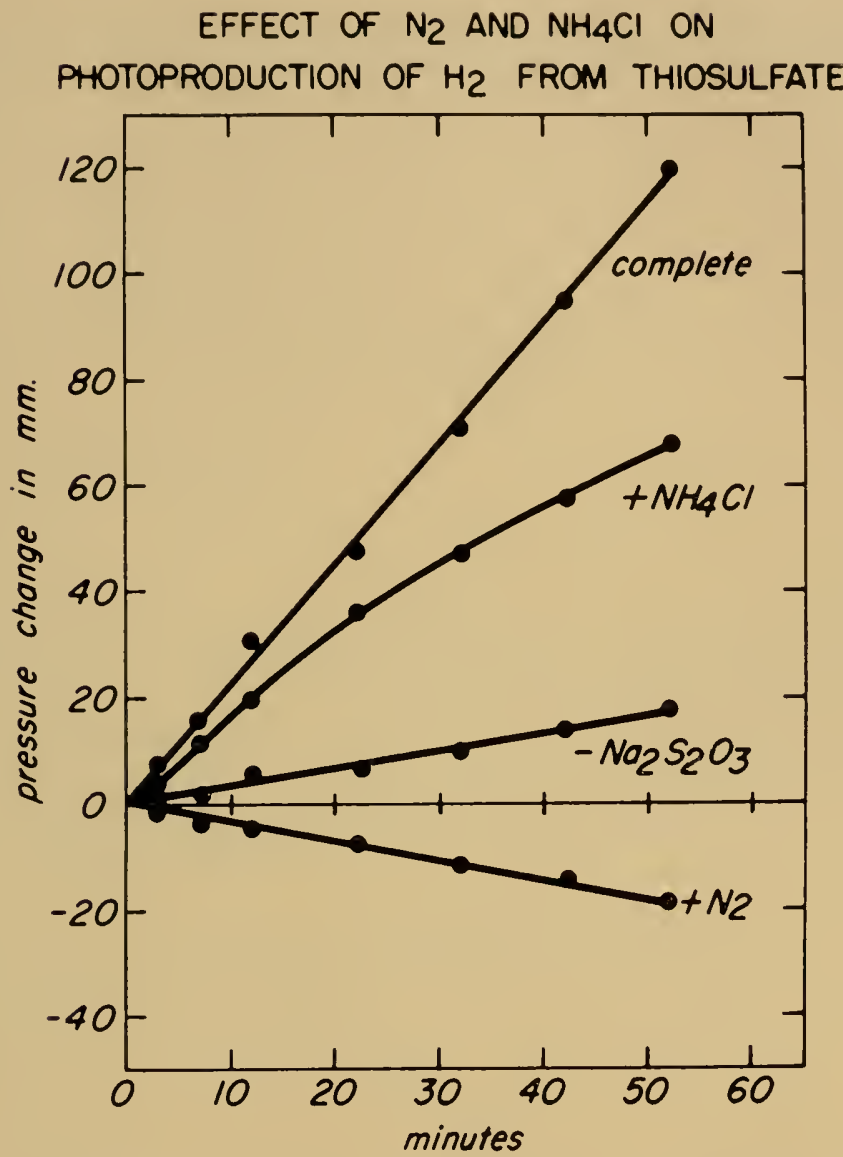

Fig. 3. Effect of $\mathrm{N}_{2}$ and $\mathrm{NH}_{4} \mathrm{Cl}$ on photoproduction of hydrogen from thiosulfate by Chromatium cells. In the $\mathrm{N}_{2}$ treatment, nitrogen gas was used instead of argon as the gas phase. 10 micromoles of $\mathrm{NH}_{4} \mathrm{Cl}$ was supplied in the $\mathrm{NH}_{4} \mathrm{Cl}$ treatment. Other experimental conditions as described for Fig. 1.

culture indicated a transitional formation of elemental sulfur. The results are explained by the following sequence of reactions:

$$
\begin{aligned}
& \mathrm{S}_{2} \mathrm{O}_{3}+2 \mathrm{H}^{+}+2 \mathrm{OH}^{-} \stackrel{\text { light }}{\longrightarrow} \mathrm{S}+\mathrm{SO}_{4}^{-}+\mathrm{H}_{2} \mathrm{O}+\mathrm{H}_{2} \\
& \mathrm{~S}+8 \mathrm{H}^{+}+8 \mathrm{OH}^{-} \stackrel{\text { light }}{\longrightarrow} \mathrm{SO}_{4}^{-}+2 \mathrm{H}^{+}+4 \mathrm{H}_{2} \mathrm{O}+3 \mathrm{H}_{2} \\
& 10 \mathrm{H}_{2} \mathrm{O} \stackrel{\text { dark }}{\longrightarrow} 10 \mathrm{H}^{+}+10 \mathrm{OH}^{-}
\end{aligned}
$$

Sum: $\mathrm{S}_{2} \mathrm{O}_{3}^{-}+5 \mathrm{H}_{2} \mathrm{O} \stackrel{\text { light }}{\longrightarrow} 2 \mathrm{SO}_{4}^{-}+2 \mathrm{H}^{+}+4 \mathrm{H}_{2}$ 
The electron flow mechanism for the photoproduction of hydrogen from thiosulfate (2) envisages the following electron transfer:

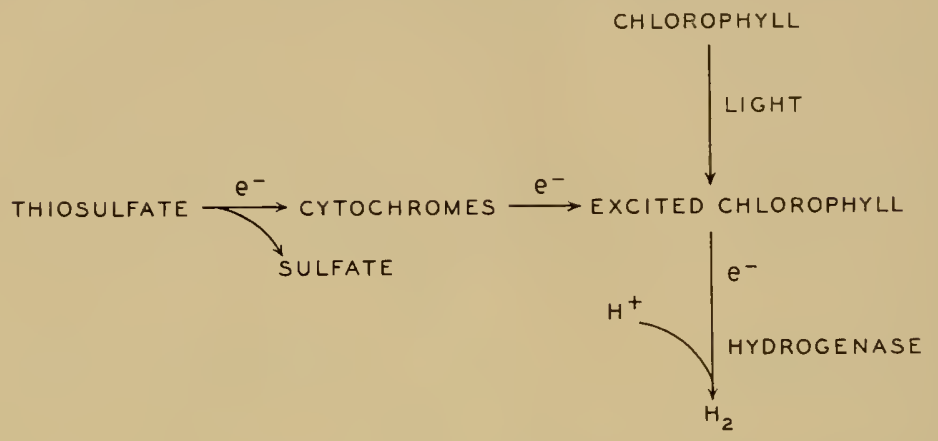

This formulation provides for a reduction of Chromatium cytochromes by thiosullate. Evidence for this reaction, in a cell-free system, is shown in Fig. 5.

PHOTOPRODUCTION OF $\mathrm{H}_{2}$ FROM THIOSULFATE

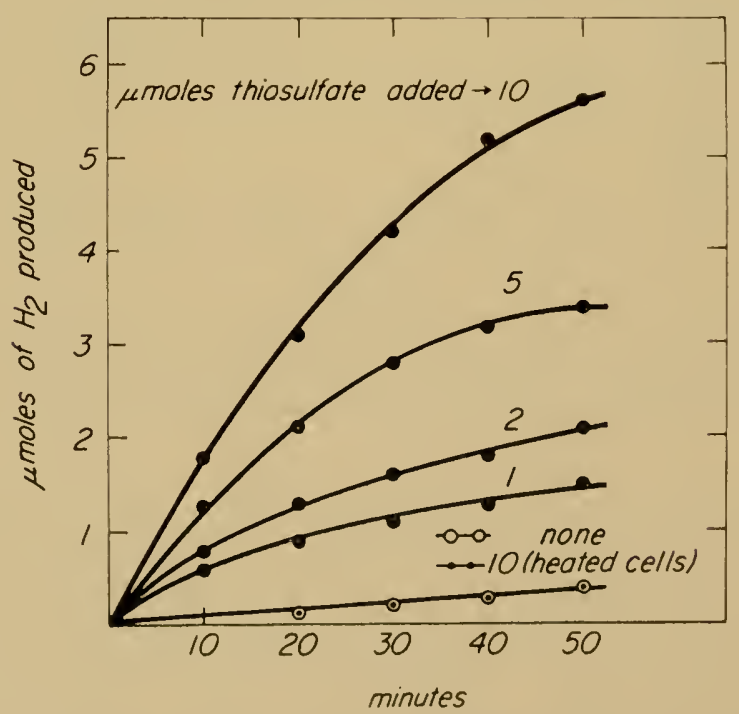

Fig. 4. Photoproduction of hydrogen by Chromatium cells as a function of added thiosulfate. A control treatment included the addition of 10 micromoles of thiosulfate to cells that had been heated for 10 minutes at $100^{\circ} \mathrm{C}$. Other experimental conditions as described for Fig. 1 . 


\section{Reduction of Chromatium $(P+S)$ \\ cytochromes by thiosulfate}

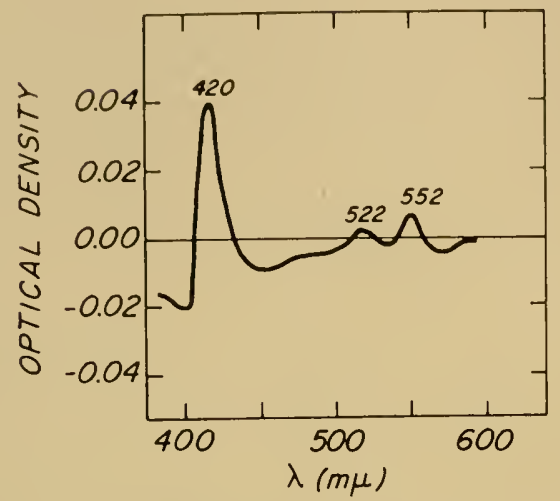

Fig. 5. Reduction of Chromatium cytochromes by thiosulfate in a cell-free system. Reaction mixture included, in a final volume of $3.0 \mathrm{ml}$ of $0.1 M$ Tris buffer, $p \mathrm{H} 7.8$, chromatophores $(P)$ containing $0.1 \mathrm{mg}$ bacteriochlorophyll and supernatant fluid $(S)$ corresponding to $0.3 \mathrm{mg}$ bacteriochlorophyll. 20 micromoles of thiosulfate were added to one of a pair of Thunberg-type cuvettes, and the resulting difference spectrum was measured in a Cary spectrophotometer after 20 minutes at room temperature. Gas phase, argon.

To summarize, illuminated Chromatium cells evolve molecular hydrogen from thiosulfate, which becomes oxidized to sulfate. The reaction is strictly light-dependent, is influenced by external $p \mathrm{H}$, and is inhibited by molecular nitrogen or ammonium ions. The reduction of oxidized Chromatium cytochromes by thiosulfate has been demonstrated in a cell-free system. These findings are compatible with the electron flow theory of photosynthesis (see Fig. 17, Arnon, this symposium).

\section{REFERENCES}

1. Armon, D. I., Nature, 167, 1008-1010 (1951).

2. Arnon, D. I. (this symposium).

3. Bregoff, H. M., and Kamen, M. D., J. Bacteriol., 63, 147-149 (1952).

4. Frenke1, A. W., Arch. Biochem, Biopliys., 38, 2I9-230 (1952).

5. Gaffron, H., and Rubin, J., J. Gen. Physiol., 26, 219-240 (1942).

6. Gest, H., Proc. Intern. Symposium on Enz. Chem., Tokyo and Kyoto, 250-256 (1957).

7. Gest, H., and Kamen, M. D., J. Bacteriol., 58, 239-245 (1949).

8. Gest, H., Kamen, M. D., and Bregoff, H. M., J. Biol. Chem., 182, 153-170 (1950).

9. Newton, J. W., and Wilson, P. W., Antonie van Leenwenhoek, 19, 7 I-77 (1953).

I0. Siegel, J. M., and Kamen, M. D., J. Bacteriol., 61, 2I5-228 (I951).

11. Treadwell, F. P., and Hall W. T., Analytical Chemistry, Vol. II, 7th ed., p. 665, John Wiley and Sons, New York (1930). 


\title{
OXIDATION-REDUCTION REACTIONS IN CHLOROPLASTS ${ }^{1,2}$
}

\author{
ANDRÉ T. Jagenhorf AND GIORgIO ForTi \\ McCollum-Pratt Institute and Biology Department \\ The Johns Hopkins University \\ Baltimore, Maryland
}

We would like to point out that a very generalized scheme for the plotosynthetic reactions in isolated chloroplasts seems sufficient to account for a large number of current experimental observations. While the skeletal scheme in itself says nothing interesting about the basic details of photochemistry in chloroplasts, at least it is useful in defining the area in which a particular reaction or inhibitor participates, under particular experimental conditions.

The possible reactions resulting from the action of light might be summarized by the five following equations:

$$
\begin{aligned}
& \mathrm{X}+\mathrm{Y}+\mathrm{H}_{2} \mathrm{O}+h_{v} \rightarrow \mathrm{XH}_{2}+\mathrm{YO} \\
& \mathrm{XH}_{2}+A \rightarrow A \mathrm{H}_{2}+\mathrm{X} \\
& \mathrm{YO} \rightarrow Y+\mathrm{I} / 2 \mathrm{O}_{2} \\
& \mathrm{YO}+A \mathrm{H}_{2} \rightarrow Y+A+\mathrm{H}_{2} \mathrm{O} \\
& A \mathrm{H}_{2}+\mathrm{I} / 2 \mathrm{O}_{2} \rightarrow A+\mathrm{H}_{2} \mathrm{O}
\end{aligned}
$$

Equation 1 is supposed to represent the initial reaction caused by light. According to most present speculations, light causes one catalyst to achieve a highly reducing potential, and another to become oxidized. In equation 1 , this is indicated by the transformation of an unspecified component $X$ to $\mathrm{XH}_{2}$, and a component $Y$ to $Y \mathrm{O}$. Water is inclicated to be a very early participant here, as suggested by van Niel (27) and by Frank and Brugger (4). Alternative suggestions in which water is not an early participant $(5,15)$ and may even be a terminal electron donor prior to oxygen evolution-(1)

${ }^{1}$ Contribution mumber 298 from the McCollum-Pratt Institute.

? Original work described here was supported in part by grant RG 3923 from the National Institutes of Health and in part by grant G 4379 from the National Science Fomdation. 
are under active consideration at the present time. However, no matter what compounds $X$ and $Y$ turn out to be, and no matter whether water is an early reactant or not, the general characteristics of equation $l$ have important experimental consequences in our reaction systenns. $X$ and $Y$ will have to be catalysts, rather than substrates, and bound firmly to the chloroplast structure. It seems likely that at any onc active center in the chloroplast there will be only one, or at most a few, of either $X$ or $Y$. It is obvious, then, that at the active center the component $X_{2}$ must be oxidized back to $X$ and IO reduced back to $Y$ in order for reaction 1 to occur a second time; and a continuous flow of electrons away from $X_{2}$ and to $Y O$ is a prerequisite for a measurable reaction.

The oxidation of $\mathrm{XH}_{2}$ is indicated in equation 2, by transfer to an added redox dye or pyridine nucleotides; any possible electron acceptor being designated as $A$. We must point out that equation 2, as the rest, is almost certain to be more complex than shown here. In particular, it seems likely that equation 2 hides an electron transport chain prior to the reduction of the added oxidant $A$; and that electron transport along this chain generates ATP. There is an alternative possibility that ATP is formed on the side of the photooxidant (l), but at the present time there does not seem to be suffcient evidence to decide between the two possible areas.

The reduction of $Y O$ to $Y$ may occur by two alternative means: reactions 3 or 4 . In the Hill reaction $Y O$ is reduced by discharging oxygen (equation 3 ); in cyclic electron transport $Y O$ is reduced by the generated reductant $A \mathrm{H}_{2}$ (reaction 4). Thus the Hill reaction consists of the reaction sequence $1,2,3$; cyclic electron transport of 1,2 , and 4 .

During the past year Venmesland and her coworkers Krogmann and Nakamoto have drawn our attention forcibly to the existence of reaction 5 , the oxidation of the added redox compound by molecular oxygen $(19,22,23)$. It is evident that when $A \mathrm{H}_{2}$ is oxidized by molecular oxygen in equation 5 , it is not available to reduce $Y O$ in equation 4 . Thus when free molecular oxygen is involved, YO must be reduced via reaction 3. The net result is that oxygen is both consumed (reaction 5) and evolved (reaction 3); and as written, the gas exchanges will cancel out. The overall sequence, under these conditions, will be reactions $1,2,3$ and 5 . This sequence can be defined as an oxygen exchange reaction, since it predicts that $\mathrm{O}$ atoms from water will be liberated into the atmosphere, and vice versa.

There are two major criteria for the existence of the oxygen ex- 
change reaction sequence $1,2,3$, and 5. Atmospheric oxygen must be present; evidently reaction 3 does not produce enough oxygen to satisfy the amount required in reaction 5. Nakamoto et al. have demonstrated an oxygen requirement for phosphorylation when FMN is the added redox cofactor $(22,23)$, and we have been able to confirm this (unpub.) on using nitrogen gas more highly purified from oxygen. Secondly, during the reaction sequence an oxygen exchange should be demonstrable between water and the atmosphere. Nakamoto et al. have observed this with FMN (23), and the amount of exchange was sufficient to account for the ATP formed. We had previously published one observation (18) in which a small exchange was observable, but was not sufficient to account for the phosphorylation occurring with FMN. It is now apparent that the earlier experiments led us to an incorrect conclusion, for technical reasons: the amount of exchange observed was limited under the conclitions then used by the rate of diffusion of oxygen between the gas and water phases. Dr. Krall has recently repeated the observations under conditions where diffusion was not limiting and has indeed seen a light- and FMN-dependent oxygen exchange reaction sufficient to account for the phosphorylation going on (pers. conmum.)

Our own experience with the variety of reaction sequences possible in chloroplasts centers around the inhibitor, CMU ( $p$-chlorophenyl dimethyl urea) $(12,14)$. Fig. I shows that it was an inhibitor for phosphorylation, but the extent of its inhibition depended on the nature of the redox cofactor. Reactions catalyzed by flavin monomucleotide (FMN) were very badly inhibited; pyocyanine as cofactor gave an intermediate inhibition; and PMS (phenazine methosulfate) was very much more resistant. All the Hill reactions, however, no matter what the Hill oxidant was, were inhibited as much as the FMN-catalyzed phosphorylation. The contrast between the Hill reactions and the PMS-catalyzed phosphorylation originally led us to propose reaction 3 as the site of inhibition by CMU.

Now that the FMIN reaction sequence seems established as an oxygen exchange reaction (eq. 1, 2, 3, and 5) it is entirely consistent for the whole sequence to be inlibited by blocking reaction 3 with CMU. At the time that inhibition of FMN was first observed, however, it seemed anomalous beciuse we thought that FMN catalyzed an electron tramsport cycle (eq. 1,2 , and $f$ ). In order to account for the FMN inhibition we devised a complicated scheme in which two oxidauts were produced in sequence, and FNN and PMS varied in the site at which they were reoxidized (12). A very similar compli- 


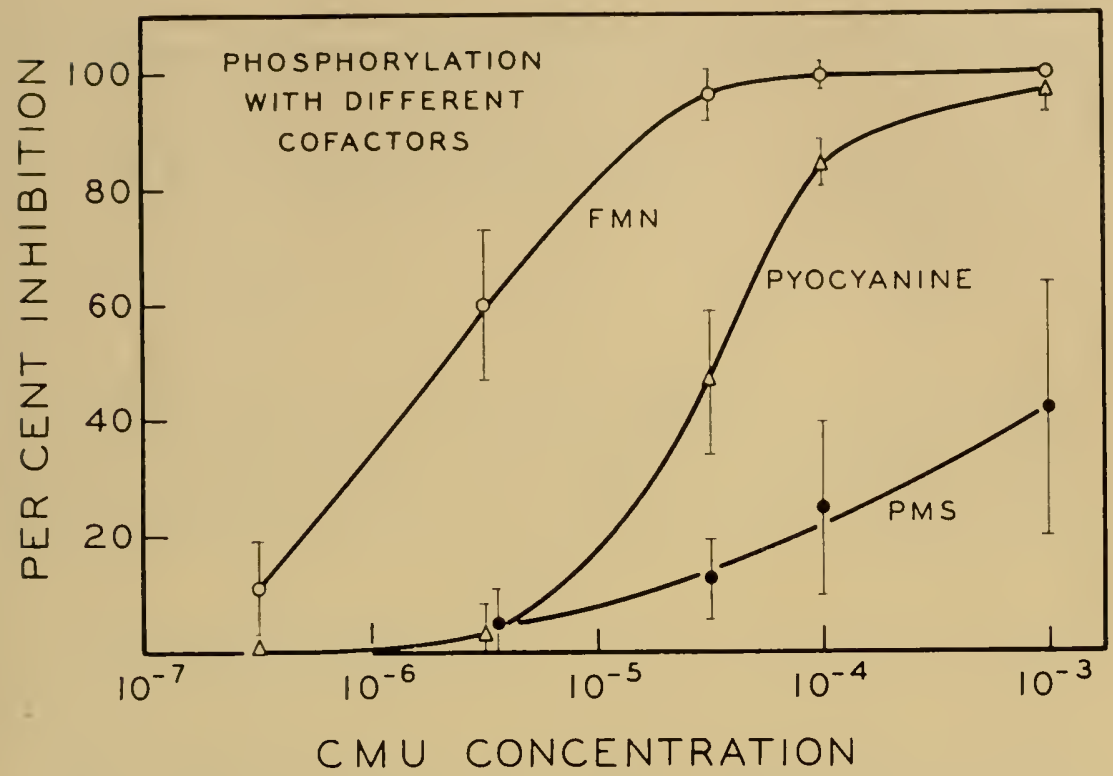

Fig. I. Inhibition of phosphorylation by CMU (p-chlorophenyl dimethyl urea) with different cofactors. FMN, PMS (phenazine methosulfale) or pyocyanine present as indicated at $3 \times 10^{-5} \mathrm{M}$. Other reaction conditions essentially those described under Table 1. The reactions were all flushed with nitrogen containing a small amount of oxygen. Each curve represents the average of from 6 to 12 experiments; the vertical lines represent twice the standard deviation. Specific activities of the control flasks ranged from 50 to $150 \mu$ moles ATP formed/mg chlorophyll/hour with FMN; from 310 to 610 for pyocyanine; from 300 to 650 for PMS.

cation was proposed by Arnon, Bové, and Whatley in connection with similar data obtained on studying chloride-deficient chloroplasts (1). In both cases the elaboration no longer seems necessary; the data do not really require us to go beyond the first five equations listed above.

The intermediate inhibition observed with pyocyanine (middle curve in Fig. 1) turned out to be an artifact, caused by a small amount of oxygen left in the nitrogen we used to eliminate air from the reaction flasks. After improving the preliminary oxygen-trapping system on our nitrogen gassing line, and increasing the length of time of flushing with nitrogen, the results shown in Table 1 were obtained. With sufficient removal of oxygen, the pyocyanine-catalyzed phosphorylation becomes resistant to CMU. This is especially true if, as in the second column, the oxygen is removed prior to addition of the CMIU. 
TABLE 1

Failure of CMu to Inhibit Anaerobic Pyocyanine-catalyzed Phosphorilation

$\%$ Inhibition by $1 \times 10^{-4} M \mathrm{CMU}$

$\mathrm{N}_{2}$ Flushing

CMU added before $\mathrm{N}_{2}$

CMU added after $\mathrm{N}_{2}$

minutes

0

2

10
96

89

53

49
90

24

10

12

Control rate was $325 \mu$ moles phosphate esterified/mg chlorophyll/hour either in air or under nitrogen. Flasks contained, in $1.5 \mathrm{ml}$ volume, Tris buffer, $p \mathrm{H} 8.0,20 \mu$ moles, $\mathrm{NaCl} 35 \mu$ moles, pyocyanine $.045 \mu$ moles, ADP $2.5 \mu$ moles, phosphate $2.5 \mu$ moles, $\mathrm{MgCl}_{2} 5 \mu$ moles, chloroplasts containing $.040 \mathrm{mg}$ of chlorophyll, and either $.05 \mathrm{ml}$ of $95 \%$ alcohol or $.05 \mathrm{ml}$ of $3 \times 10^{-3} \mathrm{M}$ CMU dissolved in $95 \%$ alcohol. Incubation time was 5 minutes at $15^{\circ} \mathrm{C}$, light intensity 4000 foot candles; residual phosphate was determined colorimetrically.

Phrasing it another way, these data show a combined inhibition by oxygen and CMU where neither alone would be inhibitory. This fact is now easily explainable, if one is aware that pyocyanine is a very readily autoxidizable dye. Thus when a small amount of oxygen is available, any pyocyanine reduced in reaction 2 will be re-oxidized in reaction 5. The existence of reaction 5, autoxidation, will compete with and essentially eliminate reaction 4 . With reaction 4 inhibited by lack of a substrate and reaction 3 inhibited by CMU, the two alternative ways for $Y O$ to be reduced are both blocked, and so reaction 1 will stop for lack of the acceptor form $Y$.

Since the reaction conditions in Fig. 1 included a small anount of oxygen, it becomes a question why PMS did not show partial inhibition by CMU the way pyocyanine dicl. In Fig. 2 it can be seen that the resistince of the PMS-catalyzed phosphorylation was actually dependent on the color of light that was used; the reaction was resistant in white light but not in red. The explanation here may be found in our observation (14) that white light under anaerobic conditions causes PMS to be reduced non-enzymatically. Red light is not absorbed by PMS, and so does not recluce it. We could also observe non-enzymatic reduction of PMS by ascorbate; and adding ascorbate, even in red light, causes the reaction to be resistant to CMU (Fig. 2). Thus when PMS is reduced non-enzynatically, the overall reaction is highly resistant to the combination of CMU and a little oxygen; when it is not being recluced non-enzymatically, then the reaction is just like pyocyanine in being sensitive to the combined inhibition. 


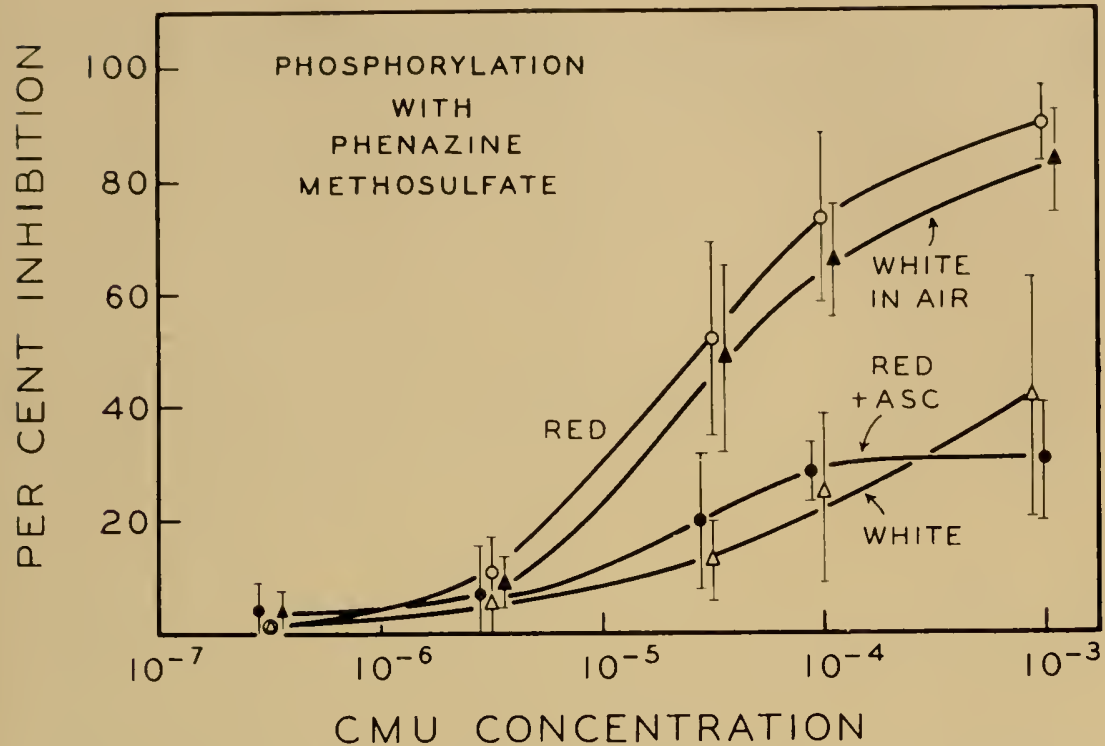

Fig. 2. Inhibition of PMS-mediated phosphorylation by C.MU under different reaction conditions. All reactions are micro-aerobic and without ascorbate unless otherwise indicated. Other conditions are described under Table 1 and Fig. 1. The curves represent the average percentage of inhibition found in from 6 to 14 experiments. Vertical lines represent twice the standard deviation. When present, $10 \mu$ moles of ascorbate were used in $1.5 \mathrm{ml}$ final volume. Red, white, color of incident light. ASC, ascorbate.

The explanation in terms of the reaction sequence listed above again becomes fairly obvious. Inhibition, as with pyocyanine, is due to a combination of reaction 3 inhibited by CMU, and oxygen (reaction 5) destroying the PMS reduced by the chloroplasts, and thus eliminating the substrate for reaction 4. Reducing PMS non-enzymatically will restore the substrate for reaction 4 , and thus permit the entire sequence 1,2 , and 4 to continue.

At this point it is possible to indicate a remarkable similarity between chloroplasts poisoned by CMU and bacterial chromatophores. In Chromatium, for instance, the phosphorylation by isolated chromatophores is poisoned by small amounts of oxygen $(10,24)$. The poisoning can be overcome by adding substrates that serve as electron donors. Similarly in CMU-treated chloroplasts, the pyocyanine- and PMS-supported phosphorylations are poisoned by small amounts of oxygen, and adding electrons from outside the system through PMS restores the phosphorylation. Bacteria are constitu- 
tionally unable to accomplish oxygen evolution, and it seems that the same general considerations discussed here will explain their reactions to the introduction of oxygen.

An advantage that we have gained from these experiments is that of an added criterion to distinguish a cyclic electron transport sequence (eq. 1, 2, and $f$ ) from an oxygen exchange reaction (eq. 1, 2, 3 , and 5). The cycle should be almost entirely resistant to inhibition by GMU, although, as we have seen, it may take highly anaerobic conditions for the full extent of the resistance to appear. Of the previous criteria, the oxygen exchange between water and $\mathrm{O}_{2}$ is difficult to measure routinely. The oxygen dependency of the exchange sequence might not be infallible if the oxygen produced in reaction 3 could be used very efficiently in reaction 5 [23].

We have been able to use these criteria for an analysis of the "endogenous" phosphorylation, i.e., the small amount of light-dependent ATP formation that occurs without any added redox cofactors (nanuscript in preparation). We found that this endogenous phosphorylation is oxygen-dependent and inhibited by CMU, and therefore cammot be a cyclic sequence of reactions 1,2, and t. Actually, in this case a net uptake of oxygen is observed (Table 2) and indicates that the reaction sequence is of the type discovered by Mehler (21), in which reaction 5 is replaced by $5 a$ :

$$
A \mathrm{H}_{2}+\mathrm{O}_{2} \rightarrow \mathrm{H}_{2} \mathrm{O}_{2}+A
$$

The full sequence here would be reactions $1,2,3$, and $5 a$. Since one mole of oxygen is taken up in $5 a$, and only $1 / 2$ mole evolved in re. action 3, a net consumption of oxygen is observable.

It now becomes a matter of some interest that the full sequence, involving both oxygen uptake and ATP formation, is very considerably stimulated by the addition of photosynthetic pyridine nucleotide reductase (PPNR) (Table 2). This enzyme is thus scen to function in the reduction of oxygen as well as TPN and heme proteins (26).

Adding relatively small amounts of ascorbic acid to the system with PPNR, but none of the usual cofactors, stimulates ATP formation and either has no eflect or in many cases inhibits the oxygen uptake (Table 3). We should emphasize that this effect can be found with $.0005 M$ ascorbate, and indeed the higher concentrations of ascorbate used previously $(28,29)$ often tend to increase oxygen uptake. Since the observed oxygen uptake does not increase upon the addition of ascorbate, it seemed possible that a cyclic sequence $(1,2$, and $f)$ might have been set up. Again, this could be ruled out because even with 
TABLE 2

Stimllation of $\mathrm{O}_{2}$ Úptake and Photomiosphorllation by Puotosyntiletic Pýridine Nucleotide Reductase (PPNR)

$\begin{array}{ccc}\text { PPNR Added } & \text { Oxygen uptake } & \text { ATP formed } \\ \text { units } & \mu \text { atoms } & \mu \text { moles } \\ 0 & 2.0 & 0.30 \\ 6.25 & 3.0 & 1.01 \\ 12.50 & 4.4 & 1.58\end{array}$

The chloroplasts, prepared in sucrose-Tris-NaCl-Versene (11), were incubated in manometric vessels at $15^{\circ} \mathrm{C}$, with a light intensity of 4500 foot-candles at vessel height. The reaction mixture was made to a final volume of $3.0 \mathrm{ml}$, containing an excess of hexokinase, and $\mathrm{NaCl} .03 \mathrm{M}$, Tris-HCl $p \mathrm{H} 8.0 .01 \mathrm{M}, \mathrm{MgCl}_{2} .005 \mathrm{M}, \mathrm{ADP} .002 \mathrm{M}$, phosphate iabeled with $\mathrm{P}^{32} .005 M$ and glucose $.025 M .0 .5 \mathrm{mg}$ of chlorophyll was present in cach flask. Reaclion time, one hour.

the appropriate concentration of ascorbate, oxygen was required and CMU inhibited phosphorylation very greatly. Thus it seems to us to be likely that ascorbate, especially in the presence of PPNR, depresses the Mehler reaction sequence (eq. 1,2, 3, and 5a) and encourages or sets up an oxygen exchange reaction (eq. 1, 2, 3, and 5) as an alternative.

A clear-cut distinction between the two types of oxygen involvement may be possible in the future. Some preliminary indication of this is found in the fact that KCN has little effect on the phosphorylation without ascorbate where $\mathrm{O}_{2}$ is consumed, but inhibits ATP formation very markedly in the presence of ascorbate (Table 3).

Of the numerous compounds which have been used as catalytic redox cofactors in photosynthetic phosphorylation, so far we can say that only PISS and its derivative pyocyanine participate in reaction 4; whereas FMN, menadione, incligo carmine, vitamin $\mathrm{K}_{5}$, and methyl and benzyl viologens run through the oxygen exchange se-

TABLE 3

Effect of $\mathrm{CN}^{-}$on Ascorbate-stimulated Photophosphorylation

\begin{tabular}{|c|c|c|c|}
\hline Additions & $\begin{array}{c}\text { Oxygen } \\
\text { consumed }\end{array}$ & $\begin{array}{c}\text { ATP } \\
\text { formed }\end{array}$ & $\begin{array}{c}\text { Ascorbate } \\
\text { disappeared }\end{array}$ \\
\hline & $\begin{array}{c}\mu \text { atoms } \\
1.3\end{array}$ & $\begin{array}{c}\mu \text { moles } \\
1.16\end{array}$ & $\mu$ moles \\
\hline $\mathrm{KCN}, M / 100$ & 3.3 & .88 & - \\
\hline Ascorbate, $.002 \mathrm{M}$ & 1.0 & 4.50 & .75 \\
\hline Ascorbate, $.002 M$ plus $\mathrm{KCN}, M / 100$ & 5.8 & 1.44 & 3.00 \\
\hline
\end{tabular}

Conditions as in Table 1. A dark control with cyanide and ascorbate present showed no oxygen uptake, ascorbate disappearance, or ATP formation. 
quence. This selectivity appears to correlate well with the selectivity seen by Kok for the spectral changes in illuminated cells (17) and would indicate that he is observing more directly what we have inferred to be reaction 4 .

Finally, we can point out the relatively large number of inhibitors or conditions that affect either a Hill reaction or an oxygen exchange reaction, but not the closed cycle, and which therefore appear to work on reaction 3. This sort of response is obtained with o-phenanthroline as an inhibitor $(22,12)$, as well as the relative of CMU, DCMU (dichlorophenyl dimethyl urea) $(19,22)$. When chloroplasts are treated with an excessive concentration of Tris, they appear to be permanently damaged in this same reaction 3 (22). A deficiency of chloride ions, especially at a high $p \mathrm{H}$, brings on the same symptoms (1). Slightly different experimental criteria (inhibition of photosynthesis, but not of photoreduction by hydrogen-adapted algae) indicate that hydroxylamine poisoning (8) and manganese deficiency (16), as well as $o$-phenanthroline (9) and DCMU (2), act at about the same site. These agents or conditions are so diverse that one becomes suspicious that reaction 3, again, may be a disguise for a series of reactions rather than a single one.

A very unusual sort of inhibition of reaction 3 was discovered by Dr. Margulies, in this laboratory. He found that if the primary leaves of the Black Valentine Bean plant were stored for 24 hours in the dark at $0^{\circ} \mathrm{C}$, their ability to photosynthesize was impaired $(20)$. Chloroplasts from these leaves could accomplish a cyclic electron flow, but not a Hill reaction or any oxygen exchange reaction. A reversal of the inhibition could be accomplished by bringing the leaves to room temperature and illuminating them for 15 minutes. Unfortunately, this reversal was not possible in vitro. Although we do not understand the mechanism of this inhibition or of its reversal, the function of light in the reversal and the speed with which it happens is reminiscent of the effect observed by Brody and Brody, in which illumination of red algae, grown in blue light, with green light induced in 20 minutes to 1 hour a tighter coupling between the accessory pigments and chlorophyll $a$ (3).

The concept that accessory pigment light absorption may be specifically necessary for reaction 3 received its first and most direct support from the experiments of Barbara Petrack (25). She was able to prepare chloroplast particles or chromatophores from blue-green algae which had lost their phycobilin pigments and retained only chlorophyll a. These particles were still able to catalyze a cyclic electron 
flow with PMS as cofactor, but were not able to perform a Hill reaction.

Strong support for this concept can now be found in the experiments of Kok and Hoch (17), in which the cyclic electron transport, forming ATP, was found to occur with spinach chloroplasts in monochromatic light at $700 m \mu$ and above. Previous action spectra had indicated that light of this wavelength, which is absorbed by chlorophyll $a$ but not by the accessory pigments, is not effective in over-all photosynthesis ( 7 ) or in the Hill reaction (6). Our previous action spectrum for phosphorylation (13) had indicated that $700 \mathrm{~m} \mu$ light was not effective for ATP formation with PMS as the cofactor. However, at that time our system was not completely anaerobic. In view of our data with CMU inhibition of PMS in red light, it seems obvious that we must have been measuring an oxygen exchange reaction rather than a true cycle. Thus, if $700 \mathrm{~m} \mu$ light does support a true electron transport cycle, it indicates that light received by chorophyll $a$ only, while sufficient to produce $\mathrm{XH}_{2}$ and $Y O$ in the present scheme, is not sufficient to reduce $Y O$ by discharging oxygen. These preliminary considerations suggest that in the future the function of the accessory pigments will be found in the steps leading to oxygen evolution, i.e., reaction 3 .

\section{ACKNowledgMents}

We would like to acknowledge the stimulation due to discussions with Dr. M. Margulies and Dr. J. Kahn in developing some of the icleas in this paper. The experimental work shown here was done with the technical assistance of Miss Marie Smith.

\section{REFERENCES}

1. Arnon, D. I., Nature, 184, 10 (1959).

2. Bishop, N. I., Biochim. Biophys. Acta, 27, 205 (1958).

3. Brody, S. S., and Brody, .11., Arch. Biochem. and Biophys., 82, 161 (1959).

4. Brugger, J. E., and Franck, J.. Arch. Biochem. and Biophys., 75, 465 (1958).

5. Chance, B., and Nishimura, M., Proc. Natl. Acad. Sri. U.S., 46, 19 (1960).

6. Chen, S. L., Plant Physiol., 27, 35 (1952).

7. Emerson, R., Ann. Rev. Plant Physiol., 9, 1 (19:8).

8. Gaffron, H., J. Gen. Physiol., 26, 195 (1942).

9. Gaffron, H., J. Gen. Physiol., 28, 269 (1945).

10. Geller, D. M., Thesis, Div. of Med. Sci., Harvard University (1957).

11. Jagendorf, А. T., and Arron, M., J. Biol. Chem., 23I, 277 (1958).

12. Jagendorf, A. T., and Arron. M., Arch. Biochem. and Biophys., 80, 246 (1959).

13. Jagendorf, A. T., Hendricks, S. B., Arron, M., and Evans, M. B., Plant Physiol., $33,72(19.58)$.

14. Jagendorf, A. T., and Margulies, M., Arch. Biochem. and Biophys. (in press).

15. Kamen, M. D., this symposium.

16. Kessler, E., Arch. Biochem. and Biophys., 59, 527 (19:5.). 
17. Kok, B., and Hoch, G., this symposimm.

18. Krall, A. R., Avon, M., and Jagendorf, A. T., Biochim. Biophys. Acta, 20, 431 (1957).

19. Krogmann, D. W., and Vennesland, B., J. Biol. Chem., 234, 2205 (1959).

20. Margulies, M., and Jagendorf, A. T., Arch. Biochem. and Biophys. (in press).

21. Mehler, A. H., Aich. Biochem. and Biophys., 33, 65 (1951).

22. Nakamoto, T., Krogmann, D. W., and Vennesland, B., J. Biol. Chem., 234, $2783(19.59)$.

23. Nakamoto, T., and Vennesland, B., Federation Proc., 19, 329 (1960).

24. Newton, J. W., and Kamen, M. D., Biochim. Biophys. Acta, 25, 462 (1957).

25. Petrack, B., Federation Proc., I8, 302 (1959).

26. San Pietro, A., this symposium.

27. van Niel, C. B., in Photosynthesis in Plants (Franck, J. and Loomis, N. E., eds.), pp. 1-500, lowa State College Press (1949).

28. Whatley, F. R., Allen, M. B., and Arnon, D. I., Biochim. Biophys. Acta, I6, $605(1955)$.

29. Whatley, F. R., Allen, M. B., and Arnon, D. I., Biochim. Biophys, Acta, 32, 32 (1959). 


\section{REFLLCTIONS ON RECENT THEORIES CONCERNING THE MECHANISM OF BACTERIAL PHOTOSYNTHESIS ${ }^{1}$}

\section{Albert W. Frenkil}

\section{Department of Botany}

University of Minnesota, Minneapolis, Minnesota

The basic features which distinguish bacterial photosynthesis from that of oxygen-producing plants have been described in great detail by van Niel $(12,18)$, and his unified theory of photosynthesis has been an effective guidepost in attempts to correlate observations on all types of photosyntheses carried out by chlorophyll-containing organisms and by particulate structures derived from them. Van Niel's theory basically envisions that oxidizing and reducing equivalents are produced as the result of the primary photochemical act; that the photochemical reductant can bring about the reduction of $\mathrm{CO}_{2}$ through suitable metabolic intermediates; and that the photochemical oxidant either dismutates to produce molecular oxygen as in plants containing chlorophyll $a$, or must react with a suitable electron donor, as is the case in photosynthetic bacteria. Cell-free preparations of photosynthetic bacteria carry out a number of light-induced reactions which, like the light-induced reactions of isolated chloroplasts, can be interpreted in terms of a modification of van Niel's theory of photosynthesis $(10)$.

Recently, the importance of the photochemically produced reductant for bacterial photosynthesis, in bringing about the reduction of metabolic intermediates, has been questioned by Stanier and coworkers (17), and by Arnon (1). Arnon argues that in bacteria we are observing a primitive photosynthetic system whose principal product is high-energy phosphate, and that the electrons which are necessary for $\mathrm{CO}_{2}$ reduction must be derived from the externally supplied reductant. Arnon's argument is based on observations concerned with light-induced phosphorylation reactions carried out by chloroplasts and bacterial chromatophores. In a number of contributions Arnon (1-3) has clescribed what he considers as two different types of phosphorylation processes carried out by illuminated chloroplasts. In "cyclic photophosphorylation" by chloroplasts the production of

${ }^{1}$ Work supported by a grant from the Graduate School of the University of Minnesota, and by grants from the National Institute of Allergy and Infectious Discases (E-2218), and the National Science Foundation (G9888). 
energy-rich phosphate is observed without a concomitant net production of molecular oxygen or the accumulation of reduced products. In "non-cyclic photophosphorylation" by chloroplasts the phosphorylation process is linked to a net reduction of added triphosphopyridine nucleotide, accompanied by the simultaneous production of molecular oxygen. Arnon then assumes that in bacteria "non-cyclic photophosphorylation" does not exist, i.e., that no photoxidant or photoreductant is formed, and he explains thereby the inability of photosynthetic bacteria to form molecular oxygen. In examining this hypothesis, it appears premature to assume that in bacterial systems light-induced phosphorylation coupled to electron flow through a "non-cyclic" electron transport system cannot be demonstrated. Experimentally, chloroplasts have been more favorable for this type of investigation because for "cyclic photophosphorylation" they require a cofactor such as FMN which is not required for the "non-cyclic" type in the presence of TPN or ferricyanide. The same type of approach has not yet been successful with bacterial chromatophores. Nevertheless it is clear that a "non-cyclic" electron transport system can be demonstrated in illuminated chromatophores $(8,9,19)$ (Fig. lB), and that phosphorylation may or may not accompany the lightinduced reduction of DPN (9), just as light-induced TPN reduction by chloroplasts also is not dependent on simultaneous phosphorylation (11). The reverse has not yet been demonstrated, namely, that phosphorylation in bacterial particles is coupled to pyridine nucleotide reduction, yet Smith and Baltscheffsky (16) have demonstrated that there exists a coupling between phosphorylation and light-induced oxidation of endogenous cytochrome $c_{2}$ in chromatophores of Rhodospirillum rubrum. In comparing the characteristics of light-induced reactions of chloroplasts and of chromatophores (Figs. 1, 2) there seems to be no question that "cyclic photophosphorylation" must be due to a flow of electrons through a "short circuit" electron transport system to which one or more phosphorylation sites are coupled; "non-cyclic photophosphorylation" must also be due to the flow of electrons, which in this case results in the reduction of pyridine nucleotides or of other suitable electron acceptors, and in the simultaneous production of $\mathrm{O} .2$ by chloroplasts, or in the simultaneous oxidation of suitable electron clonors by chromatophores. It does not appear necessary to assume that the phosphorylation sites are the same for the "cyclic" and "non-cyclic" electron transport systems. However, it appears difficult to envisage that the manner in which the electron flow is initiated by light should be different for these two processes occurring in chloroplasts. Also, there does not appear to be a good 

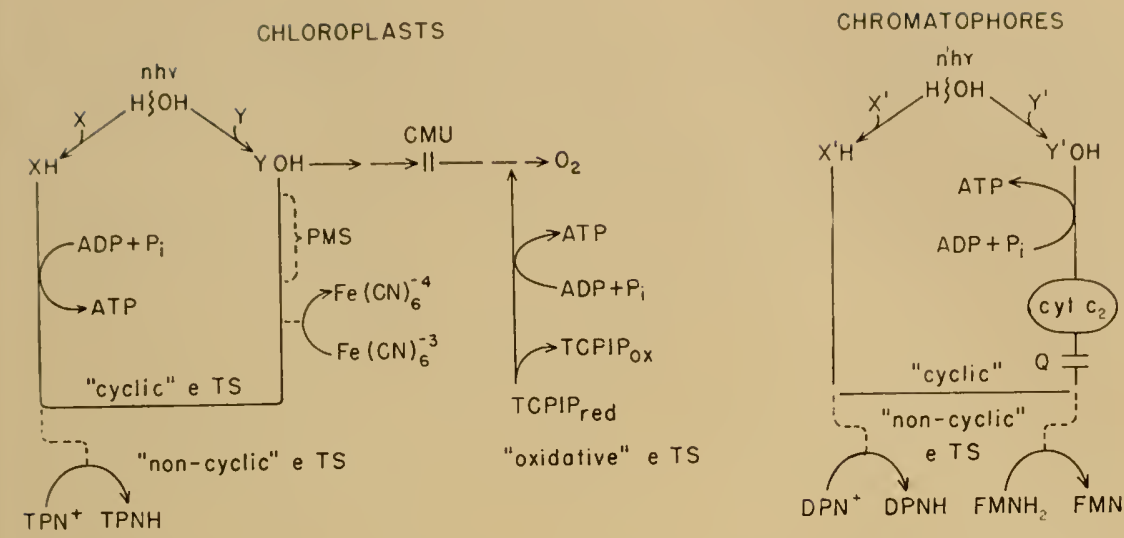

Fig 1. Comparative representation of electron transport systems and associated phosphorylation sitcs in chloroplasts and bacterial chromatophores.

Chloroplast scheme after Jagendorf (11). XH, photochemically produced reductant; YOH, photochemically produced oxidant (precursor of $\mathrm{O}_{2}$ ); CMU, p-chlorophenyl dimethyl urea; TCPIP, 2,3',6-trichlorophenol indophenol; PMS, phenazine methosulfate; e $T S$, electron transport system; "oxidative" e $T S$, cf. Krogman and Venncsland (13).

Chromatophore scheme (for Rhodospirillum mbrum). $\mathrm{X}^{\prime} \mathrm{H}, \mathrm{Y}^{\prime} \mathrm{OH}$, photochemically produced reductant and oxidant; cyt $c_{2}$, Rhodospirillum cytochrome $c$ (1916) $Q$. possible site of action of 3-hydroxy, 1-heptyl quinoline-N-oxide and of Antimycin $A$ in blocking electron transport and light-induced phosphorylation (16); site of action of these inhibitors bypassed by PMS (4).

reason for assuming that the manner in which electron flow is initiated in illuminated bacterial systems should be basically different from the mechanism which prevails in chloroplasts. The strongest argument in favor of an identical basic photochemical mechanism is the work of Larsen, Yocum, and van Niel (14) who demonstrated that the quantum efficiency for bacterial photosynthesis was close to that for photosynthesis of oxygen-producing plants, and that the quantum yield of bacterial photosynthesis was independent of the chemical potential of the exogenous hydrogen donor. It therefore appears reasonable to assume that the quantum efficiency for the production of an electron pair must thus be the same for chloroplasts as for bacterial chromatophores, but one is then faced with the well-known fact that the total light energy available to the bacterial system, per unit number of effective quanta absorbed, will be only about 75 per cent of that of the chloroplast system (based on the energy levels of the first excited state of bacteriochlorophyll versus that of chlorophyll a) (Fig. 2). Whether this difference in available energy [disregarding possible two-quantum mechanisms and energy losses which may or may not be the same for the two systems (15) ] is sufficient 


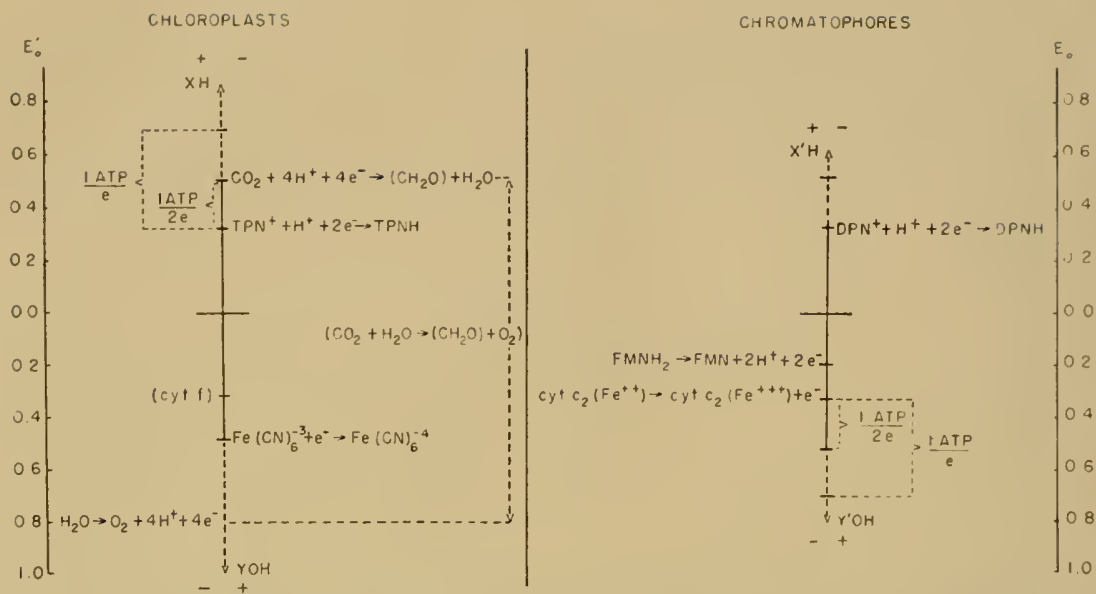

Fig. 2. Comparative representation of electron transport systems and associated phosphorylation sites in chloroplasts and bacterial chromatophores in terms of standard electrode potentials $\left(E_{n}^{\prime} ; p H 7,25^{\circ} \mathrm{C}\right)$ of known oxidation-reduction reactions (using the chemical convention of signs). Over-all reaction of photosynthesis expressed in electron volts. Distance from $\mathrm{XH}$ to $\mathrm{YOH}$ energy of quantum of $\lambda=6700 \mathrm{~A}$. equal to $1.83 \mathrm{ev}$. Distance from $\mathrm{X}^{\prime} \mathrm{H}$ to $\mathrm{Y}^{\prime \prime} \mathrm{OH}$ energy of quantum of $\lambda=8800 \mathrm{~A}$, equal to $1.40 \mathrm{ev}$. ATP/e indicates the potential span required per electron if one molecule of ATP is formed by the passage of one electron through the e TS system; ATP/2e indicates the average potential span required per electron if one molecule of ATP is formed by the passage of an electron pair through the e $T S$ system.

to account for the inability of the bacterial system to produce molecular oxygen is an open question. At any rate the argument presented here is in line with van Niel's theory, placing the main emphasis on electron flow initiated by light and considering the formation of high-energy phosphate as an important phenomenon but a secondary one which may or may not accompany the transport of electrons generated by the light reaction.

Stanier and coworkers (17) in a recent publication have presented strong evidence for the importance of light-induced phosphorylation in the metabolism of acetate by Rhodospirillum rubrum. In the absence of carbon dioxide, acetate is converted into a polymer of beta-hychroxybutyrate, ATP being required for the formation of acetyl coenzyme $A$. According to Stanier et al., the hydrogens required for the reduction of acetate to the level of beta-hydroxybutyrate are derived from the anaerobic dismutation of 1 mole of acetate for each 8 moles of acetite condensed into the beta-hydroxybutyrate polymer. These investigators, however, admit that one of the four electron 
pairs obtained from the dismutation of one molecule of acetate (Fig. $3 \mathrm{~A})$, namely, the flavin couple associated with the succinate-fumarate couple, is not of a sufficiently high chemical potential to bring about the reduction of acetoacetate to beta-hychroxybutyrate. Consequently they postulate that at this point photochemically produced reductants come into play, possibly as indicated by Fig. 3C, a reaction which has been observed with isolated chromatophores (8). Thus, Stanier and coworkers still require a photoreductant for bacterial photosynthesis, but invoke it only for one-fourth of the electron pairs required in this particular reaction system. In this connection, it may be worthwhile to consider other means by which all or part of these hydrogens could be generated. First, according to van Niel's theory all the elec-

(A)

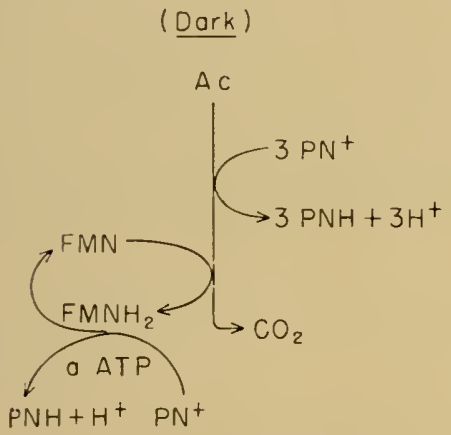

(B)

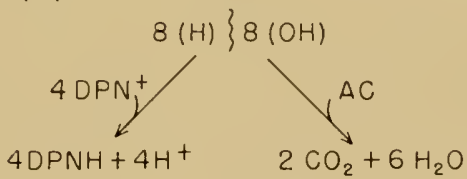

(C)

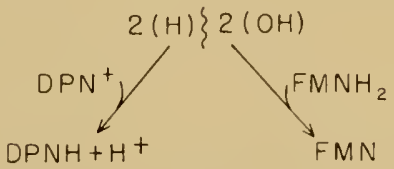

Fig. 3. Possible means of forming reduced pyridine nucleotictes in the anaerobic photometabolism of acetate (Ac) by Rhodospirillum rubrum (17).

1. Dark production of three molecules of reduced pyridine nucleotide (PNH) and of one molecule of reduced flavin $\left(\mathrm{FMNH}_{2}\right)$ from one molecule of acetate via the tricarboxylic acid cycle. The reducing equivalents of $\mathbf{F M N H}_{2}$ then may be "upgraded" to the PNH level, the required energy being supplied by a molecule of ATP (5). (For the formation of enzyme-bound DPNH in catalytic amounts, no ATP may be required; however, for the formation of reduced DPN in substrate quantities even one equivalent of energy-rich phosphate per mole of DPNH formed would appear barely adequate; two equivalents of energy-rich phosphate per mole of DPNH formed would appear to be more than sufficient).

13. Reduction of pyridine nucleotides in the light, according to scheme of van Niel. Diphosphopyridine nucleotide (DPN) reduced by the photochemical reductant $(\mathrm{H})$, acetate oxidized to $\mathrm{CO}_{2}$ and water by the photochemical oxidant $(\mathrm{OH})$, most likely by way of the tricarboxylic acid cycle. $\mathrm{x}=$ number of quanta required by the photochemical system for the production of one reducing equivalent.

C. Possible manner of "upgrading" the reducing equivalents of $\mathbf{F M N H}_{2}$ produced in (A) through a photochemical reaction. $\mathrm{FMNH}_{2}$ is oxidized by the photochemical oxidant with the simultaneous reduction of DPN to DPNH (8). 
trons required for the reduction of the substrate (presumably acetoacetate) would be derived from the photochemical reductant and acetate would be oxiclized by the photochemical oxidant, most likely by way of the tricarboxylic acid cycle (6) (Fig. 3B). The other extreme possibility would be that none of the electrons required for this reduction come from the photoreductant, but that the electron pair of the succinate-fumarate couple could be upgraded by ATP, similar to a reaction studied by Chance and Hollunger in mitochondria (5), and thus all required electron pairs would be produced in the dark (Fig. 3A). At the present time there is no clear-cut evidence which would permit one to eliminate any of these schemes (Fig. $3 \mathrm{~A}-\mathrm{C}$ ), or to permit the postulate that only one of them is operative during the photometabolism of acetate. Since cell-free preparations of Rhodospirillum rubrum can both produce ATP and bring about the reduction of DPN in the light, it appears likely that both of these light-induced processes could occur in the intact cell; but how much reduced pyridine nucleoticle is produced by the photochemical reductant and how much is formed through the anaerobic dismutation of acetate may be difficult to decide. Tracer experiments in due time may yield information which may give us a somewhat better insight into this problem.

\section{REFERENCES}

1. Arnon, D. 1., Nature, 184, 10-21 (1959).

2. Arnon, D. 1., Brookhaven Symposia in Biol., 11, $181-235$ (1959).

3. Armon, D. 1., Whatley, F. R., and Allen, M. 13., Science, 127, 1026-1034 (1958).

4. Baltscheffsky, H., and Baltscheffsky, M., Acta Chem. Scand., 12, 1333-1334 (1958).

5. Chance, B., and Hollunger, G., Federation Proc., 16, 163 (1957).

6. Elsden, S. R., and Ormerod, J. G., Biochem. J., 63,691-701 (1956).

7. Franck, J., Arch. Biochem. Biophys., 80, 378-382 (1959).

8. Frenkel, A. W., J. Am. Chem. Soc., 80, 3479-3480 (1958).

9. Frenkel, A. W., Brookhaven Symposia in Biol., 11, $276-287$ (1959).

10. Frenkel, A. W., Ann. Rev. Plant Physiol., I0, 53-70 (1959).

11. Jagendorf, A. T., Federation Proc., 18, 974-984 (1959).

12. Kluyver, A. J., and van Niel, C. B., in The Microbe's Contribution to Biology. pp. 73-92, Harvard University P'ress, Cambridge, Mass. (1956).

13. Krogman, D. W., and Vennesland, 13., J. Biol. Chem., 234, 2205-2210 (1959).

14. Larsen, H., Yocum, C. S., and van Niel, C. B., J. Gen. Physiol., 36, 161-171 (1952).

15. Rabinowitch, E., Photosynthesis, V'ol. II, part 2, Interscience Publ., New York, N. Y. (19:56).

16. Smith, L., and Baltscheffsky, M., I. Biol. Chem., 234, 1575-1579 (1959).

17. Stanier, R. Y., Doudorolf. M., Kunisawa, R., and Contopoulou, R., Proc. Nat. Acad. Sci. U.S., 45, $1246-1260$ (1959).

18. van Niel, C. B., Adrances in Enzymol., 1, 263-328 (19-11).

19. Vernon, L. P., and Ash, O. K., J. Biol. Chem., 234, 1878-1882 (1959). 
ON THE REDUCTIVE DEPHOSPHORYLATION HYPOTHESIS OF PHOTOSYNTHETIC REDUCTANT FORMATION

\author{
D. D. Hendley and B. L. Strehler
}

Gerontology Branch, National Heart Institute, National Institutes of Health, Bethesda, Maryland, and the Baltimore City Hospitals, Baltimore, Maryland

\title{
INTRODUCTION
}

Among the possible functions of ATP in photosynthesis pointed out in 1950 by Kandler (I2) and proposed in 1951 at the Second Phosphorus Symposium in relation to the demonstration of a light-induced ATP synthesis in intact Chlorella by Strehler (I6), is the production of a more reduced compound than the initial photoproduced reductant through a process analogous to the reverse of oxidative phosphorylation. It was postulated that (I) a portion of the initial oxidants and reductants produced by the photochemical apparatus recombined in a coupled phosphorylation, and (2) this was followed by a utilization of a portion of the ATP thus formed to drive electrons from the level of the photoproduced reductant to that of hydrogen carriers such as the pyridine nucleotides at the reduction level of carbohydrates. A critical test of this hypothesis was not feasible at that time because of the probable concurrent utilization of ATP in driving the carbon assimilation cycle (6), although the steadystate depression of ATP at high illuminating intensities even in the absence of $\mathrm{CO}_{2}$ was suggestive of a light-driven ATP utilization (17) . Our report in 1956 (I8) of a lack of light-induced pyridine nucleotide reduction in the presence of $\mathrm{CO}_{2}$ as measured by fluorescence assay in intact Chlorella, and its confirmation by Chance and Sager (7) through sensitive spectrophotometric measurements in a Chlamydomonas mutant further challenged the hypothesis (20) of pyridine nucleotide reduction as the primary photochemical reaction.

Several years after the demonstration of photochemical formation of ATP in intact Chlorella, Frenkel (8) and Arnon and co-workers $(2,3,1)$, in a series of elegant experiments, established unequivocally that this process occurred anaerobically in the photochemical apparatus rather than via a mitochondrial oxidative phosphorylation.

The work from Arnon's laboratory and the parallel intensive studies 
of Jagendorf $(11,4,5)$, his collaborators, and others $(15,19,9,13)$, have since elucidated many features of the process, although the exact mode of ATP formation and TPN reduction is still an open question.

In 1957 Marrè and Forti (13), on the basis of inhibitor studies, attempted to rule out the reductive dephosphorylation hypotheses set forth by Kandler and by Strehler. Subsequently Avron and Jagendorf (5) presented data which was interpreted as a lack of ATP-phosphate-exchange in illuminated chloroplasts in the presence of Hill reagents, a result having pertinence to the above question. However, even though it had been demonstrated that the Hill oxidant he employed (ferricyanide) was reduced through a coupled phosphorylation, much as was TPN, it was not shown that pyridine nucleotide reduction is unaccompanied by phosphate-ATP exchange. In the latter case the potential might well have been expected to be determinative.

The present studies were therefore undertaken in order to test more critically the hypothesis set forth in 1951. It has been found that there is indeed a light-induced incorporation of $\mathrm{P}_{i}^{32}$ into added ATP in the presence of TPN (and indeed of other Hill oxidants) and spinach chloroplasts, amounting to as much as 30 per cent of the rate of ADP photophosphorylation. The results indicate that the exchange is largely the result of a dark ATPase activity followed by photophosphorylation.

\section{Materials and Methods}

Spinach chloroplasts were prepared according to the method of Jagendorf $(11)$ in buffered sucrose solution $(150 \mathrm{ml})$ by grinding spinach leaves $(30.0 \mathrm{~g})$, macerated with scissors after a prior 30 minute illumination, for 1 minute in a chilled mortar and pestle. After filtering through cheesecloth, debris was removed at $0^{\circ} \mathrm{C}$ by centrifugation at $500 \mathrm{~g}$ for 1 minute, followed by 4 minutes at $2000 \mathrm{~g}$ to remove the chloroplasts from fragments in the supernatant liquid. They were then resuspended in sucrose medium $(4 \mathrm{ml})$, washed once by centrifugation, suspended in $3 \mathrm{ml}$ of sucrose medium, and kept at $0^{\circ} \mathrm{C}$ and in the dark until used.

At zero time an appropriate aliquot was pipetted into a reaction chamber illuminated by 2 20-watt fluorescent bulbs ( 2 inches between light and sample) at $15^{\circ} \mathrm{C}$. The experiments were teminated by adding an equal volume of $5 \%$ perchloric acid to the mixture. Samples were chilled to $0^{\circ} \mathrm{C}$.

After 30 minutes or longer, the solids were removed by centrifugation, the mucleotides were adsorbed onto activated charcoal, which 
Wats washed with water and $\mathrm{KH}_{2} \mathrm{PO}_{4}(0.005 \mathrm{M})$, then resuspended in $50 \%$ aqueous ethanol containing $0.5 \% \mathrm{v} / \mathrm{v}$ conc. $\mathrm{NH}_{3}$ and $0.2 \%$ methyl cellulose. Aliquots were plated on planchets, dried, and counted with an end-window Geiger-Miiller counter.

ATP (Sigma, sodium salt) solutions were freshly prepared for each run at $p H$ 7.5. TPNH, TPN, FMN, and PMS (phenazine methosulfate) were obtained from the Sigma Chemical Company.

\section{EXPERIMENTAL RESULTS}

A. ATP-phosphate exchange in presence of TPN. The typical rate of $\mathrm{P}_{\mathbf{i}}{ }^{2}$ incorporation into added ATP in the presence or absence of TPN is shown as a function of the time of illumination in Fig. I.

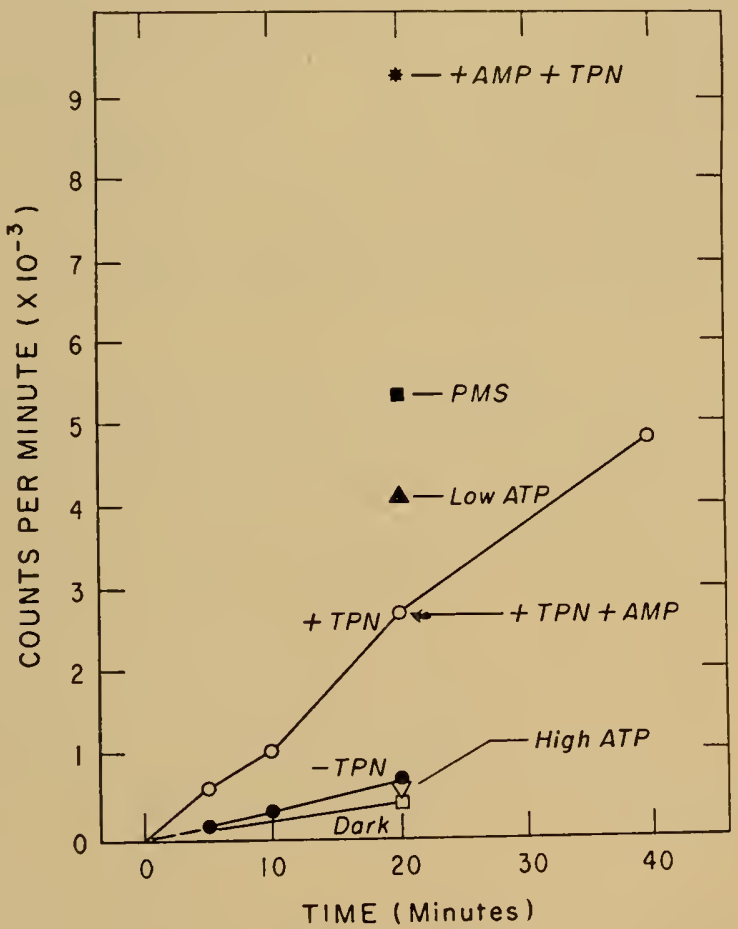

Fig. 1. Incorporation of $P_{1}^{32}$ into $A T P$ or $A M P$ moler various conditions. Each lube contained, in $\mu$ moles: $\mathrm{K}_{2} \mathrm{HPO}_{4}, 1.18$; ATP, 1.2 $(\mathbf{\Delta})$, or AMP, $1.2\left(^{*}\right)$; TPN, 0.75 (*, A. O, $\left.\nabla, \square\right)$, or PMS, (phenazine methosulfate) 0.05 (a); $\mathbf{M g C l}_{2}, 2.36 ; \mathrm{NaCl}, 11.9$; tris-HCl, $p \mathrm{H}$ 8.0, 13.3. $0.1 \mathrm{ml}$ chloroplast suspension containing $148 \mu \mathrm{g}$ chlorophyll, and $\mathrm{P}_{1}{ }^{32}, 2.8 \times 10^{5} \mathrm{counts} / \mathrm{min}$ were also added. Total volume, $1.6 \mathrm{ml}$, temp $=15^{\circ} \mathrm{C}$. Ordinate values are counts/min adsorbed on charcoal from aliquots corresponding $100.174 \mathrm{ml}$ reaction mixuture. 
Reagents are as indicated. Note particularly the points marked high and low ATP.

B. ATP-phosphate exchange in the presence of other factors. Table 1 illustrates the relative rates of $\mathrm{P}_{\mathrm{i}}^{32}$ incorporation into added ATP in the presence of other cofactors.

C. Effect of $A T P$ and TPN concentration on $P_{i}{ }^{32}$ incorporation. The data presented in Fig. 1 indicate that the exchange reaction is inhibited by higher ATP concentrations. A more detailed series of experiments is illustrated in Fig. 2, in which the relative rate of incorporation of $\mathrm{P}_{\mathrm{i}}^{32}$ into ATP is plotted against ATP concentration. The percentage turnover of added ATP is likewise shown in Fig. 2, as is the dependence of the reaction upon TPN concentration at a constant ATP concentration of $1.9 \times 10^{-4} M$. It should be noted that the inhibition of the $\mathrm{P}_{\mathrm{i}}{ }^{32}$-ATP exchange at higher levels of ATP has varied greatly in magnitude from experiment to experiment and sometimes cannot be demonstrated. The experimental conditions responsible for this variation have not been established.

The data presented in Fig. 3 indicate that ATP both stimulates and inhibits photophosphorylation of AMP, depending on its concentration. The stimulation at low levels of ATP is perhaps due to formation of ADP by myokinase.

On the mechanism of ATP-labeling. Evidence that the reaction is largely made up of a dark ATPase activity followed by photophosphorylation is given by the experiments reported in Table 2. It is apparent that incubation of ATP in the dark with chloroplasts li-

\section{TABLEE⿱日十 1}

Factors added

Relative Rate

\begin{tabular}{lccccc} 
& Exp. 1 & Exp. 2 & Exp. 3 & Exp. 4 & Exp. 5 \\
\cline { 2 - 5 } & 3.9 & 1.0 & & 6 & 2.8 \\
TPN & 9.0 & 5.1 & 5.0 & & 17.0 \\
PMS & & & 2.8 & & \\
FMN and ascorbate & & & 2.8 & & \\
Menadione and ascorbate & 1.0 & & 1.8 & & 6.5 \\
Fe +++ CN & & & 1.0 & 1 & 1.0 \\
TPNH & & & & \\
No oxidant & & &
\end{tabular}

Relative rates of $\mathrm{P}_{i}^{32}-\mathrm{ATP}$ exchange in the presence of various cofactors. In the various experiments shown each tube contained: $\mathrm{K}_{2} \mathrm{HPO}_{4}, 1.0 \times 10^{-3} \mathrm{M} ; \mathrm{NaCl}$, $1.0 \times 10^{-2} \mathrm{M} ; \mathrm{MgCl}_{2}, \times 10^{-3} \mathrm{M}$; tris- $\mathrm{HCl}, p \mathrm{H} 8.0,1.2 \times 10^{-2} \mathrm{M}$, and chloroplasts containing 60 to $132 \mu \mathrm{g} / \mathrm{ml}$. Where indicated each tube also contained the following: TPN, 7 to $9 \times 10^{-4} \mathrm{M}$; PMS, $5 \times 10^{-5} M ; \mathrm{FMN}, 2 \times 10^{-4} . M$; ascorbate, $5 \times 10^{-3} \mathrm{M}$; incnadione, saturated solution in $\mathrm{H}_{2} \mathrm{O}$ diluted $10 \times \mathrm{Fc}(\mathrm{CN})_{8}^{-3}, 1$ to $2 \times 10^{-3} \mathrm{M}$; TPNH, $1 \times 10^{-3} \mathrm{M}$. Incubations $20 \mathrm{~min}$ at $15^{\circ} \mathrm{C}$. 


\section{$P^{32}$ INCORPORATION VS.ATP, TPN CONCENTRATION}

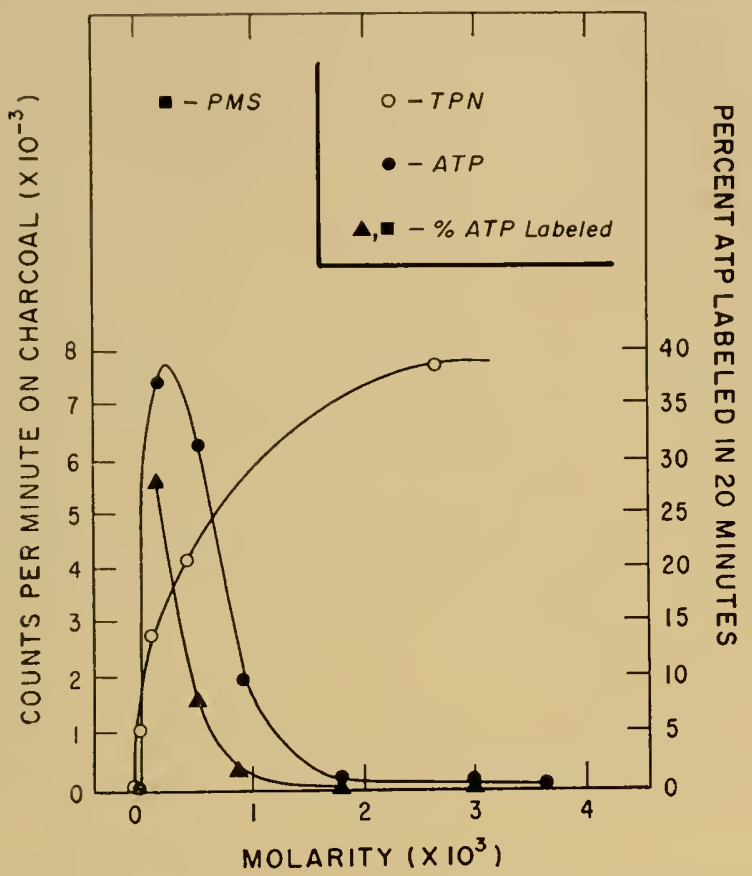

lig. 2. $P_{i}{ }^{32}$ incorporation is. ATP, TPN concentration. Expt. 1: [TPN] varied as shown. Each tube also contained, in $\mu$ moles: $\mathrm{K}_{2} \mathrm{HPO}_{4}, 1.18 ; \mathrm{ATP}, 0.2 ; \mathrm{MgCl}_{2}$, 2.36; $\mathrm{NaCl}, 11.9$; tris- $\mathrm{HCl}$ buffer $p \mathrm{H} 8.0,13.3 ; 0.1 \mathrm{ml}$ chloroplast suspension. $\mathrm{P}_{1}{ }^{32}$, I.63 $610^{5}$ counts $/ \mathrm{min}$. Vol. $=1.13 \mathrm{ml}$. Expt. 2; [ATP] varied as shown. Each tube also contained TPN. $1.0 \mu$ moles $(\bullet, \mathbf{A})$ or PMS, $0.05 \mu$ moles ( $\boldsymbol{\square}$ ); salts, buffer, and $P_{1}^{32}$ as in expt. I, $116 \mu \mathrm{g}$ chJorophyll. Vol. $=1.11 \mathrm{ml},(\mathbf{\Lambda}, \overline{\mathbf{Q}}): \%$ of ATl labeled, assuming single labeling. In both experiments left-hand ordinate represents total counts/min absorable on charcoal after $20 \mathrm{~min}$ incubation at $15^{\circ} \mathrm{C}$.

berates a component capable of being phosphorylated by a succeeding light period under appropriate conditions. In other experiments not tabulated, it was shown that the presence of ATP during the dark incubation was necessary for the stimulation of $\mathbf{P}_{i}^{32}$ incorporation in a succeeding light period.

Fig. 4 shows the effect of dark preincubation on the kinetics of incorporation of $P_{i}{ }^{32}$ in the light. After 20 minutes of dark incubation, 5 minutes of illumination results in as much $P_{i}{ }^{32}$ incorporation as does 20 minutes of illumination without a preceding dark period.

It would appear from the foregoing data that photophosphorylation of acceptor formed from ATP in the dark explains most of the 


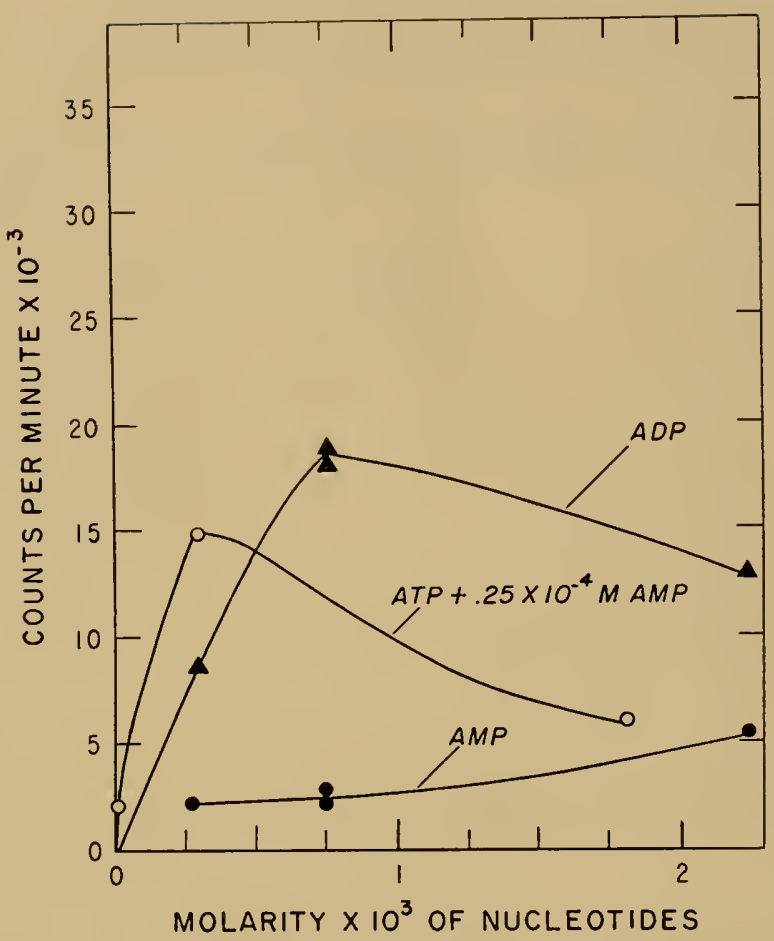

Fig. 3. Effect of nucleotide concentration on rate of $P_{i}{ }^{32}$ incorporation. Each ube contained: AMP, ADP or $0.4 \mu$ moles AMP + ATP as indicated; $\mathrm{K}_{2} \mathrm{HPO}_{4}$, 1.68 mmoles; PMS, 0.05 mmoles; $\mathrm{MgCl}_{2}, 3.38$ moles; NaCl. 17.0 $\mu$ moles; tris-HCl,

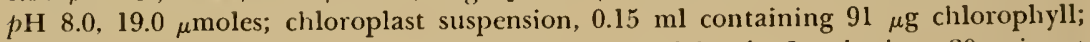
and $P_{1}{ }^{32}, 2.91 \times 10^{5}$ counts $/ m i n$. Final volume, $1.6 \mathrm{ml}$. Incubation, $20 \mathrm{~min}$ at $15^{\circ} \mathrm{C}$. Ordinate values are counts/min adsorbed on charcoal from aliquots corresponding to $0.306 \mathrm{ml}$ of reaction mixture.

incorporation of $\mathrm{P}_{1}^{32}$ into ATP. If there is in adclition a photochemical dephosphorylation of ATP, it would appear to be a small effect. The data, while thus revealing a significant ATPase activity in chloroplasts, do not support the thesis that ATP is utilized in the photoreduction of $\mathrm{TPN}$.

Of interest here is the light-dependent dephosphorylation of ATP recently reported by Petrack (14) in chloroplasts in which photophosphorylation was inhibited by high concentrations of sulfhydryl compounds. It remains to be seen whether this represents a reversal of one of the steps of photophosphorylation as suggested by the author, or perhaps the exposure of some other ATP-utilizing reaction somehow missed by the $\mathrm{P}_{1}^{32}$ incorporation experiments. 
P32 Incortoration fol lowing, Various Dakk Incuibiton Perions

Dark

Exp. conditions incubation Illumination Counts/min inin $\min$

Exp. A PMS at 0 min

+ PMS at 0 min

Exp. $13+$ PMS at 0 min, $0.05 \mu$ moles

+ PMS at 0 min, $0.05 \mu$ moles

+ PMS at 20 min, $0.05 \mu$ moles

(1)

$+\mathrm{PMS}$ at $0 \mathrm{~min}+.28 \mu$ moles

ATP at $20 \mathrm{~min}$

+ TPN, $0.2 \mu$ moles

+ TPNH, $0.2 \mu$ moles

+ TPN, $0.2 \mu$ moles

$\begin{array}{rrr}0 & 5 & 460 \\ 20 & 5 & 1830 \\ 0 & 5 & 650 \\ 20 & 5 & 1425 \\ 20 & 5 & 1530 \\ 20 & 5 & 1560 \\ 0 & 5 & 103 \\ 0 & 5 & 50 \\ 20 & 5 & 105\end{array}$

$\mathrm{P}_{\mathrm{i}}^{32}$ incorporation following various dark incubation periods.

Expt. A: Each tube contained, in $\mu$ moles: $\mathrm{K}_{2} \mathrm{HPO}_{4}$, 1.18; ATP, 0.4; PMS, 0.05; $\mathrm{MgCl}, 2.36: \mathrm{NaCl}, 11.9$ and tris- $\mathrm{HCl}, p \mathrm{H}$ 8.0, 13.3. $0.1 \mathrm{ml}$ chloroplast suspension containing $66 \mu \mathrm{g}$ chlorophyll and $\mathrm{P}_{\mathrm{i}}^{32}, 1.46 \times 10^{5}$ counts $/ \mathrm{min}$ were also added. Final volume, $1.0 \mathrm{ml}$. Counts are recorded for aliquots corresponding to $0.167 \mathrm{ml}$ of reaction mixture. Temp $=15^{\circ} \mathrm{C}$.

Expt. 13: Each cube contained in $\mu$ moles: $\mathrm{K}_{2} \mathrm{HPO}_{4}, 1.68$; ATP, 0.6; PMS, TPN TPNH, or additional ATP as indicated, $\mathrm{MgCl}_{4}, 3.38$; $\mathrm{NaCl}$, 170 ; tris- $\mathrm{HCl}, p \mathrm{H} 8.0$, 19.0. $0.15 \mathrm{ml}$ chloroplast suspension containing $100 \mu \mathrm{g}$ chlorophyll and $\mathrm{P}_{\mathrm{i}}^{32}, 1.2 \times 10^{5}$ counts $/ \mathrm{min}$ were also added. Final volume, $1.6 \mathrm{ml}$. Counts are recorded for aliquots corresponding to $0.192 \mathrm{ml}$ of reaction mixture. Temp $=15^{\circ} \mathrm{C}$.

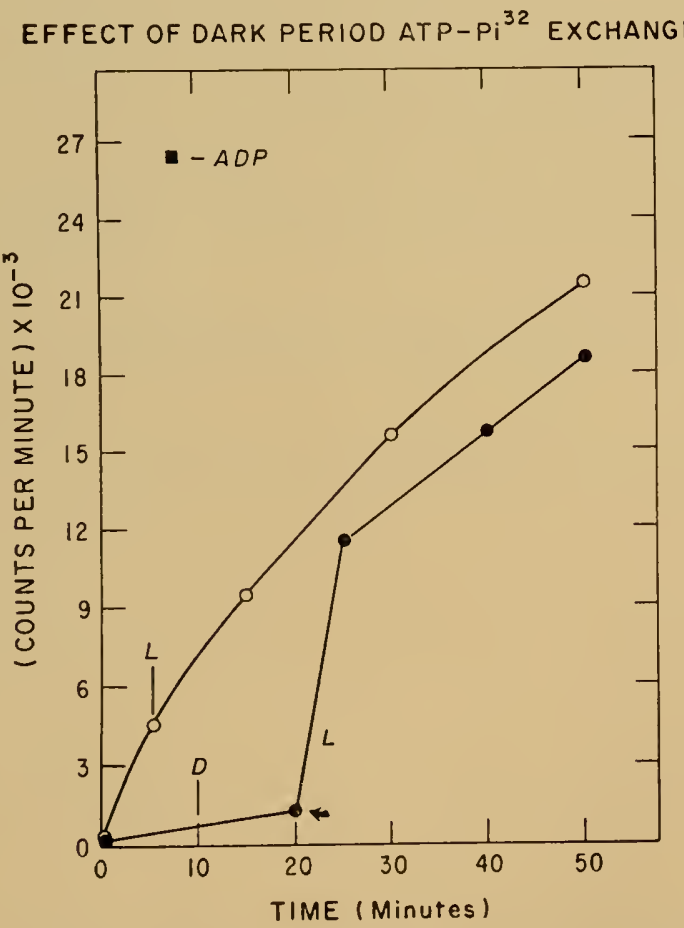

Fig. 4. Effect of clark incubation on subsequent ATP-P, exchange in the light. Each $1.0 \mathrm{ml}$ aliquot contained, in $\mu$ moles: $\mathrm{K}_{2} \mathrm{HPO}_{4}, 1.05 ; \mathrm{ATP}, 0.37$ (curres), or ADP, 0.37 (single point, as indicated); PMS, $0.30 ; \mathrm{IgCl}_{2}, 2.1 ; \mathrm{NaCl}, 10.4 ;$ tris- $\mathrm{HCl}, p \mathrm{H}$ 8.0, 11.6. Chloroplasts containing $72 \mu \mathrm{g}$ chlorophyll and $\mathrm{P}_{\mathrm{t}}^{32}, 1.15 \times 10^{5}$ counts/ min were also present. Ordinate values refer to total counts/min adsorbable on charcoal. Illumination started at zero time $(O, \mathbf{Q})$ or at $20 \mathrm{~min}(\bullet)$. 


\section{ACKNowledgments}

The authors are indebted to Mr. Malcolm Gee, Mrs. Susanne Rosche, and Mr. Samuel Crowder for invaluable technical assistance.

\section{REFERENCES}

1. Arnon, D. 1., in The Photochemical Apparatus, Its Structure and Function (Fuller, R. C., et al., eds.), Pp. 181-233, Brookhaven Natl. Lab., Upton, N. Y. (1958).

2. Arnon, D. I., Whatley, F. R., and Allen, M. B., J. Am. Chem. Soc., 76, 6324-6329 (1954).

3. Arnon, D. I., Whatley, F. R., and Allen, M. B., Biochim. et Biophys. Acta, 16, 605-608 (1955).

4. Avron, M., and Jagendorf, A., J. Biol. Chem., 234, 967-972 (1959).

5. Avron M., Jagendorf, A., Evans, M., Biochim. et Biophys. Acta, 26, 262-269 (1958).

6. Bassham, J. A., Benson, A. A., Kay, L. D., Harris, A. Z., Wilson, A. T., and Calvin, M., J. Am. Chem. Soc., 76, 1760-1770 (1954).

7. Chance, B., and Sager, R., Plant Physiol., 32, 548-561 (1957).

8. Frenkel, A., J. Am. Chem. Soc., 76, $5568-5569$ (1954).

9. Frenkel, A. W., Biochim. et Biophys. Acta, 26, 276-288 (1958).

10. Jagendorf, A. T., in The Photochemical Apparatus, Its Structure and Function. (Fuller, R. C., et al., eds.), pp. 181-235, Brookhaven Natl. Lab., Upton. N. Y. (1958).

11. Jagendorf, A., and Avron, M., J. Biol. Chem., 231, 277-290 (19.58).

12. Kandler, O., Z. Naturforsch., 5b, 423-437 (1950).

13. Marrè, E., and Forti, E., Science, 126, 976-977 (1957).

14. Petrack, B., Federation Proc., 19, (1). 329 (1960). (Abstract).

15. San Pietro, A., in The Photochemical Apparatus, Its Structure and Function (Fuller, R. C., et al., eds.), pp. 262-270, Brookhaven Natl. Lab., Upton, N. Y. (1958).

16. Strehler, B. L., in Phosphorus Metabolism, II (McElroy, W. D. and Glass, B., eds.), pp. 49I-502, Johns Hopkins l'ress, Baltimore (1952).

17. Strehler, B. L., Arch. Biochem. Biophys., 43, 67-79 (1953).

18. Strehler, B. L., in Rhythmic and Synthetic Processes in Growth (Rudnick, D.. ed.), Princeton Univ. Press (1957).

19. Vishniac, W., Biochim. et Biophys. Acta, 26, 54-64 (1958).

20. Vishniac, W., and Ochoa, S., J. Biol. Chem., 195, 75-93 (1952). 


\title{
CONTINUOUS MEASUREMENT OF PHOTOSYNTHETIC PHOSPHORYLATION WITH THE FIREFLY \\ LUMINESCENCE ASSAY SYSTEM
}

\author{
B. L. Strehler and D. D. Hendley
}

Gerontology Branch, National Heart Institute, National Institutes of Health, Bethesda, Maryland, and the Baltimore City Hospitals, Baltimore, Maryland

\section{INTRODUCTION}

In 1950, W. A. Arnold and one of the authors (24) attempted to measure ATP formation by illuminated chloroplasts, using the firefly luminescence assay method of Strehler and Totter (25). This method, which had been successfully used to measure oxidative phosphorylation in mitochondrial suspensions, is based upon the discovery by McElroy (14) that ATP will support the luminescence of firefly extracts and upon the subsequent description of the properties of the system by McElroy and collaborators $(17,16)$.

Although some stimulation of luminescence was noted when chloroplasts mixed with firefly extracts were illuminated, it was soon shown that the major effect, at least, was due to a luminescence emitted by the chloroplasts for a few seconds after they were illuminated. This photosynthetic luminescence has been intensively investigated by Arnold, Strehler, Franck, Calvin, and their collaborators (1, 3, 2, 22, 7, 10, 27) .

Subsequent experiments, using appropriate filter combinations, showed an erratic stimulation of firefly luminescence following the illumination of chloroplast-luciferase mixtures, but the reaction could not be studied systematically due to unknown sources of variability. Rather, we concentrated our efforts on the measurement of ATP changes induced in intact Chlorella $(21,23)$ and on the mechanism of photosynthetic luminescence.

Arnon's unequivocal demonstration of photosynthetic phosphorylation (6) in isolated chloroplasts and the rapid description of the properties and cofactors of the system made feasible a new attempt to measure changes of ATP level during illumination. In the present communication we describe a simple and sensitive method for the instantaneous measurement of ATP levels in illuminated chloro- 
plasts, and some properties and kinetics of the reaction under a variety of conditions. Of interest are certain experiments in which the ATP level is lowered by illumination. The stimulation of photophosphorylation by added folic acid is also reported.

\section{Material AND Methods}

(a) Chloroplasts. Washed spinach chloroplasts were prepared as described elsewhere (11) and were stored at $0^{\circ} \mathrm{C}$ in the dark until use.

(b) Firefly enzyme. Crude firefly luciferase (26) was freshly prepared by exhaustively grinding $\mathrm{I} 2$ vacuum-dried firefly lanterns (Photinus pyralis) with sea sand in a mortar and pestle, followed by extraction with $7 \mathrm{ml}$ of $0.01 M$ phosphate buffer, $p \mathrm{H} 7.4$ at $0^{\circ} \mathrm{C}$. The solution was filtered into a test tube and stored until use at $0^{\circ} \mathrm{C}$.

(c) The chloroplast and firefly enzyme mixtures were illuminated with a Bausch \& Lomb 6 v-18 amp ribbon filament microscope lamp burned at an overvoltage. The beam was collimated and projected through a water-filled heat filter and four red filters in series. The four red filters were found to be necessary to remove all traces of blue light from the illuminating beam. The reaction mixture was illuminated in a thin lucite cuvette. The blue-green fraction of light emitted within the chamber because of the luminescence of the firefly enzyme was transmitted directly to the end window of a 5819 RCA photomultiplier by two filters sandwiched between the cuvette and photomultiplier.

The direct current output of the detector was amplified by an Aminco photometer whose ontput was applied to a one-second Brown Recorcler. High voltage was furnished by a Baird Atomic Instrument, Model 312.

Details of the illuminating and measuring arrangement are illustrated in Fig. 1.

(c) ATP, ADP, AMP, DPN, TPN, DPNH, TPNH, cytochrome $c$. FMN, PMS (phenarine methosulfate), and myokinase were obtained from Sigma Chemical Company, St. Louis, Missouri. Folic acid, menadione, and $\alpha$-tocopherol were obtained from Nutritional Biochemical Corporation, Cleveland, Ohio.

\section{ExPIRIMINTAL, RESULTS}

(a) Kinctics of the reaction. In Fig. 2 is illustrated the response of the system to successive periods of light and dark. Note particularly 

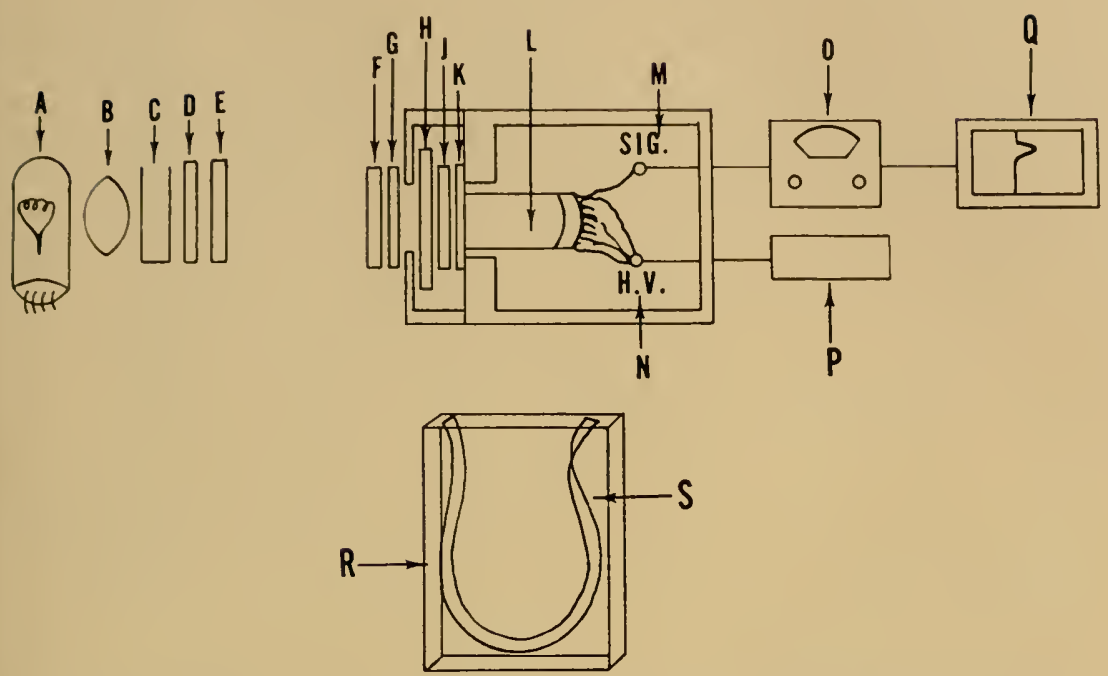

Fig. 1. Apparatus used in continuous measurement of ATP levels during photosynthetic phospliorylation. See text for detailed description.

Symbols:

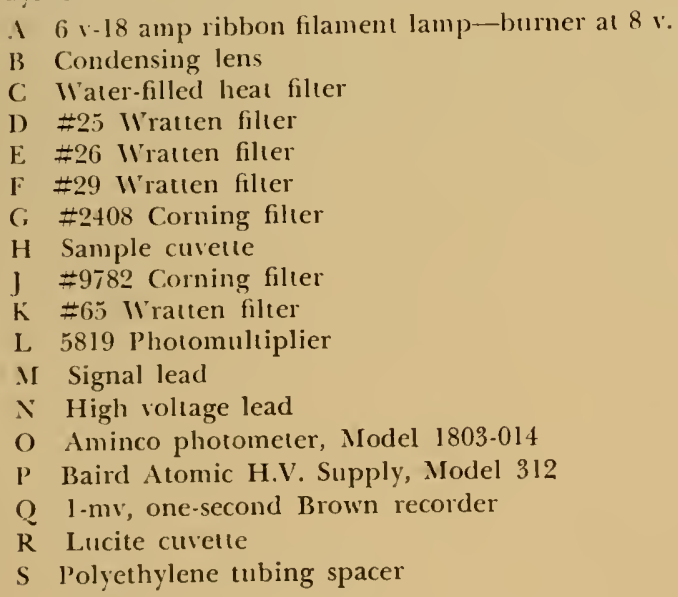

the rapid decay of luminescence following the cessation of illumination and that the steady dark level gradually increases with successive light periods.

The time course of the rise in Iuminescence on initial illumination is depicted in Fig. 3. Apparently the rate of ATP synthesis is quite high after a few seconds of illumination despite the apparent absence of ADP or ATP in the reaction mixture prior to illumination, as 


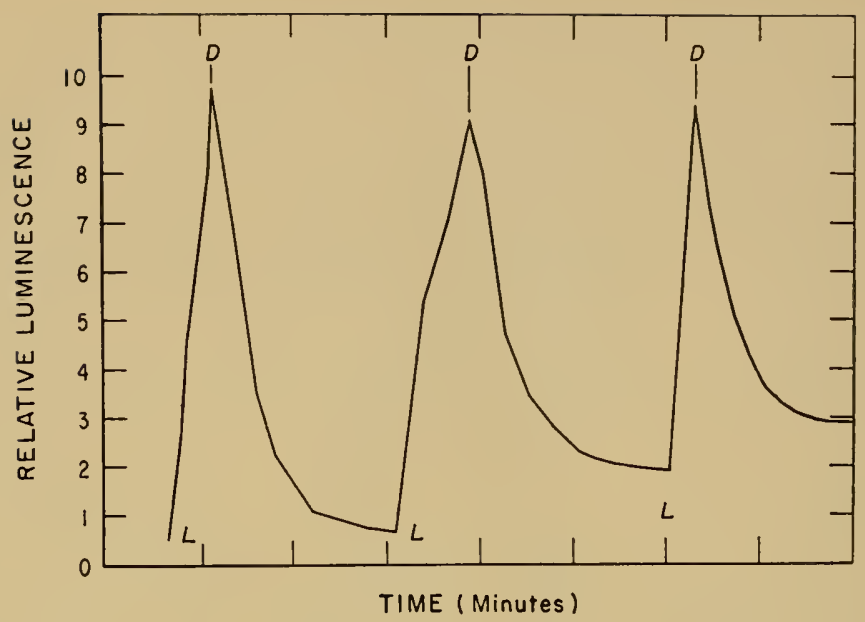

Fig. 2. Effect of successive periods of illumination and darkness on ATP levels in mixtures of firefly enzyme and chloroplasts. Reaction mixture contained:

$0.2 \mathrm{ml}$ firefly enzyme

$1.4 \mathrm{ml}$ suspension medium containing, per $\mathrm{ml}$ the following:

$1.6 \mu$ mole $\mathrm{K}_{2} \mathrm{HPO}_{4}$

$17.0 \mu$ moles $\mathrm{NaCl}$

$3.38 \mu$ moles $\mathrm{MgCl}_{2}$

$19.0 \mu$ moles tris- $\mathrm{HCl}$

buffer $p \mathrm{H} 8$

$0.1 \mathrm{ml}$ PMS $\left(5 \times 10^{-4} M\right.$ solution $)$

$0.1 \mathrm{ml} \mathrm{AMP}\left(2 \times 10^{-2} M\right.$ solution $)$

$0.2 \mathrm{ml}$ Chloroplast suspension (about $700 \mathrm{mg}$ chlorophyll/ml suspension)

All reagents taken to $p H$ 7.5-8.0 before adding to nixture; light intensity approximately $350 \mathrm{ft}$-candles. L, light; D, dark.

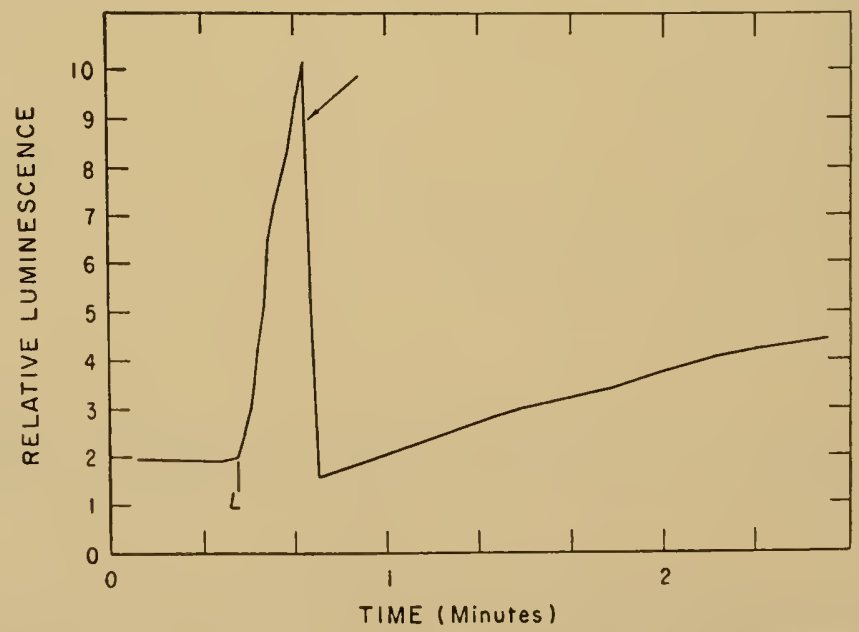

Fig. 3. Time course of luminescence during first illumination periol, without addition of . ITP or .11)P.

Sensitivity changed at arrow.

Reaction mixture, same as in Fig. 2, except that $0.1 \mathrm{ml}$ firefly enzyme was used. 
indicated by a complete absence of luminescence prior to the first illumination.

(b) Effects of myokinase. That the rapid decay in the dark period following illumination is largely due to the myokinase contained in crude furefly enzyme (15) is shown by the results depicted in Fig. 4. In this experiment excess myokinase was added at the indicated time. Note that the rate of increase of luminescence is much depressed in succeeding periods of illmmination. Moreover the decay in the subsequent dark period is also depressed or absent.

(c) Effect of colactors. Fig. 5 illustrates the effect of TPN on the rate of phosphorylation by chloroplasts. The following factors were also effective: TPNH, folic acid, cytochrome $c$, ferricyanide, PMS, and FMN (4, 5, 8, 12).

(d) Destruction of ATP by light. One of the objectives of these experiments was to determine whether illumination will cause utilization of ATP under conditions where reductive dephosphorylation (21) might have been expected to occur, as postulated by Kandler in 1950 (13) and by Strehler in 1952 (23). Although under most conclitions illumination caused an increase in ATP level as measured by luminescence, we have occasionally noticed a decrease in luminescence on exposure to light under as yet undefined conditions. Such

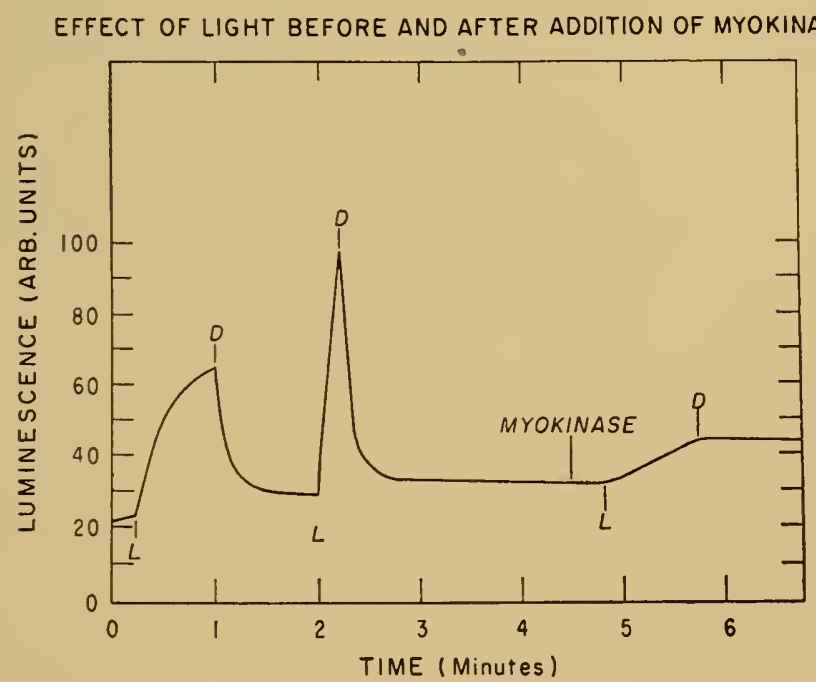

Fig. 4. Effect of addition of myokinase on luminescence during and after illumination.

Conditions as in Fig. 2, except that $0.1 \mathrm{ml}$ myokinase was added at $4.5 \mathrm{~min}$. First illumination with 0.3 O.D.U. filter in actinic beam. L, light on. D, light off. 


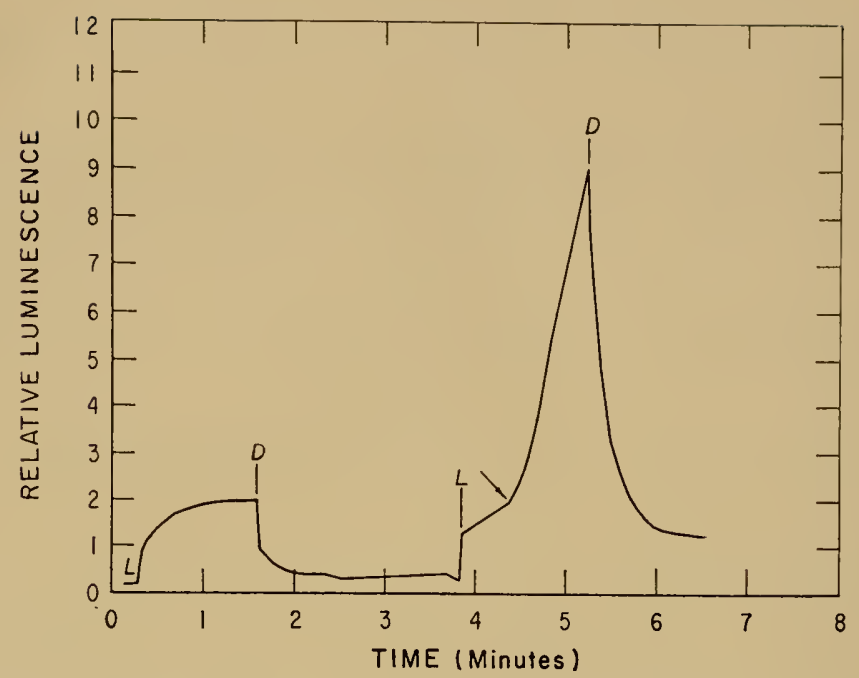

Fig. 5. Effect of addition of TPN on photosynthetic phosphorylation in mixtures of chloroplasts and firefly enzyme.

Conditions as in Fig. 2 except that TPN ( $1 \mu$ mole) was adoled at the time indicated by the arrow, in place of PMS.

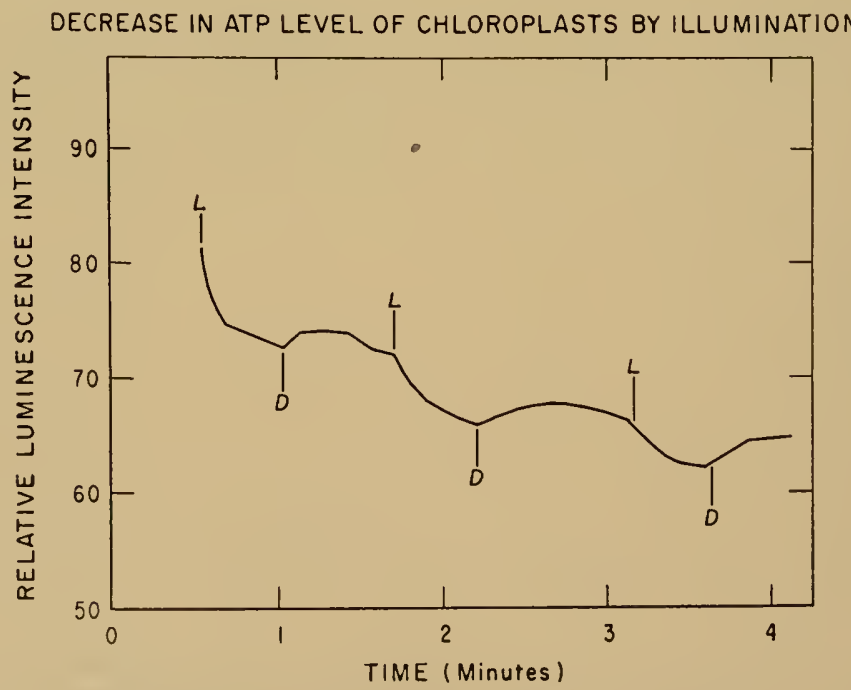

Fig. 6. Evidence that illumination causes a reversible decrease in the ATP level of mixtmes of chloroplasts and firefly enzyme.

The reaction mixtuse contained $0.2 \mathrm{ml}$ firefly emame; $0.3 \mathrm{ml}$ chloroplast sus

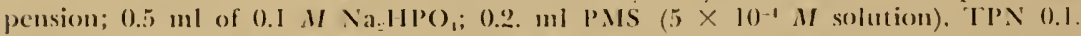
inl (1.0 $\times 10^{-2} \mathrm{M}$ solution) $0.105 \mathrm{ml}$ AMll; $0.05 \mathrm{ml}$ myokinase solution; $1.4 \mathrm{ml}$ suspension medium. The reaction medium had been illuminated for about 10 minutes prior to the beginuing of the tracing. L, light on; 1), light ofl. 
a finding is consistent with the hypothesis set forth. One such experiment is illustrated in Fig. 6. The recent funding by Petrack (24) of a light-dependent ATPase activity in chloroplasts in the presence of high levels of sulfhydryl compounds is consistent with the above results and interpretation.

\section{Discussion}

The above findings clearly demonstrate the applicability of the firefly luminescence assay to the instantaneous and continuous measurement of photosynthetic phosphorylation. Disadvantages of the method for certain applications inchude the facts that ADP will produce a high background luminescence due to myokinase action, that the rapid decay in the dark in the absence of adequate myokinase distorts the kinetics of the reaction, and that the decay of luminescence, either because of the flash phenomenon described earlier (17, 18) or the slow deterioration of the enzyme by denaturation or product inhibition (20), may contribute in some unknown manner to the kinetics. It was possible rapidly to confirm many of the original observations of Arnon et al. and of Jagendorf and his collaborators on the interaction of cofactors and Hill oxidants with the phosphorylating system. In adclition, the early kinetics of the reaction, the absence of appreciable lag under the conditions used, and the apparent efficiency of AMP as an acceptor molecule were demonstrated. The absence of induction periods may be due to the myokinase activity of the furefly enzyme, although the complete absence of luminescence prior to illumination and the rapid response cast some doubt on this explanation. In other experiments (11) we have shown that small amounts of ATP facilitate the incorporation of $\mathrm{P}_{\mathrm{i}}{ }^{32}$-label into added AMP in the light. Nevertheless, it seems possible that AMP itself is an efficient direct acceptor under the present conditions.

The stimulation of phosphorylation by folic acid may have little direct bearing on the normal mechanism, although the presence of this factor in photosynthetic tissues is suggestive of a more direct function. The mode of its action in vivo, if any, must await further study.

The negative finding with thioctic acid and thioctamide does not support the proposal of Calvin (27) that closely related cyclic dithiols participate in the early photochemical process.

Whether or not the decrease in ATP content during illumination, as reported here, supports the theory set forth in 1952 on the basis of intact cell studies (21) must await a further study of conditions 
affecting the reaction and a study of the mechanism by independent means.

\section{ACKNOWLEDGMENTS}

The authors are indebted to Mrs. Susanne Rosche and Mr. Malcolm Gee for expert technical assistance, and to the Sigma Chemical Company. St. Louis, Missouri, and to Jan and Butch Strehler for gifts of firellies.

\section{REFERENCES}

1. Arnold, W. A., and Davidson, J. B., J. Gen. Physiol., 37, 677-684 (1954).

2. Arnold, W. A., and Maclay, $\mathrm{H}$., et al., in The Photochemical Apparalus, Its Structure and Function (Fuller, R. C., eds.), pp. 1-9, Brookhaven Nat. Lab., Upton, N. Y. (1958).

3. Amold, W. A., and Sherwood, H., Pror. Nat. Acad. Sci. U. S., 43, 105-113 (1957).

4. Armon, D. I., Ann. Rev. Plant Physiol., 1, 325-350 (1956).

5. Amon, D. I., et al., in The Photochemical Apparatus, Its Structure and Function (Fuller, R. C., eds.), pp. 181-235, Brookhaven Nat. Lal)., Upton. N. Y. (1958).

9. Barltrop, J. A., Hayes, P. M., and Calvin, M., J. Am. Chem. Soc., 76, 4348-4352 6329 (1954).

7. Arthur, W. E., and Strehler, B. L., Arch. Biochem. and Biophy's., 70, 507-526 (1957).

8. Avron, M., and Jagendorf, A., J. Biol. Chem., 234, 967-972 (I959).

9. Barltrop, J. A., Hayes, P. M., and Calvin, M., J. Am. Chem. Soc., 76, 4348-4352 (1954).

10. Brugger, J. E., in Research in Photosynthesis (Galfron, H.. et al., eds.), pp. 13514], Interscience Publishers, New York (195i).

11. Hendley, D. D., and Strehler, B. L., this symposium.

12. Jagendorf, A., in The Photochemical Apparatus, Its Structure and finction (Fuller, R. C., et al., eds.), pp. 236-258, Brooklaten Nat. Lab), Lpton, N. Y. (1958).

13. Kandler, O., Z. Naturforsch., 5b, 423-437 (1950).

14. NicElroy, W. D., Proc. Nat. Acad. Sri. U. S., 33, 342-345 (1947).

15. McElroy, W. 1)., J. Biol. Chem., 19I, 547-5.57 (1951).

16. McElroy, W. D., Hastings, J. W., Coulombre, J., and Sonmenfeld, V'., Aich. Bio. chem. and Biophys., 46, 399-407 (1953).

17. McElroy, W. D., and Strehler, B. L.., Arch. Biochem., 22, 420-433 (1919).

18. McElioy, W. D., and Strehler, B. L.., Bacteriol. Rers. I8, 177-194 (19.1).

19. Petrack, B., Federation Proc., I9 (1), 329 (1960). (Al)stract).

20. Rhodes, W. C., and McElroy, W. D., Science, 128, 253-254 (1958).

21. Strebler, 13. J... in Phosphorus Metabolism, II (NcElroy, W'. D., and (;lass, B.. ecls.), pp. 491-502. The Johns Hopkins l'ress, Baltimore (19:52).

22. Strehler, B. L., Arch. Biochem. and Biophys., 34. 239-248 (1951).

23. Strchler, 13. L.. Airh. Biochem. and Biophys., 43, 67.79 (1953).

24. Streliler, B. L.., and Arnold, W. A., I. Cen. Physiol., 34, 809-820 (1951).

25. Strehler, B. L., and Totter, J. R., Arch. Biochem. and Biophys., 40, 28-41 (1952).

26. Strehler, B. 1.., and Totter, I. R., in Methods of Biochemical Analysis, V'ot I. (Glick, D., ed.), pp. 169.201, Interscience Publishers, New York (1951).

27. Tollin, G., and Calvin, M., Proc. Nat. Acad. Sci. L'. S., 43, 895.902 (1957). 


\section{THE EFFECT OF OXYGEN ON PHOTOPHOSPHORYLATION} ANI) THE EFFECT OF CARBONATE ON THE

\section{HILL REACTION ${ }^{1}$}

\section{Birgit Vennesland, T. Nakamoto, and Babette Stern}

Department of Biochemistry, University of Chicago

Chicago, lllinois

The effect of oxygen on photophosphorylation by spinach chloroplasts with FMN as a cofactor has been described previously (4). At low cofactor concentrations, the photophosphorylation is completely dependent on oxygen, but with increasing cofactor concentrations, the reaction appears to proceed as well anaerobically as aerobically. A parallel study with menadione $\left(\mathrm{K}_{3}\right)$ has shown similar results. At about $2 \times 10^{-6} M \mathrm{~K}_{3}$, optimal photophosphorylation occurs acrobically, but the "anaerobic" rate is quite small. With 10 to 40 times as much $\mathrm{K}_{3}$, the rate in nitrogen approaches that in air. With $\mathrm{K}_{3}$, air sometimes becomes inhibitory at higher cofactor concentrations. Similar studies with phenazine methosulfate and pyocyanine also show that at low cofactor concentrations, oxygen has a stimulatory effect.

The stimulatory effect of oxygen suggested that the photophosphorylation was accompanying a cyclic process in which the added cofactor is photoreduced, with $\mathrm{O}_{2}$ evolution, and is then reoxidized by molecular oxygen (Equations 1 and 2).

$$
\begin{aligned}
& \text { cofactor }+\left(\mathrm{H}_{2} \mathrm{O}\right) \stackrel{h \nu}{\longrightarrow} \text { reduced cofactor }+1 / 2 \mathrm{O}_{2} \\
& \text { reduced cofactor }+1 / 2 \mathrm{O}_{2} \stackrel{h \nu}{\longrightarrow} \text { cofactor }+\mathrm{H}_{2} \mathrm{O}
\end{aligned}
$$

This was confirmed by the demonstration that both FMN and $\mathrm{K}_{3}$ elicit a rapid oxygen exchange reaction, as measured with $\mathrm{O}^{18}$. Some representative data of Nakamoto, Krogmann, and Mayne (3) are shown in Table 1.

It is our opinion that chloroplasts which have not suffered damage to their oxygen-evolving mechanism prefer a cycling process in which

'Supported by a grant from the National Science Foundation. 
TABLE 1

Oxygen Exchange Measured with $\mathrm{O}^{\text {I8 }}$

Cofactor

$\mu$ moles $P_{\mathbf{i}}$ esterified

$\mu$ atoms oxygen

Consumed Produced

none

$2 \times 10^{-5} M$ FMN

$2 \times 10^{-5} M$ FMN

(plus $8 \times 10^{-7} M$ DCMU)

$1.2 \times 10^{-5} M \mathrm{~K}_{3}$

$1.2 \times 10^{-5} \mathrm{M} \mathrm{K}_{3}$

(plus $4 \times 10^{-7} M \mathrm{DCMU}$ )

$22 \quad 14$

$208 \quad 195$

$22 \quad 11$

$\begin{array}{lll}3 & 22 & 11\end{array}$

$\begin{array}{lll}56 & 176 & 174\end{array}$

$\begin{array}{lll}4 & 36 & 22\end{array}$

All values are per mg chlorophyll per hour. DCMU, dichlorophenyl dimethyl urea. Data of Nakamoto, Krogmann, and Mayne (3).

oxygen is evolved and reconsumed when FMN or menadione are cofactors. A truly anaerobic cycling involving neither production nor reconsumption of molecular oxygen can apparently occur also, and is most pronounced with pyocyanine and phenazine methosulfate, less so with $\mathrm{K}_{3}$, and hardly at all with FMN. The evidence for this statement is complex. One line of evidence is derived from the inhibitory effects of oxygen observed under certain circumstances. This aspect of the problem has been discussed by Jagendorf, and we add only the statement that we are in general agreement with his conchusions. Another line of evidence derives from the effect of catalase and ethanol on the photophosphorylation reactions. These reagents serve as a trap for $\mathrm{H}_{2} \mathrm{O}_{2}(1,2)$. If $\mathrm{FMN}$ or $\mathrm{K}_{3}$ are employed at concentrations which give optimal photophosphorylation rates in an atmosphere of $\mathrm{H}_{2}$, added catalase and ethanol strongly inhibit the phosphorylation. Some representative data are shown in Table 2. The cofactor concentrations here employed were sufficient to give optimal "anaerobic"

TABLE 2

Inibition of "Anaerobic" Puosphorylation by Catalase-Ethanol

\begin{tabular}{|c|c|c|c|}
\hline Cofactor & Catalasc-ethanol & Added from side arm & $-\Delta \mathbf{P}_{\mathrm{i}}$ \\
\hline $2 \times 10^{-4} M \mathrm{FMN}$ & - & $\mathbf{P}_{\mathrm{i}}$ & 2.9 \\
\hline$" \quad "$ & - & $P_{i} \cdot F M N$ & 2.9 \\
\hline$"$ & + & $\mathrm{P}_{\mathrm{i}}$ & 0.0 \\
\hline$"$ & + & $\mathrm{P}_{\mathrm{i}} \cdot \mathrm{F} M \mathrm{MN}$ & 0.4 \\
\hline $1.2 \times 10^{-4} M / \mathrm{K}_{3}$ & - & $P_{i}$ & 3.6 \\
\hline$"$ & - & $P_{i} \cdot K_{3}$ & 3.6 \\
\hline$"$ & + & $P_{i}$ & 1.0 \\
\hline
\end{tabular}

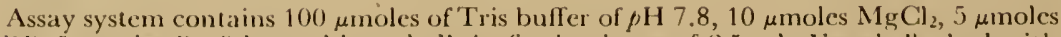
(I) $\mathrm{P}^{2}, 5$ moles $P_{1}, 0.1 \mathrm{mg}$ chloroplyyll, in final volume of $2.5 \mathrm{ml}$. Vessels llushed with $H_{2}$ (Pd inset) and preilluminated for 10 minutes prior 10 addition of contents of side-arm. Incubation time, 15 minutes; ted light; temp $=15{ }^{\circ} \mathrm{C}$. 
rates. It can be seen that in the presence of catalase and ethanol with FMN, the amomint of orthophosphate esterified is no more than that which is equivalent stoichiometrically to the FMN added. If the FMN is photoreduced prior to orthophosphate addition, there is no detectable phosphorylation whatever. With $\mathrm{K}_{3}$ there is also a marked inhibitory effect of catalase-ethanol, though the inhibition is not so striking as with FMN. (With phenazine methosulfate and pyocyanine, the inhibitory effect of catalase-ethanol is small or absent under anaerobic conditions.) Separate cxperiments show that though ethanol has a small inhibitory effect, neither catalase alone nor ethanol alone is responsible for the marked inhibitions observed here. Both reagents must be added together. When these results were first obtained, we felt uncertain about whether the inhibition by catalaseethanol should be attributed to a consumption of $\mathrm{H}_{2} \mathrm{O}_{2}$ formed by reduction of photochemically generated $\mathrm{O}_{2}$, or whether the peroxide might perhaps be an oxygen precursor. Our present views favor the former alternative, i.c., that the system is actually forming $\mathrm{O}_{2}$ and reutilizing it. The $\mathrm{H}_{2} \mathrm{O}_{2}$ may be regarded as a product of the auto-oxidation of the reduced cofactor (Equation 3). Since reaction 3 consumes more $\mathrm{O}_{2}$ than is formed in reaction 1 , cycling of the system is prevented when the $\mathrm{H}_{2} \mathrm{O}_{2}$ is removed with catalase-ethanol, unless an external oxidation is provided.

$$
\text { reduced cofactor }+\mathrm{O}_{2} \rightarrow \text { cofactor }+\mathrm{H}_{2} \mathrm{O}_{2}
$$

The question arose whether the auto-oxidation of the addled cofactor can account for all of the oxygen consumption which can be demonstrated with $\mathrm{O}^{18}$. Our first estimates indicated that this was not the case (5). That is, reactions 2 and 3 might very well be proceeding simultaneously. In order to determine what proportion of the reoxidation involves $\mathrm{H}_{2} \mathrm{O}_{2}$ formation, Nakamoto has made a set of measurements from which representative data have been assembled in Tables 3 and 4 . These measurements were carried out with different amounts of $\mathrm{O}_{2}$ in the gas phase. Catalase-ethanol does not inhibit photophosphorylation with FMN or $\mathbf{K}_{3}$ under aerobic conditions, but the amount of peroxide formed can be measured by determining the oxygen consumption manometrically. Alternatively, acetaldehyde formation can be measured, since this procedure is more sensitive, and can be shown to give equivalent information, as pointed out by Dr. Krogmann in the following paper. The FMN concentrations employed in the experiment summarized in Table 3 were sufficient to give optimal rates at the oxygen tensions employed. The 
TABLE 3

Relation of Peroxide Produgtion to Phosphorylation with FMN as Cofactor

\begin{tabular}{|c|c|c|c|c|c|}
\hline FMN & $\mathrm{O}_{2}$ & $\begin{array}{l}\text { Catalase- } \\
\text { ethanol }\end{array}$ & $-\Delta \mathrm{P}_{\mathrm{i}}$ & $\begin{array}{l}\text { Acetal- } \\
\text { dehyde }\end{array}$ & $\mathrm{P}_{\mathrm{i}} / \mathrm{CH}_{3} \mathrm{CHO}$ \\
\hline$M$ & $\%$ & & & & \\
\hline $4 \times 10^{-5}$ & 20 & - & 132 & & \\
\hline $1.6 \stackrel{\prime \prime}{\times 10^{-4}}$ & $"$ & + & 120 & 194 & 0.6 \\
\hline $1.6 \times 10^{-4}$ & 1 & - & 126 & & \\
\hline$"$ & $"$ & + & 114 & 134 & 0.9 \\
\hline $2 \times 10^{-4}$ & 0.5 & - & 126 & & \\
\hline$"$ & $"$ & + & 102 & 106 & 1.0 \\
\hline $2 \times 10^{-4}$ & ca. 0 & - & 126 & & \\
\hline$"$ & $"$ & + & 32 & 28 & 1.1 \\
\hline $3 \times 10^{-4}$ & $"$ & - & 120 & & \\
\hline$"$ & " & + & 43 & 36 & 1.2 \\
\hline
\end{tabular}

Phosphorylation and acetaldehyde formation given as $\mu$ moles per mg chlorophyll per hour.

figures in the last column show the ratio of phosphate esterified to acetaldehyde formed. Note how this ratio changes from 0.6 to 1.2 as the oxygen tension is decreased, though the photophosphorylation rate remains essentially constant.

The results of an equivalent set of measurements with $\mathrm{K}_{3}$ are given in Table 4. Here we see an even larger change in the ratio of phosphate esterified to acetaldehyde formed (from 0.7 to 5.1). That is, the relative amount of $\mathrm{H}_{2} \mathrm{O} .2$ formed is higher at the higher oxygen tensions. Most of these measurements had been completed before. we had an opportunity to study the paper of Warburg et al. (6) on the chemical mechanism of photosynthesis. The data we had obtained with $\mathrm{K}_{3}$ were very similar to those obtained in the Dahlem laboratory with naphthoquinone sulfonate, though the methods used

TABLE 4

Relation of Peroxide Production to Phosphorylation with $\mathrm{K}_{3}$ as Cofactor

\begin{tabular}{|c|c|c|c|c|c|}
\hline $\mathrm{K}_{3}$ & $\mathrm{O}_{2}$ & $\begin{array}{c}\text { Catalase- } \\
\text { cthanol }\end{array}$ & $-\Delta P_{i}$ & $\begin{array}{l}\text { Acetal- } \\
\text { dehyde }\end{array}$ & $\mathrm{P}_{\mathrm{i}} / \mathrm{CH}_{3} \mathrm{CHO}$ \\
\hline MI & $r_{n}$ & & & & \\
\hline $1.2 \times 10^{-5}$ & 20 & - & 121 & & \\
\hline $1.2{ }^{\prime \prime} \times 10^{-4}$ & $"$ & $\stackrel{t}{-}$ & $\begin{array}{l}106 \\
112\end{array}$ & 155 & 0.7 \\
\hline " & $"$ & + & 102 & 47 & 2.2 \\
\hline $1.7 \underset{\%}{\times 10^{-4}}$ & $\begin{array}{l}0.5 \\
\|\end{array}$ & $\bar{t}$ & $\begin{array}{l}107 \\
102\end{array}$ & 40 & 2.5 \\
\hline $1.7 \times 10^{-4}$ & ca. 0 & $\bar{t}$ & $\begin{array}{r}110 \\
96\end{array}$ & 19 & 5.1 \\
\hline
\end{tabular}

Phosphorylation and acctaldehyde formation given as $\mu$ moles per mg chlorophyll per hour. 
were rather different. This prompted us to see whether we might not be able to confirm some of the other findings reported by Warburg and his collaborators. liecause of its theoretical importance, the report that $\mathrm{CO}_{2}$ is required for the photoevolution of $\mathrm{O}_{2}$ seemed particularly interesting. We therelore made efforts to confirm the statement that the Hill reaction is stimulated by $\mathrm{CO}_{2}$.

For a number of practical reasons, we chose to search for a $\mathrm{CO}_{2}$ effect with a system in which spinach chloroplasts photoreduce ferricyanide in the presence of a catalytic amount of trichlorophenol indophenol. The data shown in Table 5 were obtained by Babette Stern. They are presented as evidence that $\mathrm{CO}_{2}$ (or bicarbonate) stimulates the rate of photoreduction in the particular system chosen. The incubations were carried out in double-arm Warburg vessels, and pressure changes were followed manometrically. The gas phase was air, which was either freed of $\mathrm{CO}_{2}$ by the use of base in the center-well or enriched with $\mathrm{CO}_{2}$ to a sufficient extent to give about $2 \mu$ moles of bicarbonate in the liquid medium (compared with 100 $\mu$ moles of phosphate). Each reaction mixture contained $0.2 \mathrm{mg}$ chlorophyll in the form of grana prepared by treating spinach chloroplasts with water. The grana were suspended in a phosphate-chloride medium and added to one side-arm. The other side-arm contained trichloroacetic acid, which was tipped in to stop the reaction at the end of the incubation period. This provided a check on the carbonate content of the medium. The preincubation period listed in the left

TABLE 5

Effect of Carbonate on Ferricyanide Reduction

\begin{tabular}{|c|c|c|c|}
\hline $\begin{array}{l}\text { Preincubation time } \\
\text { (min) }\end{array}$ & $\mathrm{CO}_{2}$ & $\begin{array}{l}\text { Ferricyanide reduced } \\
(\mu \mathrm{moles} / \mathrm{mg} \mathrm{chl} / \mathrm{hr})\end{array}$ & $\begin{array}{c}\mathrm{O}_{2} \text { evolved } \\
(4 \times \mu \text { moles } / \\
\mathrm{mg} \mathrm{chl} / \mathrm{hr})\end{array}$ \\
\hline 45 & - & 140 & 155 \\
\hline$"$ & + & 166 & - \\
\hline 180 & - & 54 & 55 \\
\hline$"$ & + & 82 & \\
\hline 285 & - & 25 & 10 \\
\hline$"$ & + & 53 & - \\
\hline 36 & $\bar{t}$ & $\begin{array}{l}172 \\
189\end{array}$ & 182 \\
\hline \multirow[t]{2}{*}{390} & - & 33 & 31 \\
\hline & + & 56 & \\
\hline
\end{tabular}

Reaction mixtures contained $100 \mu$ moles of phosphate buffer of $p \mathrm{H} 7.0,0.1 \% \mathrm{KCl}$, 0.1 umole trichlorophenol indophenol, $20 \mu$ moles ferricyanide, and spinach grana containing $0.2 \mathrm{mg}$ chlorophyll, in a volume of $3.0 \mathrm{ml}$. Grana added from side-arm after preincubation in dark. Temp. $15^{\circ} \mathrm{C}$; white light. 
column is the time the vessels were shaken in the bath in the dark prior to addition of the grana from the side-arm. The illumination began with the tipping in of the grana. The amount of ferricyanide actually reduced was determined by spectrophotometric examination of the trichloroacetic acid filtrates. In the case of the reaction carried out in the absence of $\mathrm{CO}_{2}$, there was reasonably good agreement between the amounts of oxygen evolved and ferricyanide reduced, at least for the samples not subjected to a prolonged preincubation. When $\mathrm{CO}_{2}$ was present, the manometric changes reflected not only the oxygen evolution but also the pressure increase due to the $\mathrm{CO}_{2}$ released by the acid formed cluring ferricyanide reduction. The figures have been omitted because the conditions were not sufficiently well-controlled to enable us to calculate $\mathrm{O}_{2}$ evolution accurately.

Note that the magnitude of the $\mathrm{CO}_{2}$ effect, if calculated as per cent stimulation, increased with the time of preincubation, although the overall rate decreased with time. The presence of dye was necessary at least to get effects of the magnitude shown in Table 5. Though the reaction mixtures containing $\mathrm{CO}_{2}$ had a somewhat lower $p \mathrm{H}$ than those without, the $p \mathrm{H}$ difference was not large, and equivalent lowering of the $p H$ was shown in separate experiments to have little effect on the photoreduction rate. We therefore regard the effects shown as specific for $\mathrm{CO}_{2}$ or bicarbonate.

Stimulation ranging from 20 to 100 per cent has been obtained in fifteen out of fifteen separate experiments conducted approximately in the manner here described. We feel reasonably confident, therefore, that this effect is reproducible, and that it can be obtained in other laboratories. We would not venture to extrapolate from these data to conclusions as far-reaching as Warburg's, but we feel encouraged to continue to explore the possibility that his conclusions may be wellfounded.

\section{REFERENCES}

I. Keilin, I)., and Hartrec, E.. J., Biochem. J., 39, 293-301 (194.).

2. Nehler, .1. H., Arch. Biochem. and Biophys., 33, 6.5.77 (19.51).

3. Nahamoto, T., Krogmann, D. W., and Nayne, B., J. Biol. Chem.. (in press).

1. Nakamoso, T., Krogmanu, 1). W., and Vennesland, B., J. Biol. Chem., 234, $2783-2788(1959)$.

5. Nakimoto, T., and Venuesland, 13., Federation Proc., 19, 329 (1960).

6. Warburg, O., Krippahl, (... Cewitz, H.-S., and Välker, W.., Z. Nalurforsch., 14b, $712-724(1959)$. 


\title{
OXIDATIVE PHOTOPHOSPHORYLATION AND THE MEHLER REACTION
}

\author{
DAVID W. KrogmaNN \\ Department of Biochemistry, Unirersily of Chicago \\ Chicago, Illinois
}

Our attention has been directed to the study of photophosphorylation in the presence of indophenol dye (5). As in the other types of photophosphorylation, undenatured chloroplasts, light, magnesium ions, inorganic phosphate, and a high-energy phosphate acceptor are necessary. In contrast to the other types of photophosphorylation, when indophenol is used as a cofactor the phosphorylation reaction is dependent on atmospheric oxygen. Several lines of evidence indicate that phosphorylation accompanies the oxidation of reduced indophenol rather than its reduction in the Hill reaction. This oxygendependent phosphorylation proceeds in the absence of any manometrically detectable oxygen consumption or evolution. The requirement for oxygen by this system could be rationalized as an uptake of oxygen accompanying phosphorylation masked by an equivalent production of oxygen by the Hill reaction.

\section{EXPERINENTS WITH IsOTOPIC OXYGEN}

Experiments with isotopically labeled oxygen were undertaken to test this interpretation. It was through the courtesy of Dr. Allan Brown that the mass spectrometer facilities at the Botany Department of the University of Minnesota Were made available to us. A detailed description of this apparatus and the method of calculation of results have been presented elsewhere $(1,2)$. In these experiments, a phosphorylation reaction mixture was incubated beneath an atmosphere enriched with the mass thirty-four isotope of oxygen. The gas phase above the reaction mixture ras continuously monitored by the mass spectrometer. The diagram in Fig. 1 is representative of the results obtained in these experiments. In the initial dark period, there is no change in the concentrations of either the mass thirty-two or mass thirty-four isotopes of oxygen. When the light is turned on, there is an immediate and sustained decrease in the con- 


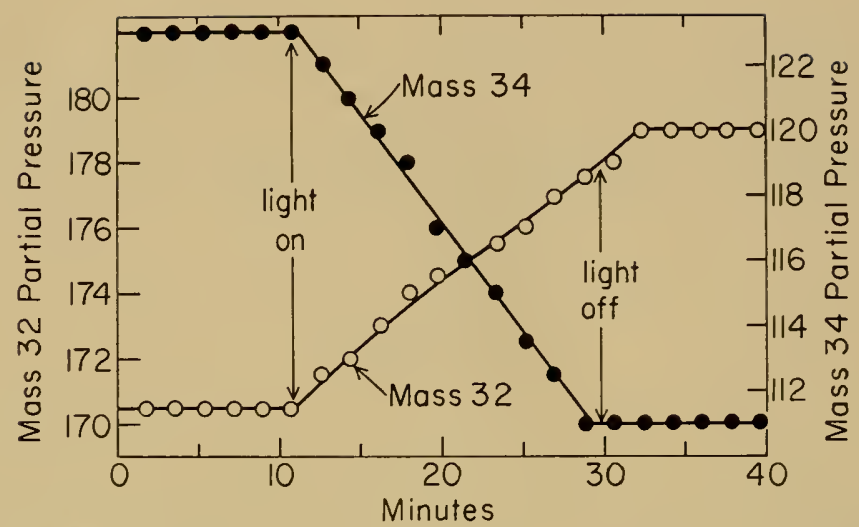

Fig. 1. Measurement of oxygen exchange with the reaction mixture for oxidative photophorylation.

centration of the mass thirty-four isotope, indicating that oxygen from the gas phase is being consumed by the illuminated chloroplasts. At the same time, an increase in the concentration of the mass thirty-two isotope in the gas phase is observed, indicating that oxygen is being produced from the aqueous phase. These rates of oxygen production and consumption are nearly equal. When the light is turned off, this oxygen exchange reaction stops. More information about the indophenol-induced oxygen exchange by illuminated chloroplasts is seen in Table 1. The rate of synthesis of ATP during these experiments is quite low because of the damaging effects on the chloroplasts of prolonged shaking during equilibration and calibration of the mass spectrometer. However, the oxygen exchange reaction is seen to be independent of the net synthesis of any considerable amount of ATP, in that the reaction is unaffected by the omission of ADP. Nevertheless, the high rate of oxygen exchange is dependent on the presence of indophenol dye. Also, there is negligible exchange activity of the complete reaction mixture if boiled chloroplasts are used.

TABLE 1

OXYGEN EXCHANGE WITH 2,3',6-TrichloRophenol INDOPHENOL

\begin{tabular}{|c|c|c|c|}
\hline $\begin{array}{l}\text { Reaction } \\
\text { Mixture }\end{array}$ & $\begin{array}{l}\mu \text { moles P } \\
\text { esterificd }\end{array}$ & 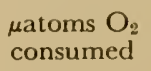 & $\begin{array}{c}\text { } \\
\text { prodoms } \mathrm{O}_{2} \\
\end{array}$ \\
\hline $\begin{array}{l}\text { complete } \\
\text { minus ADP } \\
\text { minus dye }\end{array}$ & $\begin{array}{r}12 \\
1 \\
3\end{array}$ & $\begin{array}{l}53.1 \\
58.9 \\
22\end{array}$ & $\begin{array}{l}42.6 \\
53.6 \\
14\end{array}$ \\
\hline
\end{tabular}

Values are reported as rates/mg chlorophyll/hour. 
Although the oxygen exchange reaction is not dependent on photophosphorylation, it is of sufficient magnitude to be stoichiometric with the oxygen-dependent photophosphorylation. The rate of thirty to fifty microatoms of oxygen consumed and evolved per milligram of chlorophyll per hour is to be compared to a rate of sixty to onc hundred micromoles of phosphate esterified per milligram of chlorophyll per hour in a reaction shown to have a $P / O$ ratio of two. It rould appear that phosphorylation is dependent upon an electron transport process, but that the electron transport process is not obligately coupled to phosphorylation.

\section{Hydrogen Peroxide as an Intermediate in Oxygen Exchange}

An oxygen exchange reaction by illuminated chloroplasts is by no means a new observation. Mehler has described a type of Hill reaction in which the photochemically generated reducing power of chloroplasts is used to recluce atmospheric oxygen to hydrogen peroxide (6). If catalase is present, the hydrogen peroxide is decomposed to water and oxygen. Under these circumstances, oxygen production equals oxygen consumption. We found sufficient catalase in the spinach chloroplast preparations to decompose hydrogen peroxide at a rate commensurate with the rate of oxygen exchange observed in the mass spectrometer experiments. Thus it seemed reasonable that the dye-catalyzed oxygen exchange and even the dye-catalyzed oxidative phosphorylation might be attributable to a Mehler reaction.

Mehler had devised an experiment in which the light-generated lhydrogen peroxide was trapped by the peroxidative activity of catalase in the oxidation of ethanol to acetaldehycle. This reaction sequence is outlined in Fig. 2. Under these circumstances, the reaction could be observed by net oxygen consumption or by the appearance of

\section{Mehler Reaction}

$$
\begin{aligned}
& 2 \mathrm{H}_{2} \mathrm{O} \stackrel{\text { hv }}{\longrightarrow} 2[\mathrm{H}]+2[\mathrm{OH}] \\
& 2[\mathrm{OH}] \longrightarrow \mathrm{H}_{2} \mathrm{O}+1 / 2 \mathrm{O}_{2} \\
& 2[\mathrm{H}]+\mathrm{O}_{2} \longrightarrow \mathrm{H}_{2} \mathrm{O}_{2} \\
& \mathrm{H}_{2} \mathrm{O}_{2} \text { tethanol } \stackrel{\text { catalase }}{\longrightarrow} \text { acetaldehyde }+\mathrm{H}_{2} \mathrm{O}
\end{aligned}
$$

$$
1 / 2 \mathrm{O}_{2}+\text { ethanol } \underset{\text { catalase }}{\stackrel{h v}{\longrightarrow}} \text { ucetaldehyde }+\mathrm{H}_{2} \mathrm{O}
$$

Fig 2. The Mehler reaction in the presence of catalase and ethanol. 
acetaldehyde. If indophenol dye were acting by catalyzing the reduction of oxygen to hydrogen peroxide, it should be possible, on the addition of ethanol to the reaction mixture, to observe net oxygen consumption and acetaldehyde production at a rate approaching that of the oxygen exchange observed with isotopes, and therefore commensurate with the rate of ATP synthesis.

A necessary preliminary to quantitative comparisons of the Mehler reaction with indophenol-induced oxygen exchange was to test the efficiency of the catalase-ethanol trap for hydrogen peroxide measurements. Keilin and Hartree, in first describing the peroxidative activity of catalase, found that this enzyme could use up to eighty per cent of the available hydrogen peroxide for the peroxidation of ethanol provided the hydrogen peroxide was generated in the reaction mixture (4). However, the conditions for their experiments were quite different from those employed for photophosphorylation, so it seemed imperative to check the efficiency of the catalase-ethanol trap for hydrogen peroxide under these new circumstances. The oxidation of alanine by D-amino acid oxidase was used as a hydrogenperoxide-generating system. In the presence of catalase-ethanol, oxygen consumption by this reaction should be equivalent to the amount of acetaldehyde produced. The reaction was carried out in Warburg manometer vessels at fifteen degrees in the presence of all the usual components of the oxidative photophosphorylation reaction mixture. The reaction was stopped by the addition of trichloroacetic acid, the protein removed by centrifugation and the $p \mathrm{H}$ adjusted to neutrality. An aliquot of the supernatant fluid was assayed for acetaldehyde with alcohol dehyclrogenase and DPNH (7). A similar aliquot was assayed for pyruvate-the product of alanine oxidation-with lactic dehydrogenase and DPNH (3). This second assay provided a measurement of hydrogen peroxide produced which is independent of the fate of that hydrogen peroxide-either by catalatic or peroxidatic utilization. The entire reaction sequence is outlined in Fig. 3. The second table show's the results of an experiment in which hydrogen peroxide, generated by the enzymatic oxidation of alanine, was trapped quantitatively by catalase-cthanol in a reaction mixture capable of oxidative photophosphorylation. The recovery of hydrogen peroxide by the peroxidative action of catalase is better than eighty per cent complete, as seen in a comparison of pyruvate produced with either the oxygen consumed or acetaldehyde produced. This experiment shows that the catalase-ethanol trap can give an adequate measure of hydrogen peroxide production under the circumstances of the clye-stimulated oxygen exchange. 


\section{$\mathrm{H}_{2} \mathrm{O}_{2}$ Generation and Measurement}

$$
\begin{gathered}
\text { d alanıne }+\mathrm{O}_{2} \stackrel{\text { amino acid }}{\longrightarrow \text { oxidase }} \text { pyruvate }+\mathrm{H}_{2} \mathrm{O}_{2}+\mathrm{NH}_{3} \\
\mathrm{H}_{2} \mathrm{O}_{2}+\text { ethanol } \stackrel{\text { catalase }}{\longrightarrow} \text { acetaldehyde }+\mathrm{H}_{2} \mathrm{O}
\end{gathered}
$$

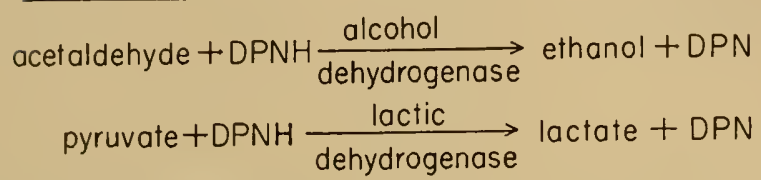

Fig. 3. Reaction scquence for the generation and measurcment of hydrogen peroxide.

The next experiment (Table 3) shows the results of a Mehler reaction assay and the lack of effect of indophenol dye on the reduction of oxygen to hydrogen peroxide by illuminated chloroplasts. The generation of hydrogen peroxide is the same in the presence or absence of dye, in contrast to the dramatic stimulation of oxygen exchange caused by indophenol in the mass spectrometer measurements. It is also apparent that there is no obvious stoichiometric relationship between the amount of hydrogen peroxide produced and the phosphate esterified.

The dye-stimulated oxygen exchange by illuminated chloroplasts does not appear to result in hydrogen peroxide formation as might be expected from Mehler's experiments. The rate of thirty to fifty microatoms of oxygen consumed and evolved per milligram of chlorophyll per hour in the presence of indophenol dye is to be contrasted to a rate of six to ten micromoles of hydrogen peroxide produced per milligram of chlorophyll per hour regardless of the presence of indophenol dye.

The final diagram (Fig. 4) represents, in mininum detail, our very tentative interpretation of the facts at hand. The dye is reduced by the Hill reaction and $\mathrm{O}_{2}$ is evolved as usual, but in such fashion

TABLE 2

RECOVERY OF $\mathrm{H}_{2} \mathrm{O}_{2}$

\begin{tabular}{lccc} 
Reaction Mixture & $\begin{array}{c}\mu \text { moles } \mathrm{O}_{2} \\
\text { consumed }\end{array}$ & $\begin{array}{c}\mu \text { moles } \mathrm{H}_{2} \mathrm{O}_{2} \\
\text { as acetaldehyde }\end{array}$ & $\begin{array}{c}\mu \text { moles pyruvate } \\
\text { produced }\end{array}$ \\
\hline complete & 2.9 & 2.6 & 3.1 \\
minus ethanol & 1.4 & 0 & 2.9 \\
minus chloroplasts & 2.4 & 2.4 & 2.8 \\
\hline
\end{tabular}


TABLE 3

Mehler Reaction and Oxidative Photophosphorylation

\begin{tabular}{lccc}
$\begin{array}{l}\text { Reaction } \\
\text { Mixture }\end{array}$ & $\begin{array}{c}\mu \text { moles } \mathrm{O}_{2} \\
\text { consumed }\end{array}$ & $\begin{array}{c}\mu \text { moles } \\
\text { acetaldehyde } \\
\text { produced }\end{array}$ & $\begin{array}{c}\mu \text { moles } \mathrm{P}_{\mathrm{i}} \\
\text { esterified }\end{array}$ \\
\hline $\begin{array}{l}\text { complete } \\
\text { minus dye }\end{array}$ & 0.7 & 0.67 & 3.8 \\
minus ethanol & 0.6 & 0.62 & 0.0 \\
minus light & 0 & 0 & 3.8 \\
\hline
\end{tabular}

that no phosphorylation occurs. The reduced dye may reduce the photochemically generated oxidant and, in so doing, permit the flow of electrons from the photochemical reductant through a phosphorylation step to oxygen. It is suggested that the product of this oxygen reduction is water, inasmuch as hydrogen peroxide production does not appear to be related to the indophenol-catalyzed reactions.

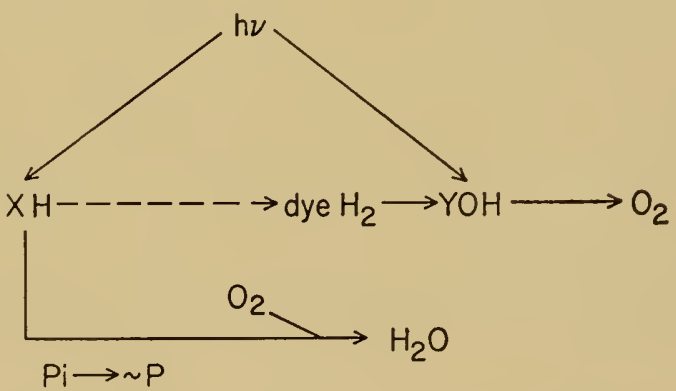

Fig. 4. Oxidative photosynthetic phosphorylation.

\section{REFERENCES}

1. Brown, A. H., Nier, A. O. C., and Van Norman, R. WI., Plunt Physiol., 27 320-333 (1952).

2. Brown, A. H., and Weis, 1)., Plant Physiol., 34, 221.234 (1959).

3. DeLuca, C., Weber, M. M., and Kaplan, N. O., J. Biol. Chem., 223, 559-567 (1956).

4. Keilin, D., and Hartrec, E. F., Biochem. J., 39, 293-301 (1945).

5. Krogmann, D. W., and Vensesland, B., J. Biol. Chem., 234, 220.5-2210 (1959).

6. Mchler, A. H., Arch. Biochem. and Biophys., 33, 6.5-77 (1951).

7. Racker, E., Methods in Enzymology (Colowick, S. P.. and Kaplan, N. O., eds.). Vol. III, p. 293, Academic Press, New York (1957). 


\title{
PHOTOPHOSPHORYLATION AND PHOTOHYDROLYSIS IN CELL-FREE PREPARATIONS OF BLUE-GREEN ALGA
}

\author{
Barbara Petrack and Fritz Lipmann \\ The Rockefeller Institute, New York City
}

Our attention was originally attracted to the blue-green algae by a paper from Calvin's group (10) in which a rapid $\mathrm{CO}_{2}$ fixation into the carbamyl group of citrulline was described. Our earlier interest in carbamyl phosphate synthesis and transcarbamylation reactions prompted us to examine this light-induced carbamylation in cellfree preparations of Nostoc muscorum and Anabaena variabilis. Although citrulline was formed rather rapidly by transcarbamylation from carbamyl phosphate to ornithine, the synthesis of carbamyl phosphate from $\mathrm{CO}_{2}, \mathrm{NH}_{3}$, and ATP, which is the more interesting reaction, was, at best, rather sluggish in these extracts. Furthermore, we soon became convinced that the effect of light on the incorporation of $\mathrm{CO}_{2}$ into citrulline was indirect and by way of photophosphorylation. We decided, therefore, to turn our attention to the more promising study of photophosphorylation in this system. Although these algae contain the green plant chlorophyll, they have many of the characteristics of a bacterial system; and photophosphorylation was, indeed, found to be similar to that in Rhodospirillum rubrum. During a study of this process a photohydrolysis of ATP was observed which strongly impresses us as being a reversal of photophosphorylation. A brief description will be given here of the general characteristics of photophosphorylation, as well as the rather novel phenomenon of photohydrolysis.

\section{Preparation of Cell-free Extracts}

In the following studies Anabaena variabilis was used exclusively. The algae were grown in continuous culture with $\mathrm{CO}_{2}$ as the only carbon source, essentially as described by Kratz and Myers (9). We are indebted to Dr. J. Myers for the cultures used as well as for considerable information on how to grow them, and to Dr. J. D. Gregory for designing the continuous culture apparatus in which they were 
grown. Rather stable extracts were prepared by grinding wet cells with 3 volumes of ahmina, followed by extraction with 5 volumes of a solution containing $40 \%$ ethylene glycol, $0.1 M$ Tris buffer $p \mathrm{H} 8$, and $0.01 M$ ascorbate. All the photophosphorylating activity was present in the $105,000 \times g$ pellet; this contains the chlorophyll and carotenoids, but is essentially free of phycocyanin. The examination by electron microscopy, kindly carried out by $\mathrm{A}$. Shatkin, showed the preparation to consist entirely of smooth vesicles varying in diameter from 30 to $300 \mathrm{~m}_{\mu}$. These appear to be derived from the peripheral lamellae seen in the electron microgram of intact cells (13).

\section{Characteristics of Photophosphorylation in Anabaena Fragments}

As in the particle suspension from $R$. rubrum described by Frenkel (4), the Anabaena system catalyzes a substrate-independent, anaerobic photophosphorylation of ADP which requires $\mathrm{Mg}^{++}$and an added electron carrier. Phenazine methosulfate (Sigma) (PMS) is most effective, although the combination of flavin mononucleotide (FMN) and menadione is also active as an electron carrier. [N.B. The name "phenazine methosulfate" should be considered a trade name. The compound is chemically better defined as methyl phenazonium methosulfate, and is derived from the reaction between dimethyl sulfate and phenazine (3).]

The experiments were carried out in an illuminated Warburg apparatus in which a bank of photoflood bulbs provides more than 7000 foot-canclles. Such strong illumination is necessary for light saturation. Anaerobic conditions are essential. Attempts to obtain phosphorylation in air, by adding various reducing agents, failed. The aerobic inhibition is largely irreversible, and is probably due to photooxidation. The removal of phycocyanin seems to be responsible for the photooxidation. This interpretation is supported by the following observations: particles prepared in $40 \%$ dextrin retain the phycocyanin, and these particles do photophosphorylate in air at about one-half the rate obtained anaerobically.

Only ADP, and not AMP, acts as a phosphate acceptor. Addition of a glucose-hexokinase trap increases the rate of phosphate utilization. During the early stages of this study inactive preparations were sometimes obtained. Such preparations strongly inhibited photophosphorylation with spinach chloroplasts, but this inhibition was overcome by bovine serum albumin (BSA), which activates these algae preparations for photophosphorylation. This effect of BSA has been described in oxidative phosphorylation (11). Since it strongly binds a 
variety of substances, however, it interfered with studies on the effect of some inhibitors upon photophosphorylation.

Sacktor, O'Neill, and Cochran (12) reported that the activation of llight muscle mitochondria by BSA appeared to depend on the presence of sulfliydryl (SH) groups on the protein. Since Vemmesland's group (8) described a variable stimulation of photophosphorylation by glutathione (GSH), we examined the relationship between the effect of BSA and GSH on photophosphorylation. Table 1 illustrates the seemingly umpredictable fluctuations of such effects. This variation does not seem to be due to preparative procedures but appears to reflect differences in the algal cells from batch to batch. Similar variable effects of GSH have been observed with microsomes, where activity lost on ageing was found to be restored by GSH (6).

In experiment 1 of Table 1 , high rates are observed in the absence of both BSA and GSH, and no further increase is found in their presence. In experiment 2, the observed rate in the absence of both is low, but either GSH or BSA will stimulate, and the effects are not additive. This would suggest that it is the SH groups on BSA that produce the activation. In experiment 3, however, GSH is unable to replace BSA as activator; but most of our preparations were activated by either compound.

Under optimum conditions, Anabaena fragments photophosphorylate at a rate of 200-400 $\mu$ moles inorganic phosphate $\left(\mathrm{P}_{\mathrm{i}}\right)$ consumed per hour per $\mathrm{mg}$ chlorophyll at $30^{\circ} \mathrm{C}$ in $\mathrm{N}_{2}$. Rates are proportional to chlorophyll concentration up to $0.3 \mathrm{mg}$ in $3 \mathrm{ml}$, and remain constant up to 30 minutes. More active preparations are obtained from 48-hour cultures than from cells grown to maximum density. The

TABLE 1

Effect of GSH and BSA on Photophosphorylation

\begin{tabular}{|c|c|c|c|c|}
\hline Experiment & $\begin{array}{l}\text { - GSH } \\
\text { - BSA }\end{array}$ & $\begin{array}{l}+\mathrm{GSH} \\
-\mathrm{BSA}\end{array}$ & $\begin{array}{l}\text { - GSH } \\
+ \text { BSA }\end{array}$ & $\begin{array}{l}+\mathrm{GSI} \\
+\mathrm{BSA}\end{array}$ \\
\hline & \multicolumn{4}{|c|}{$\mu$ moles $\Delta \mathrm{P}_{\mathrm{i}} / \mathrm{hr} / \mathrm{mg}$ chlorophyll } \\
\hline 1 & 404 & 488 & 488 & 51 \\
\hline 2 & 58 & 215 & 223 & \\
\hline 3 & 0 & 0 & 165 & 19 \\
\hline
\end{tabular}

Reaction media. Each tube contained the following in $\mu$ moles $/ 2.7 \mathrm{ml}: 20$ potassium phosphate $p \mathrm{H} \mathrm{7.7,200} \mathrm{Tris} \mathrm{buffer} p \mathrm{H} \mathrm{7.7,25}$ glucose, 2 ADP, $20 \mathrm{MgCl}_{2}, 0.4 \mathrm{PMS}$, excess hexokinase, Anabaena fragments containing approximately $0.15 \mathrm{mg}$ chlorophyll, and where shown, $1 \mu$ mole GSH and $6 \mathrm{mg}$ BSA. The incubation was for 15 minutes at $30^{\circ} \mathrm{C}$ in light and nitrogen. The reaction was stopped with $0.3 \mathrm{ml} 50 \% \mathrm{TCA}$. $\mathrm{l}_{1}$ was measured according to Fiske and Subbarow. 
preparation in $40 \%$ glycol is usually stable for one week when stored at $-15^{\circ} \mathrm{C}$. The glycol permits low-temperature storage without freezing. The washed residue is free of myokinase but contains a potent inorganic pyrophosphatase. The preparation does not appear to catalyze the Hill reaction; neither trichloroindophenol, ferricyanide, nor TPN are reduced in light, nor is oxygen evolved.

Photophosphorylation was observed over a rather wide $p \mathrm{H}$ range, from about 6.0 to 8.3, with an optimum at $p \mathrm{H} \mathrm{7.7.} \mathrm{This} \mathrm{contrasts}$ with the spinach chloroplast system, which shows a sharp optimum at $p \mathrm{H} 8.0$ and almost no activity at $p \mathrm{H} 7.0$ (2). Generally the disappearance of inorganic phosphate was measured, but photophosphorylation of ADP was also estimated spectrophotometrically by way of glucose 6-phosphate with Zwischenferment-glucose and TPN; glucose-6-phosphate (=ATP) formation was found to be equivalent to the disappearance of inorganic phosphate.

Although FMN and menadione each stimulate the chloroplast systen (16), a combination of the two appears to be necessary for the algal system. The synergistic effect between FMN and menadione is shown in Table 2. FMN, however, is not specific for this system; FAD or even riboflavin is also active. Hill reported (15) that with spinach chloroplasts the activity of PMS is due to its photooxidation to pyocyanin. In the Anabaena system, however, pyocyanin was only $10 \%$ as active as PMS, and when added together with an equal concentration of PMS, it caused a $40 \%$ inhibition.

As previously described for spinach and $R$. murum $(1,5)$, uncouplers of aerobic phosphorylation, such as dinitrophenol or dicumarol, also inhibit photophosphorylation with the algal system, but again a 10 -fold greater concentration is required. Another uncoupler of aerobic phosphorylation, the butyl ester of diiodohydroxybenzoic acid, however, causes $50 \%$ inhibition at $1 \times 10^{-5} \mathrm{M}$, and $100 \%$

TABLE 2

EFfect of FMN and Menadione on Photopiosphorýlation ‘moles $/ 3 \mathrm{ml}$

0.20

0.50

1.0

2.0

$\mu$ moles $\Delta \mathrm{P}_{\mathrm{i}} / \mathrm{hr} / \mathrm{mg}$ chlorophyll

FMN

Menadione

FMN + menadione

$\begin{array}{rrrr}0 & 17 & 36 & 36 \\ 0 & 0 & 29 & 0 \\ 72 & 96 & 68 & 22\end{array}$

The reaction conditions were the same as those in Table 1, but the PMS was replaced by FMN and menadione, as shown. 
at $1 \times 10^{-5}$ M. A similar effect has been reported by Geller with R. rubrum (5). Antinycin A does not block photophosphorylation with either PMS or the combination of FMN and menadione.

\section{Photohydrolysis of ATP}

As mentioned aheady, a stimulatory effect of GSH similar to the one reported for spinach chloroplast fragments is found with this system. Fig. 1 shows, however, that increasing the GSH concentration beyond the maximum causes increasing inhibition. The stimulatory effect of lower levels of GSH is somewhat variable and highly active preparations may not show it. At higher levels, however, all preparations are inhibited. Frequently, $100 \%$ inhibition is seen with 0.015 $M$ GSH, which appears to be a non-specific SH effect, since cysteine has similar effects.

From these observations a suspicion arose that high concentrations of GSH inhibit by producing an ATPase-like activity in the preparation. This suspicion could, indeed, be verified. With $0.015 M \mathrm{GSH}$, inorganic phosphate was released from ATP under the usual conclitions of photophosphorylation. In these experiments, ATP was added catalytically together with phosphoenol-pyruvate + pyruvic kinase (PEP-PK) as the ATP-generating system.

Table 3 shows the GSH-activated photohydrolysis of ATP. This activity requires $\mathrm{Mg}^{++}$, light, and PMS, and is lost by boiling the preparation for 3 minutes. PEP itself is not hydrolyzed. Lower rates are found when substrate levels of ATP replace the generating system. The rate of hydrolysis is optimal at $p \mathrm{H} 7.7$, the same $p \mathrm{H}$ shown to be optimal for photophosphorylation. The dark hycholysis of ATP is not stimulated by either GSH or BSA and appears to be unrelated

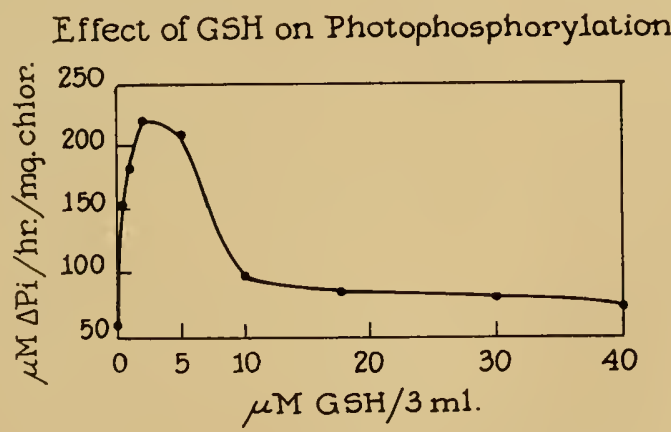

Fig. 1. The effects of glusathione on photophospliorylation in cell-free extracts of Anabaena rariabilis. Stimulation by low and inlibilion by high concentrations. 
TABLE 3

GSH-activated Photohydrolysis of ATP

$\Delta \mu$ moles $\mathrm{P}_{\mathrm{i}} / \mathrm{hr} / \mathrm{mg}$ chlorophyll

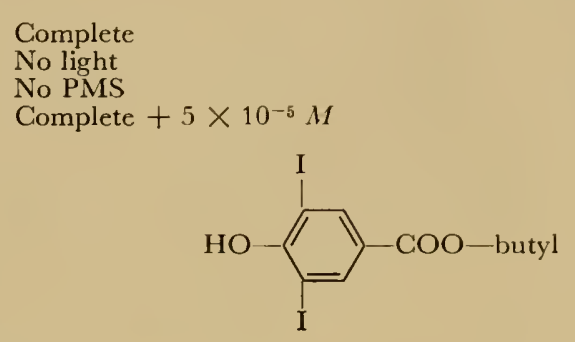

Reaction mixture. Each tube contained the following in $\mu$ moles $/ 2.7 \mathrm{ml}: 1$ ATP, 20 phosphoenol-pyruvate (PEP), $20 \mathrm{MgCl}_{2}, 40 \mathrm{GSH}, 200$ Tris buffer $p \mathrm{H} \mathrm{7.7,0.4} \mathrm{PMS,}$ $5 \mu \mathrm{g}$ pyruvic kinase, and Anabaena fragments containing approximately $0.1 \mathrm{mg}$ chlorophyll. The incubation was for 20 minutes at $30^{\circ} \mathrm{C}$ in nitrogen. The reaction was stopped with $0.3 \mathrm{ml} 50 \%$ TCA, and the $\mathrm{P}_{\mathrm{i}}$ was measured.

to the light-incluced reaction. It probably should be subtracted as a blank.

PMS may be replaced by either FMN or menadione, but then dephosphorylation is slower. As shown in Table 3, photohydrolysis is inhibited by $5 \times 10^{-5} M$ of the butyl ester of diiodohydroxybenzoic acid, a concentration that also inhibits oxidative and photophosphorylation. In contrast to photophosphorylation, the rate of hydrolysis is the same in air as in nitrogen. The high $\mathrm{SH}$ concentration used for hydrolysis seems to protect the system against photooxidation. Under optimum conditions the rate of ATP hydrolysis is $184 \mu$ moles of inorganic phosphate per hour per $\mathrm{mg}$ chlorophyll in light, and 42 in dark. This particular preparation synthesized $280 \mu$ moles ATP per hour per my chlorophyll in the light.

Cysteine may replace GSH and is even slightly more effective. Fig. 2 describes the effect of cysteine concentration on the rate of ATP hydrolysis. Its effect on photophosphorylation is also shown, and one may see the inverse relationship of SH concentration to the two light-induced reactions. SH concentrations that stimulate photophosphorylation only slightly affect photohyclrolysis, whereas SH concentrations that inhibit photophosphorylation produce maximum hydrolysis. Since the preparation contains a potent inorganic pyrophosphatase, it was necessary to consider the possibility that the ATP night be hydrolyzed to AMP and inorganic pyrophosphate which 


\section{SH Effect on Photophosphorylation and Photohydrolysis}

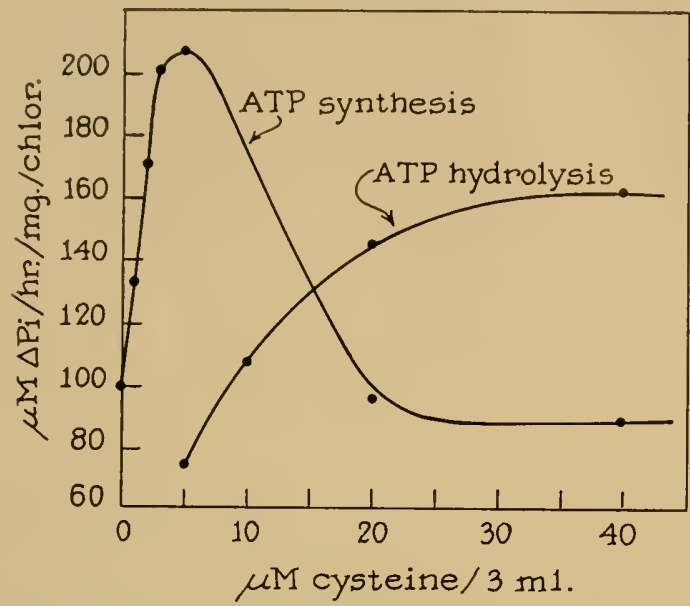

Fig. 2. The action of crsteine on photophosphorylation of ADP and photohydrolysis of ATP in cell-free extracts of Anabaena variabilis.

would subsequently be split to inorganic phosphate. The products of ATP hydrolysis were examined in an assay mixture containing substrate levels of ATP. The nucleotides contained in the TCA extract were separated by paper electrophoresis in ammonium formate buffer at $p \mathrm{H} \mathrm{3.8,} \mathrm{and} \mathrm{spotted} \mathrm{on} \mathrm{the} \mathrm{paper} \mathrm{by} \mathrm{ultraviolet} \mathrm{quenching.} \mathrm{Clearly}$ visible spots were found corresponding to ATP and ADP, but none was detected at the level of the AMP marker. It is concluded that ADP is the product of ATP hydrolysis.

The similarity between ATP synthesis and hydrolysis with respect to requirements and inhibitor effects suggests a close interrelationship between these two light-induced reactions. It seems reasonable to conclude, in fact, that the photohydrolysis is a reversal on the pathway of photophosphorylation. To support such a proposition, we felt obliged to demonstrate photohydrolysis in spinach chloroplasts, since several laboratories have reported a complete absence of ATPase in chloroplasts; the photohydrolysis was found, but it was necessary to increase the cysteine concentration to $0.08 M$. Furthermore, for this purpose, pyruvic kinase purchased as an ammonium sulfate suspension had to be dialyzed against $0.5 \mathrm{M} \mathrm{KCl}$. Due to the presence of ammonimm ion, the usual amount of pyruvic kinase completely inhibited photophosphorylation with spinach chloroplasts (7), whereas it had no effect with algal fragments. Table 4 demonstrates that 


\section{TABLE 4}

Cysteine-activated Photohydrolysis of ATP in Spinach Chloroplasts

$\Delta \mu$ moles $\mathrm{P}_{\mathrm{i}} / \mathrm{hr} / \mathrm{mg}$ chlorophyll

$\begin{array}{lr}\text { Complete } & 64 \\ \text { No light } & 5 \\ \text { No PMS } & 5 \\ \text { Complete }+5 \times 10^{-5} M & 7\end{array}$

Reaction mixture. Each tube contained the following in $\mu$ moles $/ 2.7 \mathrm{ml}$ : 1 ATP,

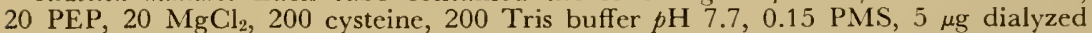
pyruvic kinase, and spinach chloroplasts containing $0.25 \mathrm{mg}$ chlorophyll. The incubation was for 20 minutes at $20^{\circ} \mathrm{C}$ in nitrogen. The reaction was stopped with $0.3 \mathrm{ml}$ of $50 \%$ TCA. $\mathrm{P}_{\mathrm{i}}$ was measured in $0.3 \mathrm{ml}$ aliquot.

cysteine-activated ATP hydrolysis in chloroplasts is dependent on light and PMS. The other characteristics of photohydrolysis also appear to be alike in the algae and spinach preparation.

The effect of cysteine concentration on photohydrolysis in chloroplasts is shown in Fig. 3. Cysteine does not increase the low dark hydrolysis, but in the light the rate of dephosphorylation increases proportionally with concentration up to $250 \mu$ moles cysteine in $3 \mathrm{ml}$. Higher concentrations were difficult to test because of interference

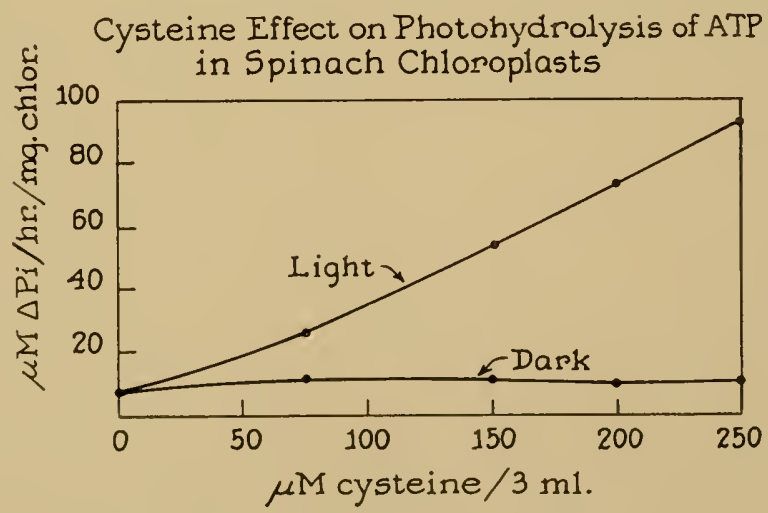

Fig. 3. Cystcinc-dependent photohydrolysis of A'TP in spinach chloroplasts. 
with the phosphate detemination. The highest rate of photohydrolysis observed in spinach chloroplasts is $100 \quad \mu$ moles $\mathrm{P}_{1} / \mathrm{hr} / \mathrm{mg}$ chlorophyll, corresponding to a specific activity for photophosphorylation ranging from 250 to 700. The unusually high SH requirement may well be a limiting factor, but this needs to be clarified. However that may be, the essential similarity between the photohydrolysis of ATP in chloroplasts and algae is apparent.

\section{Conclusions}

A photophosphorylating system was demonstrated in the bhegreen alga, Anabaena variabilis. It was hoped that the relatively greater simplicity of this preparation in comparison to chloroplasts might reveal some characteristics of the mechanism that would otherwise be masked. This view proved to be useful in finding a photohydrolysis of ATP, which could subsequently be demonstrated in chloroplasts only by forcing conditions.

The photophosphorylating activity in Anabaena has been localized in a phycocyanin-free preparation which consists of lamellar structures appearing in vesicular forms. This contrasts with the notion that phycocyanin may be essential for photosynthesis. The present study suggests that phycocyanin may function by protecting the system against photooxidation. The experiments reported by Thomas (14), in which loss of the Hill reaction was correlated with a loss of phycocyanin, were not carried out under anaerobic conditions. Furthermore, the release of oxygen during the Hill reaction may even be sufficient to inactivate the system by photooxidation in the absence of phycocyanin.

A dephosphorylation of ATP has been described in both spinach and algal preparations. Its relationship to photophosphorylation is seen in its dependence on light and on phenazine methosulfate, as well as in the inhibitory effects of diiodohydroxybenzoic acid on the two activities. Altogether, it seems likely that photohydrolysis is the reverse of photophosphorylation, and it is hoped that it may provide some new approaches to the understanding of the phosphorylating mechanism.

\section{REFERENCES}

1. Amon, D. L.. "The Photochemical Apparatus," Brookhaven Symposia in Biol., 11. p. $181(1958)$.

2. Arron, M.. Jagendorf, A. T., and Evans, M., Biochim. et Biophys. Acla, 26, $262(1957)$. 
3. Dickens, F., and Ncllwain, H., Biochem. J., xxxil, 1615 (1938).

4. Frenkel, A. W., J. Biol. Chem., 222, 823 (1956).

5. Geller, D. M., Doctoral thesis, Harvard University, Cambridge, Mass. (195i).

6. Hülsmann, W. C., and Lipmann. F., Biochim. el Biophys. Acta, 43, 123 (1950).

7. Jagendorf, A. T., "The Photochemical Apparatus." Brookhaten Symposia in Biol., Il, 236 (1958).

8. Koukol, J., Chow, C. T., and Vennesland, B., J. Biol. Chem., 2.14, 2196 (1959).

9. Kratz, W., and Myers, J., Am. J. Botany, 42, 282 (1955).

10. Linko, I., Holm-Hansen, (., Bassham, J. A., and Calvin, M. J., J. Exptl. Botany', $8,147(1957)$.

11. P’ullman, M. E., and Racker, E., Science, 123, 110.5 (1956).

12. Sacktor, B., O'Neill, J. J., and Cochran, D. G., J. Biol. Chem., 233, 1233 (19.8).

13. Shatkin, A., J. Biophys. Biochem. Cytol., 7, 583 (1960).

14. Thomas, J. B., and De Rover, W., Biochim. et Biophys. Acta, 16, 391 (195.5).

15. Walker, D. A., and Hill, R., Biochem. J., 69, 57p (1958).

16. Whatley, F. R., Allen, M. B., and Arnon, D. 1., Plant Plysiol., Suppl, 32, iii (1957). 


\title{
PHOTOCHEMICAL REIUGTION OF TRIPHOSPHOPYRIDINE NUCLEOTIDE BY ILLUMINATED CHLOROPLASTS
}

\author{
Anthony San Pietro \\ McCollum-Pratt Institute \\ The Johus Hopkins University \\ Baltimore, Maryland
}

It is becoming increasingly apparent that the elucidation of the mechanism whereby pyridine nucleotides are reduced by illuminated chloroplasts is of prime importance for an understanding of the path of hydrogen (or electrons) in photosynthesis. Furthermore, knowledge of the mechanism by which hydrogen (or electrons) is transferred from the photolytic system to the pyridine nucleotides may well further the understanding of the mechanism of photosynthetic phosphorylation. The relationship between these two processes has been the subject of considerable discussion both at this symposium and in the literature and will not be discussed here. (See the paper by Arnon for a discussion of this relationship and references.)

The ability of illuminated chloroplasts to reduce pyridine nucleotides was first demonstrated in 1951-52 by Vishniac and Ochoa (27, 28), Tolmach (24), and Arnon (2). In these experiments the formation of reduced pyridine nucleotides was demonstrated indirectly by coupling the photochemical reaction with a suitable dehydrogenase and measuring the formation of the product of the dehydrogenase system. No directly measurable reduction of pyridine nucleotides was observed in the absence of the coupling system (17). It was, therefore, suggested that the inability of pyridine nucleotides to undergo directly measurable reduction was a consequence of their low oxidation-reduction potential $\left(E_{0}{ }^{\prime}\right.$ at $p \mathrm{H} 7=-0.32$ volt $)$, since most substances that are effective as oxidants in the Hill reaction have high oxidation-reduction potentials $\left(E_{o}^{\prime}\right.$ at $p \mathrm{H} 7=+0.1$ to $0.4 \mathrm{v}$ ).

The rate of pyridine mucleotide reduction observed by Vishniac and Ochoa (28) was 0.03 to $1.6 \mu$ moles per hour per $\mathrm{mg}$ chlorophyll. A somewhat higher rate was reported by Arnon (2), who observed the erolution of oxygen in the presence of TPN, malic enzyme, pyruvate, and carbon clioxide at a rate of $7.5 \mu$ moles per hour per mg chloro- 
phyll. Faster rates of nucleotide reduction have been reported by Hendley and Conn (13), who used chloroplast fragments and the TPN-glutathione reductase from wheat germ or parsley. Recently, Vishniac $(25,26)$ has reported a light-dependent reduction of TPN in a cell-free system. In these experiments, as well as those of Hendley and Conn (13), the formation of TPNH was demonstrated indirectly by measuring the formation of reduced glutathione in the presence of glutathione reductase. In these two reports, the rate of TPN reduction was about $20 \mu$ moles per hour per $\mathrm{mg}$ chlorophyll (i.e., about $40 \mu$ moles GSH formed per hour per mg chlorophyll).

At about this same time, Jagendorf (14) demonstrated that purified chloroplasts can reduce TPN but not DPN. Both TPN and DPN were reduced when grana were used in place of chloroplasts. In the presence of grana, the rate of reduction of DPN was about 6 times that observed with TPN. It should be noted that the experimental procedure used in these experiments was similar to that employed by other investigators, namely, coupling the photochemical reaction with a suitable dehydrogenase or reductase and measuring the formation of the product of the coupling system. In the absence of the coupling system, there was no measurable reduction of pyridine nucleotides with either chloroplasts or grana.

In 1956, San Pietro and Lang (21) presented evidence that under the proper conditions photochemical reduction of pyridine nucleotides can indeed be demonstrated directly. It was shown that when DPN is incubated with chlorophyll grana in the light, either aerobically or anaerobically, DPNH accumulates in the medium. Similar results were obtained with TPN. In these studies, however, it was necessary to employ fairly high concentrations of grana and DPN. At low grana concentrations (less than 60 micrograms chlorophyll per mi) no measurable reduction of DPN occurred. At higher grana concentrations, the amount of DPNH formed was almost directly proportional to the amount of grana initially present.

Preliminary results at that time indicated that it was possible to demonstrate reduction of pyridine nucleotides at low grana concentration, provided that a soluble extract of chloroplasts was added. This finding allows direct measurement of the initial rate of photochemical reduction of pyridine nucleotides, since the reduction can be followed spectrophotometrically by measuring the increase in absorption at $340 \mathrm{~m} \mu$ of the reaction mixture without prior removal of the chloroplasts.

Soon thereafter, Arnon, Whatley, and Allen (3) reported evidence 
in agreement with our findings. They demonstrated that the reduction of TPN by illuminated chloroplasts was made possible by the addition of an aqueous extract of whole chloroplasts. The reduction process was not specific for TPN, since DPN was also reduced but at a slower rate.

Several years ago, San Pietro and Lang (22) reported the isolation from either chloroplasts or whole spinach leaves of a soluble enzyme which is required in addition to either chloroplasts or grana for the photochenical reduction of pyridine nucleotides. The enzyme was partially purified and certain of its properties studied. It was shown that the enzymatic reaction requires the intact dinucleotide structure and, in addition, was more active toward TPN than toward DPN. At that time, it was suggested that the enzyme be named photosynthetic pyridine mucleotide reductase (PPNR).

More recently, the PPNR has been purified more extensively and has been shown to be specific for TPN (19). Under the proper conditions it has been possible to obtain reduction of TPN by illuminated chloroplasts at a rate of about $180 \mu$ moles per hour per mg chlorophyll.

The apparent lack of specificity of either the partially purified PPNR or the aqueous extract of chloroplasts has been attributed, both by Arnon et al. (3) and ourselves (22), to the presence of pyridine nucleotide transhydrogenase $(5)$ in these preparations. Evidence consistent with this explanation has recently been presented by Keister and San Pietro (15). It was demonstrated that the reduction of DPN, in the presence of illuminated grana, requires both PPNR and transhydrogenase; whereas, the reduction of TPN requires only PPNR. It is possible to obtain similar results when chloroplasts are used in place of grana (16).

The transhydrogenase from spinach leaves has been purified extensively and certain of its properties studied (16). In addition, a possible role for this enzyme in photosynthesis has been proposed.

The present paper is concerned with the purification and properties of the photosynthetic pyridine nucleoticle reductase (PPNR).

\section{Determination of ACtivity}

Enzymatic activity was determined by measuring the initial rate of formation of reduced pyridine nucleotides in the Hill reaction. In this procedure the photochemical reduction process is followed directly by measuring the increase in optical density at $340 \mathrm{~m}_{\mu}$ in the following way. The reaction mixture, which contains buffer, pyridine nucleotide, enzyme, and chloroplasts, is prepared in a glass cuvette 
and mixed. The optical density of the mixture is measured against a blank which contains buffer, nucleotide, and chloroplasts before and after illumination for a certain period of time. The increase in optical density, calculated from the two measurements, serves as a measure of the enzymatic activity.

It should be noted that both the blank and the reaction cuvette are illuminated. Since the blank contains everything but enzyme, the data obtained in this manner are corrected for the slow endogenous rate of TPN reduction by chloroplasts alone in the absence of enzyme.

One unit of enzyme is defined as the amount that produces a change in optical density of 1.0 in $10 \mathrm{~min}$ at $340 \mathrm{~m} \mu$ when the reaction mixture contains $100 \mu \mathrm{g}$ chlorophyll per $3 \mathrm{ml}$. This unit of enzyme corresponds to the reduction of $4.8 \mu$ moles pyridine nucleotide per $\mathrm{mg}$ chlorophyll in $10 \mathrm{~min}$.

\section{Purification of EnZyMe}

The purification of the enzyme has been described in part previously $(19,20,22)$ and involves the following steps: (1) preparation of a crude homogenate from spinach leaves; (2) fractionation with acetone; (3) precipitation with protamine sulfate and extraction of the residue; and (4) treatment with a mixture of bentonite and Dowex-l formate followed by concentration of the enzyme with acetone.

A summary of the average yields and purifications obtained at each step for a number of different preparations of the enzyme is presented in Table 1. It is seen that the over-all recovery is 32 per cent and the increase in specific activity is about 200 -fold.

Properties of Enzime

Effect of Enzyme Concentration

Fig. 1 illustrates the relationship of the concentration of enzyme to the amount of TPNH formed. It can be seen that the test is linear

TABLE 1

Summary of Enzyme Purification

\begin{tabular}{lccc}
\hline \multicolumn{1}{c}{ Fraction } & Total units & $\begin{array}{c}\text { Specific Activity } \\
\text { units/mg protein }\end{array}$ & $\begin{array}{r}\text { Per cent } \\
\text { Recovery }\end{array}$ \\
\hline $\begin{array}{l}\text { Crude Homogenate* } \\
\text { Extract of acetone precipitate }\end{array}$ & 5000 & 0.2 & 103 \\
Protamine sulfate & 5150 & $1.5-2.5$ & 70 \\
Dowex-bentonite concentrate & 1600 & $8-12$ & 32 \\
\hline
\end{tabular}

\footnotetext{
" Spinach leares, $1000 \mathrm{~g}$, served as the starting matcrial.
} 


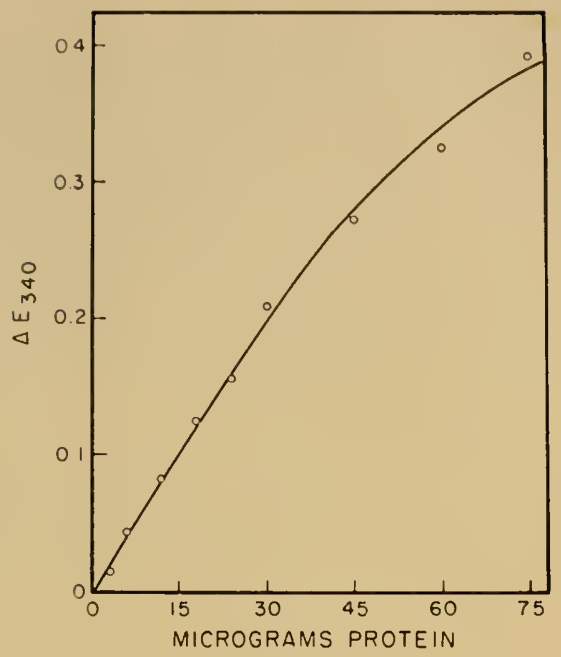

Fig. 1. Enzyme concentration (photosynthetic pyridine nucleotide reductase, PPNR) versus rate of TPN reduction in the presence of illuminated chloroplasts. Each reaction mixture contained $135 \mu$ moles of Tris buffer, $p H 7.2,0.5 \mu$ mole of TPN, enzyme, and chloroplasts equivalent to 88 micrograms of chlorophyll. Final volume, $3 \mathrm{ml}$; time of illumination, $2 \mathrm{~min}$; temperature, $24^{\circ} \mathrm{C}$.

for quantities of enzyme which reduce up to 25 per cent of the TPN initially present.

It should be noted that some six years ago Vishniac and Ochoa (28) explained the low rate of pyridine nucleotide reduction observed in their experiments by the statement that "the grana may be deficient in one or more of the factors required to transfer hydrogen from the photolytic system to the pyridine nucleotides." The results of our studies support their explanation. It is clear from the data presented in Fig. I that the rate of pyridine nucleoticle reduction is proportional to the enzyme concentration. In the absence of enzyme, there is no measurable reduction of pyridine nucleotides.

Although this is not indicated in Fig. 1, it has been possible, under the proper conditions, to observe reduction of TPN by illuminated chloroplasts at a rate of about $180 \mu$ moles per hr per $\mathrm{mg}$ chlorophyll. This rate is about 50 per cent of the maximum rate of carbon assimilation by intact leaves (18).

Nucleotide Specificity

It has been shown previously $(21,22)$ that the enzymatic reaction requires the intact dinucleotide structure. 
The nucleotide specificity of the enzyme in the presence of illuminated chloroplasts is illustrated by the data presented in Table 2. It can be seen that TPN and acetylpyridine-TPN are reduced in this system, whereas neither DPN nor acetylpyridine-DPN are reduced. In separate experiments, it has been established that neither nicotinamide mononucleotide (NMN), nicotinamicle riboside (NR) nor the $\alpha$-isomer of DPN is reduced in this system. These findings are consistent with both the postulation of Armon et al. (3) that TPN is a catalyst of photosynthetic phosphorylation (cf. Arnon, this symposium) and the observation of Jagendorf (14) that chloroplasts can reduce TPN but not DPN.

It should be noted that it is possible to demonstrate the reduction of DPN in this system provided transhydrogenase is added and the concentration of DPN is about $3-4 \times 10^{-3} M(16)$.

\section{TABLE 2}

\section{Nucleotide Specificity}

Each reaction mixture contained $300 \mu$ moles of Tris buffer, $p \mathrm{H} 7.5$, nucleotide, 2 units of enzyme, and chloroplasts equivalent to 91 micrograms of chlorophyll. Final volume was $3 \mathrm{ml}$. Illumination for 5 minutes at $24^{\circ} \mathrm{C}$.

\begin{tabular}{cccc} 
Nucleotide & Concentration & $\Delta E_{340}$ & \lrcorner $E_{365}$ \\
\hline TPN & $2 \times 10^{-4} M$ & 0.78 & \\
DPN & $2 \times 10^{-4} M$ & 0.01 & \\
Acetylpyridine-TPN* & $1.3 \times 10^{-4} M$ & & 0.90 \\
Acetylpyridine-DPN* & $1.3 \times 10^{-4} M$ & & 0.03 \\
\hline
\end{tabular}

* The millimolar extinction coefficient for the reduced acetylpyridine analogues is 9.1 (23).

Independence of Dye Reduction from Enzyme

Our earlier results (22), as well as those reported here, indicate that PPNR is required in addition to chloroplasts or grana for the photochemical reduction of TPN. These data support the hypothesis that the enzyme catalyzes the transfer of hydrogen (or electrons) from the photolytic system to TPN. Additional evidence for this hypothesis is provided by the finding that the enzyme is without effect on the photolytic activity of chloroplasts or grana when measured either spectrophotometrically with 2,3,6-trichlorophenol-indophenol or manometrically with ferricyanide. The lack of dependence on PPNR of dye reduction is illustrated by the data presented in Table 3. It is clear from these data that the rate of dye reduction observed in these experiments is maffected by the presence of PPNR at each 
of the chlorophyll concentrations tested. In separate experiments, it has been possible to demonstrate that the enzyme is completely inhibited by $p$-chloromercuribenzoate at a concentration of $10^{-5} \mathrm{M}$; this same concentration of mercurial is without effect on the dye reduction activity of the chloroplasts.

Recently, Amon, Whatley, and Allen (t) have reported that the TPN-reducing factor in chloroplast extract is not required for the reduction of ferricyanide. This factor is most probably the same as the enzyme described here.

\section{Eflect of Phosphorylation Cofactors}

It has recently been reported by Davenport (7) that the reduction of TPN, in the presence of illuminated chloroplasts, was stimulated

\section{TABLE 3}

Lack of Dependence of Dye Reduction on Photosynthetic Pyridine Nucleotide Reductase

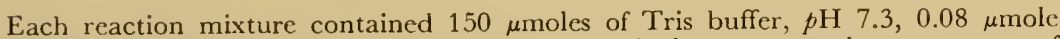
2,3,6-trichlorophenol-indophenol, chloroplasts equivalent to varying amounts of chlorophyll and, where indicated, 1 unit of PPNR. Final volume was $3 \mathrm{ml}$. Illumination for 45 seconds at room temperature.

\begin{tabular}{ccc} 
& \multicolumn{2}{c}{$-\Delta E_{620}$} \\
Chlorophyll, $\mu \mathrm{g}$ & $-\mathrm{PPNR}$ & $+\mathrm{PPN}$ \\
& & \\
1.4 & 0.04 & 0.04 \\
2.8 & 0.09 & 0.09 \\
4.2 & 0.13 & 0.12 \\
5.6 & 0.16 & 0.15 \\
7.0 & 0.19 & 0.18
\end{tabular}

$21 / 2$-fold by the presence of the phosphate acceptor system (ADP, $\mathrm{Mg}^{++}$, and phosphate) and that all the components of this system are necessary for appreciable stimulation. (See also the paper of Hill, in this symposium.) A similar effect was reported several years ago by Duysens (11); however, in a more recent report by Amesz and Duysens (l) this stimulation was not observed.

In our experiments, we have olsserved a stimulatory effect of $\mathrm{Mg}^{++}$ alone (19). This effect was not further enhanced by the addition of ADP and phosphate. We have reinvestigated this effect as well as that reported by Davenport and have confirmed both of them (Keister and San Pietro, unpub. obs.). The effect we reported is observed when the rate of TPN reduction is proportional to enzyme concentration; with excess enzyme, one observes the effect reported by Davenport $(7)$. 


\section{Effect of Chlorophyll Concentration}

The effect of chloroplast concentration on the rate of formation of TPNH is shown in Fig. 2 (lower curve). It can be seen that the rate of photochemical reduction is linearly proportional to the chloroplast concentration. Similar results have been reported previously with grana in place of chloroplasts (22). Several interpretations of this observation with grana have been discussed elsewhere and need not be repeated here (22).

It is interesting that the addition of $\mathrm{Mg}^{++}$increases the rate of TPN reduction at each of the chloroplast concentrations tested (Fig. 2 , upper curve). This effect of $\mathrm{Mg}^{++}$is not completely understood at the present time.

\section{Effect of Enzyme on Photosynthetic Phosphorylation (see paper} by Jagendorf, in this symposium, for additional information)

The effect of the enzyme on photosynthetic phosphorylation is shown in Table 4 . In these experiments both the formation of TPNH and the decrease in inorganic phosphate were determined. lt is clear from these data that the addition of enzyme stimulates both the formation of TPNH and photosynthetic phosphorylation. These results are in agreement with the findings of Arnon et al. (3) that the addition of an aqueous extract of whole chloroplasts to

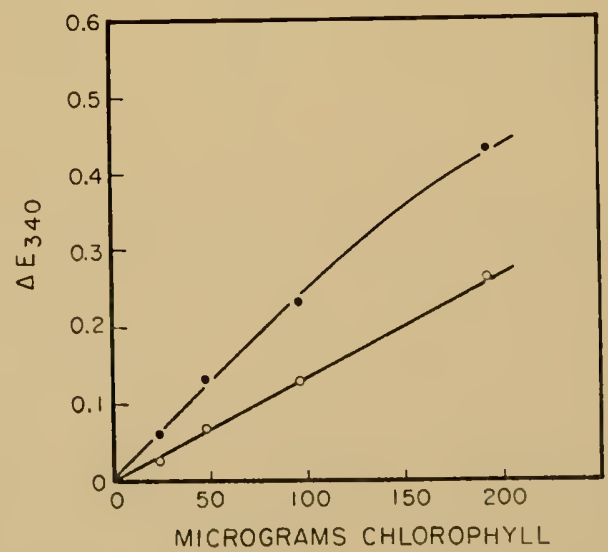

Fig. 2. Effect of chloroplast concentration on the rate of reduction of TPN. Each reaction mixture contained $150 \mu$ moles of Tris buffer, $p H 7.2,0.55 \mu$ moles of TPN, 0.4 unit of enzyme, and chloroplasts equivalent to varying amounts of chlorophyll. Final volume was $3 \mathrm{ml}$; illumination for 5 minutes at $24^{\circ} \mathrm{C}$. Lower curve, no $\mathrm{MgCl}_{2}$; upper curve, $5 \mu$ moles of $\mathrm{MgCl}_{2}$. 
TABLE 4

Effect of Pilotosintiletic Prididine Nucleotide Renugtase (PPNR) on TPN Reduction and PHotosintuletic PIIOSPIIORYiation

Each reaction mixture contained $40 \mu$ moles of Tris buffer, $p \mathrm{H} \mathrm{7.8,5} \mu$ moles of potassium phosphate, $p \mathrm{H} 7.8,10 \mu$ moles of $\mathrm{MgCl}_{2}, 70 \mu$ moles of $\mathrm{NaCl}, 8 \mu$ moles of

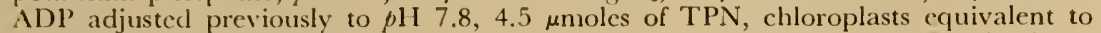
124 micrograms of chlorophyll, and, where indicated, 20 units of enzyme. Final volume was $3 \mathrm{ml}$. Illumination for 10 minutes at $22^{\circ} \mathrm{C}$.

\begin{tabular}{ccc} 
PPNR & $\begin{array}{c}\text { TPNH formed, } \\
\mu \text { moles }\end{array}$ & $\begin{array}{c}\text { Decrease in } \\
\text { inorganic phosphate } \\
\mu \text { moles }\end{array}$ \\
\hline+ & 0.4 & 0.2 \\
+ & 2.6 & 1.3 \\
\hline
\end{tabular}

washed green particles restored to the particles the capacity for photosynthetic phosphorylation.

\section{Photoinactivation}

Initial studies of the photosynthetic reduction were complicated by the observation that the rate of TPN recluction rapidly decreased with time. It was shown subsequently that the non-linearity of $\mathrm{TPN}$ reduction is the result of a chloroplast-dependent photoinactivation of PPNR (12). In the presence of a number of reducing compounds as well as ferricyanide, the photoinactivation is prevented and the reduction of TPN proceeds linearly.

\section{Physical Properties}

The purified enzyme is reddish-brown in color. An absorption spectrum of the enzyme is shown in Fig. 3. The wavelengths of the maxima are $278,327,420$, and $460 \mathrm{~m} \mu$. There is a shoulder at 290 $m_{\mu}$. To date it has not been possible to associate these spectral characteristics with any of the usual electron-transport compounds. While the nature of the redox group, if any is present, in the protein is unknown, it does not appear to be either a haem or a flavin.

It should be noted that the ratio of the absorption at 280 and 260 $\mathrm{m} \mu$ is only 1.2 .

The enzyme was found to give a single symmetrical peak in the Spinco Ultracentrifuge and Tiselius Electrophoresis. The molecular weight of the enzyme, in preliminary experiments, was found to be about 15,660 from sedimentation and diffusion data as well as by the Archibald method.

Amino acid analysis of the enzyme indicated a total of about 95 


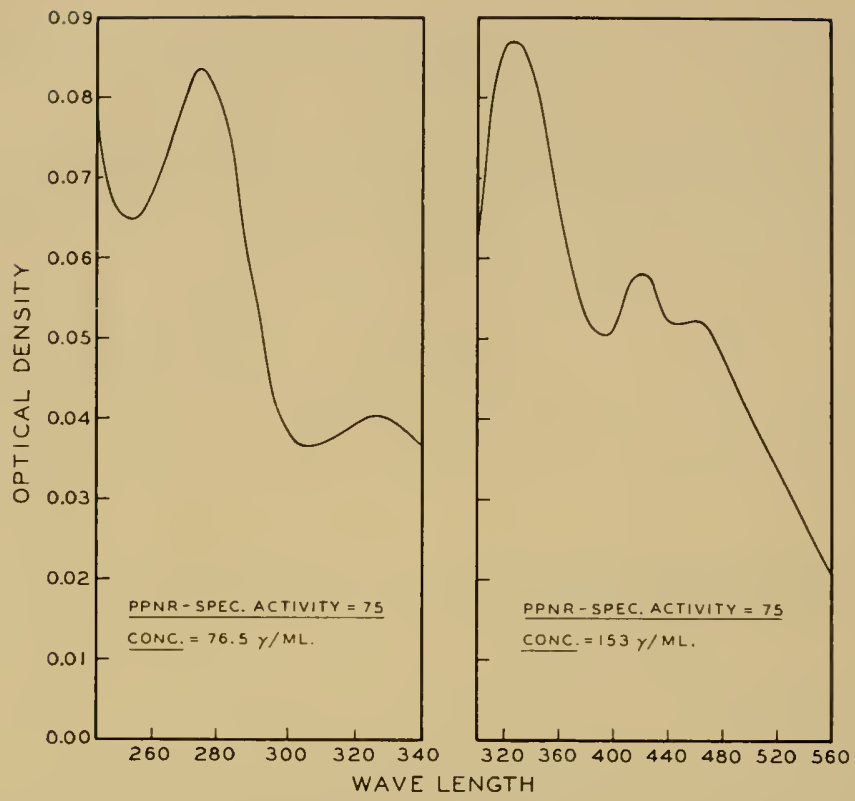

Fig. 3. Absorption spectrmm of purified photosynthetic pyridine nucleotide reductase.

amino acid residues (excluding the tryptophan content of the enzyme) and there does not appear to be any methionine in the enzyme. The minimum molecular weight calculated from amino acid analysis is about 12,000 , but this value does not include the contribution of tryptophan.

Under acid conditions, the enzyme is irreversibly bleached and the activity is completely lost. The bleaching of the enzyme is accompanied by a loss of, at least, the absorption at 420 and $460 \mathrm{~m}_{\mu}$. A similar loss in absorption in this region is observed under alkaline conditions.

\section{Relation to Methaemoglobin-reducing Factor}

Davemport and Hill (9) have recently reported the purification of a protein factor from leaves which was shown previously (10) to catalyze the reduction of methaemoglobin and metmyoglobin by illuminated chloroplasts. They have named this protein the "methaemoglobin-reducing factor." In addition to the compounds indicated above, this factor catalyzes the reduction of a number of haem-protein compounds, including cytochrome c, by illuminated chloroplasts. 
Moreorer, this factor is active in catalyzing the rechuction of TPN by illuminated chloroplasts $(6,7)$.

It is of interest, therefore, to compare the properties of PPNR with those of the purified factor which have been reported by Davenport and Hill (9). These are: "(1) the active fraction from all plants examined was pinkish orange in color. In every case absorption in the visible range increased towards the shorter wavelengths with two diffuse absorption bands at 465 and $422 \mathrm{~m}_{\mu}$. (2) The molecular weight of the protein, calculated from sedimentation and diffusion measurements, is 19,000 . (3) The chemical nature of the group conferring oxidation-reduction properties to the protein has not yet been determined but neither flavin nor haem could be detected." It should be noted that the factor does not stimulate the reduction of ferricyanide or 2,6-dichlorophenol-indophenol by illuminated chloroplasts. In addition, the minimum molecular weight from amino acid analysis is 17,400 and there is no methionine in it (8).

At the suggestion of Dr. H. E. Davenport, the ability of PPNR to catalyze the reduction of cytochrome $c$ by illuminated chloroplasts was examined, and it was found to catalyze this reduction.

In view of the similarities between PPNR and the "methaemoglobin-reducing factor," it is tempting to speculate that these two activities are associated with the same protein. The validity of this speculation must await the results of further experimentation.

In conclusion, the enzyme photosynthetic pyridine nucleotide reductase is required in addition to chloroplasts for the photochemical reduction of TPN. The evidence available to date supports the hypothesis that this enzyme catalyzes the transfer of hydrogen (or electrons) from the photolytic system to TPN. It seems reasonable, therefore, that the study of this catalytic activity will further the understanding of the mechanism for converting light energy into chemical energy.

\section{REFERENCES}

1. Amesz, J., and Duysens, L. N. M., Discussions Faraday Soc., 27, 173 (1959).

2. Arnon, D. I., Nature, 167, 1008 (1951).

3. Arnon, D. I., Whatlev, F. R., and Allen, M. 13., Nature, 180, 182 (1957).

4. Arnon, D. I., Whatley, F. R., and Allen, M. B., Science, 127, 1026 (1958).

5. Colowick, S. P., Kaplan, N. O., Neufeld, E. F., and Ciotti, M. M., J. Biol. Chem., 195, 95 (1952).

6. Davenport, H. E., Biochem. J., 73, 45P (1959).

7. Davenport, H. E., Nature, 184, 524 (1959).

8. Davenport, H. E., pers. commun.

9. Davenport, H. E., and Hill, R., Biochem. J., 74, 493 (1960). 
10. Davenport, H. E., Hill, R., and Whatley, F. R., Proc. Roy. Soc. London B, 139, 346 (1952).

11. Duysens, L. N. M., in "The Photochemical Apparatus," Brookhaven Symposia in Biol., 11, 10 (1958).

12. Giovanelli, J., and San l'ietro, A., Arch. Biochem. Biophys., 84, 471 (1959).

13. Hendley, D. D. and Conn, E. E., Arch. Biochem. Biophys., 46, 454 (1953).

14. Jagendorf, A. T., Arch. Biochem. Biophys., 62.141 (1956).

15. Keister, D. L., and San Pietro, A., Biochem. Biophys. Research Commun., I. 110 (1959).

16. Keister, D. L., San P'ietro, A., and Stolzenbach, 1. E., J. Biol. Chem., October, 1960.

17. Mehler, A., Arch. Biochem. Biophys., 33, 65 (1951).

18. Rabinowitch, E. 1.. "Photosynthesis and Related Processes," Vol. 2, 1't. 2. p. 1594, Interscience Publishers, New York (1956).

19. San Pietro, A., in The Photochemical Apparatus, Brookharen Symposia in Biology, Vol. 11, p. 262 (1958).

20. San Pietro, A., in Methods in Enzymology Vol. VI (S. P'. Colowick and N. O. Kaplan, eds.), Academic Press (in press).

21. San Pietro, A., and Lang, H. M., Science, 124, 118 (1956).

22. San Pietro, A., and Lang, H. M., J. Biol. Chem., 231, 211 (1958).

23. Stein, A. M., Kaplan, N. O., and Ciotti, M. M., J. Biol. Chem., 234, 979 (1959).

24. Tolmach, L. J., Nature, 167, 946 (1951).

25. Vishniac, W., in Research in Photosynthesis (H. Gaffron, ed.), p. 285, Inte:science Publishers, New York (1957).

26. Vishniac, W., in "The Plotochemical Apparatus," Brookhaven Symposia in Biol., 11, 54 (1958).

27. Vishniac, W., and Ochoa, S., Nature, 167, 768 (1951).

28. Vishniac, W., and Ochoa, S., J. Biol. Chem., 195, 75 (1952). 


\section{Part V}

BIOLOGICAL RESPONSES TO LIGHT 



\section{INTRODUCTORY COMMENT}

K. Hartline

Rockefeller Institute, New York 21, N. Y.

From what I have heard today, I gather that photosynthesis is now completely understood. We can leave that subject and go on to the next in this symposium on Light and Life. It is easy to persuade ourselves and probably reasonable enough that the almost complete dependence of the living world on photosynthesis will result in the evolution of useful mechanisms to bring living organisms into favorable relations with their lighted environment. Plants photosynthesize; animals consume the plants or prey on other animals which consume the plants. This notion from high school biology serves as a useful excuse for leading into our next topic in the symposium. 


\section{PHOTOTROPISM}

\section{Kenneth V. Thimann and George M. Curry ${ }^{1}$ \\ Harard Biological Laboratories, Cambridge, Massachusetts}

Phototropism stands near the base of a pyramid of light-reactions of increasing complexity, the apex of which is vision. Yet it is not quite at the base, for a still simpler reaction is light-directed free movement, shown by purple bacteria and motile cells of many aquatic plants, as well as free-swimming stages of many invertebrates. Such free movement, though sometimes erroneously referred to as phototropism, is properly called phototaxis. Phototropism is the light-directed curvature of fixed organisms (nearly always plants). It was Charles and Francis Darwin, as long ago as 1880, who showed that some of the most phototropically sensitive plants were the seedlings of the Gramineae, which have served as the major experimental material ever since. Among many other things, the Darwins showed that in these plants the zone of light perception was the tip of the coleoptile, while it was the part some millimeters below the tip which responded by curving. The recognition that the zones of perception and response are separated in space led to a sequence of studies by Rothert, Boysen Jensen, Paal, Söding, Cholodny, and Went, which established that the growth of the coleoptile is controlled by a growth hormone, termed Auxin. This substance is produced in the tip and transported thence to the lower parts, which respond by elongating. The logical consequence of such a concept is that when the plant curves, instead of growing straight upward, the curvature is clue to an excess of auxin on one side, causing that side to elongate laster than the other side. If curvature takes place towards the light (positive phototropism) this would mean that an excess of auxin must occur on the side away from the light. No sooner had this theory, applicable to all tropisms, been proposed by Cholodny (1927) than it was confirmed by Went (1928). Went allowed the atuxin coming from the tip of an illuminated oat coleoptile to diffuse out into two small agar blocks in contact respectively with the lighted and shaded sides of the plant. On assaying the auxin activity, by applying these blocks to the sides of other colcop-

'Present address: Tufıs Inisersity, Medford, Mass. 
tiles, it was found that about twice as much auxin had come from the shaded side as from the lighted side. Similar results have been obtained by several workers since. A recent more extensive repetition of these experiments (Briggs, Tocher, and Wilson, 1957) is shown in Fig. 1. The figure brings out four relevant points:

(I) illumination of the tip does not influence the total auxin production;

(2) slitting the basal end of the tip section-necessary to separate lighted and shaded halves-does not of itself affect the total auxin production;

(3) the yield of auxin from the lighted side decreases and that from the shaded side increases (confirming the older observation); but

(4) this difference does not occur when the coleoptile section is slit all the way to the top.

The first and last points show that simple destruction of the auxin by light is not the explanation of the uneven distribution, but that it depends on lateral movement of the auxin from the lighted towards the shaded side, which is demonstrated in $e$ and $f$. This conclusion had in fact been reached much earlier as a result of com-

a)

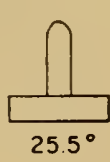

c)

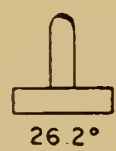

(4)

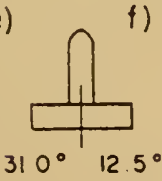

b)

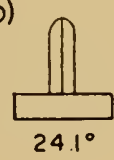

d)

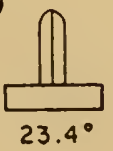$$
24.1^{\circ}
$$

LIGHT

DARK

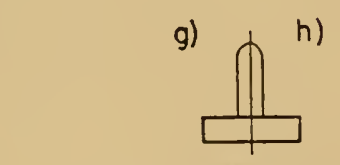

$23.0^{\circ} 24.7^{\circ}$

Fig. 1. Diffusion of auxin into agar in 3 hours from corn coleoptile tips. Each figure, the curvature produced on standard decapitated Avena by 3 corn tips. $a$, intact, dark; $b$, totally slit, dark; $c$, intact, light; $d$, totally slit, light; lower left: slit only at base; $e$, (lark side; $f$, light side; lower right, totally slit; $g$, dark side; $h$, light side. (From Briggs, Tocher, and Wilson, 1957). 
parable but simpler experiments by Boysen Jensen, in which only the curvature of slit coleoptiles was observed. Because it is of fundamental importance it will be referred to again below.

IVe may conclude, then, that the ultimate effector of phototropic curvature is a lateral diversion of auxin away from the lighted side of the plant. This is, in effect, the present state of the problem. The questions to be answered are of three types:

(a) Is there more than one kind of phototropic response?

(b) What are the photoreceptors?

(c) What kinds of processes occur between the reception of the light and the resulting asymmetric distribution of the auxin?

Before presenting the evidence which helps to answer these questions, it should be stated that the auxin has been identified, at least in the majority of plants, as indole acetic acid. It is well to mention also that although in the earlier work several different grasses have been used, our own experiments have been carried out only with the oat (Avena) which is apparently about the most sensitive member of the group, and with the fungus Phycomyces. It will be convenient to take up the second question first.

\section{Nature of the Photoreceptor in Avena}

Since phototropism is a growth response, which therefore requires intact cells, the most direct route to the study of the photoreceptor is through the spectral sensitivity of the response. Such an action spectrum should indicate the absorption spectrum of the photoreceptor, and, if obtained in sufficient detail, might serve to identify that compound.

Several studies of spectral sensitivity have been made in the past, but with insufficient detail for the drawing of reliable conclusions. All agree on one point, nevertheless: curvature is not brought about by red light. Our studies have shown, however, that oat seedlings are by no means insensitive to red light. Previous exposure of the dark-grown plants to red light of wavelength greater than $660 \mathrm{~m} \mu$ changes their sensitivity to a subsequent dose of blue or white light. What happens is that the distribution of growth rate along the seedling is changed as shown in Fig. 2. The dark-grown plants show two maxima of growth rate, one at about $6 \mathrm{~mm}$ below the tip, and a second one around the node which separates the coleoptile from the mesocotyl below. The effect of one hour's exposure to red is very greatly to increase the growth rate in the zone 4-8 $\mathrm{mm}$ below the tip and to suppress almost wholly the elongation at and below the node. This finding made it necessary to control rigidly the ex- 


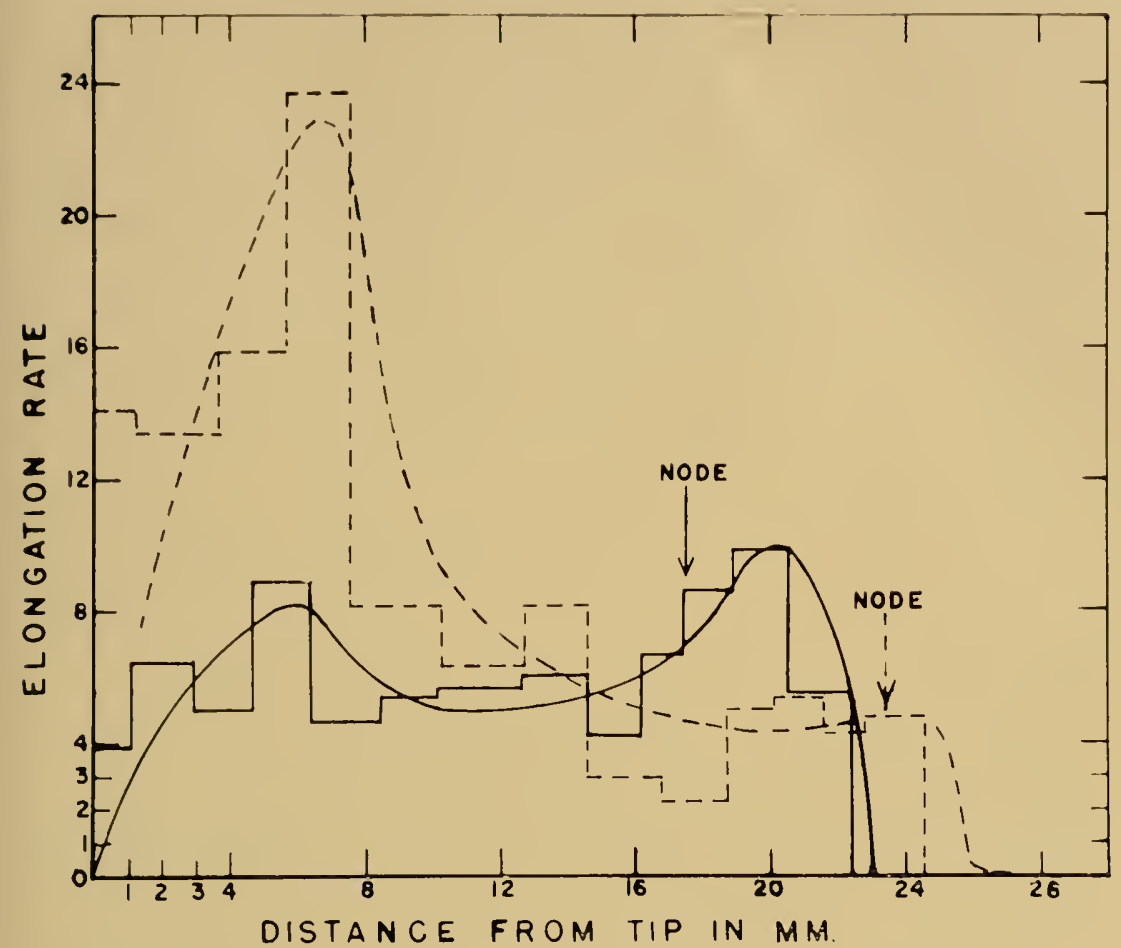

Fig. 2. Distribution of growth rate, expressed as per cent elongation per 100 minutes, along the Arena seedling. Solid line, plants kept in darkness, given red light only in first 2 hours of germination; dashed line, plants exposed to red light for one hour just before measurement. (From Curry. Thimann, and Ray, 1956).

posure of the experimental plants to red light; where possible, weak green light, which is phototropically inactive, was used, and such exposure to red as was unavoidable was carefully standardized. The treatment of the plants was of course standardized in all other respects; they were used at an age of about 74 hours from the initial soaking and selected for miform height and straightness.

When such standard seedlings are illuminated from one side with measured dosages of monochromatic blue light, results like those in Fig. 3 are obtained. As was shown as long ago as 1909 by Blaauw, curvature is proportional over a certain dosage to the product $I \times t$ (see especially Arisz, 1915). In order to cover a sufficient range of energies, $\log I t$ is plotted here, which means that the curve should flatten out asymptotically at the left. But over a satisfactory dosage range there is linear proportionality to $\log I t$. Lange (1927) found that the minimum light closage for threshold curvature was $1 / 2$ 


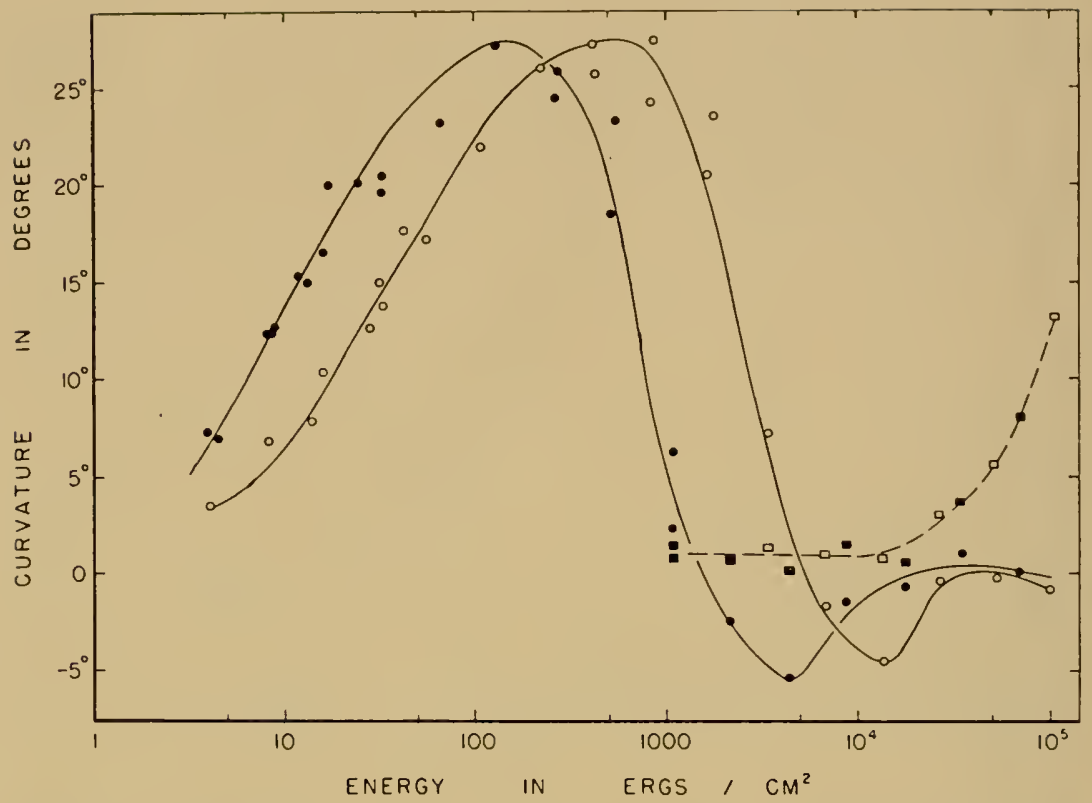

Fig. 3. The response of Atena coleoptites to a series of light dosages at two wavelengths. Each point is the mean of $10-14$ individual curvatures, measured after 100 minutes. Solid symbols, $436 \mathrm{~m} \mu$; open symbols, $365 \mathrm{~m} \mu$. Circles, tip curvature; squares, base curvature.

$\mathrm{erg} / \mathrm{cm}^{2}$, which is very well compatible with Fig. 3. Curvature becomes maximal at about $27^{\circ}$, and at higher light dosages it decreases again, finally reaching a small negative value. However, the negative curvatures occur only over limited ranges of rather high intensity and will not be cliscussed further here.

Fig. 3 shows that light of wavelength $436 \mathrm{~m} \mu$ is almost three times as effective as that of $365 \mathrm{~m} \mu$. With the same procedure a great number of wavelengths have been compared over the range of linearity, ı.e., at low energies. The mercury lines 492, 436, 408, 405, 365, and $313 \mathrm{~m} \mu$ gave the best data, and additional wavelengths were obtained by using narrow bands of the continuous spectrum from a tungsten lamp. The average of three series of experiments performed under as nearly identical conditions as possible is shown in Fig. 4.2 This, then, is the action spectrum for the positive phototropic curvature of Avena colcoptiles in the low-energy range.

"The data of three groups of experiments, in cach of which the sensitivity of the plants dillered slightly, were combined by making the sensitivities at 136 m $\mu$ identical, and calculating the relative sensitivities at all other wavelengths therefrom. 


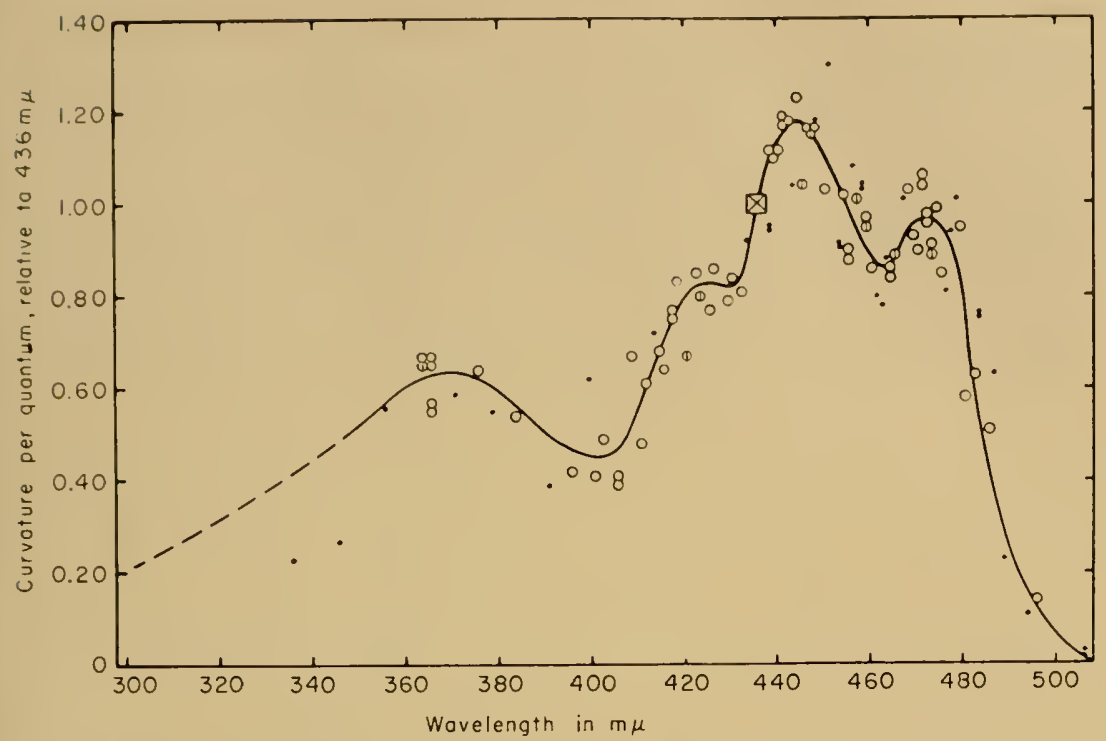

Fig. 4. Action spectrum for the positive curvature of Avena coleoptiles caused by monochromatic light sources of low intensity. Average of three series of experiments.

The spectrum in Fig. 4 has five characteristics: the main peak at $445 \mathrm{~m} \mu$, the secondary peak at about $472 \mathrm{~m}_{\mu}$, the trough separating them, the inflection at about $425 \mathrm{~m}_{\mu}$, and the broad peak in the near ultraviolet. The trough at about $460 \mathrm{~m} \mu$ has been confirmed by separate experiments in which equal energies were applied at 448 , 460 , and $472 \mathrm{~m} \mu$; the curvatures resulting were $16.02 \pm 0.40,14.82$ \pm 0.41 and $16.19 \pm 0.38$ respectively. Expressed as degrees per unit of energy the values are $1.31,1.19$, and 1.31 at 448,460 , and 472 $m \mu$ respectively. In another comparison using different doses to obtain equal curvatures, the following values were obtained: 1.37, 1.19, and 1.27 for wavelengths 443,458 , and $473 \mathrm{~m}_{\mu}$ respectively. The relative heights of the bands at 365 and $445 \mathrm{~m}_{\mu}$ were also compared in direct experiments (first perfomed by Conrad Yocum) and ratios of $0.45-0.50$ (on a quantum basis) found. These agree with the general conclusion of Blaauw (1909) and Somne (1929) that the near ultraviolet is less than half as effective as the blue. Thus the action spectrum is well established. In the meantime, and independently, Shropshire and Withrow (1958) have performed somewhat comparable experiments, and while the results differ in certain cletails, the presence of peaks at 410-415, 440-445, and $470-475 \mathrm{~m}_{\mu}$ is in agreement with Fig. 4. Their finding that the proportionality con- 
stant between curvature and $\log$ dose varies with wavelength will be discussed in a later paper.

The next question is: to what photoreceptor does this action spectrum point? For years the sensitivity of phototropism to blue light has been taken to mean that the photoreceptor may be a carotenoid, and this has been felt to be supported by the clear indications that the action spectrum has two peaks in the visible; this was particularly true of the data of Bachmann and Bergann (1930) and Johnston (1934). In Curve B of Fig. 5 the absorption spectrum of $\alpha$-carotene

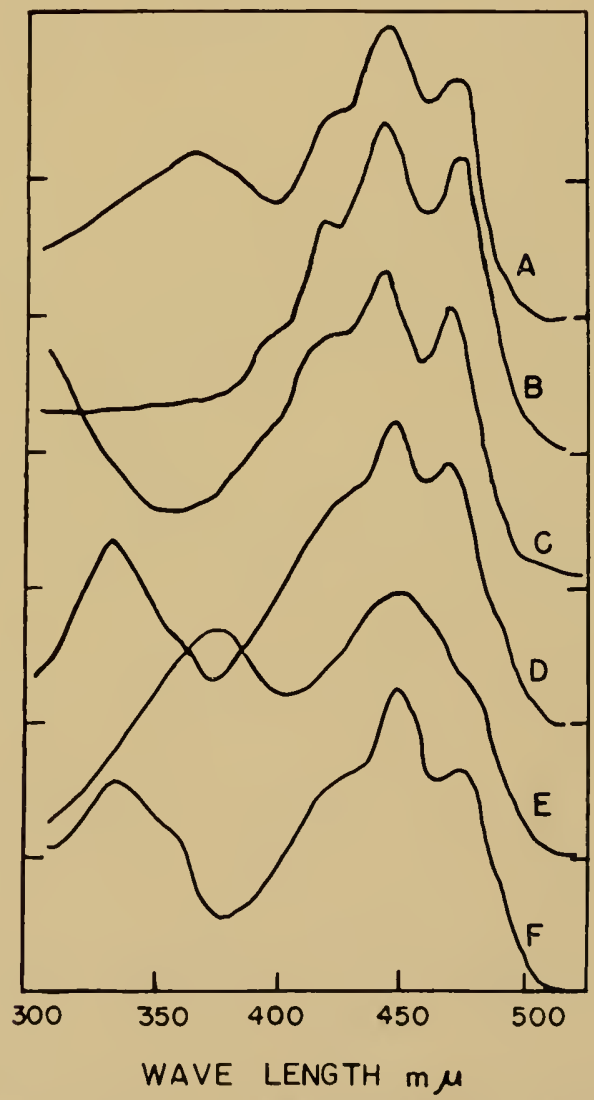

lig. 5. Action spectrum of lig. 4 (curve . 1 ) compared with absorption spectra of:-

B. $\alpha$-atotenc in hexane;

C, hexane extract of 500 coleoptile tips;

D, 9-9' cis $\beta$-carotene in hexanc; curve of Inhoffen et al. (1950):

E, riboflavin in waler;

F, 3-methyl-lumiflat in in benzene; curve of Harbury et al. (1959). 
in hexane is seen to agree exceptionally well with the observed action spectrum (Curve A) in the visible; and Curve $C$ shows that such a carotenoid is indeed extractable from the tip. However, the carotene has no absorption in the near ultraviolet. It appears that such absorption is a function of the spatial configuration of the chain of conjugated double bonds. Presence of one or more cis-configurations introduces a band, usually between 300 and $350 \mathrm{~m} \mu$. Curve D show's the absorption spectrum of $9-9^{\prime}$ cis $\beta$-carotene, synthesized by Inhoffen, Bohlmann, and Rummert (1950); the ratio of absorption in the ultraviolet to absorption at the main peak is just over 0.5 . This curve could be considered very acceptable agreement with the action spectrum were it not for the location of the cis-peak at 340 instead of $365 m \mu$. To date, in hexane solution none of the cis-peaks recorded in the literature has been found to approach within $140 \mathrm{~m}_{\mu}$ of the long wavelength peak of the corresponding all-trans form.

In 1949, however, Galston and Baker suggested that the photoreceptor for phototropism might be riboflavin; this proposal was based on (a) the general agreement between the zone of absorption in the visible by riboflavin and the range of wavelengths of phototropic sensitivity, and (b) the ability of riboflavin to sensitize the photoinactivation of indole acetic acid in vitro. The absorption spectrum of riboflavin in water is shown in Curve $\mathrm{E}$; it is evident that although it does show a peak at about $365 \mathrm{~m} \mu$, this has almost the same height as that of the main peak at $450 \mathrm{~m}_{\mu}$, while in the visible the curve differs greatly from the action spectrum for phototropism. Most of the known flavoproteins, including at least four pure flavin-enzymes, have absorption spectra very similar to that of riboflavin. However, in one or two cases, notably the dihydrothioctyl dehydrogenase of Searls and Sanadi (1959), the usually smooth curve in the visible is replaced by an asymmetric one, with the main peak at $456 \mathrm{~m} \mu$, and a clear shoulder appearing at about $485 \mathrm{~m} \mu$. This evidence of fine structure becomes greatly magnified in the ribose-free derivative lumiflavin, and especially in 3-methyl lumiflavin; when these substances are dissolved in benzene the shoulder at $485 \mathrm{~m} \mu$ actually appears as a second peak, and in addition a new shoulder appears at $425 \mathrm{~m}_{\mu}$ (curve $\mathrm{F}$ of Fig. 5). Harbury et al. (1959) have shown that this behavior is a function of the solvent; water or other hydrogen-bonding solvents suppresses the separation of the peaks, making the absorption in the visible spectrum approximate to a smooth curve, but at the same time they shift the peak in the ultraviolet up from $340 \mathrm{~m} \mu$, in benzene, to about 
$365 \mathrm{~m} \mu$ in aqueous media. In other words, the presence of the clearly defined peaks in the visible is associated with an ultraviolet peak too lar to the left; but when the ultraviolet peak is in the right place to fit that of the action spectrum the three resonances in the visible are damped out. Hence the action spectrum in the ultraviolet is not really well accounted for by any of the carotenoids or flavins known at present. However, the ratio of heights at 365 and $445 m \mu$ in the action spectrum is much nearer that of the carotenoid than that of the flavins.

Thus even the most detailed action spectra for phototropism in the visible and near ultraviolet unfortunately do not lead to an unequivocal conclusion about the photoreceptor. By analogy with vision, in which the photoreceptor is a complex of a cis-carotenoid and a protein, the participation of a cis-carotenoid in phototropism is olsviously an attractive hypothesis; however, it remains to be proven (a) that such a cis-compound is present, (b) that the solvent or combined form in which it exists has the effect of shifting the wavelength of the cis-peak about $20 m \mu$ towards the red without affecting the peaks in the visible. As against this, the possibility that the photoreceptor is a ribose-free flavin derivative in a non-polar solvent cannot be excluded, though, curiously enough, this is subject to exactly the same reservation, namely (b) above, as for a carotenoid.

There is, of course, some supporting evidence. Lactobacillus assays show that riboflavin is present throughout the coleoptile, with no particular concentration at the tip. The presence of $\beta$-carotene or lutein in the hexane extracts of the tip was shown in Fig. 5,C. The absence of the cis-peak in these extracts might be due either to a great preponderance of the trans form, or to loss of the cis-configuration as a result of extraction (cf. the paper of Wald in this symposium). Chromatographic analysis of these extracts shows at least three carotenoids present.

Since carotenoid-protein complexes do exist in plants (Nishimura and Takamatsu, 1957) it seemed worth-while to examine the absorption spectrum of the coleoptile tip in vivo and to compare this with the absorption of the protein-free extract in hexane. This was clone with the opalescent plate method of Shibata (1956), with the results shown in Fig. 6. The steeply rising "end-absorption" can be approximately corrected for by drawing a smooth curve between 390 and $52011 \mu$ and subtracting this from the overall curve, with the result shown as the lower curve. The three peaks are brought out very sharply, and it can be concluded that their positions are probably 
KENNETH I. THIMANN AND GEORGE M. CURRY 655

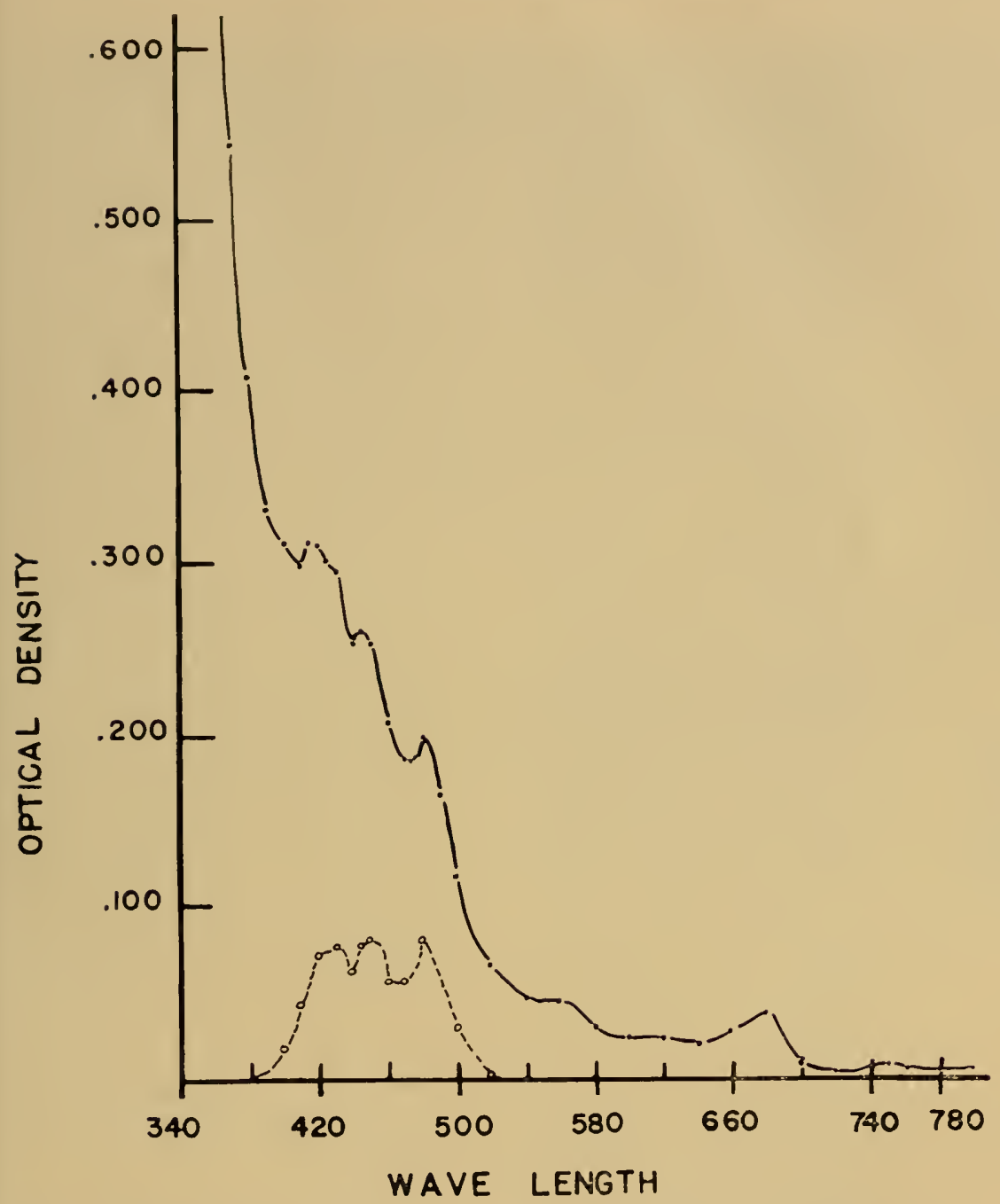

Fig. 6. Absorption spectrum in vivo of 20 Avena coleoptile tips determined with the opaque plate method. Below, curve corrected for end-absorption.

not shifted from those in the extract. Unfortunately, the end-absorption obscures the important range in the near ultraviolet.

In 1937, and again in 1955, Bünning demonstrated the presence of carotene or a lutein in the coleoptile by the microchemical method of Molisch, which leads to the formation of characteristically shaped crystals in situ. He found a high concentration of the carotenoid 
just below the tip. Mrs. Sorokin, in my laboratory, has confirmed. these observations (Fig. 7) ; excellent crystals appear from $250 \mu$ below the tip down to about $2000 \mu$; below this point, and in the extreme tip, very few crystals could be seen. Thus the carotenoid is localized close to the region of highest light-sensitivity; but again the conclusion is not unequivocal, since the highest sensitivity, according to Lange, is in the tip itself.

Several workers have clained that albino mutants of various seedlings contain little or no carotene and yet show "normal" phototropic sensitivity. However, such albinos usually contain not less than $5-10 \%$ of the carotenoid of the normal siblings, and there is no information as to the absolute quantity of photoreceptor necessary for phototropism. Furthermore, the claims of normal sensitivity have not been supported by quantitative data in the range of proportionality to light dosage, and usually rest on very long exposures. In the case of the white Helianthus mutant of Wallace and Schwarting (1954), our own experiments show that its phototropic sensitivity is indeed abnormally low. Galston and Asomaning (1960) have recently experimented with an albino mutant of Colsess barley. Its photosensitivity in the proportional range was about $30-50 \%$ of that of the potentially green (but etiolated) form, while the data for total carotenoid, mostly lutein, in the tip region of the two plants were respectively 0.7 and 1.8 , which is close to the ratio of the sensitivities. The content of FMN, on the other hand, was the same in both. They found also that both in oats and in barley pre-exposure to red light about doubles the concentration of FMN; yet in oats the photosensitivity is decreased, while in barley it is unchanged or even somewhat increased.

On balance, these varied observations favor the carotenoid as photoreceptor, and certainly it has been proved to be present, which is not the case for methyl-lumiflavin. However, more work is called for and the matter must be left there at present.

\section{Different Types of Phototropism in Avena}

The second of the major questions raised concerned the number of different types of phototropic response. As far as the Avena coleoptile is concerned an answer to this question was given long ago, when Arisz (1915) made careful measurements of the phototropic response to a graded series of light dosages. These showed that while low dosages cause a positive curvature, higher ones, if the time of exposure is not too long, may give a negative curvature. Still more 


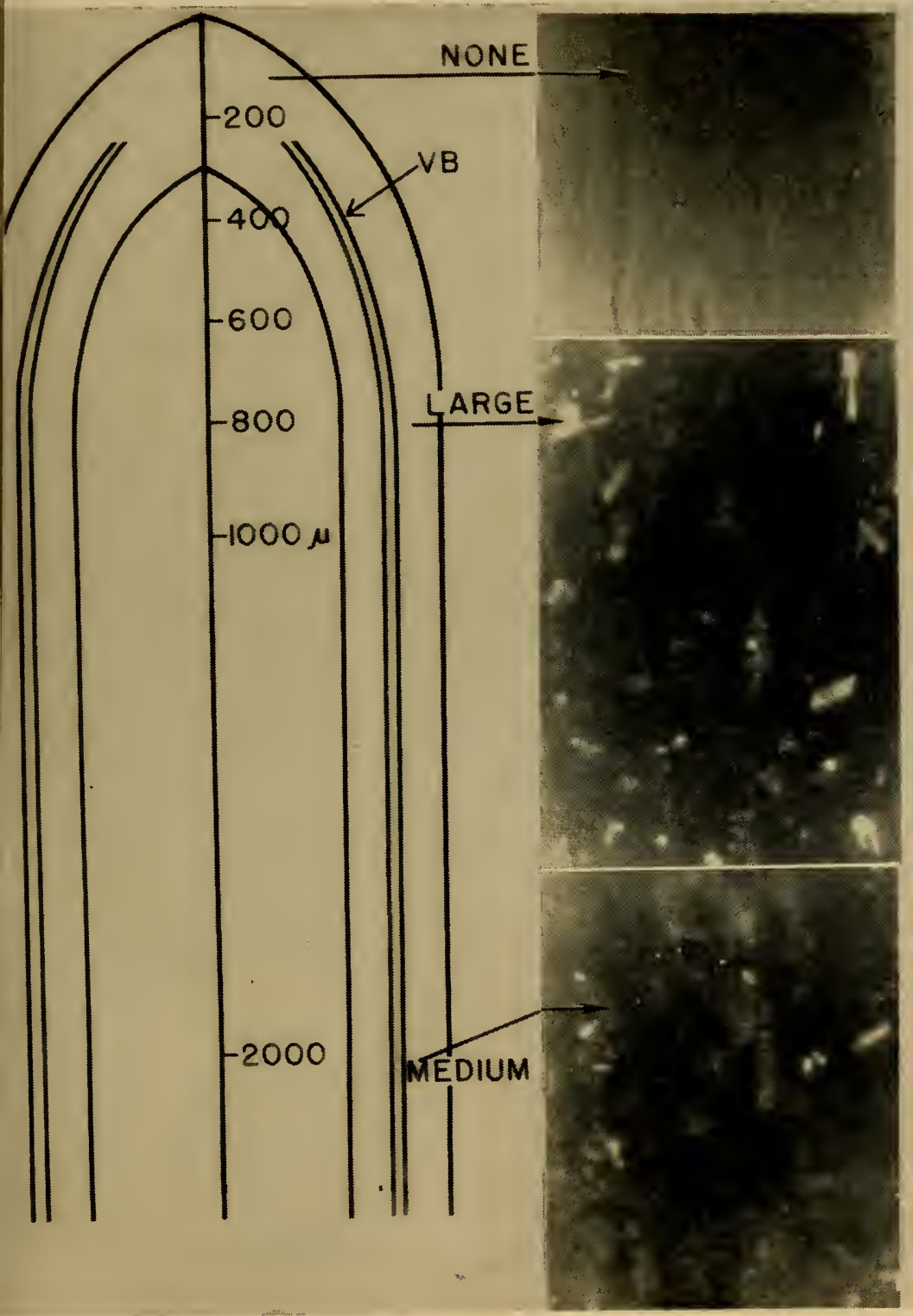

Fig. 7. Carotene crystals obtained by the Molisch method from different zones of the Avena coleoptile. VB, rascular bundle. Photographed in polarized light $\times 1000$. On right, isolated crystals $\times 1500$. 
prolonged exposure again gives a positive curvature, as can be seen above in Fig. 3. However, the curvature at very high energies is of a somewhat different type. While the response to low dosages begins slowly, is clearly centered close to the tip, and extends gradually downwards, the high-energy curvature starts earlier and is distributed along the whole length of the coleoptile, so that the plant shows no localized zone of curving. After 120 minutes the high-energy curvature appears mainly in the base. For this reason the second type has been generally called the "base response," though "high threshold response" would be a better term. It is this which mainly constitutes the normal curvatures of plants in daylight, as shown for instance by potted plants in a window.

The same type of basal curvature results when the Avena coleoptile is unilaterally illuminated with ultraviolet light. This has made it possible to determine an action spectrum for the base curvature in ultraviolet light. If the light intensity is held constant and the duration of exposure varied, the resulting curvature shows a satisfactory linear relationship with the $\log$ of the exposure. However, for the data to be meaningful, tip curvatures must be exclucled. The plants were therefore exposed behind a mask which completely shaded the most apical 2-4 mm. Using a grating monochromator and a high pressure mercury vapor lamp, the whole region down to $235 \mathrm{~m} \mu$ was explored. The results are shown in Fig. 8. There are apparently two peaks close together, at 280 and $297 \mathrm{~m} \mu$, and there is evidence of a rise again at the shortest wavelengths. Sensitivity falls off very sharply above $300 \mathrm{~m} \mu$.

It is evident, therefore, that this response differs from the tip response not only in its clistribution within the plant but in the effective photoreceptor. It is notable, for instance, that at 265 and $365 \mathrm{~m} \mu$, where flavins show major peaks, the sensitivity is low; at $365 \mathrm{~m} \mu$, indeed, it is extremely low. None of the colorless carotenoid precursors whose spectra have been recorded (e.g., phytofluene and phytoene) shows absorption matching the spectrum of Fig. 8. An attractive hypothesis (Curry, Thimann, and Ray, 1956) is that the action spectrum is the absorption spectrum of indole acetic acid (IAA) itself, but shifted $12 \mathrm{~m} \mu$ towards the visible. Both the short wavelength end and the steep fall on the long-wave side agree with this interpretation very well; there is a difference around the peak, however. The spectrum of indole acetic acid has the characteristic "pip" at about $287 \mathrm{m \mu}$, shown by most indole compounds which do not contain a side-chain double bond conjugated with those in the 


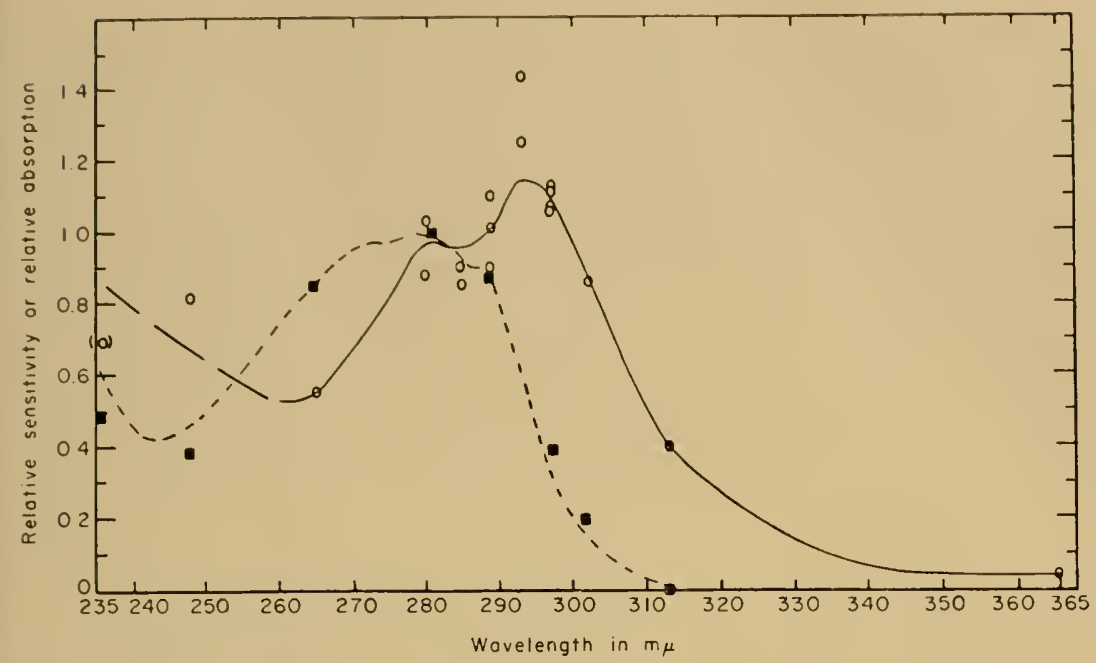

Fig. 8. Iction spectrum for the Arena coleoptile "base" curvature towards ultraviolet light (solid line). The dotted line shows the absorption spectrum of pure inclole acetic acid (1.A.). 'The black squares are points on the action spectrum for the inactivation of pure I.A in vitro. (From Curry, Thimann, and Ray, 1956).

ring (Kaper, 1957). The apparent accentuation of this "pip" in the action spectrum, together with the $12 \mathrm{~m} \mu$ shift to the right, suggests that the IAA may be in chemical combination. The hypothetical compound must still be a growth-substance, since its reaction with light modifies the growth. Furthermore, pure IAA in aqueous solution is inactivated by very high dosages of ultraviolet, and the action spectrum for this process follows closely the absorption spectrum of IAA. In particular, the value at $297 \mathrm{~m} \mu$ is about one-fourth the value at $287 \mathrm{~m} \mu$, and the peak lies near $280 \mathrm{~m} \mu$ (the black squares in Fig. 8). However, the amounts of energy required for inactivation in vitro are several thousand times as great as those needed for phototropic curvature. Therefore in the postulated compound the indole acetic acid would have to be sensitized to the action of ultraviolet.

Some support for this view was obtained as follows. If the plants are thrice decapitated 90 minutes apart so as to minimize their content of endogenous hormone, then they no longer curve toward ultraviolet light, even at a closage of $2000 \mathrm{ergs} / \mathrm{cm}^{2}$. In intact plants this close would give a maximum curvature of about $17^{\circ}$. However, if agar containing IA ( $0.5 \mathrm{mg}$ per liter) is applied between the second and third decapitations, curvatures of good magnitude can 
be obtained. If the IAA is left on only for 30 minutes the curvatures are restricted to the upper $5 \mathrm{~mm}$. Data from such a group of experiments are summarized in Table 1; clearly IAA can reinstate the lost phototropic sensitivity. This result is not due merely to reinstatement of the growth of the decapitated coleoptile, for application of the IAA after ultraviolet illumination did not cause curvature under these conditions. (With very much larger doses of white light, totalling about 500,000 ergs $/ \mathrm{cm}^{2}$, von Guttenberg (1959) has shown that IAA can produce curvature if applied after the illumination, but that is another phenomenon, to be discussed below). It is concluded that in the phototropism caused by ultraviolet light, and probably therefore in the high-threshold response in general, there is a

\section{TABLE 1}

\section{Reinstatement of Phototropic Curvature of Decapitated Coleoptiles in the Ultraviolet by Application of Indole Agetic Acid in Agar}

\begin{tabular}{|c|c|c|}
\hline & $\begin{array}{l}\text { Exposure in } \\
\text { ergs/.m } \\
297 \mathrm{~m} \mu\end{array}$ & $\begin{array}{c}\text { Curvature } \\
\text { in } \\
\text { degrees }\end{array}$ \\
\hline Intact plants, tips shielded & $\begin{array}{l}278 \\
348\end{array}$ & $\begin{array}{r}8.8 \\
10.4\end{array}$ \\
\hline Thrice decapitated controls & $\begin{array}{r}640 \\
1920\end{array}$ & $\begin{array}{l}0.5 \\
0\end{array}$ \\
\hline Thrice decapitated, IAA on for 30 minutes & $\begin{array}{r}640 \\
1920\end{array}$ & $\begin{array}{l}4 \\
7\end{array}$ \\
\hline Thrice decapitated, IAA on for 75 minutes & $\begin{array}{r}640 \\
1920\end{array}$ & $\begin{array}{l}4 \\
9\end{array}$ \\
\hline
\end{tabular}

strong possibility that IAA in some combined form is both photoreceptor and effector. Whether IAA is inactivated or only translocated in this system remains unknown.

These results provide an answer to the question as to whether there is more than one type of phototropic response in the same plant; there are at least two, making use of different photoreceptors.

\section{Phototropism in Phyconyces}

For a broader answer to the same question, one could study another member of the grasses, such as barley, whose phototropism may well differ in some respects from that of Avena, or Blaauw's old object (1915), the sunflower seedling. However, a more complete contrast seemed to be promised by leaving the flowering plants altogether and studying a fungus. The sporangiophore of Phycomyces, 
a fute, rapidly growing cylindrical cell about $150 \mu$ thick, and extending to $18 \mathrm{~cm}$ or more in height, is highly phototropic and has been studied by numerous workers. It differs from the coleoptile in several respects. Firstly, the growing zone, which extends only for 2 to $5 \mathrm{~mm}$ below a point a few tenths of a millimeter from the sporangium, is itsell the photoreceptor region, and exposure of other parts does not cause curvature. Secondly, there is no evidence for mediation of the growth by a growth hormone, although the sporangiophores have been shown to contain indole acetic acid. If droplets of IAA solution are applied to one sicle of the growing zone of a sporangiophore, they do cause curvature away from the drop, but such curvatures, which have been carefully studied in the author's laboratory by Gruen, are no larger than those caused by water alone. Thirdly, the sporangiophore is of course a single cell, very delicate and optically translucent. A suggestive similarity with Avena is that, as Blaauw showed, high light dosages, if given with high intensity, cause a reversal of sign of the phototropic curvature; at about 4000 ergs per $\mathrm{cm}^{2}$ small negative curvatures result.

Unlike those of the coleoptile, phototropic curvatures of Phycomyces are not satisfactorily proportional to the light dosage, since brief exposures, even with high intensity, produce only small curvatures. It was found, however, that the phototropism could be quantitatively studied by exposing the plants to continuous light of known intensity from one side and balancing this with a reference lamp on the opposite side. The plants were thus used as photometers. Using this system and with the same monochromator as used for Avena, the action spectrum for Phycomyces, shown in Fig. 9, has been obtained.

Fig. 9 shows a main peak at $445 \mathrm{~m}_{\mu}$, a second peak at $470 \mathrm{~m} \mu$ separated from it by a trough, indications of an inflection at $425 \mathrm{~m} \mu$, and a broad flat peak around $370 \mathrm{~m} \mu$. In all these points, as well as in the steep fall above $480 \mathrm{~m} \mu$, it shows a really striking agreement with the action spectrum for Avena. With a high degree of probability, the photoreceptors for Avena and Phycomyces in the visible and near ultraviolet are the same. This result is the more notable when one considers the botanical gap that separates Avena and Phycomyces, as well as the structural differences discussed above. It should be added that the sporangiophore, like the Avena coleoptile, contains a carotenoid (Bünning, 1937) and this is limited to the region a few millimeters below the sporangium, as has been shown by direct microspectroscopy (Abbott and Grove, 1959). 


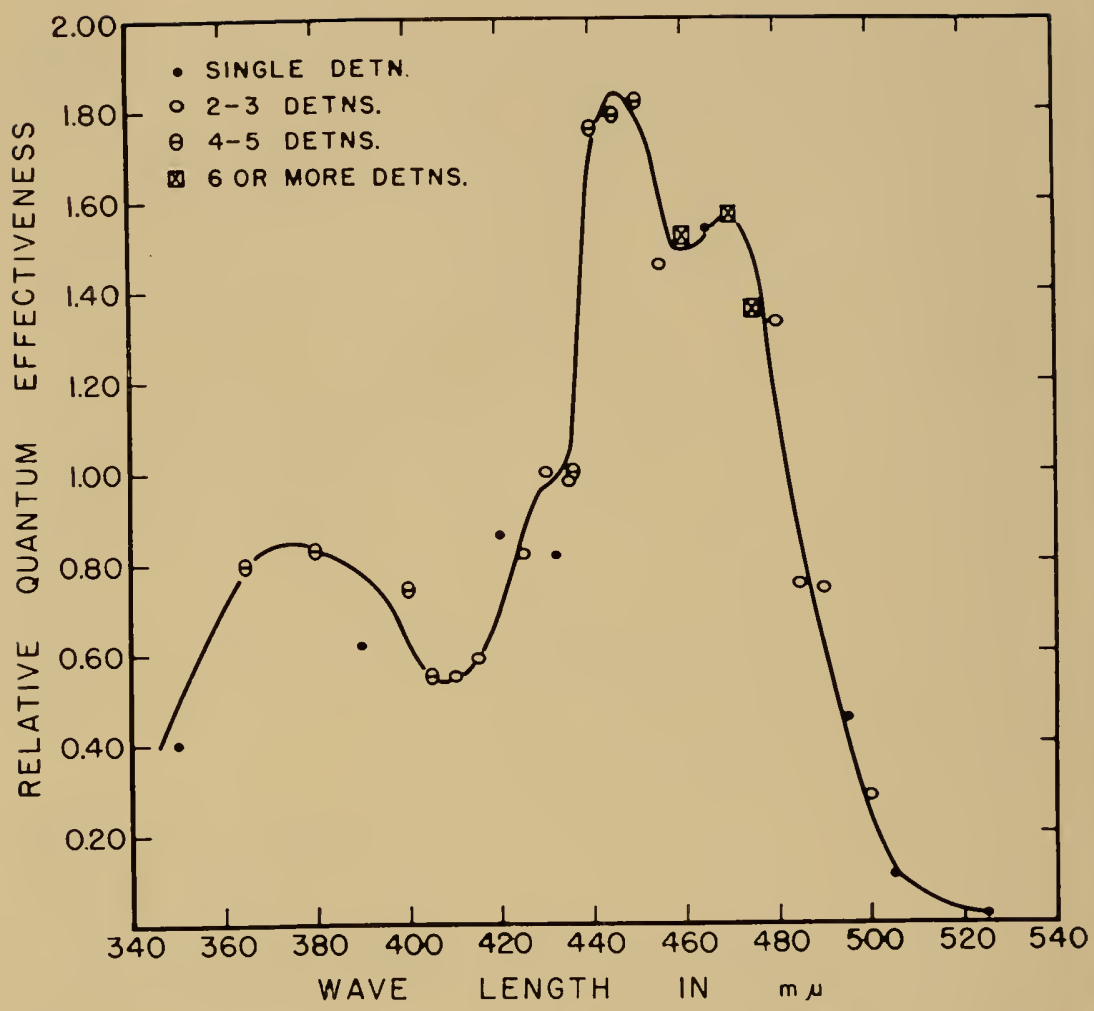

Fig. 9. Action spectrum for the positive curvature of Phycomyces sporangiophores, obtained by the balance method, using blue light as reference. (From Curry and Gruen, 1959).

There is another similarity with Avena. Phycomyces shows a second type of phototropism in the shorter-wavelength region of the ultraviolet. The curve of Fig. 9 falls towards zero at about 310 $\mathrm{m}_{\mu}$. Beyond this point the plants show negative curvature. Fig. 10 reproduces a series of pictures of a group of Phycomyces sporangiophores which are being illuminated from the right with ultraviolet. Curvature begins rapidly; by about 35 minutes some of the sporangiophores overshoot the $90^{\circ}$ and thus become exposed on their opposite flank and, as a result, show reversed curvature. The rate of curving is evidently very high.

A preliminary action spectrum for the negative curvatures in the ultraviolet has been published (Curry and Gruen, 1959), but it is not sufficiently detailed to draw conchusious as to the photoreceptor. There is a gencral similarity to the curve for Avena in the same region, 

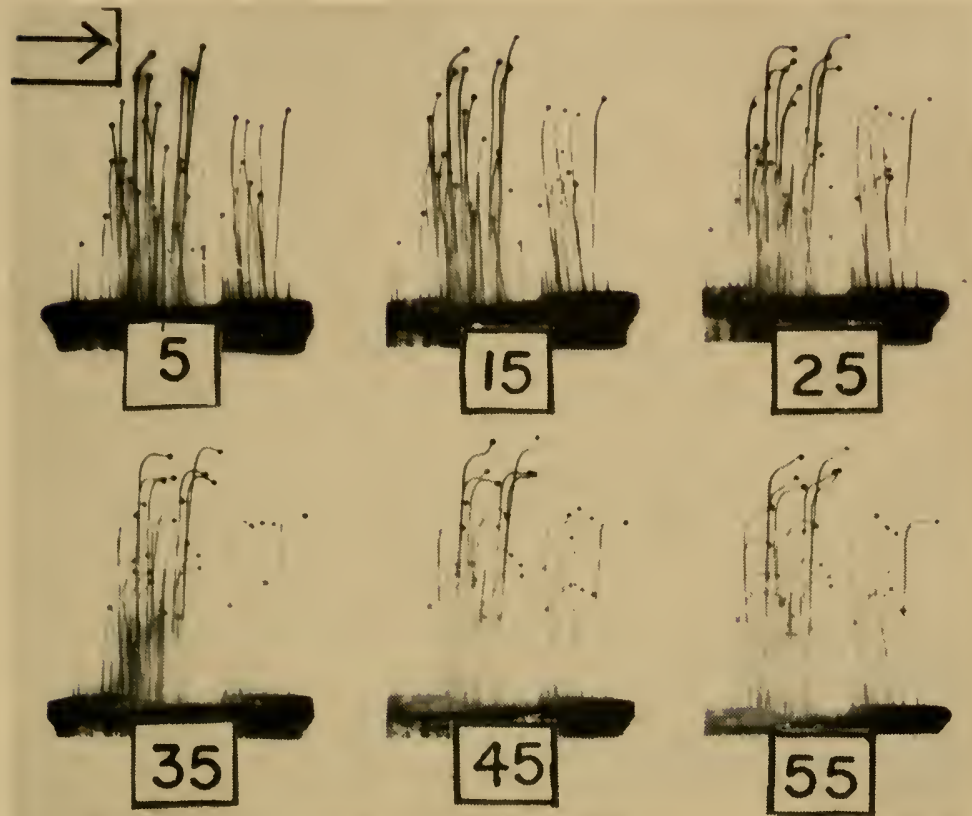

Fig. 10. Progress of negative curvatures of Phycomyces continuously illuminated from the left with $150 \mathrm{ergs} / \mathrm{cm}^{2} / \mathrm{sec}$ of $280 \mathrm{~m} \mu$. Photographed at $5,15,25,35,45$, and 5.5 minutes after illumination. (From Curry and Gruen, 1957).

the peak being near $280 \mathrm{~m} \mu$, but minor differences prevent identification of the two.

\section{Phototrolism and Light-growth Reactions}

The reversal of sign of these curvatures in the ultraviolet poses an interesting question of mechanisn. The effect of symmetrical light on Phycomyces is to accelerate its growth, as was first shown by Blaauw and has more recently been studied in detail by Delbrück and Reichardt (1956). One would expect, therefore, that unilateral illumination, by accelerating growth on the lighted side, would cause negative curvature. The fact that curvature is positive has for long been explained in terms of a "lens effect", i.e., the clear cell contents focus the light in a "bright stripe" on the opposite wall, and the resulting greater acceleration there causes positive curvature. Evilence for or against this interpretation need not be discussed here; it is sufficient to note that this concept (following Blaaww) rould explain the curvature as simply due to the difference between the growth responses of the two sides to the light. 
It should be said that from a biological viewpoint this may well be a naive view. That opposite sides of a single cell should react to an external stimulus quite independently of one another is essentially improbable. It is true that different responses to cliemical stimuli by different parts of a single cell do occur, especially in the siphonaceous algae. But to the writer it appears much more likely that some interaction between the responses would occur and that the curvature would be the result of an integrated change in the cell, rather than simply of the difference between two independent reactions. Indeed, the time relations give some support to this view, for the effect of symmetrical continuous illumination is to produce only a temporary growth acceleration, from which the growth rate returns to normal after about 10 minutes. This is ascribed by Delbrïck and Reichardt (1956) to the onset of "adaptation." However, if the illumination is unilateral, curvature begins after 4 or 5 minutes, and continues to increase for 30 minutes or more; thus, long after the light growth-reaction has died out curvature continues at a high rate. This discrepancy certainly suggests that curvature is not the simple result of a difference between light growth-reactions on opposite sides.

However, from the viewpoint of the lens theory the ultraviolet curvatures behave as expected. Brief symmetrical exposure of a sporangiophore to ultraviolet causes a growth acceleration, which begins 2 minutes after the onset of exposure and lasts only 7 to 10 minutes. As Fig. 11 shows, it can be elicited repeatedly. Such an acceleration of growth by ultraviolet would, if the lens-theory is valid, be expected to cause positive curvature just as with visible

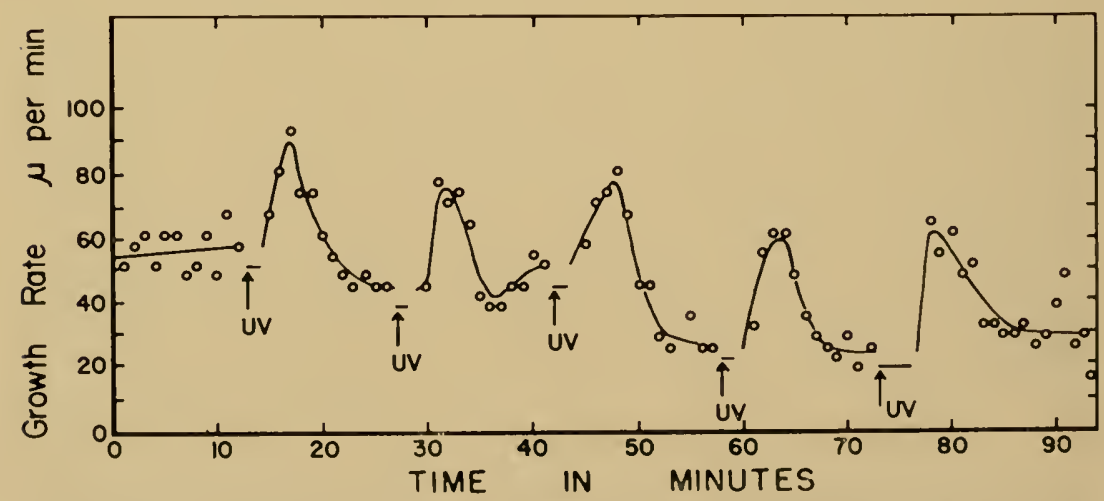

Fig. 11. Light growth reactions of a Phycomyces sporangiopluore on brief illumination with ultraviolet light. Horizontal lines show the periods of illumination. Measturements made with a lertical travelling microscope. 
light. However, Brucker (1955) found that the sporangiophore contains quantities of gallic acid, which, as Dennison (1959) has recently shown, would suffice to render it virtually opaque to light of wavelengths around $280 m \mu$. The negative curvature may therefore be ascribed to the acceleration of growth only on the lighted side.

There is at least some correlation between the light growth-reactions and phototropism in Avena too. In 1925 Went showed that there are two types of response to a brief symmetrical illumination. When the tip alone is exposed, growth decreases slowly after 20-30 minutes and returns to normal in about 90 minutes; but when the base alone is exposed the growth decrease occurs more quickly and is smaller in extent; within 30 minutes the growth rate is again normal. In each case a slight acceleration follows. These data agree roughly with the behavior of the "tip" and "base" curvatures.

\section{The Possible Mechanism of Phototropic Curvature}

The gap between the absorption of light by the photoreceptor and its result in curvature is still largely unbridged. The following points, concerned with Avena, represent only fragmentary contributions to a consistent theory.

In recent years the view has gained ground that phototropism in higher plants is due to photo-catalyzed destruction of auxin. Not only does Fig. I make this view very improbable, but further evidence against it is provided by considerations of quantum yield. For the extensive work with auxin has given a sound quantitative relation between IAA concentration and curvature which can be brought to bear on the presumed photo-destruction of IAA.

To produce a $5^{\circ}$ curvature in a decapitated Avena coleoptile an agar block containing about $10^{-5}$ grams IAA per liter must be applied. The block has a volume of $10 \mathrm{~mm}^{3}$ and hence contains $10^{-10}$ grams of IAA. During the experiment it has been shown that about one-fifth of the auxin leaves the block to enter the plant. The plant therefore receives $2 \times 10^{-11}$ grams or $1 \times 10^{-13}$ moles of IAA. This amounts to $10^{-13} \times 6 \times 10^{23}$ or $6 \times 10^{10}$ molecules and represents the difference in IAA between the convex and concave sides.

The same $5^{\circ}$ curvature can be produced by illuminating with about $2 \mathrm{ergs} / \mathrm{cm}^{2}$ of light (436 m $\mu$; cf. Fig. 3). One erg at $436 \mathrm{~m}_{\mu}=$ about $2 \times 10^{11}$ quanta, and the area of the tip is not more than $1 / 2 \mathrm{~mm}^{2}$, so that about $2 \times 10^{9}$ quanta are received by the tip. Making the generous assumption that if 10 percent of these react with auxin molecules in a one-quantum process, not more than $2 \times 10^{8}$ molecules of IAA 
could be inactivated. There must therefore be an amplification factor of at least several hundred and probably much more.

As shown above, IAA can be inactivated by ultraviolet, though much higher energies are required than those causing phototropic curvature. However, even in the ultraviolet there is no evidence for a chain reaction in the photo-inactivation of IAA; in solution or in agar the quantum yield approximates 0.2 , and when sensitized by riboflavin a value of 0.7 has been reported (Galston, 1949) .

Recent experiments of von Guttenberg (1959) add qualitative evidence against photo-destruction. Decapitated coleoptiles exposed unilaterally to very high dosages of white light, around 500,000 ergs $/ \mathrm{cm}^{2}$, give quite small curvatures; if, however, IAA in agar is applied after the exposure the curvatures may be very large, reaching $25^{\circ}$ or more. The coleoptiles could even be placed in the dark after illumination and the IAA applied as much as 8 hours later; curvatures of $15^{\circ}$ could still result. Thus direct photo-destruction is excluded, and furthermore, the probability that photo-products active enough to destroy IAA could survive in the plant for 8 hours at $25^{\circ} \mathrm{C}$ is obviously negligible.

We conclude that light does not react with the auxin itself, but that the photoreceptor brings about an effect (a) capable of surviving some time in the plant, and (b) influencing many hundreds of auxin molecules. Such an effect might well be exerted on an enzyme producing IAA. However, such an enzyme would be likely to produce auxin molecules measured in millions rather than hundreds, and in any case a modification of enzymic activity does not appear to offer any basis for the influence of light on translocation of auxin. For these reasons the following hypothesis is preferred.

If the photoreceptor is indeed a carotenoid it would be expected to be located in a plastid. Carotenoids in plants are almost invariably present in plastids. The proplasticls of etiolated Avena coleoptiles appear small, nearly circular in optical section, and with a marked "primary granum"; they are found in good numbers in the lower part of the coleoptile tip, mostly near the termination of the vascular bundles. It may be assumed, therefore, that these boclies could contain the photoreceptor.

In the case of geotropism it has long becn accepted that the receptor of gravity must be a body which can fall, and attention has been directed to a type of small starch grains termed "statoliths" (see e.g. Haberlandt, 1914). In the rertical coleoptile these can be seen to 
lie at the basil end of the cell and when the plant is turned through $90)^{\circ}$ they fall on to the lateral wall. Yocum in 1953 (unpul).) showed that the time taken in falling to the lateral side is about 2 minutes, while the exposure time for threshold geotropic response is known to be 2 to 4 minutes in the same plants. Now in shoots at least, starch is produced only in plastids, and, as far as known, starch grains are merely modified plasticls. We thus arrive at the interesting conclusion that light and gravity are probably detected by similar organelles.

The movement of auxin from cell to cell is brought about by an oxygen-dependent transport system which operates in a polar fashion, i.e., auxin molecules move from the apex toward the base. This means that the basal cross-wall, against which the statoliths lie, is the favored point of export of auxin molecules, and the two facts may be causally related, i.e., the contact of wall and particle may promote auxin movement through the wall. When the plant is horizontal and the statoliths rest against the lateral wall they now promote the movement of auxin through this wall instead, thus causing lateral translocation.

It is now proposed that unilateral light would cause migration of the plastids to the lateral wall opposite the light direction, thus favoring the transport of auxin through that wall.

Such a concept would bring phototropism into line with a hitherto unrelated light response, the movement of chloroplasts. These movements, which have been known and studied for a hundred years since they were furst recorded by Boehm (1857-9) and Borodin (1869), have so far only been observed on green chloroplasts, and it is not yet known whether the proplastids of etiolated plants behave in the same way. However, there are some suggestive parallelisms. In the first place, plastid movements show a change of sign with increasing light intensity; at low light closages they move to the illuminated walls, while at high ones they revert to the walls perpendicular to the light beam. In the second place, the low light response is sensitive only to blue light and has a peak of effectiveness at about $450 \mathrm{~m}_{\mu}$ (Zurzycka, 1951). In the third place, proplastids are strongly affected by red light treatment; the "primary granum" grows out rapidly to form the well-known lamellae of the normal chloroplast, as has been demonstrated by several electron microscopists (e.g., Mühlethaler and Frey-Wyssling, 1959). This could give a possible basis for the marked effect of red light in modifying phototropic sensitivity. 
Our own observations on the proplastids of Avena show that one hour's exposure to red light, which reduces phototropic sensitivity by a half or more, causes marked extension of the "primary granum" which can be seen clearly with the optical microscope.

That phototropism and geotropism should be caused by the movements of comparable organelles would make understandable the fact that both are accompanied by development of an enf of 10-20 millivolts across the organ. This was first observed for geotropism by Bose in 1907, and extended to the first positive phototropic curvature by Backus and Schrank (1952). In both tropisms the side which is to grow the more becomes electropositive.

An important difference between the two tropisms is that gravity can be detected by the whole coleoptile, like the high light dosages causing the second positive curvature. The first positive curvatures, on the other hand, as well as the negative curvatures, are initiated in the tip. This suggests that the plastids of the tip region differ from those below-a possibility now under study in my laboratory.

This theory could well provide for a moderate amplification of the light effect so that one quantum could alter the transport of many auxin molecules; and it would explain, as no other present theory does, how light causes asymmetrical auxin movement. As stated above, it accords with the known localization of carotenoids in plastids. The theory is applicable to fungi, too, for in them also the carotenoid is present in particles. These have been notably described in the lightsensitive zone of Pilobolus by Buller, who noted their orange-red color and referred to them as oil droplets. A study of these particles would be of the greatest interest.

In summary, phototropism is a light response of very wide distribution throughout the plant kingdom. In the higher plants the ultimate effector is asymmetric auxin distribution; in the fungi this is most probably not the case. Yet careful study of a selected higher plant and a fungus reveals that their photoreceptors for the visible spectrum are virtually identical, while in the ultraviolet both have a second type of response with, again, at least comparable photoreceptors. The correlation between phototropic curvature and the effects of symmetrical illumination on growth is far from complete, and integrative factors like those in Avena may perhaps participate in fungi also. A theory of the mechanism of phototropic curvature is outlined which unifies this phenomenon with the geotropic response and brings it into relation with the kuown movements of chloroplasts. 


\section{ACKNOWLEDGMENTS}

A number of the original experiments included in this presentation (some unpublished elsewhere) were performed in collaboration with Drs. Hans E. Ginen and Helen Sorohin. In addition some of the earliest experiments were carried out by 1)r. Conral Yocum, now of Comell University. We wish to express our best thanks to these collaborators. We are grateful also for support, over a number of years, from the National Science loundation, the Milton Fund of Harvard University, and the American Cancer Socicty. A more extensive and detailed review, including some further original work, appears as Chapter VI, Phototropism and Phototaxis, by K. V. Thimann and G. M. Curry, in Comparative Biochemistry (ed. Marcel Florkin and Harley Mason, Academic Press, New York, 1960).

\section{REFERENCES}

1. Ibbot, M. T. J., and Grove, J. F., Exptl. Cell Research, 17, 95 (1959).

2. Arisz, W. H., Rec. trat. botan. Néerl., 12, 44 (1915).

3. Isomaning, E., and Galston, A. W., Plant Phy'siol. (in press).

4. Bachmann, F., and Bergann, F., Planta, 10, 744 (1930).

5. Backus, G. E., and Schrank, A. R., Plant Physiol., 27, 251 (1952).

6. Blaauw, A. H., Rec. trav. botan. Néerl., 5, 209 (1909).

7. Blaauw, A. H., Z. Botan., 7, 465 (1915).

8. Boehm, J. A., Sitzber. kgl. Akad. JViss., 22, 479 (1857); 37, 435 (1859).

9. Borodin, A., Bull. acad. imp. sci. (Jecrograd), 13, 571 (I869).

10. Briggs, W. R., Tocher, R. D., and Wilson, J. F., Science, 126, 210 (1957).

II. Brucker, W., Flora (Jena), 142, 353 (1955).

12. Bümning, E.. Planta, 27, 148 (1937); Z. Botan., 43, 167 (1955).

13. Cholodny, N. G., Biol. Zentr., 47, 604 (1927).

14. Curry, G. M., Thesis, Harvard Unisersity (1957).

15. Curry, G. M., and Gruen, H. E., Nature, 179, 1028 (1957).

16. Curry, G. M., and Gruen, H. E., Proc. Nat. Acad. Sci. U. S., 45, 797 (1959).

17. Cury, G. M., Thimann, K. V., and Ray, P. M., Physiol. Plantarum, 9, 429 (I956).

18. Delbrïck, Ml., and Reichardt, W., in Cellular Mechanisms in Differentiation and Growth (D. M. Rudnick, ed.), Princeton University Press (1956).

19. Dennison, D. S., Nature, 184, 2036 (1959).

20. Galston, A. W., Proc. Nat. Acad. Sci. U. S., 35, 10 (1949).

21. Galston, A. W., and Baker, R. S., Am. J. Botany, 36, 773 (1949).

22. Guttenberg, H. von., Planta, 53, 412 (1959).

23. Haberlandt, G., Physiological Plant Anatomy, Trans. M. Drummond, Londou (19]4).

24. Harbury, H. A., La Noue, K. F., Loach, P. A., and Amick, R. M., Proc. Nat. Acad. Sci. U. S., 45, 1708 (1959).

25. Inhoffen, H. H., Bohlmann, F., and Rummert, G., Ann. Chem., Liebig's, 571, 75 $(1950)$.

26. Johnston, E. S., Smithsonian Inst. Publs., Misc. Collections, 92 (11), 17 pp. (1934).

27. Kaper, I. M., Dissertation, Leiden (1957).

28. Lange, S., Jahrb. wiss. Botan., 67, 1 (1927).

29. Mühlethaler, K., and Frey-Wyssling, A., J. Biophys. Biochem. Cytol., 6, 507 (1959).

30. Nishimura, M., and Takamatsu, K., Nature, 180, 699 (1957).

31. Searls, R. L., and Sanadi, D. R., Proc. Nat. Acad. Sci. U. S., 45, 697 (1959).

32. Shibata, K., Carnegie Inst. Wash. Yearbook, 55, 252 (1958); cf. Shibata, K., 
Benson, A. A., and Calvin, M., Biochim. et Biophys. Acta, 15, 46 (1954). 33. Shropshire W., Jr., and Withrow, R. B., Plant Physiol., 33, 360 (1958).

34. Sonne, C., Strahlentherapie, 31, 778 (1929).

35. Wallace, R. H., and Schwarting, A. E., Plamt Physiol., 29, 431 (1954); Wallace,

R. H., and Habcrmann, H. M., Plant Plyysiol., 33, 252 (1958).

36. Went, F. W., Proc. Koninkl. (Ned.) Akad. Wetenschap., Amsterdan, 29, 185 (1925).

37. Went, F. W., Rer. tray, botan. Néerl, 25, 1 (1928).

38. Zurzycka, A., Acta. Soc. Botan. Polon., 21, 12 (1951).

\section{DISCUSSION}

Dr. Frencn: I just wanted to ask Dr. Thimann if the action spectrum of the red effect has been made.

Dr. Thimann: No, it hasn't, I am sorry to say. It would be a very nice thing to clo.

Dr. Galston: Dr. Thimann mentioned, and quite properly, that the action spectra for phototropism do not at the moment permit us to discriminate absolutely amongst the known pigments that present themselves as candiclates for photoreceptor. I think, however, that the manner of statement of the problem led to the conclusion that it was impossible for riboflavin or one of its close relatives to be the photoreceptor. I would like to counteract this impression.

Dr. Henry Harbury and his collaborators at Yale, to whom I)r. Thimann referred, have shown that the solvent in which isoalloxazine-like molecules find themselves can shift their spectrum very markedly from a single peak (aqueous solvent) to a triple peak (benzene solvent) in the visible range. Similar things can happen, in fact, with FMN. Therefore, I believe there is every possibility that FMN, FAD or other naturally occurring molecules of the isoalloxazine type could, in fact, be the photoreceptor if only they find themselves in the proper medium, such as a lipoidal phase.

In Harbury's actual experiment, 3-methyl lumiflavin was used, which in benzene, shows 3 visible absorption peaks. If one takes FMN and puts it in water, one gets a clear single peaked spectrum. If, however, one merely adds pyridine in increasing quantities to this aqueous solution, one can find increasing evidence of a triple peak. When one has a preponderance of pyridine present, one does have a clearly triple-peaked spectrum in the visible. My only plea would be, therefore, not to disqualify the flavins because of their absorption spectra, because, depending on their state in whatever cytoplasmic body we find them, they maty have an absorption spectrum which closely resembles that of the photoreceptor.

Now, a question to Dr. Thimamn. Dr. Thimann mentioned the analytical results of my graduate student, Asomaning, on the flavins and carotenes in illuminated barley oats. I would like to ask him what he thinks of other data in the literature on the relative light sensitivity and carotene content in Phycomyces, in albine corn and in albino sunflower. Many years ago, 
Bandurski and I showed that albino com, in which Banclurski was unable to find any carotenes at all, was quite active phototropically. We did not publish the phototropic data, because the fuclian corn used has a long. unclulating first internode. and we couldn't get any good quantitative phototropism data. Secondly, Wallace and Habermann have a sunflower which they find is analytically frec of arotenes, but certainly phototropically actire. Thirdly; there is the evidence from Phycomyces grown on a medium in which the main carbon source is lactate. Growth, especially in the presence of diphenylamine, leads $t o$ a depression of the carotene level to about 1 of or less of the nomal with, however, the full retention of phototropic sensitivity. I believe it would be fair to include all of this evidence in an evaluation of the photoreceptor problem.

DR. THmanN: In general, ny answer to Dr. Galston's question is that none of these studies of albino plants is decisive. My reasons for saying this are that in general the phototropic sensitivity is spoken of as still present, but no attempts at all are made to compare quantitatively the sensitivity of these plants to those of their pigment-containing controls. An example of this is the white Helianthus, which Dr. Galston mentioned, and which we have also grown. It certainly contains very little carotenoid, and it certainly is phototropically sensitive, but only very weakly so. It is not easy to make measurements with it since it produces very large mutations. The phototropic sensitivity is a very small curvature superimposed on the large differences of the controls, so no measurements are really possible. I have corresponded with Dr. Wallace about this, because in his paper with Dr. Habermann he speaks of these plants as being normally phototropic. It turned out that he exposed these plants to light for 24 to 36 hours, so you can see what sort of energy dose they got. As a result of this they yielded a positive response. In general, this sort of thing is true. When Phycomyces is grown on diphenylamine, for instance, the amount of carotenoids is recluced to some $5 \%$ or so of that of the control. But unless one carefully measures the phototropic sensitivity, perhaps, for all we know, the phototropic sensitivity may be $5 \%$ of the control, too. So unless we have measurements of both kinds I fear those data are almost worthless.

Dr. Galstox: May 1 add one thing to that. The data which Bandurski obtained on carotenc led him to believe that there could not be more than $0.1 \%$ of the normal carotene content in the albino maize with which he worked, and yet the phototropic sensitivity was still present. Now it is completely impossible to prove that any tissue is completely free of carotene. All one can do is to set an upper limit to the carotene present. I would say that this one case at least must be taken seriously.

Dr. Tumanx: Well, I should like to see the phototropic data, and I would like to ask Dr. Galston whether he has any comparable data on the riboflavin content to indicate whether it remains normal.

Dr. Galston: In that one we did do the riboflavin assay, and it is normal. 
Dr. Commoner: Of all the natural metabolic pigments, which we studied in the ESR spectrometer, flavins and particularly FMN stand out as being exceedingly sensitive to light with respect to free radical formation. This suggests that flavins may participate very readily in light-sensitive biological processes.

DR. Gordon: If I understood correctly, you are proposing that light affects the rate of auxin transport. How is it then that light has virtually no effect on the amount of auxin obtained by basipolar diffusion into agar?

Dr. Thimann: I think this problem is too long to discuss. I will only say that I was not proposing that the total amount of auxin transported is affected. Only the direction in which it is transported is affected. If the auxin moves laterally instead of longitudinally, there need be little or no change in the amount diffusing out.

Dr. Gordon: I think it should be pointed out that, as far as I am aware, it has never been shown rigorously that light alters the direction of auxin transport. This has only been inferred from inequalities of auxin concentration.

Dr. JAGENDORF: From what I remember of the cytological work, when you illuminate cells with dim light the plasticls line up on the walls perpendicular to the illumination, but this includes the wall on the side towards the light beam and the wall on the dark side. If plasticls are localized on both walls, how do you propose that they cause a directional flow of auxin?

Dr. Thmann: Briefly, it is this way. At low light intensity the plastids line up on the wall facing the light. At high light intensities the plasticls align themselves along the walls which are parallel to the incident light. There are complicated effects varying with the dosage, but there is a directional movement at low light intensity.

Dr. WALD: In what organism does that occur?

Dr. Thimann: One of the most popular groups has been the mosses, such as Fontinalis or Mnium.

DR. WALD: Do things like this happen in the organisms you use?

Dr. Timmann: We are working on it. 


\title{
PHOTOPERIODISM, CHELATING AGENTS, AND FLOWERING OF LEMNA PERPUSILLA AND \\ L. GIBBA IN ASEPTIC CULTURE
}

\author{
William S. Hillian \\ Y'ale University, \\ New Haven, Connecticut
}

The photoperiodic control of flowering has been known for almost 40 years, but its mechanism remains obscure. Recent in-vitro and in-vivo work on the red, far-red reversible pigment (1) should help in understanding the role of light, but little is known about how this is linked to flower initiation. A possible reason for this, other than the obvious complexity of the problem, is that students of flowering physiology and photoperiodism have dealt with singularly unwieldy experimental systems. The size and slow growth of most higher plants severely limit the number and size of experiments possible in a given space and time; while plants such as Xanthium have been used for all the fundamental work so far, and have obvious advantages for the study of hormone movements, it is difficult to keep them under precisely controlled conditions. The use of duckweeds (Lemnaceae) as experimental organisms avoids many of these difficulties and provides almost unique opportunities for specifying the physical and chemical environment. Unfortunately, Saeger's (14) suggestion to this effect, made over 30 years ago, has been largely ignored; it is only within the past five or six years that photoperiodism has been demonstrated in at least two species of Lemna. The scanty work to date, however, suggests a useful new approach to photoperiodism, since it appears to indicate a close relationship between this process and metal-probably trace-metal-nutrition or metabolism. This paper provides a brief review of earlier work by others and by the writer, a report on recent work to be published in greater detail elsewhere, and a discussion of its implications for future research in this field. 


\section{Review of Flowering in the Leminaceae}

The flowering of most Lemnaceae species in nature appears to be infrequent, although the lact that the flowers are unlikely to be noticed unless deliberately looked for makes the true frequency difficult to assess. Saeger (14) collected field evidence that flowering may be at least partly controlled by the composition of the pond water. Reports of flowering induced by ultraviolet radiation and high $p \mathrm{H}$ values under poorly controlled conditions (4) have not been confirmed. In a survey of the growth of a large number of strains (clones) of various species under aseptic, controlled conditions, Landolt (11) reported flowering in several strains, usually in old, crowded cultures, but did not investigate further.

The first demonstration of photoperiodism in the Lemnaceae was that of Kandeler $(10,11)$, who found that certain strains of Lemna gibba, grown under aseptic conditions at about $30^{\circ} \mathrm{C}$, flowered as long-day plants with a critical daylength of $12-14$ hours. Two points of particular interest emerged from this work. If fluorescent light alone was used as a light-source, flowering took place rapidly only in "old" medium-medium in which the plants had grown for some time. In light containing a higher proportion of far-red radiation (e.g., supplemented with incandescent light) flowering occurred rapidly even with frequent transfers to fresh medium. A crude action spectrum suggested that the far-red also promoted flowering when given as an interruption of the dark period.

A conditional short-day response in a strain of Lemna perpusilla grown under aseptic conditions has been reported by the writer (6, $7,8)$. The term "conditional" is used here to indicate that flowering in that plant is daylength-indifferent under certain conditions but typically short-day in others. The major points can be briefly presented.

When grown in a modified Hoagland's medium, with or without sucrose and supplemented with only low (about $10^{-5} M$ ) levels of tartrate to maintain iron availability, L. perpusilla strain 6746 flowers rapidly irrespective of daylength at temperatures of $25^{\circ}-27^{\circ} \mathrm{C}$. In media supplemented with sufficient levels of certain chelating agents (c.g., ethylenediaminetetracetic acid (EI)T $A$ ), at least $10^{-5} \mathrm{M}$; citrate or tartate, at least $\left.10^{-3} M I\right)$ it responds as a typical shortday plant. Maximum flowering then takes place only under photoperiods of 10 hours or less, and none with photoperiods of 16 hours or more. Brief intermption of a long, flower-promoting dark period 
(e.g., It hours) with low energies of red light can completely inhibit its eflect. One such dark period is sufficient to induce a measurable degree of flowering.

This conversion from daylength-indifference to typical short-day behavior by high levels of chelating agents can also be viewed as a specific inhibition of flowering in long days but not in short, and occurs with little effect on vegetative growth. A short-day requirement is also found at about $29^{\circ} \mathrm{C}$ even in the medium lacking high levels of chelating agents; at or above $31.5^{\circ} \mathrm{C}$ flowering is completely inhibited irrespective of daylength, but vegetative growth continucs normally. The effect of high temperature appears to be exerted primarily on dark-period processes.

These few papers represent the work published to date on photoperiodism and flowering in the Lemnaceac. Further details will be considered later, as more recent results on both $L$. gibba and $L$. perpusilla are described.

\section{EXPERIMENTAL}

L. gibba, strains GI and G3, obtained from Dr. R. Kandeler (10, II) and L. perpusilla $6746(6,7,8)$ were grown in aseptic culture. For details of the methods employed, see $(7,8)$. Two basic media rere used, with or without $1 \%$ sucrose. For Hutner's medium, containing a high level of EDTA and used here at $8 / 10$ strength, see (9). The other basic medium, a modified Hoagland's designated M, is the same as the L medium described elsewhere (8) except that the initial $p \mathrm{H}$ is $4.2-4.3$ unless otherwise noted, and that ferric tartrate was replaced by $2 \times 10^{-5} \mathrm{M}$ of both $\mathrm{FeCl}_{3}$ and tartaric acicl. All media were autoclaved. Media, light sources and intensities, and temperatures will be specified for all data presented.

Vegetative growth is recorded as frond multiplication rate $(M R)$, which is 1000 times the increase per day in the logarithm of frond number. Thus an $M R$ of 301 represents a doubling of frond number each day, and an $M R$ of 150 a doubling every two days, and so forth. The notation $M R$ (4-6) represents the $M R$ calculated by counting fronds on the 4 th and 6 th days of the experiment. Flowering is evaluated as flowering percent $(F L \%)$, which is the percentage of fronds in which flower primordia are detectable under a 20-power dissecting microscope. $F L \%$ values are obtained by dissecting all fronds in a culture unless there are more than 100 , in which case 100 taken at random are dissected. Usually the frond number is 60 to 80 , but further details will be given as necessary. 


\section{A. Experiments with L. perpusilla $67+6$}

1. Interaction of low temperature and chelating agents. Attempts to repeat some of the results mentioned above under slightly different conditions met with difficulty in obtaining complete inhibition of flowering by chelating agents under long-day conditions. Tests of white fluorescent light intensities ranging from 10 to 800 footcandles suggested that this was not a factor of importance. Since the effect of chelating agents on photoperiodic requirements could in a sense be mimicked by high temperatures, it seemed possible that low temperatures might partially reverse it. Several experiments confirmed this possibility, indicating, for example, that levels of EDTA or citrate which would completely inhibit flowering under 16 hours of light at $27^{\circ} \mathrm{C}$ would permit some flowering at $21.5^{\circ} \mathrm{C}$. This effect, however, is small, and seemed unlikely to account completely for the difficulties experienced. The $p \mathrm{H}$ factor was the next considered, and proved to be crucial.

2. $p H$ and the action of chelating agents. Low $p \mathrm{H}$ levels, about 4.0 , had been used in the experiments in which difficulties arose. The original reason for this was the observation (8) that cultures plus or minus EDTA differed in vegetative growth mainly at higher $p \mathrm{H}$ levels, so that the use of low $p \mathrm{H}$ provided more comparable controls. However, closer attention to the known properties of chelating agents (2) suggested, and subsequent experiments showed, that the action of either EDTA or citrate in this system is extremely dependent on $p \mathrm{H}$, being greatly reduced at low values.

Results of a typical experiment are presented in Fig. 1. Cultures in 125-ml erlenmeyer flasks containing $50 \mathrm{ml}$ of medium were grown at $26^{\circ} \mathrm{C}$ under 120 footcandles of continuous cool white fluorescent light. The basic medium was $M$ with $1 \%$ sucrose adjusted to an initial $p \mathrm{H}$ of either 3.6 or 4.6, and each $p \mathrm{H}$ level contained 0 . $3.3 \times 10^{-6} M$, or $10^{-5} M$ EDTA. On the third day of the experiment, all the fronds in each culture were transferred to similar flasks of fresh medium: the $p \mathrm{H}$ changed less than 0.2 unit in these first three days. Growth was healthy in all cultures but somewhat slower at the low $p H$. All cultures (containing 40-60 fronds each) were dissected on the sixth day, giving the $F L \%$ values shown. EDTA was much more effective at $p \mathrm{H} 4.6$ than at $p \mathrm{H} 3.6$, although the $p \mathrm{H}$ made no difference in the control media. Almost superimposable results were obtained in a similar experiment with citrate at concentrations 100 times as high, and the general pattern is easily repeatable. The wide scatter in the points for EDTA at low $p H$ al- 


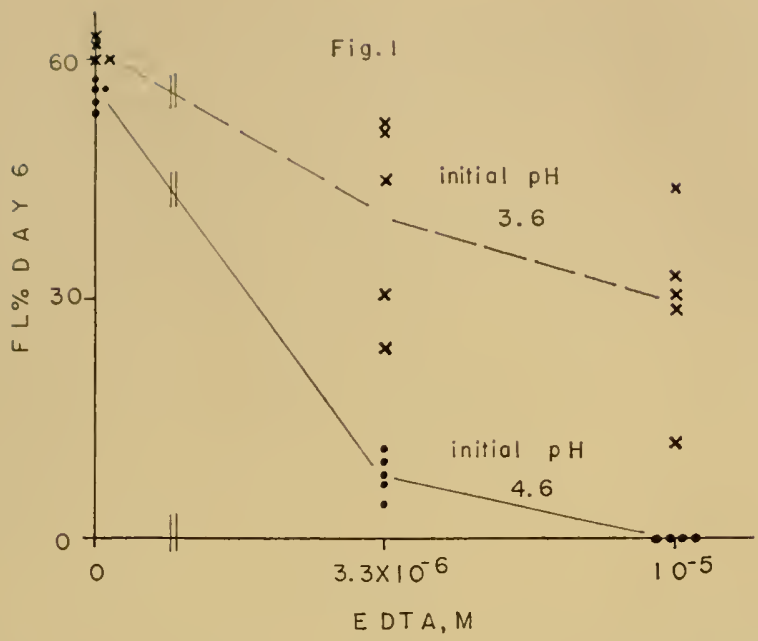

Fig. 1. Effects of two $p H$ levels on EDTA inhibition of flowering of Lemna. perpusilla $67+6$ in continuous light. Each point represents a value derived from one culture. See Experimental, A2. Exp. 6-30-59.

ways appeared, and recalls the scatter noted earlier (8) for "intermediate" levels of EDTA. It is thus clear that $p \mathrm{H}$ is a major factor in these experiments; the implications for questions of mechanism will be deferred until later.

3. Experiments on the role of iron. Frequent attempts to reverse the effects of EDTA with calcium, iron, manganese, zinc, and copper levels increased to at least 10 times those normally used, have been uniformly unsuccessful. A consideration of the levels of EDTA required in this systen, the known affinities of various chelating agents, and some of the literature on flowering suggested that EDTA and other chelating agents might affect flowering through an action on iron metabolism. There is no evidence at present to support this hypothesis directly, but it has proved possible to confirm an earlier report of work with Xanthium (15) showing that iron may play a crucial role in photoperiodic induction.

Preliminary experiments showed that flowering in cultures maintained under short days with EDTA in the medium could be almost completely inhibited by low iron concentrations which did not affect vegetative growth. Such results indicated a relatively limiting role for iron in flowering, but whether in the photoperiodic induction itself or in subsequent flower development was not clear. Experiments in which cultures are restored to normal iron conditions immediately 
after a single long night (cf. 7, section C) are more critical. One such experiment will be described.

The basic medium employed was $0.8 \times$ Hutners with $1 \%$ sucrose; the temperature was $26^{\circ} \mathrm{C}$. Photoperiods of 10 hours were given with 10 footcandles of cool white fluorescent light; for noninductive cycles, the 14-hour nights were interrupted in the middle with 50 minutes of $\operatorname{dim}\left(1.5\right.$ kiloergs $\left.\times \mathrm{cm}^{-2} \times \mathrm{min}^{-1}\right)$ red fluorescent light. At the start, series of $50 \mathrm{ml}$ erlenmeyer flasks containing $20 \mathrm{ml}$ of medium with $1 / 10,1 / 3,1$, or $3 \times$ the normal iron level were inoculated with two 3 -frond colonies from plants previously grown for three days under long-day conditions on a medium with no added iron. All cultures then received 2 non-inductive cycles followed by one uninterrupted (long) night. Within two hours of the end of the long night, all were transferred to full-iron medium, and then grown for four more non-inductive cycles, after which they were dissected. Fronds were counted on three occasions: before the first non-inductive cycle; immediately after the long night; and at dissection, when the number was 65 to 90 per flask. It was thus possible to compute $M R$ values for growth in each flask on the differing pretreatment media and growth on the full-iron medium after the long night. Means of these values with their confidence limits, and the means and ranges of the corresponding $F L \%$ values, are shown in Table 1. They indicate clearly that growth in the media with $1 / 10$ and $1 / 3$ the normal iron level before and during the long night essentially eliminated flower induction, although growth after the long night was in full iron medium. In addition, the $1 / 3$ level of iron had no significant effect on $M R$, and the $1 / 10$ level affected it only during the pretreatment. These results, and those of similar

\section{TABLE 1}

Effects of Various Iron Levels before and during a Single Long Night $(L N)$ ON Flowering $\left(F L^{C}\right)$ and Frond Multiplication Rate $(M R)$ of L. perpusilla 6746. SeE Experimental, A3. ExP. 1-14-60

Iron level (mg/L) day 0 -day 3

$59.8(3 \times$ normal $)$

$19.9(1 \times$ normal $)$

$6.6(1 / 3 \times$ normal $)$

$2.0(1 / 10 \times$ normal $)$
$\operatorname{Mr}(3-7)^{\mathrm{b}}$ at $M R(0-3)^{\mathrm{a}}$
to $\mathrm{cnd}$ of $\mathrm{LN}$
$L N$ to dissection

165.0
165.2
163.8
135.4

$$
\begin{aligned}
& 158.4 \\
& 162.0 \\
& 161.8 \\
& 154.4
\end{aligned}
$$

a Mcans of 5 culturcs. $95 \%$ confidence limits calculated by analysis of variance on all 20 values: \pm 10.1

b Means of corresponding 5 cultures. Confidence limits: \pm 8.8

c Means and ranges of 5 culturcs. 
experiments, strongly support the hypothesis that iron is crucial in photoperiodic induction, and is not related to flowering simply through its role in general metabolism.

\section{B. Experiments with L. gibba}

The effects of chelating agents on the conditionally short-day $L$. perpusilla made it desirable to see whether photoperiod-EDTA interactions also occur on the long-day $L$. gibba. The experiments were done with strain G3 except where noted, since its growth on M medium was more regular and rapid than that of the Gl chiefly studied by Kandeler $(10,11)$. Under the conditions employed, a major interaction of EDTA with photoperiod on flowering was easily demonstrated.

1. EDTA-photoperiod interaction. In exploratory experiments analogous to those possible with $L$. perpusilla, cultures of $L$. gibba G3 were placed under long (24-hour) and short (8-hour) photoperiods in M medium with or without sucrose and with or without EDTA and observed for flowering. The sucrose had little effect except to delay flowering, and to speed growth in short days. At about the 10th day, depending upon the experiment, long-day cultures with EDTA showed visible flowers and $F L \%$ values of about 30 ; none of the others showed any evidence of flowering. By the 15 th or 16 th day a low $F L \%$ value was observed in the long-day EDTA-free cultures; no flower primordia were ever observed in any short-day cultures even after the growth had proceeded beyond the frond number of flowering long-day cultures.

Most of these results are simple confirmations of Kandeler's work: no flowering under short days, and none under long days with fluorescent light until the medium becomes "old." However, the effect of EDTA in promoting flowering was large, and entirely unexpected from Kandeler's observations. Such experiments showed consistently that EDTA added to $M$ medium in concentrations from $3 \times 10^{-6}$ to $10^{-4} M$ interacts with long photoperiod to cause rapid flowering in L. gibba G3, flowering which regularly occurs before any primordia are detectable in M medium alone. A systematic investigation of this effect was thus undertaken, using the following standard conditions unless otherwise noted: the stocks were grown in M medium with sucrose under 8-hour photoperiods of 600 footcandles of mixed warm and cool white fluorescent light, at a light temperature of $26^{\circ} \mathrm{C}$ and a dark temperature of $23^{\circ} \mathrm{C}$. Experiments were performed in continuous light of the same kind and intensity at $26^{\circ} \mathrm{C}$, using M with- 
out sucrose as the basic medium. All cultures were grown in 125 $\mathrm{ml}$ erlenmeyer flasks with $50 \mathrm{ml}$ of medium.

2. $p H$ interaction with EDTA. To see whether the relation between EDTA activity and $p \mathrm{H}$ was the same as that observed for L. perpusilla, an experiment analogous to that shown in Fig. 1 was performed, using the standard conditions described above. Initial $p H$ values in the two series were 4.1 and 5.0, respectively, since levels below 4.0 proved toxic. Transfers of each entire culture to fresh medium were made on day 4 , and dissections on day 11 of the experiment. The results are shown in Fig. 2, and indicate that the action of EDTA on L. gibba flowering depends upon $p \mathrm{H}$ in the same way as that on $L$. perpusilla flowering, although here the effect is to promote rather than to inhibit. In each case, EDTA is more effective at the higher $p \mathrm{H}$. Since even by the transfer on day 4 the $p \mathrm{H}$ in the initially 4.1 medium had risen to about 4.6 , while that in the 5.0 medium had risen far less, it is clear that the data presented underestimate the true effect.

3. Relationship of the EDTA and "old" medium effects. Since the exploratory experiments indicated that the "old" medium effect was manifest in cultures left to grow sufficiently long, it was necessary to determine whether EDTA simply speeds the attainment of the "old" condition or whether it substitutes for it. For this purpose, cultures

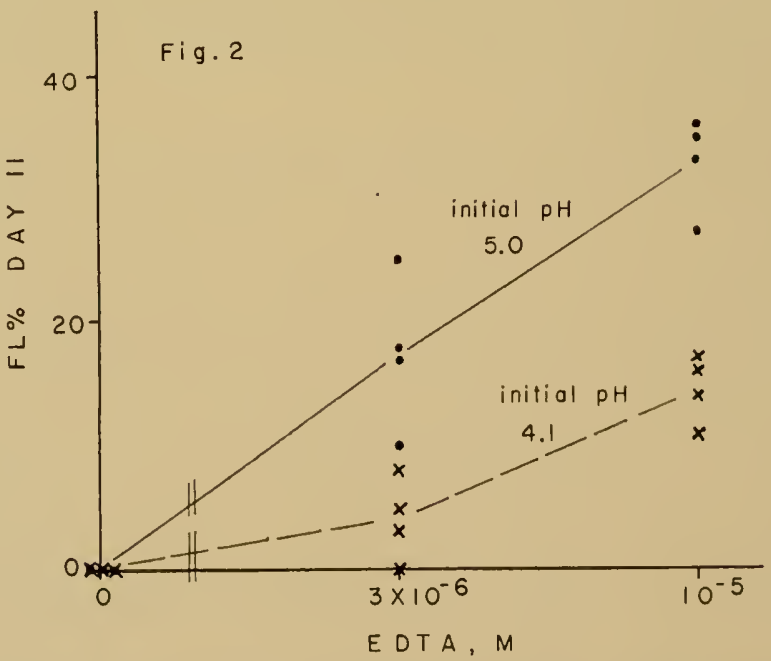

Fig. 2. Effects of two $p H$ levels on EDTA promotion of flowering of Lemna gibba G3 in continuous light. Each point represents a value derived from one culture. See Experimental, B3. Exp. 1-22-60. 
with and without EITA were subjected to two further conditions. Some were transferred in toto to fresh medium every third day, while others were left in the original medium. The results of this experiment are presented in Table 2, which shows that frequent transfer completely prevents the development of flower primordia in M medium but does not do so in the medium with EDTA. The simplest interpretation is that EITA replaces rather than hastens the "old" medium effect. Another point to be noted in Table 2 is the significantly lower frond number in the flowering as compared to the non-flowering cultures on $\mathrm{M}$ medium. In $L$. gibba, even more than in L. perpusilla (7), the onset of flowering is followed by a decrease in multiplication rate; see also Fig. 3 and caption.

Many other questions arise concerning the "old" medium effect and its relationship to that of EDTA. One is whether there is any difference between the action of the two on flowering, once the time required to render the medium "old" is eliminated. A second is whether fronds grown in long days but maintained vegetative by frequent transfers to fresh M medium are at least partially induced, so that they will be more responsive to flower-promoting conditions than short-day stocks. A third question concerns the role of growing cultures in rendering the medium "old": will simply leaving the medium unplanted under the same conditions have the same effect? A factorial experiment directed at these three questions was performed.

Four kinds of medium were used: M; M with EDTA; M made "old" by growth under long days for 12 days, followed by removal of all fronds under aseptic conditions; and M from flasks prepared and treated like the flasks of "old" medium but not planted. Into these media, two kinds of stocks were inoculated: the standard shortday stocks, or long-day stocks maintained vegetative for 12 days by

\section{TABLE 2}

Effects of Frequent Transfer on Flowering of L. gibba G3 on M Medium with OR Without $3 \times 10^{-5} M$ EDTA under Continuous Light. See Experimental, B4. Exp. A, 2-10-60

Condition

Dissected

15

15

Transfers days 3,6,9,12

With EDTA:

No transfers

Transfers days 3,6,9
Fronds/Flask ${ }^{\mathrm{s}}$

$F L \%$ Values $^{b}$

Mean \pm standard error, 4 flasks, on day of dissection.

b 100 fronds taken at random from each of 4 flasks. 
transfer to fresh M medium, like those in the second line of Table 2. The experiment was grown under standard conditions and dissected after 10 days, with the results shown in Fig. 3. The short-day stocks showed no flowering in fresh M medium, flowered in EDTA as expected, and flowered about equally in the "old" medium, thus appearing to answer the first question. The long-day stocks were similar to the short-day in their responses to M and EDTA media, but showed higher $F L \%$ values in the "old" medium. This unexpected result appears to confound the first and second question by suggesting that

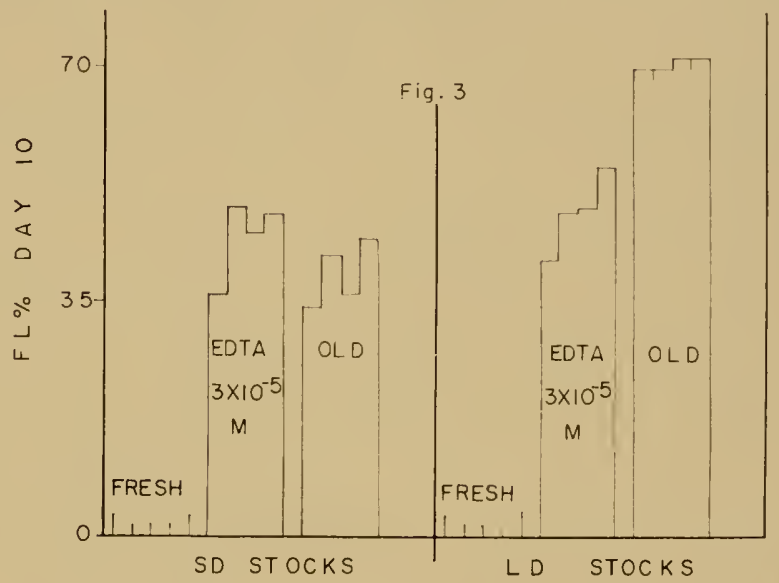

lig. 3. Flowering of $L$. gibba G3 under continuous light in fresh M medium, the same with $3 \times 10^{-5}$ M EDT $A$, or "old" M medium. Culmres started either from $S D$ stocks grown on M medium with sucrose in 8-hour photoperiod or trom LD stocks grown with frequent transfers to fresh M medium (no sucrose) in contimuous light. Divisions of bars represent values derived from one culture. Range of frond numbers per flask on day 10: Fresh, 117-146; EDTA. 95-105; old, 63-77. See Experimental, B4. Exp. 2-22-60.

EDTA and "old" medium act differently on the two stocks, or, conversely, that the two stocks respond differently to the two media. While such results require repetition and expansion, they appear sufficiently reliable to present here. It should be noted, however, that the pretreatments of the two stocks used differed in total light quantity and presence or absence of sucrose as well as in photoperiod, so that the difference need not be ascribed to the latter factor. Data for the third point in question are not included in Fig. 3, since growth in the previously umplanted but aged medium was extremely poor and iron-deficient in appearance, probably because the iron precipitated from solution cluring the 12-day period (cf. 13). It was 
thus clear that aging the medium under growth conditions but in the absence of plints did not render it "old" in the sense sought for here.

4. Other variables. In the experiments so far conducted, sucrose has little effect except to reduce flowering and to increase vegetative growth. Fluorescent light intensities from 140 to over 600 footcandles all permit rapid flowering in M medium with EDTA if given continuously. A single attempt so far to imitate the action of EDTA with levels of citrate $\left(10^{-3} M\right)$ effective on $L$. perpusilla proved unsuccessful, and further studies of this kind are required. The unexpected and at present inexplicable observation that, under the standard conditions, flowering does not occur in Hutner's medium, also requires further investigation. Although Hutner's contains a high level of EDTA, it differs from $M$ with EDTA in a number of respects such as a higher $p \mathrm{H}$, higher trace metal content, and the presence of ammonium as well as nitrate nitrogen.

The writer must here retract an earlier statement ( 7$)$, unfortunately hasty and based on a few ambiguous experiments, confirming Kandeler's observation that $L$. gibba flowering is strongly dependent on light quality. Attempts have been made during recent work to obtain rapid flowering under incandescent light, with results in all cases like those already described. Continuous incandescent light filtered through $10 \mathrm{~cm}$ of water and at intensities from 80 to 600 footcandles still permitted rapid flowering only in medium with EDT A, not in fresh $\mathrm{M}$ medium, at $26^{\circ} \mathrm{C}$. A single experiment comparing strains G1 and G3 in both incandescent and fluorescent light led to the same conclusion. Thus, results at present neither confirm nor contradict Kandeler's report; the apparent contradiction may easily be due to the use of media, temperatures, and light sources unlike those used by Kandeler.

\section{Discussion}

A considerable portion of the work reviewed and presented can be summarized in a few sentences. When L. perpusilla 6746 and L. gibba G3 are grown aseptically on a modified Hoagland's medium under fluorescent light at about $26^{\circ} \mathrm{C}$, photoperiod has no effect on the flowering of either; the former flowers, and the latter fail to flower, under all daylengths. The presence of EITA at or above a concentration of $10^{-5} M$ suppresses the flowering of $L$. perpusilla, and brings about the flowering of L. gibba, under long days (e.g., 24 hours) but not under short ( 8 hours). In a mamer of speaking, the former is converted from a daylength-indifferent to a short-day plant 
and the latter from a plant unable to flower to a long-day plant. The action of EDTA in either case is clecreased by lowering the $p \mathrm{H}$, at least in the range 3.6-5.0. With $L$. perpusilla, the EDTA effect is imitated by a considerable number of other chelating agents; with L. gibba, by medium which has become "old" from supporting the growth of plants in long day. Whether the possible reciprocal relations hold is unknown at present.

If only one or the other of the EDTA effects were known, the simplest conclusion would be that the compound merely inhibited or promoted flowering under the specified conditions, with no necessary relation to photoperiodism. Since EDTA can also modify vegetative growth, particularly in L. gibba, where it increases both root length and the gibbous character of the fronds, the effect might appear to be unspecific. Taking both systems together, however, it is difficult to avoid the conclusion that the photoperiodic mechanism itself must be intimately affected; what the effects on both species have in common is not a promotion or inhibition of flowering but the potentiation of a response to long days.

The question of how EDTA might affect the photoperiodic mechanism cannot be answered as yet. The results with $L$. perpusilla inclicate an effect through metal-chelating action, and the $p \mathrm{H}$-activity relationships for both species (Figs. 1 and 2) are in accord with this conclusion (2). Nevertheless, there are further problems of interpretation. The $p H$-activity relationship might suggest that EDTA acts in the medium itself, not within the plant; attempts to reverse the effect with increased levels of various metals, however, fail consistently, at least in $L$. perpusilla. This could indicate that it acts in the medium by increasing, rather than decreasing, the availability of one or more metals, since both are well-known effects of chelating agents (3). Further observations on $L$. perpusilla do not support this view. In a medium completely devoid of chelating agents (e.g., $M$ omitting tartrate) growth is poor, and iron-deficient in appearance. If tartrate or EDTA are used to restore iron availability and normal growth, the former is active at about $10^{-7} M$, but the latter is required at at least $10^{-5} M$ for the same effect. Yet EDTA is far more active than tartrate in affecting the photoperiodic behavior (8), so the effectiveness of the two agents in restoring iron availability does not parallel their activity on daylength requirements for flowering.

It is well known that EDTA and probably other chelators are taken up by plant tissues and have profound effects on internal metal relationships and also, perhaps indirectly, on enzyme activities of vari- 
ous kinds $(2,16,17)$. It is probably in this manner, rather than through action in the external medium, that EDTA is acting in the systcms in question. On the other hand, the $p \mathrm{H}$-activity relationship, consistent with a chelation phenomenon, is the opposite of what would be expected for the uptake of a weakly dissociated acid; it might, of course, be due to an effect of external $p \mathrm{H}$ on the internal $p \mathrm{H}$ of the tissues involved, and thus on internal chelation.

The effects on photoperiodic response might be due to an action on iron metabolism or internal iron availability. The work of Smith, McIlrath, and Bogorad (15), at least part of which is strongly confirmed by results such as those in Table 1, suggest that iron is intimately involved in photoperiodic induction. One of the first steps in the process might be a reaction of the receptor pigment itself or some closely associated compound with iron or an iron complex. This sort of reasoning, based only on the results presented here, might apply to other metals as well. Iron seems most likely simply because of the results quoted, because of its wide biological role, and its relatively high affinity for most chelating agents. The suggestion is no more than sheer speculation at present, but indicates a direction in which evidence might be sought.

One should recall that EDTA in the presence of iron can be decomposed by daylight $(2,5)$. It is questionable whether this reaction bears any relationship to the effects studied here, since, at least in $L$. perpusilla, very low energies of red light are sufficient to control the EDTA-induced short-day behavior ( 7 ).

In conclusion, a few general remarks may be made on the use of aseptically cultured species of Lemnaceae in studies of photoperiodism. The ease of controlling so many factors, which is an advantage offered by these plants, also in a sense raises difficulties. Where factors can be controlled, they must be controlled, and studied. A consideration of the work covered here suggests innumerable questions as to the nature and relationships of various nutritional, medium, light, and temperature effects, most of which have not even been discussed. A great number of experiments will be required before the responses of even the two plants mentioned are completely described, and the foundations for more intensive biochemical investigations have been laid.

In addition, there are other useful plants in this group. Preliminary experiments with a strain of $L$. paucicostata recently raised from seeds sent by Professor P. Maheshwari indicate a short-day response differing from that of $L$. perpusilla 6746 in its relation to both chelating 
agents and light. It is therefore unfortunate that work with these plants was started so recently. Had they been studied as long as Xanthium, Hyoscyamus, or Perilla, for example, the mechanism of photoperiodism would surely be better known than it is. There is no reason to believe that the Lemnaceae are less "typical" of higher plants in metabolism and responses than those mentioned, or to treat them as botanical curiosities. Only their gross morphology, size, and perhaps the resulting ease with which applied substances can be taken up, constitute the difference, making them experimental organisms with many of the advantages of those other curiosities, Drosophila and Neurospora.

\section{ACKNOWLEDGMENT}

This work was supported by National Science Foundation grant G-4433 to A. W. Galston.

\section{REFERENCES}

I. Butler, W. L., Norris, K. H., Siegelman, H. W., and Hendricks, S. B., Proc. Nat. Acad. Sci. U. S., 12, 1703-1708 (1959).

2. Chaberek, S., and Martell, A. E., Organic Sequestering Agents, John Wiley and Sons, Inc., N. Y. (1959).

3. DeKock, P. C., and Mitchell, R. L., Soil Science, 84, 55-62 (1957).

4. Hicks, L. E., Ohio J. Sci., 32, 115-131 (1932).

5. Hill-Cottingham, D. G., Nature, 175, 347-348 (1955); (Chem. Abst. 49: 100-19i).

6. Hillman, WV. S., Nature, 181, 1275 (1958).

7. Hillman, W. S., Am. J. Bot., 46, 466-473 (1959).

8. Hillman, W. S., Am. J. Bot., 46, 489-495 (1959).

9. Hutner, S. H., in Growth and Differentiation in Plants, (W. E. Loomis, ed.), lowa Sta. Coll. Press, Ames (1953).

10. Kandeler, R., Z. Botan., 43, 61-71 (1955).

11. Kandeler, R., Z. Botan., 44, 153-174 (1956).

12. Landolt, E., Ber. Schweiz. botan. Cies., 67, $271-410$ (1957).

13. Rediske, J. H., and Biddulph, O., Plant Physiol., 28, 576-593 (1953).

14. Saeger, A., Bull. Torrey Botan. Club, 56, 351-358 (1929).

15. Smith, H. J., Mcllrath. W. J., and Bogorad, I.., Botan. Gaz., I18, 174-179 (1957).

I6. Tiffin, L. O., and Brown, J. C., Science, 130, 274-275 (1959).

17. Weinstein, L. H., Meiss, A. M., Uhler, R. I.., and Purvis, E. R.. Contrib. Boyce Thompson Inst., 18, 357-370 (1956). 
COMPARATIVE STUDIES ON THE GROWTH ANI) LIGHT

SENSITIVITY OF GREEN ANI ETIOLATEI)

PEA STEM SECTIONS ${ }^{1}$

\author{
A. W. Galston and Ravindar Kaur \\ Department of Botany \\ Josiah Willard Gibbs Research Laboratory \\ Yale University \\ New Hay'en, Connecticut
}

In recent years, the stem of the common garden pea (Pisum sativum L.) has become one of the most frequently used objects in the study of plant cell growth. Both the intact stem and small excised sten sections of this plant show rapid and marked growth responses to such environmental factors as light intensity, light quality, photoperiod, and temperature, and to such chemical infuences as auxins, gibberellins, cobaltous ion, and adenine. Originally $(8,18)$, stem sections of the etiolated pea (3rd internode of 7 to 8 -day-old plants) were used exclusively in such studies, since their growth responses to auxin paralleled in many respects those of the classical Avena coleoptile. More recently $(2,7,10)$, stem sections from light-grown peas (the fifth internode of 14-day old plants) have been introduced into plant physiological reasearch. Systematic investigation of the biological and chemical properties of these two forms of the same genetic material have revealed the existence of remarkable differences in behavior which bear on our attempts to understand the nature of growth hormone action and of the morphogenetic action of light. For convenience in our later discussion, we have summarized the major points of gross morphological and physiological difference between the light-grown and etiolated pea plants in Fig. 1 and in Table 1.

Numerous questions arise from this comparison which may be of particular interest to the student of plant growth. These questions include the following: (i) Why is the auxin optimum for growth

1 Aided by grants from the National Science Foundation and the U.S. Public Health Service. Miss Mary Lyons rendered expert technical assistance with some of the section growth experiments. 


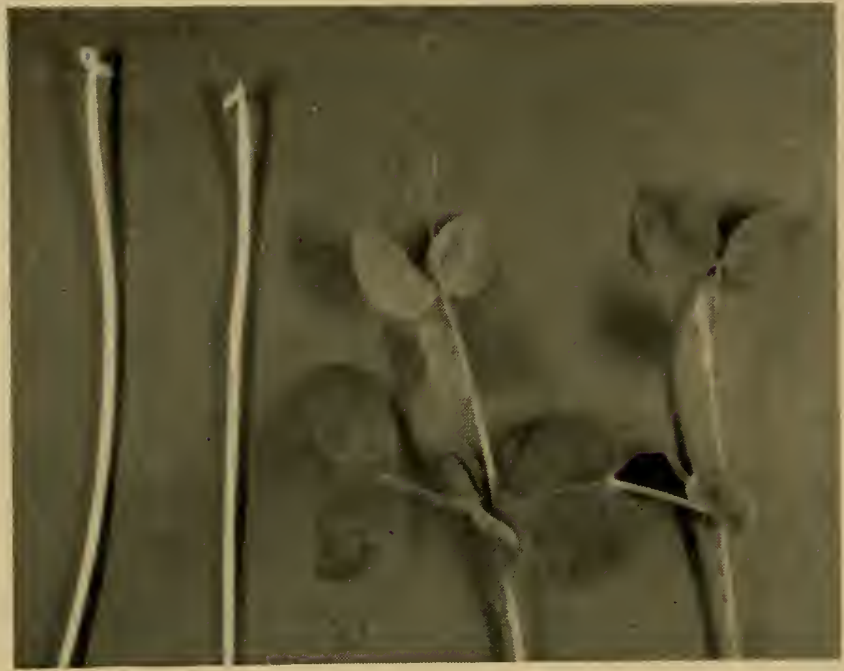

Fig. 1. Morphological differences betwen etiolated (left) and light-grown (right) pea plants.

of the green tissue a thousandfold higher than that for the etiolated tissue? (ii) Why is the gibberellin effect on growth independent of auxin in etiolated tissue and auxin-dependent in green tissue? (iii) Is there any physiological significance to the indole acetic acid (IAA) oxidase inhibitor whose level in tissue is determined by the red far-red photomorphogenic system? (12) (iv) Why are green stem sections not inhibited in their growth by the red radiations which are so effective on intact green plants and on etiolated sections? Answers to these and related questions can be obtained only when

I.IBLE I

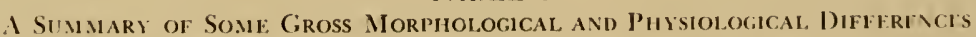
BEIWELN I)ARK-GROWX AND LIGITT-GROWX P'FA SILMS

Color

Internode length Internode width Internode cross section Nitritional habit

Sucrose optimum

Iuxin (IAA) optimum

Response to gibberellins

Response to white light Response to weak red light IA oxidase aclivily IAA oxidase inhibitor white very long narrow circular heterolrophic ca. $2 \%$ ca. $10-{ }^{6} \mathrm{M}$ relatively large independens of anxin inhibition inhibition high absent or low

\section{Light.grow'n}

green

shorler

thicker

reclangular

attotrophic or heterolrophic

cal. $1 \%$; ca. $3 \%$

$>10-1 \mathrm{M}$

relatisels smalt

dependent on atsin

promotion

none

low

high 
the companative growth characteristics of green and etiolated plants have been subjected to a much closer scrutiny than they have yet had. The investigations reported in this paper are part of a general program aimed at obtaining data which may aid in the elucidation of the problems mentioned above.

\section{Materials ANI) Methods}

Etiolated plants. All plants were obtained from seeds of Alaska peas (Pisum satirum L.) purchased from Associated Seed Growers, Inc., New Haven, Commecticut. The seeds were soaked in tap water for 4 hours in darkness and sown in carefully washed, tap-water saturated vermiculite (Mica-Grow Type B, supplied by Platt Seed Company, Branford, Connecticut) in polyethylene trays. The seedlings were allowed to grow in a darkened cabinet, in a dark room, maintained at $27 \pm 1^{\circ} \mathrm{C}$. During manipulation, they were exposed as sparingly as possible to a clin green light about $30 \mathrm{~cm}$ distant. This light source consisted of a I5-watt Sylvania green fluorescent tube wrapped with 3 layers each of green and amber DuPont cellophane, and was found to be inactive both phototropically and photomorphogenetically.

All sections were excised from 7 - to 8-day-old plants with recurved terminal buds, except for experiments in which the effect of the bud configuration on growth was studied. In general, $5 \mathrm{~mm}$ sections cut ca. $2 \mathrm{~mm}$ below the apical crook were used.

Light-grown peas. Alaska peas were sown in coarse "Zonolite" vermiculite in $4 \times 4 \times 3$ inch plastic boxes in three controlledcondition rooms maintained at ca. $23^{\circ} \mathrm{C}$ and at photoperiods of 8 , 16, and 24 hours. The plants were automatically subirrigated twicc daily with a nutrient solution consisting of $120 \mathrm{~g}$ of Hyponex (Hydroponics Chemicals Company, Copley, Ohio) per 100 l tap water. The plants received ca. 1500 foot-candles of a mixture of fluorescent and incandescent lights, and were used in the experiment at an age of $14-24$ days. All sections were excised immediately below the apical bud.

Sections $1 \mathrm{~mm}$ to $5 \mathrm{~mm}$ in length of both etiolated and green pea stems, were cut by the use of a guillotine described earlier (8). The longer sections, $10 \mathrm{~mm}$ to $20 \mathrm{~mm}$, were excised by a properly spaced double-bladed cutting tool. The sections were first randomized in distilled water, and lots of 10 sections were then floated on $5 \mathrm{ml}$ of growth medium in $5 \mathrm{~cm}$ petri dishes. The etiolated sections were permitted to grow in a completely dark growth cabinet in a dark 
room, and the light-grown sections were exposed to ca. 1200 footcandles of fluorescent light. All sections were incubated for ca. 20 hours and measured to the nearest $0.1 \mathrm{~mm}$ under a dissecting microscope equipped with an ocular micrometer. Sections initially longer than $5 \mathrm{~mm}$ were measured to the nearest $0.5 \mathrm{~mm}$ with a metric scale ruler. Fresh weights of the sections before and after growth were obtained to the nearest $\mathrm{mg}$ on a Roller-Smith torsion balance. The basic growth medium for both green and etiolated sections consisted of $0.01 \mathrm{M} p \mathrm{H}$ 6.1 $\mathrm{KH}_{2} \mathrm{PO}_{4}-\mathrm{Na}_{2} \mathrm{HPO}_{4}$ buffer, $1-2 \%$ sucrose unless otherwise mentioned, and indole acetic acid (IAA) and gibberellic acid (GA) wherever mentioned in the text. In general, etiolated sections were supplied with $10^{-6} M$ IAA and green sections with $10^{-4} M$ IAA. Both types of sections received $10^{-4} M$ GA.

\section{Experimental Results}

\section{A. Calibration of the Growth System}

Effect of section length on growth. A meaningful comparison of the growth of green and etiolated sections can be made only if optimally growing sections of both types of tissue are employed. This required a determination of the optimal length of section to be used in these experiments. To our knowledge, no such cleterminations have previously been made, despite a multiplicity of investigations on pea sections. Figs. 2 and 3 represent the percentage increase in length and fresh weight of both green and etiolated sections as a function of initial length and fresh weight of sections. It is clear that sections 3-5 mm long are close to optimal for both types of sections, with respect to both kinds of measurements. Longer sections of the etiolated sections rapidly decline in efficiency of growth, while longer sections of the green material remain close to maximally efficient. This resembles the situation in the intact plant, and probably reflects the fact that cell division is restricted to the apical region in etiolaterl peas, but is more distributed over the internode in green peas. Since many previous experiments had been performed with $5 \mathrm{~mm}$ sections, which are here shown to be close to maximally effective growth, this length was adopted as a standard.

Effect of bud configuration on growth. In both etiolated and green pea stems, the teminal bud may assume a variety of forms (Fig. 4) . In etiolated plants, the apex of the stem may be recurved $(A)$, hooked (B), or open (C). In the green plants, the terminal bud may be completely enclosed within the stipules (A), barely extruded (B), or 


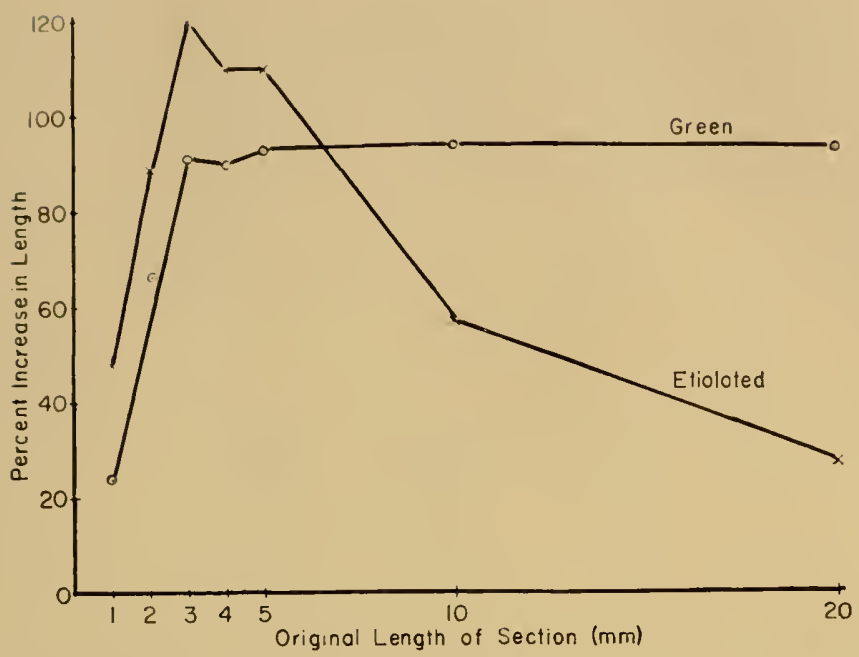

Fig. 2. Effect of the initial length on the percentage increase in length of etiolated and green pea stem sections in the presence of indole acetic acid.

significantly protruding $(\mathrm{C})$. The growth data for sections excised from these various types of plants are presented in Table 2 . It can be seen that no major growth differences appear. In general, response to auxin in etiolated plants was best in condition $\mathrm{A}$ and in green plants in conditions B and C. These configurations were therefore selected as standards in further studies.

Effect of internode length on growth. Sections may obviously be derived from internodes of varying length. Table 3 demonstrates that etiolated internodes less than $15 \mathrm{~mm}$ long are inferior to longer

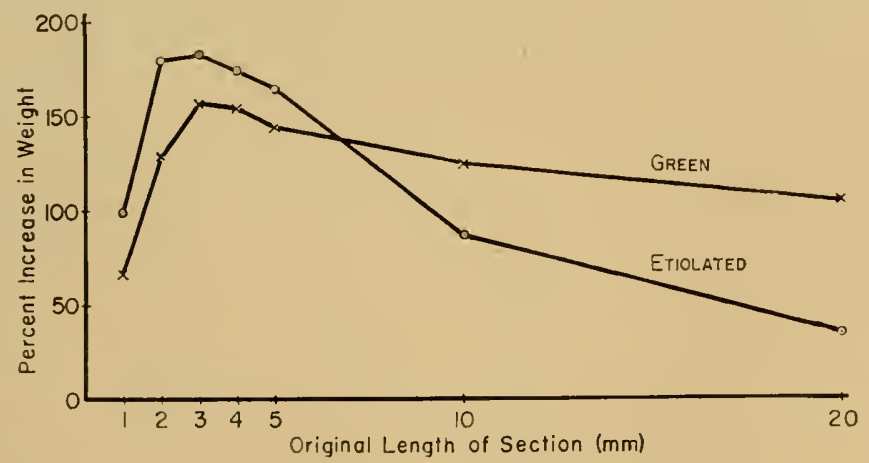

Fig. 3. Effect of the initial length on the percentage increase in fresh weight of etiolated and green pea stem sections in the presence of indole acetic acid. 
Terminal Bud Configurations
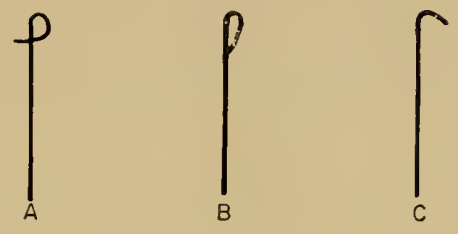

ETIOLATED
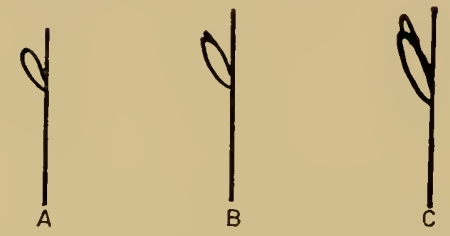

GREEN

Fig. 4. Terminal bud configurations of etiolated and green plants.

internodes as sources of tissue for growth experiments, and that green internodes shorter than $25 \mathrm{~mm}$ long are probably also inferior to longer internodes. Despite the fact that this experiment is atypical in showing depressed growth with GA, this type of result has been generally obtained in other experiments. Therefore internodes as near as possible to $30 \mathrm{~mm}$ long were selected as the most desirable material for further studies.

\section{TABLE 2}

Effect of the Configuration of the Bud on the Growth of Pea Stem Sections Incubated in the Presence and Absence of Indole Acetic Acid (IAA)

(10-6 $M$ for Etiolated, $10^{-4} M$ fOR Green Sections) and Gibberellic Acid (GA) $\left(10^{-4} M\right)$

\begin{tabular}{|c|c|c|c|c|c|}
\hline \multirow[t]{2}{*}{$\begin{array}{c}\text { Condition } \\
\text { of plant }\end{array}$} & \multirow[t]{2}{*}{$\begin{array}{l}\text { Configuration } \\
\text { bud }\end{array}$} & \multicolumn{4}{|c|}{ Average Growth of Section (mm) } \\
\hline & & Endogenous & + IAA & $+\mathrm{GA}$ & $+\underset{\text { GA }}{+ \text { IAA }}$ \\
\hline Etiolated & $\begin{array}{l}\text { A } \\
\text { B } \\
\text { C }\end{array}$ & $\begin{array}{l}2.63 \\
2.62 \\
2.31\end{array}$ & $\begin{array}{l}3.35 \\
2.84 \\
3.14\end{array}$ & $\begin{array}{l}3.50 \\
3.12 \\
3.12\end{array}$ & $\begin{array}{l}3.71 \\
3.73 \\
3.70\end{array}$ \\
\hline Green & $\begin{array}{l}\text { A } \\
B \\
\text { C }\end{array}$ & $\begin{array}{l}0.83 \\
0.77 \\
0.64\end{array}$ & $\begin{array}{l}2.89 \\
3.45 \\
3.39\end{array}$ & $\begin{array}{l}0.97 \\
1.26 \\
0.97\end{array}$ & $\begin{array}{l}3.16 \\
3.51 \\
3.79\end{array}$ \\
\hline
\end{tabular}


TABLE 3

EfFect of the Length of the Youngest Internode on the Growth of Pla Stem Sections Incubated in the Presence of Indole Acetic Acid (IAA) and Gibberellic Agid (GA). Congentrations as in Table 2

Growth of sections (mm)

\begin{tabular}{|c|c|c|c|c|c|}
\hline \multirow[b]{2}{*}{ Plant } & \multirow[b]{2}{*}{$\begin{array}{l}\text { Length of } \\
\text { internode } \\
\text { (mm) }\end{array}$} & \\
\hline & & Endogenous & + IAA & $+\mathrm{GA}$ & $\begin{array}{l}+ \text { IAA and } \\
+ \text { GA }\end{array}$ \\
\hline Etiolated & $\begin{array}{l}>15 \\
15-30 \\
30-50\end{array}$ & $\begin{array}{l}4.19 \\
4.77 \\
4.65\end{array}$ & $\begin{array}{l}4.31 \\
4.50 \\
4.51\end{array}$ & $\begin{array}{l}5.35 \\
5.56 \\
5.78\end{array}$ & $\begin{array}{l}4.53 \\
4.87 \\
4.40\end{array}$ \\
\hline Green & $\begin{array}{r}25 \\
25-50\end{array}$ & $\begin{array}{l}1.54 \\
1.53\end{array}$ & $\begin{array}{l}2.96 \\
2.98\end{array}$ & $\begin{array}{l}1.22 \\
1.00\end{array}$ & $\begin{array}{l}2.63 \\
3.15\end{array}$ \\
\hline
\end{tabular}

Effect of age of plant and of interval between harvest and sectioncutting on growth. Occasionally, eight- or nine-day-old etiolated peas had to be substituted for seven-day-old peas, and older green peas for the standard 14-day-old peas. Numerous experiments revealed that, so long as the morphology of the terminal portion of the plant was kept reasonably constant, age of the plant was not an important determinant of section growth. Age substitutions were therefore made, but only when necessary. Similarly, within the usual time limits of an experiment, the duration of the interval between harvest and sectioncutting had no effect on the growth patterns.

\section{B. Differential Growth Responses}

Sucrose optimum. Careful studies by Purves (15) have confirmed the report (8) that sucrose promotes the growth of etiolated sections, and that the optimal concentration for growth is ca. 2 per cent. Fig. 5 demonstrates that the sucrose optimum for the growth of green sections is $1 \%$ in the light and $2-3 \%$ in the dark. As previously demonstrated $(2,7)$, optimal growth in the light exceeds optimal growth in the dark, indicating the existence of some non-photosynthetic photochemical process promoting growth in green sections.

Indole acetic acid (IAA) optimum. In confirmation of previous reports $(8,18)$, the IAA optimum for increase in length of etiolated sections of various initial lengths was found to be ca. $10^{-6} M$, and for increase in fresh weight ca. $10^{-5} M$ (Figs. 6 and 7 ). In contrast, green sections show no optimum at all in the light, growth in length increasing with increasing IAA concentration beyond $3 \times 10^{-4} M$ (Fig. 8). In the dark, there appears to be a shallow optimum at ca. 


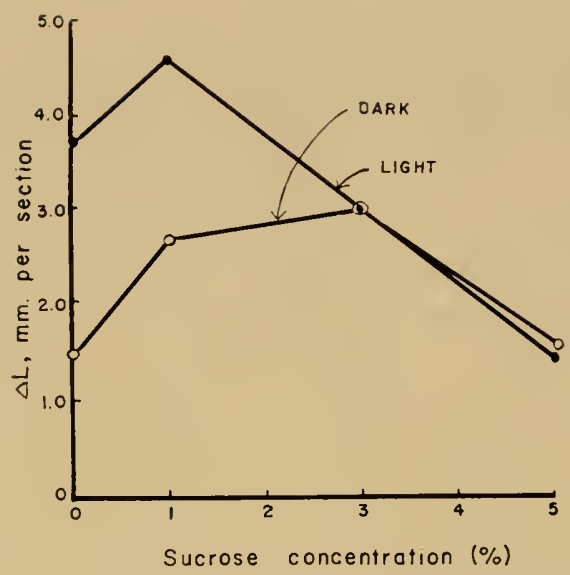

Fig. 5. Effect of sucrose concentration on the growth of green pea stem sections in light and dark.

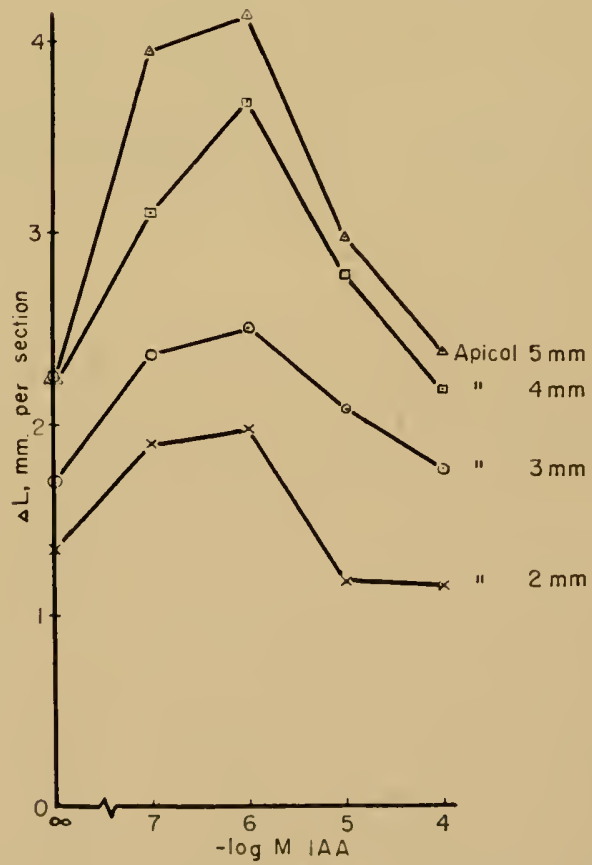

Fig. 6. Effect of indole acetic acid (IAA) concentration on the growth in length of terminal etiolated pea stem sections of various lengths. 


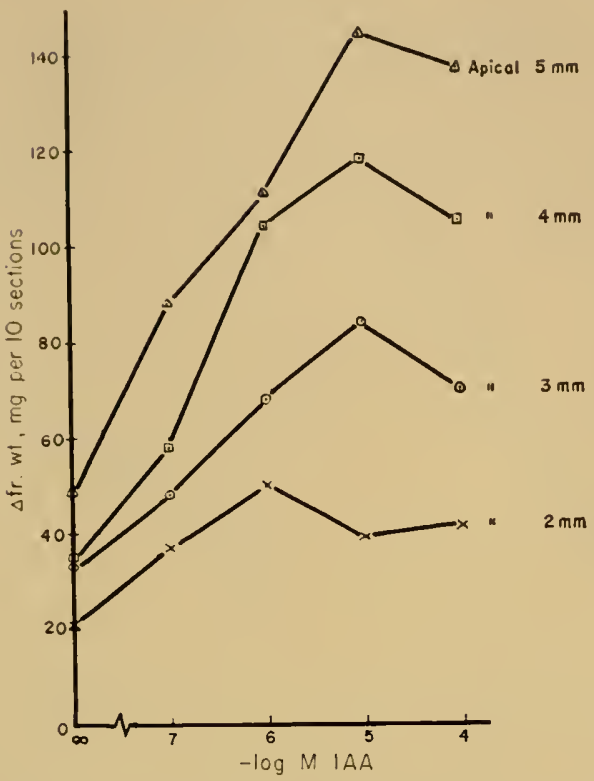

Fig. 7. Effect of indole acetic acid (IAA) concentration on the increase in fresh weight of etiolated pea stem sections of various lengths.

$10^{-4} M$. These facts seem to be independent of the photoperiod under which the plant is grown.

Gibberellic acid $(G A)$ optimum. The GA optimum for etiolated pea sections was found by Purves $(15)$ to be ca. $10^{-7}$ to $10^{-6} M$. In contrast, the GA optimum for green sections is $10^{-5} M$ or higher, depending on the photoperiodic treatment of the plants from which the sections were derived (Fig. 9).

$G A-I A A$ synergism. It has been demonstrated that GA and IAA promote growth non-synergistically in etiolated sections $(9,16,17)$ but synergistically in green sections (2). Table 4 demonstrates the effect of photoperiod on the degree of synergistic interaction. The synergism (seen in the difference between the observed and calculated values for growth in $\mathrm{GA}+1 \mathrm{AA}$ ) is readily apparent in sections from the 8-hour photoperiod plant, but the differences observed in sections from the 16-hour plants are insignificant, and in those from the 24hour plants barely significant.

Galston and Warburg (9) clemonstrated that passage of GA from base to apex through excised stem pieces of etiolated and green stems resulted in synergistic interaction with IAA subsequently applied 
LIGHT

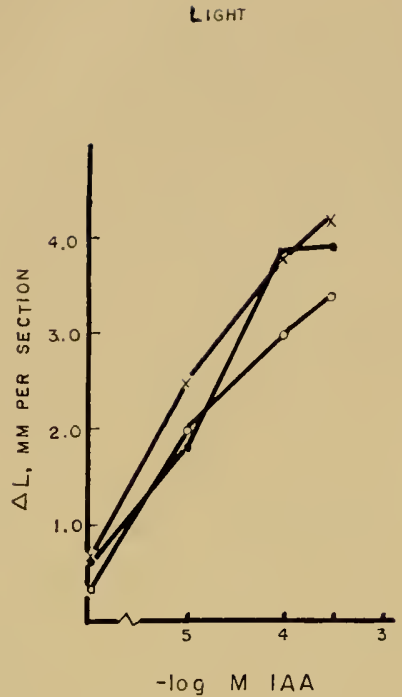

DARK

$8 \mathrm{Hr}$, Photopd. O

$16 \mathrm{Hr}$. Photopd. $x$

$24 \mathrm{Hr}$. Photopd.

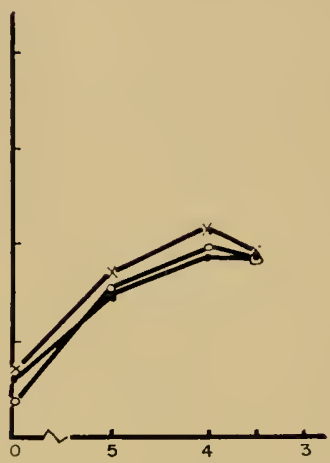

$-\log M I A A$

Fig. 8. Effect of the photoperiod of the parent plant on the indole acetic acid (IAA) optimum of green pca stem sections grown in light and dark.

to apical $5 \mathrm{~mm}$ sections excised from these treated stem pieces. While Purves and Hillman (17) could find no such induced synergism in etiolated pea stems, we have consistently been able to induce GA-IAA synergism in 16-hour and 24-hour photoperiod green stems of appropriate length by such manipulation (Table 5). Thus, $10 \mathrm{~cm}$ long stem pieces from 16-hour photoperiod plants show GA-IAA synergism, while shorter pieces do not; conversely, $2 \mathrm{~cm}$ stem pieces from 24-hour

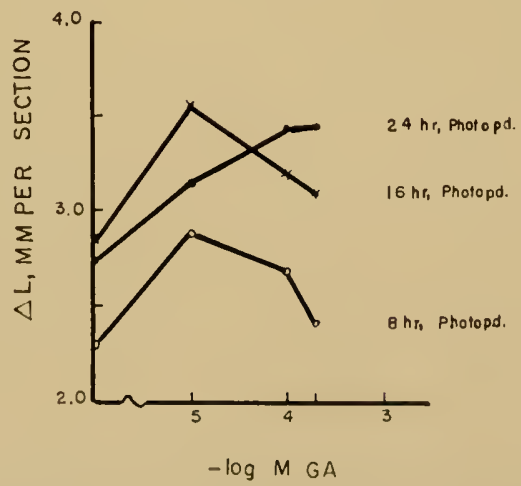

Fig. 9. Effect of the photoperiod on the gibberellic acid (GA) optimum for growth of green pea stem sections. 
TABLE 4

Erfect of the Pilotoperion of tile Parent Plant on Ga-iaA Synergism in Green Pea Stem Sections

Photopcriod Endogenous hours growth $(\mathrm{mm})$
Growth of sections ( $\mathrm{mm}$ in excess of endogenous)

\begin{tabular}{|c|c|c|c|c|c|c|}
\hline & & \multirow[t]{2}{*}{ IAA } & \multirow{2}{*}{ GA } & \multicolumn{3}{|c|}{$\mathrm{IAA}+\mathrm{GA}$} \\
\hline & & & & Calc. & Obs. & Obs. - Calc. \\
\hline 8 & 1.17 & 2.46 & 0.47 & 2.93 & 3.63 & 0.70 \\
\hline 16 & 1.63 & 2.78 & 0.72 & 3.50 & 3.69 & 0.19 \\
\hline 24 & 1.16 & 2.93 & 0.49 & 3.42 & 3.73 & 0.31 \\
\hline
\end{tabular}

photoperiod plants show GA-IAA synergism but longer pieces do not. The reasons for these curious relationships are at present completely obscure.

In previous experiments (9) it has been demonstrated that momen. tary exposure of section or stem pieces to GA induces effects almost as great as those caused by continuous exposure. Table 6 demonstrates that exposure of $5 \mathrm{~mm}$ sections from 8-hour photoperiod plants to as little as 1 minute of $10^{-4} M$ GA suffices to induce the synergism

TABLE 5

Effect of the Length of Stem Pretreated with GA on Degree of Subsequent Synergism with IAA in Green Pea Stem Sections Excised from 8-, 16-, and 24-hour Photoperiod Plants. Basal Pretreatment Time in Ga was 30 Minutes.

\begin{tabular}{|c|c|c|c|c|c|c|c|}
\hline \multirow[t]{3}{*}{$\begin{array}{l}\text { Photoperiod } \\
\text { hours }\end{array}$} & \multirow[t]{3}{*}{$\begin{array}{c}\text { Stem } \\
\text { length } \\
\mathrm{cm}\end{array}$} & \multirow[t]{3}{*}{$\begin{array}{l}\text { Endogenous } \\
\text { growth (mm) }\end{array}$} & \multicolumn{5}{|c|}{$\begin{array}{c}\text { Growth of sections } \\
\text { ( } \mathrm{mm} \text { in excess of endogenous) }\end{array}$} \\
\hline & & & IAA & GA & & $A+$ & \\
\hline & & & & & Calc. & Obs. & $\begin{array}{l}\text { Obs. - } \\
\text { Calc. }\end{array}$ \\
\hline 8 & $\begin{array}{r}2 \\
5 \\
10\end{array}$ & $\begin{array}{l}0.78 \\
0.81 \\
0.86\end{array}$ & $\begin{array}{l}2.36 \\
2.48 \\
2.32\end{array}$ & $\begin{array}{l}0.30 \\
0.26 \\
0.30\end{array}$ & $\begin{array}{l}2.66 \\
2.74 \\
2.62\end{array}$ & $\begin{array}{l}3.56 \\
3.20 \\
3.08\end{array}$ & $\begin{array}{l}0.90 \\
0.46 \\
0.46\end{array}$ \\
\hline 16 & $\begin{array}{r}2 \\
5 \\
10\end{array}$ & $\begin{array}{l}0.98 \\
1.05 \\
1.38\end{array}$ & $\begin{array}{l}2.38 \\
2.75 \\
2.41\end{array}$ & $\begin{array}{l}0.51 \\
0.43 \\
0.18\end{array}$ & $\begin{array}{l}2.89 \\
3.18 \\
2.59\end{array}$ & $\begin{array}{l}3.01 \\
3.44 \\
3.11\end{array}$ & $\begin{array}{l}0.12 \\
0.26 \\
0.52\end{array}$ \\
\hline 24 & $\begin{array}{r}2 \\
5 \\
10\end{array}$ & $\begin{array}{l}1.19 \\
1.17 \\
1.29\end{array}$ & $\begin{array}{l}2.90 \\
3.79 \\
3.48\end{array}$ & $\begin{array}{l}0.32 \\
0.43 \\
0.15\end{array}$ & $\begin{array}{l}3.22 \\
4.22 \\
3.63\end{array}$ & $\begin{array}{l}3.83 \\
3.97 \\
4.00\end{array}$ & $\begin{array}{r}0.61 \\
-0.25 \\
0.37\end{array}$ \\
\hline
\end{tabular}


TABLE 6

Effect of the Pretreatment Time of Sections in $10^{-4} M$ GA on Ga-IAA Synergism in Green Pea Stem Sections Derived from 8-hour Photoperiod Plants

Pretreatment Endogenous time $(\mathrm{min})$ growth $(\mathrm{mm})$
Growth of sections ( $\mathrm{mm}$ in excess of endogenous)

IAA $\quad$ GA + GA

Calc. Obs. Obs, - Calc.

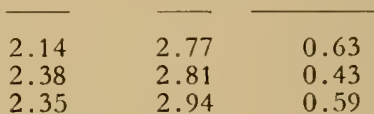

with IAA. In similar experiments with $5 \mathrm{~cm}$ sticks, 10 minutes of exposure to GA were found adequate to induce synergism.

Cobaltous ion. The cobaltous ion is known to enhance markedly the growth of etiolated pea stem sections in the presence of sucrose $(1,14)$. The optimum concentration of $\mathrm{Co}^{++}$for this effect is ca. $10^{-4} M$. With green pea sections, on the other hand, growth promotion by $\mathrm{Co}^{++}$is very slight, is independent of sucrose, and is manifested (when it occurs at all) at ca. $10^{-6} M$ (Table 7 ). This is the only instance we know in which green sections respond to lower concentrations of a growth-promoting material than do etiolated sections.

Substituted urea herbicides. Substituted ureas such as CMU (3-pchlorophenyl-1,1-dimethyl urea) and fenuron (3-phenyl-1,1-dimethyl urea) have been shown to inhibit the growth of and sometimes to kill green plants $(3,4)$ and to inhibit the Hill reaction (5) and photosynthetic phosphorylation (13). Figs. 10 and 11 show that both

\section{TABLE 7}

Effect of Cobaltous Chloride on the Growth of Green Pea Stem Sections Incubated in the Presence and Absence of Sucrose

$\mathrm{Co}^{++}$

concentration

(MI)
Growth of sections ( $\mathrm{mm}$ )

\begin{tabular}{|c|c|c|c|}
\hline \multicolumn{2}{|c|}{ Dark } & \multicolumn{2}{|c|}{ Light } \\
\hline 0 sucrose & $1 \%$ sucrose & 0 sucrose & $1 \%$ sucrose \\
\hline 1.23 & 2.80 & 2.55 & 2.92 \\
\hline 1.66 & 3.34 & 2.61 & 3.26 \\
\hline 1.70 & 3.10 & 2.34 & 3.13 \\
\hline 1.39 & 2.98 & 2.22 & 2.82 \\
\hline 1.25 & 1.73 & 1.30 & 1.73 \\
\hline
\end{tabular}




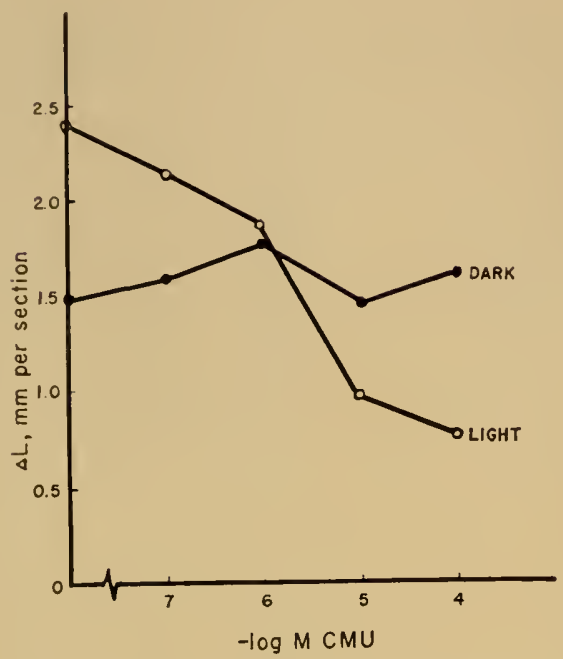

Fig. 10. Effect of $p$-chlorophenyl dimethyl urea (CMU) on the growth of green pea stem sections in light and dark,

substances are effective in inhibiting the autotrophic growth of green sections in the light, but are essentially without effect on heterotrophic. growth of green sections supplied with sucrose in the dark. Figs. 12 and 13 show that the inhibitions imposed by these substances in the

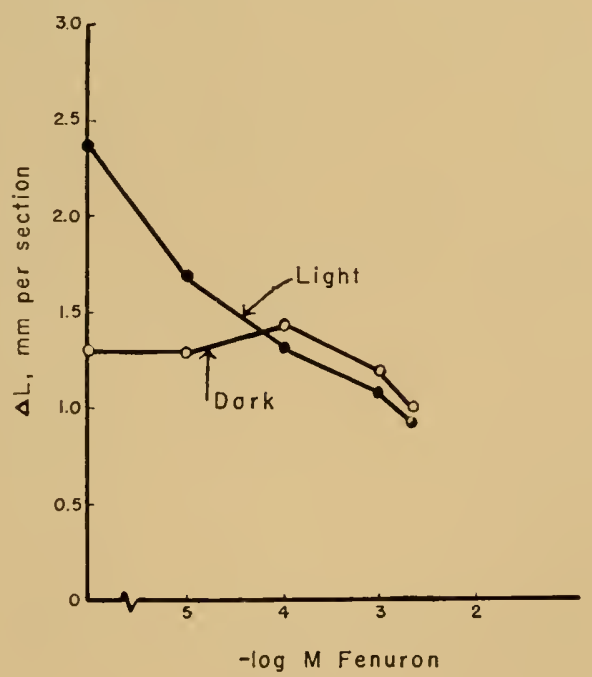

Fig. 11. Effect of fenuron (phenyl dimethyl urea) on the growth of green pea stem sections in light and dark. 


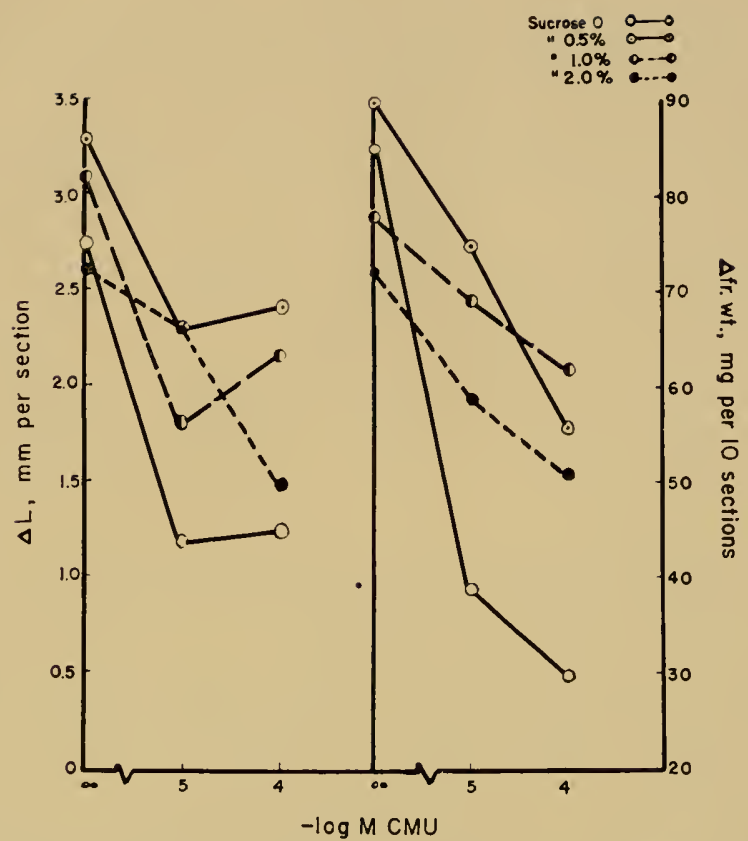

Fig. 12. Interaction of p-chlorophenyl dimethyl urea (CMU) and sucrose on the growth of green pea stem sections in light.

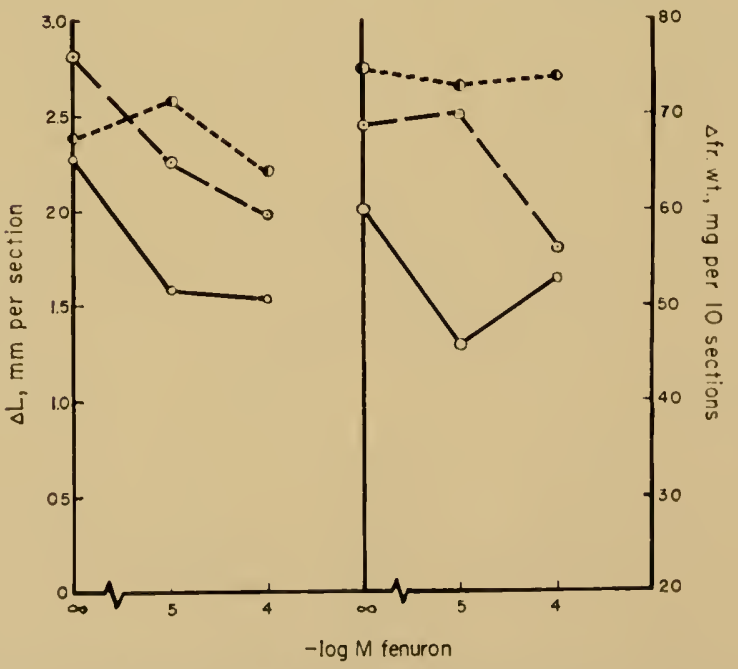

Fig. 13. Intcraction of fenuron and sucrose on the growth of green pea stem sections in light. 


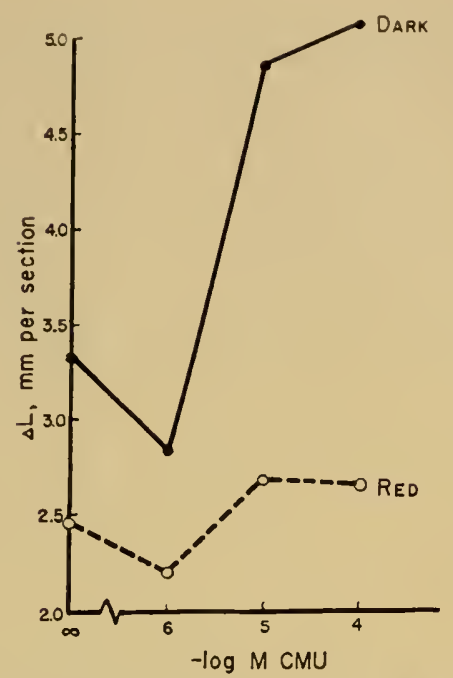

Fig. 14. Effect of $p$-chlorophenyl dimethyl urea (CMU) on the growth of etiolated pea stem sections in the dark and after a brief exposure to morphogenetically active red light.

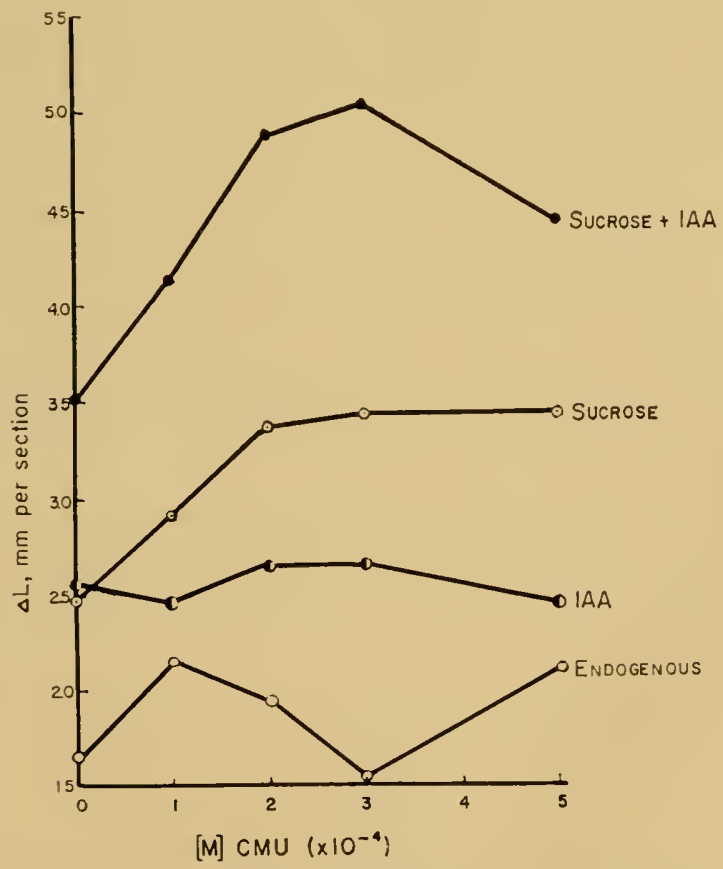

Fig. 15. Interaction of $p$-chlorophenyl dimethyl urea (CMU), sucrose, and indole acetic acid (IAA) on the growth of etiolated pea stem sections in the dark. 
light are substantially reversed by the inclusion in the medium of an appropriate level of sucrose, generally 1 per cent.

By contrast, the growth of etiolated sections in the clark is markedly enhanced by CMU (Fig. 14). The additional growth produced by CMU is essentially completely inhibited by weak red morphogenetically active light. The CMU-induced growth increase, like that of cobalt, is dependent on the presence of sucrose (Fig. 15) and is greatest when both sucrose and IAA are supplied. CMU does not alter the IAA optimum for growth (Fig. 16).

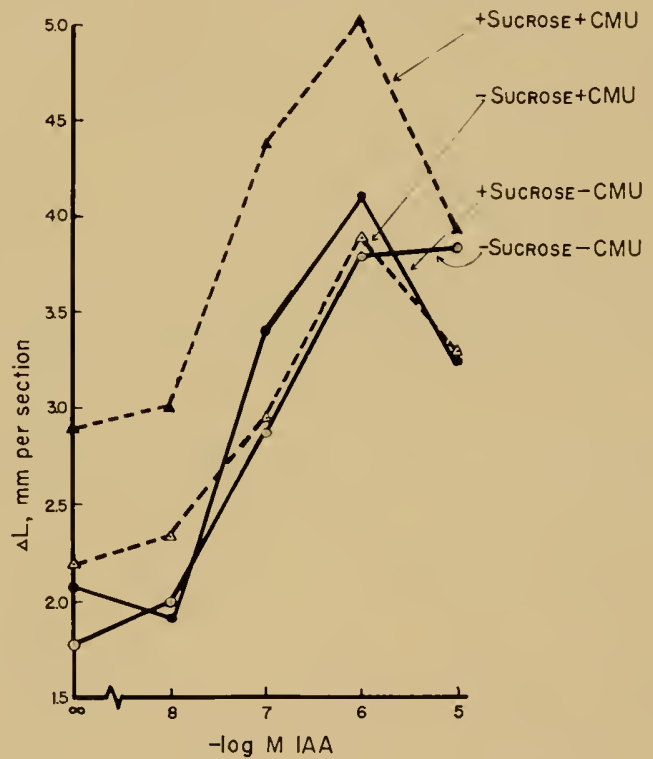

Fig. 16. Effect of $p$-chlorophenyl dimethyl urea (CMU) and sucrose on the indole acetic acid (IAA) optimum of etiolated pea stem sections.

TABLE 8

Interaction of Fenuron, Sucrose, and Indole Acetic Acid (IAA) on the Growth of Etiolated Pea Stem Sections

\begin{tabular}{|c|c|c|c|c|}
\hline \multirow[b]{2}{*}{ Sucrose } & \multicolumn{2}{|c|}{ Addenda } & \multicolumn{2}{|c|}{ Growth of sections ( $\mathrm{mm}$ ) } \\
\hline & $\begin{array}{c}\text { Fenuron } \\
5 \times 10^{-8} M\end{array}$ & 0 IAA & $10^{-7} M I \mathrm{IAA}$ & $10^{-6} M$ IAA \\
\hline- & - & 1.29 & 1.28 & 1.77 \\
\hline- & + & 1.60 & 2.31 & 2.45 \\
\hline+ & - & 3.70 & 4.23 & 3.56 \\
\hline$\dot{t}$ & + & 3.55 & 3.25 & 3.55 \\
\hline
\end{tabular}


Unlike CMIL, fenuron exerts its growth-promoting eflect on etiolated sections only in the absence of sucrose (Table 8) and at all levels of $1 \mathrm{~A}$ A tested.

Effect of photoperiod of the parent plant on growth characteristics of green sections. Sections derived [rom 24-hour photoperiod plants and supplied with sucrose grow better in the light and worse in the dark than do sections from 8-hour and 16-hour plants (Fig. 17). Therefore, they may be considered as "light-adapted," in the sense that etiolated sections are dark-adapted and are inhibited by very low total irradiances of light (11). Similarly, sections from 8-hour photoperiod plants supplied with sucrose grow better in the dark and worse in the light than do sections from 16- and 24-hour photoperiod plants. Photoperiod is however, without effect on the sucrose optimum (Fig. 18) or on the IAA optimum (Fig. 8).

Effect of morphogenetically active light. The growth of etiolated sections is reversibly inhibited by weak red light (11), this inhibition being dependent on the presence of sucrose (1). Roughly a 50 per cent inhibition of etiolated section growth can be induced by 50

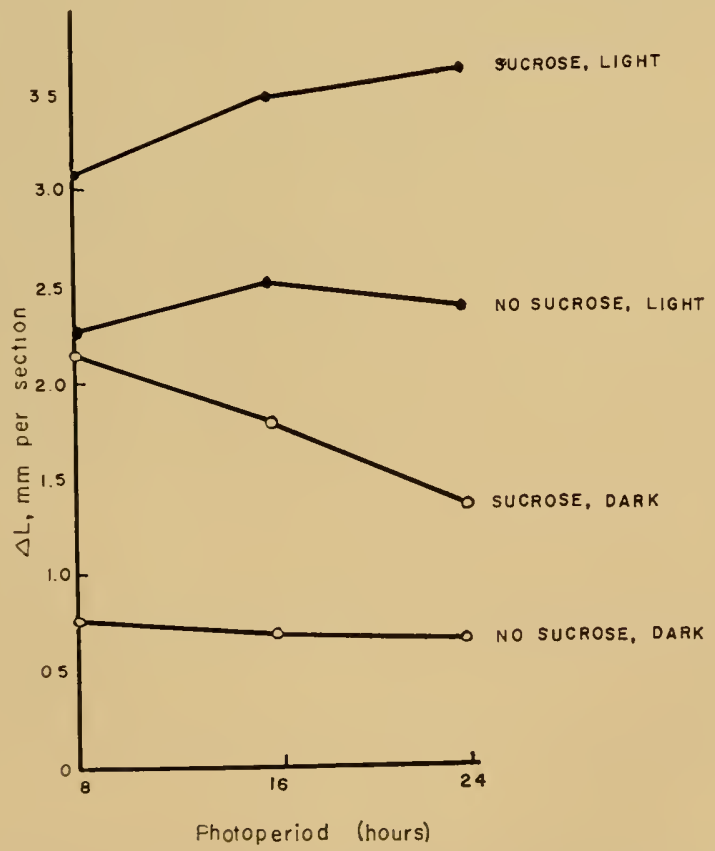

Fig. 17. Effect of photoperiod of the parent plant on the growth of green pea stem sections in light and dark. 


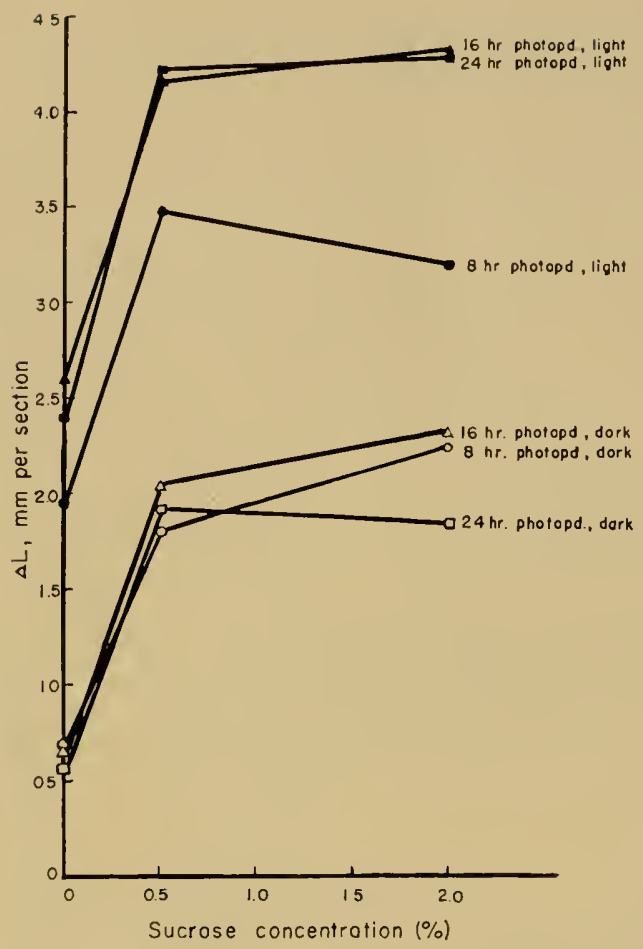

Fig. 18. Effect of the photoperiod of the parent plant on the sucrose optimum for growth of green pea stem sections in light and dark.

kiloergs $/ \mathrm{cm}^{2}$ of red light (1). All attempts to show any growth inhibition of green sections by similar light treatments have been unsuccessful. So far as we can determine, green sections are completely insensitive to photomorphogenic growth control by the red, far-red system found in etiolated sections (11) and in intact green plants (6).

\section{Conclusions}

These investigations have revealed important and extensive differences in growth characteristics to exist between green and etiolated pea stem sections. From the point of view of photobiology, the most interesting differences are the following:

(i) Etiolated sections are markedly inhibited by low irradiances with red light, while green sections are not.

(ii) Etiolated sections are markedly promoted by cobaltous ion, while green sections are essentially not. 
(iii) Etiolated sections respond optimally to very low concentrations (ca. $10^{-6} M$ ) of gibberellic acid and indole acctic acid, while green sections require ca. 100 to 1000 -fold higher concentrations of these substances for optimal growth.

(iv) The antotrophic growth of green scctions is inhibited by the substituted urea herbicides, their heterotrophic growth bcing essentially unaffected by these compounds. In sharp contrast, the (heterotrophic) growth of etiolated sections is markedly promoted by the urea herbicides.

(v) Green pea plants grown under continuous light produce sections which grow better in the light and worse in the dark than those from daily illumination regimes, including some darkness. They are thus to be considered as "light-adapted." Conversely, plants grown under short periods yield sections which grow better in the dark and worse in the light than sections from longer photoperiod plants. This implies that such sections are still partially dark-adapted, and that light induces a progressive shift from the etiolated to the fully green condition.

An explanation of the causes of any of these phenomena would bring us considerably closer to an understanding of the mechanism of the action of light on plant growth.

\section{REFERENCES}

1. Bertsch, W. F., P'h.D. thesis, Yale University (1960).

2. Brian, 1. W., and Henming, H. G., Ann. Botany, 22, 1-17 (1958).

3. Bucha, H. C., and Todd, C. W., Science, 114, 493-494 (1951).

4. Christoph, R. J., and Fisk, E. L., Botan. Gaz., 116, 1-14 (19:4).

5. Cooke, A. R., J'eeds, 4, 397-398 (1956).

6. Downs, R. J., Hendricks, S. B., and Borthwick, H. A., Botan. Gaz., 118, 199-208 $(1957)$.

7. Galston, A. W., and Baker, R. S., Plant Physiol., 26, 311-317 (1951).

8. Galston, A. W., and Hand, M. E., Am. J. Botany, 36. 85-94 (1949).

9. Galston, A. W., and Warburg, H., Plant Plysiol., 34, 16-22 (1959).

10. Hashimoto, T., Sci. Papers Coll. Gen. Educ., Univ. Tokyo, 9, 235-254 (1959).

11. Hillman, W. S., in Photoperiodism (R. B. Withrow, ed.), pp. 181-196, American Association for the Advancement of Science, Washington, D. C. (1959).

12. Hillman, W. S., and Galston, A. W., Plant Plysiol., 32, 129-135 (1957).

13. Jagendorf, A. T., Federation Proc., 18, 974-984 (1959).

14. Mliller, C. O., Plant Physiol., 29, 79-82 (1954).

I5. Purves, W. K., Ph. 1). thesis, Yale University (1959).

16. Purves, W. K., and Hillman, W. S., Physiol. Plantarum, 11, 29-35 (1958).

17. Purves, W. K., and Hillman, W. S., Physiol. Plantarum, 12, 786-798 (1959).

18. Thimann, K. V., and Schncider, C. L., Am. J. Botany, 26, 328-333 (I939). 


\title{
THE INTENSITY FACTOR IN VISION
}

\author{
W. A. H. Rushton \\ Trinity College, Cambridge;
}

Visiting Scientist to National Institutes of Health, Bethesda, Maryland

In this assembly of distinguished photochemists the fundamental visual problem which I wish to raise is "How does the eye use so effectively the quanta caught by the visual pigments of the rods and cones?" The question of the spectral sensitivity of vision is trivial, for at the chemical level it amounts simply to the statement that quanta which are not absorbed will not be seen. This expected relation was established with fair accuracy more than 50 years ago by Koenig (5) and by Trendelenburg (II), who showed that the spectral sensitivity of twilight vision corresponded to the action spertrum for the bleaching of rhodopsin. Color vision, to be sure, is no trivial question, but here the problem lies in the organization of nerve signals already generated.

What I should like to ask you is, "How are these signals generated?" For my part I have no answer, but information we have (some of it new) about the relation of the nerve signal to the light intensity, about the site where light- and dark-adaptation occur, and about the relation between the light threshold and the amount of pigment bleached. This information is here distilled into a single mathematical expression. Any theory of how the nerve signal is generated should satisfy this expression. And if it does, it will go a long way towards satisfying the relation between light intensity and vision.

\section{The Response to Light}

\section{(a) The Eye at Full Sensitivity}

The celebrated experiments of Hecht, Shlacr, and Piremne (4) measured the least number of quanta which have to be absorbed by rhodopsin in the human eye for the light to be seen. They employed two methods. In one they measured the actual energy falling upon the connea (through an artificial pupil) when a flash in optimal conditions could just be seen, and made an estimate of the fraction $(10 \%)$ which was absorbed by rhodopsin. In the other method, they 
used the statistics of quantum fluctuations and measured the frequency of secing a flash as a function of its log intensity. The two methods gave reasonable agreement, and the atuthors concluded that about 6 quanta must be absorbed in order to see, and moreover that each of these quanta was absorbed in a separate rod cell, since the probability of a double-hit upon one rod was substantially less than the probability with which subjects actually saw.

Contemporary work by van der Velden (12) and subsequent results by many authors, though diverging as to the actual minimal number of rods involved, all agree that a rod can be excited by one quantum.

Our first question, then, is: "How can a single quantum of light absorbed anywhere upon the onter segment of a rod set up some kind of excitation which travels down the cell to its fine termination $0.1 \mathrm{~mm}$ away, and there unite with similar excitations of a few other active rods to set up a nerve impulse?" All this must occur within the latent period for seeing-less than 1 second.

\section{(b) Adaptation}

It is a familiar experience that the eye can adapt rapidly to light and that it takes a much longer time to adapt to darkness. One feature of the change is the transfer of function from rods at low luminance to cones at high luminance. But, confining our attention to rod vision alone, it is a well-known fact that the visual threshold may fall 100-fold or more during the course of clark adaptation. Thus somewhere in the mechanism of vision between the light and its perception is a stage whose sensitivity varies very greatly. Where is this stage?

lt is certainly not chiefly in the brain, for records from the ganglion cells in the excised frog's eye show the same thing. Moreover, a similar effect is seen in the human electroretinogram (ERG), which is the earliest result of photolysis that can be recorded.

The records of Fig. 1 (Fuortes and Rushton, unpub.) are taken from the lateral eye of the arthropod Limulus, and they are obtained by leading from a micropipette inserted into one nerve cell in ail onmatidium. Fig. 1A shows the effect of light adaptation. First the ommatidium is exposed to a weak flash which depolarizes the cell and generates a train of impulses. Then a bright flash 4,000 times as strong is given, and finally the furst flash is repeated. The depolariration and impulse train is greatly diminished following the bright light. To what extent is the diminution due to the intense previous activity which the bright light had induced in the recorded cell? Fig. 


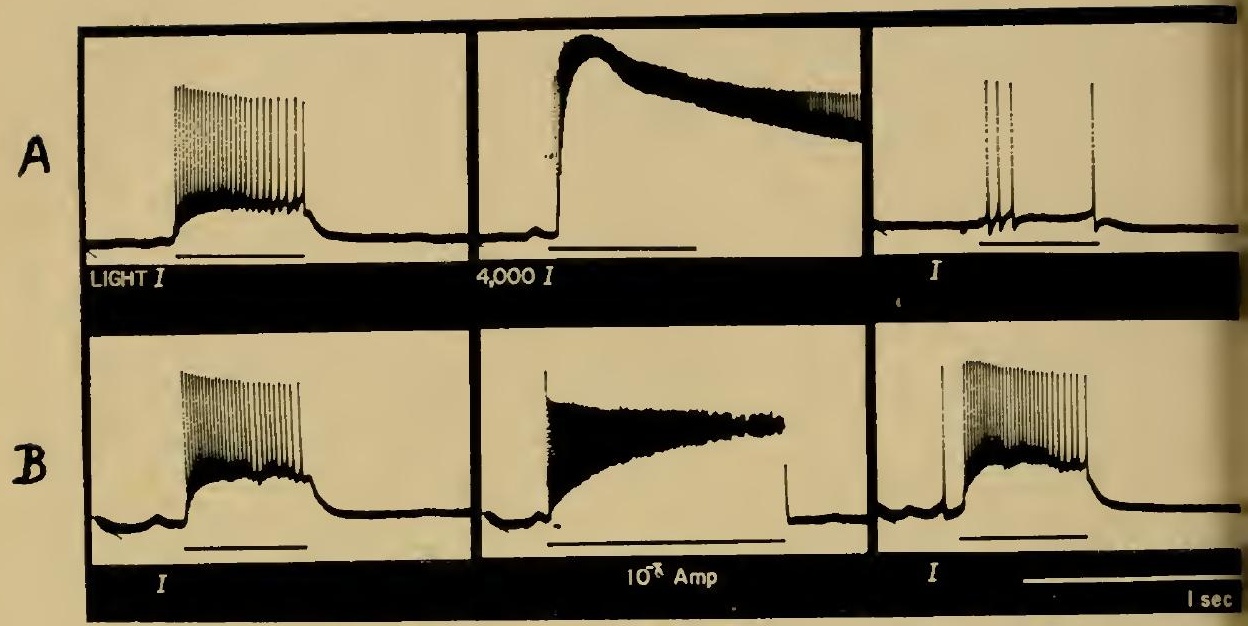

Fig. 1. Intracellular recording from a visual cell in Limulus. First and last record of each line, response of $0.5 \mathrm{sec}$ flash of light of intensity $I$. Middle record A, response of cell to bright light $4000 \mathrm{I}$ which leaves the cell insensitive to the next flash. Middle $B$ shows the discharge resulting from 1 sec of depolarization of $10-8$ amp, which does not change the sensitivity to the next flash.

1B show's a similar procedure (upon the same cell) but instead of using bright light, a strong depolarizing current was passed through the microelectrode (middle record). This lasted a second and caused a more massive discharge of impulses than did the bright light, but it had no effect upon the subsequent weak light flash. Thus light adaptation is not caused by the activity of the recorded cell. Something must have happened earlier in the train of events and altered the excitation of the cell caused by the next test flash. But how is the cell excited by the test fiash?

Fuortes (3) has measured the resistance of the cell membrane in conditions of various steady lights and steady currents passed through the microclectrode. The resistance was found to remain unchanged as a function of current but to diminish with increasing light intensity. It follows that light cannot act upon the cell by generating somewhere a current which then stimulates the cell directly, for this would not cause the fall in resistance which in fact light produces. Light clearly must stimulate by producing some agent which increases cell permeability-presumably some transmitter hormone. The effect of light adaptation is therefore either to reduce the amount of hormone produced by a given flash or to reduce the sensitivity of the cell to a fixed amount of hormone. So long as we can detect the 
homone only by its effect it is impossible to distinguish with certainty between these altematives, yet a very different kind of argument has some cogency.

It is now possible to measure the bleaching and regeneration of rod and cone pigments in the living retina of animals and man, and it is found that human cones take 8 minutes, rock 30 minutes, cat's rods 100 minutes, for the full regeneration of the pigment. But these intervals are just about the times for full return of dark adaptation. Thus the recovery of sensitivity in the dark appears closely connected with the regeneration of the visual pigment. Returning now to the question of the stimulation of the Limulus cell by transmitter hormone, it seems much more likely that during dark adaptation the hormone is increased in quantity rather than that the cell is increased in sensitivity, since the hormone is liberated in some way by photolysis and hence might well be coupled to pigment regeneration, whereas the cell sensitivity would be expected to have an entirely independent (and much faster) course of recovery.

\section{(c) The Response to Intensity}

Fuortes (3) found that the characteristic effect of light upon the Limulus cell was to lower the membrane resistance, and Fig. 2 shows the relation between the resistance change and $\log I$, where $I$ is the

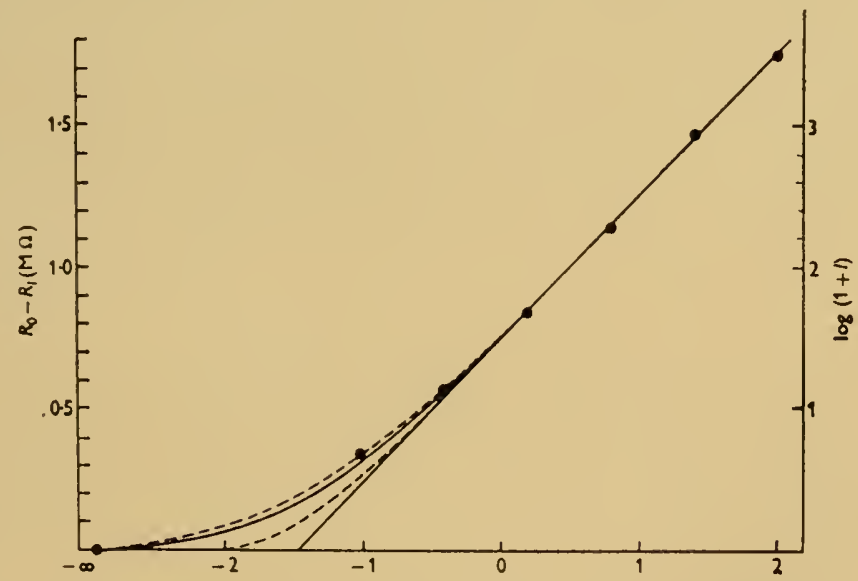

Fig. 2. Abscissae give log light intensity, ordinates (black circles) show corresponding membrane resistance (or membrane potential), of a single visual cell in limulus. The continuous curve shows function $\log \left(I / I_{D}+1\right)$. Upper interrupted curve is Stiles' log, increment threshold (man): lower is Donner and Rushton's light excitability function for the frog. 
light intensity in arbitrary units. Now the properties of the cell are such that the change in resistance is proportional to the change in membrane potential, and this in turn is a linear function of the frequency of impulses transmitted along the optic nerves in the steady state. So the relation to light shown in Fig. 2 is funclamental to the optic nerve message. It may be written:

$$
V-V_{D}=\log \left(\frac{I}{I_{D}}+1\right)
$$

where $V$ is the membrane potential in suitable units.

$V_{D}$ is the value of $V$ in the dark.

$I_{D}$ is the "dlark light" or intrinsic noise of the system. Differentiation gives:

$$
\Delta V=\Delta I /\left(I+I_{D}\right)
$$

Now Riggs and Graham (6) investigated the light increment $\triangle I$ required for a constant discharge with various background lights $I$, and found that $\triangle I /\left(I+I_{D}\right)$ was almost exactly constant over the whole range of intensities. Hence the condition for a constant discharge in these circumstances appears to be that $\triangle V$ should have a fixed value. From the careful and very extensive work of Hartline and his colleagues (mostly unpublished), it is clear that threshold responses in various changes of light are in general governed by a relation far more complex than $\Delta^{V}=$ constant. However, the simple increment threshold results of Riggs and Graham (6) seem to obey that relation, and so do the analogous measurements in man.

In psychophysical experiments the constancy of $\triangle I /\left(I+I_{1}\right)$ has been known for a century as the Weber-Fechner Law. Stiles $(9,10)$, plotting upon log scales, has shown that the relation

$$
\log \frac{\Delta I}{\Delta V}=\log \left(I+I_{D}\right)
$$

(where $\triangle V^{\prime}$ is constant) holds independendly for rods and several separate color mechanisms (upper dotted curve, Fig. 2). Donner and Rushton (2) also were led to equation (I) in order to explain the way in which the two receptors could replace each other in stimulation by light substitution (lower dotted curve, Fig. 2).

If, then, we agree that equation (2), derived in the first place from Limulus, is also the basis of intensity discrimination in vertebrates and in particular in man, we may approach the question of dark adaptation from a somewhat different aspect. For, the threshold in the 
dark is obtained from equation (2) by putting $I=0$, hence

$$
\triangle I=\triangle V \cdot I_{D}
$$

Thus the great rise in $\Delta I$ which occurs after light adaptation is clue either to a great increase in the nerve threshold $\triangle V$, or to an increase in $I_{D}$, the "dark light" of the system. This may be settled by experiment, as maly be seen by rewriting equation (2) in the form:

$$
\log \frac{\Delta I}{\Delta V \cdot I_{D}}=\log \left(\frac{I}{I_{D}}+1\right)
$$

The increment threshold curve may be obtained first in the darkadapted condition (giving the Stiles curve Fig. 2 when $\log \Delta I$ is plotted against $\log I)$. Now the measurements are repeated in a light-adapted state, and if $\triangle V$ alone is changed the curve of Fig. 2 will become shifted bodily upward by an amount equal to the change in $\log$ absolute threshold. But if $I_{D}$ is what alters, both scales will shift equally, so that the curve of Fig. 2 will move up as much as before but also equally to the right.

Published records of this experiment are disappointingly meagre and imprecise, though nearly all curves show some shift to the right. For me, however, the most convincing results are some umpublished experiments on my own cones. These were adapted to light so intense that their threshold rose 1,000 times, and the increment thresholds were nearly exactly consistent with $I_{D}$ alone changing. They diverged, however, by several 100 -fold from the expectation that only $\Delta V$ changed. And so, though further experiment is certainly needed, 1 shall assume here that $I_{D}$ alone changes in light and dark adaptation.

We thus conclude that a bright light, when extinguished, leaves the eye with an increase in the value of $I_{D}$, the intrinsic noise of the retina.

\section{The Photochemical Theory of Vision}

Hecht began his work, which was to have so immense an impact upon the science of vision, by the study of visual responses in invertebrates in the early 1920's, when it was fashionable to postulate unspecified chemical reactions to account for observed biological responses. The straightforward balanced reactions postulated for the withdrawal of the siphon of the clam Mya were found to simplify into equation (2), above, for the equilibrium condition under a steady light $I$, where $\triangle V$ now represents the amount of pigment photolysed by the flash $\triangle I$. We have seen that equation (2) describes 
the facts of increment threshold, and Hecht's theory gained immense prestige by giving so simply an accurate explanation of this relation. It is fundamentally different from the sort of explanation we have derived in our own electrophysiological approach.

According to the photochemical theory, a steady adaptation light $I$ bleaches the pigment to some fraction $1 / p$ of the initial concentration. It will now need $p$ times the original flash intensity to photolyse a fixed "threshold" amount of pigment. It follows that the pigment must be half bleached away by an intensity $I$ which causes $\triangle I$ to be twice the absolute threshold. It also follows that if $\triangle I$ is to be measured at this level, after taking a reading where $\triangle I$ was 20 times the absolute threshold, we must wait for the pigment to regenerate from 95 per cent bleached back to 50 per cent.

Neither of these conclusions is at all true. In increment threshold determinations we do not have to wait for the slow regeneration of pigment in changing from one level to another. To a first approximation, the relation of equation (2) is established instantly and remains unaffected by subsequent changes in the level of concentration of the visual pigment.

It is a remarkable thing that the chief proponents of the theory that rod function hangs upon the quantitative relations of rhodopsin bleaching appear neither to have themselves measured this rate of bleaching nor paid attention to the results of Dartnall, Goodeve, and Lythgoe (I), who did. Otherwise it would have been obvious that this goes on so slowly at a background field where $\triangle I$ is twice threshold, that even in the absence of any regeneration, the required bleaching of half the total rhodopsin would take 30 years!

The electrophysiological interpretation seems to be closer to the facts. Here the increment threshold depends upon a more or less instantaneous logarithmic transformation of the light intensity, and upon the nerve responding to a constant increment in the transformed signal. The nature of the transformation is certainly still obscure, but it is an observed fact in the Limulus eye, and even in the photosensitive ganglion of Aplysia.

There is an entirely different aspect of visual function which the photochemical theory has always related to the concentration of visual pigments. The raising of the absolute threshold as a result of cxposure to light, and its gradual fall subsequently in the dark, have long been considered to be due to bleaching and regeneration of the visual pigment. Until our own recent quantitative studies, the nature 
of this relation was never stated, nor does any evidence appear to have been offered to justify the faith of the hundreds who obtained dark adaptation curves and interpreted them as measuring in some unspecified manner the regeneration of visual pigments. Indeed, it is hard to see how the relation between the dark adaptation and the pigment levels in man could be investigated before a method became available for measuring pigments in the living eye. We have now developed such a method and have begun to investigate the relation, and it is satisfactory to be able to state that the faith of former workers was not misplaced, for clark adaptation turns out to be intimately related to the concentration of the visual pigment. What the relation is will appear in the sequel, but it is a pleasure at once to pay tribute to the brilliant intuition of Wald, who divined a formulation (Wald, Brown, and Smith, 14) that is justified by our measurements though contradicted by his own (13): Wald's contribution to this symposium, however, includes results which agree well with ours.

\section{(a) Measurement of Visual Pigments in Man}

The technique of retinal densitometry has been clescribed in detail elsewhere (Rushton 7,8 ). Here only the bare principle will be mentioned. Light from the headlights of a car reflected back from a cat's eye has passed twice through the cat's retina, and thus will have suffered absorption by the visual pigments there. When these are photolysed they become transparent (bleached), and the reflected light will be less absorbed. If the intensity of reflected light is measured on two occasions, and if the only change which has occurred between them is that the visual pigment present is more or less in amount on the second occasion, then the change in pigment density can be measured by the change in the logarithm of the reflected light. The human eye reflects only about $1 / 20,000$ of the in-going light, and various precautions must be taken on account of stray light, noise, and other unwanted fluctuations.

In practice, cone pigments may be measured upon the fovea, rod pigments $15^{\circ}$ away, with a proceclure which takes 5 to 7 seconds, bleaches less than 1 per cent, and gives a value for the fraction of total pigment present which is accurate to about 0.05 . The limit of the method is set by the steadiness with which the subject can maintain the position of eyc and head over the long periods which may be involved in a whole investigation. 


\section{(b) Relation of Increment Threshold to Visual Pigment}

Fig. 3 shows some measurements upon my own cones. The circles show the percentage of total pigment present at various times from the moment of shining upon the fovea a bright light of 60,000 trolands $(=6,000 \mathrm{~mL}$ through a $2 \mathrm{~mm}$ diameter pupil). The upper branch starts from the clark-adapted state, the lower from the completely bleached state produced by an intensely bright light kept shining until the moment of transition to $60,000 \mathrm{Td}$ at zero time. The change in pigment level is what would be expected from the fundamental kinetic equation (Rushton, 8)

$$
\frac{d x}{d t}=(1-x) I-x
$$

where $I$ is intensity in units of $40,000 \mathrm{Td}, x$ is the fraction of pigment bleached, and $t$ is time in units of 2 minutes.

The experiment was repeated, but now instead of measuring the

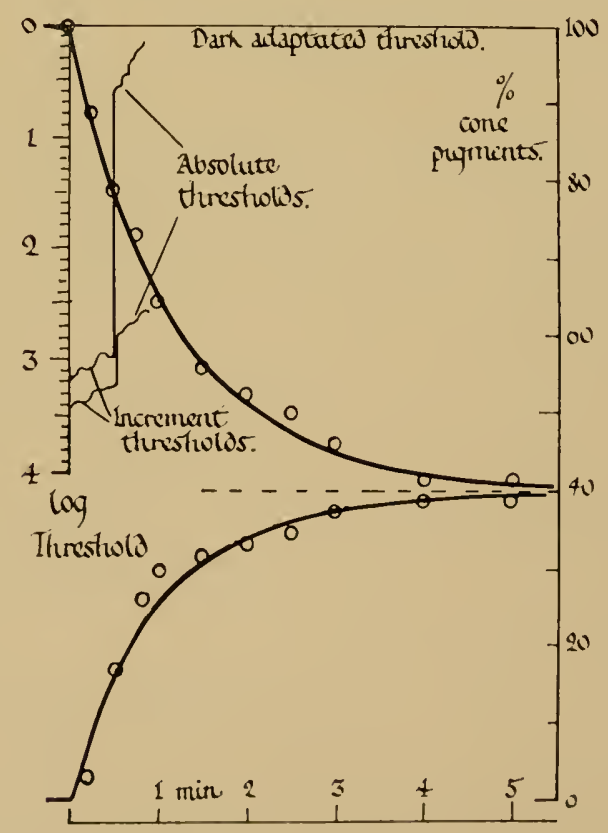

Fig. 3. White circles, right scale, percentage of cone pigment on human fovea when exposed to a light of 60,000 trolands, starting cither from darkness or from full bleaching. Tracings, left scale, log increment threshold superimposed upon 60,000 Td background for 0.5 min followed by absolute thresholds (zero background). Upper tracings start from dark, lower tracings from full bleach. 
pigment, the increment threshold was recorded. Superimposed upon the steady bright $60,000 \mathrm{Td}$ background was a small flashing light whose intensity adjusted so as to be just threshold. At the end of half a minute the $60,000 \mathrm{Td}$ was extinguished and the absolute threshold traced for the next minute or less.

These results are shown by the irregular tracings of Fig. 3. Starting from darkness, the increment threshold jumps to $3.2 \log$ units and falls back to an increment threshold of 0.6 upon extinction of the background field, the pigment being 20 per cent bleached at this moment. When, on the other hand, we start from the fully bleached state, though the increment threshold is hardly altered, the absolute threshold falls only to 2.8 units at $0.5 \mathrm{~min}$, when the pigment is still 80 per cent bleached. It is therefore plain that the absolute threshold depends very greatly upon the amount of pigment bleached, but the increment threshold very little. That, however, is precisely what would be expected from equation (2):

$$
\triangle I / \triangle \quad V=I+I_{D}
$$

For, by extracting the values of $I$ from the $\log I$ tracings of Fig. 3, we find that at $0.5 \mathrm{~min}$., when $20 \%$ of the pigment was bleached $I_{D}=4, I+I_{D}=1000$, and thus $I$ is about 1000 . On the other hand in the light-adapted trace at this moment when $80 \%$ of the pigment was bleached, $I_{D}=600$, hence $I+I_{D}$ should be 1600 and $\log$ $\left(I+I_{D}\right)$ at this point should read 3.2, as it does. Thus equation (2) predicts the 0.2 difference which is observed between the two log increment thresholds in Fig. 3.

\section{(c) Relation of Absolute Threshold to Visual Pigment}

The foregoing section makes it clear that it is not in the increment threshold but in the absolute threshold that we must look for a relation to pigment level. Is there a unique relation between the rod threshold and the level of rhodopsin at the moment, and if so, what is this relation?

Or on the contrary, do other factors intervene and in particular, does some nervous inhibition regulate the sharing of single nerve paths? For electrophysiological records from animals undoubtedly prove that rods and cones may use the same nerve, and many authors have invoked a rod-cone inhibition.

Fig. 4 is taken from an experiment by Fuortes and me and shows the time course of regeneration of rhodopsin in a $5^{\circ}$ area $15^{\circ}$ temporal to the fovea on Fuortes' left eye. The white circles give the 


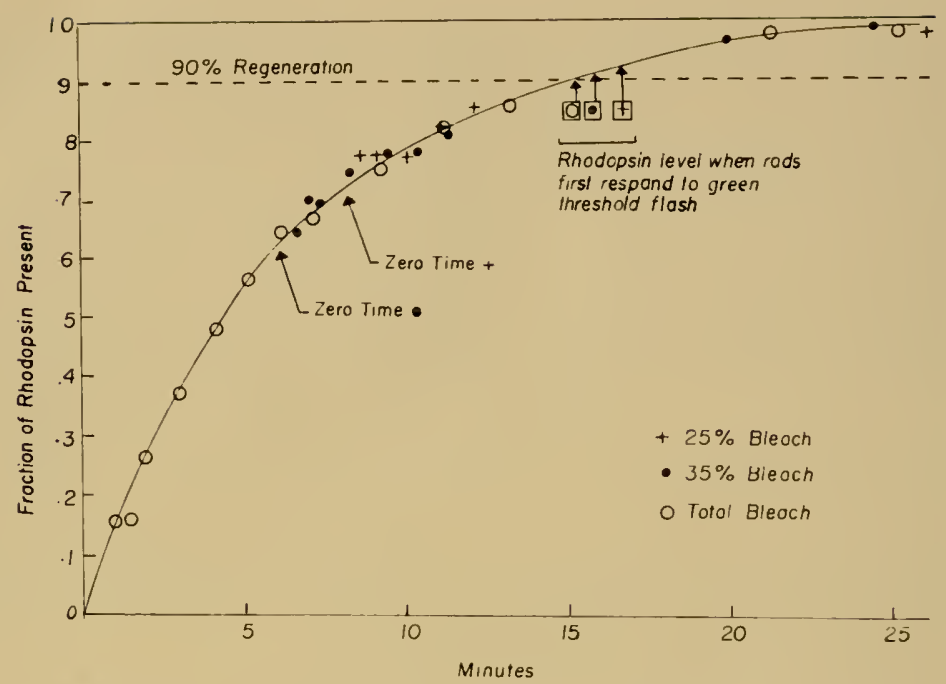

Fig. 4. Measurements of rhodopsin regeneration $15^{\circ}$ from fovea in man. Curves for $35 \%$ and $25 \%$ bleach slid to the right so that zero time comes to points indicated. Points when rods first appear in dark adaptation curve shown by vertical arrows.

result after a total bleach, black circles after $35 \%$ and crosses after $25 \%$ bleaching. The latter two sets of points have been slid to the right so that their zero times come at the points indicated, and demonstrate that the chemical course of regeneration is much the same from any level onward no matter what happened before this point.

When the pigment was seen to have regenerated to the $85 \%$ level, Fuortes shifted to a dark-adaptation apparatus and determined his threshold, using test flashes of yellow and of green (alternating each minute). These two lights lad been matched so that they were identical by rod vision, but the yellow was nearly 1 log unit brighter by cone vision.

When $85 \%$ of rhodopsin had regenerated the rods had not yet become more sensitive than the cones, for the yellow test light was found to be much the brighter. A few minutes saw the transition to rod vision, with equal efficacy for yellow and grecn. The moments when the green test reached this rod threshold curve are shown in Fig. 4. They correspond to about $90 \%$ rhodopsin regeneration in all cases. The whole question, then, of the relation of rod threshold to rhodopsin regencration is restricted to the final 10 per cent of the process, because carlier than this the rods are less sensitive than the cones. 
With a $10 \%$ range of pigment to investigate and an accuracy of measurement which at best is $3 \%$, the prospect of establishing any quantitative relation between pigment and threshold seemed small. But we were fortunate in obtaining the cooperation of an exceptionally suitable (incomplete) rod monochromat. She appeared to have nomal rhodopsin, no measurable foveal pigments, and no functional visual receptors except rods off the fovea, though she certainly had a few cones upon the fovea with which she could fixate.

Fig. 5 shows results of experiments by Fuortes, Gunkel, and me upon this subject. The black circles show the rhodopsin regeneration curve as compared with nornal (Fuortes, white circles). The irregular tracing is the dark adaptation curve traced by the subject on Gunkel's apparatus. In the absence of cones there is no early branch to hide the enormous rise in rod threshold, which in this tracing starts at 3,000,000 times the dark-adapted value. The lower part of this curve coincides with the rod portion of Fuortes's curve, obtained following a similar (total) bleach, and indicates that rod function appears at a given level at the same moment whether there are cones or not; hence cone inhibition plays no part in depressing rod function here, whatever may occur in other aspects of visual function.

The relation between threshold and pigment for the monochromat is sufficiently extended to be worth something. It is plotted in Fig. 6 and demonstrates the linear relation between pigment bleached and $\log$ threshold that Wald has long insisted upon, and which he has deduced as a consequence of his "compartment hypothesis" (13).

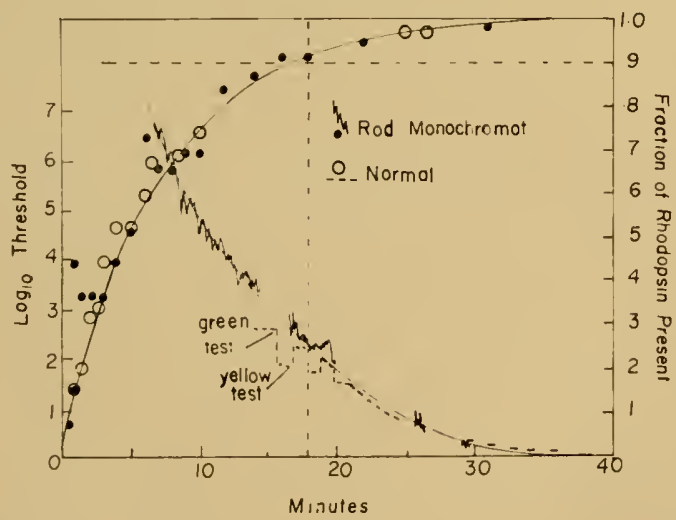

Fig. 5. Regeneration of rhodopsin in normal and rod monochromat (white and black circles, right scale). Dark adaptation curve traced by monochromat over $6.5 \log$ units (continuous irregular line, left scale). The dotcel line shows dark adaptation in the normal, changing from cones $(0)$ rods (green test) at $18 \mathrm{~min}$ and continuing along the monochromat's curve. 


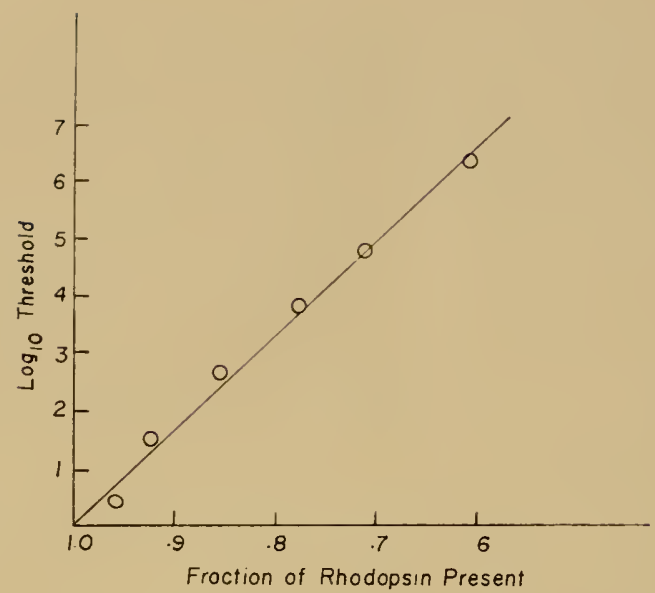

Fig. 6. Log threshold of a rod monochromat plotted against fraction of rhodopsin present.

\section{Retinal Noise and Excitation by Light}

We have seen that in light adaptation the absolute threshold rises greatly. It rises as an exponential function of the fraction $x$ of pigment bleached, and according to Fig. 6

$$
I_{D}=I_{0} \mathrm{e}^{40 x}
$$

where $I_{o}$ is the fully dark-adapted threshold.

There are two distinct ways of regarding the increase in $I_{D}$. According to one, the noise of the system has increased so that the unit of signal must be similarly increased in order to have the same significance. This is fomally equivalent to wasting signal, as when electric current is degraded into heat by shunting a galvanometer, so that it may operate at high signal strengths. A more efficient way of reducing the sensitivity of a galvanometer is by stiffening the suspension, so that no signal is lost, but sensitivity is exchanged for improved temporal resolution. Does the retina operate like this? When after light adaptation, the absohute threshold rises 100 -fold, are 99 per cent of the quanta wasted (as Wald suggests)? Or do they contribute to an increase in space-time resolution and to signal/noise reliability:

In Limulus Hartine has obtained results, which he has never published, relating the rise of threshold in light adaptation to the number of quanta used. The latter was deduced from the frequency of obtaining a response with lights of various intensities, as in the ex- 
periments of Hecht, Shlaer, and Pirenne (1). The results showed that when, as a result of light adaptation, the threshold rose 100 times, the number of quanta used also rose very greatly-perhaps 100 times. It is as though every quantum still produced an effect, but the effect was only 1 per cent of that in the dark-adapted state, so that 100 of them were needed to reach threshold.

Something of this kind is suggested by Fig. 7, which shows an intraccllular record from it cell in an ommatidium of Limulus (Fuortes and Rushton, umpub.). The three sections of this slow ink record ran consecutively. The upper line shows that in very weak constant light there is an irregular series of sudden slow potential changes, here enhanced in size by hyperpolarization of the cell. These slow pulses, first described by Yeandle (15), may be the responses to the absorption of single quanta, and may represent a contribution towards the potential $V$ upon which nerve excitation depends.
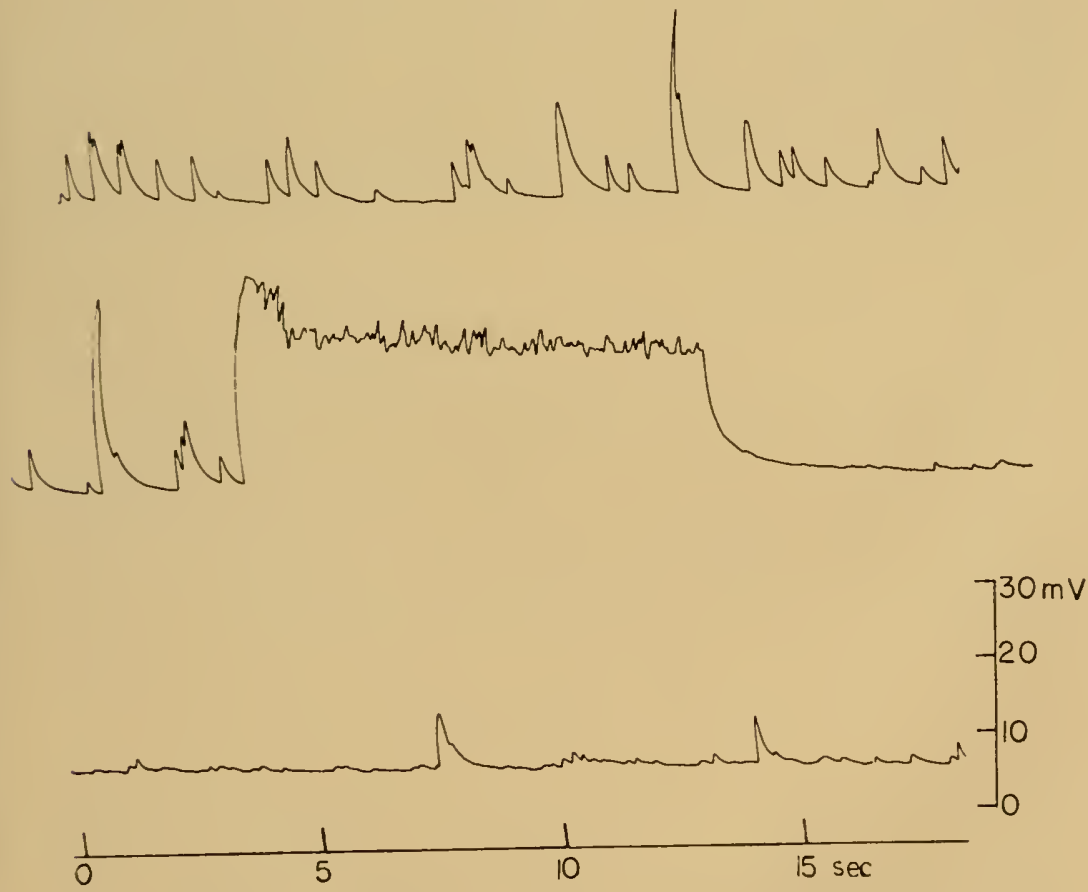

Fig. 7. Intracellular recording from visual cell of Limulus in full dark adaptation. Irregularities possibly indicating responses to single quanta. Stronger light exposed for $10 \mathrm{sec}$ in middle trace, leaving diminished responses. The three tracings follow continuously. 
During the second line a much brighter light was shone for 10 seconds, and the slow pulses are seen to be far more numerous and apparently smaller. And it could be argued that the steady potential change upon which these little pulses seem to rest is itself composed of the statistical sum of innumerable pulses. This interpretation is far from proven, but it is the sort of thing which could account for Hartline's results upon the probability of responding. The third line of Fig. 7 shows a return to the steady light of line 1, but now the preparation is light-adapted and the pulses are so small that (except for the giant pulses) they can hardly be seen.

Unless man is very much less efficient than Limulus, then, we must look for a mechanism of adaptation in which each quantum produces an effect, but in which the effect diminishes rapidly and exponentially with the fraction of pigment bleached, according to equation (4).

I do not think being confronted with this ancient arthropod should shame us into claiming a visual efficiency which we do not possess. I am not convinced that we see much better near absolute threshold with a light-adapted eye than we do with a dark-adapted eye whose threshold has been equally raised by interposing a neutral density filter. If this is so, we waste quanta after light adaptation, whatever Limulus may do. This question is not an easy one to settle but we badly need an answer.

\section{Excitation by Light}

We may now state the principal features which must be explained by any theory relating light absorption to nerve stimulation.

(a) A quantum absorbed by visual pigment almost anywhere in the rods and cones may produce an effect $0.1 \mathrm{~mm}$ away of a kind which will add to similar effects from other receptors and stimulate a nerve, and the effect occurs well within a second of the flash.

(b) Light of intensity $I$ produces a nerve signal $V$ such that

$$
V-V_{D}=\log \left(\frac{I}{I_{o}} \mathrm{e}^{-a x}+1\right)
$$

where $V_{D}$ is the value of $V$ in the dark, $I_{o}$ is the absolute clark-adapted threshold, $a$ is a constant which is about 4 for cones and 40 for rods, and $x$ is the fraction of visual pigment bleached at that time.

(c) There should be another statement relating not just the magnitude but the information content of the nerve signal to the quantun content of the light flash. But I do not know what this is. 


\section{REFERENCES}

1. Darmall. II. J. I., Coodere. C. F., and Lythgoe, R. J., Proc. Roy. Sor. London A. $164,216(1938)$.

2. 1)mucr. K. O.. and Rushtou, W. I. I1., I. Physiol., 149, 318,327 (1959).

3. Fuortes. M. G. 1., J. Physiol., 148, It (1959).

4. Hecht, S., Shlaer, S., and Pirenue, M. H., J. Cien. Physiol., 25, 819 (1912).

․ Koenig, A., S. B. Akad. Wissensch. Berlin, 577 (189.).

(6. Riggs, L. A., and Graham, C. H., J. Cellular Comp. Physiol., 26, 1 (1915).

7. Rushton, W. A. H., J. Physiol., 134, 11 (1956).

8. Rushton, W. A. H., Amm. N. Y. Acad. Sci., 74, 291 (1958).

9. Stiles, W. S., Documenta Ophthalmol., 3. 138 (1949).

10. Stiles, W. S., Proc. Nat. Acad. Sci. U. S., 45, 100 (1959).

11. Trendelenburg, W., Z. Psychol. Physiol. Simnesorg., 37, I (190)-

12. van der V'elden, H. A.. Physica, II, 179 (1914).

13. Wald, G., Science, 119. 887 (19.54).

14. Wald, G., Brown, P., and Smith, P., J. Gen. Physiol., 38, 623 (195.).

1.). Yeandle, S., Am. J. Ophthalmol., 46. (No. 3, pait 2), 82, (1958).

\section{DISCUSSION}

DR. WALD: I have a short and trivial question. What is $K$ in that last slide that you showed us?

Dr. Rushtox: What you mean is how many molecules are there in the compartment?

Dr. WALD: No, I want to know what exponent goes on intensity.

DR. Rushton: The experimental relation is $\log I_{d}=K x$, where $K$ is about 40 for rods, 4 for cones.

DR. WALD: That's the other way to look at it. That's all right.

Dr. FrANCK: I missed that point. Is the threshold you spoke of that of the rods or that of the cones?

DR. Rushtox: I was sometimes dealing with cones and sometimes with rods. To which experiment were you referring?

Dr. Franck: I want to know whether you can bleach very much of the dye present in the rods.

Dr. Rushton: Yes, we can bleach all the dye present in the rods and all the dye present in the cones. The curve starts from zero, because that was complete bleaching. However, if you press me why I know it is completely bleached, I can only say because I cannot bleach any more. That is not a very good answer, of course. If we leave the light on, we can apparently bleach everything in half a minute in bright light, for if we leave the lights on for a further 5 minutes, there is no substantial change. In half a minute we can bleach all that is bleachable.

Dr. Franck: All that can be bleached, but what percent is that of all the dye that is present? In the cones you probably bleach cverything. But is the structure in the rod such that you cam bleach everything? That is my question. 
Dr. Rushton: There is no way in which l can answer that without bleaching as far as I can and then cutting out the eye and extracting it. Or better yet, get Dr. Wald to do it, which would be a much more practical thing. But that hasn't been done. Can you answer this, Dr. Wald?

DR. WALD: I think it does almost entirely bleach. I think that when Dr. Rushton says that he has bleached it all or has bleached as much as he can, he probably bleached almost all of it. In any case, it can almost all be bleached, as Dowling has recently shown by the direct extraction and estimation of rhodopsin from the retinas of light-adapted rats (cf. Wald's paper in this volume).

Dr. Franck: I want to say why I asked this question. Could the structure of the rods and the cones be such that every part of the cones is in one way or another directly connected to the nerve, but in the rods the light is absorbed by the dye in the region where there is no nerve and then transmitted through the dye to the region of the nerve? In that case this would be similar to the photosynthetic unit in photosynthesis. In that case it would seem that only a small part of the dye would be involved in the photochemistry.

DR. RushtoN: I do not think that I have enough understanding of the molecular arrangement to make any comment which would be worth while.

DR. MacNichol: There is something which I do not understand. Is the reason that you do not get this nice simple relation between rod bleaching and sensitivity in the normal case due to some kind of neural interaction between the rods and cones?

Dr. Rushton: No, 1 think not. Dr. MacNichol asks, if 1 understand aright, why in the normal subject we cannot get evidence of rod activity early in the course of dark adaptation, and have to go to a rod monochromat to investigate it. It has often been suggested that cones inhibit rods, and if this were the reason that rods are not nomally apparent in the lightadapted state it is easy to see that a rod monochromat who lacks cones would also lack rod inhibition, and hence rod activity would be manifest. I believe that this explanation is false and that the true explanation is simpler, for if (a) there is no rod-cone interaction we may explain the results satisfactorily whereas if (b) cones inhibit rods we are led to expectations which are not fulfilled.

(a) Dark adaptation is a threshold measurement and hence will simply record the more excitable of two independent visual mechanisms. This at first is the cones, which do not saturate at high intensities and which recover much faster. The threshold of the rods, therefore, cannot be measured until it falls below that of the cones, which does not happen until about 18 minutes in the dark after a total bleach. In the rod monochromat, however, there are no cones to steal the threshold, and we obtain the full, umobstructed rod recovery curve, which as Fig. 5 shows, coincicles with the normal rod curve when that becomes visible after 18 minutes. 
(b) By a special terhique Iguilar and Stiles hate succeeded in measuring the increment theshold of nomal human rods att all luminance levels from dark adaptation to saturation. But this rod function is not likely to be alfected by conc inhibition beciuse it follows the simple Weber-fechner relation until near saturation. Horeover, our rod monochromat without cone inhibition exhibits an identical relation and saturates at the same level. And the "saturation" itself is not an inhibition by cones, for it has the rhodopsin spectral sensitivity. Thus rods, not cones, atch the light which sitturates. Does this answer your question?

1)R. MAC:Nichol.: Perfectly. 


\section{THE MOLECULAR ORGANIZATION OF VISUAL SYSTEMS}

\section{George WALD ${ }^{1}$}

Biological Laboratories of Harvard University, Cambridge, Massachusetts

Many of the fundamental properties of vision have their source in the physical and chemical properties of retinal molecules. In this paper I shall review briefly the present status of the chemistry of visual excitation, and then examine how this chemistry is reflected in visual performance.

\section{General Arrangements}

The general organization of visual systems in vertebrate eyes can be stated very simply. The outer segments of the visual receptors, the rods and cones, contain photosensitive pigments, each composed of vitamin A aldehyde (retinene) joined with a specific type of protein called an opsin. There are two vitamins $\mathrm{A}-\mathrm{A}_{1}$ and $\mathrm{A}_{2}$-differ-<smiles>CC(C=CC=C(C)CO)=CC=CC1=C(C)CCCC1(C)C</smiles>

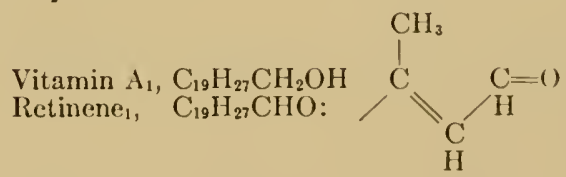<smiles>CC=CC(C)=CC=CC(C)=CC=CC(C)C</smiles><smiles>CC(C)=CC=O</smiles>

1 The experiments from our laboratory have been supported in pant with funds from the Rockefeller Foundation, the Office of Naval Research, and the Public Health Service. 
ing only in that the latter possesses an extra double bond in its $\beta$-ionone ring. From these by oxidation the corresponding aldehydes, retinene, $_{1}$ and retinenc, are derived, as shown in above structures.

There are also two main families of vertebrate opsins, one found in rods, the other in cones. The two retinenes join with the two kinds of opsin to yicld the four major pigments of vertebrate vision (44-47) :

Approx.

$\lambda_{\max }(\mathrm{m} \mu)$

Vitamin $A_{1} \stackrel{\text { DPN+ }}{\text { DPN.1I }}$ Retinene ${ }_{1} \begin{cases}+ \text { rod opsin } \stackrel{\text { light }}{\longrightarrow} \text { Rhodopsin } & 500 \\ + \text { cone opsin } \stackrel{\text { light }}{=} \text { Iodopsin } & 562\end{cases}$

(alcohol dehydrogenase)

Vitamin $A_{2} \underset{\text { DPN }}{\frac{\mathrm{DPN}^{+}}{\mathrm{DPN} \cdot \mathrm{H}}}$ Retinene, $\begin{cases}+ \text { rod opsin } \stackrel{\text { light }}{=} \text { Porphyropsin } & 522 \\ + \text { cone opsin } \stackrel{\text { light }}{\longrightarrow} \text { Cyanopsin } & 620\end{cases}$

Vitamin $\Lambda_{1}$, having been oxiclized to retinene ${ }_{1}$ by the enzyme alcohol dehydrogenase and DPN, combines with the opsin of the rods to yield the red pigment, rhodopsin, or with the opsin of the cones to yield the violet pigment, iodopsin. Similarly vitamin $A_{2}$, having been oxidized to retinene ${ }_{2}$ by the same enzyme system, joins with rod opsin to yield the purple photosensitive pigment, porphyropsin, or with cone opsin to yield the blue pigment, cyanopsin. On exposure of any of the photosensitive pigments to light, these reactions are reversed, ending in the production of a mixture of an opsin and a vitamin $A$.

All the invertebrate eyes so far examined contain similar lightsensitive pigments, formed by the combination of retinene ${ }_{1}$ with a variety of opsins (48). Vitamin $A_{2}$ and retinene ${ }_{2}$ have not yet been found in any invertebrate eye; nor as yet the alcohol dehydrogenase system that reduces retinene to vitamin $A$.

The operation of a typical vertebrate system, the rhodopsin system, is shown in more detail in Fig. 1 (27). On exposure to light rhodopsin yields a transient product, red or orange-red in color, called lumirhodopsin (54). This is the only photochemical reaction in visual excitation; all that follows consists of ordinary thermal ("dark") reactions. Lumirhodopsin is stable below about $-50^{\circ} \mathrm{C}$, but if warmed to about $-20^{\circ} \mathrm{C}$, goes over to a second intermediate, still orange-red in color, called metarhodopsin. This is stable below about $+15^{\circ} \mathrm{C}$, but if warmed above this temperature and in the presence of water, it hydrolyzes to a mixture of retinene and opsin. The 


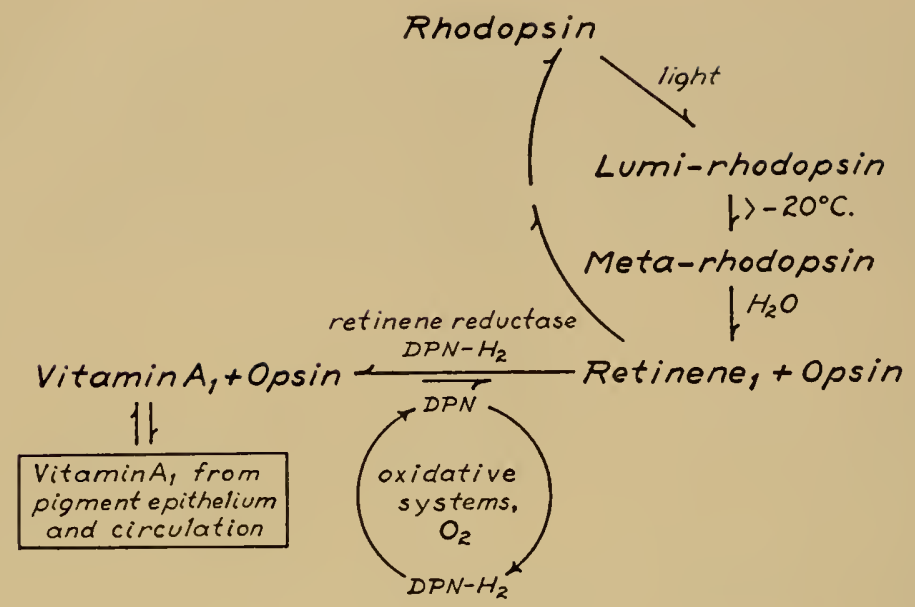

Fig. 1. Diagram of the rhodopsin system. (From Hubbard and Wald, 27).

retinene is then recluced by alcohol dehydrogenase and DPNH to vitamin $\mathrm{A}$.

The regeneration of rhodopsin depends primarily upon two reactions - the oxidation of vitamin A to retinene, and the combination of retinene with opsin-which are firmly coupled to each other (27). The equilibrium between vitamin $A$ and retinene- as between any alcohol and aldehyde in the alcohol dehydrogenase system-lies far over toward reduction, toward the alcohol (3). An appreciable oxidation occurs only in the presence of an aldehyde-trapping reagent, which displaces the equilibrium in the oxidative direction by removing the aldehyde as fast as formed. In the retina, opsin itself performs this function. The combination of retinene with opsin is a spontaneous, hence exergonic, reaction, which proceeds irreversibly in darkness, and requires no enzyne. By removing retinene to form rhodopsin, this reaction drives the endergonic oxidation of vitamin A. For this reason also, when all the opsin has been converted to rhodopsin, the oxidation of vitamin $A$ to retinene automatically ceases (27).

In the intact eye the regeneration of photopigment is aided further by two accessory processes: the migration of vitamin $A$ into the rods and cones from the pigment cpithelium, the adjoining tissue with which they lie in intimate contact; and the contimuous re-oxidation of DPN, the oxidant of vitamin $A$, by the respiratory enzymes of the retina and oxygen from the blood. Both processes help to drive 
the alcohol dehydrogenase system in the oxidative direction, speeding the formation of retinene, and hence of rhodopsin.

Exactly similar arrangencuts exist for all the other vertebrate systems. Each of the visual pigments bleaches over corresponding lumiand meta-pigments: and the alcohol deliydrogenase system and its coupling with photopigment synthesis present similar relationships in all vertebrate retinas.

\section{Cis-trans Isomerism}

To synthesize a visual pigment a specific configuration of vitamin $\mathrm{A}$ or retinene is needed. Vitamin $\mathrm{A}$ or $\mathrm{A}_{2}$ possesses four double bonds in its side-chain. These can occur in either trans or cis configuration, yielding an array of geometrical isomers (Fig. 2) .

The most stable and prevalent form of these molecules, the alltrans configuration, is not active in the synthesis of visual pigments. For this process, some cis configuration is needed (28).

We owe much of our knowledge of cis-trans isomerism in carotenoids to the penetrating studies of Zechmeister (55-57) and the associated theoretical exposition of Pauling $(33,57,34)$. Pauling had pointed out that in such an isoprenoid structure as here considered one should expect to find the cis configuration only in those double bonds adjacent to methyl groups, here numbered 9 and 13. At either of the other positions-7 or 11 -the attempt to go from the trans to the cis configuration would encounter internal steric hindrance ("molecular overcrowding") : a cis linkage at double bond 7 would bring the methyl groups on carbons 1 and 9 into conflict; and at double bond 11 , the hydrogen atom on carbon 10 would overlap with the methyl group on carbon 13 (Fig. 2). A cis linkage always introduces a bend in the chain; but at such a hindered cis linkage one should have not only a bend but a twist. The molecule could not lie flat-it could not be co-planar-and since this condition must be approximately fulfilled for resonance to occur, and resonance is one of the primary conditions of stability, one should expect that a molecule containing such a hindered cis linkage would be unstable, and hence would not be found in appreciable quantity. It was expected, therefore, that in such a molecule as vitamin $A$ one would find only the four relatively unhindered geometric isomers: all-trans, 9-cis, 13-cis, and 9, 13-dicis (Fig. 2).

Reasonable as this seemed, it has now become clear that all known visual pigments that have been analyzed contain as chromophore the sterically hindered 11 -cis isomer of retinene $e_{1}$ or retinene $e_{2}$, named 
<smiles>C=Cc1ccccc1C</smiles><smiles>CC=CC=CC=CC</smiles>

$\mathrm{H}$<smiles>C=CC=O</smiles>

all-trans Vitamin A

Retinene<smiles>CC1=C(/C=C/C=C/C(C)=C\CO)C(C)(C)CCCC1</smiles>

13 -cis (nen-a)<smiles>CC1=C(/C=C/C(C)=C/C=C/C(C)=C/CO)C(C)(C)CCC1</smiles>

$9-$ cis (iso-a)<smiles>[CH]C1CCC(C)=C(/C=C/C(C)=C\C=C\C(C)=C\CO)C1(C)C</smiles>

9,13-dicis (iso-b)<smiles>C=CC1=C(C)CCCC1(C)C</smiles>

$\mathrm{I}_{2}$<smiles>CC(C)=CC=CC=C(C)CO</smiles>

I1-ris (nen-b)

Fig. 2. Geometric isomers of vitamin 1 and retinene. I similar series of structures, differing only in possessing an extra double bond in the 3,4 position, represents vitamin $\Lambda_{2}$ and retinene ${ }_{2}$. The upper four structures represent the unhindered, and hence most probable configurations. The lowermost structure is the hindered cis isomer from which all known sisual pigments are derived. 
neo-b before its configuration was established by synthesis. This relationship has been demonstrated in both rod and cone pigments, constructed with vitamin $A$ or $A_{2}$, in vertebrate and invertebrate eyes (48) .

It is significant that one of the unhindered cis isomers of retinene also combines with opsin to form photosensitive pigments (28). This is the unhindered 9-cis isomer, which most resembles the 11-cis isomer in shape. 9-cis retinene, ${ }_{1}$ and retinene. combine with the opsins to form photosensitive pigments that greatly resemble those found naturally, but have never yet been extracted from any retina, and must as yet be regarded as artefacts. We call them the iso pigments -iso-rhodopsin, iso-porphyropsin, iso-iodopsin, and iso-cyanopsin.

The hindered 11-cis configuration of vitamin A or retinene is surprisingly stable, once formed. I think it is clear now that though Pauling's strictures were valid thermodynamically, the molecule escapes them through special kinetic circumstances. The hindered 11cis isomer represents a higher energy state than the unhindered isomers, and under true equilibrium conditions little of it should occur. It seems to be stabilized, however, by lying in a potential energy valley walled off by high potential barriers. It takes a high activation energy either to form the ll-cis isomer or to re-isomerize it, once formed. In our experience the molecule goes in either direction conveniently only as the result of absorbing light.

Under certain conditions the ll-cis isomer is indeed favored among the possible configurations of retinene. If a solution of all-trans retinene in ethyl alcohol is exposed to the light of an ordinary tungsten lamp, after about 10 minutes it has isomerized to a steady-state mixture of all possible isomers. About 25 per cent of this mixture is the hindered 1l-cis species, neo-b (6).

This being needed to make a visual pigment, what configuration of retinene emerges when a visual pigment is bleachecl? It turns out that the retinene produced by bleaching a visual pigment is inactive in its resynthesis. It is indeed the all-trans isomer. It must be reisomerized to the hindered 11-cis configuration before resynthesis can occur (28).

Fig. 3 shows one passage through such a cycle (49). On the left, a mixture of 11-cis retinene and cattle opsin combines to form rhodopsin. On the right, this rhodopsin is bleached back to retinene and opsin. To begin the experiment, a solution of cattle opsin in an absorption cell was placed in position in a Cary recording spectrophotometer. A solution of 11 -cis retinene in digitonin was added, 


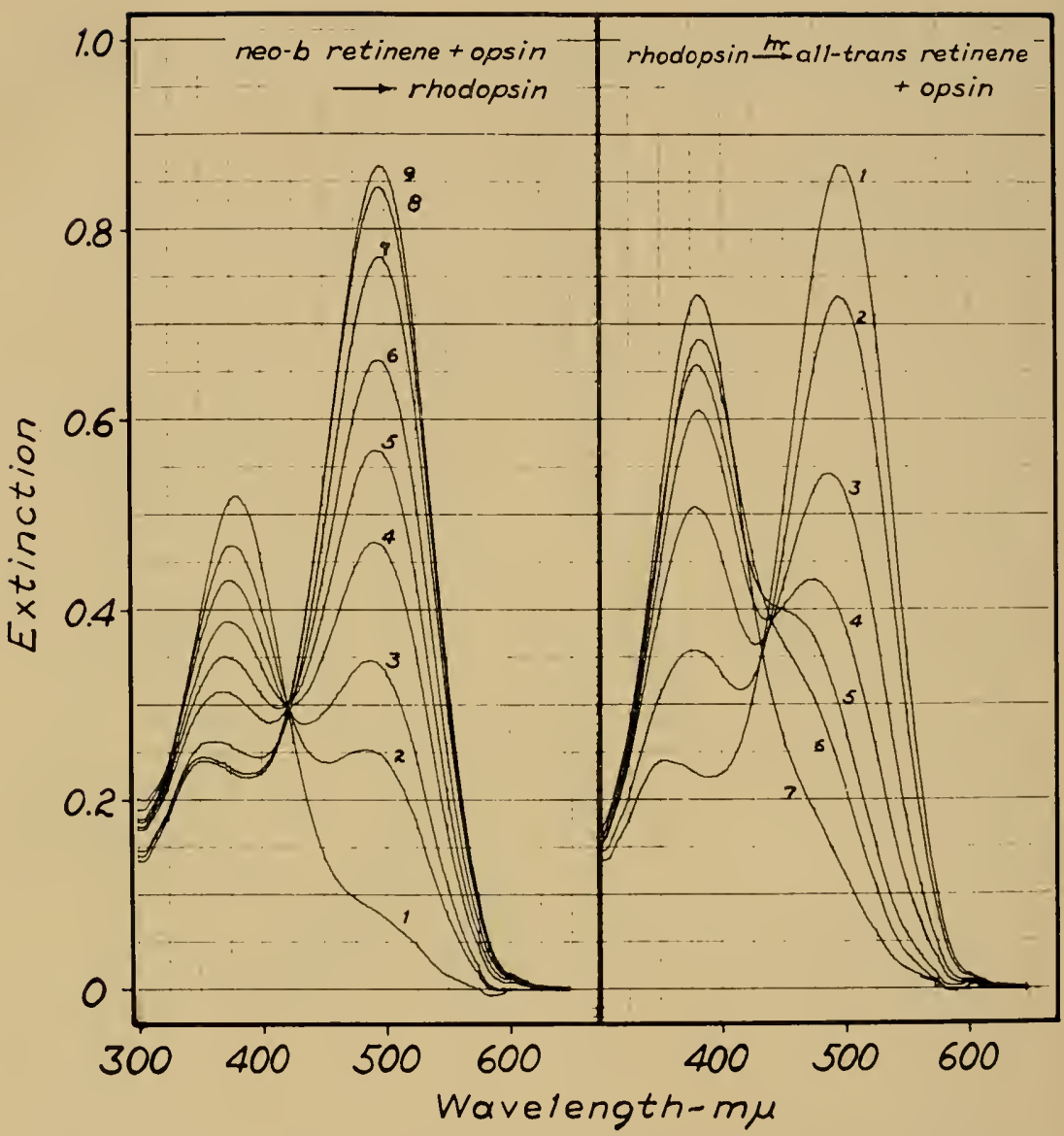

Fig. 3. Synthesis and bleaching of rhodopsin in solution, at 22.5 ${ }^{\circ} \mathrm{C}$. $p \mathrm{H} 7$. Lelt: a mixture of 11 -ris (nco-b) retinene and cattle opsin is incubated in the dark. and absorption spectra are recorded (1) at $0.3 \mathrm{min.,}$ (2) at 2.5 , (3) at 5 . (-1) at 10 , (5) at $18,(6)$ at $30,(7)$ at $60,(8)$ at 120 , and (9) at 180 minutes. The absorption band of 11 -cis retinene $\left(\lambda_{\max } 380 \mathrm{~m} \mu\right)$ falls regularly, as that of rhodopsin $\left(\lambda_{\max } 498 \mathrm{~m} \mu\right)$ rises. Right: the rhodopsin formerl at the left (I) is now exposed to light of wavelengths longer than $550 \mathrm{~m} \mu$ for various intervals, and the absorption spectra are recorded immediately afterwart. The total irradiations were: (2) 5 min., (3) 10, (4) 15, (5) 30, and (6) 120 seconds. The resirlue was exposed for 45 seconds longer to light of wavelengths longer than $4.10 \mathrm{~m} \mu$; then spectrum ( 7 ) was recorded. (From Wald and Brown, 19).

quickly stirred, and spectrum 1 immediately recorded. This shows the absorption band of neo-b retinene at abont $380 \mathrm{~m} \mu$, and a small inflection near $500 \mathrm{~m} \mu$, marking the first appearance of rhodopsin. This mixture was now left in darkness, in place in the spectrophotometer, 
and periodically its spectrum was remeasured. The retinene band declined continuously, that of rhodopsin simultaneously rising. At the end of 3 hours, only rhodopsin remained. We had purposely prepared this mixture with a small excess of opsin, and by this time it had completely removed all the 11 -cis retinene. The small band at about $350 m \mu$ in curve 9 is not the last of the retinene, but the $\beta$-band of rhodopsin.

Now curve 9 in Fig. 3 was redrawn at the right as curve 1 . The solution was exposed to orange light for 5 seconds, and the spectrum redrawn (2). Then it was exposed for 5 seconds longer, and the spectrum drawn again (3). By such repeated exposures the rhodopsin was bleached, and simultaneously retinene reformed. It looks, however, as though by this transaction we had made some retinene, for the extinction of retinene at the end of bleaching was about 1.5 times that with which we began. The reason for this is that, whereas the experiment began with 11-cis retinene, it ended with the all-trans isomer; and this has about 1.7 times the molar extinction of the hindered cis species.

The rhodopsin system, therefore, is arranged as follows (28):

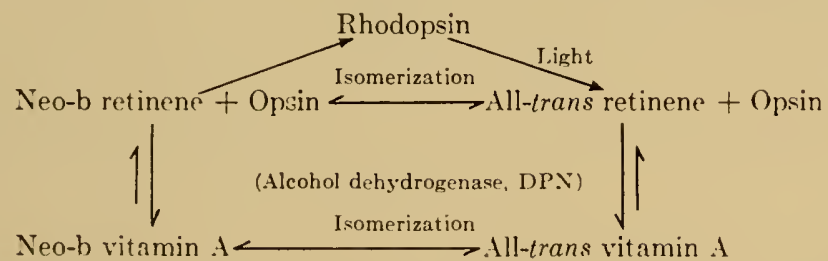

Rhodopsin on exposure to light bleaches over the orange intermediates aheady mentioned-lumi- and meta-rhodopsin-to a mixture of all-trans retinene and opsin. In order to regenerate photopigment, the all-trans retinene must be isomerized to the ll-cis configuration. While this is going on, however, much of it is reduced by alcohol dehydrogenase and DPNH to all-trans vitamin $A$; and this in turn must be isomerized to or exchanged for ll-cis vitamin A before it can reform rhodopsin.

A cycle of cis-trans isomerization is therefore an intrinsic component of the rhodopsin system. The same can be said of every other visual system that has so far been examined. In every case the visual pigment contains as chromophore the hindered 11-cis configuration of retinene or retinene ${ }_{2}$, and in every case the action of light on the visual pigment yiekds as product the all-trans configuration, which must be re-isomerized to ll-cis for resynthesis to occur. 
Much the same relationships involve the artificial isopigments, which have 9-cis retinene as chromophore. On bleaching with light, these too yield as product all-trans retinene, and again an isomerization would be needed for their resynthesis.

\section{Mechanism of bleaching.}

The recent work of Hubbard and Kropf has made it clear that these changes in the geometrical configuration of retinene represent more than a curious complication in the reactions of visual excitation. They are in fact the heart of this process. The only thing that light does in any visual system we know is to isomerize retinene. Everything else follows from this, by ordinary thermal ("dark") reactions $(21,25,26,30)$.

Retinene contains as its only reactive site the terminal aldehyde group $(-\mathrm{HC}=\mathrm{O})$. There is reasonably good evidence that in forming rhodopsin this condenses with an amino group of opsin in a Schiff base linkage: $\mathrm{C}_{19} \mathrm{H}_{27} \mathrm{HC}=0+\mathrm{H}_{2} \mathrm{~N}$-opsin $\rightarrow \mathrm{C}_{19} \mathrm{H}_{27} \mathrm{HC}=\mathrm{N}-$ opsin (1). Such a bond anchors it to the protein, but leaves unexplained why the geometric configuration of the hydrocarbon portion of the molecule is of such decisive importance. It is clear that what this means is that this shape of retinene is effective because it fits a corresponding portion of the surface of opsin. The point of fitting is that it permits a sufficiently intimate approach of the side-chain and ring of retinene to opsin to make possible strong interactions between them: the operation of van der Waals' forces and some degree of charge-displacement (21).

This interaction between 11-cis retinene and opsin is illustrated diagrammatically in Fig. 4 (26). At the left, we see rhodopsin, the aldehyde group of retinene having been joined to an amino group of opsin in a Schiff base linkage, the hydrocarbon portion of the retinene fitting closely the structure of opsin. Actually a Schiff base linkage, in itself, rather than producing the enormous shift in spectrum between retinene with its absorption maximum at about $380 m \mu$ and rhodopsin with $\lambda_{\max } 500 \mathrm{~m} \mu$, would have resulted in a loss of color. In order to account for this shift, it must be assumed that the Schiff base linkage is protonated (i.e., has attached an $\mathrm{H}^{+}$), so introducing a charge into the conjugated system which results in a large enhancement of spectrum and shift toward the red $\left(\mathrm{C}_{19} \mathrm{H}_{27} \mathrm{HC}=\mathrm{NH} \mathrm{H}^{+}\right.$. opsin). Even this, however, is inadequate, for such protonated Schiff bases of retinene ordinarily have $\lambda_{\max }$ about $440 \mathrm{~m}_{\mu}$. To bring the spectrum still further toward the red, it is assumed that a negative 


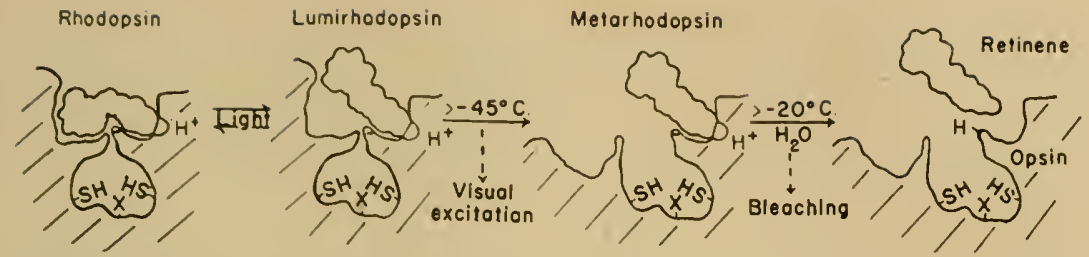

Fig. 4. The action of light on rhodopsin. The absorption of light by rhodopsin isomerizes its 11-cis chromophore to the all-trans configuration, yiclding as first product the all-trans chromoprotein lumi-rhodopsin. This labilizes the protein, upsin, which rearranges to a new configuration, yielding a second all-trans chromo. protein, metarhodopsin. This second process exposes reactive groups on opsin -two -SH gromps, and one proton-bincling group, symbolized in the figure with a negative charge-and may be responsible for triggering visual cxcitation. Vertebrate metarhodopsins are instable, and above about $-20^{\circ} \mathrm{C}$ hydrolyze to opsin and all-trans retinene, the process that corresponds to bleaching. (From Hubbard and kiropf, 26).

charge on opsin, in intimate association with the retinene side-chain, draws the positive charge up into the conjugated system, so greatly enhancing the resonance forms of the chromophore (21).

In forming the iso-pigments, the same mechanisms operate; but the fit between the retinene and opsin is less close than with the 11cis isomer, and the spectrum is correspondingly less enhanced. Not only do all the iso-pigments lie to the short-wavelength side of the corresponding visual pigments $\left(\triangle \lambda_{\max }\right.$ : $\operatorname{rod}$ pigments, $15 \mathrm{~m} \mu$; cone pigments, $50 \mathrm{~m} \mu$ ), but the special displacements of spectrum induced by cone as compared with rod opsin are much smaller (Table 1).

The absorption of a quantum of light by thodopsin or iso-rhodopsin isomerizes the retinene chromophore to the all-trans configuration. Now the retinene no longer fits the opsin; in consequence its attachment is unstable and it readily hydrolyzes off. This is the essential mechanism of bleaching (Fig. 4).

TABLE 1

ABSORP'TON MAXIMA $\left(\lambda_{\max }\right)$ OF THE VERTEBRATE VISUAL IIGNINTS AND ISO-PIGMENTS, AND THE DIFFERENCES BETI'EEN THEM ( $\Delta \lambda_{\max }$ )

\begin{tabular}{lccc} 
& \multicolumn{2}{c}{$\lambda_{\max }$} \\
\cline { 2 - 3 } & $\begin{array}{c}\text { Visual pigment } \\
(\mathrm{m} \mu)\end{array}$ & $\begin{array}{c}\text { Iso-pigment } \\
(\mathrm{m} \mu)\end{array}$ & $\begin{array}{c}\Delta \lambda_{\max } \\
(\mathrm{m} \mu)\end{array}$ \\
\hline Rhodopsin & 500 & 485 & 15 \\
Porphyropsin & 522 & 507 & 15 \\
Iodopsin & 562 & 510 & 52 \\
Cyanopsin & 620 & 575 & 45 \\
\hline
\end{tabular}


The first result of absorbing a quantum of light is to isomerize 11-cis to all-trans retinene, still in protonated Schiff base linkage to opsin. This is the transitory, still highly colored product, lumirhodopsin. The next step appears to involve changes in the configuration of opsin, including an opening of its structure, that exposes 2 to 3 sulfhydryl groups per molecule, as also one proton-binding group with $p K$ about 6.6. This is now metarhodopsin. Thereafter, given a high enough temperature and access to water, the Schiff base linkage hydrolyzes, and retinene and opsin result, the retinene in part free, in part unspecifically attached in Schiff base linkage to a variety of amino groups. It is this last reaction that constitutes bleaching.

In line with this explanation, it might be expected that any procedure that destroys the fit between retinene and opsin should bleach a visual pigment. So, for example, the denaturation of opsin, by changing its physical configuration, might have this effect, without necessarily involving the isomerization of retinene. This has proved to be true. Hubbard $(22,23)$ has shown that when rhodopsin and iso-rhodopsin are bleached by heating, about 70 per cent of the retinene emerges in the original 11- or 9-cis configuration. (The remainder has apparently been isomerized by warming in the presence of protein.) The process of thermal bleaching can be summarized as follows:

rhodopsin $\stackrel{\text { heat }}{\longrightarrow} 11$-cis retinene + denatured opsin iso-rhodopsin $\stackrel{\text { heat }}{\longrightarrow}$ 9-cis retinene + denatured opsin

The increased resonance associated with closeness of fit between the retinene and opsin is, as already said, a principal source of the stability of a visual pigment or iso-pigment. The iso-pigments, in which this fit is less close, are correspondingly less stable. Thus isorhodopsin is bleached by heat at a somewhat lower temperature, and with a slightly lower Arrhenius energy of activation than rhodopsin (23).

Visual excitation must depend upon the initial steps in such a sequence of changes as those shown in Fig. 4; that is, it must depend upon the formation of the lumi- or at most the meta-pigments (54). The hydrolysis to retinene and opsin is much too slow a process to account for excitation. Indeed, in a number of invertebrate eyes 
(squid, lobster), true bleaching hardly occurs under physiological conditions, the action of light on the visual pigments ending with the production of meta-rhodopsin (48).

It has already been said that simple exposure to light isomerizes free retinene, erentually to a steady-state mixture of all possible isomers; and that in a polar solvent about 25 per cent of this steady state mixture is 11-cis, about 25 per cent other cis isomers, and about half all-trans. Similar isomerizations can occur with retinene attached as chromophore to opsin $(25,26,30)$. If rhodopsin, for example, is irradiated at about $-60^{\circ} \mathrm{C}$, in a glycerol-water mixture, the first quantum absorbed by the molecule isomerizes 11 -cis retinene to alltrans, yielding lumirhodopsin; but the absorption of further quanta at this temperature continues to isomerize retinene, ending in such a steady-state mixture of isomers as just described. Since, however, in this case the retinene remains attached to unaltered opsin at the specific chromophoric site, the fraction of this mixture which is 11-cis is now again rhodopsin-rhodopsin made from lumirhodopsin through re-isomerization of the retinene by light. Another fraction, which is 9-cis, is now isorhodopsin, also made so by light. The remainder of the retinene is in configurations which do not serve as the chromophores of photopigments: all-trans, 13-cis, etc.; and this is the fraction-about half the total-that bleaches to retinene and opsin on warming in the dark. A mixture of rhodopsin and isorhodopsin constitutes the remaining half of the product.

A similar steady state is instituted when metarhodopsin, stabilized by holding below $-20^{\circ} \mathrm{C}$, is irradiated with visible light. The form of this steady state is shown in the following scheme, together with estimates of the quantum efficiencies $(\gamma)$ of the component photoreactions $(25,26,30)$. In this scheme the quantum yield of the isomerization from the 11-cis to the all-trans chromophore is set arbitrarily at 1 , and the others are rated accordingly.

$$
\begin{aligned}
& \text { Rhodopsin } \frac{\gamma=1}{\underset{\gamma=0.5}{-}} \text { Metarhodopsin } \frac{\gamma=0.09}{\underset{\gamma=0.3}{\longrightarrow}} \text { Isorhodopsin } \\
& \text { (11-cis) } \\
& \text { (all-trans) }
\end{aligned}
$$

When it was first found that about half the product of bleaching rhodopsin after irradiation in the cold is still in the form of photosensitive pigment, it was thought that the latter must result from some "dark" reversion process. We now realize that, on the contrary, this residue of rhodopsin and isorhodopsin is formed as de- 
scribed, by the light reaction. Similarly, it is sometimes stated on the basis of the observations just described, that the quantum efficiency of bleaching is only about $1 / 2$. In fact, the efficiency of the first quanta absorbed, which convert the visual pigment to the lumipigment-may be 1 . When the over-all efficiency of bleaching is less than 1 , it is largely because the absorption of subsequent quanta undoes to a clegree the work of the first quanta.

All these processes are summarized in the following diagram:

RE.ACTIONS INVOLVED IN BLE.ICHING; RHODOPSIN:

REACTION

1. photochemical stereol somerization of chromophore (reversiblo)

\section{2. thermal re- arrangement of opsin}

3. hydrolysis of chromophoro from opsin
PRODUCT

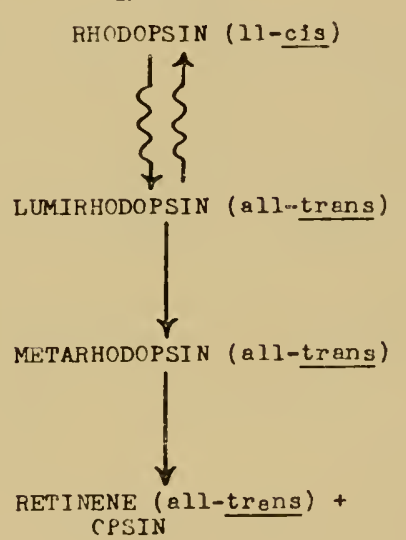

COLOR

red

red to violet

orenge to red yellow

It seems clear that-so far as we yet know-the only action of light on visual pigments or in vision is to isomerize retincue. It is not quite right to saly that light bleaches visual pigments; what it does is to isomerize retinene. Bleaching is only a secondlary consequence of the isomerization, and occurs only to the degree that the irradiation produces isomers that do not serve as the chromophores of photosensitive pigments.

\section{Carotenoids and Photoreception}

Some years ago 1 attempted to trace the widespread use of carotenoids in photoreceptor systems, beginning with the systems that govern phototropism in molds and higher plants, and ending with the role of the haplo-carotenoids (vitamins $A$, retinenes) in vision (41).

We have reached a point at which one may ask why the carotenoids have been selected for this role. I would suggest that it is because of 
their peculiar capacity for geometrical isomerization. All organic chromophores owe their color to the presence of conjugated systems of alternate single and double bonds. In all other types of natural pigment, however, the conjugated systems are largely condensed into rings, which hold the atoms rigidly in position, preventing isomerization. The carotenoids and haplocarotenoids are unique in possessing straight-chain conjugated systems, which can for this reason readily undergo extensive isomerization. One of the most general procedures for inducing isomerization is exposure to light. I think that it may be the unique capacity to undergo profound changes in shape when exposed to light that has led to the widespread selection of carotenoids for photoreception.

This thought can be carried a step further. Well-formed, imageresolving eyes are almost entirely restricted to three animal phyla: the molluscs, arthropods, and vertebrates. Each of these phyla has evolved its own characteristic types of eye, apparently in complete independence of the others. No commections, genetic, anatomical, or embryological, seem to link the eyes in any one of these phyla with those in the others.

It is highly significant, therefore, that in each case not only do the visual pigments that govern photoreception possess retinene $l$ or 2 as chromophore, but that in every case yet analyzed this is the 11-cis isomer. Why should three phyla independently have selected for vision such a fundamentally improbable, hindered cis configuration of retinene? I would suggest the reason that among all the geometric isomers of retinene, the ll-cis isomer possesses the highest photosensitivity, in the sense that it is photoisomerized to all-trans with the highest quantum efficiency, and hence at the highest intrinsic rate. The quantum efficiency for isomerization to all-trans is only about $1 / 3$ as great for the 9-cis isomer, the chromophore of the iso-pigments; and the isomerization of the 13-cis isomer, though rapid and effcient, leads to only a minor change of shape. It seems probable that its unique photosensitivity and large change of shape on isomerization are the factors that have led to the repeated selection of the 11-cis isomer for the construction of visual systems.

\section{The Problem of Visual Excitation}

Some component in the sequence of changes initiated in a visual pigment by the absorption of light accounts for visual excitation. Since, however, even in a cold-blooded animal the first electrical response to light follows the stimulus by a small fraction of a second, 
this leaves time for only the earliest steps in bleaching-the formation of the lumi- or at most the meta-pigment. One must attempt to understand what in these processes could result in a nervous impulse.

This would present a stubborn problem under any circumstances, for we do not yet understand the mechanism of any biological excitation, whether of nerve, muscle, sensory receptors, or eggs. In the eye, however, this problem takes a peculiarly stringent form, unique among all these objects, for experiments with the human eye have shown that a completely dark-adapted rod can be excited by the absorption of a single quantum of light $(19 ; 4,35,5)$.

Perhaps the simplest way to arrive at this conclusion is through such a computation as that in Table 2. This shows the numbers of quanta per second incident upon, and absorbed by the rhodopsin of a completely dark-adapted human rod at various levels of brightness, at $507 \mathrm{~m} \mu$, close to the wavelength of maximum sensitivity for rod vision. No allowance has been made in the last column for bleaching of the rhodopsin by the incident light; this would of course result in less absorption.

It should be understood that the threshold of the dark-adapted

TABLE 2

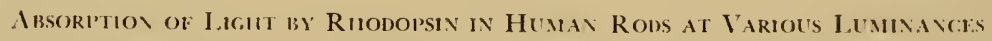

The first two columns show the flux density at the retina, corrected for the open. ing of the natural pupil (2), and allowing for an ocular transmission of 0.5 . Conrerted to quanta per sec. on the basis that for scotopic vision at $507 \mathrm{~m} \mu, 1$ lumen $=$ $5.72 \times 10^{-4}$ watt $=1.37 \times 10^{-4} \mathrm{cal} / \mathrm{sec}=1.47 \times 10^{15}$ quanta $/ \mathrm{sec}$. Cross-sectional arca of human rod outer segment, $2.54 \times 10^{-6} \mathrm{~mm}^{2}$. The outer segment contains about $4 \times 10^{7}$ molecules of rhodopsin when dark adapted, on the assimption that it absorbs about 30 per cent of incident light of wavelength $507 \mathrm{~m} \mu$ (36). In the last column. no allowance is made for the bleaching of rhodopsin, which at higher luminances would lower the numbers of quanta absorbed.

Flux Density at Rerina

\begin{tabular}{|c|c|c|c|c|}
\hline \multirow{2}{*}{$\begin{array}{c}\text { Field } \\
\text { lmminance } \\
\text { (millilamberts) }\end{array}$} & \multirow{2}{*}{$\begin{array}{l}\text { I.umens per } \\
\text { sq. mun }\end{array}$} & \multirow{2}{*}{$\begin{array}{c}\text { Quanta per } \\
\text { sec. per sq. } \mathrm{mm}\end{array}$} & \multicolumn{2}{|c|}{ Quanta per sec. per rod } \\
\hline & & & Incirlent & Ibsorbed \\
\hline 10$)^{-11}$ & $3.6 \times 10^{-13}$ & 510 & 0.0013 & 0.0004 .3 \\
\hline $10^{-5}$ & $3.5 \times 10^{-12}$ & 5000 & 0.0127 & 0.0043 \\
\hline $10^{-3}$ & $3.2 \times 10^{-10}$ & $4.6 \times 10^{5}$ & 1.17 & 0.39 \\
\hline $10^{-2}$ & $2.9 \times 10^{-0}$ & $4.18 \times 10^{n}$ & 10.6 & 3.6 \\
\hline 0.1 & $2.1 \times 10^{-8}$ & $3.1 \times 10^{7}$ & 87 & 29 \\
\hline 1.0 & $1.7 \times 10^{-7}$ & $2.38 \times 10^{4}$ & 605 & 212 \\
\hline 10 & $9.5 \times 10^{-7}$ & $1.4 \times 10^{8}$ & $3 \cdot 130$ & 1141 \\
\hline 100 & $4.8 \times 10^{-13}$ & $7 \times 10^{\circ}$ & $17,7.00$ & 5917 \\
\hline 1000 & $2.8 \times 10^{-5}$ & $1 \times 10^{10}$ & 103.000 & 34,334 \\
\hline
\end{tabular}


cye for visual fields of moderate size lies at about $10^{-6}$ millilamberts. It this luminance a single rod absorbs a quantum on the average about once in 38 minutes. At $10^{-5}$ millilamberts-about 10 times the dark-idapted threshold-a rod absorbs a quantum about once every 4 minutes: and even at $10^{-3}$ millilamberts, some 1000 times above the absolute threshold, a rod absorbs a quantum less than once in two scconds. It must be clear that if the clark-adapted rods did not serve as single quantum detectors, they could do nothing for 1ts, for above 10-2 millilamberts, when their chances of capturing quanta are improving, cones have already begun to play the primary role in vision.

A single quantum of light is absorbed by a single molecule of rhodopsin, and one must try to understand how so minuscule an event can have the relatively enormous consequence of exciting a rod. I have suggested two possible mechanisms that might enter this situation $(44,45)$.

One such mechanism rests on the realization that the membranes forming the transverse flattened sacs which constitute the outer segments of rods and cones (40) are composed in large part of visual pigment (42). For this reason the attack of light on a molecule of visual pigment might have the immediate effect of knocking a unimolecular hole in one such nembrane. One can imagine situations in which even so small a hole might have a large effect. It might result in a local depolarization, perhaps self-propagating as in other excitatory structures. Or if the sacs form a voltaic pile-essentially an electric organ-the puncture of one of them might result in a change of the generated potential sufficient to excite.

Whatever such mechanism is considered, clearly some large amplification over the initial effect is needed. Biochemically, such an amplification might be achieved through catalysis. If rhodopsin, for example, were a zymogen, a pro-enzyme, activated by the action of light, then it might catalyze the formation of many molecules of product in return for the absorption of a single photon. This would constitute one stage of amplification. If the product of this catalysis were a second enzyme, prepared to catalyze in turn a further reaction, that would constitute a second stage of amplification.

It is some encouragement to this suggestion to realize that all the examples we know of the activation of zymogens involve the breaking off from the inactive protein of a small fragment of its structure, one or a few amino acids in length, and so exposing the active catalytic center $(20,31)$. The process has been called "uncorking." 
This is what happens when pepsinogen is converted to pepsin, trypsinogen to trypsin, chymotrypsinogen to chymotrypsin. In each case the catalytic center on the zymogen molecule is covered by some small fragment of its structure. When this is removed, the enzyme can go to work.

One hardly need stress the close superficial resemblance between such a process and what we believe to be the mechanism of bleaching of a visual pigment (see Fig. 4). The intact pigment is "corked" by the close attachment of the retinene chromophore to opsin. The absorption of a photon, by opening up this attachment, may expose a catalytic center. That is, the visual pigments may in effect be zymogens, the opsins active enzymes.

If opsin were indeed an enzyme, what process may it catalyze? If we only knew what we would like to achieve in this regard, we might begin to look for it. Unfortunately, we do not yet know what is needed for an excitation, and hence where to begin to look.

Yet if either of these suggestions is relevant-whether the puncture of a membrane or the activation of an enzyme-we can be sure that either effect will persist for a time. The puncture will stay open, the enzyme will have a little time in which to act. For the retinene isomerized to all-trans by light must be re-isomerized back to the 11-cis configuration before it can recombine with opsin, bringing the action to an end. This may be the point of the isomerization cycle in vision.

\section{Photopigment Concentration and V'isual Sensitivity}

The processes that begin with the action of light on a visual pigment end in visual sensations. We do not try in general to measure visual sensations; what we usually do instead is measure the light, incident on the eye, needed to evoke a more or less constant visual response. Usually this is a threshold response of one kind or another. and the energy required to stimulate it is the energy threshold. The reciprocal of this quantity is taken as the measure of visual sensitivity.

Parallel with this external measure of sensitivity must go some internal measure, that characterizes the capacity of the visual system to respond to the stimulus. This is of course the independent variable; the amount of light required to see merely measures its status.

The visual pathways are sufficiently complex so that it might seem reasonable to suppose that endless possibilities exist for varying its sensitivity. To a degree, of course, this is so. Normally, however, the 
entire chain of processes that constitutes the visual system waits upon and hangs upon the initial events that goven excitation by light in the receptors, and in the first instance upon the absorption of light by a visual pigment.

I should like to defend the thesis that the visual sensitivity is governed primarily by the capacity of the visual pigments to absorb light, a capacity compounded from their absorption spectra and their concentrations. This is a view closely associated with the work of my former teacher, Selig Hecht, who developed and warmly supported it during his lifetime. I do not defend it on that account, but because I belicve it to be correct. It is also peculiarly important, for it takes us from wholly external dealings with a closed organism - the input of stimuli, the output of responses-to what goes on insicle, to the properties and reactions of its substances.

I have developed this view in some detail elsewhere, and should like only to review very briefly two aspects of it here: (a) that the spectral sensitivity of vision derives simply and quantitatively from the absorption spectra of the visual pigments; and (b) that the visual sensitivity and its changes in light and dark adaptation are a simple function of visual pigment concentration.

Spectral sensitivities and absorption spectra. In comparing these quantities it is best to measure the absorption spectra of the visual pigments in the retina itself or in suspensions of rods or cones, since under these conditions the spectra differ in characteristic ways from those measured in solution $(9,10,50)$. This is a significant distinction, for it involves the recognition that in the visual receptors the pigments are oriented in highly organized structures that are quasicrystalline, approximating the solid state. It must be recognized also that the spectral sensitivity with which we are concerned is that of the naked receptors, undistorted by whatever colored ocular structures may lie in front of or behind them, to screen or reflect back upon them.

When these considerations have been adequately resolved, the spectral sensitivities of the receptors appear to be virtually identical with the absorption spectra of their visual pigments. Some of the most satisfactory of such comparisons involve Granit's microelectrode measurements of rod and cone sensitivity in a variety of animals, as shown in Figs. 5 and 6.

It is clear from such measurements that the spectral sensitivity of vision, rod and cone, whether based upon vitamin $A_{1}$ or $A_{2}$, reflects simply and directly the absorption spectra of the associated 


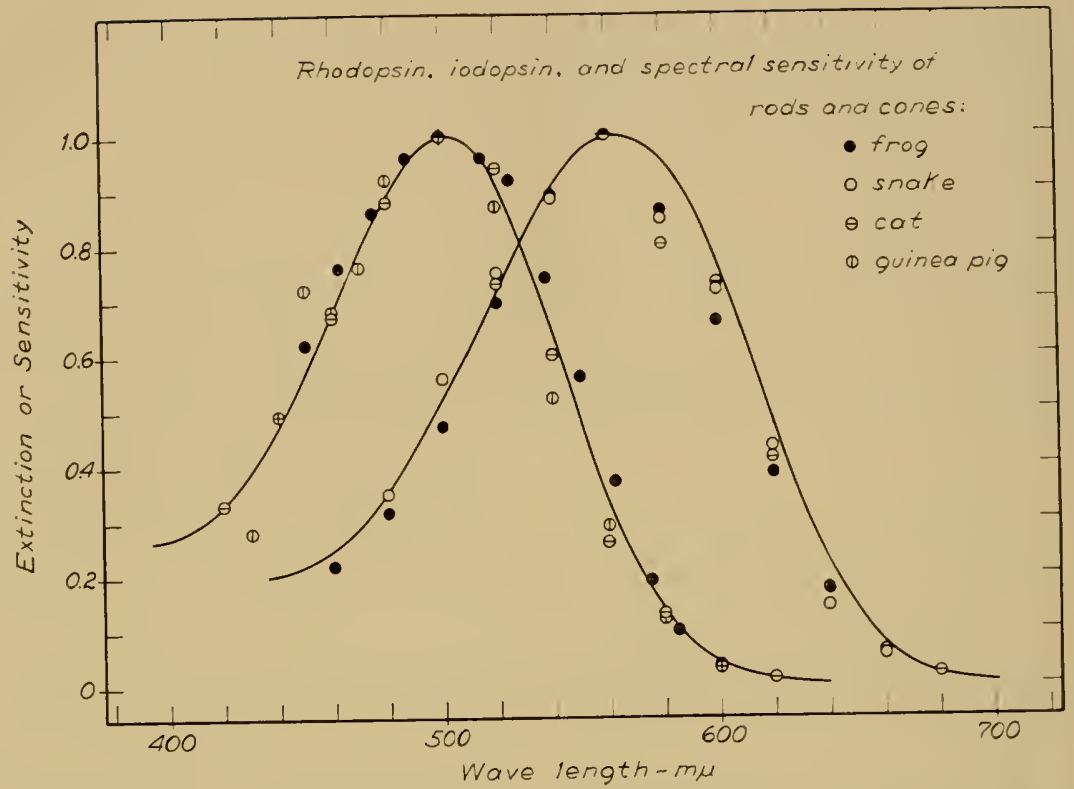

Fig. 5. The absorption spectra of chicken rhodopsin $\left(\lambda_{\max } 502 \mathrm{~ms} \mu\right.$ ) and iodopsin $\left(\lambda_{\max } 562 \mathrm{~m} \mu\right)$, compared with the rod and cone sensitivities of various animals. The lines show the absorption spectra of the visual pigments, the points electrophysiological measurements of spectral sensitivity (quantized). Rod data: frog (Granit, 1947, p. 292); cat (Donner and Granit, 1949); guinea pig (Granit, 1912b). Cone meastrements: frog (Granit, 1942a); snake (Granit, 1943a); cal (Granit, 1943b). (From Wald, Brown, and Sinith, 52).

visual pigments. By the same token the Purkinje phenomenon-the shift of spectral sensitivity toward the red in going from dim to bright light, from rod to cone vision-involves only the shift of vision from depending upon the absorption spectrum of a rod visual pigment in dim light, to depending upon the spectrum of a cone visual pigment in bright light.

Similar comparisons between the spectral sensitivity of human rod vision and the absorption spectrum of a human rod suspension (50); and between the spectral sensitivity of an invertebrate eye, that of Limulus, as measured by Graham and Hartline (16), with the absorption spectrum of its visual pigment (29), have proved equally satisfactory. I think it is safe to conclude that when the data are adequate, the spectral sensitivity of vision corresponds exactly with the absorption spectrum of the associated visual pigment.

This brings us to the second thesis, that the visual threshold, and hence its reciprocal the visual sensitivity, rises and falls during light 


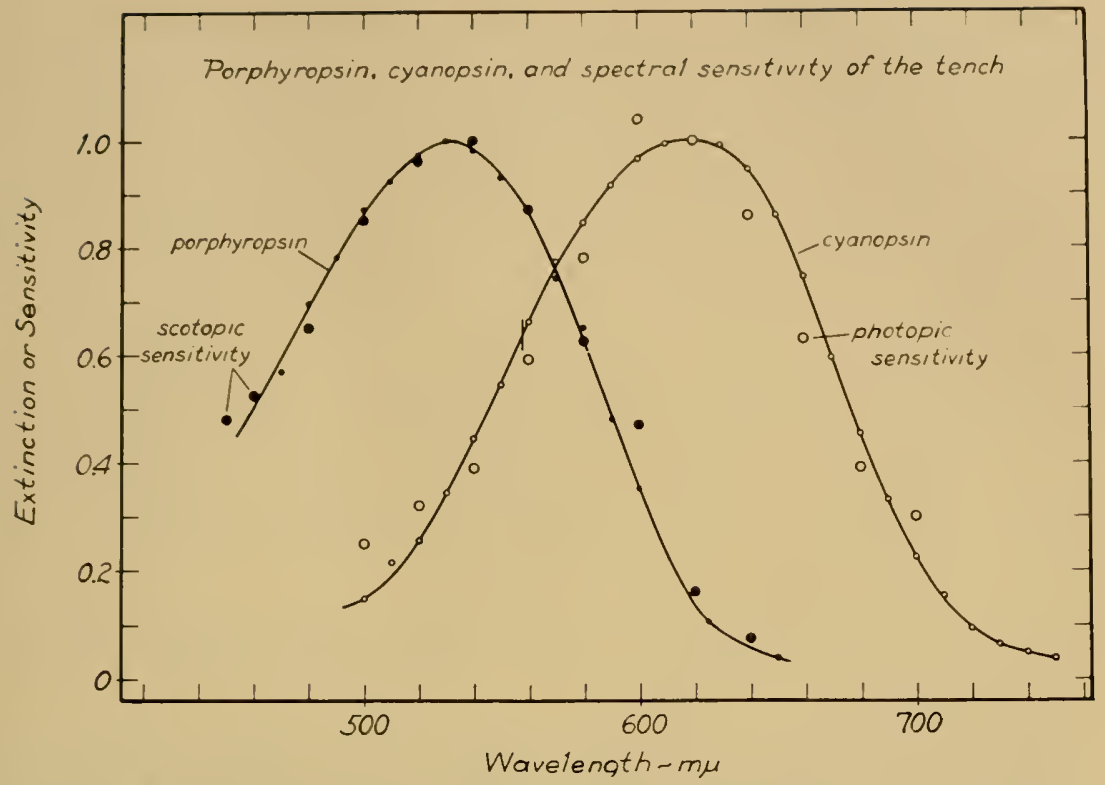

Fig. 6. Absorption spectra of porphyropsin and cyanopsin (lines, small circles) compared with the spectral sensitivities of rods and cones in a freshwater fish, the tench (large circles). The spectral sensitivities were measured electrophysiologically by Granit (1941). The absorption spectrum of tench porphyropsin is that measured by Crescitelli on a preparation from Dartnall (Crescitelli, 1958). The cyanopsin was prepared synthetically (Wald, Brown, and Smith, 52).

and dark adaptation as some simple function of the visual pigment concentration. For many years Hecht tried to infer the mathematical relationship between these quantities from sensory data alone, in the context of a priori considerations involving the physico-chemical arrangement of photoreceptor systems. He was never satisfied with the result, and after a time he stopped trying to fit theoretical curves to dark adaptation data.

I think that in recent years, as it became possible to measure the synthesis of visual pigments, first in vitro and then in vivo, it has become increasingly clear that the relation sought involves a parallelism between the logarithm of the visual sensitivity and the photopigment concentration (46). This inference was drawn first on the basis of roughly quantitative comparisons of the course of visual pigment synthesis in solution with the course of dark adaptation. So, for example, just as in duplex retinas the cones ordinarily dark adapt much more rapidly than the rods, so in extracts of chicken 
retinas the cone pigment ioclopsin was synthesized much more rapidly than the rod pigment rhodopsin (53). Frog rhodopsin is synthesized in solution relatively slowly, alligator rhodopsin with great speed; dark adaptation measured in the living animals at the same temperature was found to follow the same time relations (5l). In both cases the course of these changes resembled each other only when the logarithm of the sensitivity was compared with pigment concentration.

This argument derived strong support from the much closer comparisons made possible by Rushton's superb measurements of the rise and fall of visual pigment concentration in the living human eye, $(7,36-38)$ as related to the course of human light and dark adaptation $(46,47)$. Rushton presents a particularly handsome comparison of this type in the present volume.

I should like here to review briefly some recent measurements by Dowling in our laboratory, that I think approach this problem unequivocally $(14,15,12)$.

Normal white rats were light adapted by exposure to brilliant white light, and the recovery of both the visual threshold and the retinal content of rhodopsin were measured during subsequent dark adaptation, the threshold by means of the electroretinogram (ERG), the pigment concentration by direct extraction. In each such experiment, 5 animals, weighing about $150 \mathrm{~g}$, were light adapted for 30 minutes. One rat was anesthetized lightly with nembutal 5 minutes before the adapting light was shut off. Other animals were anesthetized periodically thereafter in the dark, each one 5 minutes before beginning the recording of electroretinograms. Thus the first animal was ready for measurement immediately on shutting off the adapting light, the others at various intervals thereafter. In all, 8 such series of measurments, complete or partial, were performed.

Fig. 7 show's the results of two such series. Light adaptation caused a rise of visual threshold of about $3.5 \mathrm{log}$ units, or about 3000 times. Afterward in darkness the threshold fell regularly, reaching the minimum level in about 2 hours. This is considerably slower than dark adaptation in man, which is completed to this degree in about 45 minutes.

On the following day, once with the same group of animals, once with a fresh batch, the same proceclure was repeated, but this time instead of measuring the ERG of the anesthetized animal, it was beheaded to yield a pair of retinas for the cxtraction and measurement of rhodopsin. 


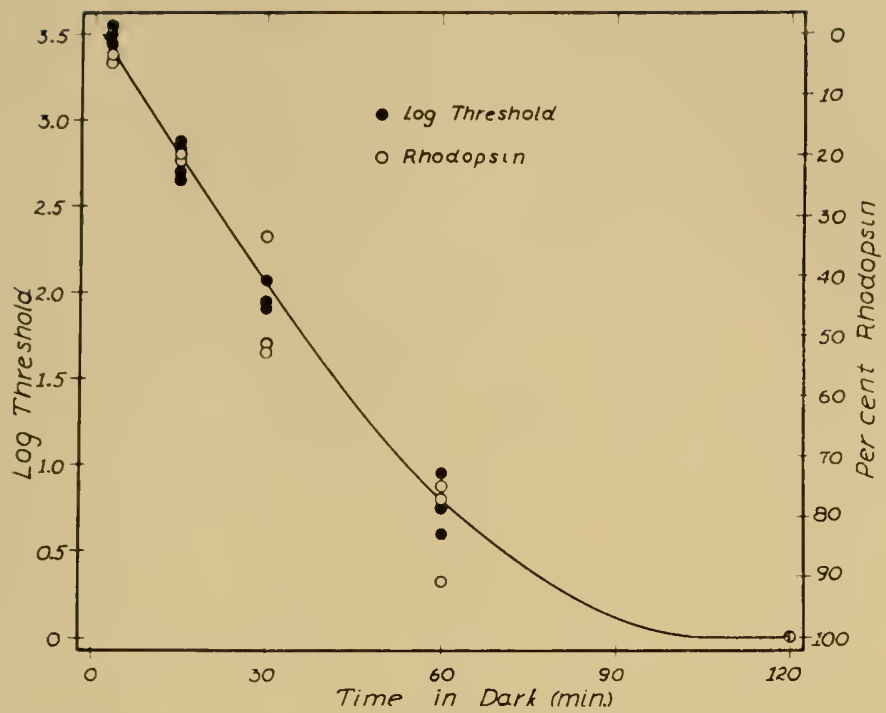

Fig. 7. Dark adlaptation in the nommal rat. Following a high light adaptation, which raised the ERG, threshold about $3.5 \mathrm{log}$ units (about 3000 times), the animals were left in clarkness. Periodically the visual (ERG) threshold was measured in one of them, or one was sacrificed to determine the rhodopsin content of the retina. During dark adaptation the $\log$ threshold (left) falls parallel with the rise of rhodopsin concentration (right). Both changes are completed in about 100 minutes (12, 14). (From Dowling).

Fig. 7 shows these results also. It is apparent that during dark adaptation, the logarithm of the threshold falls-i.e., the $\log$ sensitivity rises-in strict parallelism with the rise of rhodopsin concentration.

Fig. 8 shows the resultant, approximately linear relationship between the rhodopsin concentration and $\log$ threshold. It does so also for another kind of situation. When rats are deprived of vitamin A, the first deficiency symptom that appears is night-blindness, caused by the inability to form normal amounts of rhodopsin. In this condition the visual threshold rises, just as it does in light adaptation; and one can ask again what relationship obtains between the rise of threshold and the rhodopsin concentration. Fig. 8 shows that here again the relationship is approximately linear, and is indeed identical with that observed during dark adaptation in a normal animal.

Within the limitations of these measurements, which cover the range of rhodopsin concentrations from the maximum found in the normal dark-adapted animal (here 100) to about 5 per cent of this 


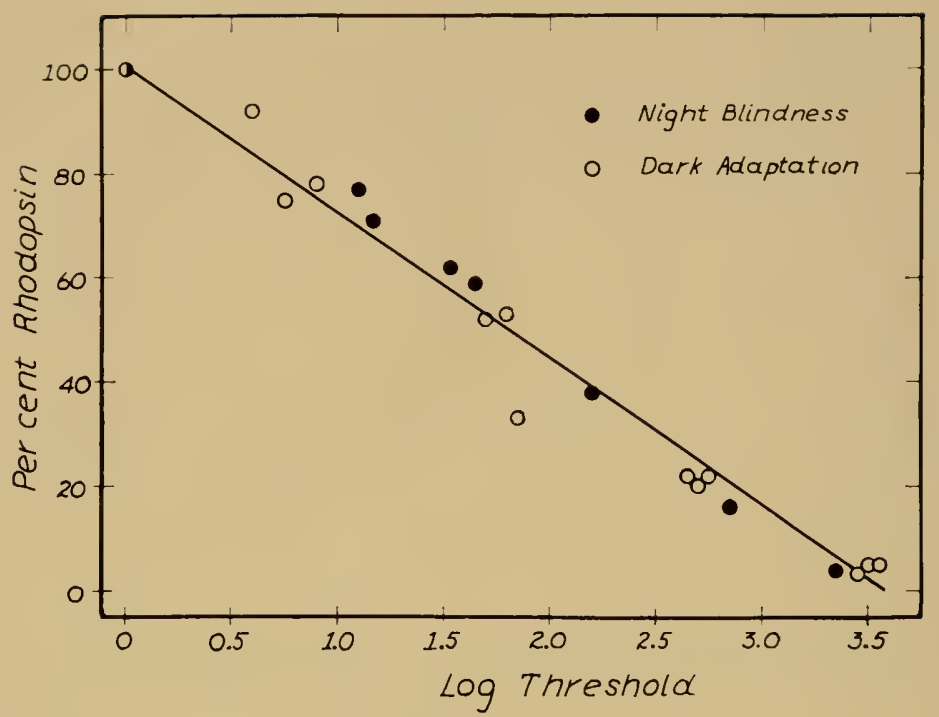

Fig. 8. Relation between the rhodopsin content of the rat retina and the logarithm of the vistal (ERG) threshold, in animals night-blind owing to vitamin A deficiency, and in normal animals during dark adaptation. The relationship is virtually identical in both cases, the $\log$ threshold or $\log$ sensitivity varying linearly with the retinal content of rhodopsin (14, 15, 12).

amount, the relationship between visual threshold and rhodopsin concentration is described reasonably well by the simple equation $(15)$ :

$$
1-\frac{R_{t}}{R_{o}}=0.28 \log \frac{I_{t}}{I_{o}}
$$

in which $I_{0}$ and $R_{0}$ are respectively the threshold and rhodopsin concentration in normal, dark-adapted animals, and $I_{t}$ and $R_{t}$ are respectively the thresholds and rhodopsin concentrations in vitamin A-deficient or partly dark-adapted animals.

This is satisfactory so far as it goes, yet clearly not the end of the story. So, for example, as the rhodopsin concentration approaches zero, one might expect the visual threshold to rise toward infinity. According to this equation, however, it rises only toward a limiting value $3.6 \log$ units above the nomal dark-adapted threshold (i.e., $\left.\log I_{n}+3.6\right)$. Dowling's measurements of rhodopsin concentration in these retinas never reached below about 5 per cent of the maximal valucs. It seems probable that at still lower concentrations, what we drew as a straight line should become convex to the origin, so as to approach infinite threshold at zero rhodopsin concentration. 
A similar adjustment may be needed in the region of 100 per cent rhodopsin. Rushton and Cohen (39) concluded on theoretical grounds, and I confirmed with direct measurements (42) that in the human eye the bleaching of the first few per cent of rhodopsin must cause a disproportionately large rise of threshold. Indeed, my measurements seemed to show that the bleaching of the first 0.6 per cent of rhodopsin raised the threshold 3300 times (42). To be sure, that was human light adaptation, and might not involve to this degree the different phenomena in another animal, shown in Fig. 8. It is possible also that the relationship between threshold and concentration is different, in the neighborhood of 100 per cent rhodopsin, for light and clark adaptation; that the bleaching and regeneration of rhodopsin involve a degree of hysteresis. In any case, any such exaggerated change of threshold with pigment concentration in this region should bend the line of Fig. 8 so as to make it concave to the origin at the upper left.

If both these changes were pertinent, the relationship shown in Fig. 8 would be changed from a straight line to "a flattened reversed $S$," approximately linear only over its middle region. That may incleed be the true condition. It is significant that as the straight line is drawn in Fig. 8, almost all the points to the left lie above it, while almost all those to the right lie below it; a flat S-shaped curve would fit the data better than the straight line that is shown. Only further measurements will decide finally between these alternatives.

\section{REFERENCES}

1. Ball, S., Collims, J. D., Dalvi, P. D., and Morton, R. A., Biochem. J., 45, 304 (1949).

2. Blanchard, J., Phys. Rer., Ser. 2, 11, 81 (1918).

3. Bliss, A. F., Arch. Biochem. and Biophys., 31, 197 (1951).

4. Bouman, M. A., Documents Ophthalmol., 4, 25 (19:50).

5. Brindley, G. S., Physiology of the Retiua and J'isual Patha'ay, Chap. I, Arnold, London (1960).

6. Brown, P. K., and Wald, G., J. Biol. Chem., 222, 865 (1956).

7. Camplell, F. W., and Rushton, W. A. H., J. Physiol., 130, 131 (1955).

8. Crescitelli, F., in Photobiology, Proc. 19th Biology Colloquimm, Oregon State College, p. 30 (1958).

9. Denton, E. J.. in V'isual Problems of Colour. Natl. Phỵs. Lab. Symp. 8, H. M. Stationery Office, London (1958).

10. Denton, E. J., Proc. Roy Soc. (London), B. 150, 78 (19:59).

11. Donner, K. O., and Granil, R., Acta Physiol. Scand., 17, 161 (1949).

12. Dowling, J. E., Nature, 1960, in press.

13. Dowling, J. E., and Wald. G., Proc. Nall. Acad. Sci. U. S., 44, 648 (19.58). 
14. Dowling. J. E., and Wald, G., in Titamin Metabolism, Pp. 185-197, Proc. IV Int. Congr. Biochemistry (19.59).

1.). Dowling, J. E., and Wald, C.., Proc. Natl. Acad. Sci. U. S., 46, 587 (1960).

16. Graham, C. H., and Hartline, H. K., J. Gen. Physiol., 18, 917 (1934-35).

17. Granit, R., Acta Physiol. Scand., 2, 334 (1941); Acta Physiol. Scand., 3, 137 (1942a); 318 (1942b); Acta Plysiol. Scand., 5, 108 (1943a); 219 (1943b).

18. Granit, R., Sensory Mechanisms of the Retina, Oxford University l'ress, London (1947).

19. Hecht, S., Shlaer, S., and Piremne. M. H., J. (ien. Physiol., 25, 819 (1942).

20. Herriott, R. M., in The Niechanism of Enzyme Action (W. D. McElroy and B. Glass, eds.), p. 24, Johns Hopkins Press, Baltimore (1954).

21. Hubbard, R., in I'isual Problems of Colour. Natl. Phys. Lab., Symposium 8, H. M. Stationery Office, London (19:58).

22. Hubbard, R., Nature, 181, 1126 (19.58).

23. Hubbard, R., J. Gen. Physiol., 42, 259 (1958-59).

24. Hubbard, R., Brown, P. K., and Kropf, A., Nature, 183, 442 (1959).

25. Hubbard, R., and Kropf, A., Proc. Natl. Acad. Sci. U. S., 44, 130 (1958).

26. Hubbard, R., and Kropf, A., Ann. N. Y. Acad. Sci., 81, 388 (1959).

27. Hubbard, R., and Wald, C., Proc. Natl. Acad. Sci. U. S., 37, 69 (19.51).

28. Hubbard, R., and Wald, G., J. Gen. Physiol., 36, 269 (1952-53).

29. Hubbard, R., and Wald, G., Nature, 186, 212 (1960).

30. Kropf, A., and Hubbard, R., Ann. N. Y'. Acad. Sci., 74, 266 (19.58).

31. Neurath, H., Gladner, J., and Davie, E., in The Mechanism of Enzyme Action (W. D. McElroy and B. Glass, eds.), p. 50, Johns Hopkins Press, Baltimore (1954).

32. Oroshnik, W., Brown, P. K., Hubbard, R., and Wald, C.. Proc. Natl. Acad. Sci. U. S., 42, 578 (1956).

33. Pauling, L., Fortschr. Chemie org. Naturstoffe, 3, 203 (1939).

34. Pauling, L., Helv. Chim. Acta, 32, 2241 (1949).

35. Pirenne, M. H., Biol. Rer's., 31, 194 (1956).

36. Rushton, W. A. H., J. Physiol., 134, 30 (1956).

37. Rushton, W. A. H., Nature, 179, 571 (1957).

38. Rushton, W. A. H., Camplocll, F. W.. Hagins, W. I., and Brindley, C, S., Optica Acta, 1, 183 (1955).

39. Rushton, W. A. H., and Cohen, R. D., Nature, 173. 30I (1951).

40. Sjöstrand, F. S., Ergeb. Biol., 21, 128 (1958).

41. Wald, G., I'itamins and Hormones, 1, 195 (1943).

42. Wald, G., Science, 119, 887 (19.54).

43. Waid, G., Amer. J. Ophthalmol.. 40. 18 (195.5).

4. Wald, G., in Enzymes: L'mits of Biological Structure and Function (O. H. Gacbler, ed.), p. 355. Academic Press, New York (1956).

45. Wali, G., Exptl. Cell Research, Suppl. 5. 389-410 (1958).

16. Wald. G., in I'isual Problems of Colour, Natl. Phys. Lab., St mupusium . Yo. \&, H. M. Stationery Office, London $\left(19^{-8}\right)$.

47. Wakl, G., in IIandbook of Physiology-Nourophysiology, Val I. p. 671, Ancrican Physiological Society, Washington (1960).

18. Wald, G., in Comparative Biochemistry (11. Florkin and H. S. Mason. cols.), Vol. 1, Chap. 8, p. 311, Academic Press. New York (1960).

19. Wald, G., and Brown, P. K., Nature, 177, 174 (1956).

50. Wald, G., and Brown, P. K.. Science, 127, 222 (1958).

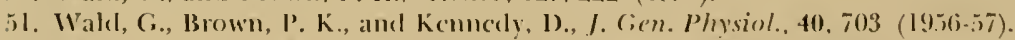

52. Wald, C., Brown, P. K., and Smith, P. H., Srience, 118, 50.5 (19.5).

53. Walel, G., Brown, I'. K., and Suith, P. H., J. (ien. Physiol., 38, 623 (19.54-5.).

5.1. Watd, G., Durell, J., and St. (icorge, R. C. C., Sriense, 111, 179 (1950).

5.5. Zechmeister, 1.., Chem. Ret's., 31, 267 (19/4). 
56. Lechuneister, 1... Experiemlia, 10, 1 (1954).

7. \%echmeister, L.. le Rosen, A. L... Schroeder, W. A., l'olger, A., and Pauling, L... J. Am. Chem. Sor. 6.5. 1910 (1943).

\section{DISCUSSION}

DR. IrNoLb: I would like to give Dr. George Wald another thorn for his lanrels. It seems to me that the most significant thing about the retina is that it has a layered structure, like a chloroplast, and that any theory of vision would have to start with this fact and make real use of it, the same way with photosyuthesis. I would suggest that this lecture which we have just heard is very fune, but it has to do simply with adjusting the sensitivity of the eyc to light. A plant doing photosynthesis, when it gets dark, can simply wait until the next morning, but animals have to run around in the evening and sometimes there are cloudy days and they need a terrific adjustment. I think this is just the chemistry of adjusting the amount of pigment to get light sensitivity. I would like to paraphrase Wald's quotation that all biology is chemistry by simply saying that it is not so. The only way that chemistry got into biology at all was that when nature went to make organisms, the only materials which occurred in large amounts were atoms and molecules.

DR. WALD: Well, I had no time to say, of course, that all chemistry is physics, and I believe that. It is one world, from ultimate particles to the Ninth Symplony. This is what Arnold is talking about. The microstructure of a rod was revealed originally by Sjöstrand. The layers which Arnold is talking about are the membranes that compose a pile of flattened sacs, of which there are 500 to several thousands in the outer segment of a rod. The whole thing is derived, as DeRobertis and others have shown, from what was originally intended to be a flagellum or cilimm-one of those things that waves back and forth at the end of a cell. Now, these layers appear to be made largely out of the visual pigments themselves. We do not know that for certain, because the great weakness in electron microscopy is that one cannot say what one is really looking at, chemically. The best present supposition is that the visual pigments constitute in large part those layers, those sacs, those membranes. If not, they would have to come in between, inside the sacs or just outside. Now, what Arnold is inviting us to do is to bring these layers into the discussion of vision, perhaps to involve them in a discussion on the level of inductive energy transfer and photoconduction. Is that your thought?

Dr. ArNolv: My thought was that by coming to this lecture, 1 might leam something about vision that would help us in the photosynthesis problem.

Dr. WALD: Well, let me say a short piece about that. First of all, I cannot tell you either where this molecule which absorbed the quantum is in the structure, or how what happens to it results in nerve excitation. But nothing is so naïve as-I have the strong temptation to end that sentence-"as a plysicist," but I won't. Nothing is so naive as the ideat that if a quantum 
is received by a rhodopsin molecule somewhere high "p in a rod outer segment, and if only you have some way of transferring the excitation down to the base, you would have solved the problem of nervous excitation. The trouble is that I do not know any better what to do with a molecular excitation at one end of a rod than at the other. To move that excitation around, when I do not know what to do with it, is sheer nonsense. I just do not yet know what wants to be done with the excited molecule, and that is that.

Dr. William Hagins came to the Faraday Society meeting on Biological Energy Transfer a year ago, and described a series of ingenious experiments in which he attempted to demonstrate inductive resonance transfer of excitation in rods. So far they have not worked. We have another experiment in mind. Hagins has told me it is not going to work. Maybe he is right.

Let me say a last thing. For heaven's sake, don't confuse vision with photosynthesis. The way to learn from comparing those two processes is not by saying how much are they alike, but how and why they are different. The job of photosynthesis is to use the absorbed energy of light to do chemical work. The more efficient it is in turning light into work, the better it is working. On the contrary, the light absorbed in vision is not used primarily to do work, but to pull a trigger. What is needed is not work, but molecular excitation. There is no evidence whatsoever that light contributes energy to the net process.

Dr. Hartune: The sad fact is that we just do not know what the primary photoreceptors do. We do not understand the excitation process. Nobody has seen for sure the immediate result of excitation of a primary visual sensory receptor, except perhaps in one case, and that is the eye of a scallop. Pecten. Limulus is more complicated, so we can only speculate about it. Until we get information about the actual responses of the primary receptor cells, we should remember that spcculation is useful but it doesn't get us very far.

DR. RABinowitcu: I think, George, you are not being fair. We really want to find out what the difference is. You see, as long as we only knew that nembranous structures existed in chloroplasts we thought that they are there to permit energy migration, and utilization of diffusely absorbed energy in one spot. When we now sec that this structure also occurs in the visual rods, we can interpret this in two ways: (1) with Arnold we can suggest that there must be energy migration also in the rods; or (2) we can sity, maybe we were altogether wrong, and layered structures in chloroplasts have no relation to energy migration. Maybe, such structures can do something quite dillerent from energy migration. That is what we would like to understand. My fecling is that, when yon have this kind of structurelarge-surface two-dimensional clements, you can achieve two things. You can improve energy migration, and you can improve chemical diffusion, because you provicle a big surface, like a frying pan, where chemical reactions can occur in good contact with whatever is on the outside. Which one is important for photosynthesis and which one in vision, we do not know. In 
any case, son do hane similar problems in vision and in plotosynthesis. You, too, do not understand how a primary process located at any one molecule in the layered structure in the visual rod can initiate nerve excitation somewhere else in the cell.

Ihe iclea that two-dimensional structures are integrating devices in both chloroplasts and visual rods seems to me sound. In one case. the mechanism may be energy migration, in the other case, chemical tramsfer. We woukl like to know which. That is why people like Bill Arnold and I are interested in this matter.

DR. WALn: Yes, it is all right as long as you keep your field of view a little wide. Hagins is the only man I know of who has tried to find these processes. We would be happy to find them too, but so far that has not succeeded. There is no logic in the position that they must be there, and that it is "p to us to find them. Biologists find lamellated structures all over the place. Mitochondria have them; but I will tell you another place where they are, very much as in rods and cones, and that is the myelin sheath of a nerve axon. In the myelin sheath the layers are arranged radially, so that they extend for long distances. Yet you know that nerve conduction does not depend, as far as we know, on processes of exciton migration or semiconduction, but it waits for the chemistry to happen.

DR. PLATT: To what degree is this ignorance about these relations of structure to chemistry due to a lack of physical micro-tools and chemical microtools for operating at this level?

Dr. Rabinowitch: You can turn the tables, and ask Bill Arnold: did you really prove energy migration in photosynthesis? The answer will be no, we did not prove it in chloroplasts either. Incidentally, in the case of the nerve sheath, a plausible purpose of concentric wrappings is easy to suggestgood insulation in the radial clirection, conductivity in the tangential direction.

DR. WALb: But the saving grace in photosynthesis is that you know what you want to do with the absorbed energy. You have a job to do. If I only had a hint of the job I want done, in terms of mechanism in visual excitation, it would be easier to go ahead. I told you a little earlier that I think that maybe the product of light acting on a visual pigment is an enzyme. Well, what do you want an enzyme to do that will produce a nervous excitation? Rushton suggested, and it has been suggested before, that it may increase the permeability of a membrane.

DR Rushtov: I just wanted to say one thing, and that is that there are two aspects in the problem which Dr. Wald raised. It is not only to turn the light sensation on, but also to turn it off. If you had this carotenoid cap on a protein and a quantum of light turns it on and lifts the cap, we know that with rhodopsin that cap will have a 50:50 chance of being back by 10 minutes later, if the light goes out.

DR. WALD: Dr. Rushton raises the very interesting and important problem of the fading of the light sensation when the light goes off. From the picture 
I have presented, one might expect that as long as products of the light reaction that are responsible for visual excitation remain-perhaps, for example, as long as the site on rhodopsin otherwise covered by II-cis retinene remains exposed-the visual sensation should continuc. This should result in a positive after-image; so that the question involves the decay of the positive after-image. Our ordinary experience of this is that it decays very rapidly; and the first rapid falling-off of the sensation, perhaps only to the point that we can just discriminate from the level of sensation when the light was on, is probably the immediate signal that the light has gone off. For the most part, little attention has been paid to the further decay of the positive after-image. The only real measurements I know are in a paper by Paul Lasareff (Pflïgers Arch. ges. Physiol., 201, 333, 1923), in which this decay was measured by Lasareff and Kravkov by two methods. The fairly complete curve of Kravkov's measurements shows the decay of the central (cone vision) after-image to take about 6 minutes; and Lasareff's measurements, showing a rapid decay still in progress after 2 minutes, are consistent with this. Ebbecke's description of the positive after-image, falling rapidly and then more slowly, lasting generally for several minutes, yet not for longer than 6 to 8 minutes after the strongest stimuli-also agrees with these measurements (U. Ebbecke, Pflügers Arch. ges. Physiol., 221, 160, 192829) .

According to these measurements and this description, the decay of the positive after-image in human cone vision follows just about the same course as human cone dark adiptation. This suggests that it may indeed be true that after shutting off the light, the visual sensation persists for as long as it takes to regenerate the visual pigment-i.e., as long as opsin remains free or uncovered by 11 -cis retinene-in the cones. That implies of course that the positive after-image associated with rod vision may last much longer, perhaps for as long as 20 to 30 minutes after shutting off the light.

Dr. FrancK: I asked this question for the same reason as Dr. Arnold and Dr. Rabinowitch. The point is, how certain are you that in this case the pigment sits between the layers?

DR. WALD: I think what Dr. Franck means to ask by his question is whether it is possible that the retincne is located in a relatively anhydrous situation in the rod structure. I would have to answer yes, that is possible, but do not forget that, for example, we need water to complete bleaching. We need the water to hydrolyze the retinene off of the opsin.

DR. FrANCK: Yes, that is my point.

DR. WALD: The next thing that happens is this. As far as we know when we move retinene, it is in the form of vitamin $A$; so one needs to have it reduced by DP'N and alcohol dehydrogenase, which of course demands clirect contact. Now, what you just last suggested is to move the excitation, and hence the bleaching and liberation of retinene out to the ends of the layers for this to happen. That is possible, but I can only say that I do not know how to go on from there. 
Dr. Francik: It is just what you wallt which makes it dillicult for me. If you have the retinene between the two lisers, do you have a diffusion of these things between these layers so quickly to the outside that all the solutes in water can act? Therefore, I think it would be simpler to assume that if it is on the inside, the energy migrates out and only the pigment on the ontside bleaches. Now, we can say that all the rhodopsin goes away and all the retinene is found some place else. I think it might not be between the layers but already outside. That is what I wanted to know. It is, on the other hand, astonishing to me that nature would not use that trick again.

Dr. WaLd: I would like to say a word about the problem that William Rushton raised, but it is not to illuminate it, just to carry the thought on a little. Certainly, a visual excitation must demand more than converting a single molecule of rhodopsin to metarhodopsin. The whole point is that an amplification is needed. I suggested an enzyme. It would be the product of that enzyme-catalyzed reaction which is presumably needed to elicit a response. One question is how long does that product stay around. How rapidly is the excitatory product removed as the basis of the off-effect in vision? I think you can make very simple models that will answer this kind of question, indeed, that will answer everything that one knows about the cessation of vision: but as to the reality of those models, that is another thing.

Dr. ARNox: I would like to make just a very brief comment apropos of photosynthesis and the problem of vision. I agree fully with Dr. Wald that in photosynthesis we do have a different problem. Light is a substrate. It is not a stimulant. But if we wish to picture it, we have excellent examples in plants where we have exactly the same problems as in vision. We have phototropism in which light is a stimulant. It seems to me we are a bit unfair to demand of Dr. Wald an explanation of the photosynthetic phenomenon. I think it would be more fair to demand of him an explanation of photostimulation.

Dr. WALD: This is what you just heard about from Dr. Thimann, and you notice again that in plant phototropism the conduction problem is one of diffusion of auxin and chemical conduction, not of exciton or electron conduction.

Dr. Arxolv: Schrödinger, in the delightful little book What is Life?, points out that a plant does not do photosynthesis to get energy. It has miles of energy around it which it could take in. It does photosynthesis to get negative entropy. And why do you see? To get information, which is essentially the same thing. The problems are not different; they are identical. And the structure is so near identical that you are just sure that you need the same kind of machinery. Biology is not all chemistry.

Dr. ArNos: I have only one brief retort, Mr. Chairman. There is plenty of energy around, but the plant cannot use it unless it is chemical.

Dr. WaLd: Neither can Bill Armold. 


\title{
SPECULATIONS ON THE INTERRELATIONS AND EVOLUTION OF PHOTIC ORGANS
}

\author{
JOHN BuCK \\ Laboratory of Physical Biology \\ National Institute of Arthritis and Metabolic Diseases \\ National Institutes of Health \\ Bethesda, Maryland
}

INTRODUCTION

Biophysicists and biochemists are probably not fully conscious of the activating effects of their emanations upon old-fashioned zoologists, even though these effects sometimes take rather bizarre forms. At the 1954 symposium on our present subject (18), for example, I remember one bemused individual, carried away by the parallels between the role of adenosine triphosphate (ATP) in fireflies and in muscle, asking whether muscles give off light during relaxation. In my case, overexposure to erudition has triggered a large release of speculative energy channeled toward possible evolutionary relations of photogeny to vision, a subject offering splendid opportunities for the exercise of free association. The direction of speculation could perhaps have been justified by the precedent set by various other contributors at more sophisticated levels, or still more strongly by the reciprocities implicit and explicit in our Symposium title and program, but in point of fact two further stimuli helped orient my thoughts. One was the existence of reports, which I had never taken time to follow up in detail, indicating that there is a provocative structural similarity between certain eyes and certain photophores. The other was the remark of a colleague, upon viewing the first record of action potentials in a photophore nerve (3), to the effect that "If you read that backwards it could be an electroretinogram." This latter item really indicates no more than that a photophore, like an eye, may have a nerve supply, but does serve to emphasize the lack of the sort of physiological information which might permit a conrparison of photophore excitation with optic nerve excitation.

Dr. Arnold has already drawn the conclusion that demonstrations of bioluminescence are necessary because no one can really believe 
that the phenomenon exists. Perhaps for the same reason bioluminescence seems to pose in exaggerated form the questions of origin and evolution, questions which of course actually apply with equal force to vision and indeed to all biology. Harvey (14) and Wald (30) have touched briefly on biochemical aspects of this subject, but the point that interests me particularly, the functional interrelations of vision and photogeny at the levels of the organ and whole organism, seens to have had little attention. One facet of the problem, however, the question of whether bioluminescence can account for the welldeveloped eyes of certain bathypelagic or abyssal forms, has been termed by Welsh and Chase (31) ". . . one of the most baffling problems associated with the biology of deep-sea animals."

\section{Photophore Anatomy}

Since the only direct indication of eye-photophore interrelation is the alleged similarity in organ structure, it will be useful to look at some of the details of the extensive literature. If we define "eyelike" as involving an external-internal sequence of a window (cornea), lens (es), photic tissue (in roughly the position of a retina), reflector (tapetum), pigment cup, and nerve, we find that organs more or less meeting this requirement occur in some deep-sea fishes (13) but reach their lighest development in deep-sea squids and shrimps. The figures $^{1}$ illustrate the structures of a few examples, along with corresponding views of certain eyes. In addition to the minimal "eyelike" structures it will be seen that the photogenic tissue is sometimes fronted by a striated "refractor" or "rod-mass," or the rodshaped indivichal photocytes themselves are packed into an oriented layer reminiscent of the arthropod retina. No photophores have a sphincter-type iris, though in some an aggregation of chromatophores, distal to the lens, simulates at least the function.

Clearly, the term "eye-like" is a loose and subjective one, not per-

"Since all the "evidence" is my interpretation of second-hand data, and since we are primarily interested in comparing major details (similarity in minor details being neither required nor anticipated), and since, in any case, apparent structural details vary with plane of section, fixation, staining technique, artistic skill of observer, and other extraneous influences, it has seemed best to me to summarize and simplify the individual pictures in semidiagrammatic fashion. I have also taken the responsibility of assigning names to structures of uncertain or disputed function. For example, light-generation in the Fig. 3 photophore has been ascribed to both the reflector (26) and to the refractor (4) as well as to the indicated tissue (7). My warm thanks are due Dr. Margaret Keister for executing the diagrams. 


\section{ILLUSTRATIONS}

All figures are median sagittal sections modified and simplified from the originals. Magnifications were usually not indicated by the original authors, but most of the organs are of the order of millimeters in diameter. No figure is given of the squid camera eye, since it is, except for its inverted retina, topographically identical with the vertebrate eye.

Code: CL, corneagenous layer; COR, comea (cuticle in arthropods); EP, epidermis or hypodermis; IR, "iris"; L, lens; LAM, lamellated ring; N, nucleus; NV, nerve; PH, photogenic ccll or layer; PIG, pigment; RET, retinal cell or layer; RFL, reflector; RFR, refractor (Stäbchen" or "rod mass" in casc of eye); TAl', tapetum.

Fig. 1. Mantle photophore of squid Abraliopsis. After Chm (6).

Fig. 2. Squid cutaneous photophore. Composite of those figured from Histioteuthis by Joubin (19), from Pterygoteuthis by Hoyle (17), and from Pyroteuthis by Mortara (22).

Fig. 3. Thoracic photophore of schizopod shrimp. Composite of those figured by Chum $(4,5)$ in several species, especially Nemaloscelis and Euphausia, and by several workers $(7,28,29)$ in Nycliphanes.

Fig. 4. Diagram of ocellus of pseudoscorpions or lateral ocellus of pedipalps. After Demoll (8). This eye has an inverted retina (refractor element behind sensory), but Demoll finds transition stages between it and the conventional type.

Fig. 5. One stemma-like unit of the eye of Xenos (Strepsiptera), after Strohm from Demoll (8). In addition to the "Stäbchen" (RFR), note the elongated lens of the type which, in other stemmata, has apparently pinched off a separate in. ternal "crystalline lens" from the cornea.

Fig. 6. Ocellus of larval wasp (Cimbex), after Redikorzew (25). In general (compare also Fig. 8) the "corneagcnous" layer of the hypodermis hypertrophies (o) greater or less extent or cuts off cells to form a refractile "Glaskörper" (crystalline cone), while the sense cells of the more proximal layer may develop distal processes ("Stäbchen"), also refractile. The two dioptric structures together are the cquivalent of cone and rod areas of an ommatidinm. The sensory elcment (retinula) may consist of two to scveral cells, as in the compound cye.

Fig. 7. Type of cyestalk and gill-chamber photophore of decapod shrimp Sergestes. Composite from drawings of Hansen (12), Kemp (20), and Terao (27).

l.ig. 8. Lateral ocellus of butterfly Zygaena, after Link (2I).

Fig. 9. Pleopod ocellus of decapod shrimp Acanthephyra, after Kemp (20). According to Denncll (9), the granular zone formed by the basal ends of the photogenic cells acts as a reflector.

lig. 10. Fyestalk section of shrimp Acanthephyra, showing part of compound eyc and a photophore of the rig. 7 type. After Kemp (20).

Fig. 11. Single photogenic unit of uropod photophore of decapod shrimp Systellaspis affinis, after Denncll (9). The distal, sublenticular cnd is to the right and the proximal end, which participates in forming the granular, nerve-connected zone (Fig. 9) is to the left. Demmell has given the different regions descriptive names, only assigning function to those indicated. He states that the photogenic unit in this species has lost the cell nucleus which he figures for the corresponding structure of Hoplophorus typus and which Kcmp saw in Acanthephyre (now Systellaspis) debilis (Fig. 9). 

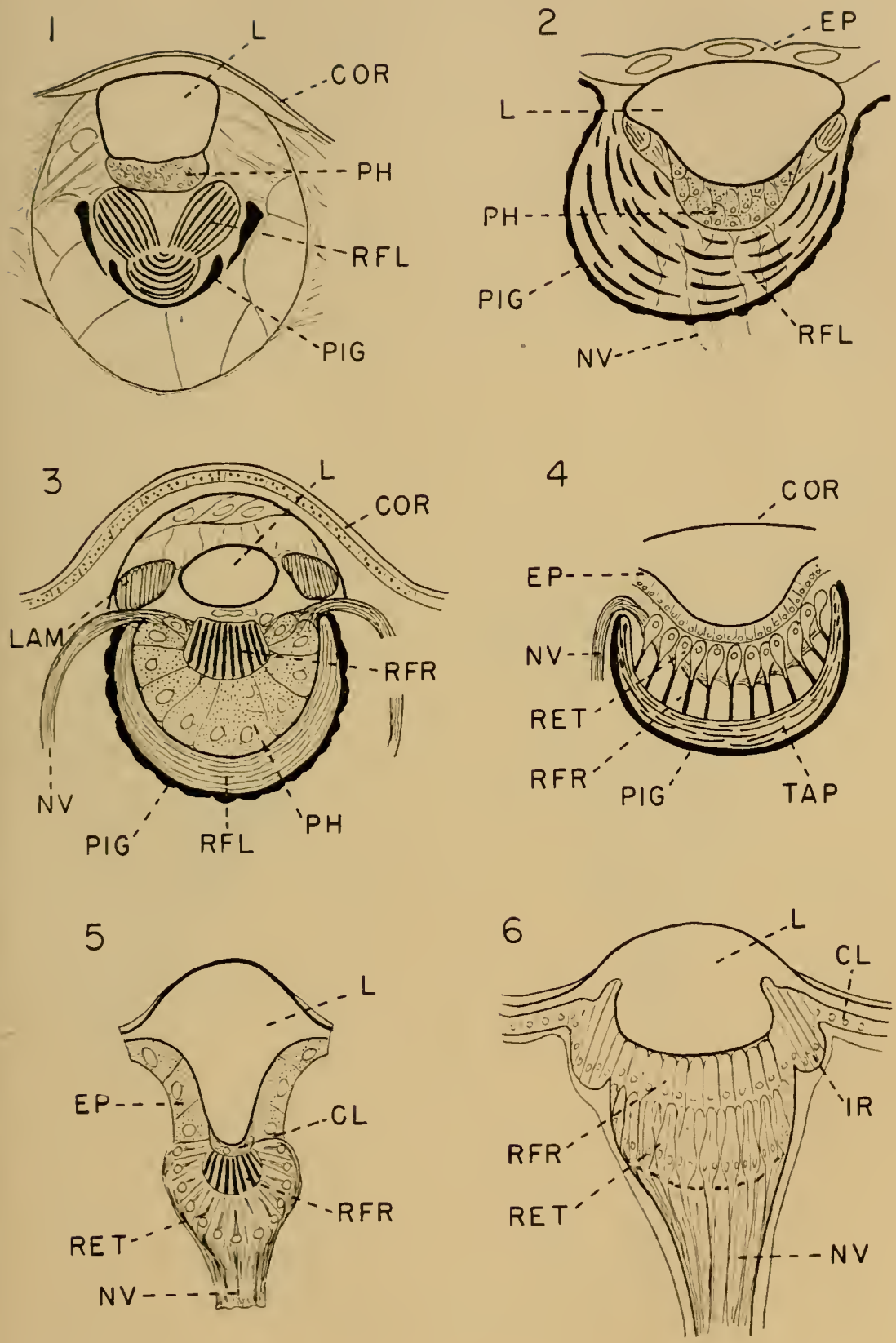

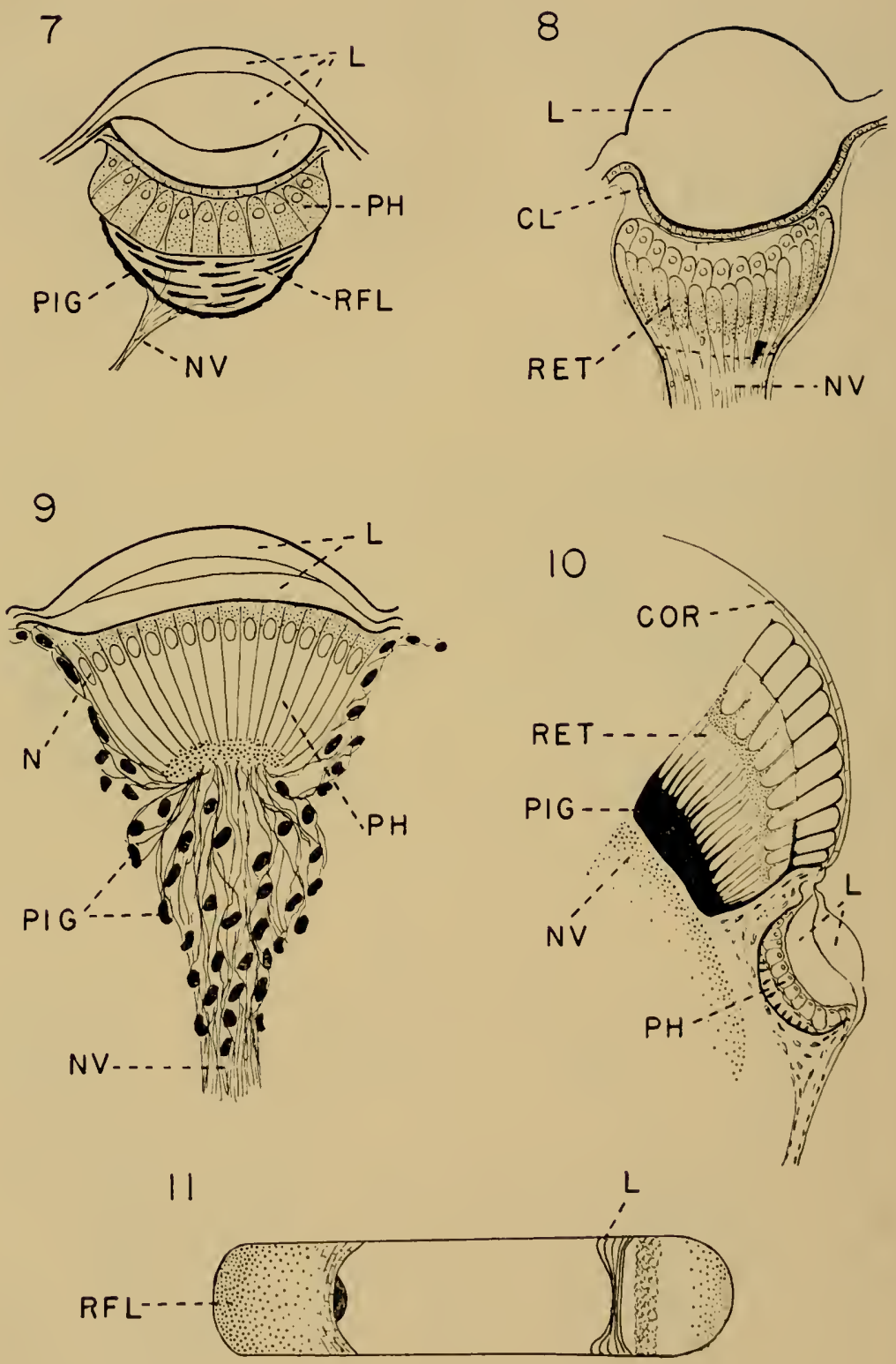
ceptibly more apt, in many instances, than the appellation "searchlight" which has also been used. Thus neither the mantle photophore of the squid Abraliopsis" (Fig. 1) nor the skin organ of certain other squids (Fig. 2) has much resemblance to the camera-type squid eye, or indeed to any known eye, though each has at least an anilog of the proper parts in proper sequence. Furthermore, even with an ostensibly eye-like structure, the eye-photophore comparison loses force if the photophore resembles the eye of some other organism rather than the animal's own, as, for exanple, the thoracic photophores of schizopod shrimps (Fig. 3) seem to mimic the camera eye of squid or vertebrate more closely than a shrimp eye. (Actually, as we shall see, there are indications of arthropod affinities in this photophore.)

Nevertheless, there do seen to be eye-photophore similarities, particularly among deepwater (bathypelagic) crustacea, that are so detailed that it seems captious to doubt that they have a more than accidental significance. In considering these it will be helpful first to recall that present-day arthropods have at least three types of eye, the ocellus, or simple eye, which has usually a single corneal lens and may form a camera-type image; the faceted or compound eye, which forms a mosaic (apposition) or superposition image as the combined product of its many individual optical units (ommatidia); and the stemma, found in larval insects and corresponding roughly to a single ommatidium. There is still disagreement as to the function and evolutionary status of the various eye types, but the variations in known structure are so great as perhaps to justify, for present purposes, the statement that the presence of an anterior lens, and the question of whether, if present, it is a corneal thickening or has separated completely from the surface cuticle, are developmental details of secondary significance. So also the question of whether, in development, the hypodermis folds in to lorm a single or doublelayered ocellar retinal cup or bulges out to form a hemispherical array of visual units (compound eye-Fig. 10) may be secondary to the fact that each resulting structure is an optical organ. This is particularly pertinent in regard to evolutionary questions, since two structures may have undergone much secondary transformation since separating from an ancestral stem-type. If eyes and photophores are

As in other fields of Zoology, there have been many taxonomic changes among the bathypelagic squids and shrimps during the past half-century. Since, however, nomenclature is a field for specialists, I have retained the species names used in the original reports. 
related, in other words, we should not expect that modern photophore and modern eye will necessarily correspond in all structural details. This is fortumate, incidentally, since I found no instance where ocellar structure and photophore structure have both been studied in the same species. This has made it necessary sometimes to compare only distantly related forms that might be expected to differ somewhat merely as a consequence of one being terrestrial and another aquatic, or because of one respiring via tracheae and another via blood gills.

Referring now to specific examples, there seem to be three somewhat different structural parallels between crustacean photophores and arthropod eyes. The first, exemplified by the thoracic photophores of schizopod shrimps (Fig. 3), has several unusual and characteristic structural features, all of which have counterparts in various arthropod eyes. For example, the thick hemispherical reflector which makes it necessary for the nerve supply to enter from the side, is almost exactly matched by the tapetum and nerve arrangement of the pseudoscorpions and pedipalps (Fig. 4). The refractor, likewise, has its counterpart in modifications of the sublenticular or "corneagenous" portion of the insect ocellar hypodermis into striated refractile structures, the "Stäbchen" of the unit eye of Xenos (RFR, Fig. 5) or in the "Glaskörper" of many ocelli (RFR, Fig. 6). The separate lens itself seems more similar to the internal "crystalline lens" of a larval insect stemma than to the separate multicellular lens of forms like Cloeon (see Hesse, (16), Fig. 15). The curious lamellated ring ("external reflector" of Trojan, 28) ${ }^{3}$ was shown by Chun (4) to be a derivative of an hypertrophied region of the hypodermis which is found in the somewhat simpler (lensless) eyestalk photophores of the same shrimps (see Chun's Fig. 1). This region in turn is paralleled by a common insect ocellar structure (the "iris" of Redikorzew (25), Fig. 6).

The second type of photophore, the eyestalk organs of decapod shrimps (Figs. 7, 10), most resemble, among eyes available for comparison, the occlli of insects, although it is difficult to match all structural features in one ocellus. Perhaps the best fits are the ocelli of a butterfly (Fig. 8) and a wasp (Fig. 6). Pigment cup and tapetum seem to be lacking in both, but are present in many other ocelli (e.g., Cloeon, Ceratopsyllis and Agrion-Hesse's (16) Figs. 15, 17, 26).

The most convincing-or perhaps most meretricious-eye-photophore similarity is between the organs found on the leg bases of many

\footnotetext{
${ }^{3}$ Physicists who have gotten into the spirit of this inquiry will recognize the similarity to a Fresnel lens.
} 
decapod shrimps (Fig. 9) and the crustacean compound eye (Fig. 10). There is, to be sure, no evidence that the rod-shaped individual "photocyte" is a multicellular structure like the ommatidium, though Demell (9) has shown that it has considerable complexity (Fig. 11), nor that the photophore cornea is faceted (some compound eyes also have a smooth cornea). However, the resemblance to an eye in general organ shape and in arrangement of units is certainly sufficiently "frappant" (4) to explain their having been originally described as eyes in the days before their light-emitting potentialities were known (sec Chun (4) for references).

In summary, although there are clearly many photophores that have only a specious structural similarity with actual eyes, it does not seem possible to deny that some photophores do have a remarkable resemblance. Further histological work is obviously needed, and there is an even worse dearth of observations on the source of lightemission, the physics of the ostensible dioptric structures, and the nature of the beams emitted (divergent, collimated, or focused). However, it is really not necessary to debate the ultimate morphological cletails of eye and photophore. It is enough to know that the sinilarity is sufficiently compelling to have deceived professional histologists into believing that both structures are eyes. Furthermore, we could scarcely expect that eye and photogenic organ should have identical structures if they have different functions. Actually, it is practically impossible to make an objective evaluation of the structural interrelations apart from their evolutionary implications, which, as we shall see, offer still more serious obstacles to acceptance. Even so, and realizing the pitfalls of vicarious evidence, 1 confess to finding the eye-photophore similarities, at least in the crustacea, extraordinarily beguiling. Considering the abundance of available possible intergrade structures (only a few of which have been touched upon), the differences in function, the differences in "host" structure and habit, and the presumably ancient origin of both types of organ, it seems almost inconceivable that entirely independent structures could by accident be so similar. However, it has to be admitted that two of the original investigators who actually worked on the material, albeit apparently without knowiedge of decapod photophores, conclucled that the schizopod photophore (Fig. 3) is an organ sui generis (4, 26).

At this point, if not before, the skeptic will quite properly ask whether it is really certain that these eye-like organs produce light. If he knows, in addition, that most of the photophores and eyes re- 
ferred to were described a half-century ago, and from preserved material, or if he knows that the skins of certain squids and crustacea are studded with refractile iridiophores, he will be even more suspicious. In point of fact, the situation is by no means as unequivocal as one could wish; but to make a long story short, I did eventually assemble enough eye-witness accounts of light production by precisely localized and identified structures to justify, in my opinion, proceeding on the assumption that there do exist eye-like organs that produce light. (There is even less evidence concerning what parts of the organs produce the light.) Actually, the uncertainties are greater for presumptive eyes, which have usually been identified only on the basis of looking like eyes and being on the head; but presumably one is justified in relying on homology in regard to such a universally present function as vision. Parenthetically it might be mentioned that the burden of speculation would not have been appreciably eased had the "photophores" actually turned out to be eyes after all, since then the rationale of their presence on tentacles, swimmerets, gillchamber wall, and perianal skin would come into question, or by demonstrating that the organs in question were neither eyes nor photophores, in which case their ostensibly dioptric structure would become even more troublesome.

\section{Significance of Eye-Photophore Similarities}

Returning, now, to the conclusion that eye-like photophore structure has a more than fortuitous significance, at least two possibilities suggest themselves. The first is that these structures are actually derived from eyes. The second is that there is some physical requirement of light-emission that demands a pigment cup-photocyte-lens-cornea sequential structure. Clearly these questions can be "answered," if at all, only in terms of possibilities or probabilities. Nevertheless, they may have some value in suggesting desirable experimental approaches to what $\mathbf{I}$ believe is a valid and interesting problem.

In relation, first, to the possibility that eye-like photophores are actually made-over eyes, mention has already been made of the fact that these organs are often in most un-eyclike locations. There are also such trifling difficulties as the necessities for assuming a conversion of photoreceptor pigments to photoemissive compounds, and sensory nerves to motor nerves. However, from the ontogenetic standpoint the possibility is at least not excluded, because it seems to be true that in spite of the cnormous variety of structure and situation among photophores, all those that have an eyc-like structure are, like 
eyes, located on the surface of the body and are presumably derived, like eyes, from the ectodem. There is also the curious fact that photophores (though not always the most eye-like ones) seem to have a much more than random tendency to occur near, or even partly in, eyes, as might be expected if the two structures are derived from common primordia. ${ }^{*}$ In so far as crustacea are concerned, the above-mentioned similarity between photocyte layer and retina could also favor the idea of common origin, because of the phylum-limited nature of this type of retinal structure, but the patent lack of similarity between retina and photocyte mass in the cephalopod molluscs weakens the case.

It is clearly risky to argue from assumed function, but two other structural features of eye-like photophores seem to me more easily understandable as relics of eye structure than as physical necessities for light-emission. These are the "refractor" or "rod bundle" and the pigment mantle. Whether the photophore produces a focused submarine beam (which I find a little hard to visualize) or diffuse illumination covering a wide angle, ${ }^{5}$ which would appear more useful for the possible functions that the organ might serve (see below), an apparently collimating device in addition to reflector, lens, and cornea seems superfluous. As to the pigment, it can hardly be argued, for example, that it is necessary to prevent the creature's light from injuring its own tissues, particularly when a reflector is present also. Furthermore, the light is of very low intensity and has a reasonably dispersed source, and many photophores, notably those buried contpletely in tissue, lack pigment entirely. Likewise, it seems not a strong argument that the pigment is necessary to prevent the light produced from entering the creature's own eyes, since the eyes are well shielded by their own pigment, and photophores are often far distant.

The second possible "explanation" of eye-like photophore structure-that it is demanded by some physical requirement of lightemission-is easily disposed of. Even if one excludes from the vast array of photogenic organs all those depending on symbiotic bacteria,

- Practically all writers on luminosity in cephalopods (e.g., 1, 6) have described the colored photophores found on the squid eyeball. Photophores imbedded in the margin of the crustacean compound eye are figured by Chun (5), Kemp (20), and Dennell (9), among others.

${ }^{5}$ Hayashi (15) has in fact described the light of the squid Watasenia as spreading out in all directions, and Trojan (28) has diagrammed the course of light through the dioptric system of the Sergestes photophore in such a way that it would also give polydirectional light (though it is not clear that he actually saw this occur), but neither observation is critical enough for our purpose. 
all those producing an extracellular luminous secretion, and all those that produce light continuously or are known to be hormonallycontrolled-most of which in any case show no resemblance to eyes whatsoever-there still remain many organs, even in deepwater crustacea and squids, that seem to function without most of the structure that one might, a priori, have anticipated in a light-emitting organ-for example, a translucent window on the outside. Only in organs actually in the integument, where the need would seem to be least, do "cornea" and lens become common.

\section{EVOLUTIONARY IMIPLICATIONS}

Even if the anatomies of sighting and lighting have no relation to one another, it may be stimulating to speculate about possible functional interrelations during evolution. Concerning this we have no shred of direct evidence, the fossil record having nothing to contribute in relation to the temporal sequence of vision and photogeny nor to the former existence of more primitive types or intermediate stages of photophore structure. However, a good deal is known or reasonably surmised about the evolution of eyes, and there is a wealth of indirect evidence about photophore evolution.

The distribution of bioluminescence in the animal kingdom leads, by conventional paths of evolutionary thought, to the conclusion that light-production must have appeared inclependently in a great number of different groups. Harvey (13) has documented this interesting deduction in great detail, emphasizing the sporadic occurrence of the faculty even among close relatives, and likening its almost random distribution to what would be obtained by throwing a handful of sand over the phylogenetic tree, with each grain marking a luminous form. The status of photophores thus contrasts strongly with that of eyes, which, though similarly polyphyletic in origin, are almost ubiquitous. From the above deduction Harvey has also drawn the corollary that the ability to produce light nust have arisen as accidental aberrations in otherwise conventional biochemical systems.

We can hardly come to grips with the evolution of photogeny without considering the question of the function of the light-that is, its selective value or use to the organism. Anthropopsychic judgments of this sort are clearly fraught with pitfalls, but at least no more so than in the case of vision, in relation to which, for example, the conventional attitude would be that envirommental light has acted over the ages as a selective agent in evoking the camera eye independently in annelids, molluscs, and vertebrates. Therefore, without any over- 
tones of teleology it will be assumed that the observed photophore structures and interelations have usefulness to the organism. In this connection it may be instructive to summarize briefly the sorts of uses for photophores that have been suggested in a variety of animals.

In view of the spectacular nature of bioluminescence it is almost unbelievable that its utility to the organism has not been unequivocally established, either observationally or experimentally, except in the single instance of the mating signals of the firefly. A similar function has been guessed at in the Japanese hotaru-ika, or "firefly squid," but it is clear that the rarity and extreme delicacy of these and other bathypelagic organisms will make direct observation of luminescent behavior under natural conditions very difficult. The suggested fishing lure utility of the barbel organs of the angler fishes is a similarly reasonable but speculative use for the organisms under consideration. Other functions, such as protection by signifying unpalatability or by frightening off predators, or as a "burglar alarm" (2) in which the victim's signal enables other members of the species to escape, seem still more hypothetical. Chun (4) claimed that the schizopod thoracic and abdominal photophores are aimed ventrally and laterally and he apparently thought that the resulting illumination would somehow aid, in conjunction with specialization of the ventrolateral regions of the compound eye, the handling of prey caught by the thoracic appendages (an operation done by "feeling" by most crustacea). One unexplained attribute of many of the shrimp photophores is that they are apparently movable, by specific muscles (e.g., $4,9)$.

In regard to cleep-sea squids and shrimps we may perhaps focus special attention on the facts that these creatures normally live for long periods in an environment of perpetual and complete absence of daylight, and that they have well-developed eyes. It rould therefore seem reasonable that one function of light-production could be in providing recognition signs to keep members of the school from straying apart forever into the vast and trackless depths. In animals breeding by sexual contact, some such mechanism for promoting gregariousness would seem to be essential for the continuation of the species. In addition, since both groups are amply well-developed, neurally, there appears to be no reason why species-specific mating signals could not develop for use during their reported diurnal or seasonal migrations to the surface at night for breeding. Dennell (10) and especially Nicol (24) have macle some interesting estimates of the distance that light from deep-sea shrimps might be visible to another 
individual of the same species. Assuming a visual threshold equal to that of dark-adapted man, and water of $90 \%$ transmission, Nicol's figure is 5 to 16 meters.

It will be seen that all the functions of bioluminescence that seem possibilities for bathypelagic squids and shrimps involve an ocular response in the same species, or, usually, in the same individual. It thus seems mandatory to assume that photosensitivity evolved before bioluminescence, and that it thereby provided the opportunity for uses which could in turn act as selective pressures for the retention and elaboration of the light-producing capacity, once it appeared. This deduction is quite in keeping with the known ancient history of vision, at least in the arthropods (trilobites and eurypterids), and would seem to be an unusually safe anchorage in proverbially shifty waters.

That is, it seems safe unless one also asks the question, "Why should abyssal squids and crustacea have developed eyes in the first place, if there was nothing down there to see?" Actually, of course, we are rescued from the egg-hen dilemma by several considerations. For example, practically all the free-swimming macrofauna are by necessity predators. Hence food-chain requirements suggest that abyssal forms must have evolved from surface or shallow-water forms that were tied originally to filter-feeding prey living on surface phytoplankton. In this environment, sunlight would have provided, we assume, an eye-evoking selective pressure.

The postulation of an eye-photophore functional association is supported by the absence of photophores in the blind deepwater shrimp Bentheuphausia (4) and by the finding of Welsh and Chase (32) that, of the sergestid shrimps they studied, the four species with photophores had the largest eyes, in accord with their suspicion (31) that deepwater crustacean eyes degenerate in the absence of photophores. An eye-photophore association is supported also by the existence of cephalopod molluscs, and of shrimps, with excellent eyes but no photogenic organs, but the complete absence of representatives with the opposite complement-photophores but no eyes. ${ }^{6}$ However, not cnough is known about crustacean distribution to permit the con-

- Though this statement may be correct, strictly speaking, the inevitable exception to the law is indicated by Nicol's statement (23) that the majority of luminescent copepods are blind. Though copepod photophores are not eye-like, and the animals are not bathypelagic, and we have already conceded that bioluminescence has no perceptible function in many animals, it is disconcerting to have an instance turn up in the crustacea. 
clusion that eyc-like photophores are limited to inhabitants of deep waters. In view of the virtual absence of photophores among tetrabranch cephalopod molluscs (i.e., the planktonic nautiloids) and octopods (the characteristically shallow-water cuttlefishes), it is also tempting to see confirmation of the iclea that bioluminescence must develop before eyed forms can venture permanently into stygian depths. However, not enough is known about squids $(1,6)$ to tell whether the oegopsids, with their good eyes and good photophores, have a characteristically deeper habitat than the myopsids, with their also excellent eyes but absent photophores.

The squicls Histioteuthys and Calliteuthys present a different type of puzzle, in that both species have enormously developed left eyes, bearing mostly rudimentary photophores, while the smaller right eyes bear a circlet of well-developed photophores (6).

Another eye-photophore interrelation that has been suggested (11), and which is perhaps not outrageously more speculative than some of the preceding, is that an organ might function simultaneously as eye and photophore. Incoming light could then serve to excite the photogenic tissue directly and the dual organ could serve as a local-reflex signalling device. This would at least make it easier to understand the presence of ostensible collimating structures in photophores, both functionally and with respect to evolution.

A third possibility, that high hydrostatic pressure is a photophoreinducer, has thus far not been possible to test, for lack of permanently dark, but shallow, marine habitats. Cave-dwelling fauna, whether eyed or blind, consistently lack photophores; but the significance of this otherwise arresting fact is vitiated by another and overriding peculiarity of bioluminescence, namely, that it is essentially absent in freshwater forms.

\section{Summation}

It has to be admitted that the number of old mysteries concerning bioluminescence has not been reduced by redescribing them, though benefit may eventually come simply from thus exposing them to a new generation of biologists, physicists, and chemists. For example, in spite of Wald's well-taken point that photosynthesis is actually closer to bioluminescence than is vision, I think it would not be amiss if biochemists stayed alert to possibilities of kinship between light production and light absorption. We surely have enough examples of swapped, shared, and made over molecular types and enzymatic 
processes in the living world not to feel that we by now know all the present potentialities of nature in this respect, let alone what may have occurred in the past. In particular it seems reasonable to believe that there was once a time when vision was not and a time. when photogeny was not. This implies that the first photoreceptive pigment molecule and the first molecule capable of being put chemically into an excited state must have originated from pre-existing types, not having these properties, that served and perhaps still serve some unrelated biological structure or function. Bioluminescence, in fact, must be presumed to have arisen separately from several such precursors.

As to the biologies of photogeny and light perception, the only (logmatic statements that can be made were made long ago, and little new has been addect except as possibilities. In a tentative way it cau be said that the burden of the evidence seems to be that species vision evolved before species photogeny. There seem to be enigmatic structural analogies between eyes and photophores, but it also looks as if eyes and photophores must have arisen independently and under quite clifferent sorts of evolutionary pressures. In the case of eyes it appears that the availability of environmental light has been a powerfut inducing pressure for the selection, or perhaps even incluction, of photosensitive systems-that is, that light presents an opportunity for or creates a need for a particular ability (vision). In photogeny, on the other hand, it seems that the ability (light-production) appeared first, and was then turned to use in conjunction with vision-that is, uses were created in response to a fortuitous endogenous ability rather than to environmental pressures.

One salient and somehow disturbing feature of bioluminescence is its accidental and incidental nature. Not only can it depend, at least in bacteria and fungi, on a single gene mutation (13), but it seemingly confers neither advantage nor disadvantage on many organisms in respect to survival, growth, nutrition, social relations, or metabolic potentialities. Perhaps we are again yielding here to Dr. Arnold's "clisbelief in biolight" if we find difficulty in reconciling ourselves to energy simply being thrown away as light instead of as heat. However, we are on slightly more secure ground if we balk at the apparently casual occurrence and significance of light procluction in so many organisms, becatuse we do know that in general few things persist during evolution unless they confer selective advantage. Probably this simply means that biologists need to look harder at organisms, and this is particularly apparent in reference to the deep-sea creatures 
we have discussed, where so strong a case can be made for a functional, if still undefuned, association of eyes and photophores. ${ }^{7}$

II have been much stimulated, in my speculations, by discussions with various colleagues. Not to imply their endorsement, however, I must mention that their attitude towarl the reasonableness of the eye-photophore comparison is perhaps epitomized by the following (paraphrased) question put by Dr. Rushton: "What would you say if 1 were to attempt to convince you that the car and the larynx are mirrol-image organs? They have the same sort of external funncl for sound collection (dispersion), the same ribrating body for sound reception (production), the same sort of devices for loosening and tightening the vibrator, and approximately the same frequency range." Actually, come to think of it, there might be something in the idea. Consider, for example, that both ear and larynx develop from gill pouches...

An additional evolutionary facet, which I had missed completely, is brought out in the following excerpt from a post-symposial letter from Dr. Wald: "l do think that you ask too close an association of photophores and eyes in the same organisms. I think that one of the most apparent uses of bioluminescence is to provide light in the deptlis for organisms that can see, and that it matters little whether these are the same or other organisms. Organisms, plant and animal, as you know, form a community; and their interrelationships are so various and complex that they quite commonly provide for one another, with ultimate advantage to themselves. It is probably a fine thing for marine bacteria that fish get along well, and this if nothing else would be a fine justification for bacteria developing luminosity."

\section{REFERENCES}

1. Berry, S. S., Biol. Bull., 38, 141-169; 171-195 (1920).

2. Burkenroad, M. D., J. Marine Research (Sears Foundation), 5, $161-164$ (1943).

3. Case, J. and Buck, J., Biol. Bull., 117, 393 (1959).

4. Chun, C., Biol. Zentr., 13, 544-571 (1893).

j. Chun C., Bibliotheca Zool., 19, 1-262 (1896).

6. Chun, C., Wiss. Ergeb. "I Ialdivia" Exped., 18, pts. 1 and 2, 1-401 (1910).

7. Dahlgren, U., J. Franklin Inst., 183, 335-434 (1916).

8. Demoll, R., Die Simesorgane der Arthropoden, Braunschweig (1917).

9. Dennell, R., Discovery Reports, 20, 307-382 (1940).

10. Denncll, R., J. Limn. Soc., 42, 393-406 (1955).

11. Grimpc, G., and Hoffman, H., Arch. Naturgeschichte, 87A, 179-219 (1921).

12. Hansen, H. J., Proc. Zool. Soc. London, 1, $52-79$ (1903).

13. Harrey, E. N., Bioluminescence. Academic Press, New York, 649 pp. (1952).

14. Harrey, E. N., Quart. Re乞. Biol., 31, 270-287 (1956).

15. Hayashi, S., Folia Anat. Japon., 5, 417-427 (1927).

16. Hesse, R., Z. wiss. Zool., 70, 347-472 (1901).

17. Hoyle, W. E., Mem. Proc. Manchester Lit. Philos. Soc., No. 46, 14 pp. (1902).

18. Johnson, F. H. (ed.), The Luminescence of Biological Systems. American Association for the Adrancement of Science, Washington, D. C. 452 pp. (1955).

19. Joubin, L., Bull. Soc. Sci. Med. de L'Ouest, 2, 49-78 (1893).

20. Kemp, S., Proc. Zool. Soc. London, 639-651 (1910).

21. Link, E., Zool. Jahirb (Anat.), 27, 213-24l (1909).

22. Nortara, S., K. Com. Talassogr. Ital., Mcm. 82, 30 pp. (1921).

23. Nicol, J. A. C., The Biology of Marine Animals. Interscience Publishers, New York. 707 pp. (1960). 
24. Nicol, J. A. C., J. Marine Biol. Assoc. United Kingdom, 37, 705-752 (1958).

25. Redikorzew, W., Z. wiss. Zool., 68, 581-624 (1900).

26. Sars, G. O., Challenger Repts., Schizopoda, 13, 1-255 (1885).

27. Terao, A., Annotationes Zool. Japon., 9, 299-316 (1917).

28. Trojan, E., Arch. mikr. Anat., 70, 177-188 (1907).

29. Vallentin, R. and Cunningham, J. T., Quart. J. Microscop. Sci., 27, 319-341 (1888).

30. Wald, G., Sci. American, 201, 92-108 (1959).

31. Welsh, J. H. and Chase, F. A., Jr., Biol. Bull., 72, 57-74 (1937).

32. Welsh, J. H. and Chase, F. A., Jr., Biol. Bull., 74, 364-375 (1938). 


\title{
THE COLOR VISION OF INSECTS
}

\author{
TIMOThy H. Goldsmith : \\ The Biological Laboratories \\ Harvard University \\ Cambridge, Massachusetts
}

Insects, as a group, have been very successful. Approximately four out of every five species of animals belong to this class, and in a significant sense this is the age of insects. Yet the sensory apparatus of insects is not at all well understood.

This review treats but one aspect of vision in insects-the discrimination of wavelengths. Even this problem, however, reaches into opposite corners of biology. We should like to know how, in terms of molecular and neurophysiological mechanisms, the visual systems of insects operate. But it is also important to ask why, in terms of animal behavior and evolutionary history, particular sensory systems exist. If in the following pages the first of these general problems appears to receive undue emphasis, it is only because the answers we have here seem somewhat less speculative.

\section{The Presence of Color Vision in Insects}

There is evidence for color vision in six of the approximately two dozen orders of insects that are recognized (Table 1). This statement does not mean that only a few insects have color vision; it indicates a dearth of comparative data. In fact, the capacity to distinguish colors may well have developed early in the evolution of insects, for both paleopterous and neopterous species are represented in Table 1.

The evidence for color vision is derived principally from three sources. Certain insects can be trained to associate food with colored

${ }^{1}$ The author is a member of the Society of Fellows of Harvard University. Present address: Department of Biophysics, University College, London; after June, 1961, Department of Zoology, Yale University, New Haven, Conn. The experiments of the author were supported in part by a grant (B2077 (A)) from the Institute of Neurological Diseases and Blindness, United States Public Health Service. 
TABLE 1

The Orders of Insegts in Which There Is Evidence for Color Vision Order Genus Method and Reference

\begin{tabular}{|c|c|c|}
\hline $\begin{array}{l}\text { Odonata } \\
\quad \text { (dragonflies) }\end{array}$ & $\begin{array}{l}\text { Aeshna (nymphs) } \\
\text { Libellula }\end{array}$ & $\begin{array}{l}\text { training }(34) \\
\text { electrophysiological }(40 a)\end{array}$ \\
\hline $\begin{array}{l}\text { Blattaria } \\
\quad \text { (cockroaches) }\end{array}$ & Periplaneta & electrophysiological $(56,57,58,59)$ \\
\hline $\begin{array}{l}\text { Coleoptera } \\
\text { (beetles) }\end{array}$ & $\begin{array}{l}\text { Chrysomela, Agelastica, } \\
\text { Geotrupes } \\
\text { Notonecta }\end{array}$ & $\begin{array}{l}\text { optomotor (48) } \\
\text { optomotor (45) }\end{array}$ \\
\hline $\begin{array}{l}\text { Hymenoptera } \\
\text { (bees, wasps, } \\
\text { ants, etc.) }\end{array}$ & Apis & $\begin{array}{l}\text { training (e.g., 16, 35, 4, 29, 9, 36) } \\
\text { electrophysiological (20) }\end{array}$ \\
\hline $\begin{array}{r}\text { Diptera } \\
\text { (flies) }\end{array}$ & $\begin{array}{l}\text { Eristalis } \\
\text { Fannia } \\
\text { Drosophila } \\
\text { Calliphora }\end{array}$ & $\begin{array}{l}\text { training }(32) \\
\text { optomotor }(48) \\
\text { observation of behavior }(24) \\
\text { electrophysiological }(2,58,59)\end{array}$ \\
\hline $\begin{array}{l}\text { Lepidoptera } \\
\text { (moths and } \\
\text { butterflies) }\end{array}$ & 21 genera & $\begin{array}{l}\text { training }(30) \\
\text { optomotor }(48)\end{array}$ \\
\hline
\end{tabular}

papers or monochromatic lights under conditions where one is confident that discriminations are based on wavelength and not on brightness, odor, or some other property of the test object. Second, many insects have a characteristic reaction-the optomotor response-to movement of vertical stripes in the visual field. When stripes of a given color alternate with gray, a shacle of gray can generally be found such that movement of the stripes does not elicit a reaction from the insect; the gray and colored stripes are then said to be of cqual "brightness." It has been observed, however, that two alternating colored stripes, each of the same brightness as a given gray, continue to evoke a response from many insects. In gencral, the more distant are the colors in the spectrum, the stronger is the optomotor reaction. Finally, in several instances electrophysiological observations on compound eyes have indicated the presence of receptors maximally scnsitive in different regions of the spectrum. In essence most of this evidence is of the following kind: when two maxima appear in the spectral sensitivity function and it is possible to alter the relative heights of the two peaks by selectively adapting the eye with colored lights, the presence of more than one visual pigment is indicated. Further, if in the same animal different regions of the spectrum evoke qualitatively different retinal action potentials-potentials whose wave forms camnot be matched by any adjustment of the intensityit is clear that more than one kind of receptor mechanism is present. These and related experiments will be considered more fully below. 
The Extent of the Spectrum Visible to Insfacts and the

\section{Regions of Maximum Sensitivity}

There have been numerous attempts to measure the spectral sensitivities of the eyes of insects, using both behavioral $(4,5,6,7,9,14$, $28,46,49,50,60,61,62,63,64,65)$ and electrophysiological (1, 2, $11,19,20,23,33,56,57,58,59)$ techniques. These experiments vary widely in sophistication, and in the following paragraphs the terms "action spectrum" and "spectral sensitivity" will be used only where the quantity measured was the relative energy for a constant effect. In addition, the term "spectral sensitivity" will be further restricted to electrophysiological measurements of the eye, as distinct from action spectra for behavioral responses of the whole organism.

Almost all attempts to measure the relative effectiveness of various wavelengths in stimulating insects, electrophysiological as well as behavioral, indicate a broad maximum in the blue-green or green regions of the spectrum and gradually diminishing effectiveness in the red. The fly Calliphora, however, possesses a second peak of sensitivity in the red $(2,58,59)$ (see Fig. 5). What is in many respects the most interesting feature of these observations is that for at least some insects the visible spectrum extends into the near ultraviolet.

\section{The Question of Sensitivity to Near Ultraviolet Light}

In 1886 Sir John Lubbock (38) showed that sunlight from which the ultraviolet had been filtered was much less effective than normal sunlight in stimulating ants to remove their pupae to the dark. The relative efficiencies of different wavelengths in eliciting behavioral responses such as phototaxis have been determined by several investigators, and where the data permit quantitative comparison, it seems that the near ultraviolet is the most effective region of the spectrum (Fig. 1).

The data reproduced in Fig. I require further comment. Bertholf (6) reported the "relative stimulative efficiency" of various wavelengths in stimulating the positive phototactic response of the fruit fly Drosophila. Unfortunately, his method was somewhat indirect; monochromatic lights were matched in stimulative effect by a white light of variable intensity. Owing to the fact that the test wavelengths varied in energy, the reciprocal of the intensity of white light required for an equal effect-the "relative stimulative effect"- has little meaning. Bertholf attempted to correct for this defect by dividing the observed values of "relative stimulative effect" by the relative energies of the monochromatic test lights, and it is this quantity, the 


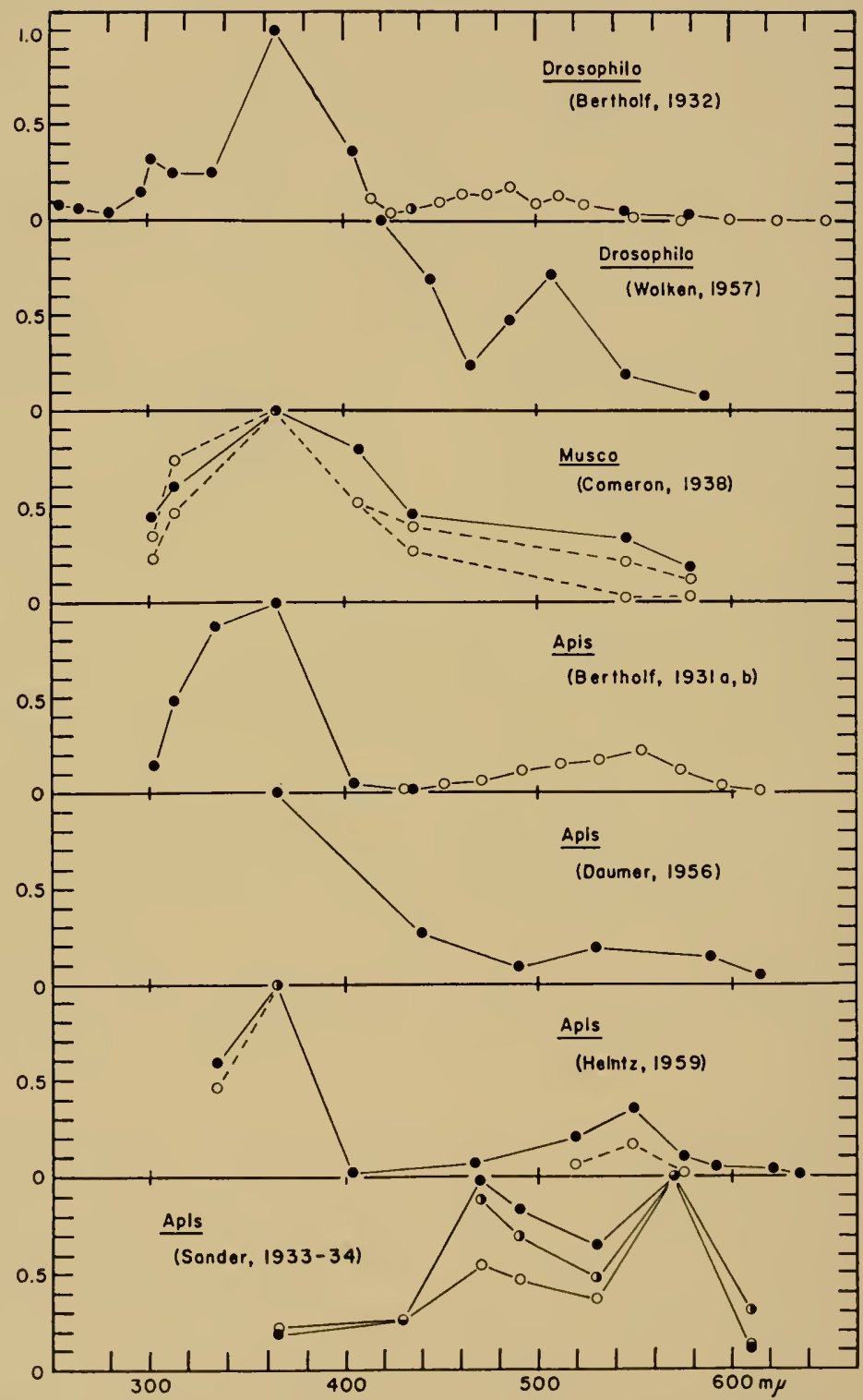

Fig. 1. The available quantitative data on the relative effectiveness of different wavelengths in evoking positive phototaxis and similar responses of adult insects. Sec the text for a description of each experiment. 
"relative stimulative efficiency," that is plotted as a function of wavelength in the uppermost graph of Fig. 1. Although this correction is surely in the proper direction, it assumes direct proportionality between phototactic effectiveness and intensity, an assumption which is almost certainly incorrect $(46,7,28)$. A further difficulty with these experiments arises from the fact that the curve is pieced together from experiments done at two different times with two different lamps. The tro halves of the curve (filled and open circles) are brought together by making equal the "relative stimulative efficiencies" at 436 $\mathrm{m} \mu$. If, however, the curves had been made to coincide at $546-550 \mathrm{~m} \mu$ or at 575-577 $\mathrm{m} \mu$ (the other points at which data were obtained with both lamps), the peak in the blue-green would be more prominent.

The phototactic response curve measured by Wolken (65) for Drosophila is an action spectrum; the ordinate is the reciprocal of the relative energy necessary for a constant phototactic effect. The curve peaks in the blue-green and starts to rise again at shorter wavelengths. Unfortunately, it does not extend to wavelengths shorter than $420 \mathrm{~m} \mu$, but already by that point it has climbed higher than the maximum at $508 m \mu$. The data of Wolken thus support Bertholf's conclusion that for Drosophila the most effective wavelengths for phototaxis lie in the near ultraviolet.

Cameron (7) performed several kinds of experiments with the housefly, Musca. The relative phototactic response to wavelengths of equal energy (filled circles, solid curve) and two minor variations of the method of "relative stimulative efficiency" (open circles, dashed curves) all gave results similar to those of Bertholf for Drosophila. None of these curves is a proper action spectrum, and (as was recognized by Cameron) the significance of the quantity "relative stimulative efficiency" is particularly uncertain.

The last four curves in Fig. 1 apply to the honeybee, Apis. Bertholf $(4,5)$ determined the "relative stimulative efficiency" of different wavelengths for phototaxis. The curve has a large peak in the near ultraviolet and a smaller peak in the green. However, the pair of objections that were made to Bertholf's experiments with Drosophila apply to these data as well.

Daumer (9) measured the lowest energies at which bees were attracted to monochromatic lights to which they had previously been trained. His is thus an action spectrum for threshold recognition of various wavelengths. Sufficient data to locate the highest maximum are not included in Daumer's paper, but it is clear that the major peak lies in the ultraviolet. 
Heintz (28) placed bees in a box, and using different wavelengths measured the number that crawled over an illuminated slit in a three-minute period as a function of intensity. The curve second from the bottom in Fig. 1 is the reciprocal of the intensity required for a constant effect-an action spectrum. Again the major peak is in the near ultraviolet, regardless of whether a small (solid curve) or a large (broken curve) effect is selected. These curves will not be found in Heintz's paper, as he chose to present his data in another form.

Sander's (46) action spectra for phototaxis of the bee are somewhat anomalous in that they show nothing of a peak in the ultraviolet. There is no ready explanation for this discrepancy. [It has been reported (62) for some beetles that when the test lights are sufficiently bright, the peak in the green is higher than the peak in the ultraviolet. Presumably Sander's three curves differ from each other because they were obtained at different levels of intensity; however, this point is not made very clear.] Somewhat perturbed by Sander's data, I have tested the relative phototactic effectiveness of green ( 546 $\mathrm{m} \mu$ ) and ultraviolet $(365 \mathrm{~m} \mu)$ lights of equal energies (20). The results of this experiment, involving just two wavelengths, support the conclusions of Bertholf (5), Daumer (9), Heintz (28), and Weiss, Soraci, and McCoy (62) in indicating that for the bee ultraviolet light is more effective than green light in stimulating positive phototaxis.

Except for the work of Sander, the conclusion is consistent that for all those species studied the near ultraviolet wavelengths are the most effective in exciting phototaxis and similar reactions. The same conclusion was reached by Peterson and Hacussler (42) and Weiss ct al. $(60,61,62,63,64)$, who studied the responses of insects to broad bands of wavelengths of equal energy. The taxonomic occurrence of this phenomenon, in so far as it is known, is summarized in Table 2. Unfortunately there are few comparative data.

With extracellular electrocles it is an easy matter to record relatively large (ca. $10 \mathrm{mv}$ or more) retinal action potentials from the eyes of insects, and recently measurements of spectral sensitivity have been made in the ultraviolet region of the spectrum by using this electrical response. Because the retinula cells lie close to neurons in the optic ganglion with which they synapse, electroretinograms (ERG) do not reflect the activities of receptor cells exclusively. This is a situation to be borne in mind, particularly in those instances where more than one kind of color receptor appears to be contributing to the retinal action potential. There are as yet no records of the activity of single units in the compound cyes or optic ganglia of insects. 
TABLE 2

Insects for Which the Near Uitraviolet Has Been Observed to be the Most Effective Region of the Spectrum in Evoking Beilavioral.

Responses Suchi as Phototaxis

Order

Hemiptera (true bugs)

Coleoptera (beetles)

Hymenoptera (bees, wasps, ants, etc.)

Diptera (fies)

Lepidoptera (moths and butterflies)
Animal and Reference
18 species $(62,63,64)$

Formica, Lasius (38)

Apis $(5,62,9,28,20)$

Macrocentrus (62)

Drosophila $(6,63,65)$

Musca (7)

Laspeyresia (42)

(also 7 of 14 larval forms tested) (64)

* Near ultraviolet and blue-green lights about equally effective in stimulating phototaxis.

A maximum of spectral sensitivity at about $340 m \mu$ in the near ultraviolet occurs in the compound eyes of the cockroach Periplaneta $(58,59)$, one fly Calliphora $(58,59)$ (see Fig. 5), and the honeybee Apis (20) (see Figs. 2 and 4 ), as well as the ocellus of the honeybee (23). In each case this maximum reflects the presence of a receptor for ultraviolet light which is one of at least two color receptors present in the eye. For example, the upper part of the eye of the cockroach has peaks of sensitivity in the green and ultraviolet regions of the spectrum. The relative heights of these maxima vary in different dark-adapted preparations and can be made to change in either direction by adapting the eye with monochromatic lights $(58,59)$. Furthermore, the time course of the retinal action potential is dependent on wavelength, being slower at the blue end of the spectrum $(56,57)$. A similar selective adaptation of the compound eye of the worker honeybee is illustrated in Fig. 2.

If the maximum in the red is ignored, the spectral sensitivity function of Calliphora (Fig. 5) is similar to the curves for the phototactic responses of other flies (Fig. 1). In the case of the honeybee, however, there is another marked difference between the spectral sensitivity function of the dark-adapted eye and the action spectrum for phototaxis. Often the spectral sensitivity curve of the bee has a single peak in the green and only a low foot extending into the near ultraviolet (Fig. 2, upper curve). If the ultraviolet maximum is present in the dark-adapted eye, it is less prominent than the peak in the green $(20)$.

This observation indicates a potential hazard in determining the properties of receptors from observations of the behavior of the entire 


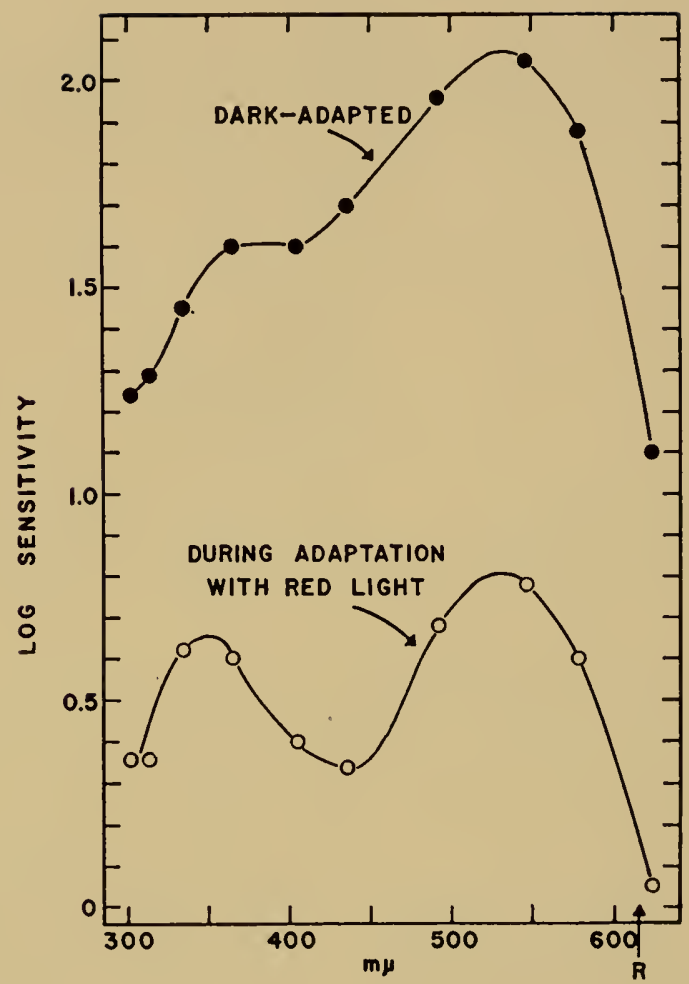

Fig. 2. Alteration of the shape of the spectral sensitivity of the worker honeybee by selective light adaptation. During a constant level of adaptation brought about by red light, the ultraviolet receptor system contributes more prominently to the spectral sensitivity function (open circles) than it does in the dark-adapted eye (filled circles). Ordinate: logarithm of the reciprocal of the relative number of quanta required to produce a retinal action potential of constant size. (From ref. 20).

animal. Although ultraviolet light is several times as effective as green in stimulating certain kinds of behavior, this does not necessarily mean that the compound cyes are several times as sensitive to the shorter wavelengths or contain several times as many ultraviolet as green receptors. In the case of the honeybee the basis for the difference in results obtained with the electroretinogram and the whole organism appears to reside in the integrative action of the central nervous system. On the other hand, differences in the ratios of the heights of the two peaks observed in different electrophysiological experiments on the same species probably reflect variations in the proportion of two types of receptors in the field from which the electrode records. 
The "preference" of an insect for one wavelength or color may be different at different times in the life cycle. Ilse has provided a clear example. Feeding cabbage butterflies (Pieris brassicae) tend to alight on blue or yellow flowers and can be attracted to blue or yellow paper models; green, on the other hand, is not visited (30). Females of the same species when laying eggs, however, seek out green stems and leaves. When paper models are again employed, the insects go to bluegreen and green while ignoring red, yellow, blue, and all shades of gray from black to white (31).

The sensitivity of insects to ultraviolet light clearly does not depend on indirect excitation of a single blue- or green-sensitive receptor through fluorescence of the ocular media. There are several reasons for rejecting this idea. (1) It is difficult to see how the sequence absorption-fluorescence-absorption could be several times more efficient than direct excitation of the visual pigment of a blue or green receptor; yet if there is neither a special ultraviolet receptor nor a single receptor maximally sensitive in the ultraviolet, this is what is implied by the phototactic response curves reproduced in Fig. 1. (2) The repeated success of a number of investigators in training honeybees to search for food at ultraviolet-illuminated feeding dishes suggests that the wavelengths shorter than $400 \mathrm{~m} \mu$ are a distinct color for bees (e.g., 35, 29, 9) . (3) The fact that the wave forms of the electroretinograms are dependent on the wavelength of the stimulus means that different regions of the spectrum exert qualitatively different effects at the level of the receptor process $(56,57,20$, 23). (4) Where electrophysiological techniques reveal the presence of two peaks in the spectral sensitivity function-in the green and the near ultraviolet-it is possible to alter their relative heights by selectively adapting the eye with lights of different wave lengths $(58,59$, 20) (see also Fig. 2).

So far this paper has considered the occurrence of color vision among insects and the question of vision in the ultraviolet. In doing so, several different kinds of evidence were introduced. It will be the task of the following two sections to examine several of these experiments in more detail and to summarize what is known about the visual systems of two species of insect.

\section{The Color Vision of the Honeybee, Apis mellifera}

Color vision has been studied more extensively in the honeybee than in any other insect. Von Frisch (16) showed that bees could be trained to collect food on either blue or yellow cards surrounded by 
an array of gray cards of all shades from black to white. Kühn (35) performed similar training experiments with monochromatic lights and demonstrated that near ultraviolet and blue-green lights also seemed to be distinct colors for bees. Subsequently Hertz (29) presented evidence that blue-green and near ultraviolet are complementary colors for bees. Bertholf (4) also reported success in training bees to different wavelengths. The difficult art of training bees to reveal their sensory capacities has most recently been extended by Daumer (9). Daumer achieved a finer control of the wavelength and intensity of stimulus than earlier workers and tested the ability of bees to discriminate monochromatic lights and known mixtures of monochromatic lights.

The following picture of the color vision system of the bee unfolds from these experiments. The spectrum visible to the honeybee extends from about $300 \mathrm{~m}_{\mu}$ in the ultraviolet to at least $650 \mathrm{~m}_{\mu}$ in the red. Beyond these limits sensitivity decreases, but between these wavelengths there exists the capacity to distinguish colors. For a time it was thought that bees could discriminate only four colors (see 17). Daumer confirmed that there are four spectral regions which bees distinguish with great reliability-near ultraviolet $\left(300-400 \quad \mathrm{~m}_{\mu}\right)$, blue-violet $(400-480 \mathrm{~m} \mu)$, blue-green $(480-500 \mathrm{~m} \mu)$, and green-yellowred (500-650 $\mathrm{m} \mu)$-but he also showed that bees can discriminate between wavelengths within these regions, although with somewhat less precision.

Daumer has also reported a series of experiments with honeybees that are closely analogous to the color-matching experiments encountered in psychophysics. Bees were trained to alight on a feeding dish illuminated from bencath. After a period of training the bees were confronted with a pair of feeding dishes, one illuminated by the training color and the other by a mixture of monochromatic lights. The mixture of wavelengths at the test dish was varied (keeping the total energy constant and equal to the training light), and when the bees went with equal frequency to the two dishes, the test and training lights were considered to be equivalent.

White light for the bee must include some ultraviolet; bees easily distinguished a training light which was "bee-white" (the full spectrum of a xenon arc) from a white test light from which the ultraviolet had been filtered. Equivalence was obtained, however, by adding an appropriate amount of monochromatic $360 \mathrm{~m} \mu$ to the test light. The important features of an experiment such as this are most easily stmmarized by a statement of colorimetric equivalence: 
("bec-white") $=15 \%(360 m \mu)+85 \%$ ("bec-white" - UV)

That is, monochromatic $360 m \mu$ call substitute for the band of wavelengths between 300 and $400 \mathrm{~m} \mu$; a mixture composed of 15 per cent $360111 \mu$ and 85 per cent white (xenon) light from which the ultraviolet has been filtered is matched for bees by the full xenon spectrum.

Similarly, the region 400 to $650111 \mu$ can be replaced by monochromatic $490 m \mu$. In an experinent in which the training light was "beewhite" and the test light a nixture of monochromatic ultraviolet and monochromatic blue-green, Daumer observed that

$$
\text { ("bee-white") }=15 \%(360 m \mu)+85 \%\left(490 m_{\mu}\right)
$$

Or, in other words, $360 \mathrm{~m} \mu$ and $490 \mathrm{~m} \mu$ are complementary colors for honeybees.

In another experiment three wavelengths were employed in the test light, and it was found that

$$
\begin{aligned}
\text { ("bee-white") = } & 55 \%(588 \mathrm{~m} \mu)+30 \%(440 \mathrm{~m} \mu) \\
& +15 \%(360 \mathrm{~m} \mu)
\end{aligned}
$$

It follows from Equations (1) through (3) that

$$
\begin{array}{r}
65 \%(588 m \mu)+35 \%(440 m \mu)=(490 \mathrm{~m} \mu) \\
=\text { ("bee-white" }-\mathrm{UV})
\end{array}
$$

and these rclations, too, are confirmed by experiment.

Mixtures of yellow and near ultraviolet lights produce new colors which honeybees do not confuse with intermediate wavelengths. The fact that these "bee-purples" exist indicates that the color vision of the bee is based on more than two types of receptor. The available behavioral data can be interpreted as indicating the presence of three classes of receptor maximally sensitive in the near ultraviolet, the blue-violet, and the green-yellow-red. The results of Daumer's experiments can be summarized in a preliminary chromaticity diagram (Fig. 3) .

As the foregoing suggests, the narrow band of wavelengths between 480 and $500 \mathrm{~m}_{\mu}$, which is seen by bees as a distinct color, is analogous in several respects to yellow in human color vision. For bees, bluegreen is the region of the spectrum in which colors are least saturated (9), and 480 to $500 m \mu$ is probably where wavelength discrimination is sharpest-where hue changes most rapidly as a function of wavelength. The color "matches" expressed in Eq. (4) suggest that the 


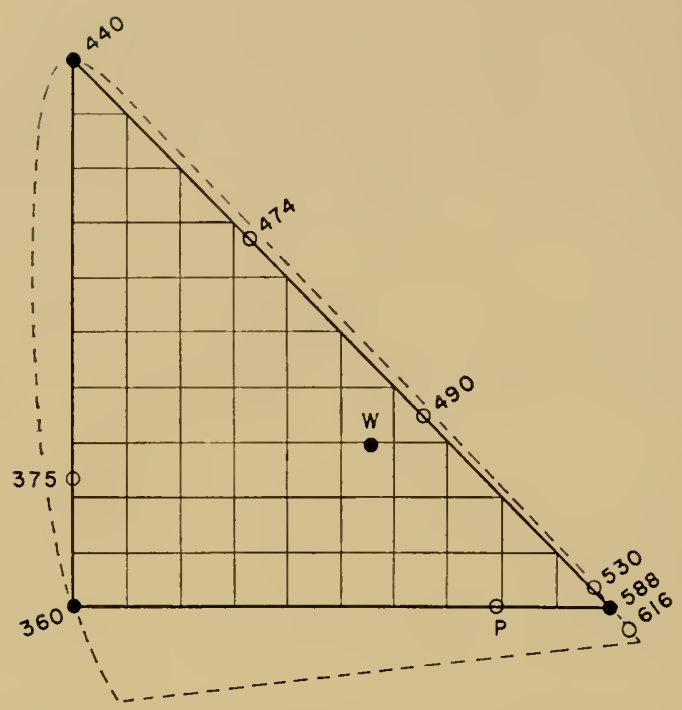

Fig. 3. Tentative chromaticity diagram for the honeybee based on the work of Daumer (9). The bchavioral experiments of Daumer lead to the prediction that any color can be matched for bees by an appropriate mixture of three monochromatic reference stimuli, e.g., ultraviolet $(360 \mathrm{~m} \mu)$, blue-violet $(440 \mathrm{~m} \mu)$, and yellow $(588 \mathrm{~m} \mu)$. Any light, therefore, can be represented in Fig. 3 by a point; for example, "W" is the white of the xenon emission spectrum. The proportions, in fractions of the total energy-the chromaticity coordinates-of the yellow and blue reference stimuli required for a colorimetric match of the light represented by the point are given by the coordinates of the point, $588 \mathrm{~m}_{\mu}$ on the abscissa and $440 \mathrm{~m} \mu$ on the ordinate. Since the sum of the three chromaticity coordinates is 1 , the fraction of the energy at $360 \mathrm{~m} \mu$ can be calculated by difference. The locus of the spectrum is given by the experimental points; however, as is suggested by the dashed line, it may actually lie somcwhat ottside the triangle, for it is questionable whether this kind of experiment is sufficiently precise to reveal the necessity for small negative values of one of the chromaticity coordinates. The complementary to $360 \mathrm{~m}_{\mu}$ is $490 \mathrm{~m} \mu$, for both wavelengths lie on a straight line passing through the white point. Similarly, the point " $P$ " is the "bee-purple" complementary to $440 \mathrm{~m} \mu$ and is composed of 79 per cent $588 \mathrm{~m} \mu$ and 21 per cent $360 \mathrm{~nm} \mu$.

responses to blue-green light occur when excitation is confined (or nearly so) to the middle and long wavelength receptors. A similar narrow region of the spectrum possessing distinct color for bees and exciting both the ultraviolet and blue-violet receptors might well exist somewhere between 440 and $375 \mathrm{~m} \mu$, but this is a region of the spectrum which has not been as adequately explored.

These behavioral experiments imply the presence of receptors maximally sensitive in the near ultraviolet, blue, and green-yellow regions 
of the spectrum. However, other methods must be employed to learn the properties of these receptors. By determining the relative energies necessary to elicit lERG's of constint size, spectral sensitivity curves have been made for both the ocellus and the compound eye of the honeybee. These experiments also reveal the presence of receptor systems maximally sensitive in different regions of the spectrum.

The electrical responses of the compound eyes of worker bees to light are complex in origin. The principal component is a maintained negativity which seems to reflect depolarization of the retinula cells. Neurons in the optic ganglion also contribute to the ERG, notably a negative off-effect which is relatively more prominent at long wavelengths and in the light-adapted eye. However, the wave forms of the responses of dark-adapted compound eyes to short $(1 / 10 \mathrm{sec}$.) flashes can usually be matched approximately at different wavelengths, and these responses have been used as a basis for spectral sensitivity measurements (20).

The dark-adapted compound eye of the worker honeybee is maximally sensitive in the green at about $535 \mathrm{~m} \mu$. Sensitivity extends into the near nltraviolet, sometimes with a second, smaller hump (20). The long wavelength maximum is shown in Fig. 4 (curve 1).

During adaptation with red or yellow light the spectral sensitivity curve is changed. Sensitivity throughout the spectrum drops, but relatively more at the long wavelength end. As the $535 \mathrm{~m} \mu$ maximm subsides, a peak appears in the near ultraviolet at about 340 to 345 $\mathrm{m}_{\mu}$ (20) (Fig. 2). The spectral sensitivity of the eye measured during adaptation with a bright yellow-green light is shown in Fig. 4 (curve 2). Measurements do not extend to longer wavelengths because under such conditions of light adaptation the shapes of retinal action potentials clearly differ at the two ends of the spectrum, and it is impossible to obtain equal effects with widely separated wavelengths.

The complementary experiment-measuring the spectral sensitivity in the presence of an ultraviolet adapting light-does not show such a striking change in the shape of the spectral sensitivity function. There are two reasons for this. First, in the dark-adapted eye the ultraviolet receptor system does not make so prominent a contribution to the ERG as does the green receptor system. Second, the sensitivity of the $345 \mathrm{~m}_{\mu}$ receptor system decreases steadily in the blue and green, whereas the $535 \mathrm{~m}_{\mu}$ receptor system maintains a significant sensitivity through the near ultraviolet. For this reason a yellow adapting light spares the ultraviolet receptor system, but an ultra- 


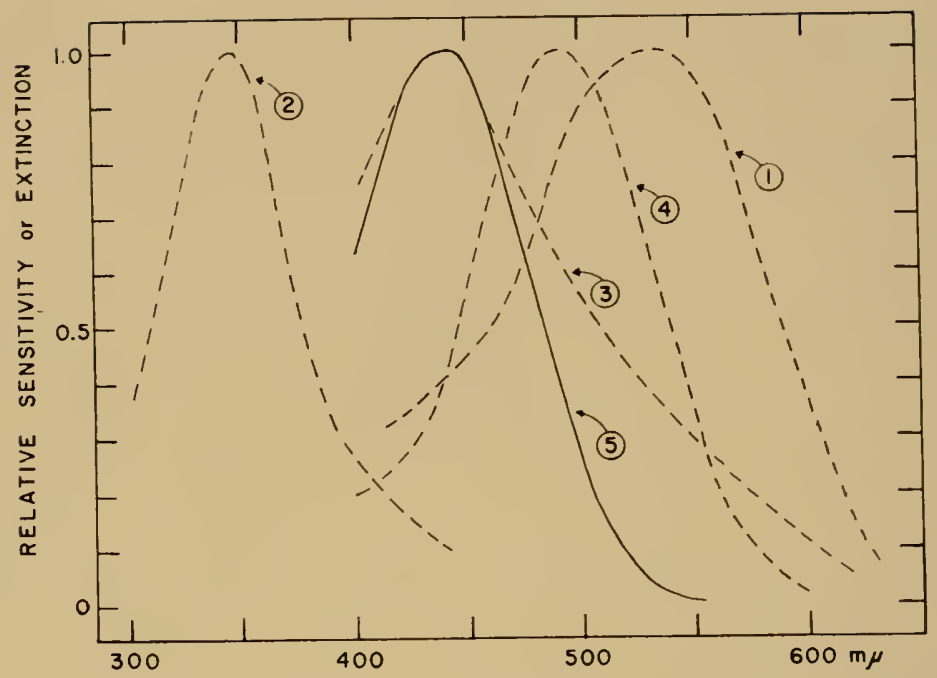

Fig. 4. The spectral sensitivities of the several receptor systems described for the honeybe (broken curves) and a visual pigment from the bee (solid curve). 1, The spectral sensitivity of the dark-adapted compound eye of the worker bec. Maximum, $535 \mathrm{~m}_{\mu}$. From (20). 2. The ultraviolet receptor system of the compound cyc of the worker bee as revealed during adaptation with a bright yellow-green light. The maximum lies near $345 \mathrm{~m}_{\mu}$. A receptor with similar spectral sensitivity is found in the compound eye of the drone and the ocellus of the worker. From (20). 3. Spectral sensitivity of the compound eyes of drones. The maximum is at about $440 \mathrm{~m} \mu$; however, as other preparations may be more sensitive at longer wavelengths, there is evidence that a grecn-sensitive receptor (curve 1 ) is also contributing to this curve. After (19). $f$, The $490 \mathrm{~m} \mu$ receptor of the ocellus of the worker bee. Ifter (23). 5, The absorption spectrum of the light-sensitive retineneprotein pigment extracted from the heads of honcybees. (Ifter 18 and 19).

violet adapting light lowers the sensitivity of both systems (20). There is some evidence that a similar situation prevails in the eye of the cockroach $(56,57,58)$.

In the compound eye of the worker honeybee there is thus evidence for two of the three color receptors postulated by Daumer. There is at this time no conclusive electrophysiological evidence to indicate the existence of a blue-violet receptor in the compound eye of the worker. However, two additional sets of observations suggest that a blue-violet receptor may be present.

In addition to the two receptor systems described for the worker bec, the compound eyc of the drone appears to possess a receptor system maximally sensitive at about $440 \mathrm{~m} \mu$ (19). The contributions of the several receptors to the spectral sensitivity function of the drone vary in different preparations, probably depending on the composition 
of the population of receptors close to the tip of the electrode. Curve 3 in Fig. 1 is an average of the spectral sensitivities (at wavelengths longer than $100(11 \mu)$ of several drones in which the contribution of the green-sensitive receptor system appeared to be small. The interesting feature of this curve is that its maximum is at about 440 $\mathrm{m}_{\mu}$.

The final piece of evidence for a blue-violet receptor is biochemical. The heads of bees contain a light-sensitive pigment formed of retinene $_{1}$ bound to a soluble protein (18). This pigment absorbs maximally at about $440 \mathrm{~m} \mu$, and on exposure to light it bleaches, liberating retinene. It is possible that it is not the only such photopigment present in the bee; it accounts for no more than half the total retinene, for in its isolation much of the retinene originally present in the heads is lost.

The absorption spectrum of the $440 m \mu$ pigment is indicated by the solid curve (curve 5) in Fig. 4. Note that one of the spectral sensitivity functions (curve 3) is also maximal at $440 \mathrm{~m}_{\mu}$. The only function known for retinene is the chromophore of visual pigments of animals. This, and the observation that in bees the retinene is found only in the heads, not the bodies, lend further support to the view that the $440 m \mu$ photopigment of the honeybee is, in fact, a visual pigment. If so, it is the only insect visual pigment that has been described to date.

The ocelli of worker honeybees also contain more than one kind of receptor. The electrical response from the ocellus is diphasic: a rapid positive deflection at "on" is followed by a slower negative wave. In the near ultraviolet region of the spectrum the threshold response consists entirely of the positive component; in the green the threshold response is the slow negative wave. At higher intensities responses evoked by widely separated wavelengths are similar and include both components of the ERG (23).

Spectral sensitivity curves based on either component have two maxima, one in the near ultraviolet, similar to the maximum described for the compound eye, and one in the blue-green at about $490 m \mu$. The latter is shown by curve $4 \mathrm{in} \mathrm{Fig.} \mathrm{4.} \mathrm{The} \mathrm{relative} \mathrm{heights} \mathrm{of} \mathrm{these}$ two peaks vary, depending on which component of the ERG the spectral sensitivity function is based (23).

The dorsal ocelli are curious organs which do not resolve images. Although in the worker honeybee they contain two kinds of receptors functioning maximally in different regions of the spectrum, there is no reason to believe that they play a critical role in the discrimination 
of color as studied in behavioral experiments by Daumer and others. They are not necessary for the "preference" for ultraviolet light exhibited in phototaxis (20).

\section{The Color Vision of the Fly, Calliphora}

Although there is no behavioral evidence to show that the blowfty Calliphora has color vision, analysis of the retinal action potential of this insect indicates the requisite sensory mechanisms for discrimination of wavelengths. As was mentioned above, the spectrum visible to Calliphora extends from the near ultraviolet through the red (Fig. 5). Autrum and Stumpf (2) found peaks of sensitivity at 540 and $630 \mathrm{~m}_{\mu}$, but these authors did not extend their measurements into the near ultraviolet. Later, measurements of Walter and Dodt (58, 59), showed peaks of sensitivity at about 340, 507, and $630 \mathrm{~m} \mu$. As was discussed above, the height of the ultraviolet maximum varied in different preparations. Both groups of workers agree that at high intensities of stimulation the $630 \mathrm{~m} \mu$ maximum is regularly more prominent, relative to the peak in the green. There remains confusion, however, about the position of the maximum in the green. Autrum (1) reported evidence that at low intensities of stimulation the peak in the green shifts to shorter wavelengths; however, such a shift cannot account for the discrepancy between Autrum's data and the curves of Walther and Dodt $(58,59)$. Judging by the relative sizes of the $630 m \mu$ maxima, the two curves of Walther and Dodt were obtained at intensities above and below those employed by Autrum and Stumpf, yet in both cases the blue-green maximum lies more than $30 \mathrm{~m}_{\mu}$ to shorter wavelengths than where it was reported by Autrum and Stumpf.

The spectral sensitivity function of Calliphora has three peaks. The relative heights of these peaks can vary, suggesting that the compound eye of Calliphora contains three types of receptor maximally sensitive in different regions of the spectrum. The experiments of Autrum and Stumpf (2) with heterochromatic flicker provide independent evidence that hetween 400 and $700 \mathrm{~m} \mu$ the compound eye of Calliphora possesses two receptor systems for differentiating color. These experiments of Autrum and Stumpf did not include the ultraviolet region of the spectrum.

According to Autrum and Stumpf, two lights of the same spectral content flickered alternately elicit a ripple in the retinal acton potential when the difference in intensity is greater than 7 per cent. When certain pairs of colored lights were alternately flickered, there 


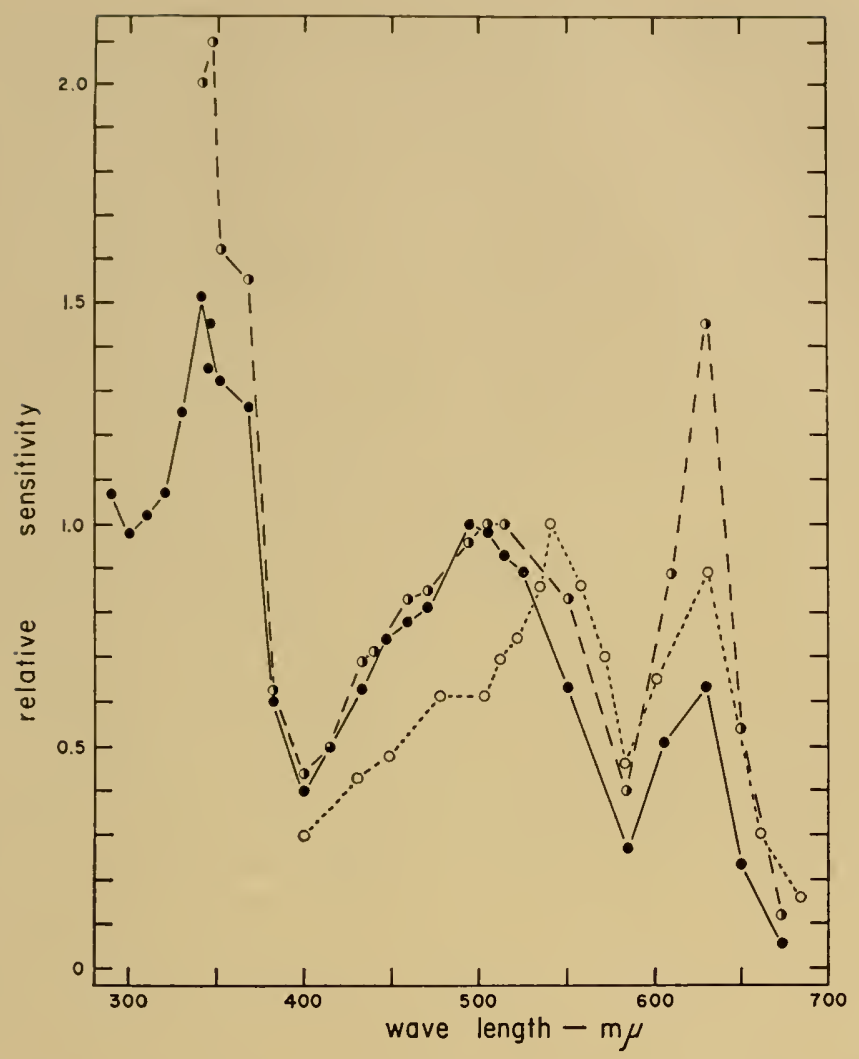

Fig. 5. The spectral sensitivity of the compound eye of the blow-fly, Calliphora. Open circles and dotted curve, from Autrum and Stumpf (2). Half-filled circles and dashed curve, from Walther and Dodt (58). Filled circles and solid curve, from Walther and Dodt (59). The height of the $630 \mathrm{~m} \mu$ peak increases (relative to the maximum in the green) with increasing intensity. The height of the ultraviolet maximum varies in different preparations, but it does not vary in a con. sistent fashion as a function of intensity.

was always a response-the oscillation could be varied in height but could not be totally abolished by altering the relative intensities of the test lights. The size of this "llicker potential" was a minimum when the test lights were the same relative intensities as was required to produce equal-size responses from the dark-adapted eye. The size of the flicker potential was used by Autrum and Stumpf as a measure of the ability of Calliphora to discriminate pairs of wavelengths.

The salient results of these experiments are briefly as follows. Yellow light (ca. $580 \mathrm{~m} \mu$ ) was not distinguished from the light of an in- 
candescent bulb, but both were differentiated from all other wavelengths between 400 and $690 \mathrm{~m} \mu$. Within the red $\left(630-690 \mathrm{~m}_{\mu}\right)$ color differentiation was very small; however, wavelengths in this region were differentiated from all shorter wavelengths, particularly blue and blue-green. Similarly, blue and green were most sharply distinguished from red, least well from closely neighboring wavelengths.

These experiments support the interpretation that the blue-green and red maxima in the spectral sensitivity function are due to the presence of two different color-receptor mechanisms. The argument is further bolstered by Autrum and Stumpf's finding of an anomalous fly which was both color-blind and lacked the $630 \mathrm{~m} \mu$ maximum.

Unfortunately, the picture is not quite so clear as the prececling paragraphs imply. Autrum (1) subsequently described the spectral sensitivity (actually height of ERG for an equal-energy spectrum) of a white-eyed mutant of Calliphora. Genetically this form differs from the wild-type at a single locus, and presumably it lacks only ommochromes, the accessory shielding pigments of the ommatidia (51). White-eyed flies possessed a single maximum of sensitivity at $520 \mathrm{m \mu}$ (again, measurements did not extend below $400 \mathrm{~m} \mu$ ). The whiteeyed flies were $10^{3}$ to $10^{4}$ times as sensitive as the wild-type, and Autrum concluded that this was because in the absence of ommochromes light which enters an ommatidium obliquely can pass laterally through the eye and stimulate adjacent ommaticlia. He further concluded that the $630 \mathrm{~m} \mu$ peak in the wild-type was clue to transmission (although perhaps multiple reflections would be more likely) of long wavelengths by the granules of red shielding pigments in the retinulae. He did not, however, relate this interpretation to his experiments with flicker potentials, particularly to the red-eyed fly that lacked the $630 \mathrm{~m} \mu$ maximum. Autrum also stated that in the wild-type fly the increasing prominence of the $630 \mathrm{~m} \mu$ maximum with increasing intensities of stimulation is a direct consequence of the "transparency" of the ommochromes to red light. He did not state, however, how he reached this conclusion.

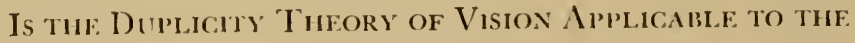

EYES OF INSECTS?

The thought has recurred mumerous times that the compound eyes of insects, like the eyes of vertebrates, possess two classes of receptors, onc functioning at low intensities but reporting no information about color, the second sensitive at higher levels of illumination and sub- 
serving color vision. In the vertebrate eye, the shift in wavelength of maximum sensitivity to longer wavelengths with increasing intensity of illumination-the Purkinje shift-is one manifestation of this duplicity.

Hanström (25) described retinula cells with long and short axons and suggested, with no supporting physiological evidence, that these wo types of cells are analogous to rods and cones. Fingerman and Brown $(14,15)$ reported a Purkinje shift in the "spectral response curve" of Drosophila. These authors used narrow bands of wavelengths as test lights, with, however, no regard for their relative intensities. The "spectral sensitivity" curves of Fingerman and Brown are therefore in reality some composite function of the emission spectrum of their lamp and the action spectrum for phototaxis. More to the point, the consistent decrease in the wavelength of maximum "sensitivity" with decreasing intensity of illumination claimed by these authors is not supported by their data. With relative intensities of $1,1 / 10,1 / 100$, and $1 / 1000$ their curves peaked at 520,540, 478, and 490-502 $\mathrm{m} \mu$, respectively.

There are several other observations which bear on this problem. Weiss (62) reported that although at low intensities $365 \mathrm{~m} \mu$ was more effective for phototaxis than the broad band of wavelengths between 470 and $528 \mathrm{~m} \mu$, at high intensities the order of attractiveness was reversed. This report stands without confirmation and warrants further investigation.

Analysis of electroretinograms recorded from the compound eye of the worker honeybee provides no evidence of a shift in spectral sensitivity analogous to the Purkinje shift in the first three logarithmic units above the threshold of the retinal action potential (20). That is, curres of magnitude of response vs. $\log$ intensity are virtually parallel at different wavelengths. Similar results have been reported for the cockroach (57). On the other hand, Autrum (1) reported that in Calliphora similar response-energy curves are not parallel and that the slopes are shallower at shorter wavelengths; therefore, the wavelength of maximum sensitivity should depend on the magnitude of the ERG's on which the spectral sensitivity curve is based. As was discussed above, the maximum was found by Autrum at $540 \mathrm{~m} \mu$. at high intensities, and the spectral sensitivity curves appeared to broaden and the peaks to shift to shorter wavelengths as the threshold of the ERG was approached. Prompted by these data, Schneider (49, 50) used a behavioral response to a striped drum illuminated with intensities which were presumably below the threshold of the retinal 
action potential and found the peak of spectral sensitivity of "Rezeptors für dlas 'Dämmerungssehen'" at $480 \mathrm{~m} \mu$. Because Autrum and Schneider measured totally different responses, it is unfortunate that Schneider did not repeat his experiment at higher intensities. His interpretation is perhaps becloucled by the fact that Walther and Dodt $(58,59)$ do not report that the maximum in the blue-green shifts about as a function of intensity.

The extension of the Duplicity Theory, with its numerous and rather specific comnotations, to the compound eyes of insects seems unwarranted on the basis of present evidence. The visual systems of insects present problems in abundance, and these will not be readily solved by forcing meager clata to fit descriptions of the vertebrate eye.

\section{The Morphology of Compound Eyes}

The anatomical identities of the specific color receptors of insects are not known. The compound eyes of insects are composed of several hundred to several thousand onmatidia. Each ommatidium is a long, slender roughly cylindrical structure composed of two concentric layers of cells. On the axis of the ommatidium is the rhabdom, presumably the site of the primary photochemical event. Surrounding the rhabdom are the cell bodies of the retinula cells, approximately eight in number. The electron microscope reveals that the rhabdom is composed of rhabdomeres, specialized medial borders of the retinula cells. The pigment cells surround the retinula cells and optically isolate the ommatidia. Distally each ommatidium is capped by a corneal facet and a transparent crystalline cone. Proximally each retinula cell is believed to send an axon through the basement membrane into complex synaptic relations with nerve cells of the optic lobe.

In 1826 Johammes Müller formulated the mosaic thcory of vision by compound cyes in which he suggested that the individual ommaticlia respond as units. This thcory has had general acceptance since the work of Exner (12). Recently, however, de Vries and Kuiper (53) report that the visual angle of an ommatidium is about eight degrees, several times wider than previously supposed. These authors also present optical evidence that the individual rhabdomeres of an ommatidium (of a fly?) can be separately illuminated, and they suggest that the basic receptor unit may be the retinula cell rather than the ommatidium. On morphological grounds this argument is reasonable for flies. The rhabdomeres of dipterans are anatomically 
distinct $(22,66,8,13,67)$, and it is generally stated that there is a nerve fiber from each retinula cell. In the four other orders of insects [Odonita (22), Orthoptera (13), Lepidoptera (13), and Hynenoptera (21)] that have been examined with the electron microscope, however, the rhabdom is a fused structure, and it is not at all certain that an excitation arising in one of the rhabdomeres would be transmitted exclusively through the corresponding retinula cell. Furthermore, counts of ganglion cells in the lamina ganglionaris of the honeybee indicate probably no more than one second-order unit lor each ommatidium (43), an observation which suggests that even if the individual retinula cells are capable of responding independently, their fibers converge extensively at the first synapse. It seems not to be the case, however, that all the receptor axons from a given ommatidium synapse with the same ganglion cell, a relation indicating that the receptive fields of the ganglion cells may be larger than single ommatidia (eg. 25).

The ommatidia of Limulus respond as units (40). However, the presence of the eccentric cell-a neuron which appears to have migrated out into the layer of sense cells (4I) - may make the horseshoe crab something of a special case. There are no comparable electrophysiological observations of individual ommatidia of insects.

Hecht and Wolf (27) found that the visual acuity of the honeybee, as determined by the optomotor response, corresponds to the interommatidial angle. Similar results have been reported for other insects by other workers. As the visual fields of adjacent onmatidia are now reported to overlap extensively (53), this correlation may be a coincidental result of sharpening of contrast based on such a neural mechanism as the inhibitory interactions between ommatidia described for Limulus (26).

There is good evidence that the receptors for color, whether they be single ommatidia, retinula cells, or groups of retinula cells, may not be distributed uniformly throughout the compound eye. According to Rokohl (45), only the clorsal-posterior ommatidia of the eye of the back-swimmer (Notonecta) subserve color vision; the rest of the eye is color-blind. Similarly, Walther (57) finds that a high sensitivity to ultraviolet light is limited to the dorsal half of the eye of the cockroach (Periplaneta), whereas a green-sensitive receptor is found in all parts of the eye. In the honeybee the spectral sensitivity depends to some extent on the position of the electrode in the eye, but the distribution of receptors seems to be different from that observed in the cockroach and has not been adequately mapped (20). 
It should be further pointed out that the distribution of color receptors may (45) or may not $(57)$ be correlated with externally visible features such as the color of the accessory pigmentation.

\section{The Significance of Sensitivity to Near Ultraviolet Light}

Unlike vertebrates, insects appear to have exploited the shortest wavelengths present in their environment. Because of a filter of ozone in the upper atmosphere, virtually no far ultraviolet light (wavelengths shorter than about $300 \mathrm{~m} \mu$ ) reaches the surface of the earth (47). Not surprisingly, the cornea of insects is transparent through the near ultraviolet but absorbs strongly below about 300 $m_{\mu}(23)$.

It has been suggested that in some apposition eyes visual acuity may be limited by the diffraction of light $(3,52)$. These calculations were for visible wavelengths, but it is possible that $340 \mathrm{~m}_{\mu}$ might have been more appropriate. However, not enough is known about the anatomical identity and distribution of color receptors in the layer of retinulae to pursue this argument further.

It is probably posing something of a false issue to ask why insects see in the ultraviolet. The appropriate question is why vertebrates do not. The visual pigments of vertebrates have a significant sensitivity to the near ultraviolet. However, the yellow lenses of vertebrates tend to prevent deep violet and ultraviolet light from reaching the visual cells $(54,55)$. As Wald $(54)$ has pointed out, this restriction of the visible spectrum was probably introduced in the vertebrate eye to relieve chromatic aberration, a problem which is not met in compound eyes.

The high effectiveness of ultraviolet in the action spectrum for phototaxis (see Fig. 1) raises an interesting question. It is curious that the small amount of ultraviolet light available to insects is apparently such a significant part of their enviromment. Some che to the nature of this problem appears in a recognition of the mutual interactions that have occurred in the evolution of insects and angiosperms. As von Frisch has said: "The colors of flowers have been developed as an adaptation to the color sense of their visitors. It is evident that they are not designed for the human eye."

Lutz (39) stiggested that variations in the amount of ultraviolet reflected from lloral parts might produce patterns of color which could not be appreciated by the human eye but which might be of the utmost importance to the plant's insect pollinators. Measurcments of the ultraviolet reflectance of petals have confirmed the 
existence of concealed patterns of color $(44,37,10)$, and recently Daumer (10) has demonstrated that honeybees are, in fact, guided by these patterns in their search for nectar and pollen.

\section{REFERENGES}

1. Autrum, H., Biol. Zentr., 74, 515-524 (1955).

2. Autrum, H., and Stumpf, 11., Z. vergleich. Physiol., 35, 71-104 (1952).

3. Barlow, 11. B., J. Exptl. Biol., 29, 667-67t (1952).

4. Bertholf, L. M., J. Agr. Research, 42, 379-419 (1931).

5. Bertholf, L. M., J. Agr. Research, 43, 703-713 (1931).

6. Bertholf, L. M., Z. v'ergleich. Physiol., 18, 32-64 (1932).

7. Cameron, J. W. M., Can. J. Research, 16 sect. D (11), 307-342 (1938).

8. Danneel, R., and Zeutzschel, B., Z. Naturforsch., J2b, 580-583 (1957).

9. Daumer, K., Z. vergleich. Physiol., 38, 413-478 (1956).

I0. Daumer, K., Z. vergleich. Pliysiol., 41, 49-110 (19:8).

11. Donner, K. O., and Krigat, G., Arkiv. Zool., 42A (14), 1-7 (1950).

12. Exner, S., Die Physiologie der fazettierten Augen von Krebsen und Insckten, Franz Deuticke, Vienna (1891).

13. Fernández-Morán, H., Exptl. Cell Rescarch, suppl. 5, 586-64t (1958).

14. Fingerman, M., and Brown, F. A., Jr., Science, 116, 17I-172 (I952).

15. Fingerman, M., and Brown, F. A., Jr., Physiol. Zool., 26, 59-67 (1953).

16. von Frisch, K., Zool. Jahrb., Abt. Allgem. Zool. Physiol. Ticre, 35, 1-182 (1914).

17. von Frisch, K.. Bees, their rision, chemical senses, and language, Cornell University Press, Ithaca, New York (1950).

18. Goldsmith, T. H., Proc. Nat. Acad. Sci. U. S., 44, I23-126 (1958).

19. Goldsmith, T. H., Ann. New York Acad. Sci., 74, 223-229 (1958).

20. Goldsmith, T. H., J. Gcn. Pliysiol., 43, 775-799 (1959-60).

21. Goldsmith, T. H., unpub.

22. Goldsmith, T. H., and Philpott, D. E., J. Biophys. Biochem. Cytol., 3, 429-440 $(1957)$.

23. Goldsmith, T. H., and Ruck, P. R., J. Gen. Physiol., 41, 1171-1185 (1957-58).

24. Hamilton, W. F., Proc. Nat. Acad. Sci. U. S., 8, 350-353 (1922).

25. Hanström, B., Z. vergleich. Physiol., 6, 566-597 (1927).

26. Hartline, H. K., Wagner, H. G., and Ratliff, F., J. Gen. Physiol., 39, 651-673 (1955-56).

27. Hecht, S., and Wolf, E., J. Ccn. Physiol., 12, 727-760 (1928-29).

28. Heintz, E., Insectes Socioux, 6. 223-229 (1959).

29. Hertz, M., J. Exptl. Biol., 16, 1-8 (1939).

30. I1se, D., Z. vergleich. Physiol., 8, 658-692 (1928-29).

31. Ilse, D., Nature, 140, 544-545 (1937).

32. Ilse, D., Nature, 163, 25.5-256 (1949).

33. Jahn, T. L., J. N. Y. Enlomol. Soc., 54, 1-8 (1946).

34. Koehler, O., V'erhandl. deut. zool. Ges. Leipzig, 29, 83-91 (1924).

35. Kühn, A.. Z. vergleich. Physiol., 5, 762-800 (1927).

36. Kuwabara, M., J. Fac. Sci. Hokkaido Univ., Ser. V'I,13, 458-46.1 (1957).

37. Lotmar, R., Z. vergleich. Plyysiol., 19, 673-723 (1933).

38. Lubbock, Sir John, Ants, bees, and wasps, a record of obscruations on the habits of the social Hymenoptera, pp. 152-J68, E. P. Dutton and Co. (1929).

39. Lutz, F. E., Ann. New York Acad. Sci., 29, 181-283 (1924).

40. MacNichoJ, E. F., Exptl. Cell Research, suppl. 5, +11-425 (I958).

40a.Mazokin-Porshniakov, G. A.. Biofizika, 4, 46-57 (19.59).

41. Miller, W. H., J. Biophys. Biochem. Cytol., 3, 42I-428 (1957).

42. Peterson, A., and Haeussler, G. J., Amm. Entomol. Soc. Am., 2I, 353-375 (1928). 
43. Phillips, E. F., Proc. Acad. Nat. Sci. Phila., 57, 123-157 (1905).

44. Richtmyer, F. K., J. Opt. Soc. Am., 7, 151-168 (1923).

45. Rokohl, R., Z. vergleich. Physiol., 29, 638-676 (1941-42).

46. Sander, W., Z. vergleich. Physiol., 20, 267-286 (1933).

47. Sanderson, J. A., and Hulburt, E. O., Radiation Biology (A. Hollaender, ed.), vol. II, Chap. 3, McGraw Hill Book Co., New York (1955).

48. Schlegtendal, A., Z. vergleich. Physiol., 20, 545-581 (I933-34).

49. Schneider, G., Verhandl. deut. zool. Ges. Tübingen, 1954, 346-35I (1954).

50. Schneider, G., Z. vergleich. Physiol., 39, 1-20 (1956).

51. Tate, P., J. Genet., 48, 338-342 (1948).

52. de Vries, H., Progress in Biophysics, 6, 207-264 (1956).

53. de Vries, H., and Kuiper, J. W., Ann. N. Y. Acad. Sci., 74, 196-203 (1958).

54. Wald, G., Documenta Ophthalmol., 3, 94-134 (1949).

55. Wald, G., J. Opt. Soc. Am., 42, 171-177 (1952).

56. Walther, J. B., Biol. Zentr., 77, 63-104 (1958).

57. Walther, J. B., J. Insect Physiol., 2, 142-151 (1958).

58. Walther, J. B., and Dodt, E., Experientia, 13, 333 (1957).

59. Walther, J. B., and Dodt, E., Z. Naturforsch., 14b, 273-278 (1959).

60. Weiss, H. B., Entomol. News, 54, 152-156 (1943).

61. Weiss, H. B., J. N. Y. Entomol. Soc., 52, $267-271$ (1944).

62. Weiss, H. B., Soraci, F. A., and McCoy, E. E., Jr., J. N. Y. Entomol. Soc., 50, $1-35$ (1942).

63. Weiss, H. B., Soraci, F. A., and McCoy, E. E., Jr., J. N. Y. Entomol. Soc., 51, 117-131 (1943).

64. Weiss, H. B., McCoy, E. E., Jr., and Boyd, W. M., J. N. Y. Entomol. Soc., 52 , $27-43$ (1944).

65. Wolken, J. J., Trans. N. Y. Acad. Sci., 19, 315-327 (1957).

66. Wolken, J. J., Capenos, J., and Turano, A., J. Biophys. Biochem. Cytol., 3, 441 448 (1957).

67. Yasuzumi, G., and Deguchi, N., J. Ultrastructure Research, 1, 259-270 (1958). 


\title{
ELECTROPHYSIOLOGICAL EVIDENCE FOR A MECHANISM OF COLOR VISION IN THE GOLDFISH ${ }^{1}, 2$
}

\author{
E. F. MACNichoL, JR. \\ M. L. WOLBARSHT \\ H. G. WAGNER
}

Thomas C. Jenkins Department of Biophysics, Johns Hopkins University, Baltimore, Maryland, and Physiology Division, Naval Medical Research Institute, National Naval Medical Center, Bethesda, Maryland

\section{INTRODUCTION}

The act of vision in the vertebrates encompasses not only the absorption of visible radiation by a photopigment and its transduction into some sort of electrochemical energy by a mosaic of photoreceptors, but also the encoding of the messages from these elements for transmission to the brain by a relatively small number of optic nerve fibers. In the brain the visual message is presumably processed further and compared with previous information stored in the memory. Signals are derived and transmitted to the various effector organs which change the organism's relationship to its environment. The objective of visual research is to understand the whole system in terms of the detailed operation of the individual functional units of which it is composed.

Certainly the logical and systematic way to begin such an investigation is at the beginning, with the light-sensitive cells of the retina. Wald (34) has given an excellent summary of what is known of the photopigments contained in some of the receptor cells and has pointed out that the pigments in other receptors have not been separated and purified successfully because of their chemical similarity and very small concentration in the retinal extracts.

These are just the pigments we need to know about if we are to understand color vision. Fortunately, Rushton (22) and his co-

${ }^{1}$ The opinions or assertions contained herein are the private ones of the authors and are not to be construed as official or reflecting the views of the Navy Department or the naval service at large.

2 This research was supported in part by National Science Foundation Grant G-7086. 
workers have developed an ingenious method for studying those pigments in the intact eye by means of retinal spectrophotometry. By this means they have identified several cone pigments in subjects having both normal and attenuated color vision, have measured the rate of bleaching and regeneration of these pigments, and have related the sensitivity of the eye to the amount of pigment present.

We still do not know how the light-induced chemical reactions of the pigment cause the excitation of the neuronal elements of the retina. This is one of the most challenging problems in visual research. Another problem concerns the mechanism of amplification of the light-induced signal and its conduction along the receptor cell. We only know that the absorption of a very few light quanta causes the release of much larger amounts of energy in the retina (31) and that the signal can be transmitted a distance of 100 microns or more, from the outer segments of the rods, in as little as a few milliseconds (14) .

In order to intercept the visual message at various points along its path, the methods of electrophysiology have been extensively used and progressively refined. Large diffuse electrodes placed on opposite sides of the retina will record a series of complex potential changes in response to light. These electrical recordings are called electroretinograms (ERG) and have been studied extensively under a variety of conditions. In the beginning, when the methods were crude, it was thought that practically all of the electrical activity of the retina originated within the receptors (15). As electrical localization methods were improved, mainly through the introduction of microelectrodes which record activity from highly localized regions, a large part of the electrical activity was found to originate in the intermediate neuronal layers of the retina. At the present time none of the recorcled activity can be said with certainty to arise within the receptors themselves, although the possibility has not been definitely ruled out $(2,3,4,21,29)$. It may be that the conductive pathway for electrochemical changes lies within the receptors instead of on their surface membranes, as is the case with ordinary neurons. Such a possibility is suggested by the fact that the receptors are ontogenetically derived from ciliated cells and that electron microscopy has demonstrated typical ciliary fibrils embedded in the cytoplasm of the rod and cone cells. Conduction might take place along these fibrils (23).

The most peripheral localized sign of electrical activity discovered in the retina to date was found some years ago by Svaetichin (24), 
in the region of the second-order neurons with which the receptors synapse. He showed that slow potential clianges in response to flashes of light could be obtained from localized regions in the isolated retinas of fishes by means of ultra-micropipette electrodes. The intraretinal potentials were of two types and were quite different from anything previously reported. One of these potentials, called by Svactichin the luminosity or " $L$ " response, appeared abruptly when the electrode reached a certain depth in the retina. The approach to this region was usually signaled by the sudden appearance of a negative resting potential of 25 to $35 \mathrm{mv}$ and large negative going (hyperpolarizing) electrical changes during illumination. This type of response is shown in the "uper record in Fig. 1, which shows the effects of equal cnergy flashes of different wavelength. The " $L$ " response has subsequently been demonstrated in a wide variety of fishes (17,

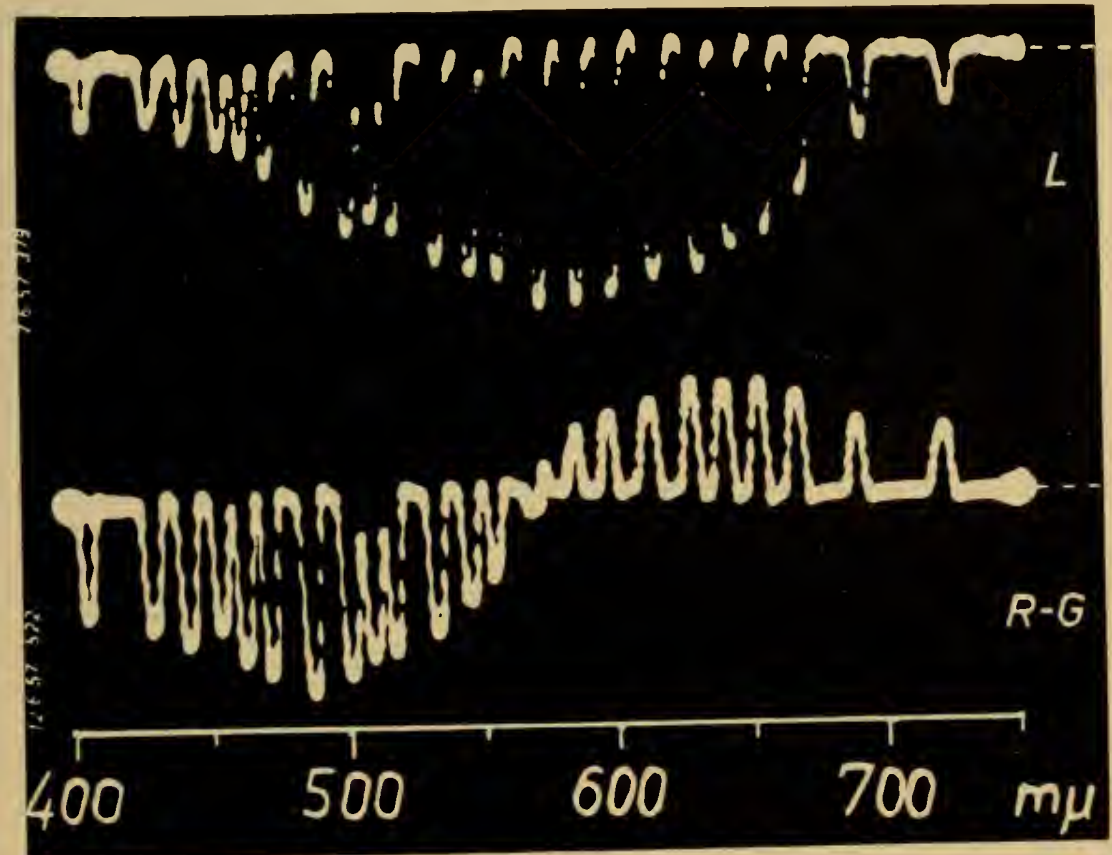

Fig. 1. Luminosity $(L)$ response (top trace) and chromatic $(C)$ response (bottom trace, R-G) recorded from the retina of a marine fish (family Gerridae). The flashes of light for stimulating the retina were obtained by rotating a wheel containing 24 interference filters adjusted by means of neutral density films to give a constant energy spectrum. The wavelength sweep was driven by a voltage obtained from a potentiometer geared to the filter wheel. A full scan required approximately 15 seconds. From Svaetichin and MacNichol (25). 
$19,20,30)$ and in the cat $(3,12,20)$. It is probably present in the retinas of all vertebrates.

The other type of intraretinal response, called the chromatic or " $C$ " response by Svaetichin, was obtained from a slightly more proximal region of the retina. It also appeared abruptly with the sudden appearance of a resting potential. When the retina was illuminated by flashes of light from the short-wave end of the spectrum, the responses were negative and indistinguishable from the " $L$ " type responses. As progressively longer wavelengths were used the responses diminished in amplitude, until a neutral point was reached at which no sustained response could be recorded. At longer wavelengths the response became positive, or depolarizing. This kind of response is shown in the lower record of Fig. 1. Stimulation at the neutral point produced no sustained potential change. Only brief negative and positive transients appeared at the beginning and end of a flash.

Not only was this type of response highly unusual, but it also indicated a possible mechanism for color vision. Furthermore, it has only been seen in certain shallow water fishes, some species of which have been demonstrated to have color vision. [See Walls (38) for a comprehensive review of behavioral studies on color vision in fishes.] On the other hand, frogs and cats, which are the animals commonly used for electrophysiological studies, appear to lack the chromatic response and also have no demonstrable color vision.

Svaetichin's experiments were repeated and confirmed in their main features by several groups of Japanese workers $(19,20,30)$. Svaetichin (24) at first suggested that these responses were recorded directly from the receptors, in this case one or the other of a pair of twin cones, and that the positive and negative potentials were the result of the differential color sensitivities of the two cells comprising the pair. Tomita (27), on the other hand, stated that the potentials arise in more proximal layers of the retina. Subsequently, investigation in which the tips of the clectrodes were localized by means of a dye-marking technique proved the correctness of Tomita's assertion $(16,17,28)$.

Fig. 2, opposite. Structure of goldfish retina (Carrassius auratus), formalin-fixed, Bodian silver-impregnated section of retina on right. Tracing illustrating main cell types in each layer at left. Abbreviations from top to bottom: P. L., pigment layer; V.C.L., visual cell layer with cones shown; O.L.M., outer limiting membrane; O.N.L., outer nuclear layer; O.P.L., outer plexiform layer showing giant horizontal cells (presumed origin of " $L$ " response); I.N.L., inner nuclear layer 


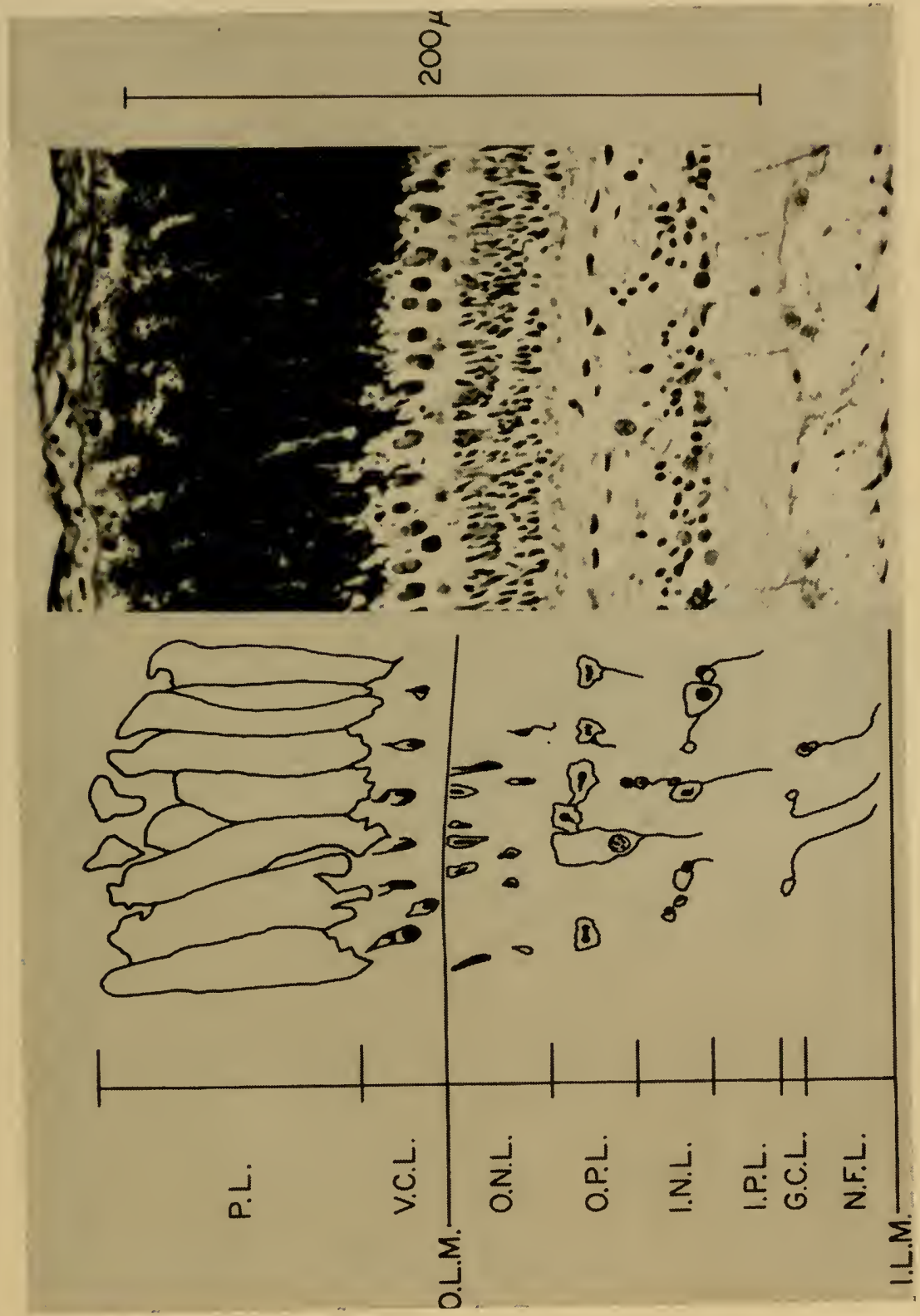

(apparent origin of " $C$ " response); 1.P.L., inner plexiform layer; G.C.L., ganglion cell layer; N.F.L., nerve fiber layer; I.L.M., inner limiting membrane. The section was kindly furnished by Mrs. A. B. Snodgress of the Naval Medical Research Institute. 
The histology of the retina of a shallow water fish is shown in Fig. 2 , together with a schematic drawing of the various cellular components. A dye-marking technique showed the " $L$ " response to appear in the outer plexiform layer in a region just below the synaptic endings of the cones (17). In fishes this region contains a large number of giant horizontal cells whose function is as yet unknown. It is possible that the " $L$ " responses are recorded from within these elements. The chromatic responses were shown to arise at a depth corresponding to the bodies of the bipolar cells in the inner nuclear layer. They could presumably have arisen from within these cells. However, Tomita (27) and Tasaki (26) have advanced persuasive arguments that both the " $L$ " and the " $C$ " responses are extracellular in origin. The situation is by no means clear, so that it is not yet possible to settle the question.

Whatever their origin may be, both the " $C$ " and " $L$ " responses are localized and presumably are related in some way to the generation of impulses in the ganglion cells. Since the axons of these cells make up the optic nerve, their discharges constitute the visual information that is transmitted to the brain. Thus, if the intraretinal responses have anything to do with color vision they must somehow correlate with the patterns of discharge in the ganglion cells. There were two preliminary requirements in selecting an experimental animal for studying this correlation. First, it should have demonstrable color vision. Second, it should be possible to obtain a " $C$ " response as rell as an " $L$ " response and a ganglion cell discharge from its retina. Recently, McCleary (18) has shown by an ingenious autonomic conditioning technique that the goldfish (Carassius auratus) appears to have a well-developed color sense. As will be shown later, both " $C$ " and " $L$ " responses could be obtained from the retinas of this species. Since the goldfish is miversally obtainable and keeps exceptionally well in the laboratory, it appeared to be an ideal experimental animal. Accordingly, the authors have unclertaken an experimental study of its retina. The following is a report of progress in this program to date. The techniques and methods used in accomplishing these studies were detailed in prior reports $(32,33)$.

\section{EXILRIMENTAL RESILTS}

Both the " $L$ " and " $C$ " responses have been recorded from the goldfish retina by means of micropipette electrodes. The responses were qualitatively the same as those described by Svactichin (24). Fig. 3 shows a typical "C" response from the goldfish retina. So far we 


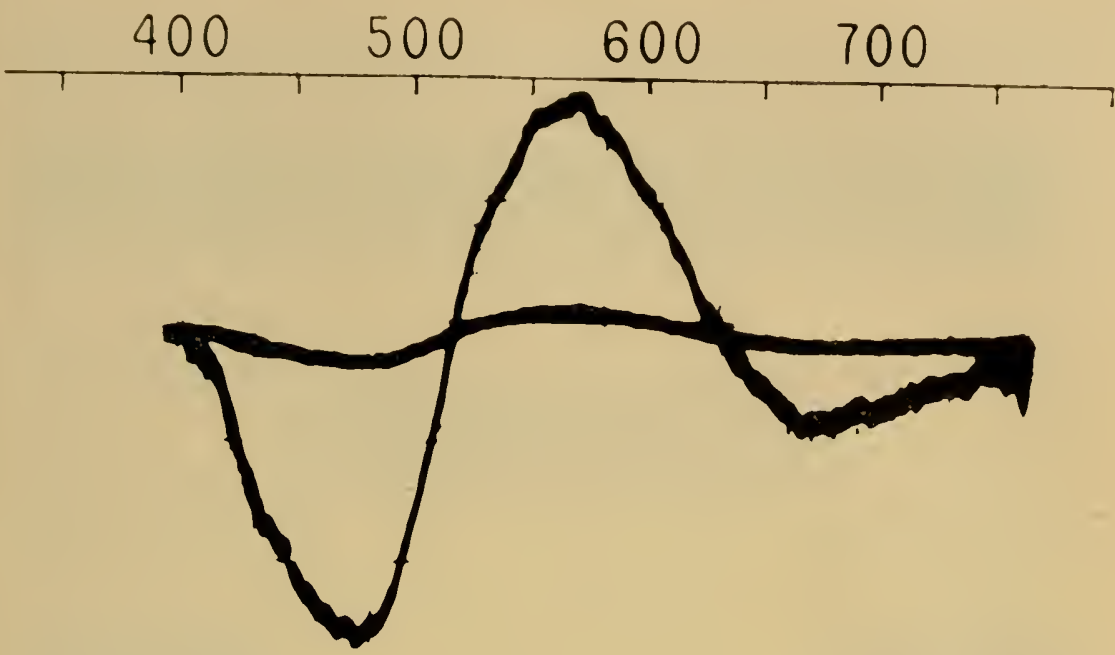

Fig. 3. Intraretinal recording from goldfish. Chromatic $(C)$ type response to a linear change in wavelength as a function of time from 400 to $750 \mathrm{~m} \mu$. The time required for this change was 0.75 seconds. The two traces are simultaneous high- and low-gain records of the same response. d-c recording was used on both channels. The upper straight line represents $0 \mathrm{mv}$, and the lower $100 \mathrm{mv}$, for the low-gain channel. The peak to peak amplitude of the high-gain response was approximately 9 millivolts. The intensity of the stimulus at $600 \mathrm{~m} \mu$ was 30 $\mu$ watts $/ \mathrm{cm}^{2}$. The stimulus was not equal energy. See Wagner, MacNichol, and Wolbarsht (32) for energies at other wavelengths and other details concerning the technique by which this record was made. In this and subsequent records, except where otherwise indicated, the stimulus was circular in outline and $5 \mathrm{~mm}$ in diameter, centered over the electrode.

have not been able to obtain " $C$ " or " $L$ " responses from the goldfish which were sufficiently stable and long-lasting for quantitative studies.

An investigation was then made of the discharges of the retinal ganglion cells. Since micropipettes are not suitable for this purpose, small, insulated metal microelectrodes were used instead. Stable, longlasting responses were obtained, making it possible to study them in considerable detail.

As shown in Fig. 4, most of the units when stimulated with light gave an "on-off" type of discharge pattern, that is, they gave a short burst of impulses when the light was turned on and a brief burst when the light was turned off. This "on-off" pattern is similar to that found 


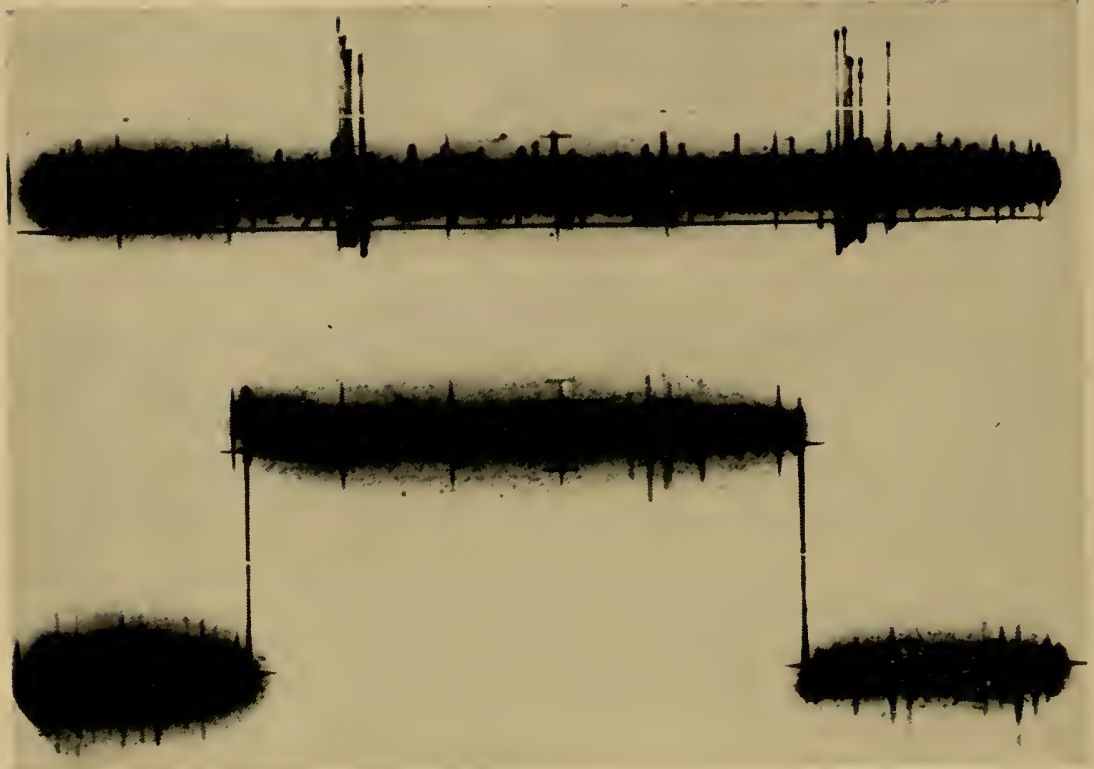

Fig. 4. Typical "on-off" response from retinal ganglion cell. The stimulus was $0.6 \mathrm{sec}$ duration, $700 \mathrm{~m} \mu$ wavelength, $0.7 \mu \mathrm{w} / \mathrm{cm}^{2}$ intensity, $2 \mathrm{~mm}$ in diameter. In this and in subsequent records, the presence of the stimulus is indicated by a step in the signal trace at the base of the record.

in the frog retina. Indeed, it is characteristic of all types of vertebrate retinas $(8,9,10,14)$. However, some units were found that gave a prolonged discharge cluring illumination, and a few which were spontaneously active in the dark were inhibited by light.

Thus far only the "on-off" units have been studied extensively. Many of these gave a response that was dependent upon wavelength in a very unusual way. If a series of flashes having a duration of 0.5 sec or so were presented at regular intervals of a second or two, the "on-off" pattern showed wavelength dependency. This is well illustrated in Fig. 5, where a typical element gave pure "on" responses in the shortwave end of the spectrum and pure "off" responses in the long-wave end. The change from "on" to "off" occurred at an intermediate wavelength. Granit (11) has reported changes in the off/on ratio of the responses of retinal ganglion cells of the cat, which suggest that a similar mechanism may be present to some extent in this animal. DeValois (7), recording from ganglion cells of the lateral geniculate of the monkey, also observed that a change in wavelength conld convert an "on" response into an "off" response. The 

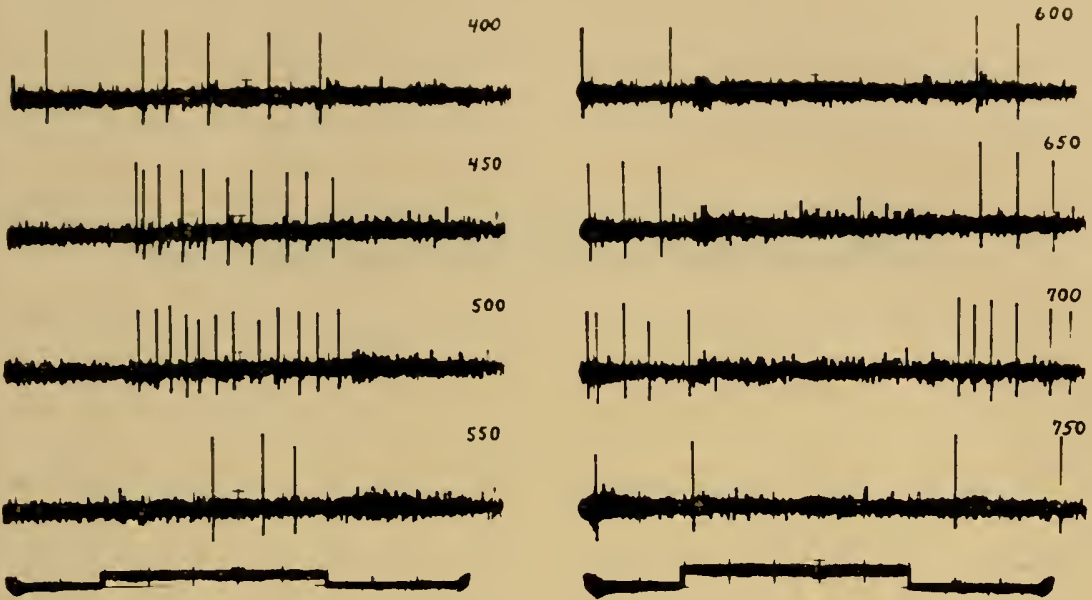

Fig. 5. Variation of response from a single ganglion cell with change in wavelength of stimulus. Each record represents the response to one of a series of stimuli $0.5 \mathrm{sec}$ in duration repeated at 2 -second intervals. Impulses occurring before the onset of the stimulus were responses to a preceding stimulus. Intensity of stimulus at $600 \mathrm{~m} \mu=55 \mu \mathrm{w} / \mathrm{cm}^{2}$. See ref. (31) for relative intensities at other wavelengths.

particular unit illustrated in Fig. 5 had a very abrupt transition. The response shifted from pure "on" to pure "off" with a wavelength change of only $10 \mathrm{ml} \mu$ (Fig. 6).

Here indeed was a pattern of optic nerve discharge that seems to follow the " $C$ " responses. At short wavelengths, where the chromatic response was large and negative, there was a vigorous "on" discharge from the ganglion cell; as the wavelength was increased the number of "on" impulses decreased just as the amplitude of the " $C$ " response decreased. Where the " $C$ " response became zero there was a transition from "on" to "off." Where the amplitude of the depolarizing phase of the " $C$ " response was greatest the number of "off" responses was also maximal. In some units (not illustrated) "on" and "off" responses were both discharged at intermediate wavelengths, with a decrease in the number of "on" responses and an increase in the number of "off" responses with increasing wavelength. The above comparison has as yet only been carried out qualitatively, because we have not been able to study the " $C$ " response in detail.

In order to investigate the processes giving rise to the "on" and the "off" response, the method of constant response was used. That is, the stimulus was adjusted so that a constant response was obtained at each wavelength rather than using a constant stimulus energy and 


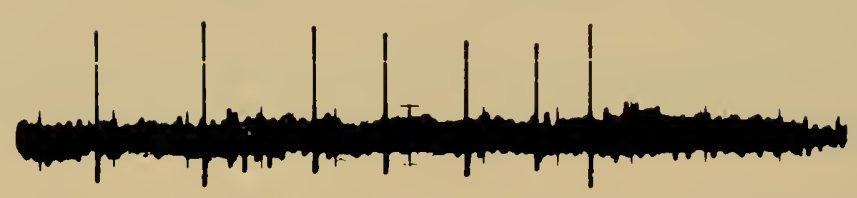

570
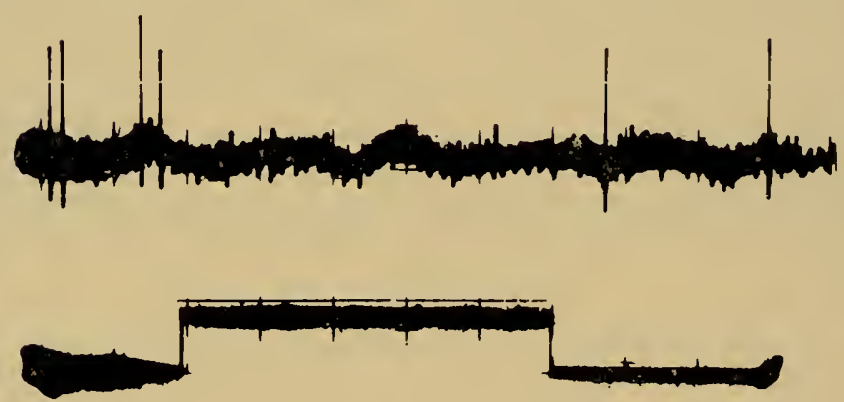

Fig. 6. Variation of ganglion cell response with a small change in wavelength. Same experimental conditions as Fig. 5. Intensity of stimulus, $55 \mu \mathrm{w} / \mathrm{cm}^{2}$.

recording the number of impulses discharged. This procedure has commonly been used in the determination of hmminosity functions and avoids certain difficulties created by the non-linear (quasi-logarithmic) nature of the response mechanism. The minimum intensity flash necessary to evoke the discharge of one impulse was the most commonly used criterion for threshold response, although the thresholds for two or more impulses were sometimes used where the presence of spontaneous activity tended to introduce an appreciable number of false positive responses. Occasionally, inhibition of pre-existing activity was used as a criterion for threshold. There seems to be no essential clifference in the results obtained with any of these response criteria.

Fig. 7 shows a threshold intensity versus wavelength plot for a typical ganglion cell. An "on" response was obtained from 400 to $610 \mathrm{~m}_{\mu}$. At $640 \mathrm{~m} \mu$ no "on" response was obtainable at any intensity; but a higher "off" threshold could be obtained out to $750 \mathrm{m \mu}$, 


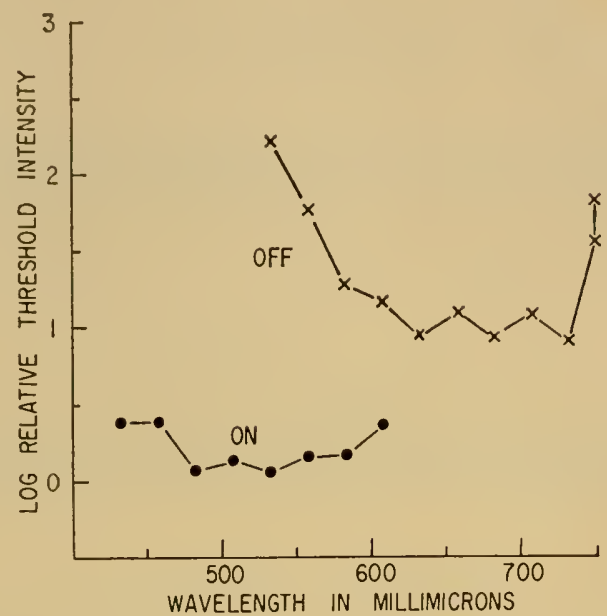

Fig. 7. Intensity necessary to elicit various types of threshold responses from a single ganglion cell at different wavelengths. The duration of the stimulus was 1.0 sec. $0 \log 11 n i t s=5.5 \times 10^{2} \mu \mathrm{w} / \mathrm{cm}^{2}$ for all wavelengths. In this and in subsequent illustrations, the thresholds of "on" responses are indicated by circles and "off" responses by crosses.

the long wavelength limit of our monochromator. Beyond $610 \mathrm{~m} \mu$ no "on" response could be obtained even at the maximum output of our stimulator, although a vigorous "off" discharge was present. An "off" response could be obtained at wavelengths shorter than $600 \mathrm{~m} \mu$ even in the presence of a vigorous "on" discharge. In this unit the "off" discharge appeared to be combined with a strong inhibitory effect on the "on" discharge. However, the "on" discharge was not associated with inhibition of the "off" discharge, which could be obtained over nearly the entire spectrum.

Further evidence that it is in fact the "off" process that inhibits the "on" response was obtained from the experiment represented in Fig. 8. Here typical blue sensitive "on" and red sensitive "off" responses were found (solid black lines). After adaptation to deep red light, the sensitivity of the "off" response was greatly decreased while the threshold of the "on" response was slightly increased (dotted lines). However, an "on" threshold could now be obtained throughout the entire visible spectrum. Thus, the effect of the adaptation to red light was both to depress the sensitivity of the "off" process and to release the "on" process from inhibition in the red end of the spectrum. After dark adaptation, the "off" response returned to its original threshold and the "on" process became slightly more sensi- 


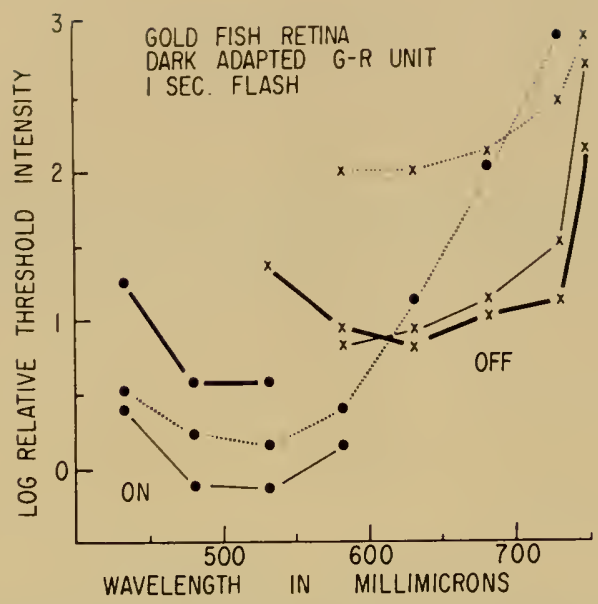

Fig. 8. Intensity necessary to elicil "on" and "off" threshold responses from a single ganglion cell at different wavelengths before, during, and after exposure to a red-adapting light. Heavy solid line indicales thresholds before adaptation, dotted lines thresholds during red-adapting light, thin solid line thresholds approximately 10 minutes after extinction of the adapting light. Test stimuli 1.0 sec in duration were repeated at 2 -sec intervals. For intensity of test stimulus 0 $\log$ units $=2.3 \times 10^{-2} \mu \mathrm{w} / \mathrm{cm}^{2}$ for all wavelengths. Energy of red-adapting light, $5.2 \mu \mathrm{w} / \mathrm{cm}^{2}$. Duration of adaptation $=27 \mathrm{~min}$. Adapting light was obtained by filtering incandescent lighı $2850 \mathrm{~K}$ color temperature through a W'ratten $898 \mathrm{~B}$ filter.

tive but was again inhibited completely in the red portion of the spectrum. Thus, inhibition appears to be an active process which always appears when there is an "off" discharge. More evidence for this assertion is shown in Fig. 9. Here the wavelength was kept constant in a region giving a low "on" threshold. As the intensity was increased, an "off" discharge was produced. When the "off" discharge became very vigorous, the "on" response was completely inhibited. Fig. 10 illustrates an experiment in which both the "off" response and the inhibition of activity during illumination were employed as threshold criteria for the long-wave process. Beyond $625 \mathrm{~m} \mu$ the thresholds obtained using these two criteria were essentially indistinguishable. Between 575 and $600 m_{\mu}$ the inhibition during illumination had a higher threshold than the "off" response, presumably because of the very vigorous "on" excitation present in this region of the spectrum. The "off" response is intimately associated with inhibition cluring illumination not only of the "on" response but of the "off" response to a preceding stimulus and of spontancous activity as well. Therefore, the "off" discharge itself is believed to be the excitatory 

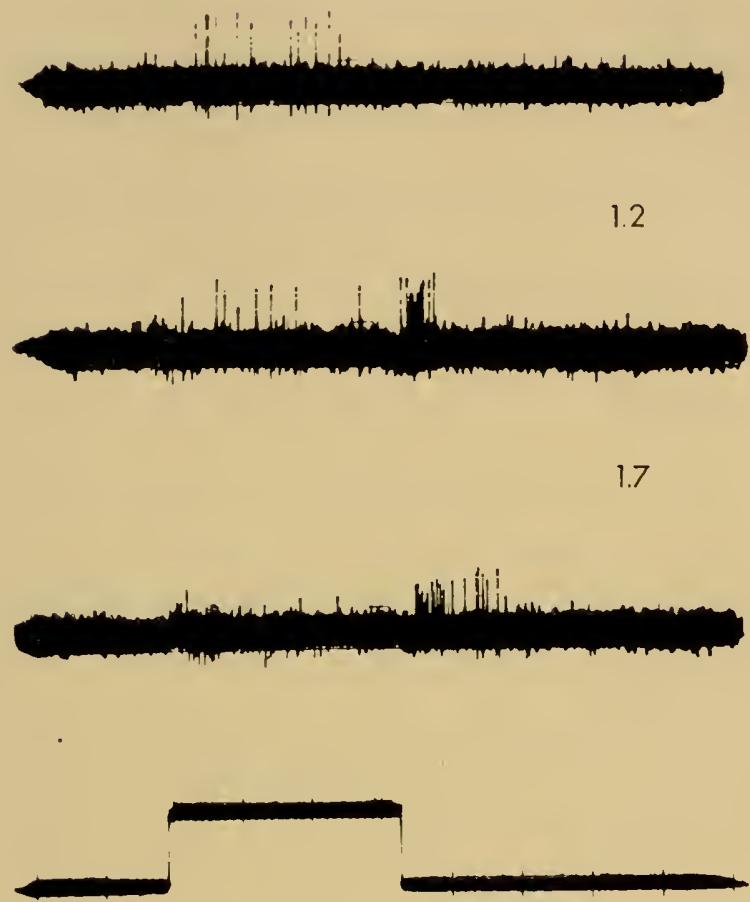

$550 \mathrm{m \mu}$

Fig. 9. Variation of ganglion cell response with change in intensity of stimulus. $0.5 \mathrm{sec}$ stimulus repeated at 2 -sec intervals. Log intensity of stimulus is in upper right of each record. $0.0 \log$ units $=1.7 \mu \mathrm{w} / \mathrm{cm}^{2}$.

effect of a sudden release of inhibition. It is neither due to excitation during darkness nor does it depend upon any pre-existing impulse activity, whether it be spontaneous or in response to light. It may resemble the classic phenomena of "anodal break excitation" and "postinhibitory rebound."

Whatever the mechanism of its production, it is evident that the "off" response represents a definite kind of message carried by many of the fibers of the optic nerve. We clo not yet understand its significance.

In the examples shown thus far, the "on" response has represented a short-wavelength sensitive process and the "off" response one with 


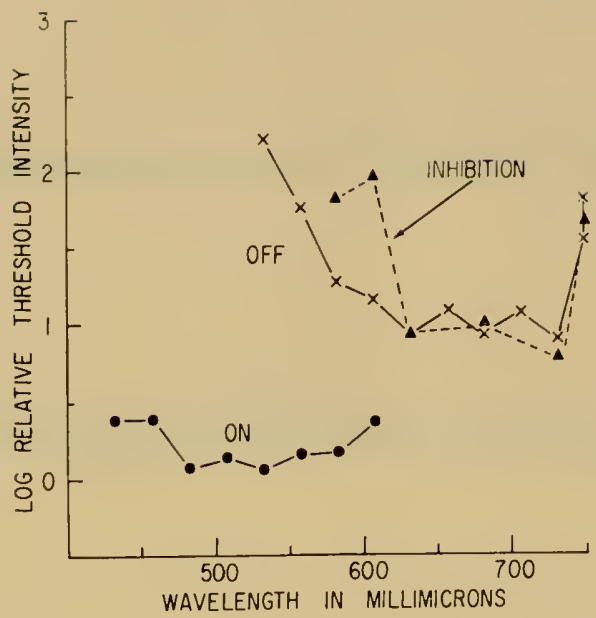

Fig. 10. Intensity necessary to elicit various types of threshold responses from a single ganglion cell at different wavelengths. Experimental conditions, scales, and symbols as in Fig 7 . Thresholds for inhibition of discharge during illumination plotted as triangles.

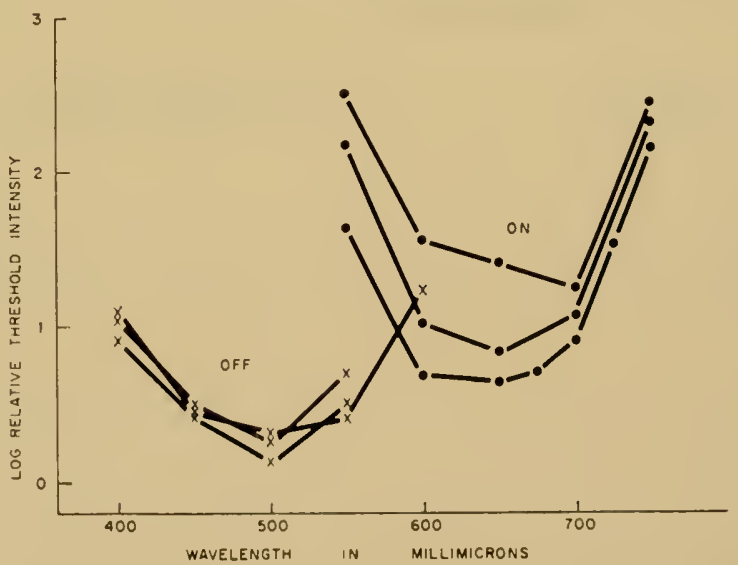

Fig. 11. Intensity necessary to elicit various types of threshold responses from a single ganglion cell at different wavelengths. Stimuli 0.5 sec in duration were repeated at 2-sec intervals. Intensity plotted on a $\log$ gुuantum scale. 0 log units $=2.6 \times 10^{11}$ quanta $/ \mathrm{sec} / \mathrm{cm}^{2}$ for all wavelengths. NOTL: During the 30 minutes necessary to determine the 3 sets of each curve the "off" components changed slightly or not at all, while the "on" component became progressively more sensitive with little change in shape, a result confrming earlier evidence of a degree of independence of the two processes. 
maximum sensitivity in the long-wave region. Many units have been found in which the converse is true. A typical response of this type is shown in Fig. 11. Still other units have been found in which the "on" and "off" responses had nearly identical spectral sensitivities. Such a unit is shown in Fig. 12.

Thus, it appears that the "on-off"-type ganglion cells giving rise to the messages in the individual optic nerve fibers can be classified into at least three subtypes: (1) a short-wave "on," long-wave "off" unit; (2) a short-wave "off," long-wave "on" unit; and (3) a unit which gives both "on" and "off" responses more or less equally throughout the spectrum.

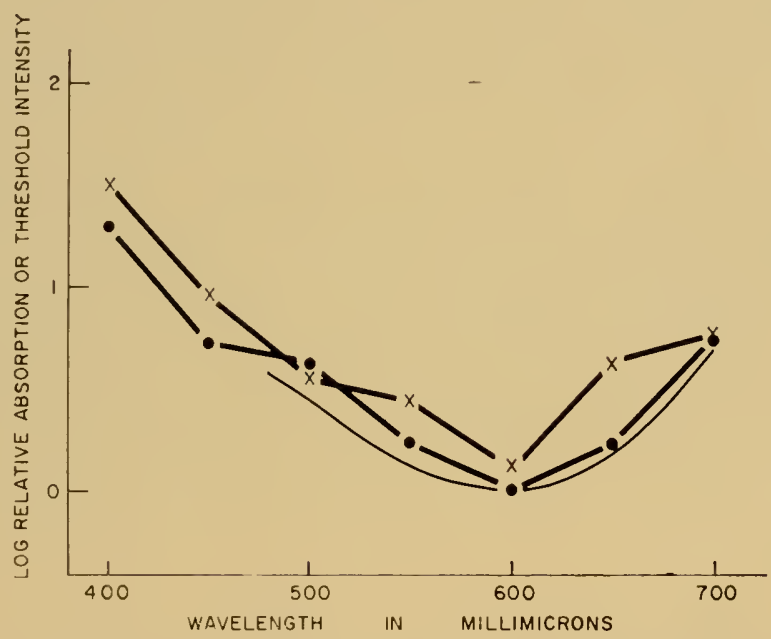

Fig 12. Intensity necessary to elicit various types of threshold responses from a single ganglion cell at different wavelengths. Experimental conditions same as those for Fig 11. Intensity plotted on a $\log$ quantum scale. $0 \log$ units $=2.6$ $\times 10^{11}$ quanta $/ \mathrm{cm}^{2} / \mathrm{sec}$. The thin line is a curve calculated from Dartnall's nomogram (5) for a photopigment having a wavelength maximum of $600 \mathrm{~m} \mu$.

\section{Discussion}

The ganglion cell spectral sensitivity curves determined by the constant response criterion discussed previously can be taken as a working indication of the action spectra of the photopigments in the primary receptors $(36,37)$. Although the action spectrum of a photopigment in a receptor may differ from the absorption spectrum of the same photopigment after it is extracted and purified, the differences tend not to be large $(1,2,6,35)$. Thus, some correlation between 
the spectral sensitivity curves of the ganglion cells in the goldfish and the absorption spectra of the various visual pigments should be possible. Furthermore, the visual pigments thus far studied in pure form have very similar absorption spectra. The whole absorption curve of any one of them can be obtained from that of any of the others by a suitable transformation which involves only the wavelength of maximal absorption as a parameter. It is likely that previously unidentified visual pigments are sufficiently similar to those already known that their absorption curves can also be calculated from this transformation. The nomogram constructed by Dartnall (5) is particularly convenient for this purpose. By its use the absorption curves of a number of hypothetical visual pigments have been obtained and compared with the sensitivity data of the retinal ganglion cells.

In Fig. 13 the sensitivity response data for the short wavelength inhibitory system (data identical to those in Fig. 11) have been fitted to a visual pigment having its wavelength maximum at $500 \mathrm{~m} \mu$. It will be seen that the agreement is satisfactory, suggesting that this may indeed represent a distinct receptor system, the response function of which is defined by a visual pigment very similar to rhodopsin. It may not be identical to rhodopsin, since extracts of the retinas of most freshwater fish contain retinene ${ }_{2}$ instead of the retinene ${ }_{1}$ chromophore characteristic of rhodopsin (36). The effect of this difference is to shift the absorption maximum of a photopigment some 20-22 $\mathrm{m} \mu$ towards longer wavelength, assuming the same opsin protein in the

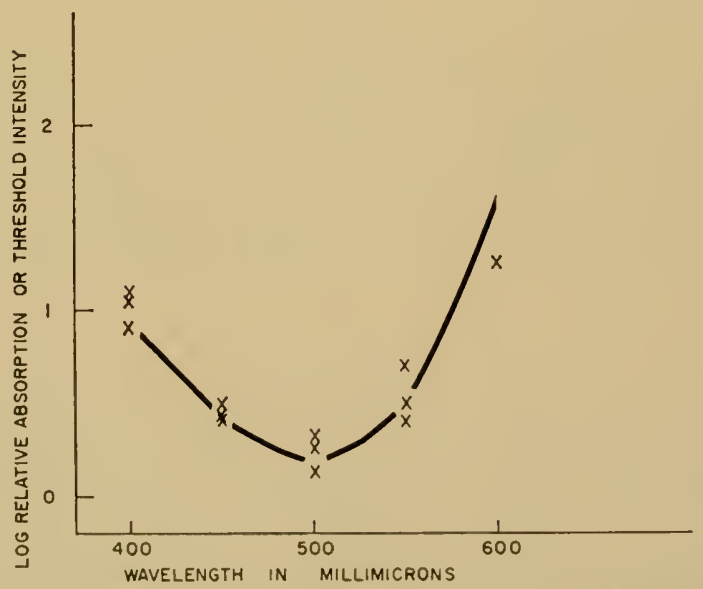

Iig. 13. Threshold detcrminations associated with short-wavelength process from Fig. 11, compared with a curve (heavy line) calculated fiom Dartuall's nomogram (5) with a wavelength maximum of $500 \mathrm{m \mu}$. Intensity scale same as in Fig. 11 . 
two situations. Different opsins conld, however, introduce much larger shifts. It is therefore conceivable that a retinene $e_{2}$ opsin photopigment with an absorption spectrum identical to that of rhodopsin could exist. No conclusion on this point can be reached on the basis of sensitivity data alone.

Fig. 12 shows the correlation that exists between the absorption spectrum of a hypothetical visual pigment with its maximum at 600 $m \mu$ and the achromatic ganglion (type 3) response system. Within the experimental error the agreement is good enough to suggest a second photopigment.

The long wavelength system showing excitation in Fig. 11 has its maximum around $650 \mathrm{~m} \mu$ and is compared in Fig. 14 to a visual pigment having a maximum at the same wavelength. The agreement here is much less satisfactory than in the previous two cases; the sensitivity response function is much narrower but is symmetrical with respect to the nomogram.

No visual pigments having maxima at $600 \mathrm{~m} \mu$ or at $650 \mathrm{~m} \mu$ have been isolated so far. However, Wald (36) synthesized a retinene ${ }_{2}$ cone opsin photopigment he called cyanopsin with a maximum at $622 \mathrm{~m} \mu$. There seems no reason to reject the possibility of other retinene-opsin photopigments having absorption maxima at other wavelengths. In fact, Hanaoka and Fujimoto (13) have identified pigments having absorption maximum at $640 m \mu$ in single cones of the carp retina by a microspectrophotometric technique. However,

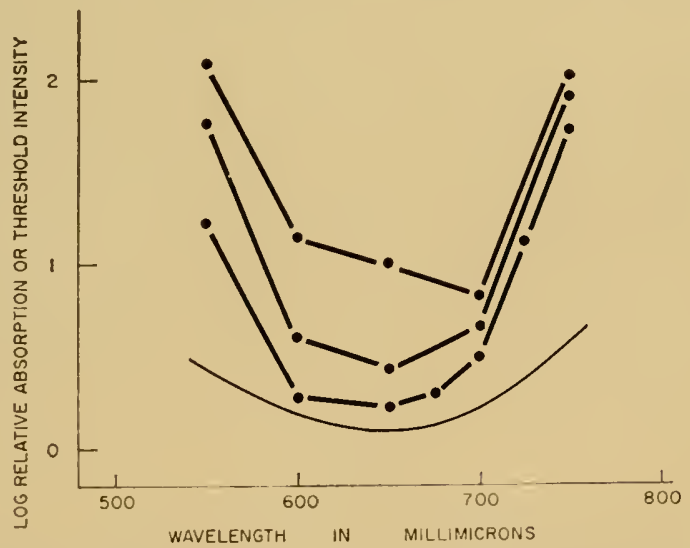

Fig. 14. Threshold determinations (heavy lines) associated with the long-wavelength sensitive process from Fig 11 compared with a curve (thin line) calculated from Dartnall's nomogram (5) with a wavelength maximum of $650 \mathrm{~m} \mu .0 \log$ units on intensity scale $=6.6 \times 10^{11}$ quanta $/ \mathrm{sec} / \mathrm{cm}^{2}$ for all wavelengths. 
the lack of agreement observed in Fig. 14 could indicate either a different kind of photopigment, or possibly, that the response function is derived from interaction of two or more receptor systems. These arguments apply equally well to the other two systems. However, the present figure demands some explanation if we are to interpret these in terms of known photopigments. A modified response could either be caused by a pigment unlike the retinene-opsin series or it could result from the interference of stable screening pigments. A third explanation is that perhaps nemonal interaction occurs in some manner in which subtraction takes place, to leave a residual having its maximum at $650 \mathrm{~m} \mu$.

No definite answer can be given now as to which of these possibilities is responsible, or if indeed some mechanism not yet considered is operative.

\section{Conclusions}

In the goldfish retina electrophysiological evidence can be found for color vision. When taken together, the chromatic (" $C$ ") and luminosity (" $L$ ") responses recorded in the intermediate layers of the retina contain sufficient information to permit the animal to distinguish various colors as well as brightness. The wavelengthsensitive "on" and "off" responses from the retinal ganglion cells should be adequate to transmit this color information to the brain. Furthermore, the sensitivity curves obtained from these responses suggest the possible presence in the receptors of three photopigments with absorption maxima at 500, 600, and $650 \mathrm{~m} \mu$. The 500 and $600 \mathrm{~m} \mu$ pigments can be adequately fitted to curves of the rhodopsin type calculated from Dartnall's nomogram (5). The $650 \mathrm{~m} \mu$ pigment has a much narrower absorption curve than the theoretical one.

At present many problems remain unsolved. The actual absorption curves of the pigments must be measured. The ganglion cell responses must be more closely correlated with the " $C$ " and " $L$ " responses. The color-discriminating ability of the goldfish must be stuclied in more detail to allow accurate comparisons with the electrophysiological data.

\section{REFERENCES}

1. Arden, G. 13., J. Pliysiol., 123, 377-385 (195i).

2. Brindley, (. S., Physiology of the retina and the visual patharay, Edward Armold, Lud., London (1960).

3. Brown, K. ' $\mathrm{l}$., and W'iesel, 'T'. N., Am. J. Ophlhalmol., 46, No. 3, Pt, 2, 91-96; (19:58).

4. Byzow, A. L., and Vtina. I. A., Biophysics (U. S. S. R.) (English Translation), 4, 64-76 (1959). 
5. 1)artmalt, 11. J. A., Brit. Med, Bull., 9, 21-31 (1953).

6. Denton, E. J., and Wyllic, J. H., J. Physiol., 127, $81-89$ (195.)).

i. De Valois, R. I.., Smith, C. J., Kitai, S. T., and Karoly, I. J., Science, 12'i, 238-239 (1958).

8. 1)omncr, K. L., J. Ploysiol., 122, 524-537 (1953).

9. Granit, R., Acla Physiol. Scomd., 2, 93-I09 (1911).

10. Granit, R., Acta Physiol. Scand., 3, $318-328$ (19.12).

11. Granic, R., Brit. J. Ophthalmol., 32, 550-55t (1948).

12. Grüsser, O. J., Natururiss., 44. 522 (1957).

13. Hanaoka, T., and liujimoto, K., Japan. J. Physiol., 7, 276-285 (1957).

14. Hartline, H. K., Am. J. Physiol., 121, 400-415 (1938).

15. Kïhne, W., and Steiner, J., Unters. Physiol. Inst. Heidelb., 4, 64-170 (1881).

I6. NacNichol, E. F.. Jr.. Nel'herson, L., and Siactichin, G., Symposium on visual problemes of colour. Paper No. 39, Tedlington, England (1957).

17. MacNichol, E. F.. Jr., and Svaetichin, G., Am. J. Ophthalmol., 46, No. 3, Pt. 2, 26-40 (1958).

18. McCleary, R. A., and Bernstein, J. J., Physiol. Zoöl., 32. 284-292 (1959).

19. Mitarai, G., and Yagasaki, Y., Amn. Rep. Research Inst. Environmental Med., Nogoya Lnivo. 54-64 (1956).

20. Motokawa, K.. Oikawa, T., and Tasaki, K., J. Neurophysiol., 20, $186-199$ (1957).

21. Ottoson, D., and Sraetichin, G., Acta Physiol. Scand., 29, Suppl. 106, 538-564 (1953).

22. Rushton, W. A. H., this symposium.

23. Sjöstrand, F. S., J. Cellular and Comp. Physiol., 42, 45-70 (1953).

24. Svaetichin, G., Acta Physiol. Scand., 39, Suppl. 134, 17-46 (1956).

25. Sraetichin, G., and MacNichol, E. F., Jr., Anm. N. Y. Acad. Sci., 74, 389-404 (1958).

26. Tasaki, K., Aich. ital. biol., 98, 81-91 (1960).

27. Tomita, T., Japan. J. Physiol., 7, 80-85 (1957).

28. Tomita, T., Murakami, M., Sato, Y., and Hashimoto, Y., Japan. J. Pliysiol., 9, 63-70 (1959).

29. Tomita, T., and Torihama, Y., Japan. I. Plysiol., 6, $118-136$ (1956).

30. Tomita, T., Tosaka, T., Watanabe, K., and Sato, Y., Japan. J. Plyysiol., 8, 41.59 (1958).

31. Wagner, H. G., and Hagins, W. A., 3rd Am. Mtg. Biophysical Society (1959) (Abstract).

32. Wagner, H. G., MacNichol, E. F., Jr., and Wolbarsht, M. L., J. Gen. Physiol., 43 No. 6 Suppl., 45-62 (1960).

33. Wagner, H. G., and Wolbarsht, M. L., Am. J. Ophthalmol., 46, No. 3, P't. 2, 46-55 (1958).

34. Wald, G., this symposium.

35. Wald, G., and Brown, P. K., Science, 127, $222-226$ (1958).

36. Wald, G., Brown, P. K., and Smith, P. H., Science, 118, 505-508 (1953).

37. Wald, G., Brown, P. K., and Smith, P. H., J. Gen. Physiol., 38, 623-681 (1954. 1955).

38. Walls, G. L., The terlebrate eye and its adaptize radiations, Cranbrook Inst. of Science, Bloom field Hills, Mich. (1912).

\section{DISCUSSION}

Dr. Buck: I would like to ask Dr. MacNichol about the off period. Was I correct in thinking that the red band continued to show spikes for a long period and then stopped when you turned the red light on?

DR. MaCNichol: If you run the experiment under conditions in which 
you keep it light-adapted, with strong illumination being presented at intervals, in some units if we just turn off all the light after awhile you just stop getting any activity. There are other units which instead of responding with a fairly steady train of impulses during the on and off situation, like I showed you in the slide, give bursts, an on-burst and an off-burst and nothing in between. There are still other units which are spontaneously active in the dark which will be excited to more activity during the flashes of light at one wavelength. This activity will be inhibited at other wavelengths. We use this on inhibition instead of the off response as a criterion for threshold, and the kind of curves you get is the same.

DR. Buck: Actually, the question I had in mind did not require such a detailed answer. I am intrigued at the idea you get impulses in the dark after red flashes. Does this mean that they are sceing red in the dark?

Dr. MACNichol: Well, you can't keep them in a rosy glow indefinitely because as they dark-adapt the situation changes. This is a condition in which the flashes are presented sufficiently frequently to keep the animal at a fairly constant level of light adaptation.

Dr. Buck: Is there any other instance known where you have this sort of negative vision?

Dr. MacNichol: Well, this isn't negative vision exactly. We have a whole population of ganglion cells, and some of these ganglion cells are excited when the light goes on at one end of the spectrum and inhibited when the light goes on at the other end of the spectrum. You have also other ganglion cells in which just the reverse takes place. I suppose as one studies the properties of this population one would find out just how this could fit into a system of color perception. We are now in the process of gathering the evidence. We would like to get a lot more evidence in before we do much speculating on this point.

Dr. Hartune: I can answer Dr. Buck's question partially. All the vertebrate retinas tested have 'on' response ganglion cells and 'off' response ganglion cells. The eye of a scallop, Pecten, has a double retina composed of two layers of primary receptors. One layer is an 'on' retina, and the other is an 'off' retina. So 'on' and 'ofl' responses may be fairly universal in visual systems.

DR. Rushtox: Donner and I found that ganglion cells could receire contributions from receptors which were shown by the Stiles-Crawford effect to be cones (yellow-sensitive) and rods (blue-sensitive). The spectral sensitivity of these ganglion cells was intermediate between that of the rods and the cones alone, and it was unaffected by adaptation to colored lights. The results followed quantitatively from the assumption that each receptor output is a lincar function of the logarithm of the light absorbed by that receptor, and that the ganglion cell is excited by the weighted mean of these two outputs.

For the effect of adaptation upon one excitability will be to shift the 
whole $\log$ sensitivity curve up or down by a certain amount. And the effect upon the other will be to shift its curve by a different amount. So the eflect upon the curve of the weighted mean (which is the ganglion sensitivity) will be a vertical shift by an amount which is the weighted mean of the two receptor shifts. It is clear that this purcly vertical shift will be appreciated as "no change in spectral sensitivity with adaptation to colored lights." And this is what Donner and I found happened with the rod-plus-cone input of our ganglion cells. This is why I feel that a spectral sensitivity which does not change in chromatic adaptation cannot exclude the possibility that it is compounded of mixed pigments and mixed receptors.

DR. MACNichol: We are very tentative about describing these pigments and I made my apologies. We did it saying that this is the best we can do at the present time. Now I am not quite clear. If you change the relative proportions of these two pigments and then sum up the logarithms, you get a curre which does not shift its maximum over?

Dr. Rushton: If the curves just go up and down you won't. If you alter the proportions at which you are going to add them, it will. But dark adaptations only send them up and down and do not alter the proportions.

Dr. Wolbarsht: Yes, but suppose one is sitting still, and the other is moving. What do you do with it then? In our data, we have two threshold curves from the same cell. Their peaks are widely separated. The relative sensitivity of one curve changes while the relative sensitivity of the other curve is standing still, yet the shape of the curve that is moving stays constant. That seems to indicate that there is no interaction.

Dr. Rushton: No.

Dr. Wolbarsht: Well, that's what we have.

Dr. Rushton: You don't prove it really.

Dr. MACNichol: I can't argue with you until I have worked this thing through. I guess we will have to work it through.

Dr. Thumans: When the light is off in the on-off experiments, is it absolute darkness?

Dr. MACNichol: It is absolute darkness. Well, it's absolute darkness as good as our shutter is. That is $7 \log$ units.

Dr. Kasha: I'd like to ask if there are any electron microscope studies on the eye retinal structure showing some kind of trend with evolutionary complexity of the animal.

Dr. HartLiNe: Yes, there are numerous ones. The arthropods show the samic general structure that Grossman has shown for the insects, the microvilli of the retinular cell surface. The scallops have laminations not unlike the vertebrate rods, again derived from cilia. There have been numerous such studies.

Dr. Linschitz: I'd like to come back to the question of energy migration in the rods. Sjöstrand's electron microscope clata show that the rod microstructure consists of a pile of laminations, each of which has a typical sand- 
wich structure, made up presumably of lipid-protein layers. There are also indications that processes extend from the edge of each layer or plate, and make contact with the base of the rod. The rhodopsin may have an important structural role. The whole pattern suggests that the lamina may themselves have some essential or rudimentary properties of nerve fibers. Changing the configuration of the rhoclopsin may casily cause changes in charge or polarization of the corresponding plate, which may be conducted latcrally along the plate and finally to the base of the rod. The sensitivity of the cell to light presumably absorbed randonly among pigment molecules may be accounted for possibly in this way.

Dr. NacNichol: Well, there are several things here. Onc is, is this clearly established? There are plenty of other pictures. also by competent electron microscopists, who say that these are individual discs. There is another point, and that is that one can follow both in the rods and in the cones the structure of the original cilia. If you cut through in the rods you see very nicely the typical 9 paired fibril structure with the two in the middle. It looks just identical with the ciliary structures you find in the sperm tail, for example. This can be detected along most of the length of the rods and also in the cones. Villegas has some very nice electron micrographs which indicate that these things go right down through the conter of the cones. Certainly, it is not inconceivable that the propagation is down through the internal fiber, which would presumably not give you a large charge separation that you can detect with certainty. But this is speculative; we have no evidence. 


\section{SUMMARY \\ Bentley Glass \\ Department of Biology, The Johns Hopkins Unirersity}

It is next to impossible to think of life originating or existing under terrestrial circumstances except through the agency, remote or immediate, of light. All organisms, as we know them, require energy in chemical form and control the storage, release, and utilization of that energy by means of complex protein molecules which function as enzymes. To acquire energy and to trap it in suitable chemical compounds is the primary business of life, most effectively done by absorbing radiant energy. An enormous range in frequency and wavelength of solar radiation bathes our earth, yet most of this is of no avail to life. The shortest wavelengths, highest in energy, cannot be trapped by living mechanisms. Such radiation destroys the enzymes and mutates the genes. The greatest part of the ultraviolet, also lethal, is absorbed by the upper atmosphere. The invisible wavelengths longer than light waves are constituted of quanta of too little energy to drive the machinery of metabolism. In the single octave of radiation with wavelengths between $3500 \mathrm{~A}$ and $7000 \mathrm{~A}$, comprising the near ultraviolet and visible portions of the spectrum, living organisms find it possible to subsist between disintegration and inertia. Life is thus ultimately a photochemical phenomenon.

True, there are chemosynthetic bacteria, which get along quite readily without light. These organisns in fact obtain energy for the reduction of carbon dioxide and the synthesis of their organic compounds by oxidizing simple inorganic compounds, either with molecular oxygen or by means of some inorganic oxidant such as nitrate, sulfate, or carbonate. But it is now widely believed that the atmosphere of the earth was originally reducing, that the oxygen of the atmosphere is entirely the product of photosynthesis over the ages, and that all oxygen was originally combined in the form of oxides. If that was so, the chemosynthetic bacteria cannot have been truly primitive, but perhaps followed or paralleled the advent of photosynthesis. (See A. I. Oparin, The Origin of Life on the Earth, 3rd ed., pp. 450-455). The first organisms, primitive heterotrophs, proba- 
bly lived at the expense of organic compounds formed through the action of ultraviolet radiation on a mixture of ammonia, methane, water, hydrogen, and carbon monoxide, in the "primeval soup."

To trap the energy of the light for useful purposes-to conduct photosynthesis-is of course the primary activity in which life depends upon light. The endeavor to understand how the absorption of a quantum of light leads through excited states of atoms to some chemical reaction and how a flow of electrons may be used in a living system to produce some reduced pyridine nucleotide and some adenosine triphosphate, which together make possible the reduction of carbon dioxide to carbohydrate, forms the essence of the modern exploration of this basic life process.

Photosynthesis makes important the orientation of the organism in the environment. The orientation of the photosynthetic organism, or its parts, to the light (phototropism and photoperiodism) is matched by the orientation manifested by heterotrophs that seek out their food through sensitivity to light (phototaxis). Predation, and the avoidance of predation, led in the course of evolution to vision; and once organisms could see, there emerged now and again some biological use for the primitive, and usually wasteful, capacity of organisms to emit light (bioluminescence). Vision, refined to a discrimination of colors, made it possible through natural selection for pigments to be utilized in a new way, as invitations to visitors, or warnings to predators; and pattern enhanced by color became the basis of myriad mutual relationships between flowering plants and insect or bird pollinators, between predators and prey, between trees and tree-dwellers, etc. Recognition of one's own species, and of sex, became instant. Social groupings sprang up, knit by visual as well as chemical signals. Eventually man stood upright, looked about, and wove his conceptions into ever more elaborate patterns. From being a light-worshipper, he became in time a seeker after light, an explorer of the nature of light and its chemical effects, of the way in which chlorophyll traps the energy of light and turns it to such use that thereby the world is fed and nourished. This Symposium presents some of the latest advances and newest insights into the biochemistry and biophysics - the biology-of light.

\section{Molecular Structure and Excited States}

The effect of light on living systems involves the absorption of quanta of radiation of appropriate wave-lengths and the promotion of photochemical reactions. Requisite to an understanding of these 
is a knowledge of the excited states of molecules producible by the absorption of energy, as well as the subsequent reactions of the energy-rich molecules at ordinary temperatures. For the most striking difference between photochemical reactions and thermal reactions, as Franck and Platzman have pointed out (Radiation Biology, A. Hollaender, ed., Vol. I, p. 193, 1954), is that the former involve "in intermediate stages electronically excited atoms, radicals, or molecules which are virtually never excited in a system in thermodynamical equilibrium at room temperature."

Transitions between different energy states of a molecule may occur by the absorption or emission of radiation, or in a racliationless transfer upon impact with another system. In a very qualitative way we can picture the electronic distribution of a molecule as follows. Electrons move only in certain orbitals, or paths around the atomic nuclei of the atoms in a molecule. The energy of an electron depends on the orbital which it occupies. When a molecule is electronically excited, one of its electrons is driven to an orbital of higher energy. The transition of an electron to a new orbital results in a reclistribution of the electronic charge over the entire molecule. Inasmuch as the chemical properties of a molecule depend not only on the energy but likewise on the charge density at various sites, electronic excitation results in the formation of an essentially novel molecule, one commonly more chemically reactive than the ground state. The transitions which are detected spectroscopically are almost exclusively those involving the most reakly bound (i.e., the valence) electrons. Particularly significant in respect to such transitions are the "lonepair" electrons of an atom, that is to say, those which are not involved in actual bonding. Such "lone-pair" electrons are found in molecules with nitrogen and oxygen atoms. Of the two electrons making up such a pair and occupying the same orbit, one of them, upon becoming excited with a requisite amount of energy undergoes an abrupt transition to a higher energy state in a different orbital. Now in general two electrons occupying the same orbital must be "paired," that is, must have opposite spins, according to the Pauli exclusion principle. But following a transition of one electron of an original pair to a higher energy state, the spins may be either opposite or alike. The former is known as a singlet state; the latter is a triplet state. The probability of clifferent possible transitions to higher energy states differs, being in some cases so low as to be "forbidden." Transitions from the ground state to a triplet state are usually such; and in actuality the triplet state is generally achieved by decay from the 
singlet state of the same electronic configuration, which usually has a somewhat higher energy level.

The decay of an excited singlet state to the ground state is known as fuorescence. It is temperature-independent and is commonly rapid (producing an afterglow of the order of $10^{-8}$ second). If the excited singlet state undergoes a transition to the related triplet state, which then decays to the ground state, phosphorescence occurs. This is temperature-dependent, and is marked by a considerably slower afterglow $\left(10^{-4}\right.$ up to 1 second). Or, if the triplet experiences a new transition to the excited singlet state, which then decays to the ground state, this too may yield phosphorescence.

The spectroscopic detection of the first excited singlet and triplet states of atoms and molecules is by virtue of their emission of ultraviolet, visible, or infrared radiation following absorption of radiation also belonging to those portions of the spectrum. The emission is largely at longer wavelengths, but may include the same wavelengths as that absorbed.) The photochemical consequences of the elevattion of atoms or molecules to higher energy states reside, however, more importantly in the radiationless transitions back to the ground state, for these more usually involve chemical reactions with other systems. These result from "collisions of the second kind"- "collisions of the first kind" being those in which translational energy is converted into excitation energy of an atom or molecule. Collisions of the second kind, among other consequences, may produce chemical changes of the model $\mathrm{A}^{*}+\mathrm{BC} \rightarrow \mathrm{AB}+\mathrm{C}$ (an exchange reaction) or $\mathrm{A}^{*}+\mathrm{BC} \rightarrow \mathrm{A}+\mathrm{B}+\mathrm{C}$, where $\mathrm{A}^{*}$ represents the activated atom or molecule and $\mathrm{BC}$ a diatomic or polyatomic molecule.

As a preliminary to understanding the electronic excited states of large molecules, which is made difficult by interactions between parts of the molecule, G. Wilse Robinson discusses the spectra and unclerlying electronic transitions of two relatively simple molecules, formaldehyde and pyridine.

The energy of a molecule is divisible into its electronic orbital and electronic spin components, as well as its translational, vibrational, rotatory, and nuclear spin motions. To treat these scparately is to ignore the important cross terms between the electronic orbital and spin and the molecular vibration, which are particularly significant in the appearance of weak but inclicative spectral lines in the visible and near ultraviolet regions.

Quantum mechanics makes possible the description of the electronic 
orbitals. Corresponding to the $s$ and $p$ orbitals of atoms are the socalled $\sigma$ and $\pi$ orbitals of molecules, with the exception that these molecular electrons extend over at least two atoms. The $\sigma$ orbitals possess a cylindrical symmetry, with high electron density along the line joining the atomic nuclei. $\pi$ orbitals have a symmetry such that the phase (or sign) of the electron is opposite on opposite sides of the line joining the atomic nuclei. In the $\pi$-bonding orbital the clectron charge density is concentrated between the nuclei. In the $\pi$-antibonding, or $\pi^{*}$, orbital, the electron charge density forms an additional node "between the nuclei in a plane perpendicular to the internuclear axis," so that electrostatic repulsion weakens the bond (Fig. 1). [There are two types of both $\sigma$ and $\pi$ orbitals, but we will be concerned chiefly with the latter.]
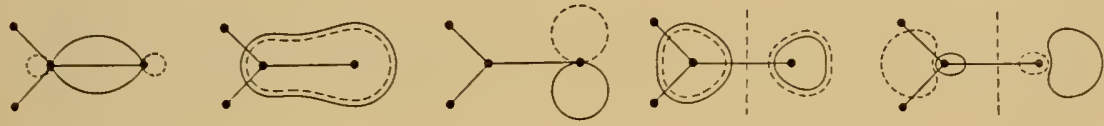

$\sigma$ $\pi$

$n_{\left(2 p_{0}\right)}$

$\pi^{*}$

$\sigma^{*}$

Fig. 1. Classification of molecular orbitals (schematic contour diagrams, formaldehyde skeleton).

The transitions most significant in the spectroscopic study of the excitation of molecules by ultraviolet or visible light are the $n \rightarrow \pi^{*}$ (non-bonding to $\pi$-antibonding), either singlet-singlet or singlettriplet, and the $\pi \rightarrow \pi^{*}$ ( $\pi$-bonding to $\pi$-antibonding). The former are important in azine or carbonyl groups of molecules. The singlettriplet $n \rightarrow \pi^{*}$ transition is characteristically very much weaker in absorption than a singlet-singlet $n \rightarrow \pi^{*}$, a relation indicating a much longer lifetime of the triplet excited state than of the comparable excited singlet state. $\pi \rightarrow \pi^{*}$ transitions occur typically in (conjugated) hydrocarbons such as ethylene, benzene, and pyridine. As the molecule increases in size the singlet-singlet transition, which occurs at $260 \mathrm{~m} \mu$ in benzene and pyridine, shifts to longer wavelengths (e.g., in carotene).

The geometry of a molecule ordinarily changes when it becomes excited since the configuration of the bonding electrons is changed. Thus, the C-C bond in benzene lengthens with the promotion of an 
electron to an antibonding orbital, and the effect is spread over all six bonds in the ring. Almost always, the chemical bonds become weaker and longer in an excited state of the molecule. A transition of one electron thus effectively reduces the strength of a triple bond to that of a double, the strength of a double bond to that of a single, and disrupts a single bond. Bond angles are also changed. The transition from a singlet state to a triplet state with the same electronic configuration has been analyzed for formaldehyde. The $n \rightarrow \pi^{*}$ singlet-singlet and singlet-triplet transitions alike involve a promotion of a nonbonding electron to an antibonding orbital affecting the $\mathrm{C}=\mathrm{O}$ bond. The bond length becomes intermediate between that of a single bond $(\mathrm{C}-\mathrm{O})$ and a double bond $(\mathrm{C}=\mathrm{O})$, and is $1.312 \mathrm{~A}$ in the triplet state and $1.326 \mathrm{~A}$ in the singlet state. There is an indication from vibrational clata respecting other molecules that in general the singlet and triplet states with the same orbital configuration have very nearly the same geometry, which may be quite different from that of other states wtih different electronic configurations. Nevertheless, it is not the geometry of the lowest triplet state that makes it so important. According, to Robinson, its significance lies rather in that it is the excited state of very lowest energy, and the only one likely to have a lifetime sufficient to convey electronic excitation to chemical reactions.

The interactions of solvent molecules with solute molecules lead to changes in the reactivity of the excited species, as well as shifts in the spectrum. In general, when the separation of the energy levels increases (a blue shift), the chemical reactivity decreases, and vice versa. These related phenomena focus attention on the nature of the solute-solvent interactions. Robinson discusses non-polar solvent interactions, charge-transfer interactions, and dipole-dipole interactions.

For non-polar solvents, attraction between solvent and solute results in shift of the electron density from both solvent and solute molecules to the intermolecular spaces between them. This effect weakens and lengthens the chemical bonds, and consequently produces a shift of the frequency spectrum toward the red. Repulsive interactions conversely produce a blue shift. The latter is less common, but can be produced, for example, by trapping the molecules in a low temperature solid. Transfer of charge is common in certain complexes, such as iodine-pyridine, which are bound by forces weaker than valence forces but greater than van der Waals' forces. As a consequence of a loss of vibrational energy by the donor molecule, a red shift is found. In the example cited, the I-I bond loses 
some $7 \mathrm{per}$ cent of its bonding electron density by transfer to the pyridine portion of the complex.

When the charge density and therefore the dipole moment of a molecule is different in the ground and excited states, then so is the dipole-dipole interaction of the solute with the solvent in the two states, and the spectrum shifts. An example is afforded by the $\mathrm{NH}_{2}$ free radical, which when trapped in crystalline rare gases undergoes a spectral shift toward the blue in argon, where the "clipoleinduced-dipole" contribution is clominant, but a shift toward the red in krypton and xenon, where it is less than the contribution of the solvent-solute dispersion effect. The effect of dipole-dipole interactions is rather larger in $n \rightarrow \pi^{*}$ transitions than in $\pi \rightarrow \pi^{*}$ transitions. The former typically show blue shifts in polar as well as non-polar solvents.

An excited molecule can lose its excess energy by emitting radiation, by undergoing a chemical reaction, or by transferring the energy to another molecule during a collision. Three conditions for ready loss of energy cluring a collision (of the "second kind") are the following: (1) The enviromment should have energy levels as far above the ground state as the excited solute is above its ground state, and spaced quite closely to correspond to the amount of energy lost by the excited molecule when it undergoes transition to a lower energy level. That is to say, many close resonances between the molecules of the environment and the excited molecules should exist in such cases. (2) A molecule will lose excitation energy to the environment more readily if for some configuration of the atoms of the molecule the energy is the same in the more and the less excited states-that is, if their potential energy surfaces (levels) intersect. (3) Some perturbation must also be present to "mix" the two states, especially when they have nearly the same energy and geometry, as in the case of an excited singlet and triplet with the same electronic configuration. The perturbation which mixes the states must be neither too large nor too small, for if it is too large the potential energy surfaces are "pushed apart" and resonance will be lacking; but if the perturbation is too small, the probability of any non-radiative transfer of energy is zero. The rate of these non-radiative transfer processes is very sensitive to the environment. In rigid media the rate can be greatly slowed down, but in gases and liquids it is very rapid $\left(10^{-4}\right.$ to $10^{-8} \mathrm{sec}$ or less). Paramagnetic molecules exhibit a well-known quenching effect on phosphorescence from the excited triplet state, which is to say, they catalyze the non-radiative energy transfer. 


\section{The Nature and Significance of $n \rightarrow \pi^{*}$ Transitions}

Michael Kasha has dealt in detail with the $n \rightarrow \pi^{*}$ transitions in polyatomic molecules, the understanding of which he has very largely elucidated himself. The application to cleoxyribose nucleic acid and to chlorophyll is of obvious importance in understanding the relation of the optical phenomena observed in molecular spectroscopy to the biological functions of these molecules. The first broad distinction in this area of work was made by Burawoy, who noted that certain absorption bands in molecules shift toward the red in solvents of higher dielectric constant, whereas others shift toward the blue under the same conditions. The former are now recognized to be $\pi \rightarrow \pi^{*}$ transitions; the latter, in most cases, $n \rightarrow \pi^{*}$ transitions.

Kasha points out that the lone-pair electrons which characteristically give rise to the lowest excited singlet states may originate either from $n$ (non-binding) orbitals which cannot conjugate with $\pi$-orbitals, or from $l$-orbitals which can do so. The carbonyl group of formaldehyde or acetone and the aza-nitrogen of pyridine supply examples of $n$-orbitals. Examples of $l$-orbitals are to be found on the nitrogen of the pyrrole ring, the amino group of the aniline molecule, or the central carbon atom of triarylmethyl radicals. Because of the conjugation of these orbitals with the $\pi$-orbitals of the molecule, only $\pi \rightarrow \pi^{*}$ transitions are to be expected. But if, in the aniline or triarylmethyl structure, there is a twist of $90^{\circ}$ at the C-N or corresponding bond, there would be no conjugation of the l-orbital with the $\pi$-orbitals; and at intermediate degrees of twisting a transition from the l-orbital to an empty antibonding $\pi$-orbital could occur. This is labeled by Kasha an $l \rightarrow a_{\pi}$ transition to distinguish it from an $n \rightarrow \pi^{*}$ transition. The spectral changes of the two types do, however, resemble one another, though their polarizations and intensities contrast sharply.

Formaldehyde has three weak absorption bands. One at $1650 \mathrm{~A}$ has been identified as a $\pi \rightarrow \pi^{*}$ transition; one at $1900 \mathrm{~A}$ as perhaps an $n \rightarrow \pi^{*}$ transition; a very weak $2900 \mathrm{~A}$ absorption band as almost certainly an $n \rightarrow \pi^{*}$ transition. In the $\mathrm{N}$-heterocyclic molecules, so important biologically, the longest wavelength singlet-singlet absorption bands are $n \rightarrow \pi^{*}$ transitions.

The molecular orbitals in which the electrons move can be classified according to their symmetry properties. According to quantum mechanics, an orbital is either symmetrical (its sign remaining unchanged upon exchange of coordinates) or antisymmetrical (its 
sign reversed) with respect to the symmetry operations characteristic of a molecule. These are the reflections, rotations, and inversions which lead to a molecule indistinguishable from the original onc. The classification of the orbitals is necessary for the evaluation of the intensity of the absorption (i.e., probability of transition) as well as the optical rotatory dispersion. The probability of a given transition is calculated from the transition moment integral, which, for an electric dipole transition-the most important in chemistry and biology-is

$$
P \sim \int_{-\infty}^{+\infty}\left(\psi_{G}^{*} E_{x, y, z} \psi_{E} d v\right)^{2}
$$

where $P$ is the probability of the transition, $\psi_{G}$ is the total wave function of the ground state of the molecule, $E_{x, y, z}$ is the electric dipole vector along $x, y$, and $z$ axes chosen to define the plane of the molecule, and $\psi_{E}$ is the total wave function of the particular excited state of the molecule.

In the examples chosen, three different kinds of promotion, $n \rightarrow \pi^{*}$, $n \rightarrow \sigma^{*}$, and $\pi \rightarrow \pi^{*}$, are considered for formaldehyde, two $\left(n \rightarrow \pi^{*}\right.$ and $\left.\pi \rightarrow \pi^{*}\right)$ for pyridine, and two $\left(n \rightarrow \pi^{*}\right.$ and $\left.\pi \rightarrow \pi^{*}\right)$ for purine.

To establish whether or not a particular transition is "allowed," that is, has a reasonable probability of occurring, one needs only to show that this integrand is of the totally symmetric species, since in that case the integrand will always be positive and the whole integral cannot be zero. Translation of the molecule along the coordinate axes is identical in symmetry properties to the electric dipole moments incluced by light waves having the same direction. The magnetic dipole moments induced by light waves have the same transformations as the rotations about the respective coordinate axes. For example, if only a translation vector along the $y$ axis causes the integrand to transform totally symmetrically, then only light with its electric vector oriented along the $y$ axis of the molecule will be absorbed (Fig. 2). Thus, in the case of formaldehyde, the $n \rightarrow \pi^{*}$ transition is forbidden, the $n \rightarrow \sigma^{*}$ transition is allowed for light polarized along the $y$ axis, and the $\pi \rightarrow \pi^{*}$ transition is allowed for light polarized along the $z$ axis. In general, transitions of $n \rightarrow \pi^{*}$ type are always polarized out of plane (i.c., along the $x$ axis), if not forbidden; whereas $\pi \rightarrow \pi^{*}$ transitions are always polarized in plane, if not forbidden. 
The optical rotatory strength of an electron transition is a scalar product of two integrals, one of them that for the transition moment integral already given and the other a corresponding integral in which $M_{x, y, z}$ (sum of the magnetic dipole vectors along the axes $x, y, z)$ replaces $E_{x, y, z}$. If there is a plane of symmetry or a center of inversion in the molecule, then the rotatory strength will be zero. If the molecule does possess optical rotatory power there is a possibility-because two different integrals are involved, only one of which is related to the intensity of the transition-that weak bands may contribute more strongly to optical rotation than strong bands. In point of fact, $n \rightarrow \pi^{*}$ bands, which are inherently weak, usually contribute strongly to optical rotation.

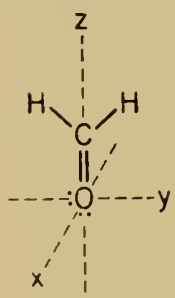

a

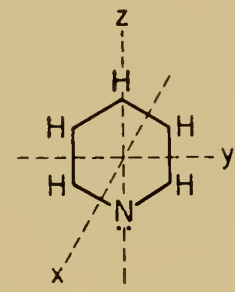

b

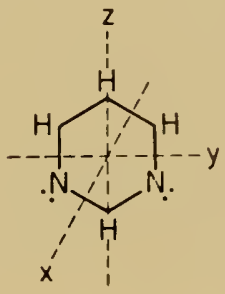

C

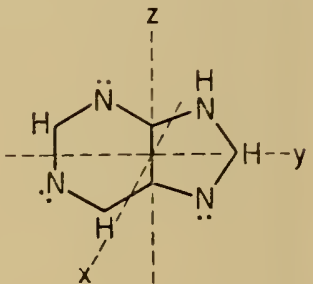

d

Fig. 2. Coordinate axes for the (a) formaldehyle, (b) pyridine.

(c) pyrimidine, and the (d) purine molecules.

The characteristics of the $n \rightarrow \pi^{*}$ transitions are summarized by Kasha as follows: (1) they are absent in hydrocarbon analogues of the molecule in question, e.g., the 2900 A band of formaldehycle is absent in ethylene; (2) they clisappear in acid media, although this observation may be obscured by the considerable shift of $\pi \rightarrow \pi^{*}$ bands under the same conditions; (3) in polar solvents they show a relatively large blue shift, whereas the $\pi \rightarrow \pi^{*}$ transitions shift toward the red end of the spectrum; (4) the blue shift is enhanced if an electron-donating group (such as $-\mathrm{NH}_{2},-\mathrm{N} R_{2},-\mathrm{OH}$, or $-\mathrm{SH}$ ) has by conjugating with the molecule increased the electron density of its $\pi$-orbitals; (5) they have a very low intensity of absorption (molar absorption coefficients of 300 to 2000 in comparison with $\pi \rightarrow \pi^{*}$ coefficients of 10,000 to 100,000$)$; (6) the $n \rightarrow \pi^{*}$ singletsinglet transitions arise only from the absorption of light polarized out of plane, whereas $\pi \rightarrow \pi^{*}$ singlet-singlet transitions are polarized in 
plane; (7) $n \rightarrow \pi^{*}$ singlet-singlet transitions usually occupy the longest wavelengths, Leing even lower in energy than the $\pi \rightarrow \pi^{*}$ transitions of N-heterocyclic molecules, although the latter have lower ionization potentials; (8) the $n \rightarrow \pi^{*}$ lowest singlet-singlet fluorescence is quenched strongly; (9) a characteristic coupling of a particular molecular vibration with the electronic excitation often occurs, as in pyridines and pyrimidines from a particular angular distortion of the ring; and (10) radical-like behavior owing to the solitary highly localized electron left in the $n$ orbital after the $n \rightarrow \pi^{*}$ promotion of its mate.

The $\mathrm{N}$-heterocyclic molecules form a group of particular interest to biologists. Among those studied so far, pyridine was first to reveal $n \rightarrow \pi^{*}$ transitions. Pyridine manifests sharp spectral lines in the 2700-2900 A region in hydrocarbon solvent. These lines are absent in the benzene spectrum and shift strongly to the blue in ethanol as solvent. Even more striking is the sharpening of the main part of the band at $2500 \mathrm{~A}$ upon changing from isopentane as solvent to ethanol; this must be due to an $n \rightarrow \pi^{*}$ transition in the $\pi \rightarrow \pi^{*}$ region of the spectrum. Diazines have $n \rightarrow \pi^{*}$ bands completely distinct from the $\pi \rightarrow \pi^{*}$ transition, e.g., pyridazine at $3500 \mathrm{~A}$ and pyrazine at about $3300 \mathrm{~A}$. Both $n \rightarrow \pi^{*}$ bands shift markedly in changing solvent from hydrocarbon to water. The $\pi \rightarrow \pi^{*}$ bands in these molecules undergo respectively a small blue shift and a small red shift under the same circumstances. Other $\mathrm{N}$-heterocyclic molecules exhibit only a long wavelength shoulder or tail on the absorption curve at 3500 $3800 \mathrm{~A}$, which may be identified as arising from an $n \rightarrow \pi^{*}$ transition by virtue of its disappearance when the solvent is changed from hydrocarbon to water. Such molecules include quinoxaline, phenazine, and quinoline. In respect to quinoline, the blue shift that accompanies the change of solvent from hydrocarbon to ethanol is scarcely observable; but there is a dramatic change in emission at low temperatures in a rigid ethanol-ether-isopentane glass solvent, and this represents, according to Kasha, an actual interchange of the $n, \pi^{*}$ and $\pi, \pi^{*}$ electronic states.

Pyrimidine in hydrocarbon solvent has a distinct $n \rightarrow \pi^{*}$ band at approximately $3000 \mathrm{~A}$, shifting strongly toward the blue in water. The $\pi \rightarrow \pi^{*}$ band at $2450 \mathrm{~A}$ shifts but slightly. Chemical substitution on the biologically important 2 and 6 positions of the ring pushes the $n \rightarrow \pi^{*}$ and $\pi \rightarrow \pi^{*}$ regions together, yet without altogether abolishing the evidence of the former. Purines, too, in hydrocarbon solvent have an $n \rightarrow \pi^{*}$ shoulder at about 3000 A which strongly shifts toward 
a shorter wavelength in water, and merges into the $\pi \rightarrow \pi^{*}$ transition bands at 2300-2700 A.

The molar absorption coefficient of deoxyribonucleic acid (DNA) at $2600 \mathrm{~A}$ undergoes a reduction of $30-50$ per cent, with little shift or change in shape of the band, when the DNA goes from a randomly coiled to a helically coiled state. A $\pi \rightarrow \pi^{*}$ excitation leads to the expectation of parallel, side-by-side stacking of the dipoles, whereas the presence of an $n \rightarrow \pi^{*}$ transition producing a long-wavelength tail in the purine and pyrimidine spectra would lead to the expectation of a head-to-tail stacking of the transition dipoles, since the $n \rightarrow \pi^{*}$ transitions in the bases will be polarized along the helical axis and perpendicular to the planes of the base pairs. One would therefore expect a small hyperchromism during the transformation from random coiling to helical coiling, instead of the hypochromism to be expected from the $\pi \rightarrow \pi^{*}$ transitions. The hyperchromic effect has been observed by Rich and Kasha. Other evidence of the $n \rightarrow \pi^{*}$ transition in DNA is obtainable from study of the rotatory dispersion curve; a Cotton effect centers on the region around 2900 A. From the agreement of the observations just summarized with expectations on the basis of the exciton model, energy transfer down the DNA helix should be efficient. The occurrence of lowest level $n \rightarrow \pi^{*}$ transitions in the purine and pyrimidine bases of DNA implies that photochemical processes in DNA will involve excited $n, \pi^{*}$ states.

The chlorophylls, as represented by chlorophyll $b$, exhibit in anhydrous benzene a long-wavelength shoulder at $6700 \mathrm{~A}$ which disappears when water or alcohol is added. At the same time, fluorescence appears. Kasha regards this shoulder as being probably attributable to an $n \rightarrow \pi^{*}$ transition, and its disappearance, like the activation of the fluorescence, as representing (in agreement with Platt) the interchange of $n, \pi^{*}$ and $\pi, \pi^{*}$ electronic states. Supported by observations made by Becker of the magnitude of the spectral shift, which accords with that to be expected from an $n \rightarrow \pi^{*}$ blue-shift, and of the spectral emission at low temperatures, these conclusions reinforce Franck's view that in photosynthesis the lowest $n, \pi^{*}$ excited states are truly important. In particular, it may turn out that wet chlorophyll (in an aqueous phase) and $d r y$ chlorophyll (in a lipid phase) may carry out complementary functions in photosynthesis.

In a paper which was unfortunately not submitted for publication, John R. Platt discussed qualitative methods of assigning electron density distributions to ground and excited states of simple organic 
molecules, and chain molecules such as cis- and trans-stilbene and various cyanines. He suggested speculatively that in the case of a cyanine molecule the attachment of a donor molecule at one end and an acceptor molecule at the other might permit the chain to serve as a conductor in a charge-transfer type of reaction.

\section{The Triplet State in Fluid Solution}

Two electronic excited states are of primary significance. The lowest excited singlet state determines the long wavelength spectrum and is the first state to be attained when light energy is absorbed. The lowest triplet state, reached only indirectly via the singlet statc, is the excited state of lowest energy, has a relatively long lifetime, and consequently a greater probability of reacting chemically. George Porter has considered the three spectroscopic methods available for studying the triplet state: (1) direct absorption from the ground state; (2) the spectrum emitted in transition from the triplet to the ground state; and (3) transition from the lowest triplet state to a higher triplet level, as a result of a further absorption of energy. The first method yields the energy differences between the lowest triplet and the ground state, and also the extinction coefficient related to the probability of the transition and the lifetime of the triplet state. The second method, involving study of the phosphorescence of the system, yields the energy of the triplet level and its lifetime. Because phosphorescence is observed only in solution in very rigid glasses or extremely viscous solutions, while workers are more interested in gaseous and fluid phases, the method is not very useful for the study of the influence of conditions upon the lifetime of the triplet state, and the occurrence of reactions involving it. Method three informs us directly of the energy of the higher triplet levels, and makes possible the direct measurement of triplet concentration as a function of time in all phases, gas, liquid, and solid.

In fluid solutions the lifetime of the triplet state is less than one millisecond; hence flash photolysis must be used to study it. In this procedure, first adapted for the study of triplet states by Porter and Windsor in 1952, the system is exposed to an electronic flash of a few microseconds duration and several hundred Joules of energy; and the transient absorption spectra are recorded by photographing with a second electronic flash or by continuous photoelectric recording of optical density at a chosen wavelength. The first experiments showed that the general absence of phosphorescence in gases and liquid solutions is owing to the occurrence under those conditions of radia- 
tionless decay at much greater rates than those of the radiative processes characterizing phosphorescence. The triplet states in liquid solvents have lifetimes of the order of $10^{-4}$ seconds. The kinetics of the transitions in a number of systems aromatic hydrocarbons and their derivatives have been studied by continuous photoelectric recording of optical density. It seems that certain modes of behavior are rather characteristic of the triplet states. In rigicl media a firstorder decay to the ground state is radiative, with a lifetime determined by the probability of transition between the two states and of the order of $10^{-4}$ up to 10 seconds. In fluid or gas phases, the nonradiative decay has a lifetime of $10^{-5}$ to $10^{-3}$ seconds, bearing little relation to the radiative lifetime. This decay is partly second-order, from collisions between two triplet molecules, and partly first-order, depending upon triplet concentration. Both processes are affected by the viscosity of the medium. The nature of the first-order process is not yet understood. The second-order reaction is a special example of the more general quenching of the triplet state by all paramagnetic species. The first such quencher to be found was oxygen, and it is so efficient that pains must be taken to degas the solutions before triplet state absorption can be observed. Nitric oxide is about as efficient a quencher as oxygen. Paramagnetic ions are quenchers and diamagnetic ions are not; but the efficiency of the former bears little relation to its magnetic susceptibility or number of unpaired electrons. Porter consequently interprets the differences between such quenchers in efficiency as arising from the varying overlap of the orbitals of the unpaired electrons in the triplet and in the quencher, rather than from magnetic field interactions. Quenching may arise also directly from triplet-triplet energy transfer between molecules when the quenching molecule is capable of a lower triplet energy level than the original molecule. Reactions of this sort conserve the electron spin and hence occur with high probability:

$$
A_{T}+B_{S} \rightarrow A_{S}+B_{T}
$$

Chemical reaction of the triplet state will also lead to its disappearance. Photochemical reactions may proceed from either the lowest excited singlet or triplet state. In relatively few cases has the precise state been identified.

The excited triplet state of a molecule is "a species with its own structure and its own physical and chemical properties ... which may be very different from the properties of the same molecule in its ground state." Redox potential and $p \mathrm{H}$ are often entirely different. 
For example, methylene blue in the triplet state oxidizes ferrous to ferric, but leuco methylene blue in the ground state is itself oxidized by ferric iron. Acidity constants of such molecules as naphthols, naphthylamines, and acridine may differ, in the first excited singlet state, by as much as $6 p K$ units from those of the ground state; but surprisingly the triplet states are not very different from the ground state in this respect. In general, the distinctive physical structure and chemical properties of the triplet state make it essential to measure them directly instead of trying to make predictions from the corresponding properties of the ground state.

\section{Excitid States of Molecules of Biological Interest}

Gregorio Weber initiated the discussion of the excited states of proteins, states that result from the absorption of radiation and are potentially capable of emitting it. Both electronic and vibrational excited states occur, but little is known of the latter. The former include both singlet and metastable states, the former with lifetimes of millimicro-seconds, the latter with much longer lifetimes of milliseconds to seconds. The singlet states are best understood. Those states arising from absorption of light of wavelength $200 \mathrm{~m} \mu$ or longer occur in three chemical species: aromatic amino acids; certain groups occurring within such molecules as heme, FMN, FAD, and carotenoids, or bound irreversibly or reversibly to proteins, as in DPNH; and certain groups artificially attached to proteins, as in various conjugates such as fluorescein conjugates.

Aromatic amino acids have two absorption bands in the 200-300 $\mathrm{m}_{\mu}$ region of the spectrum. The weaker band, at the longer wavelength, appears to represent a multipolar type of transition; the stronger band, at the shorter wavelength, a dipolar transition. When dissolved in water, each aromatic amino acid exhibits a single fluorescence emission band, at $282 \mathrm{~m}_{\mu}$ in the case of phenylalanine, at 303 $m_{\mu}$ in the case of tyrosine, and at $348 \mathrm{~m} \mu$ in the case of tryptophan. These correspond to the fluorescence bands of benzene, phenol, and indole, respectively; and actual study has been mostly of the parent compounds. In different solvents the fluorescence maximum of phenol remains virtually unchanged; but that of indole shifts to $325 \mathrm{~m}_{\mu}$ in hexane and to $340 m \mu$ in the alcohols. This shift is due to dipole interaction between solvent and excited solute molecules, and the extent of the shift depends on the dipole strength and on a quantity 
$\Delta f$ that may be calculated from the dielectric constant and refractive index of the medium. In aqueous solution the quantum yields for the three species of molecule are 0.04 for phenylalanine, 0.21 for tyrosine, and 0.20 for tryptophan. The influence of $p H$ upon the efficiency shows that protons (including those derived from the $-\mathrm{COOH}$ and $-\mathrm{NH}_{3}+$ groups of the amino acid) can quench the fluorescence of both tyrosine and tryptophan (A. White). Both anino acids are half-quenched at $p \mathrm{H} \mathrm{2.3,} \mathrm{which} \mathrm{is} \mathrm{the} p K$ of the carboxyl group; whereas esters and decarboxylated derivatives are quenched only at $p \mathrm{H}-0.55$ in the phenol series and $p \mathrm{H}+0.5$ in the indole series. In the alkaline range, members of the phenol series lose all fluorescence upon ionizing to the phenolate ion. Tryptophan and its derivatives exhibit a conspicuous increase in fluorescence at the $p \mathrm{H}$ at which the $-\mathrm{NH}_{3}+$ groups are discharged. The $-\mathrm{NH}_{2}$ form of the molecule has fluorescence efficiencies up to 0.6 which signifies that about half the time an excited indole ring can attract a proton from the $-\mathrm{NH}_{3}+$ group on the side-chain. Substances such as acetic acid and hydroxylamine, which can directly donate protons to the excited ring, consequently quench the fluorescence of molecules of this series. The fluorescence of indole and tryptophan is also quenched in alkaline solution, probably through a reaction of the excited molecule with $\mathrm{OH}^{-}$to ionize the $\mathrm{N}$ of the indole ring, followed by a nonradiative transition to the ground state. Substitution of a methyl group for $\mathrm{H}$ on the indole $\mathrm{N}$ atom blocks this quenching effect. The contribution of the ionic structure to the excited state is thus very large, and in certain naphthylamine derivatives may reach 50 per cent. Further evidence for the importance of proton carrier groups cones from studies of glycyltyrosine and glycyltryptophan, in which the $-\mathrm{COOH}$ and $-\mathrm{NH}_{3}+$ groups are farther removed from the excited ring than in the simple aromatic amino acids. In water these have very low quantum yields (ca. 0.04), which rise to about normal values of $0.2 \mathrm{in}$ a medium of high viscosity (Teale).

No direct measurements of the lifetime of the excited states in the aromatic amino acids have been made. From the oscillator strength of the molecule and liletime together with the quantum yield for the fluorescence, the lifetime of excited tyrosine is calculated to be about $8 m \mu \mathrm{sec}$, and that of tryptophan $2.5 \mathrm{~m} \mu \mathrm{sec}$.

The polarization of the emitted fluorescence, measured in viscous media, enables one to gain important information on two points: (l) by making measurements at vanishing concentration and with dif- 
ferent exciting wavelengths, the relative directions of the transition moments of the dillerent electronic bands may be determined; (2) by making measurements of changing polarization with change of concentration at a given wavelength, the transfer of electronic energy among like molecules may be estimated. The polarization spectrum is best studied in rigid solutions, such as propylene glycol at $-70^{\circ}$ C. Molecules of the phenol series exhibit two regions of the spectrum with polarization at right angles to each other. The polarization spectrum of the indole-tryptophan series is more complex. A flat maximum at $270 \mathrm{~m} \mu$ and a sharp minimum at $295 \mathrm{~m} \mu$ indicate an independent transition at a large angle. The very high maximum at or just above $300 \mathrm{~m} \mu$ is interpreted on the basis of the FranckCondon principle rather than as being an independent electron transition. N-glycyl tryptophan has an additional polarization band, at $290 \mathrm{~m} \mu$-unexplained.

The second type of polarization measurement reveals depolarizations by nonradiative transition. The reciprocal of the linear polarization of the fluorescence is a linear function of the molar concentration (so long as very thin layers, $30 \mu$ are examined). The slope constant $R_{o}$ represents the distance between parallel molecules at which there is a 50 per cent probability of transfer of the excited state from one to the other. Observed values range from $15 \mathrm{~A}$ to $40 \mathrm{~A}$, and are independent of the exciting wavelength in the case of phenol, but not in the case of indole, which shows transfer of excitation at 14 A with wavelengths below $290 \mathrm{~m} \mu$, but none that is observable above $305 \mathrm{~m} \mu$.

Proteins may be grouped into those containing tyrosine and phenylalanine but no tryptophan, and those containing tryptophan as well. Phenylalanine contributes very little to fluorescence, however. The first class of proteins includes insulin, tropomyosin, zein, ribonuclease, and several trypsin inhibitors, all of which resemble tyrosine in their fluorescence and polarization spectra, although polarization is somewhat reduced in amount. It is clear that some transfer of the excited state between tyrosine residues in the protein must take place, but the distances involved and the spatial relations are not known.

The second class of proteins includes most globular proteins. Their fluorescence spectra are characteristic of tryptophan alone (with maxima ranging from $332 \mathrm{~m} \mu$ in the case of chymotrypsin to $346 \mathrm{~m} \mu$ in the case of pepsin). From the difference one may conclude that 
the tryptophan residues in pepsin are in contact with water or a similar polar solvent, whereas in chymotrypsin they are mainly in contact with a nonpolar solvent. In human and bovine albumins, which have the lowest non-zero content of tryptophan, no treatment has revealed any contribution of tyrosine fluorescence (Teale). In fact, direct evidence of the loss of the quanta absorbed by tyrosine was found, in that between $280 \mathrm{~m} \mu$ and $300 \mathrm{~m} \mu$ the absolute yield increases as the relative proportion of quanta absorbed by tryptophan increases, while above $300 \mathrm{~m} \mu$, where only tryptophan absorbs, the yield is constant. The cause of the quenching of tyrosine fluorescence in these proteins remains obscure. The changes in quantum yield with $p H$ for bovine serum albumin (2 tryptophan residues) and human serum albumin ( 1 tryptophan residue) are interesting. Fluorescence of the former is quenched in two steps, at $p \mathrm{H} 3.5$ and $p \mathrm{H}$ 0.5 ; of the latter in one step, at $p \mathrm{H} 0.5$ (A. White). One tryptophan residue in the bovine albumin molecule must therefore be close to a proton donor (glutamic acid or aspartic acid carboxyl group); the other, like that in human albumin, must be more distant from such groups. Lysozyme has steps in the quenching of fluorescence on both acid and alkaline sides of $p \mathrm{H}$ range. Such observations clearly indicate differences in the accessibility of different tryptophan residues to protons, and exclude the possibility that protons migrate freely over the protein surface.

The polarization spectra of proteins containing tryptophan are essentially like that of tryptophan itself, but with a proportionately higher maximum at $305 \mathrm{~m} \mu$ than at $270 \mathrm{~m} \mu$. Minor differences exist in the relative magnitude of polarization at $305 / 270 \mathrm{~m} \mu$, the presence or absence of a band at $295 \mathrm{~m} \mu$, as in $\mathrm{N}$-glycyltryptophan, and the fall in polarization above $310 \mathrm{~m} \mu$. These distinctions are largely wiped out in $8 \mathrm{M}$ urea-reversibly in bovine and human serum albumin, irreversibly in rabbit muscle lactic dehydrogenase. A high $305 / 270 m \mu$ polarization ratio occurs in human serum albumin, possessing only a solitary tryptophan residue, and hence cannot be taken to signify a transfer of the excited state between tryptophan residues. It is best explained as a result of interaction between the tryptophan residue and the protein backbone. Since the fluorescence of each tryptophan residue can be quenched independently by $p \mathrm{H}$ in bovine serum albumin and lysozyme, no transfer of excited state occurs between the tryptophan residues in these proteins. 
Suclies of artificial conjugates have been limited mainly to conjugates of proteins with dimethylamino maphthalene sulfochloride (DNS). These studlies have been of use in determining the rotational relaxation times, the dielectric properties of the medium and its variation with conditions, and the "molecular expansions." Conjugates of DNS with bovine serum albumin show, by the fluorescence excitation spectra of the DNS emission, a transfer of electron energy from tryptophan residues to the attached DNS. After 20 hours of digestion with chymotrypsin, the transfer clisappears, because, in the resulting peptides, the tryptophan residues and the DNS have become separated. Analogously, expansion of a protein by penetration of methyl-ethyl carbinol at $3^{\circ} \mathrm{C}$ produces a cessation of energy transfer. This may be analyzed quantitatively by comparing the fluorescence of the native protein with that of the DNS conjugate. The characteristic distance for the transfer of electron energy from tryptophan to DNS was found by Weber to be $24 \mathrm{~A}$; and the effective average distance from a tryptophan resiche to the DNS group was $19.5 \mathrm{~A}$ in water, $23.3 \mathrm{~A}$ in $30 \%$ methyl-ethyl carbinol, and $25.8 \mathrm{~A}$ in $9.5 \mathrm{M}$ urea. If we assume an isotropic expansion of the molecular volume, its increase is 50 per cent in methyl-ethyl carbinol and 90 per cent in urea.

Sidney F. Velick used the concept of excited states, as known from absorption and emission spectra, to illuminate the nature of the relationship between protein and coenzyme in the enzyme complexes now known to be essential to enzymic activity. True, a coenzyme may alternatively be regarded either as a substrate or as a prosthetic group of the enzyme, depending upon the dissociability of the complex. But whichever is the case, it is the relations of the coenzyme in the complex to both the protein and other substrates that "determine specificity and reaction mechanism and that are the focal points for metabolic integration and control."

The pyridine and flavin nucleotides, having been most intensely studied in these respects, afford special insight into the nature of these enzyme complexes. Reduced diphosphopyridine nucleotide (DPNH) exists in the form of two isomers, $\alpha$ and $\beta$ the former biologically inactive, the latter the active form. N. O. Kaplan and his coworkers, who discovered the inactive isomer, have done much to elucidate the reasons for its inactivity. The $\alpha$ isomer differs sterically from the $\beta$ isomer at the nicotinamide riboside bond. When 
excited by light at $340 m \mu$, free $\alpha$-DPNH yields maximum emission at $465 \mathrm{~m}_{\mu}$. This $310 \mathrm{~m}_{\mu}$ band is the absorption band of the reduced nicotinamide ring, and it disappears when the DPNH is oxidized. When $\alpha$-DPNH is excited by light at $260 \mathrm{~m} \mu$, where the absorption is 95 per cent on the part of the adenine moiety of the molecule, there is very little fluorescence (Shifrin and Kaplan), although under the same conditions $\beta$-DPNH shows stronge fluorescence excitation (Weber). These observations can only mean that in the active isomer the absorbed energy is transferred from the adenine to the nicotinamide portions of the molecule, by means of a coupled oscillator mechanism, and that in the $\alpha$-isomer the transfer is impossible. In the case of the energy transfer, since the lifetime of the adenine excited state is very short, the pyridine ring must be in close juxtaposition to the adenine ring; and it may therefore be supposed that the $\alpha$-isomer is inactive because it is incapable of folding so as to bring the adenine and pyridine rings into sufficient proximity.

Following the demonstration in 1954 (Pullman, San Pietro, and Colowick) that it is position 4 on the pyridinium ring (para to the $\mathrm{N}$ atom of the ring) that is reduced by addition of $\mathrm{H}$, it was shown by a deuterium-labeling experiment that the two $\mathrm{H}$ atoms on C-4 behave differently in oxidations and reductions. Always the same $\mathbf{H}$ atom is removed in oxidations of the nicotinamide ring that has been added when reduction occurs, and this is true of non-enzymatic oxidations and reductions as much as of enzymatic ones. The explanation for the different reactivity of the two $\mathrm{H}$ atoms on C-4 probably lies in some difference in their enviromment depending on the properties of the folcled inner complex of the dinucleotide.

If it is true that free DPNH in solution has a folded conformation, this should further be evident from the effects of temperature, solvent, and structural modifications on the existence of the folded complex. Velick shows that increasing temperature does in fact reduce the relative quantum yield of DPNH fluorescence at $340 m \mu$, and that the efficiency of the excitation at wavelength $260 \mathrm{~m} \mu$ declines even more precipitously. The effects of changing the solvent are even more striking than those of temperature. In organic solvent (methyl carbitol) instead of water the emission maximum is enhanced about fourfold. The $340 \mathrm{~m} \mu$ excitation band is correspondingly enhanced, but the $260 m \mu$ excitation band completely disappears. Thus a moderate change in the diclectric constant of the 
medium has apparently completely abolished the folded conformattion of the DPNH.

The effects of structural amalogues of DPNH are illuminating. Replacement of the carboxamide group of the nicotinamide by an acetyl group, to form an acetyl pyridine group (APNH) leaves the $260 \mathrm{~m}_{\mu}$ excitation band stronger than before. This observation, as Velick suggests, seems to rule out participation of the amicle group on the carboxamide side-chain in the formation of the inner complex involved in the energy transler (but see below). Desamino DPNH, in which the amino group of adenine is replaced by $-\mathrm{OH}$, shows a weakening of the $260 \mathrm{~m} \mu$ excitation band. Shifrin and Kaplan, in their contribution to the Symposimm, report the results of substituting a thioamide group for the carboxamide group. The absorption maximum at $260 \mathrm{~m}_{\mu}$ is shifted and the $340 \mathrm{~m}_{\mu}$ band of S-DPNH is similarly red-shifted. $40-50 \mathrm{~m} \mu$ toward the red in S-DPN. The new thiocarbonyl absorption band, at $398 \mathrm{~m} \mu$, permits an examination of the function of the carboxamide group in the intramoleculer energy transfers. Shifrin and Kaplan postulate H-bonding between the thione $\mathrm{S}$ atom and the 6-amino group of adenine, as found to occur between molecules of 2-mercaptothiazole. $(\mathrm{S}=R-\mathrm{NH} \ldots \mathrm{S}=\mathrm{R}-\mathrm{NH})$. To test this, a DPN analog was formed by attaching a $-\mathrm{CH}_{2} \mathrm{CH}_{2} \mathrm{OH}$ sidechain to the adenine $\mathrm{N}$ adjacent to the adenine 6 -amino group. It proved unable to transfer absorbed energy at $260 \mathrm{~m} \mu$ to the dihydronicotinamide moiety of the molecule. The thionicotinamide analog of DPNH is very weakly fluorescent, but its absorption spectrum in different solvents affords some information about the interacting groups. A shoulder seen at $295 \mathrm{~m}_{\mu}$ in water becomes a more pronounced peak at $300 \mathrm{~m} \mu$ in propylene glycol. If, as seems probable, the $300 \mathrm{~m} \mu$ band results from an $n \rightarrow \pi^{*}$ transition, then the postulated hydrogen bond would require that a greater energy be expencled in the promotion of the $n$ electron to the $\pi^{*}$ level. This would mean a "blue shift" in the absorption band in hydroxylic solvents, exactly as observed in the change from propylene glycol to water.

When the thionicotinamicle-DPNH is exposed to strong light, aix interesting reaction occurs. The $398 \mathrm{~m} \mu$ maximum absorption disappears almost completely and a new maximum appears at $34 \mathrm{I} \mathrm{m}_{\mu}$. Also the 295-300 $m \mu$ absorption disappears, suggesting loss of the postulated thione activity. The illumination product has no enzymatic activity, reverts to the original form in the dark, and its fluo- 
rescence is enhanced by lactic dehydrogenase. That this new form $\mathrm{SH}$

is a thiol $(\mathrm{R}-\mathrm{C}=\mathrm{NH})$ was rendered probable (a) by complexing the thione $\left(\mathrm{R}-\|_{\mathrm{C}}-\mathrm{NH}_{2}\right.$ with parachloromercuribenzoate, whereupon the shift in absorption from $398 \mathrm{~m}_{\mu}$ to $341 \mathrm{~m} \mu$ upon illumination was prevented; and (b) by methylating the thione $\mathrm{S}$ atom, which is $\mathrm{S}-\mathrm{CH}_{3}$

stabilized as an analogue of the enol form $\left(\mathrm{R}-\mathrm{C}=\mathrm{NH}_{3}\right)$, and which was found likewise to have maximum absorption at $341 \mathrm{~m} \mu$. This is then a case of photo-induced tautomerism.

There is little indication that adenine and the pyridine rings interact in DPN. Aminopyridine-DPN has been prepared and its absorption characteristics studied; but since the amino group of this analogue is an electron-clonating group whereas the carboxamicle of DPN is an electron acceptor, the analogue is not a good model for DPN action.

The chief point of the foregoing considerations is that in the oxidation and reduction of DPN there is not only a change on the charge of the pyridinim ring $\mathrm{N}$ but also a change of the molecule's conformation. Yet both oxidized and reduced forms of the coenzyme occupy the same sites when bound in an enzyme complex. It must be that if one form of the dinucleoticle fits closely the other will fit less well and the binding interaction will be weaker. In most cases where the interaction has been measured, it is stronger between en yme and DPNH than between enzyme and DPN.

In discussing the reduced pyridine nucleotide enzyme complexes, Velick has compared the properties and behavior of the glyceraldehydle-3-phosphate dehydrogenase (GPD) and lactic dehydrogenase (LDH). Studies were made of the persistence and the disappearance of the $260 m_{\mu}$ excitation band, serving respectively as criteria for the closed and open conformations of the dinucleotide. These studies reveal that DPNH is bound in the folded conformation to GPD, but in L.DH and all other dehydrogenases examined it is bound in the open conformation. Correspondingly, GPD has a greater affunity for DPN than for DPNH, just the reverse of the behavior of all the other tested cnrymes. Moreover, in the GPD-DPNH complex, unlike all 
the others, fluorescence is partially quenched. The excitation spectrum is strongest at 280$) \mathrm{m} \mu$, where it is attributable almost wholly to the protein rather than the coenzyme. The emission, at $490 m \mu$, comes from the coencyme; and accordingly, there must be a transfer of excitation encrgy from the protein to the bound coenzyme. With GPD, and with GPI) alone, there is partial depolarization of the fluorescent light, as if the bound coenzyme possessed some degree of oscillatory freedom. Another distinction is that in the case of GPD the product of substrate oxidation remains bound to the enzyme until it undergoes a second reaction step, a phosphorylation, which is also influenced by the bound coenzyme. Maybe the DPNH is only rigorously fixed in position in the presence of the appropriate substrate.

Enzymes which shift the maximum absorption of bound DPNH toward the shotter wavelength end of the spectrum also shift the fluorescence emission band of the DPNH in the same direction; but no simple proportionality is to be observed, and the enzymes of this class (like LDH and umlike GPD) are not to be thought of as forming identical sorts of complexes. The relatively low affinity of the LDH class of enzymes for DPN may be greatly enhanced by nucleophilic reagents (such as cyanide, bisulfite, or hydroxylamine) which attack the pyridine C-4 atom and possibly convert it to a form resembling DPNH. These enzymes like LDH do not generate a differential reactivity of the two $\mathrm{H}$ atoms on $\mathrm{C}-4$ of the pyridine ring, since the coenzyme is bound to them in open conformation. In the case of GPI), where the stereo-specificity is present, the reactive $\mathrm{H}$ atom is most unexpectedly not the one identified as such in the free but folded DPNH. The change to an opposite stereospecificity, observed here, should have something to do with the structure of the inner complex. Perhaps, as Velick suggests, a face to face or elge to edge aligmment of the adenine and pyridine rings, an arrangement unstable in water even with the help of the postulated hydrogen bond (adenine amino to nicotinamide carbonyl oxygen), might be stabilized in the enzyme complex.

Of the analogues, desamino-DPNH is actually a better coenzyme with $\mathrm{LDH}$ than is DPNH, although desamino-DPNH has low activity in the GPD reaction. It is bound mainly in the open conformation. On the other hand, the acetyl pyridine analogue, which is perhaps bound in the folded conformation (for it occurs in that form in 
water), is nearly as efficient as DPNH in both reactions. In both reactions its fluorescence emission is shifted toward blue and is enhanced upon being bound. It is clear that each enzyme binds and polarizes the coenzyme in its own particular way.

The transfer of excitation energy from the protein to the coenzyme is well illustrated in the case of lactic dehydrogenase. Its emission seems predominantly to relate to its 28 tryptophan residues. The quantum yield of $\mathrm{LDH}$ is nearly 40 per cent, in contrast to the 10 per cent of GPD, and in terms of the light absorbed by tryptophan it actually amounts to 70 per cent. The fluorescence emission of LDH is strongly quenched by denaturation in $6 \mathrm{M}$ urea, whereas the emission of free tryptophan is enhanced under the same conditions; hence the high yield must be related to the tertiary structure of the protein. Polarization of the emitted light is about 25 per cent, or about the same as that of free tryptophan in a highly viscous medium or at a low temperature. The partial depolarization occuring in this and other large proteins therefore arises probably from vibration or oscillation of the indole side-chains. When the excitation energy passes from the protein to the bound coenzyme a new coenzyme excitation band appears and at the same time the protein emission is quenched by two-thirds, if all the binding sites for DPNH on the enzyme are occupied. In short, the data suggest that the protein emission is from tryptophan, that tryptophan residues are the donors of the energy transferred to the bound coenzyme, and that tyrosine transfers little or none of its absorbed light energy to tryptophan. The titration curves of enzyme with DPNH, using either the quenching of protein fluorescence or the emission by the excited dinucleoticle as measures, are strictly stoichiometric. It follows that each molecule of nucleotide bound quenches only the tryptophan residues within its own domain, with negligible overlap. There is no indication that these proteins are aggregates which may dissociate (except for glutamic dehydrogenase, the subunits of which are still of large molecular weight and each carry 3 or 4 binding sites for DPNH). The demarcation of "domains" strictly corresponding to indiviclual bound coenzyme molecules therefore becomes quite a problem, less difficult in the case of an enzyme with low quenching, such as GPD, than in such a case as the LDH-APNH complex, where the quenching is 80 per cent-for in the latter instance each molecule of the bound coenzyme analogue must either capture all of the energy from 
6 tryptophan residues, or $80 \%$ of the energy from the 7 resiches within its domain. From Förster's equation, the distance $R_{0}$ at which there is equal probability of a transfer between fonor and acceptor and of emission of a quantum of light is calculated to be $25 \mathrm{~A}$ for tryptophan-DPNH. If the transferred energy comes from seven different sources, it is to be expected that polarization of the excited ffuorescence would be low, as was in fact noted above. Velick however utters a caution against regarding protein quenching as a quantitative indicator of coenzyme binding in all cases.

Turning to flavin mononucleotide (FMN) and flavin adenine dinucleotide $(\mathrm{FAI})$ ), it is once again apparent that changes in the absorption spectra afford useful clues to the presence of excited states and enzyme-coenzyme interactions. To start with, the quantum yield of the fluorescence emission in the dinucleotide is only one-tenth as great as that of the mononucleotide. This fact coupled with a spectral shift to a longer wavelength in the $460 \mathrm{~m} \mu$ band, a small decrease in absorption, and a pronounced shoulder in the $460-490 \mathrm{~m} \mu$ region, lead to the suggestion that FAD, like DPNH, occurs as a folded inner complex, with perhaps a face-to-face orientation of the adenine and isoalloxazine structures and an open-chain ribotol instead of a ring ribose on the flavin. Beinert points out that the 460$490 \mathrm{~m} \mu$ shoulder, which appears in a number of flavo-proteins, either when pure or when substrate is added, may indicate the presence of a protein-coenzyme complex. A good example: D-amino acid oxidase-FAD-benzoate (Yagi). The absorption peak in the near ultraviolet $(300-400 \mathrm{~m} \mu)$ is shifted toward $370 \mathrm{~m} \mu$ by hydrogen-bonding solvents, toward $330 \mathrm{~m} \mu$ by those that do not form hydrogen bonds. An absorption band at $315 \mathrm{~m} \mu$ in the complex between yellow butyrul dehydrogenase and crotonyl-CoA can be positively assigned to the interaction of the isoalloxazine ring system with a nucleotide (Beinert), and presumably the similar band of microsomal cytochrome reductase (see below) is produced by the same type of interaction.

The folded FAD complex behaves like DPNH in respect to the independence of fluorescence from temperature and the enhancement of fluorescence in methyl carbitol as compared with water. The fluorescence excitation spectrum of FAD agrees closely with the absorption spectrum, except that at $260 \mathrm{~m} \mu$ the quantum yield is reduced. In this coenzyme most of the absorption at $260 \mathrm{~m} \mu$ is due to the isoalloxazine moiety rather than to the adenine portion of the 
molecule. The mononucleoticle is excited in this region of the spectrum even more strongly than the dinucleotide, and it may well be that all the emission from FAD comes from a small proportion in the open form which is in equilibrium with the non-emitting closed form of the molecule.

Unlike the pyridine nucleotides, FMN and FAD have strong absorption bands in the visible and near ultraviolet when they are in the oxidized rather than the reduced form, and it is the oxidized, not the reduced, forms that fluoresce. During the course of reduction, two very weak and very broad bands centered at $565 \mathrm{~m}_{\mu}$ and 880 $900 m \mu$, make their appearance, and Helmut Beinert, in his contribution to the Symposium, attributes these respectively to a semiquinone or free radical and to the climer of the semiquinone. The band in the near infrared does not occur in flavoproteins. The $560 \mathrm{~m} \mu$ band, which occurs in several flavoproteins as well as in free flavins, is clearly indicated to be a semiquinone by the presence of an electron spin resonance signal, as well as by the transient nature of the band. In several flavoproteins, however, the $560 \mathrm{~m} \mu$ band is persistent rather than transient. These cases Beinert interprets as involving a very strong binding of substrate to enzyme, with interaction of neighboring substrate radicals to make semiquinones which are undetectable by electron spin resonance spectroscopy. Or there might be interaction between adjacent flavins bound to the same enzyme molecule, with formation of an undetectable flavin biradical. Additional evidence to support the semiquinone theory is provided by R. L. Searls and D. R. Sanadi, in this Symposium. They have studied the reaction catalyzed by dihydrothioctyl (dihydrolipoic) dehydrogenase of hog heart muscle. This reaction is a reversible oxidation of DPNH by thioctamide or thioctate. The reduction of thioctate is inhibited by agents (arsenite, cadmium ions) known to inhibit the reactivity of sullhydryl groups near the active site on the enzyme. This enzyme also catalyzes the diaphorase reaction-oxidation of DPNH by 2 , 6-dichlorophenol indophenol and ferricyanide-which is activated by arsenite or cadmium ions several fold. Searls and Sanadi postulate that the thioctate reaction involves a nearby disulfhydryl group on the protein which may be oxidized to a disulfide bridge and which is not necessary for the cliaphorase reaction. A $535 \mathrm{~m} \mu$ peak in the absorption spectrum of the flavoprotein enzyme after reduction with DPNH, or with thioctate bound to $\alpha$-ketoghutaric dehydrogenase, disappears instantly upon addition of arsenite or cadnium ions. Evi- 
dence that the $535 \mathrm{~m} \mu$ peak is attributable to a semiquinoid free radical $\mathrm{FADH}^{*} \mathrm{~S}^{*} \mathrm{SH}$ was found in a model reaction: the forma-

tion from dihydrothioctate (a disullhydryl compound) and riboflavin phosphate (FMN) which had an absorption maximum of $535 \mathrm{~m} \mu$ that disappeared upon standing and was replaced by broad peaks at $565 \mathrm{~m} \mu$ and $880 \mathrm{~m} \mu$, corresponding to the flavin semiquinone and its dimer. There is also evidence that FMN will form a complex possessing a $535 \mathrm{~m} \mu$ absorption band, by reacting with tryptophan or other electron acceptors; and electron spin resonance measurements showed no signal immediately after mixing dihyclrothioctate and FMIN; but a free radical signal appeared as it turned green, some 20 minutes later. FMN plus enzyme alone turned green but gave no ESR signal. The $535 \mathrm{~m}_{\mu}$ band is thought by Searls and Sanadi to indicate the presence of a charge transfer complex, heretofore undemonstrated in biological systems. This "diaphorase" enzyme is unusual among flavoproteins in that the fluorescence of the bound flavin is enhanced rather than quenched. Velick and Beinert describe a number of other flavoprotein enzyme-coenzyme complexes, all of which seen to be characterized by the formation of a flavin semiquinone intermediate. (1) the "old yellow enzyme," the first flavoprotein ever purified, resolved, and reconstituted (Theorell, 1935), cloes not dissociate significantly under functional conditions. It oxidizes TPNH with various hydrogen acceptors, and binds one molecule of the resulting TPN per molecule of flavin, the latter seemingly also oxidized. In the absence of oxygen, an electron spin resonance signal gives notice of the formation of a flavin semiquinone intermediate. (2) Butyryl dehydrogenase, in its green form (oxidized) has a broad absorption band, with a maximum at about $720 \mathrm{~m}_{\mu}$, so far found in no other flavoprotein except the "old yellow enzyme" after treatment with ammonium sulfate at $p \mathrm{H} 10$, a treatment which also turns it green. This band probably comes from an interaction of enzyme and flavin, but its interpretation is still unclear. (3) Acyl-coenzyme-A dehydrogenases reversibly dehydrogenate fatty acyl groups linked with coenzyme A. A semiquinone type of absorption band at $570 \mathrm{~m}_{\mu}$ has been observed during the course of reduction by these FAD enzymes, although no electron spin resonance signal could be detected. (4) Lactate oxidase, by a first reaction step, forms pyruvate and peroxide which remain bound to the enzyme until they react to form acetate and $\mathrm{CO}_{2}$. There is a weak electron spin resonance signal. 
Microsomal cytochrome reductase, like many of the foregoing, occurs in organized cell structures. It catalyzes reduction of cytochrome $b^{5}$ by DPNH. A pyridine nucleotide flavoprotein complex is formed in which the flavin is fully bleached, i.e., reduced, and the pyridine nucleotide is presumably oxidized. It possesses a sulfhydryl group essential for the binding and oxidation of DPNH. In conclusion, it may be stressed that "although each flavoprotein seems to exhibit unique properties of its own, many of these are variations of a common theme."

\section{The Emission of Light by Chemical Reactions}

Two chemical reactions that emit light have features of special interest bearing on the interpretation of bioluminescence and photosynthesis. One of these is the oxidation of luminol, one of the two known reasonably efficient chemiluminescent reactions. The other is the chemiluminescence of metallo-porphyrins such as magnesium phthalocyanine and zinc tetraphenylporphine in the presence of peroxicles, a photochemical reaction resembling the reversal of the splitting of water in photosynthesis.

Henry Linschitz has summarized recent work with the porphyrin. The catalyzed reaction is a decomposition of tetralin hydroperoxide to yield $\alpha$-tetralone, water, and the electronically excited porphyrin. The luminescence intensity closely parallels the rate of decomposition of the peroxide, both being second-order reactions; and the ratio of initial rate to steady state is also the same for both. Up to the halflife of the peroxicle, about 30 molecules of peroxide are decomposed for every mole of porphyrin which disappears. This ratio is very constant, being independent of temperature over the range studied and independent also of the initial concentration ratio of peroxide and porphyrin. The chemiluminescence spectrum at $148^{\circ} \mathrm{C}$ corresponds closely to the fluorescence of the porphyrin at the same temperature in organic solvent, even to minor features of the curves. The bound metal is essential to the catalytic action of the tetraphenylporphine, copper being most effective, lead somewhat surpassing zinc, and tin being weakest.

The close agreement of the chemiluminescent and fluorescent spectra strongly suggests to Linschitz that the excited, unchanged porphyrin is itself the luminescent molecule and that it is excited during catalysis rather than in its subsequent breakdown. The metal requirement makes it reasonable to suppose that the first step in the reaction is the complexing of the peroxide at the central metal ion. 
If electron transfer is involved, as seems probable, a charge-transfer complex of metal and hydroxyl ion may be produced, in which case the excitation maly arise from removal of the $\mathrm{OH}$ and the dropping back of the electron into an excited orbital instead of the ground state. Or one may devise chain reactions that have the same result.

Linschitz states that recent experiments in his laboratory on the quenching of excited triplet states in aromatic molecules, including porphyrins, by heavy metal ions provide further evidence of chargetransfer intermediates. Ionic intemediates may thus play an important role in promoting electronic transitions, though atom transfer may also serve in the formation of intermediates in chemiluminescent reactions.

The brilliant chemiluminescence of luminol (Fig. 3) is unsurpassed among the hydrazides. Emil White points out that maleic hyclrazide and linear, rather than ring, hydrazides are but weakly luminescent.
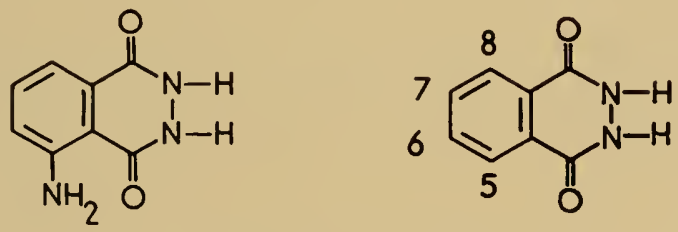

Fig. 3. Structural formula of luminol.

Studies of analogues of luminol reveal that in general a diacylhydrazine heterocyclic ring bearing two hydrogen atoms is necessary and for efficient chemiluminescence it should be attached to an aromatic ring. Substitutions at position 5 are superior to those at position 6 , and the efficiency of the substituent is related to its ability to release electrons into the conjugated system. It is the resonance effect that is involved. A similar effect of the substituent side-groups occurs in the fluorescence of these compounds and of the corresponding phthalic acids, so that their effect would seem to be exerted at the time of emission of the light. In water, the chemiluminescent system must include sodium or potassium hydroxide, hydrogen peroxide, and an oxidizing agent such as sodium hypochlorite, potassium ferricyanide, or a chelated transition metal, such as an iron or copper complex. No product of the oxiclation reaction has been identified except nitrogen; but since the quantum yield is not above 0.003 this is not surprising. Luminol is not a catalyst in the reaction but is 
consumed. Nor does correspondence of the chemiluminescence spectrum with the fluorescence spectrum serve to identify luminol as the emitter of the light, as was formerly thought. The correspondence is spurious, since the fluorescence spectrum is observed in acid solution, the chemiluminescence spectrum in basic solution, where luminol exists only as the mono anion, the fluorescence of which is essentially nil. The 3-aminophthalate ion, however, does provide a good match between chemiluminescent and fluorescent spectra at the same $p \mathrm{H}$. In addition to the above information bearing on the mechanism of the reaction, there is eviclence that free radicals are involved. Although White proposes a mechanism for the luminol reaction in water, the difficulties of studying the system are so great that he was led to try various organic solvents in place of water. With certain weakly acid, highly polar organic solvents, the most efficient of which proved to be dimethyl sulfoxide, only luminol, oxygen, and a base are required, the emission of light is superior to that in water, and the maximum emission shifts from $430 \mathrm{~m}_{\mu}$ to $480 \mathrm{~m}_{\mu}$ (Fig. 4). The products of the reaction are light, $\mathrm{N}_{2}$, and the 3-aminophthalate ion. The quantum yield was 0.1 . A possible intermediate is the hyperoxide ion shown below the main reaction in the figure. Since cyanide ions and transition metals do not noticeably affect the reaction in dimethyl sulfoxide, although they do in water, probably no free radicals are produced in the former instance. However, the simple view that the emitting molecule is excited cluring the ejection of the nitrogen, and is therefore the phthalate ion itself, runs into difficulties. The predicted agreement between the chemiluminescence of the luminol

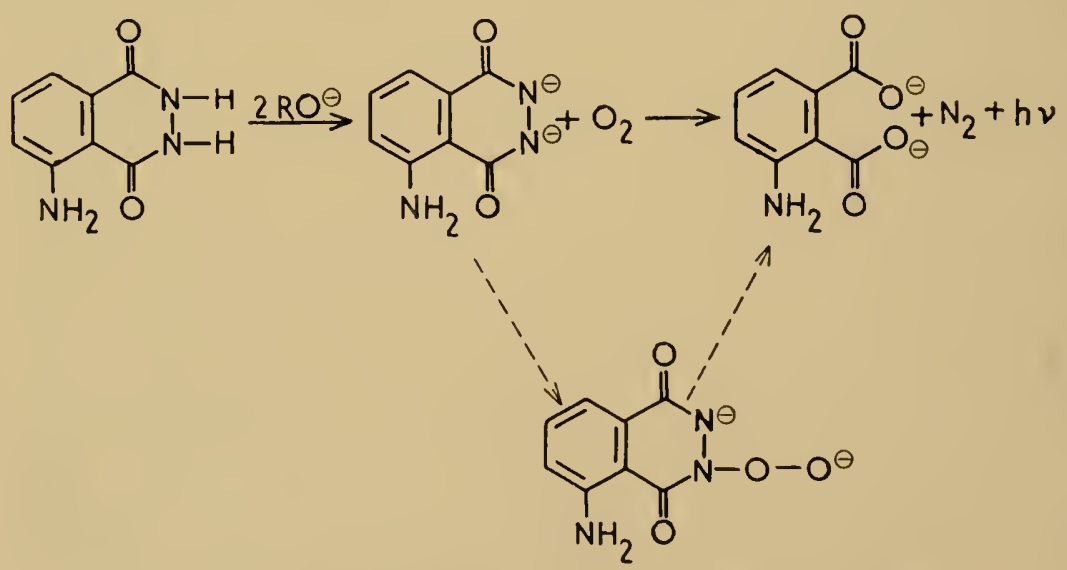

lig. 4. The chemiluminescence of luminol in dimethyl sulfoxide. 
reaction and the lluorescence maxinum of the aminophthalate ion is to be found in water, but not in dimethyl sulfate or other organic solvents.

H. H. Seliger has looked into some further aspects of the problem of chemiluminescence by luminol. On the hypothesis that the emitting species of molecule is the neutral luminol molecule, there would have to be an energy transfer from some excited oxidation product to the luminol molecule, and the quantum yield would be expected to depend on the concentration of luminol. But experiment showed that the quantum yield is constant for the concentration range from $10^{-7}$ $M$ to $10^{-4} M$. Noreover, at the very $p H$ where chemiluminescence occurs, the fluorescence quantum yield of luminol drops practically to zero. On the other hand, the chemiluminescence of luminol and the fluorescence of the aminophthalic acid ion agree in spectrum almost exactly when both are measured at the same $p H$. The quantum yield of the chemiluminescence decreases rapidly with increasing $p \mathbf{H}$, roughly parallel to the decrease in the fluorescence quantum yield of aminophthalic acid above $p \mathrm{H}$ 1l. Although the emitting molecule is not yet positively identified, these findings clearly favor the hypothesis that it is some excited oxidation product of the reaction rather than luminol itself.

\section{Bioluminescence}

Since R. Dubois' classic demonstration in 1885 that the luminescence of the firefly Pyrophorus depends upon a reaction involving separable heat-labile (protein) and heat-stable (non-protein) substances, similar "luciferase-luciferin" systems have been demonstrated in widely separated branches of the animal kingdom, as well as in certain bacteria, fungi, and dinoflagellates. Frank Johnson and his collaborators have concisely surveyed these examples, which, having evolved largely independently, show distinct differences in the biochemical systems involved, and usually, except in closely related species, manifest no cross-reactivity between the "luciferin" of one species and the "luciferase" of another. Examples of bioluminescence occur in almost every animal phylum. In most of those studied, as well as in the microorganisms and fungi, there is indirect, if not direct, evidence of an enzyme-substrate system as the mechanism in the emission of light. Yet this is not to say that the systems are identical, or even similar except in a general way.

The three best-known systems at the present time are those of the ostracod Cyridina, the North American fireflies Photinus and Photurus, 
and the bacteria Achomobacter and Photobacterium. Additional inlormation presented in this Symposium in regard to the bioluminescence of the sea pansy Renilla reniformis, the dinoflagellate Gonyaulax, and luminous fungi Armillaria mellea and Collybia velutipes provicles further comparisons with the classical examples.

The Cypridina system is the simplest, involving only luciferin, luciferase, water, and molecular oxygen. Cypridina luciferin is also autoxiclizable, without emission of light. It has been crystallized, is yellow in color, and gives out a yellow fluorescence when exposed to ultraviolet rays. Upon oxidation it yields an inactive red substance and two colorless, blue-fluorescent oxyluciferins, one of which has been crystallized, and is given the provisional structure:

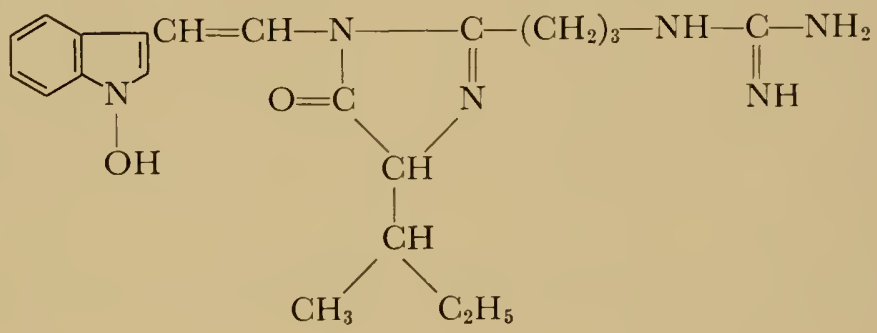

A. M. Chase and coworkers report studies on the oxidation of Cypridina luciferin at different $p \mathrm{H}$ values, and its reduction by ascorbic acid. They find that the troublesome difficulty in the preparation of Cypridina luciferin owing to its ready oxidation in the presence of dissolved oxygen is greatest at $p \mathrm{H} 9$ and can be largely avoided at a $p \mathrm{H}$ below 7 , or somewhat less reachly at a $p \mathrm{H}$ above 11. Ascorbic acid was found to be a highly effective inhibitor of this non-luminescent oxidation of luciferin. Even at $p \mathrm{H} \mathrm{8.6,} \mathrm{near} \mathrm{the} \mathrm{point} \mathrm{of} \mathrm{maxi-}$ mum instability of the luciferin, $0.01 M$ ascorbic acid almost completely prevents the autoxidation of the luciferin, and converts the course of the luminescent reaction occurring when luciferase was added from a complex to a first order relation.

Firefly luciferin and oxyluciferin, as well as the enzyme luciferase, have been crystallized and studied in considerable detail. The reduced lucilerin is yellow and has a greenish yellow fluorescence. Unlike Cypridina luciferin, it contains two sulfur atoms per molecule. In the emission of light, it loses two hydrogen atoms to form oxyluciferin, in a reaction requiring $n o t$ only luciferase and oxygen, but also ATP and magnesium ions. Pyrophosphate is split off from the ATP, and an oxyluciferin-enzyme-AMP complex is formed. 
The third general type of luminescent system, that of the luminous bacteria, tutilises a Ilavin monomucleotide as substrate, that is, as "luciferin"; and it requires, in addition to molecular oxygen, also a long-chain aliphatic aldehyde, which presumably becomes bound to the enzyme together with two molecules of reduced flavin phosphate. The function of the aldeliyde is not clear, but perhaps through peroxidation (as McElroy and Green have suggested) it provides some of the excitation energy needed in the luminescent reaction. When all essential components of the system are in limiting concentration, the total amount of light emitted is proportional to the amount of aldehyde added, with an efficiency of one guantum per 20 molecules ol aldehyde. Aldehydes with an even number of carbon atoms increase in efficiency with increasing chain lengths, but those with an odd number reach a maximum effectiveness with nine carbons (Rogers and McElroy). As in the Cypridina system, a non-luminescent oxidation of the flavin can occur in a competing reaction.

McElroy and Green, who purified the bacterial luciferase and found it to have a molecular weight of about 85,000 , showed that two molecules of reduced flavin $\left(\mathrm{FMNH}_{2}\right)$ are required in the reaction. As a possible mechanism, it is suggested that one $\mathrm{FMNH}_{2}$ molecule reacts with oxygen to form a peroxide, while the other $\mathrm{FMNH}_{2}$ molecule unites with the aldehycle which then, by reaction with the organic peroxide, becomes oxidized to a highly excited state that emits light. But it is also possible that the aldehyde is involved only in dark reactions coupled with the luminous reaction and is not a direct participant in the latter (Strehler). At present there seems no way of deciding between these possibilities.

Johnson and his coworkers do not regard either the differences in the fluorescent and chemiluminescent spectra of the varieties of luciferin found in different organisms, or the disjunct phylogenetic occurrence of bioluminescence, or the failure to obtain cross-reactions between different "luciferins" and "luciferases", or the specific requirements for different cofactors as sufficient ground for supposing the several bioluminescent systems to be entirely different in chemical nature. Although three main types of bioluminescent systems are distinguishable, they may in the long run turn out to be more similar than different. These workers are strengthened in this view by their recent finding of a luminescent cross-reaction between the luciferin and luciferase of a fish, Apogon, and the crustacean Cypridina. The absorption spectra of the respective luciferins are quite similar, but not identical: and there is a reasonable presumption that the pro- 
teins (luciferases), being from different species, are also different to some extent, even though each is capable of reacting with the luciferin of the other organism.

Other contributors to the Symposium on the subject of bioluminescence do not seem to share this view, or at least are non-committal while emphasizing the distinctiveness of the systems they have been investigating.

The luminescence of cell-free fungal preparations from Armillaria mellea and Collybia velutipies has been studied and reported by R. L. Airth. Although E. N. Harvey was not able to obtain a cellfree active luciferin-luciferase preparation from luminous fungi, it may be accomplished by mixing a cold water extract containing the luciferase with a hot water extract containing the luciferin, provided a reduced pyridine nucleotide is added. Either TPNH or DPNH will serve, but the former is some 2.5 times as efficient. The increase in light intensity during the course of the reaction is quite slow. When subjected to purification procedures, the enzyme becomes rather unstable; addition of an emulsifying and dispersing agent (Tween 80) stimulates both the initial rate of the reaction and the maximum light intensity attained. The addition of bovine plasma (or serum) albumin or of human albumin also stimulates the reaction, and reactivates the inactive enzyme, so as to produce a five-fold increase both in light intensity and in rate of the reaction. Egg albumin, gelatin, etc., will not clo this. Optimal activity is at an albumin/enzyme ratio of 2.5 to 4 . When the reaction is started without bovine albumin, and this protein is subsequently added, there is a great increase in the rate of emission of light. Airth interprets these observations in terms of an inhibition of the luciferase, which is relieved by combination of the bovine albumin with the inhibitor. Since McElroy et al. had found that irradiation with ultraviolet light markedly enhances the emission of light by the bacterial bioluminescent system, Airth tested the effect of ultraviolet on the cell-free fungal system. It reacted differently. There was, first, an inhibition of light emission during the exposure, and then, after the exposure was ended, a rapid increase up to a level some 70 per cent higher than the original level. It was demonstrated that the inhibition during the irradiation is attributable to a labile component in the hot water extract, either luciferin or oxyluciferin; while the subsequent stimulation may be an effect on the irradiated luciferase. The action of particular inhibitors suggests a metal requirement for the system. At the present time, Airth suggests that the component of the hot 
water extract is an oxylucilerin, which is reduced by the pyridine nucleoticle to the active substrate, luciferin. He emphasizes several differences between the lungal system and that found in luminous bacteria: (i) the fungal system does not respond to reduced flavin monomucleotide; (ii) there is no requirement in the fungal system for a long-chain aldehyde; and (iii) the emission maximum in the luminous mold (in vivo) is at $530 \mathrm{~m} \mu$, whereas in the bacteria it is at $480 \mathrm{~m} \mu$, and consequently the excitation energy required for the latter is greater than for the former.

The report by W. D. McElroy and H. H. Seliger reviews and extends present knowledge of the bioluminescent system of the North American fireflies. The general scheme of the firelly's luciferin-luciferase reaction system is reproduced in Fig. 5, from which it may be seen that luciferin $\left(\mathrm{LH}_{2}\right)$, reacting through its carboxyl group with ATP in the presence of luciferase, yields an enzyme-bound luciferin adenylate $\left(\mathrm{E}-\mathrm{LH}_{2}-\mathrm{AMP}\right)$ plus pyrophosphate $(\mathrm{PP})$. It is for this step that the magnesium ion is required. The enzyme-bound luciferin adenylate may undergo either of two reactions, one a simple nonradiative separation of the enzyme and the luciferin adenylate, the other an oxidation by molecular oxygen with the emission of light. The immediate product of the luminous reaction is enzyme-bound oxyluciferin adenylate (E-L-AMP). This product acts as a strong inhibitor of the luminous reaction by typing up the enzyme, to which L-AMP is tightly bound.

A study of the kinetics of the luminous reaction reveals two parts to the decay of the light intensity from the maximum flash height.

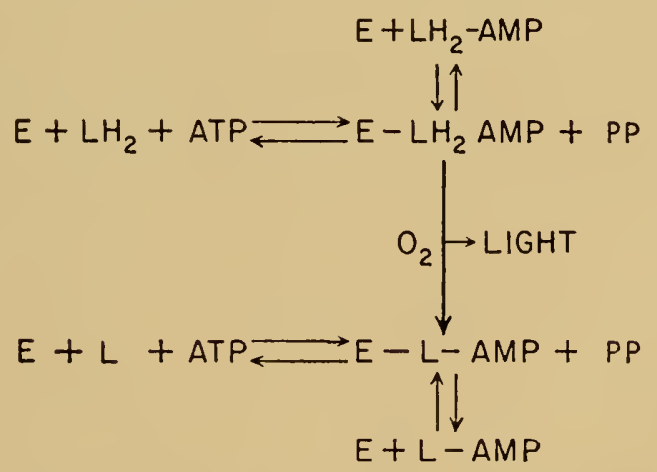

Fig. 5. Summary of the reaclions concerned with light emission in firefly extracts. $\mathrm{LH}_{2}$, luciferin; ATP, adenosine Iriphosphate; PP, pyrophosphate; E, luciferase; $\mathbf{L}$, oxyluciferin; $\mathrm{LH}_{2}-\mathrm{AMP}$ and $\mathrm{L}$-AMP, luciferyl and oxyluciferyl adenylic acid, respectively. 
There is first a rapid decrease (half-value time, $0.25 \mathrm{sec}$.) due to the initial inhibition of the enzyme through accumulation of E-L-AMP. This is followed by a slower rate of decay (half-value time, 13 seconds) governed in part by the procluction of pyrophosphate, which tends to break up the inhibitory enzyme-oxyluciferin complex and so to set free the enzyme once more. The latter effect is demonstrated by the action of pyrophosphatase, which reduces the emission of light in the system to a very low level that may be raised again through the addition of pyrophosphate. Yet pyrophosphate added to the system before the addition of ATP has an opposite effect, tending to prevent the formation of the active luciferase-lucilerin-AMP complex. The authors believe that the firefly controls its flash by using this pyrophosphate mechanism.

$\mathrm{LH}_{2}$-AMP, formed chemically by condensation of its two parts, yields, as expected, an active substrate for the luminous reaction in the presence of luciferase but without ATP or $\mathrm{Mg}^{++}$. Only free oxygen is needed. Free oxyluciferin (L) does not inhibit this reaction, but the product inhibition (i.e., by $\mathrm{E}$-L-AMP) is even more striking than in the natural reaction involving lucilerin, luciferase, and ATP. Instead of a continuous production of light, the available enzyme is rapidly bound and the emission of light ceases, although it can be reinitiated by adding more enzyme. The contrast between the two reactions (starting with $\mathrm{LH}_{2}$ or with $\mathrm{LH}_{2}$-AMP) suggests that in the natural system ATP (or hucilerin, or both) is capable to some degree of frecing the enzyme from the inhibitory product of the reaction. When the substrate, $L_{2}-A M P$, is added to enzyme, pyrophosphate, if present, will compete with oxygen and reduce the emission of light by increasing the reverse separation of the E-LH. AMP complex into enzyme, luciferin, and ATP. Magnesium ions of course greatly enhance this effect of pyrophosphate, and virtually block the emission of light completely. Further demonstration of the correcuness of the schene has been shown by incorporating P32-labeled pyrophosphate into ATP through the reaction just described; and by inhibiting lucilerase through the addition of chemically synthesized L.AMP to the luminous reaction system.

When oxyluciferin is added to enzyme and $A T P$, there is a very great decrease in its fluorescence at $544 \mathrm{~m} \mu$. This property has been uselul in the study of a hydrolytic enzyme found in the firefly which will hydrolyze both $\mathrm{LH}_{2}$-AMP and $\mathrm{L}$ - $\mathrm{AMP}$ when they are free, but not when they are bound to luciferase. The fluorescence serves an an indicator of free oxyluciferin. It is also useful in studying another 
reaction, which like one mentioned previously indicates that ATP can free the enzyme from the inhibitory product L-AMP. In a lightproducing reaction system containing lucilerin, ATP, luciferase, and magnesium, pyrophosphate will continue to be produced long alter the product inhibition of luminescence has occurred, provided pyrophosphatase is present to pull the reaction. Pyrophosphate is still lomed when oxyluciferin replaces luciferin in the system. It secms that $\mathrm{ATP}$ in some way hydrolyzes the oxylucilerin adenylate on the surface of the enzyme, so that the oxyluciferin is free to react with ATP to release pyrophosphate.

The dissociation constant of the complex E-L-AMP has becn determined, and from the linearity of the clissociation curve at various concentrations of L-AMP and of enzyme, McElroy and Seliger conclucle that the luciferase molecule has a single site for the luminous reaction and that a single molecule of L-AMP is bound at this site. Further evidence for this relationship is seen in the observation that even a thousand-fold excess of enzyme in relation to substrate does not recluce the height of the initial flash below that expected on a linear relation of flash height to enzyme-substrate ratio.

Coenzyme A has an interesting stimulatory effect upon the luminous reaction. In a system in which the luciferin concentration is greater than that of the enzyme, and which has reached the stage of slow decay in the emission of light, the addition of CoA stimulates the production of light in direct proportion to concentration. It has been shown to do this by relieving the product inhibition by reacting with the E-L-AMP to produce AMP, L-CoA, and free enzyme. The reaction is reversible. Oxyluciferyl-CoA will react with cysteine or glutathione. The stages in the reaction may be followed spectrophotometrically, since the fluorescence of L-CoA is only about $2 \%$ of that of oxyluciferin, and that of oxyluciferyl-cysteine is about $50 \%$ of that of oxyluciferin.

The effects of several factors upon the luminous reaction have been studied. In glycyl glycine buffer the $p H$ optimum is at 7.8. In phosphate buffer the intensity of the light is greatly reduced. The temperature optimum of the reaction is $25^{\circ} \mathrm{C}$. The activation energy is calculated to be $18 \mathrm{kcal}$. Increase in the oxygen concentration enhances the emission of light up to a concentration of $0.5 \%$, while lowering the $\mathrm{O}_{2}$ concentration does not materially reduce the light intensity until it falls below $0.1 \%$. Under anaerobic conditions E- $\mathrm{LH}_{2}$-AMP accumulates in the system, so that upon readmission of oxygen there is a rapid reaction marked by a flash. The duration of this oxygen 
flash is similar in shape to the normal firefly's flash but lasts much longer, and it has not been possible, to date, to duplicate the natural flash on the laboratory by altering any of the known variables. This failure is one reason for supposing that in the firefly itself the flash is controlled by pyrophosphate, acting by reversing the product inhibition of the enzyme.

The emission spectrum shows a smooth peak at $562 \mathrm{~m}_{\mu}$, the width of the band extending from 500 to $630 \mathrm{~m} \mu$. The energy requirement must therefore be at least $57 \mathrm{kcal}$ per mole. The absorption spectra of luciferin and oxyluciferin are very similar, the major peak of the latter being shifted some $20 \mathrm{~m}_{\mu}$ toward the red (in acid) in comparison with the former. This evidence indicates that the difference between the two forms is simply the addition of a double bond in conjunction with the loss of two protons from the dihydro form. The fluorescence spectra are also very similar, with maxima at $535 \mathrm{~m} \mu$ and $544 \mathrm{~m}_{\mu}$ respectively. The fluorescence spectrum of oxyluciferin exhibits a slight shoulder at $460 \mathrm{~m} \mu$ at acid $p \mathrm{H}$. The shoulder remains rather constant as the $p \mathrm{H}$ changes until it disappears in the increasingly intensified $544 m \mu$ band. The fact that the absorption maximum of oxyluciferin, like that of luciferin, shifts about $50 \mathrm{~m} \mu$ toward longer wavelength at $p \mathrm{H} 8$ and above, while the fluorescence maximum remains constant, implies that different parts of the molecule are involved in the absorption and the emission of light. The efficiency of this intramolecular transfer of energy is shown by the sharp increase in the fluorescence yield of both huciferin and oxyluciferin at $p H$ 8.4 , the point at which the hydroxyl group of the molecule undergoes ionization. The similarity of these fluorescence yield curves for the reduced and oxidized forms of luciferin indicates that both have the same ionizable groups, and the corresponding decreases in fluorescence when L-AMP and L are bound on the enzyme indicate that it is the hydroxyl group which is prevented from ionizing when oxyluciferin is bound on the enzyme surface. At no $p \mathrm{H}$ or temperature, over the range from $-196^{\circ} \mathrm{C}$ to normal temperatures, is there any fluorescence emission agreeing with the bioluminescent cmission spectrum. However, the fluorescence spectra of $\mathrm{LH}_{2}$-AMP and L-AMP are radically different from those of $\mathrm{LH}_{2}$ and $\mathrm{L}$, respectively, and $\mathrm{LH}_{2}$-AMP does have a $570 \mathrm{~m} \mu$ peak in acid that corresponds to the bioluminescence spectrum. The L-AMP fluorescence does not so correspond.

The quantum yield of the bioluminescent reaction is unity; that is, one light quantum is emitted for every luciferin molecule oxidized, 
an efliciency of 100 per cent. There is no observed change in this relation over the temperature range from $3{ }^{\circ} \mathrm{C}$ to $27^{\circ} \mathrm{C}$. Both the tight binding of the $\mathrm{LH}_{2}$-AMP and $\mathrm{L}$-AMP molecules to the enzyme, and the fact that the fluorescence of luciferase overlaps the broad absorption bands of luciferin and oxyluciferin at neutral and alkaline $p \mathrm{H}$, seem alike relevant to the high quantum yield. Perhaps the latter observation signifies that the enzyme-substrate complex forms a resonant molecule able to transfer energy intramolecularly via tryptophan residues until it is trapped at the site of emission. Physical analogies of such a process are known.

As $p \mathbf{H}$ is made more acid, the single yellow-green maximum of the bioluminescent emission spectrum becomes weaker, and the maximum emission changes to red. The quantum yield diminishes materially at the same time; it agrees rather well with the fluorescence quantum yield at various $p \mathrm{H}$ 's, except that the $p K$ is at $p \mathrm{H} 7.4 \mathrm{in}$ stead of $p \mathrm{H}$ 8.4. The previously noted inhibitory effect of inorganic phosphate on the emission of light (effect of phosphate buffer) is similar in nature to the effect of $p \mathrm{H}$ on the bioluminescence. That is, even at neutral or alkaline $p \mathrm{H}$, phosphate diminishes the yellow-green emission maximum and causes the appearance of a red emission band. These effects of phosphate and $p \mathrm{H}$ are further evidence that the enzyme significantly influences the excited state.

The mechanism of the bioluminescent reaction remains puzzling. McElroy and Seliger rule out the possibility that there might be an energy transfer from an excited product to the $\mathrm{LH}_{2}$-AMP molecule, chiefly on grounds that it is the acid fluorescence of $\mathrm{LH}_{2}$-AMP that is close to the biohmminescence emission whereas bioluminescence occurs at neutral and alkaline $p \mathrm{H}$ values, and moreover that energy transfer should vary with concentration whereas the flash height has been shown to be proportional to enzyme concentration over an enormous range. The data also eliminate the Linschitz type of mechanism, which would involve a reaction of two $\mathrm{LH}_{2}$-AMP molecules with oxygen in which one $\mathrm{LH}_{2}$-AMP is regenerated. It therefore becomes necessary to account for the approximately $60 \mathrm{kcal}$ per mole released in the oxidation of $\mathrm{LH}_{2}$-AMP by other means. Peroxidation alone is inadequate for this; but perhaps the formation of a clouble bond in the oxyluciferin, contributing as much as $30 \mathrm{kcal}$ of resonance energy, plus the formation of hydrogen peroxide through activation of oxygen in the reaction, would together be adequate.

The dinoflagellate Gonyaulax polyedra, another luminous organism, has been studied by J. W. Hastings and V. C. Bode. The cell-free 
preparations from this organism in adclition to oxygen, luciferin, and luciferase, require a high concentration of salt. Although the luciferin and lucilerase will not cross-react with enzyme and substrate, respectively, from other groups of luminous organisms, they were found to cross-react with the enzyme and substrate of another related species, Gonyaulax monilata. Gonyaulax cells, when agitated or stimulated, emit bright flashes of light lasting less than one-tenth of a second. A cell-free luciferin-luciferase preparation does not emit light until a high concentration of salt is added. The enzyme has been purified about 20 -fold. The light intensity varies directly with the enzyme concentration, but with increasing luciferin concentration a typical saturation curve is obtained. A non-luminous nonenzymatic oxidation of Gonyaulax lucilerin also occurs. The product can be reduced to active luciferin by means of cysteine or hydrogen, which is not the case when lucilerin is enzymatically oxidized. As the enzyme concentration is increased there is a much higher total amount of light from a given amount of substrate. This is interpreted as the consequence of competition between the enzymatic and nonenzymatic pathways of oxidation of luciferin. Bovine serum albumin has a marked stimulatory effect upon the emission of light, both as respects intensity and total amount. Again, this is interpreted in terms of the formation of an albumin-luciferin complex which protects the substrate from the nonenzymatic oxidation.

The salt requirement is absolute, and high concentrations, usually about $0.8 \mathrm{M}$, are required for maximal light intensity. Many salts are effective, both inorganic and organic. Others inhibit, and no pattern to explain these differences is apparent. There is evidence that the salt acts directly on the enzyme, since it affects its stability and increases its rate of denaturation by heat. Salt also increases the rate of the nonenzymatic oxidation of luciferin. The salt might, as a third possibility, alter the quantum yield of the reaction by acting on the excited state itself. To date, this has been neither demonstrated nor exclucled.

A final report on a bioluminescent system included in this Symposium was made by M. J. Cormier on the luminescence of the sea pansy Renilla reniformis, a coclenterate. Cormier strongly emphasizes the biochemical distinctiveness of this system, and holds that luminous forms of life have not only appeared sporadically during evolution but have not evolved along the same biochemical lines. At first glance, the Renilla Iuminous system appears very like that of the firefly, since in addition to lucilerin, luciferase, and oxygen, a nucleotide is re- 
quired. However, not only ATP, but also Al)P and AMP will supply this requirement, although no other nucleoticles will serve. The system was found to contain a phosphatase which will convert ATP to $\mathrm{ADP}$, and $\mathrm{ADP}$ to $\mathrm{AMP}$; but there is no phosphorylating mechanism for doing the reverse. In short, ATP here serves as a generating system for ADP and AMP, and the kinetics show that the response to ATP is much slower than that to the diphosphate and monophosphate, and has a lag period. ATP, but not ADP or AMP, requires an activating metal ion (Mg or MN) in this system. With a purified enzyme preparation loss of phosphatase activity is accompanied by a loss of ability to utilize ATP in the production of light. ADP and AMP are not affected by this loss of phosphatase activity.

The Renilla luminescent system differs from others in still another way. Normal oxygen tension will not saturate the system; flushing with oxygen causes an increase in the intensity of the light. Increasing concentration of luciferin leads to saturation; increasing enzyme concentration led to a directly proportional increase in light intensity. The $p \mathrm{H}$ optimum is at 7.5 ; the temperature optimum is at $32^{\circ} \mathrm{C}$. Strong inhibitors of bacterial lmmescence, such as cytochrome $c$, do not inhibit Renilla luciferin. It is not oxidized like the flavin substrate of the bacterial luminous system. It is not inhibited by fluoricle (except when ATP is utilized), by cyanide, or by azide, by arsenate, or by pyrophosphate. Unlike the firefly luminescence, that of Renilla is not affected by pyrophosphate or by inorganic pyrophosphatase. This is further evidence of the subsidiary role of ATP in the Renilla system. A requirement for sulfhydryl groups is indicated by the inhibitory effect on luminescence of sulfhydryl inhibitors. Metal ions, (except for zinc, which is a potent inhibitor) have no effect, but a chelating agent such as Versene proves to be a strong inhibitor of luminescence, and the inhibition cannot be reversed by adding metal ions, such as $\mathrm{Mg}^{++}$. The Versene must have a different type of action here. Unlike the Gonyanlax system, there is no requirement for salt in the luminescent system. On the contrary, sodium and potassium chlorides inhibit luminescence linearly with increasing salt concentration.

Evidence for the formation of an intermediate is found in the kinetics of a system preincubated with enzyme before oxygen is admitted. The steady-state value of the light intensity is doubled by doubling of the anaerobic preincubation time. When such a preincubated system is heated to $100^{\circ} \mathrm{C}$ for $11 / 2$ minutes, then cooled, and fresh enzyme added, the presence of a heat-stable intermediate is 
demonstrated by the instantaneous rise of luminescence to the steadystate level. Formation of the intermediate requires both ADP (or AMP) and luciferin, as well as the enzyme. The intermediate might be luciferyl phosphate. In that case, the AMP formed in the reaction might in turn react, after the luminous reaction, with the oxyluciferyl phosphate to form oxyluciferin and regenerate the ADP in a cyclic type of mechanism. For the present, this remains merely a working hypothesis.

\section{Photosynthesis}

Current studies of photosynthesis involve a number of aspects of the over-all process: the nature of the primary activation of chlorophyll and the transfer of energy from chlorophyll to other parts of the system; the transfer of absorbed light energy from accessory pigments to chlorophyll $a$; the flow of electrons in the primary reduction of pyridine nucleotides, in the Hill reaction, and in the reduction of cytochromes; the relation of photophosphorylation to photosynthesis; and the catalytic participation of oxygen in the process. The paper by Daniel Arnon on the nature of Cell-Free Photosynthesis and the Energy Conversion Process presents a number of schemes which endeavor to unify thinking about these aspects into a coherent scheme of photosynthesis as a device that evolved for producing ATP, rather than as one concerned primarily with carbon dioxide fixation. It is, however, evident alike from the other papers and from the vigorous discussion that followed these sessions that a number of key points in Arnon's scheme represent concepts either controversial or not yet fully tested. It seems best, in view of the tentative nature of many parts of this scheme, to abstain from organizing the entire consideration of photosynthesis in the Symposium around this single theory. Nevertheless, a very large portion of the general picture presented by Arnon seems likely to stand the test of lurther experimentation, and is certain to take a prominent place in this and future discussions of photosynthetic mechanisms.

\section{Chlorophyll and Organic Free Radicals}

When a quantum of light is absorbed by chlorophyll in solution, an excited state of the molecule arises, and two things may happen. Excited chlorophyll in some way promotes the oxidation of substrates by oxygen (e. g., oxidation of allylthiourea, studied by Gaffron). Also, chlorophyll in the excited state will sensitize a transfer of hydrogen 
from a reducing agent (such as ascorbic acid) to an oxidized substance (such as an azo dye). M. Calvin, in his contribution, is concerned with the conversion of electromagnetic energy into chemical energy, which does not occur in such processes as these, but does occur in photosynthesis itself.

Chlorophyll is a porphyrin with an isocyclic ring and two extra hydrogen atoms on one of the pyrrole rings; in other words, it is a dihydroporphyrin. Bacteriochlorophyll has two additional atoms on a second pyrrole ring. Protochlorophyll, which is formed in green plants growing in the absence of light, lacks the two hydrogen atoms and instead possesses a double bond in the pyrrole ring. When light is supplied to etiolated plants, the double bond is replaced by the two hydrogen atoms, that is, protochlorophyll is reduced to the ordinary dihydro form of chlorophyll. These relations, as well as more general considerations of the nature of photosynthesis, have suggested to Calvin and to others that the function of chlorophyll may be to serve as a hydrogen carrier between water and the ultimate reducing agents in $\mathrm{CO}_{2}$ fixation, among which is TPNH. Early experiments seemed to rule out the possibility that the shift from protochlorophyll was the level involved in photosynthesis, so attention was shifted to the change between the dihydro- and tetrahydro-porphyrins. The critical experiments to determine whether there is any trace of the latter (bacteriochlorophyll) in green plants have not yet been performed.

Meanwhile, model systems for studying photochemical hydrogen transfer have been studied. Calvin's group has utilized tetraphenylporphin for this purpose. Easy to synthesize as a zinc porphyrin, it contains traces of the zinc chlorin (or clihydro) form; and the spectral differences of the two porphyrins are easy to clistinguish. With a hyclrogen acceptor such as a quinone, it is easy to demonstrate a lightinduced transfer of hydrogen from zinc chlorin to the acceptor; but the reaction does not involve any storage of chemical energy, and the light serves only to supply the activation energy which is needed. However, the kinetics of the reaction do reveal that a long-lived excited state of the chlorin is involved, perhaps the triplet state. The reverse reaction, which would involve an increase in potential chemical energy, was also successfully achieved by reducing the zinc tetraphenylporphyrin with benzoin (an ene-diol) in light, but the quantum yield was very low-indeed, much less than in the opposite direction. It is noteworthy that these photoinduced reductions of zinc porphyrin do not stop at the chlorin (dihydroporphyrin) stage, but go on to a 
tetrahydro- and even a hexahydro-porphyrin state; and with more readiness than the initial stage of reduction. This result strengthened the tentative view that chlorophyll might function by shifting to and from the bacteriochlorophyll condition. Thermodynamically, the shift between chlorin and porphin (or more probably between the tetrahydro and dihydro forms) in the reduction of TPN will require a $\Delta H$ of about +20 kilocalories, and the photolysis of water to produce a reduced porphyrin and molecular oxygen will require about +26 kilocalories. The fact is that light will bring about a transfer of hydrogen atoms to a pyricline nucleotide acceptor, but in the model systems so far studied only a transfer to a very good acceptor, such as a quinone, has been obtained, and not one to so relatively poor a hydrogen acceptor as TPN. Moreover, reduction has only been accomplished with much better hydrogen donors than water. A model that will yield, in solution, similar reactions involving large energy storage is still lacking. Classic examples are the transfers of hydrogen from ascorbic acid or hydrazine to acceptors such as azo dyes (methyl red). Krasnovskii has studied these reactions extensively in the post-war period. He demonstrated that ascorbic acid reduces chlorophyll to some intermediate form, detectable by a pink color, and this intermediate will in turn reduce a hydrogen acceptor and revert to green chlorophyll. But this type of reaction is thermodymamically "downhill" and does not involve any storage of free energy. Nor, even in those cases where the reactions are to some extent photodynamically reversible, is there any extensive storage of free energy.

These difficulties have directed many students of photosynthesis to consider the possibility of an energy transfer like that in solid systems. The structure of the chloroplast may be resembling that of molecular crystals. The absorption spectrum of chlorophyll in vivo (with a peak at $6800 \mathrm{~A}$ ) is not that of chlorophyll after extraction (with a peak at $6600 \mathrm{~A}$ ); and that fact, plus the orderly arrangement of structure within the chloroplast to be seen in electron micrographs, led to the thought of photoconductivity like that in solid lattices. This shift is to be expected in molecules with $\pi$-electrons when packed into crystals, for the interaction between the $\pi$-clouds brings about a shift in the energy state toward longer wavelengths. In "crystalline" chlorophyll the peak moves even to $7200 \mathrm{~A}$.

Hunting for a convenient model of such a system to study, Calvin and his group selected phthalocyamine, a tetrapyrrole connected by nitrogen bridging atoms and hearing benzene rings on each pyrole ring (Fig. 6). Crystalline phthalocyanine laid down in a layer with a 
layer of electron acceptor (ortho-chloranil) on top of it increases in conductivity up to $10^{7}$ times in the dark and $10^{-5}$ times in the light. Electron transler was demonstrated by the presence of ESR (electron spin resonance) signals. When the light is turned on, such a system decreases in number of unpaired electrons, to juclge by the ESR signal, while the conductivity increases. The observed phenomena are interpreted as signifying a transfer of electrons from phthalocyanine to o-chloranil, so as to leave in the lattice positive ion radicals of phthalocyanine which are responsible for the increased conductivity, and to produce o-chloranil negative ion radicals carrying the immobile, trapped electrons. When $7000 \mathrm{~A}$ light is supplied, the exciton

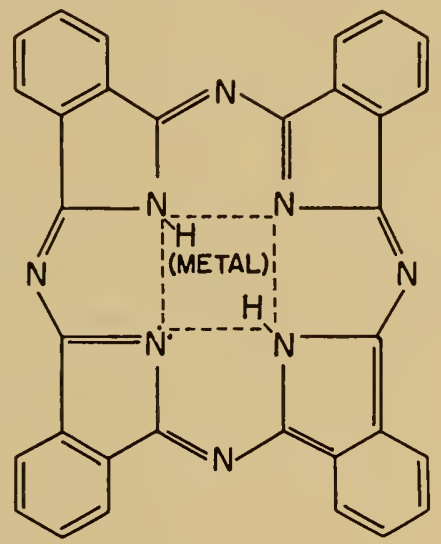

PHTHALOCYANINE

Fig. 6. Structural formula of phthalocyanine.

(combination of electron and positive hole in the lattice) can migrate until ionization occurs. The number of unpaired spins is recluced by the formation of the o-chloranil ion, but the conductivity of the phthalocyanine is increased. When light of $4000 \mathrm{~A}$ is supplied, the opposite happens. Electrons are then transferred from the o-chloranil negative ion radicals to the phthalocyanine crystal lattice; each one quickly combines with a positive hole, thus reclucing the conductivity but increasing the ESR signal. Since negative charge is trapped in the o-chloranil layer and positive charge in the phthalocyanine layer, there will be some polarization of the double layer. Light absorbed by phthalocyanine will increase this polarization, so that photochemically one can induce a separation of oxidizing power, in the form of 
the positive holes, and reclucing power, in the form of the quinone double negative ions.

That this a good model of the chloroplast system is far from substantiated, but a number of observations support such a view. The chloroplast has an electron acceptor, Coenzyme $Q_{.2 .5}$ or plastoquinone, which might function like $o$-chloranil in the model system. Unshared electrons are indeed generated and disappear in the chloroplast system. At room temperature as well as at $-150^{\circ} \mathrm{C}$, whole spinach chloroplasts give a clear ESR signal when illuminated with red light, and its generation just as rapidly at the low temperature as at a normal temperature indicates that this signal is not a product of an ordinary chemical reaction requiring activation energy. ESR signals can be obtained from chloroplast extracts, too, but not comparable with those from whole chloroplasts, either in gauss or in rapidity of decay. By using a mutant Rhodopseudomonas, which does not contain carotenoids, and comparing it with the wild type, which does, Calvin could show that the presence of the electron spin signal does not depend upon the presence of the carotenoid pigment. The action spectra for the ESR signals in the green plant chloroplasts and in Rhodopseudomonas have a maximum at $7200 \mathrm{~A}$, at the red-infrared boundary, whereas, the absorption peak falls at $6800 \mathrm{~A}$. This phenomenon corresponds to the recent demonstration by Brody of an enission peak in Chlorella at $7180 \mathrm{~A}$. Both cases indicate the presence of a previouly unknown active state-not the triplet emission, which lies still farther in the infrared. It might be the lowest $n, \pi^{*}$ state. Excitation to this state, whatever it is, would give rise to the unpaired electron.

Calvin is led to postulate a relation in the chloroplast between chlorophyll and plastoquinone such that, although the latter cannot accept an electron from chlorophyll in the ground state it can do so after the chlorophyll is raised to an excited state, by illumination at 7200 A. Transfer of a second electron to the quinone negative ion radical would be possible if the chlorophyll were excited to a still higher state (say by radiation of $6800 \mathrm{~A}$ ); and the quinone double negative ion thus produced would be sufficiently potent to reduce pyridine nucleotide. The positive chlorophyll ion would ultimately obtain electrons from water, via some donor such as iron, possibly in a cytochrome. Spatially, the chlorophyll layer might have the quinone on one side, in a lipid layer, and the cytochrome on the other side, in the aqueous phase. To quote: 
"Following the absorption of a quantum in chlorophyll, it will migrate by resonance transler to a suitable site near the quinone, at which point electron transfer to the quinone will take place. The resulting vacancy, or positive chlorophyll ion, can then migrate by hole diffusion, that is, electron transfer from nomal chlorophyll, into the vacint orbital of the neighboring chlorophyll positive ion. This process is the one in the entire sequence which most nearly resembles the properties of a semiconductor and permits the oxidizing point (the chlorophyll positive ion) to separate from the reducing point (the electrons in the quinone orbitals) by a process which is very nearly temperature-independent. The oxidizing point will make itself apparent as a chemical change, finally, when it captures an electron from a suitable reducing agent, in this case shown as a ferrocytochrome, thus producing a ferricytochrome and regenerating chlorophyll."

The virtue of this semiconductor model of the chloroplast lies, of course, in the degree to which the proposed electron translers can lead to a rapid and relatively temperature-independent separation of the electrons from the positive holes, and consequently a slowing down of the back reaction. In this respect, the solid state lattice is greatly superior to the solution.

Barry Commoner, in his paper, takes issue with Calvin in respect to the interpretation of the ESR signals. These may come from organic free radicals or semiconductors, but signals from triplets are hard to detect. That ESR signals from systems at very low temperature exclude the occurrence of ordinary chemical reactions and favor a semiconductor type of behavior in the chloroplast is denied. There is evidence that at the temperatures of liquid nitrogen in glassy solutions the absorption of light may eject an unpaired electron, and leave the organic molecule in the form of a lree radical so long as the ejected electron remains trapped in the structure (Lewis and Lipkin). This situation results in an ESR signal.

James Franck, in discussion following Calvin's paper, pointed out a number of reasons for rejecting Calvin's "solar battery" hypothesis, or at least for maintaining reservations in respect to it: (1) the quantum yield of photoionization does not seem high enough to account for the known high yield of photosynthesis; (2) the photoionization hypothesis fails to explain the characteristic relationship between the fluorescence intensity of chlorophyll and the utilization of light 
energy for photosynthesis; and (3) the hypothesis leaves unexplained the Emerson effect, whereby light of a shorter wavelength, if supplied together with the wavelength characteristically absorbed by chlorophyll a, produces a synergistic effect. According to Franck, it is more probable that the primary effect of the absorption of a light quantum is to produce a photochemical intermediate stable enough to persist through an intervening dark period and to undergo a further photochemical change when exposed to light of a shorter wavelength. Also, why does the oxygen present in aerobic cells not interfere with photosynthesis? Franck concludes that the photosynthetic oxidants are present in substrate quantities, not merely in catalytic amounts. He therefore favors the idea of a double keto-eno! transition occurring in both phosphoglyceric acid and chlorophyll at the expense of energy stored temporarily in a metastable state of the excited chlorophyll $a$. The double transition would store about 20 kilocalories of energy. In this scheme, a second step is then initiated in the form of a singlet excitation of the complex. This energy, plus the stored $20 \mathrm{kcal}$, is enough to split the water molecule and reduce phosphoglyceric acid. The $\mathrm{OH}$ group is taken up by a cytochrome. Two of the radicals formed by addition of one $\mathrm{H}$ atom to a phosphoglyceric acid molecule might dismutate to yield phosphotriose and phosphoglyceric acid, with a release of $40 \mathrm{kcal}$ of energy, available to form ATP.

Calvin pointed out that the quantum yield of photoionization in his model system was one, and that chlorophyll molecules in superabundance and not in contact with quinone molecules might transfer their energy by resonance transfer. He emphasized that the essential feature of his hypothesis was that of a charge migration through a $\pi$-system of electrons involving at least several molecules, and that it is only in this sense that the theory resembles "solid state electronic (hole) conductivity." Rabinowitch objected that if the theory were true, the absorption spectrum of chlorophyll in vivo should differ far more than it does from that of chlorophyll in molecular dispersion. But Kasha pointed out that electron overlap was the prime parameter in electronic transport of this type, and that lack of shift of the absorption spectrum is no sign that there is not adequate electronic overlap. It might be the orientation of adjacent chlorophyll molecules rather than the distance between them that is responsible for the smallness of the shift in the spectrum.

Commoner's paper points out that with a refmement in the sensi- 
tivity of the ESR spectrometer it becomes possible to tolerate samples containing liquid water and to attain scamning specels of 30 to 90 seconds. Adequate resolution of the photosynthetic ESR signals from chloroplasts of spinach and tobacco revealed the signal's complex structure. In the light, the once single ESR signal was resolved into two, and in the dark a hyperfine structure of five peaks was disclosed. One of the peaks seen in the light was a new ESR signal (I) lacking hyperfune structure. This signal had a relatively rapid onset and decay. Signal II, the dark signal, was much slower in onset and decay, and occurs in both light and dark.

Observations on living Chlorella and on green and colorless forms of Euglena further tied the two signals to the photosynthetic process. The effects of $5 \% \mathrm{CO}_{2}$, of different light intensities, and of various temperatures were studied. The interpretation reached was that there are two sequential steps ( $I$ and II) involving the formation of intermediates with unpaired electrons (i.e., of free radicals). Component I is considered the more proximal to the initial photochemical cvent, but it can scarcely be an actual light-induced excited state because it decays far too slowly. Since Component II is always present in the dark, it must also be producible in some oxidationreduction occurring in the dark. There is no evidence that Component I represents a free radical form of chlorophyll itself. By growing Chlorella in $\mathrm{D}_{2} \mathrm{O}$, a great compression of both signals is to be observed, but especially in the case of Component II. This alteraation of the ESR signal indicates that the unpaired electron is associated with hydrogen atoms, and that the hyperfine structure is due to protons. The association excludes the possibility that the unpaired electrons are conduction electrons in a semiconductor and establishes the existence of free radicals (at least Component II) in photosynthesis. Since Calvin also postulates a formation of free radicals on the basis of his phthalocyanine-quinone model, and since he does not insist on a flow of electrons but rather on a charge migration, there would seem to be no fundamental difference between Commoner and Calvin in the interpretation of the ESR signals. The basic difference of interpretation lies in the identification of the molecules that give rise to the free radicals. Calvin sees these as possibly the chlorophyll and plastoquinone; Commoner avoids iden. tifying them for the present, but excludes chlorophyll as the source of his Component $I$ and does not regard the signals as immediately related to the primary photochemical event. 
Absorption by Accessory Pigments and their Relation to Chlorophyll a

C. S. French has provided a review of the relation to photosynthesis of the so-called accessory pigments, namely, the carotenoids, phycobilins, and chlorophyll $b$. It has become increasingly apparent that these pigments are of much greater importance than was formerly thought to be the case. They are probably responsible lor a different chemical reaction to that mediated by chlorophyll $a$; and, in the green plants, chlorophyll $b$ appears to perform the same function as the more striking accessory pigments of the algae. The experiments forcing this revision of classic viewpoints include the demonstration that photosynthesis caused by one wavelength of light is enhanced by addition of a second wavelength when given simultaneously (the so-called Emerson enhancement effect), or even when given some seconds later; the analysis of chromatic transients; the relative inactivity of chlorophyll $a$ in red algae; and the occurrence and activity of several different forms of chlorophyll $a$ in green plants.

The existence of different forms of chlorophyll $a$ in living plants is inferred entirely from the occurrence of several absorption peaks in the red region of the spectrum, even though only one peak is found for chlorophyll $a$ in solution (at $662 \mathrm{~m}_{\mu}$ in ethyl ether, for instance). There is a characteristic absorption band at $682-684 \mathrm{~m} \mu$ in chlorophyll $a$ in the living leaf just after it has formed from protochlorophyll (main band, $650 \mathrm{~m} \mu$ ). The 682-684 $\mathrm{m} \mu$ band soon shifts to $670-673 \mathrm{~m}_{\mu}$ in the mature leaf. There are other absorption bands at $692-695 m_{\mu}$ and $707 m_{\mu}$. The latter band has been found especially strongly developed in the flagellates Ochromonas and Euglena. Chlorophyll $b$ absorbs principally at 430 and $644 \mathrm{~m} \mu$ in ether; carotenoids, including fucoxanthin, absorb from $500 \mathrm{~m} \mu$ into the near ultraviolet; phycoerythrin (red) and phycocyanin (blue), are strongly fluorescent and may be identified by their fluorescent spectra as well as their absorption spectra.

With a multiplicity of pigments present, the question of which ones function in photosynthesis might, one would think, be answerable by comparing the action spectrum of photosynthesis with the absorption spectra of individual pigments. Such comparison led initially to the concept that chlorophyll $a$ is the pignrent of major importance. Accessory pigments absorb light, but they were thought merely to pass the energy on to chlorophyll a, absorbing at $695 \mathrm{~m} \mu$, before the chemical changes of photosynthesis began. It was therefore surprising to discover that light at $690-700 \mathrm{~m} \mu$, although ab- 
sorbed only by chlorophyll $a$ and not by any accessory pigments, was really very ineflicient in photosynthesis. Robert Emerson and his coworkers first discovered, as mentioned above, that $650 \mathrm{~m} \mu$ light, over and above the photosynthesis it would induce by itself, acted to make simultaneously applied light at $695 \quad m \mu$ more effective. By using a background light of $695-700 \mathrm{~m} \mu$, Emerson obtained an action spectrum for the "enhancement" of the effect of the longer wavelength. In all cases this action spectrum corresponded to the fraction of light absorbed by the accessory pigment present, among those named above (for green plants this means a peak of effectiveness at $650 \mathrm{~m} \mu$, where chlorophyll $b$ absorbs most strongly). This fact meant that chloropyhll $b$ and other accessory pigments possess some more significant function than that of merely supplying extra energy to chlorophyll $a$.

French now points out that the same data can be interpreted in a broader sense, as a mutual enhancement between the pair of wavelengths-for it is equally logical to use a background light of 650 $m \mu$ and determine the efficiency of longer wavelengths in enhancing the effects of the shorter ones. These complex action spectra were directly plotted on the recording action spectrophotometer, the principle of which is to use monochromatic light slowly changing in wavelength and continuously adjusted in intensity so as to maintain a constant rate of photosynthesis as the spectrum is traversed. The results show that not only chlorophyll $b$ but also "forms" of chlorophyll $a$ with absorption peaks at about $673 \mathrm{~m} \mu$ and $684 \mathrm{~m} \mu$ can enhance the efficiency of chlorophyll $a$ absorbing maximally at 695 $m_{\mu}\left(\mathrm{C}_{\mathrm{a}} 695\right)$. This conclusion, first derived from experiments with Chlorella, was confirmed by work with Botrydiopsis, which has several forms of chlorophyll $a$ but lacks chlorophyll $b$. At present it therefore appears that the accessory pigments function principally to "activate" the $\mathrm{C}_{\mathrm{a}} 695$ form of chlorophyll $a$.

Use of the recording action spectrophotometer also confirmed the existence of the "chromatic transients" discovered by Blinks. These are consistent spikes or dips in the rate of photosynthesis caused by merely changing the color of the illuminating light, even when the steady-state rate is iclentical at the two different wavelengths. In Chlorella the action spectrum of the chromatic transient is exactly similar to the action spectrum of the Emerson enhancement effect, so that it too reflects the activation of $\mathrm{C}_{\mathrm{a}} 695$ by the accessory pigments.

Experiments done by Myers at saturating levels of light intensity surprisingly showed that at saturation with light of $700 \mathrm{~m}_{\mu}$ the photo- 
synthetic rate was far below the rate at saturation with light of shorter wavelengths. It had long been thought that at high light intensities, where the photosynthetic rate is limited by some "dark" reaction, the intensity or color of the light could have no effects; but this turns out not to be so. An action spectrum can be obtained at saturating light intensities, and recent experiments by McLeod with Chlorella show a peak of $650 \mathrm{~m} \mu$, whereas the customary action spectra obtained at low light intensities have a maximum at $680 \mathrm{~m} \mu$. French suggests that this effect is also a product of the Emerson enhancement effect, involving activation by $650 \mathrm{~m} \mu$ light of both chlorophylls a $\left(\mathrm{C}_{\mathrm{a}} 695\right)$ and $b$, whereas at $680 \mathrm{~m} \mu$ only chlorophyll $a$ is activated. At the latter wavelength only unenhanced photosynthesis would consequently be expected.

The critical problem in photosynthesis, as compared with other well-understood photochemical reactions, is to account for the way in which a reaction that seems to be a single step-the splitting of a water molecule-and yet requires more energy than is contained in a single quantum of visible light, gathers the necessary energy for the process. The several phenomena attributable to the accessory pigments seem related to this process; and since $\mathrm{C}_{\mathrm{a}} 670$ itself acts as an accessory pigment, the problem may possibly be resolved into that of understanding the respective photosynthetic roles of the different forms of chlorophyll $a$. Is the enhancement of the effect of $C_{a} 695$ through interaction within the pigment system itself? or clo the various pigments produce different chemical products that then interact? The latter is the preferred theory, because the interaction still occurs even when the beams of the two different wavelengths are separated in time, as well as because of the clifferent time course of respiration after illumination respectively at $650 \mathrm{~m} \mu$ and at $700 \mathrm{~m} \mu$. French proposes a scheme in which there are two pathways of photosynthesis: (a) a main path, depending on the forms of chlorophyll a absorbing at 673 and $681 \mathrm{~m} \mu$, which may be activated by energy transfer from the accessory pigments; and (b) the enhancement path, depending on the form of chlorophyll $a$ absorbing at $695 \mathrm{~m} \mu$, which produces a chemical product that interacts with some chemical prod uct of the accessory pigments to release oxygen. The half-lives of botl these chemical products, although not identical, are of the order of 5 seconds.

Like French, Eugene Rabinowitch and Govindjee have emphasized the need arising from the enhancement phenomena to investigate the effect on photosynthesis of combinations of monochromatic beams 
systematically varied in wavelength. The preceding explanation of the action of the accessory pigments leaves unexplained the parallelism between the action spectra of photosynthesis and the fluorescence of chlorophyll a. Rabinowitch and Govindjec, like French and his colleagues, have found clear-cut evidence of a form of chlorophyll a absorbing maximally at $670 \mathrm{~m} \mu$, not only in Chlorella but also in the diatom Navicula, which possesses fucoxanthol (peak absorption, $535 \mathrm{~m} \mu$ ) and chlorophyll $c$ (peak at $630 \mathrm{~m} \mu$ ), but which lacks chlorophyll $b$. In the blue-green alga Anacystis, Emerson found no $\mathrm{C}_{2} 670$; in fact, a negative region was present at this point in the action spectrum when monochromatic light at $670 \mathrm{~m} \mu$ was combined with light at $685-700 \mathrm{~m} \mu$. In other words, not only is there no $\mathrm{C}_{2} 670$ to activate the elfect of photosynthesis at $695 \mathrm{~m} \mu$, but the main chlorophyll component in Anacystis, $\mathrm{C}_{2} 680$, not only fails to enhance the photochemical action of $\mathrm{C}_{2} 690$ but actually counteracts it. This may be a saturation effect. Owing to the lower saturation levels in the lar-red region, the combination of two monochromatic beams may throw the process into the saturated region and consequently lower the quantum yield to a level below that of either beam alone. Further evidence of the several forms of chlorophyll $a$ is to be seen in the differential reversible bleaching of $\mathrm{C}_{\mathrm{a}} 670, \mathrm{C}_{\mathrm{a}} 680$, and $\mathrm{C}_{\mathrm{a}} 690$ by strong illumination. $\mathrm{C}_{\mathrm{a}} 680$ is considerably more sensitive, in Chlorella cells, than the two other forms.

Rabinowitch suggests, as an alternative to the scheme proposed by French, that the primary photochemical process may consist of two steps, only one of which may be effected by excited $\mathrm{C}_{\mathrm{a}} 690$, whereas $\mathrm{C}_{\mathrm{i}} 670$ can sensitize both of them. This would fit well with Franck's suggestion that $\mathrm{C}_{\mathrm{a}} 690$ leads almost exclusively to the triplet state, while $\mathrm{C}_{\mathrm{a}} 670$ leads to an excited singlet state with a long enough life to emit fluorescence. Photosynthesis would then require cooperation of a molecule in an excited singlet state with one in a triplet state, though why that should be so remains an enigma.

The quantum yield of chlorophyll $a$ in the far-red region of the spectrum has been measured by J. B. Thomas and Govindjee in the red alga Porphyridium cruentum. These algae lack chlorophyll $b$, and the phycocyanin absorption at $625 m \mu$ and phycoerythrin absorption at $500-565 \mathrm{~m} \mu$ was eliminated by passing white light through a series of filters containing extracts of the phycobilin pigments. The quantum yield of photosynthesis did not change until absorption by the phycobilin filter surpassed $20 \%$; then it decreased linearly until absorption reached $85 \%$; finally, after an even sharper drop, photosynthesis 
became no longer measurable when absorption was about $95 \%$. Nevertheless, the residual light could be combined with light in the orange range to produce photosynthesis. In the range where the quantum yield declines linearly with the decreasing amount of light absorbed by the accessory pigments in the cell, the relationship may depend on the intensity ratio of the light absorbed by the accessory pigments to the light absorbed by chlorophyll $a$. In other worls, in this range light absorbed by chlorophyll $a$ is able to produce photosynthesis only in proportion to the amount of light absorbed by the accessory pigments.

The recent results of $\mathrm{B}$. Kok and G. Hoch, and of Mary Belle Allen, show that even more forms of pigments, absorbing in the far-red at or beyond $700 \mathrm{~m} \mu$, will have to be considered in the interpretation of photosynthesis. Allen, studying the chrysomonad Ochromonas danica found strong absorption at $710 \mathrm{~m} \mu$ and fluorescence at 725 $\mathrm{m}_{\mu}$, and also in "apple green" mutants of Chlorella pyrenoidosa at $710 \mathrm{~m}_{\mu}$ absorption peak was found.

Kok and Hoch used difference spectra derived from the absorbance of samples just before and after a flash of bright white light. The changes, negative in direction, represent a bleaching of pigments, and the strongest such change occurs at a wavelength slightly longer than $700 \mathrm{~m} \mu$. It is prominent in Nostoc and Anacystis (blue-green algae) and in Porphyra, a red alga. The pigment involved is simply called "P 700" by these workers. The shortest flashes used $\left(3 \times 10^{-4}\right.$ sec) were effective and gave evidence of a direct photochemical event, leading to bleaching of the pigment. Restoration of the pigment in the clark is spontaneous and has a half-time of about 7 milliseconds. The quantum yield of the process was about unity. The activation spectrum was mainly that of chlorophyll $a$. The rapid increases and decreases in amount of $\mathrm{P} 700$ which occur when the pigment is thus exposed to bright flashes of light are superimposed on a background level of the pigment which is also modified by light. This level slowly diminishes if many successive flashes of light absorbed by chlorophyll a act upon it (a "negative light effect"). Contrariwise, the general level of $P 700$ is increased when light is absorbed by the accessory photosynthetic pigments (a 'positive light effect"). This effect, attributable to absroption by phycocyanin, is olservable only when the detecting beam (of $700 \mathrm{~m} \mu$ ) is itself strong enough to bleach a sufficient quantity of P 700 for the restorative action of the phycocyanin-absorbed energy to become evident. Bleaching of the pigment represents its oxidation; it is colored when reduced. Thus 
absorption at $700 \mathrm{~m}_{\mu}$ is increased by adding ferrocyanide or reduced phenazine methosulfate (P.MS): and the color (an be bleached again by adding lerricyanide. Ferricyanide or other dyes that are electron acceptors in the Hill reaction will, as might be expected, inhibit the light-induced oxidation of P 700 (the negative effect), since they produce more or less complete oxidation prior to the illumination. Conversely, phenazine methosulfate, which reduces $P 700$ in the dark, precludes its reduction by means of light but stimulates the extent of the oxidation occurring in the negative light-effect.

Kok and Hoch, in order to provide for a role of $\mathrm{P} 700$ in photosynthesis, propose a cyclic scheme (Fig. 7). They assume that the natural photosynthetic oxidant $X$ reacts with reduced $P 700$ in a first light-induced reaction. Since preliminary measurements indicate an oxidation-reduction potential $\left(E_{0}^{\prime}\right)$ for $\mathrm{P} 700$ of +0.46 , and that of cytochrome $f$ is +0.365 , the oxidized $P 700$ could be the agent responsible for the fast oxidation of cytochrome $f$ by light. This first phase may be short-circuited by ferricyanide and is equivalent to the Hill reaction. When all the $\mathrm{P} 700$ is oxidized, the reaction stops. In the second phase of the cycle, the oxidized P 700 becomes reduced, again in a reaction induced by the absorption of light, but this time chiefly by the accessory pigment, while oxygen is evolved. Strikingly suggestive of this possibility is the fact that, although the lightinduced oxidation of $\mathrm{P} 700$ induced by the absorption of light by chlorophyll a is quite stable as long as the particulate chloroplastprotein complex remains inact, the reduction of $\mathrm{P} 700$ (mediated

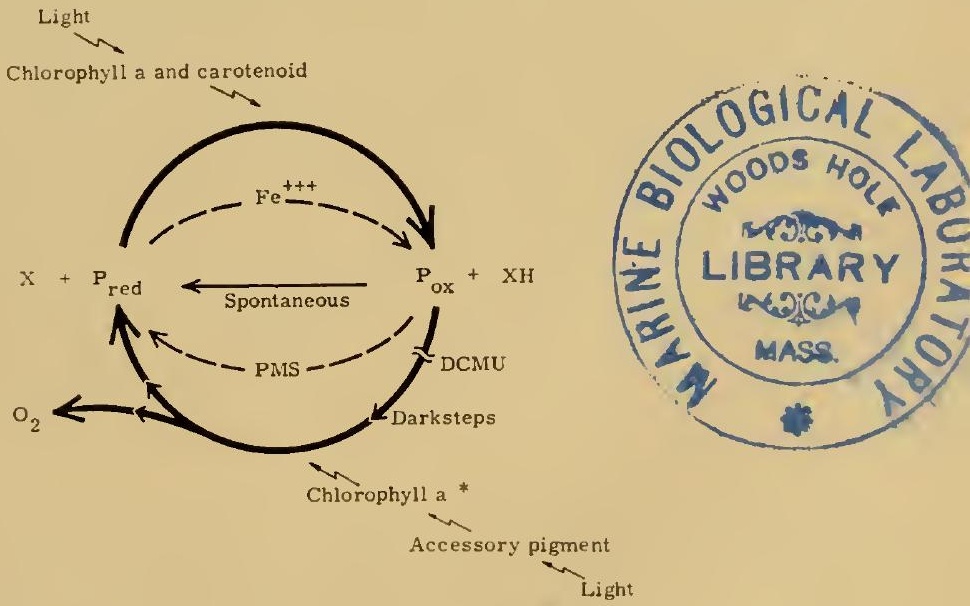

Fig. 7. Schematic summary of discussed observations. 
by the accessory pigments) is very susceptible to four conditions which also specifically inhibit the evolution of oxygen: heating, aging, and the action of hydroxylamine and of 3 (3,4-dichlorophenyl)-1,l-dimethyl urea (DCMU). This rectuction of $P 700$ by light, through electrons derived from the primary photoreductant, XH, completes the cycle. Moreover, the cyclic photophosphorylation with phenazine methosulfate, which bypasses the oxygen-evolving phase, is maximal at 710 $m_{\mu}$, a wavelength that is quite ineffective in driving complete photosynthesis or in causing the reduction of $\mathrm{P} 700$ by light.

In any event, it is clear that the assumption, made in other papers in the Symposium, that all the absorption above about $660 m \mu$ may be ascribed to "chlorophyll $a$ " in its various forms has to be modified in view of the data of Kok and Hoch showing that absorption at $700 \mathrm{~m} \mu$ is attributable to a photosynthetic pigment of distinctive functions.

\section{The Cytochromes in Photosynthesis}

R. Hill, in 1939, in his now-classic study of photosynthesis, suggested that a "respiratory pigment" might play an important part in a chemosynthetic system linked to the quantum absorption process. It is by now quite clear that this is so. The chloroplasts of the higher plants contain cytochromes, and photosynthetic bacteria likewise possess cytochromes linked to the photosynthetic system.

Two cytochromes, $f$ and $b_{i}$, have been found to be characteristic of chloroplasts. Neither of these cytochromes is iclentical with any of the mitochondrial or microsomal cytochromes, which, unlike the chloroplast cytochromes, are denatured by $80 \%$ acetone. In the light cytochromes $f$ and $b_{\mathrm{fi}}$ are present in the oxidized form. The oxidation-reduction potential $\left(E_{0}^{\prime}\right)$ of cytochrome $f$ is +0.365 , of cytochrome $b_{f i},-0.03$. Upon extraction with acetone, cytochrome $f$ becomes reduced but cytochrome $b_{6}$ remains fully oxidized. In spinach chloroplasts the ratio of $b_{i b} / f$ was found to be 1.3 . In low-temperature spectra of whole chloroplasts, in comparison with the acetone-extracted preparations, the absorption bands are shifted to slightly longer wavelengths. Illuminated chloroplasts perform the reaction:

$$
R+\mathrm{H}_{2} \mathrm{O} \rightarrow \mathrm{RH}_{2}+1 / 2 \mathrm{O}_{2}
$$

a reaction the reverse in clirection of the mitochondrial oxidations. A variety of hydrogen-acceptors, either two-electron acceptors or oneelectron acceptors, will serve. The cytochromes of the illuminated chloroplast may function as a hydrogen transport system acting 
against the themodhemical gradient. Hill and his colleagues now prefer a scheme in which wo light-driven reactions against the thermochemical gradient are comnected by an exergonic reaction between cytochomes $f$ and $b_{1 ;}$. This implies an intermediate stage when cytochrome $f$ is oxidized and cytochrome $b_{6}$ is reduced. Such a state has been observed in ilhminated pale yellow-green leaves, in agreement with this hypothesis: but it has not been observed to date in normal green leaves or in chloroplast preparations. Nor have the spectral changes to be expected during the postulated hydrogen transfer been demonstrated so far.

In general, algae and photosynthetic bacteria also possess both $f$-type and $b$ cytochromes, but their roles in photosynthesis remain unknown. Lucile Smith reports on the properties and behavior of bacterial cytochromes in the bacteria Rhodospirillum rubrum and Rhodopseudomonas spheroides. Light absorbed by the bacteriochlorophyll results in oxidation of the cytochromes (characterized by reduction in absorption of the Soret bands lying between 400 and $450 \mathrm{~m} \mu$ ), as well as changes in the absorption spectra of the carotenoid pigments which are present. The cytochromes oxidized in the light include those oxidized when oxygen is added in the dark. These cytochromes are those of the respiratory transport chain, the equivalent of the mitochondrial cytochromes of higher organisms. But in addition at least one other cytochrome is oxidized, one with an absorption peak at $420 \mathrm{~m} \mu$, corresponding to the cytochrome $c_{2}$ of these bacteria isolated by Vernon. Cell-free extracts of $R$. rubrum, which can both respire in the dark and promote rapid photophosphorylation, do not exhibit oxidation of the cytochromes upon illumination unless phosphate acceptor (ADP) is added to the system. Cytochrome $c_{2}$ is consequently implicated in the process of electron transfer associated with photophosphorylation. The action of substrates and of inhibitors also distinguishes between the respiratory and photophosphorylative cytochrome chains in the bacteria. But both chains are conceived to be very similar in properties, to include both $c$-type and $b$-type cytochromes, and to conduct phosphorylation in a manner similar to "oxidative phosphorylation." Only, in the one case, the source of electrons is the oxidizable substrate; in the other it is the photoreductant produced in the primary reaction of photosynthesis; and in the former case the transfer may be either to oxygen or to the photooxidant produced in photosynthesis, whereas in the second case only the photooxidant acts as electron acceptor.

Martin Kamen suggests that the role of the cytochromes or other 
heme proteins in photosynthesis may be related to the resonance properties of the similar tetrapyrrole structures of the chlorophyll and cytochrome molecules. If these structures are juxtaposed so that the magnesium of the chlorin lies close to the iron of the heme, the excitation of chlorophyll by absorption of a photon, leading to an excited singlet or triplet state, might be relieved by the transfer of an electron from the heme. [In discussion, Linschitz described studies of the quenching of triplet states, e.g., in porphyrin, by metal ions. Generally, complexing of the metal ion effectively blocks such quenching, but not when the metal is in a porphyrin complex. This work is being extended to study the quenching of chlorophyll triplet states by other porphyrins.] This electron transfer from heme to chlorophyll would produce a partially reduced chlorophyll radical and a "partially oxidized" heme $\left(\mathrm{Fe}^{++++}\right)$, most likely in their ground states. If so, the oxidized form of heme would probably be at least 1.5 e.v. more oxidizing than the semi-reduced chlorinogen radical, provided the energy clifference is close to that of the original excitation of chlorophyll. Kamen supposes that the heme in the higher oxidized state might have a potential of +0.9 , and hence the semichlorinogen one of -0.6 or -0.7 e.v. So strong a reducing potential could readily provide for the required electron transfer step to pyridine nucleotide $\left(E_{o}^{\prime}=-0.3\right)$, and photophosphorylation might thus be coupled to generation of TPNH from the photoreductant, here identified as the semichlorinogen radical. If substrate amounts of the pyridine nucleotide are present and are funneled off to provide for $\mathrm{CO}_{2}$ fixation, the $\mathrm{Fe}^{++++}$-heme component could withdraw electrons from other components of the system, and so lead to oxygen evolution. One would need to assume that in the photosynthetic bacteria the protein moiety of the heme protein could permit only peroxidase reactions, so that oxygen would not be evolved, but oxidizable substrate would be needed. In any case, cytochrome oxidase would have to be absent in the oxidation chain, as indeed seems to be the case in all such systems. Various other possibilities can be envisaged. As an alternative to the model set forward by Calvin, this ingenious but speculative proposal has the merit of suggesting certain kinds of experimentation that might otherwise not be carried out. In particular, a study of photochemical reactions in chlorophyll-heme systems of various kinds might be profitably carried out with the newer techniques for studying very fast reactions and detecting very short-lived internediates and radicals.

Lipid function in the chloroplast is surprisingly unknown, considering that about 30 per cent of the dry weight of chloroplasts consists 
of lipids. A. A. Benson has summarized some aspects of the problem. The lipids are in the grana and are highly oriented. Two galactolipids predominate. There is also phosphaticlyl glycerol, the predominant phosphatide. Diglycerophosphate and phosphatidyl glycerol undergo reciprocal changes in amount during illumination of cells (Chlorella). In the dark, the lipid diminishes in amount, but is rapidly replenished at the expense of the diglycerophosphate when illumination returns. The reservoirs of glycerophosphate may provicle "reducing power" which can be accumulated during illumination and afterwards serve to fix $\mathrm{CO}_{2}$ in the clark. The galactolipids are galactosyl diglycerides, and include monogalactosyl, digalactosyl, and sulfodeoxygalactosyl types. They very rapidly incorporate labeled $\mathrm{C}^{14}$ from $\mathrm{C}^{14} \mathrm{O}_{2}$ during photosynthesis and are presumably located at the phase boundaries adjacent to the transglycosylating enzymes of carbohydrate synthesis. In the chloroplast lamellae, the galactose moieties are presumably in contact with the stroma and the fatty acids in a lipid layer adjacent to a protein-rich phase. There might be two types of lipid laminae, an outer one "dominated by galactolipids in the region of carbohydrate synthesis and an inner one dominated by chlorophyll and electron transport systems in the region of oxygen production."

James Franck, who bases his views on the properties of chlorophyll $a$, which undergoes both $n \rightarrow \pi^{*}$ and $\pi \rightarrow \pi^{*}$ transitions, conceives of a sandwich-like structure, with chlorophyll lying between layers of lipid and protein and protected from fluid water, except for a small percentage of exposed molecules. Strongly protected chlorophyll molecules, when excited, pass excitation energy to the exposed chlorophyll molecules by resonance transfer. This results in a metastable triplet state. The accessory pigments transfer singlet excitation. Preumably the metastable triplet state can carry out only one of the two photochemical steps in the splitting of water; singlet excitation is needed for the other.

\section{The Relation of Photophosphorylation to Photosynthesis}

In 1954 it was established by Daniel Arnon and his collaborators that isolated chloroplast systems can in fact fix carbon dioxide, and that photosynthesis can in fact be conducted outside the living cell. Now, six years later, a vast amount of additional work on the problem, done in many laboratories, is ripe for fresh interpretation and a new synthesis of ideas. Arnon has in several recent papers attempted this labor; and in the present Symposium he has in fact 
produced a masterly synthesis, major leatures of which must be accepted. Some aspects remain in clispute and some are still hotly contested; others are at the moment more speculative than solidly supported by evidence. Be that as it may, the review is certain to be highly stimulating and should provoke a great deal of experimentation. To summarize a 78-page review of such scope is incleed not easy. Fortunately, Arnon has materially lightened the task by providing a number of diagrams of his conceptual schemes.

The great problem in photosynthesis remains that of the exact role of light. Originally, of course, light was supposed to be the direct mediator of $\mathrm{CO}_{2}$ fixation in the form of carbohydrate. The discovery in the 1880 's of the chemosynthetic bacteria by Winogradsky, and of the purple photosynthetic bacteria by Englemann, showed otherwise. But some forty years were required before it was clear that all organisms can assimilate $\mathrm{CO}_{2}$ although they differ as to the energy sources they can apply to the process. Similarly, a great lag in the undertanding of photosynthesis occurred because the evolution of oxygen was thought to be essential to the process; and not until the classic work of van Niel was it established that bacterial photosynthesis is quite similar to that in green plants except for the nature of the hychrogen donor.

Arnon states that the energy requirements for $\mathrm{CO}_{22}$ assimilation are satisfied by TPNH and ATP in combination. The work with isolated chloroplasts provided no evidence for photochemical formation of any special reductant $(\mathrm{H})$ capable of directly reducing $\mathrm{CO}_{2}$ to carbohydrate by some special photosynthetic reaction, as van Niel had postulated. Instead, the investigation of $\mathrm{CO}_{2}$ assimilation by isolated chloroplasts has "completed the chain of evidence for the view that carbohydrate synthesis is removed from the domain of photosynthetic reactions proper in which light is converted into chemical energy. In green plants the first stable, chemically defined products of this energy conversion are not intermediate of $\mathrm{CO}_{2}$ assimilation but are the two components of assimilatory power, $\mathrm{TPNH}_{2}$ and ATP."

This view of the problem may be rather less than certain on account of the recent discovery of Warburg, confirmation of which is presented by Vennesland in this Symposimm, that carbon dioxide functions catalytically in the Hill reaction. Warburg expects that in the whole cell this function of carbon dioxicle, intinately related to the light reaction and probably to chlorophyll itself, will be the starting point for carbon assinilation in photosynthesis. Until the 
full extent and implications of this discovery are known, the quantitative, or even the qualitative, requirements for $\triangle T P$ and TPNH in whole photosynthesis cammot be considered as setted.

In 1951, Vishniac and Ochoa, Tolmach, and Armon independently showed that an isolated chloroplast system can reduce pyridine nucleotides and evolve oxygen, il "pulled" by a coupled reaction that prompty utilized the reduced pyridine mucleotide which was formed. San Pietro and Lang later succeeded in isolating from chloroplasts an enzyme, which is now called photosynthetic pyridine muclcotide reductase, and which acts preferentially on TPN rather than DI'N. Use of the reductase enzyme permitted major amounts of reduced pyridine nucleotides to accumulate, and obviated the need for the pulling system. In 1954, Amon and his coworkers found that the remaining component of "assimilatory power, " $\mathrm{ATP}$, is also produced in chloroplasts. The process was shown to be distinct from the "oxidative phosphorylation" of mitochondria in two respects: no oxygen is consumed; and no chemical substrate, such as succinate, is required to provide free energy from the formation of the pyrophosphate bonds of ATP. Only light is needed to drive the process.

Although photosynthetic phosphorylation involves no net consumption of molecular oxygen, the presence of oxygen was found to be necessary for the process to proceed. In other words, oxygen behaves as a catalyst rather than a substrate in this form of phosphorylation. A prime difference is to be seen in the inability of isolated chloroplasts to form ATP in the dark by oxidizing the wellknown hydrogen donors of oxidative phosphorylation with molecular oxygen.

In work with spinach chloroplast systems, it was soon found that flavin mononucleotide (FMN) and vitamin $\mathrm{K}$ serve as catalysts of photosynthetic phosphorylation, and that at optimal concentrations of these cofactors the process becomes independent of external oxygen, although at low concentration of the cofactors oxygen remains necessary. This latter observation is regarded by Amon and his coworkers as further evidence that oxygen acts only catalytically in photosynthetic phosphorylation; and they conclude that the anaerobic situation, with optimal anounts of FMN or vitamin $\mathrm{K}$, was the more fundamental situation for photosynthesis in general, since it would apply to bacterial photosynthesis, which is anaerobic. Frankel, also in 1954, discorered photophosphorylation in Rhodospirillum rubrum, and cell-free preparations from the obligate anacrobes Chromatium and Chlorobium were likewise soon demonstrated to producc ATP 
when illuminated (Williams). Although at first the rates in the cell-free reactions were low, rates equal to those of maximum carbon assimilation in intact leaves were later obtained (Jagendorf and Avron) .

In addition to FMN and vitamin $\mathrm{K}$, magnesium ions and ascorbate serve as cofactors in the process of photophosphorylation. It was thought that FMN and vitamin $\mathrm{K}$ might provide alternative pathways in an electron transport chain, since they exhibit a differential dependence upon TPN and a differential sensitivity to the inhibitors dinitrophenol and o-phenanthroline. Further insight came with the discovery by a number of workers that the dye phenazine methosulfate, a strong reducing agent for cytochromes, will strongly stimulate photosynthetic phosphorylation. The phenazine methosulfate system more closely resemble the vitamin $\mathrm{K}$ pathway, although differing from it in certain respects. In bacterial photophosphorylation, FMN proved entirely ineffective, but vitamin $\mathrm{K}$ and ascorbate were active colactors, and phenazine methosulfate also.

These observations have led Arnon to a hypothesis about the electron flow in photo synthetic phosphorylation. The simplest view would be to assume that formation of the high-energy pyrophosphate bond of ATP is coupled with a release of free energy during electron transport, as in oxidative phosphorylation. Since the photosynthetic system does not utilize external substrate as an electron donor, the source of electrons must be sought elsewhere; and there must be a suitable electron acceptor available, too. Arnon proposes that the excitation of chlorophyll by light leads to an expulsion of an electron that has been raised to some energy level above the ground state. The resulting oxidized state of the chlorophyll $[\mathrm{Chl}]^{+}$, leaves it in position also to be the electron acceptor, the expelled electron returning stepwise via an electron carrier system and releasing free energy as it does so. The intermediate electron carriers terminate with a cytochrome which is coupled with the enzyme of the photophosphorylative process. Earlier electron carriers might be FMN or vitamin $\mathrm{K}$, the place of which could be taken non-physiologically by phenazine nethosulfate. Arnon proposes the name of "cyclic photophosphorylation" to clescribe this system, which is illustrated in Figs. 8 and 9.

The first phase of this cycle, namely, the production by light of both a high-energy electron and also of the ultimate electron acceptor, $[\mathrm{Chl}]^{+}$, is postulated on the basis of a finding by Lewis and Lipkin that in rigid media, and for a variety of substances, one of the 


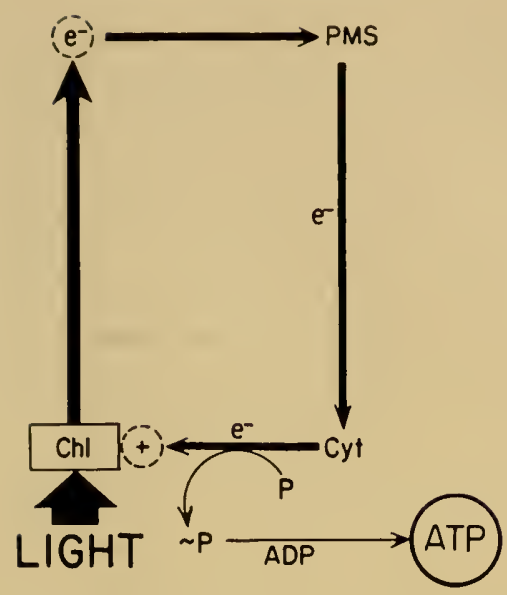

Cyclic photophosphorylation (PMS type)

lig. 8. Scheme for anaerobic cyclic photophosphorylation catalyzed by phenazine methosulfatc (PMS).

commonest results of illumination is the expulsion of an electron from an activated molecule. In the chloroplast, the resulting potential "hole" or "odd ion" might persist while the expelled electron is reaching the first intermediary electron carrier by resonance transfer or a semiconductor mechanism and is thereafter travelling by closed circuit back to the chlorophyll molecule. The evidence

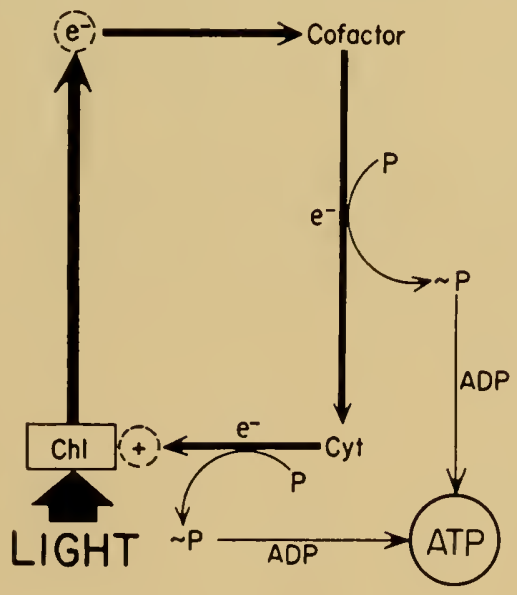

Anaerobic cyclic photophosphorylation

Fig. 9. Scheme for anaerobic cyclic photophosphorylation catalyzed by vitamin $\mathrm{K}_{3}$ or FMN. 
to support the scheme is still indirect, and rests upon the effects of chloride and ferricyanide, upon light-induced oxidations of cytochromes, and upon the effects of vitamin $\mathrm{K}$ on the reduction of the cytochromes.

With respect to chloride, it has been established that it is essential for those photosynthetic steps in which oxygen is liberated, but is not necessary for photophosphorylation. The anaerobic photophosphorylation cycle of bacteria and isolated chloroplasts proceeds without chloride ions. The experiments with ferricyanide were done to see whether an outside electron acceptor that would trap the electrons flowing in the postulated closed circuit of the cycle would in fact interfere with photophosphorylation. This was demonstrated to be the case. In both isolated chloroplasts and bacterial chromatophores, very low concentrations of ferricyanide were sufficient to abolish cyclic photophosphorylation. The effect could be reversed by reducing the ferricyanide with ascorbate; and ferrocyanide had no effect upon the system.

Both cytochrome $f$ and cytochrome $c_{2}$ have been found to be oxidized by light, the latter independently of temperature (Chance and Nishimura) and in cell-free preparations of Chromatium (Arnon et al.) The addition of vitamin $\mathrm{K}$ to a bacterial chromatophore preparation has been found, as expected on the basis of the hypothesis, greatly to accelerate the reduction of the oxidized cytochromes in the dark (but only when the preparation was aged).

At limiting light intensities, vitamin $\mathrm{K}$ is much more efficient in photophosphorylation than is phenazine methosulfate. These observations are taken by Arnon to mean that there are at least two sites of phosphorylation in the vitamin $\mathrm{K}$ (and FMN) pathways; that one of them at high light intensity becomes rate-limiting; and that this part of the pathway is bypassed by phenazine methosulfate. Fig. 9 also illustrates this conception of the existence of multiple sites of photophosphorylation in the usual anaerobic cyclic system. Other interpretations of these findings appear not yet to have been ruled out.

When chloroplasts were disrupted by sonication and purified preparations of grana were obtained, it was found that the photophosphorylating system (especially clearly when catalyzed by phenazine methosulfate) was located in the grana, together with the chloroplast cytochromes. Since the enzymes concerned with $\mathrm{CO}_{2}$ assimilation are readily clissociable during isolation of the grana and the bacterial chromatophores, Arnon has drawn the conclusion that in 
the course of biochemical crolution photosynthesis "furst emerged as a process for converting light energy into ATP and [that] this 'primitive' photosynthesis only later became a process linked to CO. reduction." The second part of his present review constitutes all examination of the evidence for this theory.

If cyclic photophosphorylation is indeed a primitive form of photosynthesis in general, then one might hope to find in nature some organism in which the only function of light in carbon assimilation, at least under some cincumstances, is that of forming ATP. Arnon's group has been unable to provide an elegant demonstration of such a situation in cell-free extracts of Chromatium. This organism, when living, utilizes either acetate or $\mathrm{CO}_{2}$ as the sole source of carbon. When acetate is assinilated, no hyrdogen source is required; in the case of $\mathrm{CO}_{2}$ hydrogen gas may serve as reductant. In the cell-free extracts of Chromatium ATP is required for the assimilation of either acetate or $\mathrm{CO}_{2}$, in the former case for activation of the carbon source in forming acetyl-CoA, in the latter case for formation of an activated intermediate (ribulose diphosphate, phosphoenolpyruvate, or 1,3-diphosphoglycerate). Evidence that the action of light in this system is solely that of supplying ATP was found by substituting exogenous ATP in the clark, and finding that assimilation went on "in the same manner," although not, it must be confessed, quite as actively. Since Chromatium is both an obligate anaerobe and also an obligate photoautotroph, it cannot be expected to make ATP by the usual oxidative phosphorylative mechanism. Thus it is highly probable that photophosphorylation is the sole source of ATP in this bacterium. Arnon cites $R$. rubrum, a facultative anaerobe, as likewise utilizing light only for cyclic photophosphorylation, and proposes that even in green plants photophosphorylation may continue under conditions when $\mathrm{CO}_{2}$ assimilation is stopped or greatly reduced, as during the midday closure of stomata.

Arnon's group was able in addition to clemonstrate that Chromatium hydrogenase can reduce DPN or TPN in the dark (although R. mbrum hydrogenase is unable to do so in a cell-free system). As in other bacterial systems, this reduction of DPN by hydrogenase required the presence of benzyl viologen. Amon regards this cellfree system (in which ATP is formed by light, DPNH by a simple dark hydrogenase) as representing the pathway in the living cell; but the requirement for benzyl viologen shows that the cell-free system is not quite identical with that in the intact cell.

Various algal species contain hydrogenases that will assinilate 
$\mathrm{CO}_{2}$ in the light with the aid of hydrogen gas (Gaffron's photoreduction). Amon suggests that this capacity is a primitive form of photosynthesis going back to a time when there was free hydrogen gas in the atmosphere and when the sole function of light was the formation of ATP by cyclic photophosphorylation.

Chromatium will utilize other hydrogen donors than hydrogen gas, and some of these, such as malate, will reduce pyridine nucleotides in the dark. But Chromatium will also use succinate and thiosulfate as electron donors, and thiosulfate and succinate are not at a potential sufficient to reduce pyridine nucleotides in a simple dark reaction. In these cases Amon favored the view that pyridine nucleotides are reduced only after the electrons derived from the substrate have passed first to the cytochrome-chlorophyll system and been raised to the required energy level by means of light.

In the paper by Losada, Nozaki, and Arnon, a study of this problem has been contributed to the Symposium. Arnon's electron flow theory of photosynthesis excludes a direct photolysis of water. It is proposed that the photoproduction of hydrogen might then come from a reduction of protons by a hydrogenase with the aid of electrons derived from the excited chlorophyll molecules, and fed to the latter by cytochromes obtaining them from the external electron donors. The primary photochemical act would suffice to raise the electrons to the reducing potential of molecular hydrogen. If this were the case, then inorganic electron donors might be substituted for the physiological organic electron donors. It was therefore decided to see whether thiosulfate would produce hydrogen gas and reduce cytochromes in illuminated Chromatium cells, but not in the dark. The reaction was in fact found to occur, and the accompanying scheme illustrates its agreement with the electron flow theory.

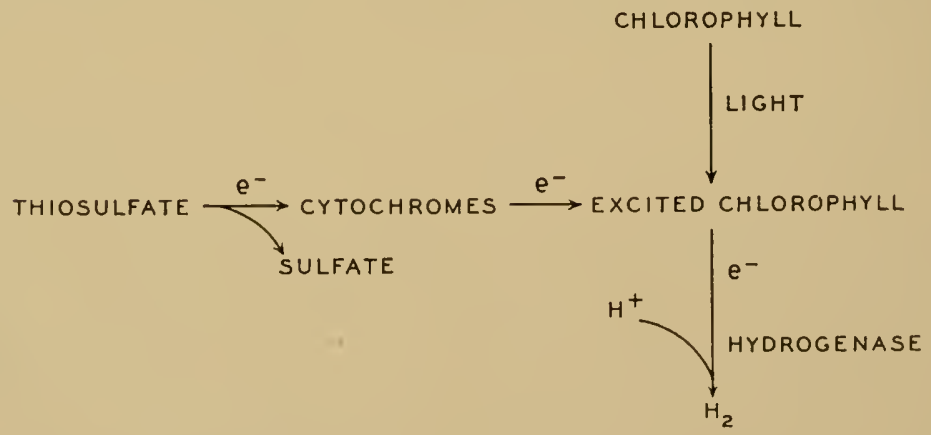

The reaction was highly dependent on $p H$, being low at $p \mathrm{H} 8$ 
and over six times as list at $p H$ 6.5. The reduction of the cyto chromes was demonstrated in a cell-free system by spectrophotometric means. The photoproduction of hydrogen gas from thiosulfite indicates that the photosynthetic bacteria are indecd able to reduce TPN and DPN in the light, and with an electron clonor, such as succinate, that is at a lower reducing potential than the pyricline nucleotides themselves.

The Chromatium hyclrogenase is also capable of reducing oneelectron viologen dyes, possessing a low reclox potential, and of evolving hydrogen in the dark from the reduced viologen. Succinate, too, is capable of reducing the cytochromes in cell-free preparations of Chromatium. The use in the production of hydrogen gas of the electrons derived from succinate or thiosulfate and raised to the reducing potential of the hydrogen electrode by the action of light of course interrupts the course of electron flow in cyclic photophosphorylation. Instead, there is a one-way flow of electrons from substrate to hydrogen gas. If this flow is also coupled to the phosphorylation mechanism, a type of non-cyclic photophosphorylation may be enrisaged (Fig. 10).

From this scheme it is possible to turn to the problem of the production of the primary reductant in photosynthesis in green plants. Arnon and his group have recently found a very direct coupling of photophosphorylation and the photoreduction of TPN. as follows:

$2 \mathrm{TPN}+2 \mathrm{ADP}+2 \mathrm{P}+2 \mathrm{H}_{2} \mathrm{O} \rightarrow 2 \mathrm{TPNH}_{2}+\mathrm{O}_{2}+2 \mathrm{ATP}$

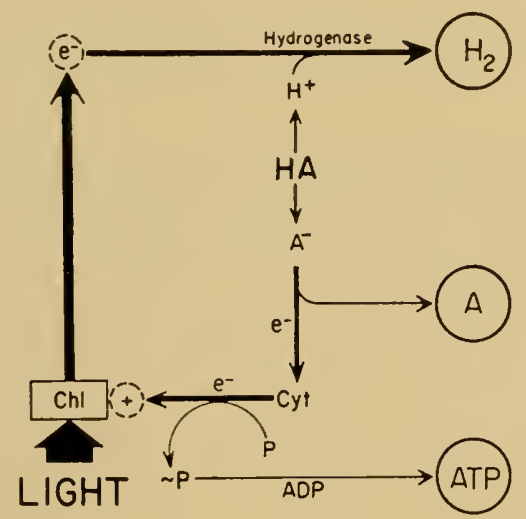

Non-cyclic photophosphorylotion (bocterio)

Fig. 10. Scheme for non-cyclic photophosphorylation in Chromatium. 
The fact that the rate of evolution of oxygen is greatly increased when it is coupled with phosphorylation inclines these workers to view the Hill reaction as an uncoupled photophosphorylation. The mechanism of the reaction above is different from the anaerobic cyclic photophosphorylation occurring in isolated chloroplasts and bacterial chromatophores, for ferricyanide in the presence of chloricle behaves here not as an inhibitor but as an electron acceptor that promotes the formation of ATP. The reaction scheme is therefore likened to the non-cyclic photophosphorylation in Chromatium, with water playing the role of electron donor in place of organic substrates. Reduced pyridine nucleoticle is produced in place of hydrogen; and the donation of electrons from $\mathrm{OH}^{-}$ions to the chloroplast cytochromes will lead to the evolution of oxygen (Fig. 11).

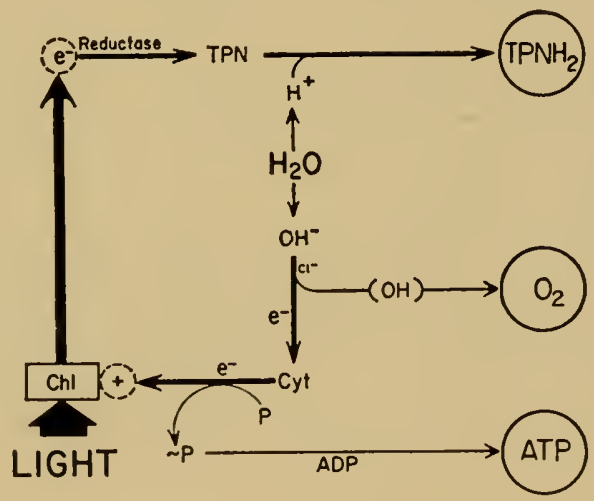

Non-cyclic photophosphorylation (chloroplasts)

Fig. 11. Scheme for non-cyclic photophosphorylation in cliloroplasts.

Still a third mechanism is postulated to occur in chloroplasts, a cyclic photophosphorylation. It arises from the previously mentioned catalytic effect of oxygen on photophosphorylation. At low light intensity, photophosphorylation is more efficient in an atmosphere of nitrogen than in air, but requires larger amounts of FMN or vitamin $\mathrm{K}$, and high concentrations of chloroplast material. The action of the inhibitors o-phenanthroline and o-chlorophenyl dimethylureal is much less effective on the chloroplast system in nitrogen than in air. Their strong inhibition of oxygen evolution by illuminated chloroplasts is therefore taken to signify that the evolution of oxygen is a step in an aerobic type of photophosphorylation, catalyzed like the anacrobic cycle by FMN and vitamin $K$. It is likely that the par- 
ticipation of oxygen in the system is wasteful of energy and is less efficient than anaerobic cyclic photophosplorylation. Perhaps it increases the over-all rate only when light is abundant and the concentrations of the cofactors are limiting. A schente for this postulated cyclic oxygen-catalyzed photophosphorylation is presented in Fig. 12. The clectron flow is comprised of two parts. In the first, the electrons from excited chlorophyll pass to oxygen and, with added protons, make water. In the second, the electrons are replaced in flow by donation from $\mathrm{OH}^{-}$ions, and oxygen is evolved. There is in consequence, as the isotope-labeling experiments require, an exchange between molecular oxygen and the oxygen of water. These

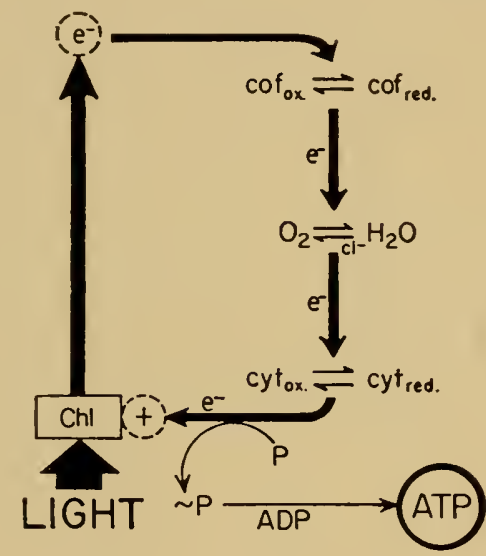

\section{$\mathrm{O}_{2}$-catalyzed cylic photophosphorylation}

Fig. 12. Scheme for oxygen-catalyzed cyclic photophosphorylation in chloroplasts.

findings are taken to mean that oxygen, when present with FMN or vitamin $\mathrm{K}$, is able to compete effectively for the electrons of cyclic photophosphorylation. The cycle can therefore be maintained only by a release of electrons in the oxygen-evolving reaction.

The ability of chloroplasts to carry out both the cyclic and noncyclic forms of photophosphorylation raises the question of the normal relation of these two processes. In isolated chloroplasts the cofactors of the cyclic process, FMN and vitamin $\mathrm{K}$, are shown experimentally to increase strongly the formation of ATP, and to abolish the accumulation of reduced pyridine nucleotide, and the evolution of oxygen. But in very much smaller, less than millimicromolar, quantities they will increase ATP formation without affect- 
ing oxygen evolution or $\mathrm{TPNH}_{2}$ accumulation, i.e., without interrupting the non-cyclic phase of photophosphorylation. Moreover, the larger quantities of FMN or vitamin $\mathrm{K}$ which suppress oxygen evolution also suppress the fixation of $\mathrm{CO}_{2}$ in carbohydrates, but the very minute concentrations which are sufficient to enhance the formation of ATP actually increase the reduction of $\mathrm{CO}_{2}$ to carbohychate. Presumably the cell maintains a well-regulated balance of cyclic and non-cyclic electron flow.

To summarize the distinctive features of Arnon's present theory, one may say, first, that the primary photochemical event is seen as an activation of chlorophyll that leads to the emission of an electron instead of the photolytic splitting of water; second, cytochromes are regarded as sufficiently oxidized to be able to withdraw electrons from hydroxyl ions, and in turn to reduce the excited chlorophyll ion $[\mathrm{Chl}]^{+}$; third, the essential products of the light phase of photosynthesis are ATP and $\mathrm{TPNH}_{2}$, and the fixation of $\mathrm{CO}_{2}$ is a reaction capable of proceeding in the dark and without chlorophyll provided ATP, reduced TPN, and the non-green portion of the chloroplast extract are present. Both cyclic and non-cyclic photophosphorylation are regarded as essential since the ATP formed in non-cyclic photophosphorylation alone is insufficient for the fixation of $\mathrm{CO}_{2}$ in the form of carbohydrates. The cyclic system demonstrably participates in $\mathrm{CO}_{2}$ assimilation when ATP and $\mathrm{TPNH}_{2}$ are present in catalytic amounts and must be constantly regenerated through utilization of the energy of light. When either type of photophosphorylation is functioning alone, only phosphoglycerate is formed; sugar phosphates appear as products only when both cyclic and noncyclic photophosphorylation are functioning in balance.

Armon suggests that these observations and postulates suggest a course of biochemical evolution of photosynthesis rather clifferent from that generally held. The beginnings of photosynthesis perhaps lay in the origin of a porphyrin that could absorb light and use the energy of these quanta in anaerobic cyclic photophosphoryla tion. If so, there rould have been initially no evolution of oxygen and no reduction of carbon dioxide. These reactions would not have been necessary in a "primeval soup" containing an abundance of preformed carbon compounds. This stage in the evolution of photosynthesis is today still exemplified by the photoassimilation of acetate by the obligate phototrophic anaerobe, Chromatium. Formation of ATP by this mechanism would have had great advantages over use of the fermentation process, the alternative means 
in the primitive oxygenless atmosphere. Just as oxidative phosphorylation during respiration is superimposed on the primitive anarebic fermentation processes of glycolysis, so the prinitive anaerobic cyclic form of photophosphorylation is retained in the photosynthesis of green plants, the later claborations which are superimposed upon the primitive process being concerned with the utiliration of ATP and the substitution of different electron donors for the most primitive one, molecular hydrogen itself. The assimilation of $\mathrm{CO}_{2}$ gained in importance as the supply of organic compounds in the enviromment was depleted. Electron donors at a lower reducing potential than hydrogen-donors such as succinate or thiosulfate-became utilizable through the use of light not only to supply ATP but also to provide electrons with high enough energy to reduce pyricline nucleotides. Finally, photosynthesis advanced in green plants to the stage where $\mathrm{CO}_{2}$ could serve as sole source of carbon and where water became the electron donor. With this achievement there appeared the evolution of oxygen, a byproduct of the removal of electrons from hydroxyl ions and reaction of the resulting $[\mathrm{OH}]$ radicals to form water and release molecular oxygen. The scheme outlined is reminiscent of Norman Horowitz' theory of the gradual lengthening of biochemical chain reactions during evolution, when exhaustion of one after another of the primeval organic components of the environment forced living organisms to add steps to existing chains until they had finally attained some inexhaustible substrate. In the case of photosynthesis, the inexhaustible supplier of electrons was water, and of carbon it was $\mathrm{CO}_{2}$. Oxygen, the byproduct, altered the composition of the atmosphere until aerobic metabolism became possible. This made oxidative phosphorylation possible. Hence mitochondria, now believed to have a common phylogenetic origin with proplastids, arose in evolution after the chloroplasts. The oxygen furthermore made possible the final accretion to the balanced photosynthetic system, the addition of oxygen-catalyzed cyclic photophosphorylation.

Certain aspects of Arnon's views have been challenged. Albert W. Frenkel, for example, believes it premature to state definitely that there are basically different photochemical mechanisms in green plants and in bacteria, that in the latter the non-cyclic stage of photophosphorylation is lacking, and also that no photooxidant and photoreductant are formed as primary products of the photochemical splitting of water. In his paper, Frenkel describes a bacterial chromatophore system from Rhodospirillum rubrum that in fact does carry 
out non-cyclic electron flow which might be coupled to phosphorylation, electrons from the photoreductant being used to reduce DPN, while reduced flavin mononucleotide, $\mathrm{FMNH}_{2}$, donates electrons to the cytochrome of the system. Frenkel also protests that light-induced reduction of TPN in green plants and of DPN in photosynthetic bacteria does not require the simultaneous generation of ATP. In bacteria, no coupling of phosphorylation and reduction of pyridine nucleotide has in fact been shown to date, although in Rhodospirillum rubrum a coupling of photophosphorylation and oxidation of cytochrome $c_{2}$ has been demonstrated (Smith and Baltchewsky). Frenkel agrees with Arnon's view that cyclic photophosphorylation is a sort of "short-circuit" electron transport system to which one or more sites of phosphorylation are coupled; but he still prefers van Niel's interpretation of photosynthesis as being initially a formation of a photoreductant and a photooxidant. Thus, in the metabolism of acetate by $R$. rubrum, where Stanier et al. have suggested that photophosphorylation is important, it is still quite possible to hold that the electron flow is initiated by light and that the formation of high-energy phosphate, though important, is a secondary phenomenon which may or may not accompany the light-induced flow of electrons. The photoreductant (XH) may be sufficient to generate all the electrons required for the reduction of pyridine nucleoticle, and the photooxidant $(Y O)$ to oxidize the acetate to $\mathrm{CO}_{2}$ and $\mathrm{H}_{2} \mathrm{O}$. Alternatively, the electrons from the succinate-fumarate pair may be sufficiently upgraded by means of energy from ATP to reduce pyridine nucleotide.

André T. Jagendorf and G. Forti also differ with Arnon's views in some respects. They summarize the possible reactions resulting from the absorption of light in five sequential equations, as follows:

$$
\begin{aligned}
& \mathrm{X}+\mathrm{Y}+\mathrm{H}_{2} \mathrm{O}+h_{\mathrm{v}} \rightarrow \mathrm{XH}_{2}+\mathrm{YO} \\
& \mathrm{XH}_{2}+A \rightarrow A \mathrm{H}_{2}+\mathrm{X} \\
& \mathrm{YO} \rightarrow Y+\mathrm{O}_{2} \\
& \mathrm{YO}+A \mathrm{H}_{2} \rightarrow Y+A+\mathrm{H}_{2} \mathrm{O} \\
& A \mathrm{H}_{2}+1 / 2 \mathrm{O}_{2} \rightarrow A+\mathrm{H}_{2} \mathrm{O}
\end{aligned}
$$

The initial reaction involves the absorption of light. As van Niel proposed, it may involve the participation of water, just as written; but whether it does or not is really irrelevant. Formation of the photoreductant and photooxidant as products will require that, if 
the process is to be repeated, and if $X$ and $Y$ are catalysts, not substrates, in amount, then there must be a flow of electrons from $\mathrm{XH}_{2}$ and to $\mathrm{YO}$. In reaction $2, \mathrm{XH}_{2}$ is oxidized by some electron acceptor such as a redox dye or a pyridine nucleotide. The reduction of $\mathrm{YO}$ to $Y$ may take place either by reaction 3 (as in the Hill reaction) or by reaction 1 (as in cyclic electron transport for which phenazine methosulfate and pyocyanine are cofactors). When $A \mathrm{H}_{2}$ is oxidized by molecular oxygen (reaction 5 ), reaction + is precluded, and $Y O$ must be reduced by reaction 3. The participation of oxygen in the sequence of reactions $1,2,3,5$ may be detected by means of $\mathrm{O}^{18}$ as an oxygen exchange reaction between the water and the atmosphere. [Ed. This corresponds in Arnon's terminology to the "oxygencatalyzed cycle."] In the present Symposium Birgit Vennesland and her associates have discussed this reaction, and have shown that both FMN and vitamin $\mathrm{K}_{3}$ elicit a rapid oxygen exchange in isolated chloroplasts. Actually, reaction 3 fails to provide sufficient oxygen for reaction 5, so that additional oxygen is required to stimulate phosphorylation with low concentrations of FMN as cofactor.

Jagendorf and Forti have worked recently with the photophosphorylation inhibitor $p$-chlorophenyldimethylurea (CMU), and have come to the conclusion that it inhibits specifically reaction 3, the oxygenevolving step. For CMU strongly inhibits the Hill reaction (steps $1,2,3$ ) and the FMN-catalyzed phosphorylation (steps 1,2,3,5), but it does not strongly inhibit an anaerobic cyclic electron flow with PMS or pyocyanine as cofactor (steps $1,2,4$ ). A number of complications appeared with PMS or pyocyanine as cofactors. Thus a small amount of oxygen present in the system makes pyocyanine-catalyzed electron flow sensitive to CMU. This was explained as an oxidation of pyocyanine by $\mathrm{O}_{2}$ (reaction 5), thereby using up $A \mathrm{H}_{2}$ (recluced pyocyanine) and blocking reaction $\mathrm{t}$. With reaction 3 simultaneously blocked by the inhibitor CMU, there would be no way open for $Y O$ to be reduced to $Y$, and so electron flow would stop. But PMS does not behave like pyocyanine, in the presence of oxygen and CMU, because in white light or upon the addition of ascorbate it is recluced non-enzymatically, thereby restoring reaction 4 . Jagendorf and Forti point out the similarity of CMU-poisoned chloroplasts to bacterial chromatophores, the cyclic electron flow and phosphorylation of which are also poisoned by small amounts of oxygen but relieved by electrondonating substrates.

Having some assurance from these studies that CMU inhibits reaction 3, workers may use this inhibitor to aid in distinguishing cyclic 
electron transport $(1,2, f)$ from an oxygen exchange reaction $(1,2,3,5)$ and in identilying the nature of electron flow in other cases. For instance, a small amount of photophosphorylation takes place in chloroplasts in the absence of any soluble redox system (FMN, vitamin $\mathrm{K}$, PMS, etc.) This "endogenous" phosphorylation is probably not attributable to cyclic electron flow, for it is dependent on oxygen and is inhibited by CMU. It may be regarded as a "Mehler reaction," a replacement of reaction 5 in the foregoing sequence by the following reaction:

$$
A \mathrm{H}_{2}+\mathrm{O}_{2} \rightarrow \mathrm{H}_{2} \mathrm{O}_{2}+A
$$

The full reaction sequence, involving oxygen uptake as well as photophosphorylation, is markedly enhanced in activity by addition of the enzyme photosynthetic pyridine nucleotide reductase (PPNR), so that this enzyme must function in the reduction of oxygen as well as that of TPN and cytochromes. Minute quantities of ascorbate, in this enzyme system, have the effect of stimulating phosphorylation without enhancing the oxygen uptake. Ascorbate therefore seems to switch the reaction system back from the Mehler reaction $(1,2,3,5 a)$ to the alternative oxygen exchange $(1,2,3,5)$.

Jagendorf and Forti cite a number of other inhibitors or inhibitory conditions that appear to act on reaction 3. These include o-phenanthroline, the chloride deficiency described by Arnon in this Symposium, and the inhibition resulting from storing bean leaves for 24 hours at $0^{\circ} \mathrm{C}$ in the dark (unpublished experiments of Margulies at Johns Hopkins University). In the same category may be placed the experiments of Barbara Petrack, which show that particles from blue-green algae containing chlorophyll $a$ but lacking phycobilin pigments can carry on cyclic electron flow reactions with PMS as cofactor, but not a Hill reaction. From this observation it seems possible that oxygen evolution (reaction 3) may depend specifically upon the absorption of light by accessory pigments. The experiments of Kok and Hoch with light of wavelength $700 \mathrm{~m}_{\mu}$ or longer likewise show that the PMS-catalyzed cyclic electron flow, but not the Hill reaction, can follow from the absorption of light by chlorophyll $a$ alone. The function of the accessory pigments again seems to be concerned with reaction 3 in the over-all photosynthetic system.

A theoretical question of importance to the interpretation of the over-all nature of photosynthesis, as Warburg has emphasized, is the question of whether carbon dioxide is required for the evolution of oxygen in the Hill reaction (equation 3). Vennesland reported work 
done by Babette Stern with a spinach chloroplast system that confirms this effect. In the presence of a catalytic amount of indophenol dye, the reduction of ferricyanide by the chloroplast system was greatly enhanced, and even doubled, by $\mathrm{CO}_{2}$ or bicarbonate; and the antount of oxygen evolved under these conditions agreed quite well with amount of ferricyanide reduced.

Further observations on the oxygen exchange reaction were contributed by David W. Krogmann. The oxygen exchange was measured by means of the mass 34 isotype of oxygen. The amount of exchange was not markedly affected by the amount of photophosphorylation in the system, but did depend on the presence of the indophenol dye. It did not result in any increase in the formation of hydrogen peroxide (Mehler reaction, equation $5 a$ ) nor in any stoichiometric relation to the amount of phosphorylation occurring. The tentative interpretation given by Krogmann is that the dye is reduced in a Hill reaction, with the evolution of oxygen, but without photophosphorylation; while a flow of electrons from the photoreductant may proceed to oxygen, with formation of water and accompanying phosphorylation.

In the blue-green alga Anabaena variabilis, photophosphorylation has been localized in a phycocyanin-free preparation containing smooth vesicles apparently derived from the peripheral lamellae seen in intact cells, according to the report by Barbara Petrack and Fritz Lipmann. The phosphorylation process is most actively catalyzed by phenazine methosulfate, but FMN and vitamin $\mathrm{K}$ (menadione), and especially a combination of the two, also will act as cofactors. Only ADP will serve as phosphate acceptor. Anaerobic conditions proved to be essential, and Petrack and Lipmann suggest that the phycocyanin normally present protects the chlorophyll system from photooxidation. Glutathione stimulates photophosphorylation in this system, up to a certain maximum above which there is inhibition owing to an ATPase-like activity. The dephosphorylation thus discovered, and found to be mediated also by cysteine, seems in all respects (requirements and inhibitions) to be a photohydrolysis that is a simple reversal of the phospliorylation process. A similar photohydrolysis of ATP was subsequently clemonstrated to occur in spinach chloroplast systems. The general significance of the photohydrolysis of ATP in photosynthetic systems will require further elucidation.

B. L. Strehler and D. D. Hendley describe the use of the firefly luminescent system to measure continuously the generation of ATP by an isolated chloroplast system. Rapid increases and decreases of 
luminescence can be observed from the assay system as the illumination is turned on and off and the ATP concentration consequently rises and falls. The rapid decay in the dark was attributed to the presence of myokinase in the crude firefly extract. The addition of TPN and other cofactors (TPNH, folic acid, cytochrome $c$, ferricyanide, PMS, and FMN) was found to enhance phosphorylation, as measured by the luminescent system. In some experiments a decrease in the level of luminescence was found upon exposure to light, in agreement with the observation reported by Petrack and Lipmann. Hendley and Strehler examine this phenomenon in terms of a "reductive dephosphorylation" hypothesis advanced independently by Kandler and Strehler in 1950 and 1951. According to that hypothesis, some portion of the ATP generated by phosphorylation coupled to photosynthesis would be used to raise the energy of electrons from the level of the photoreductant to that of pyridine nucleotides. Experiments now reported indeed demonstrate that TPN enhances the incorporation of inorganic phosphate into ATP, but the exchange reaction is inhibited by higher concentrations of ATP. Activity of ATPase in the dark and occurrence of photophosphorylation in the light account for most of the incorporation of $\mathrm{P}^{32}$ from inorganic phosphate. Preincubation in the dark, presumably by increasing the concentration of ADP, greatly speeds up the subsequent incorpora. tion of $\mathrm{P}^{32}$ into ATP in the light. These data do not support the hypothesis that some ATP produced in photophosphorylation is utilized in the reduction of TPN during photosynthesis, but neither do they rule it out.

Anthony San Pietro has successfully purified the enzyme "photosynthetic pyridine nucleotide reductase" (PPNR), which catalyzes the transfer of electrons in photosynthesis to TPN. About a 200 -fold increase in specific activity over the crude preparations has been attained. The properties of the enzyme, as now reported, include a linear relation of enzyme concentration to reduction of TPN. The nucleotide specificity of the enzyme is limited to TPN and acetylpyridine-TPN (no action on DPN, $\alpha$-DPN, or acetylpyridine-DPN, nicotinamide mononucleotide or nicotinamide riboside). The enzyme lacks effect in the Hill reaction (reduction of indophenol dye or ferricyanide). The reduction of TPN by the enzyme is not enhanced by the photophosphorylating system (ADP and inorganic phosphate), although the reverse effect, stimulation of photophosphorylation by the enzyme, does occur. There is a linear proportionality in rate of formation of TPNH to the chloroplast concentration. $\mathrm{Ig}^{++}$ions 
are a cofactor. The enzyme is inactivated by chloroplasts in the light, but reducing compounds will prevent this. It is reddish-brown in color, has a molecular weight (derived lrom sedimentation and diffusion dita) of about 14,000 , absorbs at $278,327,420$, and $460 \mathrm{~m} \mu$, and upon being bleached loses its absorption at the two higher wavelengths. It does not appear to be either a flavin or heme protein, but may well be identical with the methemoglobin-reducing protein recently isolated from leaves by Davenport and Hill.

\section{Pliototropism and Photoperiodism}

Kenneth V. Thimann and G. M. Curry begin their review of phototropism by stating that it is not quitc at the simplest level of responses of organisms to light. Phototaxis, the orientation to light of freely moving organisms, seems even simpler than phototropism, which is properly a term linited to the light-directed curvature of fixed organisms. It is not clear, however, why phototaxis is regarded as being simpler-perhaps it is because no chemical photoreceptor or mediating substances have been isolated in the case of phototaxis as they have in phototropisms. And perhaps Thimann would agree that there are various levels of complexity in both phototaxis and phototropism.

Recent studies (Briggs, Tocher, and Wilson) have refined the classic experiments with agar blocks applied to oat or corn coleoptile tips. Not only is it evident that more indole acetic acid (i.e. auxin) - about twice as much-diffuses into the agar block from the shaded as from the illuminated side of the coleoptile; but it is evident also that neither the illumination of the tip nor slitting the base of the tip modifies the total quantity of auxin produced, but that when the tip is slit all the way to the top no difference in amount of auxin on the shaded and illuminated sides can arise. Hence it is clear that the auxin is not destroyed by light, but must move away from the lighted side to the dark side.

The identity of the photoreceptor for the phototropic response has been a subject of much interest, approached experimentally by analysis of the action spectrum. Although red light will not evoke phototropic curvatures, when dark-grown plants are exposed to red light before being exposed to blue or white light, the growth rate near the tip of the coleoptile is greatly enhanced and that at the junction of coleoptile and mesocotyl is inhibited. Hence experiments are best carried out with dark-grown plants exposed to monochromatic light. The action spectrum for the positive curvature of oat coleoptiles has 
a major peak at $445 \mathrm{~m} \mu$, minor peaks at 472 and $425 \mathrm{~m}_{\mu}$, and a broad peak in the near ultraviolet $(350 \mathrm{~m} \mu)$, the latter being less than half as effective as the blue light in evoking the response. The major peaks agree well with the absorption spectrum of $\alpha$-carotene, and there is in fact a carotenoid extractable from the coleoptile and microscopically visible in polarized light within it; but $\alpha$-carotene lacks the broad absorption band in the near ultraviolet. A cisconfiguration in the molecule would produce a band between 300 and $350 \mathrm{~m} \mu$, but so far no cis-carotenoid has been found to duplicate the natural action spectrum very closely. Another view, represented by Galston, and supported by him in the general discussion, is that the photoreceptor might be riboflavin or some other flavin compound. Thimann discounts this possibility because, though riboflavin has an absorption band at $365 \mathrm{~m}_{\mu}$, this band is as strong as the major peak at $450 \mathrm{~m} \mu$, and nothing can be seen of the secondary peaks of the action spectrum for phototropic curvature. However, the ribosefree compound lumiflavin, and even more strikingly 3-methyl lumiflavin, when dissolved in benzene, exhibit absorption curves very like the action spectrum. Yet the peak in the ultraviolet is at too short a wavelength; and in water, where the band shifts to a position in agreement with the action spectrum, the details in the main part of the spectrum clisappear. Thimann accordingly prefers to look, at the present time, for some cis-carotenoid conjugated with a protein; but Galston believes that in the natural medium of the cell the absorption spectrum of a flavin might shift sufficiently to accord with the action spectrum. Moreover, he points to a number of casesalbino corn, a white sunflower, and Phycomyces grown on lactatein which the carotene content was undetectable or greatly reduced, but which nevertheless exhibit a phototropic response. Thinann questions both the complete absence of carotene and the normality of phototropic sensitivity in these cases: and he adds that pre-exposure to red light, which doubles the concentration of FMN, decreases or leaves unchanged the photosensitivity.

In addition to the positive curvature elicited by low intensities of light, there is also a negative response which is evoked by higher intensities. At very high encrgies there is again a positive curvature, but of a different kind. It appears mainly at the base, and is responsible for most of the ordinary bending of plants toward the daylight. This "base response" may also be elicited by ultraviolet illumination, and the action spectrum has peaks at 280 and $297 \mathrm{~m} \mu$. This action spectrum appears to be that of indole acetic acid (IAA) itself, shifted 
12 $m_{\mu}$ toward the visible. Pure $1 \lambda \lambda$ in aqueous solution is inactivated by ultraviolet, and the action spectrmu for the process agrees with the absorption spectrum of $\mathrm{I} \Lambda \mathrm{\Lambda}$; but the inactivation requires very high doses. In the plant, therelore, the $\mathrm{IAA}$, if it is the photoreceptor, must be greatly sensitized to the action of ultraviolet radiation. Support for this view was adduced by a demonstration that applied $I A A$ can reinstate the phototropic sensitivity lost through exposure to ultraviolet. Hence it secms likely that IAA in some combined form is the photoreceptor for the high-threshold response, as well as being the effector of it.

The phototropic response of the mold Phycomyces is particularly interesting because of its sensitivity, because the response occurs in a single cell, the sporangiophore, and because no mediation by a growth hormone has been detected, in spite of the fact that the sporangiophores contain indole acetic acid. The general similarity of the Phycomyces system to Avena is very striking. The action spectrum for Plycomyces shows close agreement with that of Avena; and the sporangiophore of Phycomyces contains a carotenoid. High closes of light reverse the direction of the curvature. The action spectrum for the negative curvature in the short ultraviolet is also like that of Avena, with a peak near $280 \mathrm{~m} \mu$.

Since symmetrically distributed light accelerates plant growth, one may wonder about the reason for a positive curvature in the usual phototropic response. The response to unilateral illumination continues in the dark for much longer than the response to symmetrical illumination. Thimann therefore is disinclined to view the curvature as simply the outcome of the clifference in growth of the two sides of a coleoptile, or, in the case of Phycomyces, of a single cell. Rather, he finds support for the lens theory, which holds that the light is focused by the clear cellular substance onto the opposite side from that which is directly illuminated. Gallic acid present in the sporangiophore would render it virtually opaque to short ultraviolet, and therefore accelerate growth only on the illuminated side, and consequently produce a negative curvature. This interpretation was supported by finding that symmetrical illumination with ultraviolet in fact produces a simple acceleration of growth of the sporangiophore.

Evidence from experiments, and considerations of quantum yield, now speak strongly against the destruction of indole acetic acid by light as being the mechanism of the phototropic response. It is especially significant that $\mathrm{IAA}$, applied as many as 8 hours after exposure of decapitated coleoptiles to very high doses of white light, will 
produce a marked curvature (although the coleoptiles otherwise would bend very litule alter such a treatment). This, to Thimann, suggests that the light cloes not react with the auxin itself but with some substance capable of lasting a considerable time in the plant and of "influencing many hundreds of auxin molecules." Although an enzyme might conceivably do this, it is hard to see how an enzyme might affect the translocation of auxin. Thimann prefers to suppose that the carotenoid proposed as the photoreceptor substance may be located within a plastid, or proplastid, in the coleoptile. He then develops a unified theory of geotropism and phototropism by suggesting (a) that the "statoliths" responsible for geotropism are the same proplastids, (b) that the movement of auxin from cell to cell is promoted by the presence of the statoliths lying against a particular wall of the cell, and (c) that unilateral light causes the plasticls to migrate to the lateral wall opposite to the clirection of the light. This theory may also unify the activity of the proplasticls and the movements of chloroplasts, which likewise are related to light intensity, spectral characteristics, and preliminary treatment with red light. In one respect, however, geotropic and phototropic responses are different. Gravity is detected by the whole coleoptile, not just by the tip. Hence, if the plastids are indeed responsible for both phenomena, those in the tip region must differ from those below. This matter is receiving investigation.

A. W. Galston and R. Kaur have presented some interesting comparisons of the effects of atuxin and gibberellic acid, applied separately or together, on etiolated and green sections of stem from the garden pea. Sections of stem 3-5 11 m long were found to be optimal for testing these effects in both green and etiolated plants. The experiments revealed that low closes of red light markedly inhibit the growth on sucrose of etiolated sections, but do not so inhibit green segments; and that the growth of the former is markedly enhanced by cobaltous ion $\left(10^{-4} M\right)$, whereas that of the green sections is not. The etiolated sections respond optimally to low concentrations of gibberellic acid and auxin $\left(10^{-6} M\right.$ or less), but green sections require concentrations 100 to 1000 times as great. The stimulation of growth by gibberellic acid and auxin is simply additive in etiolated sections, but is synergistic in green sections, especially in plants grown under an 8-hour photoperiod. The synergism is manifested after as little as I minute's exposure to $10^{-4} M$ gibberellic acid. Substituted ureas used as herbicides (CMU and fenuron) also act differentially on the green and etiolated stem segments. They will inhibit the growth of the green 
sections growing in the light antotrophically, but are of little effect on green sections growing heterotrophically on sucrose in the clark. Surprisingly, the growth of etiolated sections is markedly promoted by the same urea herbicicles, and this growth too can be inhibited by red light. The photoperiod of the donor plant has an effect on the growth of excised sections. If a green plant is grown under continuous illumination, sections from its stem will grow better on sucrose in the light and more poorly in the dark than will segments from plants exposed to 8- or 16-hour photoperiods. Conversely, under a short photoperiod such as 8 hours, the excised sections show better growth in the dark and poorer growth in the light than do segments from 16- and 24-hour photoperiod plants. One may therefore consider the plants grown under continuous illumination to be "light-adapted" and those grown under a short photoperiod to be partially "darkadapted." Explanations for these phenomena remain to be sought.

The relation of the daily photoperiod to the induction of flowering in plants has been studied for many years, but the problem has been refractory because of the laborious character of the experiments. William S. Hillman has found a nearly ideal solution that will remind a geneticist of the happy choice by Thomas Hunt Morgan of Drosophila melanogaster for genetic studies, Hillman's organism, truly small and economical to grow, is the common duckweed, Lemna, of which several species exist and permit comparisons. Most species of the Lemnaceae flower infrequently in nature. Lemna gibba may be induced to flower, as Kandeler first showed, by exposure to a longday regime (12-14 hours). Lemna perpusilla, on the contrary, is a conditional short-day plant; that is, it is indifferent to the length of the photoperiod under certain conditions but is induced to flower by exposure to a short-day period when the medium is supplemented with certain chelating agents such as ethylenediamine tetraacetic acid (EDTA), citrate, or tartrate. Exposure to red light even briefly and at low intensity during the dark period is sufficient to inhibit this short-day flowering. Alternatively, one might look on the case as one of specific long-day inhibition of flowering.

When Lemna is grown aseptically on modified Hoagland's medium, the photoperiod fails to affect either species until EDTA is added. At a concentration of EDTA above $10^{-5} M$, and under a long-day regimen, the chelating agent suppresses the flowering of L. perpusilla, which has previously been flowering; and it brings about the flowering of $L$. gibba, which has previously not been flowering. A short photoperiod (8-hour) does not change the original condition in either 
species, even when EDTA is added. Within the $p \mathrm{H}$ range 3.6 to 5.0, the action of EDTA is greater at higher $p \mathrm{H}$. In the case of $L$. perpusilla, the effect of EDTA is imitated by a variety of other chelating agents; in that of $L$. gibba, it can be duplicated by using "old" medium which has previously supported the growth of plants under a longday regimen. Although EDTA also affects vegetative growth, it seems that in respect to flowering it must affect the photoperiodic mechanism itself-but not be a simple, general inhibition or promotion of flowering. What it appears to do is to potentiate the inherent, and different, response of each species to a long-day photoperiod.

Colin S. Pittendrigh presented a stimulating review of short-term photoperiodisms in animals. These phenomena have more recently been termed "circadian" rhythms because they commonly have a period of approximately a day, and more popularly they are called "biological clocks." The paper was not submitted for publication.

\section{VISION}

\section{The Intensity Factor}

Since the studies of Selig Hecht and his associates, in 1942, on the number of quanta which must be absorbed by the rhodopsin of the rods of the vertebrate eye in order to sec, physiologists have been greatly interested in the intensity factor in vision. Hecht and his group demonstrated, by two independent methods, that it requires absorption of about 6 quanta in order to see, and that each quantum is absorbed in a separate rod cell. W. A. H. Rushton and his colleagues have pursued this type of investigation with fruitful, if still incomplete, results. It is now possible to relate the nerve signal to the light intensity, to state where light adaptation and dark adaptation occur, to relate the light threshold to the amount of pigment bleached, and to express all of this information in a single mathematical expression-one which a comprehensive theory of vision must satisfy.

As to adaptation, the reduction of the visual threshold during clark adaptation by 100 -fold or more is certainly not localized in the brain, since records taken from the ganglion cells of an excised eye and clectroretinograms themselves show the same change of sensitivity as the intact system. Records taken with a micropipette from a single nerve cell of a single ommatidimm of the horseshoe crab, Limulus, demonstrate that the same conclusion may be drawn with respect to light 
adaptation. The characteristic effect of light is to lower the membrane resistance. Niter a bright llash of light, a wak flash provokes a greatly diminished response in comparison with that which it would evoke belore the bright flash. On the contrary, a strong depolarizing current passed through the microclectrode has no effect whatsocver on the response to the subsequent weak flash. Thus the adaptation does not reside in the nerve cell itself but occurs somewhere carlier in the train of events. Any action of currents generated elsewhere and passing through the nerve cell, and assumed to lower the resistance of the cell membrane, may also be excluded, since Fuortes has found that the membrane resistance remains unchanged as a function of applied current. It would seem to follow that light must stimulate by increasing cell permeability, perhaps through the release of some chemical agent, a "transmitter hormone." If so, there is a question whether the effect of light adaptation is to reduce the amount of hormone released or to modify the sensitivity of the cell to a fixed amount of hormone. Since recovery of sensitivity in the dark, after the rod and cone pigments in the living retinas of various animal and human eyes have been bleached, corresponds closely in each case to the time required for full regeneration of the pigment, it seems more probable that the first of the suggested alternatives is the correct one.

The relation between the membrane resistance of the vistal cell in Limulus and the light intensity may be expressed by the equation

$$
V-V_{D}=\log \left(\frac{I}{I_{D}}+1\right)
$$

where $V$ is the membrane potential, $V_{D}$ is the value of $V$ in the dark, $I$ is the light intensity, and $I_{D}$ is the "dark light," or intrinsic noise of the system. By differentiation, one obtains: $\Delta V=\Delta I /\left(I+I_{n}\right)$. This righthand expression has been found experimentally (by Riggs and Graham) to be ahmost exactly constant over the entire range of light intensities; in other worcls, $\Delta I^{\prime}$ is essentially a constant for increment thresholds, and vision in Limulus, at least in its electrical aspects, obeys the classic Weber-Fechner Law-as Stiles has shown to be true of rod vision and several color mechanisms in man.

The threshold in the dark, obtained by setting $I=0$, is $\Delta I=\Delta V \cdot I_{D}$, an expression that at once makes one wonder whether the great rise in $\Delta I$ upon adaptation of the eye to light is attributable to an increase in the nerve threshold $(\Delta V)$ or to an increase in $I_{l}$, the noise of the system. Experiments by Rushton on his own cones, so adapted to light 
that the threshold was raised 1000 times, agreed precisely with the shift of the curve expected from an increase in $I_{D}$ alone, and diverged several hundred-fold from that expected if only $\Delta V$ had changed. Hence the effect of adaptation to a bright light is to increase the intrinsic noise of the retina.

Rushton points out that the photochemical theory of vision is clearly inadequate to explain the alterations of the increment thresholds during light and dark adaptation. He proposes instead an electrophysiological interpretation, in which the increment threshold should depend upon (a) a nearly instantaneous logarithmic transformation of the light intensity-a transformation the nature of which remains obscure-and upon (b) the response of the nerve to a constant increment in the transformed signal. On the other hand, Rushton points out that the photochemical theory explains very satisfactorily the rise of the absolute visual threshold upon exposure to light and its subsequent fall in the dark, as a function of the concentration of the visual pigments. Experiments using the new technique of retinal densitometry to measure the concentrations of the pigments in the living eye indeed show that dark adaptation is closely related to the pigment concentration, as Wald and others have thought. The change in pigment density can be measured by the change in the logarithm of the reflected light. Again, Rushton made measurements upon his own cones, starting both from the dark-adapted state and from the light-adapted condition. He found "that the absolute threshold depends very greatly upon the amount of pigment bleached, but the increment threshold very little"-precisely as expected. The fortunate availability of a person with rod monochromat vision permitted further conclusions. In the absence of cones, the behavior of the rhodopsin of the rods may be followed over the cntire course of the enormous rise ( 3 million times) in the rod threshold during the course of light adaptation. Rod function is not inhibited by the activity of cones, since it appears at a given level whether there are cones or not, and follows the same dark adaptation curve over the extent of rod function that is observable in normal cyes. There is a linear relation between amount of pigment bleached and log threshold, as Wald has previously proposed.

Other experiments with Limulus, recorded from a single visual cell by means of an intracellular microclectrode under a weak, steady light, revealed numerous irregular slow pulses of potential, interpreted as responses to the absorption of single quanta. The effect of adaptation to a much brighter light was to produce far more numerous, and also 
very much smaller pulses. Rushton therefore suggests that the mechanism of adaptation is such that every quantum produces an effect, but that the effect diminishes rapidly and exponentially with the proportion of pigment bleached. The theory of vision must in the future therefore reckon with the fact that a quantum of light absorbed anywhere in a rod or cone can produce an effect a tenth of a millimeter away which adds to similar effects from other receptors in stimulating a nerve, and all within a second. It must also accord with the equation

$$
V-V_{D}=\log \left(\frac{I}{I_{o}} \mathrm{e}^{-a x}+1\right)
$$

where $I$ is the light intensity, $V$ the nerve signal, $V_{D}$ the value of $V$ in the dark, $I_{o}$ the absolute dark-adapted threshold, $a$ a constant that is about 4 for cones and 40 for rods, and $x$ the fraction of visual pigment bleached. How to relate the information content of the nerve signal as well as its magnitude to the quantum content of the light remains for the present a puzzle.

\section{The Molecular Organization}

In vertebrate eyes, vitamin $A_{1}$ or $\Lambda_{2}$, oxidized to the aldehyde forms known as retinenes, and combined with specific proteins called opsins, generate the four visual pigments: rhodopsin and porphyropsin, found in rods; iodopsin and cyanopsin, found in cones. Invertebrate animals possess visual pigments of a similar type, made of vitamin $A_{1}$ and retinene $_{1}$ combined with a variety of opsins; but they lack the DPN -alcohol-dehydrogenase system that functions in vertebrates in the formation of retinenes. According to George Wald, whose comprehensive review covers the chemistry and behavior of these visual pigments, rhodopsin, the most fully studied of the four named above, changes upon exposure to light to a transient orange-red form, lumirhodopsin. Lumirhodopsin transforms into another intermediate, also orange-red, and called metarhodopsin; and metarhodopsin is hydrolyzed into retinene and opsin (Fig. 13). The regeneration of rhodopsin requires two strongly coupled reactions, the oxidation of vitamin to retinene and the union of the retinene with opsin. The second reaction is sufficiently exergonic to drive the first, and the continuous removal of the aldehyde by opsin displaces the equilibrium of the first reaction in the oxidative direction. Retinene arising from the decomposition of rhodopsin is not immediately reusable in forming rhodopsin. 


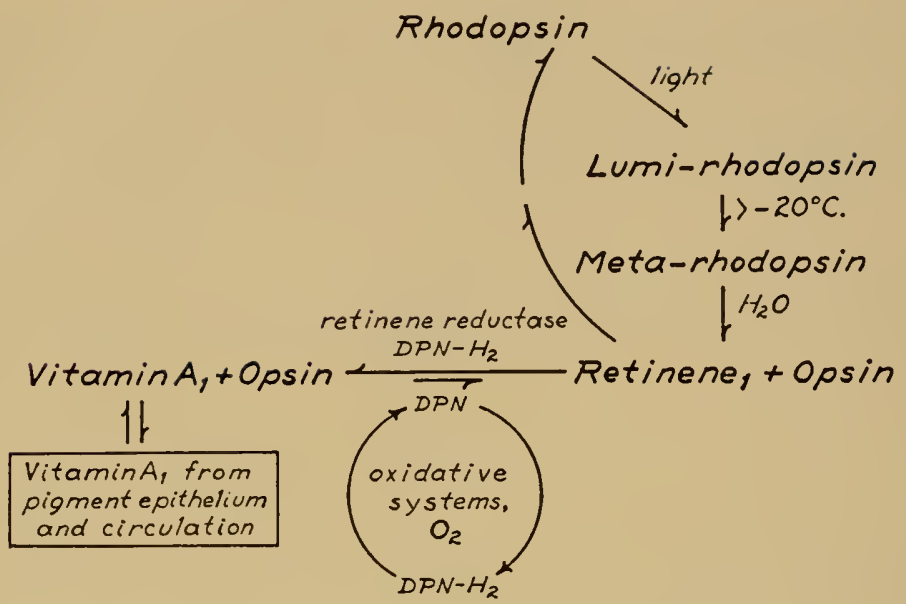

Fig. 13. Diagram of the rhodopsin system.

The vitamin A molecule has four double bonds in its side-chain, and each of these might theoretically be cis or trans in configuration (Fig. 14). Actually, only the cis forms at positions 9 and 13 are<smiles>C=Cc1ccccc1C</smiles><smiles>CC=CC=CC</smiles><smiles>CC=CCCO</smiles><smiles>C=CC=O</smiles>
all-trans Vitamin $A$

Retinene<smiles>C/C=C\C1=C(C)CCCC1(C)C</smiles><smiles>CC=CC=CC(C)=CCO</smiles>

Fig. 14. Geonctric isomcrs of vitamin 1 and retincne. A similar series of struclures, differing only in possessing an extra double bond in the 3,4 position, represents vitamin $\lambda_{2}$ and retinene. The upper structure represents the unhindered, all-trans configuration. The lowermost structure is the lindered cis isomer from which all known vistual pigments are derived. 
sterically unhindered, and consequently only the forms all-trans, 9-cis, 13-cis, and 9.13-dicis were expected. In actuality, all the visual pigments so far analyed possess instead the sterically hindered II-cis isomer. This isomer is actually remarkably stable, since it seems to lie in "a potential energy valley walled oft by high potential barriers." In other words, it requires a high activation energy either to form it or to reisomerize it. When rhodopsin or a similar pigment is bleached, however, and retinene is freed, it is in the form of the all-trans ismer; and before it can be reconverted to rhodopsin it must be reisonterized to the ll-cis configuration. A cycle of cis-trans reisomerization is therefore an integral part of the risual pignent system.

Wald emphasizes that the only thing light does in any visual systenl, so far as we know, is to isomerize retinene. All other reactions are ordinary "dlak" reactions. A suggestive scheme to account for the behavior of the system when bleached by light begins with 11-cis retinene firmly attached to a site on the opsin surface which it fits sterically, and perhaps with the aldehyde group of retinene bonded in a protonated Schiff base linkage to an amino group of the protein. Further assumptions must be made to allow for the shift of the maximal absorption so far towarl the red as it actually is (retinene, $380 \mathrm{~m} \mu$; rhodopsin, $500 \mathrm{~m} \mu$ ). Absorption of a quantum of light by rhodopsin isomerizes the retinene to the all-trans configuration. It no longer fits the opsin, hydrolyzes off, and exposes reactive groups that may trigger the visual excitation (Fig. 15).

If this explanation is correct, it would be expected that any agent that isomerizes retinene and destroys the good fit between it and the opsin would bleach the visual pigment. It turns out that this is so.
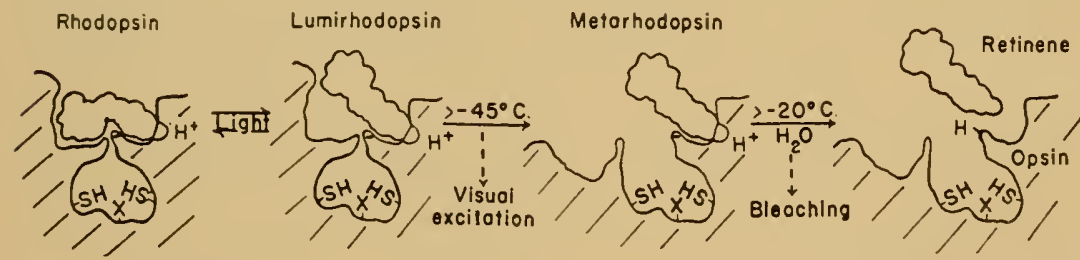

Fig. 15. The action of light on rhodopsin. The absorption of light by rhodopsin isomerizes its 11-cis chromophore to the all-trans configmation, yielding as first product the all-trans chromoprotein lumi-rbodopsin. This labilizes the protein, opsin, which rearranges to a new configuration, yielding a second all-trans chromoprolein, melatrhodopsin. This second process exposes reactive groups on opsin -two - SH groups, and one prolon-binding gromp, symbolized in the figure with a negative charge-and may be responsible for triggering visual excitation. Vertebrate metarhodopsins are unslable, and above aboul $-20^{\circ} \mathrm{C}$ hydrolyze to opsin and all-trans relinene, the process that corresponds to bleaching. 
Denaturation of the opsin by heating (Hubbard) bleaches the pigment while releasing most of the retinene still in the 11 -cis configuration.

Visual excitation, Wald suggests, must depend upon formation of the lumirhodopsin and metarhodopsin forms, since hydrolysis of the latter is far too slow to account for it. In fact, in some invertebrate eyes the bleaching ends with the production of the meta-pigment. The exposure of rhodopsin to bleaching by light leaves some rhodopsin, and the quantum efficiency of the bleaching appears to be only about .5. Actually, the quantum efficiency is close to unity. But the exposure of lumirhodopsin to light reisomerizes some of the alltrans isomer, just produced, into the ll-cis form again. On the other hand, light will not isomerize all-trans retinene in the metarhodopsin intermediate. Wald suggests that it is the special capacity of carotenoids to undergo geometrical isomerization that has led to their selection for the particular roles they play as photoreceptors in visual systems. Their straight-chain conjugated systems are readily isomerized by exposure to light. Certainly, it is remarkable that in three phylogenetically distinct groups of animals, the mollusks, the arthropods, and the vertebrates, where eyes have evolved quite independently, the 1]-cis isomer of retinene has come to occupy the same position in visual excitation. Natural selection here seems to have picked on the one geometric isomer of retinene with the largest change of shape and highest photosensitivity, that is, highest quantum efficicncy and highest intrinsic rate for photoisomerization to the all-trans form.

Visual excitation in the rods poses particularly interesting problems. At the dark-adapted threshold for rod vision (ca. $10^{-6}$ millilamberts), a single rod absorbs a quantum about once on the average in 38 minutes. Even at a luminance 1000 times above this threshold, a rod absorbs a quantum less than once in two seconds. Clearly, a single quantum, absorbed by a single molecule of rhodopsin, must be sufficient for visual excitation. The problem is to account for the amplification of effect. Wald suggests such possibilitics as the quantum's knocking a hole in the rod's outer membrane, which is largely made up of rhodopsin, and thus generating a self-propagating depolarization; or its uncorking an active site of enzymatic nature previously shielded by the retinene.

Wald's views that the spectral sensitivity of vision derives from the absorption spectra of the visual pigments, and that photosensitivity and light and dark aclaptation are functions of pigment concentration are supported by further evidence. Excellent agreement has been 
found between the spectral sensitivity of rods and cones and the absorption spectra of rhodopsin and iodopsin in the lrog, snake, cat, and guincat pig. The same holds for porphyropsin and cyanopsin in the tench. The Purkinje phenomenon-al shift of maximal spectral sensitivity toward the red-can be treated entirely satisfactorily as a shift from rock vision in dim light to cone vision in bright light. Similar observations for the human rhodopsin absorption spectrum and rod spectral sensitivity (Wald and Brown) and for the visual pigment and spectral sensitivity of Limulus (Graham and Hartline) also exist. As for the visual threshold during light and dark adaptation, Rushton's work showing that the pigment concentration varies inversely as the logarithm of the threshold sensitivity is well supported by recent studies of white rats in Wald's laboratory. Dowling finds that whether the visual threshold is varied by light and dark adaptation or by making the rats nightblind through deficiency of vitamin $A$, a very nearly linear relation between decreasing rhodopsin and increasing $\log$ threshold obtains. Wald believes that the relationship may actually be that of a flattened S-curve, approximately linear only in the mid-region; but this eventuality remains to be established.

\section{Color Vision}

Two contributions on color vision were made to the Symposium, one on spectral discrimination in insects, the other on electrophysiological evidence of color vision in the goldfish. Timothy Goldsmith reviewed the former topic. There is evidence of color discrimination in at least six orders of insects, including both primitive types such as the Odonata and Blattaria, and advanced types such as the Diptera, Hymenoptera, Coleoptera, and Lepidoptera. In some cases the evidence comes from training experiments in which color discrimination is made a guide to food; in other instances it is electrophysiological; or is based on the optomotor response; and in still other cases it is based on observation of phototaxis. [The optomotor response depends on a reaction elicited from the movement of vertical stripes in the visual field. If the stripes are alternately gray and colored, the hues can be matched so that no response from the insect is forthcoming. But it is found that a pattern of alternating stripes of two colors both of which match a given shade of gray can nevertheless evoke the insect's response. It is consequently concluded that the insect can discriminate these colors.]

Almost all insects that have been tested show a strong response to ultraviolet. Experiments utilizing phototaxis as a criterion show a 
maximum response at $340-370 \mathrm{~m} \mu$, in the cases of Drosophila, housefly, honeybee, many beetles, the cockroach Periplaneta, etc. The worker honeybee, according to Coldsmith's experiments, exhibits a marked difference in spectral sensitivity when light-adapted and when dark-adapted. Adlapted to red light, the bee exhibits two nearly equal peaks of sensitivity, at about $345 \mathrm{~m} \mu$ and $535 \mathrm{~m} \mu$. When dark-adlapted, responses of the ultraviolet receptor system are almost entirely masked by the greatly enhanced sensitivity to green light. But one must distinguish sharply between the spectral sensitivity, as measured electrophysiologically, and the behavioral response as manifested in phototaxis. It should be obvious that in color vision the behavioral response may be preferentially evoked by some wavelength of light other than that to which the eye is maximally sensitive. Ultraviolet seems most potent in eliciting behavioral responses, but the eye may be more sensitive to green or other wavelengths than to ultraviolet. Behavioral preferences even change in the same insect with the phases of the lile cycle, or with a shift in the female from feeding behavior to egg-laying.

The color vision of the honeybee has of course been the subject of prolonged study by von Frisch and others. White light for the bee must include some ultraviolet. "Bee-white" can be made from a mixture of $15 \%$ ultraviolet $\left(360 \mathrm{~m}_{\mu}\right)+85 \%$ white (xenon) or $85 \% 490 \mathrm{~m}_{\mu}$ blue-green (Daumer). It is also possible to make "beewhite" of mixtures of three monochromatic wavelengths; that is, a mixture of $55 \% 588 m \mu+30 \% 440 \mathrm{~m}_{\mu}$ can be substituted for the $490 \mathrm{~m} \mu$ blue-green in the mixture with $15 \%$ of $360 \mathrm{~m} \mu$ ultraviolet. Bee-purples also exist; and I)aumer has concluded that bees have three types of color receptors, for near ultraviolet, for blue-violet, and for green-yellow-red. The measurements undertaken to determine the relative energies required to obtain electroretinograms of constant size reveal only two of these, namely, the receptor maximally sensitive at $535 m_{\mu}$ in the green, and the ultraviolet receptor at about $345 \mathrm{~m} \mu$. Evidence for the existence of a blue-violet receptor at present comes only (a) from some measurements on the drone honeybee, which is maximally sensitive at about $440 \mathrm{~m} \mu$; and (b) from biochemical studies which indicate that bee heads (and not bee bodies) contain a retinene, photosensitive pigment bound to a soluble protein, and absorbing maximally at about $440 \mathrm{~m} \mu$. This pignent bleaches like rhodopsin, liberating retinene. The ocelli of the worker bee contain two photoreceptors very like those in the compound eyes.

Much work has also been done on the color vision of the blowfly Calliphora. Its spectral sensitivity has three peaks, at $350 m \mu, 500 m \mu$, 
and $6.30 \mathrm{~m} \mu$. There is evidence of separate receptors for the blue-green and red maxima, evidence including the discovery of a colorblind fly that lacked the 630 $m \mu$ maximum (Autrum and Stumpl). Whiteeyed mutants. however, possess a single maximum of sensitivity, at $5201 m \mu$, and a sensitivity 1000 to 10,000 times as great as that of wildtype flies. These last-mentioned findings seem unexpected and puzling, in spite of speculative explanations by Autrum and Stumpl.

Goldsmith considers the existence of a Purkinje shift in insects to be unsubstantiated. He also evaluates the "Iuplicity Theory," according to which the insect eye is supposed to have receptors varying greatly in sensitivity and threshold, like vertebrate rods and cones. Verdict: no compelling evidence.

The relation of color vision in insects to the structure of the compound eye is at present not clear. The mosaic theory of insect vision has recently been questioned (de Vries and Kuiper) on the basis of measurements indicating that the visual angle of an ommatidium is about 8 degrees, great enough to produce overlapping of visual fields. These authors propose on other evidence that the individual receptor may be the retinula cell rather than the entire ommatidium. But if that be so, convergence at the first synapse must take place, since the number of ganglion cells is about the same as the number of ommatidia. Also, fibers from different cells of an ommatidium may go to different ganglion cells. (In Limulus, however, the ommatidium probably does behave as a unit receptor.) There is good evidence that in insects the color receptors are not equally distributed throughout the eye. In the backswimmer Notonecta, only the dorsalposterior ommatidia subserve color vision. In the cockroach Periplaneta, sensitivity to ultraviolet light is restricted to the dorsal half of the eye, but the entire eye is sensitive to green.

An interesting evolutionary question relates to the ability of insects to respond to ultraviolet radiation and the inability of vertebrates to do so. The visual pigments of vertebrates are sufficiently sensitive to the near ultraviolet, but their lenses screen out the deep violet and ultraviolet rays. Is this, as Wald has suggested, a device to relieve chromatic aberration, a difficulty not met with in the insect's compound eye? However that may be, it is certain that the evolution of flower colors, pleasing as they may be to us, really has little or nothing to do with vertebrate vision, but reflects the visual capacities of insect visitors; and in the ultraviolet band of the spectrum there lie hidden many color patterns and hamonies beyond our ken.

E. F. MacNichol, Jr., M. L. Wolbarsht, and H. G. Wagner have 
combined to make a preliminary study of color vision in the goldfish, a happy choice because of its ubiquity and inexpensiveness. More detailed studies have been made of two electrical potentials found in isolated retinas of fishes by Svaetichin, a few years ago. These were called the luminosity, or $\mathrm{L}$, response and the chromaticity, or C, response, and appeared at characteristic depths when the nicroelectrodes were inserted into the retina. Tomita has identified the former as arising from the outer plexiform layer, which in fishes contains giant cells arrayed horizontally, and the latter as arising from the bipolar cells of the inner nuclear layer, or perhaps extracellularly within that layer. The problem was to see whether either response was related to color vision, and how the responses correlate with the discharges of the ganglion cells. Micropipette electrodes were used to record the $\mathrm{L}$ and $\mathrm{C}$ responses, and metal microelectrodes were used for the discharges of the retinal ganglion cells. "On-off" discharge patterns were found for most ganglion cells, just as in frog and other vertebrate retinas. The "on-off" pattern exhibited striking wavelength dependency. Some cells gave pure "on" responses in the short wavelength end of the spectrum and pure "off" responses at the long wavelength end. The shilt from one to the other was sometimes very sharp, occurring within a $10 \mathrm{~m} \mu$ clistance. Other cells exhibited the reverse sort of pattern, short-wave "off" responses, long-wave "on" responses. Assuming that this wavelength dependency of the ganglion cell's clischarge is related to the $\mathrm{C}$ response, these workers adjusted the stimulus to give a constant level of response at each wavelength and determined the threshold intensity at each wavelength. In general, they found distinct and separate threshold levels for the "on" and "off" responses, and the "off" clischarge exerted a strong inhibition on the other discharge. For example, in certain units the "on" discharge occupied the shorter wavelength end of the spectrum, the "off" discharge the longer wavelength end. In that case, the threshold was much higher for the "off" discharge, and it inhibited the "on" discharge. In other units there was a short-wave "off" response with a low threshold and a long-wave "on" response with a high threshold. A third type of ganglion cell was also found which gave both "on" and "off" responses throughout the spectrum.

The three types of sensitivity response curves from the goldfish retina have been compared with the absorption curves of a number of hypothetical pigments through the use of a nomogram prepared by Dartnall. The short wavelength inhibitory system, or short-wave "off," system fits the characteristics of a visual pignent with a maxi- 
mum absorption at 500$) \mathrm{m} \mu$, like rhockopsin, although it probably contains retinene. like the visual pigment of most other freshwater fish. The achromatic ganglion cell type that gives both "on" and "off" responses thronghout the spectrum fits nicely the characteristics of a pigment absorbing maximally at 600$) m \mu$. The long wavelength system has maximum absorption at about $650 \mathrm{~m} \mu$, but does not give a good fit to any particular hypothetical curve for a single visual pigment. No visual pigments absorbing at $600 \mathrm{~m} \mu$ or $650 \mathrm{~m} \mu$ have been isolated to date, but the carp has provided microspectrophotometric evidence of a pigment with an absorption maximum at 640 ${ }_{11} \mu$ (Hanaoka and Fujimoto). These studies, just beginning, offer promise of many interesting things to come.

\section{The Relation of Photic Organs to Eyes}

John Buck points out the existence of "a provocative structural similarity between certain eyes and certain photophores," a resemblance which it hardly seems possible to attribute to mere change or to any type of evolutionary convergence. Many organs serving in deep-sea fishes, squids, and shrimps for the production of light not only possess an external transparent layer like a cornea, a lens, photic tissue in the approximate position of a retina, a reflector layer (tapetum), a pigment cup, and a supplying nerve, but further resemble the vertebrate, molluscan, or arthropod eye in having a striated "refractor" or "rod-mass" in front of the photogenic tissue; or in having rod-shaped photocytes arranged in a layer like the arthropod retina. Three good examples among arthropods may be cited: (a) the thoracic photophores of schizopod shrimps, and the ocelli of pseudoscorpions and pedipalps: (b) the eyestalk and gill chamber photophores of decapod shrimps, and the ocelli of insects; and (c) the eyelike photophores on the bases of the legs of many decapod shrimps, and the crustacean compound eye.

Photophores are often in very uneyelike locations, yet also often occur adjacent to eyes or even within them, as if they might have been derived from some common type of rudiment. In terms of their light-emitting function, it seems especially hard to account for the presence of the pigment layer, which seems quite unnecessary, or the "refractor" structure, which seems altogether superfluous in the presence of reflector, lens, and cornea. And only when, as Buck points out, they are located in the integument, where the need would seem to be least, do photic organs commonly possess a cornea and lens. It is quite clear, furthermore, that an eyelike structure is not essential 
as a physical requirement for the emission of light. There are many photogenic structures in the animal kingdom that are not in the least eyelike; and luminous bacteria, of course, emit light with no need for accessory structures at all.

In the course of evolution biotuminescence has appeared independently in many groups, and in contrast to eyes, which are almost ubiquitous among the higher animals, photophores appear sporadically. Harvey concluded that bioluminescence generally arose as an acciclental aberration in some conventional biochemical system. The value of producing light to species which do so is not even clear in any case except the fireflies, although for cleep-sea forms it is probable that light serves as a recognition signal for schooling and mating. At least it seems to be comnected with an ocular response on the part of other individuals of the same species. If so, vision would necessarily antedate bioluminescence and would provide the basis for the selection pressure that would establish and elaborate the photogenic capacity, once it appeared. A presumptive association of eyes and photophores is strengthened by the fact that there are many cases among shrimps and molluscs of eyes without photophores, but none of photophores without eyes. And in sergestid shrimps, the four species with photophores have the largest eyes. Then have the eyelike photophores evolved from eyes? Could a structure perhaps at first function simultaneously as eye and photophore? Perhaps a comparative study of these photogenic organs might afford a better understanding of the evolutionary origin of novelties. A species with supermumerary eyes, or the capacity to produce such by a homoeotic transformation of serially homologous structures, might well find it possible to convert supernmmerary eyes to photogenic organs if there was any selective advantage in possessing such organs. The modification of eyes, vertebrate or invertebrate, into photic organs is less strange, and probably less difficult to achieve, than many other kinds of evolutionary novelty, the first origin of complex eyes, for example.

\section{Conclusion}

The current trends in the scientific investigation of the relation of light to life will probably be quite clear to any reader of the present Symposium volume. In spite of many interesting side-issues, the predominating theme appears to be the effort to harness the primary photochemical events that follow the absorption of a quantum of light-the production of excited states in molecules- to the chemical reactions known to ensue. The analysis of fluorescence and absorp- 
tion spectra and their correlation with action spectrat remain a most lrutful method of investigation. Electron spin resonance measmements and other methods of studying free radicals of mimaginably brief lifetimes are newer ways of probing these secrets. The electron microscope is heginning to provide a glimpse of the way in which line structures may make transfers of charge and electron flow possible. This is an area of biology within which physical chemistry, biochemistry, and biophysics must merge. Perusal* of this volume should convince any reader that the advancing front of science now lies in the borderlands between the older sciences, and that the inrestigator who would be successlul today must explore many fields, learn many skills, and dare to apply to a challenging problem in one area the insight he has gained in studying many. That man is lost who would "stick to his last," specializing more and more on less and less. The great problems of life and light will yield only to those whose knowledge of light suffuses their knowledge of life, whose knowledge of life quickens their knowledge of light.

*Perusul means to read thoroughly and carefully, and nol, as many of my acquaintances seem to think, to scan hastily and spottily. 



\section{AUTHOR INDEX}

Ihraliamson, E. W., 68

\irth, R. L., 262

Allen, Mary Belle, 479

Arnold, William, 749

Arnon, Daniel, 143, 309, 416, 418, 443, H41, 489, 565, 566, 570, 753

Ball, J. H., 258

Bannister, T., 67

Beinert, Helmut, 163

Benson, А. А., 392

Bessman, M. J., 64

Bishop, Norman, 389, 566

Bodle, Vernon C., 294

Bohr, Niels, 3

Bomer, Walter D., Jr., 424

Buck. John, 754, 813, 814

Calvin, Melvin, 65. 66, 196, 197, 317, 389,417

Chance, B., 107

Chase, A. M., 258, 310, 311

Commoner, Barry, 142, 356, 417, 422, 672

Cormier, Milton J., 274, 310, 311

Cornelius, C. E., 258

Curry, George M., 646

Forti, Giorgio, 566, 576

Franck, James, 66, 68, 74, 81, 105, 106, $107,386,389,421,471,472,721,722$, 751,752

French, C. S., 105, 447, 471, 472, 670

Frenkel, Albert W., 565, 58

Galston, A. W., 670, 671, 687

Goldsmith, Timothy H., 771

Gordon, Solon A., 672

Govindjee, 378,475

Haneda, Y., 206

Hartline, K., 645, 750, 814, 815

Hastings, J. Woodland, 198, 294, 310

Hendley, D. D., 593, 601

Hill, Robert, 424

Hillman, William S., 673

Hoch, George, 397, 418, 419
Jagendorf, André T., 417, 419, 423, 566 , 576,672

Johnson, Frank 11., 206

Kalckar, Herman, 65, 66

Kamen, Martin D., 444, 446, 483

Kaplan, Nathan O., 144

Kasha, Micluael, 31, 64, 65, 66, 67, 68, $75,76,196,815$

Kaur, Ravindar, 687

Kok, Bessel, 397, 416, 418, 419, 420, 423, $567,568,569$

Krogmann, Dasid W., 615

l.ederman, R. J., 258

Linschitz, Henry, 68, 76, 77, 173, 196, $197,198,446,815$

Lipmann, Fritz, 417, 443, 568, 621

Losada, . I., 570

MacNichol, E. F., Jr., 722, 795, 813, 814, 815,816

McCapra, Frank, 254, 311

McElroy, W. D., 196, 219, 307, 308, 309, $310,311,444,569$

Nakamoto, T., 609

Nozaki, M., 570

Petrack. Barbara, 569, 621

Platt, John R., 74, 75, 77, 107, 197, 473, 751

Porter, George, 69, 74, 75, 76, 77, 309

Rabinowitch, Eugene, 75, 107, 378, 390, $417,420,445,446,471,472,750,751$

Racker, E., 143, 307, 308

Robinson, G. Wilse, 11

Rushton, W. А. H., 706, 721, 722, 751, 814

Sanadi, D. R., 157

San Pietro, Anthony, 631

Searls, Robert L., 157 
Seliger, H. H., 77, 106, 107, 200, 219

Shifrin, Sidney, 144

Sie, Edward H.-C., 206

Smith, Lucile, 436

Stern, Babette, 609

Strehler, B. L., 198, 199, 306, 308, 309, $419,420,569,593,601$

Szent-Györgyi, Albert, 7

Thimann, Kenneth V., 646, 670, 671, 672,815

Thomas, J. B., 475, 567
Udenfriend, Sidney, 309

van NieI, C. B., 315, 568

Velick, Sidney F., 108, 143, 310

Vennesland, Birgit, 609

Wagner, H. G., 795

WaId, G., 444, 672, 721, 722, 724, 751, 752

Weber, G., 66, 82, 105, 106, 107, 197, 472

White, Emil, 183, 198, 199, 254, 31 I

Wolbarsht, M. L., 795, 815 


\section{A}

Msorption from the ground state: 69

Absorption from the triplet state: 69

Ibsorption spectrum of Rhodospirillum rubrum: 346

Acanthephyra: 756

Iction spectra of the Emerson effect: 381: of spinach chloroplasts, 348

Iction spectra of photosynthesis: 399; participation of accessory pigments, 455

Ictivation spectra of $700 \mathrm{~m} \mu$ absorption shifts in Anacystis cells: 406

Acyl-coenzyme-A dehydrogenases: 137

Adaptation: 707

Aestina: 772

Agelastica: 772

Ildehyde and bacterial luminescence: 251

Iminophthalic acid fiuorescence quantum yield as a function of $\mathrm{pH}: 203$

Iminopyridine derivatives, effect of solvent on absorption properties of: 154

3-Aminopyricline-DPN, fluorescence of: 155

Anacystis nidulans: 382, 399, 403, 479; enhancement effect in, 400

Anthracyl isocyanate conjugates, excited states: 82

Anti-bonding $\pi$-orbitals: 15

Apis mellifera: 772; color vision of, 779

Armillaria mellea: 262; effect of ultraviolet light on, 270

Iromatic amino acids, excited states: 82

Issimilation of $\mathrm{C}^{14}$-acetate, equivalence of ATP and light: 528

ITP-phosphate exchange in presence of TPN: 595

Arena coleoptile absorption spectrum, 655; action spectrum for positive curvature of, 651; base curvature, action spectrum for, 659; response to light dosages, 650

\section{B}

\section{Bacteria: 211}

Bacterial luminescence: 249; and aldehyde, 251; and flavin inononucleotide, 250
Bacterial phosphorylation, cofactors of: 507

Bacteriochlorophyll, chlorophyll and protochlorophyll, relation between: 318

Bacteriochlorophyll, structural formula: 319

Biological function and electronic structure: 31

Biological responses to light: 613

Bioluminescence, firefly: effect of phosphatc, 247; emission spectra, 245, 247; (quantum yield, 244, 246

Bioluminescence: fungal, 262; in Gonyaulax, ionic effects upon, 294

Bioluminescent reactions, mechanism of: 219

Blue shift in Polar Solvents: 50

Bonding $\pi$-orbitals: 15

Botrydropsis alpina, action spectra of photosynthesis: 462

Bovine serum albumin, polarization spectra of: 101

Bud configuration and growth: 690

Burawoy's $\mathrm{K}$ and $\mathrm{R}$ bands, empirical classifications: 32

\section{C}

Calliphora: 772; color vision of, 786

Carbohydrate synthesis by isolated chloroplasts: 497

Carbonyl absorption bands, interpretation of: 35

Carotenoids: 454; cis-trans isomerism, 727; excited states, 82; and photoreception, 736

Carrasius auratus, retinal structure of: 798

Caulerpa prolifera: 434

Characteristic vibrational coupling: 53

Charge density, various electronic states of the molecule: 13

Charge transfer interactions: 24

Chemiluminescence: emission spectrum of luminol, 201, 203; of luminol, 183; of luminol in climethyl sulfoxide, 192; of luminol in water, recreation scheme for, 190; in porphyrin-catalyzed decomposition of peroxides, 173; quan- 
tum yicld of luminol for various $\mathrm{pH}$ values, 204; of zinc tetraphenylporphine, decay of, 176

Chemiluminescence and fluorescence: maxima for luminol in various solvents, 194; spectra of zinc tetraphenylporphine, 178; values for the phthalazinediones, 186

Chemiluminescent reactions: 192; of luminol in water, 186; in organic solvents, 19]

Chemiluminescent $\mathrm{Zn}$-porphyrin-catalyzed decomposition of tetralin hydroperoxide, kinetics of: 177

Chlamydomonas moewnsii: 434; derivative absorption spectrum of, 451

Chlorella pyrenoidosa: 348,434 ; absorption spectrum of an "apple green mutant," 482; fluorescence spectra of, 349

Chlorin: 326

p-Chlorophenyl dimethyl urea, effect on growth of etiolated pea stem sections: 701

Chlorophyll: bacteriochlorophyll and protochlorophyll, relation between, 318; energy transfer phthalocyanine as a model for, 332; models, photochemistry of, 318; photophysical and photochemical reactions of, 317; photoscnsitized transformations, 329; scheme for reaction with a haem protcin, 484; structural formula, 319

Chlorophyll A: 448; different forms of, 378 ; three forms of, 451

Chlorophyll B: 453; visible region absorption curves of, 62

Chlorophylls: 61

Chloroplast cytochromes: 424

Chloroplast and mitochondrial cytochromes, oxidation-reduction potentials of somc: 432

Chloroplasts: $\mathrm{CO}_{2}$ assimilation by illuminated isolated, 493; decrease in ATP lcvel by illumination, 606; isolated, carbohydrate synthesis by, 497; low-tcmperature difference spectrum, 128; oxidation-reduction reactions in, 576
Chromatic transients, action spectra for: 460

Chromatium: 9

Chromatium cytochromes, reversible oxidation of: 517

Chrysomela: 772

Chymotrypsin, emission and excitation spectra: 96

Cimbex: 756

Collybia velutipies: 262

Color vision: of fly (Calliphora), 786; in goldfish, electrophysiological evidence for, 795; in honeybee, Apis mellifera, 779; of insects, presence of, 771

Configurations in formaldehyde and molecular orbitals: 14

Cresol and tyrosine, polarization spectra: 88

Cyanopsin: 725; absorption spectra of, 743

$\beta$-cyclocitral: 32 ; absorption spectra of, 33

Cypridina: 211; luciferin, 258; structure of, 213

Cytochrome $b_{6}$ some characteristics of: 425

Cytochrome f: 402; some characteristics of, 425 ; spectra of lighly purified, 425

Cytochrome pigments in photosynthetic bacteria: 436

Cytochromes: chloroplast, 424; Chromatium, reversible oxidation of, 517; light-induced oxidations of, 515; oxidation-reduction potential of some chloroplast and mitochondrial, 432; reduced, of Chromatium by thiosulfate, 575

\section{D}

Desamino-DPNH, fluorescence excitation and emission spectra of: 113

Diatomic molecule, electronic states of: 28

Diazines: 55

Difference spectra in photosynthesis: 400; of Rhodospirillum rubrum, 439; of R. spheroides, 438

Diglycerophosphatc, effect of illumination upon concentration of: 393 
Dihydrothioctate and flavin mononucicotide, spectrum of complex betwcen: 160

Dilurdrothioctyl dehydrogenase reaction: mechanism of, 157; reduction with I)PNH, effect of arsenite on spectrum of, 158 ; reduced with $a$-ketoglutaric dehydrogenase, effect of arsenate on spectrum of, 159

Dimethylaminonaphthalcne conjugates: cxcited states, 82; of proteins, 102

Dinucleotide, fluorescence quantum yields: 133

Diphosphopyridine nucleotide, properties of: 145

DNA: hypochromism in, 60; rotatory dispersion of, 61

DPN: ethylene oxide analogue of, 148; and DPNH, relative affinities of, 121

Dl'NH: alpha and beta, 146; alpha and beta isomerism at pyridine-N-riboside bond, 109; bound, fluorescence of, 119; fluorescence excitation and emission spectra of, 112; fluorescence excitation spectra of, 110, 146; fluorescence excitation spectrum in water as a function of temperature, 111; folded, conformations of, 109; polarization of, 120; spectral evidence for folded conformation, 110

Drosophila: 772

Duplicity theory of vision: 788

E

F.ffect of various agents on $700 \mathrm{~m} \mu$ shift in photosynthesis: 410

Electronic charge distribution: 13

Electronic configuration state and transitions: 44

Electronic dipole transition selection rules for several heteromolecules and state symmetries, 45

Electronic energy, storage and transportation of: 7

Electronic excited states of simple molecules: 11

Electronic free energy: 7

Electronic orbitals, states and transitions: 12 litcctronic spin resonance in chloroplast matcrials: 310 ; signal of $o$-chloranil "doped" metal-free phthalocyanine, effect of illumination on, 336; spectrum of o-chloranil "doped" phthalo. cyaninc, 335; studies of photosynthetic systcms, 356

Electronic states of a diatonic molecule: 28; and molecular orbitals, symmetry classifications of, 39

Elcctronic structure and biological function: 31

Electronic transitions, probability of: 46

Electronic and vibrational states, environmental perturbations on: 21

Emerson effect, action spectra of: 381

Emission spectra: of chymotrypsin, 96; of pepsin, 96; of spinach chloroplasts, 348

Emission spectrum: of firefly light in vitro, 238; from triplet to ground state, 69

Energy transfer: 28; effect of environment upon, 28; non-radiative, 26; in photosynthesis, 397; and quenching, 125; in solid systems, 331; between tyrosine and tryptophan, 128

Engelmann's experiment with illuminated chloroplast: 430

"Enhancement effect" in Anacystis nidulans: 400

Environmental perturbations on electronic and vibrational states: 21

Enzyme complexes: of flavin nucleotides, spectra and structure, 108; of pyridine nucleotides, spectra and structure, 108 Eristalis: 772

Escherichia coli: 496

Ethylene oxide analogue of DPN, structure of: 148

Etiolated pea stem sections, effect of pchlorophenyl dimethyl urea on growth of: 701

Etiolated plants: 689

Euphansia: 756

Eye, at full sensitivity: 706

Eyc-photophore similarities, significance of: 762

Eyes, morphology of compound: 790 
Excitation energy transfer, protein fluorescence and: 122

Excitation spectra: of chymotrypsin, 96; of pepsin, 96

Excited states: anthracyl isocyanate conjugates, 82; aromatic amino acids, 82; of artificially coupled moieties, 94; carotenoids, 82; dimethylaminonaphthalene conjugates, 82; flavin adenine dinucleotide, 82; flavin mononucleoticle, 82; fluorescein isocyanates, 82

Excited states, heme: 82

Excited states, of molecules: of biological interest, 80 ; geometry of, 18

Excited states and molecular structure: l; of proteins, 82, 95; radical-like, 54

\section{F}

\section{Fannia: 772}

Firefly: 211

Firefly bioluminescence: effect of phosphate, 247; emission spectra, 245, 247; quantum yield, 244, 246

Firefly extracts, light emission in: 220

Firefly luciferin, structure: 254

Firefly hminescence: 220

Flash photolysis: 70

Flavin adenine dinucleotide: conformation, 132; excitation spcctrum of, 133; cxcited states, 82

Flavin and flavoprotcin, complexes and semiquinoncs: 163

Flavin mononucleotide: and bacterial luminescence, 250; and dihydrothioctate, spectrum complex of, 160; emissions of, 132; excited states, 82; fluorescence quantum yields, 133; temperature and solvent effects on, 132

Flavin nuclcotides: 132; spectra and structure in cnzyme complexes of, 108

Flavins and flavoprotcins: changes in absorption spectra, 161; and semiquinonc formation, 167

Flavoprotcin oxidation-reduction, frce radical formation: 142

Flavoproteir spectrum, schematic representation of: 164

llavoproteins: flavins and semiquinone formation, 167; and molccular oxygen, 138

Flowcring in the lemnaceae: 674

Fluorescein isocyanates, excited states: 82

Fluorescence: acid quenching of tyrosine derivatives of, 84; of 3-aminopyridineDPN, 155; of bound DPNH, 119

Fluorescence: and chemiluminescence, spectra of zinc tetraphenylporphine, 178; and chemiluminescence values for the phthalazinediones, 186; emission band of glycerophosphate dehydrogenase, 125; emission bands of glycerol phosphate dehydrogenase, 123; emission bands of lactic acid dehydrogenase, 123; excitation of emission bands of lactic acid dehydrogenase, I24; excitation spectrum, 87; excitation spectrum of bovine sertm albumin, effect of digestion with chymotrypsin upon, 103; of indole and phenol derivatives, $\mathrm{pH}$ effect, 85; lifetime and polarization, 98; of Ochromonas danica, 481; of oxyluciferyl-adenylic acid, 226; oxyluciferyl-CoA, effect of cysteine on, 233; of phenol and indole derivatives by hydroxyalanine, quenching of, 86 ; polarization, 102; polarization of cmitted, 88; protein and transfer of excitation energy, 122; quenching, 53; spectra of Chlorella, 349; spectra of spinach chloroplasts, 348; yields of luciferin and oxyluciferin, 242

Fluorescence quantum yicld: of aminophthalic acid as a function of $\mathrm{pH}$. 203; of dinuclcotide, 133; of luminol as a function of $\mathrm{pH}, 202$; of flarin mononucleotide, 133

Franck-Condon principle: 13

Fucoxanthin: 459

Fungal bioluminescence: 262; effect of crystalizing borinc plasma addition. 265 ; inlubitors of, 272

\section{G}

Canglion cell: response of with change in wavelength of stimulus, 803; responsc with change in intensity, 807; 
response with small change in wavelength, so-t

Cicometry of excited states of molecules: 18

Cieotrupes: 772

(iibberellic acid: 695

Gilyceraldehyde-3-phosphate dehydrogenase: 117

Giycerol phosphate dehydrogenase: fluorescence emission bands of, 123; fluorescence emission band of. 125

$\mathrm{N}$ glycel tryptophan, polarization spectra of: 91

Gony'aulax bioluminescence, ionic effects on: 294

Gony'aulax luciferase: purification of, 298; rates of inactivation at screral different temperatures, 303

Gonyaulax luminescent reaction, oxygen requircment in: 297

(ionyaulax monilata: 294

Conyaulax polyedra: 294

Goldfish, intraretinal recording from: 801

Coldfish retina, structure of: 798

Grana, electron micrograph of: 525

\section{H}

Heme, cxcited states: 82

N-Heterocyclics: spectra of, $54 ; \mathrm{n} \rightarrow \pi^{*}$ transitions in, 37

Heteromolecules, state symmetries and electronic dipole transition selection rules for: 45

Hexahydroporphin: 326

Hill reaction, effect of carbonate on: 609

Histioteuthis: 756

Hoplophorus typus: 756

Human serum albumin, polarization spectra of: 101

Hydrogen peroxide, measurement of: 619

Hydrogenase, pyridine nucleotide reduction by: 529

Hydrogen gas, photoproduction of: 531

Hydrogen transfer reactions catalyzed by chlorophyll: 329

Hypochomism in DNA: 60

H) psochromic: 32

\section{I}

Indole: polarization of, 93; polarization spectum of, 89; potential energy, surfaces in, 90

lndole acetic acid. effect of growth: 691

Insulin, polarization spectra: 96

Interactions inoling dipole moments: 24

Intcrmolecular binding energy: 22

Internode length and growth: 691

Intraretinal recording from goldfish: 801 lodopsin: 725

Isorhodopsin: 735

\section{K}

Kinctics of a cyclic system: 408

\section{L}

Lactic acid dehydrogenase: fluorescence emission bands, 123, 124; fluorescence excitation, 124; polarization spectra of, 100

Lactic lehydrogenase-DPNH complcx, excitation of DPNH fuorescence: 119

Lemna gibba: 673

Lemna perpusilla: 673; inhibition of flowering, 677

Libellula: 772

Lifetime of the excitation: 87

Light in $\mathrm{CO}_{2}$ assimilation: 492

Light-dependent lipid metabolism: 392

Light from luciferyl-adenylic acid: 225

Light cmission: effect of inorganic pyrophosphatase and pyrophosphate on. 223; effect of pH and buffers, 235; rcsponse to $\mathrm{ATP}, 222$

Light, pigments and photosynthesis: 447 light signals from whole spinach chloroplasts: 341

Limulus: 707; intracellular recording from a visual ccll, 708

Lipid metabolism, light dependent: 392 Lipoic dehydrogenase: 138

Living organisms and physical models: 3

"Lone lair" orbitals in molecules, types of: 33

Low absorption intensity: 52 
Low transition energy: 53

Luciferase, gonyaulax: purification of, 298; rates of inactivation at several different temperatures, 303

Luciferase-luciferin reaction: 206

Luciferase-oxyluciferyl-adenylic acid dissociation of: 229

Lucifcrase, Renilla, specificity of: 282

Luciferin: absorbance spectra of, 239; cypridina, 258; firefly, structure, 254; fluorescence spectra of, 240, 244; and oxyluciferin, fluorescence yields of, 242; structure of, 213; -luciferase reaction, 206; Renilla, 275; Renilla, specificity of, 282

Luciferyl-adenylate-AMP: effect of pyrophosphate on light emission from, 225; light from, 225

Luciferyl-adenylate enzyme complex: 221

Lucigenin, structural formula: 183

L.uminescence: of dried materials, 209; effect of oxygen concentration on, 236; effect of oxygen concentration on time course of, 237; effect of salts upon, 299; cffect of temperature on, 237

L.mmincscence, bacterial: and aldehyde, 251; and flavin mononucleotide, 250

l.uminescence of Renilla reniformis: biochemistry of, 274; effect of inhibitors on, 285; effect of nucleotide concentration on, 278; effect of $\mathrm{pH}$ on, 283; effect of temperature on, 284; effect of various luciferin preparations, 282

I.uminescent cross-reaction of partially purified, highly diluted luciferase of Cypridina and Apogon: 216

Luminescent reaction in Gonyaulax, oxygen sequirement in: 297

L.uminescent systems, specificities of relationships of different: 214

L.uminol, chemiluminescence: 183, 201; cmission spectrum of, 203; quantum yicld for various $\mathrm{pH}$ valucs, 204

L.uminol, light reaction: 200

I.uminol, structural formula: 183

I.uminol fluoresceuce: 201; quantum viclel as a function of $\mathrm{pH}, 202$

L.ıuinrhodopsin: 725
Lysozyme, polarization spectra: 100

M

Metarhodopsin: 725, 735

Methaemoglobin-reducing factor: 640

$\mathrm{N}$-Methyl indole, polarization spectrum of: 89

9-Methylpurine: 59

Microsomal cytochrome reductase: 138

Molecular orbitals: classification of, 36 ; and configurations in formaldehyde, 14; and configurations in pyridine, 16; and electronic states, symmetry, classifications of, 39; symmetry species classification of, 42

Molecular structure and excited states: 1

1) $\rightarrow \pi^{*}$ transitions: characteristics of, 49; definition of, 32 ; in N-heterocyclics, 37; long wavelength shoulders and tails, 55; nature and significance of, 31 ; in polyatomic molecules, 31; singlet-singlet in pyridine, 17; singlettriplet, 16

Nematoscelis: 756

Nicotinamide riboside moiety of DPN (I) and DPNH, skeletal models of: 115

Nitzchia: 401

Non-bonding orbitals: 15

Non-radiative energy transfer: 26

Nostoc: 348,401

Notonecta: 772

Nyctiphanes: 756

O

Ochromonas danica: derivative absorption spectrum of, 451, 453; fluorescence of, $48 \mathrm{I}$

Opsiu: 725

Optical rotatory strength: 46

Orbitals: classification according to symmetry species, 40; electronic, states and transitions, 12; in heteroatom molecules, types of "lone pair," 34; molecular, symmetry species classification of, 42 
11. Orbitals of pyrimidine, schematic contour diagrams of: 13

\section{Oscillatoria: $\mathbf{1 7 9}$}

Ovalbumin, polarization spectra: 98

Oxidation-reduction potcntials of some chloroplast and mitochondrial cytochromes: 432

Oxyluciferin: absorbance spectra of, 239; absorption and fluorescence, 241; absorption and fluorescence cnergy level (liagram, 243; and E-L-AMIP, fluorescence of, 226; fluorescence spectra of, 240

Oxvluciferin activation, and reaction of cocnzyme A: 232

Oxyluciferin and luciferin, fluorescence yields of: 242

Oxyluciferyl adenylate, and oxyluciferin, fluorescence of: 226

Oxyluciferyl-CoA: 231

Oxyluciferyl-CoA decomposition, eífect of adenylic acid pyrophosphate on: 234

Oxyluciferyl-CoA fluorescence, effect of cysteine on: 233

\section{$\mathrm{P}$}

Paramagnetic substances, quenching by: 71

Pepsin, emission and excitation spectra: 96

Periplaneta: 772

l'henol, depolarization of: 92

Pholas dactylus: 207

l'hosphaticlyl glycerol: 393; effect of illumination upon concentration of, 393

Phospholipids: 392

Phosphorylation: bacterial, cofactors of, 507 ; effect of vitamin $\mathrm{K}_{3}$ and $\mathrm{FMN}$, 502; in extracts of $R$. rubrum, respiration and light-induced, 441

Phosphorylation, photosynthetic: catalysts of, 498, 505; electron flow mecluanism of, 508; and molecular oxygen, 501

Photic organs, speculations on interrelations and evolution of: 754

Photobacterium fischeri: 264 photochemical bleaching of an absorption at $700 m \mu: 401$

l'hotochemical and photophysical reactions of chlorophyll: 317

Photochemical processes in photosynthesis, function of hacm protcins: 483

photochenical reduction of triphosphopyridine nucleotide: 631

Photochemical theory of vision: 711

Photohydrolysis: of ATP, 625; of bluegreen algae, 621

Photolysis, flash: 70

Photometabolism of acctate: 591

Photoperiod: 696

Photoperiodism: 673

Photophore anatomy: 755

Photophosphorylation: 623; in Anabaena fragments. 622; anaerobic cyclic, 512; of blue-green algae, 621; by Chromatium particles, effect of inhibitors on, 508; cyclic, 511; effect of chloride, 513; effect of chloride on non-cyclic, 540; effect of $\mathrm{CN}^{-}$on ascorbate-stimulated, 583; effect of ferricyanide, 514; effect of light intensity on, 521; effect of oxygen on, 609; effect of vitamin $\mathbf{K}_{3}$ and phenazine methosulfate, 520; evidence for electron flow mechanism, 513; and Mehler reaction, 615; multiple sites in cyclic, 519; non-cyclic, 537; oxygen-catalyzed, 542; as primitive photosynthesis, 526; by purified grana, 526; in presence of PMS, sensitization of, 411 ; relation of cyclic to non-cyclic, 547; scheme for non-cyclic, 541; stimulation by photosynthetic pyridine nucleotide reductase, 583

Photophosphorylating system, structural association of chlorophyll with: 522

Photophysical effects in model systems: 331

Photophysical and photochemical reactions of chloroplyyll: 317

Photopigment concentration and visual sensitivity: 740

Photoproduction of $\mathbf{H}_{2}$ from succinate: 534

Photoproduction of molecular hydrogen 
from thiosulfate by Chromatium cells: 570

Photoreceptor in Avena, nature of: 648

Photoscnsitized transformations, chlorophyll: 329

l'hoto-spin signals in purple bacteria: 345

Photosynthesis: action spectra for chlorella, 461; action spectra, participation of accessory pigments, 455; action spectra at saturating light intensity, 463; action spectrum of, 399; and biochemical evolution, 555; Botrydiopsis alpina, action spectra, 462; ccll-frec and encrgy conversion process, 489; by Chlorella, action spectrum for, 465; dilference spectra in, 400; driven by two light reactions, 407; effect of various agents on $700 \mathrm{~m} \mu$ shift in, 410 ; energy transfer in, 397; ESR studies of, 363 ; intensity curve for, 464 ; light and pigments, 447; mechanism of bacterial, 587; in Porphyridium cruenI $\mathrm{km}$, quantum yield, 475 ; sensitization of, 398; separation of light and dark phases, 549; spectral changes in, 397; variation of coursc with wavelengths, 466

Photosynthetic bacteria: cytochrome pigments in. 436; sclscmatic representation of postulated clectron transport chains, 442

Photosynthetic pigments in plants: 448

Photosynthetic phosphorylation: 498, 603; catalysts of, 505; continuous uncasurcment of, 601; electron flow mechanism of, 508; as a function of wavelength, 413; is. light iutensity, 112; aud molecular oxygen, 501; oxidative, 620)

Photosynthetic pyridine nucleotide reductase: 199, 637; alsorption spectrum of, 6.10; relation to metha-globinreducing factor, 6.10; photoinactivation, 639; elfect of on photosynthetic phosphoryl, 638; physical properties, 639

P'hotosynthetic reductant formation: 593
Photosynthetic structure, lipid function in: 392

Photosynthetic systcms, electron spin resonance studies: 356

Phototropic curvature, mechanism of: 665

Phototropism: 646; in Avena, different types, 656; and light-growth reactions, 663; in Phycomyces, 660

Phthalocyanine as a model for chlorophyll energy transfer; 332

Phthalocyanine, structural formula: 333

Phycobilin: 476

Phycocyanin: 455

Phycoerythrin: 455

Phycomyces, phototropism in: 660

Plycomyces sporangiophores: action spectrum for positive curvature of, 662; light growth reactions of, 664

Physical models and living organisms: 3 Phytolacca americana: 492

Polarization: 52; of emitted fluorescence, 88; of indole, 93; and fluorescence lifetime, 98

Polarization spectra: of bovine serum albumin, 101; of indole, $\mathrm{N}$-methyl in. dole and tryptophan, 89; of human scrum albumin, 101; of insulin, ribonuclease, tyrosine and zein, 96; of lactic dehydrogenase, 100; of lysozyme, 100; of N-glycyl tryptophan, 91; of ovalbumin, 98; of tyrosine and cresol, 88

Porphin: 326

Porphyra lacineata: 401,479

Porphyridium cruentum: 379, 479; absorption spectra of, 45.

Porphyropsin: 725; absorption spectra of, 743

Potential energy surfaces in indole: 90

protcin fluorescence, and transfer of $\mathrm{cx}$ citation cnergy: 122

Proteins, excitation states of: 82.95

Protochlorophyll: bacteriochlorophyll and chlorophyll, relation between, 318: structural formula, 319

Pterygotenthis: 756

Purine: 58 
l'uple hacteria, photo-spin signals in: 315

Pyrazine: 55; near ultraviolet absorption curves, 56

Pyridazine, near ultraviolet absorption curves of: 56

pyridine, molecular orbitals and configurations in: 16

Pyrimidine, near ultraviolet absorption curves: 58

P'yrimidine, n-orbitals; schematic contour diagrams of: 43

l'yridine nucleotide: complex of microsomal cytochrome $b_{5}$ reductase, absorption spectrum of, 140; enzyme complexes, 117; enzyme complexes, some properties of, 119

Pyridine nucleotide reductase, photosynthetic: 499, 637

Pyridine nucleotide reduction by hydrogenase: 529

Pyridine nucleotides: interactions in, 144; spectra and structure in enzyme complexes of, 108

Pyridine ring nitrogen, isomerism at the: 115

Pyroteuthis: 756

Pyrimidine: 58

\section{Q}

Quantum yield of firefly bioluminescence: 244,246

Quantum yicld of luminol for various $\mathrm{pH}$ values, chemiluminescence: 204

Quenching by chemical reaction: 73

Quenching of fluorescence of tyrosine derivatives, acid: 84

Quenching by a molecule with a lower triplet state: 72

Quenching by paramagnetic substances: 71

Quinoxaline: 55; near ultraviolet absorption curves, 57

$\mathrm{R}$

Radiationless decay to ground state: 71 Radiative decay to ground state: 71

Radical-like excited states: 54
Renilln luciferin and luciferase, specificity of: 282

Renilla reniformis luminescence: biochemistry of, 274; effect of inhibitors on, 285; effect of nucleotide concentration on, 278; effect of pH on, 283; elfect of temperature on, 28 .

Retinal noise and excitation by light: 718

Retinenc, mechanism of bleaching: 732

Retinene $_{1}: 724$

Retinene $_{2}: 724$

9-cis retinene: 734

11-cis retinene: 734

Rhodopseudomonas spheroides: 345,436 ; difference spectra of, 438

Rhodopsin: 725; absorption spectra of chicken, 742; in human rods, absorption of light, 738; light action on, 733; in normal and rod monochromat, regeneration of, 717 ; reaction involved in bleaching, 736; regeneration measurements in man, 716; in solution, synthesis and bleaching of, 730; system, diagram of, 726

Rhodospirillum rubrum: 345, 365, 436; absorption spectrum of, 346; difference spectra of, 437, 439; effect of inhibitors on respiration, 441; ESR signals from, 342; respiration and light-induced phosphorylation in extracts of, 441

Ribonuclease, polarization spectra: 96

Rotational relaxation times: 102

Rotatory dispersion of DNA: 61

\section{S}

Scenedesmus: 401

Scmiquinone formation of flavins and flavoproteins: 167

Sensitization of photosynthesis: 398

Sensitization of photophosphorylation in presence of PMS: 411

Singlet-triplet $n-\pi^{*}$ transitions: 16

Singlet-singlet $n-\pi^{*}$ transitions in pyriline: 17

Spectra of $\mathrm{N}$-heterocyclics: 54

Spectral changes in photosynthesis: 397

Spectral sensitivities on honeybee: 781 
Spectrophotometer, recording action: 457

Spinach chloroplasts, light signals from whole: 341

State symmetries, and elcctronic dipole transition selection rules for several heteromolecules: 45

Stereochemistry of oxidation and reduction: 114

Systellaspis debilis: 756

\section{$\mathrm{T}$}

Tetragonia expansa: 492

Tetralin hydroperoxide, decomposition of: 175

Tetrahydroporphin: 326

Tetraphenylchlorin: absorption spectra, 322; kinetic analysis of photooxidation, 323; quantum yield in photooxidation, 323; structural formula of, 321

Tetraphenylporphin: absorption spectra, 322; photoreduction of, 325 ; structural formula, 321

Thiobacillus dentrificans: 496

Thiol pyridine derivatives, absorption spectra of: 152

Thione absorption band, effect of hydrogen bonding on: 150

Thionicotinamide: analogue of DPNH, spectrum of methylated product of, 152; DPN absorption spectra of, 147; DPN, oxiclized and reduced, 147; DPNH, suggested structure of, 148; DPNH, spectra of, 149

Threshold determinations: associated with short-wavelength process, 810; with long-wavelength, 811

TPN reduction and oxygen cvolution: 499
Transition moment integral: 46

Triphosphopyridine nucleotide, photochemical reduction of: 631

Triplet state in fluid solution: 69

Triplet state in solution, kinetics of: $7 \mathrm{I}$

Tyrosine and cresol, polarization spectra: 88

Tyrosine derivatives, acid quenching of fluorescence: 84

Tyrosine polarization spectra: 96

V

Vibrational and electronic states, environmental perturbations on: 21

Vicia faba: 434

Vision: duplicity theory of, 788; excitation by light, 720 ; intensity factor in, 706; photochemical theory of, 711; response to intensity, 709

Visual excitation: 737

Visual pigment: relation of absolute threshold to, 715 ; relation of increment threshold to, 714; in man, measurement of, 713

Visual sensitivity, photopigment concentration and: 740

Visual systems, molecular organization: 724

Vitamin A deficiency, visual threshold in: 746

Vitamin A and retincne, geonetric isomers: 728

Vitamin $A_{1}: 724$

$\%$

/cin, polarization spectra: 96

Zinc tetraphenylporphine chemiluminescence, decay of: 176

Zygaena: 756 




